\title{
THE HY-REDSHIFT UNIVERSE: Galaxy Formation and Evolution at High Redshift
}

A.J. Bunker and W.J.M. van Breugel

\section{October 20, 1999}

U.S. Department of Energy

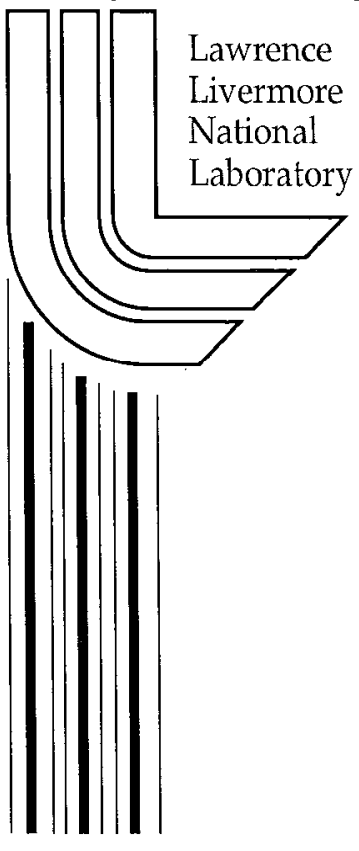





\section{DISCLAIMER}

This document was prepared as an account of work sponsored by an agency of the United States Government. Neither the United States Government nor the University of California nor any of their employees, makes any warranty, express or implied, or assumes any legal liability or responsibility for the accuracy, completeness, or usefulness of any information, apparatus, product, or process disclosed, or represents that its use would not infringe privately owned rights. Reference herein to any specific commercial product, process, or service by trade name, trademark, manufacturer, or otherwise, does not necessarily constitute or imply its endorsement, recommendation, or favoring by the United States Government or the University of California. The views and opinions of authors expressed herein do not necessarily state or reflect those of the United States Government or the University of California, and shall not be used for advertising or product endorsement purposes.

Work performed under the auspices of the U. S. Department of Energy by the University of California Lawrence Livermore National Laboratory under Contract W-7405-Eng-48.

This report has been reproduced directly from the best available copy.

Available to DOE and DOE contractors from the Office of Scientific and Techuical Information

P.O. Box 62, Oak Ridge, TN 37831

Prices available from (423) 576-8401

http://apollo.osti.gov/bridge/

Available to the public from the National Technical Information Service

U.S. Department of Commerce 5285 Port Royal Rd., Springfield, VA 22161 http://www.ntis.gov/

OR

Lawrence Livermore National Laboratory Technical Information Department's Digital Library http://www.llnl.gov/tid/Library.html 



$$
\text { Astroa... Soc... Couf series lolume } \$ 193
$$

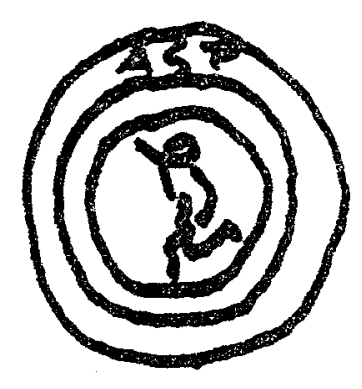

\section{THE HYOREDSHIFT UNIVERSP:

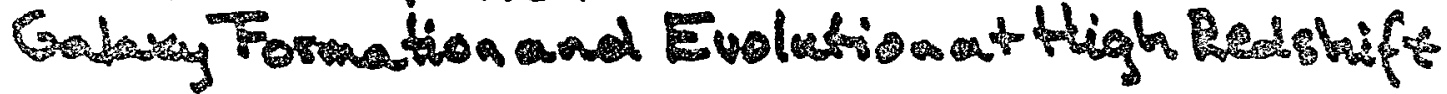

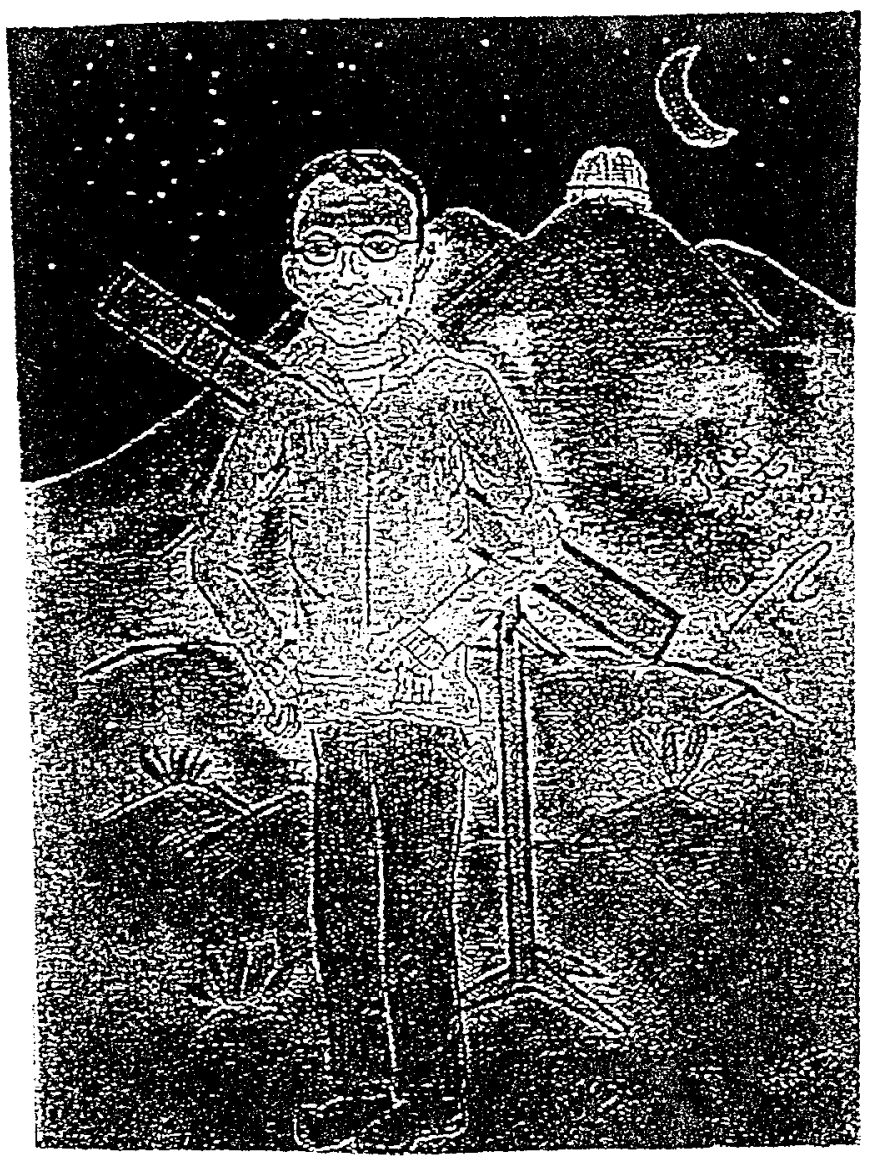

$$
\text { Andkew I. Burkes and Will J.M. van Breugel" }
$$





\section{Table of Contents}

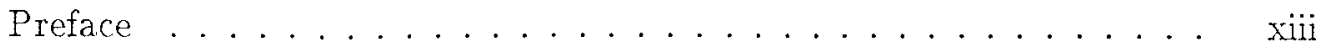

Conference participants .................. $x v$

Observations of Hyron Spinrad ................... I

I. R. Fing, J. W. Liebert and I. de Pater

Section A. Radio Galaxies at Large Redshifts

The 3CR Sample $-1962-1999 \ldots \ldots \ldots \ldots 1$

M. S. Longair

Complete Samples of Radio Sources . . . . . . . . . . . . 23

P. J. McCarthy

The Early Evolution of Radio Galaxy Hosts . . . . . . . . . . . . 34

A. Dey

Ultra-Steep Spectrum Radio Galaxies at Hy Redshifts . . . . . . . .

W. van Breugel, C. De Breuck, S. A. Stanford, H. Röttgering, G. Miley, D. Stern, D. Minniti and C. Carilli

The Vigor of Radio Astronomy at Hy Age: A Review of Faint Radio Source

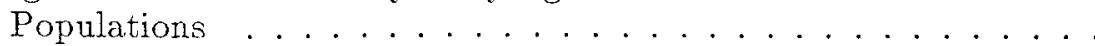

R. A. Windhorst, A. Hopkins, E. A. Richards and I. Waddington

Ionisation, Shocks, and Evolution of the Emission Line Gas of $z \sim 1$ Radio

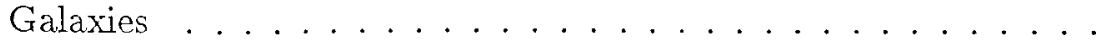

P. N. Best, H. J. Röttgering and M. S. Longair

High-z Radio Galaxies and the 'Youth-Redshift Degeneracy' . . . . . . .

K. M. Blundell and S. Rawlings

The Ages of Compact, Steep-Spectrum Quasars . . . . . . . . . . .

E. de Silva, R. Saunders, J. Baker and R. Hunstead

Star Formation and Polarization in Aligned Radio Galaxies . . . . . . .

D. S. De Young

The Nature of the Compact/Symmetric Near-IR Continuum Source in $4 \mathrm{C}$

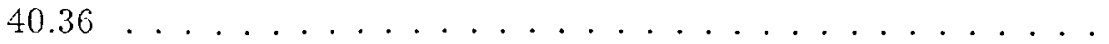

E. Egami, L. Armus, G. Neugebauer, B. T. Soifer, A. S. Evans and T. W. Murphy Jr.

No Evidence for a 'Redshift Cut-Off' for the Most Powerful Classical Double Radio Sources . . . . . . . . . . . . . . . . . M. J. Jarvis, S. Rawlings, C. J. Willott, K. M. Blundell, S. Eales and M. Lacy 
HST Imaging of Two $z>4$ Radio Galaxies . . . . . . . . . .

Infrared Spectroscopy of the Iligh Redshift Radio Galaxy MRC 2025-218 and a Neighboring Extremely Red Galaxy . . . . . . . . .

J. E. Larkin, I. S. McLean, J. R. Graham, E. E. Becklin, D. F. Figer, A. M. Gilbert, T. M. Glassman, N. A. Levenson, H. Teplitz and M. F. Wilcox

Keck Spectropolarimetry of $z \sim 2.5$ Radio Galaxies . . . . . . . . . 102 $J$. Vernet, R. A. E. Fosbury, M. Villar-Martin, M. H. Cohen, S. di Serego Alighieri and A. Cimatti

A Complete Sample of Millijanshy Radio Sources . . . . . . . . . . . 106 I. Waddington

HST Images of Radio-Selected Groups . . . . . . . . . . . . . . 110 E. L. Zirbel, A. Oemler Jr., J. Allington-Smith, R. S. Ellis and M. Dickinson

NICMOS Imaging of High-Redshift Radio Galaxies . . . . . . . . . . .

A. Zirm, A. Dey, M. Dickinson, P. J. McCarthy, P. Eisenhardt,

S. G. Djorgovski, H. Spinrad, S. A. Stanford and W. van Breugel

\section{Section B. Galaxy Stellar Populations and Scaling Relations}

Stellar Population Synthesis Models . . . . . . . . . . . . . .

G. Bruzual A.

The Formation Epoch of Massive Ellipticals, as Traced by Radio Galaxies

J. S. Dunlop

Distant Disk Galaxies: Kinematics and Evolution to $z \sim 1 \ldots \ldots$

145

N. P. Vogt

The Evolution of Early-Type Galaxies as a Function of Galaxy Mass . .

M. A. Pahre

Mid-UV Spectroscopic Dating of LBDS 53W091 . . . . . . . . . .

S. R. Heap, T. M. Lanz, T. Brown and I. Hubeny

Evolution of Spheroidal Galaxies at $z<1$ from the Deep Extragalactic

Evolutionary Probe $(\mathrm{DEEP}) \ldots \ldots \ldots \ldots$

M. Im and the DEEP Team

Determination of AGN Host Galaxy Ages . . . . . . . . . . . . 175

L. A. Nolan

CO Observations and the Tully-Fisher Relation at High Redshift . . . . 179

Y. Tutui, Y. Sofue, M. Honma, T. Ichikawa, K. Wakamatsu, I. Kazes and : ' I. Dickey.

The Low-Mass Stellar IMF at High Redshift: Faint Stars in the Ursa Minor Dwarf Spheroidal Galaxy . . . . . . . . . . . . .

R. F. G. Wyse, G. Gilmore, S. Feltzing and M. Houdashelt 
On the Age Estimation of LBDS 53W091 ........... 185

S. Ii. T. Brown, S. Heap. I. Hubeny, W. Landsman, T. Lanz and

A. Sweigart

\section{Section C. Deep Field Surveys}

The Evolution of Galaxies: A View from Toronto . . . . . . . . . . . . 191

S. Lilly

DEEP's Field Surveys: The View from Santa Cruz . . . . . . . . . 203

D. C. Hoo

The View of Galaxy Formation from Hawaii: Seeing the Dark Side of the

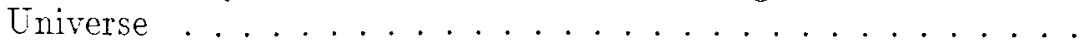

L. L. Cowie and A. J. Barger

Deep Field Surveys: The View from Pasadena . . . . . . . . . . . 224

D. W. Hogg

High Redshift Galaxies and the Hubble Deep Fields . . . . . . . 234

M. Dickinson

Galaxy counts at $450 \mu \mathrm{m}$ and $850 \mu \mathrm{m} \ldots \ldots . \ldots 246$

A. W. Blain, R. J. Ivison, J. -P. Kneib and I. Smail

High-Redshift Galaxies in the NICMOS Parallel Fields . . . . . . . . . . 250

M. R. Corbin, R. I. Thompson, E. O'Neil and W. D. Vacca

The $15 \mu \mathrm{m}$ Extragalactic Background Light from ISOCAM galaxies . . . 254

D. Elbaz, C. Cesarsky and H. Aussel

The NOAO Deep Wide-Field Survey . . . . . . . . . . . . . 258

B. T. Jannuzi and A. Dey

The Deepest Spectrum of the Universe? Constraints on the Lyman Continuum Background at High Redshift . . . . . . . . . . 262

F. R. Marleau, A. J. Bunker and J. R. Graham

Near Infrared Properties of Galaxies in the HDF-N . . . . . . . . . . . 267

C. Papovich, M. Dichinson and C. Hanley

The HDF, The Arizona View . . . . . . . . . . . . . . . 271

R. I. Thompson, R. J. Weymann and L. Storric-Lombardi

Deep Sub-millimeter Surveys: Highly Luminous Far-Infrared Sources at

High Redshift ................... 275

T. Webb, S. Lilly, S. Eales, W. Gear, L. Dune, D. Clements, D. Bond,

D. Crampton, F. Hammer and O. L. Fèvre

Section D. Galaxy Evolution in Clusters

Galaxy Evolution in Clusters: the MORPHS Study . . . . . . . . . . 281

A. Oemler $J_{r}$. 
viii

Contents

Population Gradients in Galaxy Clusters at $0.2<z<0.6 \ldots \ldots$. .

292

E. Ellingson, H. Lin, H. K. C. Yee and R. G. Carlberg

Morphological Evolution in High Redshift Galaxy Clusters . . . . . .

304

S. A. Stanford

The Star Formation History of Ellipticals from the Fossil Evidence . . .

312

A. Renzini and A. Cimatti

X-ray Observations of Groups and Clusters - A Relic of the High Redshift

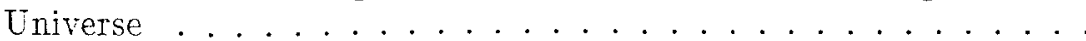

R. Mushotzhy

X-ray Emission from the Host Clusters of Five Intermediate-Redshift 3CR

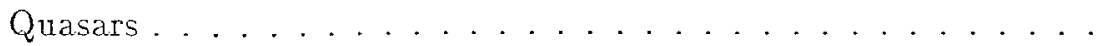

C. S. Crawford, A. C. Fabian, I. Lehmann, G. Hasinger and M. N. Bremer

The Evolution of Stellar Populations in Intermediate Redshift Clusters .

D. D. Kelson, G. D. Illingworth, M. Franx and P. G. van Dokkum

Extremely Red Galaxies in the Field of QSO 1213-0017: A Galaxy Concentration at $z=1.31 \ldots \ldots \ldots \ldots$

M. C. Liu, A. Dey, J. R. Graham, C. C. Steidel and K. Adelberger

Near-Infrared Study of Distant Cluster Galaxies . . . . . . . . . . .

T. Yamada, I. Tanaka, M. Kajisawa, T. Maihara, F. Iwamuro, H. Terada, M. Goto, K. Motohara, H. Tanabe, T. Taguchi and R. Hata

\section{Section E. Theory and Observations of Structure Formation}

Structure Formation from Numerical Simulations . . . . . . . . . . . .

R. J. Thacker and M. Davis

Fundamental Issues in Galaxy Formation . . . . . . . . . . . .

J. Silk

Galaxy Clustering in the CNOC2 Redshift Survey . . . . . . . .

R. G. Carlberg. H. I. C. Yee, S. L. Morris, H. Lin, P. Hall, D. Patton, M. Sawichi and C. W. Shepherd

Clustering Properties of Lyman-Break Galaxies . . . . . . . . . . .

M. Giavalisco

High-Redshift Quasars as Probes of Galaxy and Cluster Formation . . .

S. G. Djorgovski

Observational Tests of the Static Universe Model and the Derivation of

the Hubble Redshift . . . . . . . . . . . . . . . . . . . . 407

T. B. Andrews

The Clustering of Faint Galaxies on Small Angular Scales . . . . . . .

T. G. Brainerd, C. J. Law, J. Brauher, S. G. Djorgovshi and K. Banas

Observations of Candidate $z \sim 1.54$ Quasar Host Clusters $\ldots \ldots \ldots$

P. B. Hall, M. Sawicki, C. J. Pritchet, F. D. A. Hartwick and A. S. Evans 
Structure and Content of the 53W002 "Cluster" at $z=2.4 \ldots \ldots$. .

W. C. Keel, W. Wu, R. A. Windhorst, S. Cohen, I. Waddington and

S. Pascarelle

\section{Section F. The History of Star Formation}

Submillimeter-selected galaxies . . . . . . . . . . . .

A. W. Blain, I. Smail, R. J. Ivison and J. -P. Fneib

$15 \mu \mathrm{m}$ Fxtragalactic Surveys: Source Counts and Properties . . . . . .

D. Elbaz, C. J. Cesarshy, D. Fadda, H. Aussel, F. X. Désert,

A. Franceschini, H. Flores, M. Harwit, J. L. Pnget, I. L. Starck,

L. Danese, D. C. Foo and R. Mandolesi

Near-Infrared Emission Line Searches for High-Redshift Galdxies . . . .

A. J. Bunker

Star Forming Galaxies in the Moderately Hy-Redshift Universe . . . . .

C. C. Steidel and K. L. Adelberger

Cosmology and the Brightness of the Night Sky . . . . . . . .

P. Madau

HST/LCO Measurements of the Optical Extragalactic Background Light

R. A. Bernstein

The Extragalactic Background Light: Detections and Limits and their Cosmological Implications

E. Dwek

High- $z$ Galaxies Seen Through Cluster Lenses . . . . . . . . . . .

N. Beniter and T. Broadhurst

Completeness and Confusion in the Identification of Lyman-Break Galaxies

G. Cotter, T. Haynes, J. C. Baker, M. E. Jones and R. Saunders

Dust in High Redshift Starburst Galaxies . . . . . . . . . . . .

K. D. Gordon, T. L. Smith and G. C. Clayton

A Serendipitous Search for Hy-Redshift Ly $\alpha$ Emission: A Case Study of

Two Sources at $z \simeq 3 \ldots \ldots \ldots \ldots$

C. Manning, D. Stern, H. Spinrad and A. J. Bunker

The Halo Formation Rate and its Link to the Global Star Formation Rate

W. Percival, L. Miller and B. Ballinger

The Results From The NICMOS Parallel Imaging and Grism Survey . .

L. Yan and P. McCarthy

\section{Section G. Formation of the First Objects}

Galaxies at High Redshift $-1999 \ldots \ldots \ldots$. . . . . . . . 
Galaxies of Redshift $z>5$ : The View from Stony Brook . . . . . . .

K. M. Lanzetta, H. Chen, A. Fernández-Soto, S. Pascarelle, N. Yahata and $A$. Fahil

Galaxies at $z>5$ : The View from Hawaii . . . . . . . . . . . .

E. M. Hu, L. L. Cowie and R. G. McMahon

Searches for Quasars at $z>5 \ldots \ldots \ldots \ldots$

P. S. Osmer

Kinematics of Ionized and Neutral Gas in Damped Lya Systems . . . .

A. M. Wolfe

The First Sources of Light in the Universe . . . . . . . . . . . .

A. Loeb

Reionization of the Universe . . . . . . . . . . . . .

N. Gnedin

NICMOS Imaging of Damped Ly- $\alpha$ Absorbers at $z=2 \ldots \ldots$.

603

V. P. Kulkarni, J. M. Hill, G. Schneider, R. J. Weymann,

L. J. Storrie-Lombardi, M. J. Rieke, R. I. Thompson and B. Jannuzi

Damped Lyo Systems in Semi-Analytic Models: Sensitivity to dynamics and disk properties. . . . . . . . . . . . . . .

A. Maller, R. S. Somerville, J. X. Prochaska and J. R. Prinack

ChaMP and the High Redshift Quasars in X-rays . . . . . . . . . .

S. Mathur, H. Marshall, N. Evans, P. Green and B. Wilkes

3D Modelling of the Ly $\alpha$ Radiation from (Proto-)Galaxies . . . . . . . .

R. Wehrse

\section{Section H. Future Prospects}

Future Ground-Based Radio and Submillimeter Observations . . . . . .

R. B. Partridge

Future Observations with SIRTF and Other Mid-IR/Sub-mm Missions.

639

P. Eisenhardt

The Future Hy-Redshift Universe - A Hy-E Perspective . . . . . . . .

651

R. Mushotzky

The Future: Prospects for Observational Cosmology (and Cosmogony) -

Ground-Based Optical-IR. ............... 662

G. Illingworth

Probing $z>5$ with the Multiband Imaging Photometer for SIRTF (MIPS) 669

D. C. Hines, G. H. Rieke, F. J. Low and C. W. Engelbracht

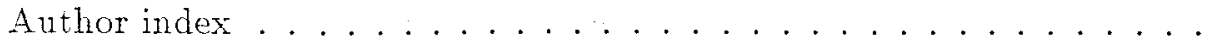

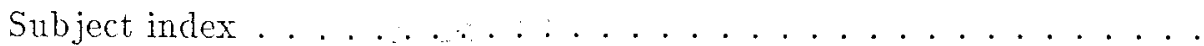




\section{Preface}

This volume contains the proceedings of the international meeting entitled "The Hy-Redshift Universe: Galaxy Formation and Evolution at High Redshift", which was held at the International House of the University of California, Berkeley, from June 21-24, 1999. The purpose was to celebrate the research interests of Hyron Spinrad for his 65 th birthday. The meeting was attended by more than 120 participants, including most of Hy's collaborators and former students, as well as colleagues from all over the world.

In their introduction, Ivan King, Jim Liebert \& Imke de Pater give a historical account of Hy's diverse scientific career which has encompassed the entire Universe, ranging from the local solar system through our own Galaxy to the furthest objects yet found. However, this meeting has specifically focussed on Hy's most recent passion - the study of high-redshift galaxies.

There were 43 invited review talks, 41 of which are included in this proceedings, giving a comprehensive snapshot of this field as of June 1999. In addition to these reviews, there were 46 contributed poster papers, which also appear in this volume.

This meeting was made possible by the hard work of the local organizing committee, chaired by Ivan King (U.C. Berkeley) and consisting of Daniel Stern, Andrew Bunker \& Maureen Barnato (U.C. Berkeley), Adam Stanford \& Wil van Breugel (LLNL). Joyce Wong helped with the financial administration, and Curt Manning oversaw the organization of the poster sessions. We gratefully acknowledge financial support from NASA, the University of California at Berkeley, and from the California Space Institute. The conference program was put together by the scientific organizing committee, under the chairmanship of George Djorgovski. The work by Wil van Breugel at IGPP/LLNL is performed under the auspices of the US Department of Energy under contract W-7405-ENG-48.

California, October 1999

Andrew Bunker \& Wil van Breugel

Cover Illustration: The artwork on the front cover is a pastel sketch of Hyron Spinrad by his wife, Bette Spinrad, entitled "The Great Observer", circa 1965. Used with the kind permission of the artist.

Scientific Organizing Committee: Arjun Dey (NOAO), Mark Dickinson (JHU), George Djorgovski (Caltech, chair), David Koo (UCSC), Simon Lilly (Toronto), Patrick McCarthy (OCIW), John Peacock (Edinburgh), Joseph Silk (UCB).

Local Organizing Committee: Maureen Barnato (UCB), Wil van Breugel (LLNL), Andrew Bunker (UCB), Ivan King (UCB, chair), Adam Stanford (LLNL, and UCD) and Daniel Stern (UCB). 


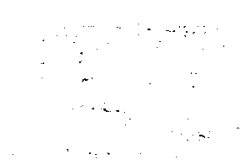

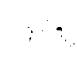

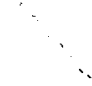




\section{Participant List}

Jay Anderson, University of California, 601 Campbell Hall, Berkeley, CA 94720-3411 〈jay@cusp.berkeley.edu〉

Thomas Andrews, 3828 Atlantic Ave, Brooklyn, NY 11224 〈tom-andrews@msn.com)

Robert Antonucci, Dept. of Physics, University of California, Santa Barbara. CA 93106 〈ski@chester.ucsb.edu〉

Lee Armus, IPAC/Caltech, MS 100-22 IPAC, Caltech, Pasadena, CA 91125 〈lee@ipac.caltech.edu〉

Joanne Baker, University of California, 601 Campbell Hall, Berkeley, CA 94720-3411 〈jcb@pluto.berkeley.edu〉

Jonathan Baker, University of California, 601 Campbell Hall, Berkeley, CA 94720-3411 〈jbaker@astron.berkeley.edu)

Amy Barger, Institute for Astronomy, University of Hawaii, 2680 Woodlawn Drive, Honolulu, HI 96822 (barger@ifa.hawa.ii.edn)

Narciso Benítez, University of California, 601 Campbell Hall, Berkeley, CA 94720-3411 〈benitezn@teddi.berkeley.edu〉

Rebecca Bernstein, Obs. of Carnegie Inst. of Washington, 813 Santa Barbara St., Pasadena, CA 91101 〈rab@ociw.edu〉

Philip Best, Leiden University, Postbus 9513, 2300 RA, Leiden, THE NETHERLANDS 〈pbest@strw.leidenuniv.nl〉

Andrew Blain, Cavendish Astrophysics Group, University of Cambridge, Madingley Road, Cambridge, CB3 0HE, UK 〈awb@mrao.cam.ac.uk〉

Katherine Blundell, Astrophysics Department, University of Oxford, NAPL, 1 Feble Road, Oxford, OX1 3RH, UK 〈kmb@astro.ox.ac.uk〉

Rychard Bouwens, University of California, 601 Campbell Hall, Berkeley, CA 94720-3411 (bouwens@astron.berkeley.edu)

Tereasa Brainerd, Astronomy Dept., Boston University, 725 Commonwealth Ave., Boston, MA 02215 〈tgb@komodo.bu.edu〉

Mike Brown, Caltech, MS 105-24 Astronomy, Pasadena, CA 91125

〈mbrown@gps.caltech.edu〉

Gustavo Bruzual A., CIDA, AP 264, Merida, 5101-A, VENEZULA 〈bruzual@cida.ve〉

Andrew Bunker, University of California, 601 Campbell Hall, Berkeley, CA 94720-3411 (bunker@bigz.berkeley.edu)

Ray Carlberg, University of Toronto, 60 St. George St., Toronto, ON M5S 3H8, CANADA (carlberg@astro.utoronto.ca)

Jennifer Carson, San Francisco State University, 1600 Holloway, San Francisco, CA 94132 〈jcarson@stars.sfsu.edu〉

Marshall Cohen, Caltech, MS 105-24 Astronomy, Pasadena, CA 91125

〈mhc@astro.caltech.edn〉 
Michael Corbin, Steward Observatory, University of Arizona, 933 N. Cherry Ave., Tucson, AZ 85721 (mcorbin@as.arizona.edu)

Garret Cotter, Cavendish Astrophysics Group, University of Cambridge, Madingley Road, Cambridge, CB3 0HE, UK 〈garret@mrao.cam.ac.uk)

Lennox Cowie, Institute for Astronomy, University of Hawaii, 2680 Woodlawn Drive, Honolulu, HI 96822-1897 〈cowie@ifa.hawaii.edu)

Carolin Crawford, Institute of Astronomy, University of Cambridge, Madingley Road, Cambridge, CB3 0HA, UK 〈csc@ast.cam.ac.uk〉

Marc Davis, University of California, 601 Campbell Hall, Berkeley, CA 94720-3411 〈marc@deep.berkeley.ern〉

Steve Dawson, University of California, 601 Campbell Hall, Berkeley, CA 94720-3411 〈sdawson@astron.berkeley.edu)

Eric de Silva, Cavendish Astrophysics Group, University of Cambridge, Madingley Road, Cambridge, CB3 OHE, UK 〈erd20@mrao.cam.ac.uk >

David De Young, NOAO, 950 N. Cherry Ave., Tucson, AZ 85719 〈deyoung@noao.edu

Arjun Dey, NOAO, 950 N. Cherry Avc., Tucson, AZ 85719 〈dcy@noao.edu〉

Mark Dickinson, Space Telescope Science Institute, 3700 San Martin Drive, Baltimore, MD 21218 〈med@stsci.edu〉

S. George Djorgovski, Caltech, MS 105-24 Astronomy, Pasadena, CA 91125 〈george@oracle.caltech.edu〉

James Dunlop, Institute for Astronomy, Royal Observatory, Blackford Hill, Edinburgh, EH9 3HJ, UK 〈jsd@roe.ac.uk)

Eli Dwek, NASA Goddard Space Flight Center, Code 685-GSFC, Greenbelt, MD 20771 〈eli.dwek@gsfc.nasa.gov〉

Eiichi Egami, Caltech, MS 320-47 Downs Lab, Pasadena, CA 91125 〈egami@monk.caltech.edu〉

Peter Eisenhardt, MS 169-327 JPL, 4800 Oak Grove Drive, Pasadena, CA 91109 〈prme@kromos.jpl.nasa.gov

David Elbaz, CEA-Saclay/Service d'Astrophysique, Orme des Merisisers, Gif-sur-Yvette, F-91190, FRANCE 〈delbaz@cea.fr 〉

Erica Ellingson, University of Colorado at Boulder, CASA CB 389, Boulder, CO 80309 (e.elling@casa.colorado.edu )

Ricardo Flores, Physics and Astronomy Dept., University of Missouri-St. Louis, 8001 Natural Bridge Rd., St. Louis, MO 63121 〈Ricardo.Flores@umsl.edu〉

Eric Gawiser, University of California, 601 Campbell Hall, Berkeley, CA 94720-3411 〈gawiser@astron.berkeley.edu)

Marc Gershow, Physics Dept., Stanford University, Stanford, CA 94305-4060 〈marcgiastanford.edu〉

Mauro Giavalisco; Space Telescope Science Institute, 3700 San Martin Drive, Baltimore, MD 21218 〈mauro@stsci.edu〉 
Tiffany Glassman. University of California, Los Angeles, CA 90025 $\langle$ glassman@astro.ucla.edu〉

Simon Glover. Institute for Astronomy, Royal Observatory, Blackford Hill, Edinburgh, EH9 3HJ , LK 〈scogaroe.ac.uk〉

Nick Gnedin, University of Colorado at Boulder, CASA CB 389, Boulder. CO 80309 〈gnedin@casa.colorado.edu〉

Karl Gordon. Dept. of Physics \& Astronomy, Louisiana State University, Baton Rouge, LA 70808 〈gordon@fenway.phys.lsu.edu)

James Graham, University of California, 601 Campbell Hall, Berkeley, CA 94720-3411 〈jrg@astro.berkeley.edu〉

Patrick Hall, University of Toronto, 60 St. George St., Toronto, ON M5S 3H8, CANADA 〈hall@astro.utoronto.ca)

Sara Heap, NASA Goddard Space Flight Center, Code 681-GSFC, Greenbelt, MD 20771 〈heap@srh.gsfc.nasa.gov〉

David Hogg, Institute for Advanced Study, Princeton, NJ 08544 〈hogg@ias.edu >

Esther M. Hu, Institute for Astronomy, University of Hawaii, 2680 Woodlawn Drive, Honolulu, HI 96822-1897 〈hu@ifa.hawaii.edu〉

Garth Illingworth. University of California, Santa Cruz, CA 95064 〈gdi@ucolick.org 〉

Myungshin Im, University of California, Santa Cruz, CA 95064 〈myung@ucolick.org)

Matt Jarvis, Astrophysics Department, University of Oxford, NAPL, 1 Keble Road, Oxford, OX1 3RH, UK (mjj@astro.ox.ac.uk)

William Keel, University of Alabama, Box 870324, Tuscaloosa, AL 35487 〈keel@bildad.astr.ua.edu〉

Daniel Kelson, Carnegie DTM, 5241 Broad Branch Road, Washington, DC 20015 〈kelson@dtm.ciw.edu〉

Ivan King, University of California, 601 Campbell Hall, Berkeley, CA 94720-3411 〈king@glob.berkeley.edu)

David Koo, University of California, Santa Cruz, CA 95064 (koo@ucolick.org)

Varsha Kulkarni, Steward Observatory, University of Arizona, 933 N. Cherry Ave., Tucson, AZ 85721 〈kulkarni@as.arizona.edu〉

Ken Lanzetta, Department of Physics and Astronomy, Stony Brook, NY 11794-3800 〈lanzetta@sbastr.ess.sunysb.edu〉

James Larkin, University of California, Los Angeles, CA 90095 〈larkin@astro.ucla.edu〉

James Liebert, Steward Observatory, University of Arizona, $933 \mathrm{~N}$. Cherry Ave., Tucson, AZ 85721 〈liebert@as.arizona.edu〉

Simon Lilly, Dept. of Astronomy, University of Toronto, 60 St. George Street, Toronto, ON M5S 3H8, CANADA (lilly@astro.utoronto.ca) 
Michael Liu, University of California, 601 Campbell Hall , Berkeley, CA 94720-3411 〈mliu@triscuit.berkeley.edu〉

Avi Loeb, Harvard-Smithsonian Center for Astrophysics, Mail Stop 51, 60 Garden Street, Cambridge, MA 02138 〈aloeb@cfa.harvard.edu)

Malcoln Longair, Cavendish Astrophysics Group, University of Cambridge, Madingley Road, Cambridge, CB3 0HE, UK 〈msl@mrao.cam.ac.uk〉

Piero Madau, Institute of Astronomy, University of Cambridge, Madingley Road, Cambridge, CB3 0HA, UK 〈pmadau@ast.cam.ac.uk〉

Ariyeh Maller, University of California, Santa Cruz, CA 95064 〈ari@cosmoK.ncscedn〉

Curtis Manning, University of California, 601 Campbell Hall , Berkeley, CA 94720-3411 〈manning@mars.berkeley.edu)

Francine Marleau, Institute of Astronomy, University of Cambridge, Madingley Road, Cambridge, CB3 0HA, UK (marleau@ast.cam.ac.uk〉

Smita Mathur, Harvard-Smithsonian Center for Astrophysics, 60 Garden St., Cambridge, MA 02138 〈smita@cfa.harvard.edu〉

Patrick McCarthy, Obs. of Carnegie Inst. of Washington, 813 Santa Barbara St., Pasadena, CA 91101 〈pmc2@ociw.edu〉

Bryan Mendez, University of California, 601 Campbell Hall, Berkeley, CA 94720-3411 〈bmendez@astron.berkeley.edu〉

R. Benton Metcalf, University of California, 601 Campbell Hall, Berkeley, CA 94720-3411 (bmetcalf@astron.berkeley.edu )

Lance Miller, Astrophysics Department, University of Oxford, NAPL, 1 Keble Road, Oxford, OX1 3RH, UK 〈L.Miller@physics.ox.ac.uk)

Leonidas Moustakas, Astrophysics Department, University of Oxford, NAPL, 1 Keble Road, Oxford, OX1 3RII, UK 〈leonidas@astro.ox.ac.uk〉

Richard Mushotzky, NASA Goddard Space Flight Center, Code 662-GSFC, Greenbelt, MD 20771 〈mushotzky@ilheavx.gsfc.nasa.gov〉

Jeffrey Newman, University of California, 601 Campbell Hall, Berkeley, CA 94720-3411 〈jnewman@astron.berkeley.edu〉

Louisa Nolan, Institute for Astronomy, Royal Observatory, Blackford Hill, Edinburgh, EH9 3HJ, UK 〈l.nolan@roe.ac.uk〉

Augustus Oemler Jr., Obs. of Carnegie Inst. of Washington, 813 Santa Barbara St., Pasadena, CA 91191 〈oemler@ociw.edu〉

Patrick Osmer, Astronomy Dept., Ohio State University, 174 W. 18th Ave, Columbus, OH 43210-1106 〈posmer@astronomy.ohio-state.edu〉

Michael Pahre, Harvard-Smithsonian Center for Astrophysics, 60 Garden Strect, MS 20, Cambridge, MA 02138 〈mpahre@cfa.harvard.cdu)

Casey Papovich, Department of Physics and Astronomy, The Johns Hopkins University, Baltimore, MD 21218 (papovich@pha.jhu.edu〉

R. Bruce Partridge, Dept. of Astronomy, Haverford, PA 19041 〈bpartrid@haverford.edu〉 
William Percival, Astrophysics Department, University of Oxford, NAPL, 1

Keble Road, Oxford, OX1 3RH, UK (w.percivall@i physics.ox.ac.uk)

Andrew Phillips, Univcrsity of California, Santa Cruz. CA 95064

〈phillips@ucolick.org )

Joel Primack, University of California, Santa Cruz, CA 95064 〈joel âucolick.org)

Steve Rawlings, Astrophysics Department, University of Oxford, NAPL, 1

Keble Road, Oxford, OX1 3RH, UK (s.rawlings1@physics.ox.ac.uk〉

Alvio Renzini, European Southern Observatory, Karl-Schwarzschild-Straße 2, Garching bei München, D 85748, GERMANY 〈arenzini@cso.org〉

Roger Romani, Physics Dept., Stanford University, Stanford, CA 94305-4060 〈rwr@astro.stanford.edu

Jose Luis Sanz, University of California, 601 Campbell Hall, Berkeley, CA 94720-3411 (sanz@mars.berkeley.edu)

Alice Shapley, Caltech, MS 105-24 Astronomy, Pasadena, CA 91125 〈aes@astro.caltech.edu〉

Joseph Silk, University of Oxford, NAPL, 1 Keble Road, Oxford, OX1 3RH, UK〈silk@astro.ox.ac.uk〉

Chris Simpson, NAOJ Subaru Observatory, 650 N. A'ohoku Place, Hilo, HI 96720 〈chris@naoj.org〉

Hyron Spinrad, University of California, 601 Campbell Hall, Berkeley, CA 94720-3411 〈spinrad@bigz.berkeley.edu〉

Tracy Spinrad, Arizona State University, Tempe, AZ 85287 〈tspinrad@asu.edu〉

S. Adam Stanford, IGPP/LLNL, L-413, P.O. 808, Livermore, CA 94551 〈adam@igpp.llni.gov >

John Stauffer, Harvard-Smithsonian Center for Astrophysics, 60 Garden St., Cambridge, MA 02138 (stauffer@cfa.harvard.edu)

Charles Steidel, Caltech, MS 105-24 Astronomy, Pasadena, CA 91125 $\langle$ ccs@astro.caltech.edu〉

Daniel Stern, University of California, 601 Campbell Hall, Berkeley, CA 94720-3411 〈dan@slothrop.berkeley.edu)

Jonathan Swift, University of California, 601 Campbell Hall, Berkeley, CA 34720-3411 〈js@slothrop.berkeley.edu)

Rodger Thompson, Steward Observatory, University of Arizona, 933 N. Cherry Ave., Tucson, AZ 85721 〈rthompson@as.arizona.edu〉

Yoshinori Tutui, Institute of Astronomy, University of Tokyo, 2-21-1 Osawa, Mitaka, Tokyo 181-8588, JAPAN 〈tutui@mtk.ioa.s.u-tokyo.ac.jp 〉

Wil van Breugel. IGPP/LINL, L-413, P.O. 808, Livermore, CA 94551 〈wil@igpp.llnl.gov〉

Joël Vernet, European Southern Observatory, Karl-Schwarzschild-Straße 2, Garching bei München D-85748, GERMANY 〈jvernet@eso.org) 
Nicole Vogt, Institute of Astronomy, Liniversity of Cambridge, Madingley

Road, Cambridge, CB3 0HA, UK (nicole@ast.cam.ac.uk)

Ian Waddington, Physics and Astronomy Dept., Arizona State University, P.O.

Box 871504, Tempe, AZ 85287-1504 (Tan.Waddington@asu.edu)

Rainer Wehrse, University of Heidelberg, Tiergartenstraße 15, D-69121

Heidelberg, GERMANY (wehrse@ita.uni-heidelberg.de)

Michael Werner, JPL, MS 264-767, 4800 Oak Grove Dr., Pasadena, CA 91109

〈mwerner(asirtfweb.jpl.nasa.gov)

Gillian Wilson, Institute for Astronomy, University of Hawaii, 2680 Woodlawn Drive, Honolulu, HI 96822-1897 (wilson@ifa.hawaii.edu )

Rogier Windhorst, Physics and Astronomy Dept., Arizona State University, P.O. Box 871504, Tempe, AZ 85287-1504 (Rogier.Windhorst@asu.edu)

Arthur Wolfe, University of California, 9500 Gilman Drive, La Jolla, San Diego, CA 92093 〈awolfe@ucsd.edu〉

Rosemary Wyse, Physics and Astronomy Department, The Johns Hopkins University, Baltimore, MD 21218 〈wyse@pha.jhu.edu〉

Toru Yamada, Astronomical Institute, Tohoku University, Aoba-ku, Sendai, 980-8578, JAPAN 〈yamada@astr.tohoku.ac.jp >

Lin Yan, Obs. of Carnegie Inst. of Washington, 813 Santa Barbara St.. Pasadena, CA 91101 〈lyan@ociw.edu〉

Howard Yee, Dept. of Astronomy, University of Toronto, 60 St. George Street, Toronto, ON M5S 3H8, CANADA 〈hyec@astro.utoronto.ca)

Sukyoung Yi, Caltech, MS 405-47, Pasadena, CA 91125 (yi@srl.caltech.edu)

Steve Zepf, Dept. of Astronomy, Yale University, P.O. Box 208101, New Haven, CT 06520-8101 〈zepf@astro.yale.edu)

Esther Zirbel, City University of New York, 2800 Victory Blvd., Staten Island, NY 10311 〈zirbel@postbox.csi.cuny.edu〉

Andrew Zirm, Physics and Astronomy Dept., The Johns Hopkins University, 3701 San Martin Drive, Baltimore, MD 21218 〈azirm@pha.jhu.edu〉 
Th.e Hy Redshift Universe

ASP Conference Series, Vol. 19S. 1999

A. J. Bunker \& W. J. M. van Breugel, eds.

\title{
Observations of Hyron Spinrad
}

\author{
Ivan R. King \\ Astronomy Department, University of California \\ Berkeley, CA 94720-3411 \\ James W. Liebert \\ Steward Observatory, University of Arizona \\ Tucson, AZ 85721 \\ Imke de Pater \\ Astronomy Department, University of California \\ Berkeley, CA 94720-3411
}

\begin{abstract}
Hyron Spinrad's career has spanned several decades, and has stretched from our neighboring planets to the remotest galaxies in the Universe, pausing in between to "enrich" our knowledge of the compositions of stars.
\end{abstract}

\section{The observer observed (by I.R.K.)}

Hyron Spinrad joined the Berkeley Astronomy Department in the Spring of 1964. Since I arrived just one semester later, I can claim to have followed his entire career at Berkeley.

Actually my first observation of $\mathrm{Hy}$ was a few years earlier, at an international symposium in Santa Barbara. He had just finished his Ph.D. thesis, which included the observation that some of the metal lines in galaxy spectra were stronger than anyone then thought they ought to be. In one of the discussion periods he presented these results (Spinrad 1962a), and they were received with doubt and distrust. In fact, this young man standing up in front of the pundits of the world and stating heretical ideas reminded me of the old carnival sideshow in which a man sticks his head through a hole in a canvas and you get a prize if you can hit him with a baseball before he gets out of the way.

Although Hy didn't yet have the interpretation quite right, this was the beginning of his work on supcr metallicity, which eventually showed that the chemical abundance picture in galaxies (and in the solar neighborhood) was more complicatcd than it had been thought to bc.

\subsection{Collaborations}

I was very much interested in this work, and actually participated in it at one stage (Spinrad et al. 1970). I co-authored three other papers with Hy (Spinrad et al. 1971; one that was only an AAS presentation; Kron, Spinrad, \& King 
1977). Interestingly, every one of them turned out to have something wrong with it. In the first paper we attributed too high a metallicity to the old open clusters M67 and NGC 188, because a distinguished colleague had lent us some standard-star spectra that were out of focus. The second paper was indeed a notable discovery (identifying Maffei 1 as a nearby elliptical galaxy), but we went too far by suggesting that it might be a member of the Local Group. The third paper (no reference) never got beyond the stage of a presentation at an AAS meeting; in it we identified the IR source IRC -20385 as a rich stellar system. Our error was to incline toward the hypothesis that it was a nearby elliptical galaxy rather than a globular cluster in the Galactic bulge. It is now catalogued as Terzan 5; except for heavy foreground obscuration, it might be the most spectacular globular cluster in the Galaxy. (As of this year, I think I understand the problem; it was not known at that time that bulge globulars have such an under-luminous red giant branch.) In the fourth paper we judged a spectral break in the green to be $\lambda 2640$, when it was really $\lambda 4000$, thus suggesting too high a redshift. The spirit was willing, but the data were weak. We were young and enthusiastic; and as Hy, a long-time fan of the Los Angeles Dodgers, would be the first to recognize, you can't win 'em all.

In any case, I have refrained from collaboration with $\mathrm{Hy}$ in the years since, and I think that without my interference he has done pretty well.

I did actually give Hy some real assistance during the early days, though. Like nearly all astronomers of that time, Hy took photographs without the tedious added effort of putting standard intensity spots on the plate. From time to time he would walk into my office and hand me a plate-usually a very interesting one that had resulted from his particular skill in hypersensitizing infrared-sensitive plates-and ask me to derive intensity values for objects on it. As an expert in scientific photography, I was sometimes able to do this. Annoying, but an interesting game.

Life with $\mathrm{Hy}$ has been interesting and often challenging-and never dull.

\subsection{Redshifts: opening up the Universe}

But Hy's career work eventually settled on the discovery and the study of galaxies with large redshifts.

The early decades of redshift exploration had been relatively quiet, with Humason's decades-old $z=0.2$ topping the list (Humason, Mayall, \& Sandage 1956) for a long time. This early era ended with a dramatic event: in his last Palomar observing run before retirement, Rudolf Minkowski found a redshift of 0.46 for the radio source $3 \mathrm{C} 295$. For nearly two decades this was the most distant galaxy known.

(After a post-retirement year at Wisconsin, Minkowshi moved to a niche that Harold Weaver created for him at the Berkeley Radio Astronomy Laboratory. He was a friend and a source of inspiration for both $\mathrm{Hy}$ and me until his death in 1976.)

The most distant galaxy did not remain the frontier of the Universe for very long. Quasars had been discovered in 1960 (reported at an AAS meeting in December of that year; published as Sandage \& Matthews 1963), and after their spectra were deciphered as redshifts (Greenstein \& Schmidt 1964), quasar redshifts soon began a dizzying climb, to levels even beyond $z=1$. 
For a long period the distant Universe was for astronomers the Universe of quasars. Their known redshifts soared to 2 and even beyond 3 , while galaxies lagged far behind, limited to Minkowshi's diminutive 0.46. Yet, spectacular as the luminosities of the quasars may be, it is the galaxies that are the fundamental building blocks of the Universe, and it is the study of their evolution that will ultimately reveal their origins. This aim became particularly frustrating when studies of the cluster around Minkowski's 3C295 showed that some evolution appeared to have taken place even in the relatively brief time that that light had taken to reach us (Butcher \& Oemler 1978), while the most distant quasars, whose nature and role in the scheme of things were mysterious, were showing us flickers of the Universe as it was when it was a fraction of its present age. If only we could study galaxies at such fabulous distances ....

During this period $\mathrm{Hy}$ had begun to reason in just this way. Pursuing his interest in the stellar mixtures that make up galaxies, he was wondering whether he could get a new insight into these populations by studying them as they were when light left them at some past era. As early as 1972 he was calculating what a local galaxy would have looked like at at the time when today's light left 3C295 (Spinrad 1972). (Studies of this sort led to Gustavo Bruzual's thesis, and the whole industry that Gustavo and others have created.) This is how Hy got into the high-redshift business, whose payoffs have been so rich that it has been his main interest ever since.

The high redshifts began slowly; the first new one (Spinrad et al. 1975) topped Minkowski by less than 0.01. But Hy then broke out of the past with a redshift of 0.75 (Spinrad \& Smith 1976), and the new era was launched. A landmark, several years later, was the passing of the psychological barrier $z=1$ (Spinrad 1982a).

In the course of this work Hy realized that radio catalogs would prove to be a good source of high-redshift galaxies, and he set out to make identifications and find redshifts for all the galaxies of the $3 \mathrm{C}$ catalog (described elegantly by Longair, elsewhere in this volume). In this he succeeded well, filling in identifications for all $3 \mathrm{C}$ objects with $|b|>10^{\circ}$, and measuring redshifts for all but one of them.

I do not mean that the use of radio galaxies to find high redshifts is an exclusive idea of Hy's. Others also thought of it at an early time (Blumenthal \& Miley 1979), and others use it today (e.g., van Breugel et al. 1999). But Hy's identification of so many $3 \mathrm{C}$ sources remains an outstanding contribution.

For more than 30 years quasars were the most distant objects known-until galaxies finally surpassed them in recent years (Franx et al. 1997). At present the highest solidly established redshifts come from two collaborations of Hy's (Dey et al. 1998, Weymann et al. 1998), although in this volume Hu and Lanzetta each discuss objects that may soon be shown to have higher redshifts. It's a very competitive game, and we all look forward to what $\mathrm{Hy}$ and his friendly competitors are going to produce in the future.

\section{Stellar abundances (by J.W.L.)}

My association with Hyron goes back thirty years to when I was a student, and both of us were young. Observing runs with Hyron featured radio galaxies as the 
main program, while my thesis was essentially done as a backup program. I got to observe in twilight, when standard stars had to be observed and one might fit in an additional ten-minute spectrum, or when the moon was too bright, or the weather too punk but the telescope could remain open. Mine wasn't the only side project. Nearly always, he would carry finding charts to take a spectrum or two for a postdoc or colleague. Or it might be to observe a flaring BL Lac for Margaret Burbidge, because her next Lich run was months later. There might be a spectrum taken of a supernova in response to a phone call or IAU circular. We might have to get the 120-inch (now the Shane 3-m) to track the nucleus of a comet. To sum up, observing with Hyron can be characterized by such words as diversity, flexibility, spontaneity, and of course enthusiasm.

The mid-70s were heady days for the Lick 120-inch. At that time the telescope was the fifth largest in the world, I think, after the Soviet 6-m, the Palomar 5-m, and KPNO and CTIO 4-meters. Thanks to Joe Wampler and Lloyd Robinson of U.C. Santa Cruz, however, the 3-m spectrograph had the best digital detector in the world - the Image Tube (or Dissector) Scanner (ITS/IDS). Thus, my spectra of white dwarfs would reveal weaker features, especially at redder wavelengths, than were possible with intensified (image-tube) photographicplate detectors. In my first thesis paper, for example, I showed that the sub-class of white dwarf called DM by Greenstein didn't exist (The Demise of the Degenerate M Stars). Thanks to Joe Wampler and Lloyd Robinson! Likewise, Spinrad slowly cranked out ever-higher $3 \mathrm{CR}$ redshifts. In an acerbic moment, Spinrad would refer to a distinguished Caltech astronomer: "If you can't beat $\mathrm{xxx} \mathrm{xxx}$, you'd better get out of the game!" And I finished an effective thesis using "backup time" in Spinrad's program.

\subsection{The dwarf carbon star and other oddities}

Besides carving out an observational thesis largely from white dwarfs which were spectrophotometric standard stars, I naturally fell into the Spinrad practice of chasing down "odd-ball" objects. One day fellow student Richard Kron came to us with such a case from the U.S. Naval Observatory at Flagstaff, where his father was Site Director. A young scientist named Conard Dahn had an object from his trig parallax program whose colors were "peculiar" compared to main sequence stars of similar absolute magnitude. Could Hyron and I get a spectrum to see what kind of nearby star this was?

We did get that spectrum, and it astonished us, because the bands sloped the wrong way. It turned out, of course, that they were not the familiar TiO, but $\mathrm{C}_{2}$. This object, $\mathrm{G} 77-61$, turned out to be the first dwarf carbon star (Dahn et al. 1977). This is now a class of binary star where the now-unseen or white dwarf companion transferred carbon-rich material to an originally normal, low-mass main sequence companion, during its carbon AGB phase. The now-visible main sequence star has a carbon-dominated spectrum. There are now about ten of these known (see Green \& Margon 1994). According to Wallerstein and Knapp (1998), dwarf carbon stars may be more numerous than the giants in a given volume of space. After the initial discovery, Dahn and I have collaborated for decades on the luminosity functions of nearby stars.

My interest in $M$ dwarf stars and in searching for the terminus of the main sequence began also with Hy and Rich Kron. In the past few years, of course, 
brown dwarfs have been discovered in abundance. As of this writing, our Two Micron All Sky Survey (2MASS) team has found several dozen of these "brownjes," as Hyron calls this new population of dim solar neighbors.

Perhaps the high point of my thesis, and my favorite object, is the white dwarf Feige 7 (L $795-\tau$ ). This was classified DC (featureless) by Greenstein, yet was too blue in photometric colors for the dominant hydrogen or helium not to show broad and strong lines. I reasoned that the object either had an unusual composition, or some other surprise awaited.

Feige 7 turned out to have both hydrogen and helium, but it was a rotating, highly magnetic object. The individual lines of each transition were split and spread by the range of magnetic field present on the stellar disk into hundreds of individual, weak components. The sensitivity of the ITS allowed many of these to be individually measured. With the help of Roger Angel at Steward Observatory, who also detected tell-tale circular polarization from the star, we figured out the interpretation (Liebert et al. 1977). Feige 7 became the central point of Angel's Pierce Prize lecture at a 1976 AAS meeting. Oh, and Feige 7 got me a job at the University of Arizona, where I have been ever since!

\subsection{Super-metal-rich stars: now come full circle}

Ivan has mentioned the high metallicity incorrectly attributed to the old open clusters M67 and NGC 188, due to the out-of-focus standard-star spectra they were lent. Another Galactic disk cluster Hyron and Ben Taylor worked on before my arrival in Berkeley is NGC 6791, for which they inferred from Lick single-channel scanner measurements an abundance several times the solar value (Spinrad and Taylor 1971). When I arrived at Berkeley, I remember having the impression that the work on "super-metal-rich" stars was controversial. Hyron and Ben had published metallicities much greater than solar for field stars like the red giant $\mu$ Leonis; others argued that this star was close to solar. Critics were skeptical that any stars existed in the Galactic disk-especially old stars, well away from the Galactic bulge region - that were appreciably more metalrich than the Sun. Although I was interested in stars and stellar evolution, my inclination was not to get involved with this.

Thirty years later, I have gotten myself involved, and the situation has changed. NGC 6791 is the richest, and among the oldest clusters of the Galactic disk. It is located at nearly the solar Galactocentric distance, more than one kiloparsec above the plane. The abundance estimates in the literature range from solar to three times solar. The problem of extrapolating from well-studied stars near and below the solar metallicity has been with us all along. However, the techniques are much improved. I got involved with Elizabeth Green and Rex Saffer (Liebert, Saffer \& Green 1994) in the study of unusually hot horizontal branch stars found in this cluster. (A metal-rich cluster was the last place one would have expected this kind of HB star, but that's a different story). Another, roughly $8000 \mathrm{~K} \mathrm{HB}$ cluster member was found, and Peterson \& Green (1998) realized that this might be the ideal object from which to determine the heavy-element abundance of the cluster (in preference to the very cool, heavily line-blanketed giants). Their study using high-resolution KPNO 4-m spectra suggested $[\mathrm{M} / \mathrm{H}]=+0.3$ to +0.5 , with the higher values for some alpha elements; and the lower value for Fe. Chaboyer, Green \& Liebert (1999) tested and pro- 
vided additional support for this determination by fitting model isochrones to both $B-V$ and $V-I$ color-magnitude diagrams available in the literature.

Despite its age (we got $8 \mathrm{Gyr}$ ) and location in the Galaxy, NGC 6791 is most definitely super-metal-rich. As for $\mu$ Leo, Ruth Peterson points out that its spectrum is virtually identical to those of the "red clump" giants in NGC 6791, so it should have similar abundances (although not all workers in the field are in complete agreement). Following the trail of Spinrad and Taylor, we have thus helped muddy again the waters of a clean chemical-enrichment picture for the Galactic disk.

\section{Solar System work (by I. de P.)}

Before he joined the Berkeley faculty, Hy had a postdoc at JPL. It was there that his career in planetary sciences got a start. In 1962 he announced the discovery of a new $\mathrm{CO}_{2}$ band on Venus in a true letter (10 sentences long) to the ApJ (Spinrad 1962b); he had identified this line on a spectrogram taken at Mt. Wilson Observatory in 1943! He concludes his letter with the statement: "the optical depth in this band is probably quite large."

In $1963 \mathrm{Hy}$ discovered water vapor in the Martian atmosphere (Spinrad, Münch, \& Kaplan 1963), a topic which is still very much alive today. At that time it was believed that the Martian polar ice-caps consisted of water-ice; the seasonal variations in the ice-caps implied a net transport of water between the poles. So Hy set out to observe this effect. He was the first to detect water vapor in the Martian atmosphere, though it was completely unrelated to a poleto-pole transport of water. (The temperature at the Martian poles is always well below freezing.) Their derived water column of $14 \pm 7$ microns of precipitable water is consistent with more recent measurements such as those by Viking, and the Very Large Array. In subsequent years, using McDonald (in collaboration with Ron Schorn) and Lick observatories, they noticed that the amount of water varied substantially over time and location on the disk, a finding which is also supported by more recent observations.

From these same Martian spectra, $\mathrm{Hy}$ and collaborators derived the $\mathrm{CO}_{2}$ abundance in Mars' atmosphere (Kaplan, Münch, \& Spinrad 1964), and from it derived the amazing result of a surface pressure as low as $25 \pm 15 \mathrm{mbar}$ ! This was the first hint that Mars's atmosphere was very tenuous, an observation that was later confirmed by an occultation experiment with the Mariner V spacecraft. Based upon their early results, Hy and collaborators further pointed out the even more startling conclusion that the atmosphere must be dominated by carbon dioxide gas, rather than nitrogen, as seen in the Earth's atmosphere. So they end their papers with the question: where is the nitrogen? Did it, perhaps, escape the gravitational attraction of the planet?

Hy's contributions to cometary research started in 1958 with a short paper (Forbes, Spinrad, \& Wood 1958) detailing their results on improving the orbit of Comet $1952 \mathrm{e}$ Harrington based upon their observations. His continuous interest

in comets, however, was not through stimulating words from his colleagues, as Hy's concluding sentence in a paper (Spinrad 1982b) on observations of the red auroral oxygen lines in nine comets testifies: "Finally, I want to thank my wife Bette for her insistence I try something else besides faint galaxies." 
Hy"s cometary research was particularly "famous" and "feared" among his students, where each of them had to struggle through masses of comet spectra. Many of these efforts did result in papers, with often novel ideas and results. A dozen years ago Hy wrote a review on "Comets and Their Composition" (Spinrad 1987). In this paper he starts out summarizing current ideas about the origin and place of formation of comets, in particular whether comets in the Oort cloud originated in the outer solar system or in the interstellar medium. Although the likelihood of the latter is deemed very low, recent results on, e.g., D/H ratios in comets Halley 1986, Hale-Bopp 1997, and Hyakutake 1996 strongly suggest that at least a fraction of the molecules contained in the icy grains from which the comets formed had their origin in cold interstellar clouds, and were incorporated as such into the forming comets.

Hy ends his paper with a speculation on the detection of distant comets, and based upon current detector technologies concludes that only giant comets might be detected at distances of say a few hundred AU. A giant comet in his terminology is one with a nucleus over $100 \mathrm{~km}$ ! At the time of Hy's review paper, the commonly accepted size for a comet nucleus was only a few $\mathrm{km}$. The discovery (by the Giotto spacecraft) that comet Halley was very dark ( $4 \%$ albedo for an icy object!) and oddly shaped with a size $8 \times 16 \mathrm{~km}$ shocked the community, so having comets several hundred $\mathrm{km}$ in diameter was unthinkable. Only 5 years later the first Kuiper-belt object was detected (Jewitt, Luu, \& Marsden 1992), a giant comet indeed. We now are familiar with the idea of giant comets, but the question of the origin of comets, and in particular cometesimals, is still largely unresolved; neither do we know how much stuff is really out there in the Kuiper belt or Oort clouds.

There is indeed much left for Hy and the rest of us to do on comets!

Acknowledgments. We are grateful to Mike Brown, Andy Bunker, and Dan Stern for assistance with background information (and to Hy himself for insisting that we give full credit to the radio-galaxy competition).

\section{References}

Blumenthal, G., \& Miley, G. K. 1979, A\&A, 80, 13

Butcher, H., \& Oemler, A. 1978, ApJ, 219, 18

Chaboyer, B., Green, E. M., \& Liebert, J. 1999, AJ, 117, 1360

Dahn, C. C., Liebert, J., Kron, R. G., Spinrad, H., \& Hintzen, P. M. 1977, ApJ, 216, 757

Dey, A., Spinrad, H., Stern, D., Graham, J. R., \& Chaffee, E'. H. 1998, ApJ, 498, L93

Forbes, J. E., Spinrad, H., \& Wood, DB. 1958, AJ, 63, 510

Franx, M., Mllingworth, G. D., Kelson, D. D., van Dokkum, P. G., \& Tran, K.-V. 1997, ApJ, 486, L75

Green, P. J., \& Margon, B. 1994, ApJ, 123, 723

Greenstein, J. L., \& Schmidt, M. 1964. ApJ, 140, 1

Humason, M. L., Mayall, N. U., \& Sandage, A. R. 1956, AJ, 61, 97

Jewitt, D., Luu, J., \& Marsden, B. 1992, IAU Circular 5611 
Kaplan, L. D., Münch, G., \& Spinrad, H. 1964, ApJ, 139, 1

Kron, R. G., Spinrad, H., \& King, I. R. 1977, ApJ, 217, 951

Liebert, J., Angel, J. R. P., Stockman, H. S., Spinrad, H., \& Beaver, E. A. 1977. ApJ, 214, 457

Liebert, J., Saffer, R. A., \& Green, E. M. 1994, AJ, 107, 1408

Peterson, R. M., \& Green, E. M. 1998, ApJ, 502, L39

Sandage, A. R., \& Matthews, T. A. 1963, ApJ, 138, 30

Spinrad, H. 1962a, in I.A.U. Symposium No. 15, Problems of Extragalactic Research, ed. G. C. McVittie, 25

Spinrad, H. 1962b, Ap.T, 135, 61

Spinrad, H. 1972, ApJ, 171, 463

Spintad, H. 1982d, PASP, 94, 397

Spinrad, H. 1982b, PASP, 94, 1008

Spinrad, H. 1987, ARAA, 25, 231

Spinrad, H., et al. (9 authors). 1971, ApJ, 163, L25

Spinrad, H., Greenstein, J. L., Taylor, B. J., \& King, I. R. 1970, ApJ, 162, 89

Spinrad, H., Münch, G., \& Kaplan, L. D. 1963, ApJ, 137, 1319

Spinrad, H., \& Smith, H. E. 1976, ApJ, 206, 355

Spinrad, H., Smith, H. E., Hunstead, R., \& Ryle, M. 1975, ApJ, 198, 7

Spinrad, H., \& Taylor, B. J. 1971, ApJ, 163, 303

van Breugel, W., De Breuck, C., Stanford, S. A., Stern, D., Röttgering, H., \& Miley, G. 1999, Ap.T, 518, L61

Wallerstein, G., \& Knapp, G. R. 1998, ARAA, 36, 369

Weymann, R. J., Stern, D., Bunker, A., Spinrad, H., Chaffee, F. H., Thompson, R. I., \& Storrie-Lombardi, L. J. 1998, ApJ, 505, L95 
Section A. Radio Galaxies at Large Redshifts 
The Hy Redshift Universe

ASP Conference Series, Vol. 193,1999

A. J. Bunker \& W. J. M. van Breugel, eds.

\title{
The 3CR Sample - 1962-1999
}

\author{
Malcolm S. Longair \\ Cavendish Astrophysics Group, \\ Cavendish Laboratory, \\ Madingley Road, \\ Cambridge $C B 3$ OHE.
}

\begin{abstract}
A brief history of the $3 \mathrm{CR}$ sample of bright radio sources is presented, as well as a summary of its remarkable properties. Two new results are discussed. First, for a number of the smaller double radio sources ( $\leq 120 \mathrm{kpc}$ ) at $z \sim 1$ which display the alignment effect, the optical line emission is excited by shocks rather than by photo excitation, consistent with the greater full-width half maxima and equivalent widths of their emission lines, as compared with the larger sources. The second concerns the kinematics of the double radio sources, as derived from their structural asymmetries. An asymmetric relativistic model is described which takes account of relativistic, intrinsic and environmental asymmetries. It is shown that the model can account for the structural properties of the sources in the $3 \mathrm{CRR}$ sample and can reconcile their kinematic and synchrotron ages.
\end{abstract}

\section{Introduction}

It is a great pleasure to pay homage to Hy Spinrad on the occasion of his $65^{\text {th }}$ birthday. Hy has played a central role in the story I have to tell. The nice thing about such occasions is that it is not only the work of the celebratee that is being recognised, but also the work of his collaborators, his colleagues and, most important of all, the generations of research students Hy has inspired this only adds to the pleasure.

I will discuss a topic very close to my heart - the $3 \mathrm{CR}$ radio sources. After a brief anecdotal history and a description of the remarkable properties of the sample, I will describe some new results on the properties of the $3 \mathrm{CR}$ sources, specifically, new optical spectroscopic studies of the $z \sim 1$ radio galaxies, work done in collaboration with Philip Best and Huub Röttgering, and a new study of the kinematics of the $3 \mathrm{CR}$ radio sources carried out in collaboration with Tigran Arshakian.

The amazing thing about this story is the incredible good luck which has attended studies of this sample of sources all the way through. It really is a remarkable sample. 


\section{A Brief Anecdotal History}

My own involvement with the sample began in the summer of 1963 when I joined the Radio Astronomy Group at Cambridge as a graduate student. I vividly recall Martin Kyle's remark to me, 'If you are going to solve the problems of radio sources and cosmology, start by doing something useful.' This meant attempting to identify the radio sources in the $3 \mathrm{CR}$ catalogue, the brightest radio sources in the northern sky selected at the low radio frequency of $178 \mathrm{MHz}$. After the problems of the $2 \mathrm{C}$ catalogue, which contained far too many faint confused sources, the more conservative 3C catalogue was published by Edge et al. (1959), but there still remained problems of source confusion. Andrew Bennett (1962) published a revised version of the catalogue from observations made with the long cylindrical paraboloid of the $4 \mathrm{C}$ aperture synthesis telescope. This had the great advantage of providing excellent angular resolution in right ascension and so resolved many of the confused sources still present in the $3 \mathrm{C}$ catalogue. Bennett's $3 \mathrm{CR}$ catalogue contained the brightest 328 radio sources north of $\delta=-5^{\circ}$ at a frequency of $178 \mathrm{MHz}$. Virtually all the sources at $|b| \geq 10^{\circ}$ are extragalactic objects.

Some of the brightest sources in the catalogue had been securely identified with bright giant elliptical galaxies/cD galaxies and then, in the period 1960-62, the sources 3C48, 196 and 286 were identified with what Matthews and Sandage (1963) termed quasi-stellar objects, soon to be abbreviated to quasar. Following Hazard's accurate position and radio structure for the source 3C273 in 1962, it was identified with a 13th magnitude quasar, still the brightest in the sky, and in the same year, Maarten Schmidt measured its redshift, $z=0.17$. All this had

just happened when I began working with Martin Ryle and Peter Scheuer.

In 1963, radio source positions were known at best to a few arcmin accuracy, but the situation was gradually improving thanks to interferometry at Cambridge and CalTech. By then it was known that the radio sources observed at low frequencies are generally extended, often possessing a double structure, and this complicated the identification procedure. Nonetheless, I had considerable success in finding new radio source identifications using the prints of the Palomar Sky Survey (Longair 1965), including the next four radio quasars (Ryle and Sandage 1964). I was particularly pleased with the identification of the quasar 3C9, since in 1965 Maarten Schmidt found it to have the largest redshift in the $3 \mathrm{CR}$ catalogue, $z=2.016$ and it remained the largest redshift object for many years.

The real breakthrough came, however, with the development of the OneMile and 5-km aperture synthesis radio telescopes at Cambridge, which enabled detailed source structures to be determined and the error circles which should be searched to be reduced ultimately to a few arcsec. 'The next bit of luck was that, during a sabbatical term at CalTech in 1972, I collaborated with Jim Gunn in using the Palomar 200-inch telescope to find identifications in conjunction with our accurate radio positions. At that time, the best we could do was to use an image tube at the prime focus of the telescope and this pushed the identifications to about 21st magnitude (Longair and Gunn 1975, Laing et al. 1978). The real breakthrough came in 1979, however, with our use of the prototype CCD camera PHUEI (reputedly standing for Palomar-Hale Universal Extragalactic 
Instrument) which enabled us to identify virtually all the remaining sources in the 3CR catalogue with very faint galaxies (Gunn et al. 1980).

Again, by great good fortune, it turned out that the optical spectra of these very faint radio galaxies contained strong narrow emission lines and Hy Spinrad and his colleagues had quite remarkable success in measuring the spectra and redshifts of many of these galaxies by the early 1980s (for example, Smith and Spinrad 1980). To my consternation, the lines were often so bright that $\mathrm{Hy}$ could point his spectrograph at the centroid of the radio emission and measure a redshift, even if the source had not been identified! Hy needed his bit of luck too. The strength of the emission lines was found to be strongly correlated with radio luminosity (McCarthy 1993) and so, in the flux density-limited 3CR sample, he had extraordinary success in measuring even the largest redshift radio galaxies.

\section{The Properties of the Sources in the 3CRR sample}

By the early 1980s, Julia Riley, Robert Laing and I realised that, with all the data then available on the radio spectra and structures of the radio sources in the sample, we could do a better job in defining a complete flux density-limited sample of sources. This revised $3 \mathrm{CR}$ sample became known as the 3 CRR sample (Laing, Riley and Longair 1983) - it has been the basis of all our subsequent statistical work. To obtain a sample of sources with complete redshift information, we restricted attention to sources away from the galactic plane, $|b| \geq 10^{\circ}$ and with $\delta \geq 10^{\circ}$. In this area of sky there are $1733 \mathrm{CRR}$ radio sources. The properties of the sources in the sample are quite remarkable.

- The sample spans a huge range of redshift, from the nearby Virgo cluster to the quasar $3 \mathrm{C} 9$ at redshift $z=2.016$. This is partly due to the fact that the luminosity function of the radio sources, $n(P) \mathrm{d} P \propto P^{-x} \mathrm{~d} P$, has slope close to the critical value of $x=2.5$, at which sources at all distances appear in a flux density-limited sample with equal numbers in equal logarithmic intervals of luminosity. In addition, the radio galaxies and quasars span the same redshift range at redshifts $z \geq 0.1$ (Fig. 1(a)).

- The sample contains within it the effects of strong cosmological evolution. The distributions of $V / V_{\max }$ for the radio galaxies and quasars in the sample are shown in Fig. 1(b), the radio sources of both classes being located preferentially towards the limits of their observable volumes. This is no more than a reflection of the steep slope of the radio source counts. Remarkably, the effects of cosmological evolution are present in the sample of the brightest 173 radio sources in the northern sky. The cosmological evolution also contributes to the fact that sources in the sample are observed in significant numbers at redshifts $\approx \gg 1$.

- The quasars and radio galaxies in the sample are in excellent agreement with orientation-based unification schemes. Following Barthel's insight (1989), every test of orientation-based unification schemes for the radio galaxies and quasars in the $3 \mathrm{CRR}$ sample we have made has been consistent with this picture. This means that, if you want to study the parent objects of $3 \mathrm{CRR}$ quasars, you need only study the $3 \mathrm{CRR}$ radio galaxies! 

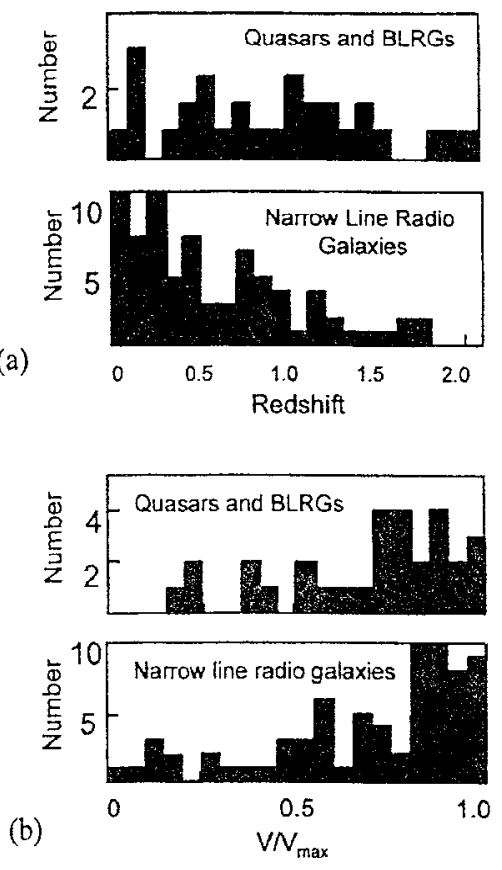

Figure 1. (a) The redshift distribution for 3CRR radio galaxies and quasars. (b) $V / V_{\max }$ distributions for $3 \mathrm{CRR}$ radio galaxies and quasars.

- The most luminous objects in the sample are producing energy in jets at a rate close to the Eddington limit for $10^{9} M_{\odot}$ black holes (Rawlings and Saunders 1990).

I find it remarkable that by studying the brightest 173 sources in any waveband, such astrophysical and cosmological riches are to be found. It is a property unique to the radio waveband.

In the late 1970s and early 1980s, Simon Lilly and I began a systematic study of the radio galaxies in the $3 \mathrm{CRR}$ sample in the infrared $J, H$ and $K$ wavebands with the UK Infrared Telescope. This had the great benefit that we were studying the underlying stellar populations of these galaxies. To our delight, we found a remarkably well-defined $\mathrm{K}$ magnitude-redshift relation which extended to redshifts $z \approx 1.8$ (Lilly and Longair 1984). The relation indicated that the radio galaxies at large redshifts were about a magnitude brighter than those at small redshifts and this could be attributed to the passive evolution of the stellar populations of the galaxies. This success encouraged us to plan major campaigns to pin down more precisely what was going on astrophysically and we were able to extend the $\mathrm{K}$ magnitude-redshift relation to redshifts $z \sim 2$. A major spanner was, however, thrown in the works by the discovery of McCarthy et al. (1987) and Chambers et al. (1987) that the optical images of the radio galaxies are aligned with their radio structures. Thus, the radio source activity must certainly influence the optical images of the galaxies and possibly their infrared properties too. 
Fortunately, the means for tackling the problem of the alignment effect were at hand. In 1977, my proposal to join the Space Telescope programme was successful and the title of my project was Optical Identifications and Studies of the Physical Properties of Extragalactic Radio Sources to the Faintest Optical Magnitudes using the Space Telescope. The proposal also stated, 'At present, I have not identified my team of co-investigators'. Seventeen years later, in the summer of 1994, the first of the observations with the refurbished HST were received and a complete sample of $283 \mathrm{CRR}$ radio galaxies at redshifts $\approx \sim 1$ was the subject of Philip Best's PhD dissertation. As he has noted, he was aged only four at the time the HST proposal had been submitted. Huub Röttgering also joined the programme during his post-doctoral fellowship at Cambridge.

The HST images of the $3 \mathrm{CRR}$ radio galaxies at $z \sim 1$ are quite spectacular. They display clearly the origin of the alignment effect. There is a remarkable correlation between the nature of the alignment effect and the size of the double radio sources (Fig. 2). In the small radio sources, $r \leq 120 \mathrm{kpc}$, the alignment effect takes the form of a string of luminous knots aligned along the radio jets, which are presumed to supply energy to the double radio source components; in the larger sources, the knotty appearance disappears and the emission is rather more diffuse and more closely associated with the galaxy (Best et al. 1996).

To cut a long and complex story short, we were able to show that the alignment effect does not have a significant effect upon the redshift- $\mathrm{K}$ magnitude relation for the $3 \mathrm{CRR}$ radio galaxies and that there is still evidence for the passive evolution of the stellar populations of these galaxies (Best et al. 1998). Equally remarkably, the stellar masses of the galaxies can be determined, taking account of the evolution of their stellar populations, and it turns out that they are remarkably independent of redshift. The typical stellar masses of the host galaxies of the $3 \mathrm{CRR}$ radio sources in the redshift interval $0.5<\approx<1.8$ lie in the range $3 \times 10^{11}$ to $10^{12} M_{\odot}$. Thus, despite the fact that they are observed at a wide range of look-back times, the selection of galaxies as $3 \mathrm{CRR}$ radio sources seems to select among the most massive galaxies which exist at these epochs.

\section{Ionisation, Shocks and Aligned Structures in the 3CRR Radio Galaxies}

To disentangle exactly what is going on in the aligned optical structures, Philip Best. Huub Röttgering and I have obtained 2-dimensional spectroscopic observations using the William Herschel Telescope of 14 of the $z \sim 13$ CRR radio galaxies which exhibit the alignment effect. More details of this programme are given in the paper contributed by Philip Best at this meeting (Best 1999, Best et al. 1999). Intriguing new results have come from the study of lines in the spectra which enable shock and photo-excitation to be distinguished. Specifically, the line ratios CIII]1909/CII]2326 and [NeIII]3969/[NeV]3426 are sensitive to the excitation mechanism, as illustrated in Fig. 3. The photoionisation models are derived from the analyses of Allen et al. (1998) and those involving shock excitation from the work of Dopita and Sutherland (1996). There is a clear separation between the regions of the diagnostic diagram in which the different excitation mechanisms are likely to be important. Two different shock models are shown. In the lower region of the diagram, the shaded area shows the region 


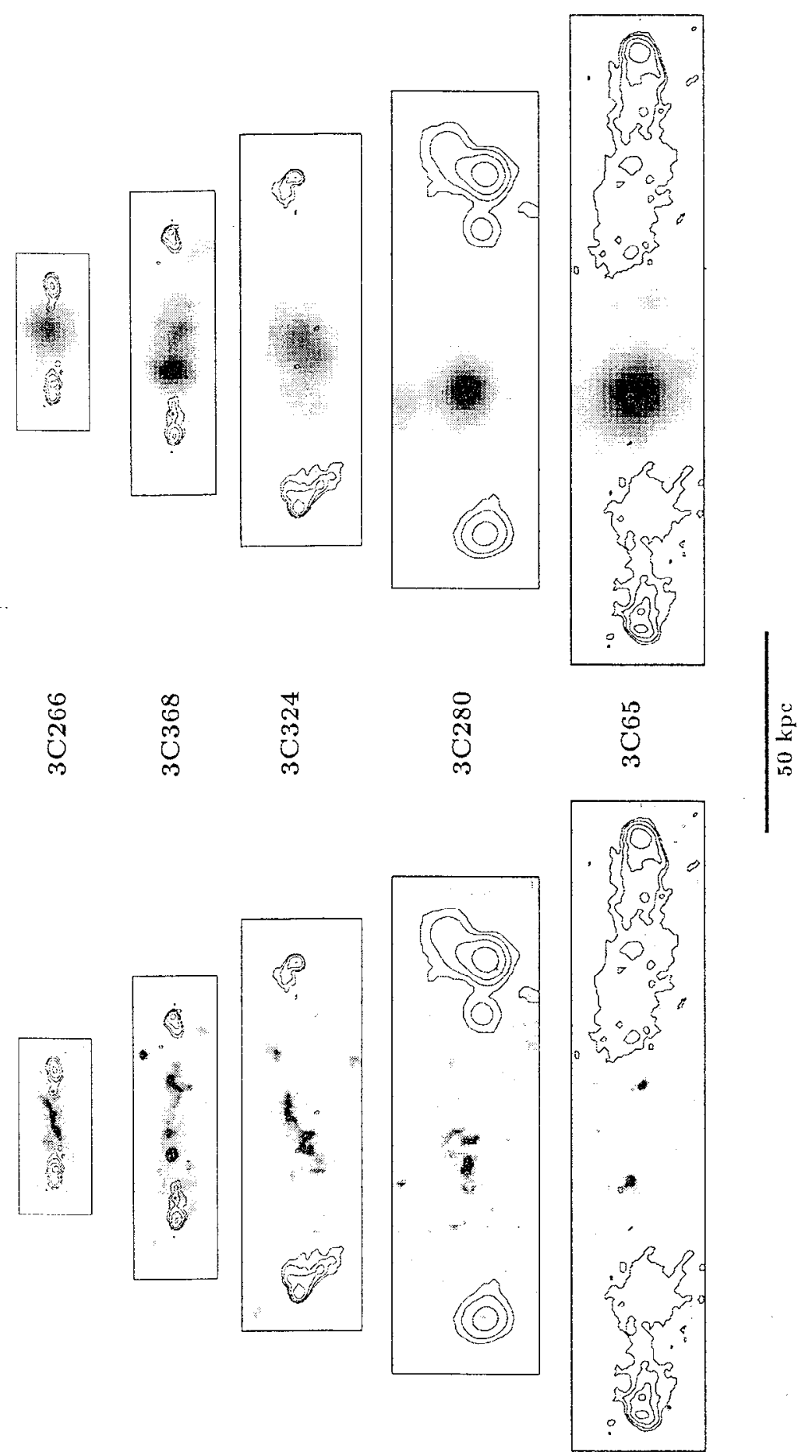

Figure 2. HST and UKIRT images of the radio galaxies $3 \mathrm{C} 266$, $368,324,280$ and 65 with the VLA radio contours superimposed. The images are drawn on the same physical scale (Best, Longair and Röttgering 1996). 


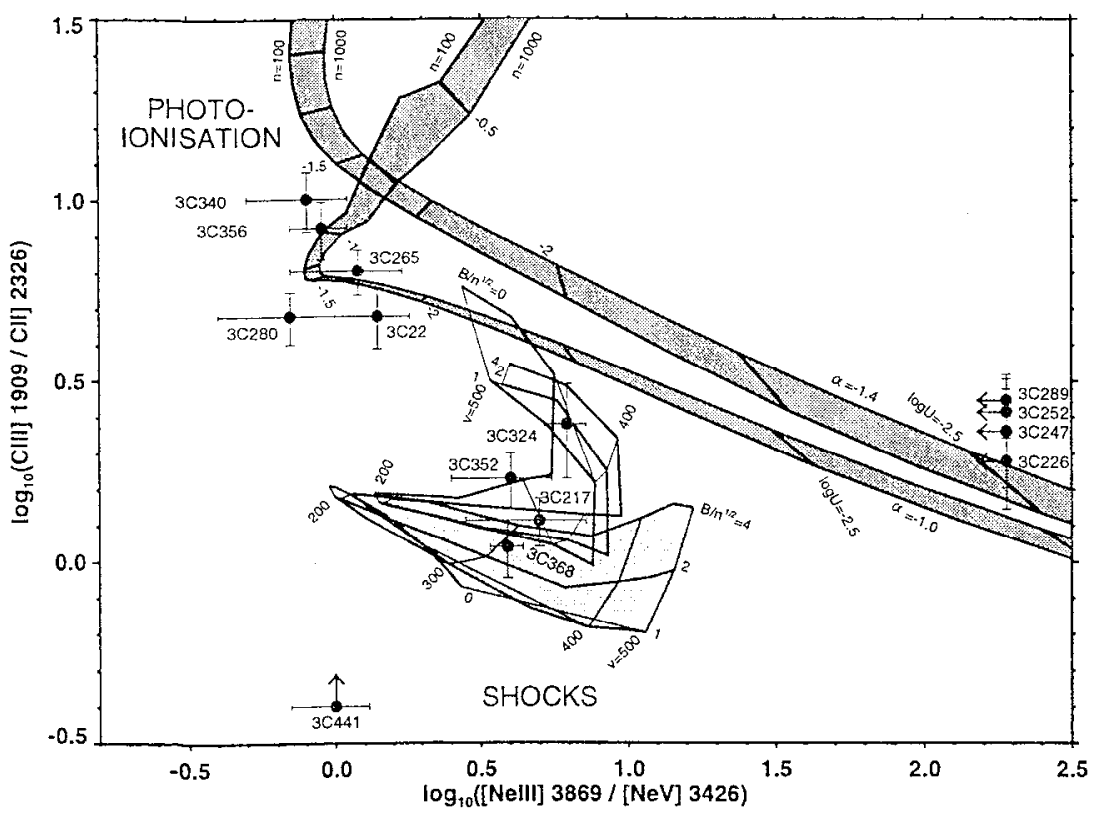

Figure 3. An emission line diagnostic diagram for $3 \mathrm{CR}$ radio galaxies at $z \approx 1$, compared with theoretical predictions. The upper shaded regions correspond to simple photoionisation models. The lower shaded region indicates the values expected for simple shock models; the upper unshaded region is the corresponding region for shock models which include photoexcitation by the radiation produced by the shock (Best et al. 1999).

occupied by shock models which do not take account of pre-ionisation of the gas upstream of the shock by the cmission produced by the shock, but this is included in the models which occupy the unshaded region above it.

It can be seen that four of the sourccs lie in the region in which the excitation mechanism is likely to be due to shocks, whereas five of the sources are more likely to be photoionised. It can be seen that the CIII]1909/CII]2326 line ratio is a particularly good discriminator for shock excitation models. What is most intriguing about these results is that the four galaxies in which shock excitation is the favoured process, $3 \mathrm{C} 217,324,352$ and 368 , are among the smaller radio sources in the sample, all of them having physical size less than $120 \mathrm{kpc}$. These are also those in which the alignment effect is associated with a compact string of knots of emission closely associated with the radio jets. To summarise the conclusions of this study,

- Small radio sources exhibit a lower degree of excitation as compared, with large sources. Sources with linear sizes less than $120 \mathrm{kpc}$ are shock ionised.

- The optical emission regions in the small radio sources have very distorted velocity profiles, large velocity widths, enhanced nebular emission and greater spatial extent than the larger radio sources. 
- The large radio sources have much smoother velocity profiles and are consistent with photoionisation being the dominant excitation mechanism.

- There are similarities in the evolution of the continuum and line properties in the sense that the small sources have knotty structures which are shock excited, whereas the large sources have small, more diffuse continuum emission regions which are photoionised.

\section{An Asymmetric Relativistic Model for Double 3CRR Radio Sources}

Another favorite game to play with the $3 \mathrm{CRR}$ sample is to attempt to determine the kinematics of the radio source structures from their observed asymmetries. The basic idea is to attribute differences in the lobe lengths (or the location of the hot-spots) to time-relay effects between the source of emission and the observer (Ryle and Longair 1967, Longair and Riley 1979). In the simplest symmetric picture, the sonrce components are assumed to be ejected from the nucleus in opposite directions at the same significantly relativistic speed $v_{0}$ and then, if the source components are ejected at an angle $\theta$ to the line of sight, the lobe ejected towards the observer should be observed to be longer than that moving away from the ohserver. A useful parameter to describe the differences in lobe lengths is the fractional separation difference (Banhatti 1980), defined as $x_{1}=\left(r_{\mathrm{j}}-r_{\mathrm{cj}}\right) /\left(r_{\mathrm{j}}+r_{\mathrm{cj}}\right)$, where the subscripts $\mathrm{j}$ and $c \mathrm{j}$ refer to the jet and counterjet direction. The reason for this is that, in many cases, a jet is observed extending from the nucleus of the radio source in the direction of one of the source components and it is natural to assume that it is the component moving towards the observer, the one-sidedness of the jet being attributed to relativistic aberration effects. In the symmetric model, $x=v / c \cos \theta$.

There are, however, three major problems with this symmetric relativistic model.

- A number of sources are now known in which the lobe advancing towards the observer, as inferred from the presence of a radio jet, is shorter than the receding lobe (Saikia 1981, 1984; Scheuer 1995).

- The velocities inferred from the relativistic symmetric model are greater than those dcrived from synchrotron ageing arguments.

- McCarthy ct al. (1991) discovered that the intensity of diffuse [OII] emission is inversely correlated with the length of the radio lobe.

The implication of these results is that there must be a contribution from intrinsic/environmental effects as well as relativistic time-delay effects. According to some analyses, the relativistic effects might be negligible. To quantify the relative importance of these effects, Tigran Arshakian and I have re-analysed the fractional separation differences $x_{1}$ for sources in the $3 \mathrm{CRR}$ sample, but now using the definition given above, which can be negative if the approaching lube is shorter than the receding lobe. The sources we have analysed are, by definition, FRII sources and so we call the sources +FRII if $x_{1}$ is positive and - FRII if $x_{1}$ 


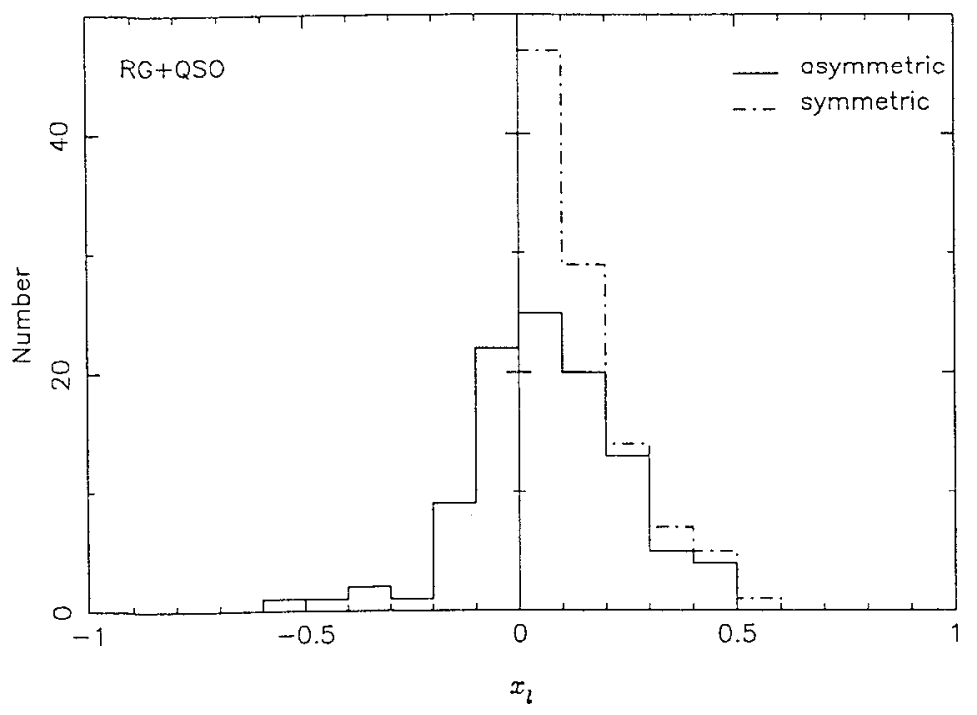

Figure 4. The distribution of the fractional separation difference $x_{1}$ for 103 FRII 3 CR radio sources according to the asymmetric (full) and symmetric (dot-dashed) models. (Arshakian and Longair 1999).

is negative. We have also introduced an asymmetry parameter $\varepsilon$ defined as

$$
\varepsilon=1-2 \frac{N(- \text { FRII })}{N(+ \text { FRII })}
$$

If $\varepsilon \approx 1$, the relativistic effect is dominant; if $\varepsilon \approx-1$, intrinsic/environmental effects are dominant, since there should be as many +FRII as -FRII sources in this case.

Of 132 FRII radio sources in the 3CRR sample, jet-sides could be identified for 103 sources and the distribution of values of $x_{1}$ is shown in Fig. 4 . The dot-dashed line shows the distribution for the symmetric model, in other words, neglecting the sign of $x_{1}$, and the solid line the results taking the sign of $x_{1}$ into account. It can be seen that there are significant numbers of -FRII sources in the sample, but there are significantly more +FRII than -FRII sources. The mean values of $x_{1}$ and $\varepsilon$ for the complete sample and for radio galaxies and quasars separately are shown in Table 1.

Table 1. Mean values of $x_{1}$ and $\varepsilon$ for the sample of 103 FRII 3 CRR sources

\begin{tabular}{ccc}
\hline & $x_{1}$ & $\varepsilon$ \\
\hline All sources & $0.070 \pm 0.018$ & $-0.07 \pm 0.22$ \\
Quasars & $0.102 \pm 0.028$ & $0.33 \pm 0.36$ \\
Radio Galaxies & $0.056 \pm 0.022$ & $-0.30 \pm 0.32$ \\
\hline
\end{tabular}

It can be seen that, for the $3 \mathrm{CRR}$ sample, both relativistic and intrinsic/environmental asymmetries must play a role. The figures suggest that relativistic 


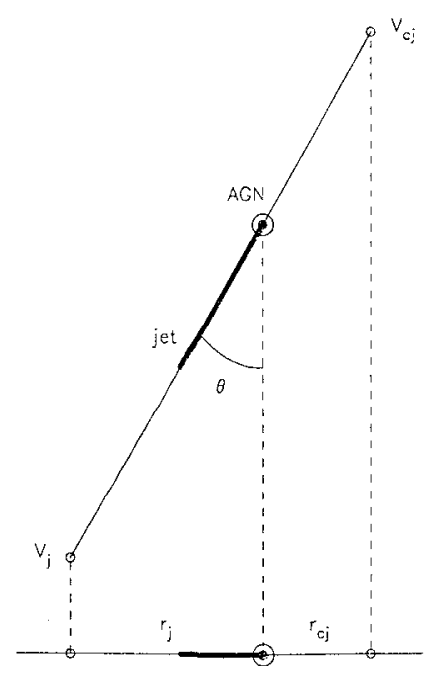

sky piane of the observer

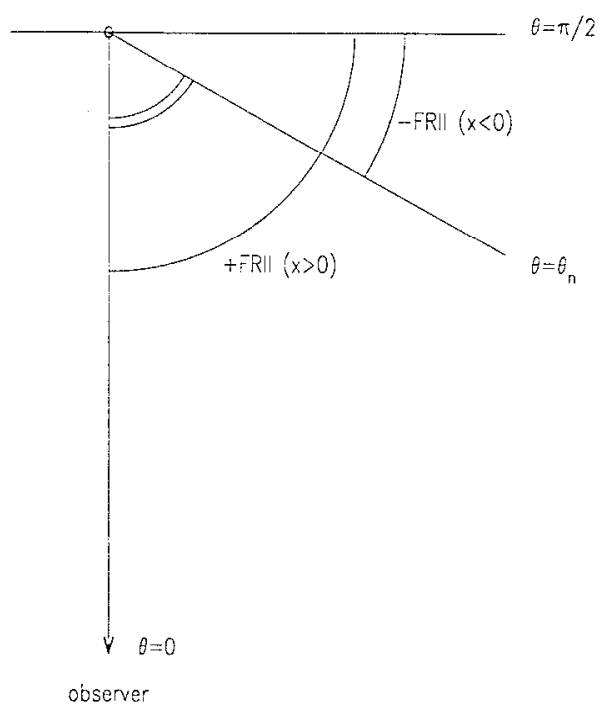

Figure 5. The asymmetric relativistic model for FRII radio sources (Arshakian and Longair 1999).

effects are more important for the quasars than they are for the radio galaxies and this is qualitatively consistent with the expectations of orientation-based unification schemes.

In order to quantify what this means physically, we have developed an asymmetric relativistic model for FRII sources. The model is illustrated in Fig. 5. The model is similar to the symmetric model, but now we allow the mean speed of the lobes in the jet and counterjet directions, $v_{\mathrm{j}}$ and $v_{\mathrm{cj}}$, to be different. 'The result is that the relation between the fractional separation difference, $v_{\mathrm{j}}$ and $v_{\mathrm{c} j}$ becomes somewhat more complex than in the symmetric model:

$$
x \equiv \frac{r_{\mathrm{j}}-r_{\mathrm{cj}}}{r_{\mathrm{j}}+r_{\mathrm{cj}}}=\frac{v_{\mathrm{j}}}{v_{\mathrm{j}}+v_{\mathrm{cj}}}+\frac{2}{c} \frac{v_{\mathrm{j}} v_{\mathrm{cj}}}{v_{\mathrm{j}}+v_{\mathrm{cj}}} \cos \theta
$$

We can write $v_{\mathrm{j}}=v_{0}+v_{\mathrm{jd}}$ and $v_{\mathrm{cj}}=v_{0}+v_{\mathrm{cjd}}$ and then introduce $\delta_{\mathrm{j}}=1+v_{\mathrm{jd}} / v_{0}$ and $\delta_{\mathrm{cj}}=1+v_{\mathrm{cjd}} / v_{0}$. Tlien, if $\delta_{\mathrm{j}}-\delta_{\mathrm{cj}} \geq 1, x \geq 0$ for all values of $\theta$. If $\delta_{\mathrm{j}}-\delta_{\mathrm{cj}} \leq 1$, two cases are possible: $x \geq 0$ for $\theta<\theta_{\mathrm{n}}$ and $x \leq 0$ for $\theta>\theta_{\mathrm{n}}$ where

$$
\theta_{\mathrm{n}}=\arccos \left(\frac{c}{v_{0}} \frac{\left|\delta_{\mathrm{j}}-\delta_{\mathrm{cj}}\right|}{2 \delta_{\mathrm{j}} \delta_{\mathrm{cj}}}\right)
$$

The value of $\theta_{\mathrm{n}}$ depends upon the value of $v_{0}$, but it can be seen that in general the intrinsic/environmental effects are expected to be greater for those sources whose axes lie closer to the plane of the sky, consistent with the observation that the latter sources are expected to be radio galaxies according to unification schemes.

In order to model the effect, we have introduced a simple Gaussian model. It is assumed that the velocity distribution of $v_{0}$ is a Gaussian with mean speed $\bar{v}_{0}$ and standard deviation $\sigma_{v_{0}}$. The distribution of the random 'disturbed' speeds 


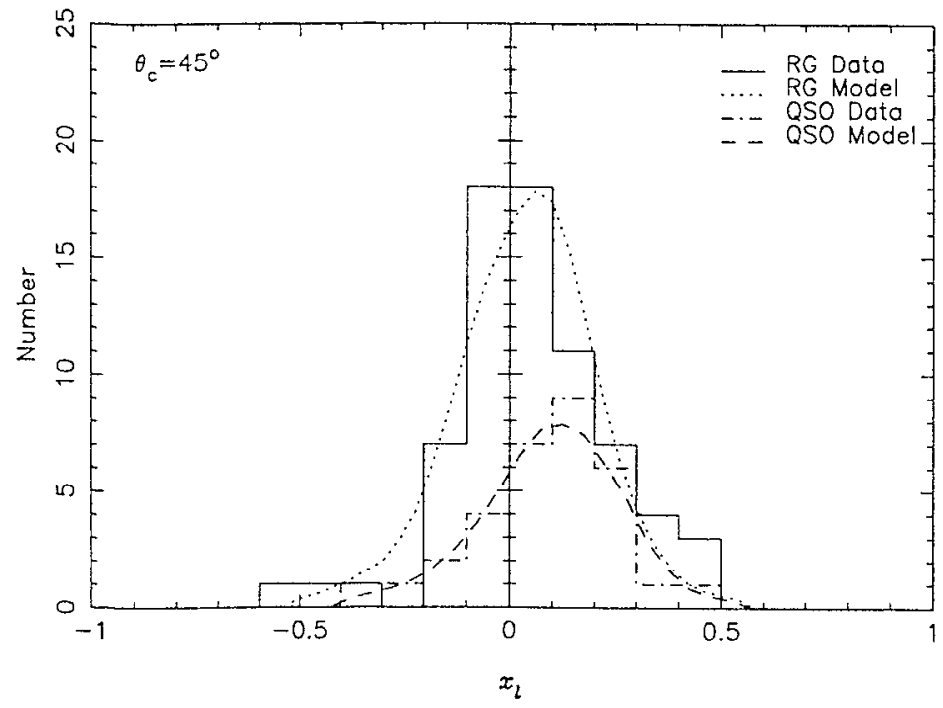

Figure 6. The predicted distributions of $x_{1}$ for $3 \mathrm{CR}$ radio galaxies and quasars, compared with the observed distributions. The bestfitting values of the parameters are given in the text (Arshakian and Longair 1999).

$v_{\mathrm{d}}$ is also assumed to be a Gaussian with the same standard deviation $\sigma_{v_{\mathrm{d}}}$ for both sides. Then, the probability distribution of $v_{1}$ is

$$
f\left(v_{1}\right)=f\left(v_{0}+v_{\mathrm{d}}\right)=\frac{1}{\sigma_{v_{1}} \sqrt{2 \pi}} \exp \left(-\frac{\left(v_{1}-\bar{v}_{0}\right)^{2}}{2 \sigma_{v_{1}}^{2}}\right)
$$

where $\sigma_{v_{\mathrm{j}}}^{2}=\sigma_{v_{0}}^{2}+\sigma_{v_{\mathrm{d}}}^{2} .10,000$ random values of these velocities were selected from the joint Gaussian distributions for each source component and the values of $\cos \theta$ were selected at random from a uniform distribution from 0 to 1 . The results are shown in Fig. 6 for the best-fitting values of the parameters, the angle of separation between the quasars and radio galaxies being $45^{\circ}$, as recommended by Barthel (1989). The best fitting values are $\bar{v}_{0}=0.11 c, \sigma_{v_{0}}=0.032$ and $\sigma_{v_{\mathrm{d}}}=0.023$. It can be seen that model can account in quantitative detail for the observed distributions of $x_{1}$ for both the radio galaxies and quasars in the 3CRR sample. We conclude that:

- The asymmetric relativistic model can account for the statistical properties of the structures of the FRII sources in the 3CRR sample.

- Kinematic and intrinsic/environmental asymmetries are of comparable importance.

- The mean speed of the lobes of $3 \mathrm{CRR}$ sources are $\bar{v}_{1} \approx(0.11 \pm 0.013) c$ with $\sigma_{v_{1}}=0.04 c$. These speeds are consistent with synchrotron ageing arguments. 
- The intrinsic speeds of the lobes and the intrinsic/environmental asymmetries increase with increasing luminosity. The intrinsic/environmental asymmetries are more important on small physical scales.

\section{References}

Allen, M.G., Dopita, M.A. and Tsvetanov, Z.I. 1998. ApJ, 493, 571

Arshakian, T. and Longair, M.S. 1999. MNRAS, (in press)

Banhatti, D.G 1980. A\&A, 84, 112

Barthel, P.D. 1989. ApJ, 336, 606

Bennett, A.S. 1962. MmRAS, 68, 163

Best, P.N., (this volume).

Best, P.N., Longair, M.S. and Röttgering, H.R.A. 1996. MNRAS, 280, 9L

Best, P.N., Longair, M.S. and Röttgering, H.R.A. 1998. MNRAS, 295, 549

Best, P.N., Longair, M.S. and Röttgering, H.R.A. 1999. MNRAS, (in press)

Chambers, K.C.,Miley, G.K. and van Breugel, W.J.M. 1987. Nature, 329, 604

Dopita, M.A. and Sutherland, R.S. 1996. ApJS, 102, 161.

Edge, D.O., Shakeshaft, J.R., McAdam, W.B., Baldwin, J.E. and Archer, S. 1959. MmRAS, 68, 37

Gunn, J.E., Hoessel, J.G., Westphal, J.A., Perryman, M.A.C. and Longair, M.S. 1981. MNRAS, 194, 111

Laing, R.A., Longair, M.S., Riley, J.M., Kibblewhite, E.J. and Gunn, J.E. 1978. MNRAS, 183,547

Laing, R.A., Riley, J.M. and Longair, M.S. 1983. MNRAS, 204, 151

Lilly, S.J. and Longair, M.S. 1984. MNRAS, 211, 833

Longair, M.S. 1965. MNRAS, 129, 419

Longair, M.S. and Gunn, J.E. 1975. MNRAS, 170, 121

Longair, M.S. and Riley, J.M. 1979. MNRAS, 188, 625

Matthews, T.A. and Sandage, A.R. 1963. ApJ, 138, 30

McCarthy, 1993. ARA\&A, 31, 639

McCarthy, P.J., van Breugel, W.J.M., Spinrad, H. and Djorgovski, G. 1987. ApJ, 321, L29

McCarthy, P.J., van Breugel, W.J.M. and Kapahi, V.K. 1991. ApJ, 371, 478

Rawlings, S. and Saunders, R.E. 1991. Nature, 349, 138

Ryle, M. and Longair, M.S. 1967. MNRAS, 136, 123

Ryle, M. and Sandage, A.R. 1964. ApJ, 139, 419

Saikia, D.J. 1981. MNRAS, 197, 11P

Saikia, D.J. 1984. MNRAS, 209, 525

Scheuer, P.A.G. 1995. MNRAS, 277, 331

Schmidt, M. 1963. Nature, 197, 1040

Smith, H.E. and Spinrad, H. 1980. PASP, 92, 553 
The Hy Redshift Universe

ASP Conference Series, Vol. 193, 1999

A. J. Bunker \& W. J. M. van Breugel, eds.

\title{
Complete Samples of Radio Sources
}

\author{
P. J. McCarthy \\ Carnegie Observatories, 813 Sarta Barbara St., Pasadena, CA 91101
}

Abstract. I review the subject of radio galaxies at intermediate and large redshift with an emphasis on complete samples.

\section{Introduction}

I introduce the subject of radio galaxies at large and intermediate redshift from the point of view of complete samples. Others in this volume (e.g. Dey, van Breugel) will review in detail some of the many subtopics of that I will only touch on. I will attempt to frame my discussion in the context of complete samples of radio galaxies, particularly since this is an area in which the work of Hyron Spinrad has been of landmark importance. I will also include some new results concerning the properties of the host galaxies from recent NICMOS observations.

By the 1960s and 70s the assembly of complete samples of radio sources had become a successful enterprise, particularly once the impact of confusion was appreciated. Surveys of interest for active galaxy work need not reach very faint flux densities, $100 \mathrm{mJy}$ at $1400 \mathrm{MHz}$ is sufficiently deep for most studies. The precise and accurate positions available from radio interferometers makes optical/near-IR identification of the sources straight forward, although they remain telescope time intensive. The $3 \mathrm{CR}$ (and its more rigorously defined variant the $3 \mathrm{CRR}$ ) is the most widely observed complete radio source sample and nearly 40 years of effort were expended in assembling complete optical identifications and redshift determinations. (see Smith, Smith, \& Spinrad 1976; Spinrad et al. 1985, Djorgovski et al.1988; Rawlings et al. 1996; Longair this volume).

\section{Complete Samples of Radio Galaxies and Quasars}

Strong source samples carry significant biases with them and the $3 \mathrm{CRR}$ has a particularly bad case. In Figure 1 I plot the run of $408 \mathrm{MHz}$ radio luminosity with redshift for several samples, including the $3 \mathrm{CRR}$. The strong and artificial correlation between power and redshift seen in each of these samples simply reflects the broad and steep radio luminosity function and the bright flux density limit of the sample. The rather dramatic changes in many of the of properties of the $3 \mathrm{CRR}$ sources with redshift (e.g. radio lobe separation, clustering environment, emission-line strength, optical morphology) clearly called for significant effort to extend the range of flux densities over which large samples were selected. Several such efforts were undertaken in the 1980s and early 90 s and while these 
succeeded in breaking the radio power-redshift degeneracy, their impact on our understanding of the differences between the high and low redshift objects has been less than one might have hoped. This is due in part to the enormous effort required in assembling the large samples of high SNR, high resolution multiwavelength observations needed for such work (e.g. Eales et al. 1997) and to the exciting results arising from the application of new techniques to the $3 \mathrm{CRR}$ and other samples (e.g. radio spectral index selection, polarimetry, sub-mm, HST etc.).

In Figure 1 I include data from the 3CRR (Laing, Riley, \& Longair 1983) and the MRC/1Jy sample (McCarthy et al. 1996; Kapahi et al. 1998). The solid and dashed lines show the locus of points corresponding to the survey depths of the MG sample (Stern et al. 1999) and the 6C and 8C surveys being carried out by the Oxford group (Eales et al. 1997). These surveys go a long way towards filling in the P-Z plane. The $1400 \mathrm{MHz} \mathrm{mJy}$ and $\mu \mathrm{Jy}$ samples (e.g. LBDS; Windhorst this volume) extend the range of powers at high redshift by a further order of magnitude or more, but I do not include them as these samples contain a mix of radio loud $A G N$ (both FRI and FRII), radio quiet $A G N$, and star forming objects. Most radio galaxy samples are selected at low frequencies, some with a view towards minimizing orientation and beaming effects, others were chosen to sample the same rest-frame wavelengths as the $z=13 \mathrm{CR}$ sample. Spinrad's work on the MIT/Greenbank sample is the exception as it is selected at $5 \mathrm{GHz}$, although with a steep spectrum selection criterion.

One of the classical motivations for the identification of complete samples of radio sources was the angular-size redshift test for cosmological world models. Even the earliest work in this area revealed the substantial impact of evolution and most subsequent efforts on the $\mathrm{kpc}$ scale were devoted towards quantifying the effects of the evolution in source sizes. There has been a considerable range in the magnitude of both the reported evolution with redshift and the dependence on source power. Parameterized as power-laws in $1+z$ and $\mathrm{P}_{408 M H z}$, the reported exponents range from 1.5 to 2.5 for the redshift dependence and -0.2 to 0.5 for the power dependence. In Figure 2 I plot the lobe separations for radio galaxies from the $3 \mathrm{CRR}$ and $\mathrm{MRC} / 1 \mathrm{Jy}$ samples along with the expected median size for the best-fitting power-law dependencies for a flat cosmology with zero $\Lambda$. Our best fitting parameters, $(1+z)^{3.5} ; \mathrm{P}_{408}^{0.5}$, are similar to those derived by Kapahi (1989) and Oort et al. (1987) for the 3CRR and LBDS samples, and significantly steeper than those inferred by Neeser et al. (1995). The higher selection frequency of the MRC sample may introduce a disproportionate contribution from smaller sources at high redshifts when compared to the $178 \mathrm{MHz}$ and $151 \mathrm{MHz}$ samples.

The recent work by Blundell and collaborators (Blundell \& Rawlings 1999; and this volume) has substantially reoriented our thinking about source sizes and reformulated the redshift-size relation as a probe of source aging rather than evolution of the population as a whole. This is an important shift in riewpoint and changes the focus of the debate regarding source sizes from cosmology to radio source physics.

\subsection{Emission-line and optical/UV morphologies}

Radio galaxies with powers at or above the break in the radio luminosity function have rich emission-line spectra. This fact and the noisy but significant correla- 


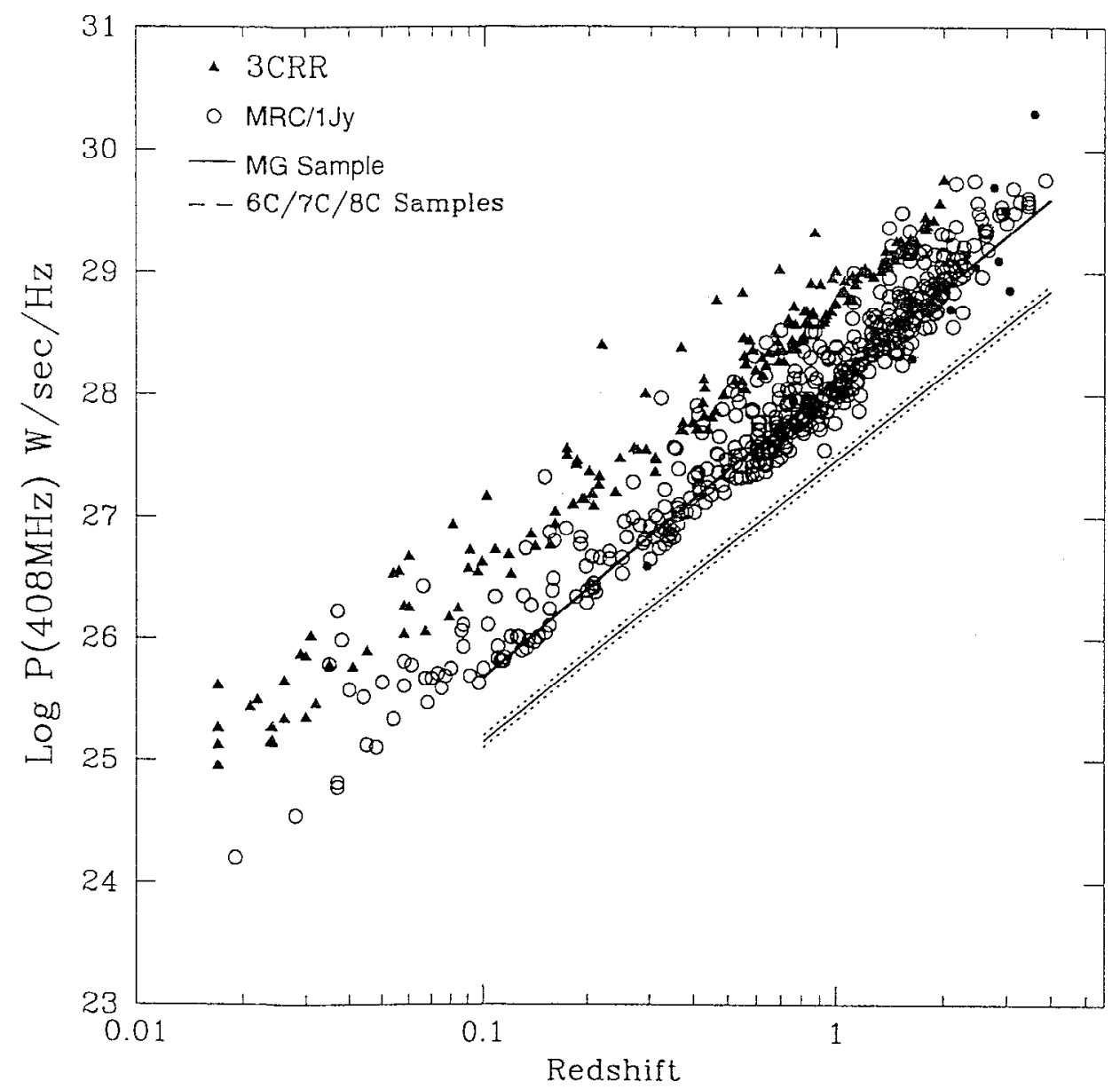

Figure 1. The run of $408 \mathrm{MHz}$ power with redshift for the $3 \mathrm{CRR}$ (triangles), MRC/1JY (open circles), MG (solid line) and 6C/8C samples (dashed lines). The curves for the MG and Cambridge samples are approximations based on their Hux density limits and a typical spectral index of -1 . 


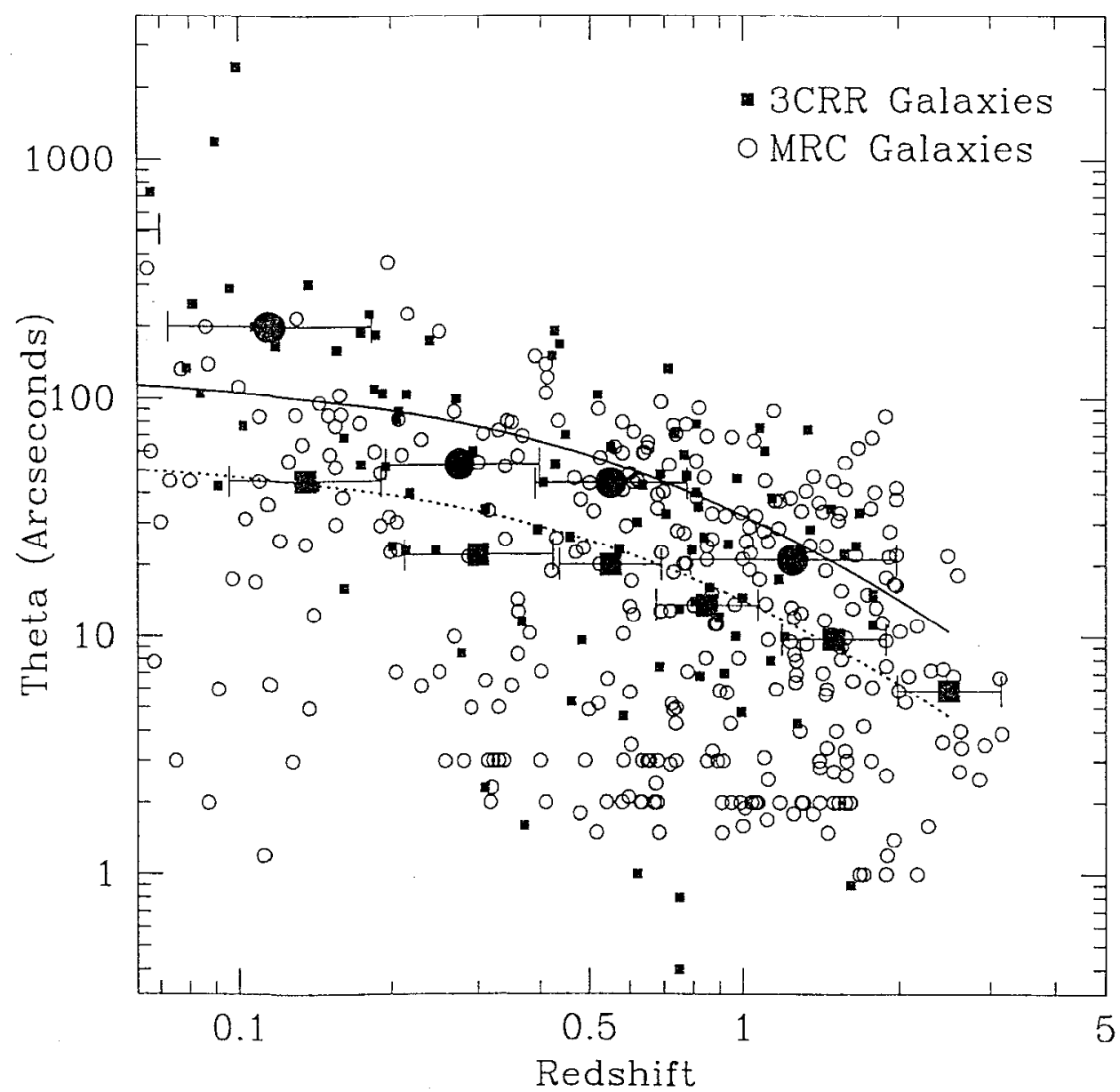

Figure 2. The angular sizes of 3CRR (filed squares) and MRC/1Jy radio galaxies (open circles). The median values in several redshift bin are shown as the large filled symbols. The curves are the expected angular sizes for the 3CRR and MRC/1.Jy flux density limits and a source whose sizes varies with redshift and power as $(1+z)^{-3.5} P_{408}^{0.5}$ for $q_{0}=0.5$. 
tion between radio power and emission-line luminosity is what made the study of radio galaxies at $z>1$ possible nearly two decades before similar work became feasible for ordinary field galaxies. There are both broad and narrow-lined radio galaxies, but nearly all have the strong forbidden lines of [OII], [OIII] and resonance lines of $\mathrm{CIV}$ and $\mathrm{H}$ Ly $\alpha$ that made redshift determinations possible event at faint levels. The narrow emission-line spectra of most $\approx>0.3$ radio galaxics have high ionization states and forbidden line ratios that are similar to those of the narrow lines in quasars, with the exception that transitions with low critical densities are more prominent in the radio galaxies.

Stern et al. (1999) have assembled a composite spectrum for the MITGreenbank sample and find evidence for a correlation between radio luminosity and ionization state, with the lower power MG sources having; on average, stronger emission from lower ionization stages of $\mathrm{C}$ when compared with the $3 \mathrm{CR}$. This is an interesting result, but perhaps more interesting is the remarkiable uniformity of the spectra among the different samples, when one considers that they have been selected over a wide range of frequencies, source sizes, core fractions etc.

The narrow line regions are spatially resolved in most cases and while some spectacular objects span more than $300 \mathrm{kpc}$, the typical extent is roughly 30 - $50 \mathrm{kpc}$. The earliest long-slit spectra revealed substantial velocity structures in the extended emission-lines and several examples of very large amplitude ( $1000-2000 \mathrm{~km} \mathrm{~s}{ }^{-1}$ ) were identified (e.g. Spinrad \& Djorgovski 1984). Baum, Spinrad, and I (McCarthy, Baum, and Spinrad 1996) have compiled the extra-nuclear velocity fields for a large fraction of the $3 \mathrm{CRR}$ sample and we find a typical amplitude at $z=1$ of $800 \mathrm{~km} \mathrm{~s}^{-1}$. At low redshifts Baum et al. (1992) find a correlation between the shape of the velocity field and the character of the emission-line spectra. Tadhunter et al. (1989) find simple symmetric velocity fields with amplitudes of $\sim 200-400 \mathrm{~km}^{-1}$, similar to the results for the high ionization objects observed by Baum et al. Baum and I (Baum \& McCarthy 1999) have looked at the systematics of the velocity fields for $0.3<z<3$ and find no clear trend with spectral properties. We see strong increases in both the line widths and amplitude of the resolved velocity fields with increasing redshift (and radio power; see section 2.0 ). We find a modest correlation between radio source size and the FWHM of the extra-nuclear emission (see Longair this volume). We note that the run of velocity amplitude with redshift shows a significant rise at $z=0.6$, with the median increasing from $\sim 300 \mathrm{~km} \mathrm{~s}^{-1}$, a value that is plausible for massive galaxy halos, to $800 \mathrm{~km} \mathrm{~s}^{-1}$, a value more appropriate for cluster potentials. The change in clustering environments at this redshift reported for both quasars and radio galaxies (e.g. Yates et al. 1991; Ellingson et al. 1991) support the idea of a change in the origin of the resolved velocity fields.

Perhaps the most dramatic change in the properties of radio galaxies between redshifts of $\sim 1$ and the present occurs in the observed-frame visible morphologies. The nature of the extended continuum and the continuum alignment effect is covered elsewhere in this volume (see e.g. Dey, Best).

\subsection{Near-IR Luminosities, Colors and Morphologies}

In contrast to the dramatic apparent evolution in the rest-frame UV and emissionline luminosities and morphologies, the near-IR is a domain of passive evolution 
and homogeneity. Our view of the rather sedate near-IR properties, however, may owe as much to the necessarily crude nature of the data as to reality itself. Out to redshifts of $1-1.5$ the near-IR colors and luminosities of the 3CRR galaxies evolve slowly and most of the apparent change in simply $\mathrm{K}$-correction (e.g. Dunlop et al. 1989). As emphasized most clearly by Lilly (Lilly \& Longair 1985; Lilly 1988) the $\mathrm{K}-\mathrm{z}$ relation for the $3 \mathrm{CRR}$ sample show a nearly constant dispersion with redshift and a degree of evolution (approximately 1 magnitude per unit redshift) consistent with simple expectations for passive evolution and a high formation redshift. Several $\mathrm{K}$-band imaging surveys from the ground have provided morphological and structural evidence for a smooth evolutionary path between distant radio galaxy hosts and present day ellipticals, the work of Best and collaborators providing the most thorough analysis (e.g. Best, Longair \& Röttgering 1998).

In Figure 3 I plot the $\mathrm{K}$-band Hubble diagram for the $3 \mathrm{CRR}, \mathrm{MRC} / 1 \mathrm{Jy}$, and $6 \mathrm{C}$ samples along with an incomplete sample of high redshift radio galaxies from van Breugel et al. (1997). I have removed a low order polynomial from the data to compress the vertical scale (this polynomial has no physical significance). The heavy open circles are the $3 \mathrm{CRR}$, the light circles are the MRC/1Jy data, the triangles the $6 \mathrm{C}$, squares are the van Breugel/NIRC sample. The filled circles and triangles are median values for the $3 \mathrm{CRR}$ and $6 \mathrm{C}$ samples, the diamonds are the MRC medians in various redshift bins, the error bars are $\pm 1 \sigma$. The first point to note is the good agreement between the 3CRR and MRC/1Jy samples. The difference between the $3 \mathrm{CRR}$ and $\mathrm{MRC}$ samples is in the range $0-0.2$ magnitudes and is less than $1 \sigma$ in each bin. The difference does appear to be systematic, with the MRC being fainter on average. The $6 \mathrm{C}$ sample shows the large, and increasing, departure from the 3CRR reported by Eales \& Rawlings (1996). At $z>1$ the $6 \mathrm{C}$ is $0.8-1.0$ magnitudes fainter than the 3CRR. The apparent discrepancy between the $6 \mathrm{C}$ and $\mathrm{MRC}$ samples is puzzling, and I can see no obvious reason why it should occur. At redshifts of roughly $1.2-1.6$ the MRC sample is significantly incomplete. Spectroscopic redshifts with CCDs is challenging in this redshift range, and it could well be that the faintest and reddest galaxies in this range have been preferentially lost from our sample of spectroscopic redshifts. At redshifts below one, I believe that the MRC is highly complete, and at $z \sim 0.5 \mathrm{I}$ am confident that we are nearly $100 \%$ complete. In this redshift range the $\mathrm{MRC}, 6 \mathrm{C}$, and $3 \mathrm{CRR}$ are all consistent to within $1 \sigma$. The small offset between the $3 \mathrm{CRR}$ and MRC could easily arise from the differences in detectors and techniques used for each, compounded with the increased fraction of extended continuum in the 3 CRR compared to weaker samples.

The solid line in Figure 3 is a minimal passive evolution Bruzual and Charlot (1993) model, the dotted line is the no-evolution $\mathrm{K}$-correction. The $6 \mathrm{C}$ points follow the no-evolution line while the MRC and 3CRR samples are consistent with passive evolution and constant stellar mass.

NICMOS offered the first opportunity for near-IR imaging with the resolution required to make definitive statements concerning the morphologies and structural parameters for the hosts at $z \sim 1$ and beyond. Zirm et al. (this volume) have imaged a sample of $3 \mathrm{CR}$ galaxies at redshifts near unity with camera 2 on NICMOS in the F160W, F165M, or F110W filters. These images reveal vastly simpler morphologies than those seen in the WFPC2 images that sam- 


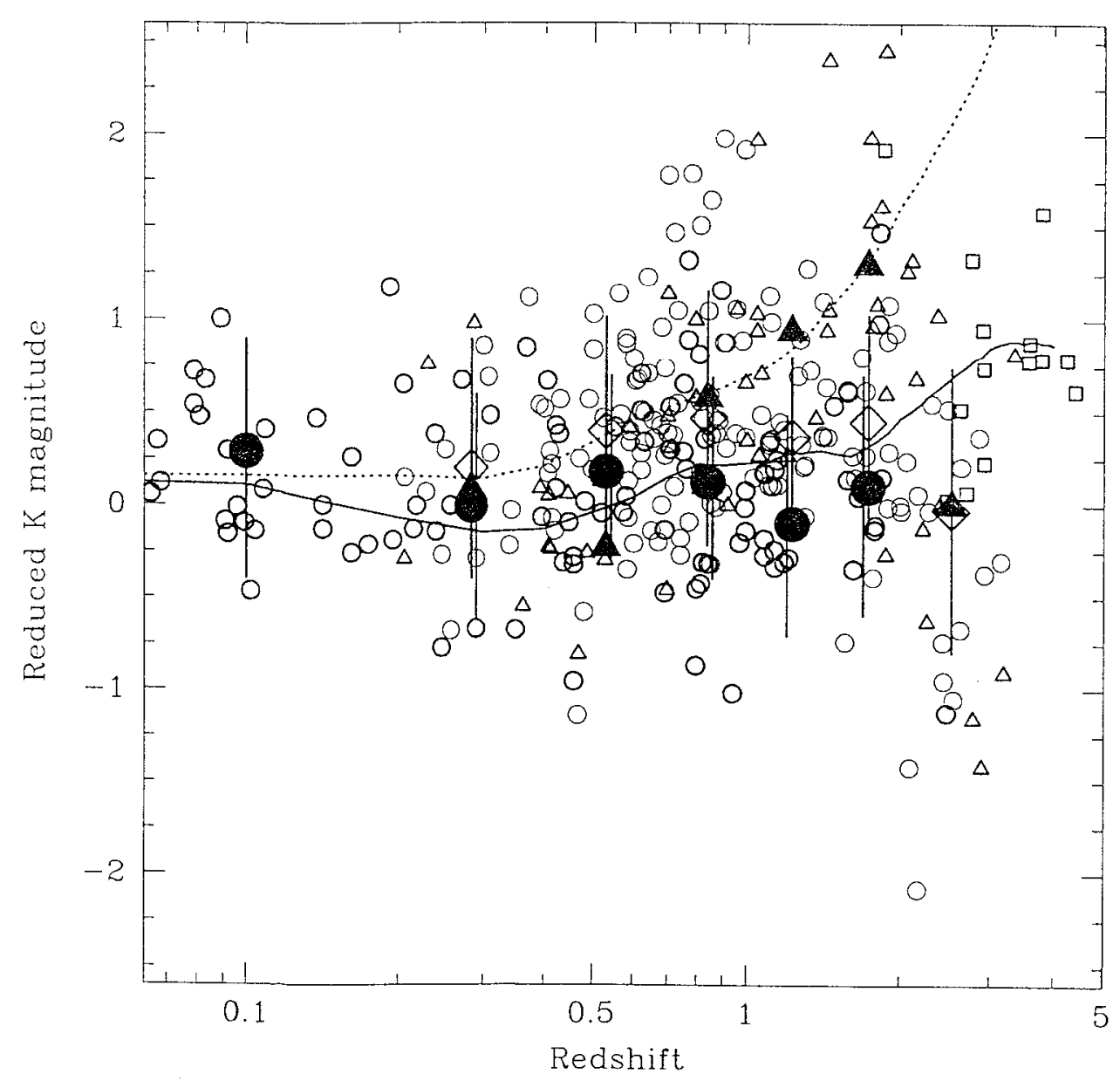

Figure 3. The K-band Hubble diagram for radio galaxies from the $3 \mathrm{CRR}, \mathrm{MRC} / 1$ - Jy surveys and a number of $z>3$ radio galaxies taken from the literature. The dotted line is a no-evolution model with $H_{0}=$ 50 and $q_{0}=0.1$; the solid line is a BC96 passive evolution model with $z_{f}=20$. A low order polynomial has be subtracted from both the data and the models to compress the scale. The heavy circles are the MRC, light circles $3 \mathrm{CRR}$, triangles $6 \mathrm{C}$ and squares are the van Breugel et al. NIRC sample. The large symbols are the medians. 
ple the continua at $\lambda<4000 \AA$. The azimuthally averaged profiles are smooth and are well represented by the $r^{1 / 4}$ law, with minor contributions from nuclear point sources. The scale lengths are smaller than those of present-day central cluster galaxies, and are more representative of $\mathrm{L}^{*}$ ellipticals (see Zirm et al., this volume, for details).

Pentericci and I (Pentericci et al. 1999) have imaged a sample of galaxies drawn from the MRC/1Jy and ESO ultra-steep spectrum (USS) samples with NICMOS. These sources have $1.7<z<3.1$ and cover the same range of radio powers as the $3 \mathrm{CR}$ sources imaged by Zirm et al. For the lower redshift end of our sample $(z<2.5)$ we are able to derive accurate surface brightness profiles and find good examples of $r^{1 / 4}$ laws for several objects. In figure 4 I show five examples. The scale lengths for four of these objects are quite small and they would be difficult to determine with significant accuracy without the spatial resolution offered by NICMOS on HST.

There are a few clear differences between the $z \sim 2$ MRC/USS and the $z \sim 1$ $3 \mathrm{CR}$ NICMOS samples. The MRC/1Jy and USS sources have a higher incidence of strong nuclear point sources ( $\sim 10 \%$ are completely unresolved). The $z>$ 2.5 objects have complex and asymmetric morphologies and clearly exhibit the alignment effect. The $z \sim 2$ NICMOS sample suffers from substantially more contamination from emission-lines than is the case for the $3 \mathrm{CR}$ sample. Even objects with negligible emission-line contributions, however, show the alignment effect (e.g. MRC 0943-242). The shorter rest-frame wavelengths clearly play a role in the changing morphologies, but there does appear to be a significant redshift-related change in the relative contribution of the aligned component. McCarthy (1999) shows that in the case of MRC 0943-242 $(z=2.93)$ the aligned component contributes $80 \%$ of the flux in the F160W band and an estimated a $75 \%$ contribution at $\mathrm{K}$. This large aligned contribution at $\mathrm{K}$ suggests that some of these objects should have significant levels of near-IR linear polarization, and this has been demonstrated by Knopp \& Chambers (1997) in a small sample of objects.

The strong nuclear point sources seen in the NICMOS images of the MRC and USS sources suggest a increasing contribution from non-stellar light in the $\mathrm{K}-\mathrm{z}$ relation at large redshifts. What is unclear from the NICMOS images alone is the relative contribution from the classic narrow-lined region (via [OIII $] 5007,4959)$ and true nuclear continuum to these point sources. Spectroscopy offers a means of quantifying the various contributions to the near-IR luminosity. Recent observations with ISAAC, the near-IR spectrometer on the VLT by di Serego-Alighieri et al. reveal broad $\mathrm{H} \alpha$ emission in a sample of three $z \sim 2.5$ radio galaxies. Two of these are in the Pentericci et al. NICMOS sample and these have strong point sources. At this meeting Larkin et al. also report the detection of broad $\mathrm{H} \alpha$ in one of the these sources (MRC 2025-218) based on early observations with NIRSPEC on the Keck $10 \mathrm{~m}$ telescope. The spectra of all three objects observed with ISAAC show strong narrow [OIII]5007,4959 emission-lines in the $\mathrm{H}$-band with line ratios that are similar to those compiled by McCarthy (1994) for the composite 3CR radio galaxy spectrum. I suspect that the point sources seen in the NICMOS images arise from direct views of the nuclear continuum in sources with modest obscuration along the line of sight. Spectroscopy of large samples with the new generation of near-IR spectrometers 

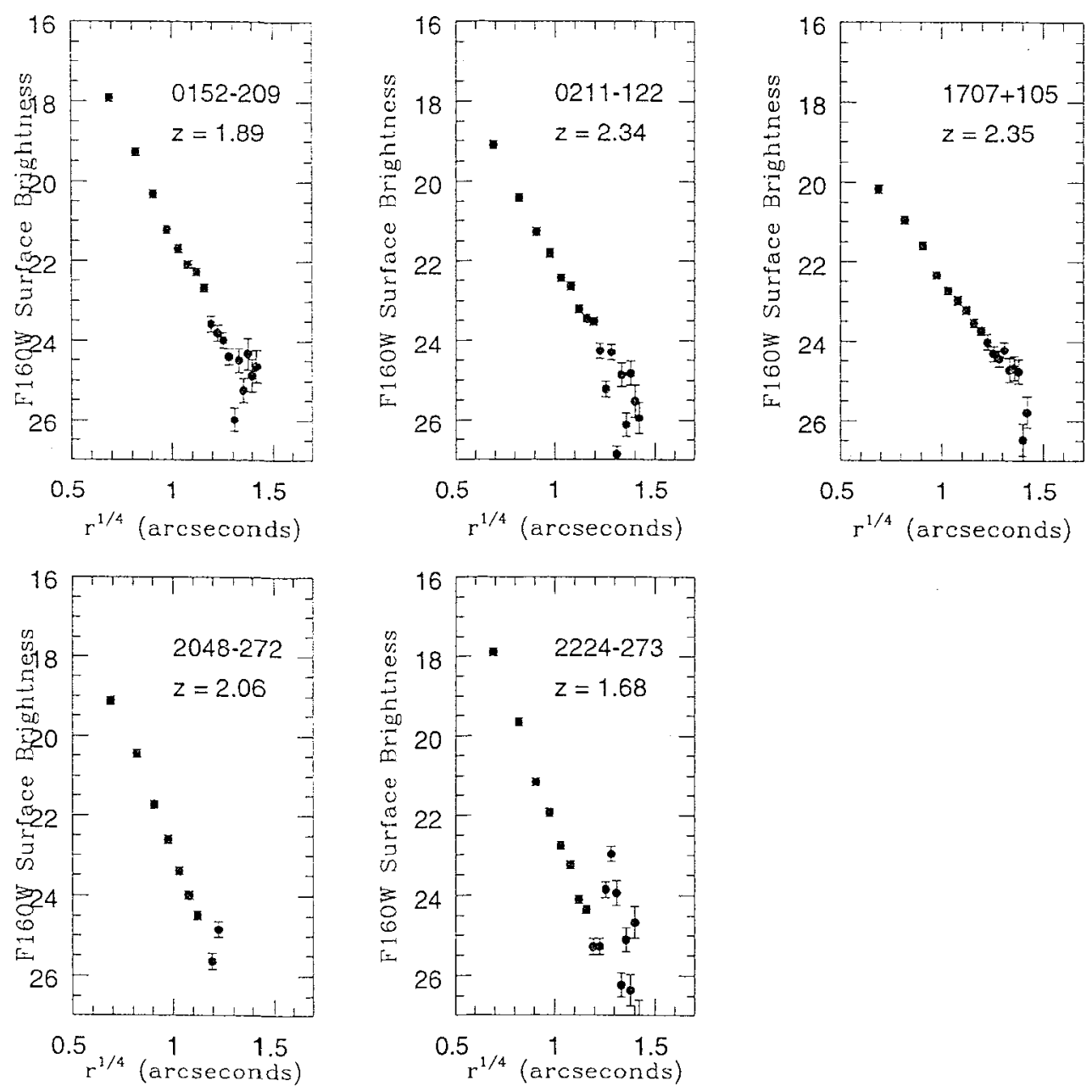

Figure 4. Surface brightness profiles for five MRC and USS galaxies with $z \sim 2$. These hosts have simple near-IR morphologies and their azimuthally averaged profiles are well represented by an $r^{1 / 4}$ law with a range of scale lengths. Details can be found in Pentericci et al. 1999. 
offers us the opportminity to examine the near-IR lines and continua of radio with the level of scrutiny previously restricted to the optical and rest-frame UV.

Acknowledgments. I thank Laura Pentericci for permission to publish Figure 4 and my European and Caltech collaborators (Fosbury; di Serego-Alighieri, Cimatti. M. Cohen, Villar-Martin) for permission to review the recent results from ISAAC.

\section{References}

di Serego-Alighieri et al. in preparation

Baum, S. A., \& McCarthy, P. in preparation

Baum, S. A., Heckman, T., \& van Breugel, W. 1992, ApJ, 389, 208

Best, P., Longair, M., \& Röttgering, H. 1998, MNRAS, 295, 549

Blundell, K. \& Rawlings, S. 1999, Nature, 399, 330

Bruzual, G. A., Charlot, S. 1993, ApJ, 405, 538

Djorgovski, S., Spinrad, H., McCarthy, P., Dickinson, M. van Breugel, W., Strom, R. 1988, AJ, 96, 836

Dunlop, J., Guiderdoni, B., Rocca-Volmerange, B., Peacock, J., \& Longair, M. 1989, MNRAS, 240, 257

Eales, S. A., \& Rawlings, S. 1996, ApJ, 460, 68

Eales, S., Rawlings, S., Law-Green, D., Gotter, G., Lacy, M. 1997, MNRAS, 291,593

Ellingson, E., Green, R., \& Yee, H. 1991, ApJ 371, 49

Kapahi, V. K. 1989, AJ, 97, 1

Kapahi, V., Athreya, R., van Breugel, W., McCarthy, P., Subrahmanya, C. 1998, ApJS, 118, 275

Kinopp, G., \& Chambers, K. C., 1997, ApJ, 487, 644

Larkin et al. These proceedings

Laing, R. A., Riley, J., \& Tongair, M. 1983, MNRAS, 204, 151

Lilly, S. J., \& Longair, M. 1984, MNRAS, 211, 833

Lilly, S. J. 1988, ApJ, 333, 161

McCarthy, P. J., Baum, S. A., \& Spinrad, H. 1996, ApJS, 106, 281

McCarthy, P., Fapahi, V., van Breugel, W., Athreya, R., Persson, S., Subrahmanya, C 1996, ApJS, 107, 19

McCarthy, P. 1999, "The Most Distant Radio Galaxies", Royal Netherlands Academy of Arts and Sciences, H. Röttgering, P. Best, \& M. Tehnert, eds., p5.

McCarthy, P. 1993, AR AA, 31, 639

Neeser, M., Eales, S., Law-Green, J. Leahy, P., Rawlings, S. 1995, ApJ, 451,-76

Oort, M. J. A., Katgert, P., Windhorst, R. A. 1987, Nature, 328, 500

Pentericci, L., McCarthy, P., Röttgering, H., Miley, G., Fosbury, R., van Breugel W. 1999, ApJ, submitted

Rawlings, S., et al. 1996, MNRAS, 279, 13 
Smith, H., Smith, E.. \& Spinrad, H. 1976, 88, 621

Spinrad, H. et al. 1985, PASP, 97, 932

Spinrad, H., \& Djorgorski, S., 1984. ApJ, 285, L49

Stern, D., Dey, A.. Spinrad, H., Maxfield, L., Dickinson, M. Schlegel, D., Gonzalez, R. 1999, AJ, 117, 1122

Tadhunter, C. N., Fosbury, R. A. E., \& Quinn, P. J., 1989, MNRAS, 240, 225

van Breugel, W., Stanford, S., Spinrad, H., Stern, D., Graham, J. 1998, ApJ, 502. 641

Yates, M., Miller, L., \& Peacock. J. 1991, MNRAS, 240, 129 
The Hy Redshift Universe

ASP Conference Series. Vol. 193,1999

A. J. Bunker \& W. J. M. van Breugel, eds.

\title{
The Early Evolution of Radio Galaxy Hosts
}

\author{
Arjun Dey \\ National Optical Astronomy Observalories. 950 N. Cherry Ave., \\ Tucson, AZ 85719
}

\begin{abstract}
High-redshift radio galaxies provide laboratories for studying the formation of massive galaxies. I briefly review recent observations which are beginning to unveil the complex processes at work during the early evolutionary stages of these systems.
\end{abstract}

\section{Introduction}

Until recently, the host galaxies of powerful radio sources reigned supreme in the study of distant galaxies: due to their large UV and optical continuum and emission line luminosities, these sources were relatively easy to detect and study at high redshift. ${ }^{1}$ Although we are now able to directly study more 'normal' galaxies (i.e., systems with continuum and line luminosities not dominated by active nuclei) at high redshift, radio galaxies remain of fundamental importance to studies of galaxy formation and evolution. This is primarily because radio galaxy hosts are the best candidates for the progenitors of present-day massive galaxies and represent strongly biased peaks in the matter distribution, an hypothesis supported at low redshift by the association of powerful radio sources with $g E$ and $\mathrm{CD}$ galaxies (Matthews et al. 1964), at intermediate and high redshifts by the tendency for these sources to reside in moderately rich cluster environments (e.g., Hill \& Lilly 1991, Dickinson 1997), and by a few direct kinematic measurements of the masses of high-redshift powerful radio galaxies (e.g., 3C265: Dey \& Spinrad 1996). In this contribution, I will briefly review the state of the observational data on high-redshift powerful radio galaxies (hereafter HzRGs) and their relevance to our understanding of the evolution of these systems.

\section{2. $\approx \sim 1$ Radio Galaxy Hosts as Elliptical Galaxies}

The $K-z$ Hubble diagram of powerful radio galaxy hosts shows a remarkably uniform sequence which is well modelled by the gradual aging of a simple stellar

\footnotetext{
'One must not interpret this to mean that the pioneering optical spectroscopic observations of distant radio galaxies were trivial: although the host galaxies are brighter than their less active peers at a given redshift, their spectroscopy still tested the limits of 1970's telescope, instrument and detector technology. It is almost eritirely due to the infections persistence (sometimes seemingly in the face of all reason!) "of Hyron ("Bulldog") Spinrad that we now have redshifts for all but one of the $3 \mathrm{CR}$ sources.
} 

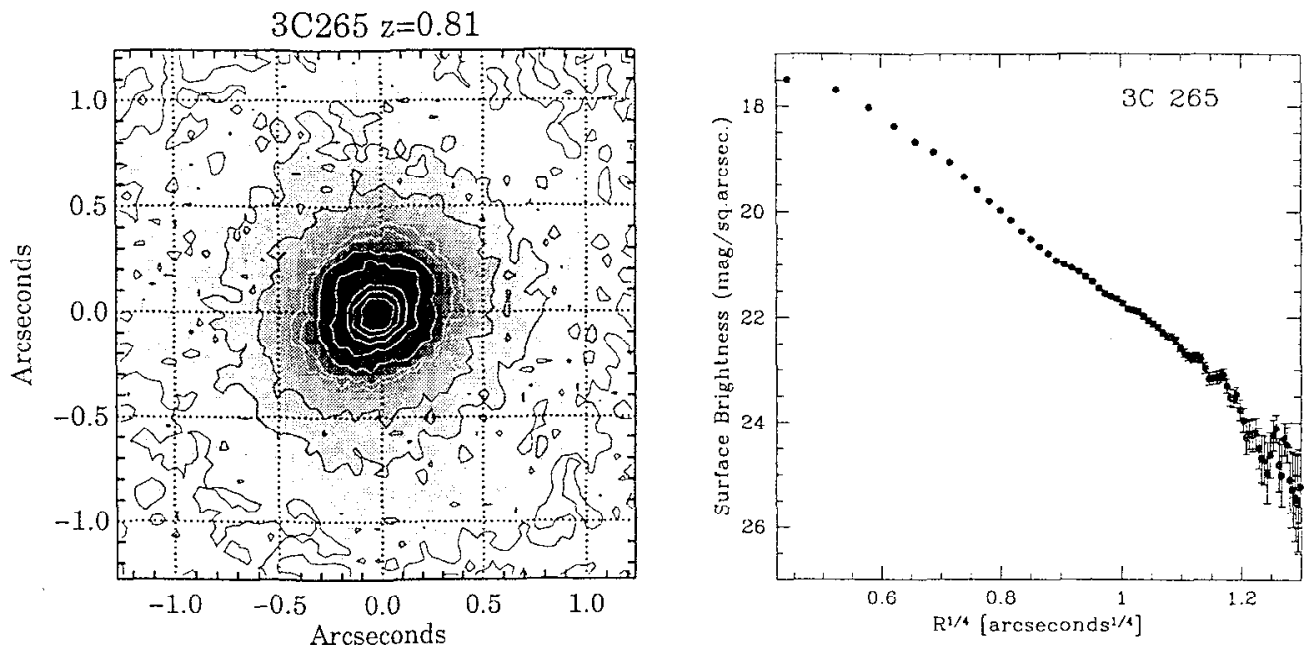

Figure 1. Some $z \sim 1$ radio galaxy hosts resemble dynamically relaxed elliptical galaxies when viewed at near-infrared wavelengths, as exemplified in the left panel by a HST NICMOS F160W image of the $z=0.811 \mathrm{HzRG} 3 \mathrm{C} 265$. The right panel shows the radial surface brightness profile of the galaxy (Zirm et al. 2000).

population formed at high redshift (e.g., Lilly \& Longair 1984, McCarthy 1993 and references therein, van Breugel et al. 1998). This has long been taken as evidence that the host galaxies are similar to elliptical galaxies. Indeed, recent $1.6 \mu \mathrm{m}$ images (which sample the rest-frame red optical light in the $z \sim 1 \mathrm{HzRGs}$ ) obtained using NICMOS on HST reveal elliptical morphologies and photometric properties more or less similar to those expected for massive ellipticals (see figure 1 and Zirm ct al. 2000, in these proceedings; see also Best, Longair \& Röttgering 1998, Rigler et al. 1992 who came to similar conclusions based on ground-based $K$-band observations).

Since the near-infrared emission is generally argued to be dominated by starlight with a negligible contribution from AGN related processes, the symmetric $H$ and $K$ band morphologies observed in the $z \sim 1 \mathrm{HzRG}$ are interpreted as cvidence that the stellar components in these systems are dynamically relaxed and possibly spectrally old. There is little direct spectroscopic evidence to support these speculations in the most radio loud HzRGs (i.e., the $z \gtrsim 13 \mathrm{CRs}$ ), since near-infrared spectroscopy has been difficult and optical spectroscopy has largely revealed that the rest-frame UV continuum emission in the $z \sim 13 \mathrm{CR}$ is dominated by AGN related processes which veil the underlying stellar population.

Nevertheless, stellar features have been detected in a few sources (e.g., Dey 1995, Dey \& Spinrad 1996, Stockton et al. 1995). Although these features are often representative of few-Gyr-old populations, the veiling of the stellar continuum by the AGN light makes it extremely difficult to estimate ages for the 


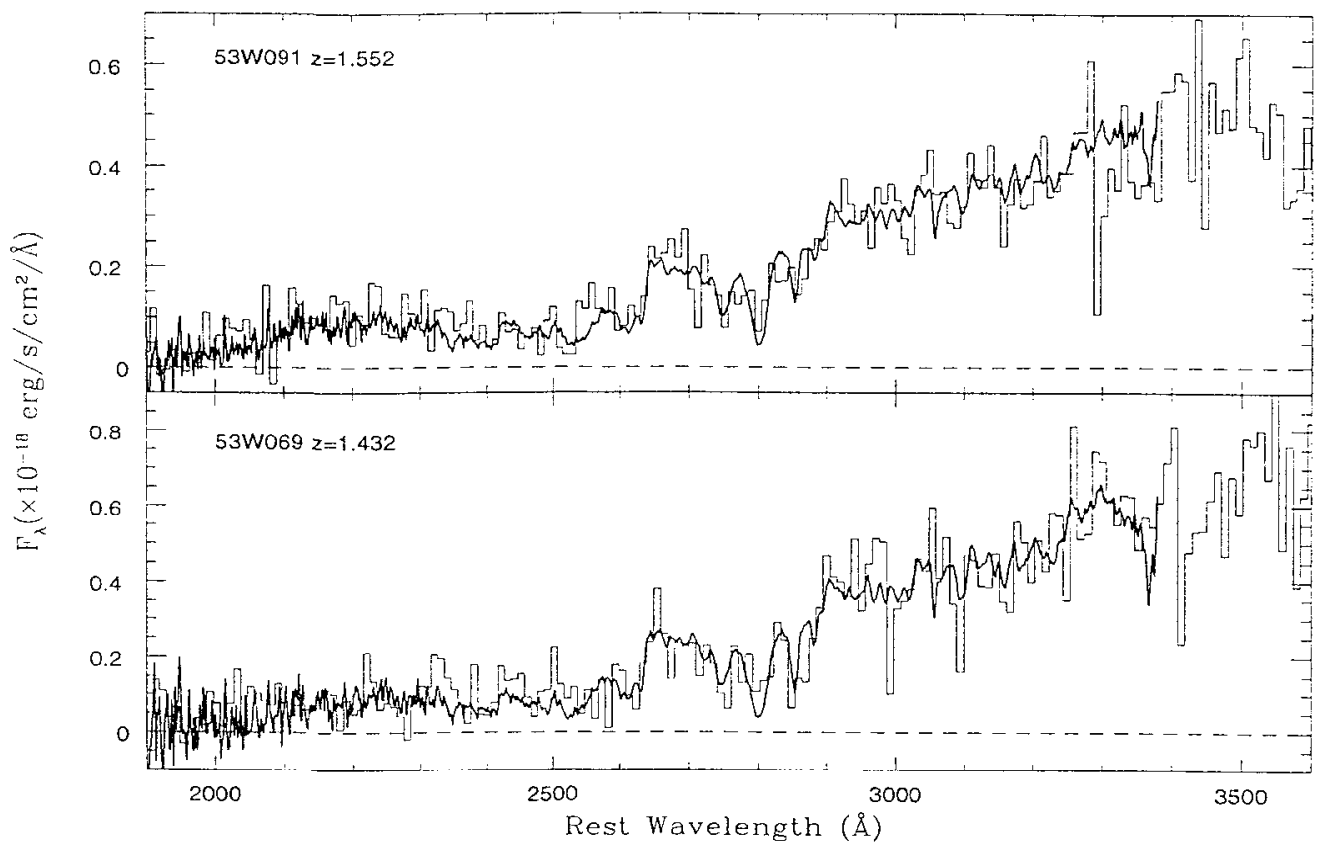

Figure 2. Spectra of LBDS 53W091 and LBDS 53W069 overplotted with the IUE spectra of late F-type main sequence stars. Since the UV light in simple stellar populations is dominated by the turn-off population, direct spectral typing suggests that these galaxies have minimum ages of around 3 Gyr (Dunlop et al. 1995, Spinrad et al. 1996, Dey et al. 2000).

$z \sim 13$ CRs. However, lower power radio sources at these redshifts can have much smaller fractional contributions of AGN light to the UV continuum: by observing two of the reddest galaxies in a sample of weak radio sources (from the Leiden-Berkeley Deep Survey), Spinrad and collaborators have demonstrated that the host galaxies of LBDS 53W069 and LBDS 53 W091 $(z \sim 1.5)$ have UV spectra similar to that of late-type F stars (Dunlop et al. 1996, Spinrad et al. 1997, Dey et al. 2000; see figure 2). Since the UV continuum emission in intermediate age simple stellar populations is dominated by the turn-off population, these spectra suggest that these galaxies have ages of at least 3 Gyr. More detailed spectral synthesis age estimates, albeit more model dependent, confirm large ages for these systems (see Dunlop's contribution in these proceedings).

The upshot of the near-infrared morphologies, $K-z$ Hubble diagram and spectral synthesis ages of $z \gtrsim 1$ radio galaxies is that these systems are both dynamically and spectrally old. Given that these are massive galaxies, these observations appear to be consistent with the simple hypothesis that the host galaxies and their stellar populations are formed in a single monolithic collapse at high redshift and consequently evolve passively. This is a speculation that bears scrutiny: the dynamical state of the galaxy reflects its merger / assembly history, which may be different from the tale told by the spectral aging of its stellar population (e.g., Kauffmann \& Charlot 1998). The advent of efficient near-infrared spectrographs on large aperture telescopes will allow direct measurements of the velocity dispersion and mean age of the stellar component in 
HzRGs, even in the more luminous systems. These observations should allow us in principle to separate the dynamic and star-formation histories of HzRGs.

\section{The Aligned UV Continuum Emission in $\approx \gtrsim 1$ Radio Galaxies}

Their near-IR morphologies notwithstanding, most optical images of the $z>$ 1 HzRGs, instead of revealing elliptical hosts, show spectacular morphologies that are spatially extended over several tens of kiloparsecs and roughly aligned with the major axis of the non-thermal radio emission (McCarthy et al. 1987, Chambers et al. 1987). These unusual alignments elicited much debate during the last decade: their existence argued for an intimate connection between the AGN and the rest-frame UV emission, and submerged the hapless radio galaxy proponents in an embarrassing morass of inexplicability. We now know that a significant fraction of the observed optical (rest-frame UV) emission from $z \sim$ $1-2$ radio galaxies is non-stellar, and dominated by scattered and reprocessed light from the active nucleus. Several imaging- and spectro-polarimetric studies (Tadhunter et al. 1988; di Serego Alighieri et al. 1989, Jannuzi \& Elston 1991, Jannuzi et al. 1995, Dey et al. 1996, Cimatti et al. 1996, 1997, Tran et al. 1998, Vernet et al. 2000) have now convincingly demonstrated that the extended, aligned UV continuum emission in most $z \sim 1-2 \mathrm{HzRGs}$ is strongly polarized, with the electric vector oriented perpendicular to the major axis of the $\mathrm{CV}$ emission. These observations provide strong evidence that much of the UV light is anisotropically radiated emission from the AGN scattered into our line of sight by dust and electrons in the ambient medium.

Not every $z \sim 1$ radio galaxy is strongly polarized. Although our observations of 3C356 (shown in the right panel of figure 3; Cimatti et al. 1997) are fairly representative of the bulk of the $\approx \sim 1-2$ radio galaxies, the aligned radio galaxy $3 \mathrm{C} 368$ at $z=1.132$ remains a notable exception (left panel of figure $3 \&$ table 1). However, even in the latter case, the extended UV continuum emission (at rest wavelengths $\lambda 2000-3500 \AA$ ) is largely accounted for by optically thin Balmer continuum emission from the line-emitting gas (Stockton et al. 1996) powered by the AGN.

There still appears to be some debate about the nature of the hidden nuclear source that is responsible (at least partially) for the UV continuum and the ionization of the gas. The acolytes of the AGN Unification Church would predict that the unseen source is a 'normal' quasar nucleus, with a spectrum similar to that observed directly in stee-spectrum, radio-loud quasars. In a few HzRGs (e.g., 3C265 - Dey \& Spinrad 1996. Tran et al. 1998; 3C324 - Cimatti et al. 1996; 3C441 - Dey 1999; 3C277.1 - Tran et al. 1998; MRC2025-218 Larkin et al. 1999) there is some evidence that the hidden nuclear source has quasar-like broad emission lines. Nevertheless, the total number of $z \gtrsim 1$ radio galaxies for which high quality data exist is small and hence concluding that all powerful radio galaxies contain quasars hidden from direct view is premature. Near-infrared spectroscopy with the new generation of large telescopes will undoubtedly provide better constraints on the spectrum of the nuclear source.

At higher redshifts $(z>3)$, the data are even more sparse: polarimetry exist for only two $z>3$ powerful radio galaxies as of this writing, and both of these are unpolarized (see table). Instead, the spectra of these galaxies show strong 

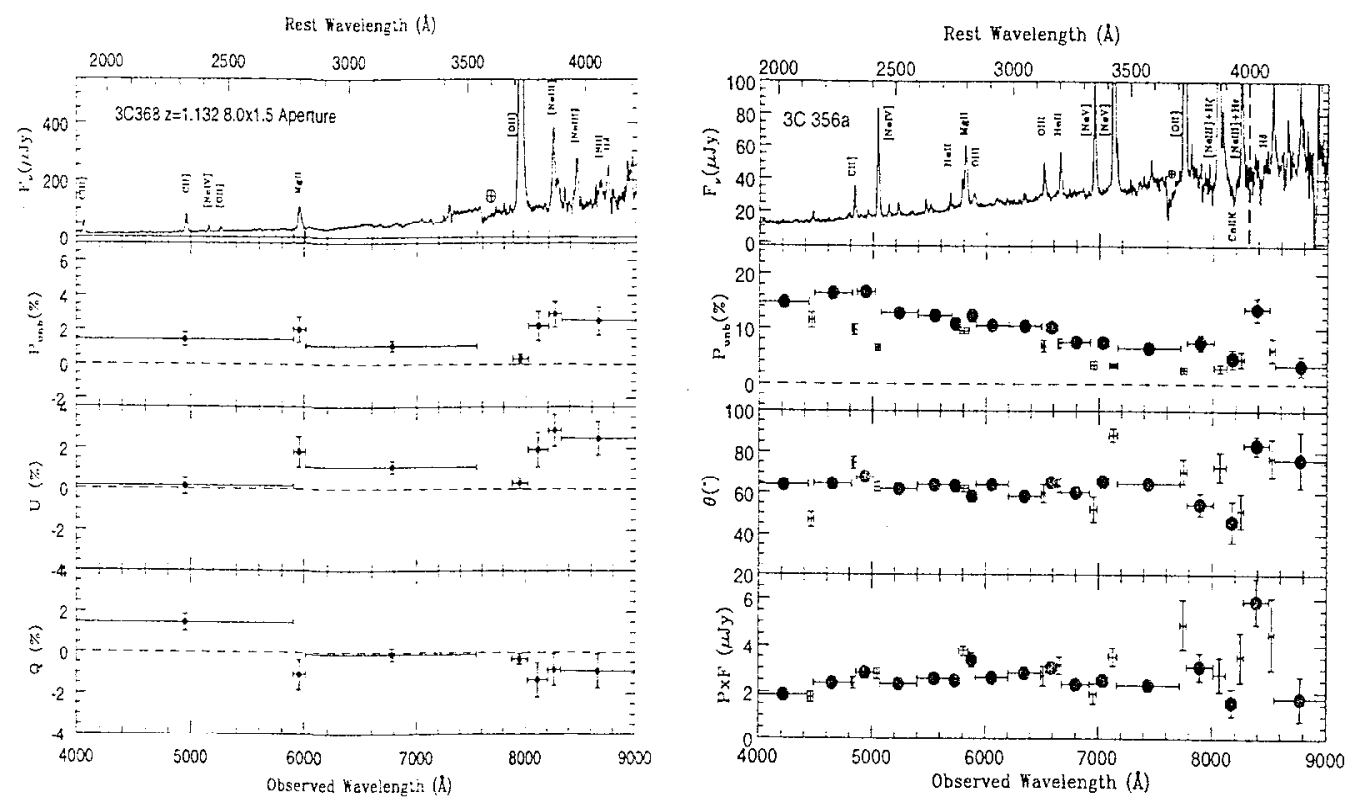

Figure 3. Most, but not all, powerful radio galaxies at $z \sim 1$ have strongly polarized UV continuum emission. 3C368 at $z=1.132$ (left panel) is a notable exception $(P<3 \%)$. The majority of sources, however, are more similar to $3 \mathrm{C} 356$ at $z=1.079$ (right panel; Cimatti et al. 1997), which exhibits large fractional polarization $(P \gtrsim 10 \%)$ and a flat polarized flux spectrum $(P \times F)$. Note the CaII $\mathrm{K}$ stellar absorption and the polarization of the $\mathrm{MgII} \lambda 2800$ emission line.

resonance absorption features typically observed in nearby starburst galaxies ( $\$ 3)$. At least in the case of 4 C41.17 $(z=3.8$; Chambers et al. 1990), the lack of polarization, the absorption lines, and the inadequacy of alternative emission processes (e.g., Balmer continuum emission) to account for the observed extended UV continum emission together imply that starlight dominates the UV light from this system (Dey et al. 1998). The case of $6 \mathrm{C} 1909+722$ is not as clear cut: although continum polarization has not yet been detected, the spectrum shows strong, broad UV resonance absorption lines similar to that observed in BAL QSOs (Dey 1997).

Lable 1 summarizes the current state of $\mathrm{HzRG}$ polarization observations. There are a few more polarization measurements of $z \sim 2.5$ radio galaxies reported by Vernet et al, in these proceedings. Although it is far too premature to draw strong conclusions from the tabulated results, there seems to be a possible decrease in the fractional polarization at redshifts $z \gtrsim 3$. There are only two sources measured at these redshifts, and we clearly need more observations.

\section{Observing the Formation of Radio Galaxies}

The advent of large aperture telescopes has rendered it feasible to study very high redshift radio galaxies in remarkable detail. The Keck Telescopes have 


\begin{tabular}{|c|c|c|c|c|}
\hline Name & $\overline{\overline{\text { Redshift }}}$ & $\begin{array}{c}\text { Rest } \\
\text { Wavelength }\end{array}$ & $\begin{array}{c}\% \\
\text { Polzn }\end{array}$ & References \\
\hline $3 \mathrm{C} 441$ & 0.706 & $2430-2770 \AA$ & $8 \%$ & $\overline{6}$ \\
\hline $3 \mathrm{C} 343.1$ & 0.750 & $2857-4343 \AA$ & $<2.2 \%$ & 13 \\
\hline $3 \mathrm{C} 277.2$ & 0.766 & $2325-2893 \AA$ & $29 \%$ & 7,13 \\
\hline $3 \mathrm{C} 265$ & 0.811 & $2200-2500 \AA$ & $14 \%$ & 10,13 \\
\hline $3 \mathrm{C} 226$ & 0.818 & $2000-2700 \AA$ & $13 \%$ & 8 \\
\hline $3 \mathrm{C} 356 \mathrm{a}$ & 1.079 & $1930-2310 \AA$ & $14 \%$ & 2 \\
\hline $3 \mathrm{C} 368$ & 1.132 & $1880-2350 \AA$ & $<1 \%$ & 6 \\
\hline $3 \mathrm{C} 324$ & 1.206 & $1915-2120 \AA$ & $12 \%$ & 1 \\
\hline $3 \mathrm{C}_{13}$ & 1.351 & $1701-2300 \AA$ & $7 \%$ & 2 \\
\hline $3 \mathrm{C} 256$ & 1.819 & $1420-1540 \AA$ & $10 \%$ & 4,11 \\
\hline TX0211-122 & 2.338 & $1950-2650 \AA$ & $15 \%$ & 9 \\
\hline $4 \mathrm{C} 00.54$ & 2.37 & $1250-1530 \AA$ & $12 \%$ & 3 \\
\hline $4 \mathrm{C} 23.56 \mathrm{a}$ & 2.419 & $1250-1520 \AA$ & $15 \%$ & 3 \\
\hline TX0830+191 & 2.572 & $1550-2140 \AA$ & $13 \%$ & 12 \\
\hline $6 \mathrm{C} 1909+722$ & 3.534 & $1270-1520 \AA$ & $<5 \%$ & 6 \\
\hline $1 \mathrm{C} 11.17$ & 3.800 & $1220-1540 \AA$ & $<2.4 \%$ & 5 \\
\hline
\end{tabular}

Fey to references: [1] Cimatti et al. (1996); [2] Cimatti et al. (1997); [3] Cimatti et al. (1998); [4] Dey et al. (1996); [5] Dey et al. (1997); [6] Dey et al. (1998); [7] di Serego Alighieri et al. (1989); [8] di Serego Alighieri (1997); [9] Fosbury et al. (1999) [10] Jannuzi \& Elston (1991); [11] Jannuzi et al. (1995); [12] Knopp \& Chambers (199i); [13] Tran et al. (1998)

already provided us with direct detections of starlight in these systems, as well as the first glimpse of the processes that may accompany the formation of these luminous systems.

There is now much circumstantial evidence that suggests that the $z \sim 4$ radio galaxy hosts are young. Spectral features typically seen in the spectra of hot, young stars have been observed in at least one $z \sim 4 \mathrm{HzRG}$ (4C 41.17; Dey et al. 1997). In addition, several HzRGs show large, spatially extended Ly $\alpha$ haloes that extend over several tens of kpc (van Ojik et al. 1997, Dey 1997). In the case of $4 \mathrm{C} 41.17$ at $z=3.8$, the halo was detected over the entire spatial extent of a $22^{\prime \prime}$ spectroscopic slit, implying a total extent larger than $200 h_{50}^{-1} \mathrm{kpc}$ (figure 4)! The origin and excitation of these large gaseous haloes is unknown, but it is possible that these reflect the large gas reservoirs from which these massive galaxies form. It is of great interest that Steidel et al. (1999) have recently discovered similar haloes (officially labelled 'Blobs' by Steidel et al.) apparently not associated with AGN, but instead loosely associated with more normal star forming galaxies at $z \approx 3$ in the SSA22 field: this is a phenomenon not restricted to the formation of $A G N$, but perhaps of massive galaxies in general.

Yet another manifestation of the youthfulness of $z \gtrsim 3$ radio galaxies is the presence of outflows. Ontflow phenomena have now been observed in nearly all $z>2$ radio galaxies for which high-resolution, high signal-to-noise ratio data exist. $3 \mathrm{C} 256$ at $z=1.8$ shows an absorption feature in its spectrum, most likely 

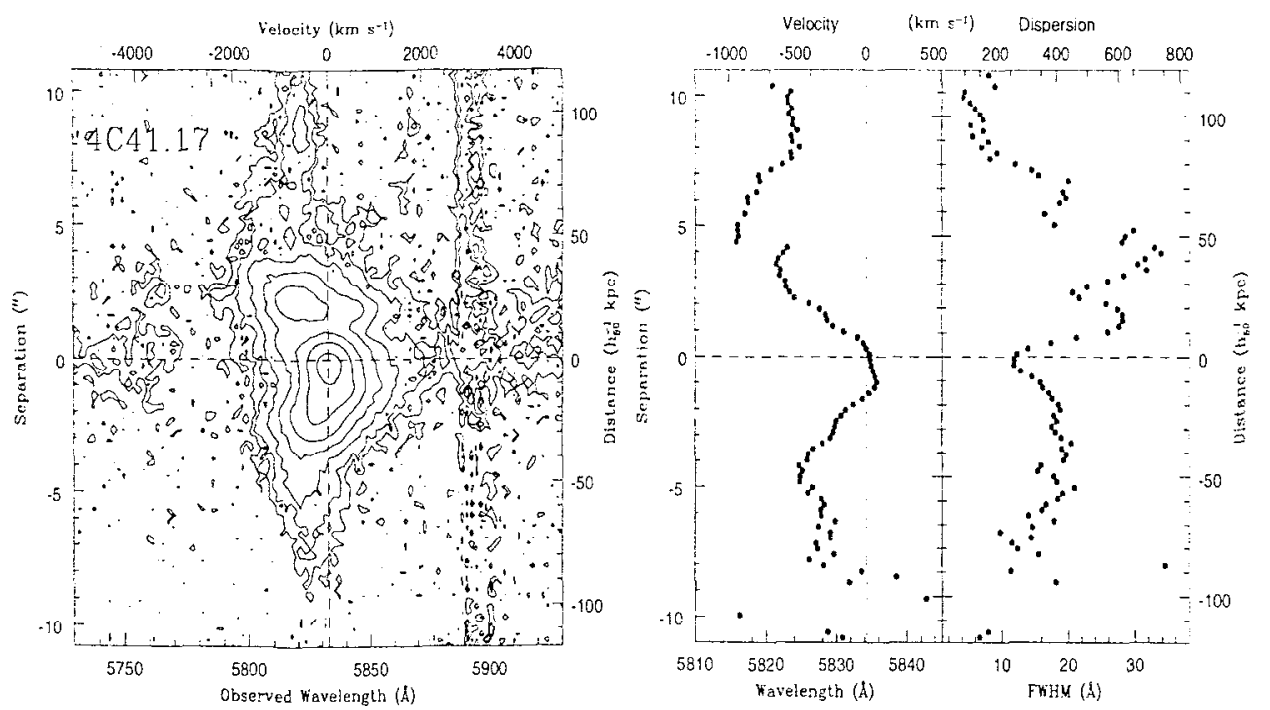

Figure 4. The Ly $\alpha$ halo of $4 \mathrm{C} 41.17$, a $z=3.8$ powerful radio galaxy, extends over more than $200 h_{50}^{-1} \mathrm{kpc}$ and shows possible uniform velocity structure suggestive of solid body rotation in the outermost regions. If taken at face value, the 'rotation' suggests a halo mass of $\sim 2 \times 10^{12} M_{\odot}$. The Ly $\alpha$ absorption on the blue-ward side of the line appears to extend over at least $70 h_{50}^{-1} \mathrm{kpc}$, and undoubtedly modifies the spatial and kinematic morphology of the Lya emission in the central regions.

CIV $\lambda 1550$ absorption arising in gas moving at a velocity of $14300 \mathrm{~km} \mathrm{~s}^{-1}$ relative to the radio galaxy (Dey et al. 1996). Absorbing gas associated with lower velocity outflows has now been observed in several HzRGs, almost all of which show asymmetric Ly $\alpha$ profiles suggesting the presence of blue-shifted absorbing gas which appears to be spatially extended over the entire emission line region (figure 4; see also van Ojik et al. 1997). In the cases for which the data exist, the outflows extend over tens of $\mathrm{kpc}$, and exhibit equivalent widths of $\sim 10 \AA$ and FWHM $\gtrsim 2000 \mathrm{~km} \mathrm{~s}^{-1}$. In many cases, $\mathrm{P}$ Cygni - like features are observed in both low- and high-ionization lines suggesting that the outflowing material has a wide range of ionization, as would be expected for a galaxian-scale outflow.

Evidence for outflows is also observed in the more normal (i.e., non-AGN) $z \sim 3$ star-forming galaxies. For example, 0000-263 D6, a 'normal' (i.e., nonAGN) star-forming galaxy at $z=2.96$, also shows P-Cygni like features associated with both the low-ionization and high-ionization lines, perhaps suggesting the presence of a galaxian wind (e.g., Spinrad et al. 1998). If the outflow phenomena are indeed ubiquitous, could they be a fundamental part of the AGN / galaxy formation process? A telling analogy may be made to the low mass star-formation process, where large-scale outflows are thought to provide a. mechanism for angular momentum loss, which is necessary to enable accretion. 


\section{An Evolutionary Tale}

Although sparse, the current results are consistent with a straw-man model in which radio galaxies form the bulk of their stars at high redshift and evolve passively thereafter. A speculative scenario for the evohtion of the host galaxies of powerful radio sources mat read something like this: At redshifts $z>5$, the accumulation of material in a deep potential well results in the formation of the first stars and the active nucleus. As the collapse progresses, the expanding over-pressured cocoon powered by the jets from the AGN trigger star formation in a clumpy medium along an axis roughly consistent with the jet axis. By redshifts of around $\sim 4$, the UV continuum luminosity of the system is dominated by the hot young stars, and the line emission is likely due to a combination of photoionization by the active nucleus and the hot stars as well as shock ionization due to the expanding radio lobes. This rapid star-forming phase is accompanied by rapid dust production, presumably in the ejecta of the SNe TI, and large galaxian scale outflows driven by stellar winds and SNe. Almost all the starformation is complete by $z \sim 3$. and the galaxy's stellar population begins to age 'passively'. By redshifts of $z \sim 1$, the stellar component is now a dynamically relaxed, few-Gyr old population. The stellar contribution to the UV continumm is therefore minimal: the turn-off population at these ages has little UV flux, and in powerful $A G N$, the UV starlight is completely swamped by the scattered light from the AGN shining off the debris from the galaxy formation process. Finally, by the modern era, the galaxian continuum luminosity is almost completely dominated by the aged stellar population.

Although speculative, this tale is roughly consistent with the observations. The rapid star-formation phase at early times would result in the UV continuum emission in high redshift $(z>3)$ radio galaxies being dominated by the (unpolarized) light from young stars, swamping any scattered light from the AGN. As the stellar population ages, it reddens rapidly and the stellar contribution to the rest frame UV emission drops precipitously, allowing the scattered light to become the dominant contributor to the UV continuum emission. The attraction of this hypothesis, at least for me, is that it explains the (possible) change in the fractional polarization with redshift and is consistent with the observed uniformity of the $K-z$ Hubble diagram and the general morphological evolution between the $z \gtrsim 3$ and $z \sim 1$ radio galaxies. In addition, it may also explain the recent sub-mm continum detections of thermal emission from warm dust in HzRGs (e.g., Hughes et al. 1998, Ivison et al. 1998). Nevertheless, in order for it to be much more than a tale, it requires the scrupulous introduction of fact. Dedicated spectroscopic and spectropolarimetric studies of HzRGs, coupled with deep high-spatial resolution imaging investigations will hopefully begin to add to our understanding and not simply confuse us further. Near-infrared spectroscopic and polarimetric observations will be crucial in this regard, since they provide us with the only direct method of studying the bulk of the stellar mass and deconstructing the AGN contribution in these systems. Given the population explosion in 8-m class telescopes, we will undoubtedly have much to disciss: when we'get together to celebrate Hy's 70 th!

Acknowledgments. I am grateful to my collaborators Hy Spinrad, Wil vain we Breugel, Andrew Zirm, Bill Vacca, Daniel Stern, Ski Antonucci, Mark Dickinson, 
Andrea Cimatti. Andrew Bunker for many stimulating discussions on the subject of radio galaxies and massive galaxy evolution. and for their large latitude in letting me present data prior to publication. In particular, I am extremely grateful to $\mathrm{Hy}$ for the many enjoyable nights spent observing these 'faint crittcrs, and for tcaching me the invaluable lesson of blindly attempting impossible observations and hoping one would get lucky. I am forever amazed that this approach pays off (at least for $\mathrm{H}_{\mathrm{y}}$ )! I thank the Local Organizing Committee and the University of California for an excellent meeting and for providing me the partial financial support that enabled me to attend this meeting. I thank NASA for their generous financial support through the Hubble Fellowship Program and grant XASA HF-01089.01-97A.

\section{References}

Best, P. N., Longair, M. S., \& Röttgering, H. J. A. 1997, MNRAS, 292, 758

Best, P. N., Longair, M. S., Röttgering, H. J. A. 1998, MNRAS, 295, 549

Chambers, K.C., Miley, G.K. \& van Breugel, W. 1987, Nature, 329, 604.

Chambers, K. C., Miley, G. K. \& van Breugel, W. J. M. 1990, ApJ, 363, 21

Cimatti, A., Dey, A., van Breugel, W., Antonucci, R. \& Spinrad, H. 1996, ApJ, 465,145 .

Cimatti, A., Dey, A., van Breugel, W., Hurt, T. \& Antonucci, R. 1997, ApJ, 476,677

Cimatti, A., di Serego Alighieri, S., Vernet, J., Cohen, M., Fosbury, R. A. E. 1998, ApJ, 499, L21

Dey, A. 1997, in 'Mass Ejection from Active Galactic Nuclei', ed. N. Arav, I. Shlosman, \& R. J. Weymann, ASP Conf. Ser. 128, 35

Dey, A. 1999, in 'The Most Distant Radio Galaxies', Proceedings of the Royal Netherlands Academy of Arts \& Sciences Colloquium, ed. XXXXXXX

Dey, A. \& Spinrad, H. 1996, ApJ, 459, 133

Dey, A., Cimatti, A., van Breugel, W., Antonucci, R. \& Spinrad, H. 1996, ApJ, 465,157

Dey, A., van Brcugcl, W., Vacca, W. D. \& Antonucci, R. ApJ, 490, 698

Dey, A. et al. 2000, in preparation

Dickinson, M. E. 1997, in 'HSI \& the High Redshift Universe', Proceedings of the $37^{t} h$ Herstmonceux Conference, eds. N.R. Tanvir, A. Aragon-Salamanca, \& J. V. Wall (World Scientific: Singapore) p.207

di Serego Alighieri, S. 1997, in 'The Early Universe with the VLT', Proceedings of the ESO Workshop at Garching, ed. J. Bergeron (Springer: Berlin) p.304

di Serego Alighieri, S., Binette, I., Conrvoisier, T. J.-L., Fosbury, R. A. E., \& Tadhunter, C. N. 198s, Nature, 334, 591.

di Serego Alighieri, S., Fosbury, R. A. E., Quinn, P. J. \& Tadhunter, C. N. 1989 , Nature, 341, 307 .

Dunlop, J. S. 1998, in these proceedings

Dunlop, J. S., Peacock, J., Spinrad, H., Dey, A., Jimenez, R., Stern; D. \&

Windhorst, R. 1996, Nature, 381, 13 
Fosbury, R. A. E., Vernet, J., Villar-Martin, M., Cohen, M. H., Cimatti, A., di Serego Alighieri, S. \& McCarthy, P. J. 1999, in 'NICMOS and the VLT: A New Era of High Resolution Near Infrared Imaging and Spectroscopy', ed. W. Freudling \& R. Hook, ESO Conference Proceedings v. 55, 190

Hill, G. J. \& Lilly, S. J. 1991, Ap.T, 367, 1

Hughes, D. H., Dunlop, J. S. \& Rawlings, S. 1997, MNRAS, 289, 766

Ivison, R. J. et al. 1998, ApJ, 494, 211

Jannuzi, B. T. \& Elston, R. 1991, ApJ, 366, L69.

Jannuzi, B. T., Elston, R., Schmidt, G., Smith, P., \& Stockman, H. 1995, ApJ, 454, L111.

Kauffmann, G. \& Charlot, S. 1998, MNRAS, 294, 705

Kinopp, G. P. \& Chambers, K. C. 1997, ApJ, 487, 644

Larkin, J. et al. 1999 , in these proceedings

Lilly, S. J. \& Longair, M. S. 1984, MNRAS, 209, 401

Matthews, T. A., Morgan, W. W. \& Schmidt, M. 1964, ApJ, 140, 35

McCarthy, P.J. 1993, ARA\&A, 31, 639

McCarthy, P.J., van Breugel, W.J.M., Spinrad, H. \& Djorgovshi, S. 1987, ApJ, 321, L29

Rigler, M. A., Stockton, A., Lilly, S. J., Hammer, F., \& LeFevre, O. 1992, ApJ, 385,61

Spinrad, H., Dey, A., Stern, D., Dunlop, J., Peacock, J., Jimenez, R. \& Windhorst, R. 1997, ApJ, 484, 581

Steidel, C. C., Adelberger, K. L., Shapley, A. E., Pettini, M., Dickinson, M. \& Giavalisco, M. 1999, ApJ, in press (astro-ph/9910144)

Stockton, A., Kellogg, M. \& Ridgway, S. E. 1995, ApJ, 443, L69

Stockton, A., Ridgway, S. \& Kellogg, M. 1996, A.I, 112, 902

Tadhunter C. N., Fosbury, R. A. E., \& di Serego Alighieri, S. 1988, in Proc. of the Como Confercnce, BL Lac Objects, ed. L. Maraschi, T. Maccacaro \& M. H. Ulrich (Berlin: Springer-Verlag), 79.

Tran, H.D., Cohen, M.H., Ogle, P.M., Goodrich, R.W., \& di Serego Alighieri, S. 1998, ApJ, 500, 660

van Breugel, W. J. M., Stanford, S. A., Spinrad, H., Stern, D., \& Graham, J. R. 1998, ApJ, 502, 614

van Ojik, R., Röttgering, H.. J. A., Miley, G. K. \& Hunstead, R. W. 1997, A\&A, 317,358

Vernet, J. et al. . 2000, in these proceedings

Zirm, A. et al. 2000, in these proceedings 
The Hy Redshift Universe

ASP Conference Series, Vol. 193, 1999

A. J. Bunker \& W. J. M. van Breugel, eds.

\section{Ultra-Steep Spectrum Radio Galaxies at Hy Redshifts}

Wil van Breugel, Carlos De Breuck ${ }^{1} \&$ Adam Stanford ${ }^{2}$

Inst. of Geophysics 89 Plartetary Physics, LLNL, Livermore, CA 94550

Huub Röttgering \& George Miley

Leiden Observatory, Leiden, The Netherlands

Daniel Stern ${ }^{3}$

Astronomy Department, University of California, Berkeley, CA 94720

Dante Minniti

P. Universidad Catolica, Santiago 22, Chile

Chris Carilli

National Radio Astronomy Observatory, VLA, Soccoro, NM 87801

Abstract. Radio sources have traditionally provided convenient beacons for probing the early Universe. Hy Spinrad was among the first of the tenacious breed of observers who would attempt to obtain optical identifications and spectra of the faintest possible 'radio galaxies' to investigate the formation and evolution of galaxies at hy redshift. Modern telescopes and instruments have made these tasks much simpler, althongh not easy, and here we summarize the current status of our hunts for hy redshift radio galaxies (HyZRGs) using radio spectral and near-IR selection.

\section{Hy Z Radio Galaxies: Why?}

The first optical identifications of (bright) radio sources with (faint) galaxies were made when Hy Spinrad was still a teenager (Windhorst 1999; these proceedings). After that it was soon realized that the 'invisible' universe of radio sources provided convenient beacons to locate very distant galaxies and thus might be used to study their formation and evolution. As so eloquently described by

\footnotetext{
${ }^{1}$ Presently at: Leiden Observatory, Leiden, The Netherlands

${ }^{2}$ University of California at Davis, CA 95616 .

${ }^{3}$ Presently at: JPL, Pasadena, CA 91109
} 
several of Hy's colleagues, collaborators and ex-students in these proceedings, he became an early key player in these distant galaxy hunts.

For most radio astronomers in those days the Universe stopped at the POSS limits. Surely, many radio sources could not be identified, but so what? It just confirmed that the Universe was bigger than the biggest optical telescope, but ... not bigger than the biggest radio telescope! Occasionally $\mathrm{Hy}$ would write letters to Leiden Observatory radio astronomers with requests of radio maps and accurate positions. When provided he would spend many hours using one of the world's then finest telescopes at Lick Observatory, to obtain optical identifications and spectra of these HyZRG candidates (presumably squeezed in between observations of standard stars for Jim Liebert; these proceedings). How foolish this seemed, to some of us (WvB).

Since then extraordinary progress in the development of optical and nearIR detectors, larger telescopes, and better selection techniques have resulted in discoveries of radio galaxies at increasingly hyer redshifts. Paradoxically this task was eased by the discovery, first by Hy and his collaborators, that the Lyo emission line was very strong in radio galaxies and could easily be detected in $<1 \mathrm{hr}$ integrations with $3 \mathrm{~m}$-class telescopes (Spinrad et al. 1985), provided that the redshifts would be hy enough $(z>1.6)$ so that Ly $\alpha$ would enter the observable optical window.

It has now become clear that HyZRGs are both a boon and a curse for students of galaxy evolution. A boon because the near-IR Hubble $K-z$ relation for radio sources appears well represented by the predicted 'passive' evolution of massive $\left(5-10 \mathrm{~L}_{\star}\right)$ galaxies with hy formation redshifts (Lilly \& Longair 1984; Eales et al. 1997; Best et al. 1998; van Breugel et al. 1999), despite the effects of $\mathrm{k}$-correction and morphological evolution (van Breugel et al. 1998). No matter the reason for this relationship, it suggests that radio sources might be used to find massive galaxies and their likely progenitors out to very hy redshift. This method was first successfully used by Lilly (1988) to identify the HyZRG B2 0902+34 at $z=3.395$. Deep spectroscopic observations of a few relatively weak radio sources (but still of the powerful double FRII class !), where the AGN do not affect the rest-frame UV as much (see below), have shown directly examples of radio galaxies at $z \sim 1.5$ with old ( $\gtrsim 3.5-4.5 \mathrm{Gyr}$ ) stellar populations with implied formation redshifts $z_{f}>10$ (e.g., Spinrad et al. 1997).

For galaxy formation studies HyZRGs are also cursed because their structures are aligned with their associated radio sources, suggesting that the collimated outflow and ionizing radiation from their $A G N$ profoundly affect their parent galaxy host appearances at UV, blue and green wavelengths (McCarthy 1999, and Dey 1999; these proceedings). HyZRGs are very well suited for studying the effects of powerful AGN on ambient dense gas, including induced star formation (e.g., Bicknell et al. 1999), and may even be used as searchlights to investigate the properties of proto-galactic material in the early Universe (Cimatti et al. 1997; Villar-Martín et al. 1997).

Recent cosmological theories are providing additional incentives to use radio galaxies as probes to study the early Universe. Within standard Cold Dark Matter scenarios the formation of galaxies is a hierarchical and biased process: Large galaxies are thought to grow through the merging of smaller systems;" 


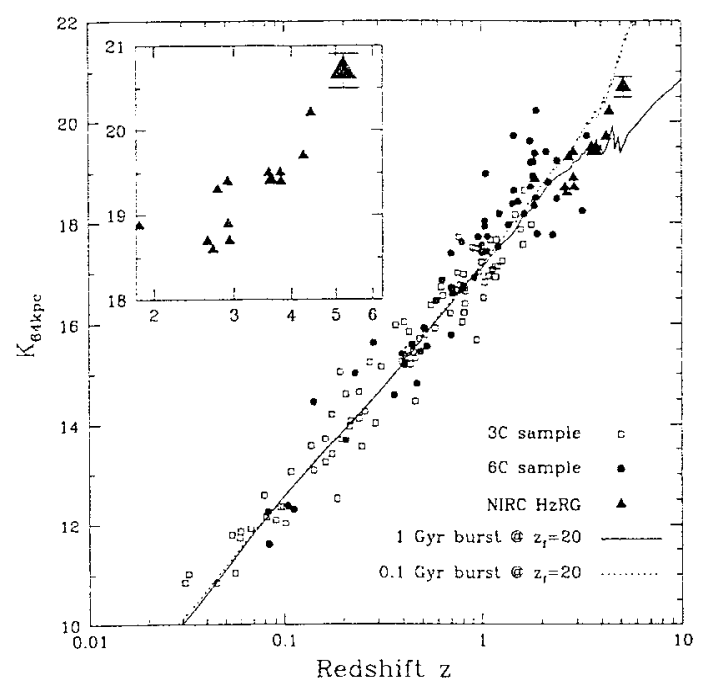

Figure 1. The Hubble $H_{-z} z$ diagram for HyZRGs. Filled triangles are Keck measurements of HzRGs from van Breugel et al. (1998), the large triangle is TN J0924-2201 at $z=5.19$, and all other photometry is from Eales et a]. (1997). Two stellar evolution models from Bruzual \& Charlot (1999), normalized at $z<0.1$, are plotted, assuming parameters as shown.

and the most massive objects form in over-dense regions, which will eventually evolve into the clusters of galaxies seen today (e.g., White 1997). It has also been suggested that the first massive black holes may grow in similar hierarchical fashion together with their parent galaxies (e.g., Kauffmann and Haehnelt 1999) or, because of time scale constraints, may precede galaxy formation and be primordial (e.g., Loeb 1993). To confront these theories it is therefore of great interest to find the progenitors of the most massive galaxies and their AGN (active massive black holes) at the hyest possible redshifts and to study their properties and cosmological evolution.

While optical, 'color-dropout' techniques have been successfully used to find large numbers of 'normal' young galaxies (without dominant AGN) at redshifts even surpassing those of quasars and radio galaxies (Weymann et al. 1998; $\mathrm{Hu}$ et al. 1999), the radio and near-infrared selection technique has the additional advantage that it is unbiased with respect to the amount of dust extinction. HyZRGs are therefore also important laboratories for studying the large amounts of dust (e.g., Ivison et al. 1998) and molecular gas (Papadopoulos et al. 1999). which are observed to accompany the formation of the first forming massive galaxies. Indeed, a significant part of the scientific rationale for building future large $\mathrm{mm}$-arrays is based on the expectation that to understand galaxy formation will ultimately require understanding of their cold gas and dusty environments. 
Table $1 . \quad$ Radio Surveys

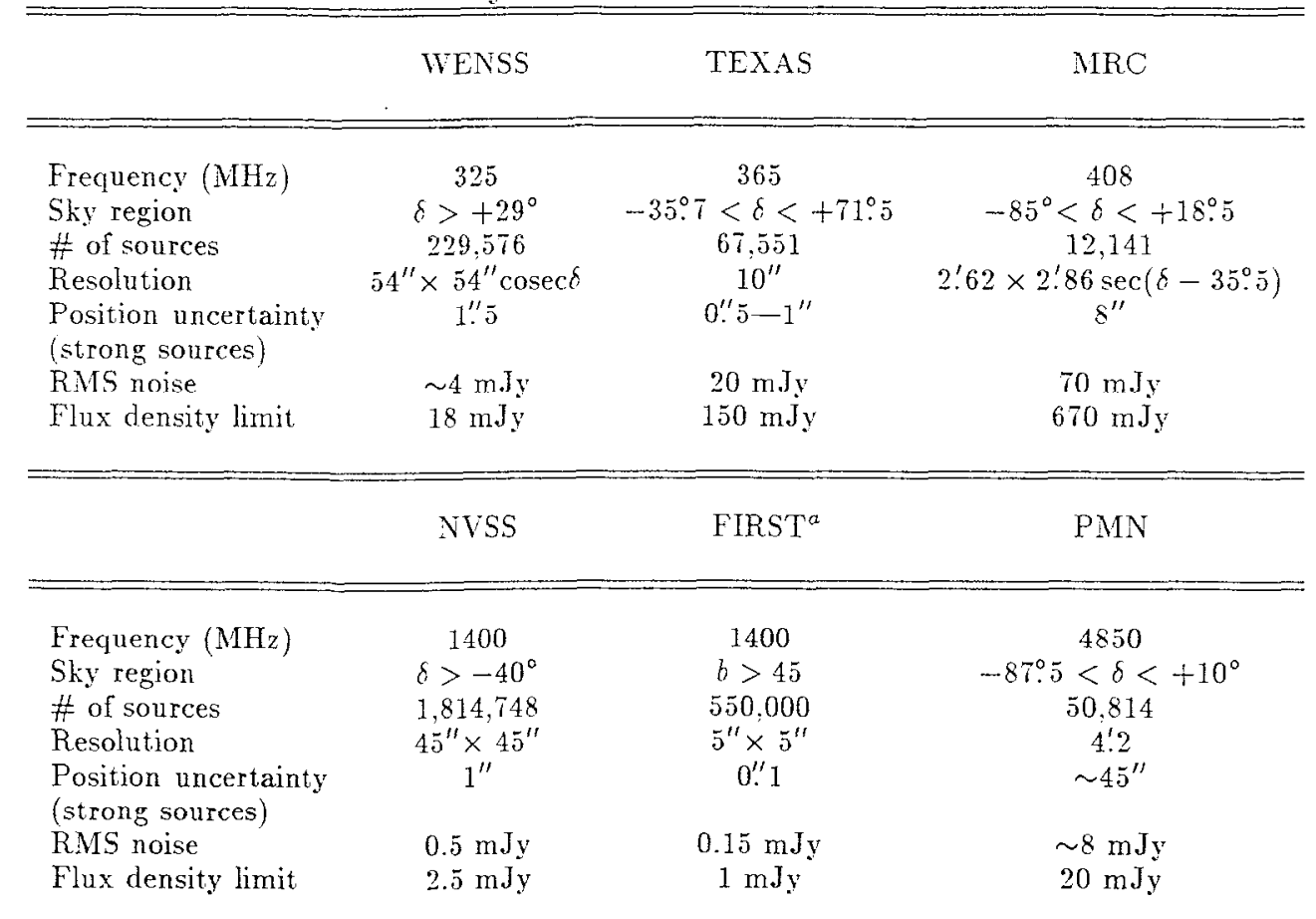

${ }^{a}$ Still in progress.

Finally, it has been claimed that the (co-moving) space densities of the most powerful radio galaxies and quasars were much hyer near $\tilde{z} \sim 2$, but that they drop off precipitously at even hyer redshifts (Dunlop and Peacock 1990; Shaver et al. 1996). However, using recently completed studies of moderately faint radio galaxies Jarvis et al. (1999; these proceedings) have argued that here is no such evidence for a redshift cut-off and that these previous results have been biased due to unknown radio $k$-correction (radio spectral index) trends and associated selection effects.

For all these reasons HyZRGs have become very useful probes of the early Universe. Unfortunately in complete, fux-limited samples the vast ma.jority of the sources will be relatively nearby, or at only modest hy redshifts. However, by employing the well-known 'red radio color' selection technique and choosing sources with ultra-steep radio spectra one can bypass most of the 'local' radio source population and efficiently identify radio galaxies at extremely hy redshift.

\section{The Ultimate Ultra-Steep Spectrum Source Sample}

With the advent of several new, large radio surveys (Table 1 ) such as the $.325 \mathrm{MHz}$ WENSS (Rengelink et al. 1997), the $365 \mathrm{MHz}$ TEXAS (Douglas et al. 1996), the $1.4 \mathrm{GHz}$ NVSS (Condon et al. 1998), the $1.4 \mathrm{GHz}$ FIRST (Becker et al. 1995), MRC $408 \mathrm{MHz}$ (Large et al. 1981) and the $4.85 \mathrm{GHz}$ PMN 


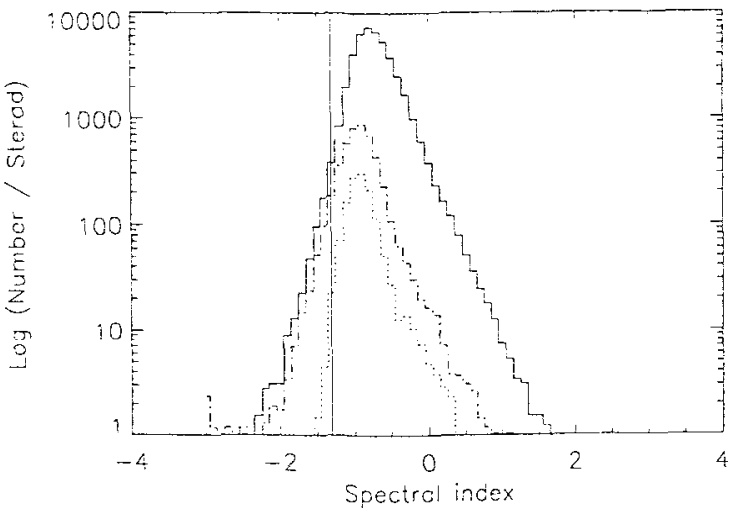

Figure 2. Logarithmic spectral index distribution for WENSS-NVSS (full line), Texas-NVSS (dot-dash line) and MRC-PMN (dotted line). The vertical line indicates the -1.3 cutoff used in our spectral index selection.

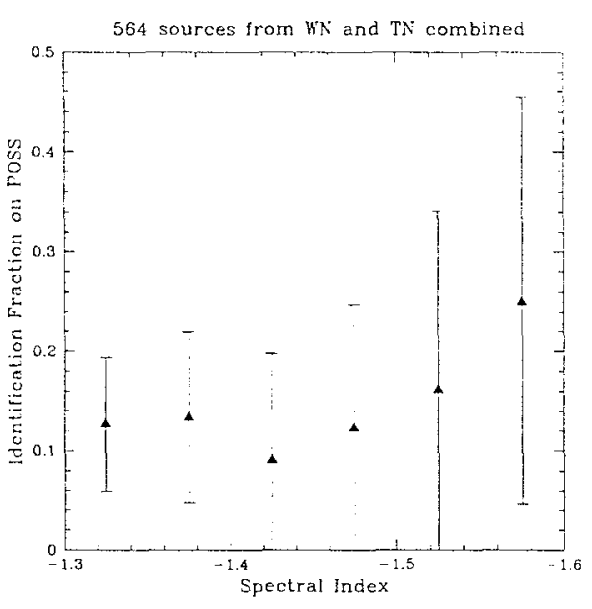

Figure 3. Identification fraction on the POSS as a function of spectral index for the combined WN and TN sample.

(Griffith et al. 1993) surveys it is now possible for the first time to define a large sample of USS sources with extremely steep radio continuum spectra $(\alpha \leq-1.3$, Fig. 2), and using $10-100$ times lower flux density limits than has been possible before (Chambers et al. 1996; Röttgering et al. 1994; Blundell et al. 1998).

Using these surveys we constructed 3 sub-samples, covering different regions of the radio sky using the deepest low and high frequency surveys available in each area (Table 2). Our largest, and most complete sample is based on the WENSS survey at $\delta>29^{\circ}$, together with the NVSS and FIRST surveys. In the remaining area covered by the northern hemisphere radio telescopes, we used the Texas survey at low frequencies, which produces a similar sample but at a higher flux density and is less complete. We also used two southern surveys to construct the first USS sample in the deep southern sky. More details about the samples are given in De Breuck et al. (2000a).

During the course of our optical and near-IR imaging and optical spectroscopy programs we have fine-tuned our selection technique. Previously it had been found that the identification fraction of radio galaxies decreases with spectral index (Tielens et al. 1979; Röttgering et al. 1995), which provided the rationale for using the USS source selection technique. With our 'hyper-steep' radio spectrum selection ( $\alpha \leq-1.3$ ) most sources remain unidentified, at least on the POSS ( $R \lesssim 20$ ). Only $\sim 15 \%$ of the sources can be identified, usually with bright galaxy clusters, as indicated by the frequent overdensity of galaxies $*$ around them, and by X-ray detections (De Breuck et al. 2000a). This identification fraction appears to be independent of spectral index (Fig. 3), in support of the idea that these are mostly foreground objects. 
This also explaincd why our initial optical imaging campaign on $3 \mathrm{~m}-4 \mathrm{~m}-$ class telescopes $(R \lesssim 24$ ) was not very successful in finding $R$-band identifications. Furthcrmore, for the typically expected $R-I \sim \sim 4$ values of HyZRGs, it would even be a challenge to detect most HyZRGs in the near-IR at Lick Observatory. Wc therefore decided to entirely skip the optical identification program and go straight to near-IR imaging at the Keck I telescope. This has produced, to date, a $100 \%$ identification rate with good photometric magnitudes to sclect HyZRG candidates using the Hubble $K-z$ diagram (Fig. 1).

We have now spectroscopically observed 30 faint USS HyZRG candidates with the following results. Only 5 of the sources have $z<2,7$ have $2<z<3$, 7 have $3<z<4$ and 2 sources have $z>4$, including one at $z>5$. At least 3 sources failed to yield redshifts, and were not detected in the continuum, despite $\sim 1$ hr integralions with LRIS, and may be at record hy redshifts, or are extremely obscured. We also found 6 sources with only a continuum detection and no emission-lines. These were all extremely compact USS sources, and may be moderately hy redshift $(1<z<3)$ BL Lac objects, 'emission-line free quasars' (c.f., Fanl et al. 1999), or even pulsars (which typically have $\alpha_{\text {radio }} \sim$ -1.6, Kaplan et al. 1998, and are faint optically; Martin et al. 1998).

Table 2. USS samples

\begin{tabular}{lcccc}
\hline \hline Sample & $\begin{array}{c}\text { Density } \\
\text { sr }^{-1}\end{array}$ & Spectral Index & $\begin{array}{c}\text { Flux Limit } \\
\text { mJy }\end{array}$ & \# of Sources \\
\hline WN & 151 & $\alpha_{325}^{1400} \leq-1.30$ & $S_{1400}>10$ & 343 \\
TN & $18^{a}$ & $\alpha_{3650}^{1400} \leq-1.30$ & $S_{1400}>10$ & 268 \\
MP & 26 & $\alpha_{408}^{4800} \leq-1.20$ & $S_{408}>700 ; S_{4850}>35$ & 58
\end{tabular}

${ }^{a}$ Due to the characteristics of the Texas survey, the TN sample is only $\sim 30 \%$ complete.

\section{The Highest Redshift Radio Galaxies}

\subsection{TN J1338-1942 at $z=4.11$}

The first $z>4$ USS radio galaxy discovered by us was TN J1338-1942. The initial identification was made with the ESO $3.6 \mathrm{~m}$ a.t $\mathrm{R}$-band, and subsequent spectroscopy with that same telescope showed that the radio galaxy has a redshift of $z=4.11 \pm 0.02$, based on a strong detection of Ly $\alpha$, and weak confirming C IV $\lambda 1549$ and He II $\lambda 1640$ (De Breuck et al. 1999a).

Subsequently we obtained a deep $K$-band image (rest-frame $B$-band) at Keck, shown in Figure 4 overlaid with a VLA radio image (De Breuck et al. 1999b). The radio source is an asymmetric double, with a very bright $\mathrm{NW}$ hotspot $\left(S_{4.7 G H z}=22 \mathrm{mJy} ; \alpha_{4.7}^{8.5 \mathrm{GHz}} \sim-1.6\right)$ coincident with the peak of the $K^{-}$-band emission. This hotspot has a very faint radio companion with a flatter spectrum $\left(S_{4.7 \mathrm{GH} z}=0.3 \mathrm{mJy} ; \alpha_{4.7}^{8.5 \mathrm{GHz}} \sim-1.0\right)$ at $1^{\prime \prime} .4$ to the $\mathrm{SE}$ which we identify as the probable nucleus. Thus the AGN and rest-frame optical (continuum and Ly $\alpha$ ) emission may not be co-centered. This resembles the $z=3.800$ radio galaxy 4C41.17 (van Brengel et al. 1999). A possible reason for this might be 


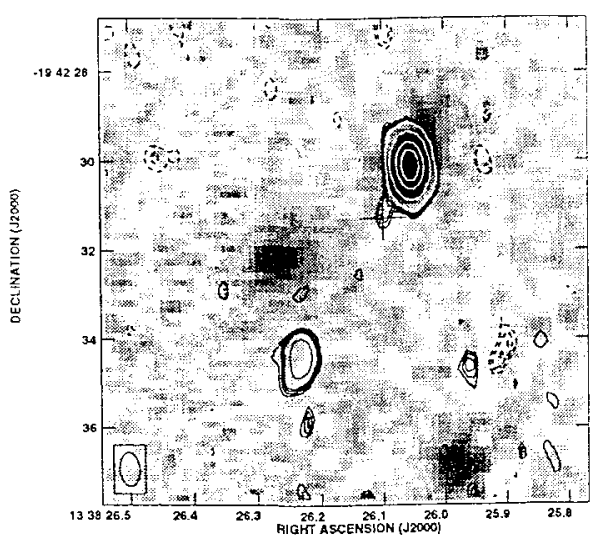

Figure 4. $4.85 \mathrm{GHz}$ VLA radio contours overlaicl on a. Keck $K$-band image of TN J1338-1942.

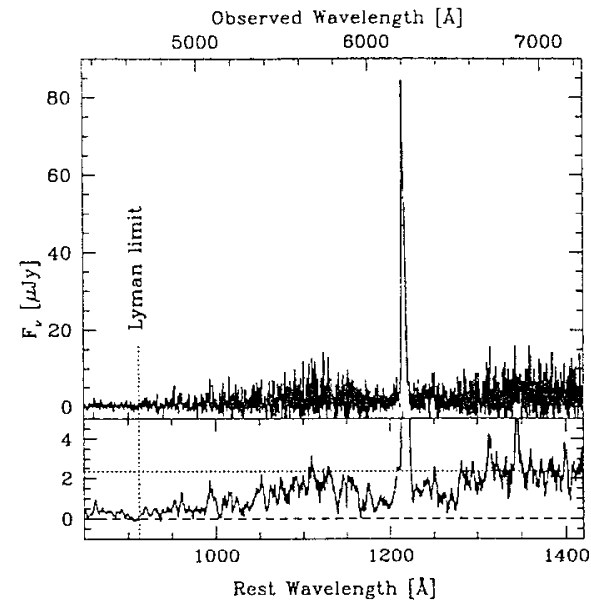

Figure 5. VLT spectrum of TN .71338-1942a.t $z=4.11$.

that the central region is obscured by dust. Such asymmetric radio sources are not uncommon. even in the local Universe, and are nswally thonght to be dne to strong interaction of one of its radio lobes with very dense gas (e.g., McCarthy et al. 1991; Feinstein et al. 1999).

A high signal-to-noise spectrum was also obtained with the VLT Antu telescope (De Breuck et al. 1999b). The spectrum is dominated by the bright Ly $\alpha$ line $\left(W_{\text {Lya }}^{\text {rest }}=210 \AA\right)$ which shows deep and broad $\left(\sim 1400 \mathrm{~km} \mathrm{~s}^{-1}\right)$ blueward absorption. The latter is probably due to resonant scattering by cold HI gas in a turbulent halo surrounding the radio galaxy and has also been seen several other HyZRGs (ran Ojik et al. 1996; Dey 1999). The continuum is relatively bright $\left(F_{1400} \sim 2 \mu \mathrm{Jy}\right)$ and if all due to young $\mathrm{O}-\mathrm{B}$ stars this would imply a total SFR of several hundred $\mathrm{M}_{\odot} / \mathrm{yr}$, resembling $1 \mathrm{C} 11.17$, and suggesting that TN J1338-1942 may be another HyZRG in which induced star formation might occur (c.f., Bicknell et al. 1999).

In Table 3 we have listed the Ly $\alpha$ properties of the known 7 most distant radio galaxies for which high quality optical slit spectroscopy data talicn with Keck or the VLT are available. We have assumed $H_{0}=65 \mathrm{~km} \mathrm{~s}{ }^{-1} \mathrm{Mpc}^{-1}$, $q_{0}=0.15$, and $\Lambda=0$. The Ly $\alpha$ fluxes are as measured i.c., uncorrected for blucward absorption. TN J1338-1942 is the most luminous Ly $\alpha$ galaxy and, after $4 \mathrm{C} 41.17$, also the brightest (in similar apertures). In all cases the brightest Ly $\alpha$ emission occurs on scale sizes of $1^{\prime \prime}-2^{\prime \prime}$, comparable to those of the brightest radio structures. $4 \mathrm{C} 41.17$ is known to have a very extended halo (Chambers et al. 1990) and the total size quoted is a lower limit, based on the deep ( 9 hrs) Keck spectropolarimetry data from Dey et al. (1997).

\subsection{TN J0924-2201 at $\tilde{z}=5.19$}

TN J0924-2201 is one of the steepest spectrum sources in our USS sample $\left(\alpha_{365 \mathrm{MHz}}^{1.4 \mathrm{GHz}}=-1.63\right)$ and therefore was one of our primary targets for near-IR identification. A deep $K$-band image at Keck showed indeed a very faint ( $K=$ 

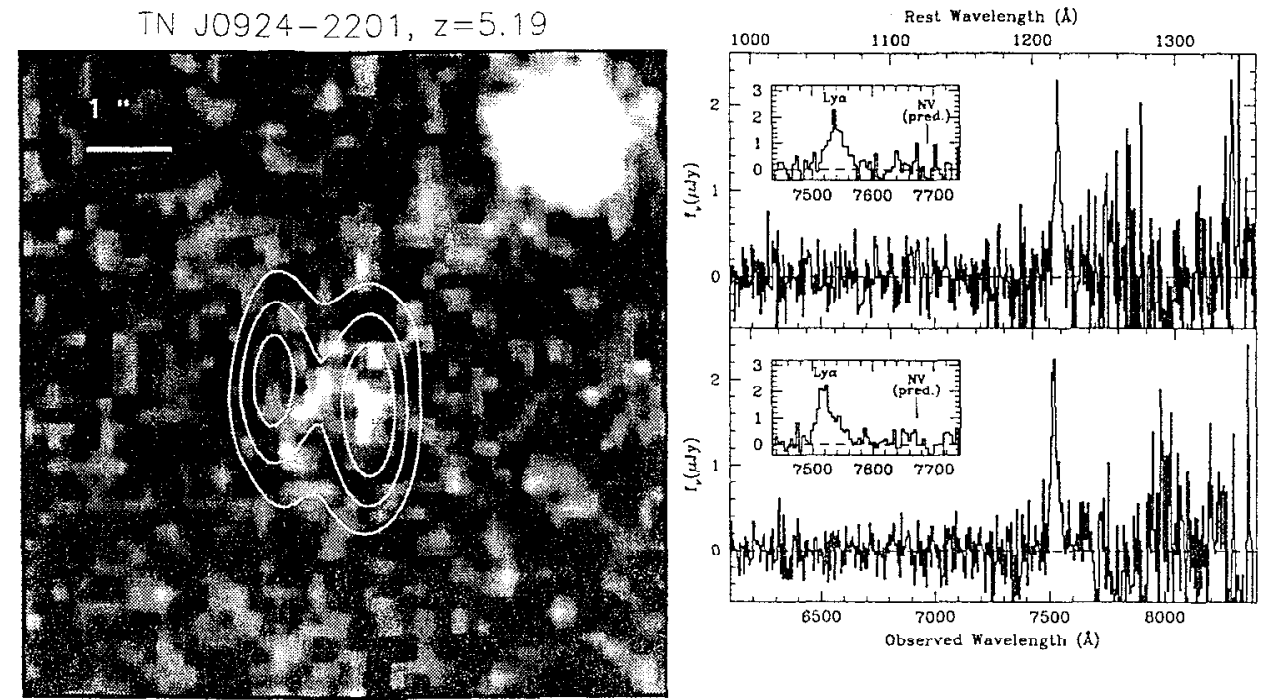

Figure 6. Keck/NIRC $\mathrm{K}$ band image of TN J0924-2201, with radio contours superposed.

Figure 7 . Keck spectra of TN J0924-2201at $z=5.19$ on two different nights.

$21.3 \pm 0.3)$, multi-component object at the position of the small ( 1.2 ) radio source (Fig. 6). The expected redshift on the basis of the $K-z$ diagram was $z>5$, and spectroscopic observations at Keck showed that this was indeed the case, based on a single emission line at $\lambda \sim 7530 \AA$ which we identified as Ly $\alpha$ at $z=5.19$ (van Breugel et al. 1999; none of the $z>5$ galaxies have more than one line detection).

Among all radio selected HyZRGs TN J0924-2201 is fairly typical in radio luminosity, equivalent width and velocity width (Table 2). It does have the steepest radio spectrum, consistent with the $\alpha-z$ relationship for powerful radio galaxies (e.g., Röttgering et al. 1997), and also has the smallest linear size. The latter may be evidence of its 'inevitable youthfulness' or a dense confining environment, neither of which would be surprising because of its extreme redshift (Blundell \& Rawlings 1999; van Ojik et al. 1997). Among the radio selected HyZRGs TN J0924-2201 appears underluminous in Ly $\alpha$, together with 8C $1435+63$, which might be caused by absorption in an exceptionally dense cold and dusty medium. Evidence for cold gas and dust in several of the most distant HyZRGs has been found from sub-mm continuum and CO-line observations (e.g., Ivison et al. 1998; Papadopoulos et al. 1999).

The second hyest redshift radio galaxy currently known listed in Table 3 is VLA J123642+621331 at $z=4.42$ (Waddington et al. 1999). This source was not USS selected and therefore provides an interesting alternate view on the possible selection effects of our method of finding HyZRGs. The source is an asymmetric double and although its radio luminosity is about a factor 1000 times lower than that of its much more luminous brothers at similar redshifts, it still qualifies as a FRII-type, though with a radio luminosity close to the FRI / FRII break at $408 \mathrm{MHz}\left(P_{408} \sim 3.2 \times 10^{26} \mathrm{~W} \mathrm{~Hz}^{-1}\right)$. Its radio spectrum is steep $\left(\alpha_{8.4 G H z}^{1.4 G H z} \sim-1.0\right.$, using the flux densities given by Waddington et al. $)$, but not 
as steep as our USS selected HyZRGs, and the Lya luminosity is a factor $5-10$ times less. Apart from the luminosity these properties are not hugely different from expected on the basis of radio selection and indicate that less extreme steep spectrum selected samples $(\alpha<-1.0)$ at much lower flux densities ( $\lesssim 1 \mathrm{~mJ}$. might be used to find many more (hundreds, thousands ?) HyZRGs at very hy redshifts (though with much lower efficiency, we suspect, than USS selected samples).

Table 3. Physical Parameters of the Hyest HyZRGs

\begin{tabular}{|c|c|c|c|c|c|c|c|c|}
\hline Name & $z$ & $L_{\mathrm{Ly} \alpha}{ }^{a}$ & $L_{365^{a}}$ & $\alpha_{365}^{1400}$ & $W_{\text {Lya }}^{\text {rest }}$ & $\Delta_{\text {Ly }{ }^{a}}$ & $\operatorname{Size}^{a}$ & Ref. $^{b}$ \\
\hline$\overline{\text { TN J0924-2201 }}$ & 5.19 & 1.3 & 7.5 & -1.63 & $>115$ & 1500 & $\overline{8}$ & WvB99 \\
\hline VLA $31236+6213$ & 4.42 & 0.2 & 0.0035 & -0.96 & $>50$ & 440 & & Wad99 \\
\hline $6 \mathrm{C} 0140+326$ & 4.41 & 16 & 1.3 & -1.15 & 700 & 1400 & 19 & DeBoo \\
\hline $8 C 1435+63$ & 4.25 & 3.2 & 11 & -1.31 & $670:$ & 1800 & 28 & Spin95 \\
\hline TN J1338-1942 & 4.11 & 25 & 2.3 & -1.31 & 200 & 1000 & 37 & DeB99 \\
\hline $4 \mathrm{C} 41.17$ & 3.798 & 12 & 3.3 & -1.25 & 100 & 1400 & 99 & Dey97 \\
\hline $4 C, 60.07$ & 3.79 & 16 & 4.1 & -1.48 & 150 & 2900: & 65 & Röt97 \\
\hline
\end{tabular}

${ }^{a}$ In units of $10^{43} \mathrm{erg} \mathrm{s}^{-1}\left(L_{L_{y} \alpha}\right), 10^{3 \epsilon} \mathrm{erg} \mathrm{s}^{-1} \mathrm{~Hz}^{-1}\left(L_{3 \epsilon \delta}\right)$, restframe $\mathrm{km} \mathrm{s}^{-1}$, kpc respectively) ${ }^{b}$ Most recent references quoted only: WvB99 = van Breugel et al. (1999); Wad99 = Waddington et al. (1999); DeB00 = De Breuck et al. $(2000 b) ;$ Spin95 = Spinrad et al. 1995; DeB99 = De Breuck et al. $(1999 a, b)$; Dey97 = Dey et al. (1997); Röt97 = Röttgering et al. (1997).

Our observations of TN J0924-2201 extend the Hubble $H-z$ diagram for powerful radio galaxies to $z=5.19$, as shown in Figure 1. Simple stellar evolution models are shown for comparison. Despite the enormous $k$-correction effect (from $U_{\text {rest }}$ at $z=5.19$ to $K_{\text {rest }}$ at $z=0$ ) and strong morphological evolution (from radio-aligned to elliptical structures), the $K-z$ diagram remains a powerful phenomenological tool for finding radio galaxies at extremely hy redshifts. Deviations from the $\pi-z$ relationship may exist (Eales et al. 1997; but see McCarthy 1999), and scatter in the $K-z$ values appears to increase with redshift, but this may in part be caused by limited signal-to-noise or emissionline contamination.

The clumpy $U_{\text {rest }}$ morphology resembles that of other HyZRGs (van Breugel et al. 1998; Pentericci et al. 1998) and if it is dominated by star light we derive a SFR of $\sim 200 \mathrm{M}_{\odot} \mathrm{yr}^{-1}$, without any correction for extinction, which may be a factor of several. TN J0924-2201 may be a massive, active galaxy in its formative stage, in which the SFR is boosted by jet-induced star formation (Dey et al. 1997; van Breugel et al. 1999; Bicknell et al. 1999). For comparison other, 'normal' star forming galaxies at $z>5$ have $10-30$ times lower SFR ( 6-20M $M_{\odot} y r^{-1}$; Dey et al. 1998; Weymann et al. 1998; Spinrad et al. 1998).

At $z=5.19$ TN J0924-2201 is currently the most distant AGN known, surpassing even quasars for the first time since their discovery 36 years ago. The presence of AGN at such early epochs in the Universe $(<1$ Gyr in most cosmogonies) poses interesting challenges to common theoretical wisdom, which 
assumes that they are massive (billion solar mass), active black holes. The question how these can form so shortly after the putative Big Bang may prove even more challenging then that of the formation of galaxies (e.g., Loeb 1993; Silk \& Rees 1998).

Acknowledgments. WvB is grateful for the many interesting conversations, advice, and wonderful collaborations he has had with Hy Spinrad and his students over the past 15 years. The work by W.v.B., C.D.B. and S.A.S. at IGPP/LLNL was performed under the auspices of the US Department of Energy under cuntract W-7405-ENG-48. W.v.B. also acknowledges support from NASA grants GO 6608, D.S. from IGPP/LLNL grant 98-AP017, and D.M. from Fondecyt Grant No. 1990440.

\section{References}

Becker, R. H., White, R. L., \& Helfand, D. J. 1995, ApJ, 450, 559

Bicknell, G., Sutherland, R., van Breugel, W., Dopita, M., Dey, A., Miley, G. 1999, ApJ, in press, astro-ph/9909218

Blundell, K. M., et al. 1998, MNRAS, 295, 265

Blundell, K. M., \& Rawlings, S. 1999, Nature, 399, 330

Bruzual, A. G., \& Charlot, S. 1999, personal communication

Chambers, K. C., Miley, G. K., \& van Breugel, W. J. M. 1990, ApJ, 363, 21

Chambers, K. C., Miley, G. K., van Breugel, W. J. M., \& Huang, J.-S. 1996, $\Lambda$ pJS, 106,215

Cimatti, A., Dey, A., van Breugel, W., Hurt, T. \& Antonucci, R. 1997, ApJ, 476,677

Condon, J., et al. 1998, AJ, 115, 1693

De Breuck, C., van Brengel, W., Röttgering, H., Miley, G. \& Carilli, C. 1999a, in 'Looking Deep in the Southern Sky' Edited by R. Morganti \& W. Couch (Springer), p. 246

De Breuck, C., van Breugel, W., Minniti, D., Miley, G., Röttgering, H., Stanford, S., \& Carilli, C. 1999b, A\&A, in press (astro-ph/9909178)

De Breuck, C., van Breugel, W., Röttgering, H., \& Miley, G. 2000a, A\&AS, submitted

De Breuck, C., valı Breugel, W., Röttgering, H., Miley, G., \& Sterı, D. 2000b, in preparation

Dey, A., van Breugel, W., Vacca, W. D., \& Antonucci, R. 1997, ApJ, 449, 698

Dey, A., Spinrad, H., Stern, D., Graham, J., \& Chaffee, F. 1998, ApJ, 498, L93

Dey, A. 1999, in 'The Most Distant Radio Galaxies', ed. H. Röttgering, P. Best \& M. Lehnert (Amsterdam: KNAW), p. 19

Douglas, J. N., et al. 1996, AJ, 111, 1945

Dunlop, J., \& Peacock, J. 1990, MNRAS, 247; 19

Eales, S., et al. 1997, MNRAS, 291, 593

Fan, X. et al. 1999, ApJ, in press, astro-ph/9910001

Feinstein, C., et al. 1999, astro-ph/9906059 
Griffith. M. \& Wright, A. E. 1993, AJ, 105, 1666

Haehnelt, M. Natarajan, P. \& Rees. M. J. 1998. MNRAS. 300, 817

Hu, E., McMahon, R., \& Cowie, L. 1999, ApJ, 522.9

Irison, R. J. et al. 1998, ApJ, 494, 211

Kaplan, D. I., et al. 1998. ApJS, 119, 75

Kauffmann. G., \& Haehnelt, M. 1999, MNRAS, in press, astro-ph/9906493

Large, M. I., Mills, B. Y., Little, A. G., Crawford, D. F., \& Sutton, J. M. 1981, MNRAS, 194, 693

Lilly, S. J. \& Longair, M., 1984, MNRAS, 211. 833

Lilly, S. 1988, ApJ, 333, 161

Loeb, A. 1993, ApJ, 403, 542

Martin, C., Halpern, J., \& Schiminovich, D. 1998 ApJ, 494, 211

McCarthy, P., van Brengel, W., \& Kapahi, V. K. 1991, ApJ, 371, 478

McCarthy, P. 1999, in 'The Most Distant Radio Galaxies', ed. H. Röttgering, P. Best \& M. Lehnert (Amsterdam: KNAW), p. 5

Papadopoulos, P. P., et al. 1999, ApJ, in press

Pentericci, L. et al. 1998, A\&A, 341, 329

Rengelink, R., et al. 1997, A\&A, 124, 259

Röttgering, H.J.A., Miley, G.K., Chambers, K.C. \& Macchetto, F. 1995, A\&AS, 114,51

Röttgering, H., van Ojik, R., Chambers, K., van Breugel, W., \& de Koff, S. 1997, A\&A, 326, 505

Shaver, P., Wall, J, Kellerman, K., Jackson, C., \& Hawkins, M. 1996, Nature. 381,139

Silk, J. \& Rees, M. 1998, A\&A, 331, L1

Spinrad, H., Filippenko, A. V., Wyckoff, S., Stocke, J. T., Wagner, R. M.d Lawrie, D. G. 1985, ApJ, 299, L7.

Spinrad, H., Dey, A. \& Graham, J. 1995, ApJ, 438, L51.

Spinrad, H., Dey, A., Stern, D., Dunlop, J., Peacock, J., Jimenez, R. \& Windhorst, R. 1997, ApJ, 484, 581

Spinrad, H. et al. 1998, AJ, 116, 2617

Tielens, A. G. G. M., Miley, G. K., \& Willis, A. G. 1979, A\&AS, 35, 153

van Brengel, W., Stanford, S. A., Spinrad, H., Stern, D., \& Graham, J. R. 1998, ApJ, 502, 614

van Breugel, W. et al. 1999, in 'The Most Distant Radio Galaxies', ed. H. Röttgering, P. Best \& M. Lehnert (Amsterdam: KNAW), p. 49

van Ojik, R., Röttgering, H. J. A., Miley, G. K., \& Hunstead, R. W. 1997, A\&A. 317,358

Villar-Martín, M., Tadhunter, C., \& Clark, N. 1997, A\&A, 323, 21

Weymann, R. et al. 1998, ApJ, 505, L95

White, S. 1997, ESO-VLT Workshop "Galaxy Scaling Relations: Origins, Evolution and Applications", astro-ph/9702214 

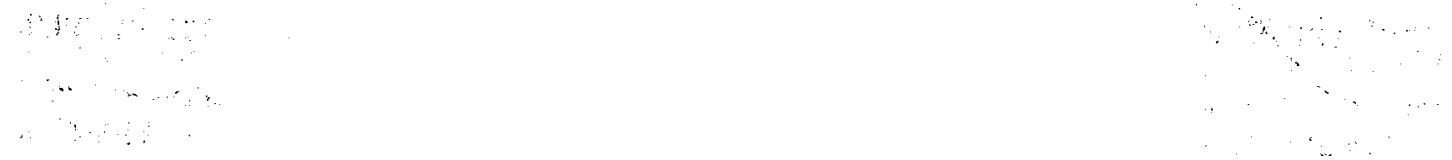
The Hy Redshift Uniwerse

ASP Conference Series, Vol. 193. 1999

A. J. Bunker \& W. J. M. van Breugel, eds.

\title{
The Vigor of Radio Astronomy at Hy Age: A Review of Faint Radio Source Populations
}

\author{
R.A. Windhorst ${ }^{1}$, A. Hopkins ${ }^{2}$, E.A. Richards ${ }^{1}$ and I. Waddington ${ }^{1}$ \\ ${ }^{1}$ Department of Physics and Astronomy, Arizona State University, \\ Tempe, AZ 85287-1504 \\ ${ }^{2}$ Australia Telescope National Facility, PO Box 76, Epping, NSW 1710, \\ Australia
}

\begin{abstract}
We present a review of faint radio source populations. First, we briefly review the life of Hy Spinrad and the history of radio source populations over the last 65 years, and note some remarkable correspondences. Next. we discuss constraints from the radio source counts over $T$ dex in flux density and $\sim 1$ dex in frequency, as well as from the angularsize and spectral-index vs. flux-density relations. We present possible extrapolations of the $\mu \mathrm{Jy}$ source counts to nanoJy levels as they may be observed with the Square Kilometer Array (SKA). We review clues to the nature and evolution of faint radio sources from deep optical identification and spectroscopy programs, as well as from HST imaging. The faint radio source population consists of a mixture of starburst, post-starburst and elliptical galaxies. The microwave radio emission at $\mu \mathrm{Jy}-\mathrm{mJy}$ levels is caused by a combination of (nuclear) starbursts and weak AGN activity. Each of these populations have probably evolved strongly as radio sources with cosmic epoch - possibly driven by the (strongly) epoch dependent galaxy merger rate. Finally, we present one example of a faint high-redshift $\mu \mathrm{Jy}$ radio source that is possibly obscured by dust.
\end{abstract}

\section{Introduction}

In a review of faint radio sources at a Conference that celebrates the $65^{\text {th }}$ birthday of Hy Spinrad, it is appropriate to compare the major events in his life with those in the history of radio astronomy, as well as those in the history of baseball. The latter two have been important and shaping aspects of the career and the life of Hy Spinrad. Table 1 attempts to give such a review. We call attention to some remarkable correspondences between these three histories - undoubtedly no coincidence if one considers the very productive scientific life of Hy Spinrad. The steady progression of finding higher and higher galaxy redshifts has been to no small extent due to the persistent and heroic efforts of Hy Spinrad, who with his collegues has measured ever larger and often record-breaking redshifts. Many of these were for samples of increasingly fainter radio sources (Fig. 1a), as several talks at this Conference showed, including a record set during the Conference for the $z=5.19$ ultra-steep radio galaxy (van Breugel et al. 1999). 
Table 1. Brief History ${ }^{a}$ of Radio Astronomy, Hy Spinrad, and Baseball

\begin{tabular}{|c|c|c|c|c|}
\hline Year & Radio Astronomy & Age & Hyron Spinrad (三 HS) & Baseball $(\equiv \mathrm{BB}$ ) \\
\hline 1931 & Jansky discovers static & & & End of BB Dark Ages \\
\hline 1933 & Jansky: Not $\odot$ but Sidereal & -1 & & All Star BB introduced \\
\hline 1934 & Jansky: =MN $18^{h}+10^{\circ} !$ & 00 & Born in Brooklyn, NY & BB player salary $\simeq$ UG today \\
\hline 1937 & Reber: MW-plare $910 \mathrm{MHz}$ & 03 & Wears first glasses & Giants fell in 5 games \\
\hline 1939 & Reber: MW-plane $160 \mathrm{MHz}$ & 05 & Uses first $\mathrm{BB}$ bat & Yankees swept Cubs \\
\hline 1944 & vandeHulst predicts $\mathrm{HI}-1420$ & 10 & looks thru ist telescope & Yankees sagged \\
\hline 1946 & Hey: Cyg-A $64 \mathrm{MHz}$ & 12 & HS moves to California & Postwar BB Recovery \\
\hline 1948 & Bolton. Ryle: Cyg-A pos $\angle 8^{\prime}$ & 14 & Learns about redshift & Robinson <batting > $=0.311$ \\
\hline 1958 & Ryle: Half Mile Telescope & 24 & Engaged, Married & Minimum Ballpark rule of \\
\hline 1959 & Ryle: source counts $>1 \mathrm{Jy}$ & 25 & Ph. D. & $325 \mathrm{ft}$ (home plate-fence) \\
\hline 1960 & Minkowski: $z(3 \mathrm{C} 295)=0.461$ & & 1960: $\exists$ Michael Spinrad & Dodgers also move to $C A$ \\
\hline 1963 & Schinidt: $z_{\max }(3 \mathrm{C} 147)=0.545$ & & 1963: $\exists$ Robert Spinrad & Dodgers win World Series \\
\hline 1965 & Penzias \& Wilson: CBR & 31 & Moves to UCB & $<$ batting $>_{\max } \simeq z_{\max }(\mathrm{gxy})$ \\
\hline 1969 & Cam, GB, Parkes $\sim 100 \mathrm{~mJ} y$ & 35 & 1969: 3 Tracy Spinrad & Will not mention 1969 ! \\
\hline 1974 & Cam,NRAO,WSRT $10 \mathrm{mJy}$ & 40 & Took kids to Lick & Dodger Pitcher $=$ Messersmith \\
\hline 1979 & Cambridge, WSRT $\sim 1 \mathrm{~mJ} y$ & 45 & trains many in audience & $\$($ Dodgers $)=0.5 \mathrm{keck}-\mathrm{I}$ \\
\hline 1980 & LBDS $\sim 0.2 \mathrm{mJy}$ starts & & Finds $z_{\max }(\mathrm{gxy})=1.01$ & $<$ batting $>_{\max }<z_{\max }(\mathrm{gxy}) !$ \\
\hline 1984 & VLA, WSRT, Cam $\sim 0.1 \mathrm{mJy}$ & 50 & Finds $z_{\max }(\mathrm{gxy})=1.8$ & Lasorda higlest paid BB mgr \\
\hline 1989 & VLA $\sim 30 \mu \mathrm{Jy}$ & 55 & $z_{\max }(\mathrm{gxy})=3.4-3.8$ & Dodger attendance $\geq 3 \times 10^{6} / \mathrm{yr}$ \\
\hline 1994 & VLA. ATCA $\sim 10 \mu \mathrm{Jy}$ & 60 & Finds $2_{\max }(\mathrm{gxy})=4.5$ & Dodgers-Braves. Short season \\
\hline 1999 & VLA, AICA 8 GHz $\sim 3 \mu \mathrm{J} y$ & 65 & finds $\begin{array}{r}z_{\max }(\mathrm{gxy})=5.3 .5 .6 \\
\text { HYFEST }(1999)\end{array}$ & $\begin{array}{r}\text { Dodgers beat Giants } \\
\text { on Friday. June 25. } 1999\end{array}$ \\
\hline $2004^{b}$ & VLA-upgrade $\sim 0.3 \mu \mathrm{Jy}$ & 70 & Finds $z_{\max }(\mathrm{gxy})=10$ & $\$($ Dodgers $) \geq V L T$ \\
\hline 2009 & SKA $12-\mathrm{hr} \sim 3 \mathrm{U}$ nJy & 75 & Finds $z_{\max }(\mathrm{gxy})=15$ & $\$$ (Dodgers $) \simeq \Sigma_{1979}^{2000}($ HST $)$ \\
\hline 2014 & SKA $1200-\mathrm{hr} \sim 2 \mathrm{I}_{\mathrm{L}} \mathrm{J}_{\mathrm{y}}$ & 80 & $\begin{array}{r}\text { Finds } z_{\max }(\mathrm{gxy})=20 \\
\text { HYFEST-II }(2014)\end{array}$ & $\begin{array}{l}\$(\text { Dodgers })>\text { NASA } \\
\text { Dodgers beat Mars }\end{array}$ \\
\hline
\end{tabular}

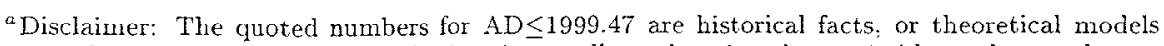
post-dictions. Like everywhere else in science, all numbers in columns 1-4 have the usual error bars attached, which are of course not specifically quoted here, like almost everywhere else in astronomy. All data in column 5 are of course error-free (see http://www totaldodgers.com/), so error-bars are not quoted here either. The rest of the paper is also error-free, hence no errors are quoted throughout.

${ }^{b}$ Disclaimer-2: The quoted numbers past $\mathrm{AD}=1999.47$ are theoretical model predictions. Like everywhere else in astronomy, these are all subject to grave errors; which are therefore not quoted. However, we note that the PREdiction made by the first author on June 21 publicly during this Conference about the Dodger victory on Friday June 25 was in fact correct. 
In general. faint radio-selected extragalactic objects are good probes of more ordinary galaxies at large distances for the following reasons: (1) unlike quasars and the powerful radio galaxies, weak radio galaxies do not generally contain significant non-thermal light in their optical spectra, neither do they have strong cmission lines (e.g. Fron et al. 1985); (2) the current VLA deep survey limits of $\sim 1 \mu \mathrm{Jy}$ can trace giant elliptical radio galaxies and quasars with radio powers in excess of the break $\left(P^{*}\right)$ in the RLF out to $z>>10$, and luminous spiral, Scyfert and actively star-forming galaxies to $z \simeq 2-5$; (3) radio sources are synchrotron sources which do not suffer from absorption by dust at high redshifts.

Radio galaxies played an important role in the study of galaxy evolution, since for a long time these were the only galaxies that could be identified easily at high redshifts. Jet-induced star formation or non-thermal radiation scattered in a reflection cone are the most probable radiation processes in the ultraluminous high redshift $3 \mathrm{CR}$ and $1 \mathrm{Jy}$ radio galaxies (Chambers et al. 1987, McCarthy et al. 1987, - 1993). The role of these processes has been studied at higher resolution with HST (e.g., Best et al. 1997, Windhorst et al. 1998). Weaker radio galaxies may be more representalive of what ordinary young galaxies would look like at those redshifts. Deep medium-band HST images showed a significant number of sub-galactic sized objects within $0.5 \mathrm{Mpc}$ from the LBDS radio galaxy $53 \mathrm{~W} 002$ at $z=2.39$ (Pascarelle et al. 1996). These $z \simeq 2.4$ objects may play an important role in the subsequent evolution of 53 W002 (Keel etal. 1999): it may have formed as an $r^{1 / 4}$-dominated galaxy during a relatively quick and sudden collapse that started at $z \simeq 3$ - possibly induced by star-formation along its radio jet (Windhorst etal. 1998). Similarly, deep HST images of faint field galaxies showed that E/SO and Sabc galaxies have evolved little since $z \lesssim 1$, but that the faint blue galaxy counts are dominated by a rapidly evolving population of late type galaxies at $z \geq 0.5-1$ (Sd/Irr's; Driver et al. 1995). The galaxy merger rate was likely higher in the past by $(1+z)^{m}$ with $m \simeq 1.5-3$ (e.g., Burkey et al. 1994), an important clue not only to understand the evolution of and formation of faint field galaxies in general (Driver et al. 1998), but also the strong cosmological evolution of (faint) radio sources in particular (Waddington et al. 1999b). We use $H_{0}=50 \mathrm{~km} \mathrm{~s}^{-1} \mathrm{Mpc}^{-1} \& q_{0}=0.1$ throughout.

\section{Constraints from $\mu \mathrm{J} y$ and $\mathrm{mJ} y$ surveys}

In the last decade, many deep radio surveys have been made at mJy and $\mu \mathrm{Jy}$ levels with e.g., the VLA, Westerbork, the Australia Telescope and Cambridge, at $1.4,4.9$ and/or $8.4 \mathrm{GHz}$. Details of the more recent surveys are given by, e.g., Becker et al. (1995), Ciliegi et al. (1999), Condon et al. (1998), Fomalont et al. (1991, 1993, 1997), Gruppioni et al. (1999b), Hopkins et al. (1998, 1999a), Richards etal. (1998), Richards (1999), White et al. (1997), Windhorst et al. $(1985,1993,1995)$. Fig. 1a shows the normalized differential source counts at $1.4,4.9$, and $8.4 \mathrm{GHz}$ for the flux range $1 \mu \mathrm{Jy} \leqslant S_{\nu} \leqslant 100 \mathrm{Jy}$, as reviewed by Hopkins et al. (1998), Richards (1999), and Windhorst et al. (1993).' The available source count data now cover about 1 dex in frequency and 7 dex in flux density. The boxes indicate statistical analyses of noise-fluctuations for $S_{8.44} \geq 2 \mu \mathrm{Jy}$ (Condon 1989; Fomalont et al. 1993; Partridge et al. 1997). The $8.4 \mathrm{GHz} \mu \mathrm{Jy}$ source counts from recent $160-\mathrm{hr}$ VLA images in the $13^{h}+43^{\circ}$ 


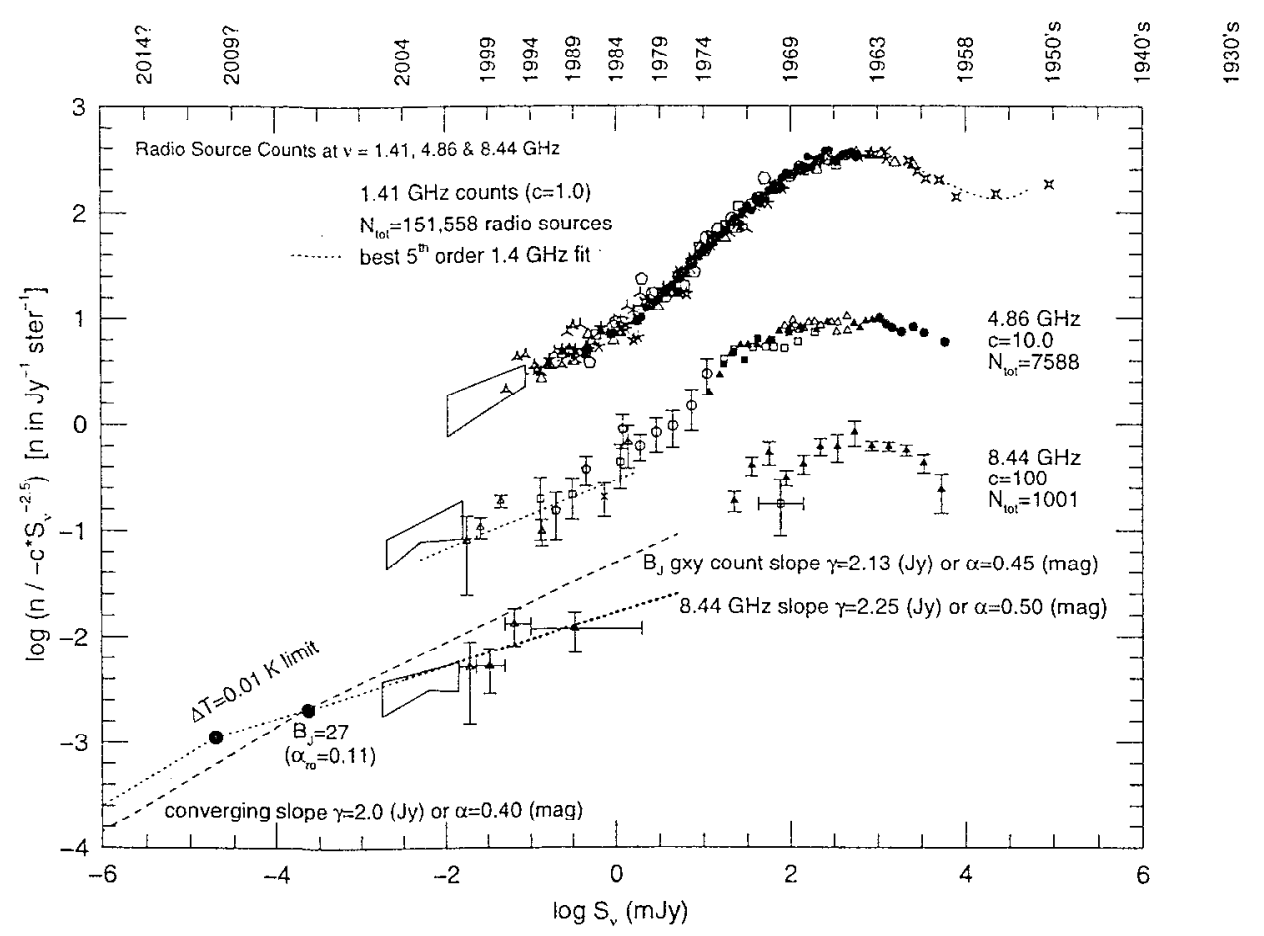

Fig. 1a. Differential source counts at $1.41,4.86$ and $8.44 \mathrm{GHz}$, normalized to a Euclidean count of $c \times S_{\nu}{ }^{-2.5}$ (Windhorst et al. 1993). The counts at all three frequencies cover a factor $10^{7}$ in $S_{\nu}$ and show an upturn between $2 \leqslant S_{\nu} \leqslant 3000$ $\mu J y$ with a similar non-converging slope $\gamma \simeq 2.2 \pm 0.2$. They must forever converge for $S_{\nu} \lesssim 100$ nanoJy $( \pm 0.5$ dex), or they would exceed the $\Delta T$ constraints.

field (Windhorst et al. 1995) and in the HDF (Fomalont et al. 1997; Richards et al. 1998) are indicated by the heavy dotted line. The light dotted lines in Fig. 1a are power-law best fits for all counts at $S_{\nu} \lesssim 2 \mathrm{mJy}$. All three frequencies show the initial steep rise between $S_{\nu} \simeq 10 \mathrm{Jy}$ and $1 \mathrm{Jy}$, the maximum excess with respect to Euclidean between $S_{\nu} \simeq 1.0 \mathrm{Jy}$ and $0.1 \mathrm{Jy}$, and continuous convergence between $S_{\nu} \simeq 100 \mathrm{mJy}$ and $3 \mathrm{mJy}$. At 1.41 and $4.86 \mathrm{GHz}$, the slope clearly changes below $S_{\nu} \simeq 3 \mathrm{mJy}$, but as yet we lack data for $3 \leqslant S_{8.44}$ $\lesssim 30 \mathrm{mJy}$. Between $S_{\nu} \simeq 3 \mu \mathrm{Jy}$ and $3000 \mu \mathrm{Jy}$, there is no significant change in slope at all three frequencies ( $\gamma \simeq 2.25 \pm 0.2$, including the fluctuation boxes), suggesting that the $\mu \mathrm{Jy}$ population covers a wide range in $\nu$. The fact that the $\mu \mathrm{Jy}$ counts sustain a nearly Euclidean slope over almost 1.0 dex in $\nu$ and 3 dex in $S_{\nu}$ (Fig. 1a) suggests that the weak radio source population likely has undergone cosmological evolution similar to gE radio galaxies and quasars.

The differential counts must converge at all frequencies for $S_{\nu} \lesssim 100$ nanoJy with slope $\gamma \leq 2.0$, because the integrated radio sky-brightness would otherwise: (1) diverge; (2) distort the low frequency CBR spectrum (Fomalont et al. 1993) [if the $8.44 \mathrm{GHz}$ counts continue below $2 \mu \mathrm{Jy}$ with $\gamma=2.25$, the $\mathrm{cm}-\lambda \mathrm{CBR}$ temperature errors would be exceeded for $S_{8.44} \leqslant 30$ nanoJy]; and (3) exceed the surface density of available field galaxies with $B_{J} \leqslant 27 \mathrm{mag}\left(\gtrsim 3 \times 10^{5} \mathrm{deg}^{-2}\right.$ , Tyson 1988) for $S_{8.44} \lesssim 300 \mathrm{nJy}$ (Windhorst et al. 1993), as indicated by the 


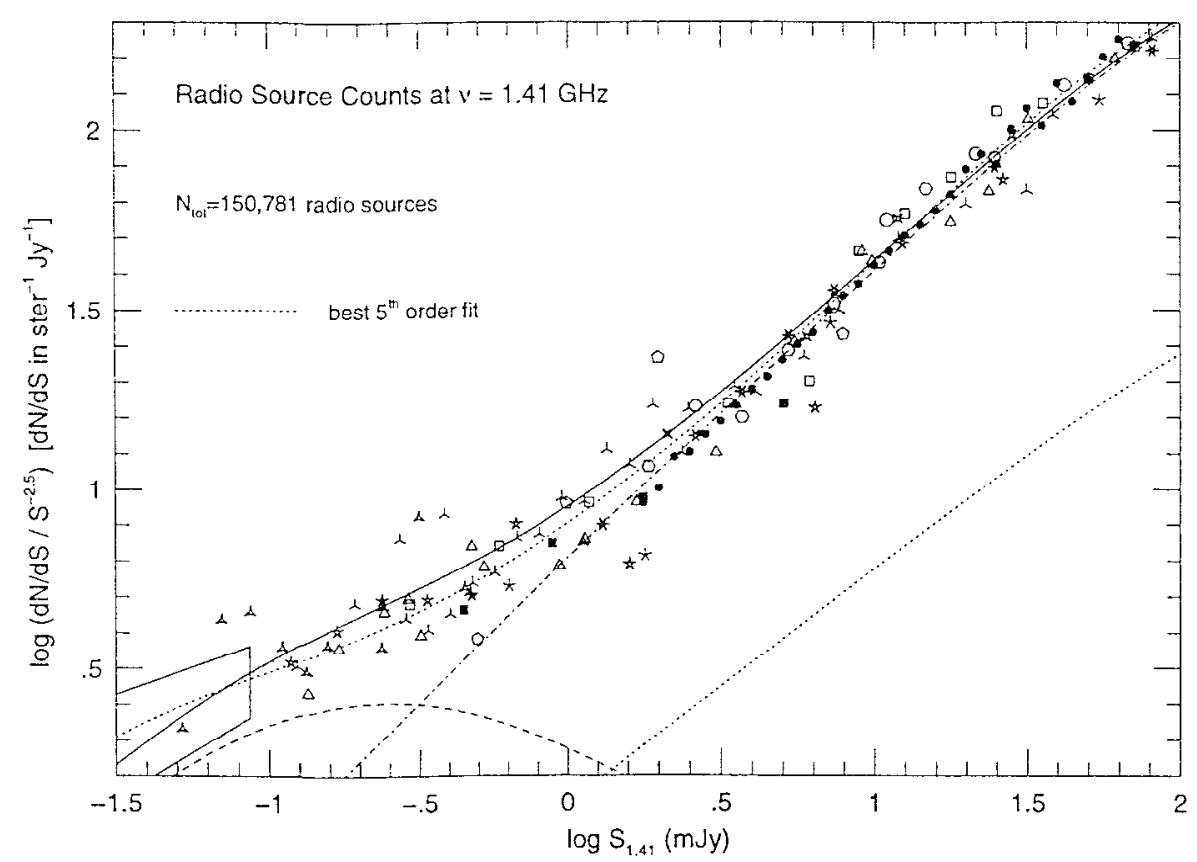

Fig. 1b. Enlargement of the differential $1.41 \mathrm{GHz}$ source counts from Fig. 1a. Models are (Hopkins etal. 1999b): steep-spectrum AGN (gE's; dot-shortdashed); flat spectrum AGN (QSR's; lower dotted line); IRAS-type starburst.s (dashed); Sum of all populations (solid). The change in slope starts around 5-9 $m J y$, but starburst galaxies do not dominate until below $\lesssim 1 \mathrm{mJy}$.

long dashed line in Fig. 1a. Somewhat stronger constraints are obtained from the measured DIRBE background (Haarsma \& Partridge 1998), which similarly cannot be exceeded by star-forming galaxies and/or radio sources, implying that the faint radio source counts must permanently turn over for $S_{\nu} \lesssim 100$ nanoJy (see also Fig. $1 \mathrm{c}$ and below).

Fig. $1 \mathrm{~b}$ shows an enlargement of Fig. 1a, but for the $1.41 \mathrm{GHz}$ counts only, which have the best statistics. All published 1.4-1.5 GHz counts were converted to $1.41 \mathrm{GHz}$ ( $c f$. Fig. 2b), normalized in the same manner, and the best possible resolution correction was applied following Windhorst et al. $(1990,1993)$, as confirmed by the FIRST survey (White et al. 1997). Following Windhorst et al. (1985, 1993), all counts from all $1.4 \mathrm{GHz}$ surveys were plotted, except: (a) in bins where a survey had insufficient statistics $(\approx 10-20$ objects/bin); (b) when not enough information was available to retrace and confirm the resolution correction; or (c) when not enough information was given to determine the counts as yet (e.g., for the NVSS). The $1.41 \mathrm{GHz}$ counts are now very well determined with statistics of $\geq 150,000$ sources from about three dozen surveys. The typical statistical precision per point is $10-20 \%$, and the global precision of the normalized differential amplitude is known to within $1-3 \%$ at most flux levels. There are no known systematics left in the $1.4 \mathrm{GHz}$ source counts at levels $\gtrsim 3 \%$.

Models for the counts from AGN (evolving giant ellipticals+quasars) and starburst+normal galaxies, and variants thereof, are plotted in Fig. 1b-1c, as described by e.g., Condon $(1989,1992)$, Hopkins et al. $(1998,1999)$, Jackson 


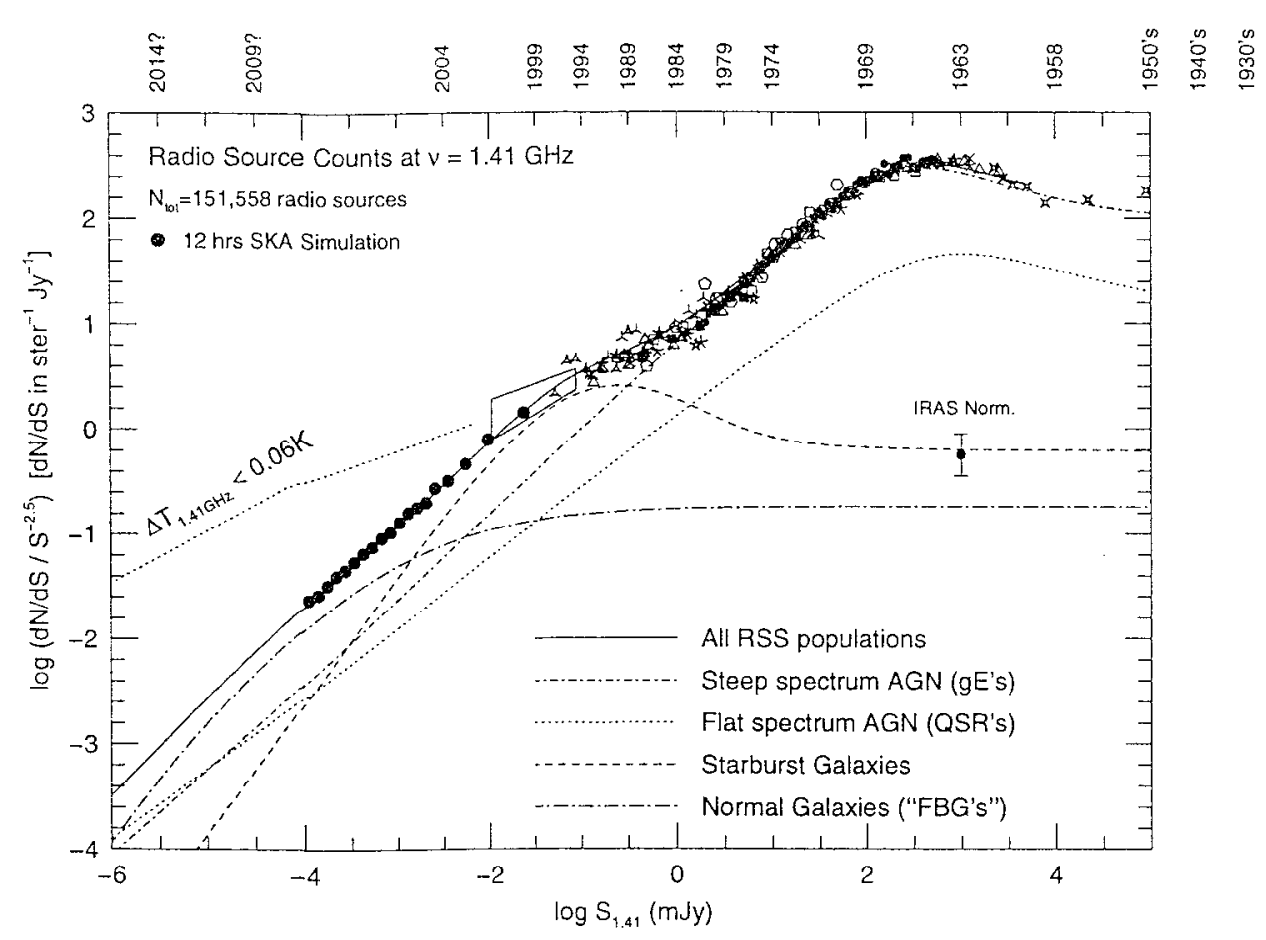

Fig. 1c. The differential $1.41 \mathrm{GHz}$ source counts from Fig. 1a. Models are as in Fig. 1b. The maximum possible contribution is shown for the normal galaxy population (dot-long-dash). Filled circles between $100 \mathrm{nJy-10 \mu Jy}$ indicate a 12-hr simulation with the Square Kilometer Array. The straight dotted lines represents the upper limit to the nJy source counts from $C B R$ constraints. 


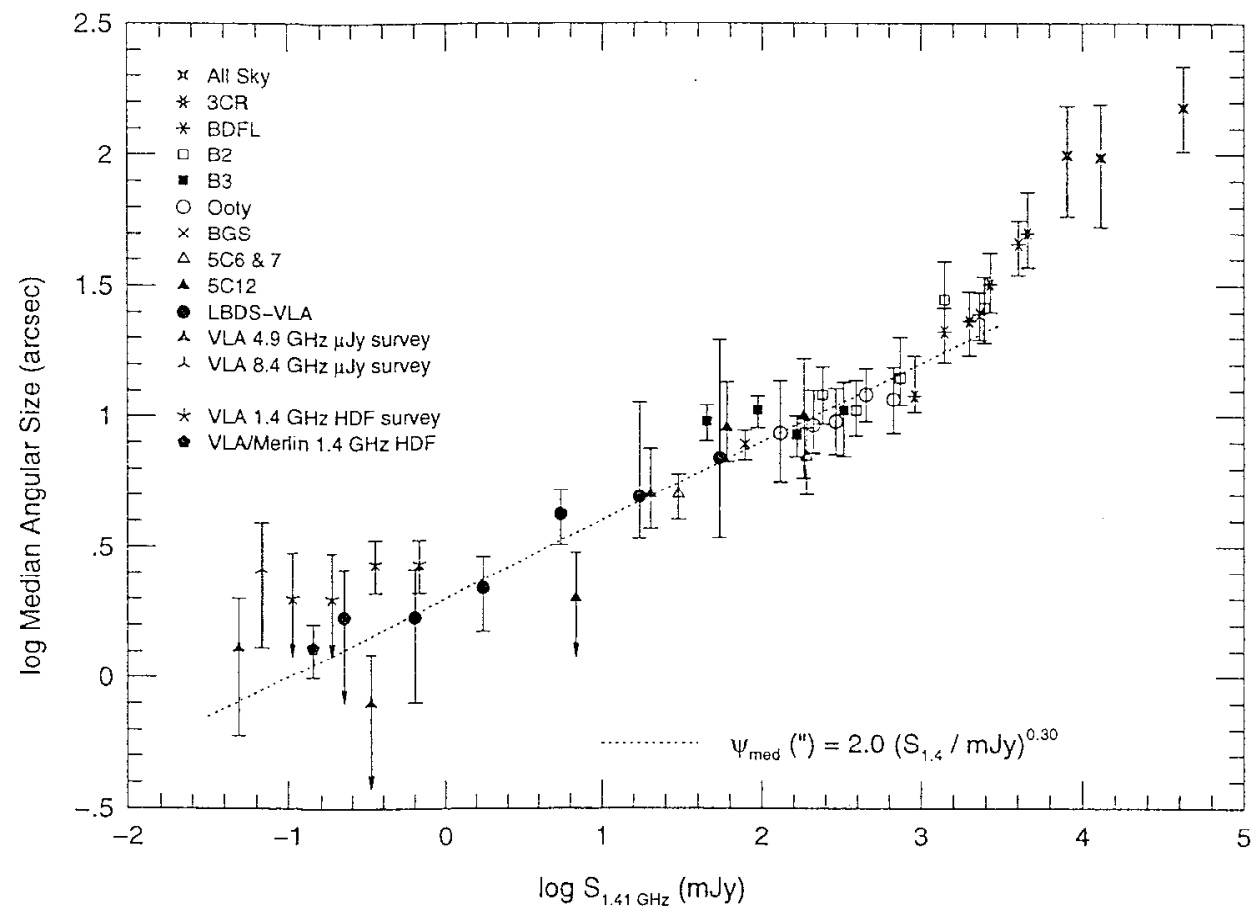

Fig. 2a. Median angular size vs. $1.41 \mathrm{GHz}$ flux for low-frequency surveys, and for the deepest VIA surveys at 8.4, 4.86 and 1.4 GHz (Richards 199.9). All flux scales were transformed to $1.41 \mathrm{GHz}$ following the $\alpha_{\text {med }}$ vs $S_{\nu}$ relation of Fig. 2b. The dotted straight line is the best fit for $0.1 \leq S_{1.4} \leq 3000 \mathrm{~m} . \mathrm{T} y$. 

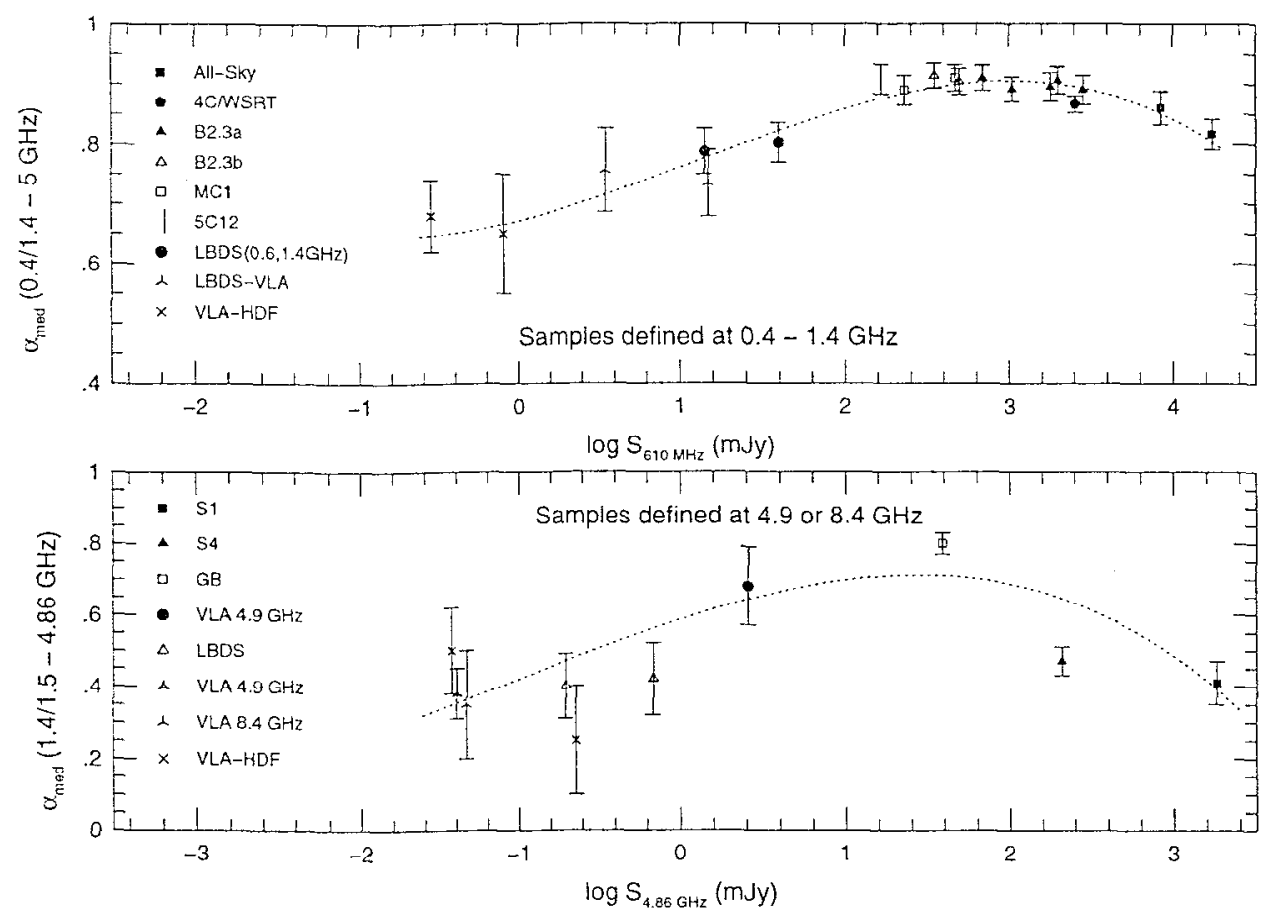

Fig. 2b.-Upper panel Median spectral index - low frequency $(0.61 \mathrm{GHz})$ flux, where $\alpha_{\text {med }}$ was measured between 0.41 (or 1.4) GHz and 4.86 (or 5.0) GHz. Lower panel Median spectral index - high frequency (4.86 GHz) flux, where $\alpha_{\text {med }}$ was measured between 1.41 (or 1.5) $\mathrm{GHz}$ and $4.86 \mathrm{GHz}$. All flux scales were converted to 0.61 or $4.86 \mathrm{GHz}$ using these same two relations. Note the similarity with the normalized differential source counts from Fig. $1 \mathrm{a}$ \&s $1 \mathrm{c}$. 
\& Wall (1999), Wall \& Jackson (1997), and Windhorst et al. (1985). It is clear that the evolving giant ellipticals+quasars ("monsters") dominate for $S_{1.4}$ $\gtrsim 0.5-1 \mathrm{mJy}$, while starburst+normal galaxies dominate for $S_{1.4} \leqslant 0.3 \mathrm{mJy}$. The likely erolving population of starburst and normal galaxies cause the remarkable upturn in the normalized differential counts below $S_{1.4} \sim 1 \mathrm{mJy}$, although this change in slope already starts at 5-9 mJy. Fig. 1c shows the extrapolation of these two evolving radio source populations to nanoJy levels, following Hophins et al. (1999b). This is relevant for e.g., the design and construction of the Square Kilometer Array that is currently being considered by an international Consortium. Hopkins et al. (1999b) also use the median angular size-1.4 GHz flux density relation (Fig. 2a) to $\mu \mathrm{Jy}$ levels, plus the extrapolation of this relation to nanoJy levels as expected from the sizes of HST disks, to predict the nanoJy source confusion limit and the nanoJy source counts. They conclude that if the sizes of faint field galaxies continues to decline at fainter optical fluxes (Odewahn et al. 1996, Cohen et al. 1999), nanoJansky surveys with SKA will likely not be confusion limited as long as it has baselines $>>100 \mathrm{~km}$. The $\mathrm{n} J y$ counts expected from a 12-hr SKA simulation are also shown in Fig. 1c. A 1000-hr HDF-like effort with SKA may reach a few nJy.

Other clues to the nature of the $\mu \mathrm{Jy}-\mathrm{m} J \mathrm{y}$ populations are obtained from the radio source sizes (Fig. 2a; Windhorst et al. 1993) and radio spectra (Fig. 2b; Fomalont et al. 1991). The dotted lines in Fig. 2a-2b are weighted leastsquares fits to the logarithmic data. High frequency $\mu \mathrm{Jy}$ samples have $\sim 40 \%$ extended steep spectrum sources, presumably synchrotron disks in intermediate redshift galaxies - with sometimes high frequency spectral steepening due to synchrotron losses. About 10-20\% are variable, and have low frequency turnovers, indicating self-absorbed compact radio cores (Oort et al. 1987). About $60 \%$ have flat spectra, $25 \%$ of which have inverted high frequency components (for $\nu \gtrsim 4.86 \mathrm{GHz}$; Windhorst et al. 1993). The median high frequency spectral index at $\mu \mathrm{Jy}$ levels is $\alpha_{\text {med }} \simeq 0.35 \pm 0.15$. The measured value of $\Theta_{\text {med }} \simeq 1-2^{\prime \prime}$ at sub-m.Jy levels corresponds to $\simeq 10-20 \mathrm{kpc}$ at $\mathrm{z} \sim 0.5-2$, which is bigger than expected for nuclear starbursts or AGN alone. Many flat-spectrum sources are extended $\left(\Theta \leq J^{\prime \prime}\right)$, and may be thermal bremsstrahlung from large-scale starformation in distant galactic disks. The median angular size of sub-mJy and $\mu \mathrm{J} y$ sources is comparable to the scale-lengths of faint HST galaxies $\left(\leqslant<1^{\prime \prime}\right.$, Odewahn et al. 1996), which is why the deepest radio (and optical!) surveys are not yet confusion limited.

\section{Nature and evolution of faint radio sources}

Through deep ground-based CCD-imaging (Fomalont et al. 1991; Neuschaefer \& Windhorst 1995; Waddington 1999; - et al. 1999b) about 400 faint radio sources were optically identified to gri $\leqslant 26-27$ mag in eight fields (Fig. 3). For most of these, accurate VLA positions were measured $\lesssim 0^{\prime \prime} 1$ (Fomalont et al. 1991; Oort et al. 1987). Several fields have systematic spectroscopy (Benn et al. 1993, Hammer et al. 1995), and similar fields were done in the South by Georgakakis et al. (1999) and Gruppioni et al. (1999). Four fields also have deep HST images that allow one to trace the morphological nature of these objects (Fomalont et al. 1997, Flores et al. 1999, Richards et al. 1999, Windhorst et al. 1998), 
in particular the HDF and 53 W002 fields. The HST morphology of mJy radio galaxies indicates primarily early-type galaxies dominated by $\mathrm{r}^{1 / 4}$-bulges with little or no color gradients, except at high redshifts (Windhorst et al. 1994). The majority of the $\mu \mathrm{J} y$ population appears to have small inner $\mathrm{r}^{1 / 4}$ bulges plus dominant exponential disks, and often distorted morphologies, indicating interactions or mergers, presumably due to the same dynamical events that also triggered their radio emission (Fomalont et al. 1997, Richards et al. 1998; Windhorst et al. 1995), suggesting that a significant fraction of the $\mu \mathrm{Jy}$ sources is cansed by (disk) synchrotron emission, enhanced by merger driven starbursts and their subsequent supernova rate (Windhorst et al. 1993). Spectroscopic studies similarly suggest that the $\mu \mathrm{Jy}-s u b-\mathrm{mJy}$ population is a mixture of starburst and post-starburst galaxies (Benn et al. 1993, Georgakakis et al. 1999), and some fraction of ellipticals and post starburst galaxies (Hammer et al. 1995; Gruppioni et al. 1999).

Fig. 3 shows the R-mag distribution of the optical IDs for eight complete samples of $\mathrm{mJy}$ and $\mu \mathrm{Jy}$ sources, in total 397 sources. The ID fraction at mJy and sub-m.Jy levels is typically $90-97 \%$ for $R \leq 26-27 \mathrm{mag}$ (Fig. 3), with the reliability and completeness of the ID sample generally exceeding $95-97 \%$. But a. $\mu . T y$ levels, up to $\sim 20 \%$ of all radio sources remains unidentified at $\mathrm{R} \leqslant 27 \mathrm{mag}$ (Richards et al. 1999). This suggests that the fraction of radio sources obscured by dust at very large redshifts is relatively small, but could be of order $10-20 \%$ for the faintest radio sources. Fig. 3 shows that in these combined samples at least 17 sources remain unidentified down to the detection limits, which are $\mathrm{R} \lesssim 26-27 \mathrm{mag}$ for the ground-based (Fig. 3) and $\mathrm{R} \lesssim 29 \mathrm{mag}$ for the HDF. This includes one - and possibly another - source in the HDF itself with $\mathrm{R} \geq 29 \mathrm{mag}$. All $\mathrm{R}$-mag distributions rise strongly for $17 \leqslant \mathrm{R} \leqslant 21 \mathrm{mag}$, indicating the strong cosmological evolution of the $\mathrm{mJy}$ and $\mu \mathrm{Jy}$ radio sources $(\$ 2)$, and gradually decline for $21 \lesssim \mathrm{R} \lesssim 27 \mathrm{mag}$, perhaps indicating a cutoff in their redshift and/or their luminosity distributions (Waddington 1999, - etal. 1999b). This is in stark contrast with the field galaxy counts that continue to rise to $B \sim 28 \mathrm{mag}$ (Driver et al. 1995, Odewahn et al. 1996, Tyson 1988, Williams et al. 1996).

Fig. 4 shows the redshift distribution for mJy, sub-mJy and $\mu \mathrm{Jy}$ samples (data from Benn et al. 1993, Hammer et al. 1995, Richards et al. 1998, Waddington 1999, -- et al. 1999b; Windhorst et al. 1994, 1995). Dotted lines show sources with measured spectroscopic redshifts, or upper limits to $z$ from inconclusive spectra. Full-drawn lines show the complete radio sample, with photometric redshift estimates for those sources without a spectroscopic redshift. The $\mathrm{m} J \mathrm{y}$ and $\mu \mathrm{Jy}$ samples are $\gtrsim 70 \%$ complete, but the sub-mJy sample is not complete for $B \geq 23$ or $z \geq 0.4$ (arrow in Fig. $4 \mathrm{~b}$ ). Bearing this in mind, the redshift distribution does not change much from $\mathrm{mJy}$ to $\mu \mathrm{Jy}$ flux levels, similar to the behavior seen in the apparent magnitude distributions in Fig. 3. Both are essentially independent of redshift for $\gtrsim 2 \mathrm{dex}$ in radio flux. Together with the steady and non-converging slope of the $\mu \mathrm{Jy}$ source counts over $\gtrsim 3 \mathrm{dex}$ in radio flux (Fig. 1a), the fact that the redshift distribution hardly changes over $\gtrsim 2$ dex in flux implies that the radio source population at flux levels $1 \mu \mathrm{Jy} \leqslant S_{\nu}, \underset{\sim}{\leqslant}$ mJy must consist of at least one fairly strongly evolving population. Given the heterogeneous optical identifications at $\mu \mathrm{Jy}-\mathrm{mJy}$ levels (see above), and given the known strongly evolving population of giant ellipticals (and quasars) which 

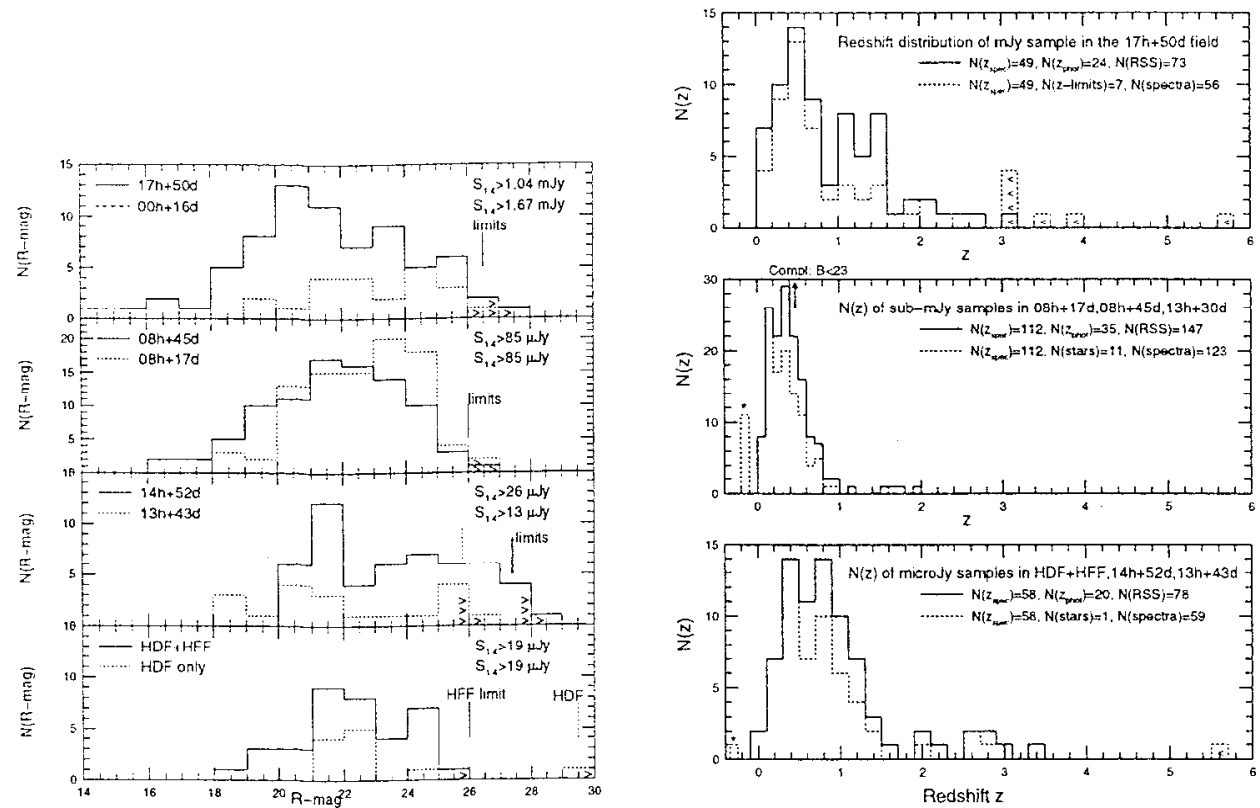

Fig. 3 [Left]. R-magnitude distribution for 397 faint radio sources from eight samples at $\mu J y-m J y$ levels with good VLA positions. Completeness limits are indicated. The 17 sources that remain unidentified with $R \geq 26-29$ mag ('>') are candidates with $z \geq 4-6$ (Fig. 5-6).

Fig. 4 [Right]. The redshift distribution for $m J y$, sub-mJy and $\mu J y$ samples, as discussed in the text. 

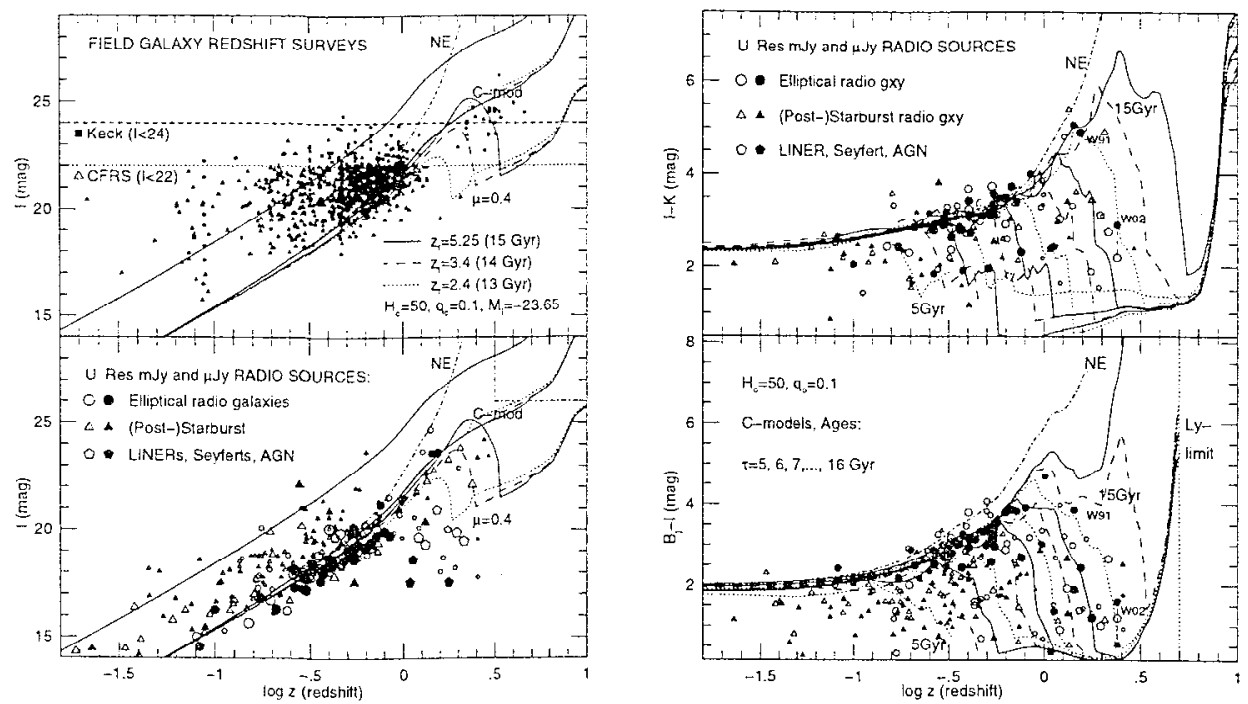

Fig. 5 [Left]. (5a.-Top): I-mag vs. $z$ for field galaxy redshift surveys; and (5b. - Bottom) for $m J y$ and $\mu J y$ samples. Circles indicate elliptical radio galaxies, triangles blue star-forming galaxies, and pentagons LINERS, Seyferts, or quasars. Filled symbols indicate extended (larger) radio sources. The symbol size increases with $\log S_{1.4}$. The box in the upper right corner of Fig. 56 indicates that - given the distribution of the brighter radio galaxies - the 17 unidentified radio sources with $R \geq 27-28$ mag (Fig. 3) are likely at $z \gtrsim 3-5$.

Fig. 6 [Right]. (6a.-Top): ( $I-K)$ and (6b.-bottom) $\left(B_{J}-I\right)$ color vs. redshift for the same $m J y$ and $\mu$ Jy samples as in Fig. 56. The spectral evolution model grids are discussed in the text. The upper envelope of the oldest high redshift (radio) ellipticals occurs al $\tau \simeq 13-14$ Gyr.

dominate the counts for $S_{\nu} \geq 3 \mathrm{mJy}$ (Fig. 1b \& 1c; Kron et al. 1985, Windhorst et al. 1985), there are most likely at least two strongly evolving galaxy populations that contribute to the radio source counts at $\mu \mathrm{Jy}-\mathrm{mJy}$ levels. This, amongst others, causes the remarkable upturn in the counts below a few $\mathrm{mJy}$ in Fig. la \& 1b.

Fig. 5b shows the I-mag versus redshift relation for $\mathrm{mJy}$, sub-mJy and $\mu \mathrm{Jy}$ samples with available redshifts (as in Fig. 4). Fig. 5a shows the same for about $800+$ faint field galaxies from recent redshift surveys (CFRS, Lilly et al. 1995; and Keck-HDF: Cohen et al. 1996, Steidel et al. 1996). Fig. 6a \& 6b show the $(\mathrm{B}-\mathrm{I})$ and $(\mathrm{I}-\mathrm{K})$ colors versus redshift diagrams, respectively, for the same radio source samples as in Fig. 5b. Optical classifications were either done with HST or from the spectra. Several model predictions have been plotted in Fig. 5-6 from the spectral evolution models of Bruzual \& Charlot (1993) for various "formation epochs", $z$ form , and assuming a best fit $M_{I}=-23.65$ mag at $z=0$. Fig. $5 \mathrm{~b}$ shows that radio galaxies are fairly luminous with a small dispersion in absolute magnitude, not only at the $3 \mathrm{CR}$ level, but also at the mJy and the $\mu$. Jy level $\left(\left\langle M_{V}\right\rangle \simeq-22.4 \pm 0.5 \mathrm{mag}\right)$. Fig. $6 \mathrm{a} \& 6 \mathrm{~b}$ shows that the (B-I) and $\left(\mathrm{I}-\mathrm{H}^{-}\right)$colors of the reddest radio galaxies at $\mathrm{z} \gtrsim 1.0$ are consistent with passively or mildly evolving giant ellipticals (circles). In order to yield colors as red as 
observed for most elliptical radio galaxies, their initial star formation should have occurred early on, about 13-14 Gyr ago, or at $z \gtrsim 3-4$ (Fron et al. 1985).

A possible sccnario is that starburst (radio) galaxies gradually transform into early type (radio) galaxies through repeated (hierarchical) merging. Ellipticals would transform most of their ISM into stars after repeated mergers so that their radio source can escape the galaxy, and so become larger, and thus seen as an extended sourcc. This merging process takes place over the redshift range $z=0.2-3$ (3-14 Gyr ago) with a peak at $z \sim 0.8-1$, presumably driven by the strongly epoch dependent ratc of (minor) morgcrs (e.g., Burkcy ct al. 1991). We note that the cosmological evolution of the radio source population follows a similar strong redshift dependence (Bcnn ct al. 1993, Waddington 1999, - et al. $1999 \mathrm{~b}$ ), and suggest that the cause of the cosmological evolution be sought in the (strongly) epoch dependent galaxy merger rate, which may have both fueled the higher $A G N^{-}$activity in the past, as well as caused stronger starburst and post-starburst activity and enhanced microwave disk emission.

\section{Searching for dusty radio galaxies at $z \geq 2-4$.}

How can we use deep radio surveys to find possible candidates for young and possibly dusty star-forming objects at $z \geq 2-4$ ? If most galaxies indeed formed through the gradual merging of sub-galactic sized fragments at $z \simeq 2-4-$ with typical sizes $\leq 0.5-1 \mathrm{kpc}$ (Odewahn et al. 1996, Pascarelle et al. 1996) - then any objects at $z \geq 5$ will appear quite small in most cosmologies. The big question is then: what is the nature and redshift of the faint radio galaxies that remain unidentified for $\mathrm{R} \approx 27-29 \mathrm{mag}$ ? Apart from intergalactic or heavy internal reddening, it is most plausible that in the areas surveyed several $d \in g^{2}$ - at least a dozen objects are indeed at $z \geq 5-6$, so that the Lyman discontinuity blanks out the UBV(I?) filters. Important clues may be obtained from Fig. 5b \& 3 . For $\mathrm{I} \lesssim 22-23 \mathrm{mag}$, the Hubble relation for $\mathrm{mJy}$ and even $\mu \mathrm{Jy}$ sources is rather well defined, and has a relatively small dispersion, especially for m $\mathrm{Jy}$ sources. A family of plausible (" $\mathrm{C}$ " to $\mu=0.4$ ) models that describes the observed color range of $\mathrm{mJy}$ and $\mu \mathrm{Jy}$ ellipticals quite well (Fig. $6 \mathrm{a} \& 6 \mathrm{~b}$ ) is shown for various formation epochs in Fig. 5b. If these models may be extrapolated to higher redshifts, they suggest that most unidentified $\mathrm{m} \cdot \mathrm{Jy}$ and $\mu \mathrm{Jy}$ radio galaxies with $\mathrm{R} \gtrsim 26.5$ (Fig. 3) are likely at $\mathrm{z} \gtrsim 3$, and quite possibly at $\mathrm{z} \gtrsim 4-10$. Given that massive early-type galaxies may have assembled very rapidly from the merging of many smaller subunits (Pascarelle et al. 1996), these objects could have already existed at $z \sim 4-10$ (Dumlop et al. 1996, Spinrad et al. 1997). The next step will be to identify all these sources and to measure their redshifts, focusing on $\lambda \gtrsim 6000-7000 \AA$.

Fig. Ta shows the WFPC2 image of one radio source in the HDF flanking fields that remained essentially unidentified at $I \geq 25.5 \mathrm{mag}$. The object is barely seen with HST/NICMOS in the J-band (Fig. $7 \mathrm{~b}$ ) with $J \simeq 25 \mathrm{mag}$, but a 6-orbit CVZ image in the H-band (Fig. $7 \mathrm{c}$ ) clearly shows an object with $\mathrm{H} \simeq 22.7 \mathrm{mag}$. It was was also seen in a deep ground-based $\mathrm{K}$-band image (Fig. $7 \mathrm{~d}$ ) with $\mathrm{K} \sim 21.1$ mag. Hence the object is very red with $(\mathrm{I}-\mathrm{K}) \gtrsim 4.4,(\mathrm{~J}-\mathrm{K}) \gtrsim 3.3,(\mathrm{~J}-\mathrm{H}) \gtrsim 1.7$ and $(\mathrm{H}-\mathrm{K}) \simeq 1.6 \mathrm{mag}$. Its NIC2 light-profle is much better fit with an exponential disk than an $r^{1 / 4}$ profile, with $r_{h l}=0$ ! 17 . A single emission-line redshift from 


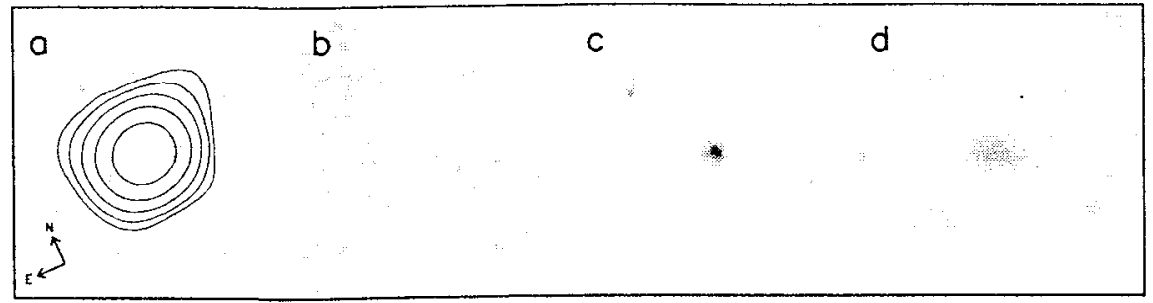

Fig. 7a [Left]: WFPC2 I-band image of an essentially unidentified radio source in the HFF (I 25.5 mag) with VLA $1.4 \mathrm{GHz}$ contour superimposed.

Fig. 7b [Middle-left]: 3-orbit CVZ NIC2 $J_{F 110 W}$-band image;

Fig. 7c [Middle-right: 6-orbit CVZ NIC2 $H_{F 160 W}$-band image, which shows a very red object at the radio position. The NIC2 H-band sky is $19.6 \mathrm{mag} \mathrm{arcsec}^{-2}$ Fig. 7d [Right]: Ground-based KPNO IRAM K-band image.

two independent Keck exposures by Hy Spinrad and Dan Stern suggests Ly $\alpha$ at $z=4.42$ (see Waddington etal. 1999a). This object is most likely a dusty starburst at $\mathrm{z} \sim 4.4$ with a disk that had just formed, and with a (major, first?) starburst that is still mostly obscured in the optical (=restframe UV; best fit $A_{V} \sim 1.7 \mathrm{mag}$ ). This would then possibly suggest a recent dissipational collapse. Several more such unidentified objects have been found in the HDF/VLA survey (Richards etal. 1999), and it would be of extreme interest to get deep high resolution near-IR images of these objects, as well as spectra in the far-red or near-IR, and to see how large a fraction of the radio-selected SFR remains optically obscured by dust (Haarsma, et al. 1999; Partridge, 1999).

Acknowledgments. We thank the NRAO and STScI staff for their continuous help in some of the projects described here. We thank our collaborators Ed Fomalont, Ken Kellermann, Bruce Partridge and Dan Stern for allowing us to quote a few unpublished results. We thank Prof. 'Iracy Spinrad from ASU for informing us about a few other unpublished facts about the life of Hy Spinrad. We thank Hy Spinrad for allowing us to publish these in Table 1 here, and for a life-long stimulation of all our careers. This work was supported by NSF grants AST88211016 \& AST9802963 and NASA grants GO-5985.01-94A, AR-6337.0396A, \& GO-7452.01.96A from STScI under NASA contract NAS5-26555.

\section{References}

Becker, R. H., White, R. L., \& Helfand, D. 1995, ApJ, 450, 559

Benn, C. R., et al. 1993, MNRAS, 263, 98

Best, P., Longair, M., \& Röttgering, H. 1997, MNRAS, 286, 785

Bruzual A., G., \& Cliarlot S. 1993, ApJ, 405, 538

Burkey, J., Keel, W., Windhorst, R., \& Franklin, B. 1994, ApJL, 429, L13

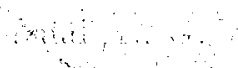

Chambers, K. C., Miley, G. K., van Breugel, W. J. M. 1987, Nature, 329, 604

Ciliegi, P. et al. 1999, MNRAS, 302, 222 
Cohen, J. G., et al. 1996, ApJL, 171, Lj

Cohen. S. H., Windhorst, R. A., Burg, C. A., Odewahn, S. C., \& Driver, S. P. 1999, AJ, submitted

Condon, J. J. 1989, ApJ, 338, 13

Condon, J. J. 1992, ARA\&A, 30, 575

Condon, J. J., Cotton, W. D., Greisen, E. W., Yin, Q. F., Perley, R. A., Taylor, G. B., Broderick, J. J. 1998, AJ, 115, 1693

Driver, S. P., Windhorst, R. A., et al. 1995, ApJL, 449, L23

Driver, S. P., et al. 1998, ApJL, 496, L93

Dunlop, J. S., Peacock, J. A., Spinrad, H., Dey, A., Jimenez, R., Stern, D., \& Windhorst, R. A. 1996, Nature, 381, 581

Flores, H. et al. $1999 \mathrm{ApJ}, 517,148$

Fomalont, E. B., et al. 1997, ApJL, 493, L5

Fomalont, E., Partridge, B., Lowenthal, J., \& Windhorst, R. 1993, ApJ, 404, 8

Fomalont, E., Windhorst, R., Kristian, J., \& Kellermann, K. 1991, AJ, 102, 1258

Georgakakis, A., Mobasher, B., Cram, L., Hopkins, A., Lidman, C., RowanRobinson, M. 1999, MNRAS, 306, 708

Gruppioni, C., Mignoli, M., \& Zamorani, G. 1999, MNRAS, 304, 199

Gruppioni, C. et al. 1999, MNRAS, 305, 297

Haarsma, D., \& Partridge, R. B., 1998, ApJL, 503, L5

Haarsma, D., Partridge, R. B., Windhorst, R. A., \& Richards, E. A. 1999, ApJ, in preparation

Hammer, F., Crampton, D., Lilly, S. J., Le Fevre, O., \& Kenet, T. 1995, MNRAS, 276, 1085

Hopkins, A. M., Mobasher, B., Cram, L., \& Rowan-Robinson, M., 1998, MNRAS, 296, 839

Hopkins, A., Afonso, J., Cram, L., Mobasher, B. 1999a, ApJL, 519, L59

Ilopkins, A., Windhorst, R. A., Cram, L., \& Ekers, R. 1999b, PASA, submitted

Jackson, C., \& Wall, J. V. 1999, MNRAS, 304, 160

Keel, W.C., Cohen, S.H., Windhorst, R.A., \& Waddington, I. 1999, AJ, in press - see also this Volume

Kron, R. G., Koo, D. C., \& Windhorst, R. A. 1985, A\&A, 146, 38

Lilly, S. J., Trcsse, L., Hammer, F., Crampton, D., \& Le Fevre, O. 1995, ApJ, 455,108

McCarthy, P. J., van Brengel, W. J. M., Spinrad, H., \& Djorgovski, S. G. 1987, ApJL, 321, L29

McCarthy, P. J. 1993, ARA\&A, 31, 639

Neuschaefer, L. W., \& Windhorst, R. A. 1995, ApJS, 96,371

Odewahn, S., Windhorst, R., Driver, S., \& Keel, W. 1996, ApJL, 472. L13

Oort, M., Katgert, P., Steeman, F., \& Windhorst, R. 1987, A\&A, 179, 41

Partridge, R. B., et al. 1997, ApJ, 483, 38

Partridge, R. B., 1999, this Volume. 
Pascarelle, S., Windhorst, R., Keel, W., Odewahn, S. 1996, Nature, 383, 45

Richards, E. A., et al. 1998, AJ, 116, 1039

Richards, E. A. 1999, ApJ, in press

Richards, E. A., et al. ApJL, submitted

Spinrad, H., Dey, A., Stern, D.. Dunlop, J., Peacock, J., Jimenez, R., \& Windhorst, R. 1997, ApJ, 484, 581

Steidel, C., Giavalisco, M., Dichinson, M., \& Adelberger, 1996, AJ, 112, 352

Tyson, J. A. 1988, AJ, 96, 1

van Brengel, W., de Breuck, C.. Stanford, S. A., Stern, D., Röttgering, H., Miley, G., 1999, ApJL, 518, L61 - see also this Volume

Waddington, I. 1999, Ph.D. thesis, (Univ. of Edinburgh) - See also this Volume

Waddington, I., Windhorst, R. A., Cohen, S. H., Partridge, R. B., Spinrad, H., \& Stern, D. 1999a, ApJL, submitted

Waddington, I., Windhorst, R. A., et al. 1999b, MNRAS, submitted

Wall, J. V., \& Jackson, C. 1997, MNRAS, 290, L17

White, R. L., Becker, R. H., Helfand, D., \& Gregg, M. D. 1997, ApJ, 475, 479

Williams, R. E. et al. 1996, AJ, 112, 1335

Windhorst, R. A., et al. 1995, Nature, 375, 471

Windhorst, R., Fomalont, E., Partridge, B., \& Lowenthal, J. 1993, ApJ, 405, 498

Windhorst, R. A., et al. 1994, ApJ, 435, 577

Windhorst, R. A., Keel, W. C. \& Pascarelle, S. M. 1998, ApJL, 494, L27

Windhorst, R. A., Mathis, D. F., Neuschaefer, L. W. 1990, in ASP Conf. Ser., Vol. 10, Evolution of the Universe of Galaxies (Edwin Hubble Centennial Symposium), ed. R. G. Kron (Provo, UT: BookCrafters, Inc.), p. 389

Windhorst, R., Miley, G., Owen, F., Kron, R., \& Koo, D. 1985, ApJ, 289, 494 
The Hy Redshift Universe

ASP Conference Series. Vol. 193, 1999

A. J. Bunker \& W. J. M. van Breugel, eds.

\title{
Ionisation, Shocks, and Evolution of the Emission Line Gas of $z \sim 1$ Radio Galaxies
}

\author{
P. N. Best and H. J. Röttgering \\ Sterrewacht Leiden, Postbus 9513, 2300RA Leiden, The Netherlands \\ M. S. Longair \\ Cavendish Astrophysics, Madingley Road, Cambridge CB3 OHE, UK
}

\begin{abstract}
From deep spectroscopic observations of a sample of powerful radio galaxies with redshifts $z \sim 1$, it is found that both the ionisation state and the kinematics of the emission line gas evolve strongly with the size of the radio source. Radio sources with small linear sizes $(\lesssim 150 \mathrm{kpc}$ ) have emission line ratios in agreement with theoretical shock ionisation models whilst those of larger sources match photoionisation predictions. The emission line gas of small sources is also more luminous and has broader line widths and more distorted velocity profiles than that of larger radio sources. The emission line properties of small radio sources appear to be dominated by the shocks associated with the radio source.
\end{abstract}

\section{Introduction}

Powerful radio galaxies possess extremely luminous extended emission line regions, often aligned along the radio axis (McCarthy et al. 1996 and references therein). The source of ionisation of this gas has been a long standing question. Robinson et al. (1987) found that optical emission line spectra of most low redshift $(z \lesssim 0.1)$ radio galaxies are well explained using photoionisation models, and a similar result was found for a composite spectrum of radio galaxies with redshifts $0.1<z<3$ (McCarthy 1993). On the other hand, detailed studies of individual sources (e.g. 3C171; Clark et al. 1998) have revealed features such as enhanced nebular line emission, large velocity widths and ionisation state minima coincident with the radio hotspots, indicating that the morphology, kinematics and ionisation of the gas in some sources are dominated by shocks.

To investigate the importance of shocks in the radio galaxy population as a whole, deep spectroscopic observations have been carried out on a sample of $143 \mathrm{CR}$ radio galaxies with redshifts $0.7<z<1.25$, previously studied in detail using the HST, the VLA and UKIRT (Best et al. 1997). Long slit spectra of the galaxies were taken, with total exposures of 1.5 to 2 hours per source, using ISIS on the WHT (see Best et al. 1999a,b for details). In this contribution; the differences between different radio galaxies in the sample are investigated. Section 2 discusses the ionisation properties of the emission line gas, and Section 3 its hinematical properties. Conclusions are drawn in Section 4. 


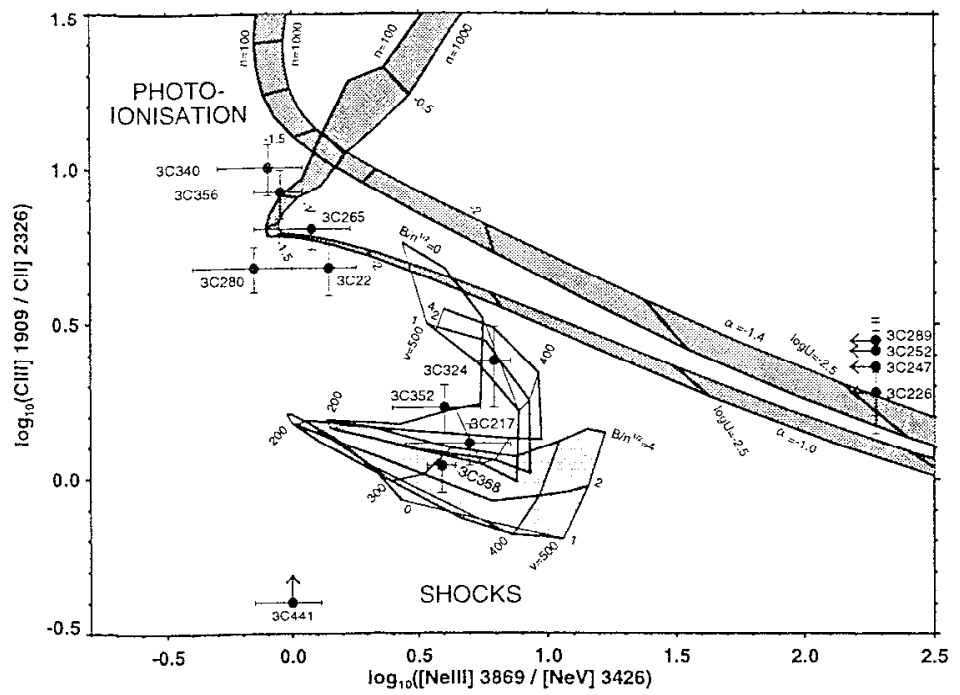

Figure 1. Emission line ratios for the $3 \mathrm{CR}$ radio galaxies, compared with theoretical predictions of photoionisation (upper shaded regions), shock ionisation (lower shaded regions), and shock ionisation including a precursor region (unshaded line region). For the five galaxies at the edges of the plot, no data is available for one of their line ratios.

\section{Ionisation of the emission line gas}

The line ratios of CIII] 1909/ CII] 2326 and [NeIII] 3869 / [NeV] 3426 have been determined for these galaxies. These ratios are particularly useful for ionisation studies because: (i) the two lines in each ratio involve the same element, and so variations in metallicity are not important; (ii) the two lines are close in wavelength, so differential extinction is minimised; (iii) the theoretical predictions of photoionisation and shock ionisation for these line ratios are very different.

Theoretical predictions for these line ratios for photoionisation models have been taken from Allen et al. (1998), assuming illumination of a planar slab of gas by a power-law ( $\alpha=-1.0$ or $\alpha=-1.4$ ) spectrum for a range of ionisation parameters and two different gas densities $\left(n=100\right.$ or $\left.1000 \mathrm{~cm}^{-3}\right)$. Shock ionisation predictions have been derived from the models of Dopita and Sutherland (1996), varying the shock velocity and the 'magnetic parameter' $B / \sqrt{n}$ (see Dopita \& Sutherland for details), and calculating the ratios both with and without the contribution of emission from pre-cursor gas ionised in front of the shock.

The observed line ratios are compared with the theoretical predictions on Figure 1. Four galaxies (3C217, 3C324, 3C352, 3C368) lie in the region of the diagram occupied by shock models, and five (3C22, 3C265, 3C280, 3C340, 3C 356) lie close to the photoionisation predictions. Interestingly, the four sources in the shock region have radio sizes smaller than $115 \mathrm{kpc}\left(\Omega=1, H_{0}=50 \mathrm{~km} \mathrm{~s}^{-1} \mathrm{Mpc}^{-1}\right)$, and the five 'photoionised sources' have larger radio sizes. This is clearer in Figure 2; there is a strong.correlation between the CIII] 1909 / CII] 2326 ratio and the radio size, with small sources having lower ionisation states. 


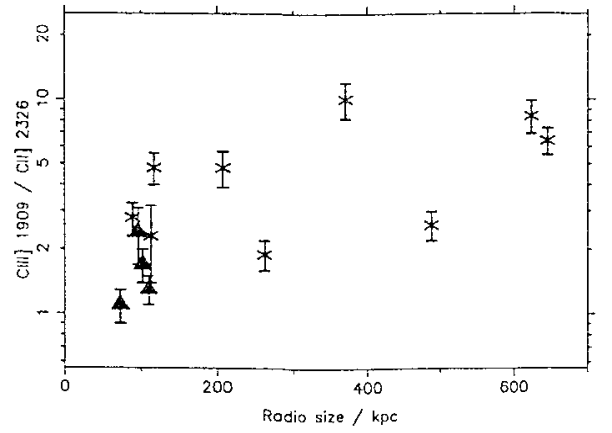

Figure 2. The correlation be: ween the CII 2326/ CII] 1909 emissism line ratio and the projectcol linear size of the radio source. The: four sources lying in the "shocks" region of the line diagnomic diagram. Figure: 1 , are ploted as fillet triangles and the remainder of the galaxiris as asterisks.

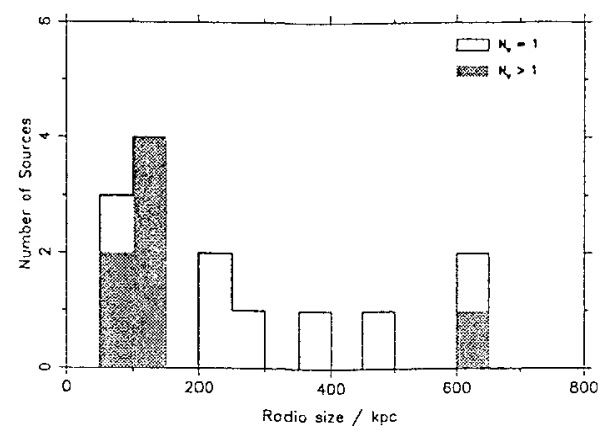

Figure 3. A histogram of the radio size distribution of the sources: separated into sources with smooth velocity profiles $\left(N_{\mathrm{v}}=1\right.$. unbhaked $)$ and these whose profiles are irregula $\left(N_{\mathrm{v}}>1\right.$, shaded $)$.

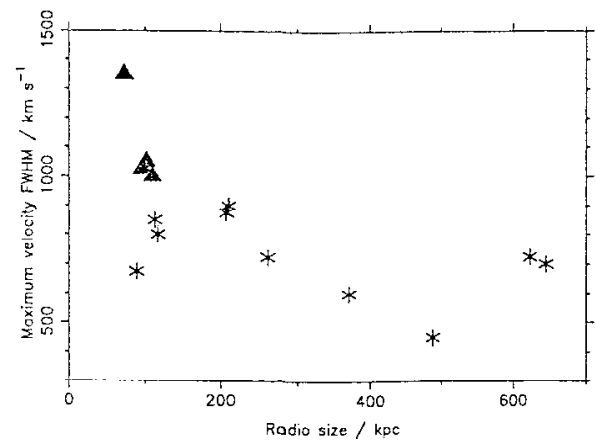

Figure 4. The inverse correlation between the maximum fWH of the [OII] 3727 emission line and the projected linear size of the ridio source. Symbots ith in Finure 2

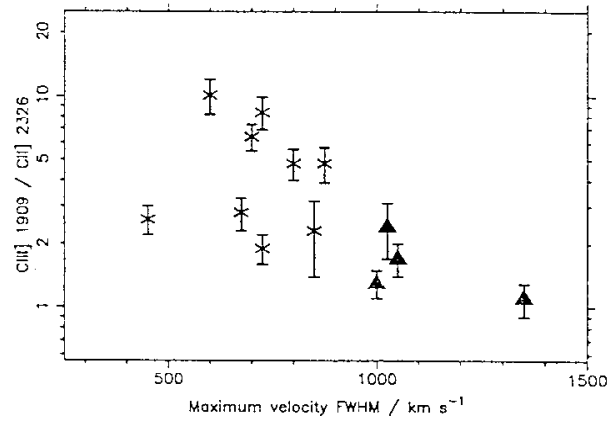

Figure 5. A plot showing the direct ronnection batwextro the ionisation state of the cmission line gas, as indicated by the OII] $2326 /$ CII] 1909 line ratio, and its kinematics in terms of the emission line maximan FWHM. Symbols as in Figure 2.

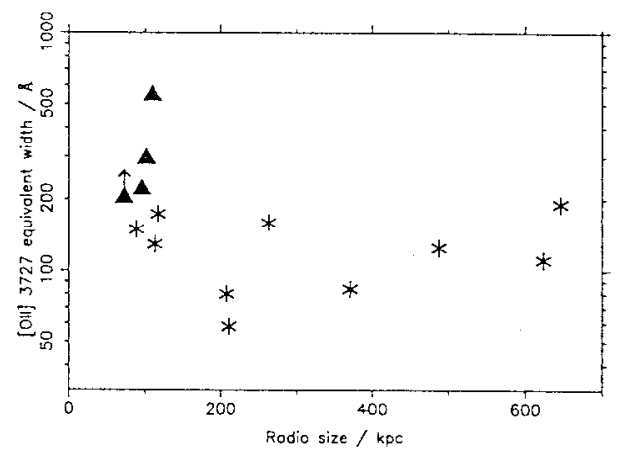

Figure 0. The decrease in the equivalent witth of the [OII] 3727 emission line with increasing size of the radio source. Symbels as in Figure 2. $3 C 368$ is ploted as a lower limit due to the contribution of the M-star to its continum level.

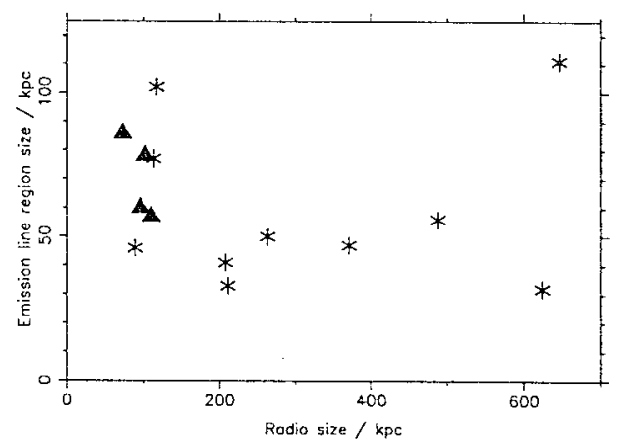

Figure 7. The variation of the linear extent of the [OM] 3727 emission line region with the size of the radio source. Symbols as
in Figure 2 . 


\section{Radio size evolution of the gas kinematics}

Nany hinematical properties of the emission line gas are also found to evolve strongly with the size of the radio source. Figure 3 shows a histogram of radio source sizes, split into those sources whose [OII] 3727 velocity profile along the radio axis is smooth. possibly representing a simple rotating halo, and those sources with distorted velocity profiles. The contrast between the small and large sources is pronounced. Figure 4 shows that the FWHM of the [OII] 3727 emission also decreases with increasing radio size; the four 'shock-dominated' sources in Figure 1 have the highest FWHM. Figure 5 shows the strong interrelation between the kinematical and ionisation properties of these sources. Figure 6 shows that the emission lines of small radio sources are typically more luminous that those of large radio sources; the distribution of equivalent widths is fairly

flat for sources larger than about $150 \mathrm{kpc}$, but small sources often have enhanced emission relative to this level. Figure 7 shows that the physical extent of the emission line region is typically larger for small radio sources.

All of these properties can be well explained in terms of the shocks associated with the radio source (for details see Best et al. 1999b). As the shocks advance through the interstellar medium, the emission line clouds are accelerated by entrainment in the shocked intercloud gas, giving rise to the distorted kinematics and large FWHM observed in the small sources. The shocks compress the clouds lowering their ionisation state, and, if the shock is radiative, then an extra source of ionising photons is also provided. This will boost the emission line luminosities of the small sources. The larger extent of the emission line regions in small sources arises since emission line clouds at radii $\gtrsim 30 \mathrm{kpc}$ only become luminous during the time when the radio shocks are passing through them and providing a local source of ionising photons.

\section{Conclusions}

Both the kinematics and the ionisation state of the emission line gas of powerful $z \sim 1$ radio sources evolve strongly with radio size; the gas properties of small sources appear to be dominated by the radio source shocks. This strong radio size evolution mirrors that of the continuum alignment effect (e.g. Best et al. 1996), suggesting that the later may also be associated with radio source shocks.

\section{References}

Allen M.G., Dopita M.A., Tsvetanov Z.I., 1998, ApJ, 193, 571

Best P.N., Longair M.S., Röttgering H.J.A., 1996, MNRAS, 280, L9

Best P.N., Longair M.S., Röttgering H.J.A., 1997, MNRAS, 292, 758

Best P.N., Röttgering H.J.A., Longair M.S., 1999a,b, MNRAS, in press

Clark N., Axon D., Tadhunter C., Robinson A., O'Brien P., 1998, ApJ, 494, 546

Dopita M.A., Sutherland R.S., 1996, ApJS, 102, 161

McCarthy P.J., Baum S.A., Spinrad H., 1996, ApJS, 106, 281

McCarthy P.J., 1993, ARAA, 31, 639

Robinson A., Binette L., Fosbury R., Tadhunter C., 1987, MNRAS, 227, 97 
The Hy Redshift Universe

ASP Conference Series. Vol. 193. 1999

A. J. Bunker 6 W. J. M. van Breugel, eds.

\title{
High- $z$ Radio Galaxies and the 'Youth-Redshift Degeneracy'
}

Fatherine M. Blundell and Steve Rawlings

Oxford University Astrophysics, Keble Road, Oxford, OX1 3RH, UK

\begin{abstract}
We discuss a unifying explanation for many 'trends with redshift' of radio galaxies which includes the relevance of their ages (time since their jet triggering event), and the marked dependence of their ages on redshift due to the selection effect of imposing a flux-limit. We briefly describe some important benefits which this 'youth-redshift degeneracy' brings.
\end{abstract}

\section{Trends in COSMIC EPOCH or trends in SOURCE AGE?}

With radio source ages of at most a few $10^{8}$ years, the huge decrease in comoving space density of luminous radio-sources from redshift $z=2$ to $z=0$ (Longair 1966, Dunlop \& Peacock 1990) is not due to a decline in luminosity of individual objects. However, it does not follow that one can ignore the luminosity evolution of the individual radio-sources and their ages in all studies of their 'cosmic evolution'.

Application of a flux-limit in any model of radio-galaxy evolution in which the luminosity $(P)$ decreases with time means that all observable high-redshift radio-galaxies must be seen when the lobes are young $\left(<10^{7}\right.$ years old; see Figure 1). This mechanism (for details see Blundell \& Rawlings 1999) is responsible for the highest-redshift members of any low-frequency survey of radio-sources (such as $3 \mathrm{C}$ ) having significantly more powerful jets and being significantly younger than the more local members. This physical mechanism plays a crucial role in explaining 'trends with redshift', without invoking any intrinsic or strong environmental differences between the radio-galaxies seen at low- $z$ and high-z; we discuss five such trends below.

(1) The linear size evolution which is observed in low-frequency flux-limited samples of classical double radio-sources (Kapahi et al. 1987, Barthel \& Miley 1988, Blundell, Rawlings \& Willott 1999) arises because the high-z sources are younger, hence tend to be shorter. Falle (1991) has shown that higher jet-powers $(Q)$ increase the rate at which radio-source lengths $(D)$ grow with time $t$. This positive dependence on jet-power of the rate at which the lobe-lengths grow contributes to the linear size evolution being as mild as it is (Blundell, Rawlings \& Willott 1999).

(2) Barthel and Miley (1988) had suggested that higher redshift environments are denser and more inhomogeneous than at low redshift since they found increased distortion in the'structures of their high- $z$ sample of steep-spectrum quasars compared with their low- $z$ sample. Young radio sources which have re- 

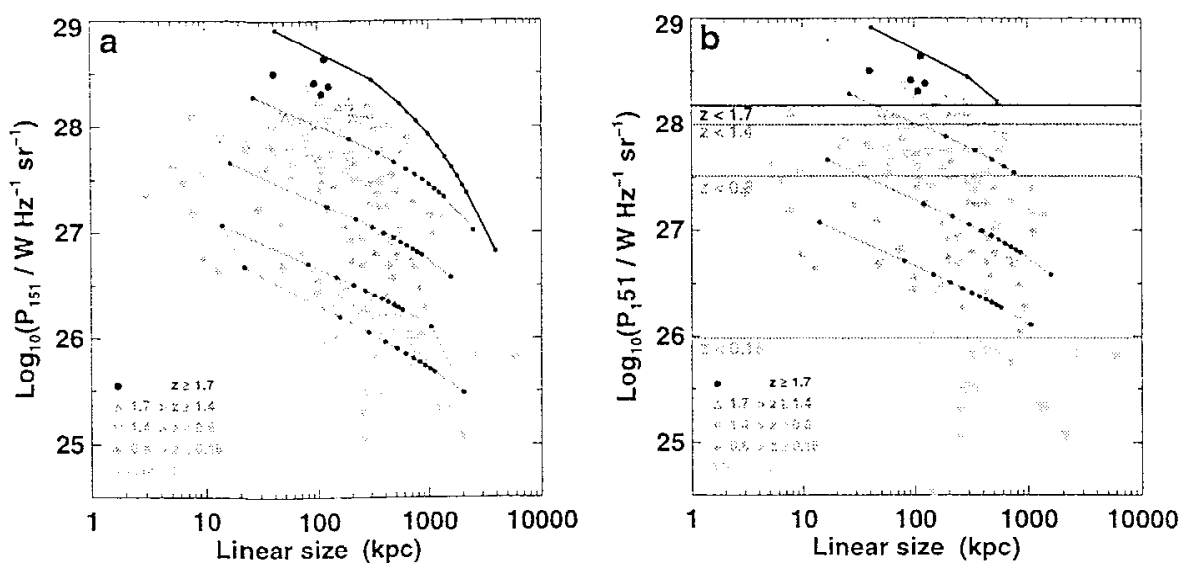

Figure 1. Overlaid on the ' $P-D$ ' plane for the $3 \mathrm{C}$ sample in a are model tracks tracing out the evolution of four example radio sources in luminosity and linear size with, from top to bottom, $Q=5 \times 10^{39}$ $\mathrm{W}$ at $z=2, Q=1 \times 10^{39} \mathrm{~W}$ at $z=0.8, Q=2 \times 10^{38} \mathrm{~W}$ at $z=0.5$ and $Q=5 \times 10^{37} \mathrm{~W}$ at $z=0.15$. The dashed line indicates how the lower track luminosity reduces by $<$ half an order of magnitude if the ambient density becomes an order of magnitude lower. In $\mathbf{b}$ the horizontal lines represent the luminosities at which the flux-limit of 12 Jy takes its effect at the different redshifts indicated. A combination of the dramatically declining luminosity-with-age of the high- $Q$ sources, their scarcity in the local Universe, together with the harsh reality of the flux-limit means that very powerful sources with large linear sizes are rarely seen.

cently undergone a jet-triggering event [assumed to be a galaxy-galaxy merger (Sanders et al. 1988)] may have the passage of their jets considerably more disrupted where there is a higher density and greater inhomogeneity in the ambient post-recent-merger environment. A general trend of denser inter-galactic environments at high-z cannot be inferred from their result.

(3) Where the alignment effect is caused by star-formation, it will be more easily triggered close in to the host galaxy or within the product of a recent merger than at distances further out sampled by the head of an expanding radio-source later in its lifetime. Where the alignment effect is caused by dustscattered quasar light, the certain youthfulness of distant radio-galaxies alleviates the near discrepancy (De Young 1998) between radio-source ages and the time-scale for which dust grains can survive in the presence of shocks caused by the advancing radio-jets. The 'youth-redshift ( $\mathrm{YZ})$ degeneracy' is consistent with Best et al's (1996) finding that the smallest sources in a sample of $z \sim 1$ radio-galaxies (all with very similar luminosities) are those which are most aligned with optical emission. Indeed Best et al. suggest this as the explanation of their observation. 
(1) Garrington \& Conway (1991) have found a tendency for depolarisation to be higher in sources with higher $z$. Objects with higher $z$ which are younger will be in much more recently merged environments with the consequence that inhomogeneities in density or magnetic field will more readily depolarise the synchrotron radiation from the lobes. Moreover, higher- $z$ sources being younger and somewhat shorter will be closer in to the centre of the potential well. The higher density in this region will enhance the observed depolarisation.

(5) Many of the highest- $z$ radio-galaxies have gas masses comparable to gas-rich spiral galaxies (Dunlop et al 1994, Irughes et al 1998) and inferred starformation rates which, in the local Universe, are rivalled only by galaxy-galaxy mergers like Arp 220 (Genzel et al 1998). If high-z objects are being viewed during a similar merging of sub-components, the associated star formation could be responsible for a significant fraction of the stellar mass in the remnant galaxy. Since the high- $z$ radio-galaxies, those which have been detected by Hughes et al (1988) using SCUBA, are necessarily young ( $<10^{\top}$ years, see Fig. 1 ), and since the whole merger must take a few dynamical crossing times, or $10^{8-9}$ years, the implication is that the event which triggered the jet-producing central engine is synchronised with massive star formation in a gas-rich system, perhaps as material streams towards the minimum of the gravitational potential well of the merging system.

The XZ degeneracy may help explain why few lower- $z$ radio-galaxies show similarly large (rest-frame) far-infrared luminosities compared to the high- $z$ population: they are being observed significantly longer after the jet-triggering event.

\section{The elusiveness of cosmic parameters}

A variant on Fig. 1 also includes the location on the $P-D$ plane of the most extreme redshift $(z>3)$ radio galaxies known. Such a figure may be found in our recent letter to Nature (Blnndell \& Rawlings 1999). When these are plotted for different cosmological models, significant though subtle differences emerge for the high- $\approx$ sources which illustrate the difficulty of distinguishing between different underlying cosmic geometries when more dramatic influences such as the $\mathrm{YZ}$-degeneracy, and variations in source environments, are at work. In rough order of importance to the distribution of sources on the $P-D$ plane, we have:

1. What is the finding frequency of the survey in the rest-frame of the source? (see Blundell, Rawlings \& Willott [astro-ph/9907418])

2. What is the flux-limit? This excludes faint/old objects at high redshift.

3. What is $Q$, the jet-power?

4. What is the ambient density into which the radio-source is expanding?

5. What density profile is the radio-source expanding into?

6. What is the cosmic geometry?

The use of double radio sources as 'standardizable' rods (e.g. Daly 1994) is beyond reach. 


\section{The benefits of the YZ degeneracy}

Since extreme-z radio galaxies are young, all with similar $Q$, they deliver the fine time-resolution required for the solution of problems which it may be difficult to study with objects like optically-selected quasars, whose ages are indeterminate: examination of the environments of distant radio galaxies provides a snapshot of the host galaxy evolutionary status just after the jet-triggering event.

At redshift $\sim 4$ we observe radio galaxies $\sim 1$ Gyr after the Big Bang and in environments which saw a jet-triggering event no earlier than $10^{7}$ years prior to that. This time-step is over an order of magnitude smaller than the dynamical crossing time of a massive galaxy, and 2 orders of magnitude smaller than the age of the Universe at the epochs probed, giving fine time-resolution essential to any study of triggering (and hence merging) rates at early cosmic epochs.

The YZ-degeneracy is unavoidable, and implies a wide and increasingly illdefined gulf between the 'luminosity function' and the 'birth function' of radiogalaxies. The luminosity function is a super-set of the radio-sources which make it above the various flux-limits. The 'birth function' measures the trigger rates of radio galaxies. We have carefully developed a model for radio-source evolution which for the first time in radio-source modelling incorporates the role played by the hotspots. This, together with a proper treatment for the interception of radio galaxies born at successively lower $z$ with our light-cone, enables us to perform Monte-Carlo simulations to constrain the birth-function of radio galaxies out to high-z (Blundell, Rawlings \& Willott, in prep).

\section{References}

Barthel, P.D. \& Miley, G.K. Nature 333, 319-325 (1988).

Best, P.N., Longair, M.S. \& Röttgering, H.J.A., MNRAS, 280, 9L-12L (1996).

Blundell, K.M. \& Rawlings, S., Nature, 399, 330-332 (1999).

Blundell, K.M., Rawlings, S. \& Willott, C.J. AJ, 117, 677-706 (1999).

Blundell, K.M., Rawlings, S. \& Willott, C.J. in 'Perspectives in Radio Astronomy' eds M.P. van Haarlem \& J.M. van der Hulst. [astro-ph/9907418]

Daly, R.A. ApJ, 426, 38-50 (1994).

De Yuung, D.S. ApJ, 507, 161-172 (1998).

Dunlop, J.S., \& Peacock, J.A., MNRAS, 247, 19-42 (1990).

Dunlop, J.S., Hughes, D.H., Rawlings S., Eales, S.A., \& Ward, M.J., Nature, $370,347-349$ (1994)

Falle, S.A.E.G., MNRAS, 250, 581-596 (1991).

Garrington, S.T. \& Conway, R.G. MNRAS, 250, 198-208 (1991).

Genzel, R., Lutz, D. \& Tacconi, L. Nature, 395, 859-862 (1998).

Hughes, D.H., Dunlop, J.S., Archibald, E.N., Rawlings, S. \& Eales, S.A. in 'The birth of galaxies' (ed B. Guiderdoni) (1998)

Kapahi, V.K., Subrahmanya, C.R. \& Kulkarni, V.K. JAA, 8, 33-50 (1987).

Longair, M.S. MNRAS, 133, 421-436 (1966).

Sanders, D.B., Soifer, B.T., Elias, J.H., Madore, B.F., Matthews, K., Neugebauer, G. \& Scoville, N.Z. ApJ, 325, 74-91 (1988). 
The Hy Redshift Universe

ASP Confcrence Series, Vol. 193, 1999

A. J. Bunker $\because$ W. J. M. van Breugel, eds.

\section{The Ages of Compact, Steep-Spectrum Quasars}

Eric de Silva and Richard Saunders

Astrophysics Group, Cavendish Laboratory, Madingley Road, Cambridge, CBз OHE, UK

Joanne Baker

Astronomy Department, University of California, Berkeley, CA 94720

Richard Hunstead

School of Physics, University of Sydney, NSW 2006, Australia

Abstract. Our results reveal a strong correlation between linear size and age for both Compact, Steep-Spectrum (CSS) and non-CSS quasars from the Molonglo Quasar Sample (MQS). CSSs $(<1 \mathrm{kpc}$ to $25 \mathrm{kpc}$ ) having ages ranging from $10^{3}$ years to $10^{5}$ and non-CSSs (30 kpc to 500 $\mathrm{kpc}$ ) having ages between $10^{5}$ years and $10^{7}$ years.

\section{Compact, Steep-Spectrum (CSS) quasars}

CSSs are radio sources with steep radio spectra - we define steep as $\alpha_{\text {radio }}>$ 0.5 where $S_{\nu} \propto \nu^{-\alpha}$. They are less than $25 \mathrm{kpc}$ in extent (the approximate size of the host galaxy) and make up about $25 \%$ of low-frequency radio-selected quasars. Complex and distorted jets and large rotation measures are common. Discussions in the literature suggest CSSs are frustrated, such that the radio jets are confined to galactic dimensions by unusually dense or clumpy ISM, or that they are simply young.

\section{CSS quasars from the Molonglo Quasar Sample (MQS)}

The MQS, descibed by Kapahi et al. (1998) contains the largest well defined sample of CSS quasars to date. As a subset of the Molonglo Reference Catalogue, the MQS is complete to $0.95 \mathrm{Jy}$ at $408 \mathrm{MHz}$. The $111 \mathrm{MQS}$ quasars have redshifts spanning the range $0.1<z<3.0$ and of these 24 are CSSs. We have complete optical identifications and follow-up data include high resolution VLA images at $5 \mathrm{GHz}$ and MERLIN images for the CSSs at $1.6 \mathrm{GHz}$, optical and infrared images (AAT, CTIO \& ESO), infrared spectroscopy (CTIO), ROSAT fluxes and $1 \AA$ resolution optical spectroscopy. Examples of a MERLIN radio map and AAT optical spectrum are shown in Figure 1. 

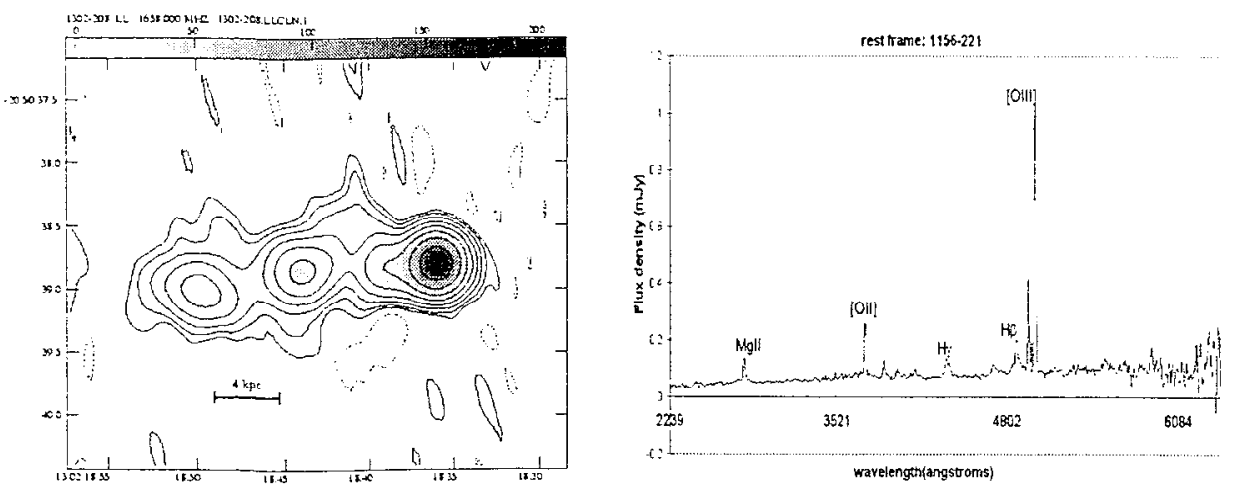

Figure 1. Typical CSS radio image and optical spectrum

\section{Jet kinetic power versus emission line luminosity}

Rawlings \& Saunders (1991) evaluated the equipartition energy $E$ of a sample of extended double radio galaxies and quasars and determined the lobe age I' of the sources in the sample from, inter alia, the curvature of their radio spectra. From these they were able to calculate the jet kinetic power $Q$ for each object in the sample using $Q=E / T$. By finding the total power radiated from the nucleus $L_{\text {tot }}$, which is mainly from UV \& optical lines and continuum, for each source, and plotting the bulk jet kinetic power $Q$ against this total narrow-line luminosity $L_{\text {tot }}$, they found a proportionality across four orders of magnitude in Q. They further found that jet formation is remarkably efficient: $Q \simeq L_{t o t}$.

\section{A method for calculating ages}

We assume that $Q \simeq L_{t o t}$ for CSSs as well as larger sources. Then $\mathrm{T}=\mathrm{E} / L_{t o t}$. We estimate $\mathrm{E}$ by the usual equipartition method, taking the line-of-sight depth of a radio component as its projected width. We assume that there are no fast protons, the filling factor is unity and $\nu_{\min }=100 \mathrm{MHz} \& \nu_{\max }=10 \mathrm{GHz}$; relaxing these assumptions makes little difference. We measured [OII] $\lambda 372.7 \&$ [OIII] $\lambda \lambda 495.9,500.7$ from optical spectra to give the total narrow line luminosity as in Rawlings \& Saunders (1991) and calculated $\mathrm{L}_{\text {tot }}$ allowing for contributions from X-ray to FIR and continuum luminosities by assuming a covering factor of $1 \%$. Finally we used $\mathrm{T}=\mathrm{E} / L_{\text {tot }}$ to give the age of the lobes and hence the approximate age of the source.

\section{Radio luminosity-linear size plane: the P-D diagram}

Figure 2 is the P-D diagram of the MQS quasars and indicates those for which we have produced age estimates. The source sizes range from sub-kpc to $\mathrm{Mpc}$. 


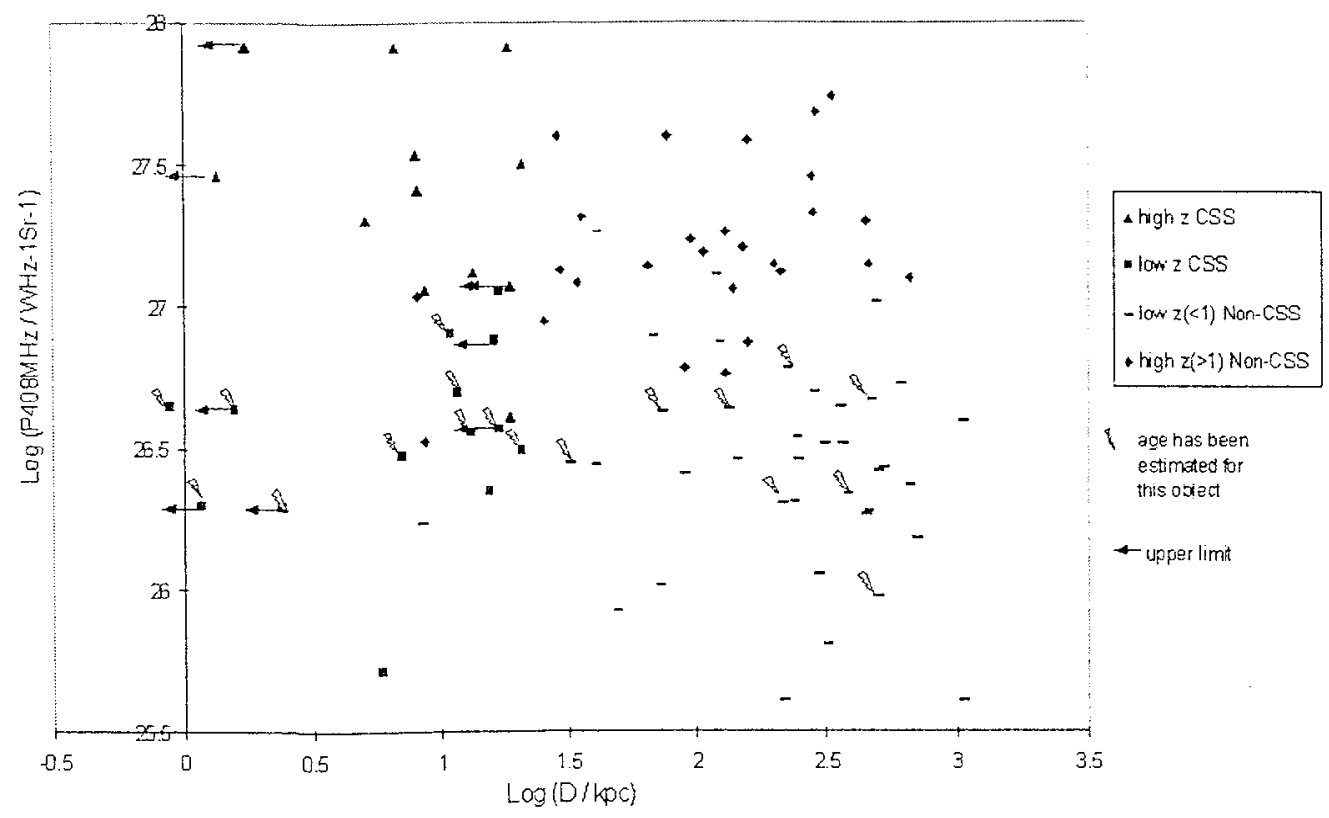

Figure 2. P-D diagram for Molonglo CSS \& non-CSS quasars.

\section{Results: ages of CSSs \& their relationship to non-CSSs}

Figure 3 shows a very tight correlation between source size and age. Clarly; CSSs are younger than the larger non-CSSs in the sample. In fact the best fit is $D \propto t^{0.7}$. There is a clcar continuity between CSS \& non-CSS sources implying that both have the same central engines. Note that if we have, say, underestimated ages by underestimating $E$ or overestimating $L_{\text {tot }}$, then this will have been done systematically and therefore change only the scaling of the plot but not affect its implications.

\section{Conclusions}

1. CSSs are young, the frustration scenario not being the dominant effect. This is consistent with the age found for $0710+439$ by Owsianik et al. (1998) and other attempts to measure CSS spectral ages such as Murgia et al. (1999).

2. CSSs evolve into larger sources as they get older.

3. CSSs have the same central engines as larger sources.

\section{Notes}

Recent work by Best \& Longair (see contribution by Best in these proceedings) implies that shock ionisation is dominant over photoionisation in compact sources. This is confirmed in the Molonglo sample, yet taking this into account 


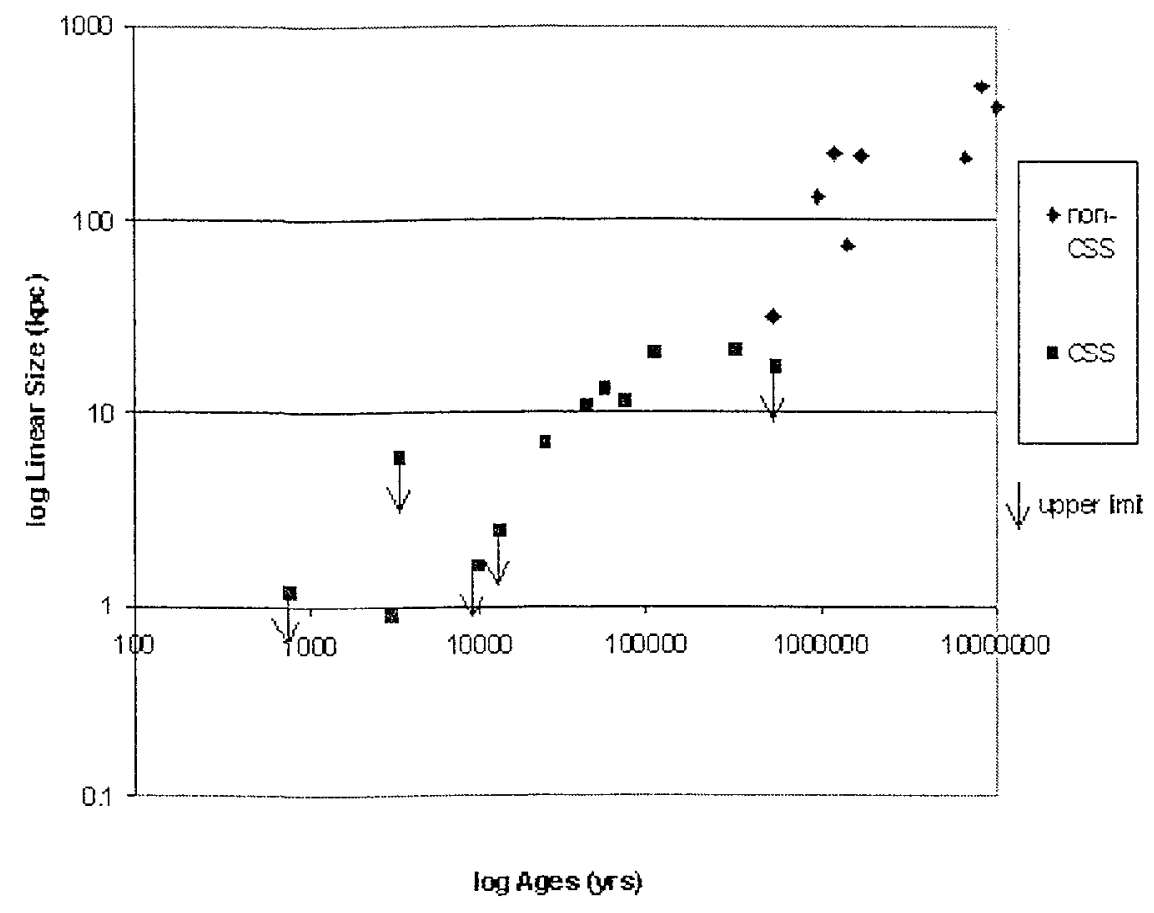

Figure 3. age vs linear size plot

makes little difference to the final values of the ages. Observations of the CSSs have just been made with MERLIN at $5 \mathrm{GHz}$. With these we will have the spectral information necessary for estimating the contribution of beamed emission as in Baker (1997) and hence the orientation of the CSS quasars.

\section{References}

Baker, J. C. 1997, MNRAS, 286, 23

Kapahi, V. K., Athreya, R. M., Subrahmanya, C. R., Baker, J. C., Hunstead, R. W., McCarthy, P. J., \& van Breugel, W. 1998, ApJS, 118, 327

Murgia, M., Fanti, C., Fanti, R., Gregorini, L., Klein, U., Mack, K.-H., \& Vigotti, M. 1999, A\&A, 345, 769

Owsianik, 1. \& Conway, J. E. 1998, A\&A, 337, 690 \& 336L, 370

Rawlings, S. \& Saunders, R., 1991, Nature, 349, 138 
The Hy Redshift Universe

ASP Conference Series. Vol. 193. 1999

A. J. Bunker \& W. J. M. van Breugel. eds.

\title{
Star Formation and Polarization in Aligned Radio Galaxies
}

\author{
D. S. De Young \\ NOAO/KPNO, Tucson, AZUSA
}

\begin{abstract}
The alignment of optical emission along the axis of radio emission in high redshift radio galaxies is now commonly observed. In many cases this aligned component can be shown to be significantly polarized, a result that has led to models for the emission that employ scattering of light emitted from the AGN. However, it can be shown that a population of pre-existing dust grains that can act as a scattering medium is unlikely to survive the passage of the shock associated with the jet that forms th radio source. For most configurations the grains are destroyed as a scattering population by sputtering processes in a time much less than the minimum radio source lifetime of $\sim 10$ million years. Thus polarization due to scattering by an in-situ population is somewhat problematic. A promising path for providing the necded grain population is that due to grain production from massive stars whose formation is triggered by the passage of the shock associatcd with the radio jet. Using a Salpeter IMF and current estimates of the dust yield from supernovae, it is shown that the rcquisite mass in dust as a scattering population can bc produced in the time required.
\end{abstract}

\section{Introduction}

The "alignment effect" occurs in high redshift radio galaxies wherein the emission line and optical continuum emission show an alignment along the axis of the radio source. It has been known for some time that in many cases - but not all - the optical continuum is polarized (e.g., Cimatti et al. 1993). A natural explanation of this is that some portion of the aligned continuum is UV light emitted by that AGN that is then scattered by an extended scattering medium extending up to $100 \mathrm{kpc}$ from the nucleus of the parent galaxy. Both electrons and dust grains have been proposed as a scattering medium (see e.g., Dey et al. 1997), and both mechanisms have some problems associated with them. The most significant problems with hot electrons is that a significant mass in electrons is required to match the observed polarization - so much so that in many cases the limits on total electron mass from $x$-ray data are violated. Because of this, the use of dust as a scattering medium has received a great deal of interest. Use of dust, in particular graphite and silicate grains, as a scattering population requires that several boundary conditions be met. Assuming an optically thin dust population with Mie scattering, Cimatti et al. derive minimum total dust masses of $\sim 10^{8}$ solar masses for a spherical dust mass distribution extending 
out to $\sim 100 \mathrm{kpc}$. This implies $\sim 10^{10}-10^{11} M_{5}$ in gas out to $100 \mathrm{kpc}$; not an unreasonable requirement. Depending upon the solid angle subtended by the radio source and the aligned optical emission, this then implies that a minimum. mass of $10^{6}-10^{7} M_{\odot}$ in dust grains as the actual scattering centers is required.

\section{Dust Survival}

The distribution of the optical emission implies that the scattering centers lie behind the tip of the jet associated with the radio "hot spots". Hence the grains must survive the passage of the strong shock associated with the radio source, and this survival must last at least as long as the age of the radio source. Radio jet propagation speeds, though usually inferred, can sometimes be measured on $\mathrm{kpc}$ scales, and in general propagation speeds of $10^{9}-10^{10} \mathrm{~cm} / \mathrm{s}$ are implied by the date (e.g., Liang, 1996). Thus for the high redshift radio galaxies with minimum sizes of $100 \mathrm{kpc}$ or more, grains must survive at least for $10^{6}-10^{7}$ yr. The effects of shocks on grains in clouds have been investigated by many authors (e.g., Tielens et al. 1994 and references therein), although always in the context of weaker and multiple SNR shocks in the ISM. Because graphite grains are the most robust, only this species need be considered; if graphite grains are destroyed, then the silicates and delicate ices will not survive as well. Initial grain sizes from $100 \mathrm{~A}$ to $2500 \mathrm{~A}$ are most relevant; use of the MRN distribution implies that almost all the grains are small. Thus grains of size $\sim 500 \dot{A}$ and smaller must survive for grains to act as effective scattering centers. The effects of shocks with the above speeds on a grain population such as this has been considered in some detail by De Young (1998) under a wide variety of conditions. This calculation shows that grains survive as a scattering population only under very special conditions; only if they are protected in dense clouds and only if the clouds are struck by slow moving shocks. Only then is the post shock flow slow enough and cool enough, with subsequent rapid grain deceleration and gas cooling, to halt grain ablation in time.

\section{How to Make Post-Shock Grains}

If the use of a pre-existing grain population to act as scattering centers seems unlikely, due either to the difficulty of placing $10^{8} M_{\odot}$ of grains out to $\sim 100 \mathrm{kpc}$ in the first place or to their subsequent destruction as described here, then the remaining options are to replenish the grain supply, to transport the grains out behind the radio source, or to produce them in situ. One can easily show that "sweeping in" more clouds with grains into the volume behind the radio source has serious problems both with grain survival and especially with the timescales - the replacement rate for clouds in virial equilibrium is too slow. Grains conld in principle be transported out from dense and dusty regions inside the galaxy via mass entrainment in the mixing layer that must surround the outflowing jet. While the mass entrainment rate for such a process is adequate (De Young 1986), the survival of grains in this environment has yet to be calculated. Of key importance is the survival of a dense cloud with grains as it is accelerated in the mixing layer with turbulent shear. In situ grain production could occur via a population of massive stars whose birth is triggered by the passage of the radio 
jet. Dwek (1998) has recently shown that about $1 M_{\odot}$ of dust can be produced per supernova event, and thus dust production can begin about $10^{6}$ I after the passage of the jet associated with the radio source. With a Salpeter IMF and upper and lower bounds on the mass function of 100 and 0.1 solar masses, the required amount of dust mass can be made by a total stellar population of $\sim 10^{9} M_{\odot}$. This number arises via using the conservative estimate that only those stars with masses in excess of 15 solar masses produce a supernova that gives rise to one solar mass of dust. This total mass in stars is consistent with models of jet induced star formation (e.g., Rees 1989, Begelman \& Cioffi 1989 , De Young 1989) and also with the estimates of star formation in high redshift galaxies derived from observations (e.g., Chambers et al. 1990). In addition, the observed dilution of the UV continuum that produces the lower polarization percentages could arise from the remnant population of young stars triggered by the passage of the radio jet.

\section{References}

Begelman, M., \& Cioffi, D. 1989, ApJ,345,L21

Chambers, K.C., Miley, G.K., \& van Breugel, W.J.M. 1990, ApJ,363,21

Cimatti, A., di Serego Alighieri, S., Fosbury, R.A.E., Salvati, M., \& Taylor, D. 1993, MNRAS,264,421

Dey, A., Cimatti, A., van Breugel, W., Antonucci, R., \& Spinrad, H. 1996, ApJ,465,157

De Young, D.S. 1980, ApJ,307,62

De Young, D.S. 1989, ApJ,342,L59

De Young, D.S. 1998, ApJ,507.161

Draine, B.T., \& Salpeter, E.E. 1979, ApJ.231,438

Dwek, E. 1998, ApJ,501,643

Liang, R. 1996, in Energy Transport in Radio Galaxies and Quasars, ed. P. Hardee, A. Bridle, \& A. Zensus (San Francisco: ASP) 241

Rees, M.J. 1989, MNRAS,239,1p

Tielens, A.G., McKee, C.F., Seab, C.G., \& Hollenbach, D.J. 1994, ApJ,431,321 

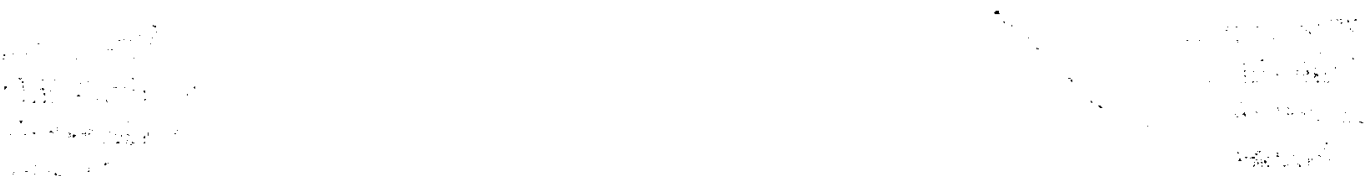
The Hy Redshift Universe

ASP Conference Series, Vol. 193, 1999

A. J. Bunker 6 W. J. M. van Breugel, eds.

\title{
The Nature of the Compact/Symmetric Near-IR Continuum Source in $4 \mathrm{C} 40.36$
}

\author{
F. Fgami ${ }^{1}$, L. Armus ${ }^{2}$, G. Neugebauer ${ }^{1}$, B. T. Soifer ${ }^{1}$, A. S. Evans ${ }^{3}$, and \\ T. W. Murphy Jr. ${ }^{1}$ \\ 1. 320-47 Downs Laboratory of Physics, California Institute of \\ Technology, Pasadena, CA 91125 \\ 2. SIRTF Science Center, IPAC 100-22, California Institute of \\ Technology, Pasadena, CA 91125
}

3. Department of Physics and Astronomy, State University of New York at Stony Brook, Slony Brook, NY 11794-3800

\begin{abstract}
Using NICMOS on HST, we have imaged the emission-line nebulae and the line-free continum in $4 \mathrm{C} 40.36$, a ultra-steep spectrum FR 11 radio galaxy at $z=2.269$. The line-free continuum was found to be extremely compact and symmetric while the emission-line nebulae seen in $\mathrm{H} \alpha+[\mathrm{N}$ II] show very clumpy structures spreading almost linearly over $16 \mathrm{kpc}$. However, this linear structure is clearly misaligned from the radio axis. The SED of the line-free continuum is very flat $\left(f_{\nu} \propto \nu^{-0.5}\right)$, suggesting that if the continuum emission is produced by a single source. it is likely to be a young bursting stellar population or scattered AGN light. However, because of the lack of a line-free optical image with a comparable spatial resolution, we cannot exclude the possibility that the observed SED is a composite of a young blue population and an old red population.
\end{abstract}

\section{Introduction}

The compact/symmetric morphology of high- $z$ radio galaxies seen in the near-IR is often taken as the evidence that radio galaxies contain an old, dynamically relaxed stellar population, whose slow passive evolution is supposed to maintain the tight $K-z$ relation over a wide redshift range (Lilly \& Longair 1984; Lilly 1989; Rigler et al. 1992). A consequence of this picture is that radio galaxies are old, and that the bulk of their mass must have been assembled at high redshifts.

On the other hand, near-IR spectroscopic studies of high- $z$ radio galaxies have shown that when the near-IR broad-band magnitudes are corrected for strong line emissions, the continuum color of some high- $z$ radio galaxies becomes extremely blue, which prompted an interpretation that some of these galaxies might be extremely young protogalaxies (Eales \& Rawlings 1993).

Here, we present high-resolution HST/NICMOS images of $4 \mathrm{C} 40.36$, an ultra-steep spectrum FR II radio galaxy at $z=2.269$ (Chambers, Miley, \& van Breugel 1988). In addition, we also present a low-resolution $(R \sim 80) H+K^{\prime}$ 


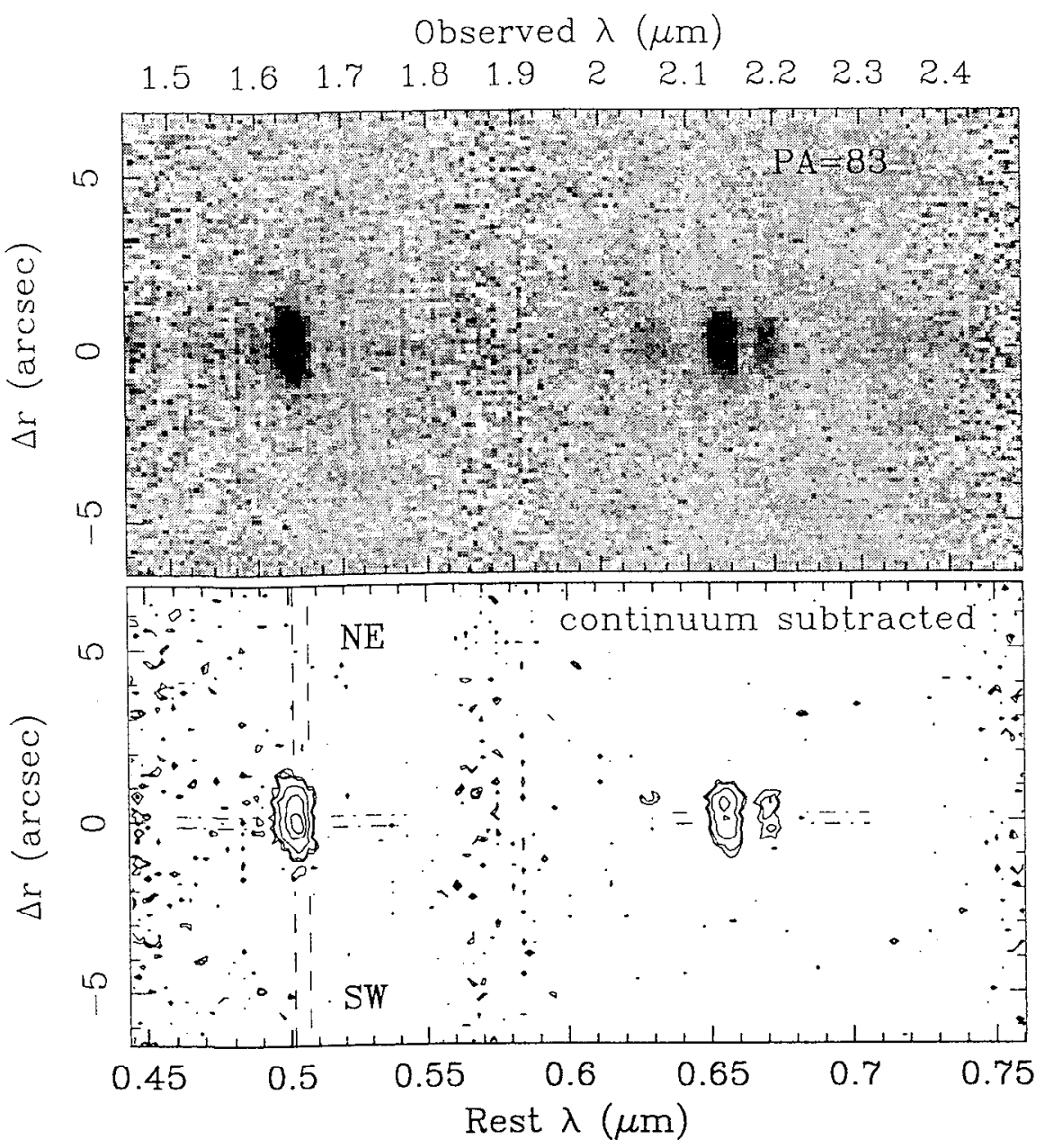

Figure 1. A near-IR NIRC low-resolution $(R \sim 80)$ grism spectrum of $4 \mathrm{C} 40.36$. The PA of the slit corresponds to that of the radio axis $\left(83^{\circ} \mathrm{E}\right.$ of $\mathrm{N}$, shown in Figure 2). The slit width is $0 . \prime 6$. The emission lines visible in the spcctrum are [O III], [O I], $\mathrm{H} \alpha+[\mathrm{N} I \mathrm{II}]$, and [S II] from left to right.

band spectrum of $4 \mathrm{C} 40.36$ taken with Keck/NIR.C. By combining the imaging data and spectrum, we are able to estimate the overall line contribution to the $H$ - and $F$-band fluxes, and obtain the morphology of the emission-line gas and the continuum in $4 \mathrm{C} 40.36$.

\section{The Strong Emission lines and Continuum Color of $4 \mathrm{C} 40.36$}

Since a number of strong restframe-optical emission lines are redshifted" into. the near-IR bands at this redshift, the flux contribution from these lines must be first cvaluated. The Keck/NIRC spectrum clearly shows the strong [O III], $\mathrm{H} \alpha+[\mathrm{N} \mathrm{II}]$, and [S II] lines (Figure 1). From this spectrum, we estimate that the line contribution is $60 \%$ in the $H$ band and $70 \%$ in the $K$ band, respectively. 

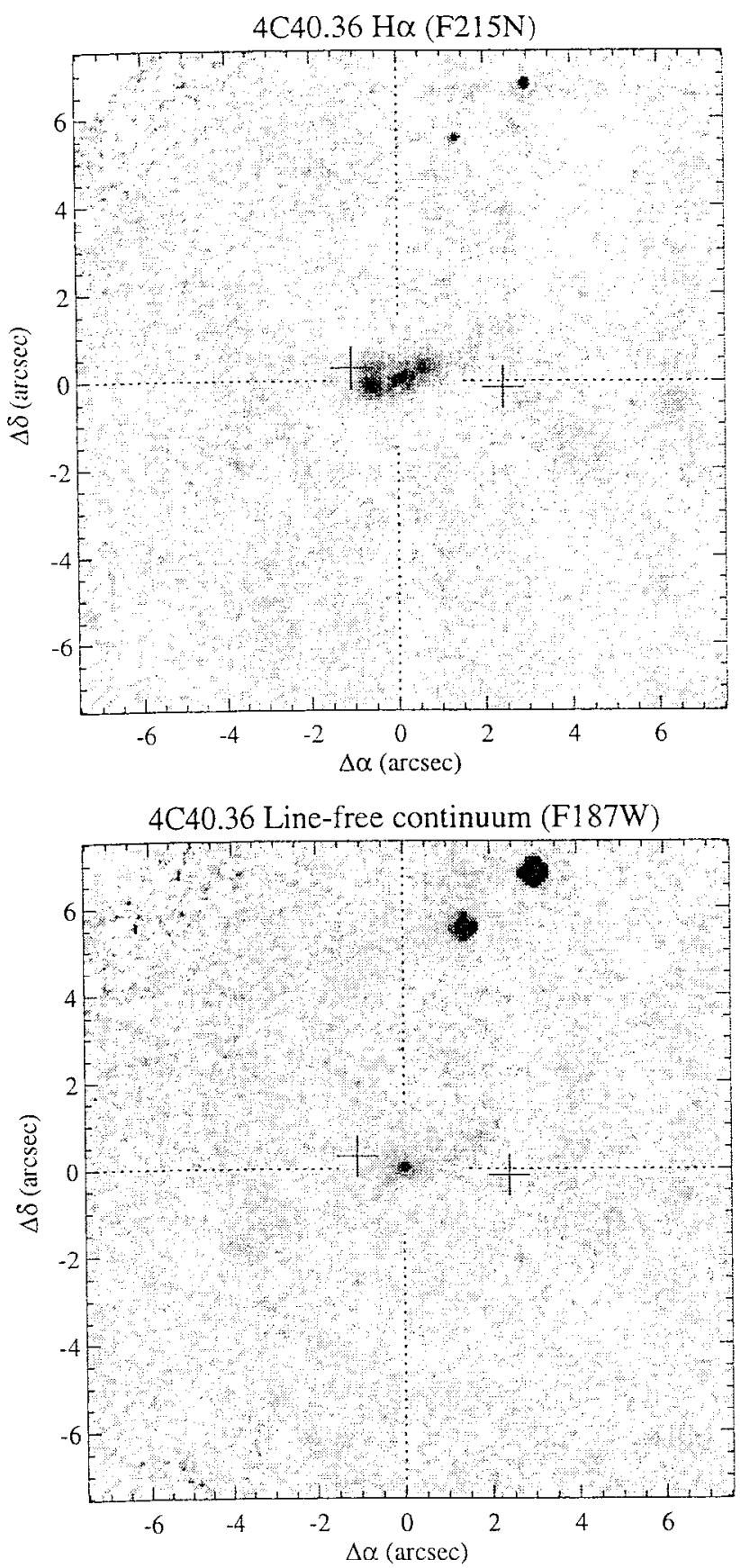

Figure 2. Upper: A narrow-band $\mathrm{H} \alpha$ image of $4 \mathrm{C} 40.36$ taken with the NIC 2 camera and the F $215 \mathrm{~N}$ narrow-band filter. The pixel scale is $0.075^{\prime \prime} /$ pixel. The crosses indicate the positions of the radio lobes; Lower: A line-free continum inage of $4 \mathrm{C} 40.36$ taken with the NIC 2 camera and the F187W wide-band filter. The core is unresolved (PSF FWHM $=2.6$ pix $\left(0.2^{\prime \prime}\right)=1.6 \mathrm{kpc}$ measured for the two stars near the top), but there is a faint circularly symmetric halo around it. 
The dominant emission line in the $K^{-}$band is $\mathrm{H} \alpha+[\mathrm{N} I \mathrm{II}]$, and the NICMOS narrow-band image of the $\mathrm{H} \alpha+[\mathrm{N}$ II]-emitting nebulae is shown in Figure 2 (upper panel). It is extended almost linearly over $2^{\prime \prime}\left(\sim 16 \mathrm{kpc} ; q_{0}=0.5, \mathrm{H}_{0}=50\right.$ km s $\mathrm{s}^{-1} \mathrm{Mpc}^{-1}$ ) with three bright knots. The eastern region shows a significant extension going up to the north. We also note that this $\mathrm{H} \alpha+[\mathrm{N}$ II] linear structure is misaligned from the radio axis, which indicates that it is not the expanding jet that is ionizing the gas.

The line-free continuum source was found to be extremely compact and symmetric (Figure 2, lower panel). It has an unresolved core (PSF FWHM = $0{ }^{\prime \prime} 2=1.6 \mathrm{kpc}$ ), and an almost circularly symmetric halo extending out to at least a radins of $\sim 4 \mathrm{kpc}$.

Based on the line-free continuum images at $1.45 \mu \mathrm{m}$ (not shown here) and $1.87 \mu \mathrm{m}$, we conclude that the continuum SED is flat ( $f_{\nu} \sim$ const. $)$ at $\sim 10 \mu \mathrm{Jy}$ within this range. Even after the line-free near-IR magnitudes are combined with the line-free $V$ and $R$ magnitudes derived by Chambers, Miley, \& van Breugel (1988), the overall SED remains very flat $\left(f_{\nu} \propto \nu^{-0.5}\right)$.

\section{Interpretation}

In the case of $4 \mathrm{C} 40.36$, the extreme blue color of the continuum seems to suggest that the source is a young bursting stellar population possibly forming a spheroid or scattered AGN light (e.g., Eales \& Rawlings 1993; Iwamuro et al. 1996). On the other hand, we cannot yet exclude the possibility that the optical continuum source and the near-IR continum source are two completely separate components. In fact, a morphological study of $z \sim 1$ radio galaxies suggests that radio galaxies seem to have both a red old stellar component, which is compact, symmetric, and provides most of the mass, and a blue young stellar component, which is luminous and often aligned with the radio axis, but involves only a small amount of mass (Rigler 1992). If this is the case, the blue color of the integrated total flux does not necessarily mean that $4 \mathrm{C} 40.36$ is a young galaxy. To remove this ambiguity, it is necessary to obtain a line-free optical (i.e., restframe UV) image with a spatial resolution comparable to that of HST/NICMOS.

\section{References}

Chambers, K. C., Miley, G. K., \& van Breugel, W. J. M. 1988, ApJ, 327, L47

Eales, S. A., \& Rawlings, S. 1993, ApJ, 411, 67

Iwamuro, F., Oya, S., Tsukamoto, H., \& Maihara, T. 1996, ApJ, 466, L67

Lilly, S. J. 1989, ApJ, 340, 77

Lilly, S. J., \& Longair, M. S. 1984, MNRAS, 211, 833

Rigler, M. A., Lilly, S. J., Stockton, A., Hammer, F., \& Le Fevre, O. 1992, ApJ, 385,61 
The Hy Redshift Universe

ASP Conference Serics. Vol. 193, 1999

A. J. Bunker \& W. J. M. van Breugel, eds.

\title{
No Evidence for a 'Redshift Cut-Off' for the Most Powerful Classical Double Radio Sources
}

\author{
Matt J. Jarvis ${ }^{1}$, Steve Rawlings ${ }^{1}$, Chris J. Willott ${ }^{1,2}$, Katherine M. \\ Blundell ${ }^{1}$, Steve Eales ${ }^{3}$ and Mark Lacy ${ }^{1}$ \\ 1 Astrophysics, Department of Physics, Keble Road, Oxford, OX13RH. \\ ${ }^{2}$ Instituto de Astrofísica de Canarias, C/ Via Lactea $s / n, 38200 \mathrm{La}$ \\ Laguna, Tenerife, Spain \\ ${ }^{3}$ Department of Physics and Astronomy, University of Wales College of \\ Cardiff, P.O. Box 913, Cardiff, CF2 3 YB
}

\begin{abstract}
We use three samples (3CRR, $6 \mathrm{CE}$ and $6 \mathrm{C}^{*}$ ) to investigale the radio luminosity function ( $\mathrm{RLF}$ ) for the 'most powerful' low-frequency selected radio sources. We find that the data are well fitted by a model with a constant co-moving space density at high redshift as well as by one with a declining co-moving space density above some particular redshift. This behaviour is very similar to that inferred for steep-spectrum radio quasars by Willott et al(1998) in line with the expectations of Unified Schemes. We conclude that there is as yet no evidence for a 'redshift cutoff' in the co-moving space densities of powerful classical double radio sources, and rule out a cut-off at $z \lesssim 2.5$.
\end{abstract}

\section{Introduction}

It is well-known that the (co-moving) space densities of the rarest, most powerful quasars and radio galaxies were much higher at epochs corresponding to $z \sim 2$ than they are now (Longair 1966). The behaviour of the space density beyond these redshifts is the subject of this paper. Dunlop \& Peacock (1990) found evidence for a 'redshift cut-off' (a decline in the co-moving space density) in the distribution of flat-spectrum radio sources over the redshift range $2-4$. Through failing to find any flat-spectrum radio quasars at $z>5$ in a large $(\approx 40$ per cent of the sky) survey, Shaver et al (1996, hereafter SH96) argued for an order-of-magnitude drop in space density between $z \sim 2.5$ and $z \sim 6$, for this class of object. As emphasised by SH96, the crucial advantage of any radioselected survey is that with sufficient optical follow-up, it can be made free of optical selection effects, such as increasing dust obscuration at high redshift. It is chiefly for this reason that the work of SH96 provides the most convincing evidence to date for the existence of an intrinsic decline in the co-moving space density of any galaxy class at very high redshift. 


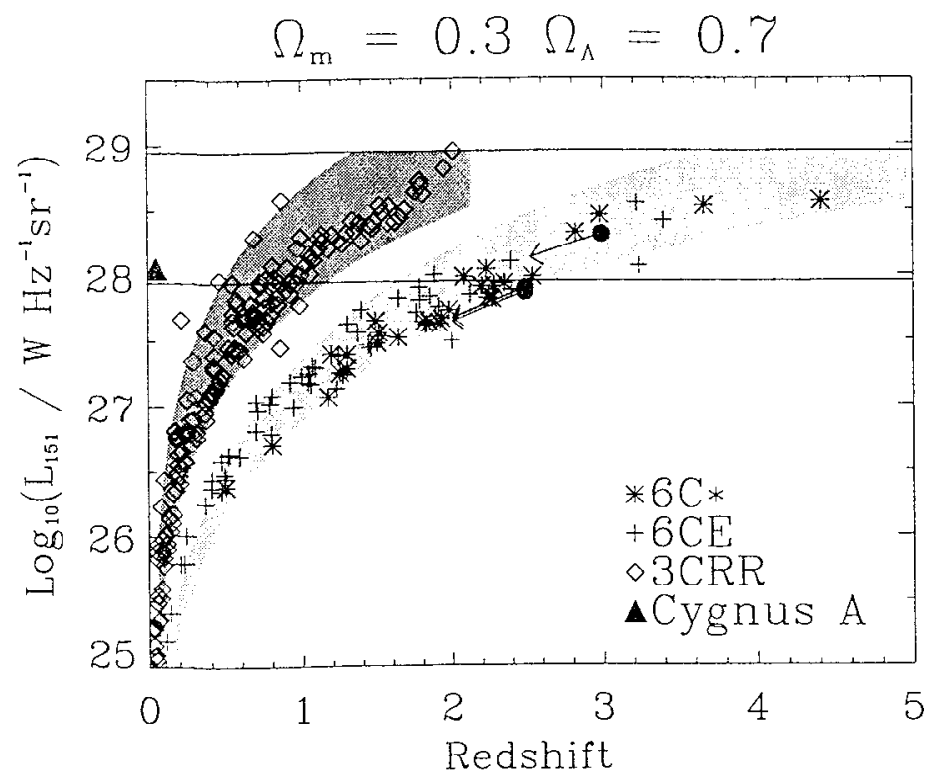

Figure 1. $L_{151}-z$ plane for the three samples used in our analysis. 3CRR (diamonds; Laing, Riley \& Longair 1983), 6CE (crosses; Eales et al 1997), 6C* (stars; Blundell et al 1998; Jarvis et al in prep.) and Cygnus-A (filled triangle). The filled circles represent the three $6 \mathrm{C}^{*}$ sources with upper redshift limits and the associated arrows show the paths of the possible redshifts and luminosities for these sources. The area between the horizontal lines is the region which contains the "most powerful' sources according to our definition. The dark grey region shows the approximate $L_{151}-z$ plane coverage of the 3 CRR sample (10.9 Jy $\leq S_{178} \leq 80.0 \mathrm{Jy}$, spectral index, $\alpha=0.5$ ) and the light grey region the $6 \mathrm{C}^{*}$ sample $\left(0.96 \mathrm{Jy} \leq S_{151} \leq 2.0 \mathrm{Jy}\right.$, with $\alpha=0.98$ and $a=1.5$ for the lower and upper fluxes, respectively). Note the area between the two shaded regions contains no sources, this is the area which corresponds to the absence of a flux-limited sample between the $6 \mathrm{CE}\left(S_{151} \leq 3.93 \mathrm{Jy}\right)$ and $3 \mathrm{CRR}\left(S_{178} \geq 10.9 \mathrm{Jy}\right)$ samples.

\section{Modelling the RLF}

We adopt a parameterisation of the RLF which is separable in $151-\mathrm{MHz}$ luminosity $L_{151}$ and redshift $z$ with a single power-law in $L_{151}$ of the form $\left(L / L_{0}\right)^{-\beta}$. We consider two cosmologies $\Omega_{M}=1, \Omega_{\Lambda}=0$ (cosmology I) and $\Omega_{M}=0.3$, $\Omega_{\Lambda}=0.7$ (cosmology II). Model A parameterises the redshift distribution as a single power-law of the form $(1+z)^{n}$. For model B the redshift distribution is parameterised as a Gaussian, giving an overall expression for the co-moving space density of $\rho=\rho_{0}\left(L / L_{0}\right)^{-\beta} \exp -\left\{\left(z-z_{0}\right) / \sqrt{2} z_{1}\right\}^{2}$ where $\rho_{0}$ and $\beta$ are the normalising term and power-law exponent respectively, $z_{0}$ is the Gaussian peak redshift and $z_{1}$ is the characteristic width of the Gaussian. Model $\mathrm{C}$ is described by the same model up to $z_{0}$ beyond which it becomes constant. 


\section{Results and Discussion}

For sources in the top-decade in luminosity of the $L_{151}-z$ plane (Fig. 1) our parametric fitting and likelihood analysis of model radio luminosity functions (Table 1) show that the data are inconsistent with a $(1+z)^{n}$ power-law in redshift (Model $\mathrm{A}$ ), but are well fitted by both models $B$ and $C$. These models are shown in Fig. 2 in the form of a $\log N / \log S$ plot. We conclude that although the relative likelihood for model $B$ is 2.5 times larger than for model $\mathrm{C}$, this is not statistically significant enough to distinguish between the two models with any confidence. This uncertainty is further compounded by the effects of assuming a mean spectral index in the model fitting. This result is in very close agreement with the RLFs for radio loud quasars modelled by Willott et al (1998) and various studies of AGN at optical (Irwin et al 1991) and X-ray (Hasinger et al 1998) wavelengths.

This is in apparent contradiction to the findings of SH96 for flat-spectrum quasars. If the relationship between the flat-and steep-spectrum populations is as described by unification models of AGN then we might expect to see similar erolution in the two populations. Thus to determine the co-moving space density of radio sources at high-redshift, an understanding of the spectral index trends, ${ }_{i}$-corrections and associated selection effects must first be achieved.

Fig. 2 also illustrates the contribution of powerful sources at high redshift to the total source count in a low-frequency survey. We see that even for the no cut-off model (Model $\mathrm{C}$ ) the fractional contribution is very small. This may render the location of the redshift cut-off virtually impossible to determine until the selection effects associated with radio surveys are fully understood.

\begin{tabular}{ccccccccc}
\hline \hline Model & Cosmology & $\log _{10}\left(\rho_{0}\right)$ & $\beta$ & $n$ & $z_{0}$ & $z_{1}$ & $P_{K S}$ & $\overline{\mathcal{L}_{R}}$ \\
\hline \hline $\mathrm{A}$ & I & -9.04 & 1.61 & $\overline{1.19}$ & - & $\overline{-}$ & 0.10 & $10^{-5}$ \\
$\mathrm{~B}$ & $\mathrm{I}$ & -7.94 & 1.98 & - & 2.59 & 0.94 & 0.33 & 1 \\
$\mathrm{C}$ & $\mathrm{I}$ & -8.18 & 1.95 & - & 1.69 & 0.54 & 0.41 & 0.4 \\
\hline $\mathrm{A}$ & II & -9.45 & 1.63 & 0.85 & -- & - & 0.12 & $10^{-5}$ \\
$\mathrm{~B}$ & II & -8.51 & 2.00 & - & 2.60 & 0.96 & 0.36 & 1 \\
$\mathrm{C}$ & II & -8.78 & 1.93 & - & 1.67 & 0.53 & 0.41 & 0.3 \\
\hline \hline
\end{tabular}

Table 1. Best-fit parameters for the model RLFs, described in the text. $P_{K S}$ is the 2-D Kolmogorov-Smirnov probability (a value above 0.2 signifies a reasonable fit to the data) and $\mathcal{L}_{\mathcal{R}}$ is the likelihood relative to model $\mathrm{B}$.

Errors for model B (cosmology I): $\Delta \log _{10}\left(\rho_{0}\right)=0.17, \Delta \beta=0.2$, $\Delta z_{0}=0.10, \Delta z_{1}=0.17$, for $68 \%$ confidence regions.

\section{References}

Blundell K.M., Rawlings S., Eales S.A., Taylor G.B., Bradley A.D., 1998, MNRAS, 295, 265

Dunlop, J.S. \& Peacock, J.A., 1990, MNRAS, 247, 19 
Eales S.A., Rarlings, S., Law-Green, D., Cotter, G., Lacy, M., 1997, MNRAS, 291,593

Ilales, S.E.G., Baldwin, J.E. \& Warner, P.J., 1988, MNRAS, 234, 919

Hasinger, G., 1998, Astron. Nachr., 319, 37

Irwin, M., McMahon, R. \& Hazard, C., 1991, in The Space Distribution of Quasars, ed. Crampton, ASPCS 21, 117

Laing, R.A., Riley, J.M. \& Longair, M.S., 1983, MNRAS, 204,151

Longair, M.S., 1966, MNRAS, 133, 421

McGilchrist M.M., Baldwin, J.E., Riley, J.M., Titterington, D.J., Waldram, E.M., Warner, P.J., 1990, MNRAS, 246, 110

Shaver, P.A., Wall, J.V., Kellermann, K.I., Jackson, C.A., Hawkins, M.R.S., 1996, Nature, 384, 439

Willott, C.J., Rawlings, S., Blundell, K.M., Lacy M., 1998, MNRAS, 300, 625

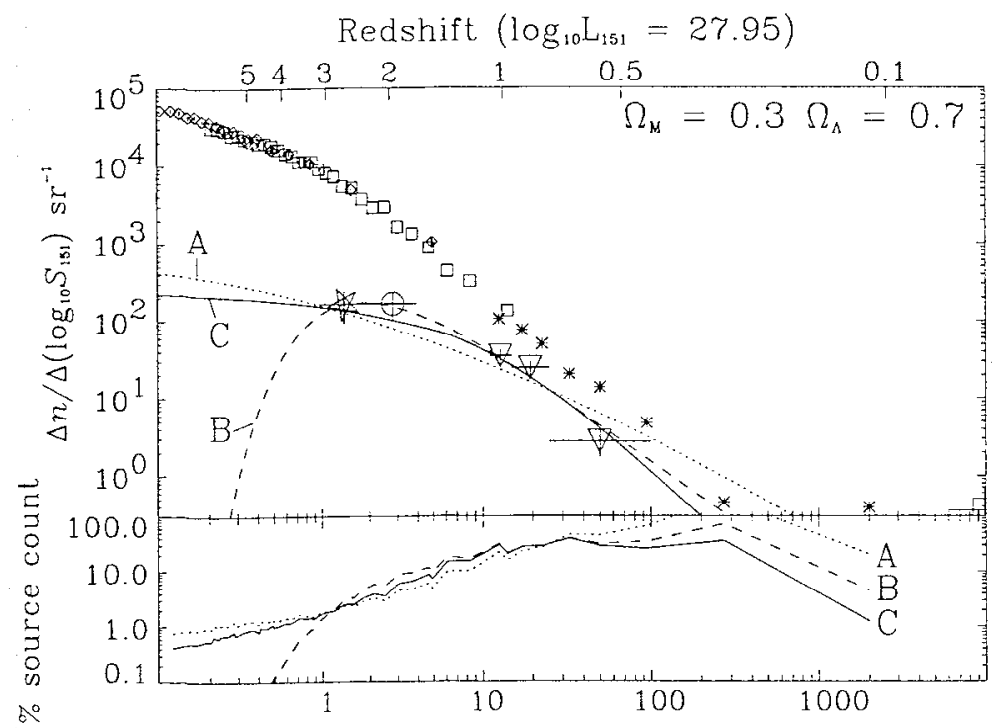

Flux density at $151 \mathrm{MHz} S_{151}(\mathrm{Jy})$

Figure 2. For cosmology II we show the areal density of the most powerful radio sources as a function of $151-\mathrm{MHz}_{\mathrm{z}}$ flux density $S_{151} / \mathrm{Jy}$ in comparison with the total sonrce connt. The $6 \mathrm{CF}$ somre count (squares) is reproduced from Hales, Baldwin \& Warner (1988) and the $7 \mathrm{C}$ source count (diamonds) from McGilchrist et al (1990). The $3 \mathrm{C}$ source (stars) count was inferred from the revised $3 \mathrm{CR}$ sample of Laing, Riley \& Longair (1983). The large open star represents the $6 \mathrm{C}^{*}$ data (note that this is a lower-limit due to spectral index and angular size selection in this filtered sample); the open circle the 6CE sample; the inverted triangles the 3 bins representing the $3 \mathrm{CRR}$ sample. The solid horizontal lines show the $S_{151}$ range of each sample. The lower panel. shows the percentage of the total source count contributed by each model. Models A, B and C are represented by the dotted, dashed and solid lines respectively. 
The Hy Redshift Universe

ASP Conference Series, Vol. 193, 1999

A. J. Bunker \& W.J. M. van Breugel, eds.

\title{
HST Imaging of Two $\approx>4$ Radio Galaxies
}

\author{
M. Lacy \\ Department of Physics, Oxford University
}

Abstract. We have imaged 8C $1435+635(z=4.25$; Lacy et al. 1994; Spinrad, Dey \& Graham 1995) and 6C 0140+326 ( $z=4.41$; Rawlings et al. 1996) in both continuum and Lya plus continuum bands with the HST. Our images show patchy distributions of continuum and line emission with a tendency for the peaks in the two types of emission to be offset. When compared to Keck $K$-band images, it seems likely that the presence of dusty neutral gas is strongly influencing the UV continuum and Ly $\alpha$ cmission. The radio and UV emission are not particularly wellcorrelated, except for a general tendency towards radio-optical alignment. It is suggested that at least some of the observed radio-optical alignment is produced as dust is cleared from the radio lobes by shocks associated by the radio source. If true, this means that $8 \mathrm{C} 1435+635$ could be hosting a luminous, galaxy-scale starburst, as suggested by submm observations.

\section{Observations}

\section{$8 \mathrm{C} 1435+635$}

This object was observed in the F814W and F622W filters (F622W includes the Ly $\alpha$ line). Using the Ly $\alpha$ flux of Spinrad et al. 1995 we have produced a "pseudo-Lyo" image by scaling and subtracting the F814W image from the F622W one. In Fig. 1 we show the F814W image, the "pseudo-Ly $\alpha$ " image and the MERLIN $5 \mathrm{GHz}$ radio map superposed on the Keck $K$-band image of van Breugel et al. (1998). (The radio-optical registration should be accurate to $\approx 1$ arcsec.) The Ly $\alpha$ peaks in the southern radio lobe, slightly offset from a faint patch of continuum emission. The central region has a (resolved) peak of UV emission surrounded by diffuse material. The $K$-band inage shows that the rest-frame blue continum is diffuse and covers the whole area enclosed by the radio lobes. The UV emission in the centre of the source is offset to the SW, but that in the sonthern lobe is closer to the major axis of the $K$-band emission. There is a suggestion of a bar of $K$-band emission perpendicular to the radio axis across the rentre of the source.

\section{$6 C \quad 0140+326$}

One orbit in each of the F675W and F658N filters was obtained on this object. Both these filters contain the Ly $\alpha$ line, and the emission in the F675W filter was found to consist entirely of line emission. In addition, NICMOS imaging of a nearby high-z field galaxy has also given us a high signal:noise NIC3 image 


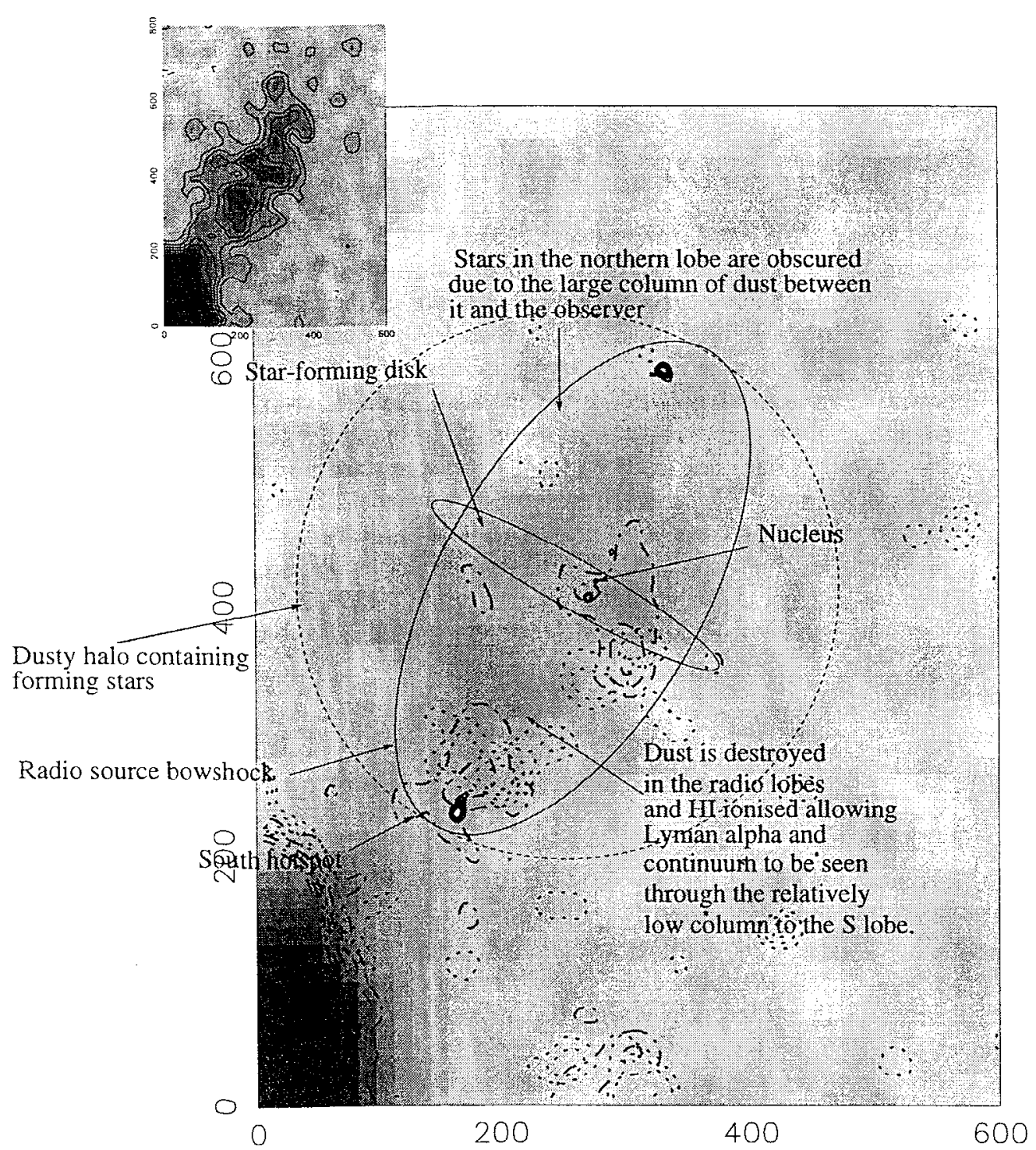

Figure 1. Overlay of the F814W image (dot-dash contonrs), the MERLIN $5 \mathrm{GHz}$ radio map (solid contours) and pseudo-Ly $\alpha$ image (dotted contours) over the smoothed $K$-band image of $8 \mathrm{C} 1435+635$. Superposed is a cartoon depicting the model discussed in the text (in which the south lobe is the closer to 11s). The image is $6 \times 8$-arcsec in size. Inset: the $K$-band image of $8 \mathrm{C} 1435+635$ smoothed with a $\sigma=0.15$-arcsec gaussian (contours and greyscale). Note the bar running across the middle of the source perpendicular to the radio axis. 


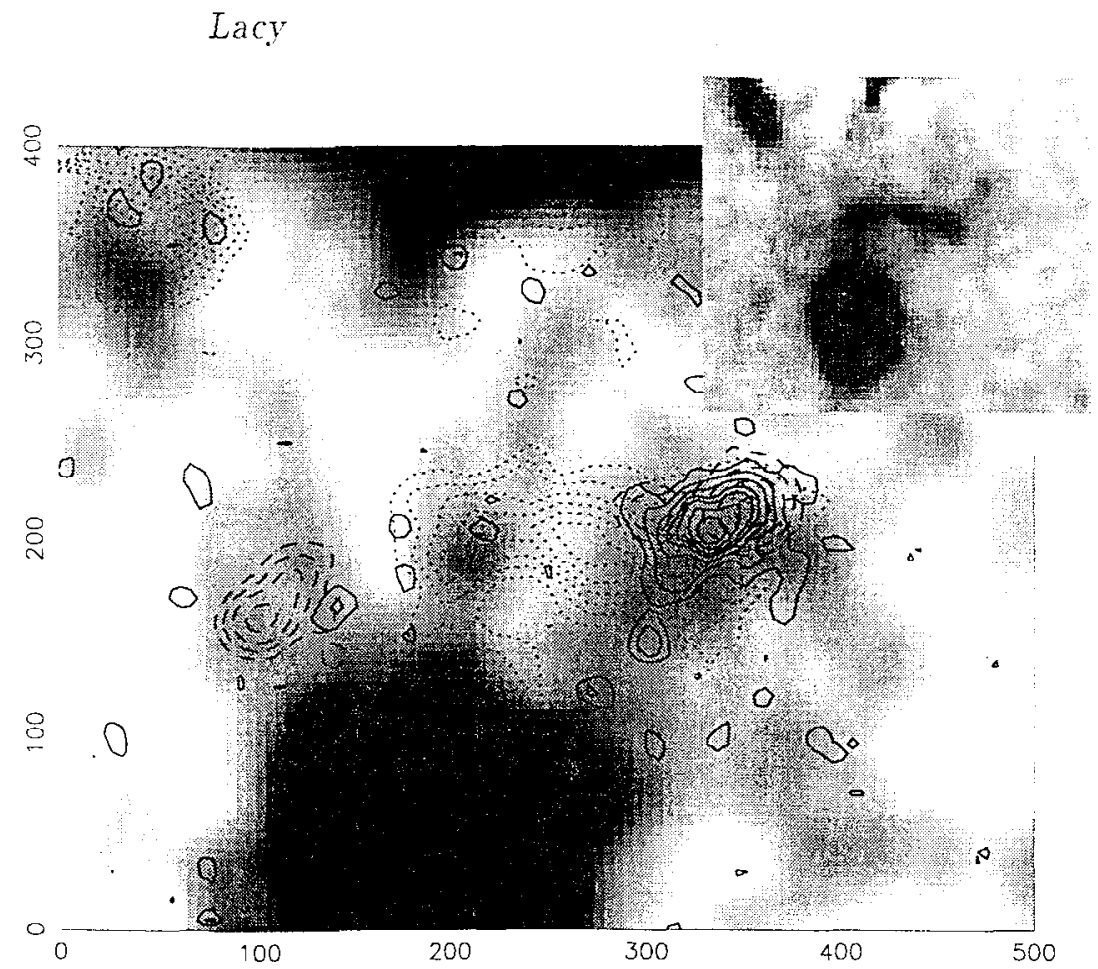

Figure 2. Overlay of the $1.5 \mathrm{GHz}$ MERLIN radio image (dashed contours), Lya emission (solid contours) and $K$-band emission (dotted contours) on a greyscale of an $I$-band WHT image of $6 \mathrm{C} 0140+326$. Radio-optical registration has been obtained by lining up the putative radio central component with the peak in the $U V / K$-band emission. The image is $5 \times 4$-arcsec in size. Inset: the NIC3 F160W image as a greyscale. In both images the bright star to the north has been subtracted.

of 6C0140. The Lya image obtained shows that the line emission is only from the westcrn end of the radio source. A UV continuum image from the WHT (Fig. 2) shows marginally-detected UV continuum from most of the area of the radio source, and the $K$-band image (van Breugel et al. 1998) is bar-shaped and aligned along the radio axis. The object is being weakly lensed by a nearby galaxy, and this is probably responsible for most of the elongation in the $K$ band, as well as the sinuous structure seen in the NIC3 image, although there is probably also some intrinsic radio-optical alignment.

\section{A galaxy-scale starburst in $8 \mathrm{C} 1435+635$ ?}

The submm detection of $8 \mathrm{C} 1435+635$ (Ivison et al. 1998) implies the presence of $\sim 2 \times 10^{8} M_{\odot}$ of dust. Although this may be concentrated in the nucleus, as is the case for local ULIGS it may alternatively be distributed on the scale of the host galaxy $(\sim 40 \mathrm{kpc}$ ), and be heated by a galaxy-wide starburst. If true, this could mean that we are seeing a giant elliptical galaxy forming according to a monolithic collapse scenario. 
Dust distributed throughout the galaxy would account for the relatively red $\mathrm{CV}$-optical colour and the patchy nature of the UV continuum emission. Distributed dust with $\mathrm{HI}$ would also explain the peculiar distribution of the Lya emission (Fig. 1) and the complicated velocity structure of the Ly $\alpha$ line (Lacy et al. 1994).

The UV flux and colour of $8 \mathrm{C} 1435+635$ is consistent with a starburst: the UV continuum flux corresponds to a star formation rate of $\approx 170 M_{\odot} \mathrm{yr}^{-1}$ before correction for reddening, if all the UV is from a starburst. The $F 814 W-I$ colour corresponds to a spectral index in $f_{\lambda}$ of $\beta \approx-1.1$. Using the models of Meurer, Heckman \& Calzetti (1999), this corresponds to an extinction at $160 \mathrm{~nm}$ of $2.2 \mathrm{mag}$ and an infrared:ultraviolet luminosity ratio of $\approx 10$. This is consistent with the far-infrared luminosity of this object as detected by SCUBA $\left(4.4 \times 10^{12} L_{\odot}\right)$, and implies a corrected star formation rate of $\sim 1200 M_{\odot} \mathrm{yr}^{-1}$. A good test of the distributed starburst theory would be to map the neutral gas directly using $\mathrm{CO}$ emission from the galaxy. This should be within the capability of the proposed large mm-array.

Of course, we must remember though that we are looking at a radio galaxy, and the radio source is likely to be influencing what we are seeing. The aligned continuum in $8 \mathrm{C} 1435+635$ could represent, for example, the result of dust scattering of a hidden quasar nucleus or inverse Compton emission from low energy electrons in the radio lobes (Spinrad et al. 1995). Nevertheless, the $K^{-}$-band emission bar across the centre of the source would seem to lie outside any plausible scattering cone, and is thercfore probably starlight.

Jet-induced star formation may also be contributing to the aligned light, and the colour gradient in the SE lobe could reflect an age gradient in the stellar population formed behind the expanding bow-shock. A colour gradient in the aligned emission could also arise if the radio source is embedded in a dusty halo of young stars, however. Shocks associated with the expanding radio source may destroy dust grains within the radio lobes, resulting in bluer emission from the end of the approaching lobe, as the column density of dust on the line of sight will be the lowest here. This model (Fig. 1) also provides a nice explanation for the lack of UV emission from the northern lobe.

Acknowledgments. I thank my collaburalors on the HST project, Hy Spinrad, Arjun Dey and Steve Rawlings. I am grateful to Wil van Breugel and Carlos de Breuck for supplying their $K$-band images of $6 \mathrm{C} 0140+326$ and 8C $1435+635$, and to Andy Bunker for putting up this poster in my absence.

\section{References}

Ivison R.J., et al., 1998, A.p.J, 494, 211

Lacy M., et al., MNRAS, 271, 504

Meurer G.R., Heckman T.M., Calzetti D., 1999, ApJ, in press

Rawlings S., Lacy M., Blundell K.M., Eales S.A., Bunker A.J., Garrington S.T., 1996, Nat, 383, 502

Spinrad H., Dey A., Graham J., 1995, ApJ, 438, 51

van Breugel W.J.M., Stanford S.A., Spinrad H., Stern D., Graham J.R., 1998; ApJ, 502, 614 
The Hy Redshift Universe

ASP Conference Serues, Vol. 198, 1999

A. J. Bunker \&. J. M. van Breugel, eds.

\section{Infrared Spectroscopy of the High Redshift Radio Galaxy MRC 2025-218 and a Neighboring Extremely Red Galaxy}

J.E. Larkin ${ }^{1}$, I.S. McLean ${ }^{1}$, J.R. Graham ${ }^{2}$, E.E. Becklin ${ }^{1}$, D.F. Figer ${ }^{1,3}$, A.M. Gilbert ${ }^{2}$, T.M. Glassmanl ${ }^{1}$, N.A. Levenson ${ }^{2,4}$, H. Teplitz ${ }^{5,6}$ and M.K. Wilcox ${ }^{1}$

${ }^{1}$ Dept. of Physics and Astronomy, University of California, Los Angeles

${ }^{2}$ Dept. of Astronomy, University of California, Berkeley

${ }^{3}$ Space Telescope Science Institute

${ }^{4}$ Dept. of Physics and Astronomy, John's Hopkins University

${ }^{5}$ LASP, Goddard Space Flight Center

${ }^{6}$ NOAO Research Associate

Abstract. This paper presents infrared spectra taken with the newly commissioned NIRSPEC spectrograph on the Keck Telescope of the High Redshift Radio Galaxy MRC 2025-218 $(z=2.630)$ and an extremely red galaxy $(\mathrm{R}-\mathrm{K}>6 \mathrm{mag})$ 9" away. These observations represent the deepest infrared spectra of a radio galaxy to date and have allowed for the detection of $\mathrm{H} \beta$, OIII (4959/5007), OI (6300), H $\alpha$, NII (6548/6583) and SII $(6716 / 6713$ ). The $\mathrm{H} \alpha$ emission is very broad (FWHM $\sim 6000 \mathrm{~km} / \mathrm{s}$ ) and strongly supports AGN unification models linking radio galaxies and quasars. The line ratios are most consistent with a partially obscured nuclear region and very high excitation. The OIII (500 $\mathrm{T}$ ) line is extended several arcseconds and shows high velocity clouds in the extended emission. The nucleus also appears spectrally double and we argue that the radio galaxy is undergoing a violent merger process. The red galaxy, by comparison, is very featureless even though we have a good continuum detection in the $\mathrm{H}$ and $\mathrm{K}$ bands. We suggest that this object is a foreground galaxy, probably at a redshift less than $\mathbf{1 . 5}$.

\section{Introduction}

Deep radio surveys have proven to by one of the best methods for finding high redshift galaxies. Most evidence points towards radio galaxies as precursors to local giant ellipticals (e.g. Pentericci, et al. 1999). Many have irregular and complex morphologies suggestive of mergers and they are often surrounded by an overdensity of compact sources; presumably sub-galactic clumps (e.g. van Brengel et al. 1998): Active galaxy unification models suggest that FRII radio galaxies are quasars with obscured broad line regions. Recent infrared spectroscopic surveys (e.g. Evans 1998) have shown that at redshifts less than 

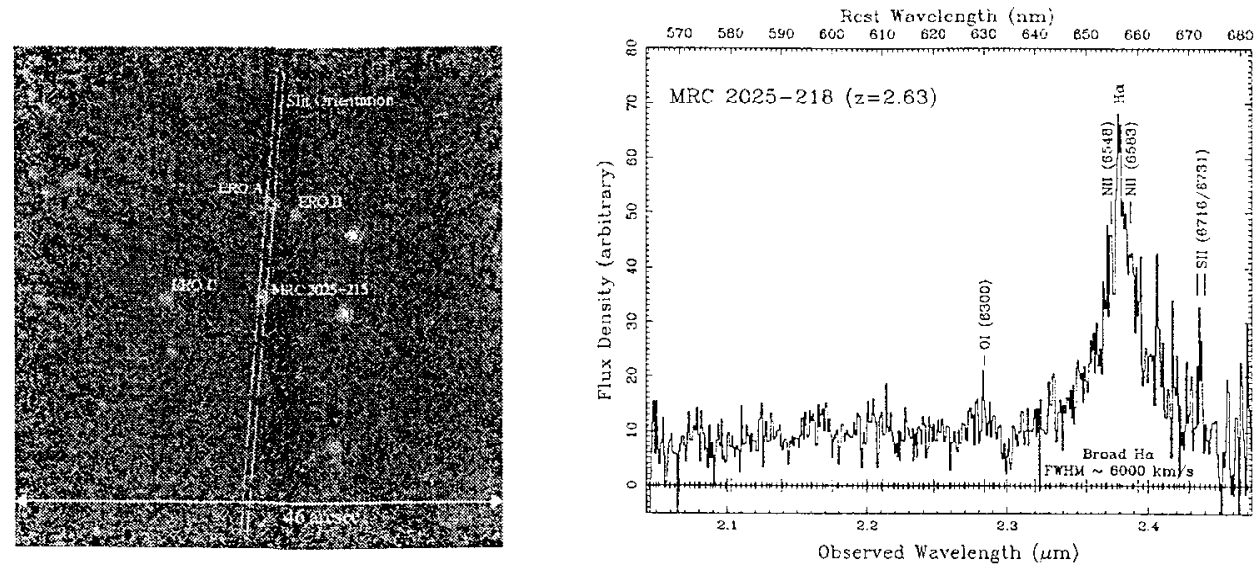

Figure 1. The $\mathrm{K}$ band image of the MRC 2025-218 field is on the left. The $\mathrm{K}$ band spectrum is on the right and is dominated by a very wide $\left(>6000 \mathrm{~km} \mathrm{~s}^{-1}\right)$ strong emission line of $\mathrm{H} \alpha$.

2.6 roughly have of the radio galaxies show evidence of an active nucleus typically wilh a Seyfert 2 spectrum.

MRC 2025-218 $(z=2.630)$ has a compact infrared and optical continuum morphology (van Breugel et al. 1998), but extended Ly $\alpha$ emission (5") aligned with its radio axis (McCarthy et al. 1992). McCarthy et al. also found three extremely red galaxies (ERO's: R-K 6 miag) within 20" of the radio galaxy. This is a large overdensity of such objects and strongly suggests and association between the ERO's and the active galaxy.

\section{Observations}

The field of MRC 2025-218 was observed on 4 Jun, 1999 (UT) with the near infrared spectrograph NIRSPEC (McLean, et al. 1998) on the Keck II Tclescopc during its commissioning. First the field was imaged in the $\mathrm{K}$ band with the slit-viewing camera. Figure 1 shows the reduced image of the field with a total integration time of 540 seconds. As shown in the figure, the slit (42" long and 0.57 wide) was placed on both the radio galaxy and the extremely red galaxy dubbed ERO-A by McCarthy et al. (1992).

Four 300 sccond exposures were taken in both the H-Band $(\sim 1.6 \mu \mathrm{m})$ and $\mathrm{K}$ Band $(\sim 2.2 \mu \mathrm{m})$. Due to vignetting at one edge of the slit, half of the exposures on the ERO wcre lost so the effective integration time on MRC 2025-215 is 20 minutes in each band but only 10 minutes on ERO-A. The seeing was 0.54 .

\section{Results}

Figure 2 shows the $\mathrm{H}$ band spectrim of MRC 2025-218. By far the most dominant line is [OIII] $(500.7 \mathrm{~nm})$ redshifted to $1.82 \mu \mathrm{m}$. This line is highlighted in the right panel of figure 2 where the complete position velocity map of this 
Larkin et al.
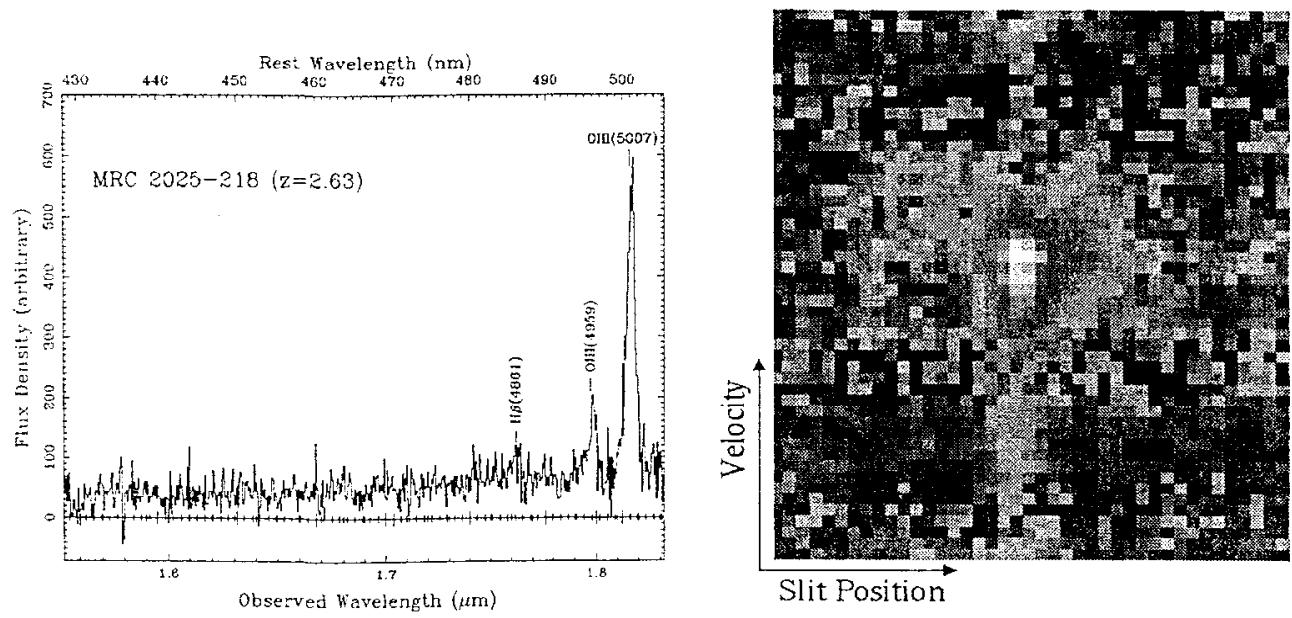

Figure 2. $\quad \mathrm{H}$ band spectrum of MRC 2025-218. The right panel is the position-velocity plot of just the OIII line at $500.7 \mathrm{~nm}$.

line is presented. The nucleus has a double peaked structure in [OIII] and $\mathrm{H} \alpha$ (see below) with a separation of $150 \mathrm{~km} \mathrm{sec}^{-1}$. Two knots appear at essential $0 \mathrm{~km} \mathrm{sec}-1$ relative velocity, but 1".8 North and 2".4 South of the Nucleus. A high speed clump appears 1 "North of the nucleus and at a redshifted relative velocity of $410 \mathrm{~km} \mathrm{sec}^{-1}$. Also detected in the $\mathrm{H}$ band spectrum is the other member of the [OIII] doublet at $495.9 \mathrm{~nm}$, and $\mathrm{H} \beta$. The ratio of [OIII] / $\mathrm{H} \beta$ is extremely large at approximately $31 \pm 6$. The $\mathrm{H} \beta$ line has a total nuclear flux of only $3.4 \times 10^{-17} \mathrm{ergs} \mathrm{cm}^{-2} \mathrm{~s}^{-1}$.

The right panel of figure 1 shows the $K$ Band spectrum which is dominated by a broad $\mathrm{H} \alpha$ emission line. The line is very non-Gaussian but has a fwhm of the broad line greater than $6000 \mathrm{~km} \mathrm{sec}{ }^{1}$. The narrow component has a similar double peaked profile as the [OIII] line at $500.7 \mathrm{~nm}$. Several other lines are also detected, but only at the few sigma level, including [OI] $(630.0 \mathrm{~nm})$, [NII] $(654.8 / 658.3 \mathrm{~nm})$ and [SII] $(671.6 / 673.1 \mathrm{~nm})$. These lines are all much weaker than $\mathrm{H} \alpha$ and the $[\mathrm{OI}] / \mathrm{H} \alpha$ ratio for the narrow components is estimated at approximately 0.1 or less.

$\mathrm{H}$ and $\mathrm{K}$ band spectra of the object labeled ERO-A were taken simultaneously with MRC 2025-218. The spectra have no significant line detections or spectral breaks. In particular no emission lines are observed at similar positions as MRC 2025-218. Given the mild $\mathrm{H}$ and $\mathrm{K}$ spectral slopes in the spectra and the large $\mathrm{r}-\mathrm{K}$ color found by McCarthy et al. (1992), we suggest that the ERO is probably at a significantly smaller redshift than the radio galaxy.

\section{Discussion \& Conclusions}

The spectrum of the HzRG MRC 2025-218 is clearly dominated by emission lines from an $A G N$. The broad $\mathrm{H} \alpha$ emission line is definitive evidence of a type I AGN. The extremely large ratio of [OIIT]/H $\beta$ is also found only in true $A G N$ and is in fact greater than in most local Seyfert 1 type galaxies. 
The double peak found in both $\mathrm{H} \alpha$ and [OIII] is highly suggestive of a double active nucleus. If the second peak were due to a star forming region it would be unlikely that the [OIII] line would be double as well. The off nucleus knots seen in [OIII] are difficult to understand. Extended [OIII] has been observed in other radio galaxies aligned to the radio axis (Armus et al. 1998). Extended Ly $\alpha$ has also been observed aligned to the radio axis (e.g. Chambers et al., 1996) but the emission mechanism is poorly understood. In the case of [OIII] it is difficult to find a mechanism for its strong production in these off nuclear sites and we believe that the most likely explanation is that we are seeing [OIII] as scattered light originally emitted by the nucleus.

The narrow line ratio of $\mathrm{H} \alpha$ over $\mathrm{H} \beta$ is roughly 8 compared with the intrinsic ratio of 3.1 observed in local $A G N$. If we assume the difference is due to dust extinction then it is consistent with obscuration of $A_{V} \sim 2$ mag. The fact that $\mathrm{H} \beta$ is not broadened and that broad $\operatorname{Ly} \alpha$ was not observed in optical spectra implies that the ccntral region is much more obscured. The presence of such an obscured broad line region in a classic radio galaxy strongly supports the AGN unification models and links radio galaxies with radio loud quasars.

The presence of 3 extremely red galaxies discovered within 20 " of the radio galaxy is very suggestive of a connection with MRC 2025-218. But if our suggestion is right that the ERO's are at a much lower redshift then their overdensity in this field and thcir general overdensity in the fields of high redshift quasars and radio galaxies may be due to weak lensing. The connection is that in flux limited surveys you are more likely to discover high redshift objects if there is an overdensity of foreground objects.

Acknowledgments. We want to thank the incredibly hard working NIRSPEC instrument team at UCLA: Maryanne Anglionto, Odvar Bendiksen, George Brims, Lealı Buchholz, John Canfield, Kim Chim, Jonah Hare, Fred Lacayanga, Samuel B. Larson, Tim Liu, Nick Magnone, Gunnar Skulason, Michael Spencer, Jason Weiss and Woon Wong. We would also like to thank the wonderful scientists and staff members of the Keck Observatory who've made the commissioning of NIRSPEC extremely productive. In particular, Tom Bida, the NIRSPEC support scientist has worked diligently to ensure the instrument's success. We also want to thank Lee Armus for useful discussions.

\section{References}

Armus, L., Soifer, B. T., Murphy, T. W., Neugebauer, G., Evans, A. S., \& Matthews, K. 1998, ApJ, 495, 276

Chambers, K. C., Miley, G. K., van Breugel, W. J. M., Bremer, M. A. R., Huang, J. S., \& Trentham, N. A. 1996, ApJS, 106, 215

Evans, A. S. 1998, ApJ, 498, 553

McCarthy, P. J., Persson, S. E., \& West, S. C. 1992 ApJ, 386, 52

McLean, I. S., et al. 1998, SPIE, 3354, 566

Pentericci, L., Röttgering, H. J., A., Miley, G. K., McCarthy, P., Spinrad, H., van Breugel, W. J. M., \& Macchetto, F. 1999, A\&A, 341, 329

van Breugel, W. J. M., Stanford, S. A., Spinrad, H., Stern, D., \& Graham, J. R. 1998, ApJ, 502, 614 
The Hy Redshift Universe

ASP Conference Series, Vol. 193,1999

A. J. Bunker \& W. J. M. van Breugel, eds.

\title{
Keck Spectropolarimetry of $z \sim 2.5$ Radio Galaxies
}

Joël Vernet and Robert A. E. Fosbury ${ }^{1}$

ESO/ST-ECF, Karl Schwarzschild strałse, 2, D-85748

Garching-bei-München, Germany

Montserrat Villar-Martín

IAP, 98 bis bvd. Arago, F-75014 Paris, France

Marshall H. Cohen

Caltech, Mail Stop 105-24,Pasadena, CA 91125, USA

Sperello di Serego Alighieri and Andrea Cimatti

Osservatorio Astrofisico di Arcetri, Largo E. Fermi 5, I-50125, Firenze. Italy

\begin{abstract}
Reflected quasar light is the dominant contributor to the rest frame ultraviolet continuum in all but one of a sample of ninc high redshift radio galaxies we have observed with the Keck spectropolarimeter. Correlations of continuum fractional polarization with the weakness of Ly $\alpha$ emission and with the NV/CIV narrow emission line ratio lead us to suggest an evolutionary scheme for massive spheroids. In this, the galaxies which host bright quasars start out with a relatively transparent, low metallicity ISM but evolve, within $\sim 1 \mathrm{Gyr}$, to dusty, high metallicity objects.
\end{abstract}

\section{Introduction}

The goal of our programme is to observe the early evolutionary phases of objects destined to become the giant ellipticals of today. In particular, we study the relationship between the formation of the $A G N$ and the accumulation of its host. galaxy. The obscuration of the quasar acts as a natural coronograph which greatly facilitates our detailed study of the host galaxy.

We have selected powerful radio galaxies at $z \sim 2.5$ because:

- the optical spectrum gives us access to the rest-frame UV emission lines from Ly $\alpha$ to CIII]

- the continuum covers the dust scaltering/extinction signature near $2200 \AA$ and the steep rise in cross section below $1400 \AA$

\footnotetext{
${ }^{1}$ Affiliated to the Astrophysics Division, Space Science Department, European Space Agency
} 
- there are number of strong absorption lines and some hot star photospheric lines visible in this spectral region

- the important diagnostic lines [OII], $\mathrm{H} \beta /[\mathrm{OIII}]$ and $\mathrm{H} \alpha / \mathrm{NII}$ are observable in the $\mathrm{J}, \mathrm{H}$ and $\mathrm{K}$ windows respectively.

We have obtained Keck/LRISp spectropolarimetry data of $9 \mathrm{HzRGs}$ with $2.3<z<3.6$. We use HST WFPC 2 and NICMOS for high resolution imaging and we have started observing a partially overlapping sample with the new ISAAC IR imager/spectrometer on the first VLT $8 \mathrm{~m}$ unit telescope.

\section{The nature of the continuum}

Most of the objects in our sample show strong continuum polarization (\%P) ranging from $\sim 7 \%$ up to about $20 \%$. Only one object $(0731+438)$ has $\% \mathrm{P}<3 \%$. In most polarized objects, $\% \mathrm{P}$ rises to the blue.

The continuum shapc is rather similar in all objects with a dip around $2200 \AA$, a flattening at below $1400 \AA$ and a power law in the $1500-2000 \AA$ range. The slope $\beta$ (with $F_{\lambda} \propto \lambda^{\beta}$ ) varies between 1.5 and 0.8 and the luminosity at $1500 \AA$ ranges from 2 to $510^{41} h_{50}^{-2} \cdot \operatorname{erg} \cdot s^{-1} \cdot \AA^{-1}$ (with $q_{0}=0.1$ ).
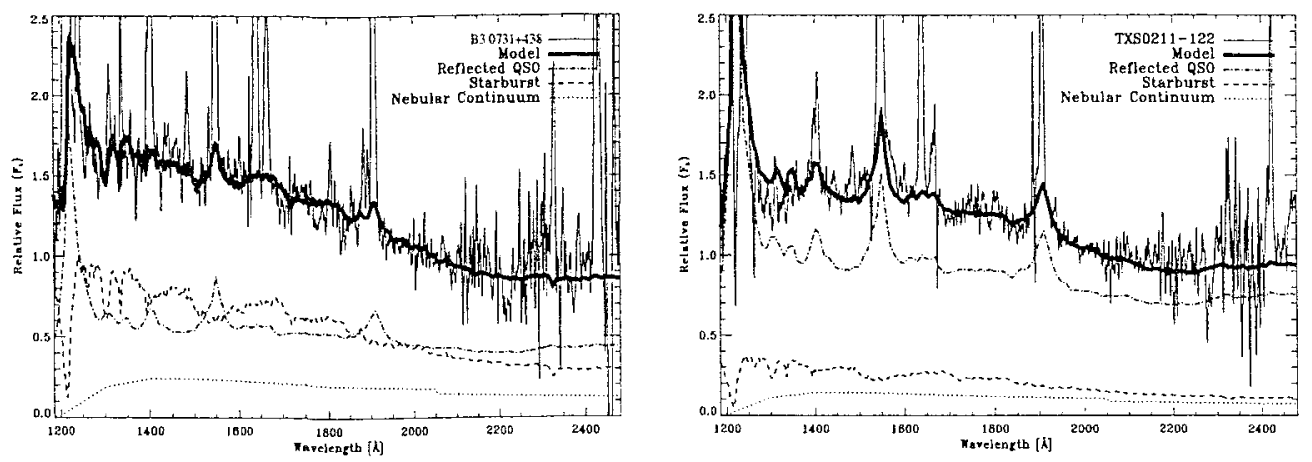

Figure 1. Three component continuum fit to $0731+438$ (left) and 0211-122 (right)

We model the continuum with 3 components:

- The nebular continuum. This is computed from the strength of the HeII recombination line at $1640 \AA$. Its contribution at $2000 \AA$ raries between $5 \%$ and $15 \%$.

- A reflected quasar. A radio loud quasar composite spectrum (Cristiani and Vio 1990) is scattered and absorbed by dust grains with standard galactic MRN distribution. The reflected light is maximized when $\tau_{\text {scut }} \sim \tau_{\text {ext }} \sim 1$ which produces an almost grey scattering. The difference between extinction and scattcring efficiencies results in the imposition of the $2175 \AA$ dust 
feature on the spectrum. This component alone provides a very good fit to the continuum and broad lines of high polarization objects (0211-122, $4 \mathrm{C} 23.56)$ in this spectral range.

- A young stellar population (YSP). This component is necessary to explain the blue continuum slope and the low polarization of objects like $0731+438$ and $4 \mathrm{C} 41.17$ (Dey et al. 1997). We use Starburst 99 (Leitherer et al.1999) continuous star formation models with solar metallicity and the standard Salpeter IMF. The age $t_{Y S P}$ and the determination of the star formation rate (SFR) are strongly dependent on the assumed distribution of dust. For $0731+438$, with $\mathrm{E}_{B-V} \sim 1$ we get $t_{Y} S P \sim 300 M y r$ and a. $\mathrm{SFR} \sim 20 M_{\odot} \cdot y r^{-1}$

The range in continuum properties suggests a variation of the relative contributions of reflected quasar and YSP and geometrical effects. The fits to the two extreme objects in our sample are displayed in fig. 1 .

\section{The emission lines}

- While CIV, HeII and CIII] show very little variation amongst the objects (see fig. 2, left), we observe a spread of nearly $1 \mathrm{dex}$ in the NV/CIV ratio.

- The continuum fractional polarization correlates with NV/CIV and anticorrelates with Lya/CIV (see fig. 3).
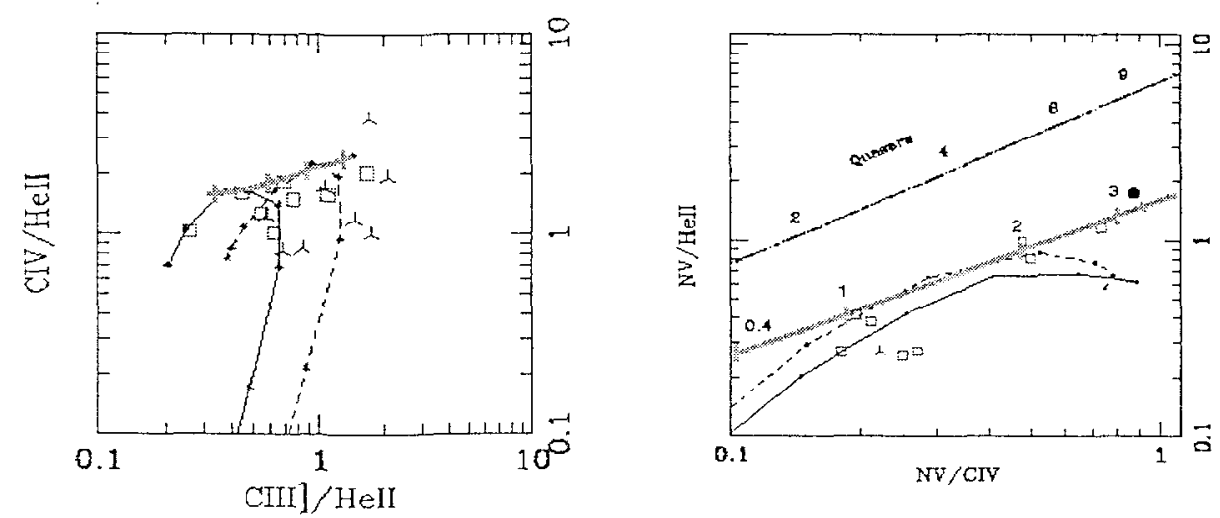

Figure 2. left: Helium carbon diagnostic diagram; right: NV/HeII vs. NV/CIV diagram. Black lines represent $U$ sequences for an ionizing spectrum of slope -1 (dashed) and -1.5 (continuous) and solar metallicity. Grey lines are metallicity sequences from 0.4 to 4 times solar with quadratic $N$ enhancement $(U=0.035$, slope -1$)$. Squares represent objects from our sample, arrows are from Stern et al. 1999, and the black dot is from Villar-Martín et al. 1999. The dot-dash line represent the quasar broad emission line super solar metallicity sequence from Hamann and Ferland 1993. 

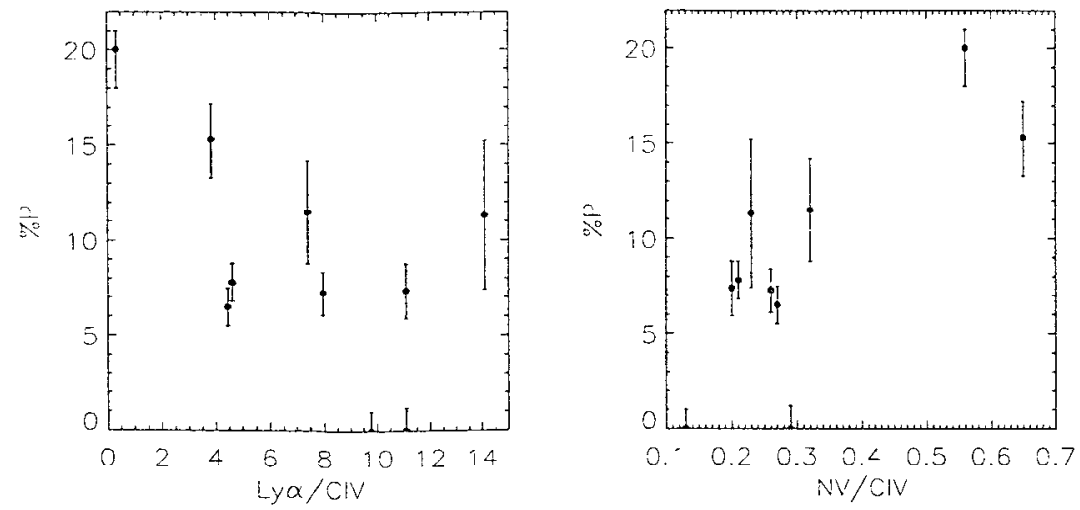

Figure 3. Correlation between Ly $\alpha / C I V$ (left) and NV/CIV (right) ratios with continuum polarization

A sequence in ionization parameter $(U)$ alone cannot explain the observed variations in a self consistent way. It would require a much larger range in $U$ to explain the NV/CIV ratio than deduced from CIV, HeII and CIII] lines. Changing the abundance of all the metals together produces effects on the electron temperature which are not observed. The best model sequence is obtained with a quadratic change in $\mathrm{N}$ abundance, while other elements are increased linearly between 0.4 and 4 times solar, consistent with a secondary $N$ production. The result is displayed in fig. 2

\section{Conclusion}

The large range in properties in our sample is consistent with an evolutionary sequence in which we witness the evolution of the ISM of forming massive spheroids.

According to our sequence, during a major merger or sequence of mergers, the massive black hole and the spheroid start forming in a relatively transparent host and may evolve into a highly obscured, IR luminous objects on a time-scale of about 1 Gyr.

\section{References}

Cristiani and Vio, 1990, A\&A, 227, 385

Dey et al. 1997, ApJ, 490, 698

Hamann and Ferland, 1993, ApJ, 418, 11

Leitherer et al, 1999, AJ, in press

Mathis, Rumpl and Nordsieck, 1977, A p.J, 323, 456

Stern et al., 1999, AJ, 117, 1122

..... Villar-Martín et al., 1999, submitted to A\&A 
The Hy Redshift Universe

ASP Conference Series, Vol. 193, 1999

A. J. Bunker \& W. J. M. van Breugel, eds.

\title{
A Complete Sample of Millijansky Radio Sources
}

\author{
I. Maddington \\ Department of Physics \& Astronomy, Arizona State University, T'empe, \\ $A Z$ 85287-1504
}

\begin{abstract}
The results of an optical and infrared investigation of a radio sample drawn from the $1.4 \mathrm{GHz}$ Leiden-Berkeley Deep Survey are presented. This is believed to be the most comprehensive sample of radio sources at millijansky flux limits that is currently available. Optical counterparts have been identified for all but four sources in the two Hercules fields, and $80 \%$ of them are identified in the near-infrared. Redshifts have been obtained for 49 of the identified sources, and photometric redshifts were computed from the gri $\mathrm{K}$ data for the remaining 20 .

The general properties of the sample are summarized. The use of this sample in measuring the $1.4 \mathrm{GHz}$ radio luminosity function is discussed. Finally, HST/NICMOS images of two old, red galaxies at $z \simeq 1.5$ are presented which show that both galaxies are dominated by an $r^{1 / 4}$ profile with a scale-length of $5 \mathrm{kpc}$.
\end{abstract}

\section{The sample}

The Leiden-Berkeley Deep Survey ('LBDS') consists of nine high latitude fields in the selected areas SA28, SA57, SA68 and an area in Hercules. They were surveyed with the Westerbork Synthesis Radio Telescope at $21 \mathrm{~cm}$ (1.412 GIIz), reaching a 5- $\sigma$ limiting flux density of $1 \mathrm{mJy}$ (Windhorst et al. 1984a). Multicolor prime focus photographic plates of the fields were used to find optical counterparts to the radio sources. Identifications were found for $53 \%$ of the sources in the full survey, whilst for the Hercules fields 47 out of 73 sources were identified (Windhorst et al. 1984b; Kron et al. 1985).

The Hercules fields were subsequently observed on the 200 inch Hale Telescope at Palomar Observatory between 1984 and 1988. Multiple observations were made through Gunn $g, r$ and $i$ lilters over six runs. After processing and stacking of the multiple-epoch images, optical counterparts for 22 of the sources were found, leaving only four sources unidentified to $r \simeq 26$. Near-infrared observations have been made of the entire subsample at $K$, yielding $60 / 73$ detections down to $\mathrm{K} \simeq 19-21$. Half of the sources have been observed in $\mathrm{H}$ and approximately one-third in J. Observations of the brighter sources were made. by Thuan et al. (1984) and by Neugebauer et al. and Katgert et al. (priv. comm.). $\mathrm{K}$-band observations of the sample were completed by the present author and collaborators at the UK Infrared Telescope.

Prior to the start of the current work, only 16 of the 73 sources in the LBDS Hercules fields had redshifts published in the literature. Another 16 sources had 

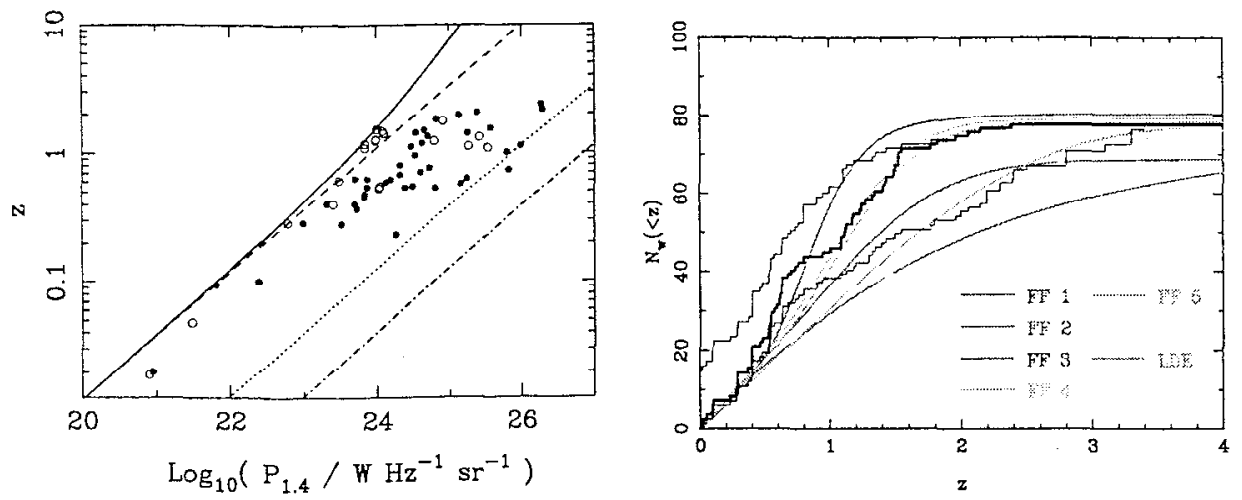

Figure 1. (a) [left] The luminosity-redshift plane for sources with $S_{1.4} \geq 2 \mathrm{mJy}$ in the LBDS Hercules fields. The flux density limits for the survey are shown for flat-spectrum (solid line, open circles) and steep-spectrum (dashed line, solid circles) sources, together with the limits for the PSR (dotted line) and 3C (dot-dash line) surveys. (b) [right] The cumulative redshift distribution for the $S_{1.4} \geq 2 \mathrm{mJy}$ sample. The bold histogram corresponds to the best-fitting photometric redshift distribution, the light histograms represent the $99 \%$ confidence limits on the photometric redshifts. The gray lines are the model luminosity functions of Dunlop \& Peacock (1990), assuming $\mathrm{H}_{0}=50 \mathrm{~km} \mathrm{~s}^{-1} \mathrm{Mpc}^{-1}, \Omega_{0}=1, \Lambda=0$.

unpublished redshifts. The author and collaborators have successfully observed a further 17 sources during the past few years, using both the $4.2 \mathrm{~m}$ William. Herschel Telescope (Waddington 1999) and the $10 \mathrm{~m}$ Keck Telescope (Dunlop et al. 1996; Spinrad et al. 1997; Dey 1997). This brings the total number of spectroscopic redshifts to 49 out of 73 sources $(67 \%)$. Photometric redshifts were calculated for the remaining one-third of the sample, using the spectral population synthesis models of Jimenez et al. (1998).

\section{The $1.4 \mathrm{GHz}$ radio luminosity function}

Dunlop \& Peacock (1990) used a sample of radio sources brighter than $0.1 \mathrm{Jy}$ at $2.7 \mathrm{GHz}$ to investigate the evolution of the radio luminosity function (RLF). They concluded that the comoving density of both flat- and steep-spectrum sources suffers a cut-off at redshifts $z \simeq 2-4$. This conclusion was drawn from the behavior of both free-form and simple parametric models (PLE/LDE), and the model-independent, banded $V / V_{\max }$ test. However, the results were crucially dependent upon the accuracy of their redshift estimates in the Parkes Selected Regions (PSR).

Figure 1a compares the flux limits of the LBDS with those of the PSR and $3 \mathrm{C}$ surveys. It is seen how the LBDS can be used to: (i) probe the faint end of the RLF out to much greater redshifts than the brighter surveys; and (ii) detect powerful radio galaxies out to very high redshifts $(z 10)$. Thus we are able to use this millijansky sample to test the reality of the redshift cut-off. In 

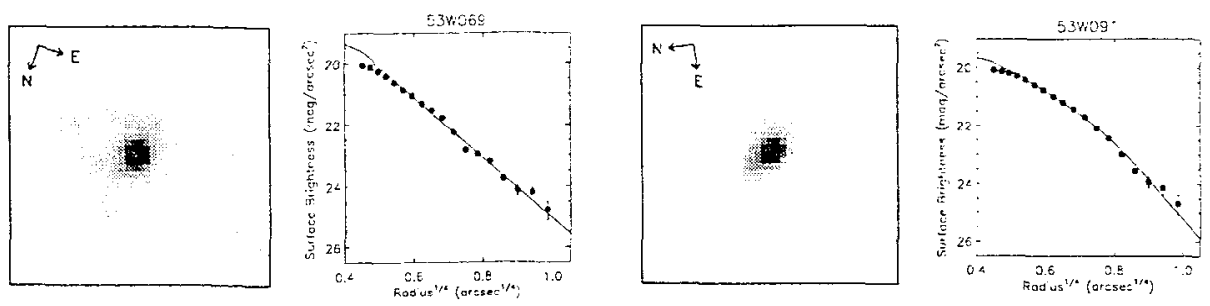

Figure 2. HST/NTCMOS F110W images and surface brightness profiles for $53 W 069$ at $z=1.432$ [left] and 53 W091 at $z=1.552$ [right]. The images are $3^{\prime \prime}$ on a side. Solid lines are the best-fitting model profiles convolved with the NICMOS PSE. AB magnitudes are used.

figure $1 b$ the cumulative redshift distribution of the LBDS Hercules sample (only those sources with $S_{1.4} \geq 2 \mathrm{mJy}$ ) is compared with the predictions of Dunlop $\&$ Peacock (1990). It is seen that two of the free-form models (FF-4 and FF-5) provide a reasonable fit to the data over all redshifts, suggesting that the high- $z$ decline in the RLF is real. The "bump" in the best-fit histogram at $0.4 \lesssim z \lesssim 1$ is due to two spikes in the redshift distribution, that may be the result of possible large-scale structures (sheets) along the line of sight.

However, a more detailed investigation of the RLF reveals that the luminosity dependence of the data does not agree with the models. The observed RLF shows some indication that it turns over at $z \simeq 0.5-1.5$, and that the redshift of this cut-off is a function of the radio luminosity. The small number of sources makes it difficult to separate the redshift and luminosity dependence of the RLF sufficiently to be certain of this trend, but work is ongoing to inprove the modeling of the data.

\section{Surface brightness profiles of two old galaxies}

Two of the most interesting individual sources in the sample are 53 W069 and 53 W091. Keck spectra of these galaxies revealed that their restframe ultraviolet light was dominated by old stellar populations at ages of 4.5 Gyr at $z=1.432$ dild 3.5 Gyr at $z=1.552$ respectively (Dey 1997; Spinrad et al. 1997). Although there continues to be some debate over the accuracy of these ages, there can be little dispute that they are two of the oldest galaxies observed at that redshift.

An important corollary to investigate was whether these galaxies were also dynamically old objects. Peacock et al. (1998) showed that in order for the number density of these objects to be consistent with the evolving power spectrum of primordial density fluctuations, then the galaxies must be 3-4 Gyr old at $z \simeq 1.5$, having collapsed at $z \simeq 6-8$. This result is independent of the ages of the sources derived from their Keck spectra. Further clues to the dynamical history of the two galaxies can be gained by looking at their morphologies. $53 \mathrm{~W} 069$ and 53W091 were observed using WFPC2 and NICMOS on the Hubble Space Telescope in Cycle 7 (Waddington et al. 1999). Data were collected in F814W and F110W: these filters bridge the $4000 \AA$ break and are thus most 
sensitive to the young and old stars in a galaxy respectively. Figure 2 shows the F110W images and surface brightness profiles for the two objects.

53 W069 has a rcgular de Vaucouleurs $r^{1 / 4}$ profile, with an effective radius of $0^{\prime \prime} 5$ or $4 \mathrm{kpc}\left(\mathrm{H}_{0}=65 \mathrm{~km} \mathrm{~s}^{-1} \mathrm{Mpc}^{-1}, \Omega_{0}=0.2, \Lambda=0\right)$. $53 \mathrm{~W} 091$ is similarly dominated by an $r^{1 / 4}$ profile of effective radius $0.5(4 \mathrm{kpc})$, however there is an additional component required to fit this galaxy. The form of this extra emission has not been unambiguously identified, but initial results suggest that it is consistent with an exponential profile of $0^{\prime \prime} 2(2 \mathrm{kpc})$ scale-length, contributing $\sim 40 \%$ of the F110W flux within a 1.5 diameter aperture.

The spectrum of 53W091 can be modeled by adding a young stellar population to the spectrum of 53W069. Similarly the surface brightness profile of 53 W091 can be modeled by adding an exponential component to the profile of 53W069. A possible interpretation is that 53W091 has a star-forming disk-like structure surrounding an otherwise passively evolving elliptical galaxy, such as 53 W069.

Acknowledgments. I thank my collaborators on this project, particularly Rogier Windhorst, James Dunlop and John Peacock. This work was supported by a research studentship from the UK PPARC; and NASA grant GO-7280.0*.96A from STScI under NASA contract NAS5-26555.

\section{References}

Dey, A. 1997, "The Hubble Space Telescope and the High Redshift Universe", N. Tanvir et al. (eds), World Scientific, p. 373

Dunlop, J.S., Peacock, J. A., Spinrad, H., Dey, A., Jimenez, R., Stern, D., Windhorst, R. A. 1996, Nature, 381, 581

Jimenez, R., Dunlop, J. S., Peacock, J. A., Padoan, P., MacDonald, J., Jørgensen, U. G. 1999, MNRAS, submitted

Kron, R. G., Koo, D. C., Windhorst, R. A. 1985, A\&A, 146, 38

Peacock, J. A., Jimenez, R., Dunlop, J. S., Waddington, I., Spinrad, H., Stern, D., Dey, A., Windhorst, R. A. 1998, MNRAS, 296, 1089

Spinrad, H., Dey, A., Stern, D., Dunlop, J. S., Peacock, J. A., Jimenez, R., Windhorst, R. A. 1997, ApJ, 484, 581

Thuan, T. X., Windhorst, R. A., Puschell, J. J., Isaacman, R. B., Owen, F. N. 1984, ApJ, 285, 515

Waddington, I. 1999, $\mathrm{PhD}$ Thesis, University of Edinburgh

Waddington, I., Windhorst, R. A., Peacock, J. A., Dunlop, J. S., Cohen, S. H., McClure, R. 1999, in preparation

Windhorst, R. A., van Heerde, G. M., Katgert, P. 1984a, A\&AS, 58, 1

Windhorst, R. A., Kron, R. G., Koo, D. C. 1984b, A\&AS, 58, 39 
The Hy Redshift Universe

ASP Conference Series. Vol. 193, 1999

A. J. Bunker \& W. J. M. van Breugel, eds.

\title{
HST Images of Radio-Selccted Groups
}

\author{
E.L. Zirbel
}

College of Staten Island

A. Oemler

Carnegie Observatories

J. Allington-Smith

Durham University

R.S. Ellis

Cambridge University

M. Dickinson

Space Telescope Science Institute

Abstract. We present high resolution HST images of six groups of galaxies surrounding powerful radio galaxies. In all groups we identify roughly as many elliptical as spiral galaxies, in addition to a sizable fraction of peculiar galaxies. Also many faint galaxies are visible. All radio galaxies have at least one close member within 3" of their nuclei.

\section{Introduction}

In the nearby universe, a strong morphology-density relation exists for galaxies, with dense cluster environments dominated by red, early-type galaxies, while the sparser field consists mainly of spirals. At higher redshift $(z \approx 0.4)$, this situation was considerably different, with many more blue galaxies present in rich clusters. To understand this evolution, we started a photometric survey of galaxies in groups and poor clusters with richness intermediate between that of rich clusters and the field. Here we present the HST images of six groups of galaxies.

\section{Observations}

We chose six groups of galaxies from our previously analyzed sample of intermediate redshift $(z \approx 0.4)$ radio-selected groups (Allington-Smith et al 1993). These groups are typical of groups in general and are not affected by the radio properties of the central radio galaxy. The table below describes the properties 
of these groups, and Figures 1 to 3 show the morphologies of the galaxies in the groups. Figures 4 to 6 show the radio galaxies in more detail.

Table 1. The Sample

\begin{tabular}{rrrrrrrr} 
Galaxy & redshift & $V_{r q}$ & $(B-V)_{r q}$ & $\log P_{408}$ & Richness & blue fraction integ time \\
\hline 3C16 & 0.405 & -22.24 & 0.90 & 27.73 & $13.2+-6.1$ & $0.68+-0.28$ & 7200 \\
3C435A & 0.471 & -22.65 & 0.64 & 27.90 & $33.5+-8.1$ & $0.21+-0.13$ & 14900 \\
$4 \mathrm{C} 27.51$ & 0.319 & -21.74 & 0.89 & 27.03 & $21.0+-6.8$ & $0.51+-0.16$ & 5200 \\
$5 \mathrm{C} 6.142$ & 0.448 & -23.19 & 0.89 & 26.00 & $23.3+-7.2$ & $0.22+-0.15$ & 11400 \\
$5 \mathrm{C} 12.251$ & 0.312 & -23.06 & 0.94 & 27.36 & $36.3+-7.2$ & $0.22+-0.10$ & 5000 \\
PKS0101+123 & 0.390 & -22.49 & 0.94 & 27.13 & $15.2+-5.8$ & $0.13+-0.20$ & 7500
\end{tabular}

\section{Discussion}

In all six groups we see a mix of disk and elliptical galaxies in addition to a substantial fraction of peculiar looking galaxies. Counter to what one would expect, the two groups with the highest fraction of blue galaxies (3C16 and $4 \mathrm{C} 27.51$ ) do NOT seem to show a relatively larger fraction of disk galaxies than the other four groups. The large fraction of faint galaxies in ALL images is particularly interesting. It is not yet clear whether or not these faint galaxies are group members.

All six radio galaxies are elliptical galaxies, although they show varying degrees of peculiarities and/or isophotal twists. Some of the radio galaxies show prominent tidal features, others much, much milder ones. All of them seem to have a small, close neighbor within 3 " of the radio galaxy nucleus.

\section{References}

Allington-Smith et al 1993, ApJ, 404, 521 

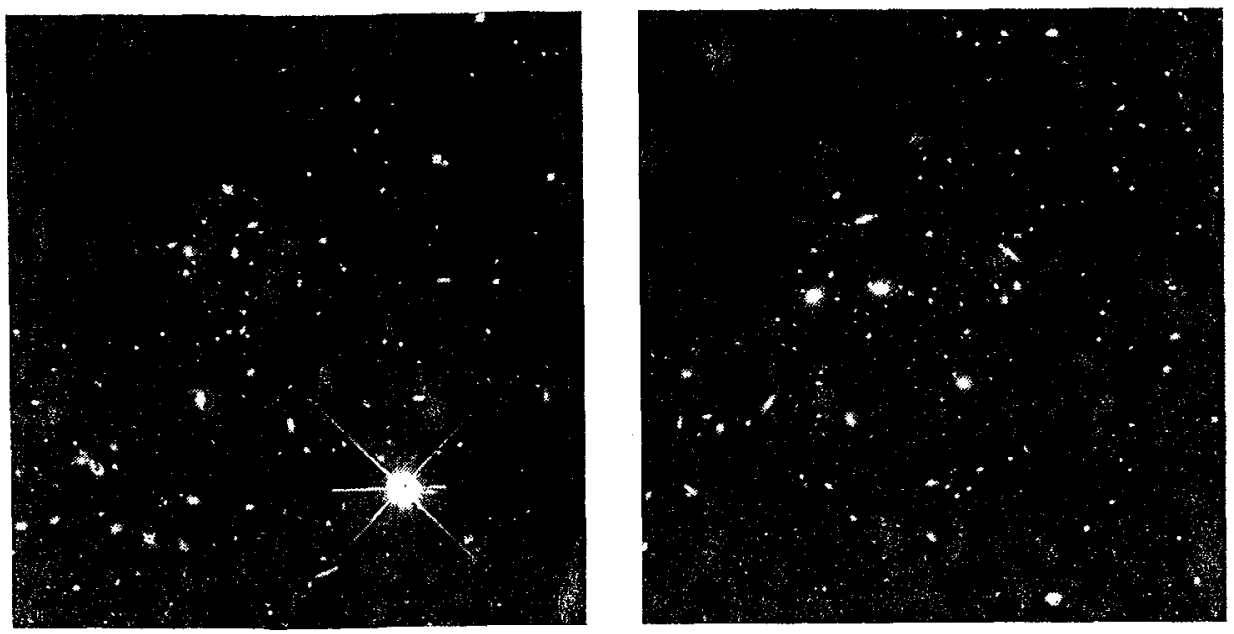

Figure 1. $3 \mathrm{c} 435$ and $5 \mathrm{c} 12$
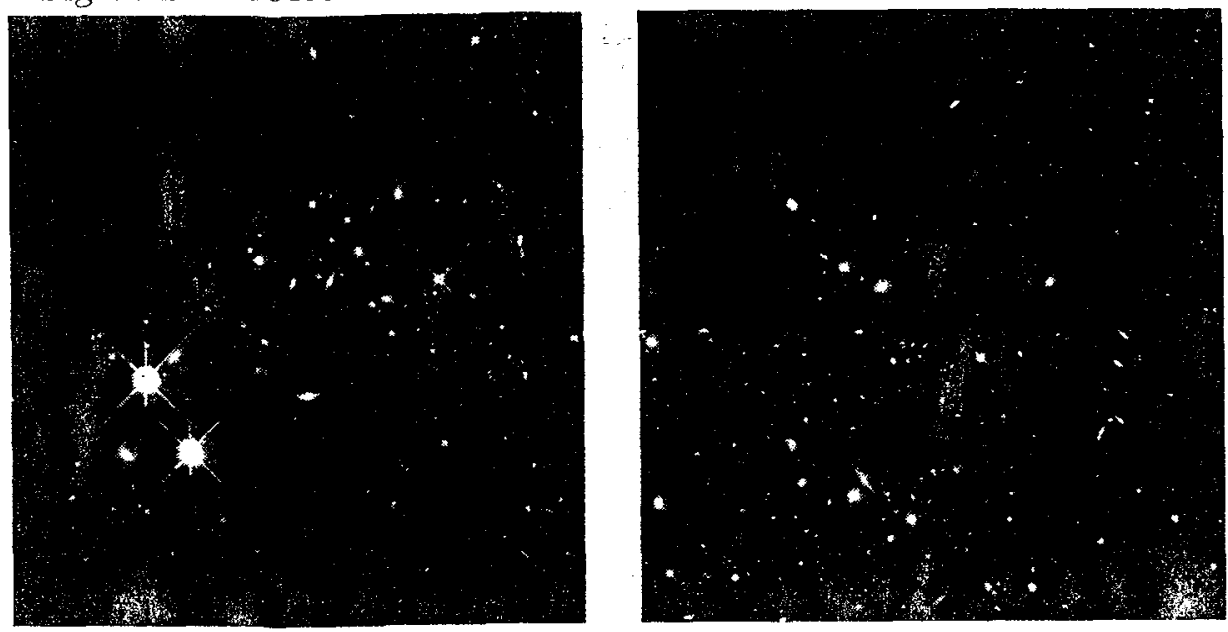

Figure 2. $\quad 4 c 2751$ and pks0101
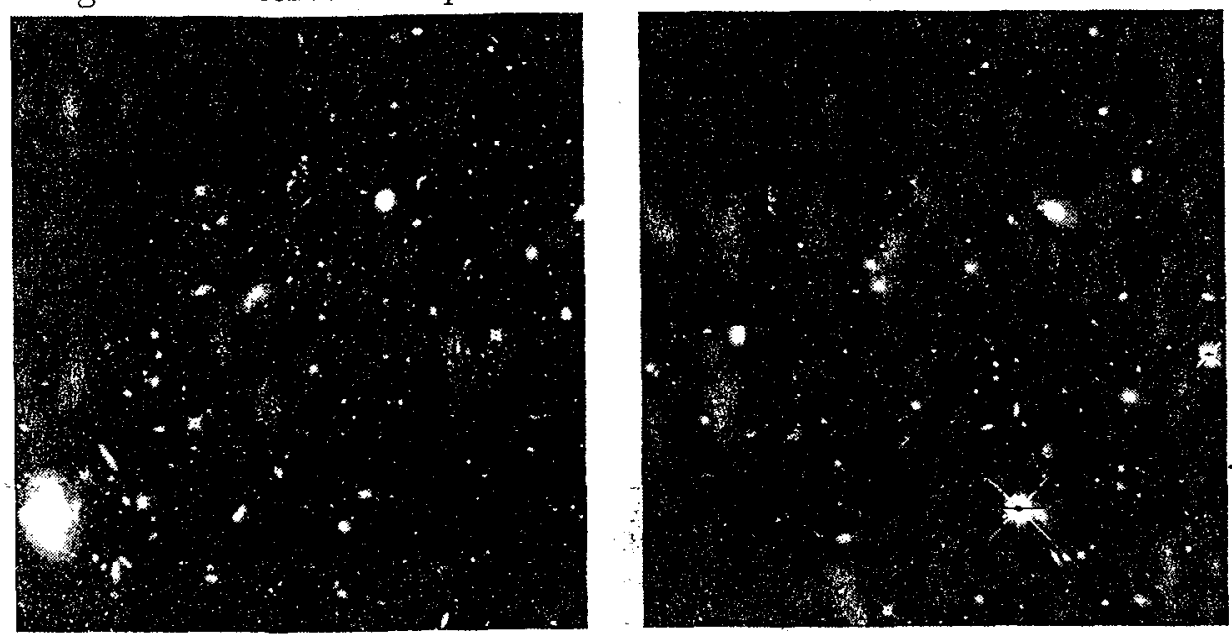

Figure 3. $3 \mathrm{c} 16$ and $5 \mathrm{c} 6$ 

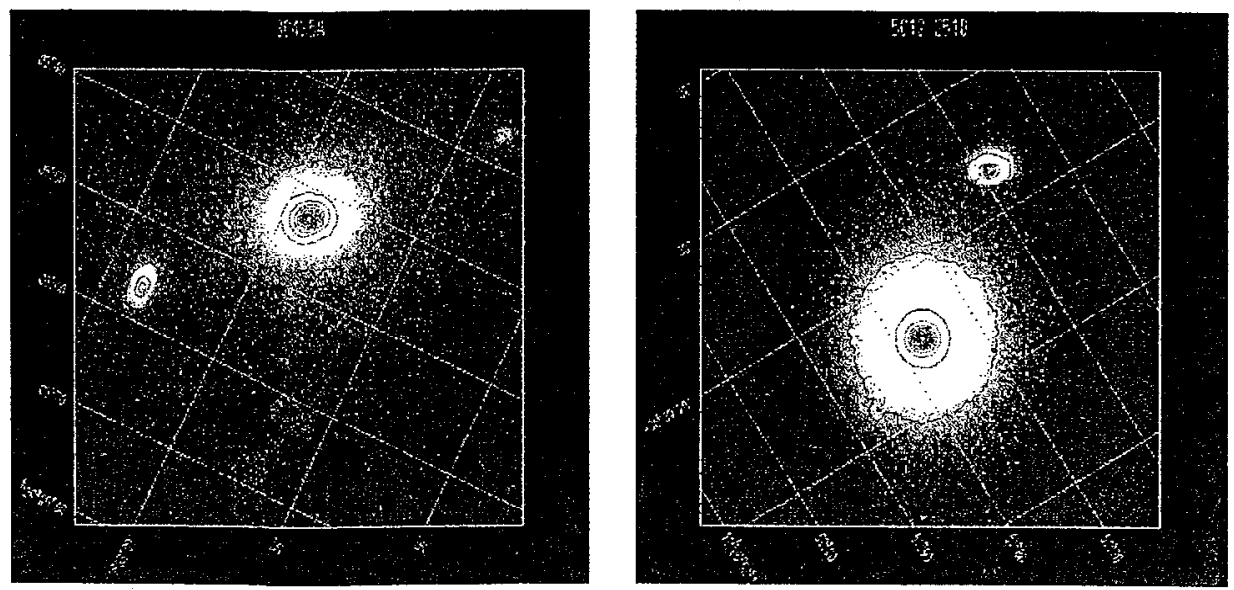

Figure 4. $3 \mathrm{c} 435 \mathrm{~A}$ and $5 \mathrm{c} 12$
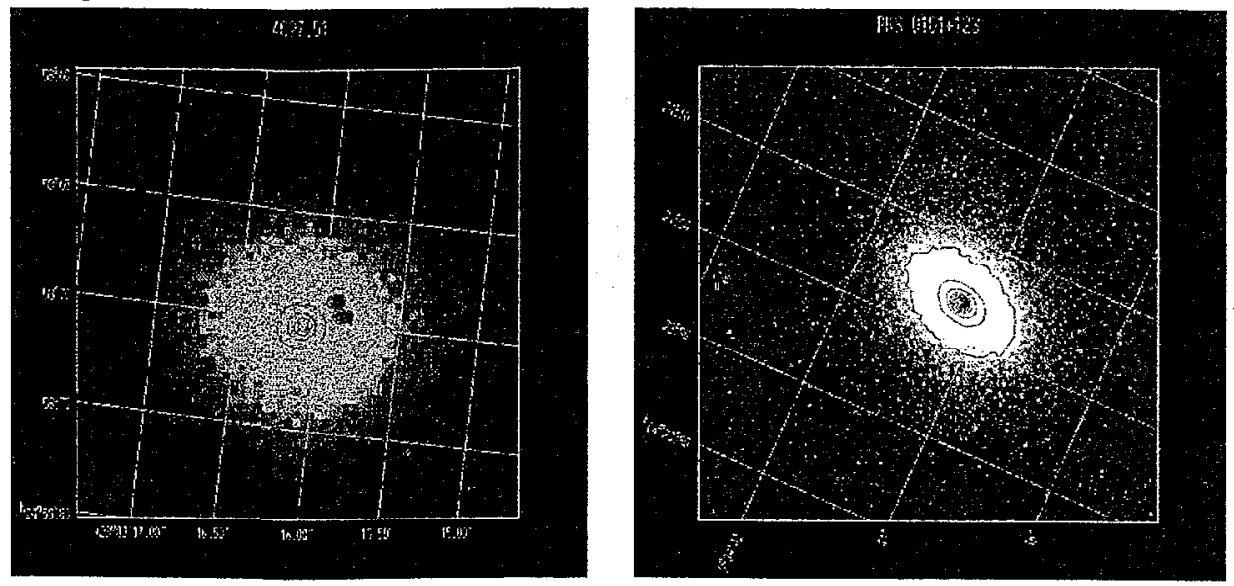

Figure 5. $\quad 4 c 2751$ and pks0101
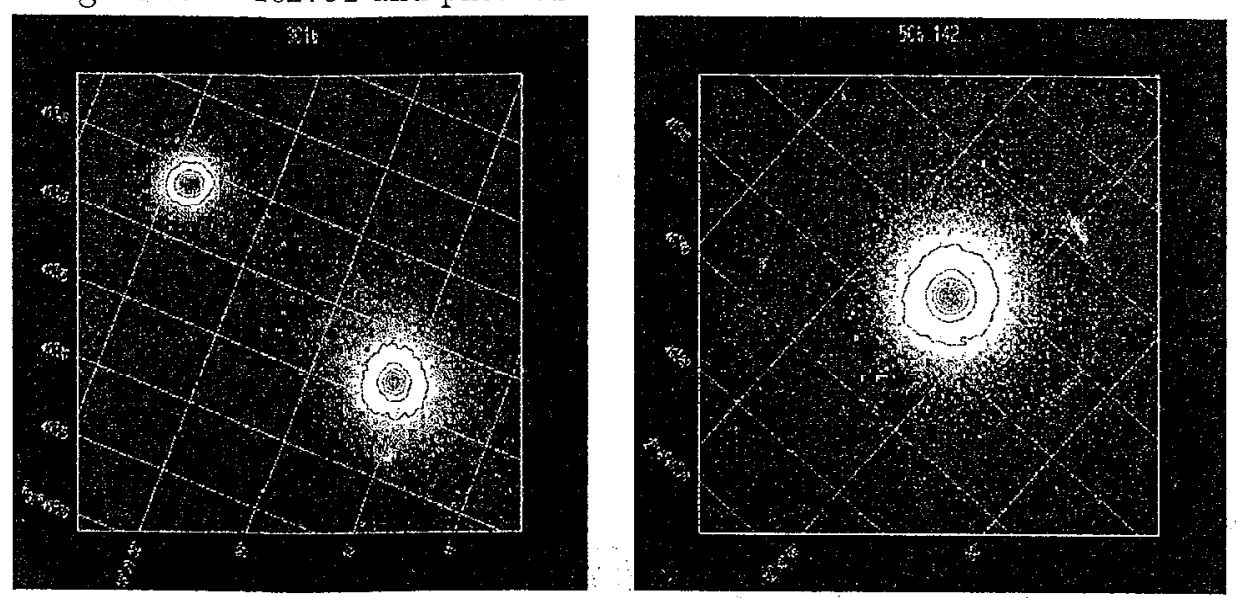

Figure $6 . \quad 3 \mathrm{c} 16$ and $5 \mathrm{c} 6$ 
The Hy Redshift Universe

ASP Conference Series, Vol. 193, 1999

A. J. Bunker \& W. J. M. van Breugel, eds.

\title{
NICMOS Imaging of High-Redshift Radio Galaxies
}

\author{
A. Zirm ${ }^{1}$, A. Dey ${ }^{2}$, M. Dichinson ${ }^{3}$, P.J. McCarthy ${ }^{4}$, P. Eisenhardt ${ }^{5}$, \\ S.G. Djorgovsk ${ }^{6}$, H. Spinrad ${ }^{7}$, S.A. Stanford ${ }^{8}$ and W. van Breugel ${ }^{8}$ \\ ${ }^{1} \mathrm{JHU},{ }^{2} \mathrm{NOAO},{ }^{3} \mathrm{STSCI},{ }^{4} \mathrm{OCIW},{ }^{5} \mathrm{JPL} / \mathrm{Caltech},{ }^{6}$ Caltech, ${ }^{7} \mathrm{UCB}$, \\ ${ }^{8} I G P P / L L N L$
}

\begin{abstract}
We have obtained near-infrared $(1.6 \mu \mathrm{m})$ images of 11 powerful $3 \mathrm{CR}$ radio galaxies at redshifts $0.8<z<1.8$ using NICMOS on board HST. The high angular resolution permits a detailed study of galaxy morphology in these systems at rest-frame optical wavelengths, where starlight dominates over the extended, aligned UV continuum. The NICMOS morphologies are mostly symmetric and are consistent with dynamically relaxed, elliptical host galaxies dominated by a red, mature stellar population. The aligned structures are sometimes faintly visible, and nuclear point sources may be present in a few cases which manifest the "unveiled" AGN that is obscured from view at optical wavelengths. Our observations are consistent with the hypothesis that the host galaxies of $z \approx 1-2$ radio galaxies are similar to modern-day gE galaxies. Their sizes are typical of $\mathrm{gE}$ galaxies but smaller than present-day $\mathrm{cD}$ and brightest cluster galaxies, and their surface brightnesses are higher, as expected given simple luminosity evolution.
\end{abstract}

\section{Introduction}

At one time, radio sources offered the only readily available means of locating galaxies at large redshifts $(z>1)$, and were thus studied as a window on the early history of galaxy evolution. Radio galaxies obey a tight near-infrared Hubble $(K-z)$ relation and are frequently associated with rich cluster environments. This suggests that there is an evolutionary sequence linking high-redshift radio galaxies to low-redshift giant ellipticals and $c D$ galaxies. The discovery that many high-redshift radio galaxies have elongated, complex UV continuum structures aligned with the radio source axis (McCarthy et al. 1987; Chambers et al. 1987) suggested that the active nucleus might affect the UV morphology and possibly even the evolutionary history of the host galaxy. It was believed that some radio galaxies might be true "protogalaxies" forming the bulk of their stars via some process induced by the radio jets. However, later studies have shown that in many cases the aligned UV continuum arises largely from scattered AGN emission (Di Serego Alighieri et al. 1989) and/or nebular continutum emission (Dickson et al. 1995).

The spectacular, complex structures seen in optical WFPC2 images of $3 \mathrm{CR}$ radio galaxies by (e.g.) Best et al. (1997) are, in many cases, AGN-related "ephemera" surrounding a more normal host galaxy. However, the brightness 


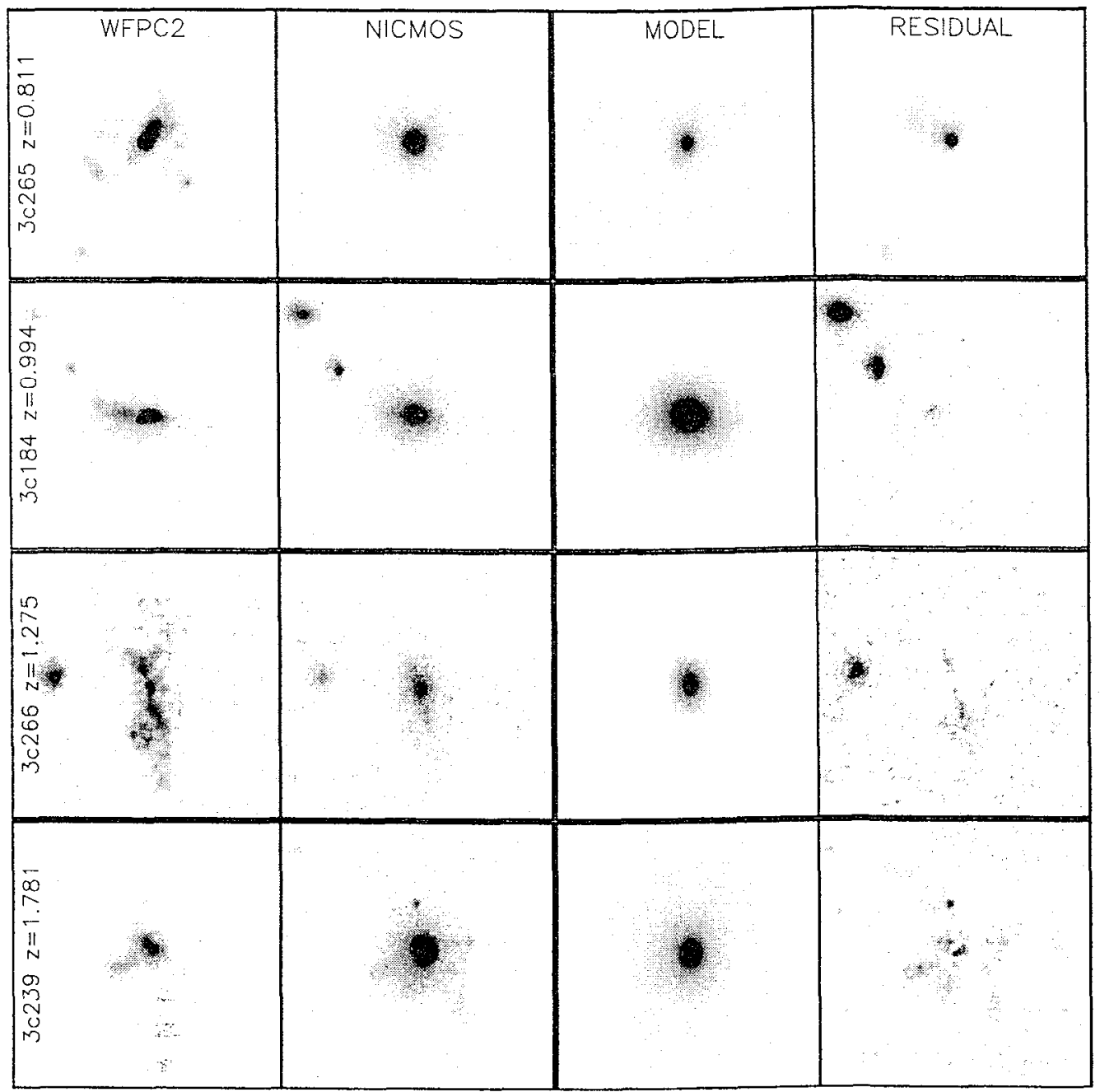

Figure 1. WFPC2 and NICMOS images of four galaxies from our NICMOS innaging sample, along with best-fitting models to the NICMOS host galaixes and the NICMOS minus model residuals.

of this aligned, blue light makes it difficult to study the properties of the underlying stellar component when observing at optical wavelengths. Observers have therefore turned to the near-IR, where the AGN-related emission is fainter and the stellar component brighter. Rigler et al. (1992), Dickinson et al. (1994) and Best et al. (1998), among others, have shown that some $3 \mathrm{CR}$ radio galaxies are rounder and more symmetric when observed in the near-IR, suggesting the presence of relatively "normal" host galaxies underlying the UV-bright, aligned Gonfinuium (but" see also Eisenhardt \& Chokshi 1990). But until now theses.

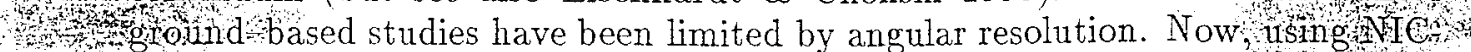
HOS on board HST, we can for the first time study the near-IR morphologies $-2+$ of high redshift galaxies with resolution comparable to that of the pioneering WFPC2 studies of these same objects. 


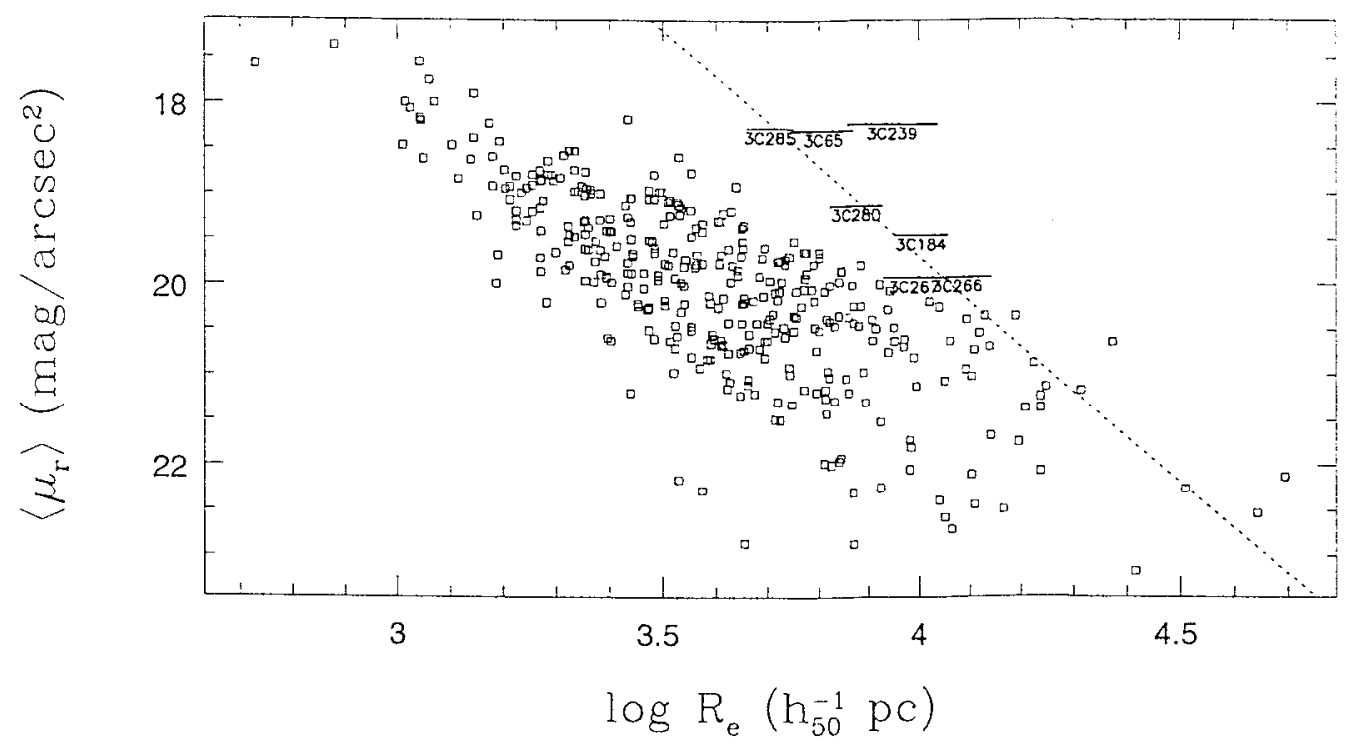

Figure 2. Rest frame Gunn $r$ size-surface brightness relation for local cluster ellipticals (small squares; Jørgensen et al. 1995) and for our NICMOS radio galaxies (lines). The lines connect the effective radii for $\mathrm{q}_{0}=0$ and $\mathrm{q}_{0}=0.5$ cosmologies. The dotted line shows the relation for constant galaxy luminosity, as expected for "standard candle" galaxies.

\section{Observations}

Our sample consists of $113 \mathrm{CR}$ radio galaxics at $0.8<z<1.8$, imaged with NICMOS Camera 2, which provides diffraction limited images at $1.6 \mu \mathrm{m}$. We used bandpasses (F160W or F165M) which avoid strong nebular emission lines, which could significantly contaminate the fluxes and affect the observed morphologies. Optical WFPC2 imaging is availablc for the whole sample: in many cases we have unusually deep and often polarimetric WFPC2 data, while in others archival data by Best et al. 1997 or other sources were used. In most cases we also have extensive ground-based supporting data (spectroscopy, polarimetry, etc.) from the W.M. Keck Observatory and other facilities.

\section{Discussion}

The NICMOS and WFPC2 images of four galaxies from this sample are shown in Figure 1. We have fit PSF-convolved models to the NICMOS images using a hybrid scheme which matches 1D surface brightness profiles and 2D PA + ellipticity information. The models and the NICMOS image residuals after model subtraction are also shown in Figure 1. In most cases, the NICMOS images show that the rest-frame optical light from powerful $3 \mathrm{CR}$ radio galaxies at $z>1$ is rounder, smoother, more symmetric and centrally concentrated than that observed at rest-frame UV wavelengths. The complex, aligned structures seen in WFPC2 images are generally much less pronounced in the near-IR, although in several cases (e.g. 3C 280, 3C 266, 3C 368) the highest surface brightness regions 
of the aligned components can still be detected. In several cases, the near-IR surface brightness peaks at the position of a local minimum in the WFPC2 images. suggesting the effects of dust lanes affecting the near-UV morphologies. A few galaxies (e.g., 3C 265) appear to have nuclear point sources in the IR, possibly showing the "unveiled" AGN.

Overall, the gross morphologies and surface brightness profiles of most $3 \mathrm{CR}$ hosts are consistent with their bcing high luminosity giant elliptical galaxies, already structurally mature. This may be true as early as $z=1.8$, although at that redshift 3C 239 appears to have "ragged edges" perhaps suggesting that it is in the process of accreting material through mergers. However, the most distant galaxy in our sample, $3 \mathrm{C} 256$ at $z=1.82$, is radically different than the others. It is elongated, aligned, diffuse, and underluminous, and thus may be the exceptional example of a young radio galaxy carly in the stages of its formation (see also Eisenhardt \& Dickinson 1992, Simpson et al. 1999).

In Figure 2, we plot surface brightness vs. effective radius (the "Kormendy relation") for 6 galaxies which are well fit by $R^{1 / 4}$-law models, converting the NICMOS photometry (rest-frame $\lambda_{0} 0.57$ to $0.88 \mu \mathrm{m}$ for our sample) to restframe Gunn $r\left(\lambda_{0} 0.65 \mu \mathrm{m}\right)$ for comparison to nearby cluster ellipticals. The galaxies are physically smaller than the largest and brightest giant cluster ellipticals at $z=0$, and have higher rest-frame surface brightnesses as would be expected given nominal luminosity evolution. Most fall on the locus of constant luminosity (see also Best et al. 1998), as might be expected given the small $K^{-}-z$ scatter. 3C 239 at $z=1.78$ is significantly more luminous for its size compared to the galaxies at $0.8<z<1.3$.

Acknowledgments. "Support for this work was provided by NASA grant GO-07454.02-96A. Thanks to Hy for getting us all into this radio galaxy mess!

\section{References}

Best, P.N., Longair, M.S., Roellgering, H.J.A., 1997, MNRAS, 292, 758.

Best, P.N., Longair, M.S., Roettgering, H.J.A., 1998, MNRAS, 295, 549.

Chambers, K.C., Miley, G.K., van Breugel, W., 1987, Nature, 329, 604.

di Serego Alighieri, S., Fosbury, R.A.E., Quinn, P.J., Tadhunter, C., 1989, Nature, $341,307$.

Dickinson, M., Dey, A., \& Spinrad, H., 1995, in Galaxies in the Young Universe, eds. Hippelein, Meisenheimer \& Roser, (Berlin: Springer), p. 164.

Dickson, R., Tadhunter, C., Shaw, M., Clark, N., Morganti, R., 1995, MNRAS, $273,29$.

Eisenhardt, P.R.M., \& Chokshi, A., 1990, ApJ, 351, L9.

Eisenhardt, P.R.M., \& Dickinson, M., 1992, in The Evolution of Galaxies and their Environments, eds. Hollenback, D., Thronsen, H., and Shull, M.

Jørgensen, I., Franx, M., \& Kjægaard, P. 1996, MNRAS, 273, 1097.

McCarthy, P.J., van Breugel, W., Spinrad, H., Djorgovski,S.,A.pJ, 321 , L29.

Rigler, M., Lilly, S.J., Stockton, A., Hammer, F., LeFèvre, O, 1992, ApJ, 385, 61.

Simpson, C., et al. 1999, ApJ, in press. 
Section B. Galaxy Stellar Populations and Scaling Relations 
The Hy Redshift Universe

ASP Conference Series, Vol. 193, 1999

A. J. Bunker \& W. J. M. van Breugel, eds.

\title{
- Stellar Population Synthesis Models
}

\author{
G. Bruzual A. \\ Centro de Investigaciones de Astronomía (C.I.D.A.), Apartado Postal \\ 264, Mérida, Venezuela
}

\begin{abstract}
I present a comparison of current population synthesis model predictions with obscrved spcctra for stellar systems of various ages and metallicities. It is argued that models built using different ingredients differ in the resulting values of some basic quantities (e.g. $M / L_{V}$ ), without need to invoking violations of physical principles. A summary of results from a companion paper is included.
\end{abstract}

\section{Introduction}

The large amount of astrophysical data that has become available in the last few years has made possible to build several complete sets of stellar population synthesis models. The predictions of these models have been used to study many types of stellar systems, from local normal galaxies to the most distant galaxies discovered so far (approaching $z$ of 4 to 5 ), from globular clusters in onr galaxy to proto-globular clusters forming in different environments in distant, interacting galaxies. Bruzual (1998, GB98 hereafter) has discussed the limitations of these models, as well as the uncertainties that are introduced into a set of models by the different choices of ingredients available in the literature. In this paper, which is complementary to GB98, I present an overview of results from population synthesis models directly applicable to the interpretation of galaxy spectra.

In §II I summarize the current status of a particular set of population synthesis models. In $\S I I I$ I clarify a possible misunderstanding on the energetics of model stellar populations that is found in the literature. In §IV I show a brief comparison of our models with Jimenez (1999) models. Conclusions are presented in $\S \mathrm{V}$.

\section{Current status of population synthesis models}

Bruzual \& Charlot (2000, BC2000 hereafter) have extended the Bruzual \& Charlot (1993, BC93 hereafter) evolutionary population synthesis models to provide the evolution in time of the spectrophotometric properties of simple stellar populations (SSPS) for a wide range of stellar metallicity. In an SSP all the stars form at $t=0$ and evolve passively afterward. The BC2000 models are based on the stellar evolutionary tracks computed by Alongi et al. (1993), Bressan et al. (1993), Fagotto et al. (1994a, b, c), and Girardi et al. (1996), which use the radiative opacities of Iglesias et al. (1992). This library includes tracks for stars with initial chemical composition $Z=0.0001,0.0004,0.004,0.008,0.02,0.05$, and 
0.10 , with $Y=2.5 Z+0.23$, and initial mass $0.6 \leq m / M_{\odot} \leq 120$ for all metallicities, except $Z=0.0001\left(0.6 \leq m / M_{\odot} \leq 100\right)$ and $Z=0.1\left(0.6 \leq m / M_{\odot} \leq 9\right)$. This set of tracks will be referred to as the Padova or $(P)$ tracks hereafter.

The published tracks go through all phases of stellar evolution from the zero-age main sequence to the beginning of the thermally pulsing regime of the asymptotic giant branch (AGB, for low-and intermediate-mass stars) and core-carbon ignition (for massive stars), and include mild overshooting in the convective core of stars more massive than $1 M_{\odot}$. The Post-AGB evolutionary phases for low- and intermediate-mass stars were added to the tracks by BC2000 from different sources (see BC2000 for details).

BC2000 use as well a parallel set of tracks for solar metallicity computed by the Geneva group (Geneva or $(G)$ tracks hereafter), which provides a framework for comparing models computed with two different sets of tracks.

The BC2000 models use the library of synthetic stellar spectra compiled by Lejeune et al. (1997a,b, LCB97 hereafter) for all the metallicities listed above. This library consists of Kurucz (1995) spectra for the hotter stars (O-K), Bessell et al. $(1989,1991)$ and Fluks et al. (1994) spectra for M giants, and Allard \& Hauschildt (1995) spectra for $\mathrm{M}$ dwarfs. For $Z=Z_{\odot}, \mathrm{BC} 2000$ also use the Pickles (1908) stellar atlas, asscmblod from cmpirical stellar data.

Regardless of the specific computational algorithm used, all evolutionary synthesis models depend on three adjustable parametric functions: (1) the stcllar initial mass function, $f(m)$, or IMF; (2) the star formation rate, $\Psi(t)$, or SFR; and (3) the chemical enrichment law, $Z(\iota)$. For a given choice of $f(m), \Psi(t)$, and $Z(t)$, a particular set of evolutionary synthesis models provides: (1) Galaxy spectral energy distribution (SED) vs. time, $F_{\lambda}(\lambda, Z(l), t)$; (2) Galaxy colors and magnitude vs. time; (3) Line strength and other spectral indices vs. time.

\section{Comparison of model and observed spectra}

\subsection{Solar metallicity}

Fig 1 shows a model fit to the average spectrum of an E galaxy (kindly provided by M. Rieke). The model sed is the line extending over the complete wavelength range shown in the figure. The observed sed covers the range from $3300 \mathrm{~A}$ to $2.75 \mu \mathrm{m}$. The residuals (observed - model) are shown at the bottom of the figure in the same vertical scale. The model corresponds to a $10 \mathrm{Gyr} Z=Z_{6} \mathrm{SSP}$ computed for the Salpeter $(1955) \operatorname{IMF}\left(m_{L}=0.15, m_{U}=125 M_{\odot}\right)$ using the $(P)$ tracks and the Pickles (1998) stellar atlas. The fit is excellent over most of the spectral range. A minor discrepancy remains in the region from 1.1 to $1.7 \mu \mathrm{m}$. The source of this discrepancy is not understood at the moment. In Fig 2 I show the same model and E galaxy sed as in Fig 1 but in different units. In addition, in Fig 2 I include the broad band fluxes representing the average of many $E$ galaxies in the Coma cluster (solid squares) from $A$. Stanford (private communication). The observed sed is the one with the lorvest spectral resolution. Figs 3 and 4 show a closer look at the same data in an enlarged scale. Again the agreement is excellent for the 3 data sets. The discrepant line in Fig 4 corresponds to the same.model shown in Figs 2 and 3 but I used the LCB9T synthetic stellar atlas instead of the empirical stellar seds. Fig 4 shows clearly 


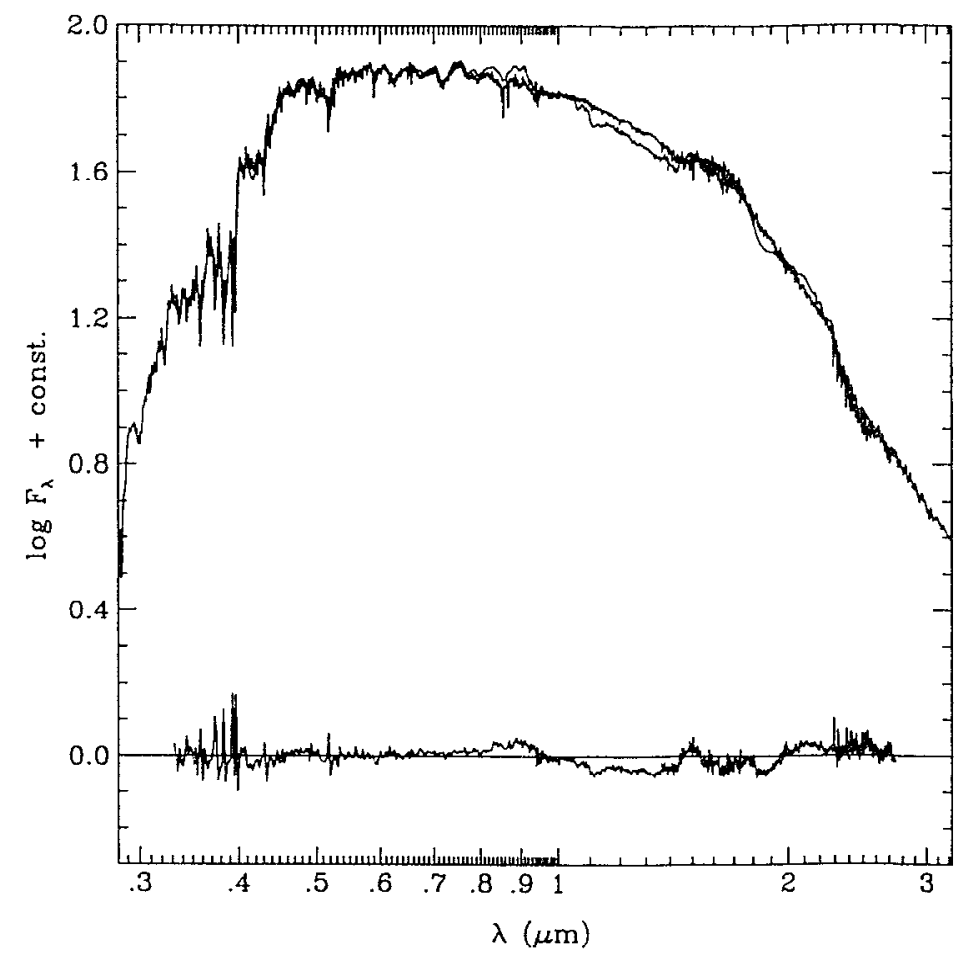

Figure 1.

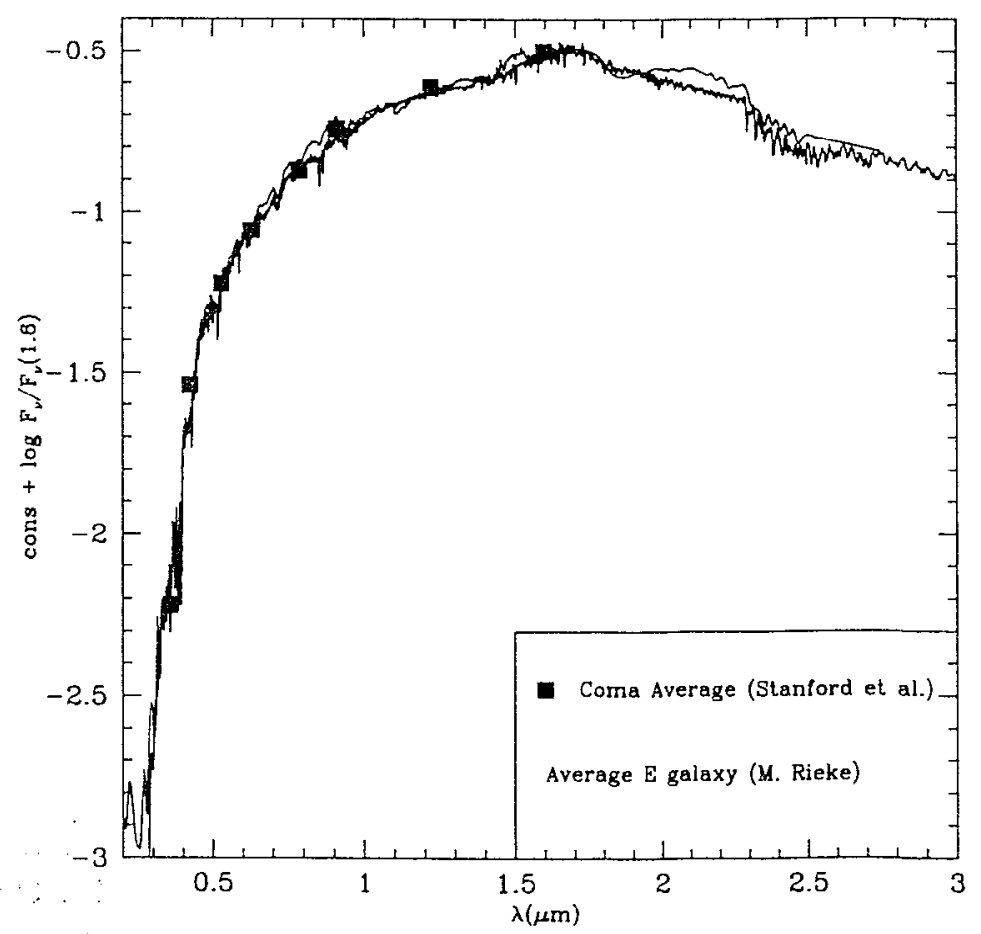

Figure 2. 


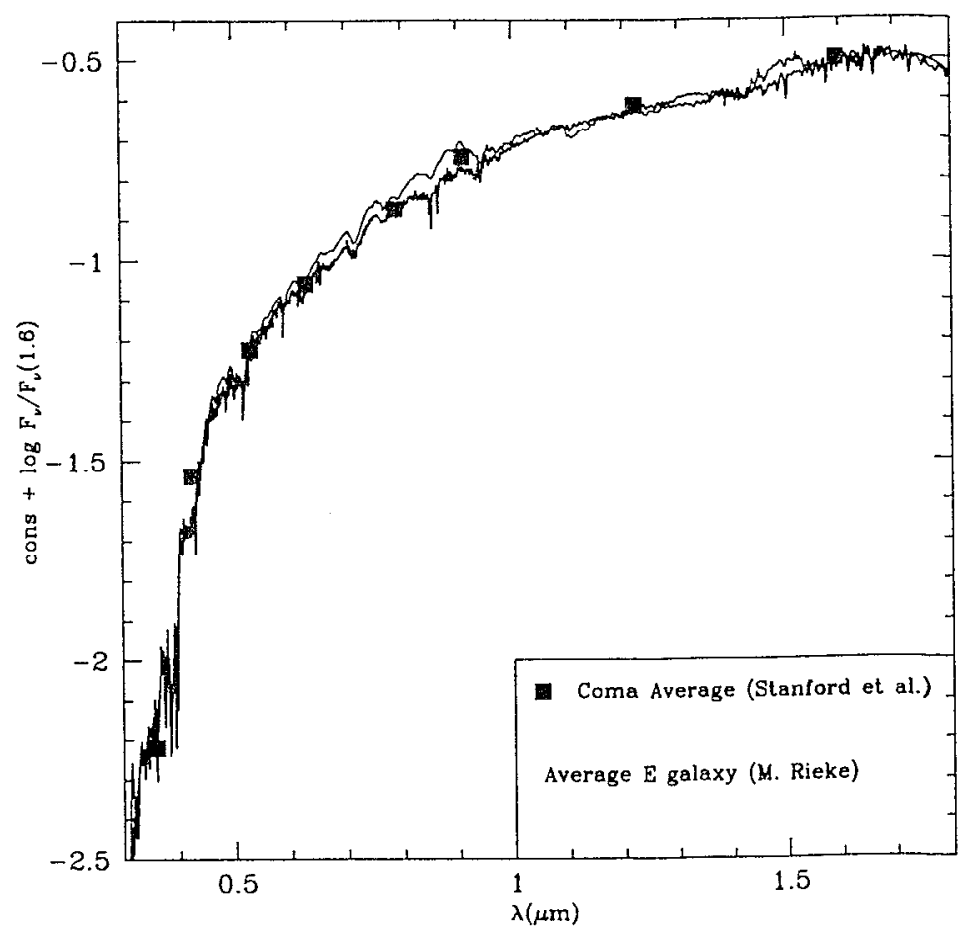

Figure 3.

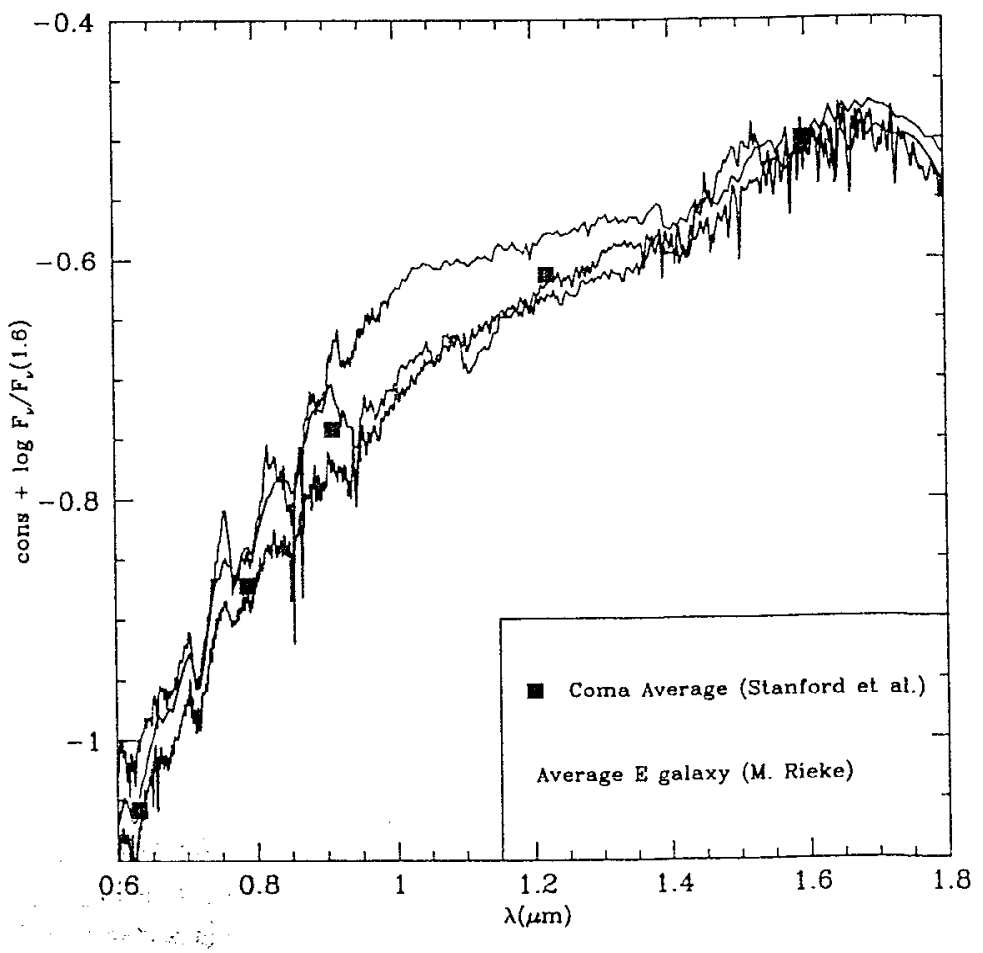

Figure 4. 


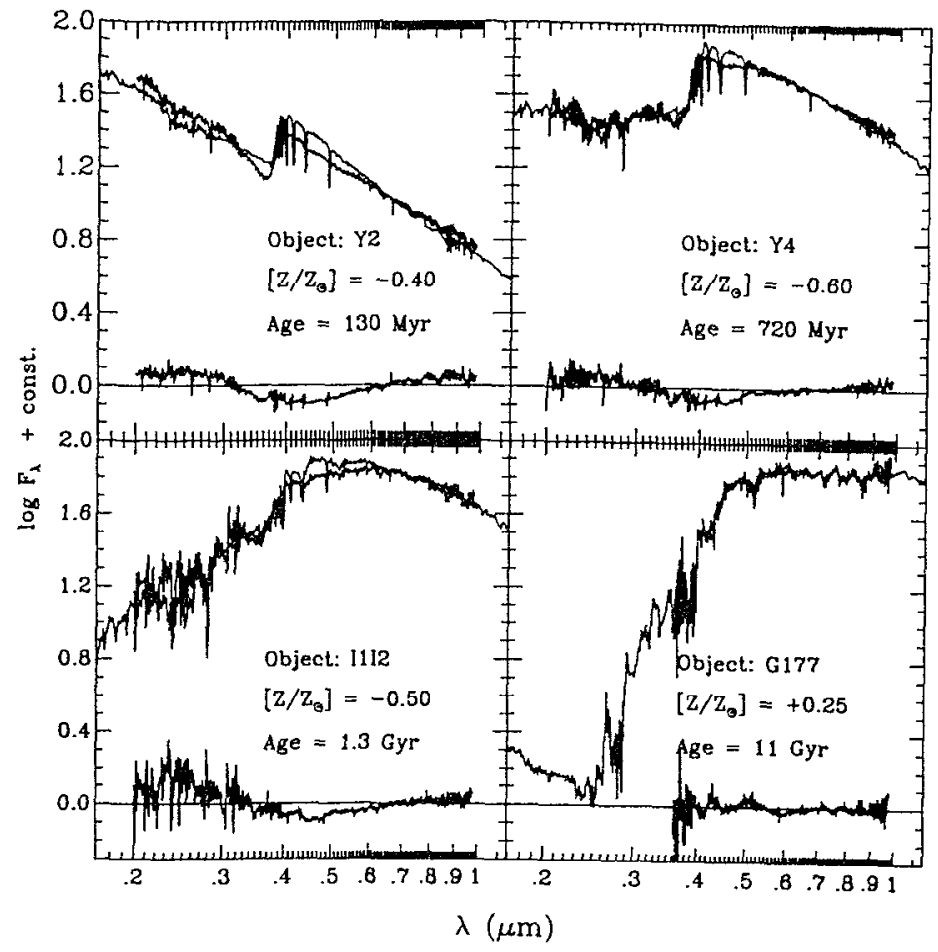

Figure 5.

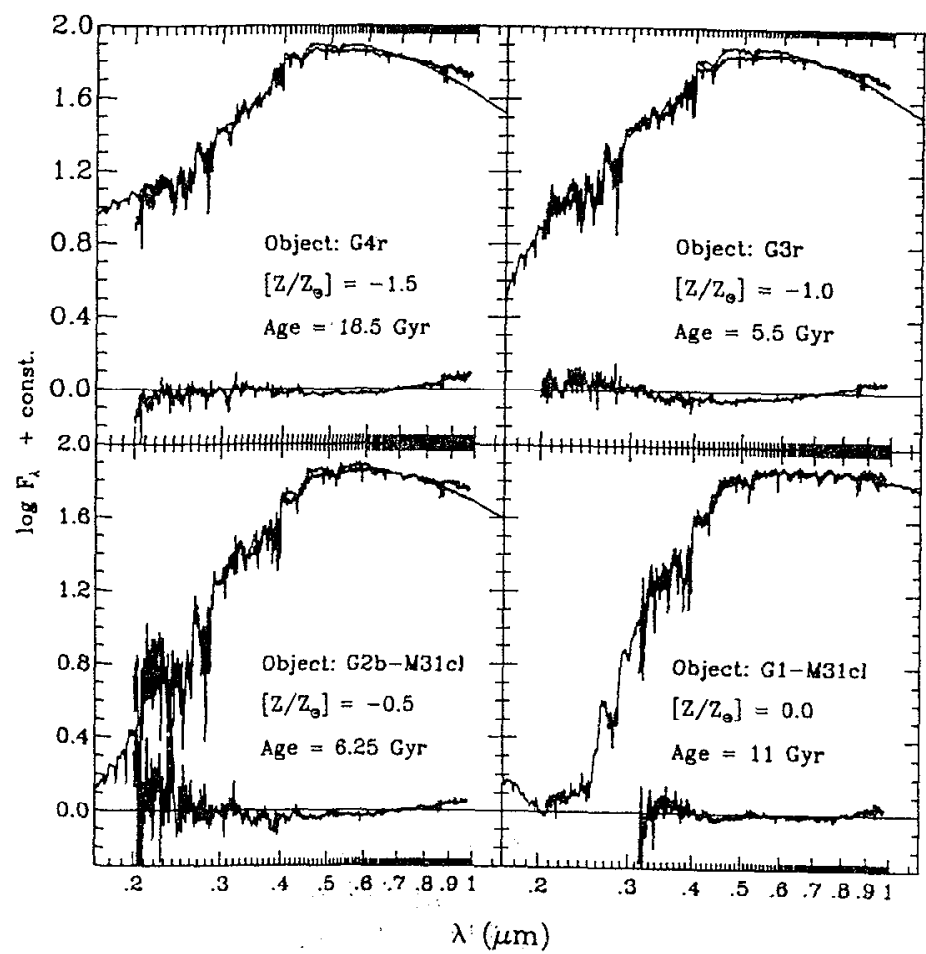

Figure 6. 
that the models based on empirical stellar seds are to be preferred over the ones based on theoretical model atmospheres. Unfortunately, complete libraries of empirical stellar seds are available only for solar metallicity.

\subsection{Non-solar metallicity}

Figs 5 and 6 show the results of a comparison of SSP models built for various metallicities using the LCB97 atlas, all for the Salpeter IMF, with several of the average spectra compiled by Bica et al. (1996). The name and the metal content of the observed spectra indicated in each panel is as given by Bica et al. The quoted age is derived from the best fit of our model spectra to the corresponding observations. The residuals (observed - model) are shown in the same vertical scale (see description to Fig 1 above for details). Even though, in detail, the fits for non-solar metallicity stellar populations are not as good as the ones for solar metallicity, over all the models reproduce the observations quite well over a wide range of $\left[Z / Z_{\odot}\right]$, and provide a reliable tool to study these stellar systems. The discrepancy can be due both to uncertainties in the synthetic stellar atlases or the evolutionary tracks at these $\left[Z / Z_{\odot}\right]$. I have used SSPs in all the fits, neglecting possibly composite stellar populations, as well as any interstellar reddening.

\section{On the energetics of model stellar populations}

There are significant differences in the fractional contribution to the integrated light by red giant branch (RGB) and asymptotic giant branch (AGB) stars in SSPs computed for different sets of evolutionary tracks. Fig. 7 shows the contribution of stars in various evolutionary stages to the bolometric light, and to the broad-band $U B V R I F L$ fluxes for a $Z=Z_{\odot}$ model SSP computed for the Salpeter IMIF $\left(m_{L}=0.15, m_{U}=125 M_{\odot}\right)$ using the $(P)$ tracks and the Pickles (1998) stellar atlas. The meaning of each line is indicated in the top central frame. Fig. 8 shows the corresponding plot for an equivalent model computed according to the $(G)$ tracks. The contribution of the RGB stars is higher in the $(P)$ track model than in the $(G)$ track model. Correspondingly, the AGB stars contribute less in the $(P)$ track model than in the $(G)$ track model. For instance, for $t>1$ Gyr, RGB and AGB stars contribute $40 \%$ and $10 \%$, respectively, to the bolumetric light in the $(P)$ track model (Fig. 7 ). These fractions change to $30 \%$ and $20 \%$ in the $(G)$ track model (Fig. 8). These differences are seeing more clearly in Fig. 9 which shows the ratio of the fractional contribution by different stellar groups in the $(G)$ track model to that in the $(P)$ track model. According to the fuel consumption theorem (Renzini 1981), these numbers reflect relatively large differences in the amount of fuel used up in the RGB and $A G B$ phases by stars of the same mass and initial chemical composition depending on the stellar evolutionary code.

Fig 10 shows the ratio of the Hux contributed by Post-.-WS to MS stars in the same bands as before for the SSP models indicated in the top central frame. From $U$ to $R$ the contribution of Post-MS to MS stars is higher with the $(P)$ tracks than with the $(G)$ tracks. The situation reverses in the $A^{-}$and $L$ bands. In the $I$ band all models are very similar. For a given set of tracks, the Scalo 


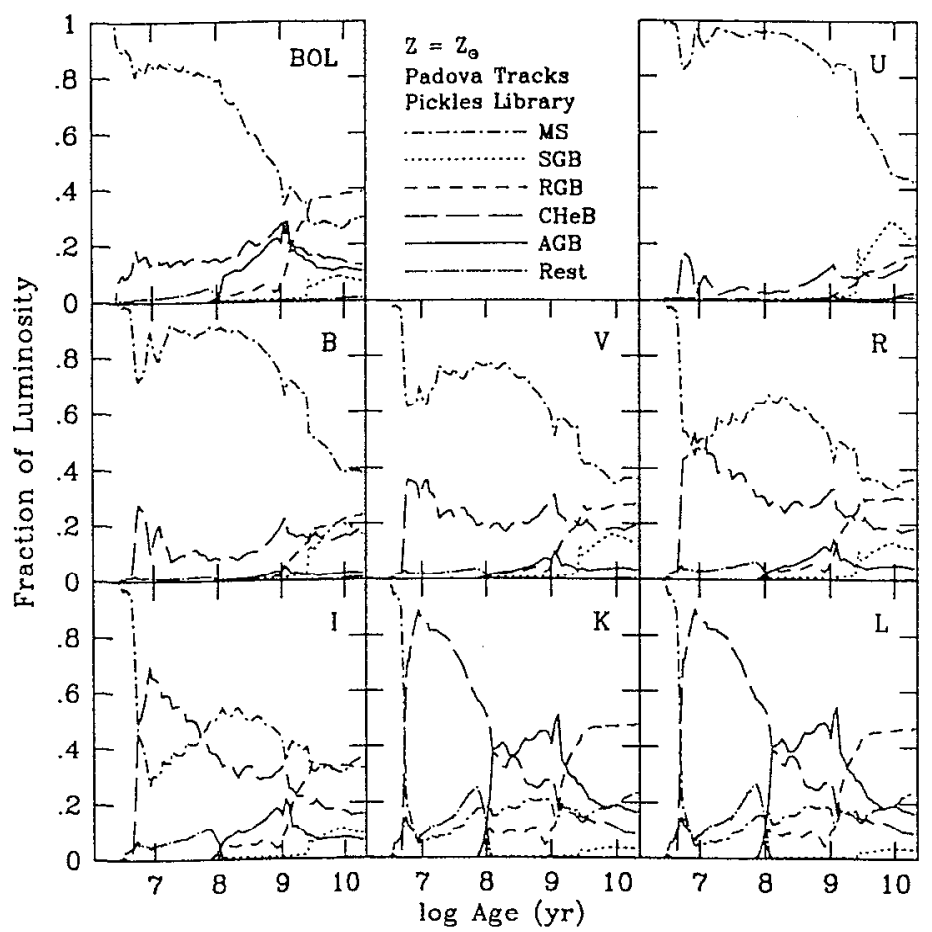

Figure $\rceil.$

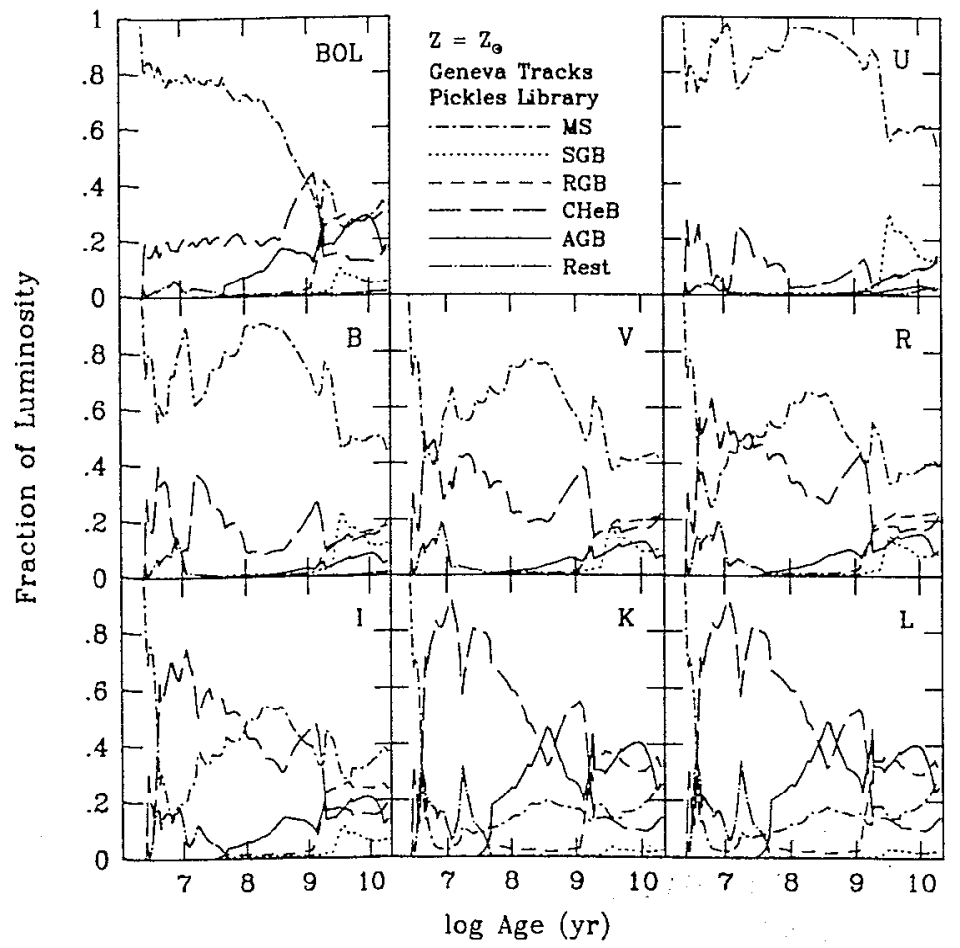

Figure 8. 


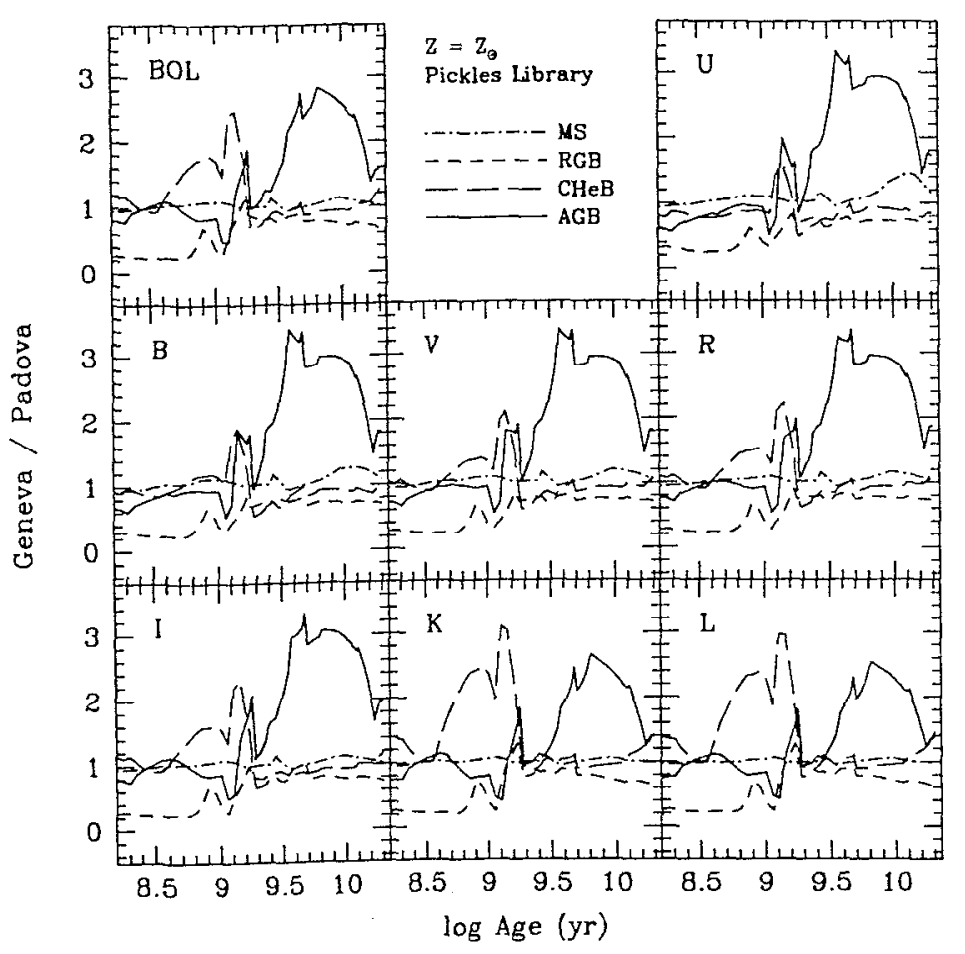

Figure 9.

(1986) IMF models have a higher Post-MS to MS contribution than the Salpeter IVIF models. The Scalo IMF is less rich in MS stars than the Salpeter IMF.

Buzzoni (1999) has argued that most population synthesis models violate basic prescriptions from the fuel consumption theorem (FCT). Fig 11 should be compared with Fig 2 of Buzzoni (1999). The line with square dots along it is reproduced form Buzzoni's Fig 2. The other lines show the dependence of the ratio of the Post-MS to MS contribution to the bolometric flux for different models. The heavy lines correspond to the Salpeter IVF models. The thin lines to the Scalo IMF models. The solid lines correspond to $(P)$ track models, whereas the dashed lines correspond to the $(G)$ track models. The $(G)$ track model for the Salpeter IMF (heavy dashed line) is in quite good agreement with Buzzoni's model for $t>5$ Gyr.

Fig 12 (after Buzzoni's Fig 1) plots the $M / L_{V}$ ratio vs. the Post-MS to MS contribution in the $V$ band. The open dots correspond to the models shown in Buzzoni's Fig 1. The solid dot is Buzzoni's model marked B in his Fig 1. The solid triangles correspond to onr $Z_{\odot}$ SSP models for various stellar atlases using the $(P)$ tracks and the Salpeter IMF. The open triangles are for the same models but for the Scalo IMF. The solid pentagons represent the $(G)$ track models for the Salpeter IMF and the open pentagons the same models but. for the Scalo INF. The three solid squares joined by a line represent sub-solar metallicity models for the $(P)$ tracks and the Salpeter IMF. The three open squares joined by a line are for identical models using the Scalo IMF. Fig 12 shows clearly that the position of points representing various models in this diagram is a strong 


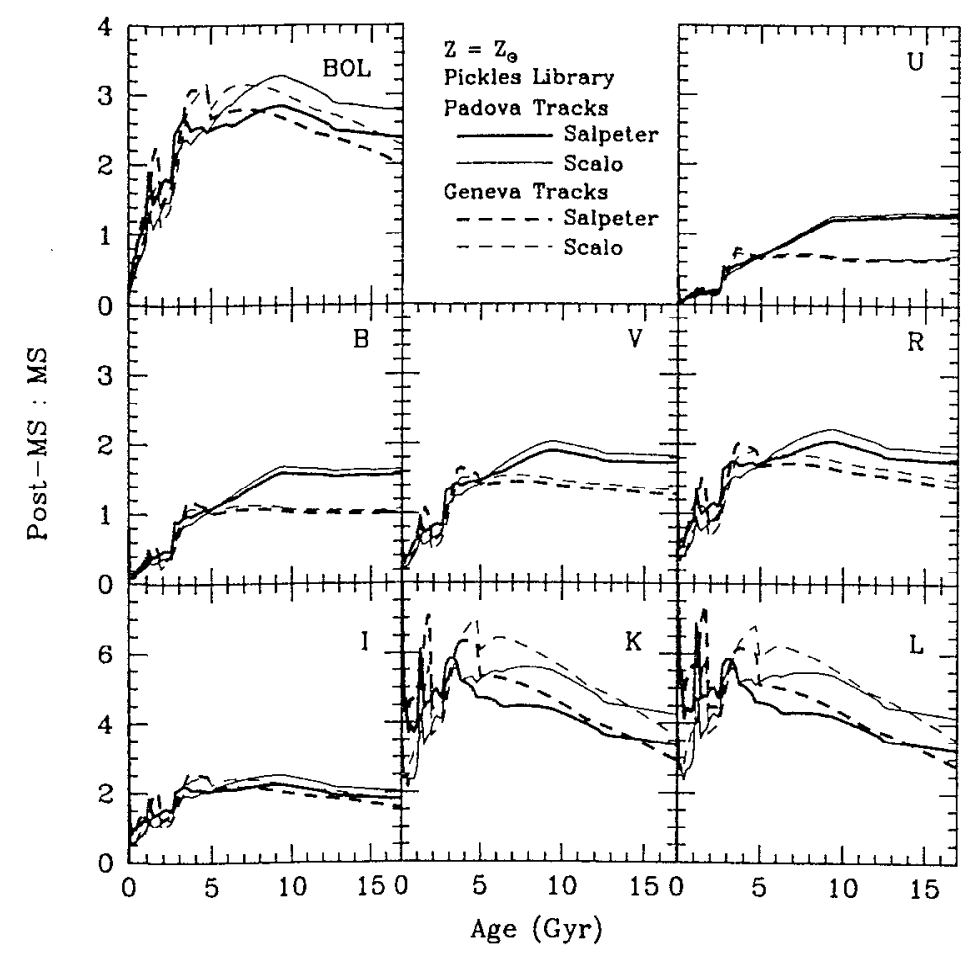

Figure 10.

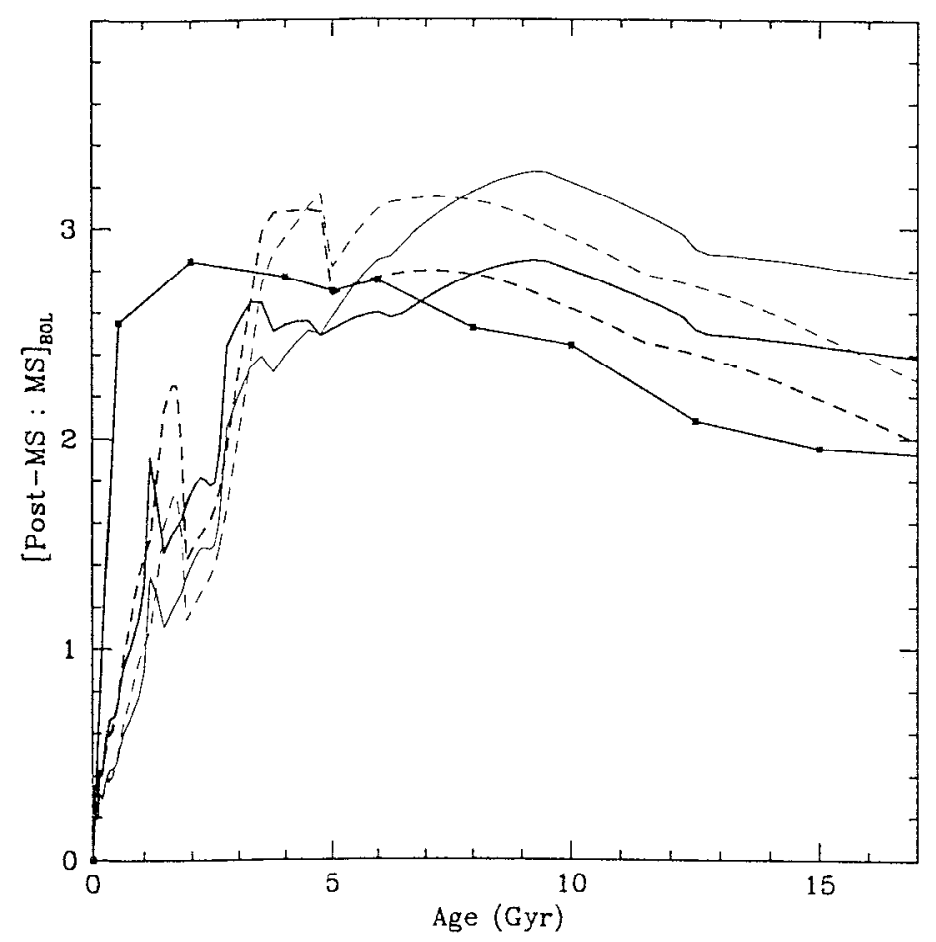

Figure 11. 


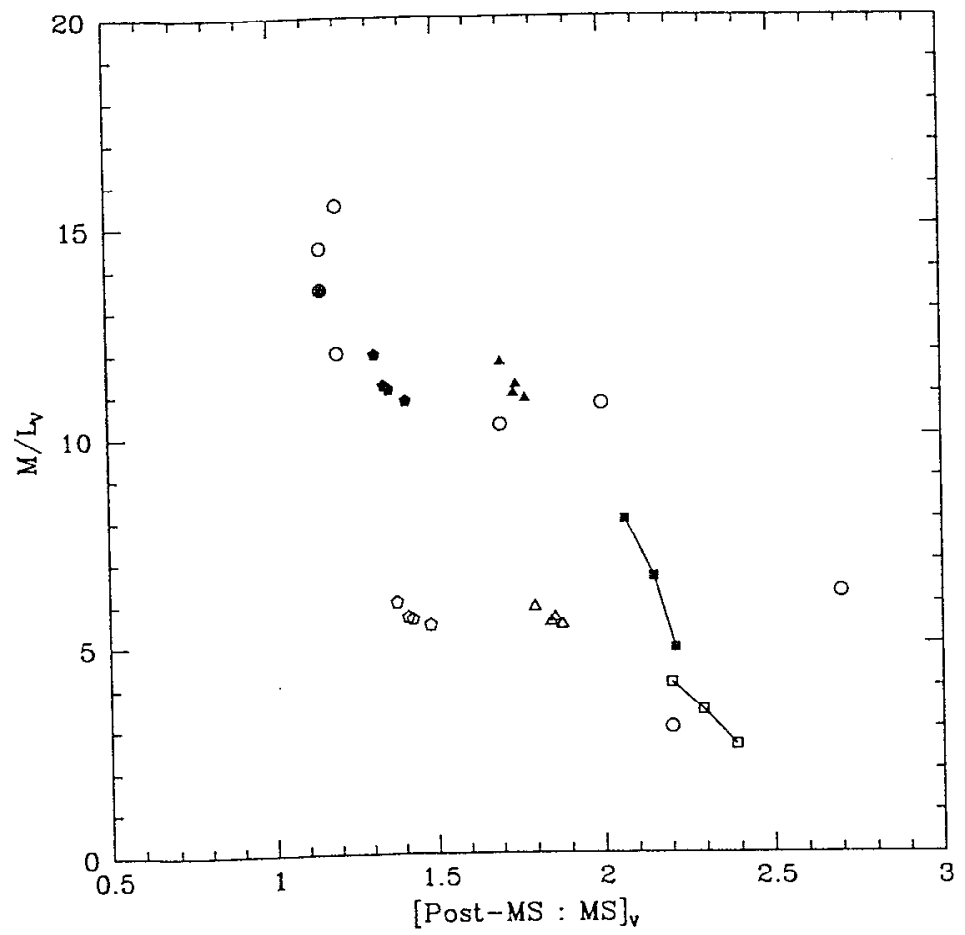

Figure 12. $\quad$ SSP, $Z=Z_{\odot}$, Salpeter IMF, 5 Gyr

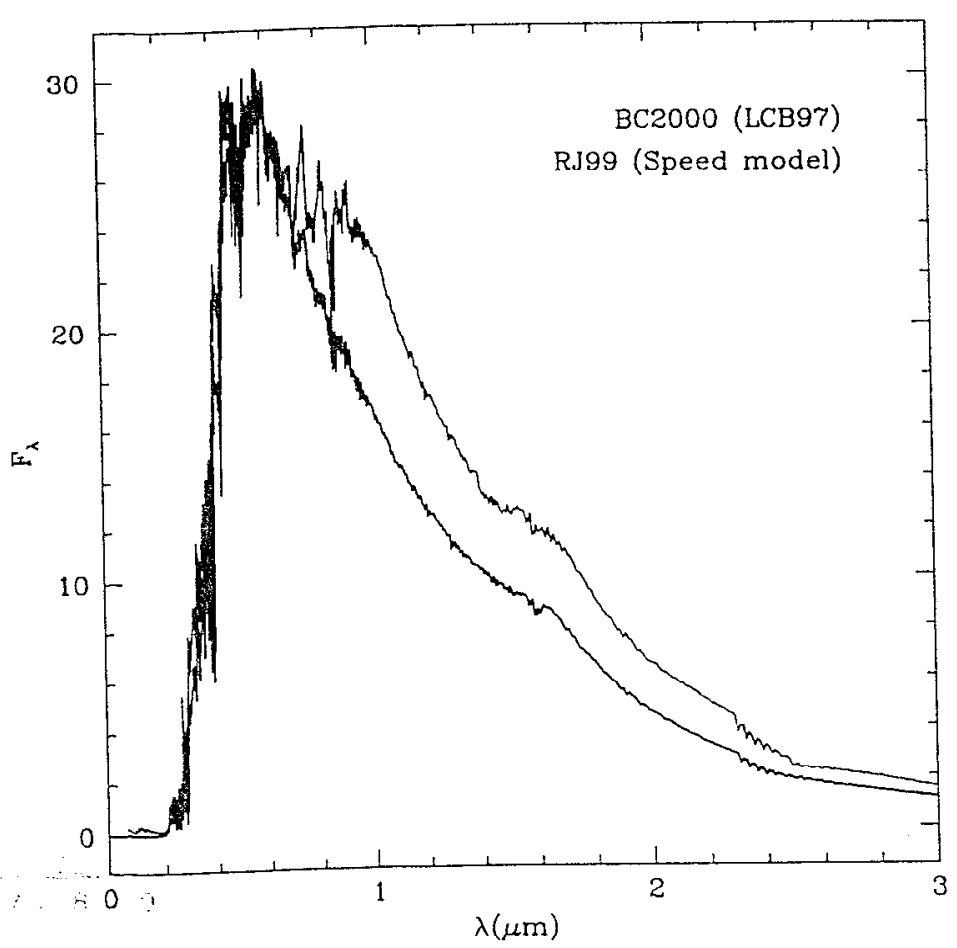

Figure 13. 
function of the stellar IMF, the set of evolutionary tracks, and the chemical composition of the stellar population. It may be too simplistic to attribute de dispersion of the points to a violation of the FCT (Buzzoni 1999).

\section{Comparison with Jimenez's models}

Fig 13 shows a comparison of the $Z_{\odot} \mathrm{SSP}$ at 5 Gyr according to the $\mathrm{BC} 2000$ models (upper line at $1 \mu \mathrm{m}$ ) and the R. Jimenez (1999, private communication) model (lower line at $1 \mu \mathrm{m}$ ). Both models were built for the Salpeter IMF and use the same stellar atlas (LCB97). The spectra are quite similar below $0.6 \mu \mathrm{m}$. At longer wavelengths the $\mathrm{BC} 2000$ model is redder than Jimenez's model. Given the similarity of ingredients used in both models, the reason for this difference must be in the evolutionary tracks. It is important to understand what is producing this difference in evolutionary rate among both sets of models, since this will have important implications in the interpretation of distant galaxies.

\section{Summary and Conclusions}

Present population synthesis models show reasonable agreement with the observed spectrum of stellar populations of various ages and metal content. Differences in results from different codes can be understood in terms of the different ingredients used to build the models and do not necessarily represent violations of physical principles by some of these models. However, inspection of Fig. 5 of GB98 shows that two different sets of evolutionary tracks for stars of the same metallicity produce models that at early ages differ in brightness and color from 0.5 to $1 \mathrm{mag}$, depending on the specific bands. The differences decrease at present ages in the rest frame, but are large in the observer frame at $z>2$. Thus any attempt to date distant galaxies, for instance, based on fitting observed colors to these lines will produce ages that depend critically on the set of models which is used. Note that from $z$ of 3 to $3.5\left(V-H^{-}\right)$in the two models differs by more than $1 \mathrm{mag}$. This difference is produced by the corresponding difference between the models seen in the rest frame at $10 \mathrm{Myr}$. From Figs. 7 and 8 (this paper) these differences can be understood in terms of the different contribution of the same stellar groups to the total $V$ and $K$ flux in the two models.

Even though at the present age models built with different IMFs show reasonably similar colors and brightness, the early evolution of these models is quite different at early ages (GB98 Fig. 7), resulting in larger color differences in the observer frame at $z>2$. Thus, the more we know about the IMF, the better the model predictions can be constrained. The small color differences seen in the rest frame when different stellar libraries of the same metallicity are used, are magnified in the observer frame (GB98 Fig. 8). When the $k$ correction brings opposing flux differences into each filter, the difference in the resulting color is enhanced. Fig. 9 (GB98) shows the danger of interpreting data for one stellar system with models of the wrong metallicity. The color differences between these models, especially in the observer frame, are so large as to make any conclusion thus derived very uncertain.

It is common practice to use solar metallicity models when no information is available about the chemical abundance of a given stellar system. Galaxies 
evolving according the $Z(t)$ laws of Fig. 10 (GB98) show color differences with respect to the $Z=Z_{\odot}$ model which are not larger than the differences introduced by the other sources of uncertainties discussed so far. Hence, the solar metallicity approximation may be justified in some instances. The color differences between the chemically inhomogeneous composite population and the purely solar case (GB98 Fig. 11), arc much smaller than the ones shown in Fig. 9 (GB98) for chemically homogeneous SSPs.

\section{References}

Allard, F., \& Hauschildt, P.H. 1995, Ap.J, 445, 433

Alongi, M., Bertelli, G., Bressan, A., Chiosi, C., Fagotto, F., Greggio, L., \& Nasi, E. 1993, A\&AS, 97, 851

Bessell, M.S., Brett, J., Scholtz, M., \& Wood, P. 1989, A\&AS, 77, 1

- 1991, A\&AS, 89, 335

Bica, E. et al. 1996, in A Data Base for Galaxy Evolution Modeling, eds. C. Leitherer et al., PASP, 108, 996

Bressan, A., Fagotto, F., Bcrtelli, G., \& Chiosi, C. 1993, A\&AS, 100, 647

Bruzual A., G. 1998, in The Evolution of Galaxies on Cosmological Timescales, ed. J. Bechman, ASP Conference Series, in press (GB98)

Bruzual A., G. \& Charlot, S. 1993, ApJ, 405, 538 (BC93)

- 2000, ApJ, in preparation (BC2000)

Buzzoni, A. 1999, in IAU Symposium No. 183 Cosmological Parameters and the Evolution of the Universe, ed. K. Sato, Dordrecht: Kluwer, p. 134

Charlot, S., Worthey, G. \& Bressan, A. 1996, ApJ,

Fagotto, F., Bressan, A., Bertelli, G., \& Chiosi, C. 1994a, A\&AS, 100, 647

- $1994 \mathrm{~b}, \mathrm{~A} \& \mathrm{AS}, 104,365$

-—. $1994 \mathrm{c}, \mathrm{A} \& \mathrm{AS}, 105,29$

Fluks, M. et al. 1994, A\&AS, 105, 311

Girardi, L., Bressan, A., Chiosi, C., Bertelli, G., \& Nasi, E. 1996, A\&AS, 117, 113

Iglesias, C.A., Rogers, F.J., \& Wilson, B.G. 1992, ApJ, 397, 717

Iglesias, C.A., \& Rogers, F.J. 1993, ApJ, 412, 752

Kroupa, P., Tout, C.A., \& Gilmore, G. 1993, MNRAS, 262, 545

Kurucz, R. 1995, private communication

Lejeune. T., Cuisinier, F., \& Buser, R. 1997a, A\&AS, 125, 229 (LCB9Ta)

- $199 \mathrm{~Tb}, \mathrm{~A} \& \mathrm{~A}$, in preparation (LCB9Tb)

Pickles, A.J. 1998, PASP, 110, $\$ 63$

Renzini, A. 1981, Ann. Phys. Fr., 6, 87

Salpeter, E.E. 1355, ApJ, 121, 161

Scalo, J.M. 1986, Fund. Cosmic Phys, 11, 1 
The Hy Redshift Universe

ASP Conference Series, Vol. 193, 1999

A. J. Bunker \& W. J. M. van Breugel, eds.

\title{
The Formation Epoch of Massive Ellipticals, as Traced by Radio Galaxies
}

\author{
J.S. Dunlop \\ Institute for Astronomy, University of Edinburgh, Royal Observatory, \\ Edinburgh, EH9 3 HJ
}

\begin{abstract}
I review the current status of an on-going investigation into the stellar and dynamical ages of the oldest known galaxies at $z>1$. The spectroscopic data, when compared with the predictions of a range of recent evolutionary synthesis models, continue to indicate that the oldest galaxies at $z \simeq 1.5$ are already $>3$ Gyr old. Moreover, comparison with new models which incorporate chemical evolution, suggests that this result may be less sensitive to the assumed metallicity than was previously suspected. Such ages should therefore be taken seriously as constraints both on theories of galaxy formation and on cosmological models. Dynamically these objects also appear well evolved; they display de Vaucouleurs surface brightness profiles, and I demonstrate that their HST-derived morphological parameters place them on the same Kormendy relation as the $z \simeq 13 \mathrm{CR}$ galaxies. Finally I consider the ages of these intermediate redshift objects in the context of our recently completed SCUBA sub-mm survey of radio galaxies spanning the redshift range $z=1 \rightarrow 4$. These data indicate that the main epoch of starformation in radio galaxies lies at $z \simeq 4$, a result which fits naturally with the ages of the oldest galaxies at $z=1.5$ within an open Universe.
\end{abstract}

\section{Introduction}

Even a very low-level burst of star-formation activity can easily obscure the true colours of the dynamically-dominant stellar population in a high-redshift galaxy. For this reason it is necessary to use the reddest (unreddened) galaxies at a given redshift to set meaningful constraints on their primary formation epoch. As demonstrated by Dunlop et al. (1996), Spinrad et al. (1997) and Dunlop (1999) the reddest $(R-K \simeq 6$ ) optical identifications of milli-Jansky radio sources have provided the best examples discovered to date of passively evolving galaxies at redshifts $z>1$. As discussed by Dunlop (1999) there can be little doubt that the red colours of 53W091 $(R-K=5.8 ; z=1.55)$ and $53 W 069(R-F=6.3: z=1.43)$ are due to evolved stellar populations rather than dust. However, the initial attempt at accurate age-dating of $53 \mathrm{~W} 091$ by Dunlop et al. (1996) has since stimulated considerable debate and controversy (Bruzual \& Magris 1997, Yi et al. 1999.. Heap et al. 1998).

In the next section I summarize the uncontroversial facts about these objects. I then consider the controversy over their precise ages and, focussing on $53 W 069$, demonstrate that there is in fact rather little disagreement between 
- the ages inferred from different evolutionary synthesis models, provided fitting is confined to the high-quality rest-frame near-UV spectra. Following this I revisit the issue of age-metallicity degeneracy before moving on to consider the morphologies of these galaxies, and their ages in the context of our recently completed major SCUBA survey of dust-enshrouded star-formation in high-redshift radio galaxies.

\section{53W091 and 53W069: uncontroversial facts}

The Keck spectra of 53W091 (Dunlop et al. 1996; Spinrad et al. 1997) and 53 W069 (Dunlop 1999; Dey et al. in preparation) both display strong spectral breaks at rest-frame wavelengths of $2640 \AA$ and $2900 \AA$ (along with several other repeatable absorption features) which prove beyond doubt that their ultraviolet spectra are dominated by starlight from $F$ stars. This is illustrated in Figure 1 ; the near-ultraviolet SED of $53 \mathrm{~W} 091$ is essentially indistinguishable from an F5V star, while that of $53 \mathrm{~W} 069$ is best described by an even redder F9V star. Since for ages less than $\simeq 5$ Gyr essentially all spectral synthesis models predict that the near-ultraviolet light of a stellar population should be dominated by main-sequence stars, this means that the main-sequence turnoff point in these stellar populations must have evolved into the mid F-star regime.

Figure 1 also shows that 53W069 appears to be a cleaner example of a genuinely coeval stellar population; the SED of 53W091 can be constructed by adding a low-level population of FOV stars (or some other comparably blue lowlevel component) to the SED of 53W069. Deriving the ages of these objects thus boils down to 'simply' determining how long it takes for the near-ultraviolet light from an evolving main sequence to impersonate the SED of an F9V star. It is worth noting that, at the time of writing, these two galaxies remain the only objects at redshifts as high as $z \simeq 1.5$ for which this type of potentially accurate age dating is possible.

\section{Age controversy}

\subsection{Model dependence}

Following the discovery of 53W091, Dunlop et al. (1996) derived an estimate for its age of $3.5 \pm 0.5 \mathrm{Gyr}$. This estimate was based primarily on comparison with a main-sequence only model of spectral evolution, with special emphasis placed on the (reddening independent) strength of the the $2640 \AA$ and $2900 \AA$ spectral breaks. The age-dating of this galaxy was then explored in more detail by Spinrad et al. (1997) who highlighted the disagreement between different models (especially if $R-K$ colour was included as a fitted quantity). but again concluded that an age of $3.5 \mathrm{Gyr}$ appeared to be the most reasonable estimate of the time elapsed since cessation of star-formation activity. Subsequently; however, both Bruzual \& Magris (1997) and Yi et al. (1999) have concluded in favour of an age as young as $1.5 \mathrm{Gyr}$.

However, it transpires that a significant fraction of this disagreement arises from the fact that Bruzual \& Magris (1997) and Yi et al. (1999) have included $R-K$ colour as an important factor in the fitting process. This dilutes much of 

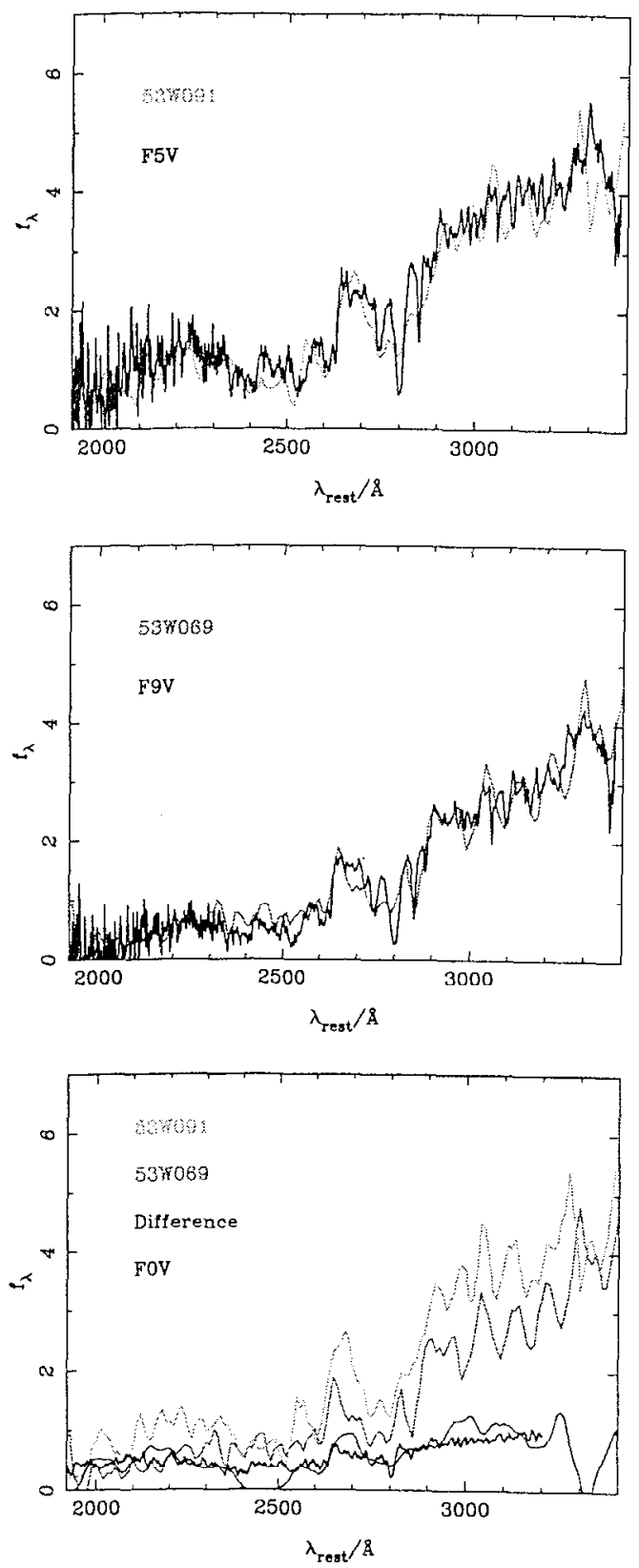

Figure 1. Top Panel: Galaxy rest-frame Keck spectrum of $53 \mathrm{~W} 091 \mathrm{com}-$ pared with an average F5V IUE spectrum. Middle Panel: Galaxy rest-frame Keck spectrum of 53W069 compared with an average F9V IUE spectrum. Bottom Panel: Comparison of the properly scaled rest-frame UV spectra of $53 \mathrm{~W} 091$ and 53 W069 with a smoothed version of the difference spectrum compared with an average FOV IUE spectrum. 

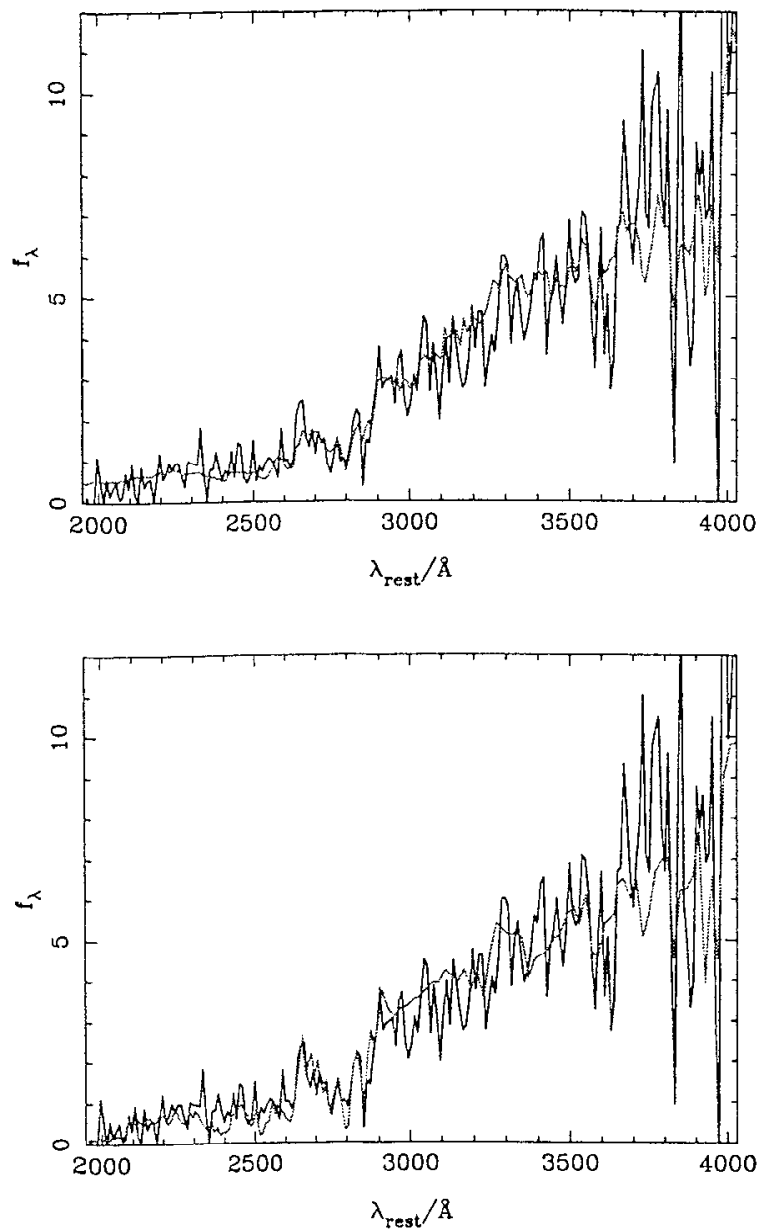

Figure 2. Upper panel: The spectrum of $53 W 069$ overlaid with the best fitting Bruzual-Charlot model, which has an age of 3.25 Gyr. Lower panel: The spectrum of $53 \mathrm{~W} 069$ overlaid with the best fitting model from Jimenez et al. (1999), which has an age of 4.25 Gyr.

the advantage offered by the detailed rest-frame near-ultraviolet Keck spectra obtained for these objects, because the evolution of $R-K$ colour at such redshifts depends not only on the main-sequence, but also on modelling of the later stages of stellar evolution, over which there remains much more controversy (due to complications such as mass loss; see Jimenez et al. 1999). In fact, if fitting is confined to the Keck spectrum, the models of Bruzual and of Yi indicate an age of $\simeq 2.5$ Gyr, while the most recent models of Jimenez indicate an age of 3.0 Gyr. Given the uncertainties, these ages are basically consistent, albeit $0.5-1$ Gyr younger than the original age quoted by Dunlop et al. (1996) (although the spectral breaks, especially the $2900 \mathrm{~A}$ break continue to favour an older age $\simeq 4$ Gyr - see Dunlop 1999).

However, as explained above, $53 W 069$ provides a better example of a clean coeval stellar population. and for this galaxy it appears that essentially all ex- 


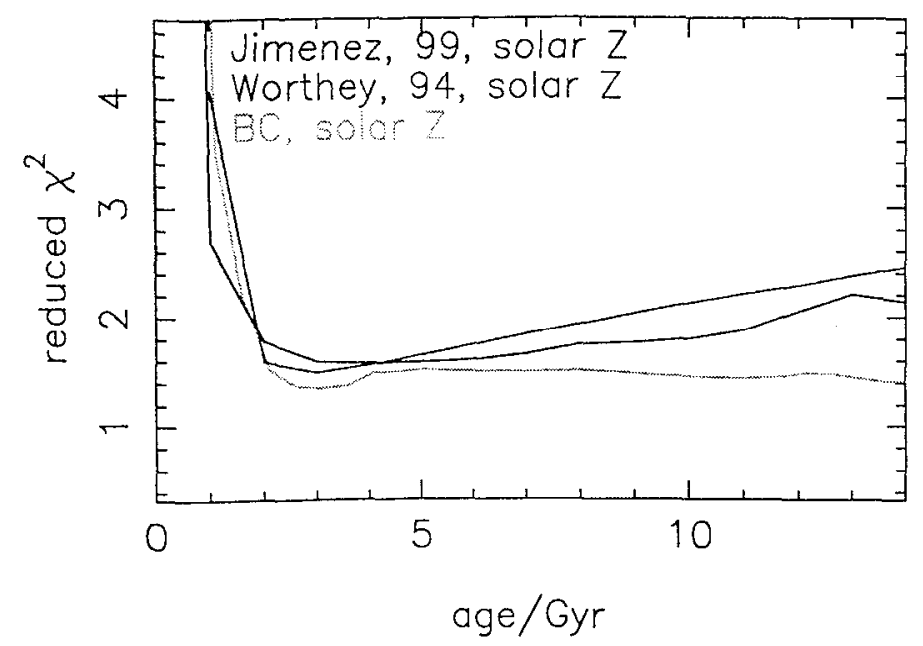

Figure 3. Reduced chi-squared versus the inferred age of $53 W 069$ derived from attempting to fit the Keck spectrum of $53 \mathrm{~W} 069$ with the evolutionary synthesis models of Jimenez et al. (1999), Bruzual \& Charlot (1993) and Worthey (1994).

isting models indicate an age $>3$ Gyr. Figure 2 shows the data fitted by the updated models of Brizual \& Charlot (1993) (inferred age 3.25 Gyr) and by the most recent models of Jimenez et al. (1999) (inferred age 4.25 Gyr).

\subsection{Age constraints for 53W069}

Figure 3 shows a plot of reduced chi-squared versus inferred age, derived from attempting to fit the models of Jimenez et al. (1999), Bruzual \& Charlot, and Worthey (1994) to the Keck spectrum of 53W069. The models of Jimenez favour an age about 1 Gyr older than the models of Bruzual \& Charlot, but it is clear from this plot that this disagreement is not very dramatic in terms of quality of fit. The key point is that all the models favour an age of 3 Gyr or greater, with ages younger than $2.5 \mathrm{Gyr}$ formally excluded. It is important to re-stress that these ages are based on instantaneous starburst models, and thus indicate the time elapsed since cessation of major star-formation activity with no additional time included for the process of galaxy/star formation. However, they do assume solar metallicity.

\subsection{Impact of varying metallicity}

A major concern with this type of spectral age dating is that, as explored by for example Worthey (1994), deduced age is approximately inversely proportional to assumed metallicity for most spectral age indicators. For the $2640 \AA$ and $2900 \AA$ breaks this age/metallicity degeneracy appears to be not quite as severe as this (Dunlop et al. 1996: Fanelli et al. 1992), but nonetheless it is clear that the inferred ages of $53 W 091$ and $53 W 069$ can be reduced to less than 2 Gyr if one 


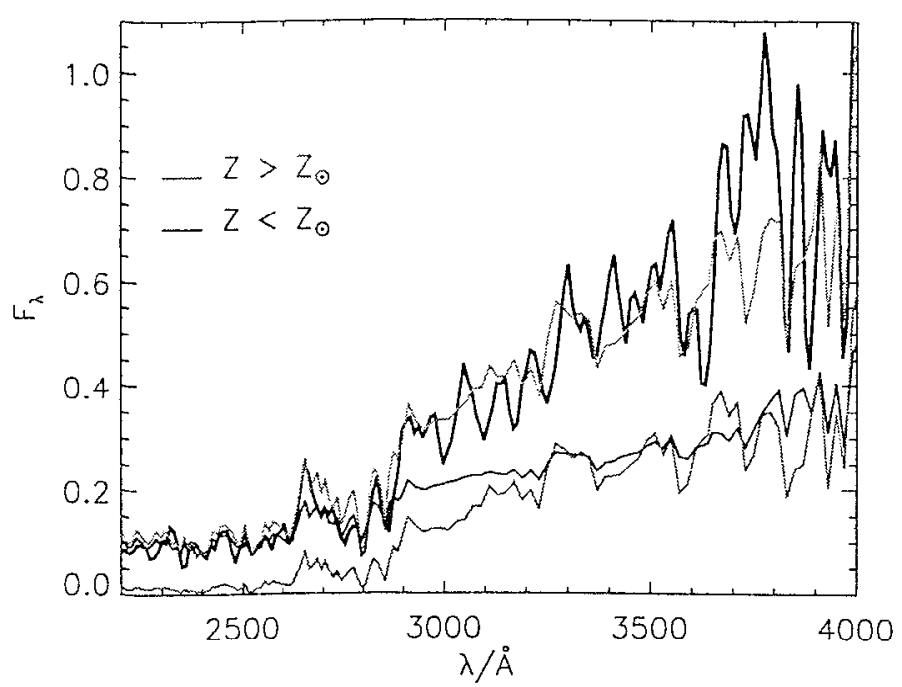

Figure 4. The Keck spectrum of 53 W069 fitted with the composite metallicity model of Jimenez et al. (1999) at an age of 4 Gyr. The mass-averaged metallicity of this model is $\simeq 2 \times$ solar, but the figure also illustrates how the sub-solar metallicity stars continue to dominate the predicted SED shortward of $3500 \AA$, leading to an age estimate which is unexpectedly robust to varying metallicity.

assumes the observed stellar population consists only of stars with twice solar metallicity.

However, new models of elliptical galaxy formation/evolution developed by Jimenez et al. (1999), and by Yi et al. (1999) suggest that adoption of this simple-minded age-metallicity degeneracy may lead to an overly pessimistic view of the accuracy to which ages can be derived. These models include chemical evolution during the initial starburst, in an attempt to produce a realistic stellar population of composite metallicity. The interesting consequence of comparing the predictions of such models to the near-ultraviolet SED of 53W069 is that the inferred age is essentially unchanged from that derived using the simple solar metallicity models, despite the fact that the mass-weighted metallicity can be as high as twice solar. The reason for this is illustrated in Figure 4 which shows the best fit obtained using the $2 \times$-solar metallicity composite model of Jimenez et al. (1999b) to 53W069, at an age of 4 Gyr. The plot shows that while the high metallicity stars dominate the red end of the spectrum, sub-solar metallicity stars continue to dominate shortward of $\lambda \simeq 3300 \AA$. with the consequence that the derived age is more robust than would have been naïvely expected. Indeed the statistical fit obtained with this 4 Gyr composite model is substantially better than that achieved with any single metallicity model.

This result, coupled with the rather good agreement between different models discussed above, suggests that an age limit of $>3 \mathrm{Gyr}$ for the oldest galaxies (and hence the Eniverse) at $z=1.5$ should be taken seriously. Such an age is also consistent with the collapse epoch of these objects as inferred from the power-spectrum analysis of Peacock et al. (1998). 


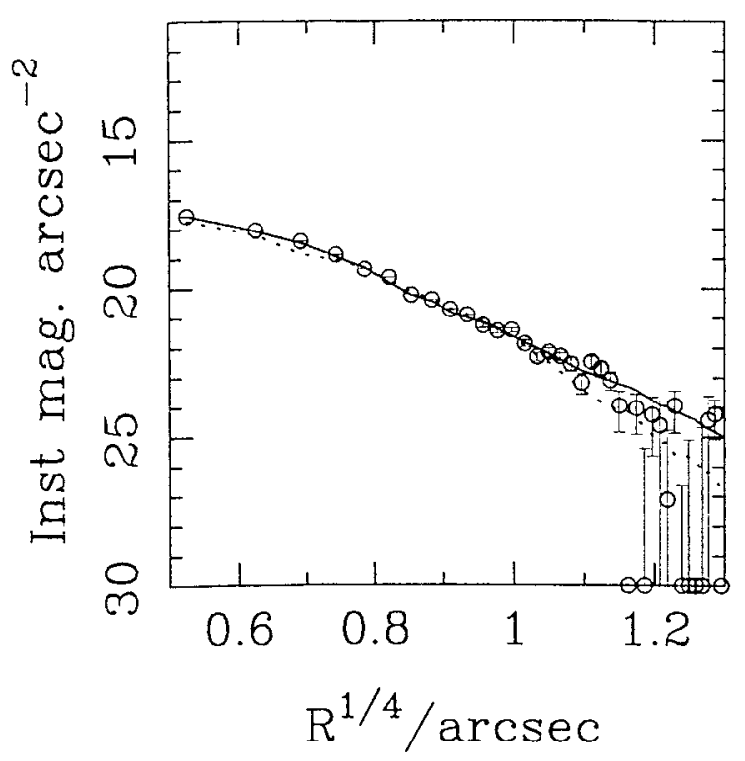

Figure 5. HST $H$-band profile of 53 W069 (circles) compared with the best fitting pure de Vaucouleurs law after convolution with the NICMOS H-band PSF (solid line) (Bunker et al. in preparation)

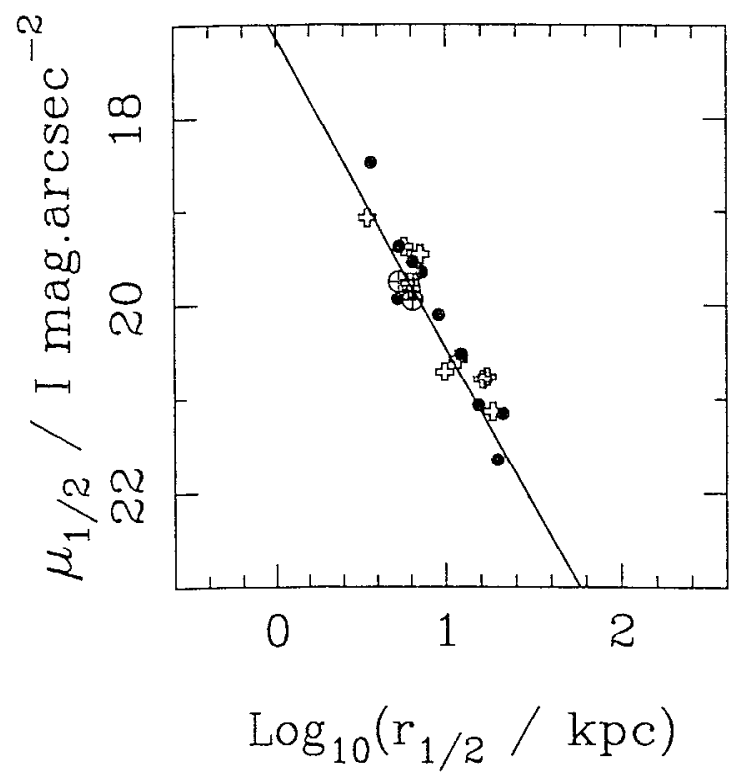

Figure 6. The Kormendy relation displayed by $3 \mathrm{CR}$ radio galaxies at $z=$ 0.2 (solid circles) overlaid with the (indistinguishable) relation displayed by $3 \mathrm{CR}$ galaxies at $z=0.9$ (crosses) after allowing for 0.6 magnitudes of passive evolution in the I-band. The solid line shows the best fitting Kormendy relation which has a slope of 3.2. The locations of 53W091 and 53W069 on this diagram are indicated by the large circle+cross symbols. They lie on the same Formendy relation, but with scalelengths a. factor $\simeq 3$ smaller than the mean scalelength displayed by the more radio-powerful $3 \mathrm{CR}$ galaxies. 


\section{Morphology and size}

HST I-band and J-band images of 53W091 and 53W069 indicate that both galaxies are dominated by a bulge component both above and below the $4000 \AA$ break (Waddington et al. in preparation). The most powerful evidence that morphologically these galaxies are dynamically evolved ellipticals comes from analysis of the deep NICMIOS $H$-band image of 53Wn91 obtained by Bunker et al. (in preparation). In Figure 5 I show the luminosity profile derived from this image; a simple de Vaucoulenrs law with no nuclear component provides a significantly better fit to the data than a disc with a nuclear contribution, and the derived scalelength is $r_{e}=3.5 \mathrm{kpc}\left(\Omega_{0}=1, H_{0}=50 \mathrm{~km} \mathrm{~s}^{-1} \mathrm{Mpc}^{-1}\right)$.

The derived scalelengths and surface brightnesses of both 53 W091 and 53 W069 are placed in context in Figure 6, which demonstrates that they lie on the Formendy relation described by $3 \mathrm{CR}$ radio galaxies (at both high and low redshift; McLurc \& Dunlop 1999), towards the lower end of the scalelength distribution of these more powerful radio galaxies. As pointed out by McLure \& Dunlop (1999), a reanalysis of the available HST data on 3CR galaxics provides no evidence for any significant dynamical evolution of massive ellipticals between $\tilde{z}=1$ : the location of 53W091 and 53W069 on Figure 6 is not unexpected given their moderate radio power (see McLure et al. 1999), and suggests that the Formendy relation for massive ellipticals may already be in place by $z \simeq 1.5$.

\section{Formation epoch inferred from sub-millimetre observations}

It is interesting to attempt to relate the dynamical and spectral passivity displayed by 53 W091 and 53W069 at $z \simeq 1.5$ to direct attempts to determine the main epoch of radio galaxy formation (and possibly all spheroid formation). There is certainly some evidence that, dynamically, radio galaxies are different at $z>3$ than at lower redshifts (van Breugel et al 1998), but the complications of radio activity have made it difficult to constrain the major epoch of star-formation activity in these objects from optical/near-infrared observations.

However, Iny collaborators and I have recently completed the first major SCUBA sub-mm survey of dust-enshrouded star-formation activity in radio galaxies between $z=1$ and $z-4$, the results of which indicate that the main epoch of star-formation activity in these objects lies at $z>3$. Previous submin detections of high-redshift radio galaxies have been at $z \simeq 4$ (Dunlop et al. 1994; Hughes et al. 1997; Ivison et al. 1998) but the extreme radio powers of the detected objects made it impossible to tell whether their inferred large dust and gas masses were primarily due to cosmic epoch, or instead related to extreme radio power. Now, as illustrated in Figure $7 \mathrm{a}$, we have achieved sufficient coverage of the $P-z$ plane to separate these effects, and by considering a slice at constant radio power, can for the first time derive the redshift dependence of sub- $m m$ emission in powerful radio galaxies. As shown in Figure $7 b$. the average sub-mm luminosity (and hence inferred gas mass and star-formation rate) rises out to at least $z \simeq 4$ ' with sub-mm luminosity growing approximately proportional to $(1+z)^{3}$. This strongly suggests that the bulk of star-formation in radio galaxies occurred at redshifts $z \simeq 4$ or higher, and that star-formation in radio galaxies, and arguably ellipticals in general. is close to completion by $z \simeq 3$. 

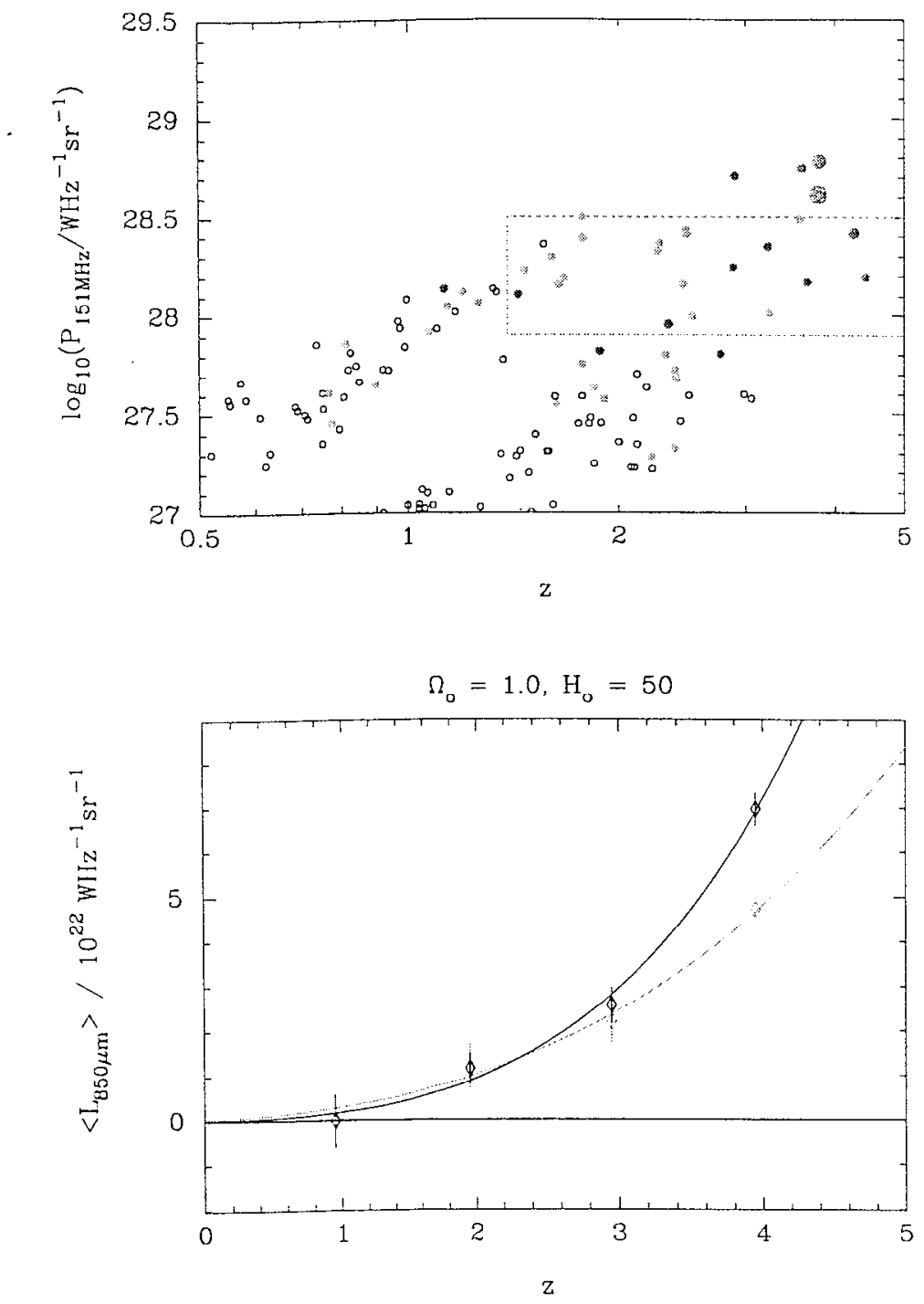

Figure 7. Upper panel: The radio-luminosity redshift plane showing the location of the $\simeq 50$ radio galaxies observed with SCUBA by Archibald et al. (in preparation). Red symbols of increasing size indicate detections of increasing brightness at $850 \mu \mathrm{m}$. Blue symbols indicate non-detections. The green box defines a subsample of objects which span the redshift range $z=1 \rightarrow 4$ at constant radio luminosity, allowing separation of the normally correlated effects of redshift and luminosity. Lower panel: Weighted mean $L_{850 \mu m}$ for the radio galaxies observed with SCUBA in redshift bins centred on $z=1,2,3,4$. The black diamonds refer to all the radio galaxies observed, while the green diamonds refer only to the subset of radio galaxies lying within the constant radio luminosity strip indicated in the upper panel. The lines are fits to the data of the form $L \propto(1+z)^{\alpha}$ to indicate the strength of the evolutionary trend. For all galaxies observed $\alpha \simeq 4$, while for those in the constant luminosity strip $\alpha \sim 3$. Such evolution is of very similar strength to that deduced for many other populations of extragalactic objects out to $z \simeq 2.5$ (Dunlop 1998). but here appears to continue out to at least $z \simeq 4$. 


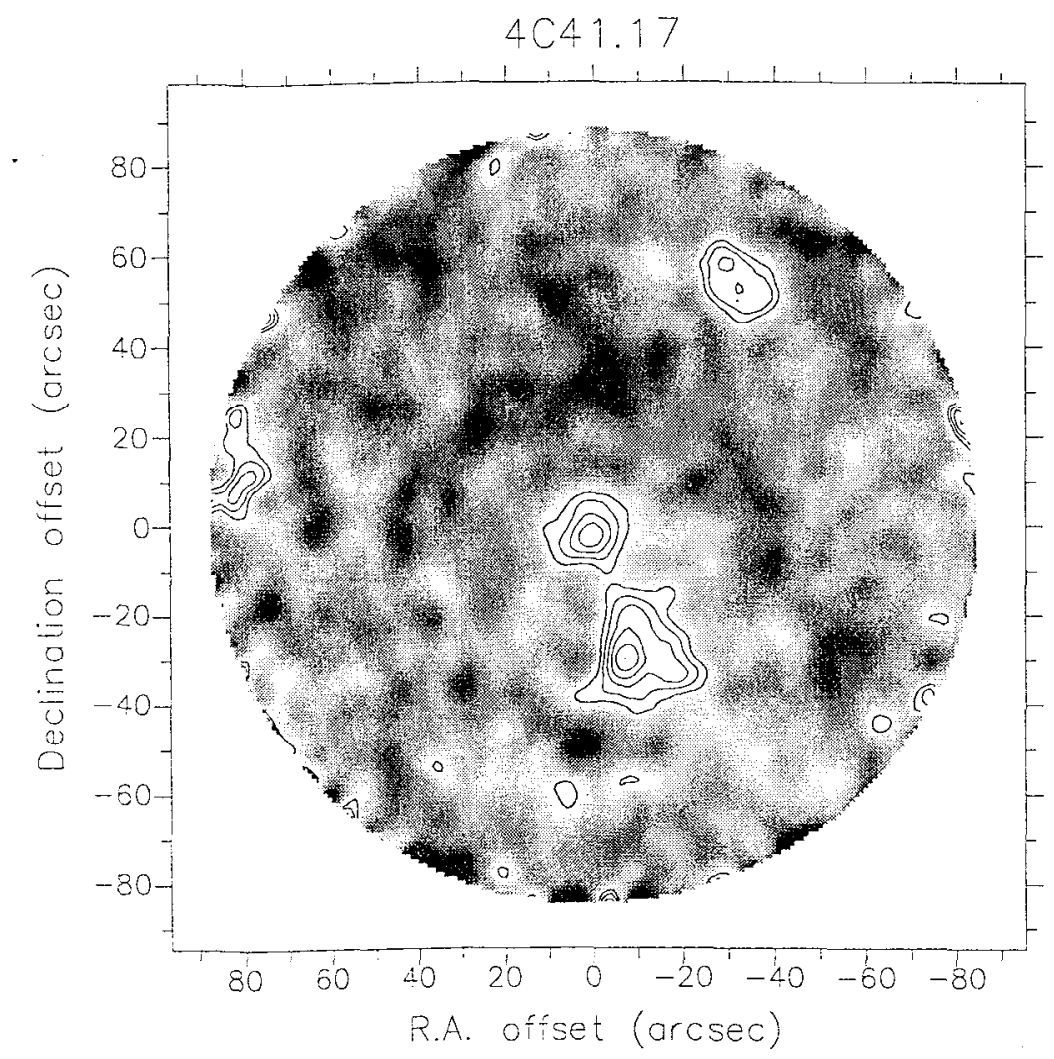

Figure 8. A deep SCUBA image centred on the $z=3.8$ radio galaxy $4 \mathrm{C} 41.17$ (Ivison et al., in preparation). The image confirms the original detection of $4 \mathrm{C} 41.17$ at $850 \mu \mathrm{m}$ by Dunlop et al. (1994) but also reveals an extended, and even brighter region of sub-mm emission to the south of the radio galaxy. This emission is clearly resolved and appears to be pointing towards the RG. Such structures are very suggestive of on-going massive star formation during the merging process by which massive galaxies are believed to be constructed. A third sub-mm source is detected to the north-west.

Einally, in Figure 8 I show a deep (10-hour) SCUBA image of the field centred on the $z=3.8$ radio galaxy $4 \mathrm{C} 41.17$. This new image reaches an $\mathrm{rms}$ sensitivity of $\simeq 1 \mathrm{mJy}$ at $850 \mu \mathrm{m}$ (Ivison et al. in preparation), and $4 \mathrm{C} 41.17 \mathrm{itself}$ is clearly detected in the centre, confirming the early single-element UKT14 detection by Dunlop et al. (1994). However, the surprising thing about this image is that, despite the fact that $4 \mathrm{C} 41.17$ is one of the most Iuminous sub$\mathrm{mm}$ sources ever detected, it is not the most luminous source in this 2.8 -arcmin diameter image. A more luminous, apparently resolved $\simeq 12 \mathrm{mJy}$ source lies to the south of the radio galaxy at a projected distance of $\simeq 200 \mathrm{kpc}$, assuming it lies at the same redshift (an assumption supported by its sub-mm colours: Irison et al. in preparation), and a third moderately bright source lies to the north.

Taken together these sources indicate a considerable excess of dust enshrouded star,formation in the vicinity of this high-redshift radio galaxy compared with that found in blank field surveys (e.g. Hughes et al. 1998, Eales et 
al. 1998). Although this is only a single field, it is strongly suggestive of violent dust-enshrouded star formation in a high-redshift proto-cluster for which the radio galaxy has acted as a signpost. Such images provide at least circumstantial support for the idea that the cosmological evolution of dust mass and SF activity plotted in Figure 7b might apply not just to radio galaxies but to spheroids in general, especially those born in rich environments (see also Renzini 1999).

\section{Conclusion}

Radio galaxies are obviously a special subset of all ellipticals, and very red/passive galaxies such as 53W091 and 53W069 form a special subset of radio galaxies. However, this does not necessarily mean they are unrepresentative of ellipticals in general. Rather, it may simply mean that it is rare to find elliptical galaxies at $z>1$ which have undergone so little star-formation activity over the $\simeq 3$ Gyrs prior to observation. As pointed out by Jimenez et al. (1999c), most realistic models of elliptical formation involve sufficient low-level secondary bursts of star-formation to frequently mask the true optical-infrared colours of a dynamically dominant stellar population at $z>1$ (cf Zepf 1997).

The apparent lack of significant dynamical growth of radio galaxies since $z \simeq$ 1 discussed above (McLure \& Dunlop 1999) may also be representative of massive ellipticals in general. Certainly there is now a growing body of observational evidence from infrared studies that cluster ellipticals (de Propris et al. 1999) and field ellipticals (Dunlop et al. 1999) are essentially all in place by $z \simeq 1$ (cf Kauffman \& Charlot 1998).

This lack of dynamical action since $z \simeq 1$, and the ages of the oldest ellipticals at $z>1$, both favour a universe with $\Omega_{\text {matter }}<1$.

Acknowledgments. This paper draws on recent results from a number of programmes, and I gratefully acknowledge the contributions of my collaborators Hy Spinrad, John Peacock, Raul Jimenez, Arjun Dey, Daniel Stern, Rogier Windhorst, Ian Waddington, Lonisa Nolan, Ross McLure, Andy Bunker, David Hughes, Elese Archibald, Rob Ivison, Steve Rawlings and Steve Eales.

\section{References}

Bruzual, A.G., \& Charlot, S., 1993, ApJ, 405, 538

Bruzual, G., \& Magris, G., 1997, in 'The Hubble Deep Field', Proc. STScI Symposium, in press (astro-ph/9707154).

De Propris, R., Stanford, S.A., Eisenhardt. P.R., Dickinson, M.E., \& Elston, R., 1999, AJ, in press (astro-ph/9905137).

Dunlop, J.S., 1998, in: 'Observational Cosmology with the New Radio Surveys', p.157, eds Bremer. M.N., et al., (astro-ph/9704294).

Dunlop, J.S., 1999, in: The Most Distant Radio Galaxies', KXAW Colloquium Amsterdam, p.il, eds. Röttgering, H.J.A., et al., Kluwer (astrph/9801114)

Dunlop, J.S., Gloag, J., \& Jimenez, R.. 1999, MNR.tS. submitted. 
Dunlop, J.S., Hughes, D.H., Rawlings, S., Eales, S.A., \& Ward, M.J., 1994, Nature, 370,347 .

Dunlop, J.S., Peacock, J.A., Spinrad, H., Dey, A., Jimenez, R., Stern, D., \& Windhorst, R., 1996, Nature, 381, 581.

Eales, S., Tilly, S., Gear, W., Dunne, L., Bond, J.R., Hammer, F., Le Fèvre, O., \& Crampton, D., 1999, ApJ, 515, 518.

Fanelli, M.N., O'Conncll, R.W., Burstein, D., \& Wu, C.-C., 1992, Ap.JS, 82, 197.

Heap, S.R., et al., 1998, ApJ, 492, L131.

Hughes, D.H., Dunlop, J.S., \& Rawlings, S., 1997, MNRAS, 289, 766

Hughes, D.H., Serjeant, S., Dunlop, J.S., Rowan-Robinson, M., et al., 1998, Nature, 394, 241.

Ivison, R.J., Dunlop, J.S., Hughes, D.H., Archibald, E.N., et al., 1998, ApJ, 494, 211.

Jimenez, R., Dunlop, J.S., Peacock, J.A., MacDonald, J., \& Jorgensen, U.G., 1999a, MNRAS, in press.

Jimenez, R., Dunlop, J. S., Peacock, J. A., Padoan, P., MacDonald, J., \& Jørgensen, U. G., 1999b, submitted to MNRAS

Jimenez, R., Friaca, A.C.S., Dunlop, J.S., Terlevich, R.J., Peacock, J.A., \& Nolan, L.A., 1999c, MNRAS, 305, L16.

Kauffman, G., \& Charlot, S., 1998, MNRAS, 297, 23.

McLure, R., \& Dunlop, J.S., 1999, MNRAS, in press (astro-ph/9908214).

Mclure, R., Kukula, M.I., Dunlop, J.S., Baum, S., O'Dea, C.P., \& Hughes, D.H., 1999, MNRAS, in press (astro-ph/9809030).

Peacock, J., Jimenez, R., Dunlop. J.S., Waddington, I., Spinrad, H., Stern, D., Dey, A., \& Windhorst, R.A., 1998, MNRAS, 296, 1089.

Renzini, A., 1999, in 'When and How do Bulges Form and Evolve?', eds. C.M. Carollo, H.C. Ferguson \& R.F.G. Wyse, CUP, (astro-ph/9902108).

Spinrad, H., Dey, A., Stern, D., Dunlop, J.S., Peacock, J., Jimenez, R., \& Windhorst, R., 1997, ApJ, 484, 581.

van Breugel, W.J.M., Stanford, S.A., Spinrad, H., Stern, D., \& Grahamm, J.R., 1998, ApJ, 502, 614.

Worthey, G., 1994, ApJS, 95, 10 i

Worthey, G., Faber, S. M.. \& Gonzalez, J. J., 1992 ApJ, 418, 947

Yi, S., Brown, T.M., Heap, S., Hubeny, I., Landsman, W., Lanz, T., \& Sweigart, A., 1999, ApJ, submitted.

Zepf, S.E., 1997, Nature, 390, 3iT. 
The Hy Redshift Universe

ASP Conference Series, Vol. 193, 1999

A. J. Bunker \&\& W. J. M. van Breugel, eds.

\title{
- Distant Disk Galaxies: Kinematics and Evolution to $z \sim 1$
}

\author{
Nicole P. Vogt \\ Institute of Astronomy, University of Cambridge, \\ Madingley Road, Cambridge, CB3 OHA, United Kingdom
}

\begin{abstract}
High resolution HST images have been combined with Keck $10 \mathrm{~m}$ telescope spectra to study the internal kinematics of 100 faint disk galaxies in the redshift range $0.2 \leq z \leq 1$. These data form a high redshift Tully-Fisher relation that spans five magnitudes and extends to well below $L^{*}$, with no obvious change in shape or slope with respect to the local Tully-Fisher relation for the bulk of the observed population. The small offset of $<0.2 B$ mag can be attributed to strong brightening of a fraction of the low mass galaxy population at redshifts $z \geq 0.5$. A comparison of disk surface brightness between local and high redshift samples yields a similar offset of $<0.3 B$ mag, one that increases more smoothly with redshift. These results provide further evidence for only a modest increase in luminosity with lookback time for this population. The change in disk size with redshift for disks of a given mass is then examined, to tost current models for the formation of galactic disks which suggest that high redshift spirals are substantially smaller than nearby counterparts.
\end{abstract}

\section{Introduction}

We stand on the threshold of an exciting new regime in the study of galaxy evolution. Relatively recent technological innovations make it possible for the first time to disentangle the effects at high redshift of the formation and evolution of galaxies from the structure of the universe in which they are found.

Within this framework, it is critical to recognize several important points. First, galaxy evolution is best studied in an extended multi-parameter space with an awareness of the interplay between correlated parameters. We must examine the luminosity, size, and mass distributions of galaxies, preferably with some knowledge of their colors, redshift-independent morphologies, and line indices and other star formation indicators. It is perilous to assume that any twodimensional projection can represent the complete picture. Second, evolution is a differential process, which may vary strongly as a function of galaxy mass (or other parameter). Third, when comparing high redshift samples to low redshift samples or to theoretical distributions, it is imperative that varied selection effects between samples be properly modelled.

While galaxy luminosity can be highly sensitive to instantaneous star formation, galaxy mass is a more stable, integrated measure of global properties. It is thus imperative to extend mass measurements to the high redshift regime, 
and there have been a number of approaches to date to this end. Studies of bright, blue galaxies have found evidence for strong brightening at redshifts as low aș $z \sim 0.3$ (Rix et al. 1997, Simard \& Pritchet 1998), and an increase in the number counts of compact, bright star forming galaxies has been seen (Phillips et al. 1997; Guzman et al. $1997 \&$ 1998). Our work has identified a population of disk galaxies with properties similar to local spirals existing as far out as a redshift $z \sim 1$ (Vogt et al. 1993, 1996, $1997 \& 1999$ ). Some intriguing attempts have also been made to derive mass estimates for $\approx \sim 3$ galaxies by measuring line widths of $\mathrm{UV}$ and blue features redshifted into the IR (Pettini et al. 1998) by examining relative velocities of components within a single potential well (Lowenthal et al. 1997), and by modeling the size and thickness of large proto-disk galaxies to produce $L_{\alpha}$ absorption features (Prochaska \& Wolfe 1998). These works serve to illustrate the potential inherent in exploiting mass measurements.

We present here mass estimates based upon rotation curves derived for 100 field galaxies at redshifts $0.2 \leq z \leq 1$, combining spatially-resolved spectra from the Keck $10 \mathrm{~m}$ telescope with inclinations and position angles determined from the refurbished Hubble Space Telescope (HST).

\section{Observations}

We have undertaken a five year (1995 through 1999) redshift survey of the Groth Survey Strip (GSS, Koo et al. 1996), a set of 28 contiguous WFPC2 pointings in the northern sky for which $F 606 W(V)$ and $F 814 W(I)$ data have been obtained. We have chosen to observe galaxies down to a limiting magnitude of $V+I \leq 48$ ( $I \leq 23.5$ for $V-I=1$, add 0.5 mag for $A B$ system), over a $40^{\prime}$ region covering $15 \mathrm{Mpc}$ by redshift $z \sim 1$. We currently have spectra for 640 galaxies, $80 \%$ of which have a well determined redshift (Phillips et al. 1999). Spectra were obtained using the Low Resolution Imaging Spectrograph (Oke et al. 1995) in multislit mode, observing up to 50 target simultaneously. Both the blue-blazed 900 line $\mathrm{mm}^{-1}$ grating ( $0.83 \AA$ per pixel) and the red-blazed (1.26 $\AA$ per pixel) 600 line $\mathrm{mm}^{-1}$ grating were used to cover the wavelength range $5000 \AA$ through $9000 \AA$, and a $2048 \times 2048$ pixel CCD with a scale of $0^{\prime \prime} \cdot 215$ per pixel.

We were able to align individual slitlets within each multislit mask along the major axes of galaxies, and thus trace spatially resolved rotation curves from strong emission lines ( $[\mathrm{O}$ II $] 3727$, $[\mathrm{O}$ III $] 5007, \mathrm{H}-\beta$, and $\mathrm{H}-\alpha$ ). Targets were prioritized for this purpose within the general redshift survey pool when they met the following criteria: an inclination angle $i \geq 30^{\circ}$, a position angle on the sky within $30^{\circ}$ of the multislit mask angle, and $I \leq 22.5$, Roughly half of our rotation curves are for fainter galaxies, however, which did not meet the magnitude cut but proved to have sufficiently strong emission to be traced successfully nonetheless. Our restriction to galaxies with inclination angles $i \geq 30^{\circ}$ serves two purposes, it removes spheroidal, early type systems which would not have strong, disk emission to trace, and it removes extremely face-on dishs. Note that there was no attempt made to discriminate on the basis of apparent morphology,

as we did not wish to perturb the sample against disk galaxies showing signs of interaction or with unusual morphology. A few elongated elliptical galaxies did meet our criteria, and were observed within the sample. 

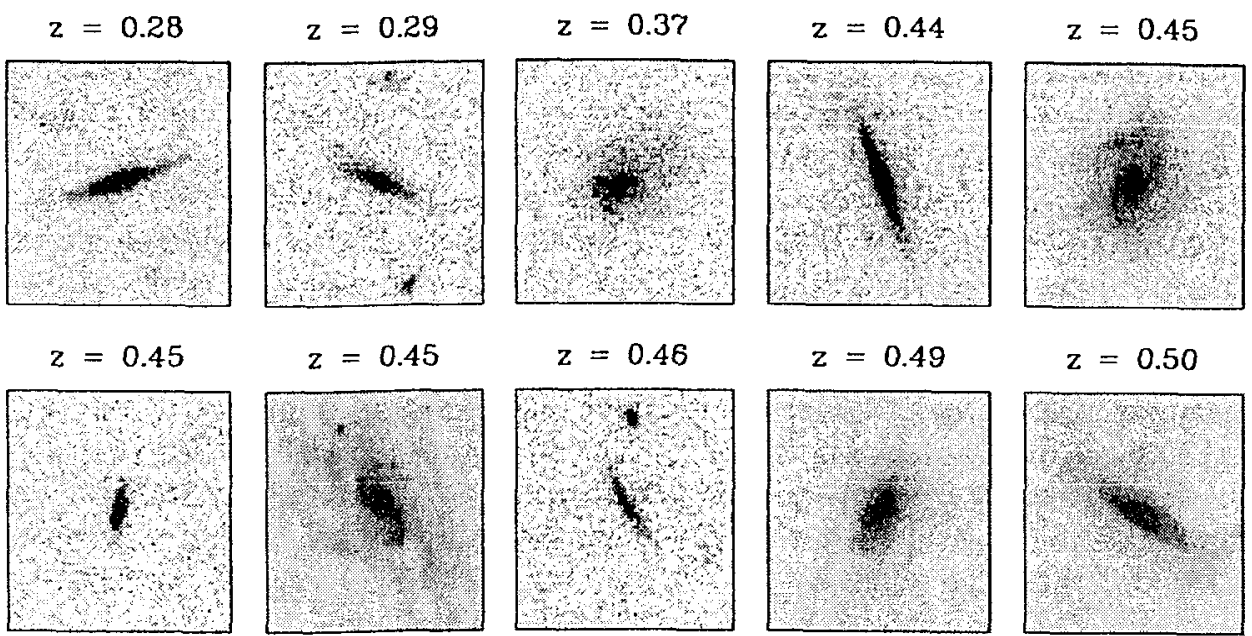

$$
z=0.50
$$
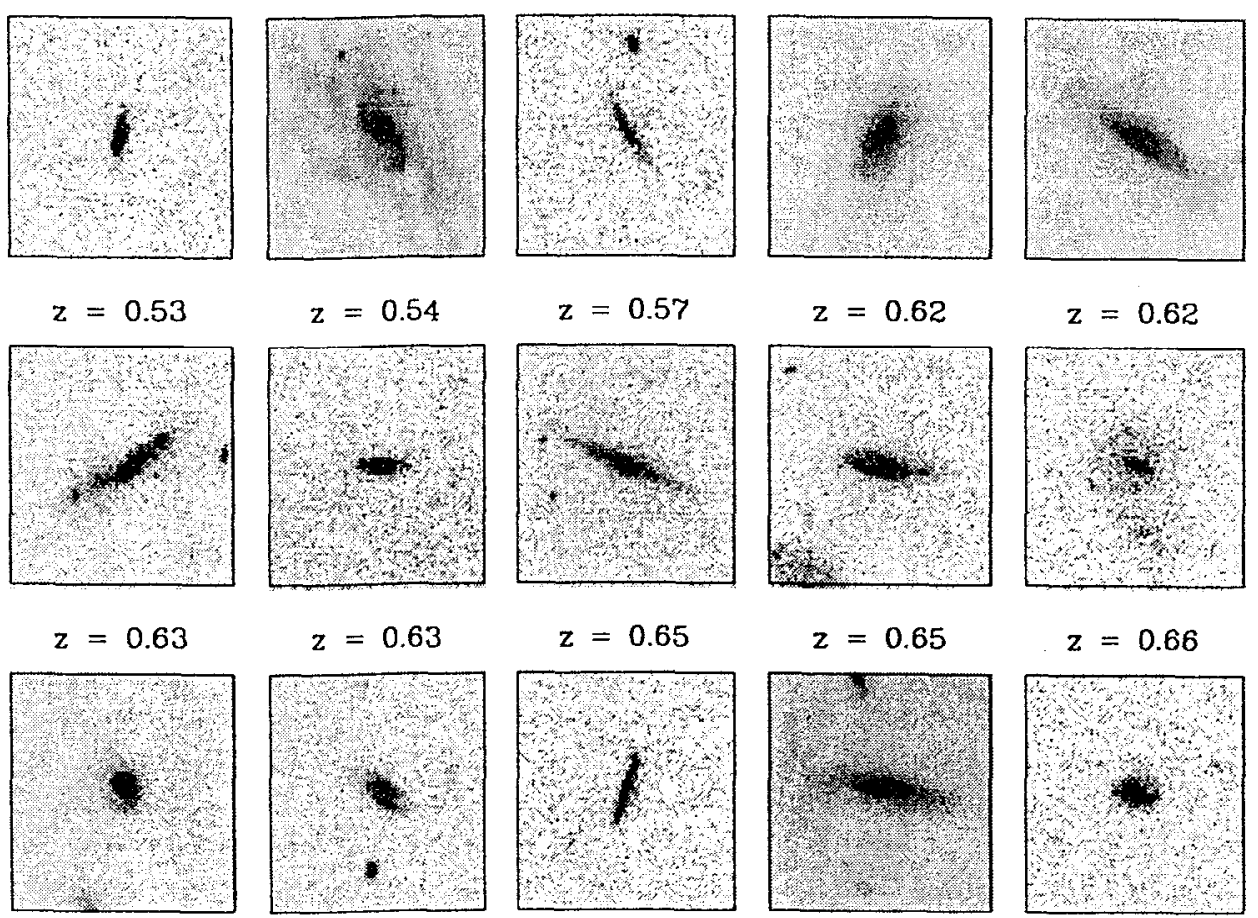

$z=0.67$
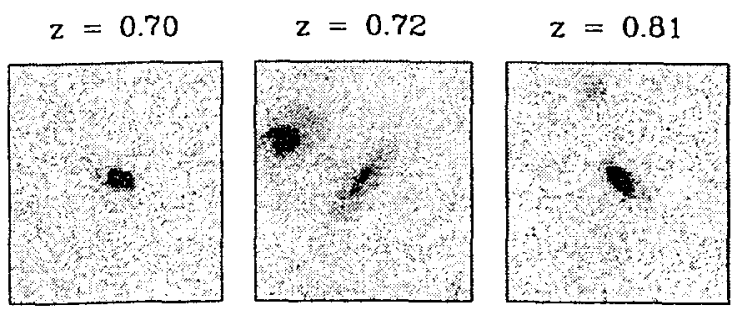

$$
z=0.66
$$
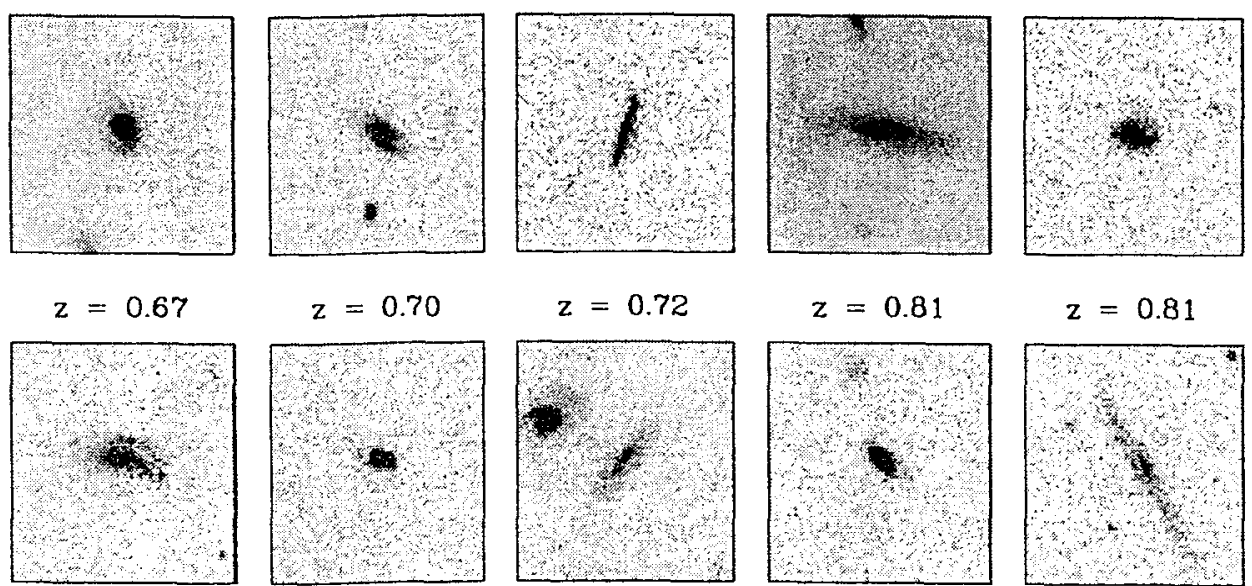

$\mathrm{z}=0.83$
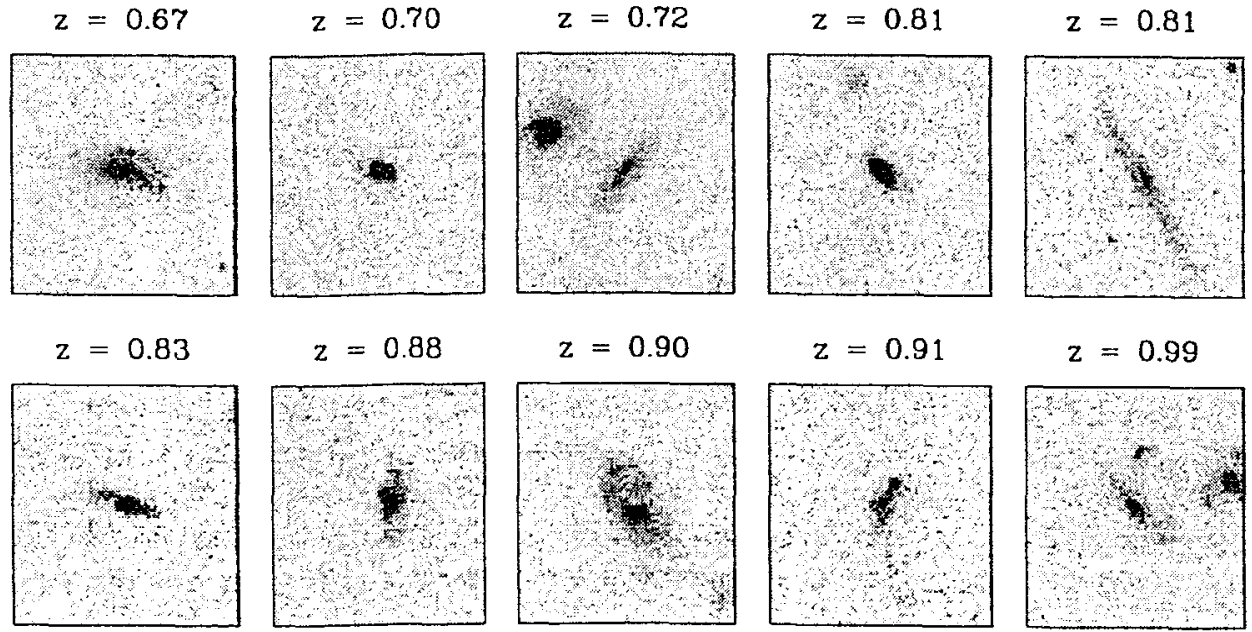
Figure 1. $\quad I 8147^{\prime \prime}$ by $7^{\prime \prime}$ images of spirals in the redshift range
$0.2<z<1$, illustrating the morphological properties of our GSS sample over a range of inclination angles between $30^{\circ}$ and $90^{\circ}$. 
Figure 1 shows a representative 30 of the galaxies for which we were able to obtain rotation curves, covering redshifts $0.2 \leq z \leq 1$. We have observed edge-on and less inclined galaxies over the entire redshift range, and find no dearth of spiral galaxies of normal appearance at high redshift.

The galaxy 104-4024 $(z=0.81)$ is one such notable large, high redshift disk. We have obtained NICMOS $H$ images for 12 of our disk galaxies within this field, including this one. By combining the HST VIH data together we were able to identify 8 small, extremely blue sources within $4^{\prime \prime}(25 \mathrm{kpc}$ at $z=0.81)$ ) of 1044024. (This figure is not reproduced here as one needs a color image in order to properly see the sources.) Given their unusually high spatial density, we believe that they lie at the same redshift and in the same potential well. Their faintness places them roughly 5 orders of magnitude below $L^{*}$, and this combined with the even distribution suggests that they may be companion satellite galaxies in orbit around the large disk galaxy.

Determining the rotation curves of these distant galaxies poses some special difficulties not encountered with nearby galaxies. The galaxies are typically not much larger than the seeing disk $\left(\sim 0.0^{\prime \prime} 8-1^{\prime \prime} 0\right)$ or the slit-width $\left(1^{\prime \prime} 0\right)$, so the resultant spectra represent a complex combination of the spatial distribution in both velocity and emission line surface brightness.

To interpret the data, we adopt a simple exponential disk model for each source, where the inclination and orientation relative to the slit matches measurements from the HST images. The velocities in the model are assumed to rise linearly with radius out to roughly one disk scale length, and then to remain flat (c.f. Persic \& Salucci 1991). The spatial distribution of the emission-line flux is assumed to follow the disk (Kennicutt 1989), with a scale-length larger by a factor of 1.5 (Ryder \& Dopita 1994). The model was then convolved with an appropriate Gaussian to approximate the seeing, masked with a model slit, and a spectral line profile calculated at each pixel along the slit. The resulting model emission line was subjected to the same analysis as the observed lines. Iterative adjustments to the circular velocity and the rise-time of the model were made until the simulated and observed emission lines matched at the velocity extremes. The model circular velocity, $V_{\text {circ }}$, was then adopted as the intrinsic terminal velocity, $V_{\text {term }}$, of the galaxy. The $1 \sigma$ errors in $V_{\text {term }}$ shown in Figures 4 and 5 were estimated by varying the inclination and position angle of each galaxy by $\pm 10^{\circ}$ and adopting the extrema. Although the model was adjusted in amplitude to fit the velocities in the outer regions of the galaxies, in most cases it provided a good fit at all galactocentric radii.

Figure 2 shows $I$-band images and observed [O II] velocity curves for several of the sample galaxies. Scales are identical and spatial axes aligned for each set of three plots. For each galaxy, the LRIS slit width and orientation are indicated on the WFPC2 image on the left, along with its $I, V-I$, and $M_{B}$ magnitudes. To the right is a $\sim 40 \AA$ section of the LRIS spectrum centered around the redshifted [O II] 3727 doublet. In the velocity information on the far right, points represent the observed [O II] velocities (plus [O III] for 284-4709), and the solid line is the model rotation curve. Error bars are internal errors derived from the line-fitting technique. 

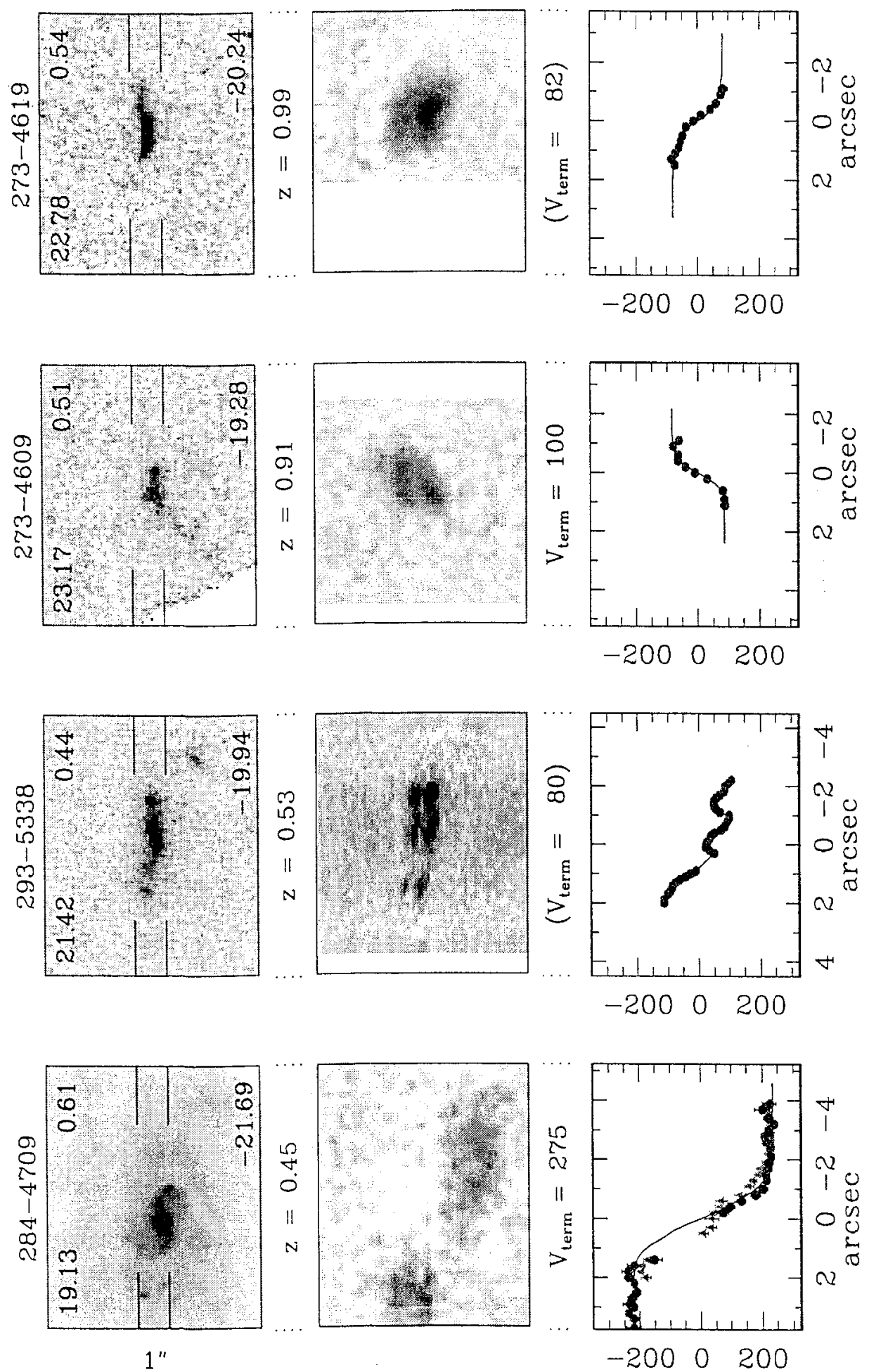

$1^{\prime \prime}$

Figure 2. I814 images, [O II] spectra, and modelled $\mathrm{km} / \mathrm{s}$ tation curves for selected galaxies within our GSS sample, chosen to illustrate several trends (see text for detailed legend). 


\section{Discussion}

Figure 3 is a plot of exponential disk scale-length $R_{d}$ vs. disk $B$ luminosity for our GSS sample, where the straight lines trace out constant surface brightness levels. We compare our data to a local sample of spirals, and a mean offset is found of $\Delta M_{B}<0.3 \mathrm{mag}$ for the total sample. The offset is found to increase smoothly with redshift when examined bin by bin $\left(\Delta M_{B} \sim 0.7\right.$ for $\left.z \geq 0.76\right)$.

We then overlay additional galaxies from our general redshift survey of the field for which the bulge fraction $B / T$ is less than $25 \%$, to check for an intrinsic size selection in our subsample of disk galaxies for which we have obtained rotation curves. For the two lower redshift bins the additional galaxies have a distribution like that of our subsample, but for redshifts $z \geq 0.76$ the mean of the general survey data lies further away from the local relation than does our subsample. This can be interpreted as a shift to higher surface brightness, or smaller intrinsic size, and is not unexpected. We require a certain spatial extent of [O II] flux along the major axis of the galaxy in order to trace ont a rotation curve, and must deconvolve the velocity profile from the effects of the seeing disk and the slit width. At redshift $z=1,1^{\prime \prime}=7 \mathrm{kpc}$ for our cosmology of $H_{0}=75$ $\mathrm{km} / \mathrm{s} / \mathrm{Mpc}, q_{0}=0.05$, and at the highest redshifts we are not able to resolve very small galaxies.

In Figure 4 we compare the data to a local Tully-Fisher relation in the restframe $B$-band, which corresponds to $V$ at $z \sim 0.4$ and to $I$ at $z \sim 0.8$ (i.e. the $k$ corrections are small). We have used a local relationship (Pierce \& Tully 1992) derived from 32 spiral galaxies in the Ursa Major cluster, based on HI velocity width measurements (corrected for turbulent broadening).

Our data form a high redshift Tully-Fisher relation that spans five magnitudes and extends to well below $L^{*}$, with no obvious change in shape or slope with respect to the local Tully-Fisher relation for the bulk of the observed population. The small offset of $<0.2 B$ mag can be attributed to strong brightening of a fraction of the low mass galaxy population at redshifts $z \geq 0.5$. We can visualize this most easily as a combination of trends. First, across all 3 redshift bands there exist galaxies which fall in the region of Tully-Fisher diagram populated by galaxies in the local universe. Second, as we move beyond redshift $z \sim 0.5$ we find a fraction of the low mass galaxies deviate from the local relation (with offsets of $\Delta M_{B} \sim 3 \mathrm{mag}$ ). Third, as we move beyond redshift $z \sim 0.5$ our selection criteria act to remove small, low luminosity galaxies from the sample. We can combine these trends to visualize a population of spirals with properties similar to those of local spirals occurring out to redshift $z \sim 1$, with a low mass, brightened fraction appearing at $\approx \sim 0.5$; both populations are truncated at the faint end of the relation for high redshift galaxies.

We next re-examine the rotation curves show in Figure 2, observing the location of the galaxies on Figure 4. Galaxies 284-4709 and 273-4609 are representative of the bulk of the galaxies. They have normal spiral morphologies, and their circular velocities place them along the local Tully-Fisher relation. The other two galaxies are quite different, have have morphologies markedly different from the rest of the sample. Galaxy 293-5339 appears like a series of pearls on a string rather than a continuous disk, and the rotation curve supports a view of three or more individual components within a single potential that have not yet: merged (each component having an individual circular velocity of $\sim 50 \mathrm{~km} / \mathrm{s}$ ). 


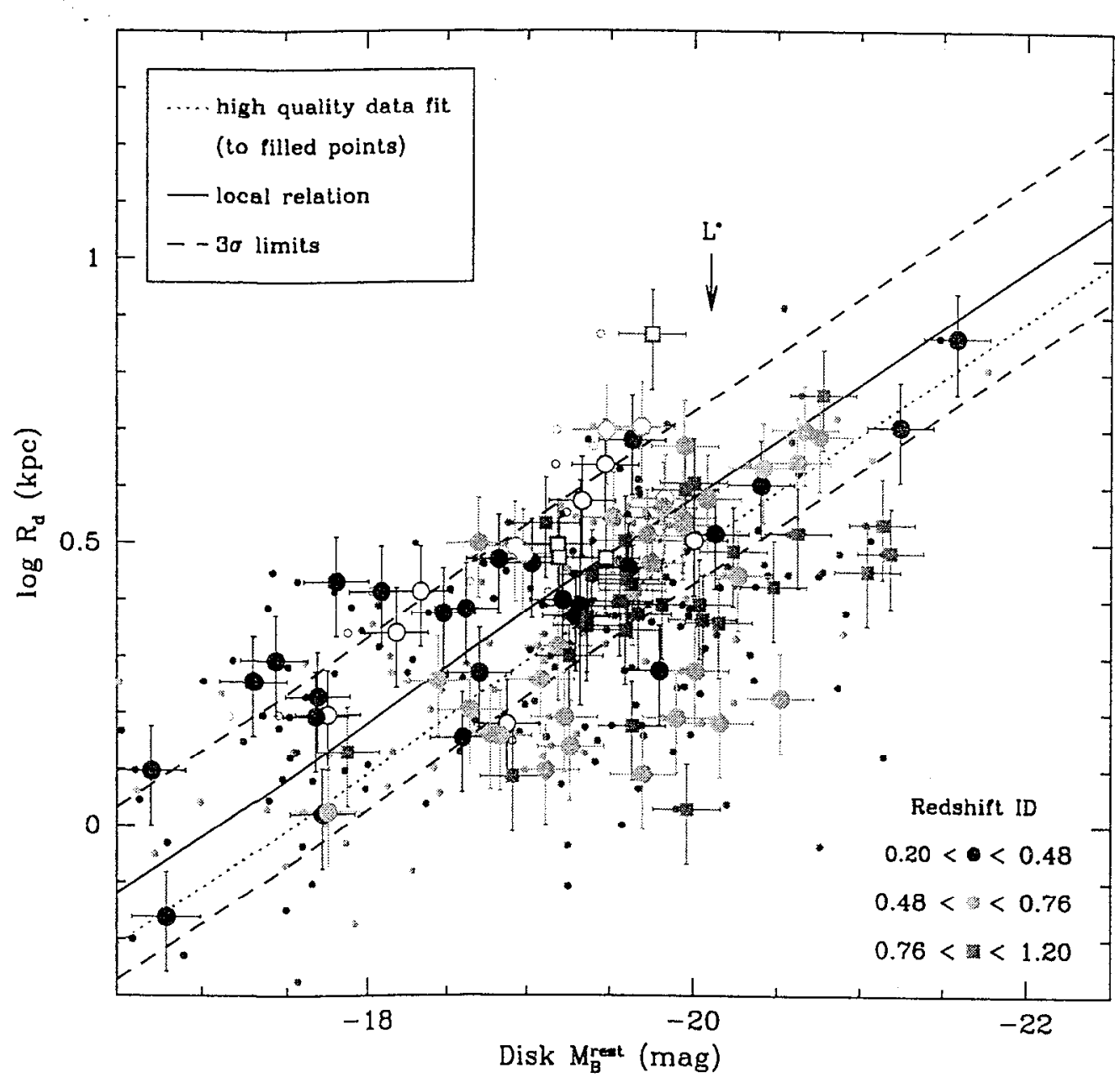

Figure 3. A high redshift surface brightness diagram plotting exponential disk scale-length $R_{d}$ vs. disk $B$ luminosity. We show data for 100 galaxies from the GSS divided into three redshift bins, with solid high quality points and hollow fair quality points. Magnitudes have been corrected for internal extinction, and disk scale-lengths have not been corrected for inclination (hollow points denote disks of inclination $i \geq 800$ ). The distribution of the high quality data (dotted line) is compared to a relationship for local spirals (de Jong 1995). We then overlay additional galaxies from our general redshift survey of the field for which the bulge fraction $B / T$ is less than $25 \%$, to check for an intrinsic size selection in our subsample of disk galaxies. 


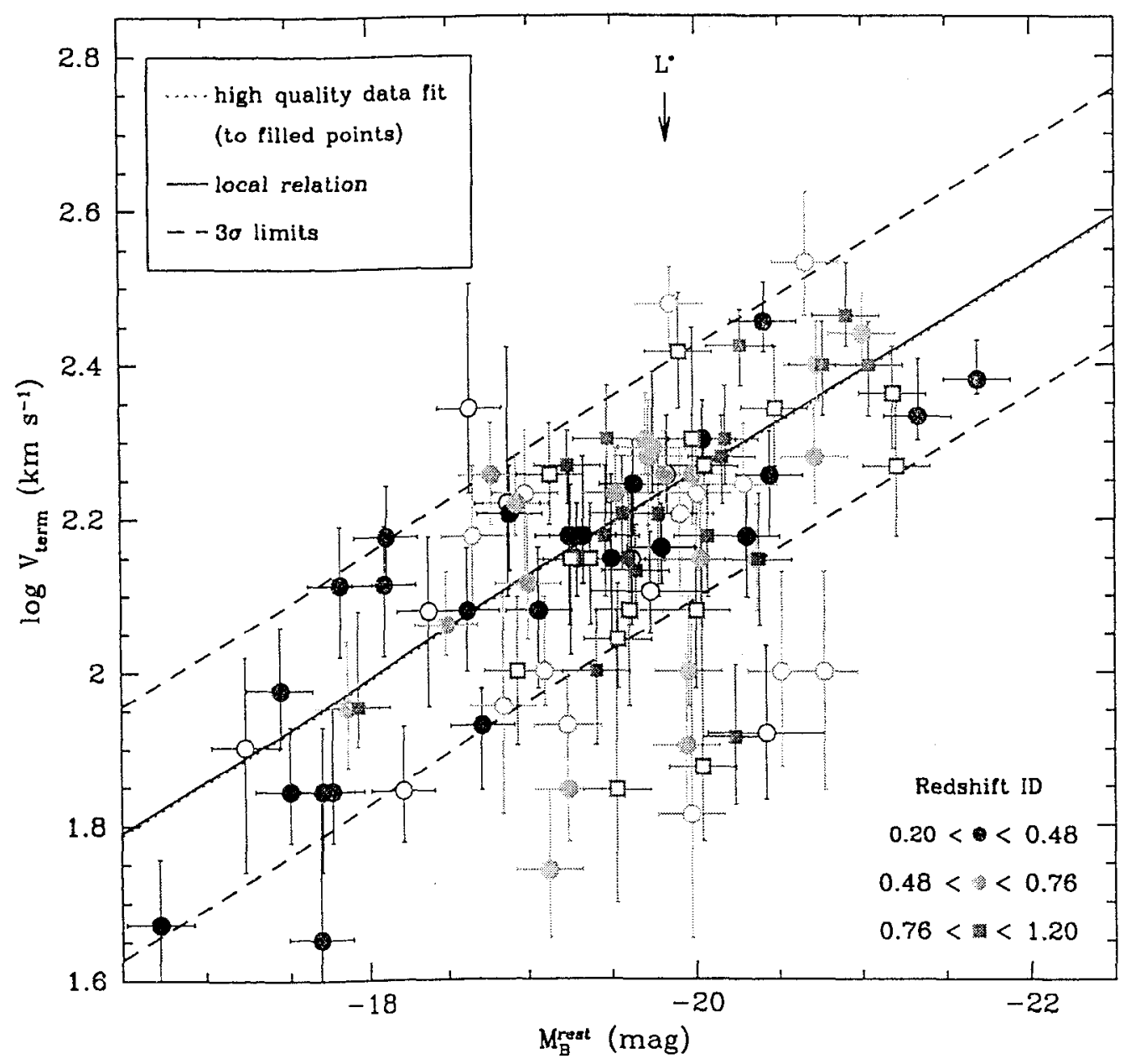

Figure 4. A high-redshift TF diagram plotting circular velocity $V_{t e r m}$ vs. total $B$ luminosity. We show data for 100 galaxies divided into three redshift ranges, with solid high quality points and hollow fair quality points. Velocities have been corrected by sin $i$, and magnitudes for internal extinction. The distribution of the high quality data is compared to a local relationship based on HI velocity width measurements for a restricted set of 32 local cluster spirals (Pierce and Tully 1992). A mean offset is found of $\Delta M_{B} \leq 0.2 \mathrm{mag}$. This offset does not vary significantly between the three redshift ranges, but at higher redshifts selection effects become more and more important. 
Galaxy 273-4619 appears to be a single component but curves like a filament rather than lying flat like a disk, with an outcrop at the upper end of the slit which is quite real. The simple model profile yields a $V_{t e r m}$ of $82 \mathrm{~km} / \mathrm{s}$, but when a contribution is removed due to the curved distribution of the flux within the slit, the remaining velocity is of order $V_{\text {term }} \sim 30 \mathrm{~km} / \mathrm{s}$. We emphasize that these galaxies (a) have a very unusual morphology, and (b) are extreme rarities in our sample, on which no morphological selection has been performed. They show an offset relative to local galaxies in this diagram, but there are also galaxies with disk morphology in this region of the Tully-Fisher diagram.

Recent years have seen the emergence of a standard model for the growth of structure - the hierarchical clustering model in which the gravitational effects of dark matter drive the evolution of structure from the near-uniform recombination epoch until the present day. Simple models for galaxy formation in the context of these CDM cosmogonies have been reniarkably successful in reproducing the properties both of the nearby galaxy population (Kauffmann, White \& Guiderdoni 1993) and of the recently discovered population of Lyman break galaxies (Baugh et al. 1998). In the last couple of years modeling of this type has been extended to predict the sizes, surface densities and rotation curves of spiral galaxy disks (Dalcanton, Spergel \& Summers 1997; Mo, Mao \& White 1998). The result is a testable scenario that predicts the basic structural properties of the disk galaxy population in any specific cosmogony of CDM type. The models also predict how the ensemble of disk galaxies should evolve with redshift. They assume that most spirals are the central galaxies of isolated halos, an assunption supported by the fact that they must have undergone minimal dynamical disturbance since the formation of the bulk of their disk stars (Tóth $\&$ Ostriker 1992). In this case the size of the disk is expected to scale with that of its dark halo, leading to the prediction

$$
R_{d} \approx 6.5 h^{-1} \mathrm{kpc}\left(\frac{\lambda}{0.05}\right)\left(\frac{V_{c}}{250 \mathrm{~km} \mathrm{~s}^{-1}}\right)\left[\frac{H(z)}{H_{0}}\right]^{-1}
$$

where $R_{d}$ is the disk scale length, $\lambda$ is the spin parameter of the $h_{a l o}, V_{c}$ is the halo circular velocity, and $H(z)$ and $H_{0}$ are the Hubble parameters at redshift $z$ and at the present epoch respectively. Disk size is predicted to scale linearly with $V_{c}$ but with substantial scatter induced by $\lambda$. At high redshift the distribution of sizes is shifted to smaller values. $\mathrm{By} z=1$ the factor is almost $0.5 \mathrm{in} \log R_{d}$ for an Einstein-de Sitter Universe, but only about 0.2 for a flat universe with $\Omega_{0}-0.3$. Thus distant disks are expected to be small and dense as compared to local disks.

Because the prediction scale linearly with redshift, it is critical to extend our measurements of circular velocities to as high a redshift as possible. Because this two-dinensional projection is not a fundamental projection for spiral galaxies, there is considerable scatter at all redshifts. Furthermore, spirals move across the plane of the diagram accurding to spiral type so we have confined our comparison to late type spirals.

Figure 5 is a plot of exponential disk scale-length $R_{d}$ vs. circular velocity $V_{\text {term }}$ for the late type (Sbc - Scd) spirals within our sample, reflecting the current limitations of this technique. We find a mean offset of $\Delta \log R_{d} \sim 0.1$. which increases with redshift $\left(\triangle \log R_{d} \sim 0.2\right.$ for $\left.z \geq 0.76\right)$. Examining the 


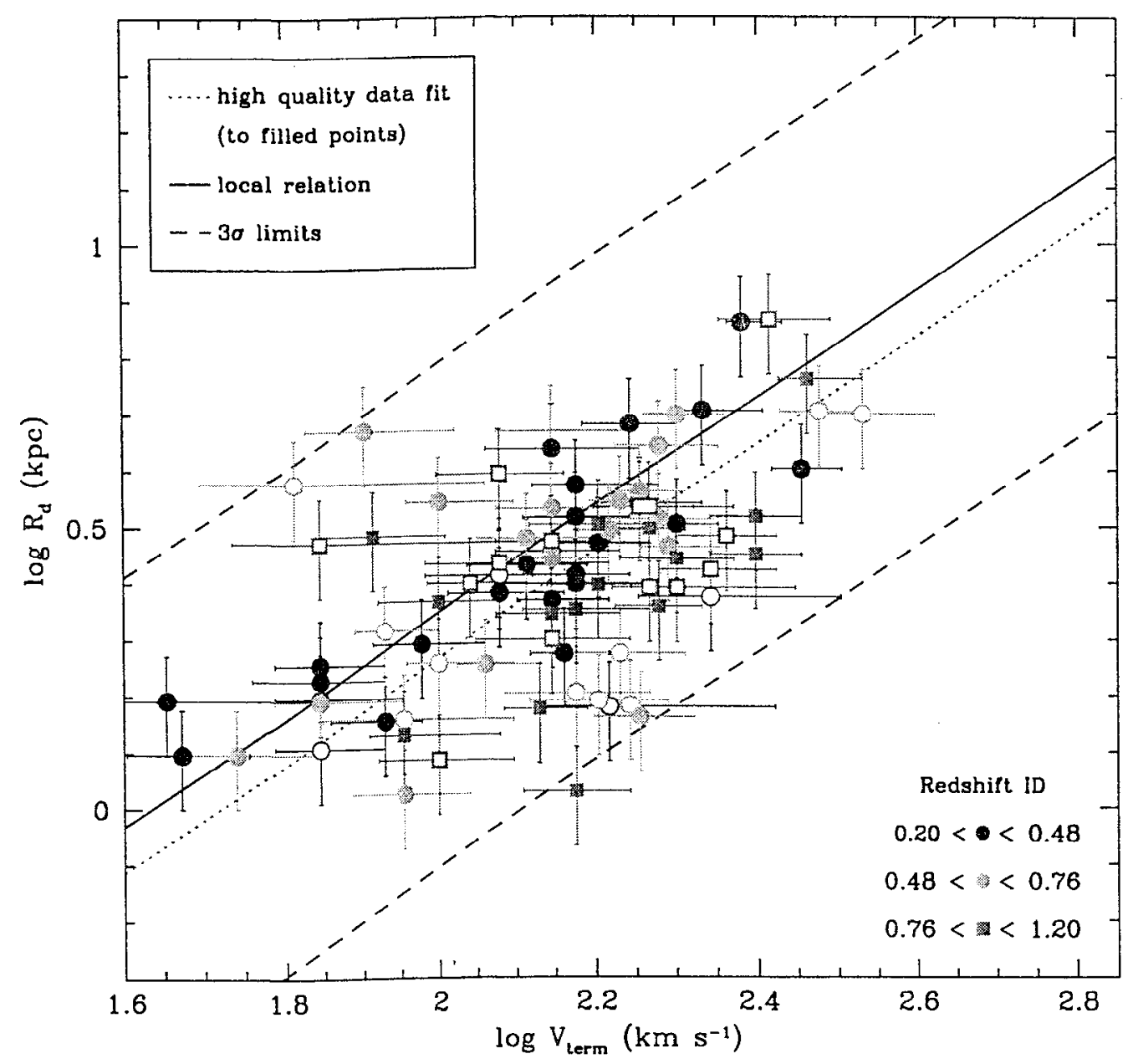

Figure 5. A high-redshift velocity-size diagram plotting exponential disk scale-length $R_{d}$ vs. circular velocity $V_{t e r m}$. We show data for 70 late type spiral galaxies divided into three redshift ranges, with solid high quality points and hollow fair quality points. Velocities have been corrected by $\sin i$, but disk scale-lengths have not been corrected for inclination. The distribution of the high quality data is compared to a local relationship (Vogt 1995. Burstein et al. 1997). A mean offset is found of $\Delta \log R_{d} \sim 0.1$, which increases with redshift ( $\Delta \log R_{d} \sim 0.2$ for $\approx \geq 0.76$ ). 
highest redshift data within our sample, we see that our data lie considerably above the predictions for an Einstein-de Sitter Universe, but agree with those for a low $\Omega_{0}$ model. However, the scatter in the data and the selection cuts are cause for concern. We are in the process of expanding the high redshift portion of this sample by additional observations out to redshift $\approx \sim 1$ with Keck+LRIS, and attempting to extend out to higher redshifts in the IR with VLT+ISAAC, to explore this further.

Acknowledgments. This work was conducted under the auspices of the DEEP (Deep Extragalactic Evolutionary Probe) project, which was established through the Center for Particle Astrophysics. Funding was provided by NSF grant AST-9529098, and NASA grants GO-07883.01-96A and AR-07532.01-96A.

\section{References}

Burstein, D. et al. 199\%, AJ, 114, 1365

Baugh et al. 1998, ApJ, 498, 50

Dalcanton, J. J., Spergel D. N. \& Summers F. J. 1997, Ap.J, 482, 659

Guzman, R. et al. 1997, ApJ, 489, 559

Guzman, R. et al. 1998, ApJ, 495, L13

de Jong, R. S. 1995, Ph.D. thesis, University of Groningen

Kauffmann, G., White, S. D. M. \& Guiderdoni, B. 1993, MNRAS, 264, 201

Kennicutt, R. C. 1989, Ap.J, 344, 685

Koo, D. C. et al. 1996, ApJ, 469, 535

Lowenthal, J. D. et al. 1997, ApJ, 481, 673

Mo, H., Mao, S. \& White, S. D. M. 1998, MNRAS, 295, 319

Oke, J. B. et al. 1995, PASP, 107, 375

Persic, M. \& Salucci, P. 1991, ApJ, 368, 60

Pettini, M. et al. 1998, ApJ, 508, 531

Phillips, A. C. et al. 1997, ApJ, 489, 543

Phillips, A. C. et al. 1999, in prep

Pierce, M. J. \& Tully, R. B. 1992, ApJ, 387, 47

Ryder, S. D. \& Dopita, M. A. 1994, ApJ, 430, 142

Rix, H.-W. et al. 1997, MNRAS, 285, 779

Simard, L. \& Pritchet, C. 1998, ApJ, 505, 96

Tóth, G. \& Ostriker, J. P. 1992, ApJ, 382, 5

Vogt, N. P. et al. 1993, ApJ, 415, L95

Vogt. N. P. 1995, Ph.D. thesis, Cornell University

Vogt, Y. P. et al. 1996, ApJ, 465, L15

Vogt, N. P. et al. 1997, ApJ, 479, L121

Vogt; N. P., Phillips, A. C., et al. 1909, in prep

Prochaska, J. X. \& Wolfe, A. M. 1998, Ap.J, 507, 113 


\section{,}


The Hy Redshift Universe

A SP Conference Series, Vol. 193, 1999

A. J. Bunker \& W. J. M. van Breugel, eds.

\title{
The Evolution of Early-Type Galaxies as a Function of Galaxy Mass
}

\author{
M. A. Pahre ${ }^{1}$ \\ Harvard-Smithsonian Center for Astrophysics
}

\begin{abstract}
The correlations known as the fundamental plane (FP) provide a powerful tool to study the evolutionary history of the early-type galaxies. The surface brightness intercept is an indicator of mean luminosity evolution of the galaxy population, while the slope of the FP is an indicator of the relative evolutionary rates of low and high mass galaxies within the population. Large galaxy samples at intermediate redshifts are required to study the latter, and selection criteria must be uniformly applicd and quantified. The first such investigation, based on $>120$ galaxies at $0.1<z<0.6$, suggests that the slope of the FP relations is subtly crolving with redshift even though the evolution of the intercepts of the FP, color-magnitude, and absorption line- $\sigma_{0}$ relations appear to favor $z_{f}>3$. The most likely scenario which could explain these effects is an evolutionary history that is a weak function of galaxy mass, such that the lowcst mass early-type galaxies are somewhat younger than the highest mass galaxies.
\end{abstract}

\section{Introduction}

The last half decade has seen a dramatic increase in our understanding of the evolutionary history of early-type galaxies in rich clusters. It is now widely accepted that the early-type galaxy population is old and formed at early epochswhether the observations are derived from HST photometric correlations alone (Barrientos et al. 1996; Pahre et al. 1996), ground-based colors and HST morphological typing (Stanford et al. 1998), HST colors and complete spectroscopic samples (van Dokkum et al. 1998a), or HST optical (van Dokkum \& Franx 1996; Kelson et al. 1997; van Dokkum et al. 1998b) and ground-based near-infrared (Pahre et al. 1999ab) imaging combined with Keck moderate dispersion spectroscopy. It is thus obvious that this revolution in evolutionary studies has been largely instrument-driven in the combination of moderate-dispersion spectroscopy on very large telescopes and high-resolution imaging either with HST or at near-infrared wavelengths from the ground.

But such studies usually suffer a common assumption in their approach: they treat early-type galaxies as a single, uniform population without inherent differences. Now that HST imaging and Keck spectroscopy can be obtained for

\footnotetext{
${ }^{1}$ Hubble Fellow.
} 


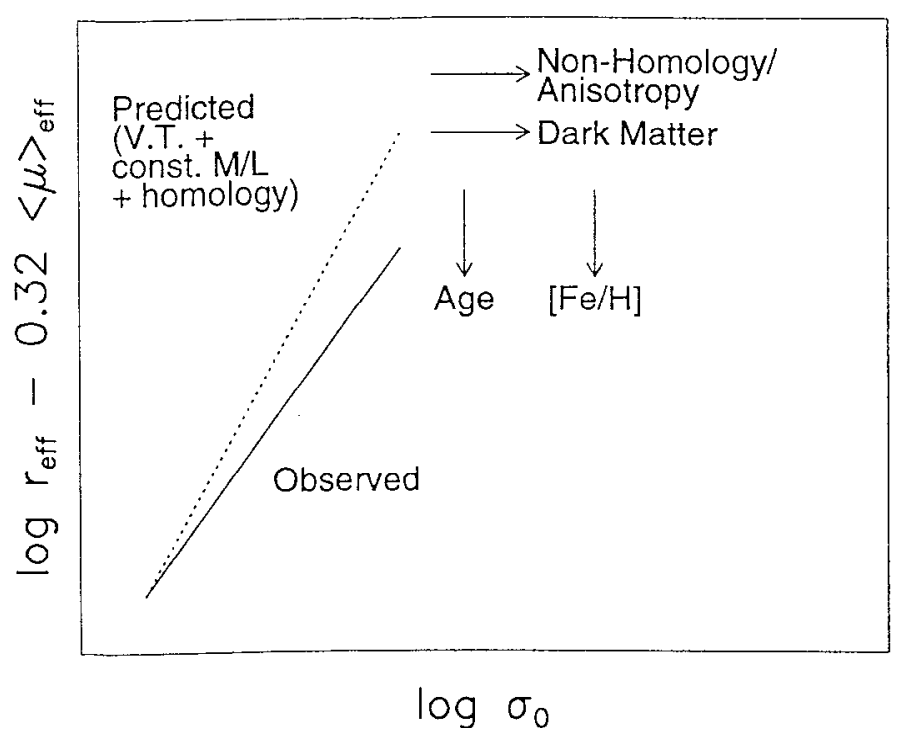

Figure 1. The effects on the slope of the $\mathrm{FP}$ when varying various physical parameters along the early-type galaxy sequence. Notice that if high mass galaxies (upper right of diagram) are made older, more metal-rich, or more dominated by dark matter (per unit luminosity) than low mass galaxics, then the slope of the FP flattens.

reasonably large samples of galaxies it is possible to relax this assumption and determine to what extent there may exist systematic differences among earlytype galaxies. These differences may take the form of age, metal abundance, dark matter content, or velocity distribution as a function of galaxy mass.

\section{The Evolutionary History Implied by Local Early-Type Galaxies}

The fundamental plane (FP) of elliptical galaxies is defined as the bivariate correlation among the observables (such as effective radius $r_{\text {eff }}$, mean $\mathrm{SB}\langle\mu\rangle_{\mathrm{eff}}$, and central velocity dispersion $\sigma_{0}$ ) that has the minimal scatter (Djorgorshi \& Davis 1987; Dressler et al. 1987). The existence of a thin FP implies a homogeneity among the physical properties of elliptical galaxies. The form of the relation provides direct insight into physical parameters that may form the basis of the galaxy sequence, such as the stellar content of the galaxies.

Age-metallicity degeneracy (Worthey 1994) prevents a clean separation of the stellar population parameters for the stellar content of early-type galaxies. It has therefore been the largest obstacle to studies of nearby galaxies in the local universe. There are added degeneracies due to possible variations in the dark matter content per unit luminosity (or stellar mass) and homology breaking in the velocity distributions among the family of early-type galaxies. These various effects are summarized schematically in Fig. 1.

Despite these challenges in deriving the evolutionary history based on ob-.... servations in the local universe, recent work in the near-infrared $K$-band-a 
wavelength nearly independent of the effects of metallicity, and hence a method of breaking its degeneracy with age-imply that age, Inetallicity, and dynamical effects (either dark matter or homology breaking) all play a role in defining the sequence (Pahre et al. 1998). If true, then the age effects should become more pronounced with redshift as the formation epoch of the youngest galaxies is approached.

\section{Evolution of Early-Type Galaxies in Clusters}

This section describes a large survey of early-type galaxies in clusters at $0<$ $z<0.6$. The data were obtained from the Palomar and Keck observatories for both imaging (optical and near-infrared wavelengths) and moderate dispersion spectra. Wide-field optical imaging was obtained for 26 rich clusters; follow-11p near-infrared imaging and optical spectroscopy were obtained for a subset of 15-30 galaxies in eight clusters.

\subsection{Homogeneous Selection Procedure}

The galaxies that are studied in this survey are selected in a quantitative and uniform manner at all redshifts. The source data are ground-based and twocolor, spanning $(U-V)_{0}$ and $(V-I)_{0}$. The color-magnitude and color-color relations are used, as well as the concentration index (Abraham et al. 1994). The method is summarized in Fig. 2 for cluster Abell $665(z=0.18)$.

\subsection{Color Evolution}

The aperture color-magnitude relation (Visvanathan \& Sandage 1977) represents a projection of the bivariate FP onto two of the three fundamental axes; the color-magnitude relation is thus a higher scatter projection of the FP. The ground-based two-color imaging data which were used to select the sample can also be used to study the color evolution of the early-type galaxies in the survey using the intercept of this aperture color-magnitude relation, evaluated at the standard condition of fixed luminosity $\left(V_{\text {tot }}=14 \mathrm{mag}\right.$ at the distance of the Coma cluster, or $M_{V}=-20.9 \mathrm{mag}$ assuming $\left.H_{0}=75 \mathrm{~km} \mathrm{~s}^{-1} \mathrm{Mpc}^{-1}\right)$.

As shown in Fig. 3, this evolution appear's to be passive following a formation epoch at high redshift. This is consistent with other results (Stanford et al. 1998), but here the redshift coverage is continuous, and the evolutionary history consistent, between the local universe (Coma cluster; Bower et al. 1992) and the intermediate redshift clusters. Note that these results only describe how the mean population is evolving, not how high or low mass galaxies might differ. ${ }^{1}$

\subsection{Evolution of Absorption Line Strengths}

The Balmer lines of hydrogen are often considered a powerful probe of age differences in the stellar content of galaxies (Worthcy ct al. 1905; Jones \& Worthey

\footnotetext{
${ }^{1}$ While Stanford et al. (1998) studied the evolution of the slope of the color-magnitude relation for HST morphologically-selected E/SO galaxies, the predicted slope evolution based on the observations in the present paper are not excluded by their data (with the possible exception of one cluster at $z \sim 0.9$ ).
} 

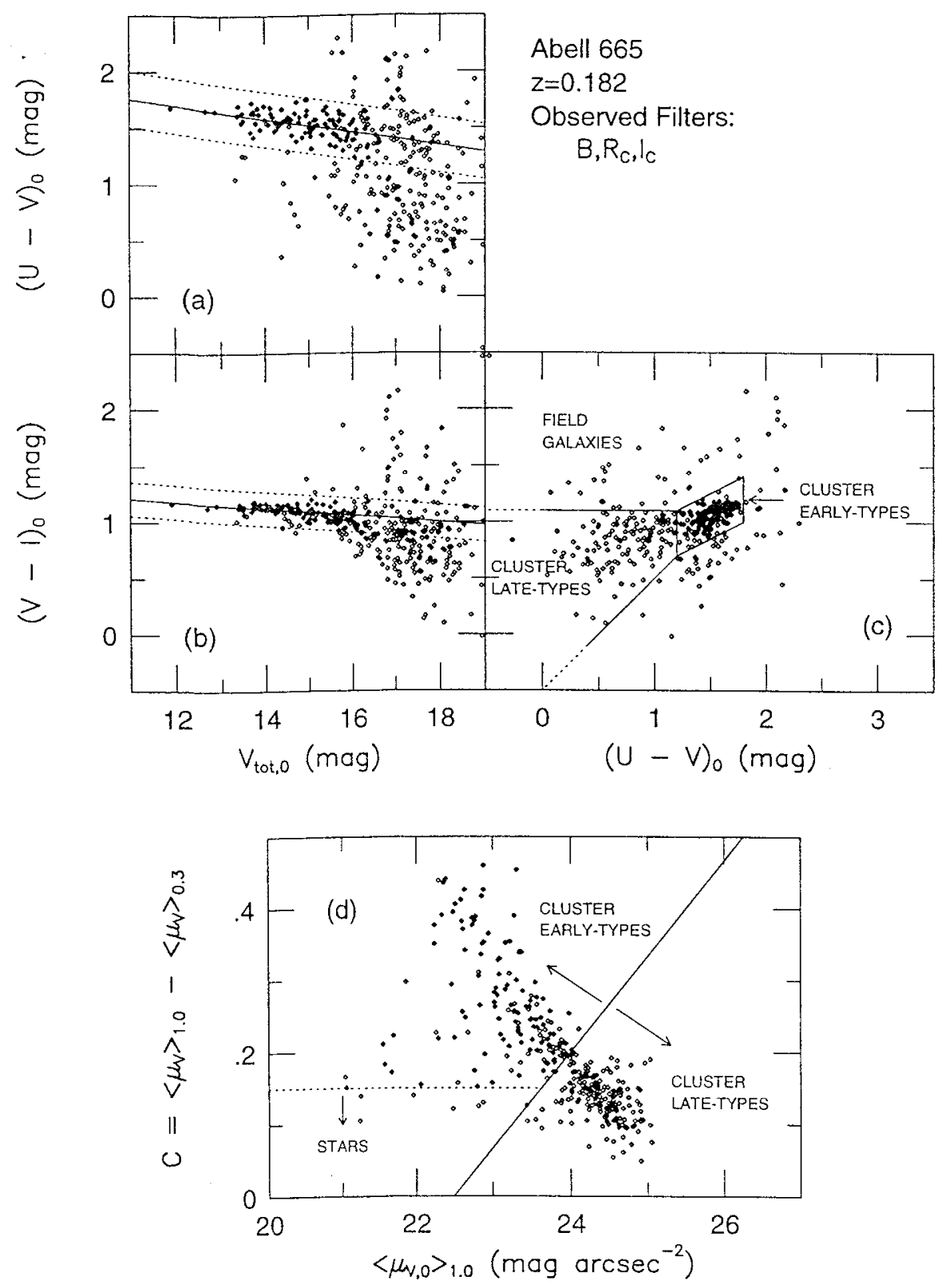

Figure 2. Example of the two-color (three-band) and morphological (concentration index) selection criteria used to constructive quantitatively uniform samples of early-type galaxies for study in clusters at $0.1<z<0.6$. The color-magnitude relations are well-defined in (a) and (b). a locus in color-color space is clearly delineated in (c), and the concentrated early-type galaxies are similarly easily identified using the concentration index in (d). (From Pahre 1999b.) 


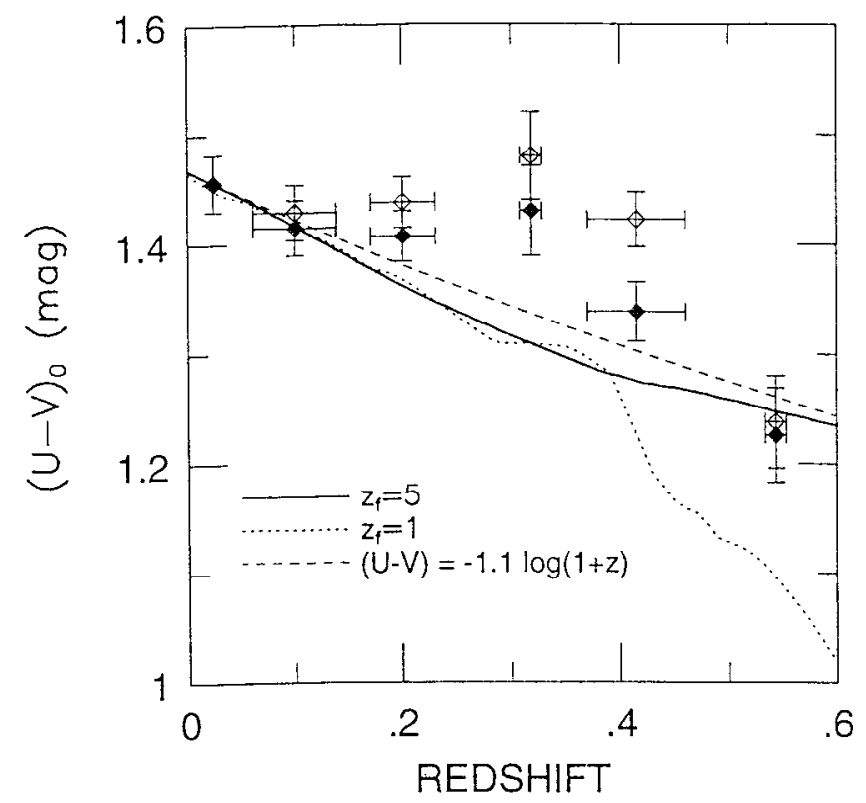

Figure 3. The color evolution in rest-frame $(U-V)$ for early-type galaxies in rich clustcrs at $0.1<z<0.6$. The clusters have been binned by redshift and a relative $k$-correction from the observed colors- ( $U$ $V)$ for $z<0.15,(B-R)$ for $0.15<z<0.5$, and $(V-I)$ for $z>$ 0.5 - into the rest-frame $(U-V)_{0}$ has been applied. Filled symbols represent a constant slope for the color-magnitude relation, while open symbols are derived by allowing the slope to vary with cluster and redshift. Evolutionary models are plotted from Bruzual \& Charlot (1996, in Leitherer et al. 1996) with formation redshifts of $z_{f}=1$ and 5 and normalized to the Coma cluster. The color evolution implies the highest formation redshifts for the mean early-type galaxy population. This evolutionary rate can be approximated by the analytical form $\Delta(U-V)=-1.1 \log (1+z)$. (From Pahre 1999b.)

1995) while the $\mathrm{Mg}_{2}$ (Mould 1978) and (Fe) indices are mostly a function of metal abundance. All these lines should evolve with redshift, although the Balmer lines will have a larger dynamical range due to their stronger age dependence.

The Palomar and Keck spectroscopy of > 100 early-type galaxies in six clusters at $z>0.1$ is shown in Fig. 4 . As seen in that figure, the $\mathrm{Mg}_{2}$ and $\langle\mathrm{Fe}\rangle$ lines weaken with increasing redshift while $\mathrm{H} \beta$ strengthens. These trends are actirally mutually consistent with one another in both the Bruzual \& Charlot (1996; as provided in Leitherer et al. 1996) and Vazdekis et al. (1996) simple stellar population models. The implied formation redshift for the mean population based on these models is $z f>3$.

\subsection{Luminosity Evolution from the FP Intercept}

The use of the FP as an indicator of early-type galaxy evolution was pioneered by Franx and collaborators (Franx 1993; van Dokkum \& Franx 1996). The 


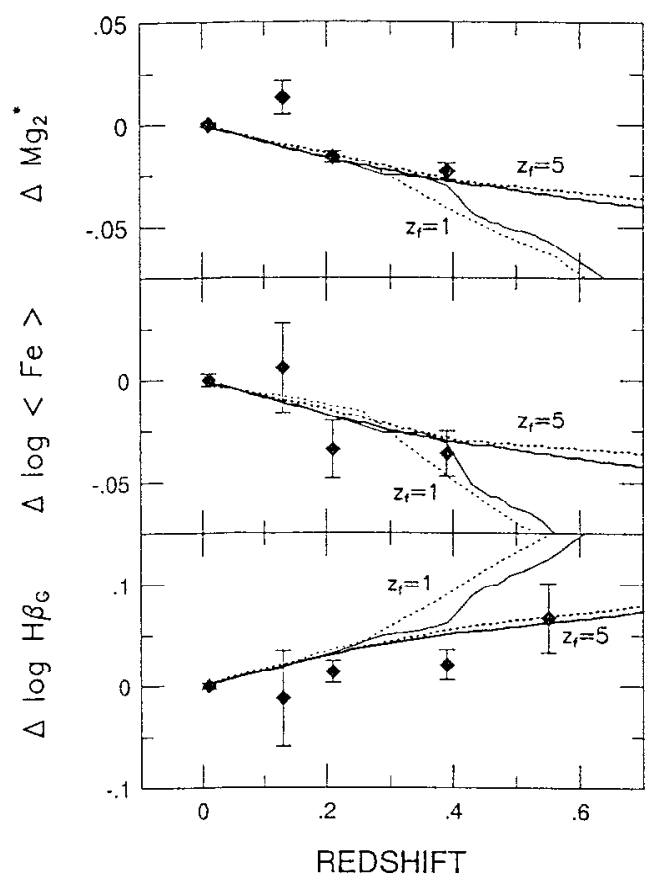

Figure 4. Evolution of the absorption line strengths of $\mathrm{Mg}_{2},\langle\mathrm{Fe}\rangle$, and $\mathrm{H} \beta_{G}$ measured from the Keck/Palomar spectra and calibrated onto the Lick/IDS system. Each index is corrected for the slope of the correlation with $\log \sigma_{0}$. Models identical to those in Fig. 3 are plotted here as solid lines; similar models from Vazdekis et al. (1996) are plotted as dotted lines. The models are fixed to the Coma cluster data at $z \sim 0$ from Jørgensen (1997). The evolutionary history of these indices implies high formation redshift for the mean early-type galaxy population. (From Pahre et al. 1999c.)

internal dynamics of galaxies at intermediate redshift galaxies were difficult to measure with $4 \mathrm{~m}$ class telescopes (van Doklkum \& Franx 1996; Ziegler \& Bender), but have become straightforward with $10 \mathrm{~m}$ telescopes (Kelson et al. 1997; van Dokkum et al. 1998b; Pahre et al. 1999a) and high throughput, multi-object spectrographs like LRIS (Oke et al. 1995). It is now possible to assemble large samples of early-type galaxies at intermediate redshifts with central velocity dispersion measurements of sufficient quality that the FP correlations can be constructed.

The FP in the near-infrared at $0<z<0.6$ is plotted in Fig. 5 for various clusters in the survey.

The SB intercept of the $\mathrm{FP}$ at any redshift must be corrected for surface brightness dimming (Hubble \& Tolman 1935), and then a $k$-correction is applied. The SB intercept is then evaluated at fixed $R_{\text {eff }}$ (in $\mathrm{kpc}$, and hence the results depend weakly on the assumed cosmology) and $\log \sigma_{0}$, which implies that 

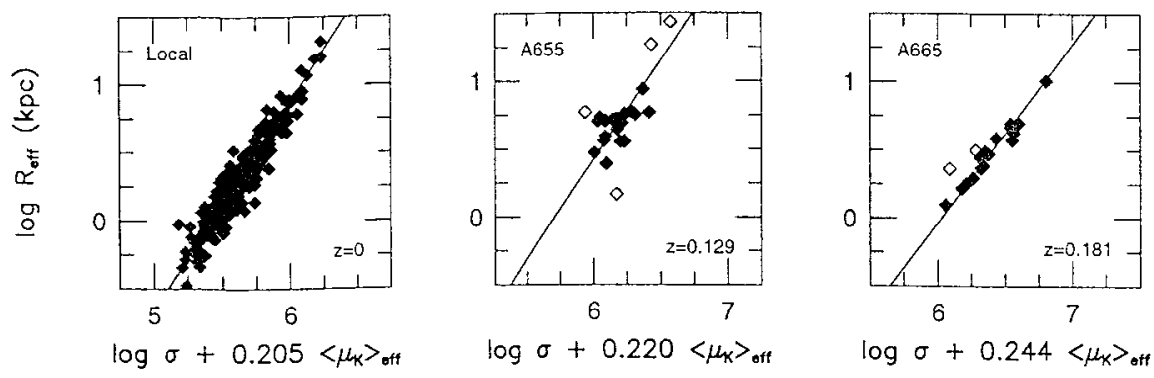

$\log \sigma+0.244\left\langle\mu_{x}\right\rangle_{\text {ott }}$
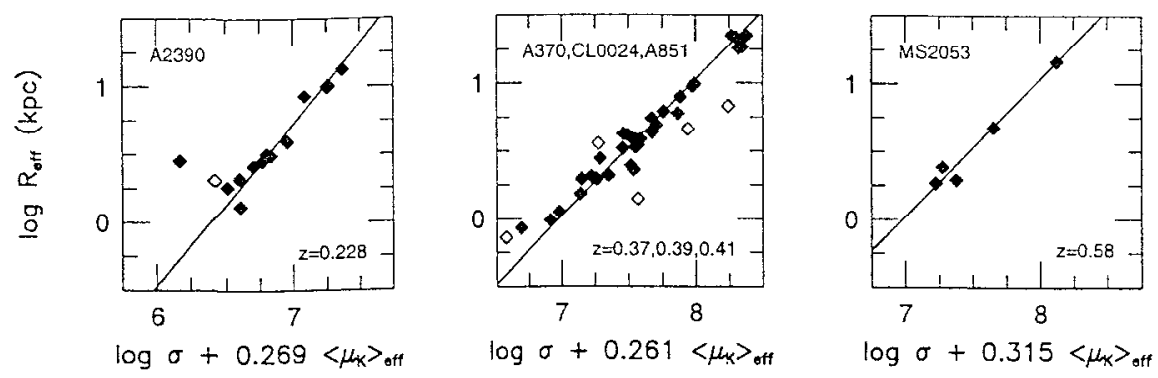

Figure 5. The EP of early-type galaxies for $0<z<0.6$ in the nearinfrared $K$-band. The local galaxy sample at $z \sim 0$ is taken from Pahre (1999a) while the higher redshift sample is taken from observations with the Keck $10 \mathrm{~m}$ and Palomar $5 \mathrm{~m}$ telescopes (Pahre et al. 1999a). The best-fitting slope is plotted in each panel, which shows the subtle effect of flattening with redshift. This is an indication that there exists differential evolutionary effects along the early-type galaxy sequence. (Figure adapted from Pahre et al. 1999c.)

the luminosity (or $M / L$ ) is evaluated at fixed mass. The SB intercept is thus corrected to a "standard condition." Any deviations of the corrected SB intercept from the local value implies evolution in the population. The evolution of luminosity at fixed mass, or the evolution of $M / L$, is plotted in Fig. 6. Various models are also plotted which show that the mean population formed at high redshift $z_{f}>3$.

\subsection{Evolution of the Slope of the FP}

While the FP at intermediate redshifts appears similar to its form at $z \sim 0$ (Fig. 5), there is a subtle change that appears in the slope of the relation. The variation of slope with redshift is quantified further in Fig. 6.

Since the slope of the FP could be a result of variations in age, metallicity, dark matter content, or homology breaking along the galaxy sequence (Fig. 1), the evolution of the slope with redshift is an indicator that age is playing a role in helping to define the slope of the correlations. Note that this does not contradict the lack of evolution of the scatter of the color-magnitude relation (Ellis et al. 1997: Stanford et al. 1998), since the scatter only implies that galaxies of any given luminosity must have a similar formation redshift. 

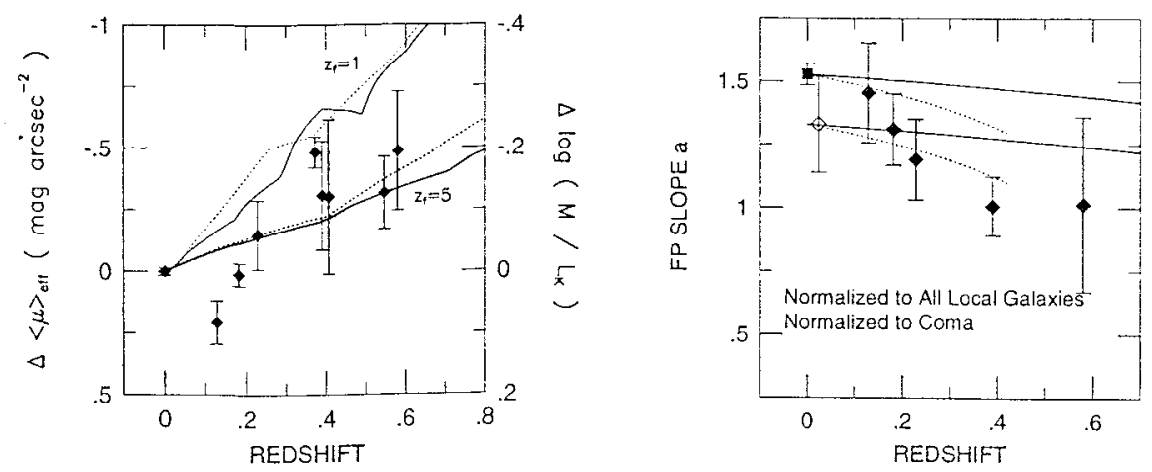

Figure 6. The evolution of the near-infrared $F P$ with redshift: [left] evolution of the surface brightness intercept (or $M / L$ ratio al fixed mass), which is an indicator of the mean evolution of the early-type galaxy population; [right] evolution of the slope, which is an indicalor of differential evolution among early-type galaxies as a function of galaxy mass. While the SB intercept appears to evolve in a manner inplying high formation redshift (as has been found in other studies), the slope evolution suggests the lowest mass early-type galaxies may have formed somewhat earlier than the highest mass galaxies. Models plotted on the right are taken from predictions based on observations of galaxies in the local universe from Pahre et al. (1998). (From Pahre et al. 1999b.)

\subsection{Possible Selection Effects}

From the above discussion, it is clear that the broad picture of the evolution of early-type galaxies in clusters is in place. The mean evolution implied by the color-magnitude relation, the absorption line strengths, and the intercept of the FP all imply that the formation redshift was $z_{f}>3$. But the far more subtle effect of the evolving slope of the FP suggests that there is more to the picture than just a single formation redshift for all early-type galaxies.

Since the vast majority of the cluster galaxies at $z \sim 0.5$ will become $z \sim 0$ early-type cluster galaxies (similar to those in Coma), it is difficult to decide which galaxies at $z \sim 0.5$ ought to fall in the evolutionary sample. For example, are $K+A$ galaxies of early-type? Since there are more bluer galaxies in higher redshift clusters due to the Butcher-Oemler effect, do these galaxies fall out of the sample at $z \sim 0.5$ while they fall into the sample at $z \sim 0$ ? Is color selection a more or less restrictive criterion than morphological selection (using HST images)?

For example, KELSON (this volume) has reported the results of a study of $\sim 50$ galaxies in a cluster at $z=0.33$. only $\sim 60 \%$ of which (to $R \leq 21 \mathrm{mag}$ ) are morphologically classified as early-type ( $E$ or S0) on the HST images. This compares to a fraction of $>80 \% \mathrm{E} / \mathrm{S} 0$ galaxies in the Coma cluster within a similar luminosity range (Terlevich et al. 1999), suggesting that a significant fraction of the galaxies are falling out of the sample between $\approx \sim 0$ and 0.3 . The "lost" galaxies are presumably on the faint end of the sequence and of 
intermediate morphological type, which underscores the importance of a detailed understanding of the sample selection criteria at all redshifts.

Such issues are crucial to address, and may hold the key for understanding subtle differences between various studies and approaches to early-type galaxy evolution. Future work will no doubt focus on these issues in far greater detail.

\section{Comparing Field and Cluster Evolution for Early-Type Galaxies}

Some models of hierarchical galaxy formation predict significant differences between early-type galaxies in the high density environment in the cores of rich clusters and those in the low density environment of the general field (Kauffmann $\&$ Charlot 1996). The general trend among such models is that the galaxies are somewhat younger in the field, because the major mergers tend to occur there at later times than in cluster cores.

Studies of possible differences between cluster and field early-type galaxies in the local universe are hampered by distance uncertainties on the FP. A recent comparative study of $\sim 100$ nearby field and cluster elliptical galaxies indicates that there are, at most, only subtle differences among the most luminous galaxies (Pahre et al. 1999d); the predicted differences are at the level of the measurement uncertainties for this local study.

Such studies may be better done at intermediate redshifts because the evolutionary effects are magnified, but the surface density of the galaxies makes them comparatively inefficient to study even with multi-object spectrographs (Treu et al. 1999; Koo 1999). Gravitational lens galaxies are typically isolated (or located in groups), and are of early-type, hence they represent other potential targets for field early-type galaxy evolntionary studies (Kochanek et al. 1999). These studies show that early-type galaxies at $z \sim 0.5$ have very similar evolutionary histories in both clusters and the field, although it is not yet clear if the results yet significantly contradict predictions of the hierarchical galaxy formation scenario.

\section{Summary}

The first large study of the evolution of the FP for $>100$ early-type galaxies at intermediate redshifts has been described. While the galaxies show a mean evolution that implies a high formation redshift, there appears to be the first sign of differential evolution along the galaxy sequence. This suggests that evolutionary rate is a function of galaxy mass; it would also be naturally explained by the lowest mass early-type galaxies being somewhat younger than the most massive galaxies.

The last few years have seen an explosion of studies of the evolutionary history of early-type galaxies. The advent of additional $8.25 \pm 1.75 \mathrm{~m}$ telescopes will continue to add to the accumulated data at an ever-increasing rate, easily dwarfing the sum of all previous studies. It will be important to use these newer data to study a number of questions which are more subtle than just the mean formation redshift of early-type galaxies: 
- How does the formation redshift (and hence age) of the galaxies vary with galaxy mass?

- How much recent star formation (like $5 \%$ by mass) could have occurred?

- Are there differences between the cluster core and the halo environments? How about between rich ( $\mathrm{X}$-ray selected) and poor clusters?

- How do E+A galaxies and the Butcher-Oemler effect factor into such evolutionary scenarios and the selection effects in these studies?

- Do field and cluster elliptical galaxies systematically differ in their ages as is predicted in many hierarchical galaxy formation scenarios?

- Given fair sample sizes and well-understood selection criteria, can the cosmological parameters be constrained?

Answers to these questions will provide us with a complete picture of the formation and evolution of early-type galaxies.

Acknowledgments. This work is supported by Hubble Fellowship grant HF-01099.01-97A from STScI (which is operated by AURA under NASA contract NAS5-26555). Thanls to the conference organizers for putting together a fun and lively meeting, and for travel support to attend it. M. A. P. wishes to thank collaborators S. Djorgovski and R. de Carvalho, who have made significant contributions to this project.

\section{References}

Abraham, R. G., Valdes, F., Yee, H. K. C., \& van den Bergh, S. 1994, ApJ, 432, 75

Barrientos, L. F., Schade, D., \& López-Cruz, O. 1996, Ap.J, 460, L89

Bower, R. B., Lucey, J. R., \& Ellis, R. S. 1992, MNRAS, 254, 601

Djorgovshi, S., \& Davis, M. 198i, ApJ, 313, 59

van Dokkum, P. G., Franx, M., Felson, D. D., Mllingworth, G. D., Fisher, D., \& Fabricant, D. 1998a, ApJ, 500, 714

van Dokkum, P. G., Franx, M., Kelson, D. D., \& Hlingworth. G. D. 1998b, Ap. 504, L17

van Dokkum, P. G., \& Franx, M. 1996, MNR.AS, 281, 985

Dressler, A., et al. 1987, ApJ, 313, 42

Ellis, R. S., et al. 1997 , ApJ, 483, 582

Franx, M. 1993, ApJ, 407, L5

Hubble, E., \& Tölman. R. C. 1935, ApJ, 82. 302

Jørgensen, I. 1997, MNRAS. 288, 161

Jones, L. A., \& Worthey, G. 1995, ApJ, 446, L31

Kauffmann, G., \& Charlot, S. 1998, MNRAS, 294. 705

Kochanek. C.. et al. 1999, ApJ. submitted (astro-ph/9909018) 
Koo, D. 1999, in Photometric Redshifts and High Redshift Galaxies, eds. R. Weymann et al. (ASP Conf. Ser.), in press (astro-ph/9907273)

Mould, J. R. 1978, ApJ, 220, 434

Oke, J. B., et al. 1995, PASP, 107, 375

Pahre, M. A. 1999a, ApJS, in press

Pahre, M. A. 1999b, AJ, submitted

Pahre, M. A., Djorgovski, S. G., \& de Carvalho, R. R. 1996, ApJ, 456, L79

Pahre, M. A., de Carvalho, R. R., \& Djorgovski, S. G. 1998, AJ, in press

Pahre, M. A., Djorgovski, S. G., \& de Carvalho, R. R. 1999a, ApJS, submitted

Pahre, M. A., Djorgovski, S. G., \& de Carvalho, R. R. 1999b, ApJL, submitted

Pahre, M. A., Djorgovski, S. G., \& de Carvalho, R. R. 1999c, ApJL, submitted

Pahre, M. A., et al. 1999d, in Cosmic Flows, ed. S. Courteau et al. in press

Stanford, S. A., Eisenhardt, P. R., \& Dickinson, M. 1998, Ap.J, 492, 461

Terlevich, A. I., Kuntschner, H., Bower, R. G., Caldwell, N., \& Sharples, R. M. 1999, MNRAS, in press (astro-ph/9907072)

Tolman, R. C. 1934, Relativity, Thermodynamics and Cosmology (Oxford: Oxford Univ. Press)

Treu, T., et al. 1999, MNRAS, in press (astro-ph/9904327)

Vazdekis, A., Casuso, E., Peletier, R., \& Beckman, J. E. 1996, ApJS, 106, 30 T

Visvanathan, N., \& Sandage, A. 1977, ApJ, 216, 214

Worthey, G. 1994, ApJS, 95, 107

Worthey, G., Trager, S. C., \& Faber, S. M. 1995, in Fresh Views of Elliptical Galaxies, ASPCS Vol. 86, eds. A. Buzzoni \& A. Renzini (San Francisco: ASP), 203

Ziegler, B. L., \& Bender, R. 1997, MNRAS, 291, 527 
The Hy Redshift Universe

ASP Conference Series, Vol. 193, 1999

A. J. Bunker \& W. J. M. van Breugel, eds.

.

\title{
- Mid-UV Spectroscopic Dating of LBDS 53W091
}

\author{
S.R. Heap, T.M. Lanz, T. Brown and I. Hubeny \\ Code 681, NASA's Goddard Space. Flight Center, Greenbelt MD 20771
}

\begin{abstract}
We have calibrated age-related spectral diagnostics in the mid-UV in terms of age and metallicity. We then make a preliminary application to dating the red galaxy, LBDS 53W091 at $z=1.55$.
\end{abstract}

\section{Introduction}

For passively evolving stellar populations younger than about 5 Gyr, the hottest stars are at the main sequence turnoff (MSTO). These stars are the only source of significant flux in the mid-UV $(2000-3200 \AA)$ spectra of such galaxies; in fact, the spectra are remarkably similar to that of a single F-star. By determining the effective temperature of the MSTO stars, we can measure the age of the population.

This technique was first applied by Dunlop et al. (1996) and Spinrad et al. (1997) to LBDS 53W091, a very red galaxy at a redshift of $z=1.55$. Observational evidence (colors, morphology, etc.) indicates that 53 W091 is a normal giant elliptical galaxy, so its red color is likely due to an aged stcllar population. Spinrad et al. (1997) used archival IUE low-dispersion spectra $(\mathrm{R}=6 \AA)$ of $F$-typc stars to calibrate the flux breaks at $2640 \AA$ and $2900 \AA$ in the spectrum of the galaxy in terms of $T_{\text {eff }}$, i.e. age.

Shortly thcreafter, Heap et al. (1998) derived a younger age of 2 Gyr for 53W091 for a solar metallicity, based on model spectra tested against a STIS spectrum of the F-typc star, 9 Comae. This obscrvation was the first in a HST program ( $\mathrm{ID}=7433)$ to observe and analyze spectra of $12 \mathrm{~F}$-type stars of known atmospheric properties and distance, and to use them to calibrate mid$\mathrm{UV}$ features in the spectra of high-redshift galaxies. Now, two years later, we have nearly completed the observing program. The analysis and modelling are underway, so we report only preliminary observational results.

\section{Observations}

The 12 program stars were selected from the sample of Edvardsson et al. (1993) in order to sample an appropriate range of $T_{\text {eff }}$ and metallicity. Their atmospheric properties ( $\mathrm{T}_{\text {eff }}, \log g$, abundances). Hipparcos distance, and ages are well known. The selected F4V - F9V stars have metallicities in the range $[\mathrm{Me} / \mathrm{H}]=-0.75$ to +0.24 , and ages from 1 to $9 \mathrm{Gyr}$. We used STIS on HST to observe the mid- $U V$ spectrum of each star at a resolving power of $R=30,000$ 
or higher. The signal-to-noise per pixel is typically about 50 before binning to lower resolution $(1 \AA$ ) for comparison to the spectrum of LBDS 53 W091.

\section{Diagnostics}

The spectral breaks, B2640 and B2900. These breaks are defined as the flux ratios in two narrow-band filters located on both sides of $\lambda=2640$ and 2900 , respectively. Both become stronger toward later spectral type. However, our studies show that a prime age diagnostic, the spectral break at $\lambda 2640$, is degenerate: young, metal-rich stars have the same B2640 as do old, metal-poor stars. This $T_{\text {eff }}-Z$ degeneracy is illustrated in Figure 1, which shows that most of the program stars have values of B2640 like that of 53 W091 (cross-hatched region), but their wide spread in ages demunstrates that this spectral feature by itself cannot constrain the age of a stellar population. The same comments apply to B2900, only this index has the further disadvantage that its strength cannot be reproduced by model-atmosphere calculations. Evidently, an important source of opacity is missing in the models.

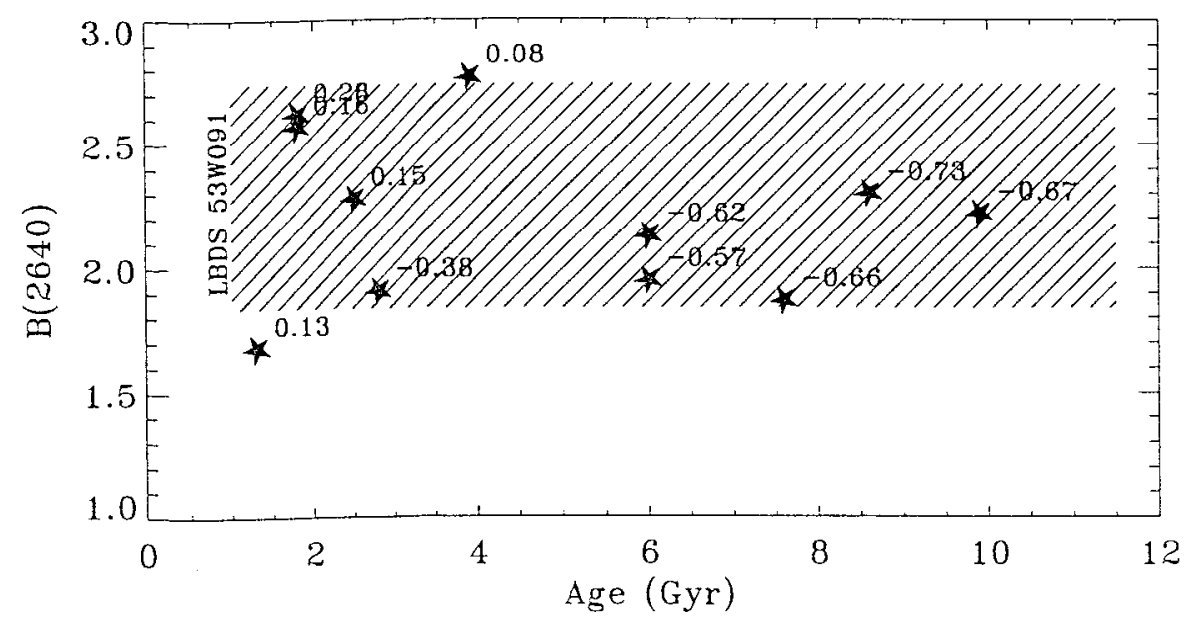

Figure 1. Measurements of B2640 in the program stars. Each star is labelled by the value of [Me/H] as derived by Edvardsson et al. (1993). The cross-hatched region shows the observed B2640 for 53 W091.

The Mg I index and mid-UV color. After a thorough analysis of F-type spectra, Lanz et al. (1999) found two spectral indices that can discriminate between effective temperature and metallicity among F-type stars. The $M g$ index, which measures the strength of the $\mathrm{Mg}$ I $\lambda 2852$ resonance line rapidly becomes weaker with increasing temperature as $\mathrm{Mg}$ becomes ionized. The mid- $\mathrm{UV}$

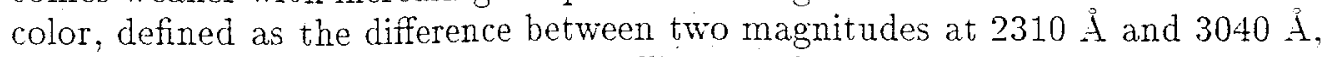
becomes "bluer" with decreasing metallicity and increasing temperature. Taken together, these two indices appear capable of breaking the $\mathrm{T}_{\text {eff }}-Z$ degeneracy. As shown in Figure 2, which plots the $\mathrm{Mg} \mathrm{I}$ index against mid-UV color. 


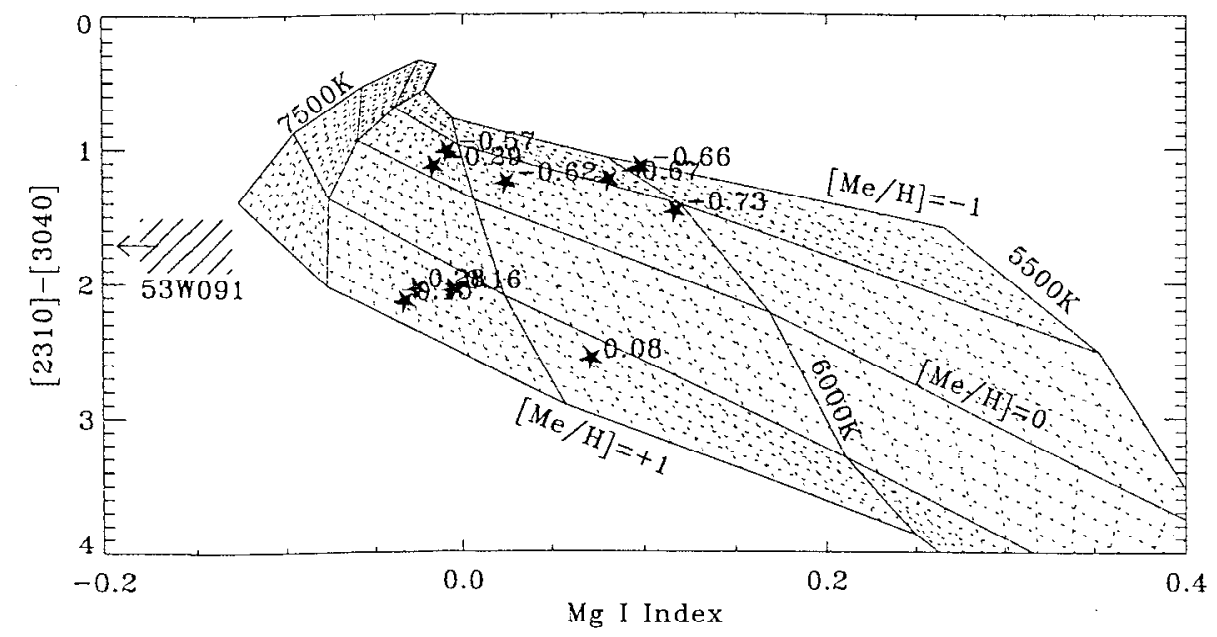

Figure 2. Calibrating $\mathrm{Mg}$ I and mid-UV color (see Lanz et al. 1999)

the high-metallicity program stars are clumped together, well away from the low-metallicity stars. A closer examination, however, shows that $T_{\text {eff }}$ is overestimated by about $200 \mathrm{~K}$, which means that the age of a stellar population will be underestimated. In addition, the metallicities of the high- $Z$ stars are overestimated (the derived metallicities of metal-poor stars are sufficiently accurate). These systematic errors will have to be accounted for before these spectral indices can be used with confidence for dating purposes. In any case, the observed $\mathrm{Mg} I$ index for 53W091 is much smaller than any of our model calculations. This is not the fault of our models, which reproduce the observed Mg I profiles of F-type stars almost exactly. Rather, the abnormally weak $\mathrm{Mg}$ I absorption in the galaxy spectrum is probably an artifact of imperfect subtraction of night-sky emission $\left(\lambda_{\text {obs }}=7273\right)$.

\section{Estimating the age of LBDS 53 W091}

Because of the ambiguity of current spectral indicators, we can only estimate the age for an assumed metallicity, or we can assume an age and solve for the metallicity. Below, we discuss both approaches.

Age for an assumed metallicity. Recent models of the chemical evolution of galaxies suggest that a giant elliptical galaxy like 53W091 should contain stars having a variety of metallicities. To pursue this point, we calculated model spectra of galaxies allowing for a composite metallicity as specified by Kodama. \& Arimoto's (1997) infall model. Following Kodama's advice, we assumed that $12 \%$ of the stars by number have a metallicity of $\mathrm{Z}=0.005,31 \%$ have a solar metallicity, and $5 \% \%$ have $Z=0.04$. Figure 3 compares the calculated spectral indices, B2640 and mid-UV color, against the observations of 53W091. Both the mid-UV color and $\lambda 2640$ break imply an age of abont 1 Gyr for 53W091, if the extinction internal to the galaxy is $A_{Y}=0.6$. If the extinction is lower: 
then the galaxy age implied by the mid-UV color can be up to $3 \mathrm{Gyr}$, in rough agreement with Spinrad et al.

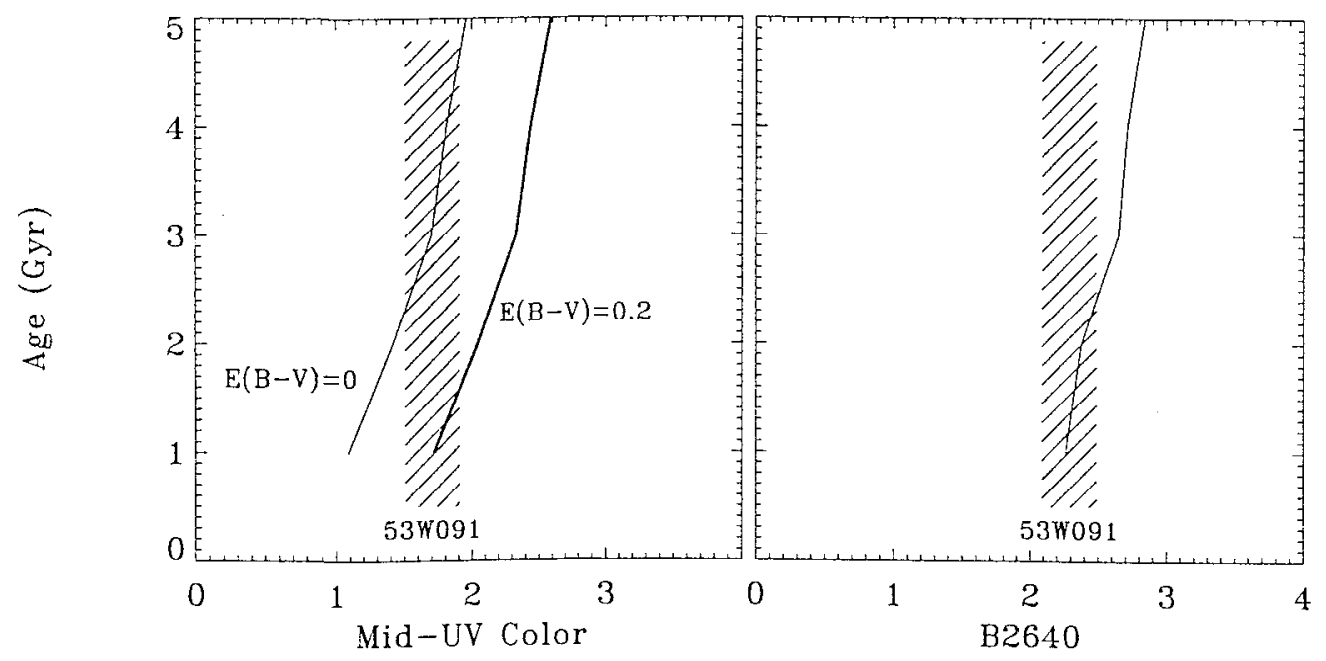

Figure 3. Observations of LBDS 53W091 vs. Kodama \& Arimoto's (1997) infall model. The isochrones we used are from Yi et al. (1999)

Metallicity for an assumed age. For currently favored cosmological parameters $\left(\mathrm{H}_{0}=67 \mathrm{~km} \mathrm{~s}^{-1}, \Omega_{m}=0.3, \Omega_{\Lambda}=0.7\right)$ and a 'reasonable' redshift of the most recent episode of star formation $\left(z_{\mathrm{SF}}\right)$, we can calculate the age of stars at $z=1.55$, which can then be used to explore possible metallicities for the galaxy. Certainly, the last burst of star formation must have occurred after the first stars in the galaxy formed, say $z_{\mathrm{SF}}=15$. At the other extreme, the last major burst of star formation might have occurred as recently as $z=3$, because some galaxies at this redshift have been observed to have star formation rates of up to $10^{3} M_{\odot} \mathrm{yr}^{-1}$. For $z_{\mathrm{SF}}=3-15$, we obtain a wide range in times, from 2.1 to $4.1 \mathrm{Gyr}$, since the last major star-forming episode. Without a better knowledge of $z \mathrm{SF}$, we cannot place constraints on metallicity.

Both approaches are unsatisfactory. We are therefore broadening our study to include other observations such as the Balmer absorption lines.

\section{References}

Dunlop J., Peacock J., Spinrad H. et al. 1996, Nature 381, 481

Edvardsson B., Andersen J., Gustafsson B. et al. 1993, A\&A 275, 101.

Heap S., Brown I., Hubeny, I., Landsman W., Yi S. et al. 1998, ApJ, 492 , L131

Lanz, T., Heap, S., T.Brown, et al. 1999. in Spectrophotometric Dating of Stars and Galaxies, ed.: I. Hubeny et al., ASP Conf. Ser., in press

Fodama T. \& Arimoto N. 1997, A\&A 320, 41

Spinrad H., Dey A., Stern D. et al. 1997. ApJ, 484, 581

Yi S., Brown T., Heap S., et al. 1999, submitted to ApJ 
The Hy Redshift Universe

ASP Conference Series, Vol. 193, 1999

A. J. Bunker \& W. J. M. van Breugel, eds.

$\because$

\title{
Evolution of Spheroidal Galaxies at $z<1$ from the Deep Extragalactic Evolutionary Probe (DEEP)
}

\author{
Myungshin $\mathrm{Im}^{1}$ and the DEEP team ${ }^{2}$ \\ UCO/Lick Observatory, UCSC, Santa Cruz, CA 95064
}

\begin{abstract}
DEEP is a multi-year survey of faint galaxies using the Keck 10n telescope and the Hubble Space Telescope (for more detail about DEEP, see an article by D.C. Koo in this conference proceedings). So far, our data consist of spectra of $\sim 1000$ galaxies with known HST morphologies. With a subset of these data, we have studied evolution of spheroidal galaxies at $z<1$, most of them very likely being E/SO's. Here, we show preliminary results of this study, which favor passive luminosity evolution and no or little number density evolution for E/SO's at $z<1$. This result is not compatible with some theoretical models based on the CDM dominated universe, where it is predicted that $50-70 \%$ of presentday $\mathrm{E} / \mathrm{S} 0$ s are formed at $z<1$.
\end{abstract}

\section{Introduction}

In hierarchical models of galaxy formation, E/S0s are formed via merger of galaxies with similar masses. The formation process requires massive $E / S 0$ s be formed relatively recently $(z<1-3)$ contrary to models which propose the formation via monolithic collapse of the proto gas clouds at high redshift (Larson 1975; Arimoto \& Yoshii 1987). Some semi-analytical models of galaxy evolution predict that more than $50 \%$ of present-day E/S0s are formed at $z<1$ when $\Omega_{m}=1$ (Baugh et al. 1996; Kauffmann et al. 1996). Here, we present the luminosity function of $170 \mathrm{E} / \mathrm{S} 0$ 's at $z<1$, constraining their luminosity and number density evolution. Final results will be presented in Im et al. (1999, in preparation).

\section{Sample Selection}

Spheroids are selected from the Groth-strip survey (Groth et al. 1995) data as well as publically available the HDF flanking field data, based upon their B/T (bulge to total light ratio) and asymmetric index $\left(A_{S}\right)$ within 2 half light radius $\left(r_{h l}\right)$ as defined in Schade et al. (1995). Therefore, we are selecting objects

\footnotetext{
${ }^{1}$ e-mail address: myung@̣ucolick.org

${ }^{2}$ For more information and current participants of DEEP; check website of DEEP at www.ucolick.org/deep/home.html.
} 
as "spheroids" if their light is centrally concentrated $(B / T>0.3)$, and their morphology is smooth $\left(A_{S} \leq 0.04-0.07\right)$. Tests on dimmed and shrunken local galaxy images from Frei et al. (1999) show that our sample selection criteria successfully picks up $\mathrm{E} / \mathrm{S} 0$ s (or $T \leq 0$ ), with almost no contamination from late-type galaxies.

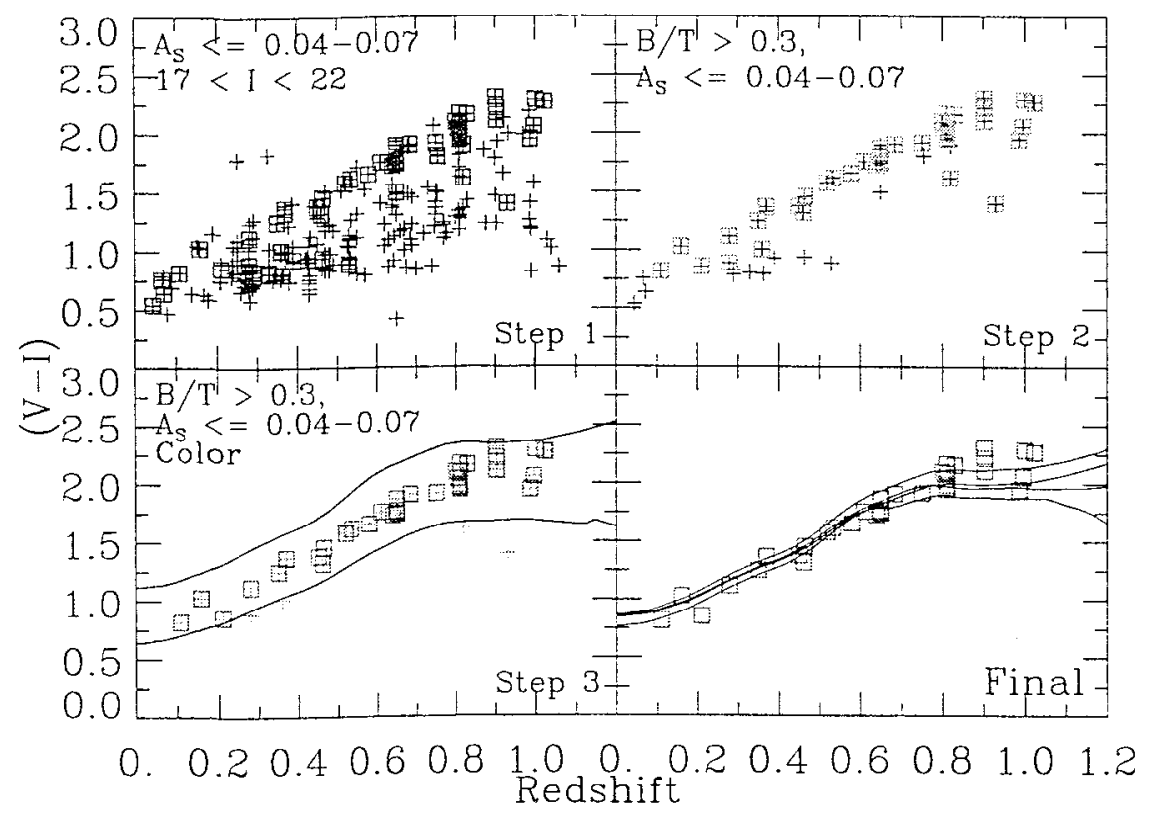

Figure 1. Step 1 through Step 4 show how spheroids are selected. Squares indicate objects selected in each step.

Fig. 1 shows how spheroids $(I<22)$ are selected from our spectroscopic sample. First, we apply an asymmetry cut to our sample (Step 1). With this the majority of blue objects are rejected. In Step 2, we apply a B/T cut with $B / T>0.3$. With this we exclude some blue-ish objects at moderate or low redshift, which are probably dwarf ellipticals (c.f., Im et al. 1995). Objects selected with steps 1 and 2 have a very tight correlation between (V-I) color and redshift, suggesting that the stellar population in these spheroids is old.

However, there are some blue spheroids which do not belong to the "red" family of spheroids. We exclude these "blue" spheroids from the sample by making an additional color cut as plotted in the step 3 panel, since we would like to study the property of passively "evolved" old spheroids. Our measurement of the velocity dispersion $(\sigma)$ shows all of these blue spheroids have $\sigma=40-$ $100 \mathrm{~km} / \mathrm{sec}$, suggesting that they are likely to be CNELG's or dwarf spheroids (Koo et al. 1995; Phillips et al. 1997; Guzman et al. 1997) rather than massive $\mathrm{E} / \mathrm{S} 0 \mathrm{~s}$. Note we find $\sigma=150-300 \mathrm{~km} / \mathrm{sec}$ for the "red" spheroids. A natural interpretation is that the "red" spheroids are progenitors of present-day E/SO's, 
while the blue spheroids are not, therefore exclusion of the blue spheroids can bè justified.

"After the color cut, we have a final sample of "red" spheroids (Final). In the "Final" panel, we also plot the color-redshift relation for a solar metalicity model with formation redshifts $z=1.1, z=5.5, z=3$, and $z=1.5$. Colors of the red spheroids suggest that the stellar populations of massive E/S0s are formed at $\tilde{z}_{\text {for }} \geq 1.5$.

\section{Luminosity function of $\mathrm{E} / \mathrm{SO}$ s at $z \sim 0.8$}
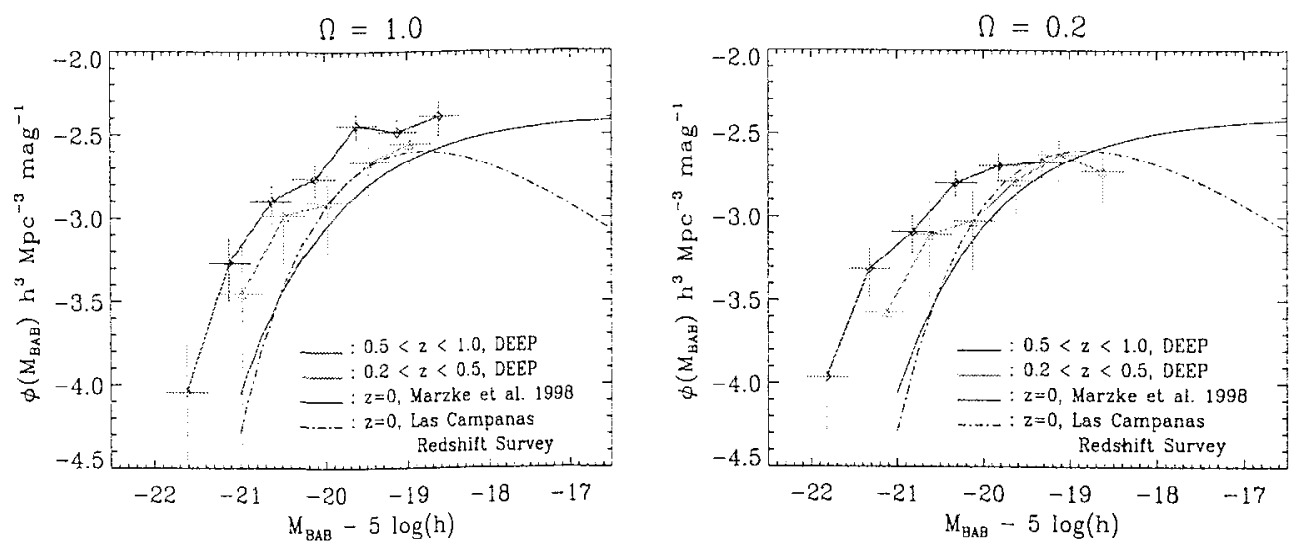

Figurc 2. LF of $170 \mathrm{E} / \mathrm{S} 0$ s at $z<1$. Note that we have used $\approx$ phot for about $60 \%$ of this sample.

In Fig.2, we plot the LF of our "red" spheroids at $0.5<z<1.0$ and at $0.2<z<0.5$. Also plotted are the local LF of E/S0s from Marzke et al. (1998), and the LF of early type galaxies from spectral classification (Bromely et al. 1998). The left figure shows the LF computed with the $\Omega_{m}=1$ and the right figure shows the $L F$ computed with the $\Omega_{m}=0.2$ and $\Lambda=0$. Magnitudes are in $B_{A B}$ for these figures, and $h=\frac{H I_{0}}{100 \mathrm{~km} / \mathrm{sec}}$. Note that faint end of the local early-type $L F$ is uncertain, but the bright end is robust. According to Fig. $2, L_{x}$ brightens by about $0.9-1.2 \mathrm{mag}$ in $\mathrm{B}$ from $z=0$ to $z \simeq 0.8$, consistent with the expected brightening from passive luminosity evolution models with formation redshift, $z>1-1.5$. This is consistent with the conclusion reached from the color of these objects as we showed in Fig.1. The 1.2 magnitude luminosity brightening at $z=0.8$ is also supported by studies of the fundamental plane of E's and the size-luminosity relation of bulges at $z \sim 0.8$ (Gebhardt et al. 1999 in prep.; Too et al. 1999 in prep.).

We also find that the number density of $\mathrm{E} / \mathrm{SO}$ s does not change significantly as a function of redshift. For $\Omega_{m}=1$. we find that the best-fit value of $\phi_{\star}$ of the LF at $0.5<z<1$ is about 1.1-1.4 times greater than $\phi_{x}$ at $z=0$. This excludes 
-strong number density evolution by as much as a factor of 2 from $z=0$ to 1 at high confidence, given that error on the measurement of $\phi_{\times}$is about $25 \%$. For an open nniverse the constraint on the number density is weaker but our data favor no or little number density evolution from $z=0$ to $z=1$, as found previously by $\operatorname{Im}$ et al. (1996).

\section{Conclusion}

- Spheroids selected with our quantitative criteria are mostly red. Sizes and $\sigma$ of these "red" spheroids are consistent with those of massive E/SOs $\left(L>0.4 L_{*}\right)$ at $z=0$. Small number of blue spheroids are found, but they have very small $\sigma$ values, therefore they are unlikely to be present-day E/SOs.

- Assuming that our "red" spheroids are present-day $\mathrm{E} / \mathrm{SO}$, we find the luminosity of $\mathrm{E} / \mathrm{SOS}$ dims by $1-1.3$ magnitudes from $z \sim 0.8$ to $z=0$.

- The number density of $E / S O s$ does not change significantly from $z=1$ to $z=0$. This is not consistent with strong number evolution as predicted by some semi-analytical models.

Acknowledgments. This research is supported by the NSF grant AT9529098. Wc are grateful to members of DEEP especially those at UC Santa Cruz for their support and contributions to this project.

\section{References}

Arimoto, N. \& Yoshii, Y. 1987, A \& A 173. 23

Baugh, C. M., Cole, S., \& Frenk, C. S. 1996, MNRAS, 283, 1361

Bromley, B. C. et al. 1998, ApJ, 505, 25

Frei, Z., et al. 1996, AJ, 111, 174

Groth, E., et al. 1994, BAAS, 26, 1403

Guzman, R., et al. 1997, ApJ, 489, 559

Im, M., Griffiths, R. E., Ratnatunga, K. U., \& Sarajedini, V. L., 1996, Ap.J, $461, \mathrm{~L} 79$

Im, M., Ratnatunga, K. U.. Griffiths, R. E., \& Casertano, S. 1995, Ap.J, 445, L15

Kauffmann, G., Charlot. S., \& White, S. D. M. 1996, MNRAS,

Koo, D. C., et al. 1995, ApJ, 440, L49

Larson, R. B. 1975, MNRAS, 173.671 283.117L

Varzke, R. O., et al. 1998, ApJ, 503, 617

Plillips, A. C., et al. 1997, ApJ. 489, 543

Schade. D. et al. 1995. ApJ, 451. L1 
The Hy Redshift Universe

ASP Conference Series, Vol. 193, 1999

A. J. Bunker \&s W. J. M. van Breugel, eds. $\checkmark$

\title{
$\because$ Determination of AGN Host Galaxy Ages
}

\author{
L. A. Nolan \\ Institute for Astronomy, University of Edinburgh, Blackford Hill, \\ Edinburgh, EH9 3RJ
}

\section{Introduction}

Understanding the host galaxies of active galactic nuclei (AGN) provides insight into the structure and evolution of active nuclei themselves, and the differences between the different classes of AGN.

This work estimates the ages of a sample of all three major types of luminous $A G N$ : radio loud quasars (RLQ), radio quiet quasars (RQQ) and radio galaxics (RG). Ages are estimated by comparing the off-nuclear spectra of the sample with evolving stellar population models. The models are composed of two single star burst stellar populations, a dominant older component and a much smaller popnlation which is young and blue. A further constraint on the ages is provided by simultaneous fitting of the $\mathrm{R}-\mathrm{K}$ colour, derived from HST and ground based imagery. The results indicate that the host galaxies of all three classes of $A G N$ investigated are best described by an evolved stellar population, of age $12-14$ Gyr.

\section{The Data and Model Spectra}

The optical $(\sim 3200 \AA$ to $\sim 9000 \AA)$ off-nuclear $(5$ arcsec) spectra of a sample of 24 powerful AGN (radio-quiet quasars, radio-loud quasars and radio galaxies) in the redshift range $0.1 \leq z \leq 0.3$ have been analyzed in an attempt to determine the age of the dominant stellar population of their host galaxies. The spectra were obtained with the Mayall 4-m and William Herschel 4.2-m telescopes, and typically consist of 2-3 hours of integration (Hughes et al, 1999). These data are complemented by $\mathrm{R}-\mathrm{K}$ colours for the host galaxies obtained from UKIRT and HST images (McLure et al, 1999), which define the basic shape of the host galaxy SED out to $\lambda \simeq 2 \mu m$. Full details of the AGN sample can be found in Dunlop et al (1993) and Taylor et al (1996).

The stellar population synthesis models used are the solar metallicity, instantaneons starburst models of Jimenez (1999) and Worthey (1994). However. at low redshift, more than one episode of star formation may have taken place. and the single starburst models may no longer be realistic, even for elliptical galaxies. Therefore, the populations fitted have two single starburst components, a dominant older population, and a much smaller young blue population. The young population adopted was the $0.1 \mathrm{Gyr}$ model of Jimenez. Further addition of an intermediate age component, $\simeq 1 \mathrm{Gyr}$, did not significantly improve the fit. 
The age of the older component, and the percentage, by mass, of the young population were parameters to be fitted. $\mathrm{R}-\mathrm{K}$ colour was fitted simultaneously with the age and composition, in order to constrain the red end of each SED. Emission lines were masked out of the fit across the spectra. A full description of the fitting process will be provided in Nolan et al (1999, in preparation).

\section{Results}
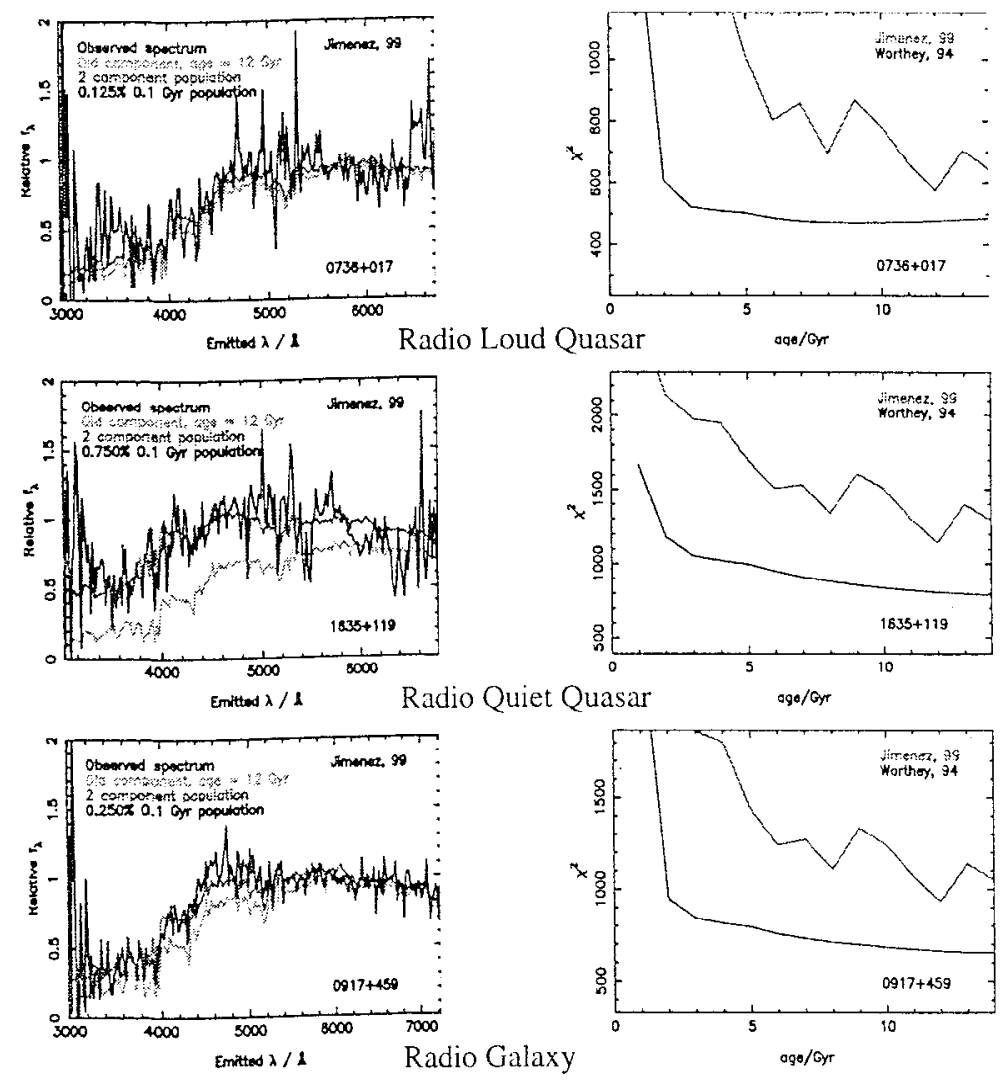

Figure 1. Model fits to the off-nuclear spectra, for an example of each AGN class, together with the corresponding $\chi^{2}$ plots. The improvement to the fit from adding a young $(0.1 \mathrm{Gyr})$ blue component can be clearly seen. There is good agreement between the best-fit ages derived using the two different models.

It can be clearly seen in Figure 1 that the addition of even a very small young stellar component reproduces the blue end of the observed spectra much more successfully than a single old population. However, at the same time the red end of the spectra combined with the $\mathrm{R}-\mathrm{K}$ colours demand an old component to describe the bulk of the underlying stars. Worthey's models become redder more rapidly than those of Jimenez, and consequently generally give a somewhat younger lower limit to the estimated ages. 
The peaks and troughs in the $\chi^{2}$ evolution of Jimenez' models are the result of real features of the population synthesis (Jimenez, private communication).

Table 1. Results from the simultaneous fitting to the AGN host sample of the two component model spectra (using the models of Jimenez, 1999 ) and $R-K$ colour. The percentage young population (percent young pop) is by mass.

\begin{tabular}{|c|c|c|c|c|c|}
\hline $\begin{array}{l}\text { IAU } \\
\text { name }\end{array}$ & $\begin{array}{l}\text { Best fit } \\
\text { age/Gyr }\end{array}$ & $\begin{array}{c}\text { Percent } \\
\text { young pop }\end{array}$ & $\bar{z}$ & $(R-K)_{f i t}$ & $(R-K)_{o b s}$ \\
\hline \multicolumn{6}{|c|}{ Radio Loud Quasars } \\
\hline $0137+012$ & 13 & 0.2 & 0.258 & 2.88 & 2.82 \\
\hline $0736+017$ & 12 & 0.7 & 0.191 & 2.89 & 3.16 \\
\hline $1004+130$ & 12 & 1.4 & 0.240 & 2.65 & 3.01 \\
\hline $1020-103$ & 5 & 0.6 & 0.197 & 2.33 & 2.29 \\
\hline $1217+023$ & 12 & 1.1 & 0.240 & 2.71 & 3.18 \\
\hline $2135-147$ & 12 & 1.6 & 0.200 & 2.50 & 2.49 \\
\hline $2141+175$ & 12 & 1.1 & 0.213 & 2.66 & 2.69 \\
\hline $2247+140$ & 8 & 0.2 & 0.237 & 2.77 & 2.81 \\
\hline $2349-014$ & 12 & 0.5 & 0.173 & 2.71 & 2.87 \\
\hline \multicolumn{6}{|c|}{ Radio Quiet Quasars } \\
\hline $0054+144$ & 8 & 1.3 & 0.171 & 2.32 & 3.12 \\
\hline $0157+001$ & 12 & 1.1 & 0.164 & 2.52 & 2.83 \\
\hline $0204+292$ & 14 & 0.2 & 0.109 & 2.47 & 2.49 \\
\hline $0244+194$ & 5 & 0.1 & 0.176 & 2.37 & 2.34 \\
\hline $0923+201$ & 12 & 0.6 & 0.190 & 2.72 & 3.24 \\
\hline $1549+203$ & 12 & 0.6 & 0.250 & 2.90 & 3.37 \\
\hline $1635+119$ & 12 & 0.7 & 0.146 & 2.57 & 3.27 \\
\hline $2215-037$ & 14 & 0.7 & 0.241 & 2.70 & 2.68 \\
\hline $2344+184$ & 12 & 0.9 & 0.138 & 2.51 & 2.57 \\
\hline \multicolumn{6}{|c|}{ Radio Galaxies } \\
\hline $0230-027$ & 1 & 0.0 & 0.230 & 2.02 & 2.08 \\
\hline $0345+337$ & 12 & 0.0 & 0.244 & 3.13 & 3.62 \\
\hline $0917+459$ & 12 & 0.2 & 0.174 & 2.79 & 3.42 \\
\hline $1215-033$ & 13 & 0.0 & 0.184 & 2.74 & 2.52 \\
\hline $1330+022$ & 8 & 0.5 & 0.215 & 2.70 & 2.79 \\
\hline $2141+279$ & 12 & 0.1 & 0.215 & 3.27 & 3.25 \\
\hline
\end{tabular}

The distribution of the estimated ages for the host galaxy sample is shown in Figure 2. All three classes of AGN have predominantly old (12-14 Gyr) stellar populations, in which the $4000 \AA$ spectral break is clearly evident. This agrees well with the results of McLure et al (1999, in preparation), who compare hostgalaxy morphologies, luminosities, scale lengths and colours in the same sample, and conclude that these are consistent with mature stellar populations.

There are a few objects with predicted ages of $<8$ Gyr. This could be because they are genuinely young populations, but equally, it could arise artificially because the spectra are contaminated with scattered nuclear light or because of the variable $\mathrm{S} / \mathrm{N}$ quality in the sample. It should also be noted that these objects have the bluest $R-K$ colours in the sample. It is possible that the 
K-band fluxes have been underestimated, and therefore an artificially young age is predicted. These possibilities will be fully discussed in Nolan et al (1999, in preparation).

It should be noted that, although best fit ages may be identified, the $\chi^{2}$ plots (as in Figure 1) suggest that these are not individually very robust. However, young ages are strongly disallowed, and the overall trend of these results strongly supports the conclusion that the host galaxies for all three classes of powerful AGN are massive ellipticals dominated by old stellar populations.

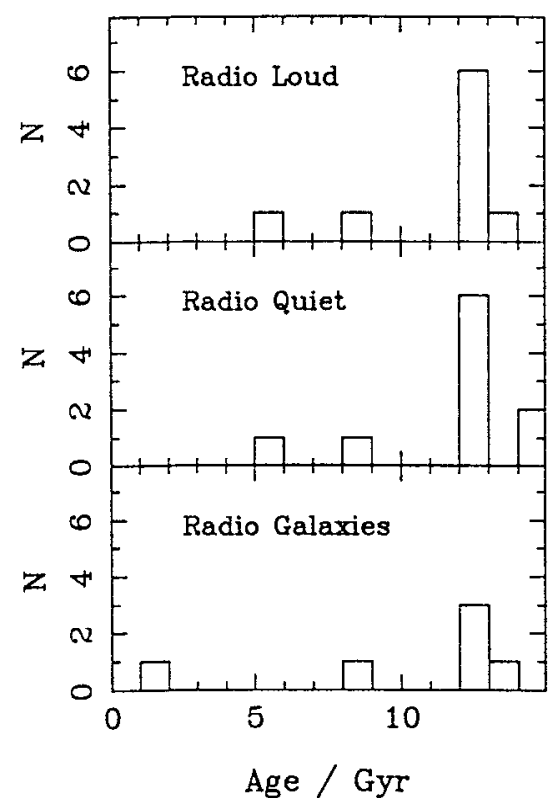

Figure 2. The age distribution of the dominant stellar populations of the sample host galaxies, from the fits of Jimenez' models. 'The populations are predominantly old $(12-14 \mathrm{Gyr})$.

\section{References}

Dunlop, J. S., Taylor, G. T., Hughes, D. H., \& Robson, E. I., 1993, MNRAS, 261,155

Hughes, D. H., Fukula, M.J., Dunlop, J.S., \& Boroson, T., 1999, in preparation

Jimenez, R., Dunlop, J. S., Peacock, J. A., Padoan, P., MacDonald, J., \& Jørgensen, U. G., 1999, submitted to MNRAS

Mclure, R. J.. Fukula, M.J., Dunlop, J. S., Baum, S. A., O'Dea, C. P., \& Hughes. D. H., 1999, in press, astro-ph/9809030

Tolan. L. A.. Dunlop. J.S., \& Kukula, M.J., 1999, in preparation

Taylor, G. T., Dunlop, J. S., Hughes, D. H., \& Robson, E. I., 1996, MNRAS, 283. 930

Worthey, G., 1994, Ap.JS, 95. 107

Worthey, G., Faber. S. M., \& Gonzalez, J. J., 1992 Ap.J, 418, 947 
The Hy Redshift Universe

ASP Conference Series, Vol. 193, 1999

A. J. Bunker \& W. J. M. van Breugel, eds.

\title{
CO Observations and the Tully-Fisher Relation at High Redshift
}

\author{
Y. Tutui ${ }^{1}, Y$. Sofue ${ }^{1}$ \\ ${ }^{1}$ Inst. of Astronomy, Univ. of Tokyo, Mitaka, Tokyo 181-8588, Japan \\ M. Honma ${ }^{2}$, T. Ichikawa ${ }^{3}$, K. Wakamatsu ${ }^{4}$, I. Kazes ${ }^{5}$ and J. Dickey ${ }^{6}$ \\ ${ }^{2}$ NAOJ, ${ }^{3}$ Tohoku Univ, ${ }^{4}$ Gifu Univ, ${ }^{5}$ Obs. Paris, ${ }^{6}$ Univ. Minnesota
}

\begin{abstract}
We performed deep CO-line observations of spiral galaxies at intermediate redshifts using the NRO $45-\mathrm{m}$ telescope and obtained the $\mathrm{CO}$ linewidths of 16 luminous IR galaxies at redshifts $c z=10,000$ - $50,000 \mathrm{~km} / \mathrm{s}$. The observed galaxies were selected by high FIR flux density and optically normal morphology to investigate the CO TullyFisher relation. When compared to other $\mathrm{CO}$ observations of intermediate redshift galaxies we find that our observations are more sensitive and reach larger dust-to-gas mass ratios.
\end{abstract}

\section{Introduction}

Previous CO observations of luminous IR galaxies at intermediate redshift ( $c z \sim$ $10,000-50,000 \mathrm{~km} / \mathrm{s}$ ) have focussed mainly on galaxies which are irregular or are merging (e.g. Mirabel et al.(1990), Sanders et al.(1991), Solomon et al.(1997), Lavezzi \& Diclicy (1998)), with the exception of the sample studied by Lavezzi $\&$ Dickey(1998). Here we present CO observations of such galaxies which have normal morphologics using long integrations with the NRO $45-\mathrm{m}$ telescope and obtained CO linewidths of 16 galaxies up to $c z \sim 50,000 \mathrm{~km} / \mathrm{s}$ (Sofue et al. 1996, Tutui ct al. 1999)

\section{CO Observations}

The $C O$ luminosities and the redshifts $c z$ of our sample are plotted in Fig. 1. Our observations are more sensitive than other surveys and are of normal galaxies. Comparisons of molecular gas masses derived from the $\mathrm{CO}$ intensities and dust masses derived from IRAS fluxes show that the galaxies in our sample have larger dust-to-gas mass ratios (see Tutui et al. 1999 for details).

We also observed one of the CO-detected galaxies in our sample (IRAS $02185+0642, c z \sim 29,000 \mathrm{~km} / \mathrm{s}$ ) using the Nobeyama Millimeter Array (NMA). The velocity field shows that the rotation is not disturbed. It supports the idea that our sample may be used to investigate the CO Tully-Fisher relation. 


\section{The CO Tully-Fisher Relation}

One of our goals is to use our CO data together with the CO Tully-Fisher relation to measure the distances to galaxies and estimate the Hubble constant. We obtained photometric observations of our sample with the CFHT 3.6-m telescope and the Okayama Astrophysical Observatory (OAO) 1.88-m telescope. From these we estimated the individual Hubble constants, or Hubble ratios, for each galaxy (Sofue et al. 1996, Tutui et al. 1999).

\section{Discussion}

Our sample contains the largest number of faint $\mathrm{CO}$-galaxies of normal type which are at intermediate redshifts. We compared their $\mathrm{CO}$ gas and dust masses, and found that our galaxies are dustier, or perhaps less luminous in $\mathrm{CO}$ than those of the other surveys. Further observations of our sample with an interferometer might reveal the $\mathrm{CO} /$ dust mass relation.

Fig. 1: Redshift $c z$ vs CO luminosities for intermediate redshift galaxies. Filled squares are our sample. The dotted line means iso-flux density. Our observations are more sensitive than other surveys.

\section{References}

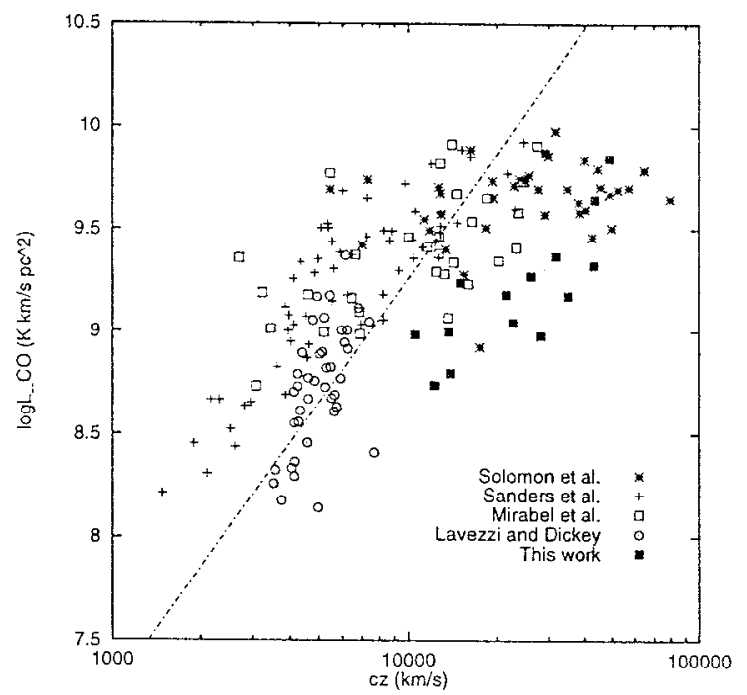

Lavezzi \& Dickey, 1998, AJ 116, 2672

Mirabel et al., 1990, A\&A 236, 327

Sanders et al., 1991, ApJ 370, 158

Solomon et al., 1997, ApJ 478, 144

Sofue et al., 1996, PASJ 48, 657

Tutui \& Sofue, 1999, A\&A in press

Tutui et al., 1999, submitted to PASJ 
The Hy Redshift Universe

ASP Conference Series, Vol. 193, 1999

A. J. Bunker \& W. J. M. van Breugel, eds.

\title{
. The Low-Mass Stellar IMF at High Redshift: Faint Stars in the Ursa Minor Dwarf Spheroidal Galaxy ${ }^{1}$
}

\author{
Rosemary F.G. Wyse ${ }^{2}$, Gerard Gilmore ${ }^{3}$, Sofia Feltzing ${ }^{4}$ and Mark \\ Houdashelt ${ }^{2}$
}

\section{Introduction}

Low-mass stars, those with main-sequence lifetimes that are of order the age of the Universe, provide unique constraints on the Initial Mass Function (IMF) when they formed. Star counts in systems with simple star-formation histories are particularly straightforward to interpret, and those in 'old' systems allow one to determine the low-mass stellar IMF at large look-back times and thus at high redshift. We present the faint stellar luminosity function in an external galaxy, the Ursa Minor dwarf Spheroidal (dSph). This relatively-nearby (distance $\sim$ $70 \mathrm{kpc}$ ) companion galaxy to the Milky Way has a stellar population with narrow distributions of age and of metallicity (e.g. Hernandez, Gilmore \& Valls-Gabaud 1999), remarkably similar to that of a classical halo globular cluster such as M92 or M15, i.e. old and metal-poor $([\mathrm{Fe} / \mathrm{H}] \sim-2.2 \mathrm{dex})$. The integrated luminosity of the Ursa Minor dSph $\left(L_{V} \sim 3 \times 10^{5} L_{\odot}\right)$ is also similar to that of a globular cluster. However, the central surface brightness of the Ursa Minor dSph is only $25.5 \mathrm{~V}-\mathrm{mag} / \mathrm{sq}$ arcsec, corresponding to a central luminosity density of only $0.006 L_{\odot \mathrm{pc}} \mathrm{p}^{-3}$, many orders of magnitude lower than that of a typical globular cluster. Further, again in contrast to globular clusters, its internal dynamics are dominated by dark matter, with $(M / L)_{V} \sim 80$, based on the relatively high value of its internal stellar velocity dispersion (Hargreaves et al. 1994; see review of Mateo 1998). Faint star counts in the Ursa Minor dSph thus allow determination of the low-mass IMF in a dark-matter-dominated external galaxy, in which the stars formed at high redshift.

\section{Observations}

We obtained deep imaging data with the Hubble Space Telescope, using WFPC2 (V-606 \& I-814). STIS (LP optical filter) and NICMOS (H-band), in a field close to the center of the Ursa Minor dSph (program GO 7419: PI Wyse, CoIs Gilmore, Tanvir, Gallagher \& Smecker-Hane; due to successive failures of HST the data acquisition phase of this project remains ongoing). The estima-

\footnotetext{
${ }^{1}$ Based on observations with the NASA/ESA Hubble Space Telescope, obtained at STScI, operated by AURA Inc. under NASA contract NAS5-26555

${ }^{2}$ Physics \& Astronomy Department, Johns Hopkins University, Baltimore, MD 21218

${ }^{3}$ Institute of Astronomy, Madingley Road, Cambridge CB3 OHA, UK

${ }^{4}$ Lund Observatory, Box 43, 22100 Lund, Sweden
} 
tion of the contamination by foreground stars and background galaxies required acquisition of similarly-exposed data for an offset field $\sim 2$ tidal radii away from the Ursa Minor dSph, at similar Galactic coordinates to the UMi field $\left(\ell=105^{\circ}, b=45^{\circ}\right)$; this field shows no evidence for Ursa Minor member stars.

\subsection{WFPC2 Data for the Ursa Minor dSph}

The WFPC2 data are discussed in more detail in Feltzing el al. (1999). They consist of $8 \times 1200$ s in each of F606W and F814W filters. Standard HST data reduction techniques were followed using the IRAF STSDAS roulines, with photometry on the reduced images using DAOPHOT and TinyTim psf's. The scatter in the zero points and photometric calibrations is $\sim 6 \%$, which is small compared to the 0.5 magnitude binning we adopt for the luminosity functions below. Identical procedures were applied to both the UMi and the offset-field datasets. The completeness of the data was determined by adding artificial stars to the original images and then re-processing them. The luninusity functions discussed below include only stars detected in both $\mathrm{V}$ and $\mathrm{I}$.

\subsection{STIS LP data for the Ursa Minor dSph \& M15}

We obtained seven 2900s exposures of each of the Ursa Minor field and the offset field. We also obtained a single exposure (1200s) of the globular cluster M15 in a field with extant deep WFPC2 V and I data (Piotto, Cool \& King 1997). The metallicity and age of the stars in M15 are very similar to those in the Ursa Minor dSph and the STIS LP magnitudes for the Ursa Minor dSph may be transformed to I-814 using the extant M15 I-814 data (courtesy of G. Piotto). Standard data reduction procedures as above were applied to our data.

\section{Faint Luminosity Functions}

\subsection{Globular Clusters}

We utilise comparisons between the data for the Ursa Minor dSph and globular clusters of the same metallicity and age (M92 and M15). A direct comparison between the luminosity functions corresponds to a comparison between the underlying stellar mass functions. However, the stellar mass function in globular clusters may be modified by both internal and external effects and thus in not all cases is the present-day mass function a good estimate of the initial mass function. The Piotto, Cool \& King (1997) data were obtained at intermediate radius within M15 and M92, where the effects of mass segregation are expected to be small and the local faint luminosity function should be a good estimate of the global faint luminosity function. Further, as discussed by Piotto \& 'Zoccali (1999), M92 and M15 have fairly steep main-sequence luminosity functions, while other globular clusters, particularly those for which external dynamical effects such as tidal shocking by the disk or bulge of the Milky Way may be important, have flatter faint luminosity functions (these differences occur fainter than the limits of the Ursa Minor dSph data). Thus we will assume that the present-day faint luminosity. functions of M92 and M15 may be taken as reasonable estimates of the initial functions. 

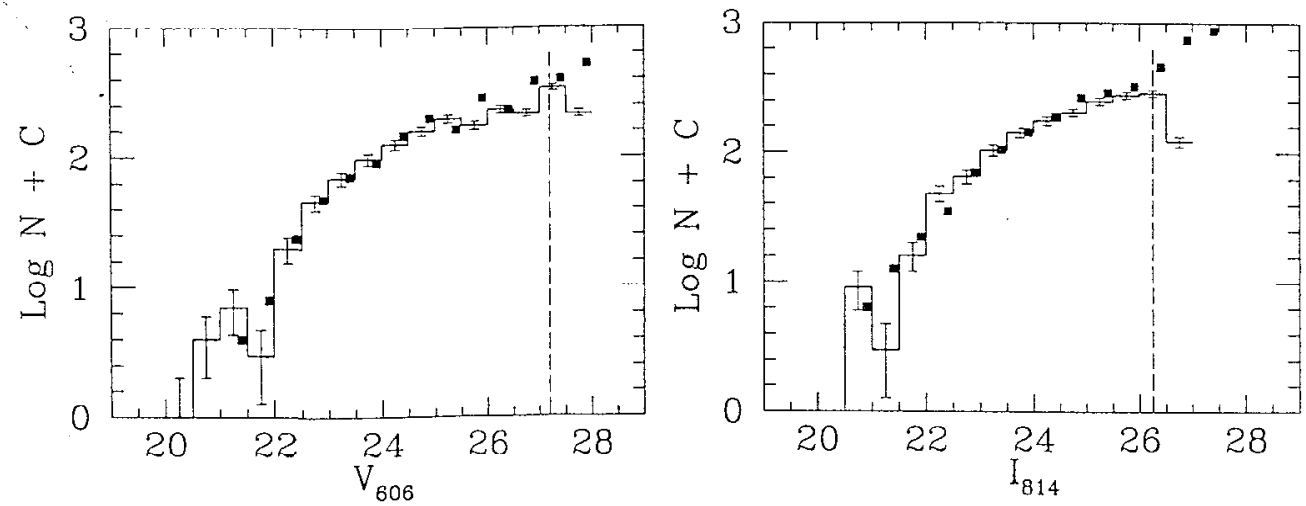

Figure 1. Comparison between the completeness-corrected Ursa Minor luminosity functions (histograms; $50 \%$ completeness indicated by the vertical dashed line) in the V-band (left panel) and the I-band (right panel) and the same for M92 (points; from Piotto et al. 1997).

\subsection{Ursa Minor Faint Luminosity Function}

The derived, completeness-corrected, Color-Magnitude Diagram-based, WFPC2 $V$-band and I-band luminosity functions for the Ursa Minor dSph are compared in Figure 1 to those for the globular cluster M92 (courtesy of G. Piotto). We find a remarkable similarity between them, down to our $50 \%$ completeness limit, which corresponds to $\sim 0.4 M_{\odot}$ (Baraffe et al. 1997 models).

A direct comparison between our STIS optical luminosity functions for U rsa Minor and for the globular cluster M15 is shown in Figure 2 (left panel; only those bins at least $50 \%$ complete are shown). Again, there is very good agreement in slopes of luminosity functions, and again our $50 \%$ completeness limit for the Ursa Minor data is around $0.4 M_{\odot}$.

\subsection{Mass Functions}

The luminosity function corresponding to a mass function of slope -1.35 (where the Salpeter slope is -2.35) may be calculated using the Baraffe et al. (1997) models. This provides a reasonable fit to the data for M[92 and for M15 (Piotto \& Zoccali 1999). Further, Zoccali et al. (1999) found this mass function to fit their data for the faint stellar luminosity function of the bulge of the Milky Way in Baade's window. Figure 2 shows our WFPC2 I-814 luminosity function for Ursa Minor, together with that derived from our transformed STIS LP data and the predictions from the Baraffe et al. models. This mass function appears to be an excellent description of the underlying IMF in the Uirsa Minor dSph too.

\section{Conclusions}

As described here, the main sequence stellar luminosity function of the Ursa Winor dSph, and implied IMF, down to $\sim 0.4 \mathrm{M} \mathrm{F}_{\odot}$, is indistinguishable from that of the halo globular clusters M92 and M15, systems with the same old age and low metallicity as the stars in the Ursa Minor dSph. However, the 
Wyse et al.
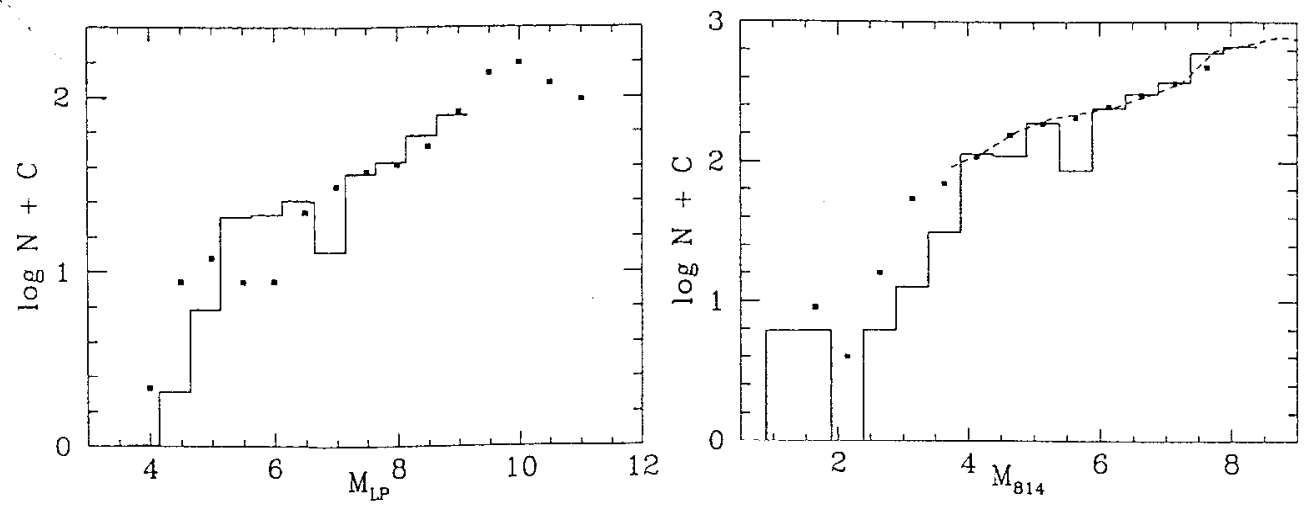

Figure 2. Left panel: comparison between the completenesscorrected STIS LP luminosity functions for the Ursa Minor dSph (solid line) and the globular cluster M15 (points). Right panel: comparison between the STIS-based I-band luminosity function of the Ursa Minor dSph (solid line), the observed I-band luminosity function (points), and the luminosity function corresponding to a power-law mass function with slope -1.35 (dashed line).

globular clusters show no evidence for dark matter, while the Ursa Minor dSph is apparently very dark-matter-dominated. Thus the low mass stellar IMF for stars that formed at high redshift is invariant in going from a low-surface-brightness, dark-matter-dominated external galaxy, to a globular cluster within the Milky Way. This luminosity function, and underlying IMF, is in good agreement with those derived for the field stars of the Milky Way bulge and is consistent with the field halo and disk (e.g. von Hippel et al. 1996), supporting the concept of a universal IMF.

Acknowledgments. RFGW thanks the Center for Particle Astrophysics, UC Berkeley, for hospitality. Support for this work was provided by NASA grant number GO-7419 from STScI, operated by AURA Inc, under NASA contract NAS5-26555. We thank J. Gallagher, N. Tanvir \& T. Smecker-Hane for discussions.

\section{References}

Baraffe, I., Chabrier, G., Allard, F. \& Hauschildt, P. 1997, A\&A, 327, 1054

Feltzing, S., Gilmore, G. \& Wyse, R.F.G. 1999, Ap.J, 516, L17

Hargreaves, J., Gilmore, G., Irwin, M. \& Carter, D. 1994, MNRAS, 271, 693

Hernandez, X., Gilmore, G. \& Valls-Gabaud. D. 1999. MNRAS. in press

Mateo, M. 1998, ARAA, 36, 435

Tiotto, G. \& Zoccali, M. 1999, A\&A, 345, 845

Piotto, G., Cool, A. \& King, I.R. 1997, AJ, 115, 1888

von Hippel, T., Gilmore, G., Jones, D. \& Tanvir, N. 1996, AJ, 112, 192

Zoccali. M.. Cassisi, S., Frogel. J., Gould, A. et al. 1999, preprint. 


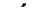


The Hy Redshift Universe

ASP Conference Series, Vol. 193, 1999

A. J. Bunker \& W. J. M. van Breugel, eds.

\title{
. On the Age Estimation of LBDS 53W091
}

\author{
Sukyoung $\mathrm{Yi}$ \\ CSA, Yonsei University, Korea; Caltech, CA; yi@srl.caltech.edu \\ T. Brown, S. Heap, I. Hubcny, W. Landsman, T. Lanz and A. Sweigart \\ NASA Goddard Space Flight Center, Greenbelt, MD 20771
}

Abstract. We present a summary of our recent paper (Yi et al. 1999).

The recent spectral analysis of LBDS 53W091 by Spinrad et al. (1997) has suggested that this red galaxy at $z=1.552$ is at least 3.5 Gyr old. This imposes an important constraint on cosmology, suggesting that it formed at $z>6.5$, assuming recent estimates of cosmological parameters. While their analysis was heavily focused on the use of some UV spectral breaks as age indicators, we have performed $\chi^{2}$ tests to the continuum of this galaxy using its UV spectrum and photometric data $(R, J, H, \& K: 2000-9000 \AA$ in rest-frame). We have used the updated Yi models ( $Y i$ et al. 1997) that are based on the Yale tracks. We find it extremely difficult to reproduce such large age estimates, under the assumption of the most probable input parameters. Using the same configuration as in Spinrad et al. (solar abundance models), our analysis suggests an age of approximately $1.4-1.8 \mathrm{Gyr}$, which is in good agreement with those of Bruzual \& Magris (1997) and of Heap et al. (1998).

The large difference in age estimate from Spinrad et al. and from this study is mainly due to the significant difference in the model integrated spectrum. Figure 1 shows the comparison between the latest Jimenez models (downloaded from Jimenez' ftp site in Nay, 1999), the preferred models in the analysis of Spinrad et al., and the Yi models, both for the solar composition. The Jimenez models shown here are the more recent ones than those used in Spinrad et al. At the time of this study, only his new models were made available to us.

In Figure 1, it is apparent that the new Jimenez ("J99") models are much bluer than the $\mathrm{Yi}$ models. Each model is denoted by the age (Gyr) and source (J: Jimenez, Y: Yi). One can easily understand why Spinrad et al. and we are achieving such different age estimates. An open diamond is an approximate relative flux of LBDS $53 \mathrm{~W} 091$ normalized at $3150 \AA$. This relative flux of LBDS 53 W091 is closely reproduced by the $1.4_{-0.1}^{+0.1}$ Gyr model when Yi's models are used or the $1.9_{-0.1}^{+0.2}$ Gyr models when the new Jimenez models are used (based on the reduced- $\chi^{2}$ tests).

Also plotted are the relative fluxes from the earlier version Jimenez ("J97") models (horizontal dashed lines) used in Spinrad et al. (1997), read off from the Figure 14 of Spinrad et al. (1997). The three dashed lines are from the top his 1. 3, and 5 Gyr models, respectively. Note that the I07 models are substantially bluer than the J99 models, which are already bluer than the Yi models. One can 

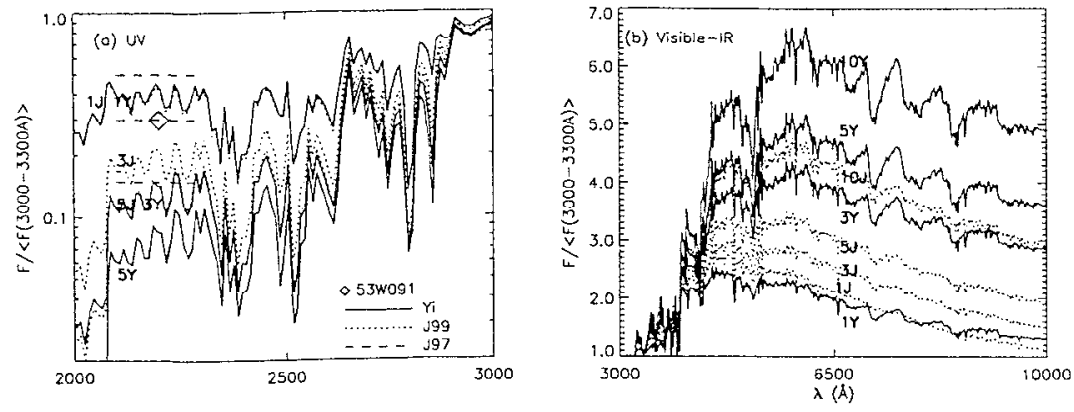

Figure 1. Comparison between the Jimenez (1999) and the Yi models.
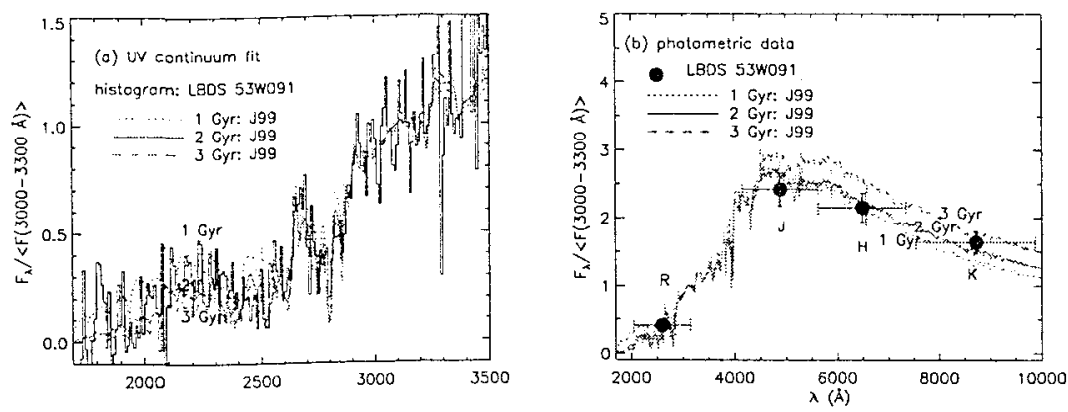

Figure 2. The new Jimenez models match the data at $1.9 \mathrm{Gyr}$ !

see that the J97 models match the LBDS 53W091 data approximately at $3 \mathrm{Gyr}$, as Spinrad et al. (1997) suggested. The Yi models are in reasonable agreement with the 1999 version Bruzual \& Charlot ("BC") models. If the new Jimenez models are the improved ones over his previous version used by Spinrad et al., all three groups now suggest rather consistent age estimates, between 1 and 2 Gyr. The fit to the UV spectrum using the new Jimenez models, which suggests an age estimate of $1.9 \mathrm{Gyr}$, is shown in Figure 2. Readers are encouraged to compare Figure 2 to Figure 14 of Spinrad et al. (1997).

Figure 1 also shows that, in the visible - IR, the difference between the J99 models and the Yi models is even larger, which contributed to the difference in the photometry-based age estimates of Spinrad et al. and Yi et al. This difference, however, is not the only cause for Spinrad et al. to get a larger age estimate, i.e., 2.5 Gyr, than ours. It was also caused by the fact that they used only $R-F$, omitting $J$ and $H$ magnitudes, in their analysis. Figure 2 shows that even the Jimenez models would have suggested a substantially smaller age if the whole photometric data had been used. When we use the new Jimenez models and the reduced $-\chi^{2}$ test on all of the four photometric data points, the best model indicates $1.9_{-0.7}^{+0.5} \mathrm{Gyr}$, which is in good agreement with the estimate from the UV analysis. Then again, all three groups that have performed continuum fits are suggesting consistent age estimates of $1-2$ Gyr (c.f., $1.5 \mathrm{Gyr}$ from $\mathrm{Yi}$ et al. 1.4 Gyr from Bruzual \& Magris). Such an agreement, at least on the age of 


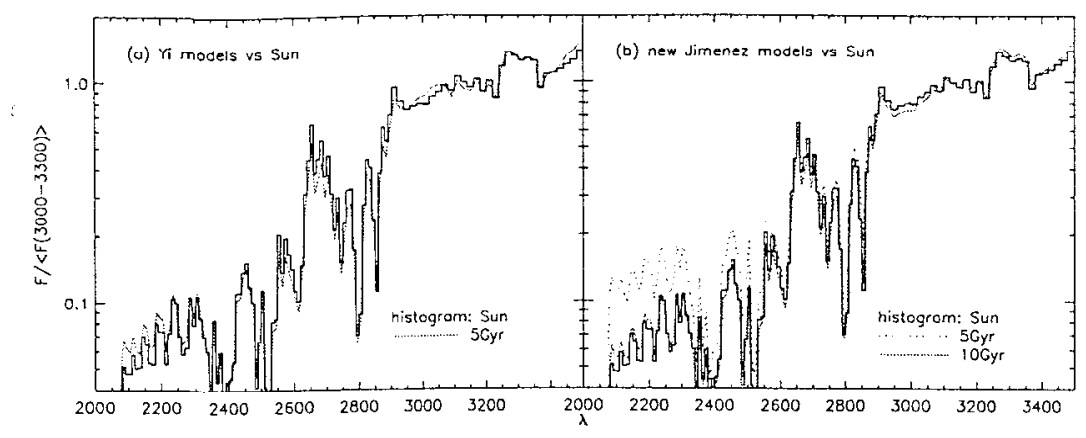

Figure 3. The solar UV spectrum is matched by the Yi models at its accepted age.

LBDS 53W091, is possible because, for small ages ( $<2 \mathrm{Gyr}$ ), the new Jimenez models differ from the $\mathrm{Yi}$ and the $\mathrm{BC}$ models only slightly.

One may then say that the age discrepancy on LBDS 53W091 has been resolved. Despite this apparent resolution, it is still quite disturbing to know that there is a significant disagreement between the Jimenez models and the $\mathrm{Yi}$ (and the BC) models at larger ages. It is extremely difficult to directly compare different population models and find a more realistic model, but it should be viable to test models by matching the observational properties of the objects whose ages are known a priori. Good examples include the sun, M32, and Galactic globular clusters. A sample test on the sun is shown in Figure 3. In a solar-age (4.5 - 4.7 Gyr), solar abundance population, most of the UV light is still produced by MS stars. Thus, such a population should exhibit a UV spectrum similar to that of the sun. Figure 3 shows the fits to the theoretical solar spectrum (2000 - 3500 $\AA$ ) from the Kurucz library using the Yi models and the new Jimenez models. The best fitting model is a $5.0 \pm 0.1 \mathrm{Gyr}$ model when the Yi models are used. This slight disagreement in the age estimate from the generally accepted solar age is perfectly expected, because we are matching a single stellar spectrum with those of composite (containing MS, red giants, and etc.) stellar populations. The proximity of this age estimate to the accepted age of the sun once again demonstrates the high reliability of the UV-based age estimates for intermediate-age, composite populations. However, when the new Jimenez models are used, much larger ages are indicated (10 Gyr giving the best fit). This was already evident in Figure 1, where the Jimenez models appeared much bluer than the $\mathrm{Yi}$ models. If the continua of G-type stars in the Kurucz library are significantly inaccurate, an effort to achieve the right age of the sun using population synthesis models and a theoretical spectrum would not be appropriate. Yet, such tests would still serve as sanity checks for the population synthesis computations. Similar tests can be performed on different objects whose ages are independently known.

We have further improved our estimates over conventional ones by adopting convective core overshoot (OS) and realistic metallicity mixtures. I'he inclusion of OS has little effect on the UV-based age estimates, but it raises the age estimates based on the visible data normalized to the UV by $20-50 \%$. Adopting realistic metallicity distributions is also important because different metallicity 
-groups dominate different parts of the integrated spectrum. If we assume that the majority of stars in LBDS 53W091 are already as metal-rich as those in nearby giant elliptical galaxies, the photometric data of LBDS 53W091 indicate up to a factor of two larger ages when metallicity mixtures are adopted.

When we use Yi's chemically composite models with OS included, the photometric data of LBDS 53 W091 indicate $1.5 \pm 0.2 \mathrm{Gyr}$, while the UV data suggest $1.9 \pm 0.2$ Gyr. The slightly larger estimates from the UV continuum fit would be consistent with this photometry-based one if we include a small amount of reddening and/or if the core of this galaxy is somewhat older or more metal-rich than its outskirt, all of which are quite plausible. It may also indicate that there is no substantial age spread among the stars in LBDS 53W091.

There is no doubt that precise age estimates of high-z galaxies would be very useful for constraining cosmology. In order to fully take advantage of the power of this technique, however, we first need to understand the details of the population synthesis, which are currently creating a substantial disagreement in age estimate. We propose to carry out a comprehensive investigation on the various population synthesis models through a series of standard tests on the objects whose ages have been independently determined. Such objects may include the sun, M32, and Galactic globular clusters. Our models (the Yi models) currently pass these tests reasonably.

Our age estimates indicate that LBDS 53W091 formed approximately at $z=2-3$. However, our smaller age estimate for this one galaxy does not contradict work that suggests galaxies generally formed at high redshifts, regardless of the rarity of massive ellipticals at $z \approx 1.5$. Furthermore, we are just beginning to expand our observations of galaxies to high redshift, and so the existence of a few old galaxies at high redshifts does yet prove any galaxy formation scenario, although it can potentially constrain cosmological parameters (in the sense that the ages of a few objects can provide lower limits on the age of the Universe at that redshift). Finding no old galaxies at high redshift would support a low $z_{f}$ for the general population. Building a larger database of observations is therefore crucial to achieve a unique and statistically significant solution.

Acknowledgments. We thank the team of the Spinrad et al. (1997) paper for providing the observed data and useful discussion. We owe special thanks to Raul Jimenez and Gustavo Bruzual for making their models available to us. This worli was supportcd by the Creative Research Initiative Program of the Korean Ministry of Science \& Technology grant (S.Y.).

\section{References}

Bruzual, G. A., \& Magris, G. C. 1997, in The Ultraviolet Universe at Low and High Redshift, AIP vol. 408 (AIP: Woodbury), 291

Heap. S. R. et al. 1998, Ap., 492, L131

Spinrad, H., Dey, A., Stern, D., Dunlop, J., Peacock, J., Jimenez, R., \& Windhorst, R. 1997, ApJ, 484, 581

Yi, S., Demarque, P., \& Oemler, A. Jr. 1997b, ApJ, 486, 201

Yi, S., Brown, T.M., Heap, S., Hubeny, I., Landsman, W., Lanz, T, \& Sweigart, A. 1999, ApJ, accepted 
Section C. Deep Field Surveys 

The Hy Redshift Universe

ASP Conference Series, Vol. 193, 1999

A. J. Bunker \& $W$. J. M. van Breugel, eds.

$\because$

\title{
The Evolution of Galaxies: A View from Toronto
}

\author{
Simon Lilly \\ University of Toronto, Toronto, Ontario, Canada
}

\begin{abstract}
I review recent progress in two areas concerned with the observational study of the formation and evolution of galaxies. Despite there still being considerable uncertainties regarding some of the most basic observational questions, I argue that changes in the galaxy population to $z \sim 1$ appear to be primarily caused by galaxies with the sizes, colours, morphologies and kinematics of irregular galaxies and not to the formation or dramatic evolution of massive galaxies which likely occurs at earlier epochs. Looking at higher redshifts, it is attractive to associate the new population of ultraluminous infrared galaxies found at high redshifts in deep sub-mm surveys with the formation of a substantial fraction of the present-day stellar population of the Universe, and in particular with the formation of the metal-rich spheroids. I conclude my suggesting that most of the available data can be incorporated into a general picture of the formation of galaxies that is testable in the near future.
\end{abstract}

\section{Introduction}

The attendees at this remarkable conference have been privileged participants in a remarkable flowering of our observational understanding of the Universe at cosmologically significant redshifts that has occurred over the last 15 years, (with much of it occurring over the last 5 years). Indeed we now have a body of data on the Universe at earlier epochs that would have been almost unimaginable 10 years ago. Nevertheless, there are still major uncertainties surrounding even the most basic observational quantities and I believe we are far from having a complete observational picture. So, we can look forward to many more years of progress driven by the remarkable suite of instruments coming on line over the next decade.

In the spirit of the slightly unusual title assigned by the organizers, I wish to start by reviewing some outstanding issues in two areas of this enterprise that I have been most closely involved with over the last five years: the characterization of the evolving field galaxy population over the interval $0.1<z<1$ and the opening up of the far-infrared/sub-millimeter waveband and the identification of a major new class of highly luminous highly obscured galaxies at high redshifts. This will represent my rather personal perspective. I will then describe what I think is a reasonable "working model" that brings these and the many other diverse phenomena that have been observed by others together. This part of the paper is necessarily considerably more speculative. 


\section{Evolution in the field galaxy population to $z \sim 1$}

\subsection{What are different types of optically-selected galaxies doing to $z \sim 1$ ?}

I think there is now no doubt that significant changes have occurred in the galaxy population since $z \sim 1$ (see e.g. Lilly et al 1995, Heyl et al 1997). Broadly speaking, these may be characterized as an increase at high redshifts in the comoving number density of blue galaxies with moderate luminosities, $M_{A B}(B) \sim-21$. In essence, the broad colour-luminosity relation which is seen at low redshifts, whereby the most luminous galaxies in $B$ are generally quite red and the bluest galaxies are generally of low luminosity, is eradicated by $z \sim 1$. This change could in principle be due to either a general brightening of the blue galaxy population (seen at low luminosities at the present-day) or to present-day luminous galaxies (which are generally red) being bluer (i.e. more vigorously forming stars) at earlier times.

Morphologies The advent of HST imaging has made it possible to add morphological and size information to the basic colour and line strength information available from ground-based redshift-survey data. Marrying the morphological studies pioneered on the MDS and later the HDF (e.g. Abraham et al 1996) to deep redshift surveys such as the CFRS has shown that galaxies with irregular and peculiar morphologies and lying at high redshifts dominate the evolution of the galaxy population to $z \sim 1$ (Brinchmann et al 1998). In contrast, the redshift distributions of galaxies classified as spirals and ellipticals are consistent with modest luminosity evolution.

Quantitative analysis of the distribution functions with redshift bears this out. In Lilly et al (1998), we claimed on the basis of some 300 objects in the HST/CFRS sample that the size function of disk scale lengths showed little change to $z \sim 0.8$, and that changes in the bi-variate size-luminosity function were dominated by the appearance of luminous galaxies with half light radii below $5 h_{50}^{-1} \mathrm{kpc}$. However, interpretation of these distribution functions is open to considerable ambiguity. For instance, a constant disk scale length size distribution function could be consistent with a growth in disk scale length with epoch if some disks were also being destroyed through merging events (see e.g. Mao, Mo and White 1998), and the crucial importance of selection effects must always be appreciated - these can enter either directly or through the application of sample selection criteria that appear at first sight to be unrelated.

Disk surface brightnesses There is a lingering uncertainty over how much surface brightness evolution has occurred in the disks of normal spiral galaxies. Lilly et al (1998) extended the earlier analysis of Schade et al (1995), and deduced that the surface brightnesses of large disks were higher at $z \sim 0 . \bar{T}$, by $0.8 \pm 0.3$ mag. We looked quite carefully at the effects of surface brightness selection cuts arising from the original apparent magnitude cut of the CFRS and concluded that $0.3 \mathrm{mag}$ of this effect could arise from this source. Our approach was to examine the effect of the selection function on local samples and on program galaxies at intermediate redshift and to point out that the numbers of large disks appeared to be roughly constant to $z \sim 0.8$ suggesting that not too many had dropped below the selection criteria. More recently. Simard et al (1999) have 
analysed galaxies from the DEEP survey and concluded that surface brightness selection cuts could conceivably account for the entire brightening effect. The basic problem of course is how to deal with an unknown number of objects which are not observed. This emphasizes to me the importance of computing comoving number densities of objects, i.e. of considering the full multi-dimensional distribution function $\phi(L, r$,$) as opposed to normalized representations of those$ distribution functions projected onto one axis.

Internal kinematics The internal kinematics of the galaxies obviously provide a very important diagnostic and there has been a large and rather confusing literature based on studies of different samples at different redshifts. The clearest picture comes from the work of Vogt et al $(1996,1997)$ on the extended rotation curves of large disk galaxies out to $z \sim 1$. These show fairly unambiguously that the Tully-Fisher relation is in place for these galaxies and that the offset relative to local samples is small. Given some uncertainty as to the selection of the galaxies in question, this is consistent with the discussion in the previous section. In terms of the smaller galaxies, which outnumber the large disks at high redshift, only integrated velocity dispersions have been measured. Guzman et al (1998) have focussed on the more compact sources while Mallen-Ornelas et al (1999) have sought to observe a sample limited primarily in colour rather than size or morphology, although there is considerable overlap. Both these studies have found rather low velocity dispersions characteristic of dwarf galaxies, $\sigma \leq$ $100 \mathrm{kms}^{-1}$, at $z \geq 0.5$. Guzman et al have argued that many of these objects will evolve into dwarf spheroidals (a hypothesis disputed by Kobulnicky et al 1999 on the basis of metallicities). Mallen-Ornelas et al have simply pointed out that these kinematics are consistent with the velocity dispersions of galaxies with similar sizes, morphologies and colours that are typically found about 2 magnitudes fainter in the present-day Universe, althongh anlologues of the same brightness can also be found, albeit at much lower comoving number density.

As anl aside it should be noted that the sizes, colours, morphologies and the optical/ultraviolet luminosities of the "small galaxies" population that increasingly duininates the $z \sim 0.7$ population are in many ways very similar to those of the $z>3$ Lyman-break selected galaxy population although there are almost no reliable mass estimates for the latter population. This similarity may or may not be significant.

The number density of elliptical galaxies An important and still-unresolved question concerns the number density of field elliptical galaxies at high redshifts. Kauffman et al (1996) claimed that the CFRS data indicated a strong decline with redshift, countering the impression given by the luminosity functions given by Lilly et al (1995) for a roughly constant number density. However, we have not been able to reproduce this result (see also Totani and Yoshii 1998). In our own re-examination of this question (Schade et al 1999) we concluded that while there was no evidence for a build-up in the numbers of ellipticals with epoch, the uncertainties were large enough that changes at the level of a factor of 2-3 could not be excluded. Part of the difficulty again lies in defining what is an "elliptical" at high redshift in the face of incomplete information (see Figs 5 and 6 of Schade et al 1999). Colour selection criteria must be very carefully applied (and in any case such a cut will include reddened spirals and exclude ellipticals 
with some modest amount of continuing star-formation) while morphological criteria are frequently ambiguous in the case of small galaxies. Im et al (1996) have used the MDS data to do a morphological selection of a very large number of ellipticals and infer on the basis of photometric redshifts that the luminosity function has showed no change in density to $z \sim 1$. At higher redshifts, both Zepf et al (1997) and Barger et al (1999) have claimed an absence of galaxies red enough to be completely dead elliptical galaxies formed at high redshift.

\subsection{The merging rate of galaxies}

Another very important (and presumably related) question is the importance of merging at $z \leq 1$ (as well of conrse at higher redshifts). Most observational studies looking at the morphological signatures of merging have found a substantial increase with redshift. (Zepf and Koo 1989, Patton et al 1998, Le Fevre et al 1999). In the Le Fevre et al study of the HST images of CFRS galaxies, we found that by $z \sim 1,15 \%$ of images shonld signs of major mergers or interactions even allowing for biases in the selection of the original sample.

The estimate given by Le Fevre et al (1999) is that typical massive galaxies have undergone of order one merger in the $0<z<1$ interval and that the merger activity was concentrated at the start of that period, $\approx \sim 1$.

\subsection{Global measures: the luminosity density of the Universe}

Several authors have examined global measures of the galaxy population such as the mean luminosity density of the Universe at ultraviolet wavelengths or in an emission line such as $\mathrm{H} \alpha$ or [OII] 3727. These quantities are relatively insensitive to the cosmology (depending only on the increment in comoving distance $\mathrm{d} \omega / \mathrm{d} z$ ) and are related, with careats concerning the effects of dust extinction, the initial Inass function, and so on, to the production of stars and heavy elements. They can thus be related to other global measurements such as the mean density of neutral gas and the mean metallicity of that gas (Pei and Fall 1995).

Most authors have found substantial increases in these quantities with redshift, with exponents, $(1+z)^{\alpha}$, in the $3<\alpha<4$ range over $0<z<1$ (Lilly et al 1996, Hammer et al 1997, Hogg et al 1998, Yan et al 1999) although Cowie et al (1999) have proposed a lower exponent than Lilly el al (1996) for the 2800 Aluminosity dependence, $\alpha \sim 1.5$. The latter discrepancy can be traced to the highest and lowest redshifts. At the lowest redshifts, we our estimate on locally determined $B$-band luminosity functions which would have to be signifcant under-estimates for the higher ultraviolet luminosity density advocated by Cowie et al to be correct (but this is possible). At $z \sim 1$ the derived luminosity density depends on the faint end slope assumed for the luminosity function Cowie et al assume a much flatter slope $(\alpha=1)$ than we did $(\alpha=1.3)$. This issue is clearly not settled beyond doubt.

A major question is whether the increase in visible/ultra-violet luminosity density can be taken as a measure of star-formation (it should be remembered that even $\mathrm{H} \alpha$ is affected by extinction). Indications from mid-IR and farr-IR surveys (Flores et al 1998b, Hughes et al 1998, Lilly et al 1999ab) suggest that the luminosity densities in these wavebands are also increasing at least as fast as indicated in the optical/ultraviolet and it therefore seems reasonable to assume that these are all due to a general rise in star-formation activity. 
Although the plot of luminosity density (and implied star-formation rate) with redshift is hailed as a significant advance in cosmology, it should also be remembered that it completely suppresses the individual identity of galaxies and thus masks completely the physical processes involved. Furthermore, it is not completely independent of cosmology. The effect of a $\Lambda$-dominated cosmology is particularly pronounced in reducing the slope of the rise and the sharpness of the change in slope at $z \sim 1$.

\subsection{Summary}

I have tried to emphasize above how uncertain our emerging picture of even the $0.1<z<1.0$ regime is. Some of the most basic descriptions of the population are still disputed, and many relevant aspects, including such basic things as metallicity, are not known to any significant degree at $z \sim 1$. The difficulties of characterizing the evolution of a diverse and continuous population of galaxies are substantial, and I believe we will have to await the detailed study of very large samples of galaxies, perhaps two orders of magnitude larger than the 1000-galaxy samples such as the CFRS, before we will have a satisfactory understanding of even this regime.

Nevertheless, I think it is true that there is little compelling evidence that the Universe at $z \sim 1$ was a dramatically different place than it is now. In particular, I think there is quite good evidence that most if not all of the presentday population of massive $\mathrm{L}^{*}$ galaxies were in place by this epoch. Consequently, the epóch of galaxy formation (certainly in terms of massive galaxies) lies at higher redshifts.

\section{The hidden phases of galaxy formation and evolution - highly luminous sources at high redshifts}

A cursory glance at the properties of present-day galaxies suggests that many galaxies may have gone through a high luminosity phase in their evolution at high redshift. In particular, the metal-rich spheroidal components of galdxies, which likely comprise a half to two-thirds of the present-day stellar mass in the Universe (Fukugita et al 1997), appear to have an age comparable to the age of the Universe and to have a structure which, unlike the disks, is consistent with a rapid and violent formation. Indeed, much of the motivation for early studies of "faint blue galaxies" was the realization by Tinsley (1980) that galaxies forming stars at $1000 \mathrm{M}_{\odot} \mathrm{yr}^{-1}$ (suflicient to make a substantial galaxy in a dynamical time of order $\left.10^{8} \mathrm{yr}\right)$ even at high redshift $(z \sim 3)$, would be readily visible at $V \sim 20$. This seminal paper motivated manly of the early redshift surveys of the faint galaxy population. Of course, no such optically-luminous galaxies have been found, despite surveys penetrating to very much fainter levels than envisaged by her. Not least the Lyman-break population (Steidel et al 1996) identified at $z \geq 2.5$ is found about 5 magnitudes fainter and have inplied starformation rates (in the ultra-violet, i.e. assuming no dust obscuration) of order $10 \mathrm{M}_{\odot \mathrm{yr}^{-1}}$. Such star-formation rates require a full (present-day) Hubble time of continuous star-formation to build up to a substantial stellar population. Very luminous slar-forming galaxies at high redshift have been conspicuous by their absence in optical/ultraviolet surveys. 
However, in the local Universe, it has been known since the IRAS mission, that the most luminous galaxies locally are heavily obscured by dust and radiate all but a few percent of their luminosity in the far-infrared, peaking around 100 $\mu \mathrm{m}$. The nature of the energy source in these ultra-luminous infrared galaxies (ULIRGs) has been long debated. Recent data from ISO (Genzel 1398) suggests that at Arp220-level luminosities $\left(2 \times 10^{12} L_{\odot}\right)$ both star-bursts and AGN contribute, with the former dominating at the roughly $3: 1$ level. These local ULIRGs are generally associated with the mergers of massive galaxies and the star-burst systems may well be associated with the production of elliptical galaxies (Sanders and Mirabel 1996 and references therein).

However, ULIRGs are very rare in the local Universe and objects with luminosities exceeding that of Arp 220 contribute only about $0.3 \%$ of the total bolometric luminosity output of the local Universe (see e.g. Sanders and Mirabel 1996 and references therein). Thus in global terms they might be considered a curiosity at the present epoch.

The discovery by two experiments onboard COBE (Puget et al 1995, Fixsen et al 1998, Hauser et al 1998) of a far-infrared/sub-millimeter background with a bolometric energy content as large or larger than that seen in the optical (Pozetti et al 1998) emphasized that obscuration by dust is an important phenomenon in the early Universe.

\subsection{Deep sub-mm selected sources and the ULIRG population at high redshifts}

It has long been appreciated that observations at millimetre wavelengths are potentially an effective way of identifying ULIRGs at high redshift, where the peak of the spectral energy distribution is redshifted into the observing band. Indeed, the highly beneficial $k$-corrections at wavelengths around $1 \mathrm{~mm}$ largely compensate for the increased distance, resulting in a flat $\mathrm{S}(\mathrm{z})$ relation over a wide redshift range. For instance, at an observing wavelength of $850 \mu \mathrm{m}$, Arp220 would be approximately as bright at $z \sim 8$ as at $z \sim 0.5$.

The SCUBA bolometer array camera on JCMT, operating at $850 \mu \mathrm{m}$ (and also, though more affected by atmospheric conditions, simultaneously at 450 $\mu \mathrm{m})$ is thus a very efficient detector of obscured high-luminosity sources at high redshifts. With long integrations, SCUBA has a sensitivity sufficient to detect sources similar to Arp 220 over a very wide rage of redshifts, and several groups (e.g. Smail et al 1997, Hughes et al 1998, Barger et al 1998, Eales et al 1999) have been undertaking surveys of small regions of sky to search for such objects.

All of these surveys have succeeded in detecting individual sources that, as a population, represent a substantial fraction of the COBE background at 850 $\mu \mathrm{m}$. At $S_{8.50} \geq 3 \mathrm{mJy}$, detectable after about 8 hours with SCUBA, 20-25\% of the background is resolved into discrete sources. Deeper exposures or the use of gravitational lensing effects suggest that at $S_{850} \geq 1 \mathrm{mJy}$, a half of the COBE background is accounted for.

There sources have Arp-220 level luminosities as long as they lie somewhere in the broad redshift interval $0.5 \cdot z<8$, which they almost certainly do. although the precise luminosities are poorly constrained at the level of a factor of 2-3 because of uncertainties in the spectral energy distribution through the peak at $100 \mu \mathrm{m}$. Even without knowledge of the redshifts of the individual 
'sources, the fact that the sub-mm background can be resolved into a small number of ultra-high redshift sources immediately tells us that such sources must be producing a significant fraction of the bolometric luminosity of the Universe at high redshifts. A reasonable estimate is that $30 \%$ of the bolometric output at high redshifts comes from ULIRGs, whereas, as noted above, the corresponding fraction in the present day Universe is only $0.3 \%$.

A major uncertainty concerns the redshifts of the sources. Identification with optical/near-IR sources is rendered ambiguous because of the poor resolution of the $15 \mathrm{~m}$ JCMT ( 15 arcsec FWHM at $850 \mu \mathrm{m}$ ). Some of the sub-mm sources are detectable as radio sources whose VLA positions make the identification unambiguous. The fraction of sources which are radio sources obviously depends on the limiting flux in both wavebands, but in our own deep survey $\left(S_{8.50} \geq 3 \mathrm{mJy}\right.$ and $S_{6 \mathrm{~cm}} \geq 16 \mu \mathrm{Jy}$ ), it is not more than $33 \%$ (Lilly et al 1999a, Richards 1999, c.f. Cowie and Barger 1999). Deep integrations with millimeter-wave interferometers have resulted in the detection of the brighter SCUBA sources in some cases disproving the claimed identification (Downes et al) and in another confirming it (Gear at al, in preparation). In our opinion, none of the deepest sub-mm surveys have been securely identified past the $50 \%$ level.

In our own survey (Eales et al 1999, Lilly et al 1999ab) we have been impressed by how many sources can be reliably identified with low redshift sources $z \leq 1$. Based on our newest unpublished results, about $20 \%$ of the sources have $z \leq 1$ ) with many more likely lying at $z \leq 3$. This limits the fraction of the sample which can lie at very high redshifts. In the past, we have estimated $\tilde{z}_{\text {median }} \sim 2$ (Lilly et al 1999ab) although we would certainly not rule out median redshifts as high as $z_{\text {median }} \sim 3$. This is consistent with the extensive spectroscopy of candidate identifications in the Smail et al cluster lens survey presented by Barger et al identification.

As is well known, the strongly beneficial $k$-corrections at sub-mm wavelengths result in a flat flux-density redshift relation, meaning that a flux-density limited sample closely approximates a volume limited sample in which the increase in comoving volume with redshift should produce a large number of very high redshift sources within samples. As we have argued elsewhere (Lilly et al 1999ab), even quite conservative limits on the fraction of sources that can lie at high redshifts place quite strong constraints on the amount of high luminosity obscured activity that can take place at high redshifts. If $50 \%$ of the activity occurs at $z>3$ then we would expect of order $85 \%$ of the $850 \mu \mathrm{m}$ background to have been produced at $z>3$ - a scenario that we can to all intents and purposes rule out. Even a model in which the luminosity density rises to $z \sim 2$ and is then constant at higher redshifts predicts that $50 \%$ of the $850 \mu \mathrm{m}$ background would come from $z \geq 4$ which is already in our view uncomfortable, but probably not ruled out. Thus it appears that much of the activity represented by these ultra-luminous sources occurs at lower redshifts, $z \sim 2$.

Another major uncertainty concerns the nature of the energy source. Based on similar objects locally, it is likely that both $A G N$ and starbursts are responsible, indeed the two phenomena are likely closely related. This would mean that this population is both supplying a significant fraction of all stars, and a large 
fraction of the central black hole mass, seen in the local Universe (see Lilly et al $1999 \mathrm{~b}$ for a discussion).

\section{Perspectives}

In seeking to synthesize the above, with also the immense amount of work on the high redshift optically-selected population, I believe the following things have been established, or at the least have not been disproved:

1. The Hubble sequence of galaxy morphologies is in place by $z \sim 1$ and the number densities of large ellipticals and spirals has not changed dramatically since that epoch.

2. The Hubble sequence of galaxy morphologies is probably not in place at $z \sim 3$, and the number densities of large ellipticals and spirals is probably much lower at this epoch than at present. I base this statement on Dickinson's beautiful NICMOS images of the Hubble Deep Field.

3. Small, irregular, vigorously star-forming galaxies of moderate mass are much nore prevalent at $z \sim 1$ (where they dominate) than they are now. The present-day descendants of these galaxies is unclear as is their relationship to the Lyman-break population at $z>3$, to which they nonetheless bear considerable similarities.

4. There is morphological evidence that merging of galaxies was a more important (and possibly dominant) phenomenon in assembling galaxies at $z \geq 1$ than in the present-day Universe.

5. Likely related to (4), ultra-luminous dust enshrouded galaxies at $z \geq 1$ contribute a Inuch larger fraction (about 30\%) of the bolometric energy output of the Universe than at the present epoch (about $0.3 \%$ ). These high redshift systems could be producing a substantial fraction of the present-day stellar population of the Universe, and could most naturally be associated with the production of the spheroids.

6. There is some preliminary evidence that the high luminosity obscured ULIRGs show a similar behaviour to the quasars in declining to the highest redshifts, $z>3$ (see Shaver et al 1996) in contrast to the luminosity function of the lower luminosity ultra-violet selected Lyman-break galaxies which seems to be roughly constant between $2<z<5$ (Steidel et al 1999).

If we accept the above (and not all will), we can sketch out the following possible story to link together these disparate strands of observational evidence. It should be emphasized that this is largely speculative at this stage.

(a) The first, as yet unobserved, stars form in small galaxies at high redshift and pollute the intergalactic medium to a uniform metallicity of about 0.01 solar.

(b) As time passes, merging increases the dark mass of galaxies and becomes increasingly important in the triggering of star formation. Star-formation in relatively small galaxies and/or steady-state star-formation at modest levels in larger galaxies would be seen as the Lyman-break population at roughly constant number density.

(c) However, as the masses and metallicities of galaxies built up, mergers would become more violent and the obscured ULIRGs would appear, dominating the star-formation in the Universe at $1<\approx<3$ and resulting in the spheroids. Quasars. probably arising under similar conditions, would follow a similar evo- 
lutionary history. These ULIRGs would be responsible for the generation of a substantial fraction of the present-day stellar population of the Universe and, most reasonably, with the massive spheroids.

(d) By $z \sim 1$ the worst of the merging would be past. Disks could survive and the familiar population of spirals and ellipticals would stabilize. The ULIRG and quasar populations would decline dramatically.

(e) Heading towards the present-epoch, star-formation in disks and in the irregular population would decline, presumably as the availability of gaseous fuel declined. Star-formation in smaller galaxies would still be modulated by the feedback between gas and stars, but would be steadier in larger disks.

In this picture, the different features of the star-formation history diagram are accounted for in terms of the merger history, and in particular the different behaviours at $z<1$ and $z>1$ may reflect phases when merging is and isn't the dominant process. One could speculate why merging might switch off at this point and it could conceivably be linked to the transition from a matter to $\Lambda$-dominated Universe in $\Lambda$-dominated cosmologies with $\Omega_{0}=0.2$.

I would argue that the above picture is broadly consistent with hierarchical models of galaxy formation. It is often remarked that the evolution back to $z \sim$ 1 , being dominated by smaller galaxies, is precisely the opposite of that predicted from hierarchical models in which we would expect small galaxies to form first and be assembled into larger ones. I personally don't find this a concern. First it should be remembered that the optically-selected population may represent considerably less than a half of the star-formation that has occurred in the Universe and that the bulk of the star-formation may be going on in the obscured high luminosity ULIRGs. Thus the evolutionary behaviour at $z<1$ could be just an after-thought, occurring after the bulk of galaxy formation was completed. This makes sense especially if the hierarchical assembly of galaxies ceased at $z \sim 1$, perhaps due to a low-density L-dominated cosmology.

Furthermore, the evolution of smaller galaxies is likely to be quite complex with feedback from star-formation play in a very important role. Thus the conversion of gas into stars within low mass haloes may well be quite episodic and not follow simple ideas of the formation of the haloes (for instance, there is as yet little evidence that a significant fraction of the blue galaxies at $z \leq 1$ are in any sense primordial). If the differential behaviour between the opticallyselected and ULIRG populations which I suspect is occurring at $z>>2$ holds up, then in fact this would be precisely the behaviour expected in hierarchical models.

This picture leads to some clear predictions that should be testable within the next few years. (a) The bulk of the present-day spheroid population should appear between $1<z<3$ even if a few examples of quiescent galaxies are found at higher redshifts; (b) The galaxies at $z>3$ should have masses significantly below those of present-day $L^{*}$ galaxies even if they are the pre-cursors of such galaxies; (c) The ultra-luminous galaxies and quasars should indeed decline in number density at the highest redshifts.

Acknowledgments. My own recent work in this field has been in collaboration with the CFRS and CUDSS teams, most notably Olivier Le Fevre, Francois Hammer. David Crampton, David Schade, Steve Eales and Walter Gear, as well as my students Gabriela Mallen-Ornelas and Tracy Webb. Their major contribu- 
tions to my own thinking are gratefully acknowledged. My research is supported by the NSERC of Canada and by the Canadian Institute for Advanced Research and their support is also gratefully acknowledged.

\section{References}

Abraham, R.G., Tanvir, N.R., Santiago, B.X., Ellis, R.S., Glazebrook, K., van den Bergh, S., 1996, MNRAS, 279, L47.

Barger, A., Cowie, L., Sanders, D., Fulton, E., Taniguchi, Y., Sato, Y., Kaware, K., Okuda, H., 1998, Nature, 394, 248.

Barger, A., Cowie, L.L., Trentham, N., Fulton, E., Hu, E.M., Songaila, A, Hall, D.,1999,AJ, 117, 102.

Barger, A., Cowie, L.L., Smail, I., Ivison, R.J., Blain, A.W., Kneib, J.-P., 1999, AJ, 117, 2656.

Blain, A., Smail, I., Ivison, R., Kneib, J.P., 1999, MNRAS, 302, 632

Brinchmann, J., Abraham, R., Schade, D., Tresse, L., Ellis, R., Lilly, S., Le Fevre, O., Glazebrook, K., Hammer, F., Colless, M., Crampton, D., Broadhurst, T., 1998, ApJ, 499, 112.

Cowie, L.L., Songaila, A., Barger, A., 1999, AJ, 118, 603.

Cowie, L.L., Barger, A., 1999, these proceedings.

Downes, D., et al., 1999, A\&A, 347, 809.

Eales, S., Lilly, S., Gear, W., Bond, J.R., Dunne, L., Hammer, F., Le Fevre, O., Crampton D., 1998, ApJ,

Fixsen, D., Dwek, E., Mather, J., Bennet, C., Shafer, R., 1998, ApJ, 508, 123.

Flores, H., Hammer, F., Desert, F.X., Cesarsky, C., Crampton. D., Omont, A., Thuan, T.X., Eales, S., Le Fevre, O., Lilly, S.J. 1998b, ApJ, 517, 148.

Fukugita, M., Hogan, C.J., Peebles, P.J.E., 1998, Ap.J, 503, 518.

Genzel, R., Lutz, D., Sturm, E., Egami, E., Kunze, D., Moorwood, A.F.M., Rigopoulou, D., Spoon, H., Sternberg, A., Tacconni-Garman, L., Tacconi, L., Thatte, N., 1998, ApJ, 498, 579.

Guzman, R., Gallego, J., Koo, D., Phllips, A., Lowenthal, J., Faber, S.M., Mlingworth, G., Vogt, N., 1998, Ap.J, 489, 559.

Haehnelt, M.G., Natarajan, P., Rees, M.J., 1998, MNRAS, 300, 817.

Hammer, F., Crampton, D., Le Fevre, O., Lilly, S., 1995a, ApJ, 455, 88.

Hanser, M., Arendt, R., Kelsall, T., Dwek, E., Odegard, N., Welland, J., Freundenreich, H., Reach, W., Silverberg, R., Modeley, S., Pei, Y., Lubin, P., Wather, J., Shafer, R., Smoot, G., Weiss, R., Wilkinson, D., Wright, E., 1998, ApJ 508, 25.

Heyl, J., Colless, M., Ellis, R.S., Broadhurst, T.J., 1997, MNRAS. 285, 613

IIogg, D.W., Cohen, J., Blandford, R., Pahre, M.A., 1998, ApJ, 504, 622.

Hughes, D., Serjeant, S., Dunlop, J., Rowan-Robinson, M., Blain, A., Mann, R., Ivison, R., Peacock, J., Efstathiou, A., Gear, W., Oliver, S., Lawrence, A., Longair, M., Goldschmidt, P., Jenness, T., 1998, Nature 394. 241. 
Im, M., Griffiths, R.E., Ratnatunga, K.U., Sarajedini, V.L., 1996, ApJ, 461, 791.

Ivison, R., Smail, I., Le Borgne, J-F., Blain, A., Kneib, J-P., Kerr, T., Bezecourt, J., Davie, J., 1998, MNRAS, 298, 583.

Kaufmann, G., Charlot, S., White, S.D.M., 1996, MNRAS, 283, L117.

Kobulnicky, H.A., Zaritsky, D., 1999, AJ, 117, 908.

Le Fevre, O., Abraham, R., Lilly, S., Ellis, R.S., Brinchmann, J., Schade, D., Tresse, L., Colless, M., Crampton, D., Glazebrook, K., Hammer, F., Broadhurst, T., 1999, astro-ph/99-090211

Lilly, S.J., Hammer, F., Le Fevre, O., Crampton, D., 1995b, ApJ, 455, 75.

Lilly, S.J., Le Fevre, O., Hammer, F., Crampton, D., 1996, ApJ, 460, L1.

Lilly, S.J., Schade, D., Ellis, R., Le Fevre, O., Brinchmann, J., Tresse, L., Abraham, R., Hammer, F., Crampton, D., Colless, M., Glazebrook, K.,

Mallen-Ornelas, G., Broadhurst, T., 1998a, ApJ, 500, 75.

Lilly, S.J., Eales, S.A., Gear, W., Dunne, L., Bond, J.R., Hammer, F., Le Fevre, O., Crampton, D., 1999a, ApJ, 518, 641.

Lilly, S.J., Eales, S.A., gear, W.K., Webb, T., Bond, J.R., Dunne, L., 1999b, astro-ph/9903157.

Madau, P., Ferguson, H., Dickinson, M., Giavalisco, M., Steidel, C., Fruchter, A., 1996, MNRAS, 283, 1388

Mallen-Ornelas, G., Lilly, S.J., Crampton, D., Schade, D., 1999, ApJ 518, L83.

Mao, S., Mo, H.J., White, S.D.M., 1998, MNRAS, $297,71$.

Patton, D.R., Pritchet, C.J., Yce, H.K., Ellingson, E., Carlberg, R.G., 1998, ApJ, $475,29$.

Pei, Y.C., Fall, S.M., 1995, ApJ, 454, 69.

Pozzetti, I., Madau, P., Zamorani, G., Ferguson, H.C., Bruzual, G., 1998, MN. RAS, 298, 1133.

Puget, J-L., Abergel, A., Bernard, J-P., Boulanger, F., Burton, W.B., Desert, F.X., Hartmann, D., 1996, A\&A, 308L, 5P.

Richards, E.A., 1999, ApJ, 513, 9.

Rowan-Robinson, M., \& Efstathiou, A., 1993, MNRAS, 263, 675

Sanders, D., \& Mirabel, I., 1996, ARA\&A, 34, 749.

Schade, D., Lilly, S.J., Crampton, D., Hammer, F., Le Fevre, O., Tresse. L., 1995, ApJ, 451, 1.

Schade, D., Lilly, S.J., Crampton, D., Ellis, R.S., Le Fevre, O., Hammer, F., Brinchmann, J., Abraham, R.G., Colless, M..M.. Glazebrook, K., Tresse, L., Broadhurst, T.J., 1999, astro-ph/9906171

Shaver, P., Wall. J.V., kellermann, K., I., Jackson, C.A., Hawkins, M.R.S.. 1996 , nature, $384,439$.

Simard, L., Koo, D.C., Faber, S.M., Sarajedini, V.L., Vogt, N., Phillips. A.C., Gebhardt, K., Mlingworth, G.D.. Wu, K.L., 1999, ApJ. 519, 563.

Smail, I., Ivison, R., Blain, A., 1997, ApJ, 490, L5.

Smail. I., Irison, R., Blain, A., Kneib. J-P, 1998, Ap.J, 507. L21. 
Steidel, C.C., Giavalisco, M., Pettini, M., Dickinson, M., Adelberger,K., 1996, ApJLetters, 462, 17 .

Steidel, C.C., Adelberger, K.L., Giavalisco, M., Dickinson, M., Pettini, M., 1999, ApJ, 519, 1.

Tinsley, B.M., 1980, Ap.J, 241, 41.

Totani, T., Yoshii, Y., 1998, ApJLetters, 501, 177.

Vogt, N., Forbes, D.A., Phillips, A.C., Gronwall, C., Faber, S.M., Illingworth, G.D., Koo, D.C., 1996, ApJLetters, 465, 15.

Vogt, N., Phillips, A.C., Faber, S.M., Gallego, J., Gronwall, C., Guzman, R., Illingworth, G.D., Koo, D.C., Lowenthal, J.D., 1997, ApJLetters, 479, 121.

Yan, L., McCarthy, P.J., Freudling, W., Teplitz, H.I., Malumuth, E.M., Weymann, R.J., Malkan, M.A., 1999, ApJ, 519, 47.

Zepf, S., and Koo, D.C., 1989, ApJ, 337, 34.

Zepf, S., 1997, Nature, 390, 377. 
The Hy Redshift Universe

ASP Conference Series, Vol. 193, 1999

A. J. Bunker \& W. J. M. van Breugel, eds.

\title{
. DEEP's Field Surveys: The View from Santa Cruz
}

\author{
David C. Koo \\ UCO/Lick Observatory, Department of Astronomy and Astrophysics, \\ University of California, Santa Cruz, CA 95064
}

\begin{abstract}
DEEP is a major redshift survey of over 50,000 $z \sim 1$ field galaxies that will use a new spectrograph (DEIMOS) on the Keck II $10 \mathrm{~m}$ telescope. The main scientific goals include understanding how galaxies form and evolve and mapping their large scale structure. Most DEEP spectra will be of quality and resolution high enough to extract rotation curves, velocity widths, age estimates, and chemical abundances. Whilc waiting for DEIMOS to complete in 2000, the Santa Cruz DEEP. team has concentrated on completing various pilot programs that combine the use of the current Keck spectrographs and of multiband HST images. I summarize highlights of our studies of early-type galaxies and bulges, spirals and disks, and compact galaxies out to $z \sim 1$ as well as high redshift $(z \sim 3)$ galaxies. We confirm that measuring kinematics is a practical technique for distant galaxies and that such information provide vital data for galaxy evolution studies. Based on these kinematic studies, the Santa Cruz team finds evidence for a wide diversity in the behavior of distant galaxies. While massive early-type galaxies and spirals appear to constitute a relatively stable population out to $z \sim 1$, lower mass compact galaxies exhibit more dramatic evolution. Our view is that galaxy evolution is complex, i.e., not solved or dominated by a single physical process such as mergers or starbursts. Due to the diversity of galaxies and of possible physical mechanisms that affect their formation and evolution, galaxy evolution studies remain in their infancy, though an explosive growth in data and theory can be expected over the next decade.
\end{abstract}

\section{Introduction to DEEP and DEIMOS}

The current suite of new $8-10 \mathrm{~m}$ class optical telescopes promises to offer new surveys of field galaxies to unprecedented faintness. Equally tantalizing are the new opportunities to combine these data with high spatial resolution measurements of size, shape, and morphology from HST and NGST, as well as in new wavebands, such as in the infrared (SIRTF) and x-ray (CHANDRA). Besides fainter redshifts, large telescopes may yield $S / \mathrm{N}$ gains and spectral resolutions high enough to enable three new, powerful diagnostics for the analysis of distant galaxies, namely internal velocities (and hence masses when combined with size), element abundances, and age estimates. These measurements are important, independent probes of galaxy properties in the early universe. Compared to the uncertainties of star formation processes needed to predict luminosities, 
the gravitational behavior of masses are much more reliably tracked by cosmological simulations of galaxy formation and evolution. Finally, because galaxy evolution and their large scale patterns are both complex (especially with uncertainties in the nature of dark matter, bias, and cosmology), field galaxy surveys with large samples will be essential to address these problems.

To meet this challenge and opportunity, the Deep Extragalactic Evolutionary Probe (DEEP ${ }^{1}$ ) team intends to undertake a new Keck survey of over 50,000 faint field galaxies that uses a new instrument (DEMMOS ${ }^{2}$ : Davis and Faber 1998), which is under construction at UCO/Lick Observatory under the direction S. Faber. DEIMOS will provide both a wide-field direct inaging capability as well as multi-object spectroscopy. Although originally designed for two optical cameras, DEIMOS has one optical camera at this time, with the second camera slot being retained for a future upgrade to a near-infrared camera. Even with one camera, DEIMOS will increase the Keck throughput (sensitivity, spectral range, resolution, area) for faint-object spectroscopy by a factor of 7 . DEIMOS has an active slit area of $16^{\prime} \times 4^{\prime}$ and focuses spectra villo a CCD array of $8 \mathrm{k} \times 8 \mathrm{k} 15 \mu \mathrm{m}$ pixels. For each field of view, slit masks containing an average of 140 slitlets will be prepared by a computer-controlled milling inachine. DETMOS is expected to be commissioned by late 2000 and fully operational by spring 2001 when the new DEEP survey begins.

DEEP is designed to provide a detailed characterization of the nature of field galaxies and their clustering statistics at redshifts $z \sim 1$. In order to overcome cosmic variance, DEEP will target over $2 \mathrm{deg}^{2}$ divided among four low-extinction fields on the sky. Each field is currently being imaged in $B R I$ by N. Kaiser and G. Luppino as part of their CFHT weak lensing campaign; these images will be used for sample selection, multicolor photometry, and image structure measurements. In each field, DELMOS spectroscopy will cover a central continuous strip of size $16^{\prime} \times 120^{\prime}$, with additional outrigger strips added to improve measurements of higher-order clustering statistics. Exposures will range from one hour for the primary strip to 3 hours for smaller subsections that will also be targeted for supplementary imaging from HST. By exploiting photometric redshifts from the $B R I$ photometry, we expect to screen out foreground galaxies with $z<0.7$, thus more than doubling the efficiency of the survey for the higher redshift regime. We will concentrate on observing $O$ [II] $3727 A$ in the spectral range $6000 \AA<\lambda<9500 \AA$, which we can study with high spectral resolution, $2.3 \AA(90 \mathrm{~km} / \mathrm{s}) \mathrm{FWHM}$, throughout the redshift window $0.61<z<1.55$. With depths of $I \sim 23$ and over 50,000 galaxies, the DEIMOS DEEP survey will thus be nearly $100 \mathrm{x}$ larger and one magnitude deeper than the pioneering Canladia France Redshift Survey (CFRS: see paper by Lilly).

DEEP will gather internal kinematic data in the form of rotation curves or linewidths. This aspect sets DEEP apart from other faint galaxy surveys. Such measurements are of special interest because, if the system is bound and if sizes and inclinations can be independently measured or estimated, the inferred grav-

\footnotetext{
'More details of DEEP's prior and planned surveys, members, publications, etc. can be found at website: http://www.ucolick.org/ deep/home.htmil

${ }^{2}$ More details on the DEep Imaging Multi-Object Spectrograph (DENIOS) can be found at website: http://www.ucolick.org/ loen/Deimos/deimos.html
} 
itational potential depth can be used to estimate the actual masses of distant galaxies. Tracking how the volume density of galaxies with different masses (as well as luminosities, sizes, structure, etc.) vary with lookback time will provide an entirely new dimension to study the rate of galaxy merging, luminosity evolution, disk and bulge structure formation, etc. Finally, if the mass, luminosity, and number evolution of galaxies can be understood and corrected, the large sample of DEEP will provide an unprecedented opportunity to measure the geometry of the universe, not only through the evolution of large scale structure but also via the classical volume test. Such volume tests are more sensitive than size or luminosity tests by factors of $\sim(1+z)^{2}$ and $\sim(1+z)$, respectively.

\section{Highlights from pre-DEIMOS DEEP Surveys}

While waiting for the completion of DEIMOS and in order to determine its expected performance, we have undertaken a number of smaller, pilot-style projects with the existing Low Resolution Imaging Spectrograph (LRIS: Oke et al. 1995) and HIgh Resolution Echelle Spectrograph (HIRES: Vogt et al. 1994). To maximize the scientific returns, we have focused our efforts in fields where HST WFPC2 images already exist, such as the Groth Survey region (J2000 14:17.5+52:30) and the Hubble Deep Field (HDF). Such HST images are crucial not only for determining the morphology of our galaxy samples, but also for obtaining reliable measures of structure, including size and inclination, which are needed to convert kinematic observations into direct measures of mass. Overall, our pre-DEIMOS pilot programs have been gratifyingly successful, not only in providing vital information to support and define the DEIMOS DEEP survey, but also in yielding a variety of important clues on the formation and evolution of distant field galaxies. The following summary of highlights from the Santa Cruz DEEP team are meant to be complementary to the contributions at this workshop on pre-DEIMOS DEEP results by $\mathrm{M}$. Im on the evolution of earlytype galaxies and by N. Vogt on the evolution of the Tully-Fisher relation for distant spiral galaxies.

\subsection{Early-Type Galaxy and Spheroid Evolution}

As reported in more detail in Im's contribution, we find no evidence for rapid changes in either the colors or number densities of luminous E/S0 and other early-type galaxies (Im et al. 2000) or of bulges/spheroidals (Koo et al. 2000) ont to redshift $z \sim 1$. Woreover, for galaxies bright enough to permit measurements of their absorption line velocity dispersions, we have obtained all needed information (surface brightness, velocity dispersion, and luminosity) to test for any significant evolution of the Fundamental Plane; none was found (Gebhardt et al. 2000). Overall, these findings support the view that most early-type systems were well in place by redshift $z \sim 1$, with little change except for the expected luminosity changes due to passive evolution of an old stellar popnlation.

\subsection{Distant Spirals and Disks}

A major theme of DEEP is the kinematics of distant galaxies. As described in more detail in Voyt's contribution, we have clearly demonstrated the feasibility of discerning emission-line rotation curves of likely spirals ont to redshifts near 
.$z \sim 1$. These results apply to galaxies as faint as $I \sim 22$ and need one to two hour exposures on Keck. To optimize the slit alignment with a galaxy's apparent major axis, the slitlets have been milled with tilts. Based on a sample of over 50 galaxies, we find little evidence for any major change $(<0.6 \mathrm{mag})$ in the zeropoint of the optical Tully-Fisher relation (Vogt et al. 1996; Vogt et al. 1997; Vogt et al 2000). We thus find that large, massive spirals, like the early-type galaxies, constitute a fairly stable population up to redshift $z \sim 1$. This conclusion is supported by recent studies that show the volume density of large disk galaxies has not changed much to $z \sim 0.8$ (Lilly et al. 1998) and by independent rotation curve work at intermediate redshifts by Bershady $(1997,1998)$ and Bershady et. al. (1998). Other kinematic studies at intermediate redshifts $(z<0.7)$ find evidence for 1 to 2 magnitudes of evolution (Rix et al. 1997; Simard and Pritchet 1998; Mallen-Ornelas et al. 1999). A full reconciliation of these discordant results has yet to be made.

Besides kinematic studies, the surface brightness evolution of disks can be studied directly through measurements of size and luminosity. Prior studies claim strong evidence for higher surface brightnesses in disks at higher redshifts (Schade et al. 1996a, 1996b; Roche et al. 1998), and our own study to fainter limits show similar apparent trends (Simard et al. 1999). However, when selection effects are taken into account, virtually all of the trends disappear, leaving only a relatively small population of luminous, compact galaxies at higher redshifts $(z>0.7)$ showing significant evolution (Simard et al. 1999). Thus again we find little evidence for substantial evolution in large galaxies to $z \sim 1$.

\subsection{Compact Galaxies}

For several years, the Santa Cruz DEEP team has been studying faint blue compact galaxies (BCG) with Keck and HST. These galaxies are generally of high luminosities, close to $L^{*}$, and thus do not belong to the blue compact dwarf $(B C D)$ class, which are instead typically of very low luminosities closer to $L^{\star} / 100$. We have two samples of sich galaxies. One of typically $B \sim 21$ was originally extracted from stellar-like QSO candidates that subsequent spectroscopy from KPNO showed to possess narrow emission lines and galaxy-like luminosities rather than broad AGN-like lines and QSO luminosities (Koo et al. 1994). The other much fainter sample of $I<23$ was from compact galaxies chosen in the HDF Flanking Fields (Phillips et al. 1997).

Our key discovery is that many of our compact galaxies, though of high luminosity, have very narrow emission lines when observed at moderate to high spectral resolution on Keck (Foo et al. 1995, Phillips et al. 1997). Indeed, for some very compact galaxies brighter than the Milky Way, we needed the HIRES to resolve velocity widths smaller than $30 \mathrm{~km}-\mathrm{s}^{-1}$ (Koo et al. 1995; Guzman et al 1996; see Fig. 1). When combined with sizes from HST, the inferred masses are typically less than $10^{10} \mathrm{M} \odot$, with resultant $\mathrm{M} / \mathrm{L}$ up to 100 times less than normal galaxies (Guzman et a1. 1996, 1997).

A major concorn is whether the emission line widths can be trusted to yicld reliable masses. Though winds, dust obscuration, and poor representation of the gravitational potential by the luminous star formation regions may reduce the correlation of the emission line widths and the galaxy's gravitational potential, the available data nevertheless show a good correlation betwecn emission-line 


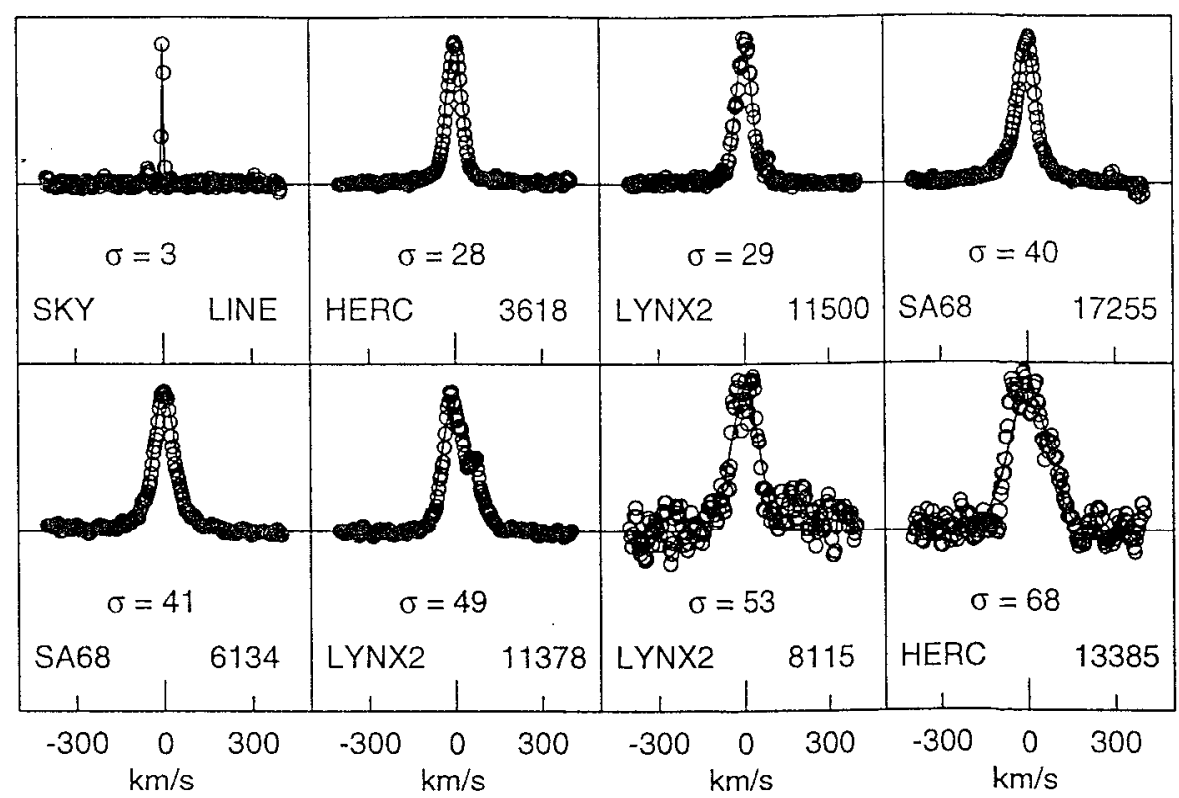

Figure 1. Emission line profiles of very luminous blue compact galaxies as observed with HIRES (Guzman et al. 1996). The velocity widths $(\sigma)$ in $\mathrm{km} / \mathrm{s}$ are as indicated where typical values for normal galaxies are closer to $200 \mathrm{~km} / \mathrm{s}$.

velocity widths and that of HI, which is assumed to trace the true galaxy potential. This has been found for normal galaxies (Kobulnicky and Cebhardt 1999) and even for very compact local HII galaxies (Telles and Terlevich 1993). Granting that these findings hold for our sample and to higher redshifts, i.e., that we are indeed measuring masses with our kinematics, the lack of correlation between luminosity and mass demonstrates the uscfulness, ncccssity, and promise of kinematics as an important new dimension to discern the evolution of different galaxy populations.

For both of our compact samples, the majority of these galaxies have luminosities, colors, surface brightnesses, sizes, star formation rates (SFR), excitations, and most importantly, velocity widths and $\mathrm{M} / \mathrm{L}$ ratios characteristic of the most luminous nearby IIII galaxies. The more massive compact galaxies form a more heterogeneous class of evolved starbursts, similar to local disk starburst galaxies (Guzman et al. 1997). Without additional star formation, the HII-like BCGs are likely to fade to resemble today's spheroidal galaxies such as NGC 205 (Koo et al. 1995: Guzman et al. 1996, 1997). By comparing the global comoving SFR densities to previous studies, we conclude that BCGs, though only $\sim 20 \%$ of the general field population, may contribute as much as $\sim 45 \%$ to the global SFR density of the universe at $z<1$. The importance of compact galaxies to galaxy evolution is supported by the CFRS team (Lilly et al. 1998; Mallen-Ornelas et al. 1999). 


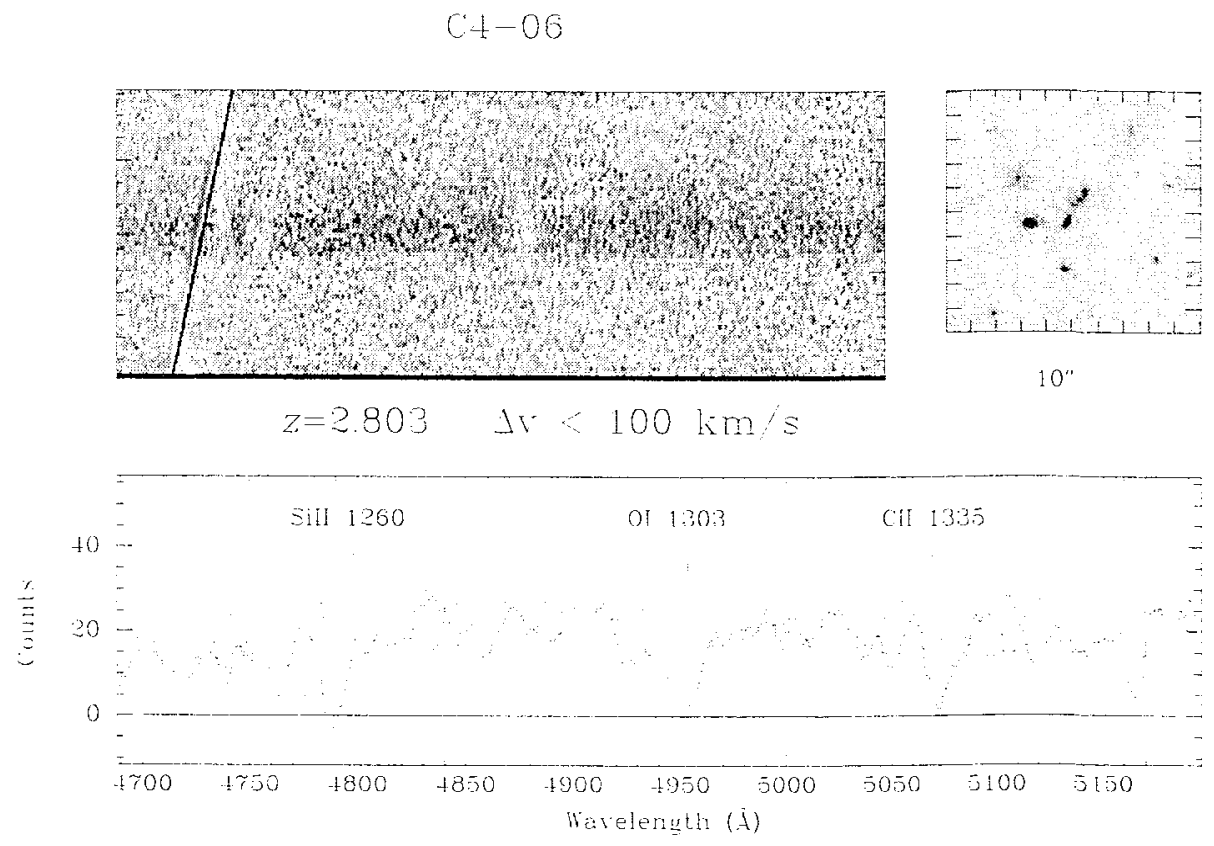

Figure 2. Upper left panel shows the 2-D sky-subtracted spectra for the extended, or possibly two-component, Lyman-drop galaxy shown in the center of the upper right panel. The bottom figure shows part of the spectrum of each component separately. A cross-correlation of the spectra yields no statistically detectable velocity shift. Adoption of an upper limit of $100 \mathrm{~km} / \mathrm{s}$ for the differential velocity, no inclination corrections, and the apparent angular separation as a measure of the actual size of the system yields an upper limit to the mass of $10^{10} \mathrm{M} \odot$. This source, C4-06, was originally discovered in the HDF by Steidel et al. (1996b) with Keck and reobserved at higher spectral resolution to derive the kinematics (Lowenthal et al. 1998). This figure was kindly provided by J. Lowenthal. 


\subsection{Redshift $\approx \sim 3$ Galaxies}

One of the major advances with Keck has been the dramatic demonstration by Steidel et al (1996a) that very high redshift $(z \sim 3)$ galaxies, originally selected on the basis of broadband colors, can be routinely confirmed spectroscopically. The DEEP team has extended the pioneering efforts of Steidel et al. (1996b) in the Hubble Deep Field (HDF) by pushing the faintness limits beyond $I \sim 25$, using redder "dropouts" to reach higher redshifts and higher levels of completeness, and adopting higher spectral resolutions to improve kinematic measurements (Lowenthal et al. 1997). Based on evidence for higher volume densities, diversity in morphologies, and emission line widths of Ly $\alpha$ being frequently narrow, we suggested that the high redshift galaxies may be a heterogenous population, including small mass systems that become dwarfs today or that later merge to form more massive galaxies (Lowenthal et al. 1997). Additional evidence for these views come from findings for non- $\mathrm{I}^{1 / 4}$ profiles in some high redshift galaxies (Marleau and Simard 1998), very narrow lines $(<100 \mathrm{~km} / \mathrm{s}$ in 4 out of five targets) seen in rest-frame optical lines as observed with an infrared spectrograph (Pettini ct al. 1998), theoretical simulations of merger induced starbursts among subclumps of massive halos (Somerville et al. 1999), and noting that the $\approx \sim 3$ galaxies have many of the physical characteristics of the most luminous of our compact galaxies seen at $z<1$ (Lowenthal et al. 1998). However the original view that these galaxies are only the cores of massive galaxics (Stcidel et al. 1996a) is strongly supported by their strong clustering (Steidel et al. 1998) and various other simulations (e.g. Baugh et al. 1998).

A key discriminant among the possible nature and descendents of these high redshift galaxies is mass. Although Lyman-drop galaxies are generally too small $\left(\sim 0.2^{\prime \prime}\right)$ to yield rotation curves with present ground-based systems, some appear to have multiple or cxtended components, which then allow spatially rosolvcd kinematics to be gathered. So far, we have results for only for two galaxies, with one consistent with a very low mass of less than $10^{10} M_{\odot}$ (see Fig. 2), while the other has a more typical mass of $310^{11} M_{\odot}$ for luminous galaxies (Lowenthal et al. 1998). Given the unknown inclination of the plane of their relative motions and their true sizes or separations, more data are clearly needed to obtain the needed statistics to assess whether Lyman drop galaxies are generally large or small mass systems.

\section{Summary and Future Plans}

Our DEEP pilot programs with LRIS and HIRES clearly show the need and power of kinematics for galaxy surveys, since we have evidence that $\mathrm{M} / \mathrm{L}$ may vary by over a factor of 100 for galaxies. Moreover, our findings suggest that galaxy evolution is complex, and probably not mainly the result of one physical process such as merging or starbursts. For larger galaxies, both of early type or of spirals, we find that they have largely been a stable population out to redshift $z \sim 1$. In contrast, we find that compact galaxies, whether to redshift $z \sim 1$ or those seen as Lyman-drop galaxies at $z \sim 3$. constitute an important population for our understanding of galaxies, since they both contribute significantly to the evolution of the star-formation rate density. Yet the nature of these galaxies and 

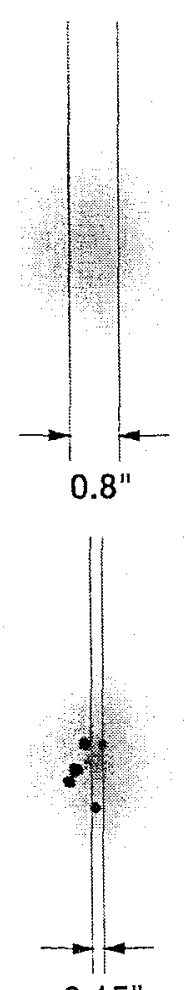
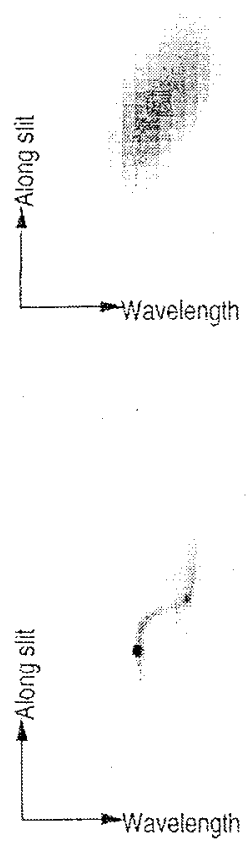

Figure 3. Simulations of an emission line rotation curve with and without using adaptive oplics on the Keck 10-m Telescope. The target has an exponential disk with a scale length of $0.2^{\prime \prime}$, inclination of $45^{\circ}$, a maximum velocity in the rotation curve of $40 \mathrm{~km} / \mathrm{s}$, and 6 knots of intense star formation. The upper left panel shows a $0.8^{\prime \prime}$ slit over the image under seeing of $0.6^{\prime \prime}$. The upper right panel shows the expected emission line using the DEIMOS instrument (Davis and Faber 1998) with $0.12^{\prime \prime}$ pixels in the vertical direction and $0.32 \AA$ pixels in wavelength. The lower two panels show the vast improvement in both imaging and spectroscopy with adaptive optics. The system is assumed to have a Strehl of 0.33 with a $0.05^{\prime \prime}$ core; a much narrower slit of $0.15^{\prime \prime}$ is adopted with a hypothetical spectrograph having $0.03^{\prime \prime}$ pixels in the vertical direction and a spectral resolution of $0.08 \AA$ per pixel. The star formation knots are clearly visible in the direct image and those within the narrow slit are seen in the spectra. This simulation was produced and generously provided by A. C. Phillips from efforts associated with the new NSF Science and Technology Center for Adaptive Optics. based at University of California, Santa Cruz. 
their link to galaxies today are unclear. In particular, whether those at $z<1$ are progenitors of low-mass spheroidals today or those at $z \sim 3$ are progenitors of more massive, evolved early-type galaxies at $z \sim 1$ remain important but are only among many unsolved puzzles in our understanding of galaxy formation and evolution.

The study of galaxy evolution is still very much in its infancy, but over the next decade, we will see an explosive growth in new data. Particularly valuable for galaxy evolution studies will be the completion of several large redshift surveys. These include the $2 \mathrm{dF}$ redshift survey (Folkes et al. 1999) and the Sloan Digital Sky Survey (SDSS: Loveday 1998), which will firmly set the foundation for the local properties of diverse galaxy types; DEEP, which will aim for about 50,000 galaxies at $z \sim 1$ with good kinematic information; and the VIRMOS survey on the VLT (Le Fevre et al 1998), which, though with limited kinematic data, will access both optical and near-IR. In principle, the kinematic surveys can be used to estimate the masses of galaxies on larger scales than actually observed in the luminous portions of galaxies, by measuring the distribution of relative velocities of galaxy pairs at different separations. More direct measures of dynamical mass on larger scales are likely to come from weak lensing surveys, several of which are already underway; some will take advantage of photometric redshifts to vastly improve the $\mathrm{S} / \mathrm{N}$ by discriminating between foreground and background sources. Moreover, adaptive optics (see Fig. 3) along with NGST will yield spatially resolved kinematics of very high redshift and even the most compact galaxies. Finally, planned enhancements to submm, $\mathrm{mm}$, and radio telescopes should provide direct measures of the amount and motions of various forms of gas in distant galaxies.

Acknowledgments. DEEP was initiated by the Berkeley Center for Particle Astrophysics (CAPA) and has been supported by various NSF, NASA, and STScI grants over the years, including NSF AST-9529098 and STScI AR07532.01-96. The senior members of DEEP have managed the project, but I would like to give special thanks to our talented pool of more junior astronomers (see DFFP URI for names), without whom the results presented here would not have been possible. R. Guzman, A. C. Phillips, and J. Lowenthal are especially thanked for providing the figures.

\section{References}

Baugh, C. M., et al. 1998, ApJ, 498, 504

Bershady, M. A. 1097, PASP Conf., 117, 537

Bershady, M. A. 1998, PASP Conf., eds. Merrit, Valluri, Sellwood, in press.

Bershady, M. A., et al. 1998, PASP Conf., 152, 253

Davis, M., and Faber, S. M. 1998, Wide Field Surveys in Cosmology, eds. S. Colombi, Y. Mellier. B. Raban (Editions Frontieres), 333

Folkes, S, et al. 1999, MNRAS, 308, 459

Gebhardt, K. et al. 2000, ApJ, in preparation

Guzman, R., et al. 1996, ApJ, 460, Lj

Guzman, R., et al. 1997, Ap.J, 489, 559 
Guzman, R., et al. 1998, ApJ, 495, L13

$\mathrm{Im}, \mathrm{M}$. et al. 2000, ApJ, in preparation

Kobulnicky, H. A., and Gebhardt, K. 1999, Ap.J, submitted

Koo, D. C., et al. 1994, ApJ, 427, L9

Koo, D. C., et al. 1995, ApJ, 440, L49

Koo, D. C., et al. 1999, ApJ, in preparation

Le Fevre, O., et al. 1998, in Wide Field Surveys in Cosmology, eds S. Colombi, Y. Mellier, B. Raban (Editions Frontieres), 327

Lilly, S. J., et al. 1998, ApJ, 500, 75

Loveday, J., Pier, J. 1998, in Wide Field Surveys in Cosmology, eds. S. Colombi, Y. Mellier, B. Raban (Editions Frontieres), 317

Lowenthal, J. D. et al. 1997, ApJ, 481, 673

Lowenthal, J. D., Simard, L., Koo, D. C. 1998, PASP Conf. , 146, 110

Marleau, F. R., and Simard, L. 1998, ApJ, 505, 585

Mallen-Ornelas, G., et al. 1999, ApJ, 518, L83

Oke, B., et al. 1995, PASP, 107, 375

Phillips, A. C., et al. 1997, ApJ, 480, 543

Pettini, M., et al. 1998, ApJ, 508, 539

Rix, H.-W., et al. 1997, MNRAS, 285, 779

Roche, N., et al. 1998, MNRAS, 293, 157

Schade, D., et al. 1996a, ApJ, 464, 63

Schade, D., et al. 1996b, ApJ, 465, 103

Simard, L., Pritchet, C. 1998, ApJ, 505, 96

Simard, L., et al. 1999, ApJ, 519, 563

Steidel, C. C., et al. 1996a, ApJ, 462, L17

Steidel, C. C., et al. 1996b, AJ, 112, 352

Steidel, C. C., et al. 1998, ApJ, 492, 428

Somerville, R. S., et al. 1999, MNRAS, Astro-Ph/9806228

Telles, E., and Terlevich, R. 1993, Ap\&SS, 205, 49

Vogt, S. et al. 1994, Proc. Soc. Photo-Optical Inst. Eng., 2198, 362

Vogt, N. P. et al. 1996, ApJ, 465, L15

Vogt, N. P. et al. 1997, ApJ, 479, L121

Vogt, N. P. et al. 2000, ApJ. in preparation 
The Hy Redshift Universe

ASP Conference Series, Vol. 193, 1999

A. J. Bunker \& W. J. M. van Breugel, eds.

$\because$

\title{
The View of Galaxy Formation from Hawaii: Seeing the Dark Side of the Universe
}

\author{
L.L. Cowie and A.J. Barger \\ Institute for Astronomy, University of Hawaii, 2680 Woodlawn Drive, \\ Honolulu, HI 96822
}

\begin{abstract}
The strength of the submillimeter background light shows directly that much of the energy radiated by star formation and AGN is moved to far infrared wavelengths. However, it is only as this background at $850 \mu \mathrm{m}$ has been resolved with direct submillimeter imaging that we have seen that it is created by a population of ultraluminous (or near ultraluminous) infrared galaxies (ULIGs) which appear to lie at relatively high redshifts $(z>1)$. Mapping the redshift evolution of this major portion of the universal star formation has been difficult because of the poor strbmillimeter spatial resolution, but this difficulty can be overcome by using extremely deep $\mathrm{cm}$ continuum radio observations to obtain precise astrometric information since the bulk of the brighter submillimeter sources have detectable radio counterparts. However, with this precise position information available, we find that most of the submillimeter sources are extremely faint in the optical and near infrared $(I>>24$ and $K=21-22$ ) and inaccessible to optical spectroscopy. Rough photometric redshift estimates can be made from combined radio and submillimeter energy distributions. We shall refer to this procedure as millimetric redshift estimation to distinguish it from photometric estimators in the optical and near IR. These estimators place the bulk of the submillimeter population at $z=1-3$, where it corresponds to the high redshift tail of the faint $\mathrm{cm}$ radio population. While still preliminary, the results suggest that the submillineter population appears to dominate the star formation in this redshift range by almost an order of magnitude over the mostly distinct populations selected in the optical-ultraviolet.
\end{abstract}

\section{Introduction}

The cosmic far infrared (FIR) and submillimeter (SMM) background, which is the cumulative rest frame FIR emission from all objects lying beyond uur Galaxy, has recently been detected by the FIRAS and DIRBE experiments on the COBE satellite (Puget et al. 1996; Guiderdoni et al. 1997; Schlegel et al. 1998; Fixsell et a1. 1998; Hauser et al. 1998) and found to be comparable to the total unobscured emission at optical/UV wavelengths. This result shows directly that much of the energy released by the totality of star formation and AGN radiation through the lifetime of the universe has been dust absorbed and reradiated into the restframe FIR. This in turn implies that. to obtain a full accounting of the history 


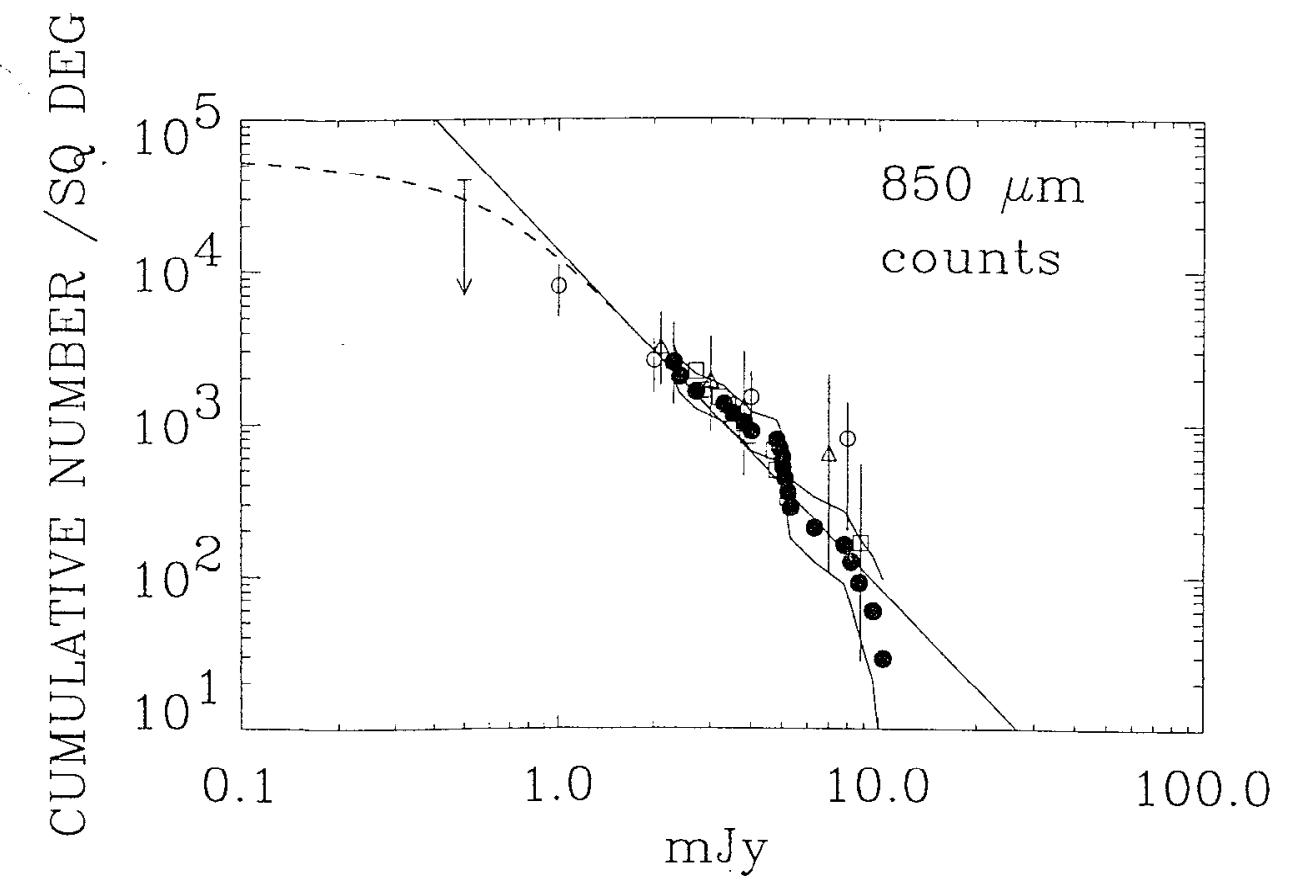

Figure 1. The $850 \mu \mathrm{m}$ source counts from Barger, Cowie, and Sanders 1999 (solid circles) with $1 \sigma$ error limits (jagged solid lines) are well described by the power-law parameterization in Eq. 1 with $a=0.4-1.0, \alpha=3.2$, and $N_{0}=3.0 \times 10^{4} \mathrm{deg}^{-2} \mathrm{mJy}^{-1}$ (solid line). The dashed curve shows a smooth extrapolation of this fit to match the EBL measurements using the value $a=0.5$. Counts from Blain et al. (1999) (open circles), Hughes et al. (1998) (open triangles), and Eales et al. (1999) (open squares) are in good agreement with our data and the empirical fit.

of the universal star formation, we must turn our attention to this dark side of the universe.

\section{Resolving the submillimeter background}

The first stage in this process is to locate the individual objects giving rise to the background. Resolution of the extragalactic submm background at $850 \mu \mathrm{m}$ became possible almost simultaneously with the measurement of the background when the Submillimeter Common User Bolometer Array (SCUBA; Holland et al. 1999) was installed on the 10-m James Clerk Maxwell Telescope (JCMT) on Mauna Kea. SCUBA's sensitivity and area coverage enabled the sources producing the submm background to be directly imaged for the first time. The current count determinations determined from blank field surveys and from cluster lensed fields (Smail. Ivison \& Blain 1997: Barger et al. 1998, 1999b; Hughes et al. 1998: Blain et al. 1999; Eales et al. 1999) are shown in Figure 1. Barger, Cowie and Sanders have shown, using optimal fitting techniques combined with 
Monte Carlo simulations of the completeness of the count determinations, that the cumulative counts are well fit by a power law above $2 \mathrm{mJy}$. In addition, they showed that, in order to match the background and fit to the very limited (one source!) information at fainter magnitudes from the lensed sample (Blain et al. 1999), a differential source count law

$$
n(S)=N_{0} /\left(a+S^{3.2}\right)
$$

was reasonable. Here $S$ is the flux in $\mathrm{mJy}, N_{0}=3.0 \times 10^{4}$ per square degree per $\mathrm{mJy}$, and $a=0.4-1.0$ is chosen to match the $850 \mu \mathrm{m}$ extragalactic background light. The 95 percent confidence range for the power law index is from 2.6 to 3.9. The extrapolation suggests that the typical SMM source producing the bulk of the background lies at around $1 \mathrm{mJy}$, and the direct counts show that roughly 30 percent of the $850 \mu \mathrm{m}$ background comes from sources above $2 \mathrm{mJy}$.

Provided only that the redshifts lie near or above $z=1$ (see below), the farinfrared (FIR) luminosity is approximately independent of the redshift. Thus, if we assume an Arp 220-like spectrum with $T=47 \mathrm{~K}$ (e.g., Barger et al. 1998), the FIR luminosity of a characteristic $\sim 1 \mathrm{mJy}$ source is in the range $4-5 \times 10^{11} h_{65}^{-2} \mathrm{~L}_{\odot}$ for a $q_{0}=0.5$ cosmology $\left(T-15 \times 10^{11}\right.$ for $\left.q_{0}=0.02\right)$. The FIR luminosity provides a measure of the current star formation rate (SFR) of massive stars (Scoville \& Young 1983; Thronson \& Telesco 1986), SFR $1.5 \times 10^{-10}\left(L_{F I R} / \mathrm{L}_{\odot}\right) \mathrm{M}_{\odot} \mathrm{yr}^{-1}$; a $1 \mathrm{mJy}$ source would therefore have a star formation rate of $\sim 70 \mathrm{~h}_{65}^{-2} \mathrm{M}_{\odot} \mathrm{yr}^{-1}$ for $q_{0}=0.5$, placing the 'typical' submm source at or above the high end of extinction-corrected SFRs in optically-selected galaxies (Pettini et al. 1998). If we were to allow the dust temperature to go as low as $30 \mathrm{~K}, L_{F I R}$ and the corresponding SFR would be $\sim 4$ times smaller.

\section{Direct attempts at a redshift distribution}

The identification of the optical/near-infrared counterparts to the SCUBA sources is made difficult by the uncertainty in the $850 \mu \mathrm{m}$ SCUBA positions and by the intrinsic faintness of the counterparts. Barger et al. (1999c) presented a spectroscopic survey of possible optical counterparts to a flux-limited sample of galaxies selected from the $850 \mu \mathrm{m}$ survey of massive lensing clusters by Smail et al. $(1997,1998)$. The advantage of a lensed survey is that the clusters magnify any background sources, thereby providing otherwise unachievable sensitivity in the submm, and easing spectroscopic follow-up in the optical. In the Barger et al. survey, identifications were attempted for all objects in the SCUBA error-boxes that were bright enough for reliable spectroscopy; redshifts or limits were obtained for 24 possible counterparts to a complete sample of 16 SCUBA sources. The redshift survey produced reliable identifications for six of the submm sources: two high redshift galaxy pairs ( $a z=2.8 \mathrm{AGN} /$ starburst pair (Ivison et al. 1998) and a $z=2.6$ Lyman-break-like pair (Ivison et al. (1993)), two galaxies showing $A G N$ signatures $(z=1.16$ and $z=1.06)$, and two $c D$ galaxies (cluster contamination). The galaxy pairs were later confirmed as the true counterparts through the detection at their redshifts of $\mathrm{CO}$ emission in the millimeter (Frayer et al. 1998, 1999). Because AGN are very uncommon in optically selected spectroscopic samples, it is also probable that the $A G N$ identifications are correct, and they place a rough lower limit of about 20 percent 
on the fraction of the submillimeter sources which have AGN characteristics. These results suggest that, excluding the cluster objects, about a quarter of the submillimeter sources can be spectroscopically identified.

However, two of the submillimeter sources in the sample have no counterparts to $I$ around 26 and, while the remaining eight sources have optical galaxies within the large error circles, these are rather normal objects which may simply be chance projections. We shall show in the next section that this is very probably the case. These missing sources may be at higher redshifts, or be more dust obscured, than the spectroscopically identifiable sources. Furthermore Smail et al. (1999), using deep near IR and optical imaging of the fields, have recently detected two extremely red objects which may be the counterparts of two of these sources, rather than the nearby bright spiral galaxies which Barger et al. observed as the most likely counterparts to the SMM sources. This result also suggests that many of the optical source identifications may be suspect.

\section{Positional determination from $\mathrm{cm}$ continuum radio observations}

To proceed further we need accurate astrometric positions, and these are most easily obtained using $\mathrm{cm}$ radio continuum observations. Because of the well known radio-FIR correlation, both the $\mathrm{cm}$ data and the submillimeter observations are linearly dependent on the star formation rates in the galaxy (Condon 1992), though the ratio of the $850 \mu \mathrm{m}$ flux to the $\mathrm{cm}$ radio flux rises rapidly as a function of redshift because of the opposite signs of the $\mathrm{K}$-correction in the two wavelength ranges. (We discuss this further in the next section.) Because of the redshift dependence, a cm flux-limited sample will contain a high proportion of lower redshift objects, while the $850 \mu \mathrm{m}$ sample will choose out primarily the high redshift objects.

The flanking field region of the Hubble deep field (the HFF) is well suited to looking at the radio versus submillimeter selection. Eric Richards (1999) has recently obtained an extremely deep VLA $20 \mathrm{~cm}$ image of this region, with a relatively uniform $(1 \sigma=8 \mu \mathrm{Jy})$ sensitivity over the whole flanking field region, which can be combined with the deep ground based optical and near IR (NIR) imaging of the HFF (Barger et al. 1999a). Richards et al. (1999c) find that roughly two thirds of the $5 \sigma$-selected $20 \mathrm{~cm}$ population have relatively bright optical/NIR counterparts while the remaining third are very faint. Barger, Cowie, and Richards (1999) have observed a complete subsample of the radio-selected objects with the LRIS on the Keck II 10m telescope, and find that nearly all the objects with $K<20$ can be spectroscopically identified, with a maximum redshift of around 1.2; however, almost none of the fainter objects were identified.

From a total sample of 70 radio selected galaxies in the $\mathrm{HFF}$ region, Barger et al. (1999c) chose the 16 with $K>21$ for follow-up with SCUBA. However, because they used the jiggle map mode which provides approximately a 5 square arcminute field around the target. a large fraction of the remaining radio sources $(35 / 54)$ were also serendipitonsly measured. 14 of the 16 targeted blank field sources were observed. Even. with relatively shallow SCUBA observations ( $3 \sigma=$ $6 \mathrm{mJy}$ at $850 \mu \mathrm{m}$ ) a very large fraction of the blank field radio sources were detected in the submillimeter, as is illustrated in Figure 2. Of the 14 targeted sources, 5 are detected above $6 \mathrm{mJy}$ while by contrast none of the 35 optical/NIR 

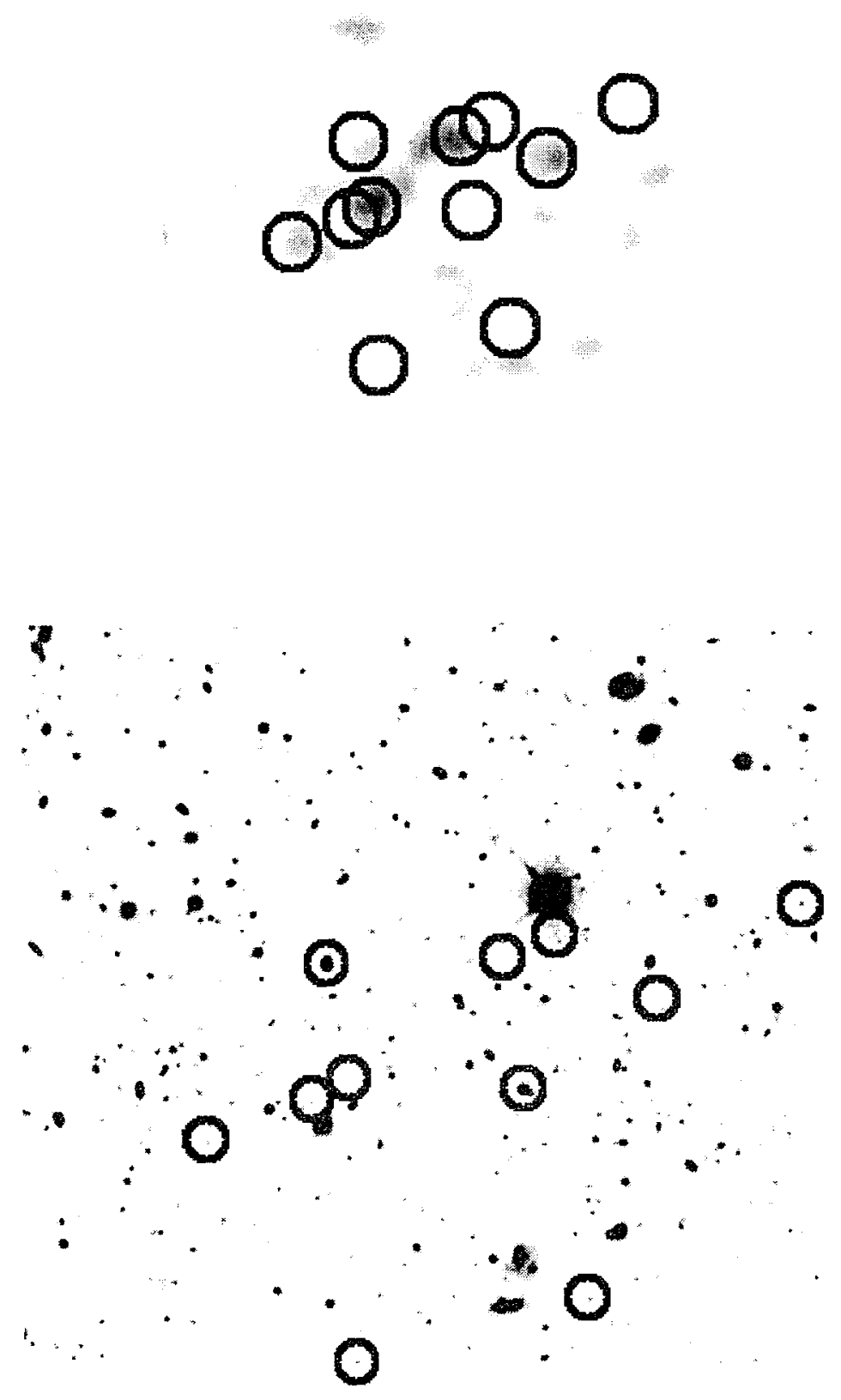

Figure 2. Radio sources in the HFF: the figure shows an overlay of the $20 \mathrm{~cm}$ radio sources in a small region of the $\mathrm{HFF}$ on a SCUBA $850 \mu \mathrm{m}$ image on the top and on a near IR image of the region on the bottom. ( $N$ is to the right and $\mathrm{E}$ to the top in these images.) In general it is the radio sources which are faint in the optical and near IR that are detected in the submillimeter. 


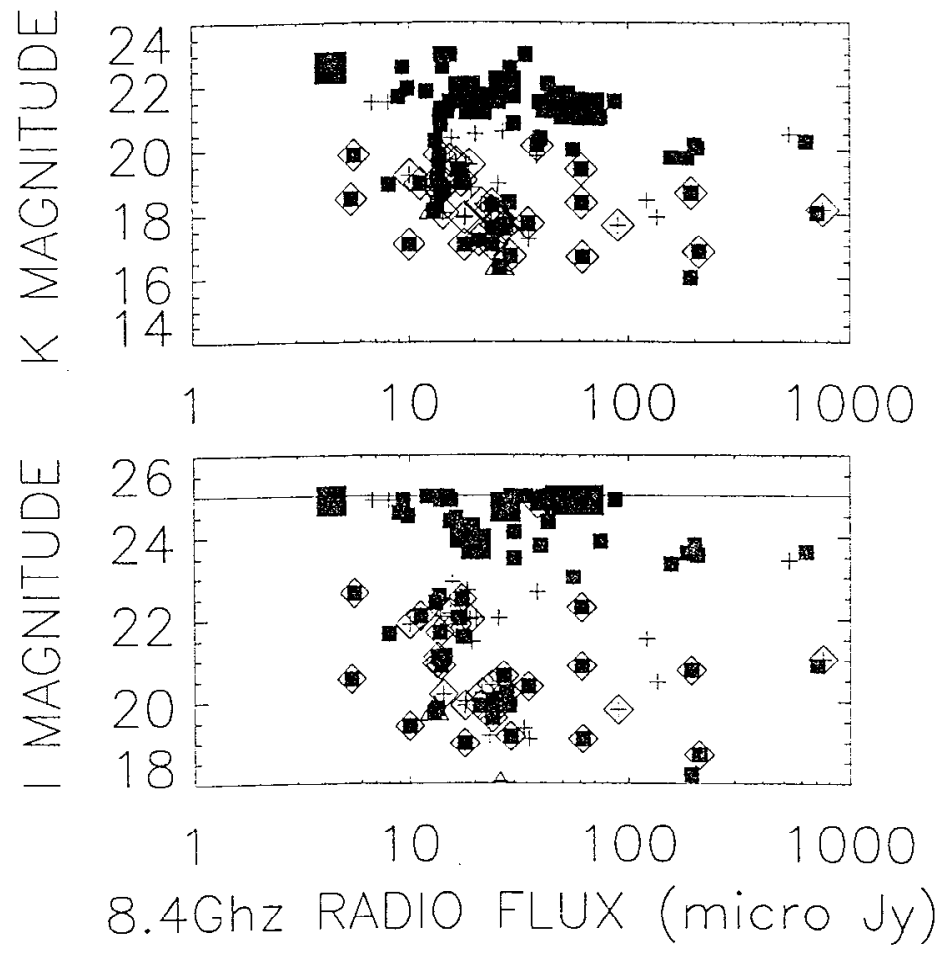

Figure 3. The optical and NIR magnitudes of the radio sources in the HFF and the SSA13 field versus $8.4 \mathrm{GHz}$ flux: the whole radio population is shown as crosses with sources which only have $20 \mathrm{~cm}$ fluxes extrapolated to $8.4 \mathrm{GHz}$ assuming a synchrotron spectrum. Sources with known redshifts are shown with open diamonds and all lie at $z<1.2$ except for two quasars in the SSA13 field (Windhorst et al. 1995) which are shown as triangles. Sources which have been observed in the $850 \mu \mathrm{m}$ but not detected at the typical $6 \mathrm{mJy}(3 \sigma)$ level are shown as the small squares while those detected at $850 \mu \mathrm{m}$ are shown as the large squares.

bright sources were detected. In the observed fields, which covered slightly more than half of the HFF, a further two sources brighter than $6 \mathrm{mJy}$ were discovered which were not in the radio sample. Even if there are further non-radio-detected submillimeter sources at the same level in the remaining unobserved portions of the HFF. it appears that the radio selection is turning up the majority of the bright submillimeter sources.

The fact that many of the bright submillimeter sources can be identified with the optical/VIR faint radio sources in this way has the extremely important corollary that many of the $850 \mu \mathrm{m}$-selected sources have extremely faint optical/NIR counterparts. This is illustrated in Figure 3, where we show the $F^{-}$ and I magnitudes of radio-selected sources in the HFF, and also in the SSA13: field (Richards et al. $1999 \mathrm{c}$ and references therein) where a similar submillimeter survey has been carried out (Cowie, Barger, and Richards 1999). Extremely deep F observations with NIRC on the Keck I $10 \mathrm{~m}$ can yield detections of nearly all 
the $850 \mu \mathrm{m}$ detected radio sources, and these are found to lie in the $\pi=21-22$ range. However, many of the sources are not detected in the $I$ band at the $2 \sigma$ limit of $I=25$ for the HFF, and, for the HDF850.2 source in the HDF proper (Hughes et al. 1998), the source is not seen to $I=29$. The brightest sources lie in the $I=24-25$ range.

\section{Millimetric redshift estimation}

While it is clear from the work described in section 3 that a fraction of the submillimeter sources have optical and NIR counterparts that are bright enough for spectroscopic identification, the results of section 4 show that a very large fraction simply cannot be identified in this way. At the current time the small numbers of objects suggest that perhaps a quarter of the sources (of which a fairly large fraction have AGN characteristics) are bright in the optical and spectroscopically identifiable, while the remainder fall into the optical/near IR faint category. For this latter category of objects we will have to rely on photometric estimates using the shape of the spectral energy distribution in the radio and submillimeter, and the submillimeter to NIR ratios (Carilli and Yun 1999, Blain et al. 1999).

Carilli and Yun have suggested the use of the $850 \mu \mathrm{m}$ to $20 \mathrm{~cm}$ flux ratio as such a redshift indicator. Because of the opposing spectral slopes of the synchrotron spectrum in the radio and the black body spectrum in the submillimeter, the submillimeter to radio ratio rises extremely rapidly with redshift, as is shown in Figure 4, which is take from Barger, Cowie, and Richards (1999) where a much more extensive discussion may be found. The primary uncertainty in this quantity lies in the dust temperature dependence, which in the local ULIG sample produces a range in the ratio of about a multiplicative factor of 2 relative to Arp220.

We can test the estimator in a variety of ways. In Figure 4 we have shown the average submillimeter to radio ratios for the objects in the HFF with known spectroscopic redshifts. While none of these sources is individually detected, the average values are consistent with a null result at low redshifts but a strongly significant positive detection for the sources near $z=1$, which is extremely

consistent with the Arp220 ratio. Individual submillimeter sources with spectroscopic identifications are also broadly consistent with the expected ratios, though there is a suggestion that, as might be expected, those with the AGN characteristics have slightly lower ratios, although still within the broad general range.

The optically/near IR faint radio sources in the HFF with submillimeter detections are shown as the horizontal lines in the figure. The redshift estimator places them in the same broad general $z=1-3$ range as the typical spectroscopically identified sources. (For AGN we may be systematically underestimating the redshifts). Radio sources without $850 \mu \mathrm{m}$ detections probably lie at lower redshifts while the $850 \mu \mathrm{m}$ sources without radio counterparts may represent the high end redshift tail. 


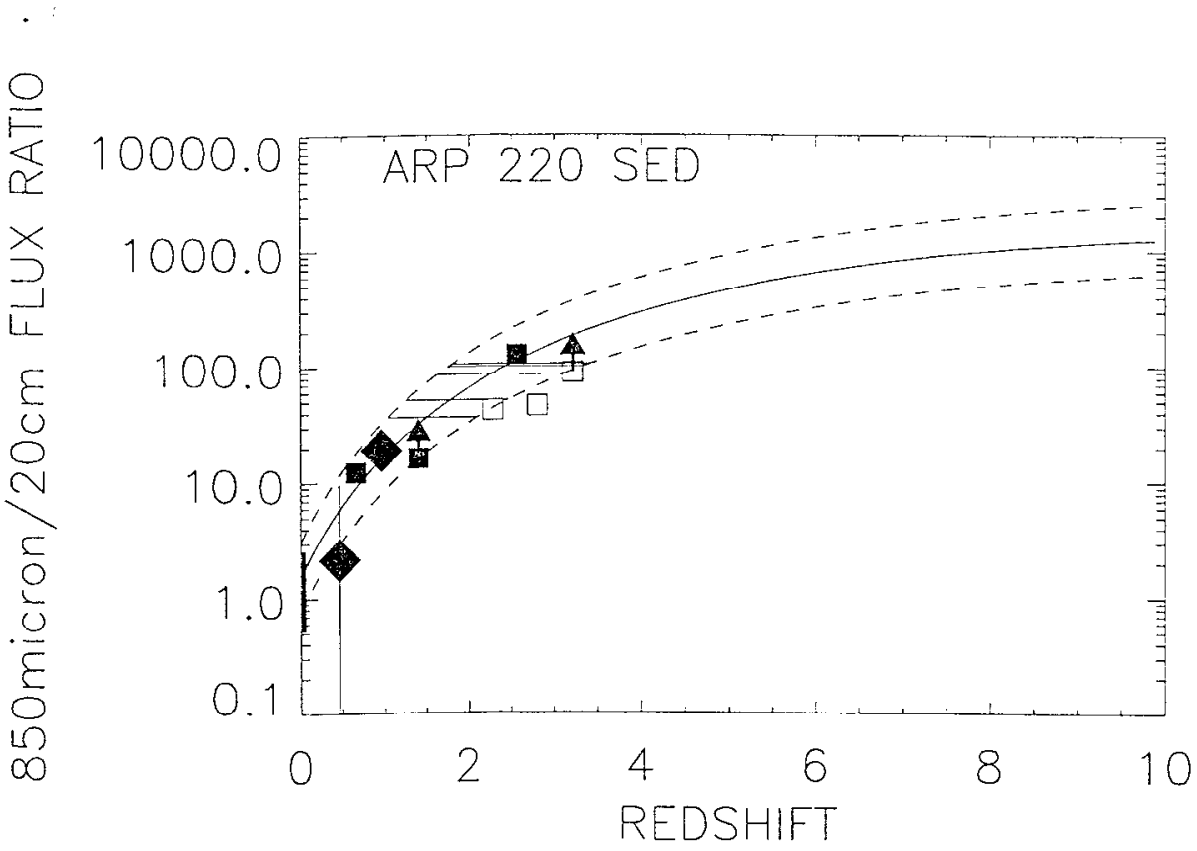

Figure 4. Millimetric redshift estimation: The solid curve shows the ratio of the $850 \mu \mathrm{m}$ to $20 \mathrm{~cm}$ flux that a non evolving ARP220 would have as a function of redshift. The solid bar at low redshift shows the range of the $850 \mu \mathrm{m}$ fluxes to the $8.4 \mathrm{GHz}$ flux at low redshift extrapolated to $20 \mathrm{~cm}$ assuming a synchrotron spectrum with the data taken from Rigopoulou et al. (1996). This suggest that the ULIGS have a range of about a multiplicative factor of 2 relative to ARP220 which is shown by the dashed lines. The average submillimeter to $20 \mathrm{~cm}$ ratio of the galaxies which have spectroscopic redshifts and also have been observed in the submillimeter are shown as the large diamonds with $1 \sigma$ errors. The lowest point is consistent with a null detection but in the higher redshift bin there is a strong positive detection consistent with an ARP220 ratio. Individually detected objects from Lilly et al. (1999) and Ivison et al. (1999) are also consistent within the error spread though those with $A G N$ characteristics (open symbols) appear to fall low in the figure. The solid line show the best guess for the redshift range (typically $z=1-3$ ) for the optically/NIR faint galaxies which are detected in the submillimeter. Radio objects which are not detected in the submillimeter are likely to lie at lower redshifts than this while submillimeter detected objects which are not seen in the radio are potentially at higher redshift. 


\section{Discussion}

We can summarise the results as follows. Roughly 30 percent of the $850 \mu \mathrm{m}$ background is already resolved and the slope of the counts is sufficiently steep (a power law index of -2.2 for the cumulative counts) that only a small extrapolation to fainter fluxes will result in convergence to the background. Thus, the typical submillimeter source contributing to the background seems to be in the 1-2 mJy range. Because of the direct correspondence between flux and luminosity at these wavelengths we may identify the sources with ULIGs or near ULIGs. About a quarter of the sources have optical counterparts which are bright enough to be spectroscopically identified, and a large fraction of these show AGN characteristics, though at least one is ail extremely bright pair of Lyman break galaxies. However, many of the remaining sources are extremely faint in the optical and NIR $(K=21-22)$. Redshift estimation for these sources using the submillimeter to radio ratios places the bulk of them in the same $z=1-3$ range as the spectroscopically identified sources.

It is interesting to consider where this population fits in the overall history of the universal star formation. One uncertainty in doing this is the question of what fraction of the SMM light is powered by AGN rather than star formation. It has long been debated whether the dust-enshrouded local ULIGs are powered by massive bursts of star formation induced by violent galaxy-galaxy collisions or by $A G N$ activity. A recent mid-infrared speclroscopic survey of 15 ULIGs by Genzel et al. (1998) found that 70-80 per cent of the sample are predominantly powered by star formation and $20-30$ per cent by a central AGN. Thus, while the spectroscopic follow-up studies of the gravitationally lensed submm sample (Barger et al. 1999c; Ivison et al. 1998) discussed in section 3 indicate that at least 20 per cent of the sample show some AGN activity, we shall assume in the following discussion that a substantial fraction of the submu light arises from star formation.

Several groups (Smail et al. 1998; Eales et al. 1999; Lilly et al. 1999; Trentham, Blain, \& Goldader 1999; Barger, Cowie, \& Sanders 1999) have suggested that the submm sources are associated with major Inerger events giving rise to the formation of spheroidal galaxies. The approximate equality of the optical and submm backgrounds supports this hypothesis; present-day spheroidal and disk populations have roughly comparable amounts of metal density, and thus their formation is expected to produce comparable amounts of light (Cowie 1988). Since the volume density of local ULIGS is very low (approximately $10^{-6} \mathrm{~h}_{65}^{3} \mathrm{Mpc}^{-3}$ for objects with bolometric luminosities above $5 \times 10^{11} \mathrm{~h}_{65}^{2} \mathrm{~L}_{0}$ e.g. Sanders and Mirabel 1996) it appears that the star formation rate in this population must have been much higher in the past and have declined very steeply after $z=1$, which may also be consistent with this interpretation. For a cumulative source density of $4.0 \times 10^{4} \mathrm{deg}^{-2}$ required to reproduce the EBL with $1 \mathrm{mJy}$ sources $(\langle N\rangle=\mathrm{EBL} /\langle S\rangle$ with $\langle S\rangle \sim 1 \mathrm{mJy})$ and redshifts in the $1-3$ range, the average space density is $5 \times 10^{-3} \mathrm{~h}_{6.5}^{3} \mathrm{Mpc}^{-3}$ for a $q_{0}=0.5$ cosmology $\left(10^{-3}\right.$ for $\left.q_{0}=0.02\right)$. This space density is rather insensitive to the upper cut-off on the redshift distribution, dropping by only a factor of $\sim 2$ or 3 if we extend the volume calculation to $z=5$. For comparison, the space density of present-day ellipticals is about $10^{-3} \mathrm{~h}_{6,5}^{3} \mathrm{Mpc}^{-3}$ (Marzke et al. 1994). Within the still substantial uncertainty posed by the dust temperatures, the 
. estimated star formation rate from submm sources in the $z=1-3$ range is $\sim 0.3 \mathrm{~h}_{6.5} \mathrm{M}_{\odot} \mathrm{yr}^{-1} \mathrm{Mpc}^{-3}$ for $q_{0}=0.5$, which is nearly an order of magnitude higher than that observed in the optical, $0.04 \mathrm{~h}_{65} \mathrm{M}_{\odot} \mathrm{yr}^{-1} \mathrm{Mpc}^{-3}$ (e.g., Steidel et al. 1999) suggesting that at these redshifts it is the submillimeter light which marks the bulk of the star formation.

Acknowledgments. We would like to thank our collaborators Eric Richards, Dave Sanders, Ian Smail, Rob Ivison, Andrew Blain and Jean-Paul Kneib.

\section{References}

Barger, A.J., Cowie, L.L., Sanders, D.B., Fulton, E., Taniguchi, Y., Sato, Y., Kawara, K., Okuda, H. 1998, Nature, 394, 248

Barger, A.J., Cowie, L.L., Irentham, N., Fulton, E., Hu, E.M., Songaila, A., Hall, D. 1999a, AJ, 117, 102

Barger, A.J., Cowie, L.L., Sanders, D.B. 1999b, ApJ, 518, L5

Barger, A.J., Cowie, L.L., Smail, I., Ivison, R.J., Blain, A.W., Kneib, J.-P. $1999 \mathrm{c}, \mathrm{AJ}$, in press

Barger, A.J., Cowic, L.L., Richards E.A. 1999, AJ, to be submitted

Blain, A.W., Kneib, J.-P., Ivison, R.J., Smai1, I. 1999, ApJ, 512, L87

Carilli, C.L., Yun, M.S. 1999, ApJ, 513, L13

Condon, J.J. 1992, ARA\&A, 30, 575

Cowie, L.L. 1988, in The Post-Recombination Universe, N. Kaiser \& A.N. Lasenby, Dordrecht: Kluwer, 1

Cowie, L.L., Barger A.J., Richards E.A. 1999 AJ, to be submitted

Fixsen, D.I., Dwek, E., Mather, J.C., Bennett, C.L., Shafer, R.A. 1998, Ap.J, 508,123

Eales, S. et al. 1999, ApJ, 515, 518

Frayer, D.T., Ivison, R.J., Scoville, N.Z., Yun, M., Evans, A.S., Smail, I., Blain, A.W., Kneib, J.-P. 1998, ApJ, 506, Li

Frayer, D.T., Ivison, R.J., Scoville, N.Z., Evans, A.S., Yun, M., Smail, I., Barger, A.J., Blain, A.W., Kneib, J.-P. 1999, ApJ, 514, 13L

Genzel, R. et al. 1998, Ap. 498,579

Guiderdoni, B., Bouchet, F.R., Puget, J.-L., Lagache, G., Hivon, E. 1997, Nature, 390,257

Hauser, M.G. et al. 1998, 508, 25

Holland, W.S. et al. 1999. MNRAS, 303, 659

Hughes, D.H. et al. 1998. Nature, 394, 241

Ivison, R., Smail, I., Le Borgne, J.-F., Blain, A.W., Fineib, J.-P., Bézecourt, J., Kerr. T.H., Davies, J.K. 1998; MNRAS. 298, 583

Ivison, R. et al. 1999, MNRAS, submitted.

Lilly, S.J. et al. 1999, ApJ. 518, 641

Marzke, R.O.. Geller, M.J., Huchra, J.P., Corwin. Jr., H.G. 1994, AJ, 108. 437 
Pettini, M., Kellogg, M., Steidel, C.C., Dickinson, M., Adelberger, K.L., Giavalisco, M. 1998, ApJ, 508, 539

Puget. J.-L., Abergel, A., Bernard, J.-P., Boulanger, F., Burton, W.B., Desert, F.-X., Hartmann, D. 1996, A\&A, 308, L5

Richards, E.A., Kellermann, K.I., Fomalont, E.B., Windhorst, R.A., Partridge, R.B. 1998, AJ, 116, 1039

Richards, E.A. 1999a, ApJ, 513, 9L

Richards, E.A. 1999b, ApJ, in press

Richards, E.A., Fomalont, E.B., Kellermann, K.I., Partridge, R.B., Windhorst, R.A., Cowie, L.L, Barger, A.J. 1999c, ApJ, submitted

Rigopoulou, D., Lawrence, A., Rowan-Robinson, M. 1996, MNRAS, 278,1049

Sanders, D.B., Mirabel, I.F. 1996, ARA\&A, 34, 749

Schlegel, D.J., Finkbeiner, D.P., Davis, M. 1998, ApJ, 500, 525

Scoville, N., Young, J.S. 1983, ApJ, 265, 148

Smail, I., Ivison, R.J., Blain, A.W. 1997, ApJ, 490, L5

Smail, I., Ivison, R.J., Blain, A.W., Kneib, J.-P. 1998, ApJ, 507, 21L

Smail, I., Ivison, R.J., Kneib, J.-P., Cowie, L.L., Blain, A.W., Barger, A.J., Owen, F.N., Morrison, G.E. 1999, MNRAS, in press, [astro-ph/9905246]

Steidel, C.C., Adelberger, K.L., Giavalisco, M., Dickinson, M., Pettini, M. 1999, [astro-ph/9811399]

Thronson, H.A., Telesco, C.M. 1986, A P.T, 311, 98

Trentham, N., Blain, A.W., Goldader, J. 1999, MNRAS, 305, 61

Windhorst, R.A., Fomalont, E.B., Kellermann, K.I., Partridge, R.B., Richards, E., Franklin, B.E., Pascarelle, S.M., Griffiths, R.E. 1995, Nature, 375, 471 


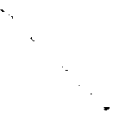


The Hy Redshift Universe

ASP Conference Series, Vol. 193, 1999

A. J. Bunker \& W. J. M. van Breugel, eds.

\title{
. Deep Field Surveys: The View from Pasadena
}

\author{
David W. Hogg ${ }^{1}$ \\ Institute for Advanced Study, Old.en. Tane, Princeton NJ 08540, USA
}

\begin{abstract}
The Caltech Faint Galaxy Redshift Survey has 1200 faint galaxy redshifts in two regions of the sky, with median redshift $z=0.6$. Spectral classifications of the galaxies is correlated with colors but not strongly with morphologies. No strong evolution of galaxies is found in their number density or characteristic luminosities or clustering properties. Strong evolution is found only in the prevalence and strengths of emission lines. This can be interpreted as a dramatic drop in average star formation rates since redshift unity. There are several hints of significant large-scale structure on scales of many leiss of $\mathrm{Mpc}$.
\end{abstract}

\section{Introduction}

The title of this talk was assigned by the conference organizers. Since this author now lives in Princeton, the title might more accurately contain an "of" than a "from." Furthermore, the group represented in this contribution is just one of a wide range of galaxy survey groups working in the several academic and laboratory institutions in Pasadena!

Hy Spinrad's publications show a steady stream of discoveries, many of which (though certainly not all of which!) are high-redshift galaxies, including $z=0.469$ in $1975, z=0.840$ in $1979, z=3.215$ in 1985 , and now $z=5.34$ just last year (Spinrad et al 1975; Smith et al 1979; Djorgovski et al 1985, 1987; Dey et al 1998; Spinrad et al 1998). As of this meeting, the Caltech Faint Galaxy Redshift Survey (J. G. Cohen, principal investigator) has two samples totalling $\sim 1200$ faint galaxy redshifts (median $z=0.6$ ), selected in the $R$ and $K$ bands, in two regions of the sky (Cohen et al 1996a, 1999a, 1999b, 1999c). Although the numbers of galaxies in the samples may be bigger, the current effort in Pasadena is directed at those redshifts Hy left behind back when this author was trying to convince his father to let him take the day off from elementary school to go see Toronto's new baseball team, the Blue Jays. One unfortunate consequence of Hy's work is that the meaning of the compound word "high redshift" changes on an annual basis, the definition being, roughly, "the redshift of Hy"s most recent galaxy." For this reason, the work described here will be termed a study of galaxy evolution from "intermediate redshift" to the present day.

Our work is motivated by the usual interests: How are the faint galaxies. seen at intermediate redshift related to galaxies we observe in the Milky Way

\footnotetext{
${ }^{1}$ Hubble Fellow
} 
and the local Universe? How have galaxies evolved with cosmic time? Can we confirm or refute predictions of structure and galaxy formation models? Are the observations consistent with all cosmological world models? Unfortunately, as will be seen below and in other contributions to this volume, the progress in definitively answering these questions (over, say, Hy's scientific career) has been significantly less marked than the technological advancement in, for example, our ability to observe extremely faint galaxies at a wide range of wavelengths. It is also less marked than Hy's progress in pushing the redshift limit.

There is one somewhat unusual, basic issue which was originally, and continues to be, a motivator for this faint galaxy research: There are a huge number of faint galaxies out there. The local density of galaxies (down to a few magnitudes below the characteristic luminosity $L^{*}$ ), multiplied by the comoving volume of the Universe, is only a few $10^{9}$, maybe $10^{10}$. But if we believe the Hubble Deep Field (HDF; Williams et al 1996), there are nearly $10^{11}$ faint galaxies observed all-sky, and that number appears to be increasing at a factor of nearly of 2 for every magnitude fainter the observations go. Some galaxies are expected to fade away, some are expected to merge into larger ones, some of those faint sources we count as separate may in fact be joined already but by galaxy appendages which have yet to "turn on." But it is safe to say that no convincing and complete story exists on what happens to those sources; many of the lines of evidence (e.g., searches for faded galaxy remnants near the Milky Way, measurements of merging rates from close pair statistics, evolution in the Tully-Fisher and Fundamental Plane relations, stellar population and kinematic studies of local galaxies) are ambiguous at best, contradictory at worst.

In this contribution, emphasis will be placed on only three aspects of the Caltech Faint Galaxy Redshift Survey: (1) The scale of a practical survey assembled over a few years by a small group (new to the field but with good Keck access), (2) the strong evolution in the distribution of spectral properties of galaxies from redshift unity to the present, which is not accompanied by nearly as strong an evolution in luminosities, and (3) apparently weak evolution in clustering properties.

Unless mentioned otherwise, the adopted world model is $H_{0}=60 \mathrm{~km} \mathrm{~s}^{-1} \mathrm{Mpc}^{-1}$ (or $h=0.6$ ), $\Omega_{M}=0.3$, and $\Omega_{\Lambda}=0.0$. All magnitudes are Vega-relative (sorry $\mathrm{Hy}$ !).

\section{The catalogs}

The survey has redshifts in two regions of the sky, one centered on 005323 +123358 (J2000), known as the "J0053+1234 region" the other centered on $123649+621258$ (J2000), known as the "HDF region."

The J0053+1234 region: Redshifts were initially obtained in a $2.0 \times 7.3 \mathrm{arcmin}^{2}$ field, in which identifications of 139 galaxies and 24 Galactic stars were made among 195 sources to $2.2 \mu \mathrm{m}$ flux limit $K \leq 20 \mathrm{mag}$ (Cohen et al 1999a). The survey is 84 percent complete to $K=20 \mathrm{mag}$. The redshifts go to $z=1.44$, with median redshift $z=0.58$. For adequate studies of clustering it is preferable to cover transverse spatial baselines of at least $10 \mathrm{Mpc}$; in the $.5053+1234$ region, six patches of sky ("subfields") were observed, distributed over a $1.2 \times 1.2 \mathrm{deg}^{2}$ 
area on the sky, surrounding the $J 0053+1234$ field. The configuration of the subfields is shown in Figure 1. The subfields contribute another 590 redshifts to the J0053+1234 region, selected at $22 \leq R \leq 23.5 \mathrm{mag}$, but they do not yet represent a complete, densely sampled catalog, given limited telescope time.

The Hubble Deep Field region: Redshifts have been obtained and compiled for 610 galaxies in a sky region centered on the HDF. The sources in this region are selected in the $R$ band, to $R=24 \mathrm{mag}$ in the deep HST-imaged portion of the HDF, and to $R=23 \mathrm{mag}$ in a circular field of 8 arcmin diameter centered on the deep portion. By these criteria, the HDF survey is 92 percent complete; perhaps the highest completeness level ever for a survey of this type. This completeness is very important for inferring evolutionary effects at intermediate redshift, as will be seen below. The imaging data, source selection procedures, and redshift catalog are described elsewhere (Hogg et al 1999; Cohen et al 1999c). The HDF survey has a similar number of galaxies to the J0053+1234 field, with the advantage that the HDF survey is of a simple solid angular shape, but with the disadvantage that pairs of galaxies in the HDF sample do not span as large a range of angular separations.

The spectroscopic observations were taken with the Low Resolution Imaging Spectrograph (LRIS; Oke et al 1995) on the 10-m W. M. Keck Telescope. They have a resolution corresponding to $\approx 200 \mathrm{~km} \mathrm{~s}^{-1}$. The redshifts and galaxy spectral classifications were determined as described elsewhere (Cohen et al 1999a, 1999c); galaxies will be referred to in what follows as "absorption" if their spectra are dominated by absorption lines, "emission" if by emission lines, and "intermediate" when both appear.

Just as an aside: at intermediate redshift, the broadband colors of galaxies are well correlated with spectral type (which is based on narrow features such as breaks and lines) just as they are in the local Universe. However, there is not nearly the strong correlation between spectral type and classic morphological type. It is not clear how convincing this latter result will become before the advent of widely accepted quantitative morphological classification.

\section{Galaxy spectral properties}

Figure 2 shows the magnitudes and redshifts of the galaxies in the HDF sample as a function of spectral type. The redshifts are plotted in terms of the comoving volume in the survey area to which they correspond, so a constant horizontal density of points in the plot corresponds to vanishing evolution in number density with redshift. The Figure shows a drop-off of number density at $z>1$. This is due to the selection problem that visual-band spectral features leave the LRIS spectroscopic window at redshifts just beyond unity (and are among sky lines at unity). The selection function is computed elsewhere (Hogg et al 1998b), but it is clear that the 8 percent incompleteness of the HDF sample must almost entirely be in this $z>1$ range; i.e. the sample is very close to perfectly complete at $z<1$.

Perhaps the most striking aspect of Figure 2 is the vertical striping. These stripes represent clumps in redshift space which we interpret to be galaxy groups; see discussion below. The existence of these groups makes it difficult, with only 


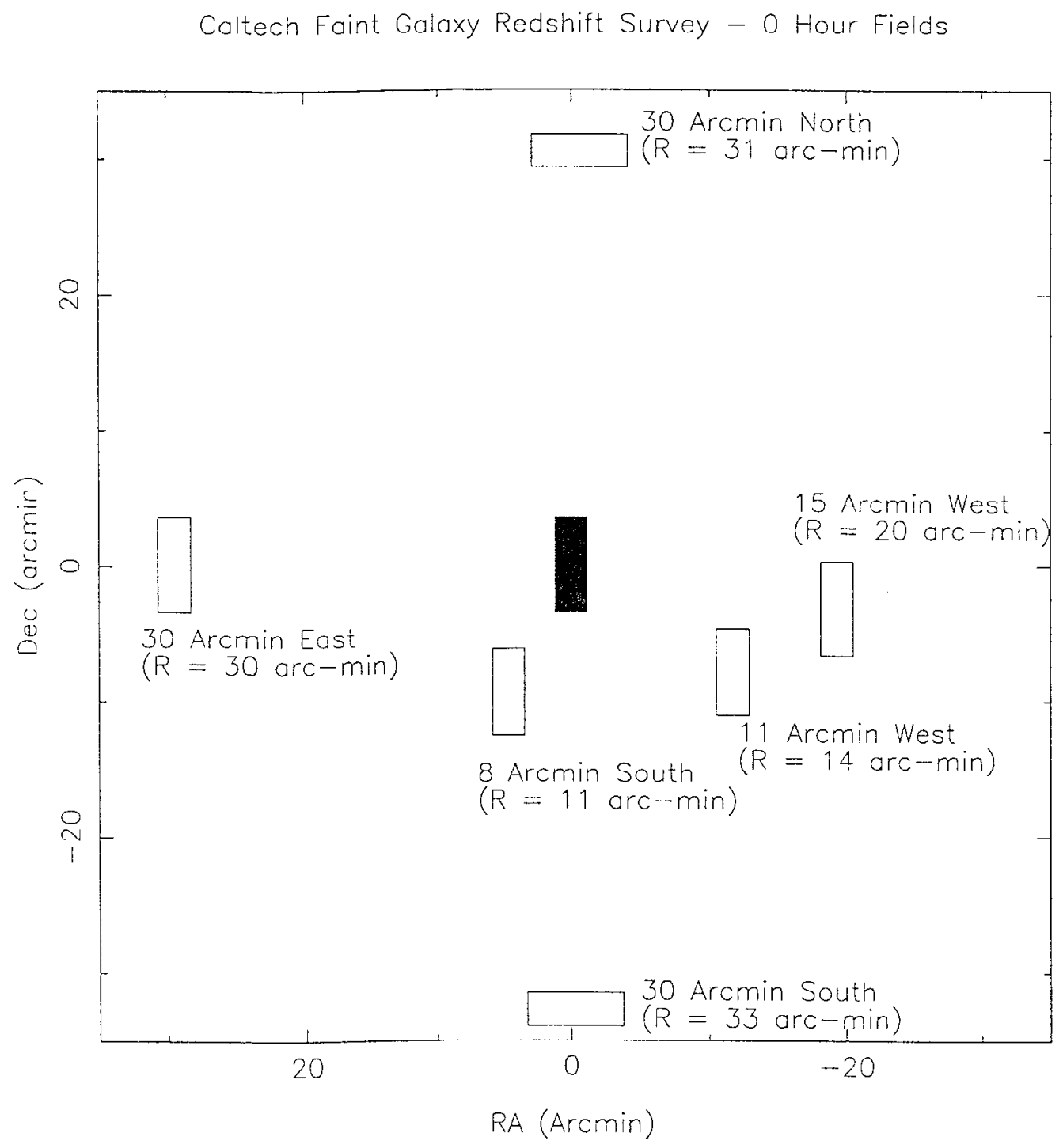

Figure 1. The layout of subfields in the J0053+1243 region. 


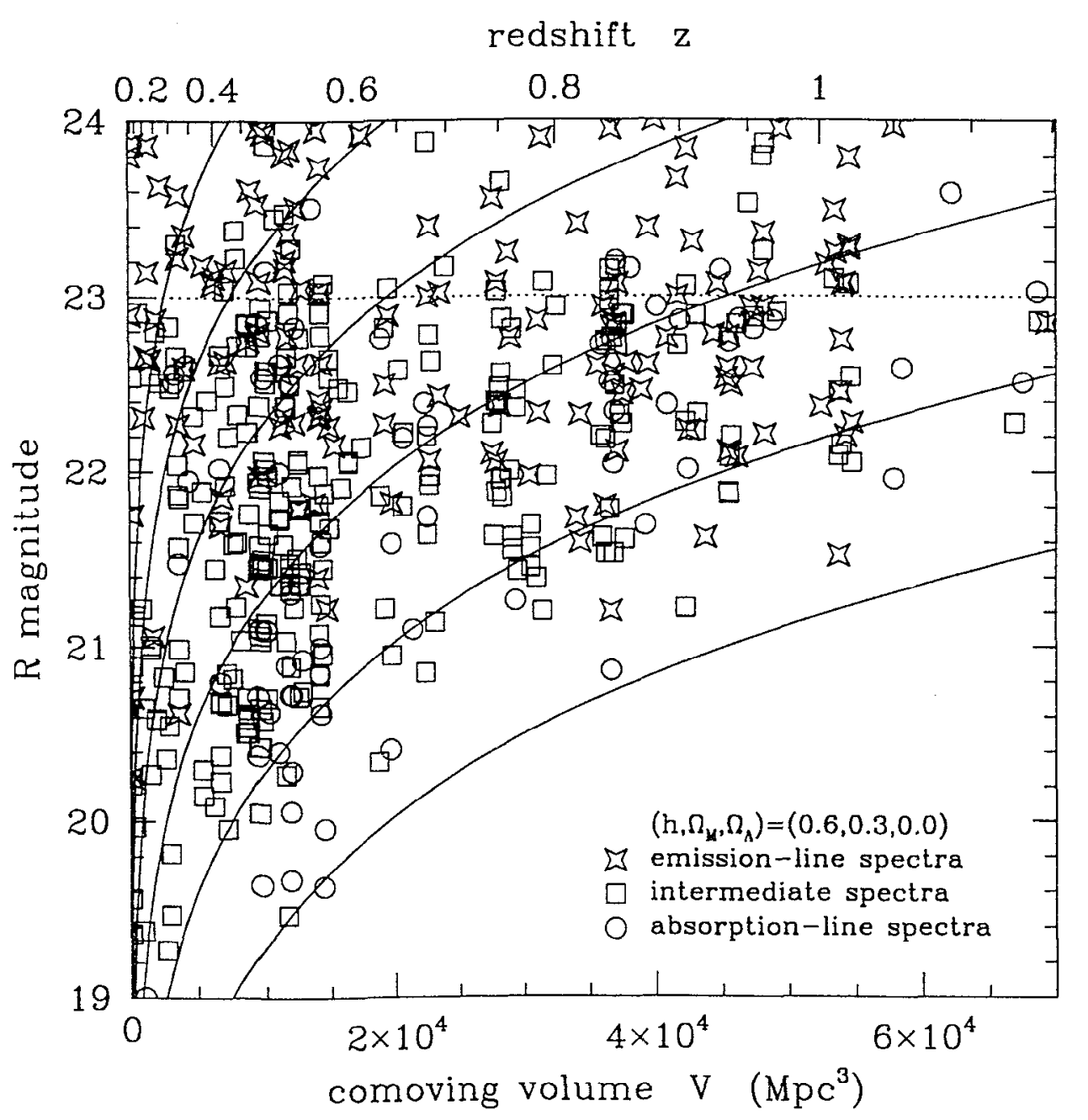

Figure 2. The apparent magnitudes and redshifts of the HDF sample, with redshift plotted as comoving volume. Different symbols represent galaxies with different spectral classifications. The dotted line shows the $R=23 \mathrm{mag}$ completeness limit for the entire 8 arcmin diameter circular field; the HST-imaged portion of the field is complete to $R=24 \mathrm{mag}$. Solid curves show loci of galaxies with fixed intrinsic luminosity (and spectral energy distribution constant in $\nu f_{\nu}$ ). 
a single field, to make clear statements about evolution in number density. The number density appears high at $z \sim 0.5$ in the Figure in part because, as can be seen, there are a few of these groups in the HDF which appear in this redshift region (Cohen et al 1996b). It may also appear high at $z \sim 0.5$ because at that redshift a wide range of intrinsic luminosities is being probed by the survey (and the luminosity function is dwarf-rich, or "steep" by that redshift; Lilly et al 1995; Hogg 1998a). When many fields are combined, there is no strong evidence for evolution in number density, especially at bright intrinsic luminosities (Lilly et al 1995).

Figure 2 is remarkable in the lack of evolution it shows in the characteristic luminosity $L^{*}$. If galaxies really are significantly younger at $z \sim 1$, why aren't they either brighter (because the stellar populations are younger), or fainter (because they have not yet been assembled from their component sub-galaxies)? The Figure argues for early formation, contrary to the predictions of most adiabatic CDM-like models, even with low matter density (e.g., Davis, this volume, and references therein). This weak (or no) evolution in $L^{*}$ is a multiply confirmed result (e.g., Songaila et al 1994; Lilly et al 1995; Hogg 1998a).

One mode of evolution which is clearly taking place in the Figure is in the fraction of emission-line dominated galaxies, especially at intrinsically bright magnitudes, where they are rare at low redshift (cf, Cowie et al 1996). This is not a selection effect; although it is much easier to get redshifts for sources which have emission lines, the sample is 92 percent complete (as defined above) and therefore there cannot be a huge contamination from sources with no redshifts. Especially since most of the unidentified 8 percent will of necessity be at $z>1$. This result, which would be impossible without good completeness, illustrates the considerable uncertainty in deriving evolutionary results from incomplete surveys; one of this group's obsessions is true redshift completeness.

A quantitative analysis of the emission lines in these samples shows a factorof-ten decline in the emission-line luminosity density of the Universe with cosmic time since $z \approx 1$ (Hogg et al 1998b). This is in good agreement with all other measures of star formation or hot star activity (e.g., Madau, this volume, and references therein; Yan, this volume) and is commonly interpreted as a dramatic decline in the star formation rate of the Universe (but see a somewhat dissenting view by Cowie, this volume). It is to be strongly emphasized that this change in apparent star formation activity is the only convincing (i.e., many-sigma, and multiply confirmed by qualitatively different methods) evolutionary effect known for normal field galaxies. Many other claims abound, but most are only a few sigma, and many require the comparison of qualitatively different data sets. It remains something of a puzzle how star formation rates can vary so dramatically since $z \sim 1$ while characteristic luminosities hold constant.

\section{Galaxy groups at intermediate redshift}

All redshift surveys, local to high redshift. show striking and undeniable clustering. Groups, redshift spikes, filaments, walls and clusters are prominent. Nowhere is this more true than in surveys of the type described here (e.g., Crampton et al 1995: Cohen et al 1996a, 1996b. 1999b). Striking galaxy struc- 
tures are found along every line of sight in abundance, from redshift $z=3$ (Steidel et al 1998) to the present day.

In a typical LRIS $\left(2 \times \bar{r} \operatorname{arcmin}^{2}\right)$ pencil-beam survey to redshift unity, there are five or six statistically significant peaks in the redshift distribution, with velocity dispersions in the hundreds of $\mathrm{km} \mathrm{s}^{-1}$ (Cohen et al 1996a, 1996b, 1999b). These five or six structures generally contain roughly half of the galaxies. What is now apparent from intercomparison of the seven fields in the J0053+1234 region is that the structures do not span the large angular separations between the subfields. Their proper extents are therefore on Mpc scales. The structures are too abundant (roughly $6 \times 10^{-4} \mathrm{Mpc}^{-1}$ in this $h=0.6$ cosmology) and too small in velocity dispersion to be large clusters of galaxies; they are galaxy groups. Virial estimates put their masses at a few $10^{13} M_{\odot}$. Given the great uncertainties, these numbers are not far off typical numbers for groups in the local Universe.

A tantalizing observation is that the galaxies comprising the groups show evidence of being related. Within a group, the colors and absorption feature strengths show far narrower distributions than the samples show as wholes (Cohen et al 1999b). It is not yet clear that this is a generic feature of these groups, but certainly the richest gromps show this effect. The natural interpretation is that galaxies in groups are coeval, or affected by one another's evolution in some important way. It is not clear that this fits into models of structure formation which form galaxies fairly late and involve constant infall of smaller mass concentrations into larger.

The distribution of the peaks along the line of sight is very clearly not poissonian; the peaks appear to be "self-avoiding." Since the typical separations are many tens of comoving Mpc, this observation argues-perhaps weakly-for some power on very large scales.

\section{Correlation lengths}

Unfortunately, the conventional measurement of galaxy clustering is the twopoint correlation function, an extremely blunt tool. The tool is blunt because a 200-galaxy pencil beam which shows undeniable, significant, strong redshift peaks (containing often over half of the galaxies in the pencil) will often show a barely significant two-point correlation function. In the absence of widelyused alternatives, however, the correlation function is still the most relevant for comparison of observed galaxy clustering with models of structure formation.

In what follows, the three-space correlation function $\xi(r)$ is not directly computed. Rather, a projected correlation function $\omega\left(R_{\perp}\right)$ is found through the angular correlation of galaxies close in redshift, very similar to the technique employed by the CHRS (Le Fevre et al 1996). The technique was complicated by the fact that the different individual subfields in the J0053+1234 region have somewhat different galaxy selection criteria, making the construction of the "random" catalog non-trivial. The correlation function estimates were compared with three-space correlation function $\xi(r)$ of the form $\left(r / r_{0}\right)^{-1.8}$. Results are shown in Figure 3. Error bars on the points are one-sigma, computed by: bootstrap resampling the $\mathrm{D}$ catalog, keeping track of the entire covariance matrix. It is important to note that these bootstrap-resampling error bars do not 


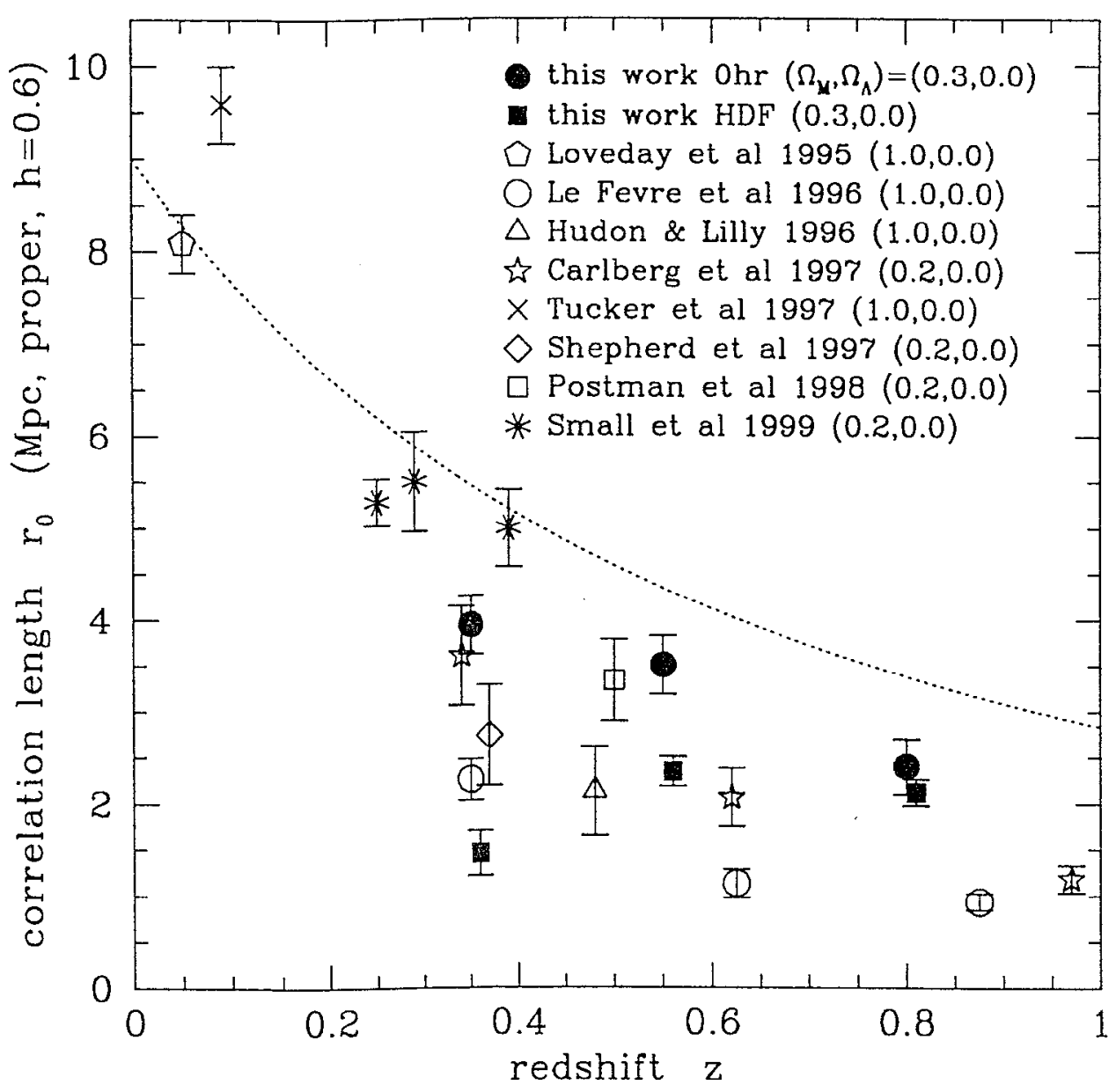

Figure 3. The best-fit correlation lengths $r_{0}$ in this work and from other studies. For this work, the fits were performed with exponent $\gamma$ fixed at 1.8. The dotted line shows the "stable clustering" prediction (eg, Peebles 1993) for $\gamma=1.8$ with $r_{0}=9.0 \mathrm{Mpc}$ at $z=0$. The results of the other work have been transformed to $h=0.6$ and proper coordinates. It should be noted that the other work is heterogeneous, with galaxies selected in many different wavs, in different magnitude and redshift ranges, with different assumed or fit values of $\gamma$, and in different cosmographic models. See the original references for details. 
include uncertainties due to sample variance, which, with a sample this small, are almost certainly large; compare the J0053+1234 and $\mathrm{HDF}^{\prime}$ results. The error bars do, however, include contributions from the covariances of the individual data points in the correlation function estimates. These covariances play a significant (and oft ignored) role in setting the uncertainties (Hogg et al in preparation).

The HDF sample shows weaker overall clustering than the J0053+1234 sample. This conld be due in part to the fact that the HDF was selected to be "empty"; i.e., devoid of large, bright galaxies, quasars, or radio sources (Williams et al 1996). Since this clustering analysis probes the non-linear regime, such selection criteria can strongly bias the results. This explanation is supported by the much lower clustering amplitude in the lowest redshift bin (Figure 3), which is the bin most affected by avoidance of bright galaxies. This emphasizes that field-to-field variations cannot be ignored.

When the galaxies are separated by spectral type, the absorption-dominated galaxies show stronger correlations at small separations. Of course the sample of absorption galaxies is small (less than one third of the overall sample), so sample variance may make this result somewhat uncertain. One indication that this may be important is that the points are not well-fit by a power-law correlation function. In fact, the correlation function shows apparently significant power at large radius (tens of $\mathrm{Mpc}$ ) among the absorption-dominated galaxies, a further hint that there is important power on very large scales (Hogg et al in preparation).

\section{Conclusion}

All the evidence-luminosities, number densities, group abundance and properties, clustering-points to early formation of galaxies and small amounts of evolution. The most strong, multiply confirmed, convincing evidence for evolution comes in the study of star formation rates. The Universe appears to be making stars at a rate much lower now than it was at redshift unity. It will be very exciting to see if this fits in with the fossil evidence in the stellar populations of the local group and nearby.

Acknowledgments. It is a pleasure to thank Hy Spinrad, Ivan King, and the other conference organizers for a fun and stimulating meeting. This work was all done in collaboration with Judy Cohen, the principal investigator of the Caltech Faint Galaxy Redshift Survey, and Roger Blandford. It is based primarily on observations made at the W. M. Keck Observatory, which is operated jointly by the California Institute of Technology and the University of California. Financial support was provided under NSF grant AST 95-29170 and Hubble Fellowship grant HF-01093.01-97A from STScI, which is operated by AURA under NASA contract NAS 5-26555. This research made use of the NASA ADS Abstract Service and the SM software.

\section{References}

in:

Carlberg R. G., Cowie L. L., Songaila A., Hu E. M., 1997, ApJ, 484, 538 
Cohen J. G., Hogg D. W., Pahre M. A., Blandford R., 1996a, ApJ, 462, L9

Cohen J. G., Cowie L. L., Hogg D. W., Songaila A., Blandford R., Hu E. M., - Shopbell P., 1996b, ApJ, 471, L5

Cohen J. G., Hogg D. W., Pahre M. A., Blandford R. D., Shopbell P. L., Richberg K., 1999a, ApJS, 120, 171

Cohen J. G., Blandford R. D., Hogg D. W., Pahre M. A., Shopbell P. L., 1999b, ApJ, 512, 30

Cohen J. G., Hogg D. W., Blandford R. D., Cowie L. L., Hu E., Songaila A., Shopbell P., Richberg K., 1999c, ApJ submitted

Cowie L. L., Songaila A., Hu E. M., Cohen J. G., 1996, AJ 112839

Crampton D., Le Fèvre O., Lilly S. J., Hammer F., 1995, ApJ 45596

Dey A., Spinrad H., Stern D., Graham I. R., Chaffee F. H., 1998, ApJ 498 L93

Djorgovshi S., Spinrad H., McCarthy P., Strauss M. A., 1985, ApJ 299 L1

Djorgovski S., Strauss M. A., Spinrad H., McCarthy P., Perley R. A., 1987, AJ 931318

Hogg D. W., 1998a, PhD thesis, California Institute of Technology

Hogg D. W., Cohen J. G., Blandford R., Pahre M. A., 1998, ApJ 504622

Hudon J. D., Lilly S. J., 1996, ApJ, 469, 519

Le Fèvre O., Hudon D., Lilly S. J., Crampton D., Hammer F., Tresse L., 1996, ApJ, 461, 534

Lilly S. J., Tresse L., Hammer F., Crampton D., Le Fèvre O., 1995, ApJ 455 108

Loveday J., Maddox S. J., Efstathiou G., Peterson B. A., 1995, ApJ, 442, 457

Oke J. B. et al, 1995, PASP 107375

Postman M., Lauer T. R., Szapudi I., Oegerle W., 1998, ApJ, 506, 33

Shepherd C. W., Carlberg R. G., Yee H. K. C., Ellingson E., 1997, ApJ, 479, 82

Small T. A., Ma C.-P., Sargent W. L. W., Hamilton D., 1999, astro-ph/9901194

Smith H. E., Junkkarinen V. T., Spinrad H., Grueff G., Vigotti M., 1979, ApJ 231307

Songaila A., Cowie L. L., Hu E. M., Gardner J. P., 1994, ApJS 94461

Spinrad H., Smith H. E., Hunstead R., Ryle M., 1975, ApJ 1987

Spinrad H., Stern D., Bunker A., Dey A., Lanzetta K., Yahil A., Pascarelle S., Fernández-Soto A., 1998, AJ 1162617

Steidel C. C., Adelberger K. L., Dickinson M., Giavalisco M., Pettini M., Kellogg M., 1908, ApJ 492428

Tucker D. L., Oemler A. Jr., Kirshner R. P., Lin H., Shectman S. A., Landy S. D., Schechter P. L., Müller V., Gottlöber S., Einasto J., 1997, MNRAS, 285, L.5 
The Hy Redshift Universe

ASP Conference Series, Vol. 193, 1999

A. J. Bunker \&s W. J. M. van Breugel, eds.

$\because$

\title{
- High Redshift Galaxies and the Hubble Deep Fields
}

\author{
Mark Dickinson \\ Space Telescope Science Institute, Baltimore MD
}

\begin{abstract}
The Hubble Deep Fields continue to be a valuable resource for studying the distant universe, particularly at $z>2$ where their comoving volume becomes large enough to encompass several hundred $L^{*}$ galaxies or their progenitors. The original HST images have been supplemented by deep observations at other wavelengths using a wide variety of ground-and space-based instruments. Here, I present some results from our near-infrared observalions of the HDF-North with NICMOS. I illustrate the photometric and morphological properties of galaxies at $2<z<3.5$, and consider whether there are other galaxies at those redshifts that have been missed by UV-based Lyman break selection. Using the well-characterized $z \sim 3$ galaxy population as a point of reference, I discuss color-selected galaxy candidates at $4.5<z<8.5$, as well as one remarkable object which might (or might not) be at $z>12$.
\end{abstract}

\section{Introduction}

For the past four and a half years, the Hubble Deep Field North (HDF-N) has served as the most exquisitely deep, high angular resolution optical census of the distant universe. It has also been my major occupation (or obsession) since leaving Berkeley and Hy Spinrad's tutelage. The images offer an opportunity to have a good, close look at the galaxy population at redshifts where once upon a time only $\mathrm{Hy}$ and a few, fearless others dared to tread. It is important (if somewhat pedantic) to consider what the HDF is actually good for. One WFPC2 field covers $5 \mathrm{arcmin}^{2}$, and probes a very small co-moving volume at $z<1$, enough to hold only $\sim 12-30 L^{\times}$galaxies, depending on the cosmology. Given small number statistics and concerns about clustering, the central HDF is therefore not the best place study massive galaxies in the "low" redshift universe, despite the fact that most of cosmic time and most bright galaxies with spectroscopic redshifts are at $z \lesssim 1$. There is far more volume at high redshift: 10.5 to $40 \times$ more at $2<z<10$ than at $z<1$ for plausible cosmologies, room enough for several hundred $L^{*}$ galaxies or their progenitors. For this reason, and also because it is most in the spirit of Hy's interests, I will concentrate here on galaxies at $z>2$, using new data which may help us to understand their properties, as well to continue the search for still higher redshift objects.

At $z>1$, the rest-frame optical light from galaxies shifts out of the optical bandpasses and into the near-infrared. Thus in order to compare $z>2$ galaxies to their local counterparts, and to search for still more distant objects at $z \gg 5$, it is important to extend the wavelength baseline. The HDF-N was observed 
in the near--IR from the ground in several different programs (e.g., Hogg et al. 1997; Barger et al. 1998; and our own KPNO $4 \mathrm{~m} J H \mathrm{~K}$ imaging, Dickinson 1998). The depth and angular resolution (typically $\sim 1^{\prime \prime}$ ) of these data are a poor match to that of the optical WFPC2 HDF images. 'T'wo programs therefore targeted the HDF-N with NICMOS on board HST, providing much deeper images with high angular resolution. The NICMOS GTOs (Ihompson et al. $1999)$ imaged one NIC3 field $\left(\sim 51^{\prime \prime} \times 51^{\prime \prime}\right)$ for 49 orbits each at F110W $(1.1 \mu \mathrm{m})$ and $\mathrm{F} 160 \mathrm{~W}(1.6 \mu \mathrm{m})$. Our own program mosaiced the complete HDF-N with a mean exposure time of 12600 s per filter in F110W and F160W. Sensitivity varies over the field of view, but the mean depth is $A B \approx 26.1$ at $S / N=10$ in an 0.7 diameter aperture. The drizzled PSF has $F W H M=0.22$, primarily limited by the NIC3 pixel scale. Because most galaxies have spectral energy distributions (SEDs) which brighten (in $f_{\nu}$ units) at redder wavelengths, our images detect whalf of the galaxies from the WFPC' $\mathrm{HDF}$, despite their short exposure times. We have also reanalyzed the KPNO $K_{s}$ images (see the contribution by Casey Papovich to this volume). These are not as deep as one would like, which is unfortunate because they provide the only access to rest-frame optical fluxes for objects at $3<z<4.4$, but they are the best data presently available.

I will use $A B$ magnitudes here throughout, and notate the six WFPC2 + NICMOS bandpasses by $U_{300}, B_{450}, V_{606}, I_{814}, J_{110}$ and $H_{160}$. I will often refer to $z>2$ galaxies as Lyman break galaxies, or LBGs, after the color selection technique used to identify them.

\section{Color selection and photometric redshifts}

Lyman break color selection requires three bandpasses ( 2 colors): one below the Lyman limit and/or Ly $\alpha$ forest breaks at the redshift of interest, and two above. Galaxies enter a color selection box at redshifts where one of these breaks reddens one color, and exit at some higher redshift where the breaks redden the other. In reality, galaxies do not all have the same intrinsic SEDs, nor is the opacity of the IGM (or the galaxies' ISM) uniform. This, along with photometric errors, introduces scatter so that objects move into and out of the box at somewhat different redshifts depending on their intrinsic SEDs (cf., Steidel et al. 1999). For these reasons, the method's selection efficiency is a function of redshift, intrinsic galaxy SED, and magnitude, i.e., $f(z, S E D, m)$, and is not a uniform top-hat in $z$. The advantages of the 2-color selection method are simplicity of application and relative robustness. With a large enough sample of spectroscopic calibrators, as we now have from our ground-based survey, and with realistic Monte Carlo simulations to understand detection efficiencies and photometric errors, one can reliably model $f(z, S E D, m)$, and derive the intrinsic distribution of galaxy colors and luminosities (e.g., Adelberger et al., in prep.).

As an alternative. full-up photometric redshifts fit the SED of each galaxy using all available bands. If they are well calibrated over the full range of galaxy types, phot-zs avoid the "hard edged" boundaries of the 2-color selection technique, i.e. there is no strict "redshift selection tunction." Phot-z fitting can also potentially identify objects whose intrinsic colors place them outside the conventional 2-color boundaries. Of course the photometric $z$ 's will have uncertainties, both random and occasionally systematic (i.e., due to poorly matched 
templates or multiple $\chi^{2}$ minima in $z_{p h o t}$ ). Thus galaxies may scatter in with some error distribution $\delta z_{p h o t}(m, S E D)$. Both color selection and photometric redshift fitting are occasionally subject to degeneracies, when objects at very different redshifts have similar observed colors, especially given real photometric errors. For example, galactic subdwarfs are the main contaminant for our ground-based $U_{n} G \mathcal{R}$ color-selected survey, while $\mathrm{M}$ dwarfs have have opticalto-near-IR colors which resemble those of $z \sim 4.5$ galaxies (cf. experience from QSO searches). Early type galaxies at $z \sim 0.8$ contaminate our $G \mathcal{R} i$ selection for $z \sim 4$ galaxies (Steidel et al. 1999) and must be weeded out spectroscopically. ${ }^{1}$ In general, however, the successes of both methods have been impressive.

In this discussion, I will occasionally use both 2-color selection and photometric redshifts. For the latter, I take phot-zs fit to our 7 -band HDF photometry by Budavari et al. (1999) using an "adaptive template" method (see also Csabai et al. 1999). The details of the technique are not important here: essentially, it is a modification of an otherwise straightforward SED template fitting scheme, like that used by many other groups.

\section{Galaxy SEDs and colors at $2<z<3.5$}

Figure 1 shows a compendium of photometry for spectroscopically confirmed HDF galaxies at $2<z<3.5$, all shifted to the rest frame and normalized to a common flux at $\lambda_{0} 2000 \AA$. The shaded envelope is defined by local UV-tooptical starburst galaxy spectral templates from Kinney et al. (1996), which span a broad range in optical/UV extinction. The HDF LBGs scatter between these envelopes. As an ensemble, the galaxies have relatively blue (but not flat spectrum) UV continua, with a flux increase and spectral "inflection" around the Balmer/4000 $\AA$ break region that indicates the presence of older ( $A$ and later) stars which apparently contribute a significant fraction of the rest-frame optical light. Considered individually, the large majority are reasonably well fit by the Kinney et al. "SB1" or "SB2" templates $(0<E(B-V)<0.21)$ : A few match "SB3," but very few approach the more heavily reddened SEDs. There is partially true by definition/selection, of course, since we are considering the brighter objects for which redshifts were successfully measured, and which were selected for spectroscopy by their UV colors.

Using a NICMOS-selected catalog, we may then ask what galaxies might be missed altogether by Lyman break color selection keyed to the rest-frame UV light. In particular, one might expect some red high redshift galaxies, either because they are not actively forming stars or because of extinction, that would "drop out" of the dropout samples. I restrict the analysis to $H_{160}<26$, where we believe our catalogs are highly complete, uncontaminated by spurious sources, and where the NICMOS photometry has $S / N \gtrsim 10$. The typical $L B G$ at $H_{160} \approx 26$ has $V_{606} \approx 27$, the practical limit for $H D F U_{300}$-dropout selection using standard 2 -color criteria, but red galaxies with similar rest-frame optical luminosities might be fainter or absent in the UV. At $H_{160}<26$ there

\footnotetext{
${ }^{1}$ These objects are less of a problem for conservative $B_{450} V_{606} I_{814}$ color selection criteria in the HDF due to the small photometric errors in the WFPC2 data.
} 


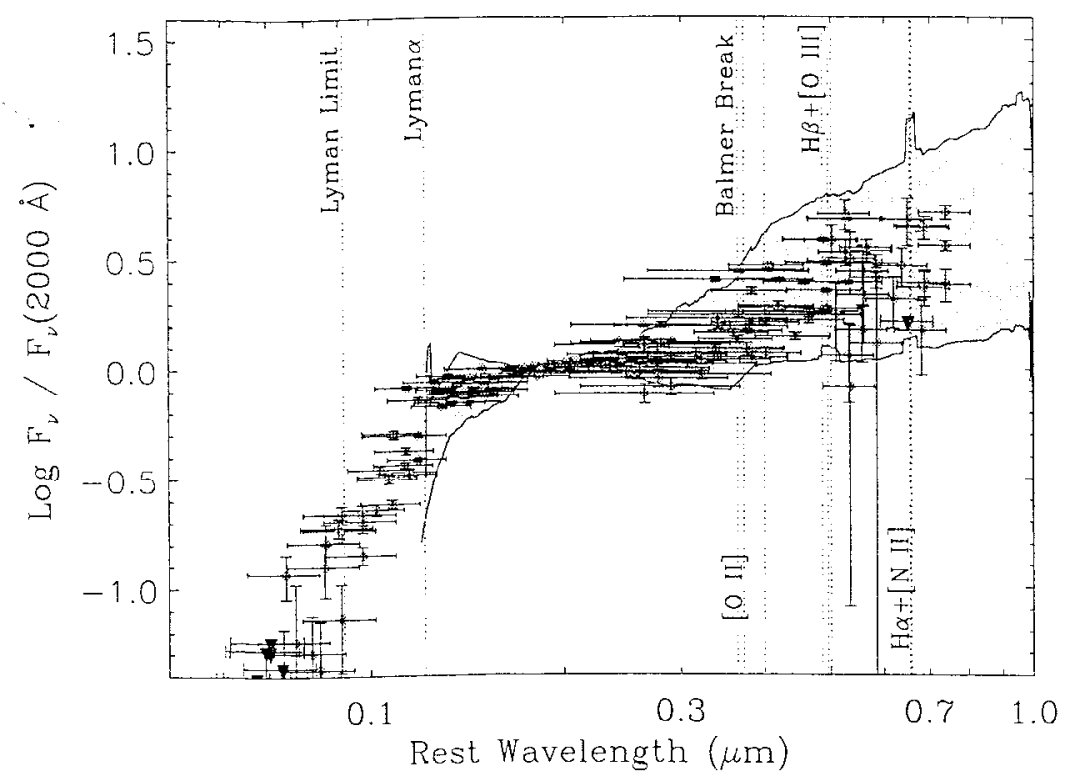

Figure 1. Photometry for $27 \mathrm{HDF}$ galaxies with spectroscopic redshifts, shifted to the rest frame and normalized at $2000 \AA$. The shaded region is that spanned by the library of empirical starburst templates from Kinney et al. (1996). This SED sequence is primarily defined by reddening up to $E(B-V) \lesssim 0.7$; the blue envelope is set by NGC1605.

is only one object which is undetected with $S / N<2$ in $V_{606}$ or $I_{814}$ (both, in this case): the so-called "J dropout" object HDFN-JD1 $\left(H_{160}=25.2\right)$, which I will discuss further in $\S 6$. In fact, this is the only NICMOS-selected object with $H_{160}<26$ and $S / N\left(I_{814}\right)<6.5$. Two other objects have $S / N\left(V_{606}\right)<3$; both are $z \gtrsim 5$ " $V_{606}$-dropout" candidates identified by Lanzetta et al. (1996) and Fernández-Soto et al. (1998), one of which (3-951) was spectroscopically confirmed at $z=5.34$ (Spinrad et al. 1999). Thus the only possible candidate for a NICMOS-selected, "UV-invisible" galaxy at $z \sim 3$ is HDF N-JD1.

Next let us consider UV-bright objects which might nevertheless have been missed by the LBG color criteria, using the 7 -band photometric redshift estimates for all galaxies. In principle, these may identify plausible candidates at $2<z<3.5$ that otherwise fall outside a given set of UV color criteria, as long as their intrinsic SEDs are "recognizably similar" to those of galaxies at lower redshift which define the templates used for the phot $-z$ fitting. In practise, the calibration of the HDF phot-zs at $z \sim 3$ is based on the spectroscopic redshifts in this range, which are almost all for galaxies that were selected by 2 -color dropout methods, and so there may be a bias for the phot-zs to work best for objects most similar to those selected by the standard dropout methods. Nevertheless, there are few better alternatives.

Figure 2 shows the $z_{\text {phot }}$ distributions for all $\mathrm{HDF}$ galaxies with $21<H_{160}<$ 26, and for those that meet the $U_{300}$ "dropout" criteria $\left(U_{300}-B_{450}\right)>\left(B_{450}-\right.$ $\left.V_{606}\right)+1$ (Dickinson 1998). There are 43 objects with $2<z_{\text {phot }}<3.5$ which do not meet the LBG color criteria. Nearly all are at $2<z_{\text {phot }}<2.2$ or 


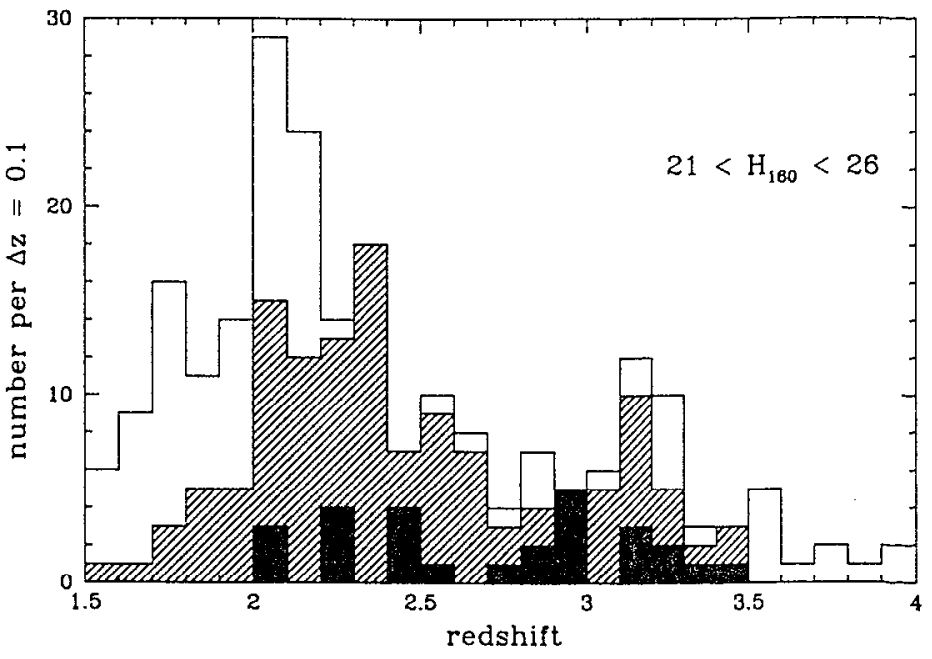

Figure 2. Photometric and spectroscopic redshift distributions in the HDF at $1.5<z<4$. The open histogram shows the $z_{p h o t}$ distribution from Budavari et al. (1999). The hatched histogram indicates galaxies which obey the Lyman break criteria defined in the text, while the filled histogram marks spectroscopic redshifts.

$3.2<z_{\text {phot }}<3.5$, and lie just outside color selection boundaries defined here: either slightly too blue at low $z$, and some slightly too red at high $z$. This is expected, as discussed in $\S 2$ and in Steidel et al. (1999). Only 7 objects fall at intermediate photometric redshifts, $2.5<z_{\text {phot }}<3.1$, and most of these are also just outside the color selection box. Some are quite interesting, including a $\mu \mathrm{Jy}$ radio source with very red $J_{110}-H_{160}$ colors which may be a dusty starburst or a fading post starburst galaxy at $z \sim 2.6$. Others may be scattered out of the box by photometry errors (especially in $U_{300}$ ), or might not be at the indicated $z_{\text {phot }}$. Overall, there is no evidence for a substantial population (by number) of galaxies at $2<z<3.5$ that are missed by UV Lyman break color selection but which are detectable in the NICMOS data.

\section{Morphologies}

The NICMOS $H_{160}$ images sample rest-frame wavelengths in the optical $B$-band or redder out to $z=2.8$. This is about the midpoint of the redshift range where traditional " $U_{300}$-dropout" Lyman break selection is most efficient. Therefore, at $2<z<2.8$ we may use the NICMOS data to study the morphologies and photometry of LBGs at wavelengths where long-lived stars, if they are present, may dominate the light from the galaxy, and where dust obscuration should play a significantly lesser role than it does in the ultraviolet. For higher redshift LBGs, the NICMOS $H_{160}$ bandpass slips into the rest-frame ultraviolet.

Figure 3 compares WFPC2 and NICMOS images of a set of HDF-N LBGs with spectroscopic redshifts $2<z<3$. The NICMOS data have somewhat poorer angular resolution $\left(00^{\prime \prime 2} 2\right.$ compared to 0 "' 14 for WFPC2), but otherwise 

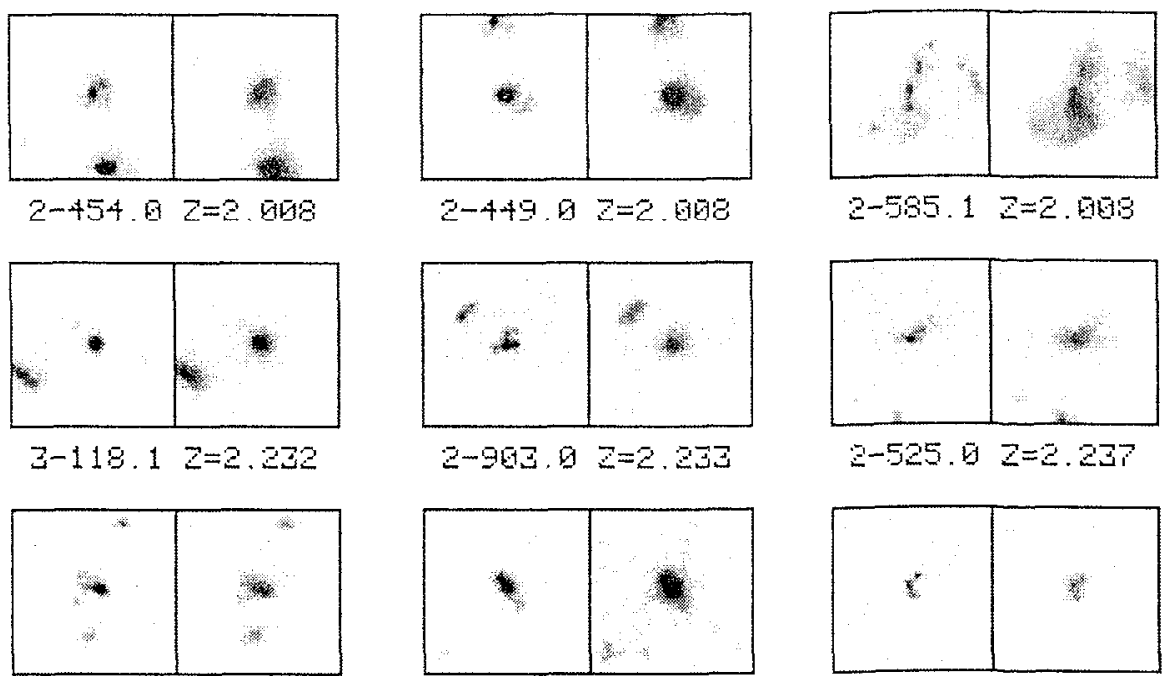

$z-\theta z, 1 \quad z=2.267$

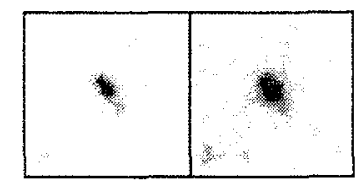

$4-445.92=2.268$
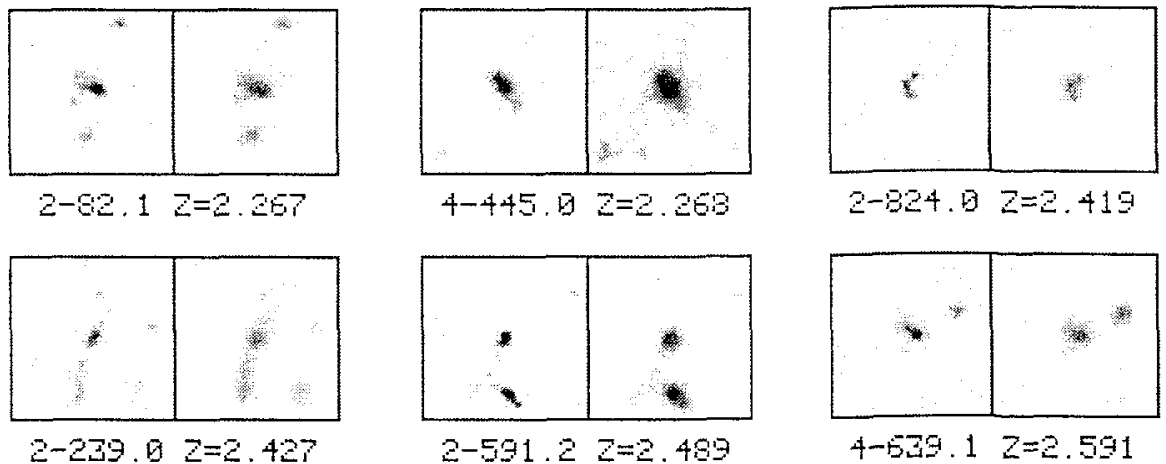

$2-239.02=2.427$
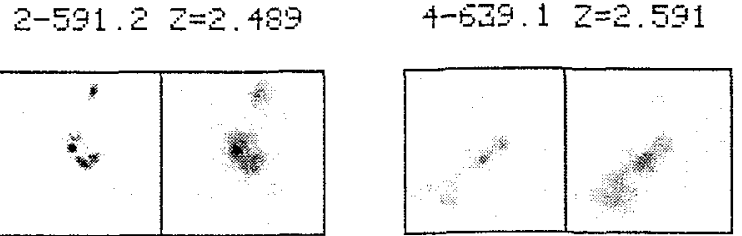

$4-52.82=2.931$

$4-55517=2903$
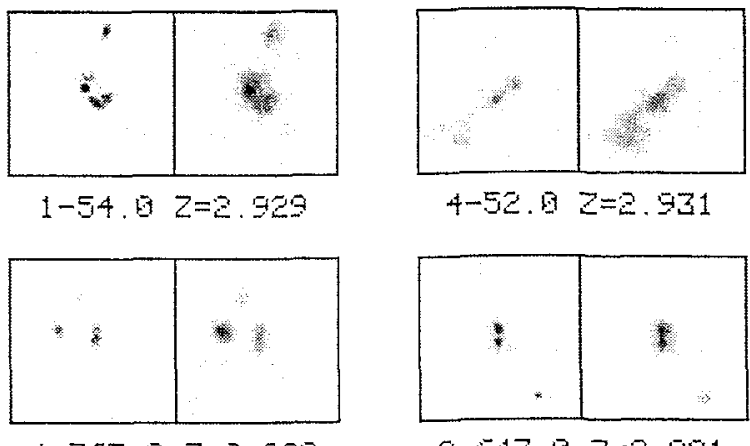

$4-299.5 \quad 2=2.959$

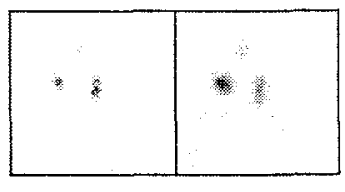

$4-363.0 \quad 2=2.900$

$2-6+3.8 \quad 2=2.991$

Figure 3. A montage of WFPC2 (left) and NICMOS (right) images of Lyman break selected galaxies with spectroscopic redshifts $2<z<$ 3. For this figure, the WEPC2 images have not been convolved to match the PSF of the NICMIOS data. 
in some specified color-color box to the actual number of similar objects that are found in the HDF data set.

Figure 4 shows a series of 2 -color diagrams for the HDF, each using combinations of three adjacent bandpasses, from $U B V$ (i.e., $z \sim 3$ selection) through $I J H$ (i.e., $z \sim 7$ selection). In each case, I define somewhat arbitrary selection boxes based on the expected location of high redshift galaxies in color-color space, then count the galaxies in those boxes and compare this to the "no evolution" (NE) model predictions (Figure 5).

The $U_{300}$-dropout counts agree well with the models by construction, since the input luminosity function is partially based on HDF data. The $B_{450^{-}}$ dropouts fall somewhat below the NE predictions. This is just the original Madau et al. (1996) result revisited: the HDF-N appears to have fewer galaxies at $z \sim 4$ than at $z \sim 3$. Steidel et al. (1999), who surveyed larger solid angles in several fields, suggest that the bright end of the $z \sim 4 \mathrm{LF}$ is actually compatible with that at $z \sim 3$. The HDF may just be an anomaly, indicating the importance of field-to-field fluctuations, or perhaps there are differences in the faint end LF slopes. For the $V_{606}$-dropouts, there $\sim 7$ candidates with $J_{110}<26.5$ (including the two with spectroscopic confirmation), compared to a prediction of $\sim 17$. Careful inspection of their images and SEDs suggests that they are all very plausible $4.5 \lesssim z \lesssim 6$ candidates. There are no $I_{814}$-dropout candidates with $H_{160}<26$, and only two with $H_{160}<26.5$, one of which clearly has $B_{450}$ and $V_{606}$ flux and thus is probably not at $z>6$. The models predict 9 to 13 objects to this magnitude limit. Some of the fainter objects may be real $z>6$ galaxies, but on visual inspection many are rather dubious, with very low $S / N$. At these magnitudes, we are reaching or passing the useful depth limits of the $\mathrm{HDF}$ for this purpose. It may be that cosmological surface brightness dimming is the strongest limiting factor. We have examined this by taking the $\mathrm{HDF}$ images of $z \sim 2.5 \mathrm{LBGs}$, artificially shifting them to higher redshifts, and reinserting them into the NICMOS data to assess their detectability. The highest surface brightness objects should be detectable to $z \approx 8$ at the depth of our GO NICMOS images, and more easily in the GTO field which reaches 1 magnitude deeper, but a general census may indeed be woefully incomplete, a point that has also been stressed by Ferguson (1998) and by Ken Lanzetta at this meeting.

\section{An object at $z \gtrsim 12$ ?}

Although the few $I$-dropout candidates in the HDF-N are very faint, paradoxically there is a comparatively bright, easy to spot " $J$-dropout" object which we call HDFN-JD1, shown in Figure 6. Lanzetta et al. (1998) identified five candidate objects in the KPNO $K_{s}$ images which were invisible in the WFPC2 data. Of these, one has a very robust NICMIOS $1.6 \mu \mathrm{m}$ detection $\left(H_{160} \approx 25.2\right.$ ). but is at best only marginally $(<2 \sigma)$ detected at $1.1 \mu \mathrm{m}$ and shorter wavelengths. These marginal optical detections, if real, would be important, as they would most likely exclude the most exotic hypothesis for this object. i.e., that it is a galaxy or QSO at $z \gtrsim 10$. It will be difficult, however, to ubtain much deeper optical data needed to provide a more firm measurement. The red $H_{160}-K_{s}$

color of HDFN-JD1 suggests $z \approx 12.5$ under the high redshift LBG hypothesis, with the Ly $\alpha$ forest partially suppressing the $H_{160}$ flux. We obtained $H$-band 

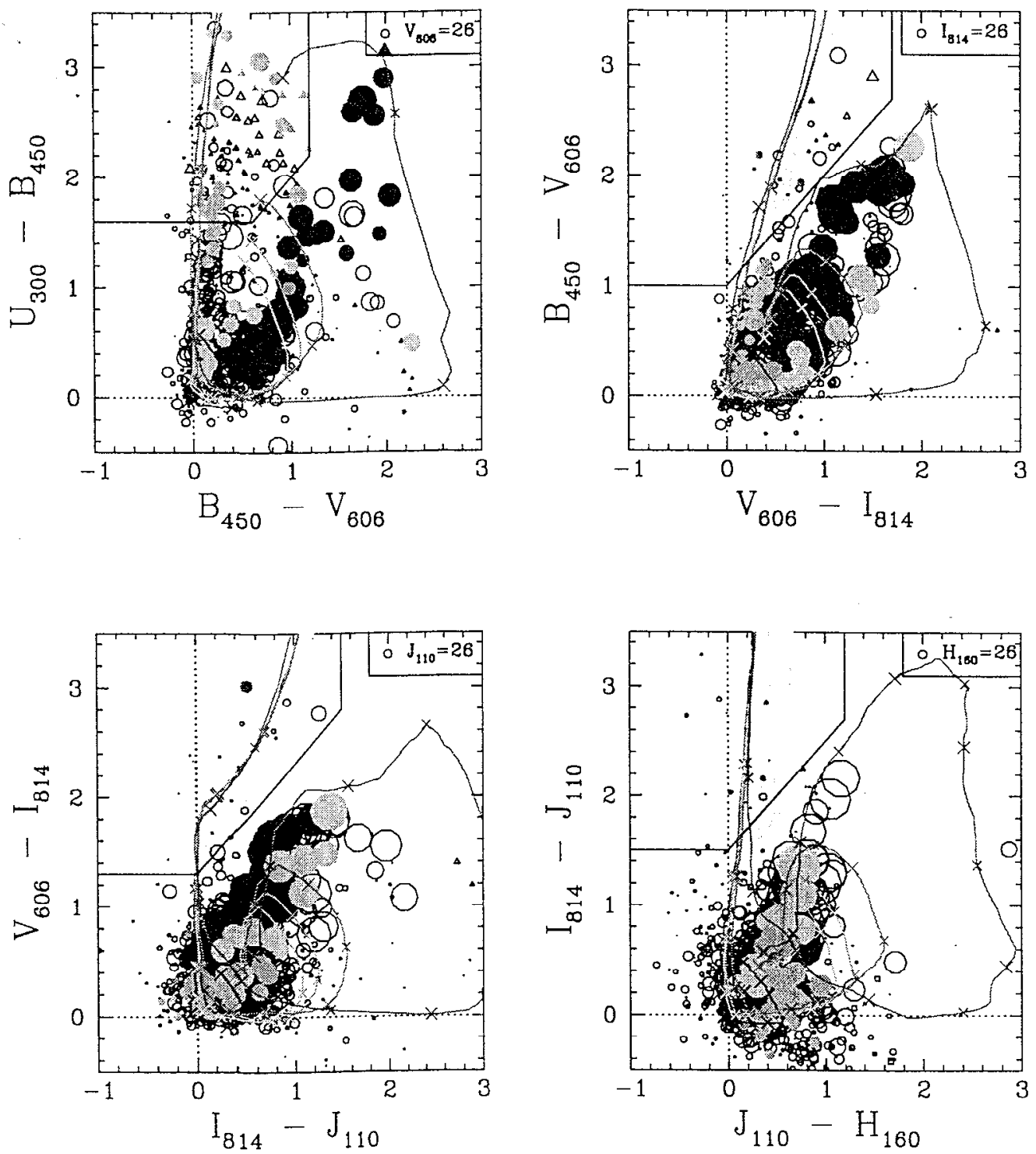

Figurc 4. 2-color diagrams for combinations of adjacent $\mathrm{HDF}$ WFPC2+NICMOS filters. Known and suspected stars, plus a few bright galaxies, have been excluded for clarity. Symbol size scales with magnitude; triangles mark $1 \sigma$ lower color limits. The lines show the nominal color-vs $z$ tracks for various unevolving galaxy SEDs, and the shaded region shows the approximate range of colors expected for galaxies in redshift ranges appropriate to each color pair (UBV: 2-3.5; $B V I: 3.5-4.5 ;$ VIJ: 4.5-6; IJH: 6-8.5). Filled symbols indicated objects with spectroscopic redshifts. The color selection boxes used for the comparison with Monte Carlo simulations are indicated. 

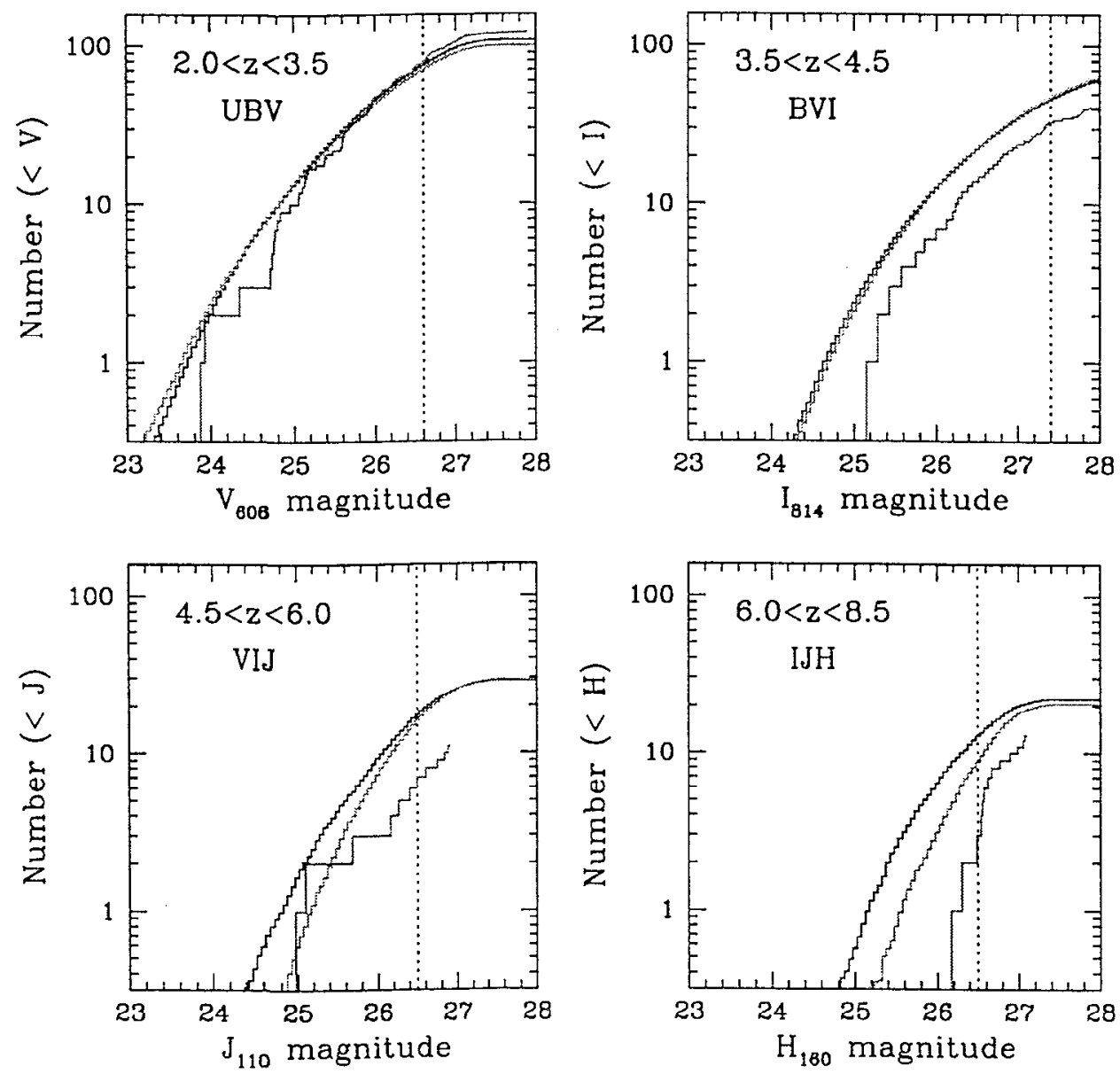

Figure j. Cumulative number counts of objects satisfying the high redshift color criteria shown in Figure 4, for each color combination. The irregular, jagged histograms are from the HDF data, and the "smoother" histograms are predictions from the Monte Carlo simulations which assume that the galaxy population at high redshift is like that observed at $z \approx 3$. Models were computed for two $\Lambda=0$ cosmologies: $\Omega_{M}=1.0$ and 0.2 (the open cosmology is the lower model in the VIJ and $I J H$ plots). The vertical dashed line indicates a rough "confidence limit" in magnitude for each plot, below which the data should become significantly incomplete and/or contaminated by spurious sources. 


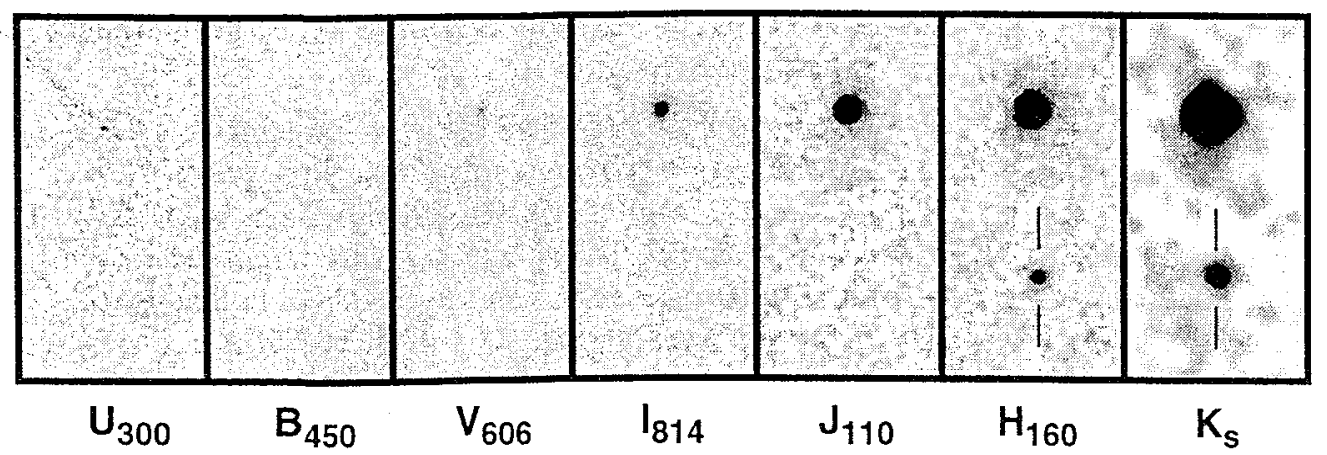

Figure 6. HST and Keck images of HDFN-JD1 at $0.3-2.16 \mu \mathrm{m}$. The field of view of each panel is $4^{\prime \prime} \times 8^{\prime \prime}$. HDFN-JD1 is identified by tick marks in the $H_{160}$ and $K_{s}$ panels. The NIRC $K_{s}$ image has been smoothed by a Gaussian with FWHM $=0$ "' 38 .

spectra of HDFN-JD1 with CRSP at the KPNO $4 \mathrm{~m}$, and to our surprise saw a moderately convincing "ghost line" (in Spinrad terminology) at $1.65 \mu \mathrm{m}$ with $S / N \approx 4$ that could plausibly agree with Ly $\alpha$ at that very large redshift. The line did not reproduce, however, in a subsequent reobservation at higher dispersion (see Dickinson et al. 2000 for the spectra and further discussion).

If this is not a Lyman break object, then it may be either a heavily reddened galaxy or AGN at arbitrary redshift (but most likely $z>2$, given the colors), or possibly a maximally old elliptical galaxy at $3<z<4$, although it is probably too red for that without additional obscuration from dust or an unusual IMF. If it is really at $z \approx 12.5$, then it is either a galaxy whose unobscured star formation rate (computed from the UV luminosity) is several hundred $M_{\odot} /$ year, or an AGN, possibly part of the population responsible for re-ionizing the universe. If so, however, such objects are rare at $2<z<13$, with a space density several hundred times lower than that of present-day $L^{*}$ galaxies, and it is unlikely that most of today's galaxies began their life in such a way.

Overall, these new infrared data demonstrate both the promise and challenges which lie ahead for opening up the $z>6$ universe to scrutiny. The NICMOS HDF images are the deepest, wides -field near-IR data presently available. They do include plausible candidates for galaxies at $6<z<9$, but they are relatively few and are may all be too faint for spectroscopy. While the one NICMOS-detected $z>12$ candidate is surprisingly bright, it may also be too faint for confirmation. Surface brightness dimming may limit our ability to see much more with NICMOS, and ground-based near-IR imaging may never go deep enough. Wider field near-IR imaging with the HST WFC3 infrared channel (coming circa 2003) may offer the best survey tool until NGST, but a substantial investment of HST time will be needed to survey adequate solid angles to sufficient depth. These data are offering a first glimpse into the so-called "dark agés," and giving hope that there may be luminous things there to find and study, but the effort will not be easy. However, galaxies at $z>1$ seemed all but unreachable when Hy Spinrad started chasing them, but with his wonderful persistence he has helped take us out to $z=5$ and beyond. With similar per- 
sistence, I suspect that we will continue to explore the universe at still higher redshifts as long as there are galaxies left to find.

Acknowledgments. I thank my HDF/NICMOS collaborators for their contributions and permission to show results in advance of publication, particularly Casey Papovich for preparing Figure 1 and Chris Hanley for help with data reduction. Thanks to the Hyfest LOC for throwing a great party and for financial support, and especially to the editors of this volume for their considerable patience. This work was supported by NASA grant GO-07817.01-96A.

\section{References}

Barger, A.J., Cowie, L.L., Trentham, N., Fulton, E., Hu, E.M., Songaila, A., \& Hall, D. 1999, AJ, 117, 102

Budavari, T., Szalay, A.S., Connolly, A.J., Csabai, I., \& Dickinson, M. 1999, in Photometric Redshifts and the Detection of High-Redshift Galaxies, eds. R. Weymann, L. Storrie-Lombardi M. Sawicki \& R. Brunner, (San Francisco: ASP), in press (astro-ph/9908008)

Csabai, I., Szalay, A.S., Connolly, A.J. Budavari, T., 1999, A.J, in press (astro$\mathrm{ph} / 9910389)$

Dickinson, M. 1998, in The Hubble Deep Field, eds. M. Livio, S. M. Fall \& P. Madau (Cambridge: Cambridge Univ. Press), 219

Dickinson, M., et al. 2000, ApJ, in press (astro-pl//9908083)

Ferguson, H.C. 1998, in The Hubble Deep Field, eds. M. Livio, S. M. Fall \& P. Madau (Cambridge: Cambridge Univ. Press), 181

Fernández-Soto, A., Lanzetta, K.M., \& Yahil, A. 1999, ApJ, 513, 34

Giavalisco, M., Steidel, C.C., \& Macchetto, D. 1996, ApJ, 470, 189

Hogg, D.W., Neugcbaner, G., Armus, L., Matthews, K., Pahre, M.A., Soifer, B.T., \& Weinberger, A.J. 1997, AJ, 113, 2338

Kininey, A.L., Calzetti, D., Bohlin, R.C., McQuade, K., Storchi-Bergmann, T., \& Schmitt, H.R. 1996, ApJ, 467, 38

Lanzetta, K.M., Yahil, A., \& Fernández-Soto, A. 1996, Nature, 381, 759

Lanzetta, F.M., Yahil, A., \& Fernández-Soto, A. 1998, A.J, 116, 1066

Madau, P., Ferguson H. C. Dickinson, M., Giavalisco, M., Steidel, C.C., Fruchter, A.S. 1996, MNRAS, 283, 1388

Richards, E.A., Kellerman, K.I., Fomalont, E.B., Windhorst, R.A., \& Partridge, R.B. 1998, AJ, 116, 1039

Spinrad, H., Stern, D., Bunker, A., Dey, A., Lanzetta, K.M., Yahil, A., Pascarelle, S., \& Fernández-Soto, A. 1998, AJ, 116, 2617

Steidel, C.C., Adelberger, K.L., Giavalisco, M., Dickinson, M., \& Pettini, M. 1999, ApJ, 519, 1

Thompson. R.I., Storrie--Lombardi, L.J., Weymann, R.I., Rieke, M., Schneider, G., Stobie, E., \& Lytle.D. 1999b, A.J, 117, 17

Weymann, R.J., Stern, D.. Bunker. A., Spinrad, H.. Chaffee, F.H., Thompson. R.I., \& Storrie-Lombardi. L.J. 1998, ApJ. 505, L95 
The Hy Redshift Universe

ASP Conference Series, Vol. 193, 1999

A. J. Bunker \& W. J. M. van Breugel, eds.

$\checkmark$

\section{. Galaxy counts at $450 \mu \mathrm{m}$ and $850 \mu \mathrm{m}$}

A. W. Blain

Cavendish Laboratory, Madingley Road, Cambridge, CB.3 OHE, UK

R. J. Ivison

Department of Physics 83 Astronomy, University College London, Gower Street, London, WC1E 6BT, UK

J.-P. Kneib

Observatoire Midi-Pyrénées, 14 Avenue E. Belin, 31400 Toulouse, France

Ian Smail

Department of Physics, University of Durham, South Road, Durham, $D H 1$ зLE, UK

Abstract. Surveys of the distant Universe have been made using the SCUBA submillimeter(submm)-wave camera at the JCMT. 450- and 850$\mu \mathrm{m}$ data is taken simultaneously by SCUBA in the same 5 -arcmin ${ }^{2}$ field. Deep $850-\mu \mathrm{m}$ counts of high-redshift dusty galaxies have been published; however, at $450 \mu \mathrm{m}$ both the atmospheric transmission and antenna efficiency are lower, and the atmospheric noise is higher, and so only upper limits to the $450-\mu \mathrm{m}$ counts have been reported so far. Here we apply the methods used by Blain et al. (1999) to derive deep $850-\mu \mathrm{m}$ counts from SCUBA images of lensing clusters to the $450-\mu \mathrm{m}$ images that were obtained in parallel, in which four sources were detected. We present the first $450-\mu \mathrm{m}$ galaxy count. This analysis has only just become possible because of the volume of data and the difficulty of calibration are both greater for the $450-\mu \mathrm{m}$ array. In light of recent work, in which the identification of two of the galaxies in our sample was clarified, we also update our deep $850-\mu \mathrm{m}$ counts.

\section{Introduction}

Submm-wave surveys are sensitive to high-redshift dusty galaxies. By exploiting the gravitational lensing effect of rich foreground clusters, the efficiency of these surveys is enhanced as compared with those made in blank fields (Blain 1998). In addition, follow-up observations are significantly easier in the lensed fields as compared with true blank fields because of the typical magnification, a factor of 2.5, of the detected sources at all wavelengths (Ivison et al. 1998, 1999; Frayer et al. 1998, 1999; Smail et al. 1998, 1999a,b). 
Surveys using the $850 / 450-\mu \mathrm{m}$ SCUBA (Holland et al. 1999) have provided deep $850-\mu \mathrm{m}$ galaxy counts and upper limits to the $450-\mu \mathrm{m}$ counts (Smail, Ivison \& Blain 1997; Barger et al. 1998,1999a; Hughes et al. 1998; Blain et al. 1999; Eales et al. 1999). Here we use the detections of four high-redshift dusty galaxies in the $450-\mu \mathrm{m}$ SCUBA lens survey data (Smail et al. 1998) to yield the first galaxy count at a wavelength of $450 \mu \mathrm{m}$.

The relative number counts at different wavelengths depend on the distribution of both the redshifts and dust temperatures of the submm galaxy population. It is possible to impose more rigorous constraints on the form of evolution of high-redshift dusty galaxies if accurate galaxy counts are available at several submm wavelengths.

\section{Obtaining counts at $450 \mu \mathrm{m}$}

Recently we published counts of galaxies detected at a wavelength of $850 \mu \mathrm{m}$ through the cores of seven massive cluster lenses (Smail et al. 1998) to a depth of $0.5 \mathrm{mJy}$ (Blain et al. 1999). We used accurate mass models of the foreground lenses, which are constrained using the properties of lensed arcs detected in deep optical images and the spectroscopic redshifts of multiply-imaged background galaxies (for example Kneib et al. 1993; Bézecourt et al. 1999), to reconstruct the background sky. The robustness of the method was verified by extensive Monte-Carlo simulations. Here we apply the same method to the $450-\mu \mathrm{m}$ SCUBA maps of the seven lensing clusters observed in the survey; Cl0024+16, A370, MS0440+02, Cl0939+4i/A851, A1835, A2390 and Cl2244-02. The data was taken in a range of (generally exceptional) atmospheric conditions, and so the thresholds for the detection of a $450-\mu \mathrm{m}$ source vary from cluster to cluster. The $3 \sigma$ flux density limits for detcction arc about $60,30,60,20,20,60$ and $60 \mathrm{mJy}$ in each cluster respectively. Four sources were detected: one behind A370 (SMIM J02399-0136, the brightest source in the sample, with a 450$\mu \mathrm{m}$ flux density of $85 \pm 15 \mathrm{mJy}$ ); two behind A1835 (SMM J14009+0252 and SMM J14011+0252; Ivison et al. 1999); and onc behind Cl0939+47/A851 (an extremely red object or ERO - SMMJ09429+4658; Smail et al. 1999a). The $450-\mu \mathrm{m}$ counts that result from the analysis, which is described in detail in Blain et al. (1999), are listed in Table 1 and Fig. 1. The $450-\mu \mathrm{m}$ count at about $10 \mathrm{~m} . \mathrm{Jy}$ is equivalent to the $850-\mu \mathrm{m}$ surface density at $3 \mathrm{mJy}$.

\section{Updating the $850-\mu \mathrm{m}$ counts}

In our earlier analysis of the 17 sources detected in the SCUBA lens survey to yield $850-\mu \mathrm{m}$ counts, we first removed 2 submm sources identified with cluster $\mathrm{CD}$ galaxies from the sample (Edge et al. 1099). We also removed one other submm galaxy from the sample. which was identified with a spiral galaxy in the foreground of the cluster Cl0939+47/A851, and identified one further submm galaxy with a spiral galaxy falling into MS0440+02. We now know that these two SCUBA detections are more likely to be identified with EROs discovered in our deep near-infrared follow-up images (Smail et al. 1999a) than with the lowredshift spiral galaxies. Here we repeat the earlier analysis of the counts (Blain et al. 1999), but now include these two galaxies as lensed high-redshift background 
Table 1. New $450-\mu \mathrm{m}$ and updated $850-\mu \mathrm{m}$ integral counts of galaxies. Our previous direct $850-\mu \mathrm{m}$ counts are listed for comparison. Our Monte-Carlo method (Blain et al. 1999) yields a $450-\mu \mathrm{m}$ count of the form $N(>S)=\Pi\left(S / S_{0}\right)^{\alpha}$ as a function of flux density $S$, with $K=530 \pm 300 \mathrm{deg}^{-2}, \alpha=-1.8 \pm 0.5$ and $S_{0}=20 \mathrm{~m} . \mathrm{Jy}$.

\begin{tabular}{cccc}
$\begin{array}{c}\text { Wavelength } \\
(\mu \mathrm{m})\end{array}$ & $\begin{array}{c}\text { Flux density } \\
(\mathrm{m} J \mathrm{y})\end{array}$ & $\begin{array}{c}\text { Count } \\
\left(10^{3} \mathrm{deg}^{-2}\right)\end{array}$ & $\begin{array}{c}\text { Previous count } \\
\left(10^{3} \mathrm{deg}^{-2}\right)\end{array}$ \\
\hline 450 & 10.0 & $2.1 \pm 1.2$ & $\ldots$ \\
& 25.0 & $0.5 \pm 0.5$ & $\cdots$ \\
850 & 0.25 & $51 \pm 19$ & $\ldots$ \\
& 0.5 & $27 \pm 9$ & $22 \pm 9$ \\
& 1.0 & $9.5 \pm 3.3$ & $7.9 \pm 3.0$ \\
& 2.0 & $2.9 \pm 1.0$ & $2.6 \pm 1.0$ \\
& 4.0 & $1.6 \pm 0.7$ & $1.5 \pm 0.7$ \\
& 8.0 & $0.92 \pm 0.53$ & $0.8 \pm 0.6$ \\
& 16.0 & $0.34 \pm 0.34$ & $\ldots$
\end{tabular}

sources. The updated $850-\mu \mathrm{m}$ counts that result are shown in Table 1 and Fig. 1. The new results are within the $1 \sigma$ errors of our previous analysis of the $850-\mu \mathrm{m}$ counts, and are modified at only the $10 \%$ level if the redshift distribution of the source population is assumed to be given by the results of either Barger et al. (1999b) or Smail et al. (1999b).

We emphasize that three of the $850-\mu \mathrm{m}$ galaxies used in this analysis have flux densities less than $3 \mathrm{mJy}$, after correcting for lensing magnification. Further, two of these sources have flux densities less than the blank-field confusion limit for identification (about $2 \mathrm{mJy}$ ), and one has a sub-mJy flux density.

The lower limits to the background radiation intensity $\left(\nu I_{\nu}\right)$ obtained from the flux densities of the detected galaxies are $(5.0 \pm 1.5) \times 10^{-10}$ and $(1.1 \pm$ $0.6) \times 10^{-9} \mathrm{~W} \mathrm{~m}^{-2} \mathrm{sr}^{-1}$ at 850 and $450 \mu \mathrm{m}$ respectively, $94 \%$ and $34 \%$ of the COBE-FIRAS values (Fixsen et al. 1998).

\section{Conclusions}

We present the first count of galaxies at the short submm wavelength of $450 \mu \mathrm{m}$, and update our deep $850-\mu \mathrm{m}$ counts in the light of improved optical identifications (Smail et al. 1999a). The relative counts of galaxies at 450 and $850 \mu \mathrm{m}$ are consistent with both the spectral energy distributions and the forms of evolution of distant dusty galaxies that were derived and discussed in our modeling papers.

Acknowledgments. We are glad to be able to help acknowledge Hy's 65 years. AWB thanks the conference organizers for their hospitality and for support during the meeting, and also Jon Lacey and Jackie Davidson for good food before and after. The JCMT is operated by the Observatories on behalf of the UI PPARC, the Netherlands SRON and the Canadian NRC.

\section{References}

Barger, A. J., Cowie, L. L.. Sanders, D. B., et al., 1998, Nature, 394, 248 

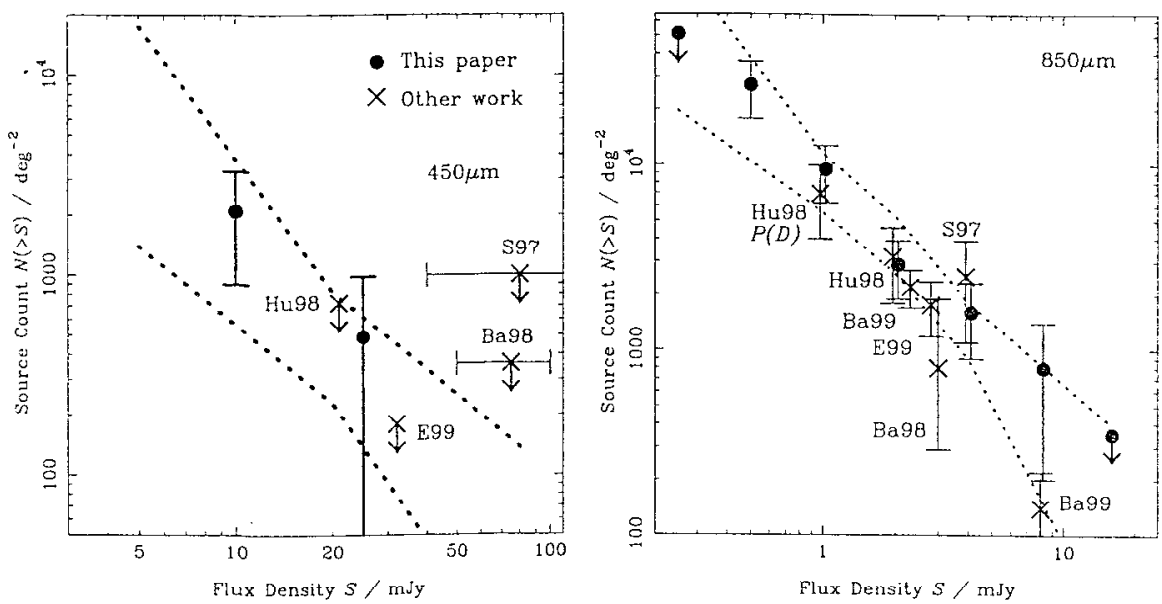

Figure 1. Left: the $450-\mu \mathrm{m}$ counts of galaxies. The direct and MonteCarlo counts derived here are shown by the solid points and dotted lines respectively. Right: the $850-\mu \mathrm{m}$ counts of galaxies including the updated SCUBA lens survey counts. To avoid complicating the figure the direct counts obtained by Blain et al. (1999; see Table 1) are not shown. The associated Monte-Carlo results are shown by the dotted lines. Ba98/Ba99 - Barger et al. (1998, 1999a); E99 - Eales et al. (1999); Hu98 - Hughes et al. (1998); S97 - Smail et al. (1997).

Barger, A. J., Cowie, L. L., \& Sanders, D. B., 1999a, ApJ, 518, L5

Barger, A. J., Cowie, L. L., Smail, I., et al., 1999b, AJ, 117, 2656

Bézeconrt. J., Kneib, J.-P., et al., 1999, A\&A, 347, 21

Blain, A. W., 1998, MNRAS, 290, 553

Blain, A. W., Kneib, J.-P., Ivison, R. J., \& Smail, I., 1099, ApJ, 512, L87

Edge, A. C., Ivison, R. J., Smail, I., et al., 1999, MNRAS, 306, 599

Eales, S. A., Lilly, S. J., Gear, W. K., et al., 1999, ApJ, 515, 518

Holland, W. S., Robson, E. I., Gear, W. K., et al., 1999, MNRAS, 303, 659

Hughes, D., Serjeant, S., Dunlop, J., et al., 1998, Nature, 394, 241

Ivison, R. J., Smail, I., Le Borgne, J.-F., et al., 1998, MNRAS, 298, 583

Ivison, R. J., Smail, I., Barger, A. J., et al., 1999, MNRAS, submitted

Fixsen, D. J., Dwek, E., Mather, J. C., et al., 1998, Ap.J, 508, 123

Frayer, D. T., Ivison, R. J., Scoville, N. Z., et al., 1998, ApJ, 506, L7

Frayer, D. T., Ivison, R. J., Scoville, N. Z., et al., 1999, ApJ, 514, L13

Kneib, J.-P., Mellier, Y., Fort, B., Mathez, G., 1993, A\&A, 273, 367

Smail, I., Ivison, R. J., Blain, A. W., 1997, Ap.J, 490, LJ

Smail, I., Ivison, R. J., Blain, A. W., Kneib J.-P., 1998, Ap.J. 507, L21

Smail, I., Ivison, R. J., et al., 1999a, MNRAS, in press (astro-ph/9905246)

Smail. I., Ivison, R. J., et al., 1999b, ApJ, submitted (astro-ph/9907083) 
The Hy Redshift Universe

ASP Conference Series, Vol. 19.8, 1.999

A. J. Bunker \& W. J. M. van Breugel, eds.

\title{
. High-Redshift Galaxies in the NICMOS Parallel Fields
}

\author{
Michael R. Corbin, Rodger I. Thompson and Earl O'Neil \\ NICMOS Group, Steward Observatory, The Uninersity of A rizona, \\ Tucson, AZ, 85721; mcorbin@as.arizona.edu \\ William D. Vacca \\ Institute for Astronomy, 2680 Woodlawn Dr., Honolulu, HI, 96822; \\ vacca@minera.ifa.hawaii.edu
}

\section{Introduction}

The search for high-redshift galaxies in the near-IR is important for several reasons, including that it extends the range at which such objects may be photometrically identified to $z>7$. Near-IR images of galaxies in the approximate range $1<z<4$ also cover their rest-frame optical emission, which should better trace their underlying structure than that of the rest-frame ultraviolet. An opportunity to study a large number of high-redshift galaxies in the near infrared and at high spatial resolution has been presented by the archive of parallel images obtained by the Hubble Space Telescope NICMOS instrument during its initial lifetime. The NICMOS parallel images taken in the high-resolution cameras ( 1 and 2) cumulatively cover an area several times larger than the Hubble Deep Field, and reach a depth that should be sufficient to detect the recently discovered population of star-forming galaxies at $z \sim 3$ (Steidel et al. 1996).

\section{Results}

We have identified 98 high-redshift galaxy candidates in the NICMOS Camera 1 and Camera 2 parallel images under the criteria that the objects have an extended structure but a small angular size $\left(<2.5^{\prime \prime}\right)$. A representative sample of twelve objects is shown in Figure 1 . We find a wide range of morphologies, including apparently normal spirals, ellipticals, and peculiar galaxies, some of which appear to be in the process of merging. In the case of the peculiar galaxies, by observing in the near-IR we can be more confident that we are seeing their genuine structure, as opposed to that revealed in the rest-frame ultraviolet by isolated regions of star formation.

The NICMOS parallel images were taken in the F110W, F160W and F222M filters, which are analogs of the $J, H$ and $K$ bands. We obtained deep $V$ and $R$ band images of the fields containing 60 of the candidate objects using the Steward Observatory 30 " and 61 " telescopes. The combined data were used to estimate photometric redshifts of the objects by fitting Bruzual \& Charlot (1993) population synthesis model spectra for the case of an instantaneous burst of star formation. Four examples are of these fits are shown in Figure 2. We find a range in these photometric redshifts of $z \sim 0.2-5$, with a peak between $z \sim 1-2$. 


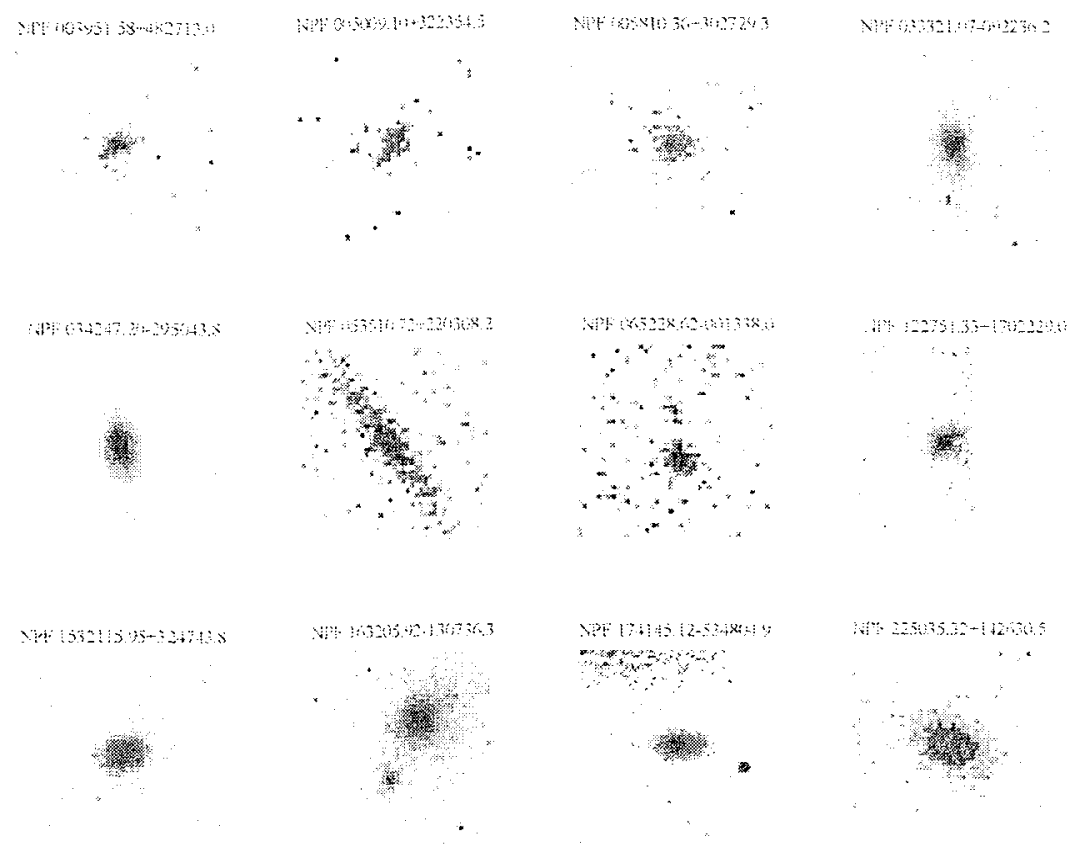

Figure 1. Twelve candidate high-redshift galaxies discovered in the NICMOS Camera 1 and Camera 2 parallel fields. Images are in the F160W filter, and are $3^{\prime \prime}$ square. 

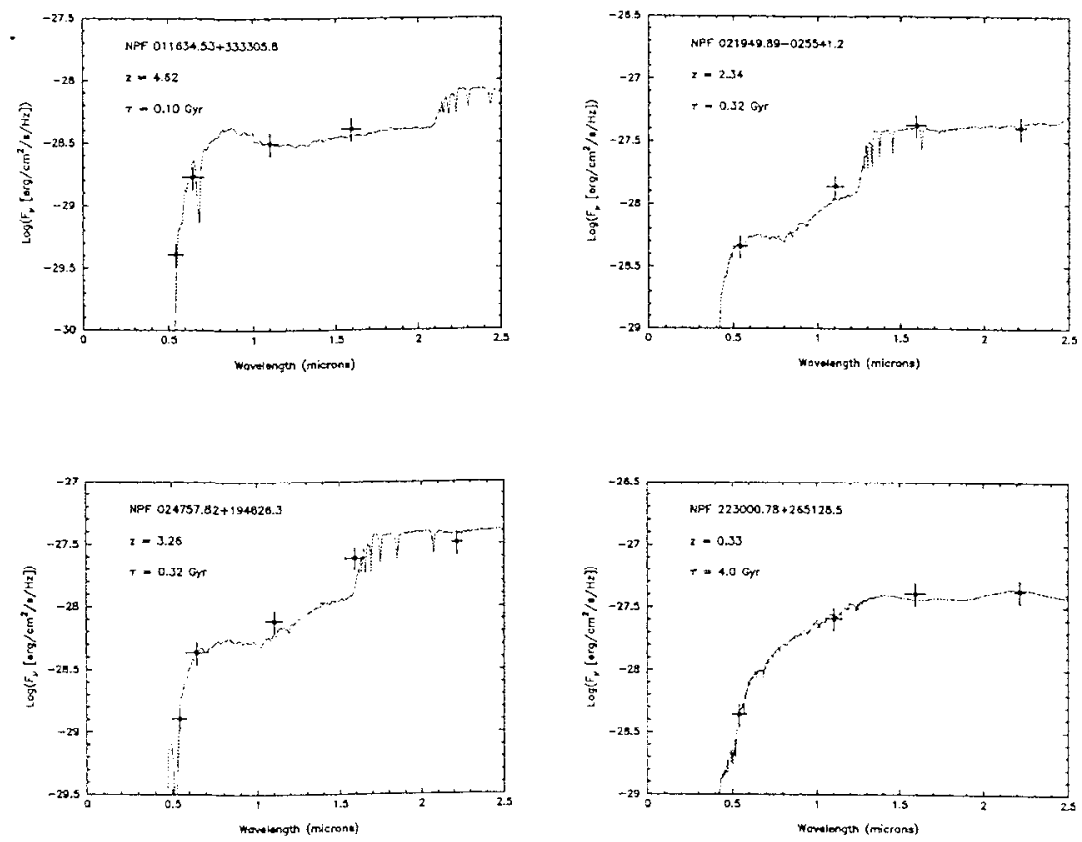

Figure 2. Observed-frame spectral energy distributions of four objects from our sample, along with the best-fitting Bruzual \& Charlot instantaneous burst model and the associated redshift and age. The object NPF 024757.82+194626.3 appears to be a spiral, while the other objects appear to be ellipticals.

This distribution of redshift values also differs with morphological type, with the number of spirals peaking below $z \sim 1$, the number of peculiar galaxies peaking at $z \sim 1-2$, and the number of elliptical galaxies staying roughly constant with redshift to $z \sim 5$.

\section{Discussion}

Our results are very consistent with those for more complete samples of highredshift galaxies, including the Hubble Deep Field and the Hubble Medium Deep Survey (see, e.g., Glazebrook et al. 1995; Driver et al. 1998; Im et al. 1999). Specifically, these studies also find that the fraction of peculiar galaxies rises sharply with redshift, while the fraction of spirals declines. In all samples, elliptical galaxies show no evidence of strong density evolution. These results support top-down models of galaxy formation such as Balland. Silk \& Schaeffer (1998) in which more massive galaxies such as ellipticals form first. The peculiar galaxies we find also appear similar to recent numerical simulations of disk galaxies in the process of formation (Contardo, Steinmetz \& Fritze-von Alvensleben 1998). 
If their redshifts are spectroscopically confirmed, the elliptical galaxies we find at $z>4$, including NPF $011634.53+333305.8$ (Figure 2), would represent the youngest ellipticals yet discovered.

\section{References}

Balland, C., Silk, J. \& Schaeffer, R. 1998, ApJ, 497, 541

Bruzual, G. \& Charlot, S. 1993, ApJ, 405, 538

Contardo, G., Steinmetz, M. \& Fritze-von Alvensleben 1998, Ap.J, 507, 497

Driver, S.P., Fernandez-Soto, A., Couch, W.J., Odewahn, S.C., Windhorst, R.A., Phillipps, S., Lanzetta, K, \& Yahil, A. 1998, ApJ, 496, L93

Glazebrook, K., Ellis, R., Santiago, B. \& Griffiths, R. 1995, MNRAS, 275, L19

Im, M., Griffiths, R.E., Naim, A., Ratnatunga, K.U., Roche, N., Green, R.F. \& Sarajedini, V. 1999, ApJ, 510, 82

Steidel, C.C., Giavalisco, M., Dichinson, M. \& Adelberger, K. 1996, ApJ, 462, L17 
The Hy Redshift Universe

ASP Conference Series, Vol. 193, 1990

A. J. Bunker \& W. J. M. van Breugel, eds.

\title{
The $15 \mu \mathrm{m}$ Extragalactic Background Light from ISOCAM galaxies
}

\author{
D. Elbaz ${ }^{1,2}$, C. Cesarsky ${ }^{1,3}$, H. Aussel ${ }^{1,4}$ \\ ${ }^{1}$ CEA-Saclay, SAp/DAPNIA/DSM, ${ }^{2}$ UCSC, Physics Department, \\ Santa Cruz, CA 95064, USA \\ ${ }^{3}$ European Southern Observatory, Karl-Schwarzchild-Straße, 2 D-85748 \\ Garching bei München, Germany \\ ${ }^{4}$ Osservatorio di Padova, Vicolo Osservatorio, 5, I35122 Padova, Italy
}

\begin{abstract}
We present the contribution of the galaxies detected in the ISOCAM extragalactic surveys to the extragalactic background light at $15 \mu \mathrm{m}$ and we discuss its repercussion on the cosmic infrared background (CIRB) measured by the COBE satellite as well as their consistency with other surveys at $175 \mu \mathrm{m}$ (ISOPHOT) and $850 \mu \mathrm{m}$ (SCUBA).
\end{abstract}

\section{Contribution to the $\mathbf{1 5} \mu \mathrm{m}$ Extragalactic Background Light}

The number counts resulting from the ISOCAM $15 \mu \mathrm{m}$ extragalactic surveys have been presented in Elbaz et al. (1999, these proceedings and paper accepted in A\&A; hereafter Paper I). By integrating the differential number counts presented in the Fig. 2 of Paper I, one can derive the $15 \mu \mathrm{m}$ extragalactic background light (EBL) produced by these galaxies and use it as a lower limit to the total $15 \mu \mathrm{m} \mathrm{EBL}$. We performed this calculation by taking into account a large number of surveys overlapping in flux density, hence including large scale fluctuations in the error bars, for a total of 614 galaxies in both the Northern (Lockman Hole, Hubble Deep Field-North, A2390) and Southern (Marano Field, HDF-South) hemispheres. We find that the sum of all galaxies brighter than $S_{15 \mu m}=0.05$ mJy produce a $15 \mu \mathrm{m} \mathrm{EBL}$ of: $2.2 \pm 0.95 \mathrm{nW} \mathrm{m}^{-2} \mathrm{sr}^{-1}$ (see Fig. 1).

This is already as high as $\sim 60 \%$ of the B band EBL $\left(3.7_{-0.5}^{+0.7} \mathrm{nW} \mathrm{m}^{-2} \mathrm{sr} \mathrm{H}^{-1}\right)$ and $\sim 30 \%$ of the $V$ or I EBL (respectively $6.7_{-0.9}^{+1.2}$ and $7.8_{-0.9}^{+1.6} n W m^{-2} s r^{-1}$ ) measured in the HDF-N by Pozzet ti et al. (1998), while for 1000 galaxics brighter than $I(A B)=29$, only 3 are brighter than $0.1 \mathrm{mJy}$ at $15 \mu \mathrm{m}$. This ratio must be compared to the one found by Soifer \& Neugebauer (1991) in the local universe $(z<0.2)$ where the light radiated by galaxies in the full infrared range, from 8 to $1000 \mu \mathrm{m}$. makes only $30 \%$ of the optical light, with a $12 \mu \mathrm{m}$ contribution of $\sim 10$ $\%$ only. The bulk of the $15 \mu \mathrm{m}$ EBL measured by ISOCA.M comes from galaxies of a few $100 \mu \mathrm{Jy}$ with a median redshift around $z \sim 0.7-0.8$, as inferred from a sub-sample of 83 galaxies with spectroscopic redshifts from the HDF-N (Aussel et al. 1999a,b) and the CFRS-1415 field (Flores et al. 1999). Hence, the first conclusion suggested by this lower limit to the $15 \mu \mathrm{m} \mathrm{EBL}$ is that the ratio of the mid-IR over optical light globally emitted by galaxies has strongly decreased 


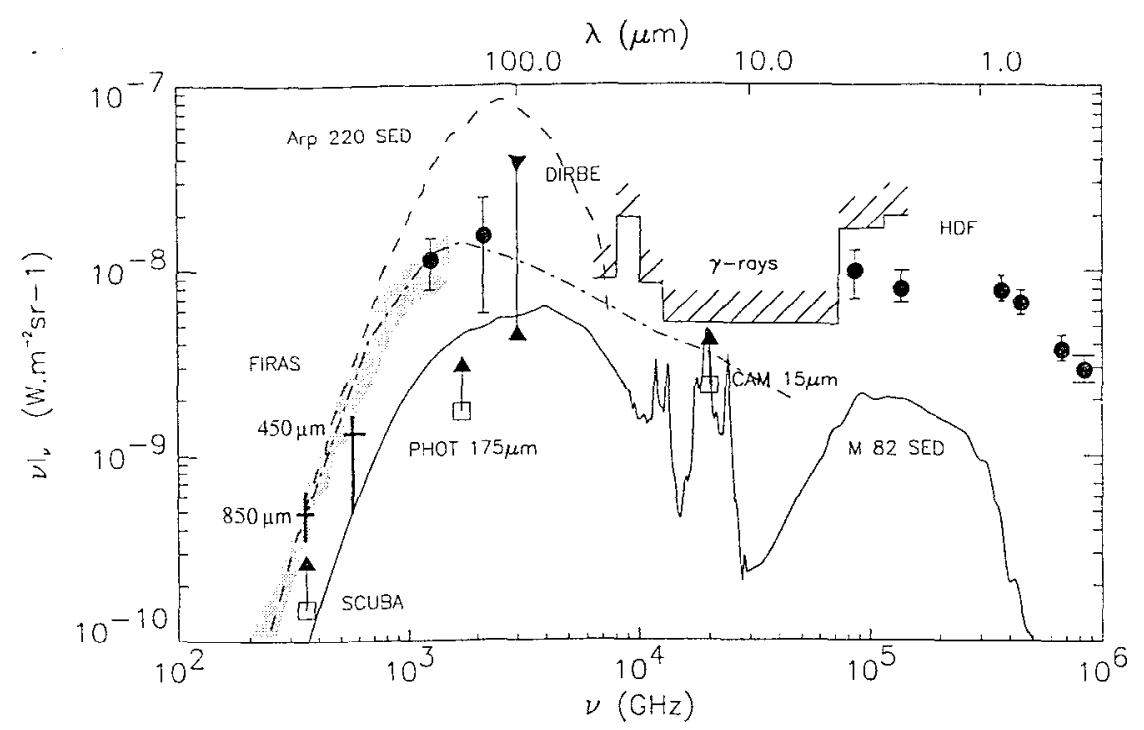

Figure 1. Cosmic background from UV to $\mathrm{mm}$. Open squares give the lower limits from ISO (ISOCAM-15 $\mu \mathrm{m}$ and ISOPHOT-175 $\mu \mathrm{m}$ ) and SCUBA-850 $\mu \mathrm{m}$ (the two thick crosses correspond to the recent values at 450 and $850 \mu \mathrm{m}$ from Blain et al. in these proceedings). The optical-UV points are from Pozzetti et al. (1998). The COBE FIRAS (grey area) and DIRBE $140 \& 240 \mu \mathrm{m}$ (filled circles) data are from Lagache et al. (1999).

from $z \sim 0.7$ to $z<0.2$. Consequently, the luminous infrared galaxies discovered by IRAS (Sanders \& Mirabel 1996) can hardly be considered anymore as peculiar cases producing a tiny fraction of the energy content of the universe. The same conclusion can be derived from the shape of the number counts presented in the Paper I. Direct observations of the far-IR to sub-mm EBL from COBE (references in Paper I), as well as the integration of the EBL produced by the $850 \mu \mathrm{m}$ SCUBA galaxics (Blain et al. in these proceedings) or $175 \mu \mathrm{m}$ ISOPHOT galaxies (ISOPHOT, Puget et al. 1999, Dole et al. 1999), also reach the same conclusion. We will address in the following the question of the consistency of these different measurements with the ISOCAM $15 \mu \mathrm{m}$ results.

\section{Comparison to the COBE background}

Assuming that the median redshift of $z \sim 0.7-0.8$ previously quoted applies to the bulk of the $15 \mu \mathrm{m}$ galaxies, one can extrapolate the contribution of these galaxies to the EBL at any other wavelength with the assumption of a given 
SED. We showed two examples of such a calculation by overplotting SEDs in the Fig. 1, where we normalized each galaxy SED to the $15 \mu \mathrm{m}$ EBL measured by IS.OCAM above $0.05 \mathrm{mJy}$. The dashed line peaking around $100 \mu \mathrm{m}$ is the SED of the extreme ultra-luminous IR galaxy Arp220 $\left(L_{b o l}=210^{12} L_{\odot}, H_{o}=\right.$ $\left.70 \mathrm{~km} \mathrm{~s}^{-1} \mathrm{Mpc}^{-1}\right)$. Such an extreme SED can already be rejected since it would produce a $140 \mu \mathrm{m}$ EBL in excess with respect to the COBE-DIRBE value (Hauser et al. 1998, Lagache et al. 1999). Another argument against Arp220like SEDs can be derived from the number counts plots as presented in Paper I. A SED like the one of the more moderate infrared starburst galaxy M82 $\left(L_{b o l}=410^{10} L_{\odot}\right)$ would give a contribution of about one third of the DIRBE $140 \mu \mathrm{m}$ EBL. Only Seyfert 2 galaxies could both be detected by ISOCAM at such redshifts and exhibit a much flatter SED than the one of M82, and hence produce a smaller contribution to the $140 \mu \mathrm{m}$ EBL. The only argument against Seyfert 2's being the dominant population of galaxies detected by ISOCAMI surveys comes from the study of nearby ultra-luminous IR galaxies by Genzel et al. (1998), who showed that only 20-30\% of the energy radiated by these galaxies came from the active nucleus. Since this contribution gets weaker for the lowest luminous objects and that ISOCAM galaxies have typical luminosities lower than $10^{12} L_{\odot}$ (see Paper I), our guess is that accretion is not the main source of energy of these galaxies but instead star formation. But this question will require ground-based and X-ray follow-ups to be definitively answered. If we assume that our guess is right, then the galaxies detected at $15 \mu \mathrm{m}$ by ISOCAM could produce an important contribution to the global cosmic IR background.

\section{The consistency of the ISOCAM results with ISOPHOT \& SCUBA}

We would like to briefly comment on the results that were obtained nearly simultaneously by the far-infrared photometer on-board ISO, ISOPHOT at $175 \mu \mathrm{m}$, and by the sub-millimeter camera mounted on the JCMT telescope, SCUBA at $850 \mu \mathrm{m}$. All three instruments, ISOCAM, ISOPHOT and SCUBA, seem to have separately unveiled a strong evolution in a wavelength range where dust emission dominates over other sources of radiation, of a population of luminous infrared galaxies which produces a strong contribution to the cosmic infrared background (CIRB). We wish to show that even though few galaxies have been detected at all three wavelengths, these results are consistent but that each instrument probe a different region of the luminosity function due to $k$-correction and sensitivity limits. In the Fig. 2, we show that a $210^{11} L_{\odot}$ galaxy with a spectral energy distribution (SED) similar to that of M82, but 5 times more luminous, would be detected at $15 \mu \mathrm{m}$ up to $z \sim 1.2$, but not at $175 \mu \mathrm{m}$ or $850 \mu \mathrm{m}$, whereas it would be the reverse for a $210^{12} L_{\odot}$ galaxy with a larger optical depth like Arp 220. On the contrary, galaxies with such a large far-IR over mid-IR ratio would easily be detected at $850 \mu \mathrm{m}$ above $3 \mathrm{mJy}$, by SCUBA. They would be detected at $z>0.5$ above $\sim 100 \mathrm{mJy}$ at $175 \mu \mathrm{m}$ by ISOPHOT if they were typically three times brighter than Arp220. In a region of $1 / 4$ of square degree in the Marano Field surveyed by both ISOCAM at $15 \mu \mathrm{m}$ and ISOPHOT at $175 \mu \mathrm{m}, \sim 50 \%$ of the $175 \mu \mathrm{m}$ galaxies have $15 \mu \mathrm{m}$ counterparts with flux densities around a few hundred $\mu \mathrm{Jy}$. Such a high $175 \mu \mathrm{m}$ over $15 \mu \mathrm{m}$ flux density ratio can only be reproduced by a SED like the one of Arp220 and with redshifts below 1 . The little 

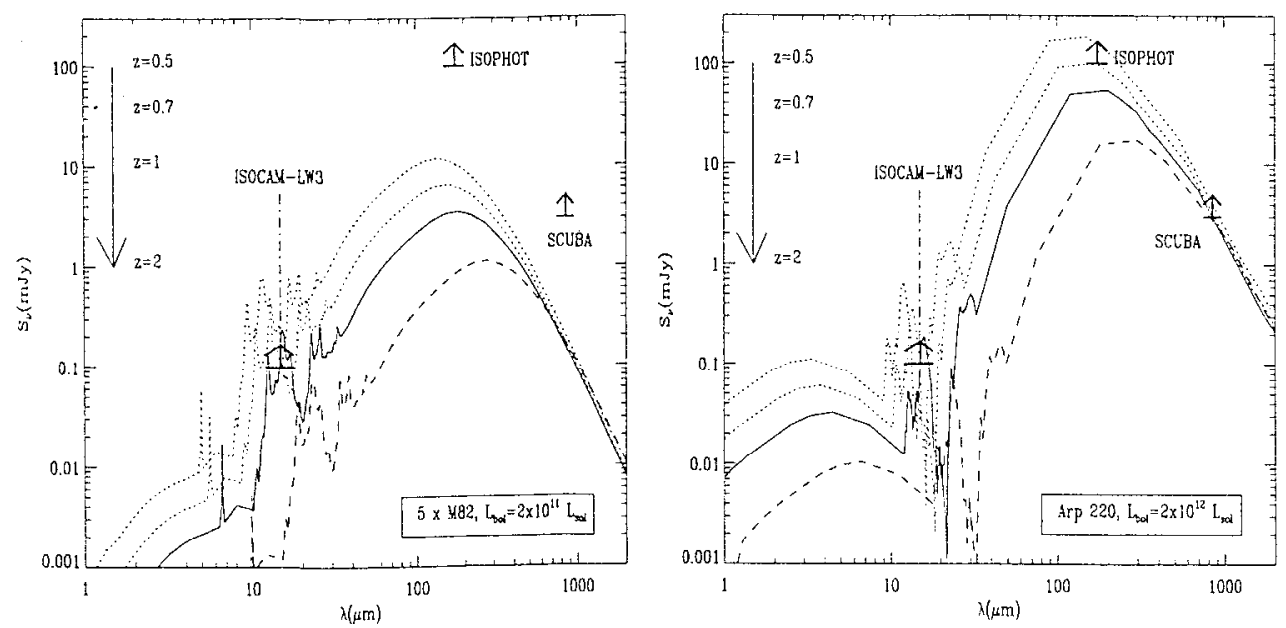

Figure 2. Detection limits at $15 \mu \mathrm{m}$ (ISOCAM), $175 \mu \mathrm{m}$ (ISOPHOT) and $850 \mu \mathrm{m}$ (SCUBA) for a $210^{11} L_{\odot}$ galaxy with the SED of M82 (5 times brighter)(left) and Arp 220 (right) as a function of redshift.

overlap between the source lists of these three instruments when they observed the same fields (SCTBA-CAM: HDF, Hughes et al. 1998; CFRS, Lilly et al. 1999 ) would then naturally be explained by a different selection effect: SCUBA selects the heavily extincted galaxies at the bright end of the luminosity function while ISOCAM selects more moderate galaxies and ISOPHOT is intermediate between the two.

\section{References}

Aussel H., Cesarsky C., Elbaz D., et al., 1999a, A\&A 342, 313

Aussel H., et al., 1999b, in preparation

Dole H., Lagache G., Puget J.L., et al., 1998, to appear in 'The Universe as seen by ISO', Cox P., Kessler M.F. (eds.), Unesco, Paris

Flores H., Hammer F., Thuan T., et al., 1999, ApJ 517. 148

Genzel R., Iutz D., Sturm E., Egami E., Funze D., et al., 1998, ApJ 498, 579

Hauser M.G., Arendt R.G., Kelsall T., et al., 1998, ApJ 508, 25

Hughes D. H., Serjeant S., Dunlop J., et al., 1998, Nature 394, 241

La.gache G.. A bergel A., Boulanger F., et al., 1999, A\&A 344, 322

Lilly S.J., Eales S.A., Gear W.K.P., et al., 1999, Ap.J 518, 641L

Pozzetti L., Madau P., Zamorani G., Ferguson II.C., Bruzual G.A., 1998, MNRAS, 298, 1133

Puget J.-L., Lagache G., Clements D.L. et al., 1999, A\&A 345, 29

Sanders D.B., Mirabel I.F., 1996, Ann. Rev. Astron. Astrophys. 34, 749

Soifer B.T., Neugebaner G.. 1991. A.J 101. 354 
The Hy Redshift Universe

ASP Conference Series, Vol. 193, 1999

A. J. Bunker $\& \mathcal{G} W . J$. M. van Breugel, eds.

$\because$

\title{
. The NOAO Deep Wide-Field Survey
}

\author{
Buell T. Jannuzi and Arjun Dey \\ National Optical Astronomy Observatories, 950 N. Cherry Avenue, \\ Tucson, AZ 85719
}

\begin{abstract}
The NOAO Deep Wide-Field Survey is a deep optical and near-IR ( $\left.B_{W} R I J H K\right)$ imaging survey. We are mapping an unprecedentedly large area (two $9 \square^{\circ}$ regions) to faint flux limits $\left(B_{W} R I \geq 26 \mathrm{AB}\right.$ mag. and $J, H=21 ; K=21.4 \mathrm{AB}$ mag; $5-\sigma$ detection limits in a $2^{\prime \prime}$ diameter aperture). The primary goal of the survey is the study of the evolution of large-scale structure from $z \sim 1-4$. The images should also enable investigation of the formation and evolution of the red-envelope galaxy population and the detection of luminous, very distant $(z>4)$, star-forming galaxies and quasars.
\end{abstract}

\section{Survey Goals}

We have completed the first two years of observations for a deep wide-field optical and IR imaging survey designed to investigate the extent, nature, and evolution of large scale structure at redshifts $z \approx 1-4$; to identify luminous $z \gtrsim 4$ star-forming galaxies; and to find the cosinologically important $z>1$ redenvelope galaxy population (e.g., old ellipticals and dusty protogalaxies). While there are tantalizing hints of small-scale cluslering in the $z>2.5$ starburst population (e.g., Steidel et al. 1996), the detection of large-scale structures at $z>1$ will provide unique constraints on hierarchical clustering theories and galaxy formation models. In addition, there is growing evidence that massive elliptical galaxies form first at $z>4$ (in apparent conlradiclion to hierarchical scenarios) and are already old $(>4 \mathrm{Gyr})$ at $z>1.5$, providing a strong lower bound to the age of the Universe (e.g., Dunlop et al. 1996, Spinrad et al. 1997, Francis et al. 1997). Our survey will probe the evolution of the large scale clustering properties of the $z>1$ galaxy population, is designed (with an IR component) to identify the $z \sim 1$ luminous red galaxy population, and will trace the evolution of luminous star-forming galaxies to $z \sim 5$. The survey occupies a unique region of parameter space (Figure 1), providing both optical (Figure 2) and IR coverage over a large area and to great depth in the optical bands.

\section{Survey Design}

We are observing two independent $9 \square^{\circ}$ fields, selected for their low IRAS cirrus emission, low $N_{\mathrm{HI}}$, lack of very bright stars, and the availability of radio data from the VLA FIRST Survey for these fields. Both fields have $f_{100 \mu m}<$ 


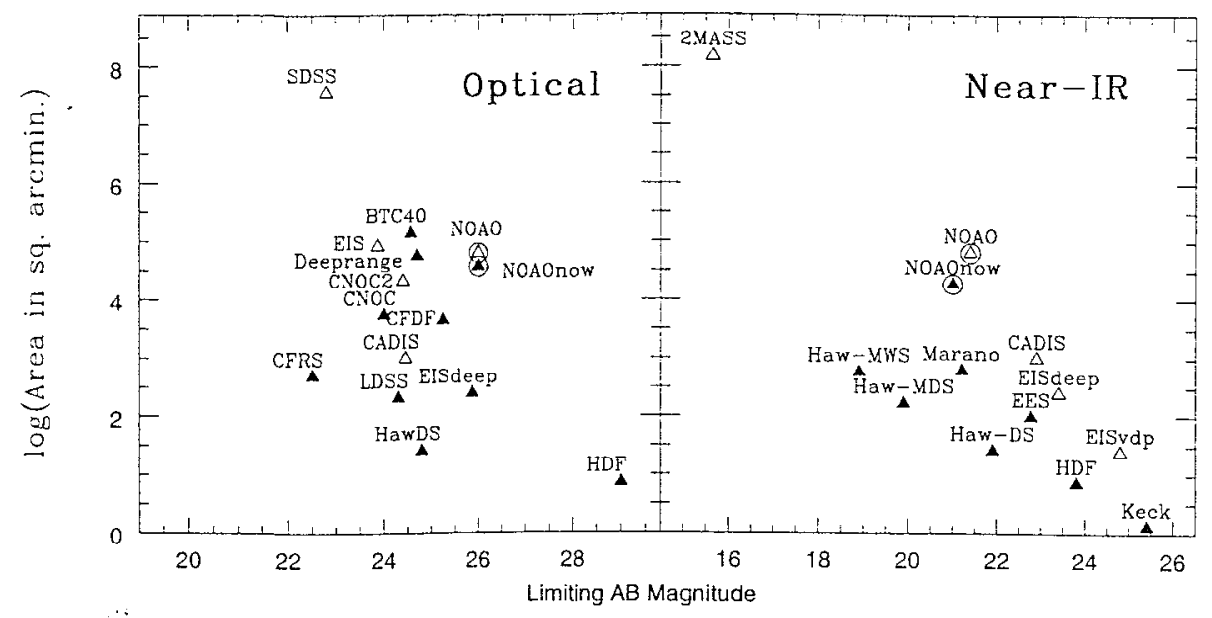

Figure 1. A comparison of the NOAO Survey with selected recent and proposed surveys of the sky. The survey detection thresholds (in a $2^{\prime \prime}$ dia. apt.; $A B$ units) shown are for the deepest band, which provides a comparison for the surveys' sensitivities to a flat-spectrum starforming galaxy. Completed surveys are represented by filled triangles and those planned or in progress by open symbols. The points labeled "NOAOnow" represent the status of the NOAO Survey through the end of our Semester 1998B observations.

$1.4 \mathrm{MJy} / \mathrm{sr}, N_{\mathrm{HI}} \lesssim 1.75 \times 10^{20} \mathrm{~cm}^{-2}$, corresponding to $E(B-V)<0.04$. The northern field is $3^{\circ} \times 3^{\circ}$ centered at (J2000) $R A=14: 32: 06, D E C=+34: 16: 48$ and the southern field is $2^{\circ} \times 4.5^{\circ}$ centered at (J2000) $R A=02: 10: 00, D E C=-04: 30: 00$. The optical imaging is currently being done with the new wide-field $\left(36^{\prime} \times 36^{\prime}\right)$ MOSAIC cameras on NOAO's $4 \mathrm{~m}$ telescopes. The IR imaging is being done with the Ohio State/NOAO Imaging Spectrograph (ONIS) at the KPNO $2.1 \mathrm{~m}$. Lising a 2-quadrant InSb detector, this imager provides a $2.9^{\prime} \times 5.8^{\prime}$ field of view.

Attempts to study the evolution of the high-z galaxy population must begin with a survey that reaches well beyond the depths probed by recent wide-field surveys (Table 1). The successes in detecting $z>3$ galaxies by their Lyman break (Steidel et al. 1996; Madau et al. 1996) suggest that this method can be extended to higher redshifts by looking for ' $B$-band dropouts'. Our initial data demonstrates that we are able to find such candidate objects (Figure 3). Our optical detection limits ensure identification of the $>L^{*}$ star-forming galaxy population at $z \gtrsim 3.5$. The IR component of the survey is critical to the identification of the old, high- $z(>1)$ elliptical galaxy population (missed by most current optical surveys), and to the discrimination against low- $z$ contamination in our search for 'B-band dropouts'. The IR limits allow the detection of the entire (passively?) evolving $L \geq L^{*}$ elliptical galaxy population out to $\approx \lesssim 2\left(K^{-}=21.2 \mathrm{AB}\right.$ mag. for a $\approx=1.5$ unevolved $M_{B}=-21$ elliptical: $H_{0}=65, q_{0}=0.1$. The planned depth should also permit the study of the Galactic halo stellar populations, the coolest high-latitude white dwarfs to $1.5 \mathrm{kpc}$, young (bright) field brown dwarfs (like GL229B) to about $75 \mathrm{pc}$, distant supernovae, and distant radio sources. 


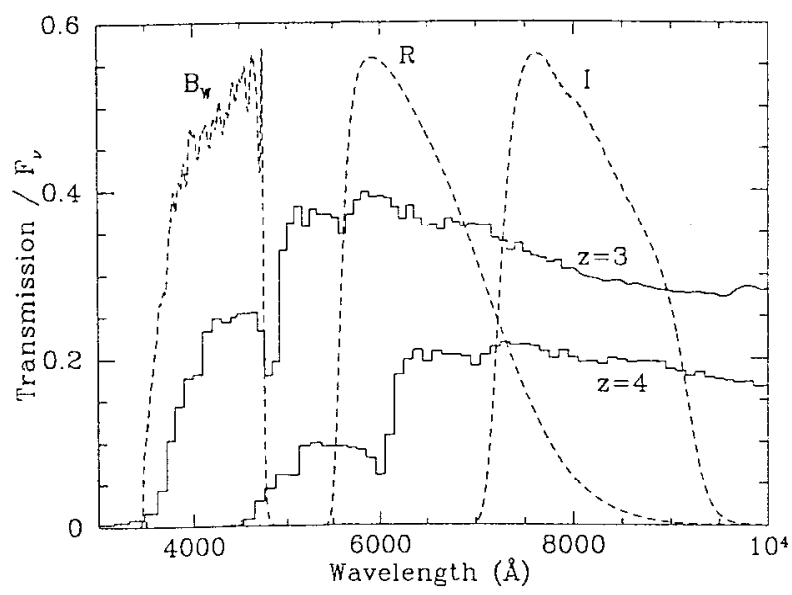

Figure 2. The optical filters used in the survey are all from the Kitt Peak Harris set with the exception of the custom $B_{W}$ filter. The effective transmission curves (including the effects of the quantum efficiency of the MOSAIC CCDs and the throughput of the $4 \mathrm{~m}$ primefocus corrector) for each filter are shown superimposed on the spectra of $z=3$ and $z=4$ star-forming galaxies. The galaxy spectra were constructed from a redshifted Bruzual-Charlot model spectral energy distribution, with the intervening cosmic absorption estimated using Madau et al. (1996) approximation.

The large areal coverage of the survey $\left(2 \times 9 \square^{\circ}\right)$ is critical to the realization of many of our scientific goals. It allows the detection of large scale structure in the forming galaxy population at early epochs and the study of the passivelyevolving red-envelope galaxy population at $z>1$. The redshift distributions of galaxies are clustered to $z \sim 1$, with measured $\sigma_{v}$ of coherent structures comparable to that of the Great Wall (Cohcn ct al. 1996a, 1996b). A $3^{\circ} \times 3^{\circ}$ survey area corresponds to $\approx 94 \times 94 \mathrm{Mpc}^{2}$ at $\approx \sim 2\left(H_{0}=65, q_{0}=0.1\right)$, which will allow us to well-sample the clustcr-cluster correlation length and connect it with shallower (low-z) wide-field surveys (e.g., 16 $\square^{\circ}$ Postman et al. $1998 I_{5 \sigma}=$ 23.7 ; i.e., to $z \sim 1$ ). In addition, the large arca of the survey allows us to find rare objects. An example are luminous galaxies at high- $z$ (red, gEs at $z>1.5$ and luminous forming galaxies at $z>1$ ), which are rare enough (e.g., there are no $L \geq L^{\times} z>4$ candidates in the $\mathrm{HDF}$ ) that large areal coverage is required to find them. Finally, since our fields lie within completed FIRST survey (Becker, White, \& Helfand 1995) regions, we will identify almost all the radio sources in these regions $\left(\sim 90 / 0^{\circ}\right.$ with $\left.S_{2 U c m}>1 \mathrm{mJy}\right)$ and produce a large sample of faint high-z radio-loud QSOs candidates. The $18 \square^{\circ}$ area should yield $\sim 1004<z<5$ radio-quiet QSOs and the IR observations will allow us to search for the elusive red QSO population (Webster et al. 1995) and characterize their space density. 

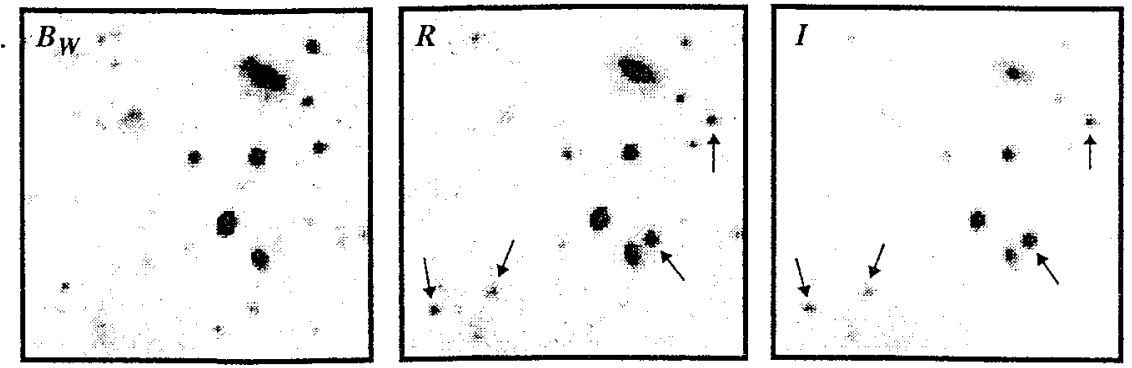

Figure 3. Shown in these panels is a $40^{\prime \prime} \times 40^{\prime \prime}$ sub-region of our survey (approximately $0.5 \mathrm{~h}_{50} \mathrm{Mpc}$ on a side for $z \sim 3$ ) in which several candidate high redshift objects ( $B_{W}-$ "drop-outs") can be seen.

Table 1. Survey Detection Limits

\begin{tabular}{|c|c|c|c|c|}
\hline $\begin{array}{c}\text { Observed } \\
\text { Band }\end{array}$ & \multicolumn{2}{|c|}{$\begin{array}{c}5-\sigma \text { Detcction in } \\
2^{\prime \prime} \text { dia. apt. }\end{array}$} & $\begin{array}{c}\text { 1- } \sigma \text { surface brightness } \\
\text { limit per } \square^{\prime \prime}\end{array}$ \\
\hline & AB mag. & Vega-mag. & AB mag. & Vega-mag. \\
\hline$B_{W}$ & 26.6 & 26.6 & 29.0 & 29.0 \\
$R$ & 26.0 & 25.8 & 28.4 & 28.2 \\
$I$ & 26.0 & 25.5 & 28.4 & 27.9 \\
$I$ & 21.0 & 20.2 & 23.4 & 22.6 \\
$H$ & 21.0 & 19.6 & 23.4 & 22.0 \\
$K$ & 21.4 & 19.5 & 23.8 & 21.9 \\
\hline
\end{tabular}

Acknowledgments. We the NSF for support of this work through its funding of the NOAO under a cooperative agreement with the AURA, Inc.. We thank all our Co-I's on this project and in particular I. Dell'Antonio, J. Rhoads, and F. Valdes for assistance with material presented in this contribution. Our survey web page (http://www.noao.edu/noao/noaodeep/) contains complete information about the survey.

\section{References}

Becker, R. H., White, R. L. \& Helfand, D. J. 1995, ApJ, 450, 559

Cohen, J. et al., 1996a, ApJ, 462. L9

Cohen, J. et al., 1996b, ApJ, 471, L5

Dunlop, J. et al., 1996, Nature, 381, 581

Francis, P. J., Woodgate, B. E. \& Danks, A. C., 1997, ApJ, 482, L25

Madau, P. et al., 1996, MNRAS, 283, 1388

Postman, M., Lauer. T., Szapudi, I.. \& Oegerle, W. 1998, Ap.J, 506. 33

Spinrad, H. et al., 1997, ApJ, 484, 581

Steidel, C. C. et al. 1996, ApJ, 462. L17

Webster, R. L. et al., 1995, Nature, 375, 469 
The Hy Redshift Universe

ASP Conference Series, Vol. 19.3, 1.999

A. J. Bunker \& W. J. M. van Breugel, eds.

\title{
The Deepest Spectrum of the Universe? Constraints on the Lyman Continuum Background at High Redshift
}

\author{
Francine R. Marleau ${ }^{1}$ \\ Institute of Astronomy, Madingley Road, Cambridge CB3 OHA, UK \\ Andrew J. Bunker ${ }^{2}$ and James R. Graham \\ Department of Astronomy, Univcrsity of California at Berkeley, \\ 601 Campbell Hall, Berkeley CA 94720, USA
}

\begin{abstract}
We describe an ongoing experiment to search for the metagalactic Lyman-continuum background at $z \approx 2-3$. We are obtaining one of the deepest optical spectra ever, using LRIS/Keck-II to search for the fluorescent Ly- $\alpha$ emission from optically thick HI clouds. The null results of our pilot study (Bunker, Marleau \& Graham 1998) placed a $3 \sigma$ upper bound on the mean intensity of the ionizing background of $J_{\nu 0}<2 \times 10^{-21} \mathrm{erg} \mathrm{s}^{-1} \mathrm{~cm}^{-2} \mathrm{~Hz}^{-1} \mathrm{sr}^{-1}$ at $z \approx 3$. This constraint was more than two orders of magnitude more stringent than any previously published direct limit. Our results excluded the possibility that decaying relic neutrinos are responsible for the meta-galactic radiation field. We have recently greatly extended our search, obtaining a 15-hour spectrum which is sensitive to UV background fluxes below $10^{-21} \mathrm{erg} \mathrm{s}^{-1} \mathrm{~cm}^{-2} \mathrm{~Hz}^{-1} \mathrm{sr}^{-1}$ $(3 \sigma, z \approx 2.3)$. We describe how the results of this study can be used to constrain the quasar luminosity function and the contribution of highredshift star-forming galaxies to the ambient ionizing background.
\end{abstract}

\section{Introduction}

The meta-galactic nltraviolet (UV) background has a central astrophysical rôle. The absence of a Gunn-Peterson trough at $z \approx 5$ implies that the inter-galactic medium (IGMI) must have been highly ionized by the UV background at even greater redshift. This Lyman continuum background is responsible for maintaining the Ly $\alpha$ forest clouds in a highly-ionized state, and may also canse the sharp edges of HI disks in nearby spirals (Dove \& Shull 1994). It is widely believed that the meta-galactic UV flux is the integrated light of QSOs, or hot massive stars in young galaxies, or both. However, this ionizing background has never been directly detected. We present here the interim results of an observational program designed to achieve this goal.

\footnotetext{
1email: marleauqast.cam.ac.uk

${ }^{2}$ NICMIOS Postdoctoral Researcher
} 


\section{Seeking the Ultraviolet Background}

Hogan \& Weymann (1987) proposed that long-slit spectroscopy of "blank sky" should reveal patches of fluorescent Ly $\alpha$ emission, excited by the meta-galactic ionizing background, from the population of clouds whose absorption produces the Ly $\alpha$ forest in QSO spectra. A measurement of the surface brightness of this fluorescent emission puts limits on the incident ionizing flux at high redshift. Gould \& Weinberg (1996) present a detailed treatment of the transport of Ly $\alpha$ in clouds with $N_{\mathrm{HI}}=10^{17}-10^{20} \mathrm{~cm}^{-2}$. For optically thick clouds $\left(\tau_{912}>6\right.$, $N_{\mathrm{HI}}>1 \times 10^{18} \mathrm{~cm}^{-2}$ ) the flux of Ly $\alpha$ photons from recombination cascades is equal to 0.6 times the flux of incident ionizing photons; this fraction is robust and independent of cloud geometry. The Ly $\alpha$ photons are absorbed and re-emitted until scattering from an atom with a velocity $(v-\bar{v})= \pm 4 \sigma$, at which point it can escape. A typical Ly $\alpha$ cloud with a velocity dispersion of $\sigma=30 \mathrm{~km} \mathrm{~s}^{-1}$ ( $\mathrm{Kim}$ et al. 1997) would have double-peaked fluorescent Ly $\alpha$ line with a width of $240 \mathrm{~km} \mathrm{~s}^{-1}$. Hence, moderate resolution spectroscopy $\left(\lambda / \Delta \lambda_{\mathrm{FWHM}} \gtrsim 1000\right)$ of an optically thick cloud, known to exist from a QSO absorption system, gives a direct measurement of the energy in the ionizing background. Moreover, since there are a few Lyman-limit systems per unit redshift interval at $z \sim 2$, the same long-slit exposure would also detect tens of serendipitous clouds making it possible to make a two-dimensional (2D) map of the Lyo forest.

\section{Our Survey}

The calculations of Gould \& Weinberg (1996) suggest that it should be possible to detect fluorescent Ly $\alpha$ emission from optically thick Ly $\alpha$ clouds at $z \sim 3$ with a deep ( $>10$ hour) long-slit spectrogram on a 10-m telescope. Motivated by this, we have embarked on an extremely sensitive spectroscopic search with the Keck II Telescope. Our pilot study (detailed in Bunker, Marlean \& Graham 1998) showed that it is possible to reach the required line fluxes with the Low Resolution Imaging Spectrograph (LRIS. Oke et al. 1995). Over the past year, we have greatly extended our program, obtaining a total of 15 hours of integration time. This constitutes one of the deepest optical spectra ever obtained.

The data for our extended program was taken towards the quasar DMS 2139.00405 (Hall et al. 1996) and a $1^{\prime \prime}$-wide long-slit was used with a blue-blazed grating of resolving power $\lambda / \Delta \lambda_{\text {FWHM }} \approx 2000$. Our observations sample the wavelength range $3750-5500 \AA$, corresponding to Ly $\alpha$ in the redshift range $2.1<z<3.5$. Our most recent data was obtained using multiple parallel slits with a $10 \%$ bandwidth filter to cover a much larger solid angle while concentrating on those redshifts $(z \approx 2.4)$ where our sensitivity to $J_{\nu 0}$ is greatest (see Fig. 2).

\section{Data Reduction and Analysis}

The details of the data reduction are presented in Bunker, Marleau, \& Graham (1998). Great care was taken in removing the spectrum of the night sky while preserving any cosmological signal (expected to be spatially-extended line emission of low surface brightness, with a velocity spread $\leftarrow-500 \mathrm{~km} \mathrm{~s}^{-1}$ ). Rather than doing the sky-subtraction in the usual manner by fitting a polynomial to 
each column (which might subtract the extended emission we are looking for), we first rectified the sky lines using a distortion matrix. We then subtracted off a high signal-to-noise ratio (SNR) sky spectrum from each detector row, scaled to the slit illumination at that point along the slit. We search for extended Ly $\alpha$ emission by smoothing along the spatial axis of the background-subtracted composite 2D spectrum (e.g., Fig. 1b) on various scale lengths between the size of the seeing disk $\left(\lesssim 1^{\prime \prime}\right)$ and the length of the slit $\left(3^{\prime}-7^{\prime}\right)$. To amplify any signal present in our data, we calculate the $2 \mathrm{D}$ power spectrum. This may potentially reveal the combined signal for a population of clouds that are too faint to be detected individually (see the simulation in Fig. 1d). Finally, we use the SExtractor algorithm (Bertin \& Arnouts 1996) to catalogue objects and we determine the completeness of our search method via artificial-clouds experiments.
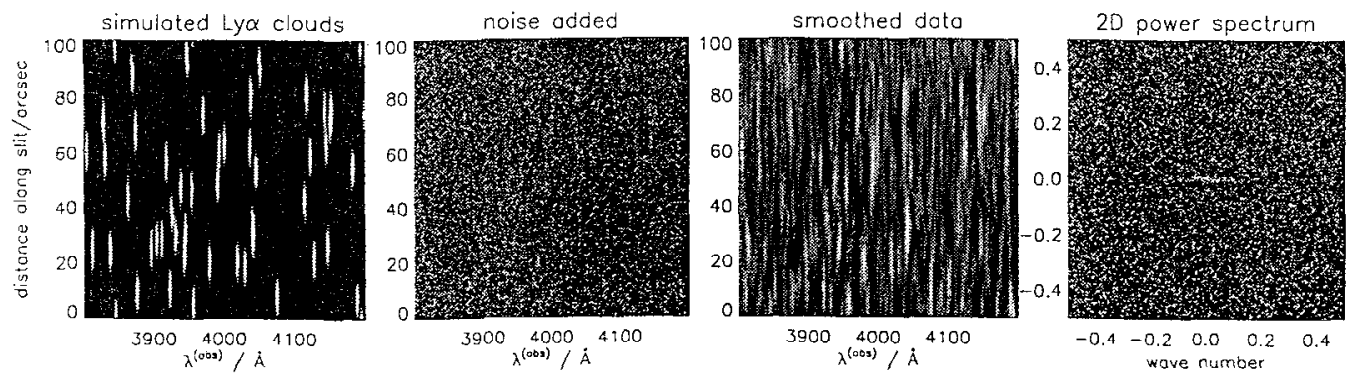

Figure 1. A simulation of an LRIS long-slit 2D spectrum containing 50 clouds, each with a FWHM in the dispersion direction of $250 \mathrm{~km} \mathrm{~s}^{-1}$ and a length of $10^{\prime \prime}$ (Fig. 1a). Noise is added to the image with the peak of a cloud corresponding to $\mathrm{SNR}=1$ per resolution element (Fig. $1 \mathrm{~b}$ ), which renders individual clouds invisible to casual inspection by eye. However, when the noisy image is convolved with a 2D Gaussian kernel of comparable size to the clouds, their signal is revealed (Fig. 1c). The 2D power spectrum of the noisy image is calculated in order to combine the signal of the population of clonds (the central peak in Fig. 1d). Simulations show that when single clouds are not visible, the combination of many clouds is enough to make the detection possible.

\section{Our Limits on the UV Background}

Our pilot study (Bunker, Marleau \& Graham 1998) failed to find Lyo fluorescence from optically-thick clouds. A lack of Ly $\alpha$ emission constrains the UV flux. The upper limit on the ambient UV background at $2.7<z<3.1$ (where our constraints on $J_{\nu 0}$ are most stringent) is equivalent to a flux at the Lyman limit of $J_{\nu 0}<2.0 \times 10^{-21} \mathrm{erg} \mathrm{s}^{-1} \mathrm{~cm}^{-2} \mathrm{~Hz}^{-1} \mathrm{sr}^{-1}$ (Fig. 2). This assumes optically-thich clouds with dimensions $\approx 10^{\prime \prime}$ - the size of such systems derived from $\mathrm{Mg}$ II QSO absorber studics (Steidel \& Dickinson 1995). This limit on $J_{\nu 0}$ is almost two orders of magnitude lower than any previous direct limit (Lowenthal et al. 1990; Martínez-González ct al. 1095) but is still three times above the expected contribution of known QSOs for $q_{0}=0.5$ (Haardt \& Madau 1996). This implies that the completeness of optical QSO catalogs is better than $30 \%$ 
and that the contribution to $J_{\nu 0}$ at $z \approx 3$ from star-forming galaxies (Songaila et al. 1990) cannot exceed twice that from known QSOs. We calculate that the escape fraction of Lyman continuum photons from star-forming galaxies at these redshifts must therefore be less than 10-50\% (depending on the dust obscuration of the rest-UV continuum for $z \approx 3$ galaxies). Based on our extended program, our sensitivity thus far on the ambient $U V$ background at $z \sim 2.5$ probes down to a flux at the Lyman limit of $J_{\nu 0}<10^{-21} \mathrm{erg} \mathrm{s}^{-1} \mathrm{~cm}^{-2} \mathrm{~Hz}^{-1} \mathrm{Sr}^{-1}$ (see Fig. 2).

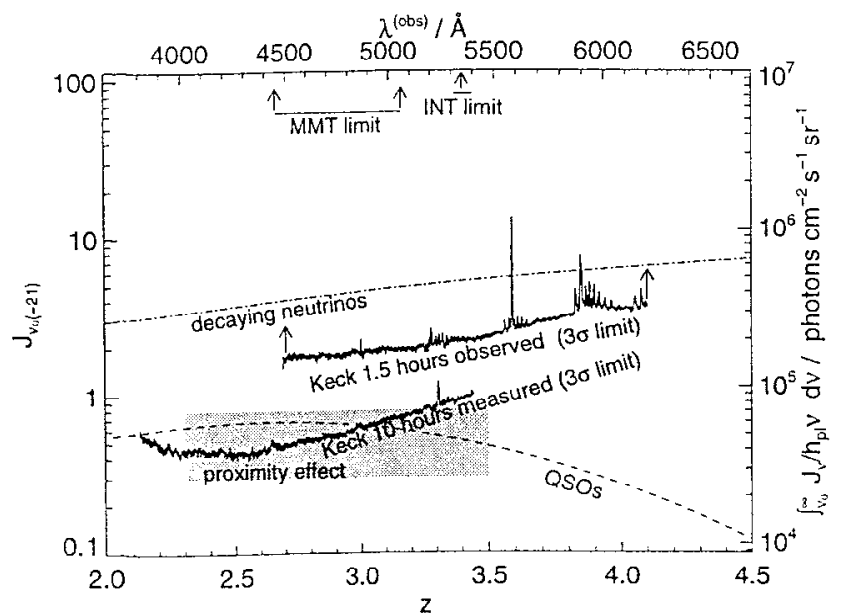

Figurc 2. Our $3 \sigma$ upper-limits on the flux density at the Lyman edge, $J_{\nu 0(-21)}=J_{\nu 0} / 10^{-21} \mathrm{erg} \mathrm{s}^{-1} \mathrm{~cm}^{-2} \mathrm{~Hz}^{-1} \mathrm{sr}^{-1}$, as a function of redshift (solid line) from our 1.5 hour pilot study (Bunkcr, Marleau \& Graham 1998). The null results of this preliminary search mean that the region above the heavy solid curve is excluded. Also shown arc the previous $3 \sigma$ upper-limits from the INT (Martínez-González 1995) and the MMT (Lowenthal et al. 1990), converted to the same assumed projected cloud size $\left(\approx 10^{\prime \prime}\right)$. We also plot the $3 \sigma$ sensitivity reached in our recently extended program: we have obtained a 15 hour spectrum with LRIS/Keck-II (dotted curved), and we are currently processing this enlarged data set to look for the signature of the ionizing back ground, reprocessed as Ly $\alpha$. The shaded region is the lower-limit on $J_{\nu 0}$ from the QSO proximity effect (Espey 1993). The estimatcd contribution to $J_{\nu 0}$ from the luminosity function of known high-z QSOs is also shown as the dashed line (Haardt \& Madau 1996). The equivalent Lyman continuum flux from decaying relic neutrinos is plotted as a dot-dash line, adopting the parameters of Sciama (1998) and assuming these neutrinos form the bulk of the dark matter in an $\Omega_{\mathrm{M}}=1 \mathrm{Uni-}$ verse. Such a scenario is strongly ruled out by the null results of our pilot study, as we would have detected Ly $\alpha$ fluorescence from the flux of ionizing decay photons. 


\section{Conclusion}

A search with LRIS/Keck-II is used to constrain the fluorescent Ly $\alpha$ emission at $z \approx 2-3$ from the clouds which produce the higher-column-density component of the Ly $\alpha$ forest. The null results of a pilot study by Bunker, Marleau \& Graham (1998) provided the best upper limit yet on the ionizing UV background of $J_{\nu 0} \lesssim 2 \times 10^{-21} \mathrm{erg} \mathrm{s}^{-1} \mathrm{~cm}^{-2} \mathrm{~Hz}^{-1} \mathrm{sr}^{-1}(3 \sigma$ limit at $2.7<z<3.1)$. We have now extended our integration time to 15 hours, altaining a $3 \sigma$ sensitivity of $J_{\nu 0}<10^{-21} \mathrm{erg} \mathrm{s}^{-1} \mathrm{~cm}^{-2} \mathrm{~Hz}^{-1} \mathrm{sr}^{-1}$ at $z \approx 2.3$ - one of the deepest optical spectra ever obtained. We are currently conducting a power-spectrum analysis to detect the signature of the ionizing background at high redshift.

Acknowledgments. We thank the organizing committee for an enjoyable and informative meeting. We are grateful to everyone at the Keck observatory for their assistance with our program. FRM is supported by an IoA postdoctoral research fellowship and AJB acknowledges a NICMOS postdoctoral fellowship (grant NAG 5-3043). JRG is supported by the Packard Foundation.

\section{References}

Bertin, E., \& Arnouts, S. 1996, A\&AS, 117, 393

Bunker, A.J., Marleau, F.R., \& Graham, J.R. 1998, AJ, 116, 2086

Charlot, S., \& Fall, S.M. 1993, Ap.J, 415, 580

Dove, J.B. \& Shull, J.M. 1994, ApJ, 423, 196

Espey, B.R. 1993, ApJ, 411, 59

Gould, A., \& Weinberg, D.H. 1996, ApJ, 468, 462

Haardt, F., \& Madau, P. 1996, ApJ, 461, 20

Hall. P.B, Osmer, P.S., Green, R.F., Porter, A.C., \& Warren, S.J. 1996, ApJ, 462,614

Hogan, C.J., \& Weymann, R.J. 1987, MNRAS, 225, 1

Kim, T.-S., Hu, E.M., Cowie, L.L., \& Songaila, A. 1997, AJ, 114, 1

Lowenthal, J.D., Hogan, C.J., Leach, R.W., Schmidt, G.D., \& Follz, C.B. 1990, ApJ, 389, 1

Martínez-González, J.I., González-Serrano, L., Cayón, L., Sanz, J.L., \& MartínMirons, J.M. 1995, A\&A, 303, 379

Oke, J.B., Cohen, J.G., Carr, M., Cromer, J., Dingizian, A., Harris, F.H., Labrecque, S., Lucinio, R., Schall, W., Epps H. \& Miller, J. 1995, P.ASP, 107,375

Sciama. D.W. 1998, A\&A, 335, 12

Songaila, A., Cowie, L.L. \& Lilly, S.J. 1990, ApJ, 348, 371

Steidel, C.C., \& Dickinson, M. 1995, in "Wide Field Spectroscupy of the Distant Universe", eds. Maddox, S.J. \& Aragon-Salamenca, A.

Stengler-Larrea, E., Boksenberg, A., Steidel. C.C., et al. 1995, ApJ, 444.64 
$\because$ 
The Hy Redshift Universe

ASP Conference Series, Vol. 193, 1999

A. J. Bunker \& W. J. M. van Breugel, eds.

\title{
Near Infrared Properties of Galaxies in the HDF-N
}

\author{
Casey Papovich \\ Department of Physics and Astronomy, Johns Hopkins University, \\ Baltimore, MD 21218
}

Mark Dickinson and Chris Hanley

STScI, Baltimore, MD 21218 (for the NICMOS HDF-N GO Team)

\begin{abstract}
We present results from a complete HSI/NICMOS map of the Hubble Deep Field North (HDF-N). We briefly illustrate the photometric properties of the galaxy population with near-infrared (NIR) color-magnitude and color-redshift distributions. Fainter than $H_{160}=$ 23.5 , the large majority of galaxies have very blue optical-NIR colors. We illustrate the NICMOS counterparts to VLA $8.5 \mathrm{GHz}$ and SCUBA $850 \mu \mathrm{m}$ sources. 'The optical-IR properties of objects near the SCUBA positions are unremarkable, and no new candidate counterparts are detected for the "blank field" SCUBA or VLA sources.
\end{abstract}

\section{Introduction}

The HDF-N (Williams et al. 1996) has provided an invaluable resource for the study of galaxies at high redshifts. The original WFPC2 HDF-N observations provided four-band photometric coverage with high resolution from $0.3-0.8 \mu \mathrm{m}$. Optical rest-frame light from galaxies at $z>1$ is shifted out the WFPC2 passbands into the near-infrared (NIR). This population includes many (or even most) of the HDF galaxies and it becomes difficult to reliably compare the morphological and photometric properties to low-redshift galaxies without supplementary observations in the near-infrared.

The installation of NICMOS on board HST permitted NIR observations with high resolution and strong sensitivity. Two complementary programs studied the HDF-N with NICMOS. The NICMOS IDT (Thompson et al. 1998) observed a single NIC3 field with the $J_{110}$ and $H_{160}$ filters with long ( $>100 \mathrm{ksec}$ ) exposures. Our GO program mosaicked the entire HDF using eight pointings with a $12.6 \mathrm{ksec}$ exposure per filter, reaching (on average) $S / N \approx 10$ at $H_{160}=26.1$ (we use $A B$ magnitudes throughout) in a 0.7 diameter aperture. The data reduction and analysis will be presented elsewhere (Dickinson et al. 1999, in prep.). The images are $\approx 2.1$ and 2.8 magnitudes deeper than the best available ground-based data at $J$ and $H$. Our IR-selected catalog contains $\approx 1700$ objects, compared to the $\approx 3200$ objects detected by Williams et al. (1996). We have also extended the catalog photometry to $2.2 \mu \mathrm{m}$ where possible using a KPNO $K$-band image (e.g., Dickinson 1998). 


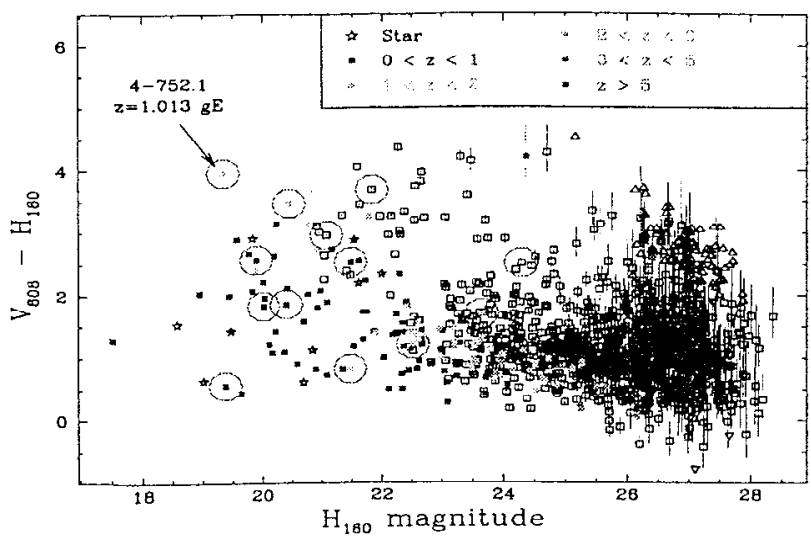

Figure 1. $V_{606}-H_{160}$ vs. $H_{160}$ for the NICMOS HDF-N. Circles indicate the identified counterparts to 13 VLA $8.5 \mathrm{GHz}$ HDF sources, and filled squares have spectroscopic redshifts as indicated.

\section{Statistical Photometric Properties of Galaxies}

We illustrate the photometric properties of the HDF/NICMOS sample with $V_{606}-H_{160}$ color-magnitude (Figure 1 ) and color-redshift (Figure 2) diagrams. An interesting split in the galaxy population is seen in Figure 1: At $H_{160} \lesssim 23.5$, galaxies span a wide range of color with a "red envelope" which is nicely visible in the color-redshift diagram (note the $z=1.01$, gE radio galaxy $4-742.1$ [VLA 3644+1133]). At $H_{160} \gtrsim 23.5$, the color range collapses and is dominated by the archetypal, "faint blue galaxies" even for a NIR-selected sample. The blue galaxies span a wide redshift range, $0 \lesssim z \lesssim 4$.

\section{Near-IR Counterparts to Radio and Far-IR sources}

The HDF-N has been the target of longer-wavelength surveys including the VLA (Richards et al. 1998) and SCUBA (Hughes et al. 1998). These wavelength regions are important as tracers of star forming activity, either directly (cm) Or through dust reprocessing ( $5 u b-\mathrm{mm}$ ).

In Figure 3 we show NICMOS $H_{160}$ images of the VLA $8.5 \mathrm{GHz}$ sources within the NICMOS field. In general, the NIR morphology of the radio sources is similar to the optical, although $3641+1142$ (redshift uncertain) transforms Iadically from "fragments" in the optical images to a unified bulge + arms disk system in the near-IR. Richards et al. (1998) reported one source, $3646+1226$, with no optical counterpart, nor is there one in the NICMOS data. 3651+1221 has a very red counterpart in the NICMOS data. This was uncataloged from optical data due to confusion with the foreground spiral, although there is faint optical flux. This object has a strong discontinuity, $J_{110}-H_{160}=1.6$, which may be the Balmer/4000A break, and which corresponds to the photometric redshift, $\tilde{z}_{\mathrm{ph}}=2.6$, from Budavari et al. (1999, in prep.).

Due to the negative $H$-correction of the far-IR, SCUBA observations are, in principle, sensitive to star-forming galaxies out to $\approx \sim 10$, where galaxies 


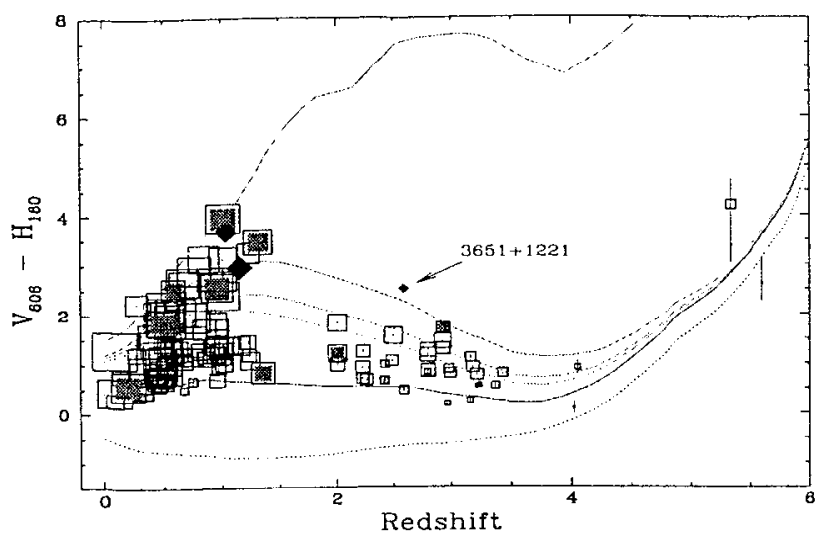

Figure 2. $\quad V_{606}-H_{160}$ vs. redshift. The VLA sources are plotted as filled squares, or diamonds where only photometric redshifts are available. The curves are fiducial tracks for model galaxies. The reddest curve is a passively evolving elliptical formed at $z=15$, while the bluer curves are various non-evolving, late-type galaxies; the bluest is an unreddened, young (10 Myr) starburst.

would be invisible to the optical HDF. At any redshift, starbursts may be dusty and red, and indeed some faint, non-HDF, sub-mm sources have been identified with very red objects in infrared images (Dey et al. 1999; Smail et al. 1999). However, the NICMIOS images (Figure 4) reveal no new counterparts for the "blank field" HDF SCUBA sources, and the plausible counterpart candidates do not have particularly unusual colors.

Acknowledgments. We thank our collaborators on the NICMOS GO HDF$\mathrm{N}$ tcam and our colleagues at JHU and STScI. Support was provided by NASA through grant GO-07817.01-96A from STScI, which is operated by AURA under NASA contract NAS5 26555.

\section{References}

Dey, A., et al. 1999, ApJ, in press; astro-ph/9902044

Dickinson, M. 1998, in The Hubhle Deep Field, eds. M. Livio, M. Fall, \& P. Madau, Cambridge: Cambridge Univ. Press, 219

Dickinson, M. 1999, in After the Dark Ages: When Galaxics werc Young, cds. S. Holt \& E. Smith, American Institute of Physics Press, 122

Downes, D. et al. 1999, A\&A, submitted; astro-ph/9907139

Hughes, D. H. et al. 1998, Nature, 394, 241

Richards, E. A., et al. 1998, ApJ, 116, 1039

Smail, I., et al. 1999, MNRAS; in press; astro-ph/9905246

Thompson, R. I., et al. 1999, AJ, 117, 17

Williams, R. et al. 1996, AJ, 112, 1355 


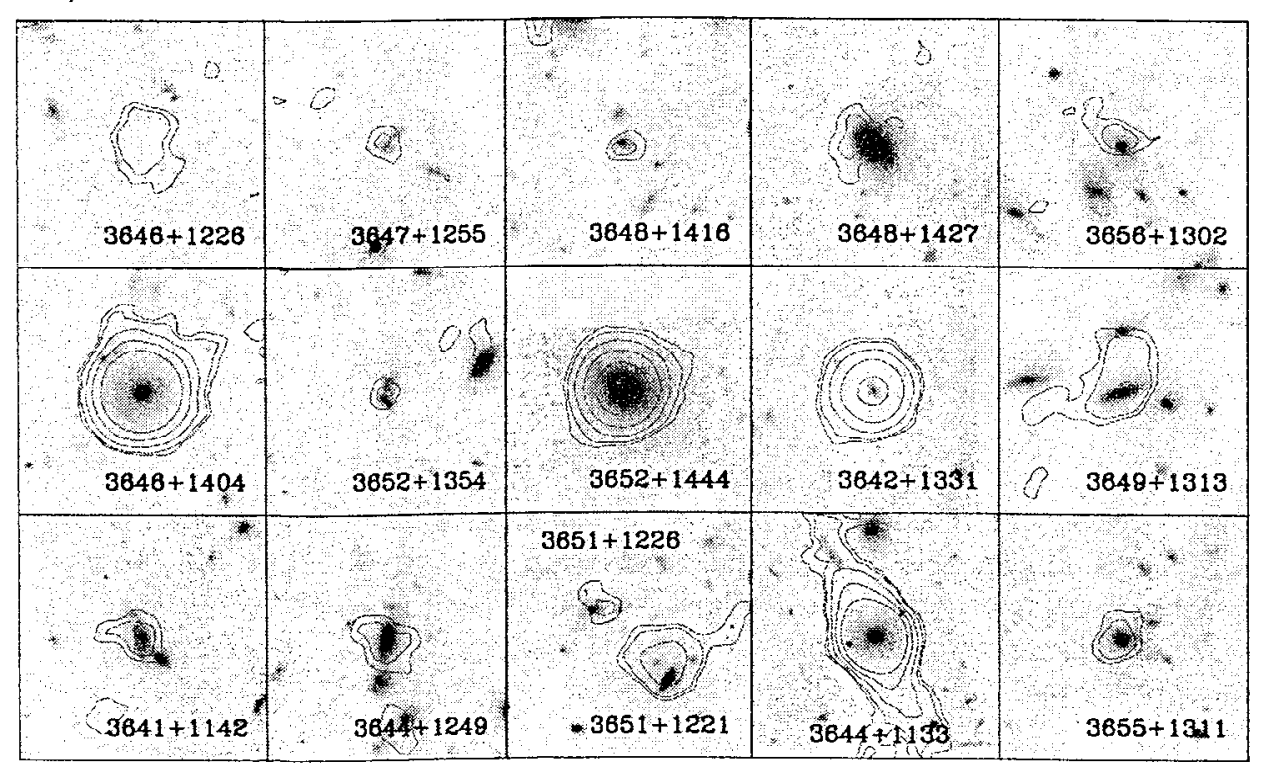

Figure 3. Gray-scale NICMOS $I_{160}$ images for 16 of the 17 VLA 8.5 GHz sources within the NICMOS HDF-N field of view, excluding $3651+1321$ ( $z=0.199$ spiral) for lack of space. Each cutout is $10^{\prime \prime} \times 16^{\prime \prime}$, except $3644+1133$ which is $24^{\prime \prime} \times 24^{\prime \prime}$. Contour levels correspond to the 8.5 $\mathrm{GHz}$ data at $2 \sqrt{2} \sigma, 4 \sigma$, and then increase by factors of 2 .

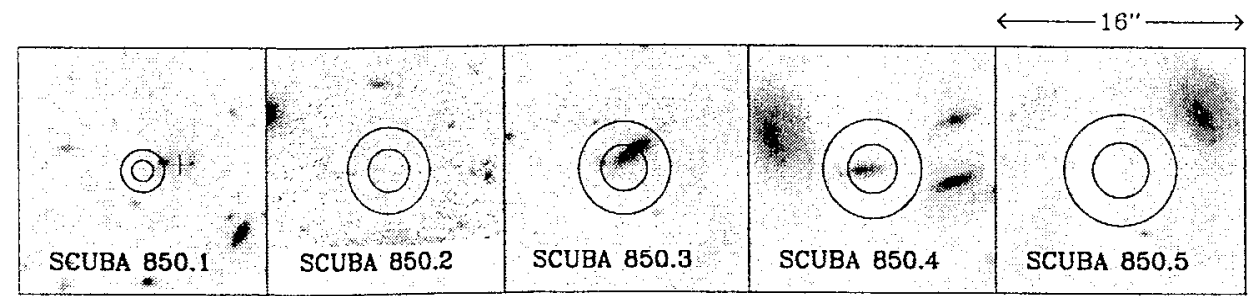

Figure 4. Gray-scale NICMOS $H_{160}$ images showing fields of the SCUBA $850 \mu \mathrm{m}$ sources. The circles show the $1 \sigma$ and $2 \sigma$ SCUBA position uncertainties from Hughes et al. (1998). The cross for 850.1 shows the IRA.M $1.3 \mathrm{~mm}$ position from Downes et al. (1999) 
The Hy Redshift Universe

ASP Conference Series, Vol. 193, 1999

A. J. Bunker \& W. J. M. van Breugel, eds.

\title{
. The HDF, The Arizona View
}

\author{
Rodger I. Thompson \\ Steward Observatory and Department of Astronomy, University of \\ Arizona, Tucson, AZ 85721
}

Ray J. Weymann and Lisa Storrie-Lombardi

Carnegie Observatories, Pasadena CA 91101

\begin{abstract}
We present an initial analysis of the star formation rates in the deep NICMOS field of the Hubble Deep Field. The analysis utilizes template photometric redshift and extinction techniques on the combination of six optical and near infrared fluxes available for this region from observations with both NICMOS and WHPC2. Our results are consistent with a constant star formation rate for a redshift range of 1 to 6 .
\end{abstract}

\section{Introduction}

The history of star formation in the universe is a matter of intense current study. Early observations eg. Madau et al. 1996 found an order of magnitude increase in star formation from the present epoch to a redshift range of approximately 1.5 then a decrease in star formation out to a redshift of 4 . Since that analysis used the $1500 \AA$ flux as a star formation indicator it is subject to uncertainty due to extinction. Far infrared studies in the HDF eg. Hughes et al. 1998 detected several far infrared sources and suggested that significant amounts of star formation can be hidden by dust obscuration. The combined NICMOS and WFPC observations cover a factor of 5 (0.3 - 1.6 microns) in wavelength, therefore, it is possible to determine both the redshift and the extinction by photometric means. The data for this analysis comes from the HDF observations of Williams et al. 1996 and Thompson et al. 1999.

\section{Methodology}

Since there are no good polynomial fits for very high redshift galaxies and near infrared fluxes we utilize the template fitting method of photometric redshifts. We take 11 different spectral energy distribution templates that span the range from early galaxies to very recent starbursts. The templates also include two low metallicity templates. Each template is then numerically redshifted and extincted to provide a very large grid of templates. The extinction law is taken from Calzetti et al. 1994.

The observed fluxes are then matched against the template fluxes to determine the fit with the minimum chi squared value. The redshift and extinction 
- for that template is then considered the correct redshift and extinction for the source.

The chi squared value for the fit is given by

$$
\chi(z, E)^{2}=\sum_{i=1}^{6} \frac{\left(f_{i}-A \cdot f \bmod (z, E)_{i}\right)^{2}}{\sqrt{\sigma_{i}^{2}+f_{i}^{2}}}
$$

where $\mathrm{i}$ is the flux band, $f_{i}$ is the observed flux, $\operatorname{fmod}(z, E)$ is the template flux numerically altered for the redshift $z$ and extinction $E$ and $A$ is the normalization that minimizes the chi squared value given by

$$
A=\sum_{i=1}^{6} \frac{f_{i} \cdot f \bmod (\tilde{z}, E)_{i}}{\sigma_{i}^{2}+f_{i}^{2}} / \sum_{i=1}^{6} \frac{\left(f \bmod (z, E)_{i}\right)^{2}}{\sigma_{i}^{2}+f_{i}^{2}}
$$

Note that in addition to the usual errors given by $\sigma_{i}$ we also divide through by the flux $f_{i}$. This takes into account that at larger fluxes the main error is a systematic fraction of the flux rather than the formal $1 \sigma$ error from the noise.

\section{Star Formation Rates}

Following Madau, Pozzetti and Dickinson 1998 we determine the star formation rate from the $1500 \AA$ flux by

$$
S F R=8.0 \times 10^{27} \cdot U V_{1500} M_{\odot} / y r
$$

In our case we utilize the UV flux from the unextincted template since we wish to correct for the extinction effects. The star formation rate as a function of redshift for the NICMOS deep observations of the HDF appear in Figure 1.

The error bars on this figure are determined from an analysis of the effect of photometric errors on the star formation rate not by number statistics. This is appropriate since we are measuring the star formation rate that exists in the NICMOS region of the HDF and are not trying to extrapolate it to other regions of the sky. Except for the excess near a redshift of 2 the star formation rate is roughly constant at all $z$ values out to 6 . The bump at redshift 2 is probably due to the very narrow line of sight passing through a $z=2$ cluster of galaxies. We do not try to determine the star formation rate at low redshifts due to the very small number of galaxies in our sample at low redshifts. The inclusion of extinction raises the rate by roughly a factor of two at all redshifts.

\section{Conclusions}

Our observations do not show a decrease in the star formation rate as a function of $z$ out to a redshift of 6 . This conclusion is not due to the inclusion of extinction but extinction does reduce the rate determined by optical means by about a factor of two at all redshifts. These results are consistent with the recent results of Steidel_et al. 1999 that find no fall off in star formation rate out to a redshift of 5 . 
STAR FORMATION RATE VERSUS REDSHIFT

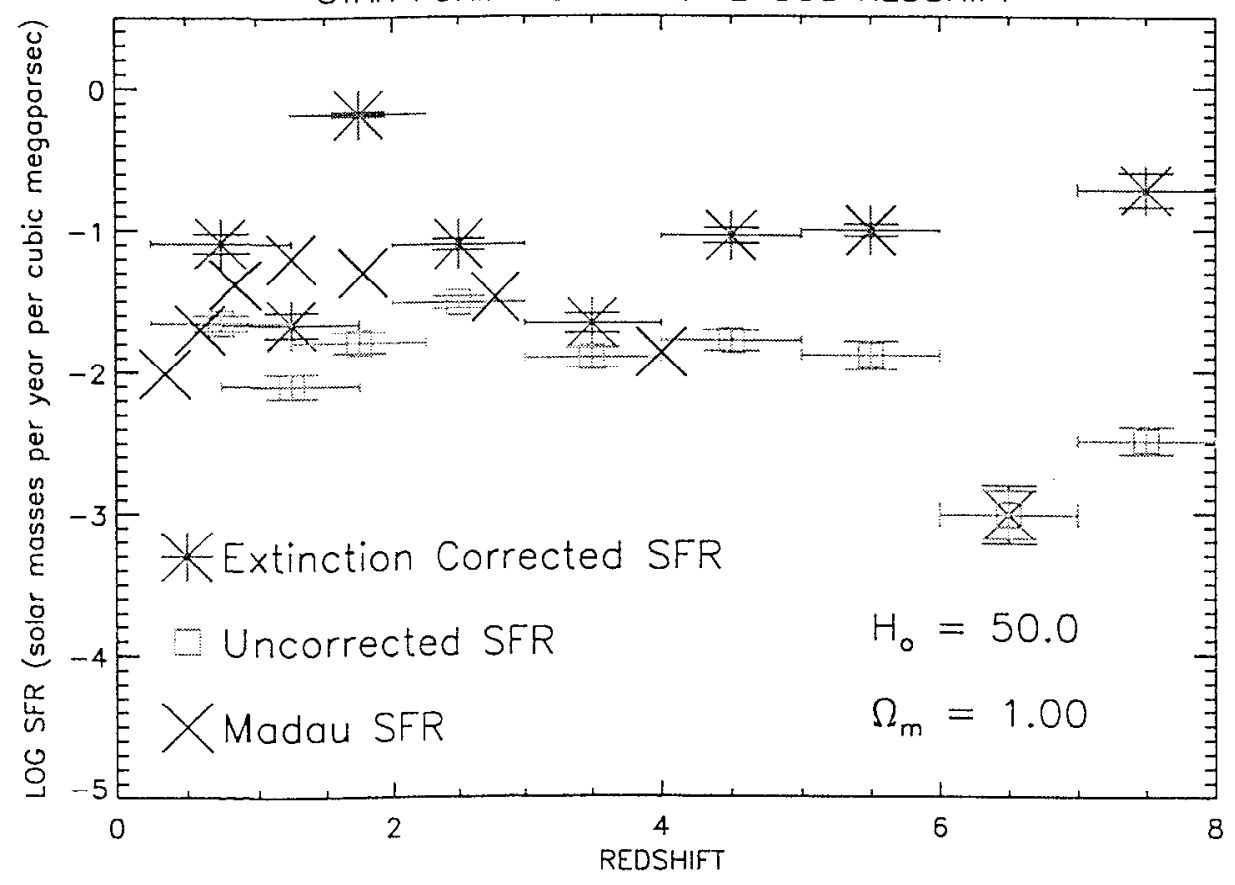

Eigure 1. Star formation rate per comoving volume as a function of redshift 
Acknowledgments. This work is supported in part by NASA grant NAG 5-3042. This work utilized observations with the NASA/ESA Hubble Space Telescope, obtained at the Space Telescope Science Institute, which is operated by the Association of Universities for Research in Astronomy under NASA contract NAS5-26555.

\section{References}

Calzetti, D., Kinney, A. L., and Storchi-Bergmann, T. 1994, Ap.J, 429, 582

Hughes, D., Sergeant, S., Dunlop, J., Rowan-Robinson, M., Blain, A., Mann, R.G., Ivison, R., Peacock, J., Efstathiou, A., Gear, W., Oliver, S., Lawrence, A., Longair, M., Goldschmidt, P., and Jenness, T. 1998, Nature, 394, 241

Madau, P., Ferguson, H.C., Dickinson, M.E., Giavalisco, M., Steidel, C.C., and Fruchter, A. 1996, MNRAS, 283, 1388

Steidel, C.C., Adelberger, K.L., Giavalisco, M., Dickinson, M., Pettini, M. 1999 preprint, ApJ, 519, 1

Thompson, Rodger I., Storrie-Lombardi. Lisa, J., Weymann, Ray J., Rieke, Marcia, J., Schneider, Glenn, Stobie, Elizabeth, and Lytle, Dyer 1999, AJ, 117,17 .

Williams, R.E., Blacker, B. Dickinson, M., Dixon, W.V.D, Ferguson, H.C., Fruchter, A.S., Giavalisco, M., Gilliland, R.L., Heyer, I. Katsanis, R., Lcvay, Z. Lucas, R.A., McElroy, D.B., Petro, L., Postman, M. Adorf, H-M., and Hook, R.N. 1996, AJ, 112, 1335 
The Hy Redshift Universe

ASP Conference Series, Vol. 193, 1999

A. J. Bunker \& W. J. M. van Breugel, eds. $\checkmark$

\title{
Deep Sub-millimeter Surveys: Highly Luminous Far-Infrared Sources at High Redshift
}

Tracy Webb and Simon Lilly

Department of Astronomy, University of Toronto, 60 St. George St., Toronto, Ontario, Canada, M5S $3 \mathrm{H} 8$

Steve Eales, Walter Gear, Loretta Dune and Dave Clements

Department of Physics and Astronomy, Cardiff University, P.O. Box

913, Cardiff, United Kindom, CF2 $3 Y B$

Dick Bond

Canadian Institute for Theoretical Astrophysics, University of Toronto, 60 St. George St., Toronto, Ontario, Canada, M5S $3 \mathrm{H} 8$

David Crampton

Dominion Astrophysical Observatory, National Research Council of Carıada, Victoria, British Colombia, Canada, V8X \&M6

François ITammer and Olivier Le Fèvre

$D A E C$, Observatoire de Meudon, Meudon, France, 92195

\begin{abstract}
We present results from the Canada-UK deep sub-millimeter survey with the JCMT. We have resolved $20 \%$ of the far-IR/sub-mm background (to $3 \mathrm{mJy}$ ) and have identified a population of objects which appear similar to Arp220 although significantly more luminous. These objects span a redshift range of $z \sim 0.5$ to $z \sim 3$, peaking at $z \sim 2$. At these redshifts these objects are responsible for $\sim 30 \%$ (to $1 \mathrm{mJy}$ ) of the bolometric background and are therefore an extremely important phase of galary evolution.
\end{abstract}

\section{Introduction}

Over the last few years we have made great strides forward in our understanding of the evolution of the universe. However, the current picture is based primarily on optical/UV studies while recent results from COBE (Hauser et al 1998, Fixsen et al 1998, Dwek et al 1998) have shown that the bolometric energy density in the far-IR/sub-mm background is at least as large as that of the optical/UV/near-IR background (determined from integrated galaxy counts (Pozzetti et al 1998)). The far-IR/sub-mm background is produced by the thermal emission from low-temperature dust that has absorbed and reprocessed op- 
tical/UV radiation. Understanding the sources responsible for this background is essential to a complete view of the evolution of the universe.

The recent commissioning of the SCUBA bolometer array on the James Clerk Maxwell Telescope (JCMT) has made deep sub-mm surveys possible for the first time and there are a number of independent surveys currently underway (e.g. Smail et al (1997), Hughes et al (1998), Barger et al 1998 and ourselves). These surveys have the advantage that at $850 \mu \mathrm{m}$ the $k$-corrections are such that the flux from an Arp220-like object (i.e. thermally radiating dust) remains constant between redshifts of approximately $z=1$ to $z=8$, for $\Omega=1$. Thus, deep sub-mm surveys are not limited by the usual correlation between distance and luminosity.

\section{The Canada-UK Deep Sub-millimeter Survey}

Our group is mapping two $8 \times 8$ arcmins $^{2}$ regions of the CFRS redshift survey $(1417+52,0302+00)$ to a detection limit of $3 \mathrm{mJy}($ at $3 \sigma)$. Because we are working at low signal-to-noise we create a mosaic of one-hour pointings such that each location in the map is observed nine separate times, but never with the same area of the array, producing cosmetically clean maps with uniform noise properties. We have completed just over one half of the survey area (Eales ct al 1999, Lilly et al 1999) and we expect to have two $6 \times 8 \mathrm{arcmins}^{2}$ regions complete to $3 \sigma$ by the end of the year 2000 .

A great deal of data already exists for these CFRS fields. The CFRS survey determined spectroscopic redshifts for thousands of galaxies down to a limiting magnitude of $\mathrm{I}_{A B} \sim 22.5$. As well, these fields have been observed with HST, ISO (Flores et al 1998ab) and the VLA (Fomalont et al 1992, Hammer et al 1995). Thus, many sources alrcady have morphological information, spectroscopic or photometric redshifts and information about their spectral energy distributions availablc. Our group has several initiatives currently underway increase the amount of information available for each source. We plan to determine spectroscopic and photometric redshifts for the remaining optical identifications without redshifts (with CFHT and UKIRT). Deep infrared imaging will improve the photometric redshifts and provide morphological information as will more HST imaging. Of extreme importance are observations at $1.3 \mathrm{~mm}$ with the IRAM intcrforomcter which will greatly reduce the positional uncertainties of the sources and secure our identifications.

\section{The Identifications}

The 15 arcsecs beam of the JCMT makes the optical identifications difficult. We have adopted a probabilistic method in which we estimate the probability that a sub-mm source is randomly associated with a galaxy selected from a population with surface density $n$ and located a distance $d$ from the source. 'This probability is given by:

$P=1-\exp \left(-\pi n d^{2}\right)$

Thus, for a given source we can only estimate the probability that the identification is correct. It is the distribution of $P$ values for the entire sample; rather than for a single source which is meaningful. That is, the distribution of 
-probabilities must be compared to what is expected from randomly associated populations.

For faint galaxy populations, which have a high surface density, the success of this method is limited. However, the surface density of radio sources is very low and are identified with at least one half of the sub-mm sources. Colour and morphological information can also be used to secure an identification as many of the identifications appear disturbed with merger-like morphologies and red colours.

\section{Results}

We have completed approximately one half of our survey area and have detected $\sim 30$ sources. Twelve of these have been published in Eales et al (1999) and Lilly et al (1999) and the remaining sources are in various stages of identification. The redshifts of the identified sources span a range of $z=0.5$ to $z=3$, peaking at $\mathrm{z} \sim 2$ and they are extremely luminous (i.e. $\mathrm{L}_{I R}>3 \times 10^{12} \mathrm{~L} \odot$ ). As well, the spectral energy distributions, when multi-wavelength data is available, indicate that these sources are very similar to Arp220.

The internal energy source responsible for these high luminosities is still unknown. However, if we assume it is star formation then these objects have star formation rates of $>600 \mathrm{M}_{\odot} /$ year. This massive star formation rate coupled with the large amounts of dust present and the predominance of disturbed morphologies makes it attractive to associate these sources with formation of the metal-rich spheroids of today, through highly dissipative mergers of gas-rich galaxies. It is also possible that these objects are powered by black holes, which could account for the large number of black holes in the local universe and may indicate that the formation and evolution of black holes is closely linked to that of the spheroids.

At $3 \mathrm{mJy}$ we have resolved $\sim 20 \%$ of the far-IR/sub-mm background, with $\sim 50 \%$ resolved at $1 \mathrm{mJy}$ (Hughes et al 1998, Blain et al 1999). Thus, these super-Arp220 objects are responsible for $\sim 30 \%$ of the bolometric background. In fact, the bolometric luminosity density in the infrared of these objects is greater than the bolometric luminosity density in the UV of the entire UV selected population. A comparison with the local universe, in which Arp220-like objects contribute $\sim 0.3 \%$ to the bolometric light, indicates that these ultra-luminous infrared galaxies are much more important at $\mathrm{z} \sim 2$ than today. Approximately one third of the sources are classified as empty-field sources (i.e. they have no optical counterpart) and may lie at $z>3$. However, because $f_{\nu} \propto \nu^{3.5}$ for these dusty objects, the flux is weighted by $(1+z)^{3.5}$, so that the production of the background is not directly proportional to the production of mass. Thus, even if these sources are producing $30 \%$ of the resolved background they are likely producing less than $10 \%$ of the stellar (or black hole) mass.

\section{References}

Barger. A.. Cowie, L., Sandars, D., Fulton, E., Taniguchi, Y., Sato, Y., Katware, K., Okuda, H. 1998, Nature, 394. 248 
Blain, A., Kneib, J.P., Ivison, R., Smail, I. 1999 ApJ, 512, 87

Dwek, E., Arendt, R., Hauser, M., Fixsen, D., Kelsall, T., Leisawitz, D., Pei, Y., - Wright, E., Mather, J., Moseley, S., Odegard, N., Shafer, R., Silverberg, R., Welland, I. 1998, ApJ, 508, 106.

Eales, S., Lilly, S., Gear, W., Dunne, L., Bond, R.J., Hammer, F., Le Fèvre, O., Crampton, D. 1999, ApJ, 515, 518

Fixsen, D., Dwek, E., Mather, J., Bennet, C., Shafer, R. 1998, ApJ, 508, 123

Flores H., Hammer,F., Desert, F.X., Cesarsky, C., Thuan, T.X., Crampton, D., Eales, S., Le Fevre, O., Lilly, S., Omont, O., Elbaz, D. 1998a

Flores H., Hammer,F., Desert, F.X., Cesarsky, C., Crampton, D., Omont, A., Thuan, T.X., Eales, S., Le Fèvre, O., Lilly, S. 1998b

Hauser, M., Arendt, R., Kelsall, T., Dwek, E., Odegard, N., Welland, J., Freundenreich, H., Reach, W., Silverberg, R., Moseley, S., Pei, Y., Lubin, P., Mather J., Shafer, R., Smoot, G., Weiss, R., Wilkinson, D., Wright E., 1998, ApJ, 508, 25

Hughes, D., Serjeant, S., Dunlop, J., Rowan-Robinson, M., Blain, A., Mann, R., Ivison, R., Peacock, J., Efstathiou, A., Gear, W., Oliver, S., Lawrence, A., Longair, M., Goldschmidt, P., Jeness T., 1998, Natıre, 394, 241

Lilly, S. ApJ, 518, 6411999

Pozzetti, L., Madau, P1, Zamorani, G., Ferguson, H.C., Bruzual, G., 1998, MÑRAS, 298, 1133

Smail, I., Irison, R, Blain, A., 1997, ApJ, 490, Lj 
Section D. Galaxy Evolution in Clusters 
The Hy Redshift Universe

ASP Conference Series, Vol. 193, 1999

A. J. Bunker \& W. J. M. van Breugel, eds.

\title{
- Galaxy Evolution in Clusters: the MORPHS Study
}

\author{
Augustus Oemler, Jr. \\ Carnegie. Obseruatories, 813 Santa Barbara St., Pasadena, CA 91101
}

\begin{abstract}
The MORPHS group has studied 10 rich clusters of galaxies in the range $0.37 \leq z \leq 0.56$, using HST imaging and ground-based spectroscopy. The principal conclusions of this study are: (1) The elliptical galaxy populations of clusters were fully in place prior to $z=0.5$; the disk galixies are a much more loosely bound population, probably produced by later infall onto the E-galaxy core. (2) The S0 population has increased dramatically since $z \sim 0.5$, due to the transformation of spiral galaxies. (3) Gravitational interactions and starbursts play an important role in this process, but other factors, operating only in clusters, are necessary to complete the transformation to S0's.
\end{abstract}

\section{Introduction}

The MORPHS collaboration (A. Dressler, R. Ellis, I. Smail, B. Poggianti, W. Couch, H. Bulcher, R. Sharples, A. Barger, and A. Oemler) formed with the goal of making significant progress in understanding cluster galaxy evolution. It has been known for many years that star formation was much more prevalent in clusters at $z \sim 0.5$ than it is today (Butcher \& Oemler 1978), and that much of this star formation occurred in bursts (Dressler \& Gunn 1982). The first cycles of HST observations showed that the spectral peculiarities indicative of starbursts are often accompanied by morphological peculiarities: many cluster galaxies at $z \sim 0.5$ show strong distortions suggestive of mergers or tidal interactions (Couch et al. 1994, Dressler et al. 1994).

It was tempting at this point to consider the problem solved: galaxy evolution in clusters is driven by dynamical interactions; these interactions precipitate starbursts, and the combined effects of dynamical shocks and rapid star formation drive galaxies towards the early end of the IIubble sequence. This is a tidy picture, but the supporting evidence has been no more than suggestive. Furthermore, mergers are an awhward means of driving cluster galaxy evolution: the merger rate in a high-velocity cluster environment should be very low, and the outcome of major mergers is generally expected to be ellipticals, while the dominant galaxy population in nearby clusters is S0's.

Given the sparse and fragmenlary data, it was clear that many more were needed, ideally a large, homogeneous data set covering many clusters with a wide range of properties. The MORPHS siudy includes HST and ground-based observations of 10 clusters of galaxies in the range $0.37 \leq z \leq 0.56$. Their selection was not based upon a rigorous system. Many were included because they had been previously observed; they were observed before because they were 
among the few high-redshift clusters known at the time. However, this set of clusters does have the virtue of spanning a wide range of structural properties and richness; therefore they sample most of the parameter space occupied by clusters.

The HST data consists of WFPC2 images of the fields listed in Table 1. They cover the central regions of all clusters except Abell 370, and images of

Table 1. The Cluster Sample

\begin{tabular}{llcl}
\hline \multicolumn{1}{c}{ Cluster } & Region & $z$ & \multicolumn{1}{c}{ Description } \\
\hline Abell 370 & outer & 0.37 & rich, concentrated \\
C11447+23 & center & 0.37 & rich, irregular \\
C10024+16 & center & 0.39 & very rich, concentrated \\
Cl0939+47 & center & 0.39 & rich, mod. irregular \\
& outer & & \\
C10303+17 & center & 0.42 & mod. rich, irregular \\
3 C295 & center & 0.46 & mod. poor, concentrated \\
C10412-65 & center & 0.51 & mod. poor, irregular \\
Cl1601+42 & center & 0.54 & mod. poor, mod. irregular \\
Cl0016+16 & center & 0.55 & very rich, concentrated \\
C10054-27 & center & 0.56 & mod. rich, concentrated \\
\hline \hline
\end{tabular}

fields $3^{\prime}-5^{\prime}$ from the centers of Abell 370 and Cl0939+47. Exposure times range from 4 to 23 kiloseconds, and are- mostly- in either the F555W, F702W, or F814W bands. Six of the fields have observations in at least 2 bands, allowing us to derive colors. From these data we have obtained photometry of about 7000 galaxies, and morphologies of about 1800 galaxies brighter than $r \sim 23.5$. We have also obtained ground-based spectroscopy of 657 galaxies ( 424 cluster members and 233 field galaxies), of which 275 (204 cluster/71 field) are within the HST fields and therefore have morphological types. The imaging and spectroscopic data sets are described in more detail in Smail et al. (1997) and Dressler et al. (1999). Most of the analysis described below is contained in two other papers, Dressler et al. (1998) and Poggianti et al. (1999). Unless otherwise noted, all of the discussion below refers to the subset of objects for which we have spectroscopy, and can, therefore, assign membership to either clusters or field. Although the field objects have a wide range of redshift, most are within a few tenths of those of the clusters. It seems reasonable, then, to consider them as representative of the field population at the same epoch as that observed in the clusters.

\section{Galaxy Properties}

Morphological types were assigned to galaxies within the HST fields by visual inspection, using the standard Hubble system. We also assigned two additional parameters, the first describing the degree of apparent disturbance of the galaxy, and the second our best guess for its nature: I- tidal interaction with a neighbor; $\mathrm{M}$-merger, $\mathrm{T}$-tidal tail with no obvious interacting galaxy, and $\mathrm{C}$-chaotic. The 


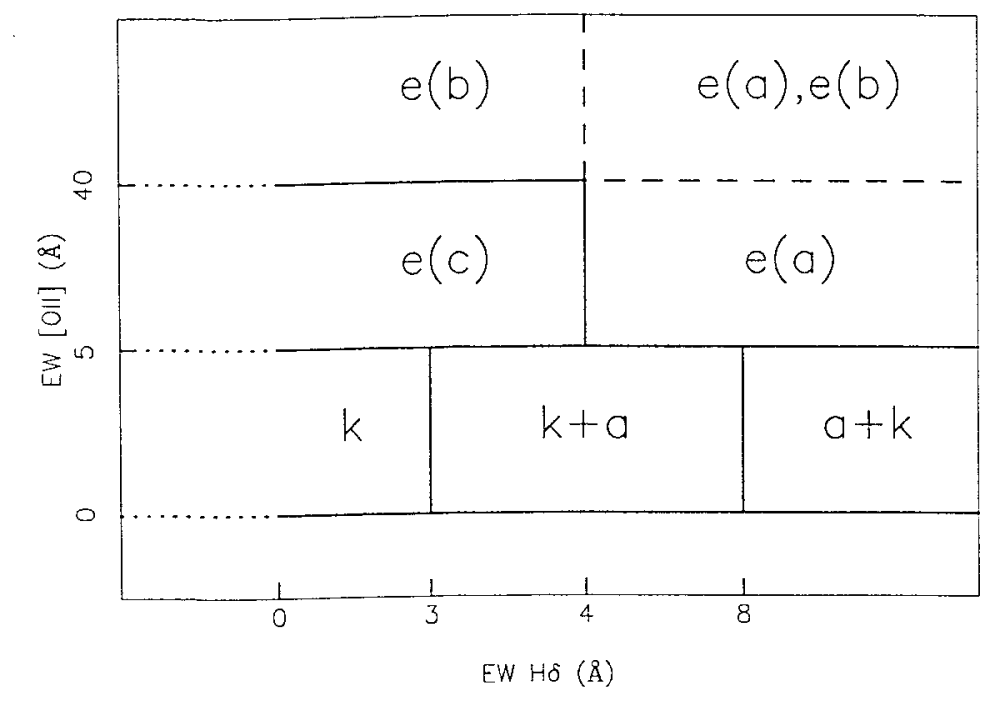

Figure 1. The spectral classification system

overwhelming majority of objects can be assigned a standard Hubble class. The majority of these could be considered "normal", but only when judged against a rather looser standard of normality than that applied today. As has been noted before, (e.g. Oemler et al. 1997) most disk galaxies look less symmetric and less well-organized than nearby examples of their Hubble type.

A significant fraction, about $18 \%$ of the cluster members, show signs of strong dynamical disturbance. We have labeled most of these (those denoted, I, $T$, or $M$ types) as mergers or tidal interactions. However, as a group their properties are rather different than nearby tidal encounters and mergers. Extended tidal features are quite uncommon, and tidal bridges between pairs of cluster galaxies almost nonexistent. It is plausible that such extended features cannot long survive in the violent cluster environment. However it is also possible that we are simply misinterpreting the origin of the observed peculiarities. For example, it has been suggested (Smail et al. 1999) that dust is responsible for the unusual light distributions in some of these objects. In my opinion, however, the light distributions of most do not look like dust is responsible.

Galaxies with spectroscopy have been divided into classes based on the strengths of the [OII] $\lambda 3737$ and $\mathrm{H} \delta$ lines. This has become standard practice (Couch \& Sharples 1987); [OII] is a reasonable measure of the present star formation rate, while $\mathrm{H} \delta$ is an indicator of the star formation over the past billion years or so. Figure 1 defines our classification system, which is based, in large part, on observations of local galaxies (Kennicutt 1992). An additional type, e(n), was assigned to galaxies whose emission lines suggested an AGN. The $k$-type spectra are typical of old, metal-rich stellar populations, like those seen in nearby $E$ and $S 0$ galaxies. The galaxies called " $k+a$ " and " $a+k$ " have balmer lines which are too strong to be consistent with a purely old population. These 
objects have been called, variously, "E+A", " $H \delta$-strong", or "poststarburst" objects. Spectral modelling has demonstrated that such strong balmer lines can only occur in the aftermath of a rapid drop in the star formation rate. While the weakest examples can be explained by the truncation of a steady level of star formation, the stronger examples can only be explained as the aftermath of a starburst. All of the other spectral types, e(c), e(a), and e(b), require continuing star formation. The $\mathrm{e}(\mathrm{c})$ galaxies span the range of normal star formation seen locally (Kennicutt 1992). Luminous normal galaxies are not seen with $\mathrm{EW}([\mathrm{OII}]) \geq 40 \AA$, thus the e(b) galaxies were assumed to be starbursts. The e(a) galaxies, with emission lines and strong balmer lines, are most easily understood as a starburst whose aftermath has continuing star formation. The populations of each type in the clusters and field are summarized in Table 2.

With this classification scheme we can neatly pigeonhole every galaxy according to its star formation history, and construct a simple picture in which "normal" spiral galaxies begin as e(c)'s. Some process precipitates a starburst, moving the galaxy into the e(b) class. If star formation continues after the burst, the galaxy moves into the e(a) class and later back to the e(c) class, "normal" once again. Alternatively, if the event removes the galaxy's gas supply, star formation will end with the burst, and the galaxy will move first to the $a+k$, then the $k+a$ class, finally settling down as a $k$ type.

Table 2. Proportions of Spectroscopic Types

\begin{tabular}{lrr}
\hline Type & $N_{\text {cluster }}$ & $N_{\text {field }}$ \\
\hline $\mathrm{k}$ & 186 & 36 \\
$\mathrm{k}+\mathrm{a}$ & 60 & 7 \\
$\mathrm{a}+\mathrm{k}$ & 18 & 0 \\
$\mathrm{e}(\mathrm{a})$ & 44 & 37 \\
$\mathrm{e}(\mathrm{c})$ & 64 & 74 \\
$\mathrm{e}(\mathrm{b})$ & 20 & 39 \\
$\mathrm{e}(\mathrm{n})$ & 7 & 3 \\
\hline \hline
\end{tabular}

Unfortunately, a little examination reveals problems with this picture. We should expect the e(b) phase to be the most luminous; galaxies should fade as star formation ends and they move through the $a+k$ and $k+a$ phases. Figure 2 presents the luminosity functions of cluster members of each spectroscopic type. The e(b) galaxies are, in fact, fainter than any of the other types. Indeed, since the sample is very incomplete fainter than $M_{V} \leq-19.0$, the data suggests that the e(b)'s are much fainter than any other group.

However, their faintness suggests an alternate interpretation. There is one nearby population of normal, non-bursting galaxies with very strong emission lines: low-metallicity dwarf irregulars. Most of the e(b) galaxies with HST derived morphological types have been classified as very late spirals or Irr's. Furthermore, analysis of the ratio $([\mathrm{OII}]+[\mathrm{OIII}]) / \mathrm{H} \beta$ shows that their metallicities are quite low, consistent with those expected for dwarf irregulars. Thus, rather than being starbursts, apparently most of the e(b)'s are merely the low- 


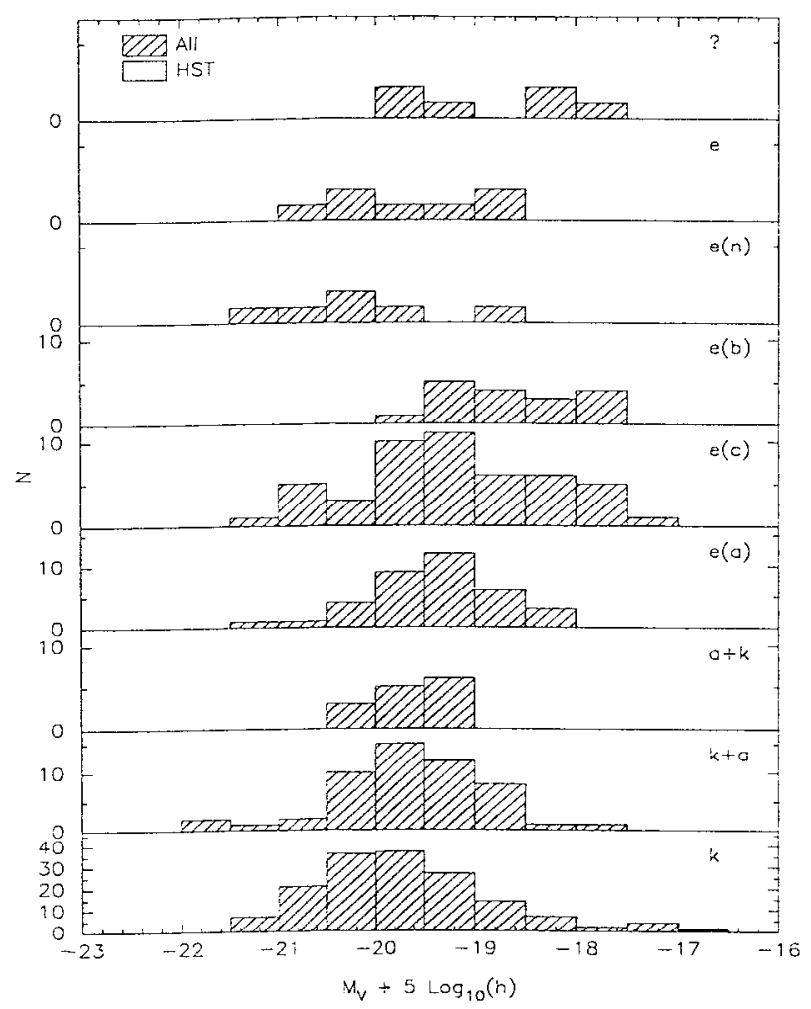

Figure 2. The luminosity functions of the spectroscopic types

luminosity, low-metallicity extension of the sequence of normal galaxies represented by the e(c)'s.

Where, then, are the slarburst progenitors of the numerous post-starburst galaxies seen in Table 2? Several lines of evidence suggest that they lie amongst the e(a)'s. Poggianti \& Wu (1999) have found that the majority of very luminous IR galaxies have e(a) spectra. Excluding those with Seyfert I spectra, $62 \%$ are $\mathrm{e}(\mathrm{a})$ 's, $28 \%$ are e(c)'s, and only $10 \%$ are e(b)'s. Similarly, annong a sample of disk galaxies in Cl0939+26 observed to have strong radio continuum emission Smail et al. (1999) found 1 or 2 e(a)'s and $4 a+k$ and $k+a$ 's, but no e(b)'s. Finally, a substantial fraction of high-redshift ISO sources are found to have e(a) spectra (vid. Poggianti \& W11 1999).

Why should starbursts produce weakish emission and strong balmer absorption lines? There is evidence that $e(a)$ galaxies are very dusty. The ratios of far infrared to $H \alpha$ flux and of $H \alpha$ to [OII] line strengths (Poggianti \& Wu 1999 ) and the relation between optical continu1um color and radio continuum flux (Morrison 1999) all imply very high dust extinction. If the lifetime of a starburst is long compared to that of OB stars, and if the dust preferentially enshrouds the regions of current star formation (see, e.g. Calzetti 1997), the A stars formed earlier in the burst will be less obscured than the HII regions, producing the characteristic features of the e(a) spectrum. 
It is worth noting that, since the emergent optical/near IR flux is dependent on the complex geometry of the dust as well as on its reddening law, there is no.way of correcting the optical/near IR flux for extinction. Thus, optical observers are left with a perhaps reliable indicator of the presence of a starburst, the e(a) spectral type, but with no reliable measure of its strength. Without either radio continuum or far-infrared observations, we cannot hope to perform any quantitative analysis of the star formation rate in these galaxies, or in any population of galaxies in which such starbursts are an important component.

\section{Galaxy Populations}

With reliable measures of structure and stellar content in hand, some very interesting patterns emerge when onc cxamincs the distributions of morphological and spectroscopic types. Although both the cluster and field galaxies span the same range of Hubble types as do ncarby populations, the distribution of types is markedly different, as may be seen from Table 3 , where the $z \sim 0.5$ numbers

\begin{tabular}{|c|c|c|c|c|}
\hline \multirow[b]{2}{*}{ Type } & \multicolumn{2}{|c|}{$z \sim 0 . \overline{5}$} & \multicolumn{2}{|c|}{$z \sim 0.0$} \\
\hline & Cluster & Field & Cluster & Field \\
\hline $\mathrm{E}$ & $36 \%$ & $13 \%$ & $28 \%$ & $10 \%$ \\
\hline So & $17 \%$ & $3 \%$ & $47 \%$ & $20 \%$ \\
\hline Spiral & $47 \%$ & $84 \%$ & $25 \%$ & $70 \%$ \\
\hline
\end{tabular}

are from our sample, and the $z \sim 0.0$ numbers are from Dressler (1980). As expected, the fraction of cluster spirals is higher at high than low redshift, and higher in the field than in the cluster. However, the relative proportions of $E$ and SO galaxies are rather surprising. The $E$ fraction in the high $z$ clusters is quite high, higher even than it is today. This does not require that ellipticals be destroyed between $z=0.5$ and today; one expects the elliptical fraction in clusters to be steadily diluted by an infalling field population which contains very few ellipticals. However, it does suggest that most or all of the elliptical population was in place by $z=0.5$, rather than being formed-by mergers for example- during intervening epochs. This is also consistent with what we think we know about the ages of the stellar populations in cluster ellipticals; studies based on their colors (Ellis et al. 1997), luminosities (Barger et al. 1998) fundamental plane (Kelson et al. 1999), and line strengths (Lrager 1997, Trager \& Dressler 1999), among others, have all deduced an early epoch of formation for their stellar populations.

There is also strong kinematical evidence that the ellipticals represent a population which is distinct from disk types. While the velocity dispersions of the various subsets of disk galaxies, including S0's, are not significantly different from each other. that of the ellipticals is much lower. In the $z \sim 0.5$ clusters $\sigma_{d i s k} / \sigma_{E}=1.49 \pm 0.10$, suggesting that the ellipticals represent a much more tightly bound population than the disk galaxies, as they would if the disk galaxies arose from later infall onto a preexisting core of predominantly elliptical 


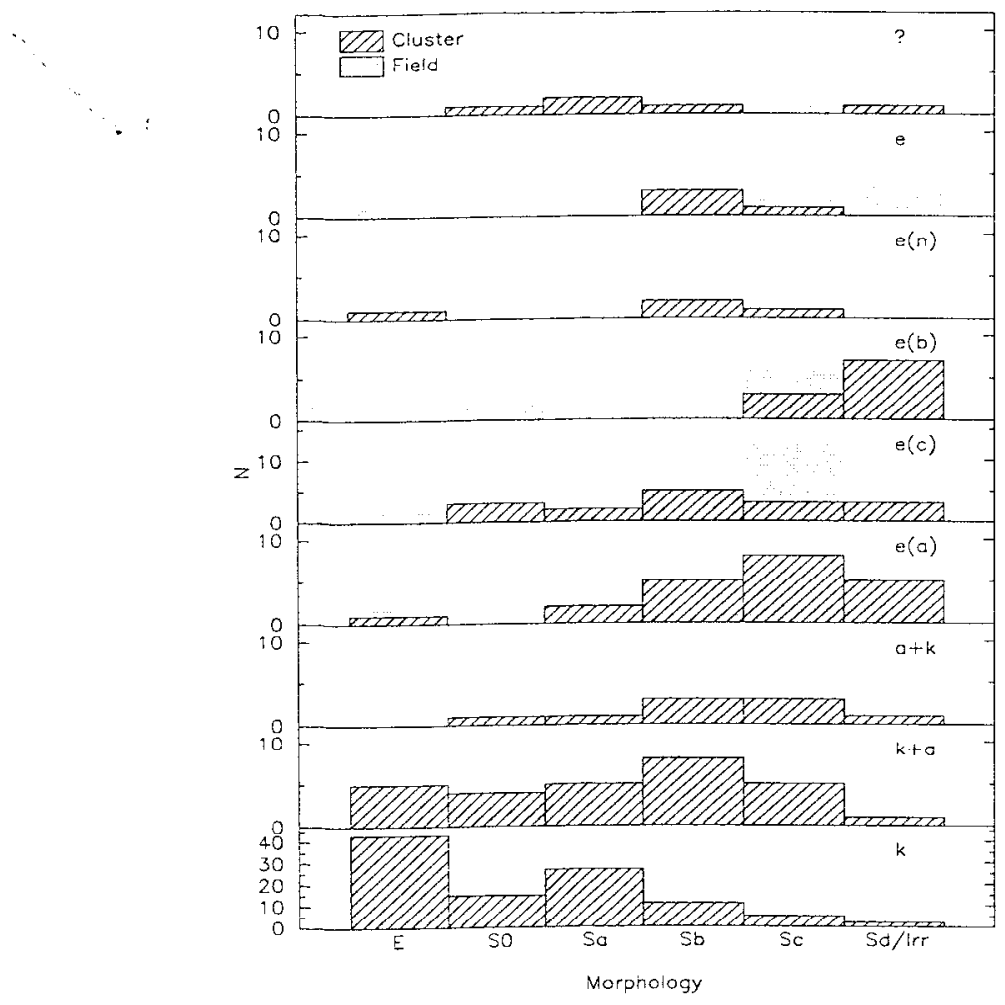

Figure 3. The distribution of morphological types for each spectral type

galaxies. The radial distribution of the morphological types within the clusters is consistent with this; the central concentration of the ellipticals-at least within the concentrated clusters- is much higher than that of any of the other types.

In contrast to the E's, S0's are not very numerous at $z \sim 0.5$, either in clusters or the field, suggesting that many or most have been formed since that epoch. This is, of course, consistent with the old idea that cluster S0's have been formed by the transformation of spirals. Almost direct evidence of such transformation may be seen in Figure 3, which summarizes the mix of spectral and morphological types in the cluster and field populations. Nearly all of the post-starburst $a+k$ and $k+a$ objects are disk galaxies. It is very hard to avoid the conclusion that these are objects currently making the transition from starforming spiral to quiescent So. At least some of the very numerous k-type spirals may be the next and penultimate stage of this transformation. It should be noted, however, that the transformation of spirals into SO's is not just a cluster phenomenon. While about half of the cluster spirals present at $z=0.5$ have become S0's today, the same has happened to about one quarter of the field spirals.

Direct evidence on the ages of S0's is contradictory. Ellis et al. (1997) find no evidence for a larger color spread for S0's than E's at $z=0.5$, but van Dokkum et al. (1998) find that S0's in the outer parts of a $z=0.33$ cluster 
- are bluer than either ellipticals of the central S0's. Jones et al. (1999) find no evidence from their spectra that the stellar populations in $z=0.5 \mathrm{~S} 0$ 's are any younger than those in E's, but Trager et al.(1999), using very high signal-to-noise Keck spectra, do see such a trend.

One conclusion implicit in the above is that the evolution of a galaxy's stellar population occurs on a faster timescale that the evolution of its morphology. The existence of post-starburst and k-type spirals implies that galaxy disks can retain the characteristic features of spiral galaxies well after all star formation has ceased. Indeed, we cannot assume that $k$-type spirals will ever evolve into S0's on their own. It is possible that the morphological transformation to S0 is an independent event, requiring other processes, rather than merely the inevitable outcome of gas depletion. Resolving this point is essential if we are to understand So formation.

If spirals are being transformed into S0's, in either one or two steps. what are the evolutionary processes driving that transformation? Whatever they may be, starbursts are apparently often involved, given the association we have just made between proto-S0's and poststarbursts. Dynamical disturbances are also implicated, given the connection between disturbed morphologies and starburst spectra. However, major mergers are not an attractive solution, for the reasons mentioned at the beginning of this article. Some hint about what is happening may be derived from Table 4, which presents the distribution of spectral types

Table 4. Spectral Types of Spiral Galaxies

\begin{tabular}{lrrrr} 
& \multicolumn{2}{c}{$\approx \sim 0.5$} & \multicolumn{2}{c}{$z \sim 0.0$} \\
\multicolumn{1}{c}{ Type } & Cluster & Field & Cluster & Field \\
\hline Normal & $20 \%$ & $58 \%$ &. & $67 \%$ \\
k-type & $40 \%$ & $24 \%$ &. & $18 \%$ \\
Starburst & $14 \%$ & $16 \%$ &. & $18 \%$ \\
$\mathrm{a}+\mathrm{k}$ & $6 \%$ & $0 \%$ &. & $0 \%$ \\
$\mathrm{k}+\mathrm{a}$ & $20 \%$ & $2 \%$ &. & $7 \%$ \\
\hline \hline
\end{tabular}

of spiral galaxies at low and intermediate redshifts. I have taken e(c) and e(b) spectra to be "normal" and e(a)'s to be starbursts. The low redshift field data are a bit of a cheat. The distribution of spectral types are from Hashimoto's (1999) analysis of the Las Campanas Redshift Survey spectra. Because there are no reliable morphological types for these galaxies, I have assumed that $30 \%$ of the galaxies were E's and S0's, and that all had k-type spectra. The remaining galaxies were assumed to be spirals. Although a bit rough, the result is unlikely to be far wrong. There are insufficient spectral data to construct numbers for low redshift clusters.

This table contains several quite striking results. Firstly; the $z=0.5$ clusters, in contrast to the field at both epochs, contain few spirals with normal star formation. (Fragmentary data on nearby rich clusters suggests the same is true today.) One must conclude that a spiral galaxy cannot long survive undisturbed in the very stressful environment of a rich cluster. Secondly, starbursts, manifested as e(a)'s, are quite common in both field and cluster. at low and 
higher redshift. These data, at least, provide no evidence for an evolution in the starburst rate of galaxies since $z=0.5$, nor for an environmental dependence of the starburst rate. These are rather suprising results, given the expectation of conventional wisdum (see, however, Carlberg's article in this volume). Since the e(a) spectral class we are using as a starburst indicator is an imperfect measure of starburst strength, I would hesitate to draw very strong conclusions from this result.

Nevertheless, this result does imply that starbursts are common everywhere, which makes it particularly interesting that, poststarbursts are common only in clusters (ninst of the $z \sim 0 \mathrm{k}+\mathrm{a}$ field objects are very marginal examples). This suggests that the different environment of chuster and field affects profoundly the after-starburst history of galaxies, directing field galaxies back towards normal star formation, and cluster members away. It also suggests, however, that the starburst $\rightarrow$ poststarburst route is not the only route through which S0's are produced, since the S0 population has been growing over time in the field as well. This should serve as a reminder that there are many possible ways in which a disk galaxy can loose its gas. Perhaps the simplest, gas exhaustion through star formation, is sufficient to account for the increase in field S0's.

\section{Conclusions and Worries}

One can summarize the picture emerging from the above discussion as follows. The cure of a cluster forms at early epochs. Whatever their original properties, the galaxies in this core have evolved into ellipticals before $z=1$. Infall from a more loosely-bound population of field galaxies, composed predominantly of disk galaxies, steadily adds to the cluster population at later times. Galaxy-galaxy interactions within the field and infalling populations produce a high rate of starbursts. Among the field galaxies, the starburst phase is often transitory, after which galaxies return to their previous state, but in clusters other processesram pressure stripping; perhaps- remove the remaining gas from the starbursts, setting them on an evolutionary path towards S0's. Additional processes, such as galaxy harassment, may play a role in completing that transformation.

This little stury is consistent with most or all of the observations of $z \sim 0.5$ clusters, but there are lots of loose ends. What kinds of interactions precipitate the starbursts, and how can they be as prevalent in clusters as in the field, as they must given the relative starburst rates? Can any stripping process be so efficient at removing the gas from cluster starbursts? A very old and still unresolved worry: do cluster S0's really look like stripped field spirals? Are their luminosities and bulge-to-disk ratios consistent with this origin? If not, does this support the idea that the transformation of spiral into S0 is a lwo-step process, the first removing the gas and the second modifying the disk+bulge structure? Hashimoto and collaborators (Hashimoto et al. 1998, Hashimoto \& Oemler 1999) have recently presented evidence for this in local galaxy populations. The morphology-density relation seems, in fact, to be two distinct relations: galaxy structure and star formation rate seem to be influenced by different environmental factors. If this is so, one must ask why the two independent transformations so frequently go hand in hand. One final awkward fact: at $z=0 . \bar{z}, \sigma_{d i s k} / \sigma_{E}=1.49 \pm 0.10$. Analysis of the data on nearby clusters 
in Dressler \& Shectman (1988) shows that today $\sigma_{\text {disk }} / \sigma_{E}=1.10 \pm 0.06$. How can this be? It is hard to imagine any process that could radically alter the kinematics of the cluster populations without destroying the morphology-density relation.

It is clear that our story has lots of gaps. It is less clear how to fill them, though some things are obvious. One key lies in the infalling population, which is the link between the original spiral-dominated field population and the spiralpoor population forming in the cluster core. Only the cores have been wellstudied so far, but the outer parts of the clusters must be where much of the action is. HST observations of these regions, and of the smaller groups which are the form in which the infalling population is assembled, are the essential next step.

The other key will be more elusive. We have little evidence of the transforming processes actually at work: some suggestive galaxy distortions and some starbursts. It will be hard to do better with what we can hope to obtain: low signal-to-noise snapshots of one instant in the history of a cluster population. Even nearby it has been exceedingly difficult to obtain unambiguous evidence of, for example, stripping of disk gas in clusters. It may be that the best we can do is build a circumstantial case from more extensive higher-redshift data, buttressed by more and better observations of processes occurring in nearby galaxies.

Acknowledgments. Thanks to my MORPHS collaborators, who actually did all the work that I have written about in this article.

\section{References}

Butcher, H., \& Oemler, A. 1978, ApJ. 219, 18

Calzetti, D. 1997, AJ, 113, 162

Couch, W.J., \& Sharples, R.M. 1987, MNRAS, 229, 423

Dressler, A. 1980 ApJS, 42, 565

Dressler, A., \& Gunn, J.E. 1982, ApJ, 270, 7

Dressler, A., \& Shectman, S.A. 1988, AJ, 95, 284

Dressler, A., Oemler, A., Butcher, H.R., \& Gunn, J.E. 1984, ApJ, 430, 107

Dressler, A., Oemler, A., Couch, W.J., Smail, I., Ellis, R.S., Barger, A., Butcher, H.R., Poggianti, B.M., \& Sharples, R.M. 1997, ApJ, 490, 577

Dressler, A., Smail, I., Poggianti, B.M., Butcher, H.R., Couch, W.J., Ellis, R.S., \& Oemler, A. 1999, ApJS, 122. 51

Ellis, R.S., Smail, I., Dressler, A., Couch. W.J., Oemler, A., Butcher, H.R., \& Sharples, R.M. 1997. ApJ. 483, 582

Oemler, A., Dressler, A.. \& Butcher. H.R. 1997, Ap.J, 474. 561

Hashimoto, Y., Oemler. A. Jin, Y., and Trcker, D.L. 1998, ApJ, 499, 589

Hashimoto. Y. \& Oemler, A. 1999 ApJ, 510, 609

Hashimoto, Y. 1999, private communication

Jones, L., Smail, I., \& Couch. W.J. 1999, Ap.J, in press 
Kelson, D., Hlingworth, G.D., van Dokkum, P.G., \& Franx, M. 1999, Ap.J, in press

Kennicutt, R.C. 1992, Ap.JS, 79, 255

Morrison, $1999 \mathrm{PhD}$ thesis

Poggianti, B.M., Smail, I., Dressler, A., Couch, W.J., Barger, A.J., Butcher, H.R., Ellis, R.S., \& Oemler, A. 1999, ApJ, 518, 576

Poggianti, B.M., \& Wu, H. 1999, in preparation

Smail, I., Dressler, A., Couch, W.J., Ellis, R.S., Oemler, A., Butcher, H.R., \& Sharples, R.M. 1997, ApJS, 110, 213

Smail, I., Morrison, G., Gray, M.E., Owen, F.N., Ivison, R.J., Kneib, J.-P., \& Ellis, R.S. 1999, preprint.

Trager, S. 1997, PhD thesis, University of California

Trager, S., \& Dressler, A. 1999, in preparation

van Dokkum, P.G., Franx, M., Kelson, D.D., Mlingworth, G.D., Fisher, D., \& Fabricant, D. 1998, ApJ, 500, 714 
The Hy Redshift Universe

ASP Conference Series, Vol. 193, 1999

A. J. Bunker 6 W. J. M. van Breugel, eds.

\title{
$\checkmark$
}

. Population Gradients in Galaxy Clusters at $0.2<z<0.6$

\author{
E. Ellingson \\ Center for Astrophysics and Space Astronomy, Univ. of Colorado, \\ Boulder, CO, 80309
}

H. Lin

Steward Observatory, Univ. of Arizona, Tucson, $A Z, 85721$

H.K.C. Yee and R.G. Carlberg

Dept. of Astronomy, Univ. of Toronto, Toronto, Ontario, M5S $3 H 8$, Canada

\begin{abstract}
We present a principal component analysis of galaxy spectra from the CNOC sample of rich X-ray luminous clusters at $0.18<z<$ 0.55. Composite radial distributions of different stellar populations show strong gradients as a function of cluster-centric redshift. The composite population is dominated by evolved populations in the core, and gradually changes to one which is similar to coeval field galaxies at radii greater than the virial radius. We do not see evidence in the clusters for an excess of star formation over that seen in the coeval field. Within this redshift range, significant evolution in the gradient shape is seen, with higher redshift clusters showing steeper gradients. This results in larger numbers of younger galaxies seen towards the inner regions of the clusters-in effect, a restatement of the Butcher-Oemler effect. Luminosity density profiles are consistent with a scenario where this phenomenon is due to a decline over time in the infall rate of field galaxies into clusters. Depending on how long galaxies reside in clusters before their star formation rates are diminished, this suggests an epoch for maximal infall into clusters at $z>0.7$. We also discuss alternative scenarios for the evolution of cluster populations.
\end{abstract}

\section{Introduction}

The evolving populations in galaxy clusters offer a unique opportunity to observe galaxy evolution in action, and particularly the effects of environment on star forming galaxies. Present-day rich clusters have strikingly different populations from galaxies in poorer environments, suggesting that some mechanism is at work transforming normal field galaxies into the cluster population. Many recent investigations have focused on the details of how this transformation occurs (Couch \& Sharples 1987, Barger et al., 1996, Poggianti et al., 1999, Balogh et al., 1999). The emerging picture is that there may be a population of galax- 
ies which were formed very early in the cluster's history, corresponding to the ellipticals often seen in cluster cores. (e.g. Bower, et al., 1992, Ellis, et al., 1997). Subsequent generations of infalling field galaxies have had their star formation disrupted, possibly with an associated starburst. As this transformation progresses, these galaxies might be identified with normal-looking spirals, then galaxies with strong Balmer absorption spectra, and finally $\mathrm{SO}$ galaxies which have retained some of their disk structure but have ceased active star formation.

Most of these insights have been obtained by observing distant clusters, as higher redshift clusters have been shown to contain a higher fraction of starforming galaxies- first described as the Butcher-Oemler effect (Butcher \& Oemler 1984). Ostensibly, the processes which create present day clusters are at an earlier stage of their work at these epochs, and these clusters provide a time sequence for observing the growth of the cluster structure. Here we describe an investigation into the relationship between galaxy evolution and cluster structure, based on a well-defined and fairly homogeneous sample of intermediate redshift clusters, and coeval field galaxy measurements. In an approach complementary to that of looking at galaxy properties in detail, we instead focus on building smooth composite spatial distributions within the cluster of the various stellar populations. With these distributions, it is possible to chart the relationship between the evolution of galaxy populations and the growth and evolution of the cluster.

\section{The CNOC Cluster Sample}

The CNOC (Canadian Network for Observational Cosmology) Cluster Redshift, Survey targeted 16 rich X-ray luminous galaxy clusters with $0.17<z<0.55$. Deep Gunn $g$ and $r$ imaging and multi-slit spectroscopic observations from the Canada-France-Hawaii $3.6 \mathrm{~m}$ telescope were used to map the cluster sample to radii of $1-3 h^{-1} \mathrm{Mpc}$ from the cluster cores (see Yee et al., 1996). Wavelength coverage was $\sim 3500-4500 \AA$ in the rest frame of each cluster, with resolution of about $15 \AA$. A total of 1200 cluster galaxies were spectroscopically identified. Particular care was taken to quantify selection effects and the completeness of the sample as a function of galaxy magnitude, color, redshift and position. The wide field coverage and careful attention to the empirical selection functions are crucial for building an accurate portrait of cluster structure.

Dynamical and spatial analyses of the clusters (Carlberg et al., 1996, Carlberg, Yee \& Ellingson 1997. Carlberg et al., 1997a,b) yielded cluster masses, mass-to-light ratios and density profiles. Lewis et al., (1999) presented the X-ray gas profiles from ROSAT HRI and PSPC observations of much of the sample. Balogh et al., (1997, 1999) analyzed spectral line indices for cluster galaxies. Here we combine measurements of the stellar populations in the cluster galaxies with the dynamical and X-ray properties of the clusters to address the issue of galaxy evolution in terms of the spatial distribution of different populations.

\section{Principal Component Analysis}

Principal component analysis ( $\mathrm{PCA}$ ) provides a sensitive method for measuring the strengths of different stellar populations from spectroscopic data. This tech- 


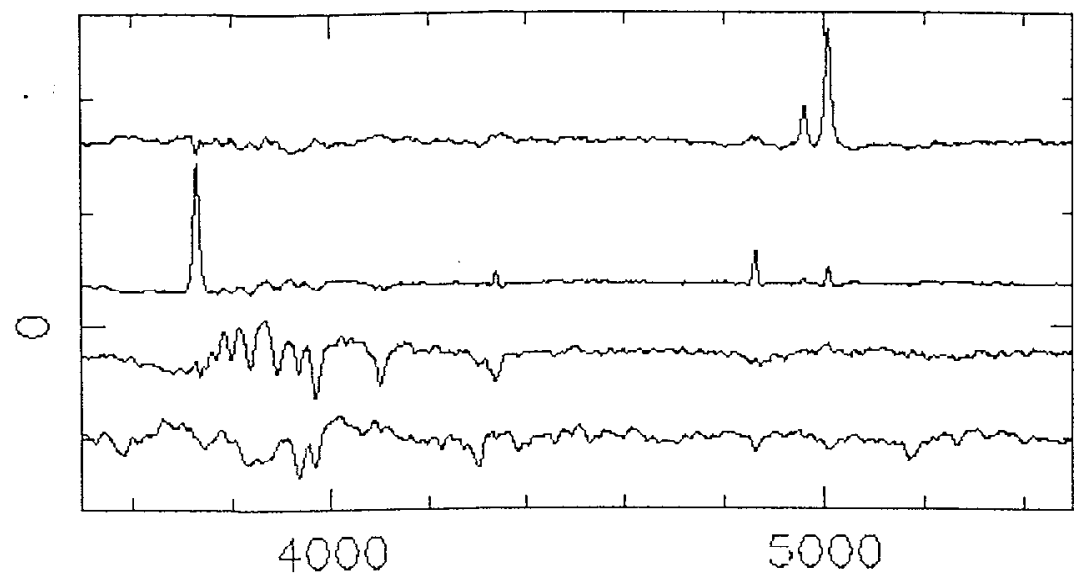

Rest Wavelength

Figure 1. Principal Component Vectors derived from the Las Campanas Redshift Survey. Four vectors are defined: two which are combined to form our "Emission" vector, "Balmer," and "Elliptical".

nique has been used by other groups (e.g. Connolly et al., 1995, see also Zaritsky, Zabludoff \& Willick 1995) to determine galaxy populations. It is espccially applicable to the fairly noisy high-redshift data of the CNOC survey, because the cntire spectrum, rather than a narrowly defined range of line indices, contributes to the measurement. We project each spectrum as the sum of four orthonormal galaxy "vectors." These vectors are derived from relatively high quality galaxy spectra in the Las Campanas Redshift Survey (Shectman et al., 1996) at low redshift. Figure 1 shows the four original vectors, which correspond roughly to two "Emission line" components ([OII] and [OIII], which we combine to a single vector because our spectra do not extend to the $\mathrm{H}-\beta /$ [OIII] region), a "Balmer component" featuring strong Balmer absorption lines indicative of intermediate age stars, and an "Elliptical" spectrum showing the standard old-population features. The power in each of the three components provides a measurement of the stellar population.

The combination of these three PCA components are adequate to separate most types of normal galaxies. Figure 2 shows PCA components for galaxies in the Kennicutt Spectral Atlas of low redshift galaxies (Kennicutt, 1992). Normal elliptical and spiral galaxies are well separated on this plot." Note that the Kennicutt Atlas does not populate the region of the plot with large Balmer values and low emission line values. In this region we plot high signal-to-noise ratio examples of $\mathrm{K}+\mathrm{A}$ galaxies from the CNOC clusters. 


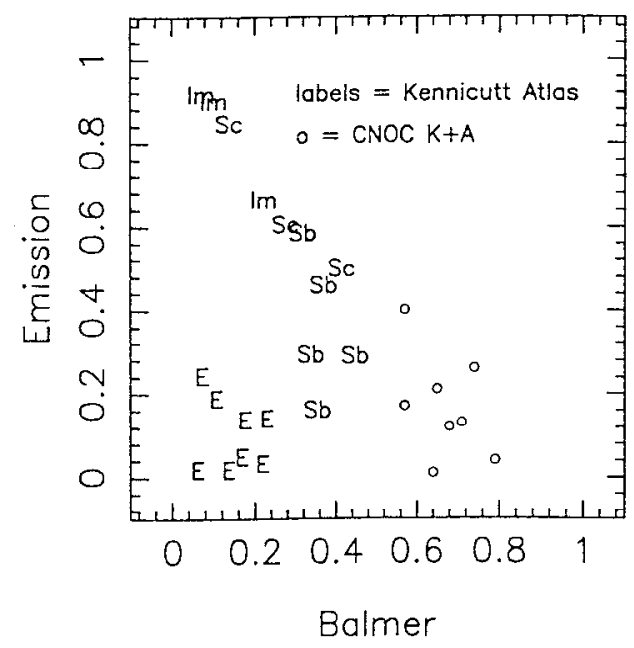

Figure 2. PCA Decomposition of Galaxies from the Kennicutt Spcc tral Atlas. Morphological types of the low redshift galaxies are labeled. Open circles denote $\mathrm{K}+\mathrm{A}$ galaxies from the CNOC clusters. The normalization of the sum of the three components to 1 means that non-zero data occupy a triangular region on this plot.

\section{Population Gradients in Galaxy Clusters}

A composite average population gradient from 15 clusters was constructed from galaxies with $\mathrm{k}$-corrected $M_{r}<-19$ ( $\mathrm{Ho}=100 \mathrm{~km} \mathrm{~s}^{-1} \mathrm{Mpc}^{-1}$, qo=0.1). Cluster membership was delineated by a redshift within 3 times the line-of-sight velocity dispersion from the mean redshift. Galaxy positions were scaled by a characteristic radius, $r_{200}$, determined for each cluster by Carlberg et al., (1996), to normalize for differing cluster richness, and so that values at a given $r / r_{200}$ represent those at a fixed overdensity. Typically, $r_{200} \sim 0.75-1.5 \mathrm{~h}^{-1} \mathrm{Mpc}$ for these cluster's, and virial radii are $1.5-2 \mathrm{r}_{200}$. These dynamical parameters are well determined for these clusters by 30-200 cluster members each, and have been found to yield results consistent with $\mathrm{X}$-ray determinations of the cluster masses (Lewis, et al., 1999). The CNOC cluster MS0906+11 was omitted because it shows strong binary substructure in both $\mathrm{X}$-ray and velocity data, and robust values for dynamical parameters were not possible.

Weights for each galaxy were calculated as inverses of the completeness functions for magnitude, color and position within each cluster field based on deep two-color galaxy photometry in the fields (see Yee et al., 1996 for details on completeness functions). Using weights such as these ensures that our spectroscopic sample yields a result representative of the entire cluster.

A clear spatial gradient is seen, with the elliptical-like population declining from the cluster core towards the outer parts of the cluster, and an accompanying increase in emission line galaxies (Figure 3). There are between 20 and 294 galaxies in each bin; uncertainties were estimated via bootstrap techniques. A self-consistent correction for projection effects was tested, assuming an average 


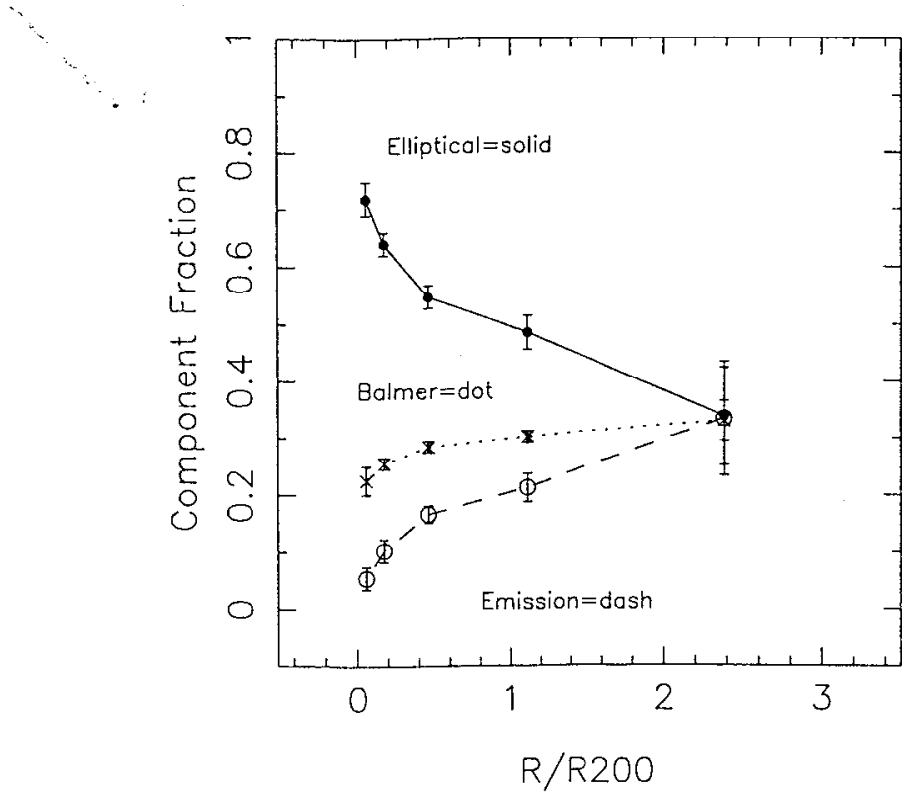

Figure 3. Composite radial gradients from 15 clusters based on the vectors shown in Figure 1. Normal field galaxies at these redshifts have values approximately equal to $[0.30,0.40,0.30]$ for Elliptical, Emission and Balmer, respectively.

spherical galaxy distribution, but is not included in the figures shown here. This correction tends to steepen the gradient very slightly but is very small, generally raising the innermost point for the older populations just a few percent.

Morphological and spectral gradients similar to this are seen in low- $z$ clusters (e.g. Oemler, 1974; Dressler, 1980; Whitmore et al., 1993). Similar gradients over large scales at higher redshifts have also been noted by Abraham et al., (1996), Couch et al., (1998) and Dressler et al., (1997). Balogh et al., (1997) traced the star formation gradients in this CNOC sample via measurements of the [OII] emission line and also found a similar trend. Such gradients are consistent with the infall of field galaxies in hierarchical clustering models of cluster growth (e.g. Gunn \& Gott, 1972; Kauffmann et al., 1995a,b) and suggest that galaxies which have more recently fallen into the cluster potential preferentially inhabit its outskirts.

The PCA components can be transformed to measure a galaxy's match to any specific stellar population. In Figure 4 we plot a weighted composite for the sample, where the components are redefined. "Cluster" values were chosen to match ellipticals in the Kennicutt Atlas (1992), and agree with the reddest galaxies in these clusters. Spectral synthesis models (Charlot \& Bruzual, 1993) indicate that any population more than about $3 \mathrm{Gyr}$ old will yield similar PCA values. The new "Field-like" vector is calculated separately for each cluster, based on the average for field galaxies at these same magnitudes at the cluster redshift from the CNOC2 Field Redshift Survey (Carlberg et al., 1998). On average, values are similar to a present-day Sbc, but with additional power in 


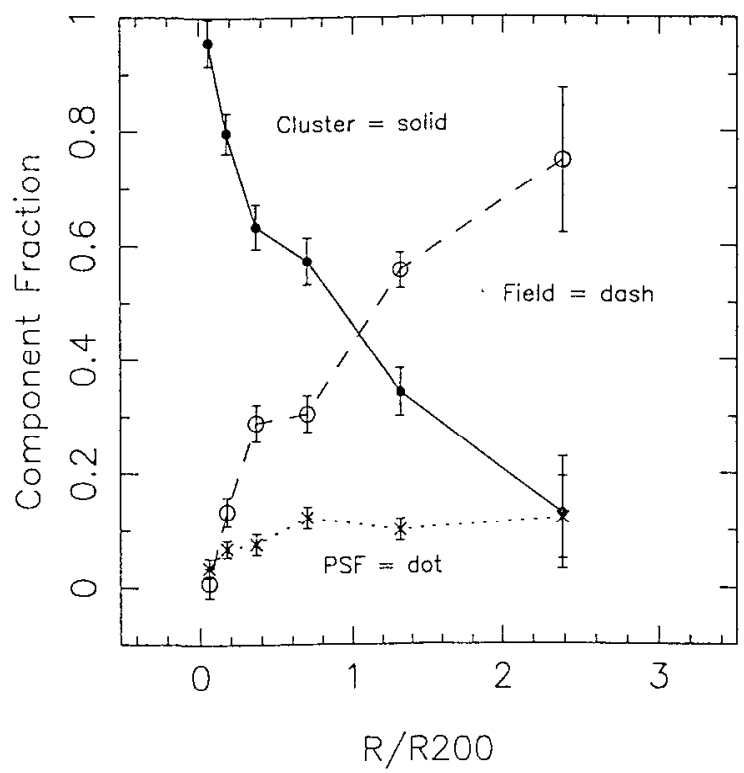

Figure 4. Composite radial gradients in transformed coordinates (see text).

Balmer absorption lines. There is a mild increase in the "Emission" fraction between $z=0.2$ and $z=0.6$. "Post-Star-Formation (PSF)" values are determined from a spectral synthesis model of a galaxy whose star formation is constant for 4 Gyr and then is abruptly stopped. The new vector corresponds to the time of the maximum Balmer component, about $0.5 \mathrm{Gyr}$ after the truncation, and is in general agreement with PCA values from the strong $K+A$ spectra of galaxies seen in the clusters.

Note that these components are not strictly orthonormal, though they are normalized to a sum of unity, as before. Thus, the equivalent of total flux is conserved, but the component values are dependent on the choice of all three vectors. While the "Cluster" and "Field-like" components are robustly determined empirically, the "PSF" component is more arbitrarily defined. Choosing a slightly different recipe for this component will raise or lower all three values slightly.

Despite this potential degeneracy, the overall cluster structure is well illustrated by this transformation. Old population galaxies preferentially occupy the cluster interior, and a smooth gradient towards younger populations is seen until the properties of cluster galaxies (defined from their redshifts) asymptotically approach those of the coeval field population at $2-5 r_{200}$, well outside of the virial radius.

The post-star-formation component appears relatively flat, with a component fraction of about $10 \%$. This is larger than the fraction of identifiable $\mathrm{K}+\mathrm{A}$ galaxies seen in the same dataset by Balogh et al.,;(1999), but smaller than the fractions seen by Poggianti et al.. (1999) in the MORPHS sample of clusters at similar redshifts. The PCA technique, of course, is sensitive to smaller fractions 


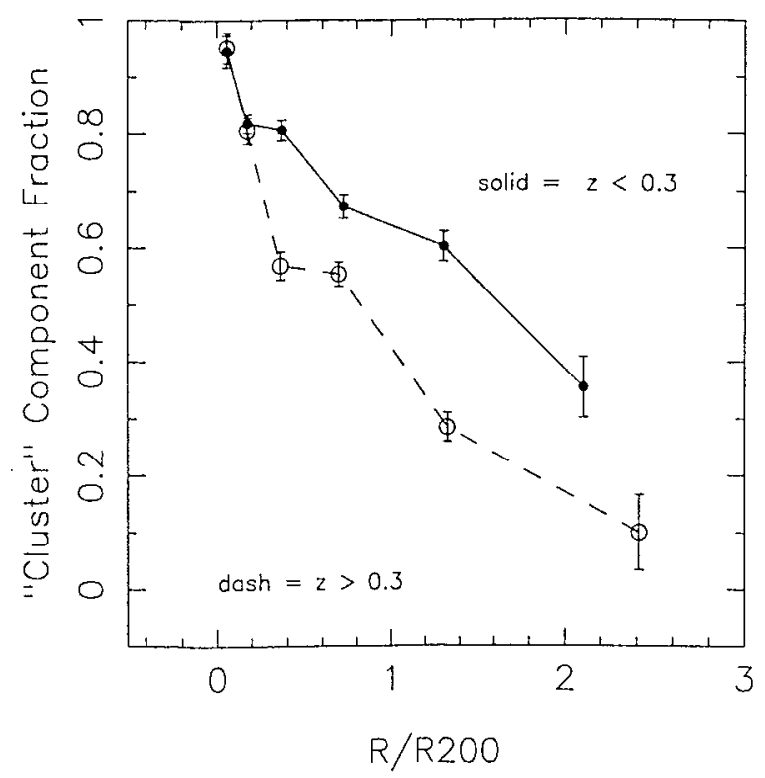

Figure 5. Composite radial gradients in the "Cluster" component as a function of cluster redshift.

of galaxy light in the post star formation state, so higher fractions are expected than for studies which count only galaxies with extreme signaturcs. Thus the comparison with Balogh et al., appears reasonable. However, the comparison with the MORPHS clusters is more troubling, and may signify truc differences in cluster populations for X-ray selected clusters such as the EMSS sample, versus the optically selected MORPHS clusters. One possibility is that the former sample is likely to be more relaxed and centrally concentrated, whereas optically selected clusters may include objects with more recent infalling structures, which may explain a higher fraction of galaxies which have recently had their star formation truncated.

In Figure 5 we examine the trend in population gradients in clusters over our redshift range of 0.18 to 0.55 . Plotted are the "Cluster" components versus radius for samples at $0.18<z<0.3$ and $0.3<z<0.55$. The PCA values, weights and unccrtaintics are calculated as beforc. While the cluster cores appear to be similarly dominated by old populations, a significant change in the cluster gradicnt is seen bctween the two redshift bins. At lower redshift, the old population appears to dominate to larger radii, whereas at high redshifts, the ficld galaxies are more noticeable in the inner portions of the cluster. This steepening of the population gradient can be thought of as a more detailed restatement of the Butcher-Oemler effect, which describes the increasing blue fraction in higher redshift clusters, typically measured inside of $0.5 \mathrm{~h}^{-1} \mathrm{Mpc}$. 


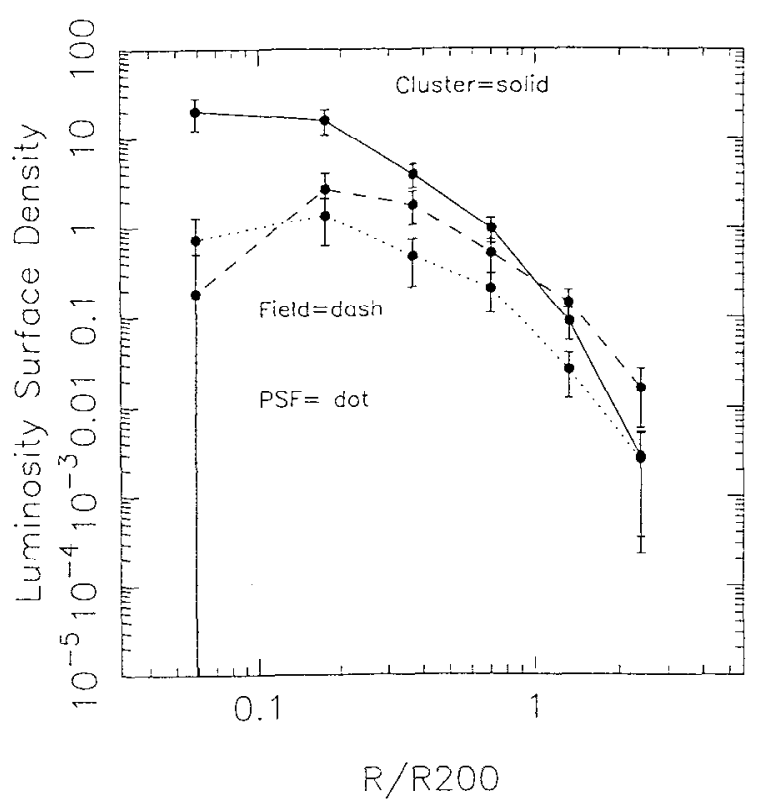

Figure 6. Composite luminosity density profiles from 15 clusters based on the transformed vector coordinates. The profiles show an increasing spatial extent from "Cluster" to "Post-Star-Formation" to "Field-like."

\section{Luminosity Density Profiles}

From the principal component analysis, relative luminosity surface density profiles can also be constructed, to trace the total light associated with the various stellar populations. Figure 6 shows the profiles for the transformed vector coordinates describer above. The fractional gradients seen in Figures 3 and 4 are shown here to be caused by light distributions which increase in extent from older to younger. This is quite consistent with what is expected from the accretion of field galaxies into the cluster. At first, infalling galaxies should have a relatively flat distribution, and as the galaxies become dynamically incorporated into the cluster, their distribution will appear more virialized, with a tighter profile. This dynamical evolution occurs in tandem with spectral evolution, causing the observed population gradients.

In Figure 7 we illustrate a possible explanation for the evolution in cluster populations and population gradients in clusters by constructing luminosity profiles as a function of cluster redshift. Figure 7 a shows the relative luminosity surface density for the old "Cluster" component for $0.18<z<0.3$ and $0.3<z<0.55$. The two curves are normalized to have the same total number of galaxies with $0.5 \mathrm{~h}^{-1} \mathrm{Mpc}$ in both bins. It appears that the old population has not changed in shape significantly over this timescale, which is in agrce ment with the idea that these galaxies are in a stable dynamical equilibrium. This population contains light both from any elliptical galaxies which may have formed early in the cluster history, as well as once star forming galaxies which have had their star formation truncated 3 Gyr or more ago. 

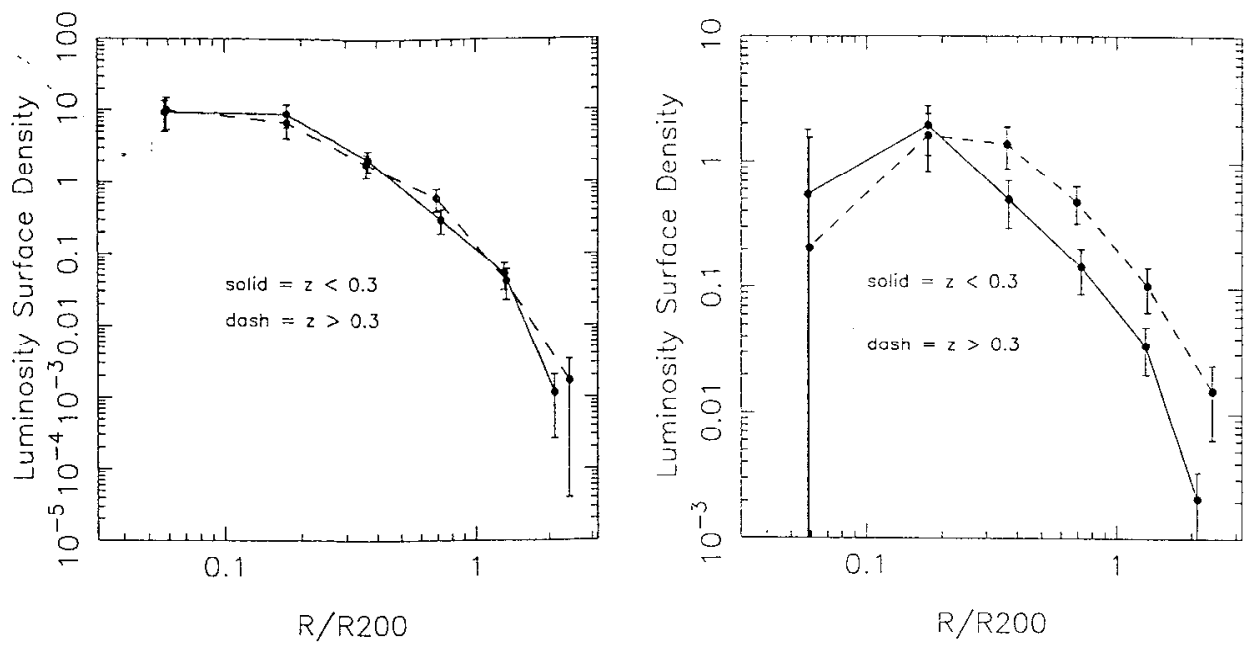

Figure 7. Composite luminosity density profiles from 15 clusters as a function of redshift. Figure 7 a (left) shows profiles for the "Cluster" component for clusters with $0.18<z<0.30$ (solid) and $0.30<z<0.55$ (dash). Figure $7 \mathrm{~b}$ (right) shows the profiles for the sum of the "Fieldlike" and "PSF" components and the same redshift bins.

Figure $7 \mathrm{~b}$ shows the sum of the "Field-like" and "Post-Star-Formation" light profiles, representing galaxies which are newer introductions to the cluster potential. These curves are normalized identically to those in Figure 7a. Here the Butcher-Oemler effect is again obvious, with a higher density of younger populations being seen at higher redshifts. Outside the core region $\left(>0.25 \mathrm{r}_{200}\right)$ these curves also appear to be similar in shape, suggesting that dynamical state (i.e. time since entry to the cluster potential) and age of the stellar populations are fundamentally linked. This is a reasonable assumption if the mechanism which transformed the population is due to the cluster environment. The main difference in these curves is then a simple vertical shift, and these results can thus be interpreted as a decline in the infall rate of new galaxies into the cluster.

Linking this interpretation to cosmological models remains uncertain because of the unknown timescales of both virialization and population evolution in these environments. For example, the mechanism which affects ongoing star formation in clusters may operate quite gradually, and the "field" galaxies we see in the cluster may have been introduced to the cluster a billion or more years earlier than the observed epoch. The observed epochs are at $z \sim 0.23$ and $z \sim 0.43$; a rough estimate of at least 1 billion year delay between infall and the start of the spectral transformation would place the epoch of maximal infall into clusters at $z>0.7$, in agreement with predictions from a low-density universe.

\section{Other Scenarios for Galaxy Evolution in Clusters}

An alternate viewpoint to a declining infall rate is that some physical property of the cluster environs has evolved over these timescales, driving the observed 


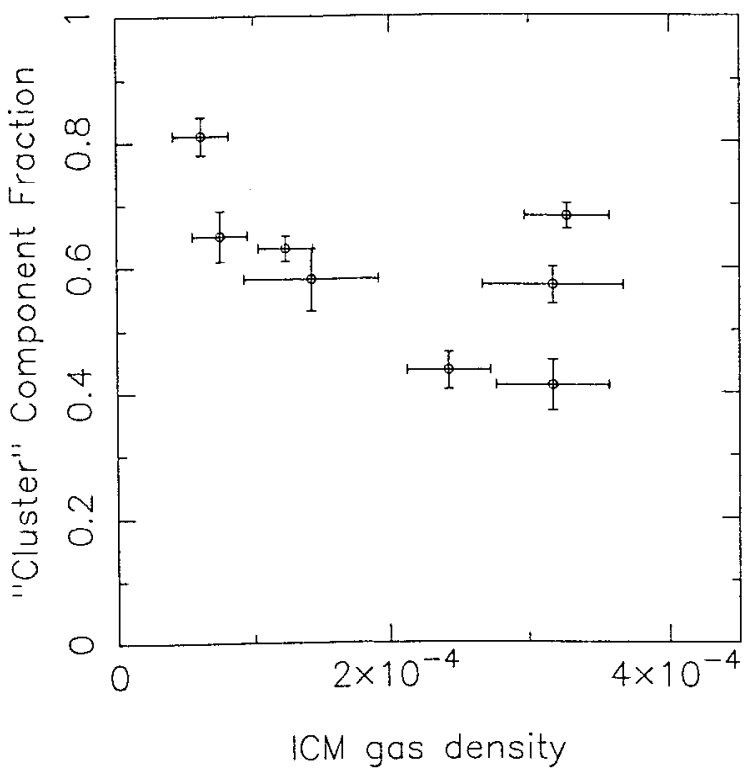

Figure 8. "Cluster" component value versus $\mathrm{X}$-ray gas density, (units of $\mathrm{cm}^{-3}$ ) as measurcd at $0.5 \mathrm{r}_{200}$. direct link between cluster population evolution and X-ray gas evolution would predict a positive correlation. Nonc is scen, suggesting that other factors drive the observed evolution.

evolution in the populations. Possibilities include evolution of the infalling field population, differing rates or efficiencies of galaxy-galaxy interactions, and evolving gas densities in the cluster, which would affect the efficiency of ram-pressure stripping of gas from infalling galaxies. We have essentially removed the effects of field galaxy evolution by constructing vector components which are based on the observed field population at the same magnitudes and redshifts as the clusters.

Global evolution in the $\mathrm{X}$-ray gas is also probably not a strong driving force in the observed evolution. All of the clusters in our sample are luminous X-ray clusters, and there does not appear to be a strong correlation between the X-ray gas and the galaxy populations. Figure 8 shows the old "Cluster" component for 8 clusters versus azimuthally averaged $\mathrm{X}$-ray gas density, each measured at $0.5 \mathrm{r}_{200}$ from the cluster core. While there is some variation in each parameter, they do not appear positively correlated, as would be expected if gas density evolution were driving the overall population changes. (Note that gas stripping may still be responsible for the transformation of individual galaxies, while a cosmologically changing condition drives the population evolution.)

Another possibility is that at higher redshift, infalling galaxies have a larger reservoir of interstellar gas, and hence are more resilient to stripping. If this were the case, we would expect to see that the "Field-like" population would have a tighter, more evolved spatial distribution at higher redshift than at lower redshifts. Figure $T$ cannot quantitatively rule out this possibility; however the data appear to be more consistent with the distributions keeping the same shape. 
with a simple vertical shift, rather than the higher redshift distributions having a higher peak towards the inner regions due to a dynamically relaxed but spectroscopically unevolved population.

Galaxy-galaxy interactions remain a possible mechanism for galaxy evolution in clusters, although morphological evidence for this remains mixed (e.g. Oemler et al., 1997). Since the effects of cluster richness and dynamics are normalized in this analysis, any evolutionary effect must come from either a cosmological change in the clustering of galaxies as they fall into the cluster (i.e. a declining rate of infalling pairs or small groups which might preferentially inhabit the regions near clusters), or in the resiliency of galaxies at continuing to form stars in the wake of a collision.

\section{Conclusions}

We have carried out a principal component analysis of galaxies in 15 clusters at intermediate redshift to investigate the relationship between cluster population and global spatial/dynamical structure. Composite population gradients show a smooth transition from the infalling field galaxy population to the older populations seen in the cluster core. The gradients are corrected for the evolution of the field population and cluster richness. Evolution between $z=0.6$ and 0.2 is manifest in a flattening of this gradient at later epochs, consistent with the Butcher-Oemler effect. This phenomenon is most consistent with scenarios where the mechanism which truncates star formation in individual galaxies remains constant, but the cluster population evolution is driven by a declining rate of infall into the clusters. Scenarios in which galaxies at earlier times are more resilient to destructive environmental effects of the cluster may also be possible.

Acknowledgments. EE acknowledges support provided by the National Science Foundation grant AST 9617145. HL acknowledges support provided by NASA through Hubble Fellowship grant \#HF-01110.01-98A awarded by the Space Telescope Science Institute, which is operated by the Association of Universities for Research in Astronomy, Inc., for NASA under contract NAS 5-26555.

\section{References}

Abraham, R. G., et al., 1996, ApJ, 471, 694

Barger, A., et al., 1998, ApJ, 501, 522

Balogh et al., 1997, ApJ, 488, L75

Balogh et al., 1999, in press

Bower, R. G., Luccy., J. R., \& Ellis, R. S., 1992, MNRAS, 254, 601

Butcher, H., \& Oemler, A. 1984, ApJ, 285, 426

Carlberg et al., 1996, ApJ, 462, 32

Carlberg et al., 1997a, ApJ, 476, L7

Carlberg et al., 1997b ApJ, 479, L19

Carlberg, R., et al. 1998, astro-ph/9805131

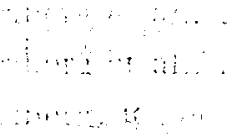

Carlberg, R. G., Yee, H. K. C., \& Ellingson, E. 1997, ApJ, 478, 462 
Charlot, S. \& Bruzual G. 1993, ApJ, 405, 538

Coleman, G.D., Wu, C.C., \& Weedman, D.W. 1980, Ap.JS, 43, 393

Connelly et al., 1995, AJ, 110, 1071

Couch et al., 1998, ApJ, 497, 188

Dressler, A. 1980, ApJ, 236, 351

Dressler, A., 1997, ApJ, 490, 577

Ellis, R., et al., 1997, ApJ, 483, 582

Gunn, J. E., \& Gott, J. R. 1972, ApJ, 176

Kauffmann, G. 1995a, MNRAS, 274, 153

Kauffmann, G. 1995b, MNR 1 S, 274, 161

Kennicutt, R. 1992, ApJS, 79, 255

Lewis, et al., 1999, ApJ, 517, 587

Oemler, A. 1974, ApJ, 194

Poggianti, B., et al., 1999, ApJ, 518, 576

Richstone, D., Loeb, \& Turner, E. L. 1992, ApJ, 393, 477

Whitmore, B. C., Gilmore, D. M., \& Jones, C. 1993, ApJ, 407, 489

Yee, H. K. C., Ellingson, E., \& Carlberg, R. G. 1996, ApJS, 102, 269

Zaritsky, D. Zabludoff, A. I., \& Willick, J. A., 1995, AJ, 110, 1602 
The Hy Redshift Universe

ASP Conference Series, Vol. 193, 1999

A. J. Bunker \& W. J. M. van Breugel, eds.

\title{
Morphological Evolution in High Redshift Galaxy Clusters
}

\author{
S.A. Stanford \\ Physics Department, University of California at Davis
}

\begin{abstract}
I review recent research on the galaxy populations in moderate and high redshift clusters. Most studies using the high resolution capability of $H S T$ find that the fractions of late-type and disturbed galaxies increase with redshift up to $z \sim 0.5$. I present new results on galaxy morphological types determined from $H S T$ imaging of $4 z>1$ galaxy clusters in $K$-band selected samples. The trends seen at lower redshifts appear to continue past $z \sim 1$, with an increasing number of disturbed galaxies and a decreasing number of early-type galaxies, on average after a statistical field correction, in clusters at $z \sim 1.2$. These results suggest that some elliptical galaxies in high redshift clusters are being constructed at $z<1.2$.
\end{abstract}

\section{Introduction}

Early-type galaxies completely dominate the core region of the nearby Coma cluster in the central $\sim 1 \mathrm{Mpc}$. There are almost no late-type disk galaxies nor disturbed systems. Naturally the question arises: were clusters always dominated by ellipticals and S0s? Clearly if we want to study the evolution of galaxy populations in clusters, the only choice for an observer is to compare clusters at a variety of redshifts. Its important to remember that there may be at least two major problems with this approach. First, clusters in the present epoch may be fundamentally different from the clusters we find at higher redshifts because of the growth in large scale structures on such mass scales. Second, currently we have no complete and well-defined samples over a sufficiently large range in $z$. Great progress has been made recently to construct well-defined cluster samples, particularly using the Rosat archive (e.g. Rosati et al. 1998), but even in these samples much work remains to be done on spectroscopic membership within the individual clusters.

With these caveats in mind, let us consider one of the most distant galaxy clusters known, 3C 324 at $z=1.206$. This cluster was discovered by Dickinson et al. by virtue of a large number of very red galaxies around the central radio galaxy which have the $R-K$ colors of $\mathrm{E} / \mathrm{S} 0 \mathrm{~s}$ at $z \sim 1.2$ (Dickinson 1996). The cluster was the target of a deep HST WFPC2 exposure, which roughly samples the rest frame $U$ band and covers the central $\sim 1 \mathrm{Mpc}$, that shows a large number of very peculiar galaxies, at least some of which are no doubt in the cluster. So a simple comparison of the galaxy populations in the cluster around $3 \mathrm{C} 324$ with those in Coma suggests that there have been large scale morphological transformations of cluster galaxies since $z \sim 1$. 


\section{Moderate-Redshift Galaxy Clusters}

Now this is not exactly news to anyone who has been working in the field. With the advent of WFPC2 in $H S^{\prime} I$, a number of studies were conducted of the galaxy populations in moderate-redshift clusters, mostly at $\approx<0.5$, which have already found similar results regarding a change in the morphological types of galaxies in clusters. I'd like to briefly review these results, which have been the work mostly of various permutations of the MORPHS group involving Dressler, Oemler, Ellis, Couch, and collaborators. Their WFPC2 imaging of the core regions of about 10 clusters at $0.3<z<0.6$ has shown a number of very interesting changes occurring in the galaxy populations of clusters. First, the well-known ButcherOemler effect is primarily due to the disappearance of normal late-type galaxies

in clusters at the present epoch (Oemler et al. 1997). A secondary cause of the Butcher-Oemler effect is a decline since $z \sim 0.5$ in the number of disturbed galaxies. Third, the fraction of ellipticals remains constant with redshift, while that of the S0s declines dramatically to the present epoch (Dressler et al. 1997). Taken together, these results may be understood by a model for the evolution of cluster galaxies wherein ellipticals formed at $z>2$ as the core of a cluster, followed by the infall of late-type galaxies from the field which are converted into So galaxies by interactions with each other and with the intracluster medium (Couch \& Sharples 1987).

\section{High Redshift Clusters}

Next let us examine the highest redshift clusters known to see what happens to the trends described above in the morphological distribution of galaxies within clusters. At $z>1$ very few clusters are known, and even fewer have been imaged by HST. So we must work with what we have if we wish to determine the morphological types of galaxies in clusters at these redshifts. I will present here results on four such clusters for which my collaborators and I have obtained HST imaging. One of these clusters was found serendipitously in a near-IR field survey, while the other three have been found by targetting known high- $z$ radio galaxies. ClG J0848+4453 was confirmed to be at $z=1.27$ by Keck spectroscopy of galaxies in a compact group of very red galaxies found in the imaging survey of Elston et al. Subsequently, it was discovered that a deep Rosat exposure had been obtained of this field and that a faint X-ray source coincides with the spectroscopic members (Stanford et al. 1997). Although not appreciably extended in the PSPC data, the X-ray source is probably due to hot gas in the intracluster medium, indicating an $L_{x}=1 \times 10^{44} \mathrm{ergs}^{-1}$. To date, 14 members have been spectroscopically confirmed in this cluster, many of which fall within a well-defined early-type sequence, which, by comparison with the predicted no-evolution color-mag lines based on early-type galaxies in the Coma cluster, indicates color evolution on the order of e.g. $0.5 \mathrm{mag}$ in $R-K$.

Although large telescopes such as Keck and now the VLT are capable of obtaining spectroscopic redshifts of galaxies in clusters at $z \sim 1.3$, such work is very difficult and slow. Until such time as complete spectroscopic identification of magnitude-limited samples are available, we can use statistical techniques to correct $K$-band selected galaxy samples in $z>1$ clusters for field contamination. 


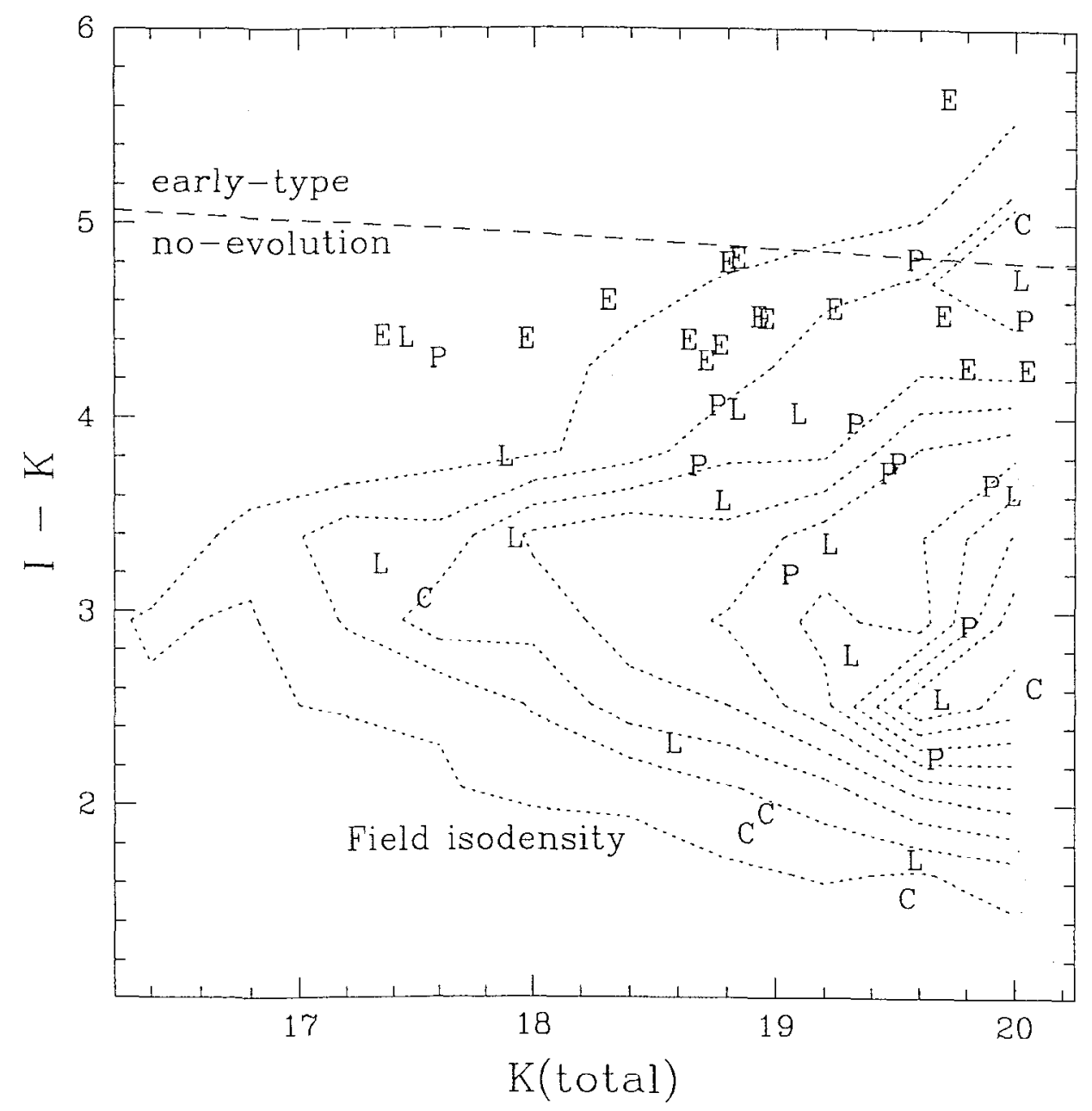

Figure 1. $\quad I-K$ vs $K$ diagram for ClG J0848+4453 at $z=1.27$. Each galaxy is represented by the letter corresponding to its morphologicar type, where E stands for early-types, L for late-types, $P$ for peculiar, and $\mathrm{C}$ for compact. The dashed line is the predicted no-evolution colors for early-type galaxies. The dotted lines represent contours of the isodensity of field galaxies. 
We have made use of the Elston et al. survey which covers $100 \operatorname{arcmin}^{2}$ down to $K=21$ in the $B R I z J K$ bands to perform a correction in color-magnitude space rather than just by $K$ magnitude. By calculating the density of field galaxies at any one position in a color-magnitude diagram, we can assign a probability to each galaxy detected in a cluster field as to its likelihood of being a cluster member. The technique is illustrated in Fig. 1 for 0848+4453. Morphological types in a $K<20.1$ sample were determined from $H S T$ imaging using the broad categories of early-type (E), late-type (L), peculiar/disturbed (P), and compact (C). Overlaid in the $I-K$ vs $K$ diagram are contours of isodensity of objects in the Elston et al. field survey. Its clear by visual inspection of Fig. 1 that the E types in the red sequence are very likely to be cluster members (which we know to be true in many cases because of the available spectroscopy for these objects at $K<19$ ), while the bluer objects at fainter $K$ have a stronger probability of being field galaxies. Weighting by these probabilities enables us to calculate the morphological fractions of likely cluster members in $0848+4453$.

We have performed a similar analysis on the other three $z>1$ clusters for which we have $H S T$ imaging. These include $3 \mathrm{C} 324,3 \mathrm{C} 210$ at $z=1.169$, and 3C 267 at $z=1.140$. The results are shown in Fig. 2, Fig. 3, and Fig. 4. In these color-magnitude diagrams we again see the well-defined early-type galaxy sequence, except for in 3C 267 where very few such galaxies are found. This may be related to its optical appearance as an irregular cluster which may be early in its formation. The morphological fractions for the galaxies in $K^{*}<20$ samples, after weighting by the field probability, were then determined for each of the radio galaxy clusters.

After averaging over all 4 of the $z>1$ clusters, the morphological fractions for the three main groups are shown in Table 1 . We have also included the fractions for $z<1$ clusters as determined from $H S T$ imaging of the core regions of the clusters reported on in Stanford, Eisenhardt, \& Dickinson (1998), after averaging over similar $K$-selected samples and grouping in the listed redshift bins. The trends found at $z<1$ in the declining numbers of early-type galaxies and increasing numbers of late-type and disturbed/peculiar galaxies appear to continue towards higher redshifts. While we did not attempt to classify ellipticals and SOs separately in the $z>1$ clusters, it is of interest to attempt to constrain the fraction of ellipticals in the highest redshift clusters. Even if all the earlytypes are ellipticals in the $z \sim 1.2$ clusters, then the number of ellipticals appears to be increasing with decreasing $z$, which suggests that ellipticals are still being created in clusters at $z<1$.

Table 1. Galaxy Morphological Percentages in Clusters

\begin{tabular}{c|ccc}
\hline redshift & Early & Late & Disturbed \\
\hline present & 85 & 12 & 2 \\
0.35 & 65 & 25 & 10 \\
$\cdots$ & 67 & 21 & 12 \\
0.55 & 49 & 27 & 20 \\
0.8 & 31 & 25 & 31 \\
\hline
\end{tabular}




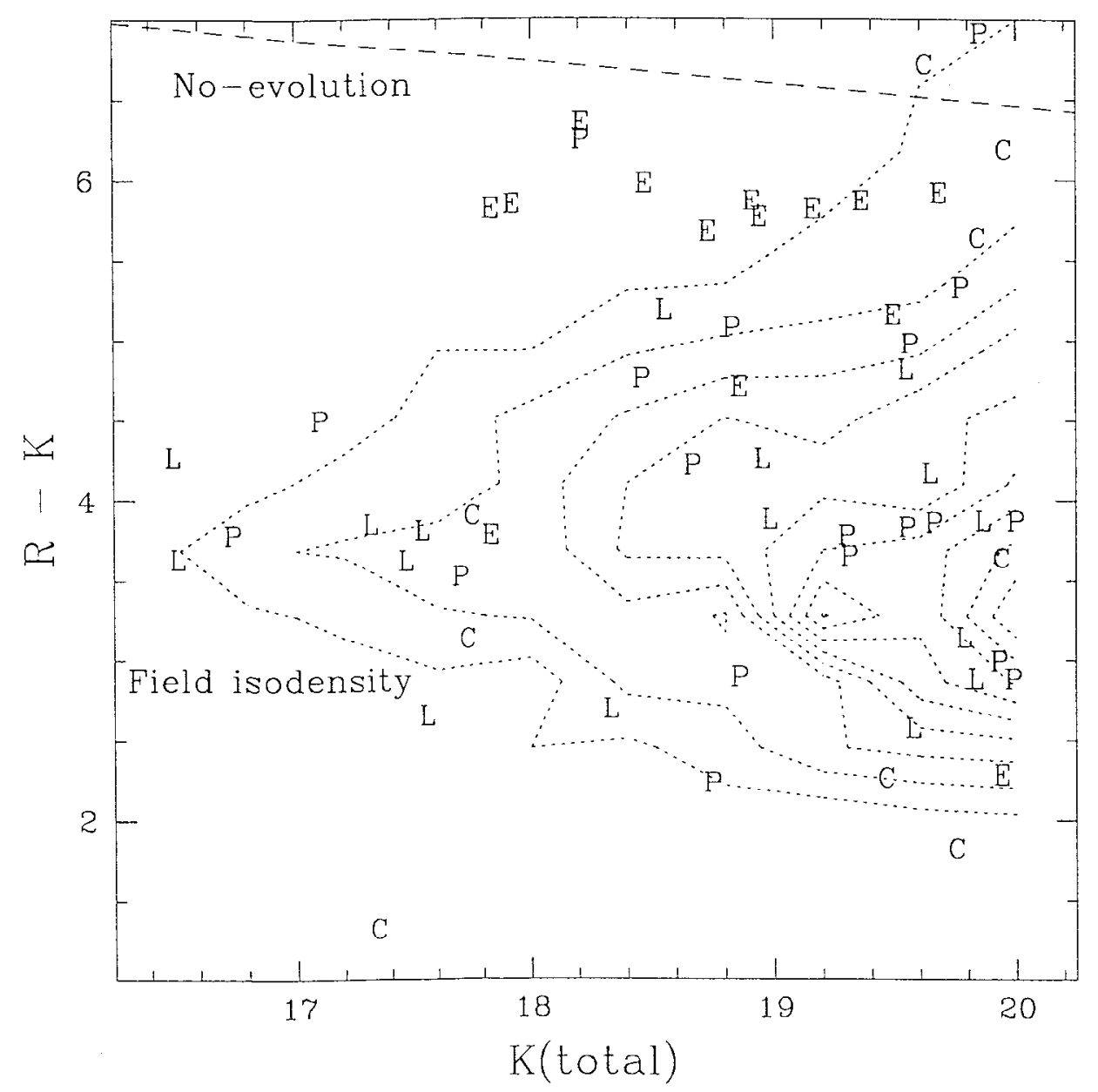

Figure 2. $R-K$ vs $K$ diagram for 3 C 324 at $z=1.206$, with the same symbols and lines as in Figure 1. 


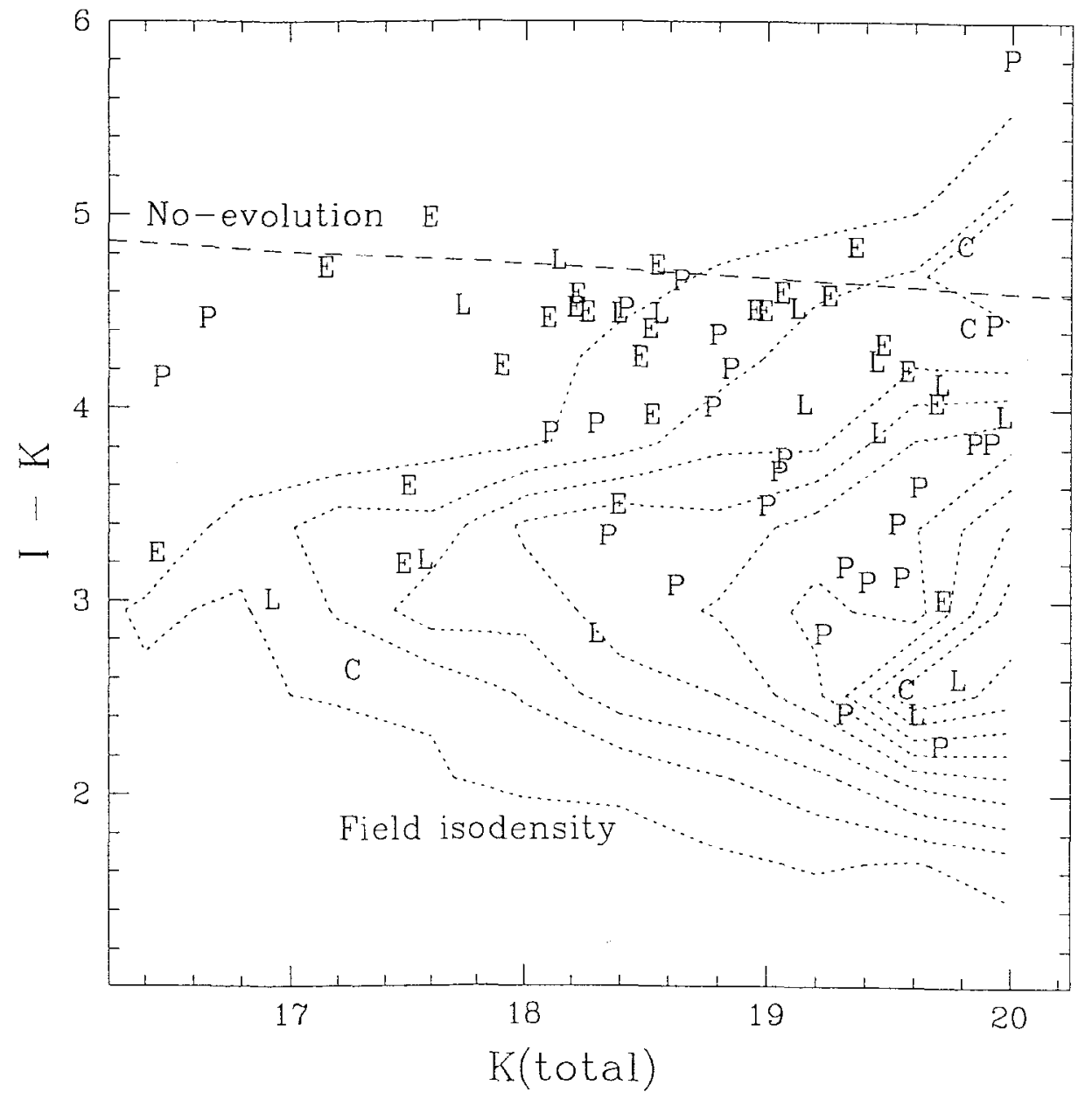

Figure 3. $I-K$ vs $K$ diagram for 3 C 210 at $z=1.169$, with the same symbols and lines as in Figure 1. 
The Hy Redshift Universe

A.SP Conference Series, Vol. 193, 1999

A. J. Bunker \& W. J. M. van Breugel, eds.

\title{
X-ray Emission from the Host Clusters of Five Intermediate-Redshift 3CR Quasar's
}

\author{
C.S. Crawford, A.C. Fabian \\ Institute of Astronomy, Madingley Road, Cambridge CBз OHA \\ I. Lehmann, G. Hasinger \\ Astrophysikalisches Institut Potsdam, An der Sternwarte 16, D-14482 \\ Potsdam
}

M.N. Bremer

Dept. of Physics, University of Bristol, Tyndall Avenue, Bristol

\begin{abstract}
We report the direct detection of extended X-ray emission around five powerful $3 \mathrm{CR}$ quasars at $0.4<z<0.75$. The ROSAT HRI images of the quasars have been corrected for spacecraft wobble and compared with an empirical point-spread-function. The spatial profiles of the extended component are consistent with thermal emission from the intracluster medium of moderately rich host clusters to the quasars. The bolometric luminosities of the clusters are in the range $\sim 6 \times 10^{44}-3 \times 10^{45} \mathrm{erg} \mathrm{s}^{-1}$, assuming a temperature of $4 \mathrm{keV}$. The inner regions of the intracluster medium are, in all cases, dense enough to be part of a cooling flow.
\end{abstract}

\section{Introduction}

The clearest way to determine the presence of a cluster of galaxies is to detect thermal X-ray emission from its hot intracluster medium. A certain degree of success has been achieved in detecting and spatially resolving the $\mathrm{X}$-ray emission around distant $(0.5<z<2$ ) radio galaxies using ROSAT (Crawford \& Fabian 1993; Worrall et al 1994; Crawford \& Fabian 1995, 1996a,b; Dickinson 1997; Hardcastle et al 1998; Carilli et al 1998; Dickinson et al 1999; Hardcastle \& Worrall 1999). The inferred bolometric luminosity of the X-ray sources associated with the radio galaxies is $\sim 0.7-18 \times 10^{44} \mathrm{erg} \mathrm{s}^{-1}$ (assuming $H_{0}=50 \mathrm{~km} \mathrm{~s}^{-1} \mathrm{Mpc}^{-1}$ ), easily compatible with that expected from moderately rich clusters of galaxies around the radio sources.

In the case of radio quasars, however, the $\mathrm{X}$-ray detection of the spatially extended environment is complicated by the presence of bright spatially-unresolved $\mathrm{X}$-ray emission from the active nucleus. We have obtained ROSAT HRI data to spatially resolve the extended emission from the intracluster medium around each of a small sample of intermediate-redshift radio-loud quasars. The detection of such a component is. however, complicated by the wobble of the spacecraft 
during the observation which leads to smearing of the point-spread function $\left(\right.$ PSH $^{\circ}$. We have corrected for this using the new technique derived by Harris et al. (1998).

\section{Radial profile analysis}

We extract a background-subtracted radial profile from the wobble-corrected Xray source associated with each quasar. We assume that the spill-over of quasar nuclear light will follow the instrumental PSF, which is modelled by a good analytical characterization obtained empirically from observations of bright stars. For each quasar we first fit the profile by the HRI PSF alone; we then fit this PSF in combination with each of two models chosen to represent any extended emission (a broken power-law or a projected King law). The fits show a significant improvement for those models that include a component of extended emission. In all this analysis we necessarily assume that any extended component is both centred on the quasar and derive its properties such as scale and luminosity assuming that it is at the redshift of the quasar. A summary of the properties of the spatially extended component is shown in Table 1.

Table 1. Quasar sample and summary of results.

\begin{tabular}{lcrrr}
\hline Quasar & $z$ & $\begin{array}{r}\mathrm{R} \\
(\mathrm{kpc})\end{array}$ & $\%$ & $\begin{array}{r}L_{\text {bol }} \\
\left(10^{43} \mathrm{erg} \mathrm{s}^{-1}\right)\end{array}$ \\
\hline 3C215 & 0.412 & (King) 33 & 26 & 89 \\
& & (BPL) 49 & 19 & 68 \\
3C254 & 0.734 & 94 & 17 & 141 \\
3C275.1 & 0.555 & 270 & 24 & 62 \\
3C281 & 0.602 & (King) 38 & 64 & 276 \\
& & (BPL) 48 & 47 & 213 \\
3 C334 & 0.555 & 49 & 17 & 117 \\
\hline \hline
\end{tabular}

Columns 3-5 present the average best-fit properties of the extended X-ray component, deduced from fitting the X-ray radial profile. Two sets of values are given for 3C215 and 3C281 as the two models assumed (PSF+King law and a PSF +broken power-law) give consistently different parameters. $R$ is the core radius of the cluster, $\%$ shows the fraction of the total luminosity represented by the extended component, and $L_{b o l}$ is its bolometric luminosity.

\section{Discussion}

We plot the bolometric luminosity of the extended cluster component against quasar redshift in Fig 1, along with that associated with distant $3 \mathrm{C}$ radio galaxies (from Crawford \& Fabian 1996b, Carilli et al 1998), and the clusters surrounding the two nearby FR II radio galaxies Cygnus A (Ueno et al 1994) and 3C295 (Henry \& Henriksen 1986). The inferred bolometric luminosities of the extended components around the quasars are compatible with those of moderately rich clusters of galaxies at low redshift. They are, however, more luminous than 


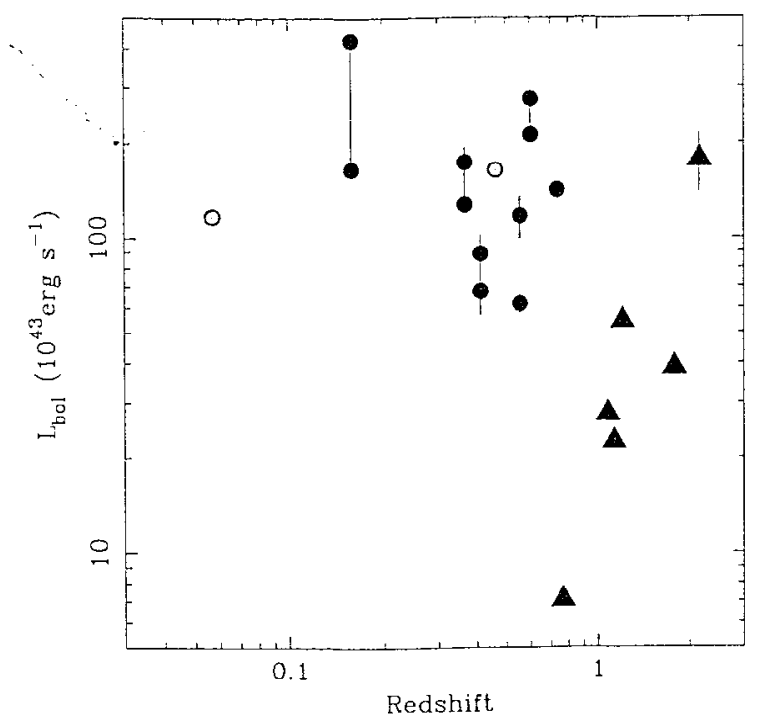

Figure 1. The bolometric luminosity of the extended component of emission associated with the quasars plotted against redshift (circle markers). The open circles mark the luminosities of the host clusters of the nearby FR II radio galaxies Cygnus A and 3C295, and the luminosities of the $\mathrm{X}$-ray source associated with the distant radio galaxies are also plotted (triangle markers).

the extended X-ray emission detected around more distant $3 \mathrm{CR}$ radio galaxies. Whether this indicates evolution, a problem for radio galaxy/quasar unification, or is a result of small number statistics awaits the compilation of a complete sample.

All the extended models imply a central cooling time considerably shorter than a Hubble time. We therefore explore the properties of the implied cooling flows occurring around these quasars by deriving some approximate parameters from the fits to the profiles. We attribute all the $\mathrm{X}$-ray luminosity of the extended component within radius $R$ to thermal bremsstrahlung from gas with electron density $n$ (where $n \propto r^{-1}$ ) at a temperature of $4 \mathrm{keV}$. The cooling time of the gas at $R$ is then between about 1-3 billion yr. We then estimate the cooling flow radius $r_{\mathrm{CF}}$ at which the cooling time is $10^{10} \mathrm{yr}$ and obtain a rough indication of the mass deposition rate within that radius from the ratio of the mass of gas within $r_{\mathrm{CF}}$ to $10^{10} \mathrm{yr}$. The derived values range over $100-500 \mathrm{M}_{\odot} \mathrm{yr}^{-1}$, although they should be regarded as uncertain by at least a factor of 2 .

Inverse-Compton scattering of quasar radiation could still contribute to our extended component of X-ray emission (Brunetti et al 1997). If a significant process, the $\mathrm{X}$-ray source would appear asymmetric and lop-sided, as (back) scattering by the electrons in the more distant radio lobe should be stronger than in the nearer lobe. The quality of our current data is insufficient to show any significant asymmetry, and so we are unable to assess the contribution from this process. Observations of these sources with Chandra and XMM should clarify its relative importance to the total emission. 


\section{Conclusions}

The powerful radio-lond quasars presented here are surrounded by luminous extended X-ray emission. The spatial properties of the emission are consistent with a thermal origin from an intracluster medium. The radiative cooling time of the gas within $\sim 50 \mathrm{kpc}$ of the quasars is only a few billion years or less, indicating the presence of strong cooling flows of hundreds of $\mathrm{M}_{\odot} \mathrm{yr}^{-1}$. The high pressure of that gas is sufficient to support the extended optical nebulosities seen around many of these quasars and may play a rôle in shaping the properties of the radio sources, such as structure, depolarization and possibly even fuelling and evolution (Fabian \& Crawford 1990).

A small number of luminous clusters without central AGN have so far been found beyond redshift 0.5 , and the discovery of a cluster at $z \sim 0.8$ with luminosity $10^{45} \mathrm{erg}^{-1}$ (Donahue et al 1998) provides strong evidence for a low density universe. We have shown that the study of the environment of powerful radio-loud quasars is a promising way of extending the discovery of similarly luminous clusters both in numbers and to higher redshifts.

Acknowledgments. CSC and ACF thank the Royal Society for financial support. This work has been supported in part by the DLR (format DARA $\mathrm{GmbH})$ under grant 50 OR 94035 (GH and IL).

\section{References}

Allen S.W., 1998, MNR.AS, 296, 392

Brunetti G., Setti G, Comastri A., 1997, AaA, 235, 898

Carilli C.L., Harris D.E., Pentericci L., Röttergering J.J.A., Miley G.K., Bremer M.N., 1998, ApJ, 494, L143

Crawford C.S., Fabian A.C., 1993, MNRAS, 260, L15

Crawford C.S., Fabian A.C., 1995, MNRAS, 273, 827

Crawford C.S., Fabian A.C., 1996a, MNRAS, 281, L5

Crawford C.S., Fabian A.C., 1996b, MNRAS, 282, 1483

Dickinson M., 1997 HST and the High Redshift Universe, eds, Tanvir N., AragonSalamanca A., Wall J.V., published by World Scientific.

Dickinson M. et al 1999, preprint.

Donahue M., Voit G.M., Gioia I., Lupino G., Huges J.P., Stocke J.T., 1998, ApJ, 502, 550

Fabian A.C., Crawford C.S., 1990, MNRAS, 247, 439

Hardcastle M.J., Lawrence C.R., Worrall D.M., 1998, Ap.J, 504, 713

Hardcastle M.J. Worrall D.M., 1999, MNRAS in press (astro-ph/9907034).

Harris D.E., Silverman J.D., Hasinger G., Lehmann I., 1998, AaA Suppl, 133, 431

Henry J.P., Henrikisen M.J., 1986, ApJ, 301, 689

Ueno S., Ioyama K., Nishida M., Yamanchi S., Ward M.J., 1994, ApJ, 431, L1

Worrall D.M., Lawrence C.R., Pearson T.J., Readhead A.C.S., 1994, ApJ, 420, L1T 
The Hy Redshift Universe

ASP Conference Series, Vol. 193, 1999

A. J. Bunker \& W. J. M. van Breugel, eds.

\title{
The Evolution of Stellar Populations in Intermediate Redshift Clusters
}

\author{
Daniel D. Kelson \\ Dcpt. of Torrcstrial Magnetism, Carnegie Institution of Washington, \\ 5241 Broad Branch Rd., NW, Washington, DC 20015 \\ Garth D. Mlingworth \\ UCO/Lick Observatory, Dept. of Astronomy and Astrophysics, UCSC, \\ Santa Cruz, CA 95064 \\ Marijn Franx and Pieter G. van Dokium \\ Leiden Observatory, P.O. Box 9513, NL-2300 RA, Leiden, The \\ Netherlands
}

\begin{abstract}
The $M / L$ ratios and absorption line-strengths of distant cluster galaxies can be used to directly study their stellar populations, determine their redshift of formation, their scatter in ages, and any dependence of their ages on such internal properties such as velocity dispersion or mass. Comparing the zero-point the fundamental plane in the $z=0.33$ cluster CL1358+62 to that in Coma, we conclude that the redshift of formation for the stars in massive cluster $\mathrm{E} / \mathrm{S} 0 \mathrm{~s}$ was $z \gtrsim 2$. The fundamental plane in CL1358+62 has the following form: $r_{e} \propto \sigma^{1.31 \pm 0.13}\langle I\rangle_{e}^{-0.86 \pm 0.10}$, indicating that the last epoch of star-formation has very little dependence on galaxy mass. The scatter in the CL1358+62 fundamental plane is also very low, equivalent to a scatter in ages of $\sim 15 \%$. We have also analyzed the $M / L_{V}$ ratios of galaxies of type $\mathrm{S} 0 / \mathrm{a}$ and later. These early-type spirals follow a different plane from the $\mathrm{E}$ and $\mathrm{S} 0$ galaxies, with a scatter that is twice as large as the scatter for the E/SOs. These residuals also correlate with the residuals from the color-magnitude relation. Preliminary analysis of the absorption line-strengths of the CL1358+62 early-types indicate that they are well-described by uniformly old, single-burst stellar populations with metallicity varying as a function of velocity dispersion.
\end{abstract}

\section{Introduction}

The fundamental plane (FP) is an empirical relation between galaxy half-light radius, $r_{e}$, surface brightness, $\langle I\rangle_{e}$, and central velocity dispersion, $\sigma$ for earlytype galaxies. Locally,

$$
r_{e} \propto \sigma^{1.24}\langle I\rangle_{e}^{-0.82}
$$

in Gunn $r_{g}$ (Jørgensen et al. 1996). Under the assumption of homology, this implies $M / L_{r_{g}} \propto M^{1 / 4} r_{e}^{-0.02}$. The fundamental plane is very thin, with an $r m s$ 
scatter of $\pm 23 \%$ in Coma in $M / L_{V}$ ratio for a given galaxy mass, $M$ ( $J \varnothing$ rgensen et al. 1993). With its low scatter, the FP is a very powerful tool for measuring the evolution of galaxy $M / L$ ratios as a function of redshift, $M$, etc.

Because reasonable stellar populations evolve as $M / L_{V} \propto t^{\kappa}$ (Tinsley \& Gunn 1976), evolution in the properties of the fundamental plane directly probe the star-formation histories of cluster galaxies. Measurements of the evolution of the FP zero-point, scatter, and slope can be used to constrain the mean epoch of star-formation, the spread in early-type galaxy ages; and the dependence of galaxy age on, e.g., $M$.

We exploit high resolution Keck spectroscopy for velocity dispersions and line strengths, and WFPC2 imaging for the structural parameters, colors, and morphologies. Details can be found in Kelson et al. (1999abc).

\section{The Fundamental Plane of Early-Type Galaxies in CL1358+62}
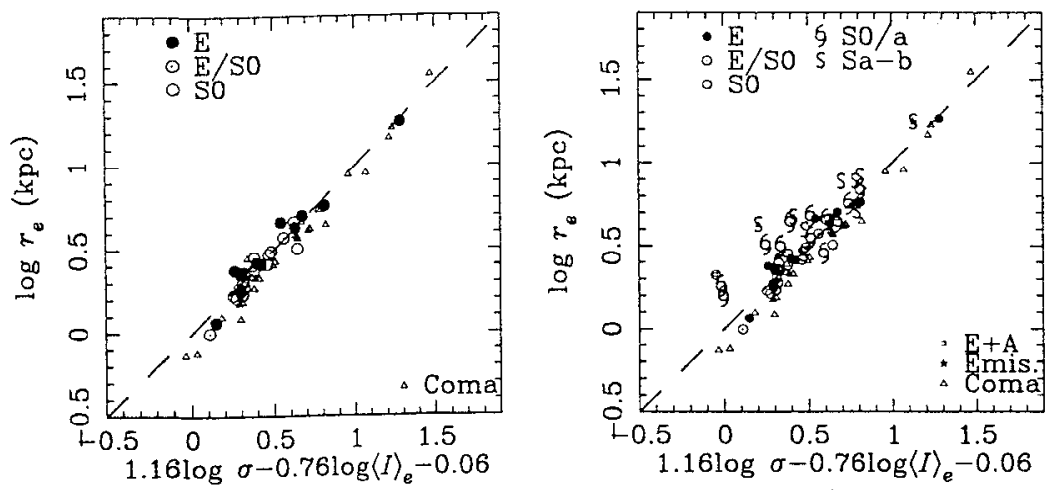

Figure 1. The left-hand panel shows the fundamental plane of E/S0s in CL1358+62. The early-type galaxies clearly form a tight relation. The intrinsic scatter is $14 \% \mathrm{in} r_{e}$. In the right-hand panel, the full sample of 53 galaxies is shown, including the early-type spirals.

The left-hand panel of Figure 1 shows the tight fundamental plane relation in the $z=0.33$ cluster CL1358+62. The $30 \mathrm{E} / \mathrm{S} 0 \mathrm{~s}$ in this sample are fit by the following plane in Johnson $V$ (Kelson et al. 1999c):

$$
r_{e} \propto \sigma^{1.31 \pm 0.13}\langle I\rangle_{e}^{-0.86 \pm 0.10}
$$

This sample has comparable depth to the FP samples of Coma, ensuring that the comparison of these FP exponents with those determined in nearby clusters is reasonably free from selection effects. We draw the following three conclusions:

- Mild evolution in the EP zero-point, of $\Delta M / L_{V}=-0.13 \pm 0.03\left(q_{0}=0.1\right)$, indicates a mean redshift of formation of $\left\langle z_{f}\right\rangle \geq 2$ for the stars in chister E/SOs.

- The scatter in the color-magnitude relation and FP suggest a $1-\sigma$ scatter in ages of $15 \%$. For $q_{0}=0.05$ and $\left\langle z_{f}\right\rangle=2$, $\pm 1-\sigma$ is equivalent to $1.5 \lesssim z_{f} \lesssim 3.5$.

- No evolution in the FP slope is seen. indicating that the mean luminosity weighted ages of the stellar populations do not depend significantly on $\sigma$ or $W$ :

$$
\log M /\left.L\right|_{z=0.33}-\log M /\left.L\right|_{z=0} \propto(0.01 \pm 0.23) \log \sigma-(0.16 \pm 0.16) \log r_{e}
$$




\section{The Spiral Galaxies of CL1358+62}

Our sample contains many galaxies of type S0/a through Sb. These early-type spirals are shown with the E/S0s in the right-hand panel of Figure 1. The spirals show large scatter about the FP and have systematically higher surface brightnesses for a given $\sigma$ and $r_{e}$. The scatter for the early-lype spirals is twice as large as that for the E/SOs. For the spirals, the FP residuals correlate strongly with residual from the color-magnitude relation (see Kelson et al. 1999c).

Correcting the surface brightnesses of the spirals for their young stellar populations using their residuals from the color-magnitude relation, one obtains a new plane for the spirals which follows that of the E/SOs. We conclude that after several Gyr, these spirals, including the low-mass E+As, may evolve into present-day, low-mass S0s and appear in nearby FP samples.

\section{Absorption Line Strengths of Early-Type Galaxies in CL1358+62}

The CL1358+62 spectra are of sufficiently high $S / N$ that one can accurately measure absorption line strengths, and use stellar population synthesis models, such as those of Vazdekis et al. (1996), to constrain the systematic variation of the stellar populations along the FP and color-magnitude relations.

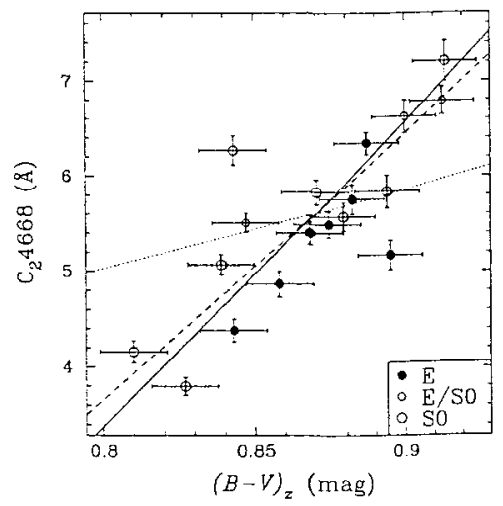

Figure 2. Measurements of the Lick/IDS $\mathrm{C}_{2} 4668 \mathrm{~A}$ index are plotted against restframe $(B-V)$ color for the E/S0 galaxies. The Vazdekis et al. (1996) single-burst stellar population models predict the slope shown by the dashed line if metallicity is the sole cause for variations in these colors and indices. The dotted line shows that expected for age alone. The best-fit slope in our preliminary analysis, shown by the solid line, is nearly identical to the slope defined by metallicity variations at fixed age (Vazdekis et al. 1996).

In Figure 2, we show that the sequence of $\mathrm{E} / \mathrm{S} 0$ galaxies in CL1358+62 is defined by a tight correlation between $\mathrm{C}_{2} 4668 \AA$ and restframe $(B-V)$ color. The best-fit slope of the correlation is nearly identical to that predicted for a sequence of single-burst stellar population models of constant age, in which only metallicity varies. Even with these preliminary measurements, this conclusion is reinforced by the correlations of the metal-sensitive $\mathrm{C}_{2} 4668 \AA$ index with the high-order Balmer lines. In the first panel of Figure 3 , the $\mathrm{C}_{2} 4668 \AA$ index is shown to systematically correlate with the $\mathrm{H} \delta_{A}$ index in a manner which is also consistent with the line of constant age. The scatter in these two diagrams is consistent with the small scatter in ages that has been estimated using the FP and color-magnitude relations (Kelson et al. 1999c, van Dokkum et al. 1998).

The latter two panels show the correlations of $\mathrm{C}_{2} 4668 \mathrm{~A}$ and $\mathrm{H} \delta_{A}$ with central velocity dispersion, indicating that metallicity is strongly correlated with the depth of the galaxy potential. The solid lines are the best-fit slopes, with the $\pm 1-\sigma$ uncertainties indicated by dotted lines. Using the Vazdekis et al. (1996) models, these correlations with velocity dispersion imply $W / L_{V} \propto M^{0.14 \pm 0.02}$. 
Preliminary analysis using Monte Carlo simulations of real data (Kelson 1999) indicate that this correlation can produce an observed FP of the approximate form: $r_{e} \propto \sigma^{1.3}\langle I\rangle_{e}^{-0.8}$, though the precise shape depends on sample selection criteria, and the underlying distribution of bulge-to-disk ratios.
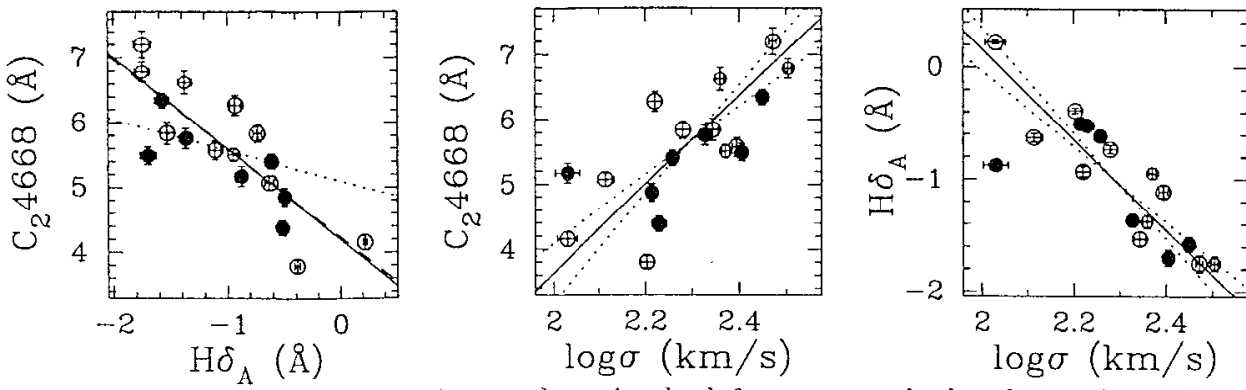

Figure 3. Using the $\mathrm{E} / \mathrm{S} 0$ galaxies we show, in the left-most panel, the observed correlations of the metal-sensitive $\mathrm{C}_{2} 4668 \AA$ Lick/IDS index with the $\mathrm{H} \delta_{A}$ index of Worthey \& Ottavianni (1997). The two right-hand panels show these two indices plotted against central velocity dispersion for the same galaxies.

\section{Summary}

We have found that the slope of the fundamental plane has remained constant with redshift. Together with the absorption line strengths and restframe colors, these data suggest that early-type galaxies in clusters have very uniform stellar population ages, and chemical abundances which are strongly determined by the depths of their potential wells, assuming that the stellar populations of cluster E/SOs can be adequately modeled by simple single-burst models. The implied correlation of metallicity with $\sigma$ appears to be the physical basis behind both the color-magnitude relation and the fundamental plane, though further work is required to fully understand the nature of the selection biases and other systematic effects.

\section{References}

Jørgensen I., Franx M., \& Kjærgaard P. 1993, ApJ, 411, 34

Jørgensen I., Franx M., \& Kjærgaard P. 1996, MNRAS, 280, 167

Kelson, D.D., et al. 1999a, ApJ, sirbmitted

Kelson, D.D., et al. 1999b, ApJ, in press

Kelson, D.D., et al. 1999c, ApJ, in press

Kelson, D.D. 1999, in preparation

Tinsley, B.M. \& Gunn, J.E. 1976, Ap.J, 203, 52

van Dokkum, P.G., et al. 1998, Ap.T, 500, 714

Vazdekis, A., et al. 1996, ApJS, 107, 306

Worthey, G. \& Ottavianni, D.L. 1997, ApJS, 111, 377 
The Hy Redshift Universe

ASP Conference Series, Vol. 193, 1999

A. J. Bunker \& W. J. M. van Breugel, eds.

\title{
Extremely Red Galaxies in the Field of QSO 1213-0017: A Galaxy Concentration at $z=1.31$
}

\author{
Michael C. Lin \\ Department of Astronomy, University of California, Berkeley, CA 94720 \\ Arjun Dey ${ }^{1}$ \\ National Optical Astronomy Observatories, Tucson, AZ 85710 \\ James R. Graham \\ Department of Astronomy, University of California, Berkeley, CA 94720 \\ Charles C. Steide ${ }^{2}$ and Kurt Adelberger \\ Palomar Observatory, Caltech 105-24, Pasadena, CA 91125
}

\begin{abstract}
We have discovered an excess of extremely red objects (EROs) surrounding the $z=2.69$ quasar QSO 1213-0017 (UM 485). Optical/IR colors for these galaxies are consistent with $z=1-2$ ellipticals, and there are at least 5 galaxies with spectroscopic redshifts at $z \approx 1.31$. Keck optical spectra for 3 of the red galaxies show rest-frame UV breaks resembling local elliptical galaxies. Our initial results suggest a coherent structure in redshift, possibly arising from a massive galaxy cluster.
\end{abstract}

\section{Introduction}

The nature of extremely red objects (EROs) remains an open question in understanding the faint galaxy population at $z>1$. First identified by Elston et al. (1988), the identity of these objects, defined by their extreme optical/IR colors $(R-K \geq 6)$, has proven elusive due to the optical faintness of the population. The few EROs with spectroscopic redshifts form a motley $z \gtrsim 1$ collection, including both ultraluminous dusty star-forming galaxies like HR 10 (Graham \& Dey 1996; Dey et al. 1999) and passively-evolving luminous ellipticals like LBDS 53W091 (Dunlop et al. 1996). Both of these types of systems are of great utility in learning about the formation of galaxies and clusters of galaxies.

We are conducting an on-going study of EROs, using ground-based optical and near-IR imaging to assemble large samples amenable to statistical stud-

\footnotetext{
${ }^{1}$ Hubble Fellow

${ }^{2}$ Visiting Astronomer, Kitt Peak National Observatories, National Optical Astronomy Observatories, which is operated by Associated Universities for Research in Astronomy, Inc., under cooperative agreement with the National Science Foundation.
} 


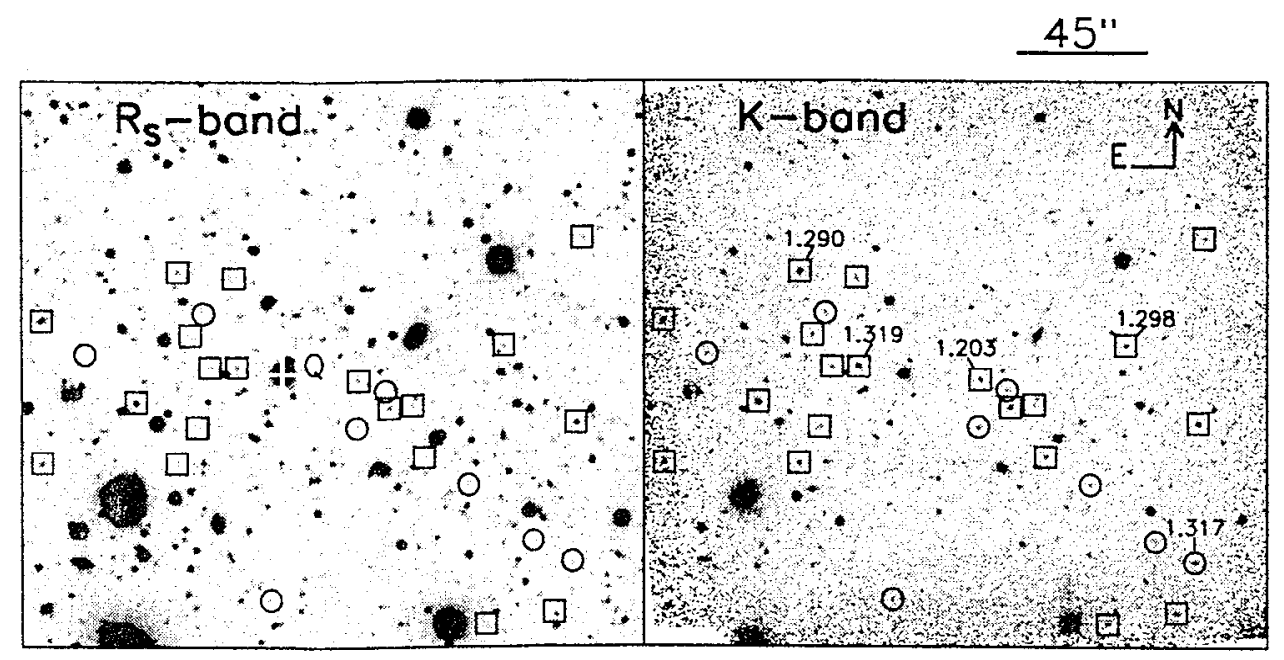

Figure 1. $R_{S}$ and $K$-band images of the field. Each is $3.5 \times 3{ }^{\prime} 1$. Objects with $R_{S}-K>6$ are circled and those with $R_{S}-K=5-6$ are marked with a square. The $z=2.69$ quasar is marked with a white cross and a "Q". Objects with spectroscopic redshifts are labelled by their redshift.

ies and Keck optical/near-IR spectroscopy to determine redshifts and physical properties. Deciphering the identity of EROs from broad-band colors alone is ambiguous - spectroscopy is essential. In addition, given the population's apparent heterogeneity, a reasonably large sample of objects needs to be studied to understand the nature and relative abundances of the subsets, instead of the spectroscopy of individual EROs which has been done to date.

We have discovered a concentration of EROs in the field of QSO 1213-0017, a quasar at $z=2.69$. In this proceedings, we highlight some of our results. The complete analysis and its details are presented in Liu et al. (1999).

\section{Optical/IR Imaging: An Excess of EROs}

Figure 1 shows optical and IR images obtained from KPNO of the 1213-0017 field. A population of objects with red $\left(R_{S}-K>5\right)$ colors are seen, with the brightest ones being $K \approx 18$. These are as red as the expected appearance of $\approx z 1$ passively evolving ellipticals. Most of the sources are resolved in HST $F 814 W$ imaging so they are certainly galaxies and not M stars. We also have $J$ and $H$-band images for a subset of the galaxies; their optical/IR colors are also consistent with $z=1-2$ ellipticals (Liu et al. 1999).

To gauge the significance of these red galaxies, we compare with field ERO counts of Thompson et al. (1999). After accounting for the difference between our $R_{S}$ filter $\left(\lambda_{c} \approx 6930 \AA\right)$ and the $R_{C A D I S}$ filter of Thompson et al. $\left(\lambda_{c} \approx\right.$ $6480 A$ ). we find 4 galaxies with $R-\Pi^{-}>6$ and $\Pi \leq 19$ in the deepest $6.2 \operatorname{arcmin}^{2}$ of our images. Compared to the 0.24 objects expected from the field counts, this is a factor of $\approx 15$ excess, with a factor of 6 being the $95 \%$ confidence value. 


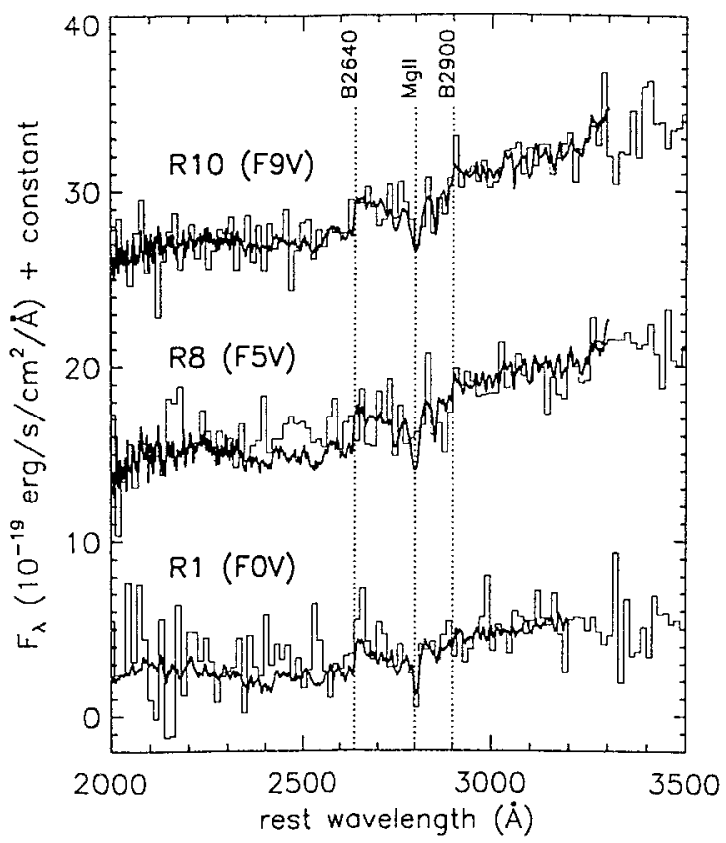

Figure 2. Spectra of the 3 continuum-break galaxies in the 12130017 field, averaged in 7 pixel bins. The $R 8$ spectrum is offset by +13 along the $y$-axis and the R10 spectrum by +26 . Overplotted with the thick lines are IUE spectra of $F$ dwarfs, which have been normalized to the galaxy flux longward of $3000 \AA$.

\section{Keck Optical Spcctroscopy}

We identified spectroscopic features for 5 of the 1213-0017 red galaxies (Table 1). Two of them are emission line galaxies. Galaxy R6 shows a single narrow emission line which we identify as [O II] at $z-1.203$. The spectrum of $R 7$ reveals the presence of an active galactic nucleus, showing strong lines of $[\mathrm{O}$ II] and C II] with intrinsic widths of $\approx 1800 \mathrm{~km} \mathrm{~s}^{-1}$.

The other 3 red galaxies are devoid of strong emission lines, but they do show continuum breaks identifiable as the rest-frame mid-UV breaks at $2640 \AA$ and $2900 \AA$ (Figure 2). At least two of the red galaxies may also have Mg II in absorption. The UV break features are characteristic of the oldest stellar populations, such as Galactic and M31 globular clusters and the cores of local elliptical galdxies. They have also been identified in high-redshift ellipticals, e.g., LBDS 53W091 at $z=1.55$. For old stellar populations, this spectral region is expected to be dominated by light from stars near the main sequence turnoff so, in principal, the strengths of these features can be used to infer the age of the population - at least the portion emitting the bulk of the mid-UV flux by basically measuring the turnoff mass (e.g., Spinrad et al. 1997). We do not attempt this, but we do point out that real variations exist in the UV colors and spectral break amplitudes of R1, R8, and R10, suggesting heterogeneous stellar populations and star formation histories even though they are at a common location in the Universe and have similar $K$-band luminosities. 
Table 1. Spectroscopic Redshifts in the 1213-0017 Field

\begin{tabular}{lcclcl} 
Id \# & $K(\mathrm{mag})$ & $R_{S}-K$ & morphology $^{a}$ & $z$ & spectral features \\
\hline R1 & & & & & \\
R6 & 18.30 & 6.14 & - & 1.317 & B2640, MgII, B2900 \\
R7 & 18.89 & 5.69 & difuse & 1.203 & [O II] \\
R8 & 18.04 & 5.56 & compact+disk? & 1.319 & [O II], C II], [Ne IV], C III]? \\
R10 & 18.94 & 5.02 & E & 1.298 & B2640, MgII?, B2900, D4000? \\
& 18.19 & 5.98 & edge-on disk & 1.290 & B2640, MgII, B2900, D4000? \\
Mg Il & & & & 1.3196 & \\
& & & & 1.5534 & \\
\hline
\end{tabular}

${ }^{a}$ As seen in HST $F 814 \mathrm{~W}$ images. Sources with a blank line "-_" were not observed.

${ }^{b} \mathrm{Mg}$ II absorption system in the spectrum of QSO 1213-0017 (Steidel \& Sargent 1992).

\section{A Coherent Structure at $z=1.31$}

We have found a significant excess of EROs in this field, and of the 5 galaxies with spectroscopic redshifts, 4 lie close together. Moreover, the spectrum of QSO 1213-0017 shows Mg II absorption at $z=1.3106$, similar to R7's redshift. It is unlikely that $\mathrm{R} 7$ is the absorber as it is $15^{\prime \prime}$ from the QSO $\left(65 h^{-1} \mathrm{kpc}\right.$ for $\Omega=1, \Lambda=0$ ), too large compared to cxpccted sizes of absorbers (Steidel 1993). In addition, our HST images show some faint galaxies closer to the QSO, making them better candidates for the absorber. We therefore have a total of 5 galaxies close in redshift and within $\approx 3 h^{-1} \mathrm{Mpc}$ on the sky. Their unweighted mean redshift is 1.309 with a standard deviation of $1810 \pm 580 \mathrm{~km} \mathrm{~s}^{-1}$ and a full range of $3800 \mathrm{~km} \mathrm{~s}^{-1}$ in the mean rest frame.

This is the highest redshift concentration of old, rcd galaxies published to date which has been spectroscopically confirmed. Further data are needed to gauge if this is a genuine massive cluster, both $\mathrm{X}$ ray images to scarch for hot intracluster gas in a deep potential and more redshifts to ascertain the velocity distribution. Finally, we point out that galaxics R8 and R10 lic at $z=1.290-1.298$ while the other 3 are at $z=1.317-1.320$, i.e., there is a "gap" of $2500 \mathrm{~km} \mathrm{~s}^{-1}$ in the mean rest frame, though there is no clear segrcgation on the sky. These may be two separate physical entities (e.g., filaments/sheets/subclusters), but this speculation lies bcyond the available data.

\section{References}

Dey, A. et al. 1999, ApJ, 519,610

Dunlop, J. et al. 1996, Nature, 381, 581

Elston, R., Rieke, G. H., \& Rieke, M. J. 1988, ApJ, 331, Li7

Graham, J. R. \& Dey, A. 1996, ApJ, 471, 720

Liu, M. C. et al. 1999, in preparation

Spinrad, H. et al. 1997, ApJ, 484,581

Steidel, C. C. \& Sargent, W. L. W. 1992, ApJS, 80, 1

Steidel, C. C. 1993, in The Environment and Evolution of Galaxies, 263

Thompson. D. J. et al. 1999, ApJ, in press (astro-ph/9907216) 
The Hy Redshift Universe

ASP Conference Series, Vol. 193, 1999

A. J. Bunker \& W. J. M. van Breugel, eds.

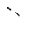

\section{. Near-Infrared Study of Distant Cluster Galaxies}

T. Yamada, I. Tanaka, M. Kajisawa

Astronomical Institute, Tohoku University, Sendai, 980-8578 Japan

T. Maihara, F. Iwamuro, H. Terada, M. Goto, K. Motohara, H. Tanabe, T. Taguchi, R. Hata

Department of Physics, Faculty of Science, Kyoto University, 606 Jupan

Abstract. We present the results of our near-infrared observations of galaxy clusters at $z \sim 1.1-1.2$ : the cluster near the radio-loud quasar $\mathrm{B} 21335+28$ at $z=1.086$, and the cluster near the radio galaxy 3C 324 at $z=1.206$.

\section{Introduction}

It has been well established that giant elliptical galaxies in clusters are formed at high redshift at $z>2$ and have evolved almost passively at intermediate redshift at $z<1$. On the other hand, a fraction of cluster galaxics show cvidence of starformation activity, which evolves with redshift, and the evolution of the entire cluster galaxy population seems more complex. If wo go to higher redshift, $z>1$, these evolutionary effect would be seen more conspicuous and we can put stronger constraints on their formation scenario. Near-infrared obscrvations arc essential in studying galaxy clusters at $z>1$, both in identifying the clusters and in studying the photometric properties of galaxies selected homogencously.

\section{The $\mathrm{B} 21335+28$ Cluster}

Yamada et al. (1997) identified a concentration of galaxies with very red opticalNIR color near the radio-loud quasar B2 $1335+28$ at $z=1.086$. Tanaka et al. (1999) studied the more detailed photometric properties of the galaxies in the field and established an existence of a rich cluster at $z \sim 1.1$. The colors of the cluster galaxies are widely distributed and the strong color-magnitude sequence is not recognized except some red galaxies. Tanaka et al. (1999) argued that this wide color distribution may be due to differences in star formation activity occurring in a significant fraction $(75 \%$ of $K<19)$ of the cluster galaxies. The color-magnitude sequence is, however, recovered for the relatively small number of quiescent galaxies that shows no UV excess compared to the passive-evolution models. The color distribution of the galaxies with $I^{-}<19$ in the cluster region is shown in Figure 1 (left) and the recovered C-MI relation is shown in Figure 1 (right). 


\section{3C324 and the Clusters at $\mathrm{z}=1.2$}

We observed the field of the radio galaxy $3 \mathrm{C} 324$ and the associated clusters (Dickinson 1997) with the SUBARU telescope equipped with the CISCO instrument. Figure 3 (left) shows the obtained $K^{\prime}$-band image with $\sim 2 \times 2 \operatorname{arcmin}^{2}$ f.o.v. The detection completeness is $\sim 70 \%$ at $K=21.5$ in a $3000-s e c$ exposure. We have compared the data with the archived HST WFPC2 deep images taken with the $F 702 W$ and the $F 450 W$ filters. The clustering of the red galaxies around 3C 324 is conspicuous. Detailed results about the luminosity and color distribution of the galaxies will be published elsewhere.

Thanks to the good seeing (0.3-0.4 arcsec FWHM of the stellar images), we can combine the SUBARU $K^{-\prime}$-band and HST WFPC2 images without seriously degrading them. The host galaxy of $3 \mathrm{C} 324$ is clearly resolved in the $K^{\prime \prime}$-band image. It shows a moderately luminous elliptical galaxy with a smooth light distribution. Figure 3 (right) shows the isophotal contour map of the host. The light profile is well represented by the de Vaucoulours's law with effective radius of $1.3 \mathrm{arcsec}(8.9 \mathrm{Kpc}$ at $z=1.21$ ), which is significantly smaller than previously published in Best et al. (1998). The peak of the NIR light distribution coincides with the radio core as well as the "gap" of the optical knotty structures. We see the heavily obscured powerful $A G N$ from an almost edge-on view. Those parts of the host galaxy which avoid the 'aligned' optical structure have very red opticalNIR colors, R-K $\sim 6$, which implies that the optical-NIR light of the galaxy is dominated by an old stellar population. However, the total luminosity of the galaxy is $\sim 1 \mathrm{mag}$ fainter than the prediction of the passive-evolution models, as found for the intermediate-redshift radio galaxies (Aragón-Salamanca et al. 1998).

Acknowledgments. TY and IT thanks A.Aragón-Salamanca, T.Kodama, K.Ohta, T.Miyaji, and N.Arimoto, the co-authors of the paper of B21335+28 cluster (Tanaka et al. 1999) for kindly permitting us presenting the pre-published results. Authors thank the SUBARU telescope team for kindly permitting us to present the results obtained during the telescope-commissioning period.

\section{References}

Aragón-Salamanca, A. et al. 1998, MNRAS, 297, 427

Best, P. et al. 1998, MNRAS, 295, 549

Tanaka, I. et al. 1999, ApJ, in press.

Yamada, T. et al. 1997, ApJ, 487, L125 

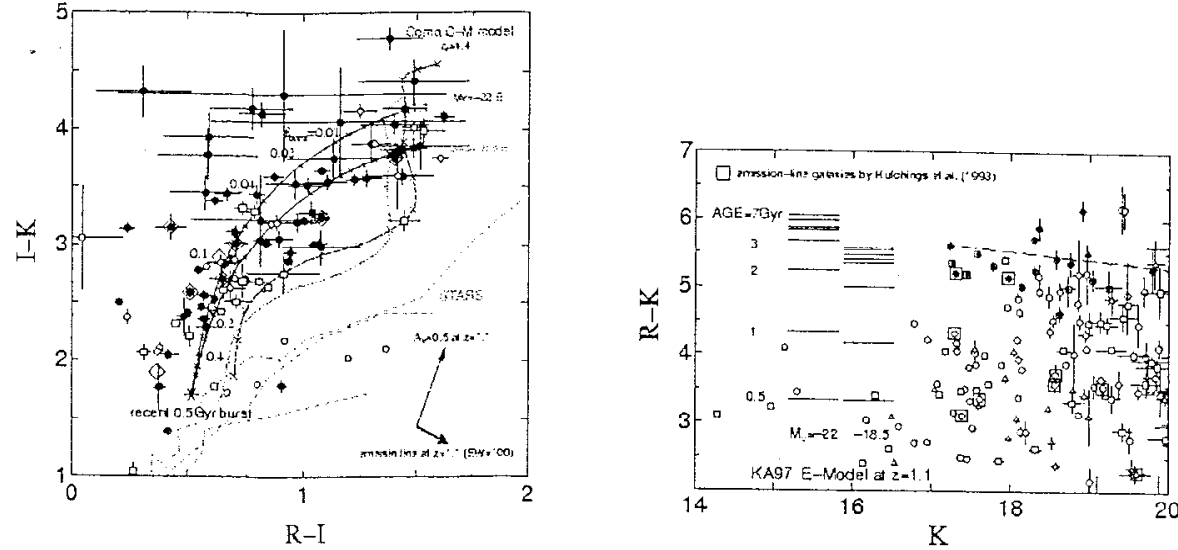

Figure 1. Left. $I-K$ versus $R-I$ two-color diagram for all objects brighter than $K=19$ in the field of B21335+28 cluster. Filled and open symbols represent the objects in the cluster region and the adjacent field regions, respectively. The passive-evolution and the old+starforming model tracks (thin lines) are plotted. Right. The "recovered" $\mathrm{C}-\mathrm{M}$ sequence for the quiescent cluster galaxies. The symbols with small filled circles show the galaxies having colors consistent with the passive evolution model and without $U V$ excess. The dashed line shows the predicted $\mathrm{C}-\mathrm{M}$ relation at $z=1.1\left(z_{f}=4.4\right)$.
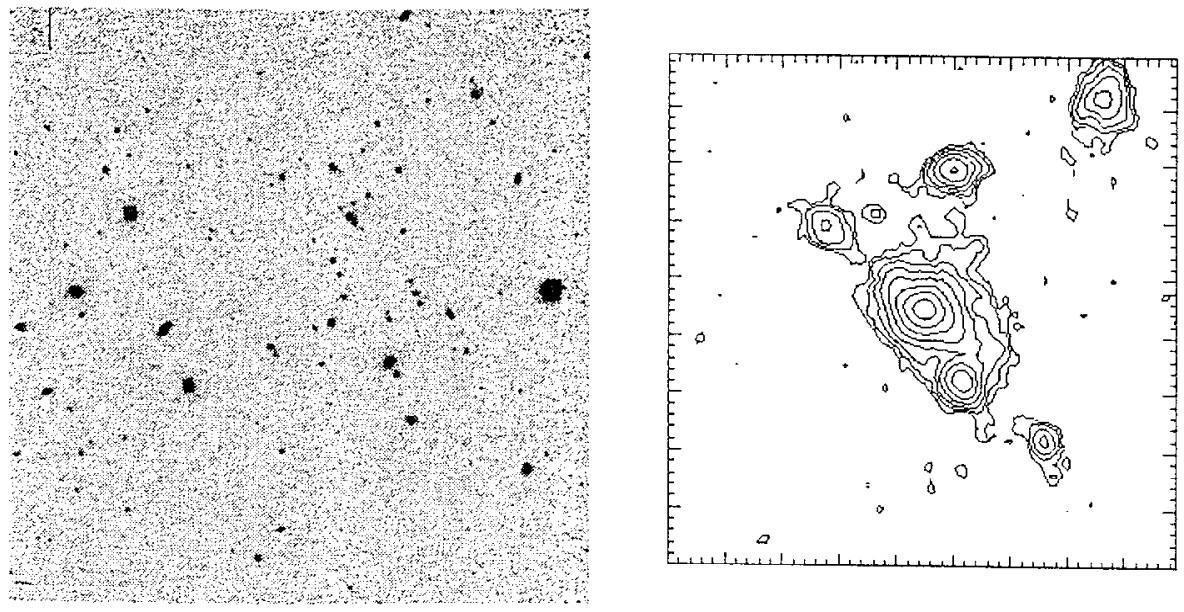

Figure 2. Left. $K^{\prime \prime}$-band image of the 3C324 region taken with the SUBARU telescope. Right. Ishophoto contours of the radio galaxy 3 C324. The f.o.v. of the figure is $\sim 10 \times 10$ arcsec. The lowest contour corresponds to the 1-sigma of the shy fluctuation. 
The Hy Redshift Universe

ASP Conference Series, Vol. 193, 1999

A. J. Bunker \& W. J. M. van Breugel, eds.

\title{
- Structure Formation from Numerical Simulations
}

\author{
Robert J. Thacker and Marc Davis \\ Astronomy Department, University of California, Berkeley, CA 9/420
}

\begin{abstract}
We present a review of the current status in simulating the evolution of structure formation. First a general overview of results from gravitational n-body calculations is given and then the inclusion of hydrodynamics and star formation is discussed. Brief results from the highest resolution hydrodynamic simulation of galaxy formation yet performed are also presented.
\end{abstract}

\section{Introduction}

The evolution of structure in the Universe has been a topic of great activity for the past twenty years. Since the late 70 's, and especially since the invention of inflationary models (Guth 1981) cosmologists have discussed models of structure formation based on causal initial conditions specified by considerations of the very early Universe. These small amplitude fluctnations must have evolved into the highly structured Universe observed today, and the origins of galaxies and all large-scale structure should, in principle, be explained by a. nnified physical mechanism.

Linear theory considerations can be understood by analytic means, but the strongly nonlinear density fluctuations that characterize galaxies and clusters of galaxies cannot be fully understood without resorting to numerical methods to solve the highly coupled equations of self-gravitation. The introduction of well-motivated models of structure formation fortunately arose just at the time when computing power was becoming sufficient to allow detailed simulations of the non-linear phases.

\section{Collisionless large-scale structure simulations}

The earliest and still the bulk of simulation activity is the study of the growth of structure without inclusion of any gas-dynamical complications. On scales larger than individual galaxies, this should be sufficient, as the gas is incapable of cooling and is a small constituent in the mass budget. In the simulations, the Universe is assumed to be dominated by dark matter with initial conditions specified by some more or less platisible modei at an epoch when linear theory is applicable. Most models have assumed random phase, Gaussian initial conditions, which is particularly easy to realize by means of the Zeldorich approximation (Efstathiou et al. 1985). 
Output from these simulations consists of pretty pictures and details of the statistical properties of the clustering of the dark matter. Unfortunately, only galaxies are directly observable in our Universe, and one usually assumes that galaxies form within the dark matter halos seen in the models. Even more unfortunately, inferring the position and abundance of galaxies within the dark matter halos is as much an art as a science. This is especially true of the early simulations which had too little mass resolution to detect separate galaxies within common dark matter halos (e.g. Davis et al.1985), but as computing power has improved, the definition of many separate halos in common envelopes has improved dramatically (e.g. Moore et al.1998, Pearce et al.1999).

One of the key features that we originally hoped to explain with the aide of collisionless simulations is the remarkable power law behavior of the galaxy two-point correlation function, $\xi(r)$, which is observed to have a power law slope, $\gamma \approx-1.8$, for three decades of length scale. No simulation to date, for any set of initial conditions, has ever reproduced a power law mass correlation. function with anywhere near this precision. In many models the slope of the mass correlations slowly steepens with time, while the amplitude grows rapidly with time, more or less as expected from analytic considerations.

In contrast, the correlation properties of the dark matter matter halos within the models is largely static, and the precise behavior of the inferred evolution of the "galaxies", $\xi_{g g}(r)$, within the simulations depends sensitively on the details of how large halos are subdivided into multiple galaxies, and on the merging history of these small halo (e.g. Colin, Carlberg, and Couchman 1997). It is inevitable, therefore, that the "bias" $b$ in the galaxy distribution, the ratio of the galaxy correlations to the underlying matter correlations, $b^{2} \equiv \xi_{g g}(r) / \xi(r)$, will be a function of time. The non-power law nature of the matter correlations within the simulations further implies that the bias must be scale dependent (e.g. Jenkins et al. 1998). Currently favored low density cosmological models have a mass correlation function that is quite steep on small scales, $\gamma \approx-2$, (Davis et al. 1985, Primack et al.1998, Jenkins et al. 1998), so that these models can work only if the galaxy distribution is anti-biased relative to the mass, $(b<1)$.

These results are disappointing, as they imply that more complex physical processes must be invoked to explain what had originally been considered to be an easy question. Semi-analytic models combined with large N-body simulations, as described below, provide a plausible explanation for power-law correlations, involving mass to light ratios for galaxies that have a deep minimum at a luminosity $L \approx L_{*}$. That is, the efficiency of converting mass to (luminous) galaxies is quite low for low mass halos, as well as for very large halos (i.e. clusters of galaxies) but is higher for halos of order $10^{12} M_{\odot}$. Whether these explanations hold as the simulations continue to improve remains to be seen.

Another well defined property of the observed galaxy distribution is the relative velocity dispersion of pairs of galaxies as a function of their separation, $\sigma_{12}(r)$. Although this quantity is quite sensitive to the presence of rare, rich clusters of galaxies, redshift surveys have now progressed to the point that the estimates of the statistic are stable (e.g. Marzke et al.1995, Jing et al.1998). Yet no N-body simulations, for any reasonable values of the cosmological parameters, has been able to reproduce this simple statistic, if the galaxy bias is scale

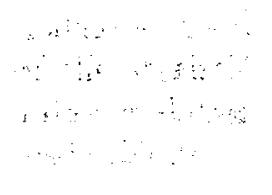


independent (Gelb \& Bertschinger 1994, Jenkins et al.1998, Jing et al.1998). Typically the pair dispersion is too hot relative to the observed dispersion of $\sim 500-600 \mathrm{~km} / \mathrm{s}$ (at $1 h^{-1} \mathrm{Mpc}$ ). Jing et al. (1998) have demonstrated that an antibias in the galaxy distribution, in the sense of reducing the number of galaxies per unit mass within more massive clusters, does improve the match to the observations. This is again a major disappointment, as it eliminates this powerful measure as a diagnostic of cosmological models.

\section{Collisionless simulations of galaxy formation}

As a crude initial study, ignoring all physics beyond gravitation makes sense, since this alone can eliminate many models of galaxy formation. The initial simulations of 10 years ago seemed a reasuriable match to galaxies, with rotation curves that appeared to be flat. It was noted at the time that halos would lose their identity as they merged into larger structures, an effect due to two body heating of the massive particles within (Steinmetz \& White 1997). As the mass resulution of simulations improved in this decade, this problem became less serious, so that dark matter halos orbiting within clusters of galaxies in the current generation of cluster simulations are seen to be stable for at least several crossing times (Moore et al. 1998, 1999).

An important step during the past decade was the realization that the profile of dark matter halos was remarkably universal, with $\rho \propto r^{-1}$ at small scale, and $\rho \propto r^{-3}$ at large scales (Navarro, Frenk, \& White 1996, 1997 (NFW), Huss et al.1999, Moore et al.1998). This type profile emerges both in high and low density cosmologies, although the scaling constants are model dependent. The profile is not sensitive to the details of the small-scale power spectrum (Huss et al.1999, Moore et al.1999). With the Inore precise recent simulations, the inner slope of the core is actually closer to $r^{-1.5}$ rather than the original NFW profile. A current controversy is whether in fact this steep density law at small scales is truly universal, or is somehow a numerical artifact that is not present in higher Inass simulations, as claimed by Kravtsov et al.(1998). This is a purely technical point that should be resolved soon, but so far no other groups have been able to reproduce the Kravtsov et al. result.

This is an important issue because, although $1 / r$ dark matter density profiles are a good description of clusters of galaxies and perhaps of luminous galaxies, they are a poor description of the cores of dwarf galaxies (e.g. Burkert \& Silk 1997, Moore et al. 1999). Many dwarf galaxies contain abundant dark matter and appear to be in solid body rotation in their central regions, consistent with constant density dark matter. To date, nobody has explained how collisionless dark matter could produce a core radius and constant central density in a dark matter halo. Phase space constraints, such as exist in Hot Dark Matter models, are not applicable in the case of cold dark matter. This could be a fundamental problem with the entire cold dark matter paradigm, but then again, it is far from clear what would replace this paradigm.

Yet another controversy is the degree of substructure present in the high resolution simulations. Recent work by the University of Washington group on the formation of the Local Group of galaxies has demonstrated that the final group has as many small halos orbiting the system as are present in a 
cluster of galaxies. In fact the final output of the simulations are essentially indistinguishable. If this is correct, the Milky Way would have not one LMC orbiting it, but hundreds of LMC-sized halos in orbit. Perhaps these objects contain few baryons are so are not luminous, but as they orbit the Milky Way they would heat the disk excessively. This problem is serious but could be made to disappear if there exists a small scale cutoff in the initial power spectrum of density perturbations (as in Warm Dark Matter). Such a cutoff must have been imposed in the early Universe. One way to do this, although rather ugly, is to let the bulk of the dark matter be comprised of particles with a mass in the range of hundreds to thousands of electron volts.

Are these two crises in the small-scale clustering of dark matter related? Can both problems be understood in terms of our current model of cold dark matter, or does the solution to these problems require that the dark matter be more than collisionless dust? Suggestions of self-interacting dark matter have occasionally surfaced (e.g. Carlson et al. 1992, Spergel and Steinhardt 1999), but we are presently very far for a compelling physical motivation for abandoning the cold dark matter paradigm that has been such a successful standard for the past 15 years.

\section{Hydrodynamic simulations of galaxy formation}

A full treatment of galaxy formation must necessarily include physical processes beyond self-gravitation. The baryonic gas will have an isotropic pressure, and is not collisionless. It can furthermore suffer shocks, and most importantly, it can radiate energy and thereby cool. The gas will cool to a disk and eventually form stars, whereupon feedback from UV photoionization and supcrnovae explosions will drastically modify the cooling of the gas as well as pollute the environment with heavy elements. The gas will almost certainly be multi-phase, and magnetic fields are undoubtedly important as well. Given the difficulties of understanding star formation in our own galaxy, it is hardly surprising the cosmologists have barely begun to work out the details of high redshift galaxy formation.

\subsection{Why simulating galaxy formation with gas is a difficult problem}

In the hierarchical clustering picture, a large proportion of the mass is resident in low mass halos at early epochs. The cooling time for baryons within these halos is much shorter than the free fall time (White \& Rees 1978). Consequently, a significant fraction of the baryons condense into cold clumps at very early epochs. If star formation is directly related to the fraction of cold gas then rapid star formation will occur at high redshifts (White \& Frenk 1991). Consequently, it is difficult to see how disc systems, which require baryons to dissipate convergent motions and correlate them into spin, can form. Feedback is believed to be the only method of averting this "cooling catastrophe", since the effects of photoionization are linited to systems with $v_{c}<50 \mathrm{~km} \mathrm{~s}^{-1}$ (Efstathiou 1992, Thoul \&. Weinberg 1996). So far only toy model simulations (such as redistributing the gas in collapsed halos once formed, Sommer-Larsen et ul. 1998) have been shown to produce reasonable results since the star formation is reduced by the redistribution phase. As yet no self-consistent simulation, namely one that predicts the 
star formation rate and from that a distribution of $\mathrm{SN}$ events, has succeeded in forming a galaxy with a structure similar to those observed.

Galaxies formed in CDM halos in simulations have too little angular moInentum (AM) relative to those observed. This fact was discovered at the birth of the simulation field (Navarro \& Benz 1991). It is partly a consequence of the overcooling problem, and there appear to be two mechanisms at work. Firstly, the 'Barnes Mechanism' (Barnes 1992) progressively removes orbital angular momentum from dense gas cores in merging dark malter lialos. If the gas can be supported higher in the halo and allowed to cool at later epochs, this problem can be avoided (Weil et al.1998). The second mechanism is due to the bar instability in disc formation: in the presence of a bar, gas is vigorously funneled inward leading to strong shocking and a build up of a large central concentration of gas that has little angular momentum (Dominguez-Tenreiro et al. 1998). These results are strongly at odds with the fundannental tenet of the work by Fall \& Efstathiou (1980), namely that the specific angular momenta of the two components should be approximately equal during galaxy formation.

\subsection{Hydrodynamic methods}

Since galaxy formation occurs in the highly non-linear regime, mode coupling can lead to short wavelength modes interacting strongly with longer modes. Galaxy formation is thus observed to be affected by long range tidal fields which, therefore, must be accounted for within simulations (Kofman \& Pogosyan 1995). Consequently, a prerequisite for 'accurate' depiction of galaxy formation is dynamic range of $10^{4}$. Fixed grid hydrodynamic methods are limited since the largest current grids are of order $10^{3}$. Moving-mesh methods rapidly suffer entanglement problems as the mesh becomes severely distorted due to the non-linear nature of the dynamics. Adaptive grid/multi-grid methods, such as Adaptive Mesh Refinement (AMR, Berger \& Olgier 1984), are far more promising although, as yet, only exploratory studies have been conducted (Bryan \& Kepner 1999). The main drawback of AMR is that storing all the sub-grids requires a large amount of memory which is not always available.

Particle based hydrodynamic algorithms (principally Smoothed Particle $\mathrm{Hy}-$ drodynamics, SPH, Gingold \& Monaghan 1977, Lucy 1977) are a very popular alternative to mesh methods. Because particle methods are inherently Lagrangian they offer a number of advantages over Eulerian methods, such as concentration of the calculation in high density regions, and little effort is required to combine them with particle based gravitational solvers. These facts, combined with the simplicity of the SPH algorithm make it particularly appealing to cosmologists. The drawbacks of the SPH algorithm include poor shock-capturing compared to modern grid techniques and also poor representation of low amplitude sound wave phenomena. Although SPH was initially developed for applications involving colliding gas polytropes, it has been applied to many different fields (see Monaghan 1992, for an overview). Technical details relating to the numerical implementation of SPH are discussed in Thacker (1999, chapter 2).

\subsection{Incorporating star formation and feedback from supernovae}

Regardless of the fact that star formation is sub-resolution-by orders of magnitude in length and mass-it has an enormous effect on the dynamics of galaxy 
formation simulations. The simple conversion of collisional matter to collisionless can change the resultant morphology of an object from disk-like to elliptical.

As a first approximation, star formation algorithms can be divided into three groups. The first group contains algorithms which rely upon experimental laws to derive the star formation rate (Mihos \& Hernquist 1994, for example). The second group contains those which predict the SFR from physical criteria (Katz 1992, for example). The third group is comprised of algorithms which do not attempt to predict the SFR, but instead set a density criterion for the gas so that when this limit is reached the gas is converted into stars (Gerritsen \& Icke 1997, for example). Analysis of a large selection of galaxies by Kennicutt (1998) has provided a strong argument for the adoption of the first method.

The redistribution of gas following a feedback event is discussed in terms of 'blow-away' where the gas is unbound from the halo in which it resides and 'blow-out' when it remains bound, leading to 'fountain-like' behavior. Given that feedback occurs on sub-resolution scales, it is difficult to decide upon the scale over which energy should be returned. For fixed grid simulations the minimum cell size presents itself, whilst for SPH simulations either a single particle or the SPH smoothing scale are possibilities. Katz (1992) was the first to show that simply returning energy to the ISM in SPH simulations by smoothing the energy over neighbors is ineffective: radiative losses from the optically thin plasma removes the energy almost immediately. Alternative algorithms that keep the energy present in the ISM for longer have been considered by Gerritsen (1997) and Thacker \& Couchman (1999, in prep, also see Thacker 1999, chapter 3). Allowing the energy to persist on Myr timescales, to correlate with the lifetime of large associations of stars, produces significant heating in the ISM, but does not yet produce fountain behavior. The inclusion of feedback leads to a new phase in the temperature-density distribution of gas: a hot-dense phase. If blow-away is present this phase is connected to the hot low density phase.

\subsection{Problems inherent in the numerics}

What to do at the high resolution end in SPH simulations?

This is a difficult issue to address since the picture is not as simple as grid based simulations, where a minimum rell size is reached. In particle based simulations the hydrodynamic resolution must match that of the gravitational solver to avoid unwanted energy transfers between the media (Bate \& Burkert 1997). This places a lower limit on the smoothing scale. However, in doing so a very significant slow down in the algorithm occurs as the SPH particles become clustered. Some authors avoid this by allowing the gas resolution scale to become as small as it needs, leading to 'incorrect' densities ${ }^{1}$, as well as the problems previously mentioned. This is undesirable, particularly in the context of star formation where algorithms take the gas density as an input parameter. Further, both the Courant and acceleration criteria on the time-step mean that as $h \rightarrow 0, \delta t \rightarrow 0$, and thus simulations take tens of thousands of time-steps (Navarro \& Steinmetz 1997). An alternative solution of allowing the resolution

\footnotetext{
${ }^{1}$ Allowing $h-0$ can lead to density estimates two orders of magnitude higher than a properly smoothed estimate. When this density is input into a $n=1.5$ Schimdt Law the resulting SFR is three magnitudes larger than the correctly smoothed version.
} 
scale used to reach a set minimum, but searching over a reduced list of neighbors, has been discussed (Navarro \& White 1993). So far this method has not been tested in detail against the method which calculates over the full neighbor list.

Does an effective Jeans' Mass need to be resolved?

Tt is widely known that in simulations of star formation the Jeans' Length (Mass) must always be resolved (Truelove et al.1998, Bate \& Burkert 1997). This is because star formation proceeds as the fragmentation of initially smooth clouds, for which numerical noise can be a problem, unless sufficient pressure support/resolution is assured. This result is callsed by small truncation errors in the solution growing rapidly because of the attractive nature of the gravitational force. There may not be a problem for hierarchical clustering models since growing modes are present on all length scales in the simulation. The short scale modes have amplitudes that should swamp any error in the larger modes. Further, there may well be subtle dependence on the way the short-range forces are implemented in the gas and gravity. So far, Owen \& Villumsen (1997) have conducted studies on cosmological simulations and argue that the Jeans' Mass must be resolved (although their gravitational and hydrodynamic solvers do not have the same resolution). Tittley and Couchman (1999, in prep) have found that, provided the analyzed objects have formed from a sufficient number of mergers, there is negligible difference between simulations that resolve the Jeans' Mass and those which do not.

How much resolution is enough?

Most SPH work to date has not had sufficient resolution to address the formation process accurately regardless of the object under simulation. Accuracy studies (Stcinmetz \& Muller 1993, Thacker et al.1998) have shown that at least 10,000 particles are necessary to resolve radial shocks. Additionally, in a hierarchical clustering cosmology, a lack of resolution acts much like a fecdback procoss: since the halos are not resolved the gas does not cool as quickly as it should (Weinberg et al.1997). This means that disk formation is more casily achieved at lower resolution. Fixed grid approaches also lack resolution since very often only $30^{3}$ or so zones of a $256^{3}$ simulation are actually resident in the object of interest. As already discussed, AMR investigations are still being awaited.

Just how resolution dependent are star formation algorithms?

Star formation algorithms are explicitly dependent upon the density of gas in a simulation and are thus dependent upon numerical resolution. The hope is that this dependence can be parameterized relative to the underlying resolution. Elizondo et al. (1999a) are the only group to look at resolution dependence. Their conclusions are that global properties, such as total luminosity and rotational velocity, are comparatively unaffected by resolution. However, since at higher resolution the cooling catastrophe means more gas is available for star formation, objects become systematically bluer as star bursts become more prominent. This is roughly in line with what would be expected but ideally it should be quantifiable. So far no SPH researchers have tested resolution in detail. 


\section{The Star Formation Rate in Disks}

Simple dimensional arguments siggest that the star formation rate in a galaxy should scale as

$$
S F R \propto \rho_{g a s} t_{d y n}^{-1} \propto \rho_{g a s}^{3 / 2} .
$$

In disks, the observed rate of star formation per unit area is (Kennicutt 1998)

$$
\Sigma_{S F R}=(2.5 \pm 0.7) \times 10^{-4}\left(\Sigma_{g a s} / 1 M_{\odot} p c^{-2}\right)^{1.4 \pm 0.15} M_{\odot} \mathrm{yr}^{-1} \mathrm{kpc}^{-2} .
$$

The proportionality observed agrees with the naive expectation. The in triguing and difficult question however is what determines the coefficient in the expression for the star formation rate, i.e. what is the efficiency of star forma. tion? One can define efficiency in this context as the fraction of gas turned into stars per dynamical time.

The consensus view is that feedback is responsible for the low observed efficiency of star formation. The star formation efficiency (SFE) indeed is low. One measures the SFE directly by counting stars in giant molecular cloud complexes, or by globally averaging the gas content and star formation rate in the Milky Way galaxy. The observed SFE is only a few percent.

A simple model for feedback appeals to wind from protostellar outflows in molecular clouds and to supernovae and $O B$ stellar winds reaching more globally into the interstellar medinm. One example has been recently developed by Scalo and Chappell (1999). Outflows sweep up shells that undergo mutual interactions before being completely decelerated by the ambient gas. Continuing star formation is assumed to occur in the dense shells or shell fragments, once a critical swept-up column density is exceeded. The stars undergo dynamical relaxation in a 2-D simulation. A molecular cloud complex is viewed as a network of interacting gaseous filaments.

This theory of star-forming molecular clouds make some notable predictions, including the velocity and mass distribution of molecular cloud clumps. Nongaussian distributions are predicted, and are observed. The distribution of young stars is also predicted, and perhaps most impressively, the observed two-point correlation function of T-Tauri stars (Larson 1995, Simon 1997, Nakajimita et al. 1998) can be interpreted in terms of the feedback model.

While this particular model is specific to molecular clouds, one can readily imagine that global star formation is regulated in an analogous manner. In this case, the feedback from star formation is via supernovae and OB stellar winds. The feedback is modulated by the porosity of a two-phase interstellar medium (Silk 1997). Supernovae, in particular, generate a low density $\sim 10^{6}$ $\mathrm{K}$ diffuse phase in approximate pressure balance with the cold HI gas, which in turn surrounds and pressurizes the $H_{2}$ complexes. Although the supernovaedriven bubbles may initially be spherical, they rapidly become highly irregular as the swept-up shells interact with each other. In a limiting case, the fraction of the hot phase is of order fifty percent and the porosity is of order unity. $\left(f_{\text {hot }}=1-e^{-P}\right.$ ). Here there is strong feedback between star death and star formation, the latter occurring only in the dense, swept-up shells, In another limiting case, the hot phase fraction and porosity are both small, and feedback is unimportant. 
What is not so obvious is the sign of the feedback. It is mostly negative, due to the three-dimensionality, of the disk. The hot phase gas is expelled from the disk in chimneys and other less regular regions of outflow, and entrains neutral gas at a rate that is several times that expended in star formation. Such an expulsion rate is expected if $P \sim 1$ and is indeed observed for nearby dish galaxies in the form of high latitude, $H \alpha$ emitting gas (Martin 1999). This suggests that porosity provides negative feedback to star formation. The converse might be true in local situations where the hot gas overpressures cold clouds and induces collapse. However one might expect the reality of three-dimensional disk modelling, hitherto not yet accomplished for a multiphase disk, to favour outflows and hence negative feedback.

Adopting the hypothesis of porosity-driven negative feedback, one can discern two limiting regimes. If $f_{\text {hot }} \sim 0.5$, the feedback occurs only in actively starforming galaxies, such as the Milky Way. The hot gas fraction is self-limiting, so that $P \sim 1$ since by definition

$$
\begin{aligned}
P & =(4-\text { volume of } \mathrm{SN} \text { bubble })(\mathrm{SN} \text { rate per unit volume) } \\
& \approx p_{a m b}^{-1.4}(S F R) .
\end{aligned}
$$

The inferred star formation rate agrees with the empirical result for disks, together with an additional dependence as $\sim v_{\text {turb }}^{3}$. Enhancement of turbulence, as might occur in a merger, therefore stimulates the star formation rate.

A more dramatic effect occurs if $f_{\text {hot }} \sim P \ll 1$. In this limit, the SN bubbles do not propagate far enough to be volume-filling. This would apply in a very gas-rich environment, as expected after a bar or a merger has driven gas into the central region or in a protogalaxy. The suppression of feedback is likely to lead to a starburst. One would expect this limit to apply for example to the intense star formation rates found in ultra-luminous infrared galaxies.

\section{Spheroid Formation}

Disk star formation has an underlying theory of the instability of cold selfgravitating disks to provide the basic infrastructure. Formation of spheroids takes us into unknown territory with regard to estimating the properties of the associated star formation. The observed stars are cold, although there is considerable debate on whether the mean age is closer to 3 or 10 billion years, and there is little recent star formation. The consensus view is that massive spheroids formed via disk mergers, while low mass spheroids, and especially the spheroids of late-type spirals, formed via secular dynamical evolution of disks. In the latter case, the star formation largely occurs in the disk phase.

With regard to massive spheroid formation, one can conceive of two alternative hypotheses for star formation. There may have been a sequence of gas-rich mergers, as suggested by hierarchical structure formation theory, in which bursts of star formation accompanied each stage of the merging sequence. Another possibility is that strong feedback from star formation suppressed most of the star. formation during the early, low mass mergers, and that the bulk of the star formation occurred during the last, major merger. One can therefore choose between two extreme scenarios: a sequence of small bursts of star formation, 
or a relatively monolithic star formation burst. Both scenarios have testable predictions for observational cosmology.

The hierarchical star formation model predicts that there should be young components present in subpopulations of the spheroid, and that one should observe merging at modest redshift. The monolithic model requires that the major star formation event be dust shrouded, otherwise the predicted luminous starbursts would be in conflict with optical searches. Indeed, there are submillimeter and far infrared observations of ultraluminous starbursts at high redshifts that are candidates for spheroid formation. There are HST observations of aggregations of sub-galactic, star-bursting objects that are plausible candidates for ongoing merging associated with induced star formation (Pascarelle, Windhorst and Keel 1998).

Both pathways have some observational support, and it is likely that the entire range of merger-induced star formation, from minor to major starbursts, plays a role in massive spheroidal formation in differing environments. A useful probe of star formation history is provided by studying the stellar populations of spheroids at low redshift, $z \sim 1$, where high resolution morphological and spectral data is attainable. One can, by judicial use of the color-magnitude diagram and of metal line indices, attempt to resolve the degeneracy between age and metallicity of old stellar populations. Study of individual galaxy clusters has provided a first step in this direction, since a cluster provides a relatively uniform sample of nearly coeval galaxies, or at least, of galaxies with a similar environmental history and set of protogalactic initial conditions. It is especially interesting to study clusters at $z \sim 1$, and compare them with nearby clusters, since the reduced time available since formation helps highlight and break the degeneracies between age and metallicity.

It has been found for example, that the color-magnitude diagram in a galaxy cluster at $z=0.55$ can be projected into metallicity-age space to show that the observed galaxies span a range of about a factor of 2 in metallicity $(0.1 \gtrsim[Z] z-0.2)$ and 10 in formation redshift $\left(1 \lesssim z_{f} \lesssim 10\right)$, with a systematic trend in decreasing metallicity and more recent formation epoch at lower luminosity (Ferreras, Charlot and Silk 1999). A combination of metallicity and age spread is required to explain the low dispersion and slope of the colormagnitude diagram for early-type galaxies. Neither a pure age or metallicity sequence suffices. Application of simple chemical evolution models with various combination of inflow, outflow or enhanced yield favours models with variable outflows as being able to span the range of observed (metallicity, age) parameter space in individual clusters (Ferreras and Silk 1999). Such an outflow interpretation, applied to all luminous spheroids, provides a source of heavy element enrichment that can account for the observed enrichment of the intracluster gas, which is known empirically to be associated with the spheroidal components of cluster galaxies.

An age sequence yields predictions about the variation with epoch of massto-light ratio. This has been studied via the fundamental plane in clusters. to $\approx \sim 1$, and can be independently used to constrain the formation epoch of the old stellar population (Jorgensen et al. 1999). Line indices can also provide a potentially powerful means of breaking the degeneracy between metallicity and age, via for example use of $\mathrm{H} \beta$ and $\mathrm{Mg}$ or $\mathrm{Fe}$ indices, although conversion to 
actual metallicity values is sufficiently uncertain that comparison with model predictions has not yet been widely implemented.

\section{The High Redshift Universe}

Hy Spinrad has pioneered low signal-to-noise determinations of record redshifts for galaxies. At present, there are three galaxies known with $z>5$, and according to Spinrad (private communication), the surface density of galaxies at $z \sim 5$ is $\sim 2( \pm 1)$ galaxies per square arc minute and per unit redshift. The relevant continuum magnitude limit is $M_{A B}<28$ at rest frame $1500 \mathrm{~A}$. These observations, and especially their extrapolation to even higher redshift, provide an interesting discriminant of cosmological models.

The number dersity of objects at high redshift is determined by the available comoving space density. This varies dramatically at $z>5$, and even more dramatically at $z>10$, between a critical density universe $\left(\Omega_{m}=1\right)$ and a low density universe. Especially at the higher redshifts, one can easily distinguish a flat, low density universe with a cosmological constant (e.g. $\Omega_{m}-0.3, \Omega_{\Lambda}=0.7$ ) from an open universe $\left(\Omega_{M}=0.3\right)$. The latter model has a density of galaxies above a specified mass ( say $10^{11} M_{\odot}$ ) at $z>10$, that exceeds that in the flat, low model by $\sim 100$ and that in the critical density model by $>10^{6}$. These differences are likely to overwhelm the uncertainties in converting galaxy mass into luminosity.

A simple approach to predicting galaxy number counts is as follows (Robinson and Silk 1999). Take the galaxy star formation rate to be $\epsilon v_{c i r c}^{3} G^{-1}$, and fit this to empirical data (adopting a Press-Schechter model for the mass function of galaxies as a function of circular velocity $v_{c i r c}$ and epoch) on the galaxy luminosity function at $z=0$ and the cosmic star formation history. Parameters in such a model include the shape and normalization of the initial power spectrum as well as feed-back, which is most simply incorporated as a low circular velocity cut-off below which formation is suppressed. There is still considerable freedom in high redshift predictions of galaxy counts, but one can utilize the data on counts of Lyman break galaxies at $z \approx 3$ and 4 , where there are measurement of the galaxy luminosity function, to fully constrain the model. One finds that at $z=5$, the observed surface density of galaxies is accounted for in low density models, but cannot be accommodated in a critical density universe. At $z=10$, the predicted number of galaxies above $m_{A B}=28$ varies between $\sim 0.5\left(\Omega_{m}=0.2, \Omega_{\Lambda}=0\right)$, $0.001\left(\Omega_{m}=0.3, \Omega_{\Lambda}=0.7\right)$ and $\sim 10^{-10}\left(\Omega_{m}=1\right)$, all per unit redshift and per square arc-minute. Above $m_{A B}=30$, the respective predictions are $3,0.1$ and $10^{-5}$. these are central values for the model normalised to data at $z \lesssim 5$, and one expects the galaxy formation model uncertainties (probably less than an order of magnitude) to be overwhelmed by the cosmological model uncertainties. The prospects are bright for doing cosmology at $z=10$, although it may require the NGST to provide the candidate galaxies.

\section{Summary}

Galaxy formation will :only be understood phenomenologically. The physics is far too complex for us to anticipate any fundamental understanding of the con- 
version of early universe baryons into late epoch stars. As observations improve, we can hope to better constrain the IMF. I have cited theoretical arguments that suggest we should at least be open to possible IMF variations in ultra-luminous starbursts at very low metallicities, and at very high redshifts. Signatures of IMF variations include anomalous abundance ratios, enhanced Type II supernova rates, and high mass-to-light ratios in regions dominated by luminous matter. One can search for these signatures both in exotic environments and in the early universe.

The SFR is directly measurable, in that it is associated with massive star formation. The correction for the unobserved low mass stellar component will remain difficult to establish but can be indirectly investigated by measuring mass-to-light ratios. Feasible issues that can be addressed include a possible dependence of global SFR on gas velocity dispersion as measured by $H \alpha$ linewidths and any dependency of SFR on environment, whether gas-rich or metal-poor. The efficiency of the SFR can be quantitatively evaluated in starbursts, given dynamical measurements and studies of the gas reservoir. The indication from the interpretation of the elliptical/SO color-magnitude diagram in clusters which suggests that outflows are ubiquitous in early galaxy evolution can be pursued by studying the dependence of outflows and winds on host galaxy characteristics.

The epoch of galaxy formation can be probed indirectly by studying the ages of the oldest stellar populations at $z \sim 1$. Breaking of the metallicity-age degeneracy via use of colours and line indices is already indicating high formation redshifts. As more and more galaxies are discovered at ever higher redshifts, one can only push the formation redshift back to epochs where the challenge of the cosmological models dominates the uncertainty in galaxy formation modelling. Exploration of the high redshift universe is barely beginning.

Acknowledgments. I am grateful to Ignacio Ferreras, James Robinson and Marco Spaans for many discussions of galaxy formation issues, and for allowing me to cite results from our ongoing collaborations.

\section{References}

Ballesteros-Paredes, J., Vazquez-Semadeni, E. and Scalo, J. 1999, ApJ, 515, 286.

Bangh, C. et al. 1999, preprint astro-ph/9907056.

Ellis, J. et al. 1999, Astroparticle Physics in press.

Ferreras, I. and Silk, J. 1999, ApJin press.

Ferreras, I., Charlot, S. and Silk, J. 1999, ApJ, 521, 81.

Fukugita, M., Hogan, C. and Peebles, P. 1998, ApJ, 503, 518.

Gawiser, E. and Silk, J. 1998, Science, 280, 140.

Hughes, D. et al. 1998, Nature, 394, 241.

Jorgensen, I. et al. 1999, MNRAS in press.

Kennicutt, R. 1998, ApJ, 498, 541.

Martin, C. 1999, ApJ, 513, 156.

Pascarelle, S., Windhorst, R. and Keel. W. 1998, AJ, 116,2659.

Robinson, J. and Silk, J. 1999, in preparation. 
Scalo, J. and Chapell 1999, ApJ, 510, 258.

Schramm, D. and Turner, M. 1998, RMP. 70, 303.

Spaans, M. and Silk, J. 1999, in preparation.

Weinberg, D. et al. 1997, ApJ, 490, 564. 
The Hy Redshift Universe

ASP Conference Series, Vol. 193, 1999

A. J. Bunker \& W. J. M. van Breugel, eds.

\title{
Galaxy Clustering in the CNOC2 Redshift Survey
}

\author{
R. G. Carlberg ${ }^{1,2}$, H. K. C. Yee ${ }^{1,2}$, S. L. Morris ${ }^{1,3}$, H. $\operatorname{Lin}^{1,2,4,5}$, P.

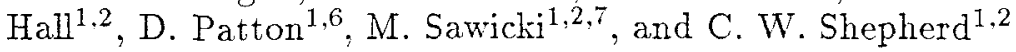

\begin{abstract}
The correlation evolution of a high luminosity subsample of the CNOC2 redshift survey is examined. The sample is restricted to galaxies for which the $\mathrm{k}$ corrected and evolution corrected $\mathrm{R}$ luminosity is $M_{R} \leq-20 \mathrm{mag}$, where $M_{*} \simeq-20.3 \mathrm{mag}$. This subsample contains about 2300 galaxies. In consort with 13000 galaxies in a similarly defined low redshift sample from the Las Campanas Redshift survey we find that the comoving correlation can be described as $\xi(r \mid z)=\left(r_{00} / r\right)^{\gamma}(1+z)^{-(3+\epsilon)}$ with $r_{00}=5.08 \pm 0.08 h^{-1} \mathrm{Mpc}, \epsilon=0.02 \pm 0.23$ and $\gamma=1.81 \pm 0.03$ over the $z=0.03$ to 0.65 redshift range in a cosmology with $\Omega_{M}=0.2, \Lambda=0$. The measured clustering amplitude, and its evolution, are dependent on the adopted cosmology. The evolution rates for $\Omega_{M}=1$ and flat low density models are $\epsilon=0.9 \pm 0.3$ and $\epsilon=-0.5 \pm 0.2$, respectively, with $r_{00} \simeq 5 h^{-1} \mathrm{Mpc}$ in all cases.
\end{abstract}

\section{Introduction}

The measurement of the evolution of galaxy clustering is a direct test of theories for the formation of structure and galaxies in the universe. Within CDM-style structure formation theories galaxies form exclusively within dark matter halos whose clustering evolution should be slower than the underlying dark matter. Clustering evolution is of empirical and pragmatic interest on scales comparable to the size of galaxies themselves, since clustering leads to galaxy-galaxy merging of gas and stars which is a physical driver of galaxy evolution. On somewhat larger scales galaxy groups and clusters contain hot gas which exerts evolution pressures on galaxies.

\footnotetext{
${ }^{1}$ Visiting Astronomer, Canada-France-Hawaii Telescope, which is operated by the National Research Council of Canada, le Centre National de Recherche Scientifique, and the University of Hawain.

${ }^{2}$ Department of Astronomy, University of Toronto, Toronto ON, M5S 3H8 Canada

${ }^{3}$ Dominion Astrophysical Observatory, Herzberg Institute of Astrophysics, , National Rescarch Council of Canada, 5071 West Saanich Road, Victoria, BC, V8X 4M6, Canada

${ }^{4}$ Steward Observatory, University of Arizona, Tucson, AZ, 85721

${ }^{5}$ Hubble Fellow

${ }^{6}$ Department of Physics \& Astronomy, University of Victoria, Victoria, BC, V8W 3P6, Canada

'Mail Code 320-47, Caltech, Pasadena 91125, USA
} 
The theoretical groundwork to interpret the quantitative evolution of dark matter clustering and the qualitative trends of galaxy clustering evolution is largely in place for hierarchical structure models. Although the details of the mass buildup of galaxies and the evolution of their emitted light are far from certain at this time, clustering of galaxies depends primarily on the distribution of initial density fluctuations on the mass scale of galaxies. N-body simulations of ever growing precision and their theoretical analysis (Davis et al. 1985, Efstathiou et al. 1985) have lead to a good semi-analytic understanding of dark matter clustering into the nonlinear regime. Normal galaxies, which are known to exist near the centers of dark matter halos with velocity dispersions in the approximate range of 50 to $250 \mathrm{~km} \mathrm{~s}^{-1}$, will not have a clustering evolution identical to the full dark matter density field (Kaiser 1984).

The observational measurement of clustering at higher redshifts is still maturing, with no published survey of the size, scale coverage, or redshift precision of the pioneering low redshift CfA survey (Davis \& Peebles 1983). There are two redshift surveys extending out to $z \simeq 1$, the Canada France Redshift Survey (Lilly et al. 1995) and the Hawaii $\mathrm{K}$ survey (Cowie et al. 1996). Their redshift precision is insufficient for kinematic studies, however both quantify the substantial evolution in the luminosity function. Measurement of the correlation evolution of the galaxies in these surveys found a fairly rapid decline in clustering with redshift (LeFèvre et al. 1996, Carlberg et al. 1997) although neither analysis took into account the evolution of the luminosity function or was able to quantify the effects of the small survey volumes.

\section{The CNOC2 Sample}

The Canadian Network for Observational Cosmology field galaxy redshift survey (CNOC2) is designed to investigate nonlinear clustering dynamics and its relation to galaxy evolution on scales smaller than approximately $20 h^{-1} \mathrm{Mpc}$ over the $0.1 \leq z \leq 0.7$ range. There is substantial galaxy evolution over this redshift range (Broadhurst, Ellis \& Shanks 1988, Ellis et al. 1996, Lilly et al. 1995, Cowie et al. 1996, Lin et al. 1999). Investigating the clustering evolution of a population requires some basic ability to recover its progenitor hosts at higher redshift.

The observing and catalogue design procedures are adaptations and extensions of those for the CNOC cluster survey (Yee, Ellingson \& Carlberg 1996, Yee et al. 1997). The CNOC2 survey is contained in four patches on the sky subtending a total sky area of about 1.55 square degrees. The sampled volume is about $0.5 \times 10^{6} h^{-3} \mathrm{Mpc}^{3}$, roughly comparable to the low redshift "CfA" survey used for similar measurements at low redshift (Davis \& Peebles 1983) which had 1230 galaxies in the "semi-volume limited" Northern sample from which the correlation length was derived. The CNOC2 limiting magnitude of $R=21.5$ mag efficiently covers the redshift range targeted, 0.1 to about 0.7 . The survey layout for the high luminosity subsample analyzed here is shown in Figure 1.

Photometry is obtained in the UBVRI bands, with the $\mathrm{R}$ band fixing the sample limit at $21.5 \mathrm{mag}$. The $R$ filter stays redward of the $4000 \AA$ break over our redshift range. The other bands provide information useful for determining appropriate $k$-corrections and separating galaxies into types of different evolutionary state ( an issue not considered in this paper). 

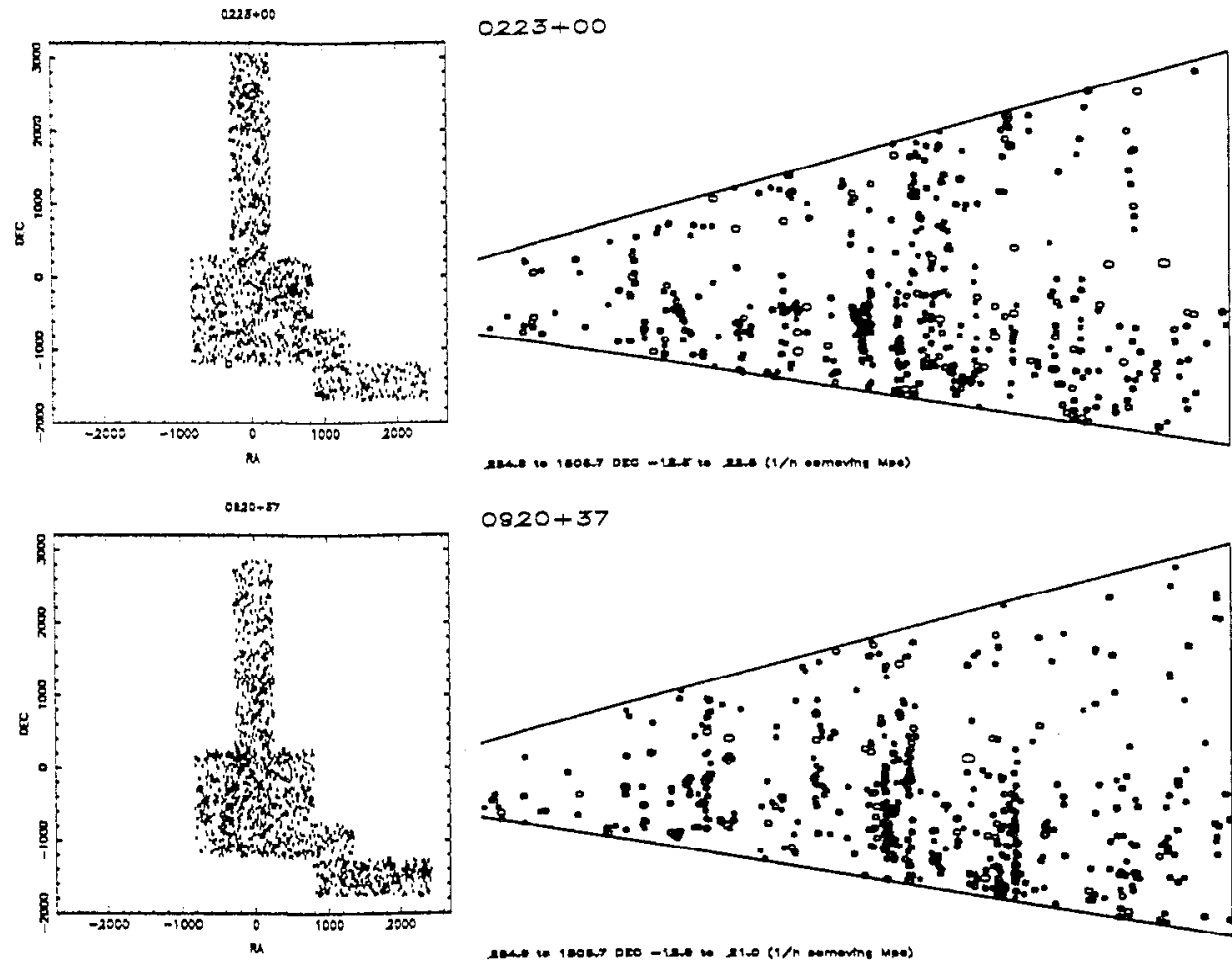

$0820+37$
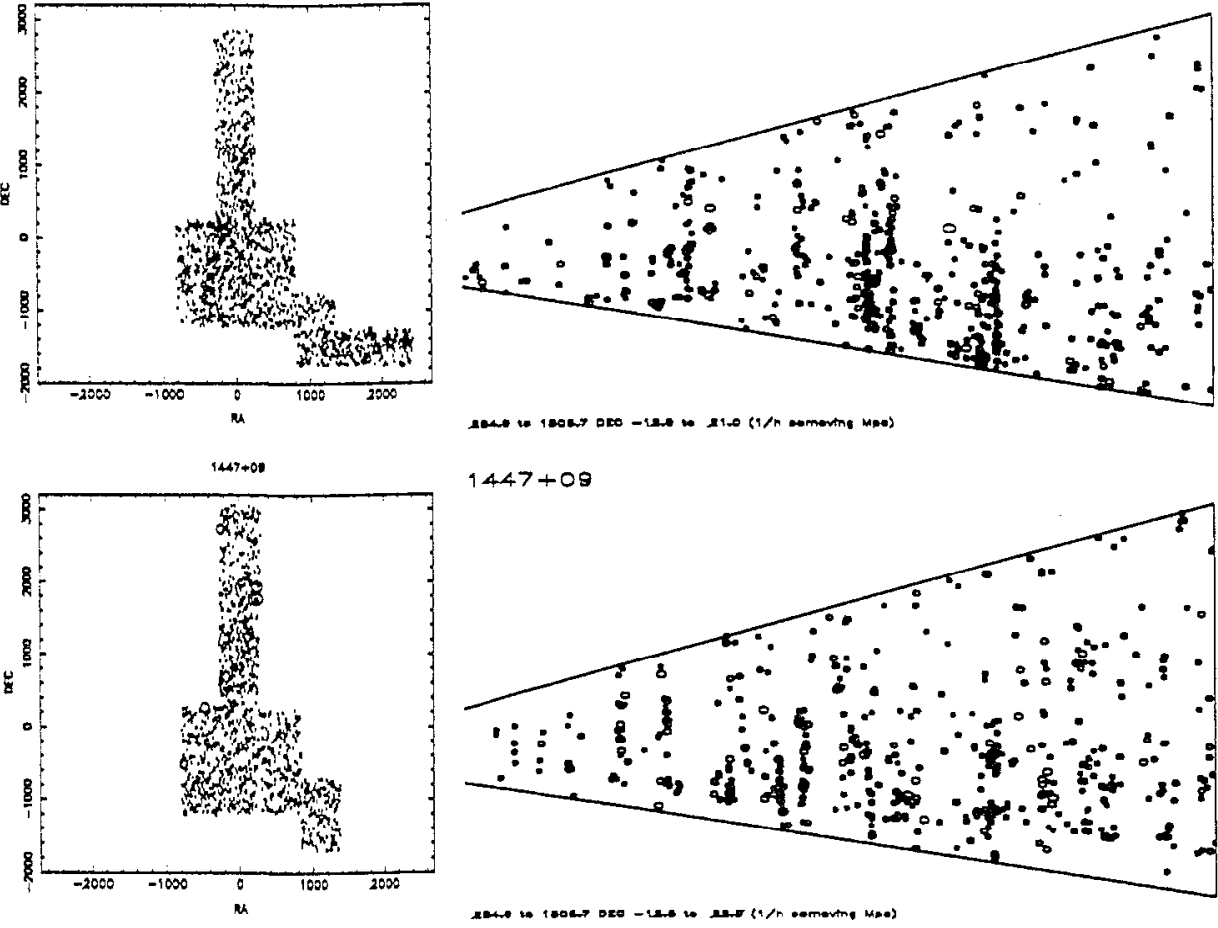

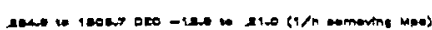

$1447+09$
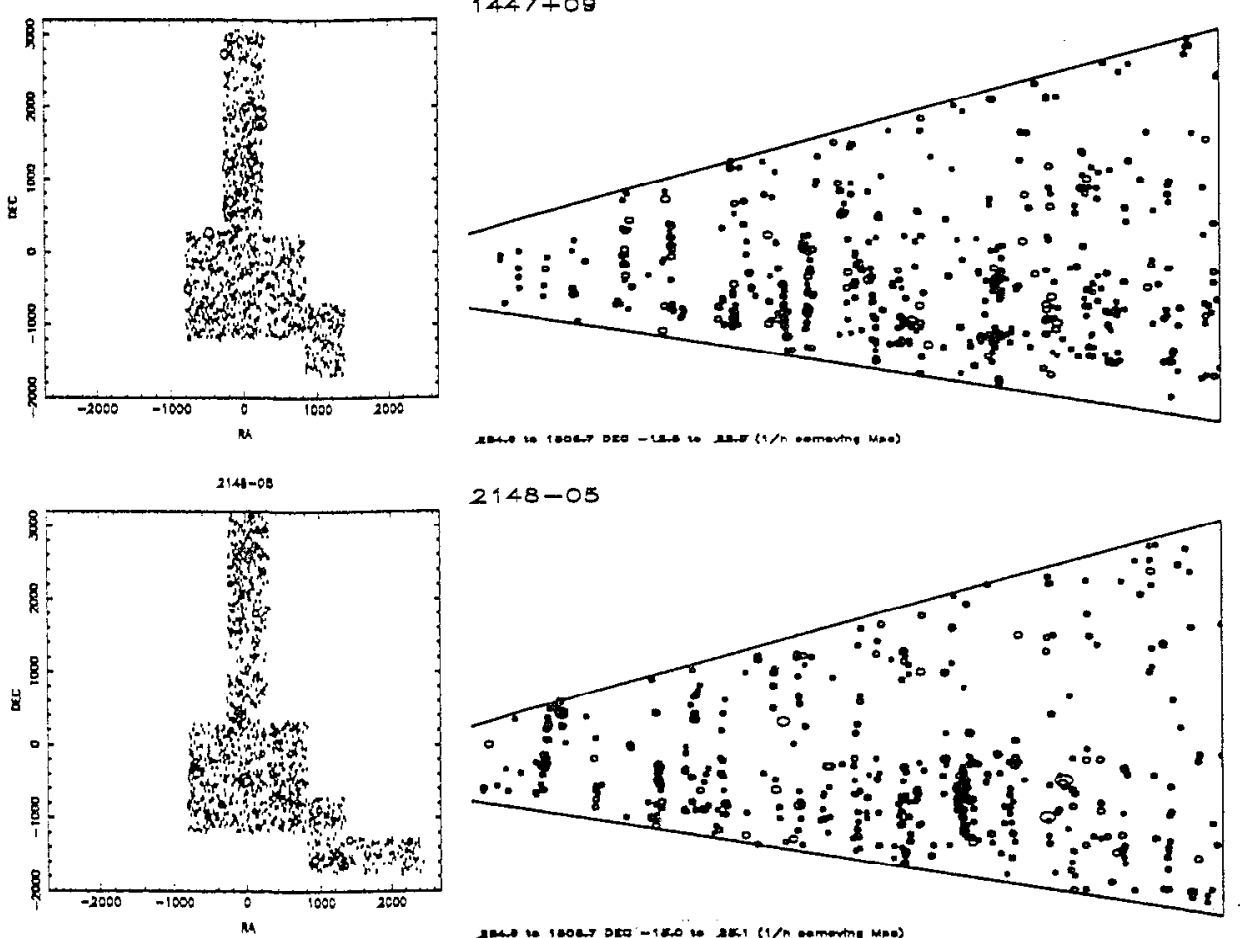

Figure 1. The layout of the high luminosity redshift sample, $M_{R}^{k e} \leq$ $-20 \mathrm{mag}$, sample on the sky (left) and in co-moving distance (redshifts from 0.1 to 0.65 ) versus declination in comoving coordinates (right). 
At low redshift we use the Las Campanas Redshift Survey (LCRS) to provide a directly comparable sample. The LCRS is an $R$ band selected survey (Schectman et al. 1996) that covers the redshift range 0.033 to 0.15 , with $\mathrm{R}$ band flux between 15.0 and $17.7 \mathrm{mag}$. The same correlation analysis programs were used with for the LCRS and CNOC2 data. The only differences are that the LCRS data are not $\mathrm{k}$ corrected and is only analyzed for a single cosmological model, $q_{0}=0.1$.

\subsection{The High Luminosity Subsample}

The evolution of correlations with redshift is sensitive to luminosity evolution. That is, usually the goal is to compare the evolution of the correlations of the same "primary" galaxies, which have likely evolved in luminosity as a result of stellar evolution of the existing stars, star formation, and merging of companion galaxies. Over the redshift range discussed here these effects are significant, but basically perturbative on a stable underlying population. In the absence of a luminosity correction one will be adding in galaxies of lower intrinsic mass with increasing redshift. Lower luminosity galaxies are less correlated (Carlberg et al. 1998, Loveday et al. 1995) which if over-represented in the higher redshift will lead to an erroneously rapid correlation evolution. From previous studies we concluded that the luminosity function can be approximated as evolving such that the absolute magnitude varies as $M(z)=M(0)-Q z$ with $Q \simeq 1$ (Lin et al. 1999). It should be noted that the this approach is a statistical correction designed to identify the same population at two redshifts and will work even for an evolving rate of bursting star formation, provided that evolution has no significant mass dependence. This assumption should be adequate for our high luminosity subsample (Lilly et al. 1995, Lin et al. 1999) but may fail for at lower luminosities. The volume density of galaxies makes no difference to the correlations. For the correlation analysis here we will use galaxies with $M_{R}^{k, e} \leq-20 \mathrm{mag}$, which defines a volume limited sample over the $0.1 \leq z \leq 0.65$ range. The resulting sample (for $q_{0}=0.1$ ) contains 2285 galaxies.

The LCRS data are evolution corrected with the same $Q$ as the CNOC2 data, although at a mean redshift of about 0.1 , this makes very little difference. The resulting low redshift sample contains 12467 galaxies that are used in the correlation analysis.

\section{Real Space Correlations}

The CNOC2 sample is designed to measure nonlinear clustering on scales of $10 \mathrm{~h}^{-1} \mathrm{Mpc}$ and less. The clustering is quite naturally measured in terms of the two-point correlation function, $\xi(r)$, which measures the galaxy density excess above a random distribution, $n_{0}$, at distance $r$ from a galaxy, $n(r)=n_{0}[1+\xi(r)]$ (Peebles 1980). Measurement of the real space function $\xi(r)$ is not straightforward with redshift space data in any case, and yet more difficult with only a few hundred galaxies per redshift bin. The projected real space correlation function removes the peculiar velocities of redshift space at the cost of making a choice for the length of the redshift column over which the summation is done. Noting that the correlation function measures the fractional variance in galaxies in different 
volumes we check as much as possible that our evaluation of the correlation does not artificially add or remove variance which will bias the result.

\subsection{The Projected Real Space Correlation Function}

The correlation function is a real space quantity, whereas the redshift spacc separation of two galaxies depends on their peculiar velocities as well as the physical separation. Although the peculiar velocities contain much useful infor. mation about clustering dynamics, they are mainly a complication for the study of configuration space correlations. The peculiar velocities are eliminated by integrating over the redshift direction to give the projected correlation function,

$$
w_{p}\left(r_{p}\right)=\int_{-R_{p}}^{R_{p}} \xi\left(\sqrt{r_{p}^{2}+r_{z}^{2}}\right) d r_{z}
$$

(Davis \& Peebles 1983). If $R_{p}=\infty$ and $\xi(r)$ is a power law, $\xi(r)=\left(r_{0} / r\right)^{\gamma}$, then Equation 1 integrates to $w_{p}\left(r_{p}\right) / r_{p}=\Gamma(1 / 2) \Gamma((\gamma-1) / 2) / \Gamma(\gamma / 2)\left(r_{0} / r_{p}\right)^{\gamma}$ (Peebles 1980). However, in a practical survey summing over the entire redshift extent of the survey leads to little increase in the signal and growing noise from fluctuations in the field density. Here we are focussed on the non-linear correlations, $\xi>1$ which suggests we use $R_{p}$ of several correlation lengths. A range of $R_{p}$ and the resulting errors in $r_{00}$ (see Equation 2 below) are displayed in Figure 2. We select $R_{p}=10 \mathrm{~h}^{-1} \mathrm{Mpc}$ as a conservative choice, having the largest (and most stable) errors. Similar results are obtained for any $10 h^{-1} \mathrm{Mpc} \leq$ $R_{p} \leq 50 h^{-1} \mathrm{Mpc}$, but with increasing statistical fluctuations, most notably inl the estimated errors of the fitted correlations.

The choice of a statistical estimator of the correlation function depends on the application. With point data the basic procedure is to determine the average number of neighboring galaxies within some projected radius and redshift distance $R_{p}$. We estimate $w_{p}\left(r_{p}\right)$ using the simplest estimator, $w_{p}=D D / D R-1$ (Peebles 1980), which is accurate for the nonlinear clustering examined here and faster than methods which include the RR sum. The DD and DR sums extend over all four patches in CNOC2 and the six slices of LCRS, so that patch to patch variations in the mean volume density become part of the correlation signal. The patch to patch variation is used to estimate the error.

Estimated projected correlation functions, in co-moving co-ordinates using $R_{p}=10 h^{-1} \mathrm{Mpc}$, are calculated for the LCRS galaxies bounded by redshifts $[0.033,0.15]$ and seven redshift bins for the CNOC2 data, $[0.10,0.20,0.26,0.35$, $0.40,0.45,0.55,0.65]$. The first CNOC2 redshift bin is the least populated with 185 galaxies and the fourth bin has the most with 602 galaxies. Relatively narrow redshift bins helps to reduce any problems associated with the detailed shape of the variation of $n(z)$ over the redshift range of the bin. To fit the data to a specified function requires error estimates at each point.

The correlations are fit to the projection of the power law correlation function, $\xi(r)=\left(r_{0} / r\right)^{\gamma}$ taking the fractional errors to be $1 / \sqrt{D D}$, the weighted counts in each $r_{p}$ bin. The fits use only the $0.16 \leq r_{p} \leq 5.0 h^{-1} \mathrm{Mpc}$ range where there are minimal complications from slit crowding and small correlations: : All results here are derived using co-moving co-ordinates, and normalized to a Hubble constant $H_{0}=100 \mathrm{~km} \mathrm{~s}^{-1} \mathrm{Mpc}^{-1}$. The results displayed in Figure 3 are derived assuming a background cosmology of $\Omega_{M}=0.2, \Omega_{\Lambda}=0$. 


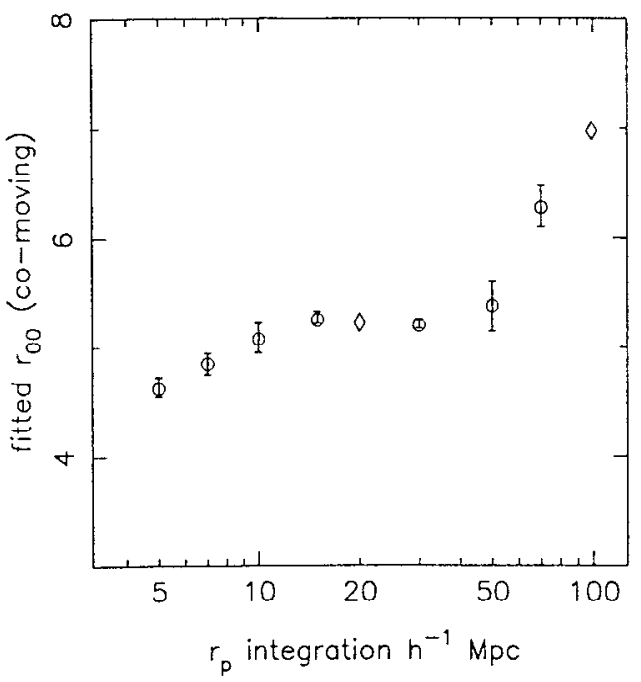

Figure 2. The derived $r_{00}$ (see Eq. 2) as a function of the integration length, $R_{p}$ used to define $w_{p}\left(r_{p}\right)$. The $90 \%$ confidence intervals are shown. The diamonds are plotted when $\chi^{2}$ exceeds the formal level for $90 \%$ confidence. This most likely arises because the variances used to calculate $\chi^{2}$ are estimated from the dispersion of the four patches, which will sometimes lead to erroncously small variances and hence large $\chi^{2}$ values.

\subsection{Errors of the Correlations}

A straightforward approach to random error estimates is to take advantage of our sample being distributed over a number of separate patches. In each patch we fit four for CNOC2 and six for LCRS. The other points are the results for the four individual patches CNOC2 fields (the individual LCRS slices are so similar that they are not displayed). The solid points give the result from the combined data, along with the estimated error.

As the size of a correlation survey grows there is a systematic increase in the derived correlation length. This is is illustrated in Figure 3. The straight mean of the CNOC2 $r_{0}$ is $3.2 h^{-1} \mathrm{Mpc}$, the median is $3.4 h^{-1} \mathrm{Mpc}$, and the mean of $r_{0}^{\gamma}$ is $3.5 h^{-1} \mathrm{Mpc}$, whereas the four patches analyzed together find $r_{0}$ is nearly $4.0 h^{-1} \mathrm{Mpc}$. This raises the question as to whether the correlations have converged within the current survey. We note that the expected variation from patch to patch for the given volumes with narrow redshift bins is about $45 \%$, which is consistent with the difference between a correlation length of 3.5 and $4.3 h^{-1} \mathrm{Mpc}$. In the combined sample with bigger bins we expect that there could be as much as $10 \%$ of the variance missing, which would boost the correlation lengths by another $5 \%$. 


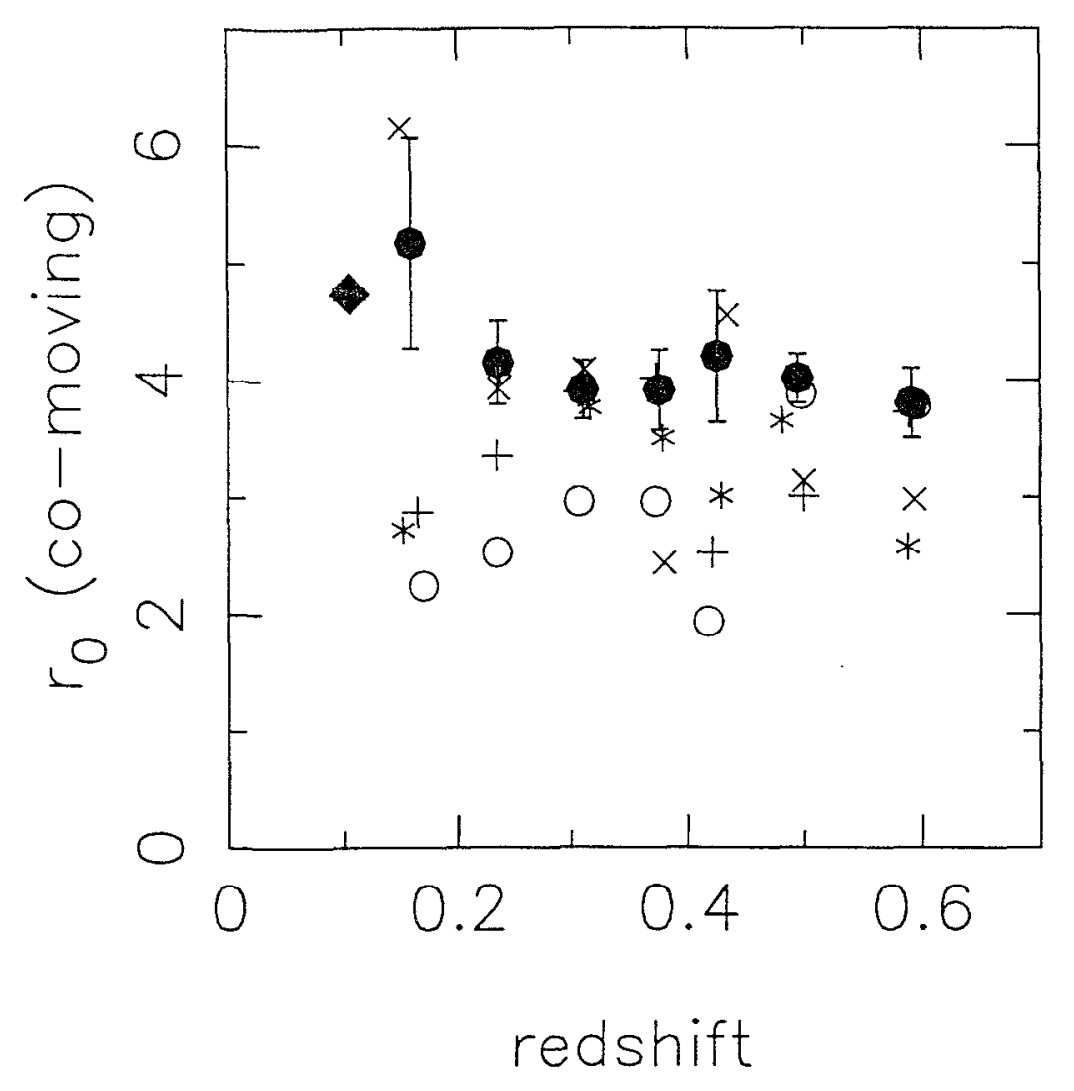

Figure 3. The correlation lengths (normalized to $\gamma=1.8$ ) as a function of redshift for $\Omega_{M}=0.2, \Omega_{\Lambda}=0$. The darkened diamond is from the LCRS with $q_{0}=0.1$. The errors are estimated from the variance of the four CNOC2 sky patches (shown with plus, asterisk, circle and cross symbols for the $0223,0920,1417$ and 2148 patches, respectively) and the six LCRS slices (not shown since they are small). The solid points are the result of the combined correlation analysis of the four fields and are in general always larger than the mean of the individual fields, since they include the field to field variance. 


\section{The Evolution of Galaxy Clustering}

The evolution of correlations is conveniently described with the "epsilon" model,

$$
r_{0}(z)=r_{00}(1+z)^{-(3+\epsilon-\gamma) / \gamma}
$$

where all lengths are in co-moving units. The parameter $\epsilon$ measures the rate of growth of the mean physical density of neighboring galaxies. If $\epsilon=0$ then there is no change in the physical density with redshift. Positive $\epsilon$ indicate a decline of clustered density with increasing redshift. The derived $r_{0}(z)$ along with the estimated errors, $\sigma\left(r_{0}(z)\right)$ are fit to the $\epsilon$ model with a formal $\chi^{2}$ which reports both the suitability of the model and the parameter confidence interval.

The correlation lengths for the CNOC2 and LCRS analyzed in precisely the same way for our standard $R_{p}=10 h^{-1} \mathrm{Mpc}$ and $Q=1$ are shown in Figure 3 . It is immediately clear that there is relatively little correlation evolution of this sample. It must be borne in mind that the sample is defined to be a similar set of galaxies with luminosities larger than about $L_{*}$, with luminosity evolution corrected, that accurately approximates a sample of fixed stellar mass with redshift. Samples which admit lower luminosity galaxies, or do not correct for evolution, or are selected in bluer pass-bands where evolutionary effects are larger and less certainly corrected, will all tend to have lower correlation amplitudes.

The $\epsilon$ model fits to the measured correlations are shown in Figure 4. The results are clearly a strong function of the cosmological world model. However, the strength of the correlations is truly impressive for any of the cosmologies. In the case of the low density flat model, the distances to the highest redshift galaxies are sufficiently large that the mean luminosity of the highest redshift galaxies is larger than those at lower redshifts. The best fit $\epsilon$ values are $0.02 \pm 0.23$ for $\Omega_{M}=0.2, \Omega_{\Lambda}=0$,

At redshifts beyond 0.1 or so, the choice of cosmological model has a substantial effect on the correlation estimates. Relative to high matter density cosmological models, low density and $\Lambda$ models tend to have larger distances and volumes, which causes the correlations to be enhanced. The LCRS data are analyzed only within the $q_{0}=0.1$ model. The correlations for three cosmologies, flat matter dominated, open, and low density $\Lambda$, are shown in Figure 3 . The evolution rates for the flat and flat low density models are $\epsilon=0.9 \pm 0.3$ and $\epsilon=-0.5 \pm 0.2$, respectively, with $r_{00} \simeq 5 h^{-1} \mathrm{Mpc}$ in all cases. These are marked with plus signs in Figure 4.

The effects of alternate values for the luminosity evolution are shown in Figure 4 with crosses indicating the results for $Q=0$ and $Q=2$, with the adopted value being $Q=1$. The absolute magnitude limit remains $M_{R}=-20$ $\mathrm{mag}$ in all cases. In the absence of any allowance for luminosity evolution, $Q=0$, galaxies of lower current epoch luminosity are increasingly included with redshift. Since lower luminosity galaxies are generally less correlated, that leads to an artificially large $\epsilon$, the effect over this redshift range being very roughly $\Delta \epsilon \approx 0.5 \Delta Q$. 


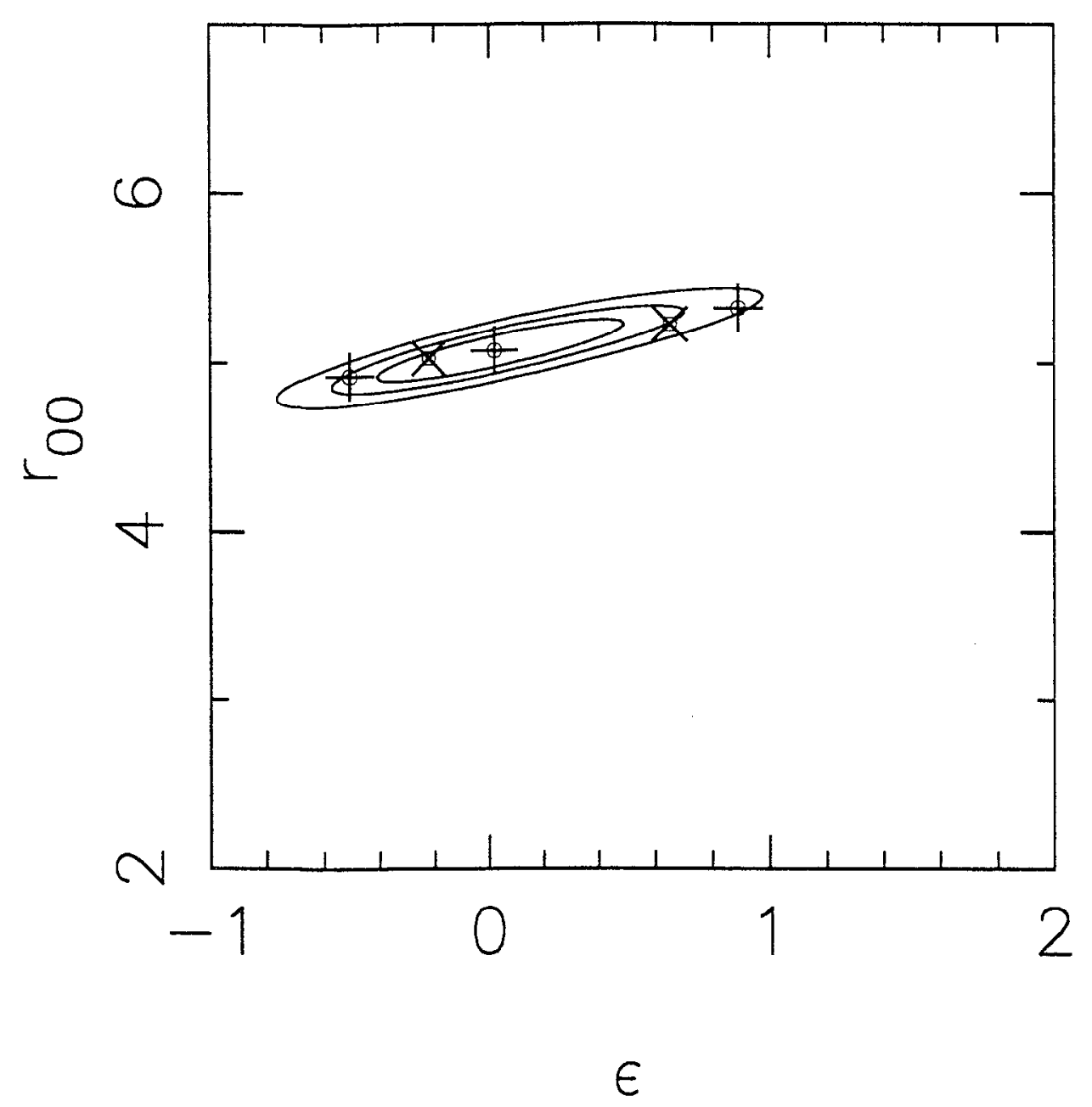

Figure 4. The $\chi^{2}$ confidence levels for fits to the $\epsilon$ model for the $\Omega_{M}=0.2, \Omega_{\Lambda}=0$ model. The contours are for $68 \%, 90$ and $99 \%$ confidence. The plus signs mark the results for $\Omega_{M}=1, \Omega_{\Lambda}=0$, peaking at $\epsilon \simeq 0.6$ and $\Omega_{M}=0.2, \Omega_{\Lambda}=0.8$ peaking at $\epsilon=-0.4$. The $x$ 's show the results for no evolution correction, $Q=0$, in which case $\epsilon=0.65$, and $Q=2$, evolution, where $\epsilon=-0.3$. 


\section{Conclusions}

The primary conclusion is that correlations evolve very weakly, if at all, with redshift. The main correlation results of this paper are contained in Figures 3. Out to redshift 0.65 the correlation evolution of high luminosity galaxies, $k$ and evolution corrected, can be described with the double power law model. $\xi(r \mid z)=\left(r_{00} / r\right)^{\gamma}(1+z)^{-(3+\epsilon)}$, with $r_{00}=5.08 \pm 0.08 h^{-1} \mathrm{Mpc}, \epsilon=0.02 \pm 0.23$ and $;=1.81 \pm 0.03$. There is no evidence in the current data for a change in the slope of the correlation function with redshift. The clustering results are consistent with galaxies being located in dark matter halos (Colin, Carlberg \& Couchman 1997, Baugh et al. 1998, Colin et al. 1999) but are also consistent with "light-traces-mass" in low density universes where the mass clustering evolves very slowly with redshift.

Environmental factors clearly play a major role in the development of galaxies, as most clearly seen in the morphology-density relation (Dressler 1980. Hashimoto et al. 1998). We have established here that the neighborhood density, at separations between 0.1 and about $5 h^{-1} \mathrm{Mpc}$, is essentially non-evolving with increasing redshift. The implication is that any evolution in the population cannot be attributed to a significant change in the environmental density of neighboring galaxies. In a flat low-density model there is a slight rise of the mean density of neighbors into the past. The high luminosity galaxies examined here do not as a population evolve much over the $z \leq 1$ range (Lilly et al. 1995, Cowie et al. 1996, Lin et al. 1999) which could be the outcome of either no change in the environment, or, these galaxies being relatively insensitive to environmental change.

Acknowledgments. This research was supported by NSERC and NRC of Canada. We thank the CFHT Corporation for support, and particularly the telescope operators for their enthusiastic and efficient control of the telescope. HL acknowledges support provided by NASA through Hubble Fellowship grant \#HF-01110.01-98A awarded by the Space Telescope Science Institute, which is operated by the Association of Universities for Research in Astronomy, Inc., for NASA under contract NAS 5-26555.

\section{References}

Baugh, C. M, Benson, A. J., Cole, S., Frenk, C. S., \& Lacey, C. G. 1998, MNRAS, submitted (astro-ph/9811222)

Broadhurst, T. J., Ellis, R. S., \& Shanks, T. 1988, MNRAS, 235, 827

Carlberg, R. G., Cowie, L. L., Songaila, A., \& Hu, E. M. 1997, ApJ, 483, 538

Carlberg, R. G., Yee, H. K. C., Morris, S. L., Lin, H., Sawicki, M., Wirth, G., Patton, D., Shepherd, C. W., Ellingson, E., Schade, D., Pritchet, C. J., \& Hartwick, F. D. A. 1998, Phil. Trans. Roy. Soc. Lond. A. 357, 167 •

Colin, P., Carlberg, R. G., \& Couchman, H. M. P. 1997, ApJ, 390, 1

Colin, P., Klypin, A., Kravtsov, A., \& Khokhlov, A. 1999, ApJ, submitted (astro-ph/9809202) 
Cowie. L. L., Songaila, A., Hu, E. M., \& Cohen, J. G. 1996, AJ, 112, 839

Davis, M. \& Peebles. P. J. E. 1983, ApJ, 267, 465

Davis, M.. Efstathiou, G., Frenk, C. S., \& White, S. D. M. 1985, ApJ, 292, 371

Dressler, A. 1980, ApJ, 236. 351

Efstathiou, G.. Davis, M., White, S. D. M., \& Frenk, C. S. 1985, ApJS, 57, 241

Ellis, R. S., Colless. M., Broddhurst, T., Heyl, J., \& Glazebrook, K. 1996, MNRAS, 280.235

Hashimoto, Y., Oemler, A., J., Lin, H., \& Tucher, D. L. 1998, ApJ, 499, 589

Jenkins, A., et al. 1998, ApJ, 499, 20

Kaiser, N. 1984, ApJ, 284, L9

LeFèrre, O, Hudon, D., Lilly, S. J. Crampton, D., IIammer, F., \& Tresse, L.1996, ApJ, 461, 534

Lilly, S. J., Tresse, L., Hammer, F., Crampton, D., \& Le Fevre, O. 1995, ApJ, 455,108

Lin, H., Yee., H. K. C., Carlberg, R. G., Morris, S. L., Sawicki, M., Patton, D., Wirth, G., \& Shepherd, C. W. 1998, ApJ, 518, 533

Loveday, J., Maddox, S. J., Efstathiou, G., \& Peterson, B. A. 1995, ApJ, 442 , 457

Jenkins, A., et al. 1999, ApJ, 521, L99

Peebles, P. J. E. 1980, Large Scale Structure of the Universe (Princeton University Press: Princeton)

Schectman, S. A., Landy, S. D., Oemler, A., Tucker, D. L., Lin, H., Kirshner, R. P., \& Schechter, P. I. 1996, ApJ, 470,172

Yee, II. K. C., Ellingsoin, E., \& Carlberg, R. G. 1996, ApJS, 102, 269

Yee, H. K. C., Sawicki, M., Carlberg, R. G., Lin, H., Morris, S. L., Patton, D. R., Wirth, G. D., Shepherd, C. W., Ellingson, E., Schade, D., \& Marzke, R. in the proceedings for JD 11 "Redshift Surveys in the 21st Century" at the 23rd IAU General Assembly, Kyoto, 1997 (astro-ph/9710356) 


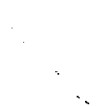


The Hy Redshift Universe

ASP Conference Series, Vol. 193, 1999

A. J. Bunker \& W. J. M. van Breugel, eds.

\title{
Clustering Properties of Lyman-Break Galaxies
}

\author{
M. Giavalisco \\ Space Telescope Science Institute, Baltimore, MD 21218
}

\begin{abstract}
The high efficiency of the Lyman-break technique has made clustcring of star-forming galaxies at high redshifts a powerful instrument to test fundamental ideas on galaxy formation. The discovery that Lyman break galaxies are strongly clustered in space, and that the clus tering strength is a function of their UV luminosity sets important empirical constraints on the yet largely unknown relationship betwecn dark matter and star formation activity. The former provides direct support to the notion that visible galaxies form in highly biased dark matter halos. The latter implies a tight correlation between the mass of the halos and the star-formation rates of the galaxies, suggcsting that star formation in high redshift systems is a process regulated more by the local gravity than by "external" random occurrences such as interactions.
\end{abstract}

\section{Introduction}

If gravity has assembled the cosmic structures, then a general result is that the clustering of virialized systems differs from that of the mass distribution, and depends on the properties of the systems themselves, with more massive systems being less abundant and more strongly clustered in space (Press \& Schechter 1974; Mo \& White 1996). The formalism of biased galaxy formation (Kaiser 1984; Bardeen et al. 1986) provides a simple description of the clustering of dark matter structures, and $N$-body simulations have confirmed the overall robustness of this treatment (Jing 1999). However, while modeling the dark matter poses relatively little problems, understanding the relationship between star formation and the properties of the halos has proved to be significantly more challenging, and has traditionally been a fertile source of free parameters, because the physics of star formation still remains theoretically poorly understood and empirically largely unconstrained (see e.g. Kennicutt 1998; Silk 1997).

The study of the clustering of forming galaxies at high redshifts, recently made possible by the large and complete samples obtained with the highly efficient Lyman-break technique (Steidel et al. 1998; Giavalisco et al. 1998; Adelberger et al. 1998), offers a new avenue of investigation. One of the advantages of this kind of studies is that optical observations probe the rest-frame far-UV spectrum, which only depends on the instantaneous rate of star formation (Kennicutt 1998) of the galaxies (modulo the obscuration by dust) and not on their previous evolutionary history, allowing one to probe in a relatively direct way the relationship between dark matter and the formation of stars on galactic scales. The idea is that if star formation is controlled by some simple property 

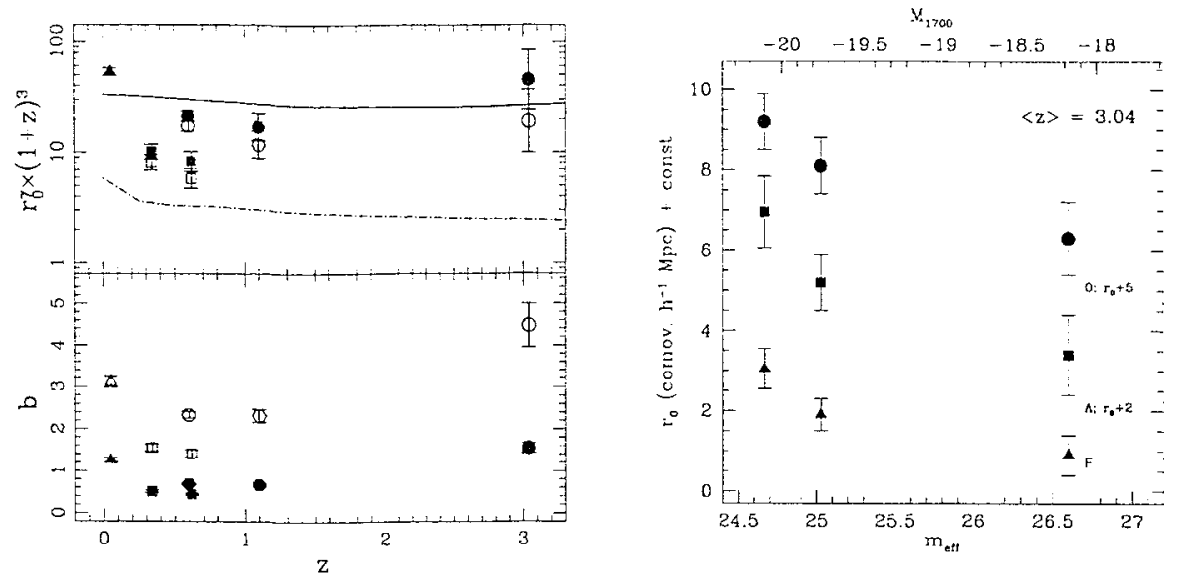

Figure 1. (a), Left. The upper panel shows the strength of galaxy clustering as a function of redshift. Filled symbols are for $(\Omega=0.2 ; \Lambda=$ 0 ), open symbols for $(\Omega=1 ; \Lambda=0)$. Triangles are APM data (Loveday et al. 1995), squares are CFRS data (Le Fèvre et al. 1996), hexagons Keck K-band data (Carlberg et al. 1997), and circles are the LBG data. The continuous and dashed lines are the expectations from the $\mathrm{CDM}$ theory with $\Gamma=0.25, \sigma_{8}=1.0$ and 0.5 for the two cosmological models, respectively. The bottom panel shows the corresponding linear bias as a function of redshifts for the same data sets. (b), Right. The dependence of the spatial correlation length of Lyman-break galaxies with their UV luminosity in the three adopted cosmological models (see text). For clarity, we added a constant to the values of $r_{0}$, as described in the figure.

of the hosting halos, one can hope to use the clustering of Lyman-break galaxies (LBGs) to learn something useful about the connection between the halos and the forming galaxies, and, possibly, the cosmology. If, on the other hand, star formation is intrinsically a highly stochastic process, with the UV luminosity of the galaxies being the product of the random vagaries interactions, merging or dust obscuration, then this hope will remain frustrated. In either case the data will be able to put constraints on the mechanisms of galaxy formation.

\section{The Clustering of Lyman-Break Galaxies}

One of the most interesting results that came from the studies of the properties of LBGs is that these sources are strongly clustered in space, with a spatial correlation length that directly compares to that of present-day galaxies (Steidel et al. 1998; Giavalisco et al. 1998; Adelberger et al. 1998). Figure 1a illustrates this point by comparing the clustering strength of LBGs to that of galaxies in the local and intermediate-redshift universe from various surveys.

This is very difficult to explain in terms of the clustering of the mass for any reasonable assumption on the background cosmology, if the normalization of the power spectrum (e.g. CDM) has to match the present-day distribution of 
galaxies and clusters (Eke et al. 1996). A more physically plausible explanation is that LBGs are highly biased tracers of the mass, and the values of the bias parameter $b$ that can be derived from the observed correlation function (assuming the a power spectrum) are indeed significantly larger than those found in the local universe (see the bottom panel of Figure 1a). The strong clustering of the LBGs is probably one of the most compelling evidence of the reality of galaxy biasing in general, if one is prepared to accept the gravitational instability as the primary physical mechanism responsible for the formation of cosmic structures.

The models of biased galaxy formation (Kaiser 1984; Bardeen et al. 1986; Mo \& White 1996) provide a physical mechanism for such a strong bias by associating the formation of visible galaxies to collapsing dark matter halos. An important and testable prediction in this case is that the LBGs follow the same clustering pattern expected for the halos, for example the same dependence of the correlation length (or the bias parameter) with the mass or the volume density of the objects, with more massive and less abundant objects being more strongly clustered (biased) in space. Very interestingly, this prediction seems confirmed by the observations, as we shall now discuss.

We studied the dependence of the clustering strength of LBGs with the volume density using three samples of different depth, two of them derived from our ground-based survey and one from the Hubble Deep Field. The brightest sample consists of all the galaxies from the deepest and largest fields that have spectroscopic redshifts. This sample has limiting magnitude $\mathcal{R} \lesssim 25.0$, and we used the count-in-cell technique to measure its correlation length (Adelberger et al. 1998). A fainter sample includes all the color selected LBGs with $\mathcal{R} \leq 25.5$ with and without spectroscopic redshifts, again coming from the deepest and largest fields of the survey. This sample takes advantage of the high efficiency of the color selection of $U$-band dropouts ( $\gtrsim 95 \%$ ), and because its redshift distribution $N(z)$ is very well known from about 500 redshifts, we could take advantage of its size, about twice that of the spectroscopic sample, and measure the spatial correlation function by de-projecting the angular one with the Limber transform (Giavalisco et al. 1998). The same technique has also been applied to the third and faintest sample, which has been extracted from the HDF catalog (including the North and South pointings), and has limiting magnitude $V_{606}=$ 27.0 (Giavalisco et al. 1999).

Figure $1 \mathrm{~b}$ plots the comoving correlation length $r_{0}$ of the three samples for three adopted cosmological models $[(\Omega=0.2, \Lambda=0),(\Omega=0.3, \Lambda=0.7),(\Omega=$ $1, \Lambda=0)$ ] as a function of both the effective magnitude and the corresponding absolute magnitude (in the $\Lambda$ cosmology), and shows the main result of this study, namely that the clustering strength of Lyman-break galaxies is a function of their UV luminosity at $\lambda \sim 1700 \AA$. The detection is significant, with the null hypothesis of no dependence of $r_{0}$ with $L_{U V}$ being rejected at the $\sim 99.23 \%$ confidence level. Remembering that the UV luminosity is a good tracer of the instantaneous rate of star formation of the galaxies (modulo the obscuration by dust), another way to state the result is that LBGs with more intense star formation are also more strongly clustered in space. 


\section{Lyman-Break Galaxies and Dark Matter Halos}

The fact that the clustering strength of the LBGs varies as a function of the UV luminosity has interesting consequences. In the scenario of gravitational instability it would imply that LBGs are tracers of dark matter halos and that there is a direct correlation between the mass of the halos and the star formation rate of the galaxies. To elucidate these points and to verify whether or not the the relationship between the clustering strength and volume density of these galaxies is consistent with the predictions of gravitational instability, we used the Press-Schechter formalism (PS, Press \& Schechter 1974) to compute the clustering strength (or, equivalently, bias), and mass function of dark matter halos.

The formalism reproduces very accurately the results from $\mathrm{N}$-body simulations for relatively massive halos, namely $M \lesssim 0.1 M^{\star}$, but it underestimates the clustering strength and overestimates the abundances of smaller halos. However, corrections have been recently proposed (e.g. Jing 1999) that improve the agreement with the simulations, and the formalism can be used to provide a relatively accurate description of the clustering properties of halos (relative to the simulations, that is) over the mass range relevant to the formation of galaxies and larger structures. We adopted the implementation discussed by Mo \& White (1996) with the corrections by Jing (1999). We used a CDM power spectrum with spectral index $n=1$ and transfer function by Bardeen et al. (1986), and normalized its amplitude at $z=0$ to that of clusters (Eke et al. 1996), using $\sigma_{8}=1.0,0.9$ and 0.5 in our three adopted cosmologies, respectively.

Ideally, to compare the predicted properties of dark matter halos to those of the LBGs one would like to study the bias (which we will adopt from now on as a measure of the clustering strength) as a function of the mass. Unfortunately, the masses of LBGs are not yet arailable from the observations and, furthermore, the only galaxies that are accessible to our investigation are those whose UV SED satisfies the selection criteria of the samples. Therefore, one cannot directly compare halo properties to galaxy properties, but one needs first to model the relationship between the mass of the halos and the net UV luminosity (i.e. including the effects of the dust obscuration) of the LBGs and predict the clustering strength and the luminosity function of LBGs (we will refer to these as the "model LBGs").

The problem with the described approach is that the mechanisms of star formation and the amount of dust obscuration in nascent galaxies are too difficult to model in a realistic way. However, we can constrain this uncertainty by an appropriate choice of the observables. The goal is to find a model which, combined with the PS mass function, can simultaneously reproduce the clustering properties and the luminosity function of LBGs. To do this at best, on the one hand one needs observables that have a pronounced dependence on the mass-UV luminosity relationship to maximize the sensitivity. On the other hand one also wants other observable that depends only weakly on this relationship in order to minimize the effect of the uncertainties of the model and carry out a meaningful comparison with the data.

Observables of the first type include the luminosity function and the relationship between the mean bias $\langle b\rangle$ of a sample of LBGs and its limiting luminosity $L_{l}$. Given the halo mass function, these observables constrain the 
mass-UV relationship by simultaneously reproducing the luminosity distribution, the abundances and the clustering strength of the LBGs.

An observable of the second type is the function that links together the mean bias of a sample and its volume density (we shall refer to it as the clustering segregation). If there is little scatter of UV luminosity among galaxies hosted in halos of equal mass then the shape of the clustering segregation of the model LBGs is very close to that of the halos and independent on the shape of the massUV luminosity relationship. Of course, the UV luminosity of LBGs hosted in halos of given mass (and hence of given volume density and bias) does depend on the mass-UV luminosity relationship. The latter sets the flux limit of the sample that one needs to reach in order to observe those halos, but it does not affect the shape of the clustering segregation.

Let's now discuss the adopted model for the mass-UV luminosity relationship. We can imagine that the UV luminosity $L_{U V}$ of $L B G$ s hosted in halos of mass $M_{h}$ is a random variable, whose mean and variance depend on $M_{h}$ and are described by some continuous function $\left\langle L_{U V}\right\rangle=\mu\left(M_{h}\right)$ and $\sigma_{U V}^{2}=s\left(M_{h}\right)$. We have modeled $L_{U V}$ as the product of a random variable $A$ with the function. $\mu\left(M_{h}\right)$ :

$$
L_{U V}=A \times \mu\left(M_{h}\right),
$$

where the mean value of $A$ is, by definition, equal to 1 . For simplicity, we have assumed the distribution function of $\log (. A)$ to be gaussian and considered the cases of both a constant variance $\sigma_{U V}^{2}=\sigma_{0}^{2}$, and of a variance that depends on $M_{h}$ as

$$
\sigma_{U V}^{2}=\frac{\sigma_{0}^{2}}{1+\left(M / M_{\sigma}\right)}
$$

(in the logarithm space). We have also used a power law model for $<L_{U V}>$ :

$$
\phi\left(M_{h}\right)=\epsilon \times M_{h}^{\alpha} .
$$

Given the PS halo mass function $n\left(M_{h}\right)$, it is straightforward to compute the expected observables for the model LBGs. The mean bias of a flux-limited sample of LBGs with $L \leq L_{0}$ is given by (Catclan et al. 1998):

$$
<b_{e}\left(L_{0}\right)>=\frac{\int_{-\infty}^{+\infty} p\left(M_{h} \mid L_{0}, \sigma_{U V}^{2}\right) b\left(M_{h}\right) d M_{h}}{\int_{-\infty}^{+\infty} p\left(M_{h} \mid L_{0}, \sigma_{U V}^{2}\right) d M_{h}}
$$

where $p\left(M_{h} \mid L_{0}, \sigma_{U V}^{2}\right)$ is the probability that a halo with mass $M_{h}$ is included in the sample. The luminosity function is given by

$$
n\left(L_{U V}\right) d L_{U V}=\iint d M_{h} d A n\left(M_{h}\right) \times f(A),
$$

where $f(A)$ is the distribution function of $A$ and the double integral is extended over the domain of $M_{h}$ and $A$ such that $L_{U V}=A \phi\left(M_{h}\right)$.

We performed a multi-dimensional fit to the data, minimizing the sum of two $\chi^{2}$, one relative to the $\langle b\rangle$ vs $L_{U V}$ relationship and the other to the luminosity function. The results are shown in the top and middle panels of Figure 2 and Figure 3a, for the three adopted cosmological models. 

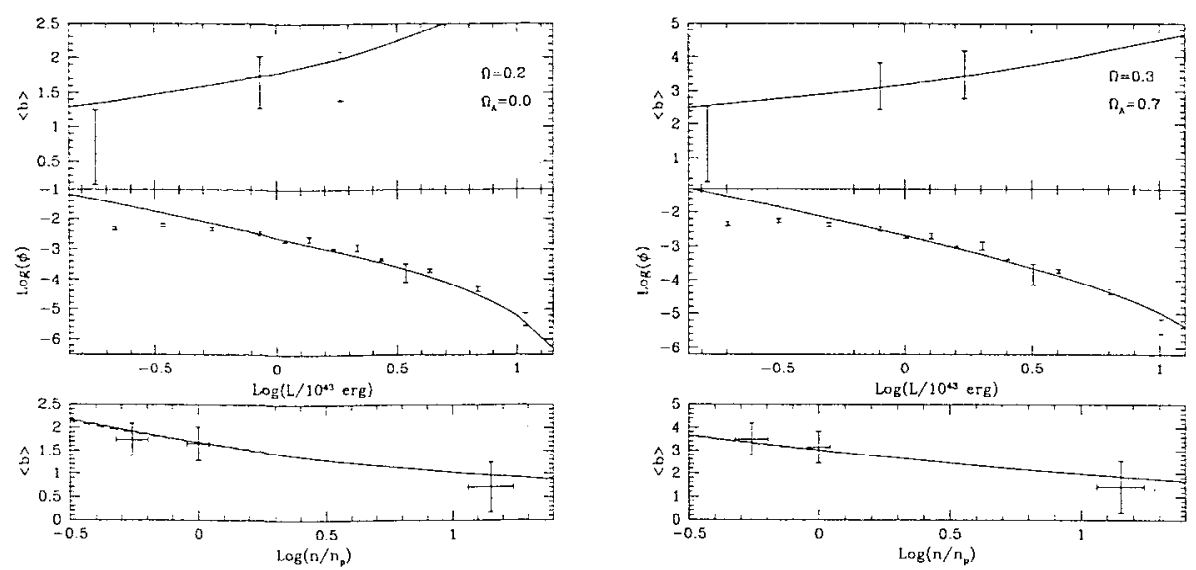

Figure 2. (Top panels). The $\langle b\rangle$ vs $L_{U V}$ relationship. The curve is the best fit to the data. (Middle panels). The luminosity function. The curve is the best fit to the data. (Bottom panels) The clustering segregation of observed $z \sim 3$ LBGs (data points), model LBGs (continuous line) and halos (dashed line). The dotted lines above and below the continuous line in the plot to the right show the clustering segregation predicted at $z=4$ and $z=2.5$, respectively.
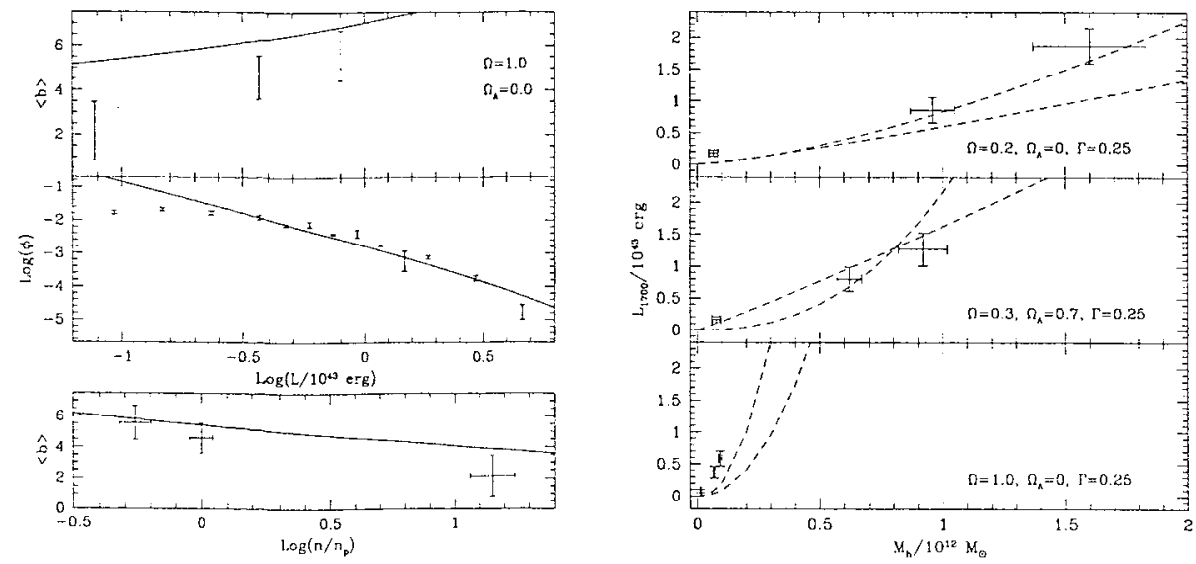

Figure 3. (Left). As in Figure 2. (Right). The mass-UV lumi-: nosity relationship derived from the fit (dashed curves) and from the clustering segregation (data points). See text for explanation. 
The random errors on the fitted four parameters of the model, i.e. $\epsilon$, a. $\sigma_{0}^{2}$, and $M_{\sigma}$ obtained from the bootstrap technique are typically smaller than the variations that correspond to adopting a fixed or a variable variance, or to different choices of the relative weights of the $\chi^{2}$ of the two fitted functions. The curves in Figure $3 \mathrm{~b}$ shows extreme cases of the derived mass-UV luminosity relationship in the three adopted cosmologies, together with the the points implied by the clustering segregation (see later). This suggests that the choice of the model is far from being optimal, although the overall shape of the luminosity function and $\langle b\rangle$ vs $L_{U V}$ relationship are reproduced (note that some of the data themselves might be affected by systematic errors). However, in all cases the fits returned a small value for the variance, with $\sigma_{0}^{2} \lesssim 0.5$ in every fit (corresponding to $\approx 50 \%$ of the value of the luminosity).

With the variance in the estimated range, the chistering segregation of the model LBGs is virtually identical to that of the halos, as it can be seen in the top panels of Figure 2 and Figure 3a, where they are both shown together with the data points of the observed LBGs. More importantly, it does not depend on the assumptions on the shape of the mass-UV luminosity relationship. The agreement between the data and the model is surprisingly good. Note that in this case one can plot the luminosity of the galaxies versus the mass of the halos that have equal bias and volume density to compare with the fitted models, which we have done in Figure $3 \mathrm{~b}$.

\section{What does all this mean?}

Several interesting conclusions can be drawn from the analysis above. First. the Press-Schechter mass function and the adopted model for the mass-UV luminosity relationship are capable to simultaneously reproduce in broad terms the overall features of the clustering of LBGs, namely the shape of the $\langle b\rangle$ vs $L_{l}$ relationship, and thcir UV luminosity function. The agreement between the models and the data appears to be better in the open and $\Lambda$-flat cosmologies than in the Einstcin de Sittcr one, where the $\langle b\rangle$ vs $L_{l}$ function scems poorly modeled, although the discrepancy is not statistically significant, given the still large unccrtainties on the clustcring measures. At this stage it is best not to read too much into this, since it is impossible to discriminate intrinsic effects from the inadequacies of the model and the still largc errors.

Second, fitting the model to the data consistently returns a relatively small variance in the mass-UV luminosity relationship, in all cases smaller than $\approx 50 \%$ of the value of the average. This shows that halo masses and star formation rates are tightly correlated, implying that the star formation rate is controlled to a large extent by the halo mass. The overall magnitude of the variance does not depend much whether a variable variance with mass or a constant one is used. When the variable one is used, the fit returns a "half-variance mass" $M_{\sigma}$ equal to $\sim 10^{12}, 0.5 \times 10^{12}$ and $10^{11}$ in the open, $\Lambda$ and EdS model, respectively. This is consistent with the idea that when the local gravity becomes relatively small (smaller than the mass of a present-day $L^{*}$ galaxy), the external environment (interactions, merging, etc.) starts to have larger a effect on the star formation activity of the galaxies. The picture that seems to emerge is that star formation 
in LBGs is an ordered process, with the mass of the halos (and hence local gravity) as one of the primary parameters that control the star formation rate.

As Figure 3b shows, the mass scale of the halos implied by the clustering segregation varies with the cosmology. Bright LBGs have masses $M \gtrsim 10^{12} \mathrm{M}_{\odot}$ in the open and $\Lambda$-flat cosmology - approximately the mass of present-day bright galaxies -, while they are an order of magnitude smaller in the Einsteinde Sitter model. Thus, although the results on the clustering segregation are largely degenerate with the cosmology, it is clear that measuring the mass of the LBGs will break such degeneracy. It is interesting to note that regardless of the cosmology, however, the mass implied for the LBGs of the HDF sample are always small, $\approx 10^{10} \mathrm{M}_{\odot}$, suggesting that at magnitudes $\mathcal{R} \sim 27$ LBGs are mostly associated with small, sub-massive systems.

To some extent, it is intriguing that the clustering segregation of the halos in the chosen CDM cosmology seems to agree so well with that observed for the LBGs. The agreement seems better in the open and $A$ cosmologies than in the Einstein-de Sitter one, although a formal $\chi^{2}$ test shows that the difference is not statistically significant. Better clustering data will allow us to test if these differences are significant and, in particular, test the effects of the various assumptions on the power spectrum. Also, knowing the $\langle b\rangle$ vs. $L_{l}$ function with higher accuracy will result, in combination with the luminosity function, in better constraints on the mass-UV luminosity relationship. More importantly, larger samples are much needed to quantify the effects of systematic errors stemming from the largely unquantified cosmic variance in the measure of the correlation function, particularly the HDF one. Deep ground-based surveys for LBGs from 8-m telescopes will be very effective in providing HDF-like samples over much larger areas.

In summary, the observed scaling relations of the clustering strength, abundances and UV luminosity of LBGs agree well with the predictions of the gravitational instability theory and biased galaxy formation if the CDM spectrum of perturbations is used. This implies that LBGs flag the sites of massive dark matter halos and that their mass is tightly correlated to the the star formation rates. The implication is that local gravity in these system is a primary parameter that controls the star formation activity. The important point is that this conclusions depend weekly on the assumptions about the still poorly known physics of star formation in young galaxies and its relationship with the properties of the dark matter. Studying large scale structure by using galaxies ultimately requires to understand galaxies themselves, as the recent extension of these studies to high redshifts has dramatically highlighted, for example, when the high bias of the LBGs was observed. This once more reiterates that the problem of structure formation and galaxy formation are intimately coupled and very difficult to separate. Interestingly, however, while the clustering of high redshift galaxies (or at least what we know of it now) has turned out not to constitute the simple and powerful cosmological test that was initially hoped, it has given us a much deeper insight into the mechanisms of galaxy formation and shown that our basic ideas about the relationship between visible galaxies and dark halos are fundamentally correct. 


\section{References}

Adelberger, K. L., Steidel, C. C., Giavalisco, M., Dickinson, M. E., Pettini, M., \& Kellogg, M. 1998, ApJ, 505, 18, A98

Bardeen, J. M., Bond, J. R., Kaiser, N., Szalay, A. S. 1986, ApJ, 304, 15, BBKS

Catelan, P., Matarrese, S.. \& Porciani, C. 1998, ApJ, 502, L1

Eke, V. R., Cole, S., \& Frenk, C. S. 1996, MNRAS, 282, 263

Giavalisco, M., Steidel, C. C., Adelberger, K. L., Dichinson, M. E., Pettini, M., \& Kellogg, M. 1998, ApJ, 503, 543, G98

Giaralisco, M., Adelberger, K. L., Steidel, C. C., Dickinson, M. E., \& Pettini, M. 1999, submitted to ApJ

Kaiser, N. 1984, Ap.J, 284, L1

Kennicutt, R. C. 1998, ARA\&A, 36, 189

Jing, Y. P. 1999, ApJ, irı press, astro-ph/

Mo, H. J., \& White, S. D. M. 1996, MNRAS, 282, 347

Peacock, J. A. 1997, MNRAS, 284, 885

Press, W. H., \& Schechter, P. 1974, ApJ, 187, 425

Silk, J. 1997, ApJ, 481, 703

Steidel, C. C., Adelberger, K. L., Dickinson, M. E., Giavalisco, M., Pettini, M., \& Kellogg, M. 1998, ApJ, 492, 428, S98

Steidel, C. C., Adelberger, K. L., Giaralisco, M., Dickinson, M. E., \& Pettini, M. 1999, ApJ, 519, 43 
The Hy Redshift Universe

ASP Conference Series. Vul. 193, 1999

A. J. Bunker \& W. J. M. van Breugel, eds.

\title{
High-Redshift Quasars as Probes of Galaxy and Cluster Formation
}

\author{
S. G. Djorgorski
}

Palomar Observatory, Caltech, Pasadena, CA 91125, USA

\begin{abstract}
Quasars at large redshifts provide a powerful probe of structure formation in the early universe. Several arguments suggest that the formation of ellipticals and massive bulges may have involved an early quasar phase. At very large redshifts, such structures are likely to be found at the highest peaks of the density field, and would thus be highly biased tracers: the earliest (massive) galaxy formation may have occurred in the cores of future rich clusters. Preliminary results from our search for clustered protogalaxies around quasars at $z>4$ support this idea. Quasars at even larger redshifts may be an important contributor to the reionisation of the universe, and signposts of the earliest galaxy and cluster formation.
\end{abstract}

\section{Introduction}

Hy Spinrad always hated quasars (Spinrad 1979). In those paleolithic days (i.e., before 1987 or so) it was not yet obvious that a powerful quasar lurks in the heart of every one of Hy's beloved radio galaxies, but in some sense this does not really matter: in the work of the Spinrad School of Observational Cosmology, AGN have been used simply as means to find stellar populations at large redshifts, in order to probe their formation and evolution. This is in principle a viable and sound approach.

In the simplest view, the very existence of luminous quasars at large redshifts suggests the existence of their (massive?) host galaxies, at least in the minds of a vast majority of astronomers today. At $z>4$, this has some very interesting and non-trivial implications for our understanding of galaxy and structure formation (Turner 1991).

At a slightly more complex level, the observed history of the comoving number density of quasars may be indicative of the history of galaxy formation and evolution: the same hind of processes, i.e., dissipative mergers and tidal interactions, may be fueling both bursts of star formation and AGN activity. The peak seen in the comoving number density of quasars around $z \sim 2$ or 3 (Schmidt, Schneider \& Gunn 1995) can then be interpreted in this context: the ostensible decline at high redshifts may be indicative of the initial assembly and growth of quasar central engines and their host galaxies; whereas the decline at lower redshifts may be indicative of the decrease in fueling, as galaxies are carried apart by the universal expansion, as many of the smaller pieces are being consumed, and as the gas is being converted into stars. Qualitatively similar 
predictions are made by virtually all models of hierarchical structure formation (see, e.g., Cataneo 1999, or Kauffmann \& Haehnelt 1999).

Due to their brightness, quasars are much easier to find (per unit telescope time) than galaxies at comparable redshifts. It then makes sense to use quasars as probes, or at least as pointers of sites of galaxy formation.

Quasars have been used very effectively as probes of the intergalactic medium, and indirectly of galaxy formation, through the studies of absorption line systems. A vast literature exists on this subject, which is beyond the scope of this review; for good summaries, see, e.g., Rees (1998a) or Rauch (1998).

A good review of the searches for quasars and related topics was given by Hartwick \& Schade (1990). Osmer (1999) provides a modern update. Some of the issues covered in this review have been described by Djorgovski (1998) and Djorgovski et al. (1999).

\section{Quasars and Galaxy Formation}

Possibly the most direct evidence for a close relation between quasars and galaxy formation is the remarkable correlation between the masses of central black holes $(\mathrm{MBH})$ in nearby galaxies, and the luminosities ( $\sim$ masses) of their old, metalrich stellar populations, a.k.a. bulges (Kormendy \& Richstone 1995, Magorrian et al. 1998), with MBH's containing on average $~ 0.6 \%$ of the bulge stellar mass. The most natural explanation for this correlation is that both MBH's and the stellar populations are generated through a parallel set of processes, i.e., dissipative merging and assembly at large redshifts. Quiescent MBH's are evidently common among the normal galaxies at $z \sim 0$, and had to originate at some point: as they grow by accretion, their formation is the quasar activity. Quasars may thus be a common phase of the early formation of ellipticals and massive bulges.

Quasar demographics support this idea. Small \& Blandford (1992), Chokshi \& Turner (1992), and Haehnelt \& Rees (1993) all conclude that an average $L_{\times}$-ish galaxy today should contain an MBH with $M_{\bullet} \sim 10^{7} M_{\odot}$ or so. These estimates (essentially integrating the known $A G N$ radiation over the past history of the universe) are fully consistent with the actual census of $\mathrm{MBH}$ (quasar remnants) in nearby galaxies.

Two other pieces of fossil evidence link the high- $z$ quasars with the formation of old, metal-rich stellar populations. First, the analysis of metallicities in QSO BEL regions indicates super-solar abundances (up to $Z_{Q} \sim 10 Z_{\odot}$ !) in quasars at $z>4$ (Hamann \& Ferland 1993, 1999; Matteucci \& Padovani 1993). The only places we know such abundances to occur are the nuclei of giant elliptical galaxies. Furthermore, abundance patterns in the intracluster xray gas at low redshifts are suggestive of an early, rapid star formation phase in protoclusters populated by young ellipticals (Loewenstein \& Mushotzky 1996).

A nearly simultaneous formation of quasars and their host galaxies, or at least ellipticals and bulges, is consistent with all of these observations, and it fits naturally in the general picture of hierarchical galaxy and structure formation via dissipative merging (see, e.g., Norman \& Scoville 1988, Sanders et al. 1988, Carlberg 1990, Hernquist \& Mihos 1995, Mihos \& Hernquist 1996, Monaco et al. 1999, Franceschini et. al. 1999, etc.). 
An extreme case of this idea is that quasars are completely reducible to ultraluminous starbursts, as advocated for many years by Terlevich and collaborators (see, e.g., Terlevich \& Boyle 1993, and references therein). Most other authors disagree with such a view (cf. Heckman 1991, or Williams \& Perry 1994), but (nearly) simultaneous manifestations of both ultraluminous starbursts and AGN, perhaps with comparable energetics, are clearly allowed by the data. It is thus also possible that the early AGN can have a profound impact on their still forming hosts, through the input of energy and momentum (Theuchi \& Norman 1991, Haehnelt et al. 1998).

\section{Quasar (Proto) Clustering and Biased Galaxy Formation}

Producing sufficient numbers of massive host galaxies needed to accommodate the observed populations of quasars at $z>4$, say, is not easy for most hierarchical models: such massive halos should be rare, and associated on average with $\sim 4$ to 5 - $\sigma$ pealss of the primordial density field (Efstathiou \& Rees 1988, Cole \& Kaiser 1989, Nusser \& Silk 1993). It is a generic prediction that for essentially every model of structure formation such high density peaks should be strongly clustered (Faiser 1984). This is a purely geometrical effect, independent of any messy astrophysical details of galaxy formation, and thus it is a fairly robust prediction: the formation of the first galaxies (some of which may be the hosts of high- $z$ quasars) and of the primordial large-scale structure should be strongly coupled.

Quasars provide a potentially useful probe of large-scale structure out to very high redshifts. The pre-1990 work has been reviewed by Hartwick \& Schade (1990). A number of quasar pairs on tens to hundreds of comoving kpc scales has been seen (Djorgovski 1991, Kochanek et al. 1999), and some larger groupings on scales reaching $\sim 100 \mathrm{Mpc}$ (Crampton et al. 1989, Clowes \& Campusano 1991), but all in heterogeneous data sets. Analysis of some more complete samples did show a clustering signal (e.g., Iovino \& Shaver 1988, Boyle et al. 1998). The overall conclusion is that quasar clustering has been detected, but that its strength decreases from $z \sim 0$ out to $\tilde{z} \sim 2$, the peak of the quasar era, presumably reflecting the linear growth of the large-scale structure. However, if quasars are biased tracers of structure formation at even higher redshifts, associated with very massive peaks of the primordial density field, this trend should reverse and the clustering strength should again start increasing towards the larger look-back times.

The first hints of such an effect were provided by the three few-Mpc quasar pairs found in the statistically complete survey by Schneider et al. (1994), as pointed out by Djorgovshi et al. (1993) and Djorgovski (1996), and subsequently confirmed by more detailed analysis by Kundic (1997) and Stephens et al. (1997). A deeper survey for more such pairs by Kennefick et al. (1996) did not find any more, presumably due to a limited volume coverage. La Franca et al. (1998) find a turn-up in the clustering strength of quasars even at redshifts as low as $z \sim 2$. It would be very important to check these results with new, large, complete samples of quasars over a wide baseline in redshift.

More recently, observations of large numbers of "field" galaxies at $z \sim 3-3.5$ by Steidel et al. (1998) identified redshift space structures which are almost 
certainly the manifestation of biasing. However, the effect (the bias) should be even stronger at higher redshifts, and most of the earliest massive galaxies should be strongly clustered. A search for protoclusters around known high-z objects such as quasars thus provides an important test of our basic ideas about the biased galaxy formation.

Intriguingly, there is a hint of a possible superclustering of quasars at $z>4$, on scales $\sim 100 h^{-1}$ comoving Mpc (cf. Djorgovski 1998). The effect is clearly present in the DPOSS sample (which is complete, but still with a patchy coverage on the sky), and in a more extended, but heterogeneous sample of all QSOs at $z>4$ reported to date. The apparent clustering in the complete sample may be an artifact of a variable depth of the survey, which we will be able to check in a near future. Or, it could be due to patchy gravitational lensing magnification of the high-z quasars by the foreground large-scale structure; again, we will be able to test this hypothesis using the DPOSS galaxy counts. But it could also represent real clustering of high-density peaks in the early universe, only $\sim 0.5-1$ Gyr after the recombination. The observed scale of the clustering is intriguing: it is comparable to that corresponding to the first Doppler peak seen in CMBR fluctuations, and to the preferred scales seen in some redshift surveys (e.g., Broadhurst et al. 1990; Landy et al. 1996). More data are needed to check on this remarkable result.

\section{Quasar-Marked Protoclusters at $\mathrm{z}>4$ ?}

Any single search method for high-z protogalaxies (PGs) has its own biases, and formative histories of galaxies in different environments may vary substantially. For example, galaxies in rich clusters are likely to start forming earlier than in the general field, and studies of galaxy formation in the field may have missed possible rare active spots associated with rich protoclusters.

We are conducting a systematic search for clustered PGs, by using quasars at $z>4$ as markers of the early galaxy formation sites (ostensibly protocluster cores). The quasars themselves are selected from the DPOSS survey (Djorgovski et al. 1999, and in prep.; Kennefick et al. 1995). They are purely incidental to this search: they are simply used as beacons, pointing towards the possible sites of early, massive galaxy formation.

The first galaxy discovered at $z>3$ was a quasar companion (Djorgovski et al. 1985, 1987). A Ly $\alpha$ galaxy and a dusty companion of BR 1202-0725 at $z=4.695$ have been discovered by several groups (Djorgovski 1995, Hu et al. 1996, Petitjean et al. 1996), and a dusty companion object has been found in the same field (Omont et al. 1995, Ohta et al. 1995). Hu \& McMahon (1996) also found two companion galaxies in the field of BR 2237-0607 at $z=4.55$.

We have searches to various degrees of completeness in about twenty QSO fields so far (Djorgovski 1998; Djorgovski et al., in prep.). Companion galaxies have been found in virtually every case, despite very incomplete coverage. They are typically located anywhere between a few arcsec to tens of arcsec from the quasars, i.e., on scales $\sim 100+$ comoving kpc. We also select candidate PGs by using deep $B R I$ imaging over a field of view of several arcmin, probing $\sim 10$ comoving Mpc ( cluster size) projected scales. This is a straightforward extension of the method employed so successfully to find the quasars themselves 


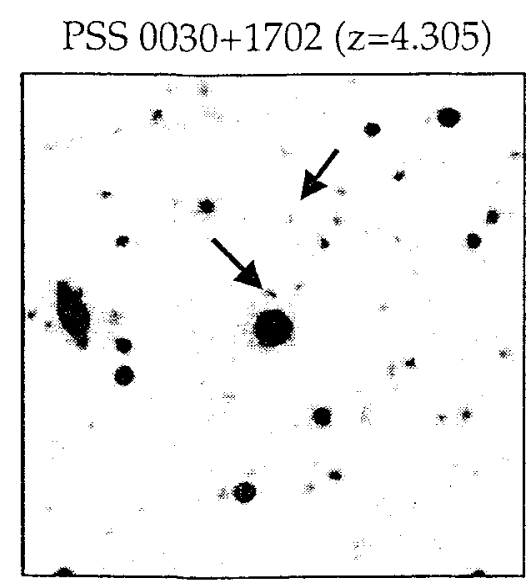

PSS $0248+1802(z=4.465)$

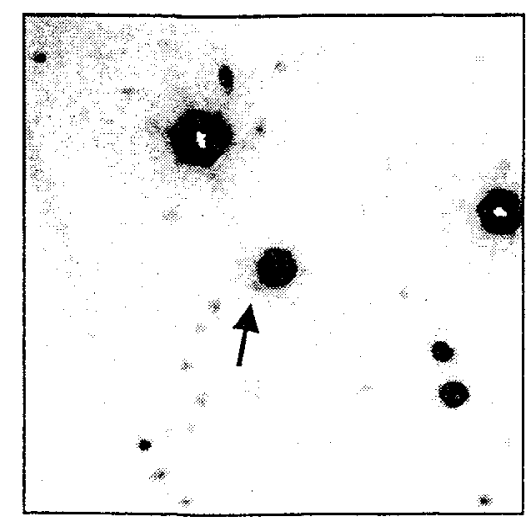

PSS 0117+1552 ( $z=4.275)$

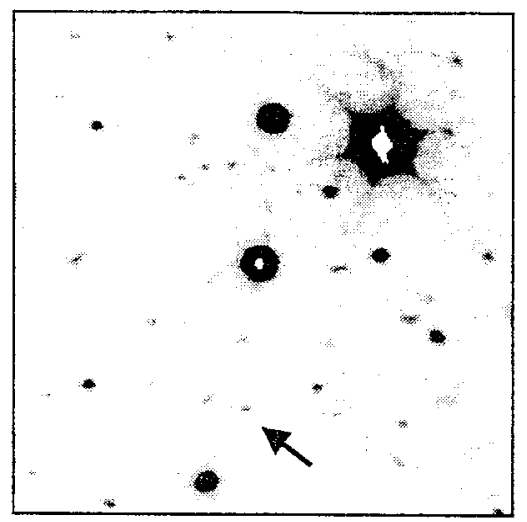

PSS $1721+3256(z=4.031)$

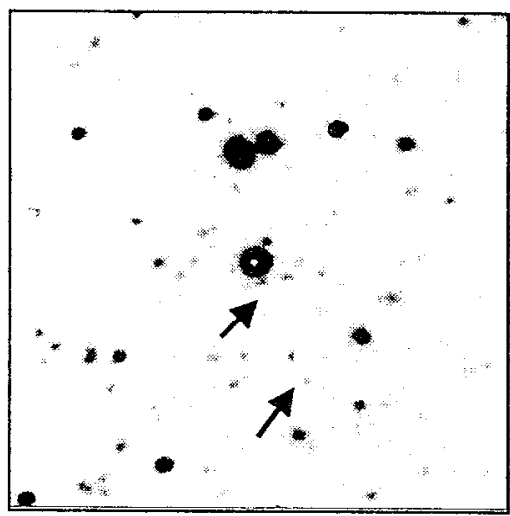

Figure 1. Examples of clustered companion protogalaxies in the fields of 4 DPOSS quasars at $z>4$. These are deep, $R$-band Keck images centered on the quasars. The fields shown are 54 arcsec square. Some of the spectroscopically confirmed companions are labeled with the arrows.

at $z>4$ (at these redshifts, the continuum drop is dominated by the Ly $\alpha$ forest, rather than the Lyman break, which is used to select galaxies at $z \sim 2-3.5$ ). The candidates are followed up by multislit spectroscopy at the Keck, which is still in progress as of this writing.

As of the mid-1999, abont two dozen companion galaxies have been confirmed spectroscopically. Their typical magnitudes are $R \sim 25^{\mathrm{m}}$, implying continuum luminosities $L \leq L_{*}$. The Iy $\alpha$ line emission is relatively weak, with typical restframe equivalent widths $\sim 20-30 \AA$, an order of magnitude lower than what is seen in quasars and powerful radio galaxies, but perfectly reasonable for the objects powered by star formation. There are no high-ionization lines in their spectra, and no signs of AGN. The SFR inferred both from the Ly $\alpha$ line, and the UV continumm flux is typically $\sim 5-10 M_{\odot} / \mathrm{yr}$, not corrected for the extinction, and thus it conld easily be a factor of 5 to 10 times higher. 
Overall, the intrinsic properties of these quasar companion galaxies are very similar to those of the Lyman-break selected population at $z \sim 3-4$, except of course for their special environments and somewhat higher look-back times.

There is a hint of a trend that the objects closer to the quasars have stronger I $\alpha$ a line emission, as it may be expected due to the QSO ionization field. In addition to these galaxies where we actually detect (presumably starlight) continuum, pure Lya emission line nebulae are found within $\sim 2-3$ arcsec for several of the quasars, with no detectable continuum at all. The Ly $\alpha$ fluxes are exactly what may be expected from photoionization by the QSO, with typical $I_{\text {Lyo }} \sim$ a few $\times 10^{43} \mathrm{erg} / \mathrm{s}$. They may represent ionized parts of still gaseous protogalaxy hosts of the quasars. We can thus see and distinguish both the objects powered by the neighboring QSO, and "normal" PGs in their vicinity.

The median projected separations of these objects from the quasars are $\sim$ a few $\times 100 h^{-1}$ comoving $\mathrm{kpc}$, an order of magnitude less than the comoving r.m.s. separation of $L_{*}$ galaxies today, but comparable to that in the rich cluster cores. The frequency of QSO companion galaxies at $z>4$ also appears to be an order of magnitude higher than in the comparable QSO samples at $z \sim 2-3$, the peak of the QSO era and the ostensible peak merging epoch. However, interaction and merging rates are likely to be high in the densest regions at high redshifts, which would naturally account for the propensity of some of these early PGs to undergo a quasar phase, and to have close companions.

The implied average star formation density rate in these regions is some 2 or 3 orders of magnitude higher than expected from the limits estimated for these redshifts by Madau et al. (1996) for field galaxies, and 1 or 2 orders of magnitude higher than the measurements by Steidel et al. at $z \sim 4$, even if we ignore any SFR associated with the QSO hosts (which we cannot measure, but is surely there). These must be very special regions of an enhanced galaxy formation in the early universe.

It is also worth noting that (perhaps coincidentally) the observed comoving number density of quasars at $z>4$ is roughly comparable to the comoving density of very rich clusters of galaxies today. Of course, depending on the timescales involved, there must be some protoclusters without observable quasars in them, and some where more than one AGN is present (an example may be the obscured companion of BR 1202-0725).

\section{Towards the Renaissance at $z>5$ : the First Quasars and the First Galaxies}

The remarkable progress in cosmology over the past few years, reviewed by several speakers at this meeting, has pushed the frontiers of galaxy and structure formation studies out to $z>5$. Half a dozen galaxies, two QSOs (cf. Fan et al. 1999), and one radio galaxy are now known at $z \geq 5$, with the most distant confirmed object at $z=5.74$ ( $\mathrm{Hu}$ et al. 1999). Remarkably, there is no convincing evidence yet for a high- $z$ decline of the comoving star formation rate density out to $z>4$ (Steidel et al. 1999). Moreover, the universe at $z \sim 5$ appears to be already fully reionised (Songaila et al. 1999, Madau et al. 1999), implying the existence of a substantial activity in a population of sources at even higher redshifts. 
These observational results pose something of a challenge for the models of galaxy formation. Essentially in all modern models, the first subgalactic fragments with masses $\geq 10^{6} M_{\odot}$ begin to form at $z \sim 10-30$, and the universe becomes reionised at $z \sim 8-12$ (see, e.g., Gnedin \& Ostriker 1997, MiraldaEscude \& Rees 1997, or Rauch 1998 and references therein). This corresponds to a time interval of only about $\sim 0.5-1 \mathrm{Gyr}$ for a reasonable range of cosmologies.

What is not known is what are the first or the dominant ionisation sources which break the "dark ages": primordial starbursts or primordial AGN? This is one of the fundamental questions in cosmology today, and it dominates many of the discussions about the NGST (see, e.g., Rees 1998b, Haiman \& Loeb 1998, or Loeb 1999). Optical searches for quasars at $z>5$ have been reviewed by Osmer (1999). There are exciting new prospects of detecting such a population in $\mathrm{x}$ rays using CXO (Haiman \& Loeb 1999). The value of such quasars as probes of the earliest phases of galaxy and structure formation during the reionisation era a.t $z \sim 10 \times 2^{ \pm 1}$ cannot be overstated.

Some numerical simulations suggest that an early formation of quasars, at $z \sim 8$, say, is viable in the framework of the currently popular hierarchical models with dissipation (cf. Katz et al. 1994). It is even possible that a substantial amount of QSO activity may predate the peak epoch of star formation in galaxies (Silk \& Rees 1998). A catastrophic gravitational collapse of a massive primordial star cluster may be the most natural way of forming the first MBHs, but a variety of other mechanisms have been proposed (e.g., Loeb 1993, Umemura et al. 1993, Loeb \& Rasio 1994, etc.). Future observations will tell whether such primordial fireworks marked the end of the dark ages in the universe.

Acknowledgments. It is a pleasure to acknowledge the work of my collaborators, R. Gal, R. Brunner, R. de Carvalho, S. Odewahn, and the rest of the DPOSS QSO search team. I also wish to thank the staff of Palomar and Keck observatories for their expert assistance during our observing runs. This work was supported in part by the Norris Foundation and by the Bressler Foundation. Ivan King and his LOC crew brought this meeting into existence; thank you all. Finally, many thanks to Hy for introducing me to the joys of low-S/N astronomy and letting me play with the big toys: it was fun (most of the time)!

\section{References}

Boyle, B., Croom, S., Smith, R., Shanks, T., Miller, L., \& Loaring, N. 1998, preprint [astro-ph/9805140]

Broadhurst, T., Ellis, R., Koo, D., \& Szalay, A. 1990, Nat, 343, 726

Cataneo, A. 1999, MNRAS in press [astro-ph/9907335]

Carlberg, R. 1990, ApJ, 350, 505

Chokshi, A., \& Turner, E. 1992, MNRAS, 259, 421

Clowes, R., \& Campusano, L. 1991, MNRAS, 249, 218

Cole, S. \& Kaiser, N. 1989, MNRAS, 237, 1127

Crampton, D., Cowley, A., \& Hartwick, F.D.A. 1989, ApJ, 345, 59

Djorgovski. S., Spinrad, H., McCarthy, P., \& Strauss, M. 1985, ApJ, 299, L1 
Djorgovshi, S., Strauss, M. , Perley, R., Spinrad, H., \& McCarthy, P. 1987, AJ, 93,1318

Djorgovski, S. 1991, in The Space Distribution of Quasars, ed. D. Crampton, ASPCS, 21, 349

Djorgovski, S., Thompson, D., \& Smith, J. 1993, in First Light in the Universe, eds. B. Rocca-Volmerange et al., Gif sur Yvette: Eds. Frontières, p.67

Djorgovski, S.G. 1995, in Science with the VLT, eds. J.R. Walsh \& I.J. Danziger, Berlin: Springer Verlag, p. 351

Djorgovski, S., Pahre, M., Bechtold, J., \& Elston, R. 1996, Nat, 382, 234

Djorgovski, S.G. 1996, in New Light on Galaxy Evolution, IAU Symp. 171, eds. R. Bender \& R. Davies, Dordrecht: Kluwer, p.277

Djorgovski, S.G. 1998, in Fundamental Parameters in Cosmology, Rec. de Moriond, eds. Y. Girand-Herand et al., Gif sur Yvette: Eds. Frontières, p. 313 [astro-ph/9805159]

Djorgovski, S.G., Odewahn, S.C., Gal, R.R., Brunner, R., \& de Carvalho, R.R. 1999, in Photometric Redshifts and the Detection of High Redshift Galaxies, eds. R. Weymann et al., ASPCS in press [astro-ph/9908142]

Djorgovski, S.G., Gal, R. R., Odewahn, S. C., de Carvalho, R. R., Brumner, R., Longo, G., \& Scaramella, R. 1999, in Wide Field Surveys in Cosmology, eds. S. Colombi el al., Gif sur Yvette: Eds. Frontières, p.89 [astro$\mathrm{ph} / 9809187]$

Efstathiou, G., \& Rees, M. 1988, MNRAS, 230, P5

Fan, X. et al. (the SDSS collaboration) 1999, AJ, 118, 1

Franceschini, A., Hasinger, G., Miyaji, T., \& Malquori, D. 1999, MNRAS in press [astro-ph/9909290]

Gnedin, N., \& Ostriker, J. 1997, ApJ, 486, 581

Hachnclt, M., \& Rees, M. 1993, MNRAS, 263, 168

Haehnelt, M., Natarajan, P., \& Rees, M. 1998, MNRAS, 300, 817

Haiman, Z., \& Loeb, A. 1998, ApJ, 503, 505

Haiman, Z., \& Loeb, A. 1999, ApJ, 521, L9

Hamann, F., \& Ferland, G. 1993, ApJ, 418, 11

Hamann, F., \& Ferland, G. 1999, ARAA in press

Hartwick, F.D.A, \& Schade, D. 1990, ARAA, 28, 437

Heckman, 'I. 1991, in Massive Stars in Starbursts, eds. C. Leitherer et al., STScI Symposium No. 5, Cambridge: Cambridge Univ. Press, p.289

Hernquist, L., \& Mihos, C. 1995, ApJ, 448, 41

Hu, E., McMahon, R., \& Egami, E. 1996, ApJ, 459, L53

Hu, E., \& McMahon, R. 1996, Nat, 382, 231

Hu, E., McMahon, R., \& Cowie, L. 1999, ApJL in press [astro-ph/9907079]

Theuchi, S., \& Norman, C. 1991, ApJ, 375, 479

Iovino, A., \& Shaver, P. 1988, ApJ, 330, L13

Kaiser, N. 1984, ApJ, 284, L9

Fatz, N., Quinn, T., Bertschinger, E., \& Gelb, J. 1994, MNRAS, 270, L71 
Kauffmann, G., \& Haehnelt, M. 1999, MNRAS in press [astro-ph/9906493]

Kennefick, J.D., Djorgorshi. S.G., \& de Carvalho, R. 1995. AJ, 110, 2553

Kennefick, J.D., Djorgorski. S.G., \& Meylan, G. 1996, AJ, 111, 1816

Kochanek, C., Falco, E., \& Muño, J. 1999, ApJ, 510, 590

Formendy, J., \& Richstone, D. 1995, ARAA, 33, 581

Kundic, T. $1997, \mathrm{ApJ}, 482,631$

La Franca, F., Andreani, P., \& Cristiani, S. 1998, ApJ, 497, 529

Landy, S., Shectman, S., Lin, H., Kirshner, R., Oemler, A., \& Tucker, D. 1996, ApJ, 456, L1

Loeb, A. 1993, ApJ, 403, 542

Loeb, A., \& Rasio, F. 1994, ApJ, 432, 52

Loeb, A. 1999, this volume

Loewenstein, M., \& Mushotzky; R. 1996, ApJ, 466, 695

Mada.u, P., Ferguson, H., Dickinson, M., Giavalisco, M., Steidel, C., \& Fruchter, A. 1996, MNRAS, 283, 1388

Madau, P., Haardt, F., \& Rees, M. 1999, ApJ, 514, 648

Magorrian, J. et al. 1998, AJ, 115, 2285

Matteucci, F,. and Padovani, P. 1993, ApJ, 419485

Mihos, C., \& Hernquist, L. 1996, ApJ, 464, 641

Miralda-Escude, J., \& Rees, M. 1997, ApJ, 478, L57

Monaco, P., Salucci, P., \& Danese, L. 1999, MNRAS in press [astro-ph/9907095]

Norman, C., \& Scoville, N. 1988, ApJ, 332, 124

Nusser, A., \& Silk, J. 1993, ApJ, 411, L1

Ohta, K., Yamada, T., Nakanishi, K., Kohno, K,. Akiyama, M., \& Kawabe, R. 1990, Nat, 382, 426

Omont, A., Petitjean, P., Guilloteau, S., McMahon, R., Solomon, P., \& Pecontal, E. 1996, Nat, 382, 428

Osmer, P. 1999, this volume

Petitjean, P., Pecontal, E., Vals-Gabaud, D., \& Charlot, S. 1996, Nat, 380, 411

Rauch, M. 1998, ARAA, 36, 267

Rees, M. 1998a, in Structure and Evolution of the Intergalactic Medium from QSO Absorption Systems, eds. P. Petitjean \& S. Charlot, Gif sur Yvette: Eds. Frontieres, p. 19

Rees, M. 1998b, preprint [astro-ph/9809029]

Sanders, D., Soifer, B.T., Elias, J., Neugebaner, G.. \& Matthews, K. 1988, ApJ, $328, \mathrm{~L} 35$

Schmidt, M., Schneider, D., \& Gunn, J. 1995, AJ, 110, 68

Schneider, D., Schmidt, M., \& Gunn, J. 1994, AJ 107, 1245

Silk, J., \& Rees, M. 1998, A\&A, 331, L1.

Small, T., \& Blandford, R. 1992, MNRAS, 259, 725

Songaila, A., Hu, E., Cowie, L., \& McMahon, R. 1999, ApJL in press

Spinrad, H. 1979, private communication 
Steidel, C., Giavalisco, M., Pettini, M., Dichinson, M., \& Adelberger, K. 1996 , ApJ, 462, L17

Stcidel, C., Adclberger, K., Dickinson, M., Pettini, M., \& Kcllogg, M. 1998, ApJ, 492, 428

Steidel, C., Adelberger, K., Giavaliscu, M., Dichinson, M., \& Pettini, M. 1999, ApJ $, 519,1$

Stephens, A., Schneider, D., Schmidt, M., Gunn, J., \& Weinberg, D. 1997, AJ, 114,41

Terlevich, R., \& Boyle, B. 1993, MNRAS, 262, 491

Turner, E. 1991, AJ, 101, 5

Umemura, M., Loeb. A., \& Turner, E. 1993, ApJ, 419, 459

Williams, R., \& Perry, J. 1994, MNRAS, 269, 538 
The Hy Redshift Universe

ASP Conference Series, Vol. 193, 1999

A. J. Bunker \& W. J. M. van Breugel, eds.

\title{
Observational Tests of the Static Universe Model and the Derivation of the Hubble Redshift
}

\author{
Thomas B. Andrews \\ 3828 Atlantic Ave, Brooklyn, NY 11224
}

\begin{abstract}
The static universe model is tested and compared with the $q_{0}=0.5$, expanding universe model based on the apparent magnitudes of 1st-rank elliptical galaxies and type Ia supernovae and the angular sizes of 1st-rank elliptical galaxies. Using new physics, a gravitational process is proposed for the Hubble red-shift, time-dilation and the anomalous acceleration of the Pioneer spacecraft toward the sun.
\end{abstract}

\section{Observational Tests}

With the HST and the new large ground telescopes, it is now possible to distinguish observationally between different models of the universe. As an example, the static universe model is tested and compared with the $q_{0}=0.5$, expanding universe model. In addition, a gravitational process based on new physics is proposed for the Hubble red-shift, time-dilation (supernovae light curve) and the anomalous acceleration of the Pioneer spacecraft (Anderson 1998).

The observational tests are based on the apparent magnitudes of 1st-rank elliptical galaxies (Kristian 1978), fundamental plane analysis of elliptical galaxies in clusters (Schade 1997), type Ia supernovae (Riess 1998) and the effective angular sizes of 1st-rank elliptical galaxies. Graphs of the four tests are shown in figure 1. The observed slope and the standard deviation of the slope for the static universe model are shown on each graph. In all tests, the static universe model fits the data significantly better than the expanding universe model.

The apparent magnitude, $m$, for the static universe model is given by

$$
m=M+5 \log r+2.5 \log (1+z)+42.38-5 \log \left(H_{o} / 100\right) .
$$

Normally $m$ would be plotted versus $\log (z)$. Instead, $m^{\prime}=m-2.5 \log (1+z)$ is plotted versus $\log (r) . r$ is the normalized distance given by $r=s / R=\ln (1+z)$ where $s$ is the Euclidean distance and $R(\approx 20$ billion light years $)$ is the mean interactive radius of the universe. For supernovae, $m^{\prime}=m-5 \log (1+z)$ is plotted since the apparent magnitude of supernovae is increased by $2.5 \log (1+z)$ due to time-dilation of the supernovae light curve. In both cases, the plot of $\mathrm{m}^{\prime}$ versus $\log (r)$ is theoretically linear with slope 5 .

The theoretical relation between the effective angular radius, $\theta$ (sec), the effective radius, $R_{E}$ (Kpc), and $r$ for the static universe model is

$$
\theta=R_{E} /(14.05 r)\left(H_{o} / 100\right) .
$$


$R_{E}$ is assumed constant. This is a critical assumption which is confirmed by observations (van Dokkum 1998) showing that high-z 1st-rank elliptical galaxies have the same velocity dispersions as those at low- $z$. Therefore, all 1st-rank elliptical galaxies must have nearly the same mass and, since size is correlated with mass, approximately the same effective radius. Plotting $\log (\theta)$ vs $\log (r)$. the observations are a good fit to the theoretical slope of -1 . This is especially true for the distant radio galaxy (Pascarelle 1996) at $z=2.39$, a distance in the static universe model of about 24 billion light years.

\section{New Physics}

New physics is proposed based on a wave system paradigm. Assume the universe is a pure wave system with mass density and tension parameters proportional to the local intensity of the wave modes. From eigenvalue theory, the frequency is minimized when there is complete constructive interference between the normal modes of the system. Then, it is hypothesized that the constructive interference peaks are the elementary particles.

The constructive interference peaks could be extremely large in intensity due to the cross-product amplitude terms. However, because there is a split in frequency due to the interactions between modes, the cross-product terms become equally positive and negative (electrostatic forces) and cancel at the particles. Since this leaves the constructive interference peaks only with the squared amplitude terms, the energy of particles is purely gravitational.

In the wave system, $F=-p / c(d I / d s)$ where $p$ is the linear size of the particle, $s$ is the distance between two particles and $I$ is the intensity of a single mode. From this force law, Newton's law of gravitation (Andrews 1998) can be derived only if the intensity varies as $1 / s$. This result implies that the wave modes propagate circularly in a plane. Solving the classical wave equation for the radial normal modes, assuming the wave parameters of a single mode are proportional to the intensity $1 / s$ and the normal modes are harmonic, the normal modes are uniquely determined by the Bessel equation of half-order with $1=0$. Surprisingly, these are the normal modes of the universe.

However, an equally important result is that the total intensity, $I_{T}$, at a local particle comes primarily from distant particles because the intensity depends on $1 / s$, instead of $1 / s^{2}$. Thus, assuming the universe is static, Euclidean and infinite,

$$
I_{T} \propto 4 \pi \int_{0}^{\infty} s \exp (-s / R) d s=4 \pi R^{2}
$$

The exponential in the integral occurs because the intensity of the wave modes is reduced by interactions with mass particles in the propagation path. However, normal modes which interact cannot maintain the exact intensity relation, $1 / \mathrm{s}$, required of the Bessel equation and, therefore, cannot propagate. Then, assuming two particles interact via a single wave mode, the exponential determines, in effect, the proportion of particles at different distances which interact with local particles. As a result, a local particle does not interact with all particles in the universe but only with a fixed set of particles. 


\section{Hubble Red-Shift Process for Photons and Mass Particles}

Based on the new physics, the Hubble red-shift is analogous to a photon rising in the gravitational field of a large mass. To make the analogy exact, substitute for the large mass, a fixed set of particles which interact with the photon. Then, since the photon is composed of progressive modes propagating in the same direction, the intensity at the photon originates from the fixed set of particles in the infinite half-sphere behind the mass particle which emitted the photon.

Given $E=p I_{T} / c$, a three-dimensional computer simulation of the red-shift process shows that the energy decrease is $d E=-E(d s / R)$ where $E$ is the energy of a photon. Integrating the equation, $\ln \left(E / E_{o}\right)=-s / R$ or $E=E_{o} \exp (-s / R)$. Then, from the relation $f=E / h$, the red-shifted frequency is $f=f_{o} \exp (-s / R)$.

The mass particle, on the other hand, is a constructive interference of progressive modes propagating in both directions. Consequently, the mass particle also interacts with the progressive modes from the fixed particle set towards which the mass particle is moving. Since the energy decrease from this set of particles is $1 / 2$ the photon energy decrease, the total decrease in energy for a mass particle is given by $d E=-1.5 E(d s / R)$.

This mass particle red-shift is applicable to the anomalous accelerations toward the sun of the Pioneer spacecraft. To derive the acceleration, set $F=$ $d E / d s=m a, E=m c^{2}$ and $R=c / H_{o}$. Then, $a=-1.5 c H_{o}$. For $H_{o}=59(5)$ $\mathrm{km} / \mathrm{sec} / \mathrm{Mpc}\left(\right.$ Tammann 1999),$a=-8.6(0.7) \times 10^{-8} \mathrm{~cm} / \mathrm{sec}^{2}$. This compares with the anomalous accelerations of $-8.09(0.20) \times 10^{-8} \mathrm{~cm} / \mathrm{sec}^{2}$ for Pioneer 10 and $-8.56(0.15) \times 10^{-8} \mathrm{~cm} / \mathrm{sec}^{2}$ for Pioneer 11 . Alternatively, $H_{0}$ may be determined from the anomalous accelerations of the Pioneer spacecraft.

\section{Time-Dilation Process}

The light curve of supernovae represents a very low frequency modulation of the luminosity. Since all frequencies are reduced by the Hubble red-shift, this low frequency modulation is, therefore, red-shifted to a lower frequency. This increases the period of the supernovae light curve at the observer by the factor $(1+z)$. Then, because the light is spread-out over time (time-dilation), the apparent magnitude is increased by $2.5 \log (1+z)$.

\section{References}

Anderson, J.D. et al 1998, Phys.Rev.Lett, 81, 2858

Andrews, T.B. 1998, Causality and Locality in Modern Physics, Klnwer Academic Publishers, The Netherlands, 135

Kristian, J., Sandage, A., and Westphal, J.A. 1978, ApJ, 221, 383

Pascarelle et al 1996, ApJ, 456, L21

Riess, A.G. et al 1998, AJ, 116, 1009

Schade, D., Barrientos, L.F. and Lopez-Cruz, O. 1997, ApJ, 477, L17

Tammann, G.A. and Reindl, B. 1999, astro-ph/9903220

van Dokkum, P.G. et al 1998, ApJ, 504, L17 

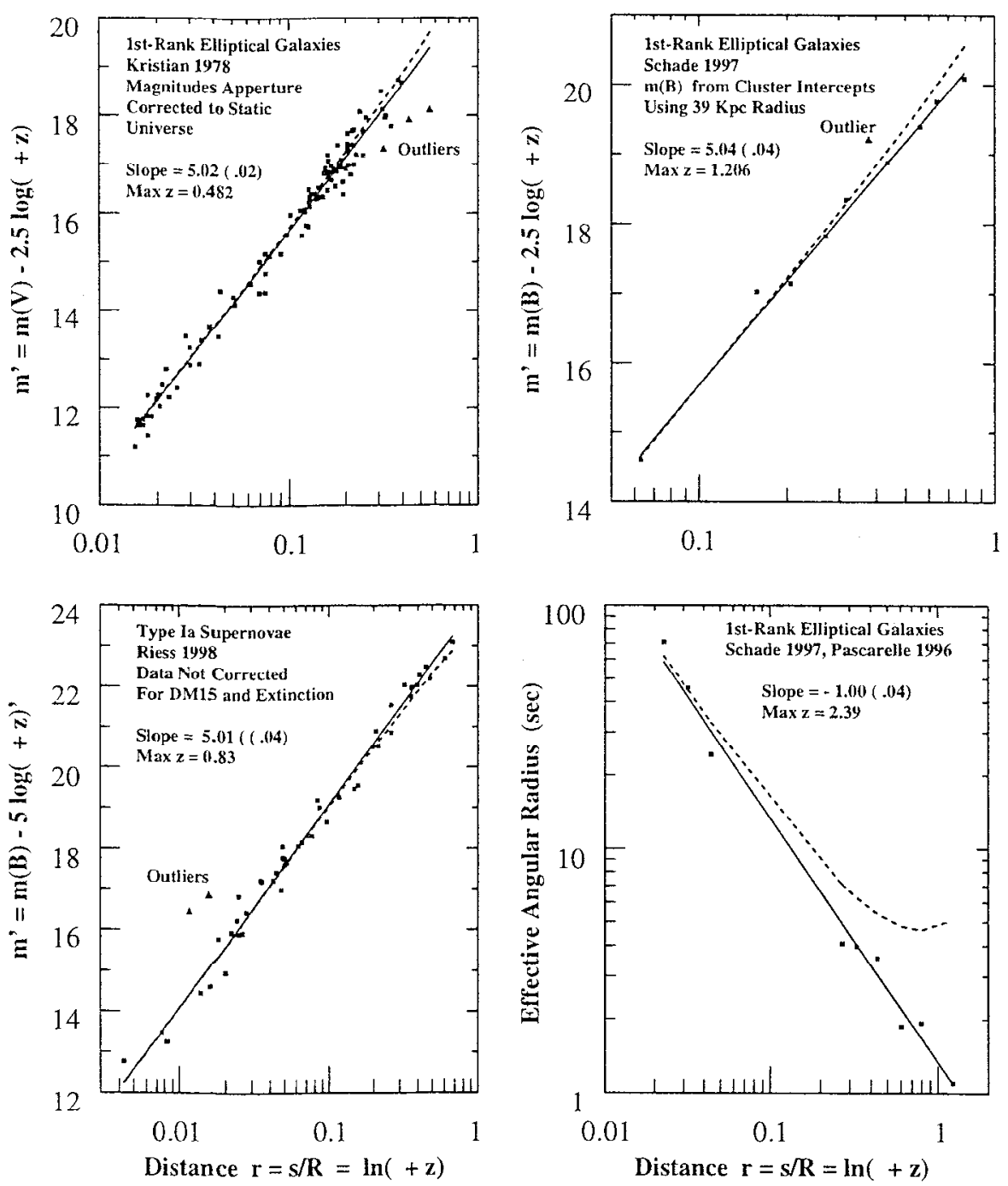

Figure 1. Four observational tests of the static universe model are shown. The solid line represents the theoretical relation for the static universe model and the dashed line the relation for the $q=0.5$, expanding universe model.

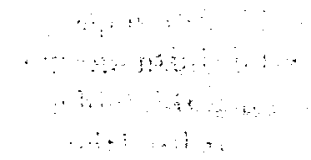


The Hy Redshift Universe

ASP Conference Series, Vol. 193, 1999

A. J. Burkier \& W. J. M. van Breugel. eds.

\title{
The Clustering of Faint Galaxies on Small Angular Scales
}

Tereasa G. Brainerd and Casey J. Law

Boston University, Dept. of Astronomy, Boston, MA 02215

James Brauher

IPAC, California Institute of Technology, Pasadena, CA 91125

S. G. Djorgovski and Ken Banas

California Institute of Technology, 105-24, Pasadena, CA 91125

\begin{abstract}
We present a preliminary measurement of the angular clustering of faint $(R \leq 25)$ field galaxies in which we concentrate on the behavior of $\omega(\theta)$ on small angular scales $\left(\theta \lesssim 10^{\prime \prime}\right)$. The galaxies are strongly clustered and $\omega(\theta)$ is well-characterized by a power law of the form $A_{\omega} \theta^{-\delta}$. The best-fitting value of the power law index, $\delta$, is, however, steeper than the fiducial value of $\delta=0.8$, indicating that there are more pairs of galaxies separated by $\theta \lesssim 10^{\prime \prime}$ in our sample than would be otherwise expected. Using the best-fitting form of $\omega(\theta)$, we estimate that $\sim 10 \%$ of the galaxies are in physically close pairs (separations $\$ 21 h^{-1} \mathrm{kpc}$ ). This is a factor of order 2 larger than local galaxy samples but comparable to galaxy samples with $\langle z\rangle \sim 0.4$. The mean redshift of our galaxies is of order 0.95 , and, therefore, our result suggests that there was little or no evolution in the merger rate of galaxies between $z \sim 1$ and $z \sim 0.4$.
\end{abstract}

\section{Introduction}

The angular clustering of faint $R$-selected field galaxies has been studied extensively (e.g., Efstathiou et al. 1991; Roche et al. 1993, 1996; Brainerd, Smail \& Mould 1995; Hudon \& Lilly 1996; Lidman \& Peterson 1996; Villumser, Freudling \& da Costa 1996; Woods \& Fahlman 1997), and a prime motivation of these studies has been to investigate the nature of the fain field population. In particular, it is possible to infer the effective correlation length of the sample and the rate at which clustering evolves from a combination of the amplitude of the angular autocorrelation function, $\omega(\theta)$, and the redshift distribution of the faint galaxies, $N(z)$. These observations can then be used to link properties of the faint field population with samples of local galaxies. While the exact interpretation remains controversial, it is generally accepted that overall $\omega(\theta)$ is fitted well by a power law of the form $\theta^{-0.8}$ (although see Infante \& Pritchet (1995) for evidence of a flattening in the power-law coefficient at faint limits).

Here we investigate the clustering of faint galaxies and focus on the behavior of $\omega(\theta)$ at small angular separations. We obtain a clear measurement of $\omega(\theta)$ on 
scales of $\theta<10^{\prime \prime}$ whereas previous investigations have been largely limited to scales of $\theta \gtrsim 20^{\prime \prime}$. Additionally, we use the clustering properties of the galaxies to estimate the number of pairs of galaxies that are physically close to each other in space (separations of $\lesssim 21 h^{-1} \mathrm{hpc}$ ).

\section{Observations}

The data consist of deep $R$-band imaging of 11 independent fields that were obtained in good conditions with the Low Resolution Imaging Spectrograph on the $10-\mathrm{m}$ Kech-I telescope. Each of the $6^{\prime} \times 8^{\prime}$ fields is centered on a high redshift quasar with high galactic latitude; however, the presence of the quasar in the field is irrelevant to the present investigation (i.e., the presence of a small group of galaxies at the redshift of the quasar will not influence the results below). The galaxy catalogs are complete to $R=25.0$ and the apparent magnitudes of the galaxies have been corrected for extinction. In order to reduce the stellar contamination in the object catalogs, only objects with $R \geq 21$ are considered in the analysis below. There is, of course, some residual stellar contamination of the galaxy catalogs at faint limits and we estimate that to be: $\sim 16 \%(21.0 \leq$ $R \leq 24.0), \sim 13 \%(21.0 \leq R \leq 24.5), \sim 11 \%(21.0 \leq R \leq 25.0)$. The integral constraints vary little from field to field due to the use of the same detector in all cases as well as the lack of very large, bright galaxies in the fields.

\section{Analysis and Results}

To compute the angular clustering of the faint galaxies we use the Landy \& Szalay (1993) estimator:

$$
\omega(\theta)=\frac{D D-2 D R+R R}{R R}
$$

where $D D, D R$, and $R R$ are the number of unique data-data, data-random, and random-random pairs within a given angular separation bin. Regions of the frame where faint galaxy detection was either lower than average or impossible (e.g., due to the presence of bright stars and galaxies) were masked out when computing $D R$ and $R R$. Raw correlation functions (uncorrected for stellar contamination or the integral constraint) were determined for each of the fields, from which a mean correlation function was computed.

The resilts for the mean raw correlation function are shown in Figure 1, where the error bars show the standard deviation in the mean. From top to bottom, the panels show the results for objects with $21.0 \leq R \leq 24.0,21.0 \leq$ $R \leq 24.5$, and $21.0 \leq R \leq 25.0$, respectively. Also shown are the formal best-fitting power laws of the form $\theta^{-\delta}$ (solid lines) and the best-fitting power laws of the form $\theta^{-0.8}$ (dashed lines). The power laws in the figure have been suppressed by the appropriate integral constraints and no correction for residual stellar contamination has been applied.

The number of pairs of galaxies that we observe to be separated by $\theta \sim 3^{\prime \prime}$ is larger than the number predicted by the fiducial $\theta^{-0.8}$ - power law (i.e., the power law that is typically obtained from measurements that have been performed on 


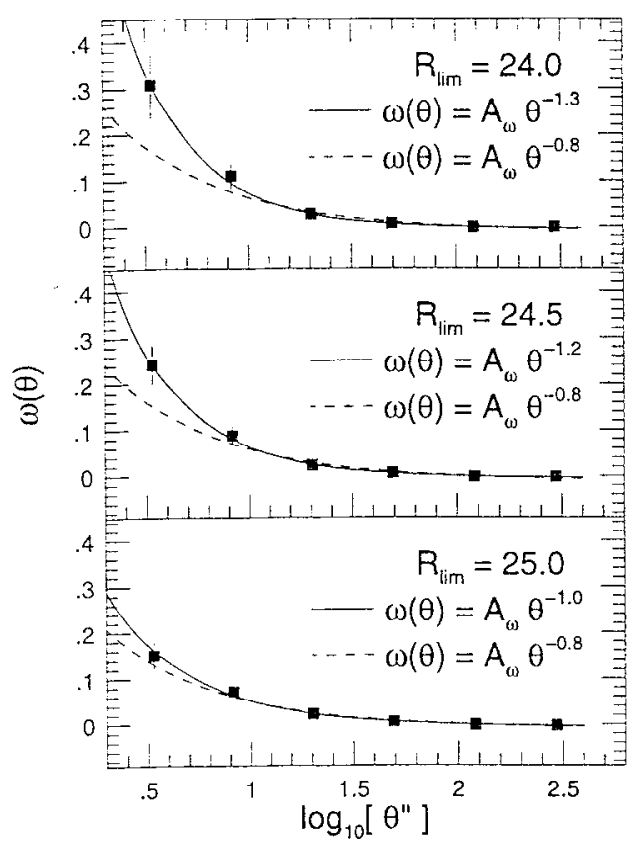

Figure 1. Mean correlation functions computed using the 11 independent estimates of $\omega(\theta)$ that were obtained in each magnitude bin.

scales of $\theta \gtrsim 10^{\prime \prime}$ ). This is consistent with the results of Carlberg et al. (1994) and Infante et al. (1996) who both found $\omega(\theta)$ to have a higher amplitude on small angular scales $\left(\theta \lesssim 6^{\prime \prime}\right)$ than a simple inward extrapolation of $\omega(\theta)$ as measured at large angular scales. As yet, however, it is unclear whether the steepening of $\omega(\theta)$ is due to the existence of a population of "companion" galaxies (which are not seen at the present epoch) or luminosity enhancement (e.g., due to interactions) of intrinsically faint galaxies that are in pairs.

In the absence of significant luminosity enhancement, we can estimate the number of pairs of galaxies that are physically close to each other simply by using the following probability:

$$
P=\int_{\beta}^{\theta} 2 \pi \rho \alpha \exp \left(-\pi \rho \alpha^{2}\right) d \alpha
$$

(e.g., Burkey et al. 1994), where $\rho$ is the number density of galaxies brighter than the faintest member in a pair of galaxies that is a candidate for close physical separation, $\theta$ is the observed angular separation between the galaxies, and $\beta$ is the smallest separation observed between all detected galaxies $\left(\beta \sim 1^{\prime \prime}\right.$ in our data). Using Eqn. (2) we compute the number of pairs of galaxies for which $P \leq 0.05$ and $P \leq 0.10$ in our data. Additionally, we use Monte Carlo simulations (in which the magnitudes of the galaxies are shuffled at random) to calculate the number of pairs of galaxies that would have $P \leq 0.05$ and $P<0.10$ simply by chance. The latter step allows the removal of random superpositions from the estimate of the "true" number of close pairs in the sample. 
Below $\theta \sim 3^{\prime \prime}$ there are fewer pairs of galaxies with $P \leq 0.05$ and $P \leq 0.10$ in the actual data than are expected in a random distribution (i.e., based on the Monte Carlo simulations), indicating that we are undercounting the very closest pairs due to blending of the images. Using our measured $\omega(\theta)$, however, we can correct the faint pair counts on scales $1^{\prime \prime} \leq \theta \leq 2^{\prime \prime}$ and estimate the fraction of galaxies in our sample that are in truly close physical pairs.

Based on a simple extrapolation of the CFRS redshift distribution, we expect that the mean redshift of our galaxies is $\sim 0.95$ and, hence, if $\Omega_{0}=1$, physical pairs of galaxies that are separated by $\theta \leq 5^{\prime \prime}$ will be within $21 h^{-1} \mathrm{kpc}$ of each other. The best-fitting power law form of $\omega(\theta)$ (corrected for stellar contamination and the integral constraint) then yields an estimate of the pair fraction at this physical separation of $\sim 10 \%$ for $P \leq 0.10$, which agrees with the results obtained by Carlberg et al. (1994) for the fraction of galaxy pairs with separations $\$ 19 h^{-1} \mathrm{kpc}$ at $\langle z\rangle \sim 0.4$. This suggests, therefore, that little evolution in the merger rate of galaxies occurred between $z \sim 1$ and $z \sim 0.4$.

\section{Future Work}

A significant amount of work remains to be done on this project, including a clustering analysis of 7 additional independent fields and a more rigorous study of the number of pairs of faint galaxies located at close physical separation.

Acknowledgments. Financial support under NSF contract AST-9616968 (TGB) and a Boston University Presidential Graduate Fellowship (CJL) are gratefully acknowledged. The observations were obtained at the W. M. Keck Observatory, which is operated jointly by the California Institute of Technology and the University of California. Data analysis was performed exclusively on the Origin2000 at Boston University's Scientific Computing \& Visualization facility.

\section{References}

Brainerd, T.G., Smail, I. \& Mould, J.R., 1995, MNRAS, 275, 781

Burkey, J.M., Keel, W.C., Windhorst, R.A., \& kranklin, B.E., 1994, ApJ, 429, $\mathrm{L} 13$

Carlberg, R. G., Pritchet, C. J., \& Infante, L. 1994, ApJ, 435, 540

Efstathiou, G., Bernstein, G., Katz, N., Tyson, J.A., \& Guhathakurta, P., 1991, ApJ, 380, L47

Hudon, J.D. \& Lilly, S.J., 1996, ApJ, 469, 519

Infante, I. \& Pritchet, C.J., 1995, ApJ, 439, 565

Infante, L., de Mello, D. \& Menanteau, F. 1996, ApJ, 469, L85

Landy, S.D. \& Szalay, A.S., 1993, ApJ, 412, 64

Lidman, C.E. \& Peterson, B.A., 1996, MNRAS, 279, 1357

Roche, N., Shanks, T., Metcalfe, N., \& Fong, R., 1993, MNRAS, 263, 360

Roche, N., Shanks, T., Metcalfe, N., \& Fong, R., 1996, MNRAS, 280, 397

Woods, D. \& Fahlman, G.G., 1997, ApJ, 490, 11

Villumsen, J.V., Freudling, W., \& da Costa, L.N., 1996, ApJ, 481, 578 
The Hy Redshift Universe

ASP Conference Series, Vol. 193. 1999

A. J. Bunker \&6 W. J. M. van Breugel, eds.

\title{
Observations of Candidate $z \sim 1.54$ Quasar Host Clusters
}

\author{
Patrick B. Hall
}

Department of Astronomy, University of Toronto, 60 St. George Street, Toronto, ON MSS $3 \mathrm{H8}$, Canada

Marcin Sawicki

Caltech, Mail Stop 320-47, Pasadena, CA 91125

C.J. Pritchet, F.D.A. Hartwick

Department of Physics and Astronomy, University of Victoria, Victoria, $B C$ V8W 3P6, Canada

Aaron S. Evans

Dept. of Physics and Astronomy, State University of New York at Stony Brook, Stony Brook, NY 11794-3800

\begin{abstract}
We present new data on several $z=1.54$ radio-loud quasar fields from a sample of 31 at $z=1-2$ in which we have previously identified an excess population of predominantly red galaxies. Narrow-band $\mathrm{H} \alpha$ observations detect five candidate galaxies at the quasar redshifts in three fields totaling $10.156 \square^{\prime}$, a surface density $\sim 30$ times higher than in previous surveys, even targeted ones. SCUBA observations of three fields detect at least one candidate quasar-associated galaxy. Many galaxies with SEDs indicating considerable dust are not detected, but the limits are only sufficient to rule out hyperluminous infrared galaxies. Finally, quantitative photometric redshifts and SED fits are presented for one " $J$ band dropout" galaxy with $J-K>2.5$ which is confirmed to be very dusty $(E(B-V) \simeq 0.5)$ and background to the quasar at $\geq 99.9 \%$ confidence.
\end{abstract}

\section{Introduction}

It is of considerable interest to identify structures of galaxies at $z>1$ to study the evolution of both galaxies and galaxy clusters. Radio-loud quasars (RLQs) are obvious signposts around which to search for clusters at $z>1$. In Hall \& Green (1998; hereafter HG98) we presented imaging of 31 RLQs at $z=1-2$ which revealed an excess population of predominantly red galaxies. Most candidate excess galaxies' SEDs are consistent with them being at the quasar redshifts and red due to high age or metallicity, but some are consistent with being heavily dust-reddened galaxies, and/or background galaxies at $z>2.5$. Here and in Hall etal. (1999) we present new observations of these fields and further analyses of existing data which strengthen many of our previous conclusions. 


\section{Narrow-band $\mathrm{H}_{\sigma}$ Imaging}

In the past few years, narrow-band surveys have typically yielded a few detections per survey of $\mathrm{H} \alpha$ emitters at $z>1$ (see Teplitz, McLean \& Malkan 1999). Our RLQ fields make promising targets for narrow-band searches for $\mathrm{H} \alpha$ emission at the quasar redshifts. We would hope to detect galaxies whose SEDs suggest they are dust-reddened and thus possibly actively star-forming and not to detect galaxies whose SEDs suggest they are old and dust-free.

We observed $Q 0835+580$ with IRTF using a circularly variable filter (CVF), Q 2149+212 with CFHT using a special narrow filter, and Q 2345+061 with both. There is a $>3 \sigma$ detection in each of the three fields and two $3 \sigma$ detections in the Q $2345+061$ field. Q $0835+580(\mathrm{H} \alpha 1)$ is an unremarkable faint blue galaxy with $\mathrm{SFR}_{\mathrm{H \alpha}}=14.7 \pm 2.5 \mathrm{M}_{\odot} \mathrm{yr}^{-1}$. Based on $U$-band data for this field, we estimate $\mathrm{SFR}_{F U V}=5.3 \pm 0.3 M_{\odot} \mathrm{yr}^{-1}$, in good agreement with $\mathrm{SFR}_{\mathrm{H} \alpha}$ given the various uncertainties (e.g. no correction for dust extinction has been made to either value). None of the nine very red galaxies within $20^{\prime \prime}$ of $\mathrm{Q} 0835+580$ were detected in $\mathrm{H} \alpha$, strengthening the case for them being red due to age or metallicity instead of dust (if they are at the quasar redshift).

In the Q $2345+061$ field, the candidate $\mathrm{H} \alpha$ emitter seen with IRTF (SFR Ha $=4.9 \pm 0.1 M_{\odot} \mathrm{yr}^{-1}$ ) is not confirmed with CFHT, but the IRTF CVF is three times wider than the CFHT narrow-band filter. If the $\mathrm{H} \alpha$ excess observed with IRTF is real, the line must lie outside the CFHT filter. Conversely, the two $3 \sigma$ detections $\left(\mathrm{SFR}_{\mathrm{H} \alpha}=1.8 \pm 0.4 M_{\odot} \mathrm{yr}^{-1}\right.$ ) are seen with $\mathrm{CFHT}$ but not IRTF. Hoever, given the relative widths of the filters, lines of the strength seen in the CFHT data could be present but lost in the noise in the IRTF data.

Five detections over $10.156 \square^{\prime}$ in these three fields (all among our top ten cluster candidates) gives a surface density of $0.5_{-0.2}^{+0.3} \operatorname{arcmin}^{-2}, \sim 30$ times higher than previous shallower surveys, even targeted ones. The deep CFHT H $\alpha$ images show that there are only three quasar-associated galaxies with star formation rates of $>2 M_{\odot} \mathrm{yr}^{-1}$ within fields $\sim 1 \mathrm{Mpc}$ wide centered on $\mathrm{Q} 2149+212$ and Q 2345+061. This is a lower limit which neglects extinction and the velocity dispersion of the clusters, but it illustrates the potential of deep wide-field narrow-band data in studying star formation rates in high redshift clusters.

\section{Sub-millimeter Mapping}

The presence of a number of galaxies with SEDs strongly indicative of substantial dust reddening in our RLQ fields (see HG98) suggested that they might be detectable sub-mm sources. Thus we observed the fields of $\mathrm{Q} 0835+580$, Q $1126+101$, and Q 2345+061 with SCUBA on the JCMT. The reduced jiggle maps were cross-correlated with the beam map and correlation coefficients measured; a high value was required to accept any potential source as real.

$\mathrm{Q} 2345+061$ was detected at $2.8 \sigma$, and $\mathrm{Q} 1126+101$ at $3.4 \sigma$. Only one other source, dubbed Q $1126+101$ (SM1), is securely detected, but the limits on our relatively short exposures can only rule out luminosities $\geq 10^{13} \mathrm{~L}_{\odot}$ for galaxies at the quasar redshifts. Q $1126+101$ (SM1) has two possible counterparts. The closest is a candidate quasar-associated red galaxy with $K=19: 4, r-K=5.7$, $z-J=3.4$ and $J-K=2.7$. The next closest has a moderate $r-K$ and with 
$H^{-}=17.7$ is almost certainly foreground to the quasar. Photometric redshifts and spectral types for the objects may help determine which ID is most plausible.

\section{Photometric Redshifts and Spectral Types}

We are calculating photometric redshifts and spectral types for objects in $z>1$ quasar fields with multicolor imaging data to verify the existence of excess galaxies at the quasar redshifts or beyond, investigate such galaxies' SEDs, and remove secure foreground objects from consideration for future spectroscopy. To compute photometric redshifts, a solar metallicity GISSEL model with synthetic Kurucz spectra (Bruzual \& Charlot 1996) was calculated for ages 0-20 Gyr and $z=0-4$ assuming either an instantaneous burst or a constant SFR and with dust added using the Calzetti (1997) extinction law for ten values of $E(B-V)$ from 0 to 1.6. Fluxes were computed and compared to observations to construct $\chi^{2}$ contour plots in age- $z$ space for each value of $E(B-V)$ for each SFR scenario.

We present preliminary results on one object. Q $1126+101$ (425) was studied since it is the brightest "J-dropout" $(J-K>2.5)$ in its field and it has a very red $z-J>2.7$. For either a constant SFR or instantaneous burst (the latter being shown in Figure 1), the lowest $\chi^{2}$ is given by $E(B-V) \sim 0.5$. With $z_{p h}=3.5 \pm 0.5$, the object is background to the $z=1.54$ quasar at $>99.9 \%$ confidence for all $E(B-V)<0.7$. For a constant SFR, the very red colors of $\mathrm{Q} 1126+101$ (425) require $E(B-V)>0.5$ at any $z$ to remain younger than the universe in any reasonable cosmology. The required dust is consistent with its $J-K$ color being redder than that of HR10, the prototypical dusty ERO (Extremely Red Object). However, the prediction of HG98 that this particular object would be at the quasar redshift seems to be erroneous. Instead, it appears to be a member of the other $J$-band dropout population proposed in HG98, namely galaxies at $z \geq 2.5$ which have red $J-K$ colors due to the redshifted $4000 \AA$ break.

\section{Conclusion}

These and other observations (Hall etal. 1999) are good evidence for galaxy overdensities around $z>1$ RLQs, and for a population of red galaxies at $z \geq 2.5$. If confirmed by spectroscopy with 8 -m class telescopes, it may prove worthwhile to extend RLQ host cluster searches to $z>2$ by searching for " $J$-dropout" galaxies.

\section{References}

Bruzual A., G., and Charlot, S. 1996, in "A Data Base for Galaxy Evolution Modeling," eds. C. Leitherer et al., PASP, 108, 996

Calzetti, D. 1997, in "The Ultraviolet Universe at Low and High Redshift," eds. W. H. Waller, M. N. Fanelli, and A. C. Danks (AIP: New York), 403.

Hall, P. B., and Green, R. F. 1998, ApJ, 507, 558 (HG98)

Hall, P., Sawicki, M., Martini, P., Finn, R., Pritchet, C., McCarthy, D., Osmer, . . . . P., Evans; A., Lin, H., and Hartwick, F. 1999, in preparation

Teplitz, H., McLean, I., and Malkan, M. 1999, ApJ, 520, 469 

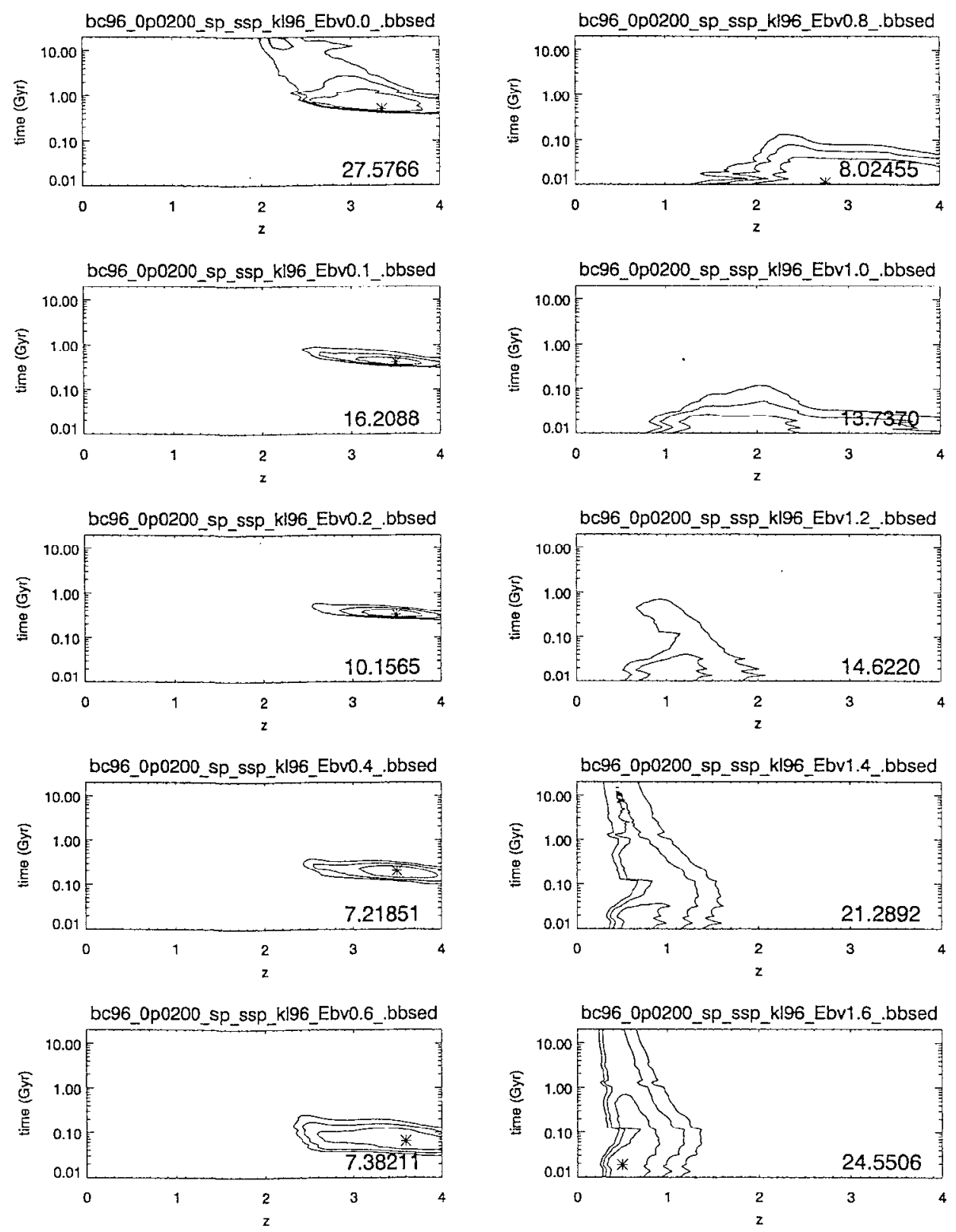

Figure 1. $\chi^{2}$ contour plots for instantaneous burst fits to the SED of object Q 1126+101 (425). Each panel corresponds to a different $E(B-V)$, starting with 0 at top left and increasing downward to 0.6 at the bottom of the first column and 1.6 at the bottom of the second. The asterisk shows the point with minimum $\chi^{2}$ in each panel. From . smallest to largest, the error contours enclose the 90,99 and $99.9 \%$ probability regions respectively. Note that the contours are drawn for each panel independently relative to the minimum unreduced $\chi^{2}$ for that panel, which is printed in the bottom right corner. 
The Hy Redshift Universe

ASP Conference Series, Vol. 193, 1999

A. J. Bunker \& W. J. M. van Breugel, eds.

Structure and Content of the 53W002 "Cluster" at $z=2.1$

\author{
William C. Keel ${ }^{1}$ and Wentao Wu \\ Department of Physics and Astronomy, University of Alabama, \\ Tuscaloosa, AL 35487
}

Rogier A. Windhorst ${ }^{1}$, Seth Cohen ${ }^{1}$, and Ian Waddington ${ }^{1}$

Department of Physics and Astronomy, Arizona State University, Tempe, AZ 85287

Sebastian Pascarelle

Department of Earth and Space Science, SUNY Stony Brook, Stony Brook, NY 11794

\begin{abstract}
We report new observations of the spatial distribution and multiband colors of the objects in the group or cluster surrounding the radio galaxy $53 \mathrm{~W} 002$ at $z=2.39$. New Lyman $\alpha$ emission candidates span an area $5.5^{\prime}(2.5 \mathrm{Mpc})$ across, including two spectroscopically confirmed QSOs. The non-AGN members have broadband spectral energy distributions most like short bursts of duration less than $800 \mathrm{Myr}$. Their radial distribution is inconsistent with a relaxed King distribution at the $90 \%$ level, implying that this system is not yet virialized and may not have fully decoupled from the Hubble flow.
\end{abstract}

\title{
1. Introduction
}

HST imagery by Pascarelle et al. (1996) showed 18 candidate Lyman a emitters in the field of the radio galaxy 53W002. Three are bright AGN, and Keck spectroscopy by Armus et al. 1999 shows that some of the fainter ones are apparently star-forming objects with narrow Lyman $\alpha$. We called them "subgalactic" since their emitted-UV images have effective radii of $0.1^{\prime \prime}$ ( $1 \mathrm{kpc}$ or so) and less.

We have now added to the data several new aspects:

1. Wider-field ground-based surveys in intermediate-passband filters, using the KPNO 4 m Mayall telescope, for additional bright emission objects to trace the grouping's extent.

2. HST NICMOS images, measuring the spectral shape into the emitted optical regime and seeking any redder envelopes of the compact star-forming objects.

${ }^{1} V$ isiting Astronomer, Kitt Peak National Observatory, NOAO, operated by AURA, Inc. under cooperative agreement with the National Science Foundation 
3. Narrow-band imaging in redshifted $\mathrm{H} \alpha$ at the NASA Infrared Telescope Facility, to measure the Lyman $\alpha / \mathrm{H} \alpha$ ratio and look for any similar but heavily reddened objects.

\section{Results - Summary}

1. There are 11 more candidate emitters spanning a $5.5^{\prime}$ area (about $2.5 \mathrm{Mpc}$ proper length for $\mathrm{H}_{0}=80 \mathrm{~km} \mathrm{~s}^{-1} \mathrm{Mpc}-1$ and $q_{0}=1 / 2$ ), including two new QSOs at $z=2.39$ These are shown in Figure 1 .

2. The star-forming objects have broadband spectra much like models of young unreddened starbursts (or young constant-SFR objects), at ages less than 200 inillion years.

3. Even existing limits on $\mathrm{H} \alpha$ emission from the star-forming members are low enough to show that destruction of Lyman $\alpha$ must be less effective than in nearby galaxies

4. We don't see any redder halos around these objects.

5. Three of the AGN show extensive emission-line structures, consistent with anisotropic photoionization ("ionization cones"), and one shows a very extended and complex 50-kpc Lyman $\alpha$ envelope.

6. The spatial distribution of candidates is inconsistent with a relaxed Kinglike profile (at the $90 \%$ level).

7. Based on additional fields observed at $z=2.4$ and 2.55 , this grouping represents about a $5 \mathrm{x}$ overdensity and such groups have covering factor $<0.04$ for $\Delta z=0.15$.

Wo describc the results on characteristic ages of the stellar populations and on the distribution of the grouping below. Full details on the spatial distribution and newly identificd QSOs are in Keel et al. (1999).

\section{Spectral Shapes: Ages of Star-Forming Objects}

We compare the broadband spectra of all the objects in this field at $z>2$ to various models and templates. The comparisons are:

Constant star-formation rate, taken from the Bruzual/Charlot (1993) code at ages 0.1-0.8 Gyr. These are good fits to the slopes we observe from 1200-5000 $\AA$ in the emitted frame.

Aging starbursts, with very short bursts seen in passive evolution thereafter. They are too red in the emitted UV to match our data, except for bursts so young as to look identical to the constant-SFR cäse.

Nearby starbursts, using two nuclear starbursts (NGC 1614 and 7714) with fairly high metallicity and very different Balmer decrements, from the Kinney et al. (1996) spectral atlas. These include effects of internal dust empirically, and are also not nearly as blue as we see for the $z>2$ objects. 


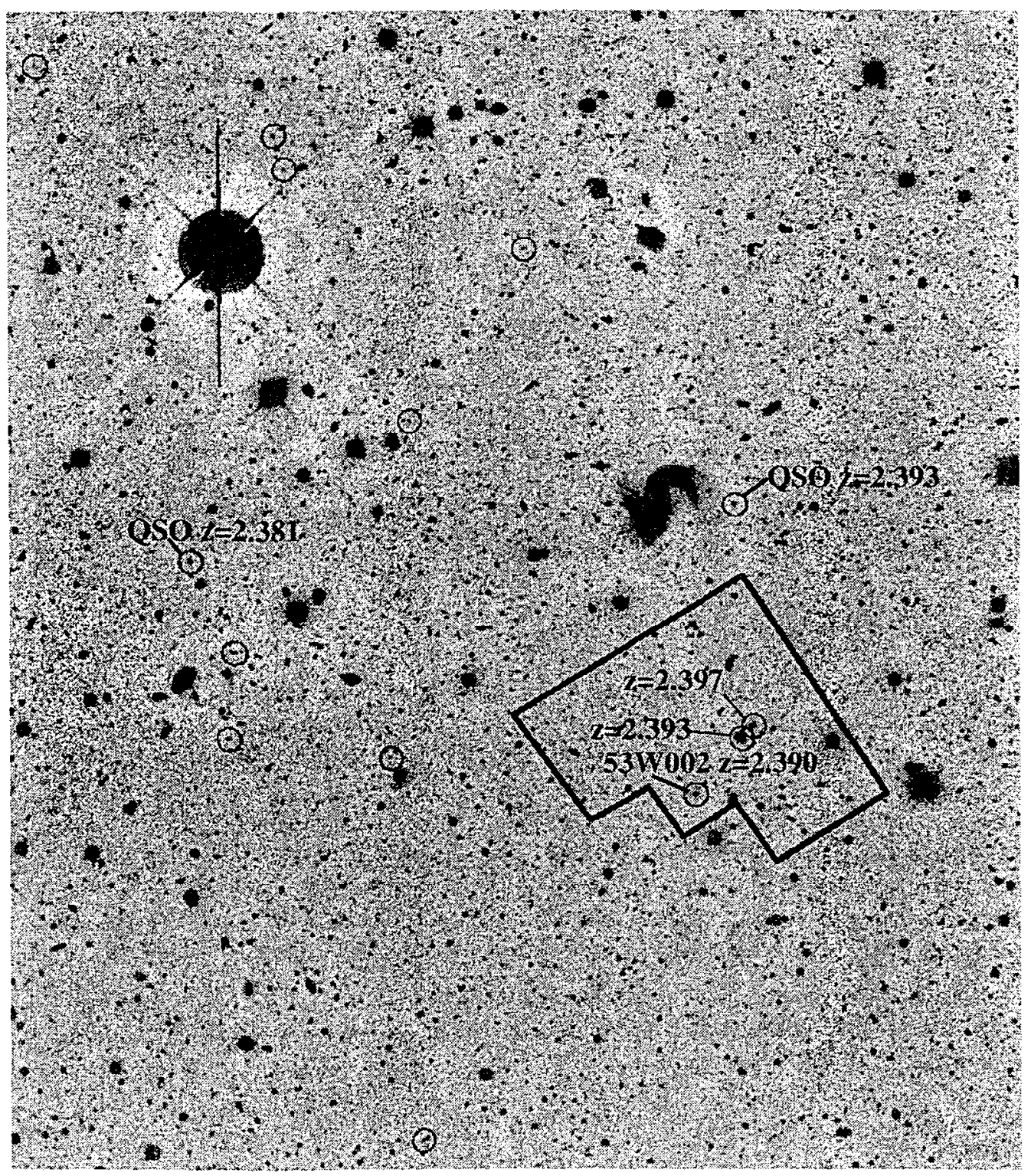

Figure 1. Part of the 4m PFCCD field, an 8-hour stacked of exposures in the F413M medium-band filter, showing the known and new Lyman $\alpha$ emission candidates in the 53W002 field. Known $z$ values and the earlier WFPC2 field are marked. This image contains all the. new candidates, and is a $5^{\prime} \times 6^{\prime}$ section from the $14.3^{\prime}$ field observed. 
In each case, [O III] would be more prominent in the F160W than $\mathrm{F} 175 \mathrm{~W}$ bands because of different filter widths. $\mathrm{H} \alpha$ may be an issue in the IRTF $\mathrm{K}$-band data for some objects; it is known to be a $30 \%$ contributor for the bright AGN.

The conclusion? We re seeing these objects within a few hundred million years of the onset of significant star formation. Near-IR spectroscopy should tell just how much or how little pre-existing population there was. The blue color and small implied ages fit well with the large Lyman $\alpha / \mathrm{H} \alpha$ ratio required by IRTF narrow-band data. Are these only the currently bursting subset of a much richer population?

\section{Extended Lyman $\alpha$ Emission}

Three of the AGN show extended, elongated structures in line emission. While they were detected with WFPC2, the better surface-brightness sensitivity from the ground reveals new aspects. They extend to distances as large as $50 \mathrm{kpc}$ in one case. Two are well aligned with small-scale features seen in HST imagery, while the largest (object 18 of Pascarelle et al. 1996) shows a large asymmetric spray of gas not obviously related to the inner structure. Photon-counting arguments suggest that in at least object 18 , anisotropic photoionization is the culprit, so that we may speak of ionization cones.

\section{Size and structure of the group}

We measure the radial distribution of the candidate group/cluster members using the curve of growth of radius of the smallest circle including $N$ members as a function of $N$, compared to the Monte Carlo behavior of the same sample size extracted from a King model of known core radius. This frees us from needing a priori knowledge of the center (which such sparse sampling doesn't provide). The observed distribution is flatter than a King profile, whether normalized at the inner or outer end. Error bounds were evaluated from $10^{5}$ Monte Carlo trials. However we scale them, the data don't fit at the $90 \%$ level. At this confidence level, the grouping appears not to be viralized (yet?).

Acknowledgments. This work was supported by NASA through HST grants GO-07450* and allocation of IRTF timc. WCK acknowledges the role of the Delta SkyMiles program.

\section{References}

Armus, L. et al. 1999, in preparation

Bruzual A., G., \& Charlot, S. 1993, ApJ, 405, 538

Keel, W.C., Cohen, S.H., Windhorst, R.A., \& Waddington, I. 1999, AJ, submitted

Kinney, A.E., Calzetti, D., Bohlin, R.C.,McQuade, K., Storchi-Bergmann, T., \& Schmitt, H.R. 1996, ApJ, 467, 38

Pascarelle, S.M., Windhorst, R.A., Keel; W.C., \& Odewahn, S.C. 1996, Nature, 383,45 
Section F. The History of Star Formation 

The Hy Redshift Universe

ASP Conlerence Series, Vol. 193, 1999

A. J. Bunker \& W. J. M. van Breugel, eds.

\title{
Submillimeter-selected galaxies
}

\author{
A. W. Blain \\ Cavendish Laboratory, Madingley Road, Cambridge, CBз OHE, UK \\ Ian Smail \\ Department of Physics, University of Durham, South Road, Durham, \\ DH1 3LE, UK
}
R. J. Ivison
Department of Physics 89 Astronomy, University College London, Gower Street, London, WC1E 6BT, UK

J.-P. Kneib

Observatoire Midi-Pyrénées, 14 Avenue E. Belin, 31400 Toulouse, France

\begin{abstract}
The first generation of submillimeter(submm)-wave surveys arc bcing carried out using the $150 / 850 \mu \mathrm{m} \mathrm{SCUBA}$ camcra at the JCMT on Mauna Kea. These surveys are potentially sensitive to galaxies at very high redshift, and the galaxies that have been detected so far appear to contribute the greater fraction of the $\mathrm{mm} / \mathrm{submm}$-wave background radiation intensity measured by $C O B E$. In order to understand this now population of galaxies, individual examples must be studied in detail across many wavebands; in particular their redshifts must be determined. Wc discuss the potential selection effects at work in submm-wave surveys and describe the spectral energy distributions (SEDs) of galaxies selected or luminous in the submm waveband. We also describe the general procedure for, and cmphasize the difficulty of, identifying optical counterparts to submm-selected galaxies. Finally, we summarize what is known about the redshifts of these galaxies and the source of their luminosity.
\end{abstract}

\section{Introduction}

The first submm-wave surveys, designed to detect the redshifted restframe farinfrared(IR) radiation from warm dust in distant galaxies (Blain \& Longair 1993), have recently been carried out (Smail, Ivison \& Blain 1997; Barger et al. 1998, 1999b; Hughes et al. 1998; Blain et al. 1999b, 2000; Eales et al. 1999). These surveys are uniquely sensitive to high-redshift galaxies, and have been possible since the sensitive SCUBA camera (Holland et al. 1999) was commissioned at the JCMT in 1997. SCUBA is an imaging and photometric instrument, optimized to observe a 5 -arcmin ${ }^{2}$ field simultaneously in the 450 - and $850-\mu \mathrm{m}$ 
atmospheric transmission windows. The images produced have a resolution of about 15 arcsec at $850 \mu \mathrm{m}$ and 7 arcsec at $450 \mu \mathrm{m}$.

The intensity of $\mathrm{mm}$, submm and far-IR background radiation has been measured recently using data from the FIRAS and DIRBE instruments on the COBE satellite (Puget et al. 1996; Fixsen et al. 1998; Hauser et al. 1998; Schlegel, Finkbeiner \& Davis 1998; Finkbeiner, Davis \& Schlegel 1999). The results of the first galaxy surveys in the far-IR waveband, carried out using the ISO satellite, have also been reported recently (Kawara et al. 1998; Puget et al. 1999).

In this paper we review certain aspects of the existing submm-wave surveys, concentrating on the selection technique and the procedures for following up the submm-wave detections in other wavebands. We discuss whether any selection effects could act to prevent any types of high-redshift dusty galaxies being detected efficiently in submm-wave surveys, and compare all the welldetermined SEDs of submm-selected galaxies with those of other dusty galaxies and active galactic nuclei (AGNs). We discuss the difficulties of identifying the galaxy responsible for the detected submm emission, and show an example, the SCUBA-selected galaxy SMM J09429+4658 (Smail et al. 1999a), in which very deep multiwaveband images of the fields around the centroid of the SCUBA source were required in order to make progress in the identification. Finally, we discuss our current state of knowledge about the redshift distribution of the galaxies detected in SCUBA surveys, which is crucial for distinguishing between different models of galaxy evolution (Blain et al. 1999a,c), about the connections between the high-redshift SCUBA sources and the properties of dust in lowredshift galaxies, and about whether the SCUBA galaxies are powered by starformation activity or by AGN accretion. Unless otherwise stated we assume an Einstein-de Sitter world model with Hubble's constant $H_{0}=50 \mathrm{~km} \mathrm{~s}^{-1} \mathrm{Mpc}^{-1}$.

\section{Submm selection effects: spectral energy distributions (SEDs)}

The reason why a large fraction of the galaxies detected in submm-wave surveys are at high redshifts is the steep long-wavelength slope of the dust emission spectrum, which can be described well by a Rayleigh-Jeans thermal spectrum, modified by an emissivity function $\epsilon_{\nu}$. An emissivity with the form $\epsilon_{\nu} \propto \nu^{\beta}$, where $\beta \simeq 1.5$ provides a reasonable fit to the data, and so the submm-wave emission spectrum of a dusty galaxy $f_{\nu} \propto \nu^{\alpha}$, where $\alpha \simeq 3.5$. This steep spectrum leads to a strong negative $K$-correction, which at redshifts $z>0.5$ can be sufficient to overcome the geometric dimming due to the inverse square law, and lead to a flat flux density-redshift relation in the submm waveband. The SED is also defined by a dust temperature $T_{d}$. Low-redshift spiral galaxies generally have dust temperatures of about $15-20 \mathrm{~K}$, but there is evidence, from both individual high-redshift dusty galaxies and the background radiation spectrum, that hotter temperatures, $T_{d} \simeq 40 \mathrm{~K}$, are typical of the submm-selected galaxy population (see Section 4), at least as a luminosity-weighted mean temperature.

The strong submm-wave $K$-correction is illustrated in Fig. 1 . We show the flux density-redshift relation predicted for a dusty galaxy with a fixed bolometric luminosity in the restframe far-IR waveband for a variety of dust temperatures $T_{\mathrm{d}}$ and dust emissivity indices $\beta$. The SED template used is assumed to have a power-law slope $f_{\nu} \propto \nu^{-1.7}$ on the Wien side of the dust emission spectrum in the 

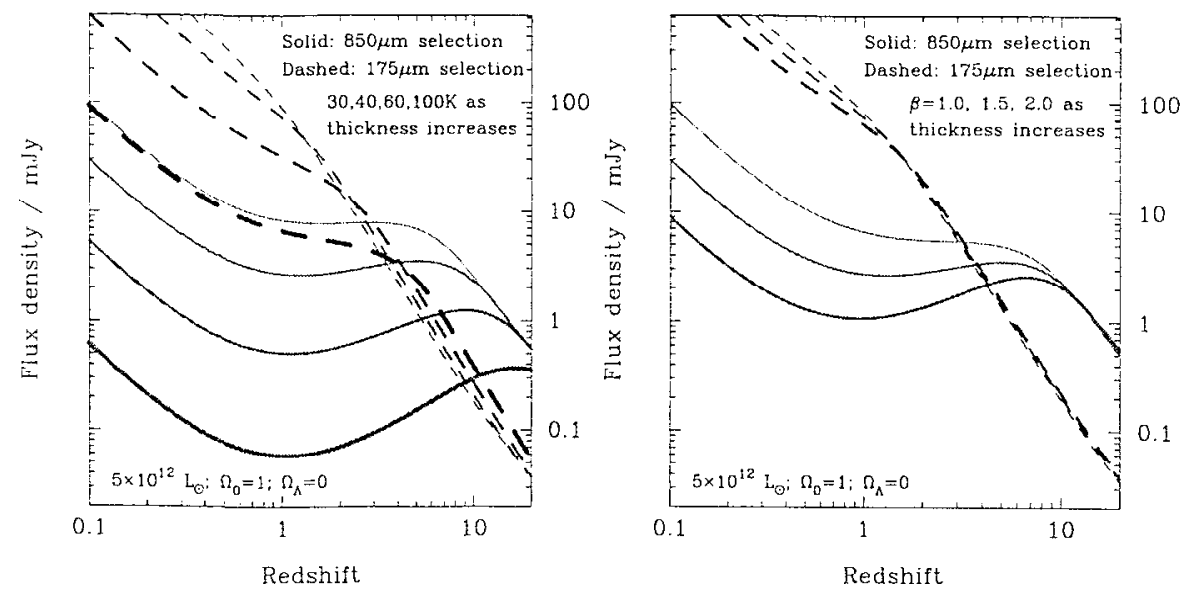

Figure 1. Flux density-redshift relations for dusty galaxies with fixed bolometric luminosities at wavelengths of 850 and $175 \mu \mathrm{m}$ in the submm and far-IR wavebands. Left: the emissivity index $\beta-1.5$ is fixed and the dust temperature $T_{\mathrm{d}}$ varies. Right: $T_{\mathrm{d}}=38 \mathrm{~K}$ is fixed and $\beta$ varies.

mid-IR waveband. This power-law slope takes account of the contribution made to the SED at shorter wavelengths by the populations of hotter dust grains in the interstellar medium (ISM) of the galaxy, and provide a good representation of very deep $15-\mu \mathrm{m}$ counts of galaxies determined using the ISO satellite (Altieri et al. 1999), see Blain et al. (1999a,c) for more information.

It is clear from the left-hand panel of Fig. 1 that the dust temperature has a significant effect on the detectability of a dusty galaxy with a fixed bolometric luminosity, both when observed close to the peak of the SED at $175 \mu \mathrm{m}$ in the far-IR waveband and at $850 \mu \mathrm{m}$ in the submm waveband. Hotter galaxies produce less submm-wave flux density per unit bolometric luminosity, and are thus less likely to be detected in the submm waveband. This effect is illustrated by a comparison of the $850-\mu \mathrm{m}$ flux densities, dust temperatures and bolometric luminosities of APM $08279+5255$ (Lewis et al. 1998) and IRAS F10214+4724 (Lacy et al. 1998). Although the $850-\mu \mathrm{m}$ flux density of APM $08279+5255$ is only a factor of 1.5 times greater than that of IRAS F10214+4724, because $T_{\mathrm{d}}>100 \mathrm{~K}$ in $\mathrm{APM} 08279+5255$ but only $\simeq 80 \mathrm{~K}$ in IRAS F10214+4724, the inferred luminosity of APM $08279+5255$ exceeds that of IRAS F10214+4724 by an order of magnitude.

It is interesting to note that the dust temperature that best accounts for the properties of the faint submm galaxy population (Blain et al. 1999a,c; Trentham et al. 1999) is close to $40 \mathrm{~K}$, which also seems to be typical of the dust in highredshift quasars and radio galaxies (Benford et al. 1999; Ivison et al. 1998a) and in IRAS galaxies (Lisenfeld, Isaak \& Hills 1999). It is possible therefore that an additional population of high-redshift galaxies could exist with hotter dust temperatures, that are underrepresented in submm-selected samples. Note that such a population would probably be too distant to have been detected using the ISO satellite at $175 \mu \mathrm{m}$. 


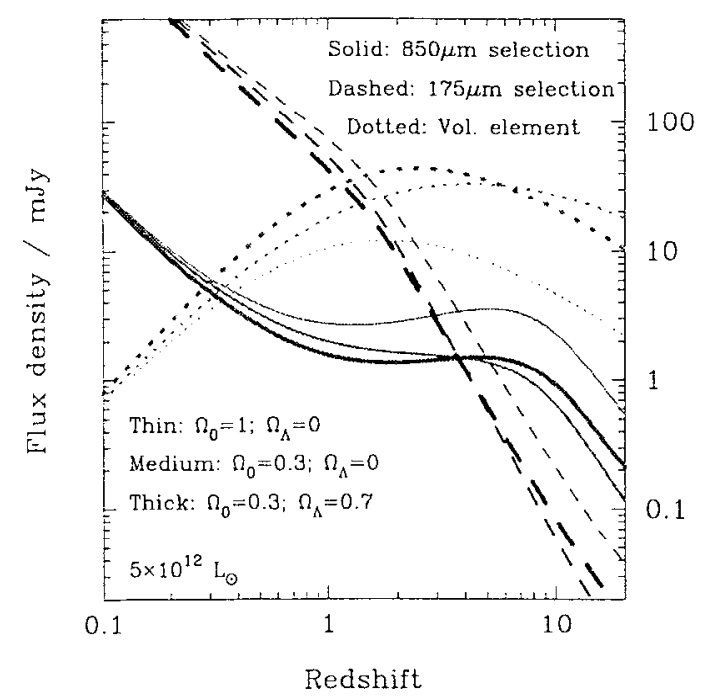

Figure 2. The flux density-redshift relation for a dusty galaxy with a dust temperature of $38 \mathrm{~K}$ and an emissivity index $\beta=1.5$ in three different cosmological models. The relative size of the volume element as a function of redshift is also shown in each model, in arbitrary units.

The effect of varying the emissivity index of the dust spectrum is shown in the right-hand panel of Fig. 1. The value of the index has little effect on the detectability of galaxies at a far-IR wavelength of $175-\mu \mathrm{m}$, which is close to the restframe peak of the SED; however, the effects are more significant at a submm wavelength of $850 \mu \mathrm{m}$. Low-redshift galaxies would be more likely to be detected in a SCUBA survey if the emissivity index $\beta$ is low.

\section{Submm selection effects: cosmology}

Because submm-wave surveys probe the Universe at $z \geq 1$, the effects of different world models on the observability of dusty galaxies could be significant. The effects are illustrated in Fig. 2 , in which the flux density-redshift relation for a dusty galaxy with a fixed template SED and a fixed bolometric luminosity is compared in three different world models. The $K$-correction is less dramatic in the two $\Omega_{0}<1$ models as compared with the Einstein-de Sitter model, and so in these two models, the intrinsic luminosity of a high-redshift dusty galaxy detected at a certain $850-\mu \mathrm{m}$ flux density will be greater. However, the effect of the world model on the interpretation of submm-wave source counts is not very significant. This is because the volume element is also larger in the two $\Omega_{0}<1$ models, and so, in order to avoid overpredicting the measured source counts, the evolution of either the space density or luminosity of the population of submm-selected galaxies must be less dramatic in these models. The effects of an increased luminosity per unit flux density and a less dramatic form of evolution will largely counteract each other, and so the general conclusions about evolution are unchanged. 
When accurate counts and redshift distributions are available for submmselected galaxies, and their SEDs are known, it will be possible to investigate the effects of different cosmological models. However, at present the uncertainties due to the SED are greater than those due to the world model.

\section{The SEDs of submm galaxies with known redshifts}

In Fig. 3 we compare the observed restframe SEDs of luminous dusty galaxies with known redshifts and two template SEDs derived for use in galaxy evolution models. The Guiderdoni et al. (1998) model was compiled from a large sample of SEDs of IRAS galaxies, and the Blain et al. (1999c) Inodel was derived from the counts of IRAS and ISO galaxies at 60 and $175 \mu \mathrm{m}$. Observed SEDs for several classes of submm-luminous source are shown: low-redshift IRAS galaxies, for which SEDs are available right across the submm, far- and mid-IR wavebands; submm-selected galaxies, of which four have known redshifts; lensed quasars; and both optical- and radio-selected high-redshift AGN. The optical-selected extremely red object (ERO) HR10 (Dey et al. 1999) is included as a submmselected galaxy, as it would have been identified in the current surveys.

Both of the model spectra shown in Fig. 3 appear to provide reasonable descriptions of most of the galaxies, and all of the submm-selected ones. The exceptions are the sources that are knowil to be strongly lensed by foreground galaxies and some of the high-redshift active galaxies, in which the dust temperature is considerably higher than $40 \mathrm{~K}$. However, it is reasonable to have a greater mid-IR luminosity from these objects, due both to intense heating by an AGN and to differential magnification across the Inucleus of the source. Both of these processes would be expected to increase the dust temperature required to fit the observed SED (Eisenhardt et al. 1996; Blain 1999a). In general, an SED model with $T_{\mathrm{d}} \simeq 40 \mathrm{~K}$ and $\beta \simeq 1.5$ seems to describe the observed SEDs of subinn-selected dusty galixies reasonably well.

A lower value of $\beta=1$ was assumed by Eales et al. (1999) to help explain the significant fraction of galaxies at $z<1$ identified by Lilly et al. (1999) in their SCUBA survey of the CFRS fields. Very few galaxies are expected to be detected at these low redshifts based on models with $\beta=1.5$ (Blain et al. 1999c), and in deep multiwaveband follow-up observations of fifteen background sources in the fields of seven lensing clusters (Sinail et al. 1997, 1998, 1999a) none are found at $z<1$. The most appropriate value of $\beta$ will be clarified by further observations, which will also reveal whether some of the low-redshift galaxies in the CFRS fields could be mis-identified.

\subsection{The population of submm-luminous galaxies at low redshifts}

Faint submm-wave surveys are currently only possible in small fields, and so probe very high-redshift pencil beams. It is also interesting to investigate the abundance, distribution and properties of dust in low-redshift galaxies, which reflects the evolutionary processes at work, both star formation and chemical evolution; in the ISM of galaxies throughout their history. Dunne et al. (1999) have recently reported the results of a survey of typical low-redshift IRAS galaxies, for which they obtained 450- and $850-\mu \mathrm{m}$ submm-wave SCUBA photometry to investigate the dust temperature and spectral index. Note, however, that 


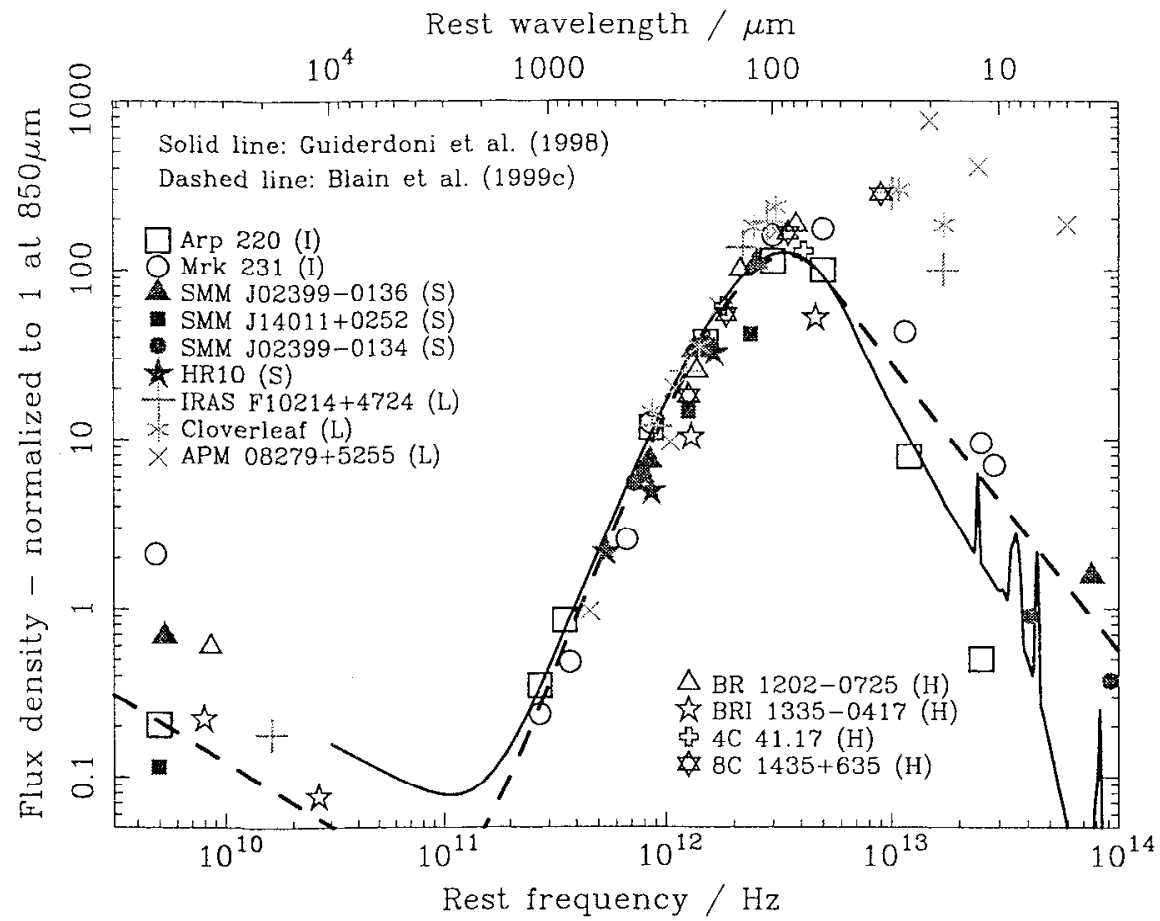

Figurc 3. The SEDs of a range of dusty galaxics with known redshifts (points), and two model spectra, that are chosen to accord with IRAS and ISO data (lines). There are four types of galaxies; low-redshift IRAS galaxies (I), sub-mm luminous galaxies (S), distant sources lensed by a foreground galaxy (L), and high-redshift radio-loud and radioquiet $\mathrm{AGN}(\mathrm{H})$. The SED models include a dust emissivity index $\beta \simeq$ 1.5 and a dust tcmperature $T_{\mathrm{d}} \simeq 40 \mathrm{~K}$. Only the lensed galaxies and quasars, and some of the high-redshift AGN depart significantly from the template $S E D s$ in the restframe mid-IR waveband.

at $z=0$ the SEDs of even normal spiral galaxies, with dust temperatures as low as $20 \mathrm{~K}$, are probed by SCUBA quite a long way down the Rayleigh--Jeans slope of the dust SED, and that their bolometric luminosity is constrained by the 60- $\mu \mathrm{m}$ IRAS data. The results will be interesting, but should have no dramatic consequences for the interpretation of the high-redshift SCUBA surveys, in which the detected galaxies are both observed at significantly shorter restframe wavelengths, closer to the peak of their restframe SED, and are much more luminous.

\section{The identification of optical counterparts}

Unusually, because of the flat flux density-redshift relation illustrated in Fig. 1, a measurement of the submm-wave flux density of a distant galaxy can be translated into a luminosity directly, independent of redshift, subject to an uncertain value of the dust temperature and emissivity index. However, a crucial step in 
understanding the nature of submm-selected galaxies is still the determination of their redshifts, individually and as a population. A problem arises because the SCUBA beam is $15 \operatorname{arcsec}$ in size at $850 \mu \mathrm{m}$, and so there could easily be more than 5 plausible faint optical counterparts within 3 arcsec of the centroid of a SCUBA detection in images as deep as the Hubble Deep Field (HDF) see Hughes et al. (1998) and Downes et al. (1999). Identifying the correct optical counterpart will in general be very difficult, especially as a priori submm-selected galaxies would be expected to have large internal extinctions and thus to be optically faint: see for example the source SMM J14009+0252 (Ivison et al. 1999) and the brightest source in the SCUBA map of the HDF (Downes et al. 1999), which currently have no plausible optical counterparts.

Sensitive radio observations are very useful for improving the chances of making a correct association, as the maximum resolution of the VLA at $1.4 \mathrm{GHz}$ is about 1 arcsec. Any submm-selected galaxy should be detectable in a sufficiently deep radio image, based on the the observed low-redshift radio-far-IR correlation (Condon 1992), which links the synchrotron radio emission from supernova remnants and the submm-wave dust emission powered by young highmass stars. The consequences for locating submm sources and estimating their redshifts are discussed by Carilli \& Yun (1999), Blain (1999b) and Carilli et al. (1999). This technique has been applied to a submm-selected sample by Smail et al. (1999b) and to a radio-selected, $K$-band faint sample by Barger, Cowie \& Richards (1999a). Note, however, that extremely deep radio observations are required in order to detect the current population of submm-selected sources. Only seven of Smail et al.'s fifteen SCUBA galaxies have 1.4-GHz detections, the rest only upper limits (Smail et al. 1999b).

Plausible optical counterparts can be identified by overlaying the submm image with as many deep radio, near-IR and optical images as are available. Optical or near-IR spectroscopic redshifts are then required for the candidates. With such intrinsically faint sources, this is in general difficult, even using the largest telescopes (Ivison et al. 1998b; Barger et al. 1999c). The likelihood of an association being correct can be assessed based on photometric or positional arguments (Hughes et al. 1998; Smail et al. 1998); however, the final confirmation of the correct identification comes from the detection of molecular gas in interferometric mm-wave observations (Frayer et al. 1998, 1999). At 90-GHz the OVRO Millimeter Array has a resolution of several arcsec, a few times finer than SCUBA, and a bandwidth of $1 \mathrm{GHz}$. Hence, if the redshift of a plausible counterpart is known to better than $1 \%$, ideally from near-IR spectroscopy of low-excitation lines, then the array can be tuned to the frequency of an appropriate $\mathrm{CO}$ rotation line at that redshift. If the line is detected at the position of the optical counterpart, then the presence of a large mass of molecular gas coincident in position and redshift ties together the optical and submm emission and confirms the identification.

In only two cases has this procedure been completed, for SMM J02399-0136 (Ivison et al. 1998b; Frayer et al. 1998) at $z=2.8$ and SMM J14011+0252 (Ivison et al. 1999; Frayer et al. 1999) at $z=2.6$. Another source, SMM J02399-0134, has a very good candidate at $z=1.06$ (Barger et al. 1999c), with a blue ring galaxy morphology, distorted by the magnification of the chister Abell 370, and a coincident 15- $\mu \mathrm{m}$ ISO detection (Soucail et al. 1999). 


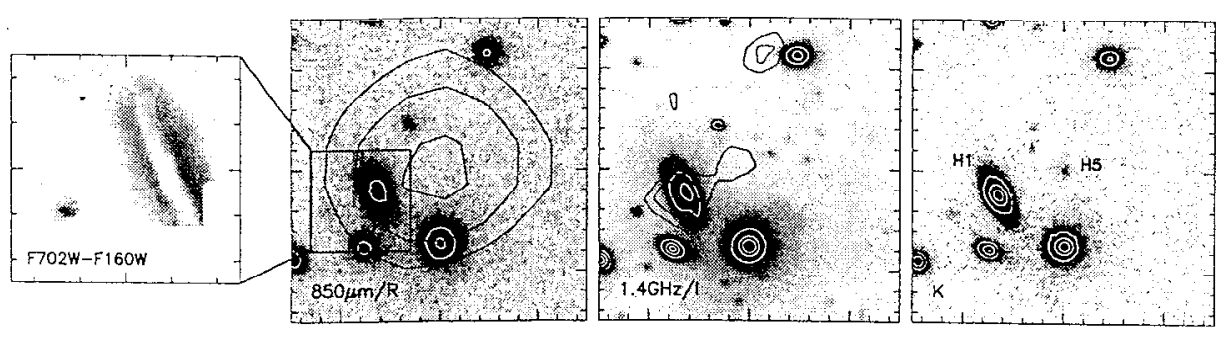

H'igure 4. A multiwaveband view of the field of the submm galaxy SMM J09429+4658 (Smail et al. 1999a). The three larger images are 30 arcsec on a side and are centred on the position of the centroid of the 850- $\mu \mathrm{m}$ detection. From left to right: An HST difference image showing the dust lane in the spiral galaxy $H 1$; SCUBA $850-\mu \mathrm{m} s u b m m$ emission (black contours; 3,6 and $9 \sigma$ ) overlaid on a Hale 5-m Gunn$r$ image; VLA 1.4-GHz radio emission (black contours) overlaid on a deep Keck-II $I$-band image; and a UKIRT $K$-band image. The relative radio and optical astrometry is accurate to less than 0.4 arcsec.

With fluxes at or below the faintest detectable levels of radio and optical flux density, many of the counterparts of SCUBA sources necessarily remain enigmatic. The typical achromatic magnification by a factor of 2.5 experienced by background sources in the fields of rich clusters, and the associated expansion of the background sky by the same factor, has proved to be a great advantage for making follow-up observations.

\subsection{SMM J09429+4658: a case study}

The SCUBA source SMM J09429+4658, for which an array of high-quality data is available, has proved to be a very interesting case study in making identifications of submm sources. In Fig. 4 we show all the excellent multiwaveband data that has been compiled for this galaxy in the field of the $z=0.41$ cluster $\mathrm{Cl} 0939+4713 / \mathrm{A} 851$. A partial two-colour HST image, a Hale $R$-band image, a very deep 1.4-GHz VLA map, a deep Keck-II I-band image and a UKIRT Kband image are available (Smail et al. 1999a). These images are extremely deep: after correcting for lens magnification, the $1 \sigma$ sensitivities are $3.6 \mu \mathrm{Jy}_{\mathrm{y}}$ beam ${ }^{-1}$ at 1.4- $\mathrm{GHz}$ and $I=27$.

In the initial search for optical counterparts to the SCUBA galaxies (Smail et al. 1998), the dusty spiral galaxy $\mathrm{H} 1$ at $z=0.33$ in the foreground of the cluster was identified as a possible counterpart, despite being 7 arcsec away from the SCUBA centroid and having no strong optical line emission (Barger et al. 1999c). When a deep 1.4-GHz VLA radio map was obtained, two radio sources were found within the SCUBA positional uncertainty: $\mathrm{H} 1(5 \mathrm{~T} \mu \mathrm{Jy})$ and a new source $\mathrm{H} 5(36 \mu \mathrm{Jy})$, which has no optical counterpart but is aligned exactly with the SCUBA centroid. If $\mathrm{H} 1$ is the true counterpart, then unless its dust temperature is less than about $13 \mathrm{~K}$, it lies well below the far-IR-radio correlation at $z=0.33$; however, if $\mathrm{H} 5$ is the true counterpart, then its radio-submm flux ratio is typical of a $40-\mathrm{K}$ dusty galaxy at $z \geq 3.5$ (Blain 1999b; Smail et al. 1999a). Unfortunately H5, lies off the edge of the HST image, and it has no 
optical counterpart in a very deep Keck-II $I$-band image. Only the acquisition of a deep UKIRT $K$-band image revealed a source at the $\mathrm{H} 5$ position. Keck NIRSPEC spectroscopy and, if a spectroscopic redshift is obtained, mm-wave observations of redshifted $\mathrm{CO}$ emission from $\mathrm{H} 5$ will hopefully confirm the identification over the 1999-2000 winter. The follow-up observations of this galaxy illustrate the importance of obtaining very deep multiwaveband data in order to identify SCUBA galaxies, especially radio and near-IR images.

\section{The redshift distribution of submm-selected galaxies}

The determination of the redshift distribution of submm-selected galaxies is crucial in order to constrain models of galaxy evolution (Blain et al. 1999c). There is considerable degeneracy between different models of the population of distant dusty galaxies that can all account for both the observed spectrum of $\mathrm{mm}$, submm and far-IR bachground radiation and for the $450-$ and $850-\mu \mathrm{m}$ counts of galaxies. The degeneracy can be broken most easily and definitively by determining the redshift distribution of the submm galaxies. Several different approaches have so far been taken to try and tackle this difficult problem.

Smail et al. (1998) made plausible identifications of optical sources in deep $H S T$ and ground-based images of the fields of submm-selected galaxies behind seven rich clusters of galaxies. The lack of $V$-band dropont sources indicated that $80 \%$ of the proposed counterparts were probably at $z<5$. Using Keck-II spectroscopy of this incomplete sample, Barger et al. (1999c) found a median redshift $\bar{z} \simeq 1.7$ for these counterparts. However, as illustrated by the detection of two EROs in the fields (Smail et al. 1999a), which were unknown at the time, this is likely to be a lower limit, as correct counterparts are more likely to be missing from the sample at higher redshifts. $\bar{z} \simeq 2.5-3.0$ is indicated by a Carilli $\&$ Yun analysis of the radio-submm flux density ratios of the SCUBA galaxies derived from very deep VLA images of the fields (Smail et al. 1999b).

Hughes et al. (1998) identified plausible faint optical counterparts to the brightest sources in the SCUBA $H D F$ image, and deduced from photometric arguments that the redshifts of the sources were in the range $2<z<4$. Working from a deep 1.4-GHz VLA image, Richards (1999) suggested a systematic offset of the SCUBA astrometry, and a lower typical redshift. A subsequent high-resolution mm-continuum map of this source made using the IRAM interferometer (Downes et al. 1999) has provided a more accurate position, ruling

out both of the counterparts suggested by Hughes and Richards, but suggesting that the source is gravitationally lensed by an elliptical galaxy. A very high redshift is potentially indicated for this source by the lack of a detectable optical counterpart in the $H D F$ at this exact position.

Using a variety of optical, mid-IR and radio data, Lilly et al. (1999) investigated the SCUBA sources detected in the CFRS fields (Eales et al. 1999), and found a significant fraction $(\simeq 30 \%)$ of plausible counterparts at $z<1$. This is a lower redshift distribution than reported in other surveys. Misidentification of some of these $z<1$ galaxies remains a possibility; further follow-up observations should resolve this apparent discrepancy.

Recently, Barger et al. (1999a) made SCUBA maps of the areas around the $1.4-\mathrm{GHz}$ radio sources as faint as $40 \mu \mathrm{Jy}$ in the HDF flanking fields with 
no near-IR counterparts down to $K=20.5$. By using Carilli \& Yun's radiosubmm redshift estimator they derived redshifts in the range $1<z<3$ for all six of the galaxies they detected using SCUBA. When the area of the submm maps and the number of detections are compared, it seems likely that there is a significant overlap between this population of near-IR-blank faint radio sources and the bright ( $>8 \mathrm{mJy}$ ) SCUBA galaxies. However, this radio-near-IR selection technique will tend to bias the sample as compared with a submmselected sample, by enhancing the fraction of radio-loud objects and $A G N$.

At present, there are only two submm-selected galaxies with certain redshifts, SMM J02399-0136 and SMM J14011+0252 at redshifts $z=2.8$ and 2.6 respectively. This information alone suggests that the submm-selected population as a whole is probably at a significant redshift. Other techniques based on statistical arguments, tend to support the idea that $2 \leq \bar{z} \leq 4$ for the SCUBA galaxies, and that there is probably a tail of sources at higher redshifts.

\section{The power source of submm-selected galaxies}

It is clear from the observed SEDs of submm-selected galaxies that thermal emission from warm dust grains is being detected. However, it is an open question whether the power source heating the grains is the radiation from young stars or from the accretion disk of an AGN. The form of the broad-band submm and far-IR SED provides no information about this question. However, at shorter mid-IR wavelengths the slope of the continuum SED may tend to be harder in sources which are heated by AGN, for example compare the different slopes of Arp 220 and Mrk 231 in Fig. 3. Also, the properties of PAH spectral features observed in nearby IRAS galaxies by the ISO satellite (Lutz et al. 1999), can provide an indication of the power source, because the large molecules that produce them should not survive in the hard UV continuum radiation field expected in the core of an AGN. Lutz et al. (1999) conclude that $70 \%$ of the far-IR radiation from these galaxies appears to be powered by star formation.

At present, direct observations of high-redshift submm-selected galaxies and of the X-ray background tend to support the continuation of this relative contribution out to high redshifts. One of the two best-studied SCUBA-selected galaxies (SMM J02399-0136; Ivison et al. 1998) shows clear signs of AGN activity, but the other (SMM J14011+0252; Ivison et al. 1999) does not. Barger et al. (1999c) see evidence for high-excitation optical line emission, typical of AGN activity, in about $20 \%$ of their relatively shallow Keck spectra of plausible optical counterparts to submm-selected galaxies (Smail et al. 1998). Note, however, that these are the easiest optical emission lines to detect, and so the AGN fraction in a complete sample may differ. A fraction of the optical counterparts targeted using the Keck will also certainly have been misidentified, leaving the true optical counterparts to the SCUBA galaxies unobserved. Almaini, Lawrence \& Boyle (1999) show that it is most plausible to explain the relative intensity of hard and soft X-ray background radiation by a population of high-redshift dust-enshrouded AGN, which can account for 10-20\% of the SCUBA sources.

It is not yet definite, but it seems likely that the majority of the emission making up the far-IR background and the counts of submm galaxies is powered by young high-mass stars, with a less significant contribution from AGN. 


\section{Conclusions}

1. A significant population of high-redshift galaxies with powerful dust emission has been discovered since the commissioning of SCUBA, the first submm-wave bolometer array camera.

2. The surface density of these galaxies is such that a significant fraction of the diffuse extragalactic background radiation detected by the $C O B E$ satellite has already been resolved into discrete sources.

3. The SEDs of the SCUBA galaxies and other submm-luminous distant galaxies are generally consistent with a dust tempcraturc $T_{\mathrm{d}} \simeq 40 \mathrm{~K}$.

4. If there is a population of hotter submm-luminous sources, then these galaxies are less likely to be detected by SCUBA. At present there is little evidence that there is a significant population of such sources.

5. It is difficult and time consuming to identify and detect the current population of submm-selected galaxies in other wavebands. Only two such galaxies have so far been identified beyond reasonable doubt.

6. It seems likely at present that star-formation activity dominates AGN accretion as a power source for submm-selected galaxies in the approximate proportion 2 or $3: 1$. The AGN-powered subset will hopefully soon be detected in high-resolution Chamulu and $X M M \mathrm{X}$-ray observations.

Acknowledgments. The work described here is largely based on the results of the SCUBA Cluster Lens Survey, which has been lead by the authors, with collaborative support from Any Barger, Jocelyn Bézecourt, Len Cowie, Aaron Evans, Dave Frayer, Allon Jameson, Jean-Francois Le Borgne, Malcolm Longair, Glenin Morrison, Frazer Ower, Nick Scoville, Paul van der Werf and Min Yun. AWB thanks the conference organizers for their hospitality and for support during the meeting, and Chris Carilli for helpful comments. This research has made use of the NASA/IPAC Extragalactic Database (NED) which is operated by JPL, Caltech, under conlract with NASA.

\section{References}

Almaini, O., Lawrence, A., \& Boyle, B. J., 1999, MNRAS, 305, Lð9

Altieri, B., Metcalfe, L., Kneib J.-P., et al. 1999, A\&A, 343, L65

Barger, A. J., Cowie, L. L., Sanders, D. B., et al., 1998, Nature, 394, 248

Barger, A. J., Cowie, L. L., \& Richards, E. A., 1999a, (astro-ph/9907022)

Barger, A. J., Cowie, L. L., \& Sanders, D. B., 1999b, Ap.J, 518, L5

Barger, A. J., Cowie, L. L., Smail, I., et al., 1999c, AJ, 117, 2656

Benford, D. J., Cox, P., Omont, A., et al., 1999, Ap.J, 518, L65

Blain, A. W., 1999a, MNRAS, 304,669

Blain, A. W., 1999b, MiNRAS, in press (astro-ph/9906438)

Blain, A. W., \& Longair, M. S., 1993, MNRAS, 264, 509 
Blain, A. W., Jameson, A., et al., 1999a, MNRAS, in press, (astro-ph/9906311)

Blain, A. W., Kneib, J.-P., Ivison, R.J., \& Smail, I., 1999b, ApJ, 512, L87

Blain; A. W., Smail, I., Ivison, R. J., \& Kneib, J.-P., 1999c, MNRAS, 302, 632

Blain, A. W., Ivison, R. J., et al., 2000, this volume (astro-ph/9908024)

Carilli, C. L., \& Yun, M. S., 1999, Ap.J, 513, L13

Carilli, C. L., et al., 1999, conference proceeding, (astro-ph/9907436)

Condon, J. J., 1992, ARA\&A, 30, 575

Dey, A., Graham, J. R., Ivison, R. J., ct al., 1990, ApJ, 519, 610

Downes, D., Neri, R., Greve, A., et al., 1999, A\&A, 347, 809

Dunne, L., et al., 1999, MNRAS, submitted

Eales, S. A., Lilly, S. J., Gear, W. K., et a1., 1999, ApJ, 515, 518 :

Eisenhardt, P., Armus, L., Hogg, D. W., et al., 1996, ApJ, 461, 72

Finlbcincr, D., Davis, M., \& Schlegel, D., 1999, ApJ, in press, (astro-ph/9905128)

Fixsen, D. J., Dwek, E., Mather, J. C., et al., 1998, ApJ, 508, 123

Frayer, D. 'I', Ivison, R. J., Scoville, N. Z., et al., 1998, ApJ, 506, Li

Frayer, D. T., Ivison, R. J., Scoville, N. Z., et al., 1999, ApJ, 514, L13

Guiderdoni, B., Hivon, E., et al., 1998, MNRAS, 295, 877

Hauser, M. G., Arendt, R. G., Felsall, T., et al., 1998, ApJ, 508, 25

Holland, W. S., Robson, E. I., Gear, W. Ǩ., et al., 1999, MNRAS, 303, 659

Hughes, D., Serjeant, S., Dunlop, J., et al., 1998, Nature, 394, 241

Ivison, R. J., Dunlop, J. S., Hughes, D. H., et al., 1998a, ApJ, 494, 211

Ivison, R. J., Smail, I., Le Borgne, J.-F., et al., 1998b, MNRAS, 298, 583

Ivison, R. J., Smail, I., Barger, A. J., et, al., 1999, MNRAS, submitted

Kawara, K., Sato, Y., Matsuhara, H., et al., 1998, A\&A, 336, L9

Lacy, M., Rawlings, S., \& Serjeant, S., 1998, MNRAS, 299, 1220

Lewis, G. F., Chapman, S. C., Ibata, R. A., et al., 1998, ApJ, 505, L1

Lilly, S. J., Eales, S. A., Gear, W. K. P., et al., 1999, ApJ, 518, 641

Lisenfeld, U., Isaak, K. G., Hills, R., 1999, MNRAS, in press (astro-ph/9907035)

Lutz, D., Spoon, H. W. W., Rigopoulou, D., et al., 1999, ApJ, 505, L103

Puget, J.-L., Abergel, A., Bernard, J.-P., et al., 1996, A\&A, 308, L5

Puget, J.-L., Lagache, G., Clements, D. L., et al., 1999, A\&A, 345, 29

Richards, E. A., 1999, ApJ, 513, L9

Schlegel, D. J., Finkbeiner, D. P., \& Davis, M., 1998, ApJ, 500, 525

Smail, I., Ivison, R. J., \& Blain, A. W., 1997, ApJ, 490, L5

Smail, I., Ivisurl, R. J., Blain, A. W., \& Kneib J.-P., 1998, Ap., 507, L21

Smail, I., Ivison, R. J., et al., 1999a, MNRAS, in press (astro-ph/9905246)

Smail, I., Ivison, R. J., et al., 1999b, Ap.J, submitted (astro-ph/9907083)

Soucail, G., Kneib, J.-P., Bézecourt, J., et al., 1999, A\&A, 343, L70

Trentham, N., Blain, A. W., \& Goldader, J., 1999, MNRAS, 305, 61 
The Hy Redshift Universe

ASP Conference Series, Vol. 193, 1999

A. J. Bunker \&s W. J. M. van Breugel, eds.

\section{$15 \mu \mathrm{m}$ Extragalactic Surveys: Source Counts and Properties}

D. Elbaz ${ }^{1,2}$, C.J. Cesarsky ${ }^{1,3}$, D. Fadda ${ }^{1}$, H. Aussel ${ }^{1,4}$, F.X. Désert ${ }^{5}$, A. Franceschini $^{6}$, H. Flores ${ }^{1}$, M. Harwit ${ }^{\top}$, J.L. Puget ${ }^{8}$, J.L. Starch ${ }^{1}$, L. Danese $^{9}$, D.C. Koo ${ }^{10}$ and R. Mandolesi ${ }^{11}$

${ }^{1}$ CEA-Saclay, SAp/DAPNIA/DSM, ${ }^{2}$ UCSC, Physics Department, Santa Cruz, CA 95064, USA

${ }^{3}$ European Southern Observatory, Karl-Schwarzchild-Straße, 2 D-85748 Garching bei München, Germany

${ }^{4}$ Osservatorio di Padova, Vicolo Osservatorio, 5, I35122 Padova, Italy

${ }^{5}$ Observatoire de Grenoble, BP 53, 414 rue de la piscine, 38041 Grenoble Cedex 9, France

${ }^{6}$ Dipartimento di Astronomia, Università di Padova, Vicolo dell'Osservatorio, 5, I35122 Padova, Italy

${ }^{7} 511$ H Street S.W., Washington, DC 20024-2725; also Cornell University, USA

${ }^{8}$ Institut d'Astrophysique Spatiale, Université Paris XI, F-91405 Orsay Cédex, France

${ }^{9}$ SISSA, via Beirut 2-4, T-34013 Trieste, , Ttaly

${ }^{10}$ UCO/Lick Obs., University of California, Santa Cruz, CA 95064, $U S A$

${ }^{11}$ Bologna Radio Astronomy Institute, via Gobetti 101, 40129 Bologna, Italy.

Abstract. We present the results of the five mid-infrared $15 \mu \mathrm{m}$ (12$18 \mu \mathrm{m}$ LW3 band) ISOCAM Guaranteed Time Extragalactic Surveys performed in the regions of the Lockman Hole and Marano Field. The roughly 1000 sources detected, 614 of which have a flux above the $80 \%$ completeness limit, guarantee a very high statistical significance for the intcgral and differential source counts from $0.1 \mathrm{mJy}$ up to $\sim 5 \mathrm{mJy}$. By adding the ISOCAM surveys of the HDF-North and South (plus flanking ficlds) and the lensing cluster A2390 at low fluxes and IRAS at high fluxes, we cover four decades in flux from $50 \mu \mathrm{Jy}$ to $\sim 0.3 \mathrm{Jy}$. The slope of the differential counts is very steep $(\alpha=-3.0)$ in the flux range 0.4$4 \mathrm{mJy}$, hence much above the Euclidean expectation (no expansion, no curvature) of $\alpha=-2.5$. When comparcd with no-evolution models based on IRAS, our counts show a factor $\sim 10$ excess at $400 \mu \mathrm{Jy}$, and a fast convergence, with $\alpha=-1.6$, at lowcr fluxes. 
They prolongate the IRAS image of the infrared universe four orders of magnitude fainter in the mid-IR. Being limited to redshifts below . $z \sim 1.6$ due to $k$-correction, the following results may not seem to reveal the nature of the 'high redshift universe' highlighted during this conference. However, we wish to demonstrate that they reveal a fundamental constituent in the understanding of galaxy evolution, namely the dust, which affects not only a few local ultra-luminous galaxies but probably most of the past star formation. The following text originates from Elbaz et al. (1999), except for the discussion of the nature of the $15 \mu \mathrm{m}$ galaxies.

\section{Introduction}

Deep galaxy counts as a function of magnitude, or flux density, should, in principle, give a constraint on the geometry of the universe $\left(\Omega_{0}, \Lambda_{0}\right)$. In fact, their departure from the Euclidean expectation (no expansion, no curvature) is dominated by the e-correction (intrinsic evolution of the galaxies) and by the $k$-correction (redshift dependence). The understanding of galaxy evolution therefore is a key problem for cosmology. Number counts present the advantage of setting strong constraints on galaxy evolution models, without suffering from the peculiar behavior of each individual galaxy. In the local universe, galaxies radiate mainly in the optical-UV energy range (Soifer \& Neugebauer 1991). If this remained true over the whole history of the universe, then one could follow the comoving star formation rate of the universe as a function of redshift by measuring the optical-UV light radiated by galaxies (Lilly et al. 1996, Madau et al. 1996).

This scenario changed considerably after the detection of a substantial diffuse cosmic infrared background (CIRB) in the $0.1-1 \mathrm{~mm}$ range from the COBE-FIRAS data (Puget et al. 1996, Guiderdoni et al. 1997, Hauser et al. 1998, Fixsen etal. 1998, Lagache et al. 1999) and at $140-240 \mu \mathrm{m}$ from the COBE-DIRBE data (Hauser et al. 1998, Lagache et al. 1999). Surprisingly the mid-IR/sub-mm extragalactic background light is at least as large as that of the UV/optical/near-IR background (Dwek et al. 1998, Lagache et al. 1999), which implies a stronger contribution of obscured star formation at redshifts larger than those sampled by IRAS (limited to $z<0.2$ ). In order to understand the exact origin of this diffuse emission and its implications for galaxy evolution, it needs to be resolved into its individual galaxies which requires direct observations in the IR/sub-mm range (since correcting for dust extinction is a complex and non secure way).

Since dust emission starts to dominate the spectral energy distribution (SED) of most galaxies around $6 \mu \mathrm{m}$, the galaxies responsible for the CIRB must also appear in the mid-IR (i.e. $5-20 \mu \mathrm{m}$ for ISOCAM) as long as their redshift is smaller than $z \sim 1.6$. This is particularly true for luminous infrared galaxies (LIGs), which exhibit a bolometric luminosity greater than $10^{11} \mathrm{~L}$. mostly radiated in the infrared domain. While the detection of such galaxies was limited to the closeby universe with IRAS $(z<0.2$, Sanders \& Mirabel 1996) becanse of its sensitivity, ISOCAM (Cesarsky et al. 1996), the camera on-board ISO (Fessler et al. 1996), with a thousand times better sensitivity and sixty 
times better spatial resolution, provides for the first time the opportunity to perform cosmologically meaningful surveys limited only by the disappearance of dust emission due to $k$-correction above $z \sim 1.6$, instead of sensitivity. Deep surveys have been carried out on small fields containing sources well known at other wavelengths: the HDF North (Serjeant et al. 1997, A11ssel et al. 1999, Désert et al. 1999) and the CFRS $1452+52$ (Flores et al. 1999). This has yielded a small but meaningful sample of sources ( 83 galaxies) with a positional accuracy better than 6", sufficient for most multiwavelength studies. Optical counterparts are easily identifiable for these sources, precisely because their maximum redshift is limited by the $k$-correction to $z \sim 1.6$, and that they appeared to be intrinsically bright $\left(L_{b o l} \sim\right.$ few $\left.10^{11} \mathrm{~L}_{\odot}\right)$ and massive $\left(M \sim 10^{11} M_{\odot}\right.$, estimated from their optical and near-IR magnitudes) galaxies (Aussel et al. 1999a,b, Flores et al. 1999). Flores et al. (1999) estimate, from their sample of 41 sources, that accounting for the IR light from star forming galaxies may lead to a global star formation rate which is 2 to 3 times larger than assessed from UV light only.

To obtain reliable source count diagrams, better statistics and a wider range of flux densities are required. For this reason, we have performed several cosmological surveys with ISOCAM, ranging from large arca shallow surveys to small area-ultra deep surveys. These surveys were obtained in the two main broad-band filters LW2 $(58.5 \mu \mathrm{m})$ and LW3 $(12-18 \mu \mathrm{m})$, contcred at $6.75 \mu \mathrm{m}$ and $15 \mu \mathrm{m}$ respectively. This paper only presents the source counts at $15 \mu \mathrm{m}$, because the sample of galaxics detected in the $6.75 \mu \mathrm{m}$ band is strongly contaminated by Galactic stars, whose secure identification requires ground-based follow-up observations. Including the surveys over the two Hubble dcep fields, almost 1000 sources with flux densities ranging from $0.1 \mathrm{mJy}$ to $10 \mathrm{mJy}$ were detected, allowing us to establish detailed source count diagrams.

We will not discuss the modeling of these source counts, since the strong evolution they revealed can hardly be reproduced by published models. However, we will discuss their compatibility with those obtained in the far-infrared with ISOPHOT, at $175 \mu \mathrm{m}$, and in the sub-millimeter with SCUBA, at $850 \mu \mathrm{m}$. In a second paper, published in these proceedings, we discuss these results in terms of extragalactic background light.

\section{Description of the Surveys}

The five ISOCAM Guaranteed Time Extragalactic Surveys (Cesarsky \& Elbaz 1996) are complementary. They were carried out in both the northern (Lockman Hole) and southern (Marano Field) hemispheres, in order to be less biased by large-scale structures. These two fields were selected for their low zodiacal and cirrus emission and because they had been studied at other wavelengths, in particular in the X-ray band, which is an indicator of the AGN activity of the galaxies. Their features are summarized in the Table 1 below.

Only one of the 'Marano' maps was scanned at the exact position of the original Marano Field (Varano, Zamorani, Zitelli 1988), while the 'Marano FIRBACK' (MFB) Deep and Ultra-Deep were positioned at the site of lowest galactic cirrus emission, because they were combined with the FIRBACK ISOPHOT survey at $175 \mu \mathrm{m}$ (Puget et al. 1999, Dole et al. 1999). Since the importance 
Table 1. $15 \mu \mathrm{m}$ ISOCAM deep surveys.

\begin{tabular}{|c|c|c|c|c|c|c|}
\hline $\begin{array}{c}\text { Survey } \\
\text { Name } \\
\text { (1) }\end{array}$ & $\begin{array}{c}N_{o b s} \\
(2)\end{array}$ & $\begin{array}{c}\text { Area } \\
\left(\mathrm{am}^{2}\right) \\
(3)\end{array}$ & $\begin{array}{c}\mathrm{S}_{80 \%} \\
(\mathrm{mJy}) \\
(4)\end{array}$ & $\begin{array}{c}t_{\text {int }} \\
(\min ) \\
(5) \\
\end{array}$ & \# gal & $\begin{array}{c}\text { Slope } \\
(7)\end{array}$ \\
\hline Lockman Shallow ${ }^{(a)}$ & 3 & 1944 & 1 & 3 & 80 & $-2.1 \pm 0.2$ \\
\hline Lockman Deep ${ }^{(a)}$ & 6 & 510 & 0.6 & 11 & 70 & $-2.4 \pm 0.3$ \\
\hline MFB Deep ${ }^{(a)}$ & 18 & 710 & 0.4 & 15.4 & 144 & $-2.4 \pm 0.2$ \\
\hline Marano $\mathrm{UD}^{(a)}$ & 75 & 70 & 0.2 & 114 & 82 & $-1.5 \pm 0.1$ \\
\hline $\operatorname{MFB} \cup D^{(a)}$ & 75 & 90 & 0.2 & 114 & 100 & $-1.5 \pm 0.2$ \\
\hline HDF North ${ }^{(b)}$ & 64 & 27 & 0.1 & 135 & 44 & $-1.6 \pm 0.2$ \\
\hline HDF South ${ }^{(a)}$ & 64 & 28 & 0.1 & 168 & 63 & $-1.4 \pm 0.1$ \\
\hline$A 2390^{(c)}$ & 100 & 5.3 & 0.05 & 432 & 31 & $-1.2 \pm 0.6$ \\
\hline
\end{tabular}

Comments: Col.(1) Survey name with reference: (a) in preparation, (b) Aussel et al. (1999), (c) Altieri et al. (1999); Col.(2) maximum number of pointings on the same sky position (redundancy); Col.(3) the total area covered in square arcminutes; Col.(4) the flux at which the survey is at least $80 \%$ complete; Col. $(5)$ the corresponding integration time per sky position (in minutes); Col.(6) the number of galaxies whose flux is over the $80 \%$ completeness threshold; Col.(7) the slope of the fit to the integral $\log N-\log S . A 2390$ completeness limit includes the corrections for lensing magnification.

of the Galactic cirrus emission in hampering source detection is much larger at $175 \mu \mathrm{m}$ than at $15 \mu \mathrm{m}$, the quality of the two $15 \mu \mathrm{m}$ ultra deep surveys in the Marano Field area is equivalent. In addition, very deep surveys were taken with ISOCAM over the areas of the HDF North (Serjeant et al. 1997) and HDF South. In this paper, we include the HDF North results from Aussel et al. (1999), and show for the first time ISOCAM counts on the HDF South field.

\section{Data Reduction and Simulations}

The transient behavior of the cosmic ray induced glitches, which makes some of them mimic real sources, is the main limitation of ISOCAM surveys. We have developed two pipelines for the analysis of ISOCAM surveys in order to obtain two independent source lists per survey and improve the quality of the analysis. PRETI (Pattern REcognition Technique for ISOCAM data), developed by Starck et al. (1999), is able to find and remove glitches using multi-resolution wavelet transforms. It includes also Monte Carlo simulations to quantify the false detection rate, to calibrate the photometry and to estimate the completeness. The 'Triple Beam-Switch' (TBS) technique, developed by Désert et al. (1999), treats micro-scanning or mosaic images as if they resulted from beamswitching observations. All the surveys have been independently analyzed using both techniques and the source lists were cross-checked to attribute quality coefficients to the sources. PRETI and TBS agree at the $20 \%$ level in photometry (corresponding to the photometric accuracy of both techniques), with an astrometric accuracy smaller than the pixel size (due to the redundancy). PRETI allowed us to attain fainter levels in deep surveys, whereas in the shallow surveys, where the redundancy is not very high, a very strict criterion of multiple detections had to be applied. Finally, we have made Monte Carlo simulations by 


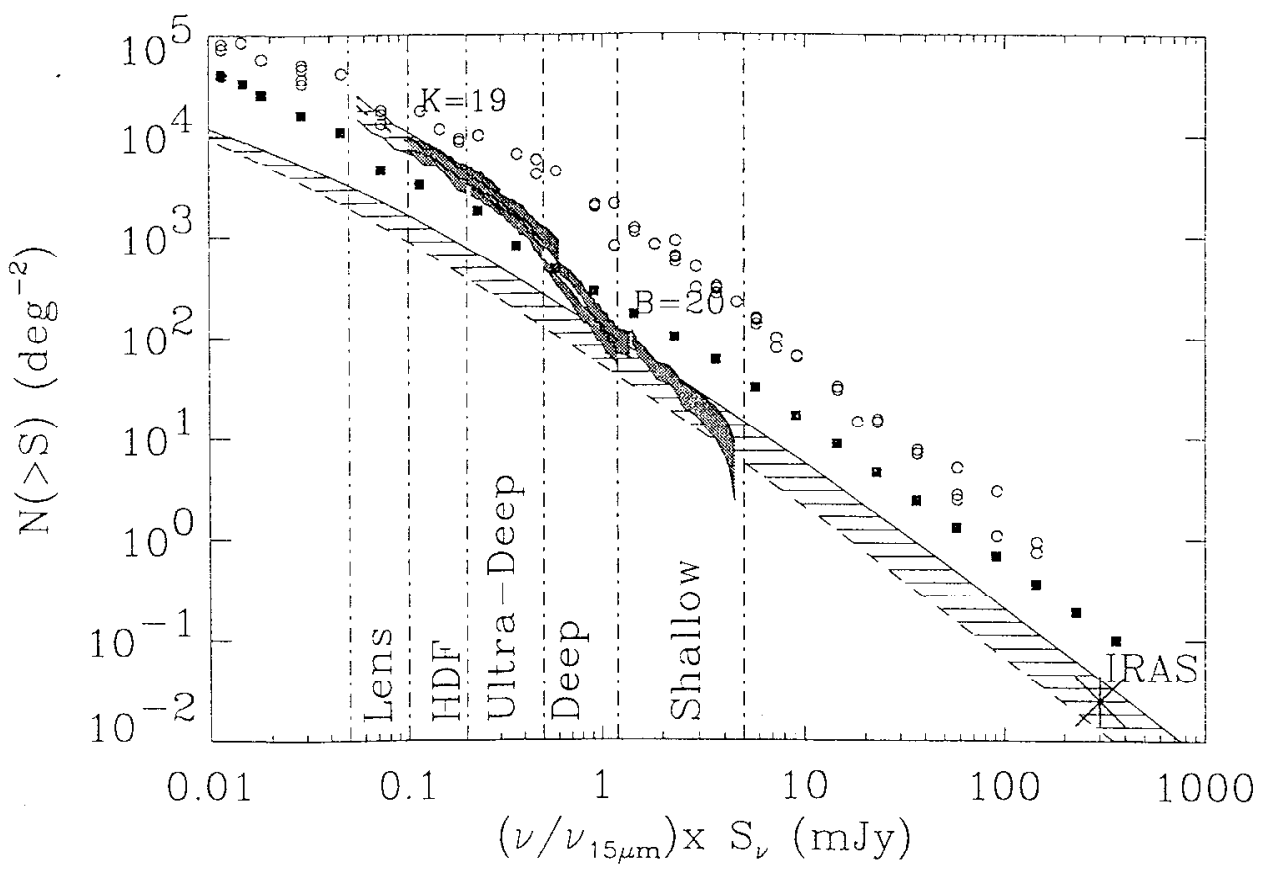

Figure 1. Integral counts, i.e. the number of galaxies, $N$, detected at $15 \mu \mathrm{m}$ above the flux S(mJy), with $68 \%$ confidence contours. $\mathrm{K}$ counts (Gardner et al. 1993) and B counts (Metcalfe et al. 1995), multiplied by the ratio $\nu / \nu_{15}$ to represent the relative energy densities at high fluxes, are overplotted with open circles and filled squares, respectively. The hatched area materializes the range of possible expectations from models assuming no evolution and normalized to the IRAS $12 \mu \mathrm{m}$ local luminosity function (LLF). The upper limit was calculated on the basis of the LLF of Rush, Malkan \& Spinoglio (1993), as Xu et al. (1998) and shifted from 12 to $15 \mu \mathrm{m}$ with the template SED of M82; the lower limit uses the LLF of Fang et al. (1998) and the template SED of M51.

taking into account completeness and photometric accuracy to correct for the Eddington bias and to compute error bars in the number count plots.

\section{The ISOCAM $15 \mu \mathrm{m}$ source counts}

Figures 1 and 2 show respectively the integral and the differential number counts obtained in the five independent guaranteed time surveys conducted in the ISOCAM $15 \mu \mathrm{m}$ band, as well as the HDF surveys. The contribution of stars to the $15 \mu \mathrm{m}$ counts was corrected. It is negligible at fluxes below the m. Iy level as confirmed by the spectro-photometric identifications in the ISOHDF-North ( 1 star out of 44 sources), South ( 3 stars over 71 sources), and CFRS $1415+52$ ( 1 star over 41 sources ranging from $\sim 0.3 \mathrm{~m} . J y$ to $\sim 0.8 \mathrm{mJy}$ ). In the Lockman 


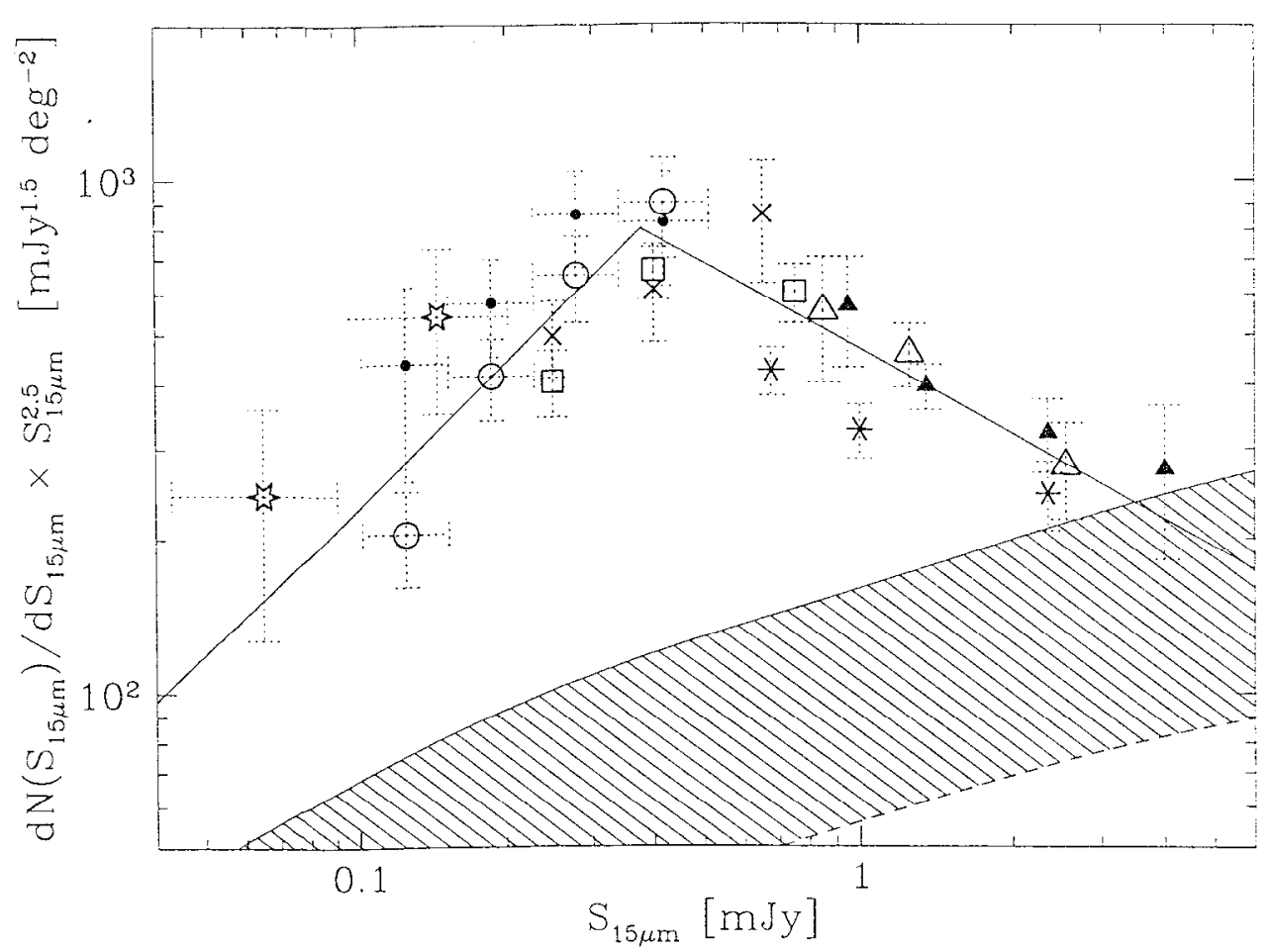

Figure 2. Differential Number Counts of $15 \mu \mathrm{m}$ Galaxies, with $68 \%$ error bars. The counts are normalized to a Euclidean distribution of non-crolving sources, which would have a slope of $\alpha--2.5$ in such a universe. Data points: A2390 (open stars), ISOHDF-North (open circles), ISOHDF-South (filled circles), Marano FIRBACK (MFB) UltraDeep (open squares), Marano Ultra-Deep (crosses), MFB Deep (stars), Locliman Dcep (open triangles), Lockman Shallow (filled triangles). The hatched area materializes the range of possible expectations from modcls assuming no evolution (see Fig.1).

Shallow Survey $\left(\mathrm{S}_{15 \mu \mathrm{m}}>1 \mathrm{mJy}\right)$, about $12 \%$ of the sources were classified as stars from their optical-mid IR colors (using the Rayleigh-Jeans law).

We have also represented the counts from the ISOHDF-North (from Aussel et al. 1999), ISOHDF-South, and, at the lowest fluxes, the counts obtained from the A2390 cluster lens (down to $50 \mu \mathrm{Jy}$, corrected for the lensing effect; Altieri et al. 1999, see also Metcalfe et al. 1999). We have only included the flux bins where the surveys are at least $80 \%$ complete, according to the simulations.

The first striking result of these complementary source counts is the consistency of the eight $15 \mu \mathrm{m}$ surveys over the full flux range. Some scatter is nevertheless apparent; given the small size of the fields surveyed, we attribute some of it to clustering effects.

The two main features of the observed counts are a significantly supereuclidean slope $(\alpha=-3.0)$ from 3 to $0.4 \mathrm{~m}$ Jy and a fast convergence at flux den- 
sities fainter than $0.4 \mathrm{mJy}$. In particular, the combination of five independent surveys in the flux range $90-400 \mu \mathrm{J}$ shows a turnover of the normalized differential counts around $400 \mu \mathrm{Jy}$ and a decrease by a factor $\sim 3$ at $100 \mu \mathrm{Jy}$. We believe that this decrease, or the flattening of the integral counts below $\sim 400 \mu \mathrm{Jy}$, is real. It cannot be due to incompleteness, since this has been estimated from the Monte-Carlo simulations (see Section 3.). The differential counts can be fitted by two power laws by splitting the flux axis in two regions around $0.4 \mathrm{~m}$.Jy. In units of $\mathrm{mJy}^{-1} \mathrm{deg}^{-2}$, we obtain, by taking into account the error bars:

$$
\frac{d N(S)}{d S}=\left\{\begin{array}{cccc}
(2000 \pm 600) & S^{(-1.6 \pm 0.2)} & \ldots & 0.1 \leq S \leq 0.4 \\
(470 \pm 30) & S^{(-3.0 \pm 0.1)} & \ldots & 0.4 \leq S \leq 4
\end{array}\right.
$$

In the integral plot (Fig. 1), the curves are plotted with $68 \%$ confidence contours based on our simulation analysis. The total number density of sources detected by ISOCAM at $1 \tilde{j} \mu \mathrm{m}$ is $(4.2 \pm 1.1) \operatorname{arcmin}^{-2}$ down to $60 \mu \mathrm{Jy}$ (accounting for lensing) or (2.35 \pm 0.3$) \mathrm{arcmin}^{-2}$ down to $100 \mu \mathrm{Jy}$, using only direct flux derisity measurements.

\section{Discussion of the number counts}

We have presented the $15 \mu \mathrm{m}$ differential and integral counts drawn by several complementary ISOCAM deep surveys, with a significant statistical sampling (993 galaxies, 614 of which have a flux above the $80 \%$ completeness limit) over two decades in flux from $50 \mu \mathrm{Jy}$ up to $5 \mathrm{mJy}$. The differential counts (Fig. 2), which are normalized to $S^{-2.5}$, the expected differential counts in a non expanding Euclidean universe with sources that shine with constant luminosity, present a turnover around $S_{15 \mu m}=0.40 \mathrm{mJy}$, above which the slope is very steep ( $\alpha=-$ $3.0 \pm 0.1$ ). No evolution predictions were derived assuming a pure $k$-correction in a flat universe $\left(q_{0}=0.5\right)$, including the effect of Unidentified Infrared Band emission in the galaxy spectra. In the Figures, the lower curve is based on the Fang ct al. (1998) IRAS $12 \mu \mathrm{m}$ local luminosity function (LLF), using the spcctral template of a quiescent spiral galaxy (M51). The upper curve is based on the Rush, Malkan and Spinoglio (1993) IRAS-12 $\mu \mathrm{m}$ LLF, translated to $15 \mu \mathrm{m}$ using as template the M82 spectrum. We consider that, in the absence of a well established LLF at $15 \mu \mathrm{m}$, thesc two models can be considered as upper and lower bounds to the actual no-evolution expectations; note that the corresponding slope is $\sim-2$. The actual number counts are wcll above these predictions; in the $0.3 \mathrm{mJy}$ to $0.6 \mathrm{~m}$.Jy range, the excess is around a factor 10: clearly, strong evolution is required to explain this result.

In Fig. 1, we have overplotted the integral counts in the $K$ (from Gardner et al. 1993) and B (Metcalfe et al. 1995) bands, in terms of $\nu S_{\nu}$. For bright sources, with densities lower than $10 \mathrm{deg}^{-} 2$, these curves run parallel to an interpolation between the ISOCAM counts presented here and the IRAS counts; the bright $\mathrm{K}$ sources emit about ten times more energy in this band than a comparable number of bright ISOCAM sources at $15 \mu \mathrm{m}$. But the ISOCAM integral counts present a rapid change of slope around 1-2 mJy, and their numbers rise much faster than those of the $K$ and $B$ sources. The sources detected by 


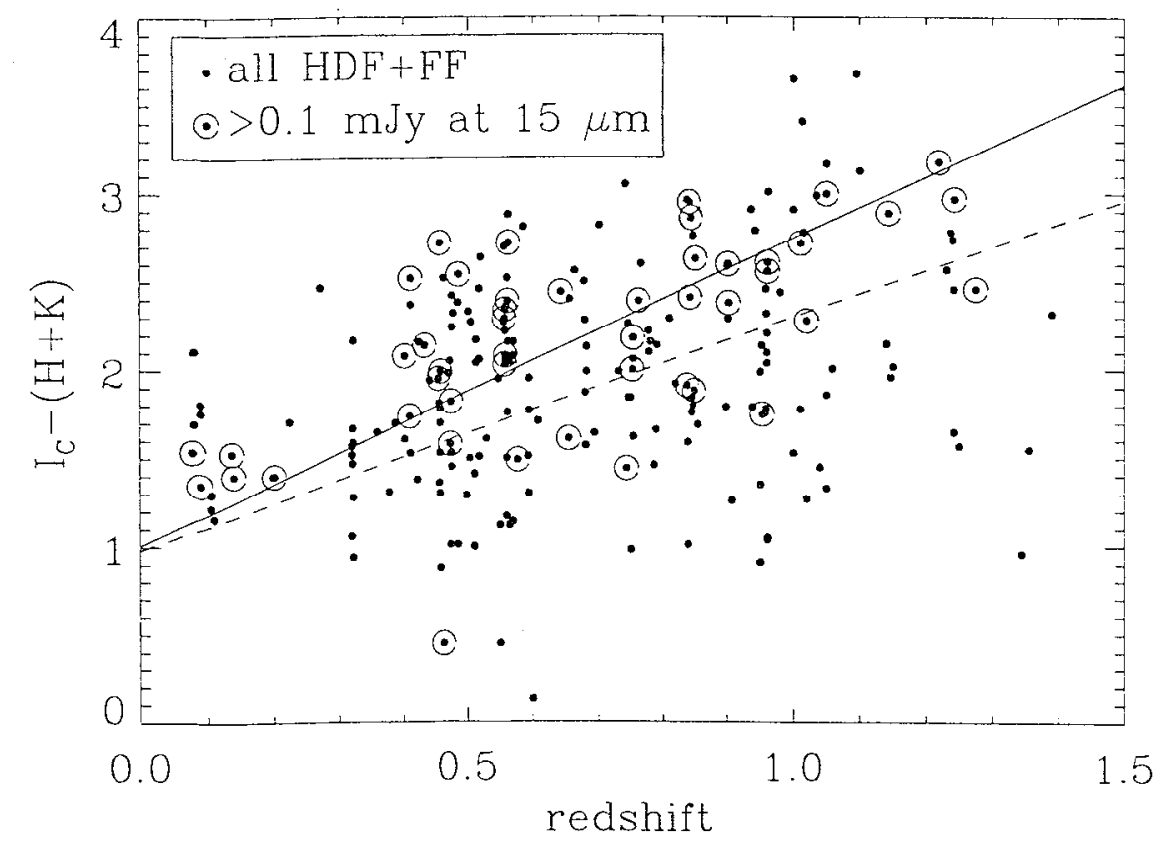

Figure 3. I(Kron-Cousins)- $(\mathrm{H}+\mathrm{K})$ color (from Cowie et al. 1999) versus redshift for field galaxies (dots) and galaxies detected above 0.1 $\mathrm{m} . \mathrm{Jy}$ at $15 \mu \mathrm{m}$ by ISOCAM (dots surrounded by circles), in the Hubble Deep Field North plus its Flanking Fields. Plain line: linear fit to the colors of ISOCAM galaxies. Dashed line: fit for all galaxies.

ISOCAM are a sub-class of the $K$ and $B$ sources which harbor activity hidden by dust, and their proportion is higher among the faint sources.

\section{Discussion of the nature of the $15 \mu \mathrm{m}$ galaxies}

We believe, according to the results obtained on the HDF and CFRS fields (Aussel et al. 1999; Flores et al. 1999), that the sources responsible for the 'bump' in the $15 \mathrm{~km}$ counts are not the faint blue galaxies which dominate the optical counts and have a median redshift around $z \sim 0.6$ (Pozzetti et al. 1998). Indeed, as shown in the Fig. 3, the galaxies from the HDF-N plus Flanking Fields whose $15 \mu \mathrm{m}$ flux density is greater than $0.1 \mathrm{mJy}$ (sensitivity limit of ISOCAM) harbour a $I_{C}-(H+K)$ color distribution very similar to that of the whole sample of galaxies for which we have access to both the $\mathrm{I}_{C}-(\mathrm{H}+\mathrm{h})$ colors and spectroscopic redshifts (from Cowie et al. 1999, where the index $\mathrm{C}$ is for Kron-Cousins). Aussel et al. (1999, PhD thesis and paper in preparation) showed that the sub-sample of galaxies with known spectroscopic redshift keeps the same color properties than the full sample of HDF +FF galaxies, hence we did not include a selection bias in the color distribution. 


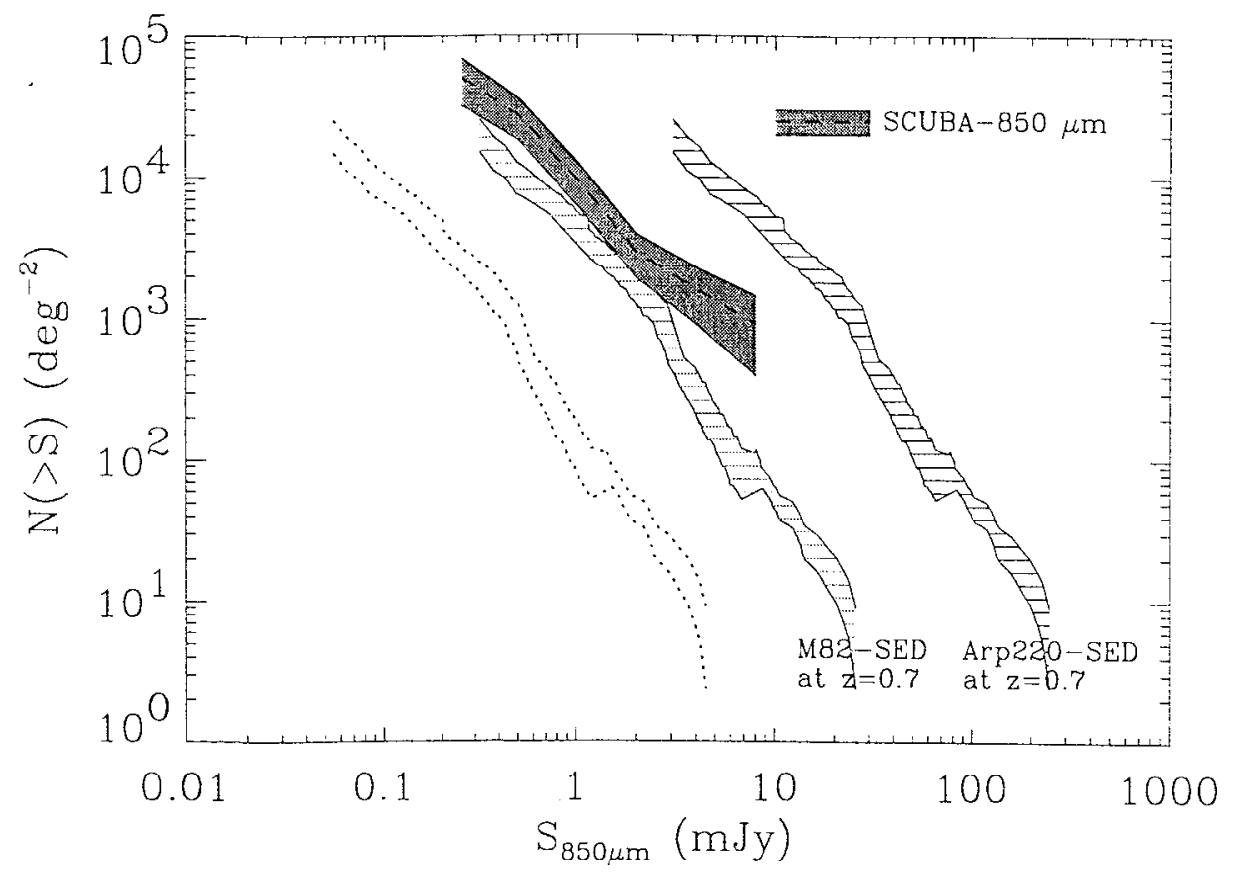

Figure 4. Integral counts at $850 \mu \mathrm{m}$ from SCUBA (Blain et al., these proceedings). From the $15 \mu \mathrm{m}$ ISOCAM counts (dotted lines), one can extrapolate the contribution of the ISOCAM galaxies at $850 \mu \mathrm{m}$ for a given redshift distribution and spectral energy distribution (SED). Two examples are shown here using the SEDs of M82 and Arp 220, and assuming that all ISOCAM galaxies have the same redshift, $\mathrm{z}=0.7$, corresponding to their median redshift in the HDF and CFRS fields.

The median colors are $\mathrm{I}_{C}(\mathrm{H}+\mathrm{K})=2.3$ for the $15 \mu \mathrm{m}$ galaxies and $\mathrm{I}_{C^{-}}$$(\mathrm{H}+\mathrm{K})=2$ for all galaxies. However, the linear fit to the $\mathrm{I}_{C}-(\mathrm{H}+\mathrm{K})$ versus redshift plot of the two samples of galaxies in Fig. 3 shows that the 'dusty' galaxies tend to redden slightly faster with increasing redshift, than the natural reddening of the whole population of field galaxies which is due to $k$-correction. Nevertheless, this difference is not strong enough to allow one to separate the infrared galaxies from the whole sample like for Lyman-break galaxies (even accounting for the other set of optical colors existing for the HDF galaxies). The origin of this reddening with redshift is not clear but it is probably due to a selection of the galaxies suffering from more extinction, hence emitting more in the infrared, at larger redshifts.

From the full sample of $15 \mu \mathrm{m}$ galaxies with known redshift and optical-near IR magnitudes, we found that these galaxies are massive $\left(\sim 10^{11} U_{\odot}\right)$ and that their emission occurs essentially in the IR and could account fo: a considerable part of the star formation in the universe at $z<1.5$ (Flores et al. 1999). Their median redshift is $z \sim 0.8 \mathrm{in}$ a sample of 42 galaxies brighter than $0.1 \mathrm{mJy}$ in the $\mathrm{HDF}+\mathrm{FF}$ (Aussel et al. 1999) and $\mathrm{z} \sim 0.7$ in a sample of 41 galaxies brighter than 
$0.35 \mathrm{mJy}$ in the CFRS-1415 field (Flores et al. 1999). The assessment of their bolometric luminosity requires the assumption of a spectral energy distribution, which is largely uncertain since the ratio of the far-IR over mid-IR flux densities is highly variable among galaxies. However, one can set limits to the bolometric luminosity using the following arguments. If all galaxies had extreme SEDs like the one of Arp220, they would produce a contribution to the SCUBA. $850 \mu \mathrm{m}$ number counts (see Blain et al., these proceedings) much above observations (see Fig. 4). We show in a second paper presented in these proceedings about the $15 \mu \mathrm{m}$ extragalactic background light (EBL) that Arp220-like SEDs can also be rejected for over-producing the $140 \mu \mathrm{m}$ EBL with respect to the one measured by DIRBE on-board COBE. The light radiated in the far-IR by these galaxies however cannot be much lower than that emitted at $15 \mu \mathrm{m}$, since only galaxies luminous in the IR can be detected at $z>0.5$ with a $15 \mu \mathrm{m}$ flux density larger than $0.1 \mathrm{~m} . \mathrm{Jy}$ (the origin of the emission cannot be stellar without requiring excessive masses). We therefore estimated the mean bolometric luminosity of these galaxies to be of the order of a few $10^{11} L_{\odot}$. Hence the population of galaxies producing the $15 \mu \mathrm{m}$ number counts excess is very distinct from the one which dominates the deep optical counts, known to be made of low mass galaxies with blue luminosities, although at a similar redshift. In other words, the star formation activity responsible for the light emitted by the $15 \mu \mathrm{m}$ galaxies is not the other face of the same star formation activity already quantified from the optical surveys, but instead should be considered as a second component, which was previously missed.

Linking luminosity to distance, we predict a rapid change of the luminosity function with increasing redshift, which can only be confirmed by a complete ground-based spectro-photometric follow-up. We should be able to follow the evolution of the luminosity function from $z \sim 0.2$ to 1.5 with the large number of galaxies detected in the Marano Field surveys. The combination of the intensity of the $\mathrm{H}_{\alpha}$ emission line (redshifted in the J-band) with the IR luminosity could set strong constraints on the star formation rate. Finally, emission line diagnostics, combined with hard X-ray observations with XMM and Chandra, would allow us to understand whether the dominant source of energy is star formation or AGN activity.

\section{References}

Altieri B., Metcalfe L., Kneib J.P., 1999, A\&A 343L, 65

Anssel H., Cesarsky C., Elbaz D., et al, 1999a, A\&A 342, 313

Aussel H., Cesarsky C., Elbaz D., et al.. 1999b, In "The Universe as seen by ISO", eds.: P.Cox, M.F.Kessler, ESA Publication Division, ESTEC, Noordwijk, NL

Cesarsky C.. Abergel A., Agnèse P., et al., 1996, A\&A 315, L32

Cesarsky C.J., Elbaz D., 1996, IAUS, M. C. Kafatos, Y. Kondo (eds.), Kluwer Acad. Pub., Dordrecht, vol. 168, 109.

Cowie.L.L., Clowe D., Fulton E., et al., 1999, A.J, in preparation

Désert F.X., Puget J.L., Clements D., et al., 1999, A\&A 342, 363 
Dole H., Lagache G., Puget J.L., et al., 1999, in Cox, P., Kessler, M.F. (eds.), The Universe as seen by ISO, ESA SP-427, p. 1031, astro-ph/9902122

Dwek. E., Arendt R.G., Hauser M.G., et al., 1998, Ap.J 508, 106

Elbaz D., Cesarsky C.J., Fadda, D., et al., 1999, to appear in A\&A Letter

Fang F., Shupe D., Xu C., Hacking P., 1998, Ap.J 500, 693

Fixsen D.J., Dwek E., Mather J.C., et al., 1998, Ap.J 508, 123

Flores H., Hammer F., Thuan T., et al., 1999, ApJ 517, 148

Franceschini A., et al., 1999, in preparation

Gardner J.P., Cowie L.L., Wainscoat R.J., 1993, ApJ 415, L9

Guiderdoni B., Bouchet F, Puget J.L., et al., 1997, Nat 390, 257

Hauser M.G., Arendt R.G., Kelsall T., 1998, ApJ 508, 25

Kessler M., Steinz J., Anderegg M., et al., 1996, A\&A 315, L27

Lagache G., Abergel A., Boulanger F., et al., 1999, A\&A 344, 322

Lilly S., Le Fèvre O., Hammer F., et al., 1996, ApJ 460, L1

Madau P., Ferguson H., Dickinson M., et al., 1906, MNRAS 283, 1388

Marano, B., Zamorani, G., Zitelli, V., 1988, MNRAS 232, 111

Metcalfe L., Altieri B., McBreen B., et al., 1999, In "The Universe as seen by ISO", eds.: P.Cox, M.F.Kessler, ESA Publication Division, ESTEC, Noordwijk, NL

Metcalfe N., Shanks T., Fong R., et al., 1995, MNRAS 273, 257

Pozzetti L., Madau P., Zamorani G., Ferguson H.C., Bruzual G.A., 1998, MNRAS, 298, 1133

Puget J.I. , Abergel A., Bernard J.P., et al., 1996, A\&A 308, L5

Puget J.L., Lagache G., Clements D., et al., 1999, A\&A 345, 29

Rush B., Malkan M.A., Spinoglio L., 1993, ApJS 89, 1

Sanders, D.B., Mirabel, I.F., 1996, Ann. Rev. Astron. Astrophys. 34, 749

Serjeant S., Eaton N., Oliver S., et al., 1997, MNRAS 289, 457

Soifer B.T., Neugebauter G., 1991, AJ 101, 354

Starck J.-L., Aussel II., Elbaz D., ct al., 1090, A\&AS 138, 365

Xu C., Hacking P., Fang F., et al., 1998, ApJ 508, 576 

The Hy Redshift Universe

ASP Conference Series, Vol. 193, 1999

A. J. Bunker \& W. J. M. van Breugel, eds.

\title{
Near-Infrared Emission Line Searches for High-Redshift Galaxies
}

\author{
Andrew Bunker ${ }^{1}$ \\ Dept. of Astronomy, University of California, Berkeley CA 94720 \\ email: bunker@ast.cam.ac.uk
}

\begin{abstract}
In this article I review recent developments in near-infrared emission line searches for star-forming galaxies at high redshift. Using the $J_{-}, H$ - \& $K_{-}$-bands we can potentially chart the history of star formation over the range $1<z<5$ using the prominent rest-optical nebular emission lines alone, filling in the "redshift desert" at $z \sim 1-3$ where most common emission lines lie outside the optical bands. Studying the rest-frame optical at $z \sim 2$ also allows a fair comparison to be made with the local Universe - the rest-optical lines are vastly more robust to extinction by dust than the rest-UV, with the resonantly-scattered Ly $\alpha$ line particularly unreliable. I discuss the recent history of near-infrared emission-line searches using narrow-band imaging and spectroscopy, and look to the future in this era of $10 \mathrm{~m}$ telescopes: such work gives us the potential to push to $\tilde{z}>10$, the next frontier in the Hy-Redshift Universe.
\end{abstract}

\section{Introduction}

This conference celebrates some of the research interests of Hyron Spinrad on his 65 th birthday. Hy's career has been synonymous with the discovery of the most distant galaxies, and in recent years this field has undergone a renaissance. Galaxies at $>90 \%$ of the look-back time are now being successfully hunted with a broad armory of observational techniques. In this article I will review recent developments in near-infrared emission-line searches.

The observational study of galaxy evolution aims to follow the star formation, gas consumption, metal enrichment and merging rates of the components which have combined to form today's galaxies. In the last four years, our knowledge of high redshift 'normal' (non-AGN) galaxies has blossomed. The photometric selection technique of Steidel, Pettini \& Hamilton (1995), using the intrinsic Lyman limit continuum break at $\lambda_{\text {rest }}=91.2 \mathrm{~nm}$ and the blanketing effect of intervening Lyman- $\alpha$ absorbers below $\lambda_{\text {rest }}=121.6 \mathrm{~nm}$, has proven a robust way to select star-forming galaxies through broad-band optical imaging at $z \approx 3$ (the ' $U$-drops', Steidel et al. 1996a,b) and now at $z \approx 4$ (the 'B-drops', Steidel et al. 1999 and Chuck Steidel's contribution to this conference proceedings). In addition, after many years of largely fruitless searches for Ly $\alpha 121.6 \mathrm{~nm}$ (e.g., Pritchet \& Hartwick 1990; de Propris et al. 1993: Thompson, Djorgovski

\footnotetext{
${ }^{1}$ Present address: Institute of Astronomy, Madingley Road, Cambrilge, CB3 OHA, England
} 
$\&$ Trauger 1995), a number of field galaxies have now been identified at $z \sim 3-6$ through this emission line (e.g., Dey et al. 1998; Hu, Cowie \& McMahon 1998: Chen.et al. 1999; Steidel et al. 2000 - see also the articles by Ken Lanzetta, George Djorgovski, Esther Hu and Hy Spinrad in this volume).

Observations in the optical are now, at last, revealing large numbers of high-redshift galaxies. Why, then, should we be interested in searches in the near-infrared at all when the optical is so 'easy' by comparison (in terms of the low sky background and the mature detector technology)? The motivation of moving to the near-infrared is three-fold:

- to fill in the redshift desert at $z \sim 1-3$, where most common emission lines lie outside the optical;

- to study the rest-frame optical at $z \sim 2$ in order to make a fair comparison with local Universe - the rest-optical lines are vastly more robust to extinction by dust than the rest-UV, with Ly $\alpha$ particularly unreliable;

- the near-IR gives us the potential to push to the highest redshifts yet $(z>8)$.

In this article, I will concentrate on near-infrared search techniques for actively star-forming galaxies. In their contributions to this proceedings, Adam Stanford, Peter Eisenhardt and Jim Dunlop discuss infrared methods of finding and studying high-redshift galaxies with evolved stellar populations, both in clusters and in the field. Andrew Blain and Len Cowie describe far-infrared/sub-mm surveys. Thronghout $\mathrm{I}$ will consider a cosmology with a vanishing cosmological constant $\left(\Lambda_{0}=0\right), H_{0}=100 h \mathrm{~km} \mathrm{~s}^{-1} \mathrm{Mpc}^{-1}$ and $q_{0}=0.5$ unless otherwise stated.

\section{Line Emission as an Indicator of Star Formation}

Most high- $z$ emission line searches have focussed on Ly $\alpha$, and until the recent advent of $10-\mathrm{m}$ telescopes have been uniformly unsuccessful. With the benefit of hindsight, it appears the large path-length for the resonant Ly $\alpha$ line in typical neutral hydrogen columns of $10^{24} \mathrm{~m}^{-2}$ greatly enhances the absorption cross section with only modest dust (e.g., Chen \& Neufeld 1994), depending sensitively on the geometry and hinematics of the gas. This selective quenching of Ly $\alpha$ is observed in low-z star-bursts (e.g., Funth et al. 1998), and at high redshift Ly $\alpha$ emission is typically weak in the Lyman-limit selected galaxies of Steidel et al. $(1996 \mathrm{ab}, 1999)$ - indeed this line is actually seen in absorption in half their sample. Put simply, the substantial effort in Ly $\alpha$ searches has yielded mainly upper limits of essentially no interest for constraining the underlying starformation rates, as the extinction of this line is extremely difficult to quantify.

A natural progression is to look for lines of longer rest-frame wavelength, less affected by extinction than the rest-UV and immune to the effects of resonant scattering. The Balmer hydrogen recombination emission lines, $\mathrm{H} \alpha \lambda 656.3 \mathrm{~nm}$ $\& H \beta \lambda 486.1 \mathrm{~nm}$, and the collisionally de-excited forbidden lines of oxygen, [O III] $\lambda \lambda 495.9,500.7 \mathrm{~nm} \&[\mathrm{O} \mathrm{II}] \lambda \lambda 372.6,372.9 \mathrm{~nm}$, are fairly good indicators of the intrinsic rest- $U V$ continuum from the hottest, most massive and shortestlived stars $\left(M z 10 M_{\odot}, T_{\text {MS }} \lesssim 10 \mathrm{Myr}, T_{\text {eff }} z 30,000 \mathrm{~K}\right)$, and hence the nearinstantaneous star formation rate (SFR). The most fruitful indicator of star 

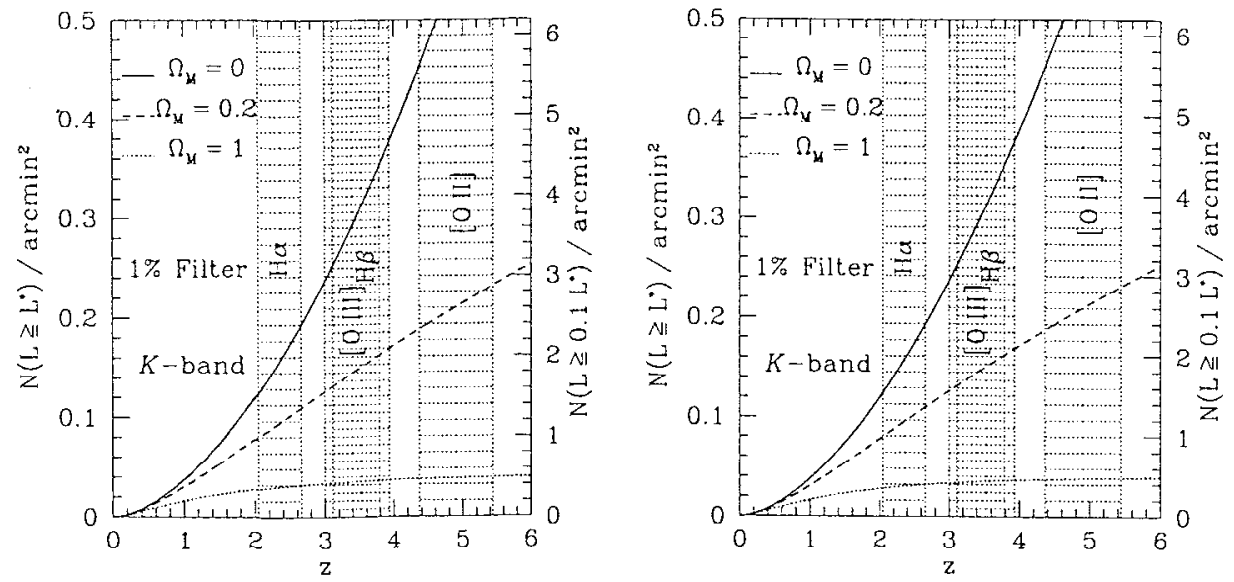

Figure 1. Left: predictions of the number density of field galaxies within a $1 \%$ band-pass in the case of no evolution in the co-moving number density. 'l'he surface density brighter than $L^{*}$ (left scale) or $0.1 L^{*}$ (right scale) is plotted. The redshift intervals covered by four prominent restframe-optical emission lines in the $K$-band atmospheric window are indicated. Using the $J$ - and $H$-bands too allows complete coverage for $1<z<5$.

Right: The predicted (unobscured) line fluxes as a function of redshift for several emission lines indicative of star formation. The thick lines indicate the range of redshifts at which these appear in the $K$-window of the near-infrared (from Bunker 1996). The Kennicutt (1983) relation between $\mathrm{H} \alpha$ luminosity and star formation rate is adopted, and $[\mathrm{O} \mathrm{II}]$ is assumed to have a comparable intensity to $\mathrm{H} \beta$ (Kennicutt 1992), and [O III] in the most actively star-forming systems rivals $\mathrm{H} \alpha$ (e.g., Mannucci \& Beckwith 1995), although the scatter is large. The left-hand axis shows the line fluxes for a star formation rate of $25 h^{-2} M_{\odot} \mathrm{yr}^{-1}$, which may be typical of an $L^{*}$-galaxy progenitor, and the right-hand axis is for $1 h^{-2} M_{\ominus} \mathrm{yr}^{-1}$.

formation at modest redshifts has been the $[\mathrm{O} \mathrm{II}]$ line. However, there is a 'deadzone' between $z \approx 1.3$ (beyond which $[\mathrm{O} \mathrm{II}]$ leaves the optical) and $z \approx 2.7$ (below which Lyman-break photometric selection is ineffective). Yet some theories currently in vogue predict this to be the crucial epoch of maximal merger activity and star formation. Using the atmospheric transmission windows in the near-infrared - the $J(1.1-1.4 \mu \mathrm{m}), H(1.5-1.7 \mu \mathrm{m})$ and $K(2.0-2.4 \mu \mathrm{m})$ pass-bands - gives access to the full redshift range $1<z<5$ through the four prominent rest-frame optical nebular emission lines. The conversion of the nebular line luminosities to star formation rates is also better understood than that for the sub-mm continuum, which is an indirect measure of UV flux reprocessed as thermal grain emission in the far-infrared, which depends on a number of factors including the temperature of the dust, and changes by as much as a factor of four over the range $T_{\text {dust }}=30-50 \mathrm{~K}$ (Barger et al. 1999). 


\section{Search Strategies for Emission Lines}

A search for line emission objects does not constitute a magnitude-limited survey - indeed, blank-sky narrow-band imaging and long-slit spectroscopy are quite unsuited to this, as the sensitivity to line emission is much better than to continuum. The goal of such searches is to trace star formation over a range of redshifts through the nebular emission lines, and the selection criteria should be designed to discriminate actively star-forming galaxies from the bulk of the population. While faint magnitude-limited redshift surveys can address this to some extent, the issue is muddied: selecting on broad-band magnitudes is not the same as selecting on star formation activity. Even the rest-frame UV is suffers badly and unpredictably from dust extinction, and below $121.6 \mathrm{~nm}$ it is gradually eroded by intervening absorbers. There may also exist a population of star-forming galaxies with very large equivalent width emission lines which would be missing from magnitude-limited surveys on account of their faint continua, despite high star formation rates (e.g., Curt Manning's poster paper in this volume).

When constructing a survey for high-redshift star-forming galaxies, there are six main considerations which must be balanced (e.g., Koo 1986; Pritchet 1994):

- Redshift Coverage: Does the survey range cover redshifts where star formation activity is thought to be high? Is the spread in look-back time adequate to test various models?

- Solid Angle: Is sufficient area on the sky covered to intercept several galaxies?

- Clustering: What density enhancement above the average for the field can be expected by targeting the search on known objects at high- $z$ ?

- Discrimination Against Foreground Objects: How effectively can the high-redshift sheep be separated from low-redshift goats? Is there any foreground contaminant population?

- Sensitivity: Can faint enough line fluxes be attained to reach cosmologically interesting star formation rates?

- The Scientific Goals: How cleanly is the star-forming population at a particular redshift isulaled?

Unlike broad-band imaging and spectroscopy, narrow-band work samples a very small wavelength range (and so a small dispersion in redshifts). This has the advantage of reducing the background noise by cutting the spectral extent of sky the detector is exposed to, but has the major drawback that little depth in redshift space is probed (although targetted searches for clusters may offset this disadvantage: Nauro Giavalisco and Ray Carlberg discuss the evolution of clustering properties at high- $z$ in their articles in this proceedings). Untargetted ("blank-sky") long-slit spectroscopy can cover a larger redshift range, but over a very restricted solid angle. Although covering a large volume, and despite recent advances in photometric redshift estimation from multi-waveband colours, broad-band imaging does not offer precise redshift information. It is also relatively insensitive to line emission due to the high background - as is also the case with ground-based slitless spectroscopy (but see $\$$ j.1.). 
One of the advantages of high- $z$ searches for $\mathrm{H} \alpha$ in particular is the lack of lower-redshift interlopers to mimic this line emission: should an object with a single emission line be detected in a $K$-band search, the most conservative interpretation would be $\mathrm{H} \alpha$ at $\approx \approx 2-2.5$; as there are no strong emission lines at rest-wavelengths longward of $656.3 \mathrm{~nm}$, it is unlikely that the redshift is actually less than two (and with plausible higher-redshift degeneracies of $\mathrm{H} \beta$ or [O III] $500.7 \mathrm{~nm}$ at $z \sim 3.5$ or [O II] $372.7 \mathrm{~nm}$ at $z \sim 5)$. This is in stark contrast to searches for Ly $\alpha$, where there is frequent confusion between the high-redshift (Ly $\alpha$-line) interpretation, with a continum break at the line attributed to the $\mathrm{H}$ I forest absorption, and a low-redshift galaxy with [O II] $372.7 \mathrm{~nm}$ emission accompanied by the $4000 \AA+$ Balmer continum break. Neither Ly $\alpha$ nor [O II] have strong nearby lines with which to differentiate the two interpretations through low-dispersion spectroscopy (see Stern et al. 2000 for a detailed discussion).

\section{Near-Infrared Narrow-Band Imaging}

Infrared searches using narrow-band filters (typically " $1 \%$ filters" with a velocity width of $\approx 3000 \mathrm{~km} \mathrm{~s}^{-1}$ ) have become popular in the last seven years. The technique is to search for objects which have excess flux in the narrow-band filter when compared to an off-band, which could be attributable to an emission line being redshifted into the bandpass (see Figs. $2 \& 3$ ). In practice, a broadband filter is almost commonly used as the 'off-band', as the sensitivity to the continuum is much greater than with simply using an adjacent narrow-band filter (Pat Hall's paper in this volume describes a QSO companion detected by this technique).

\subsection{Pilot Studies on $4 \mathrm{~m}$-Class Telescopes}

One of the first surveys was undertaken on the 3.8-m UK Infrared Telescope (UKIRT) by Parkes, Collins \& Juseph (1994). This involved $J$-band imaging with IRCAM-1 (a $62 \times 58$ pixel InSb array), intended to search for Lya at very high redshift $(7 \lesssim z \lesssim 9)$ but also potentially sensitive to $\mathrm{H} \alpha$ at $\approx \approx$ 0.5-0.9 (Collins, Parkes \& Joseph 1996). However, there were no confirmed line-emission candidates within the $3 \mathrm{arcmin}^{2}$ area surveyed to a limiting $\mathrm{flux}^{1}$ of $10^{-18}-10^{-19} \mathrm{Wm}^{-2}$. Reaching a comparable flux limit in the $K_{\text {-band, }}$ Thompson, Djorgovski \& Beckwith (1994) surveyed $0.7 \mathrm{arcmin}^{2}$ with a similar array on the Palomar $5-\mathrm{m}$. This search was targetted on [OII] $\lambda 372.7 \mathrm{~nm}$ from objects clustered around three $z z 4$ QSOs, but did not detect any line-emission companion galaxies.

These pilot studies demonstrated that potentially significant cosmological volumes and star formation rates could be probed at high- $\tilde{z}$ through nearinfrared narrow-band imaging. However, it was also clear that deeper surveys covering larger areas were required to unveil any population of $z>2$ star-forming galaxies.

\footnotetext{
${ }^{1}$ Throughout, I convert the limits quoted for various surveys to a $3 \sigma$ threshold in a $3^{\prime \prime}$-diameter aperture.
} 

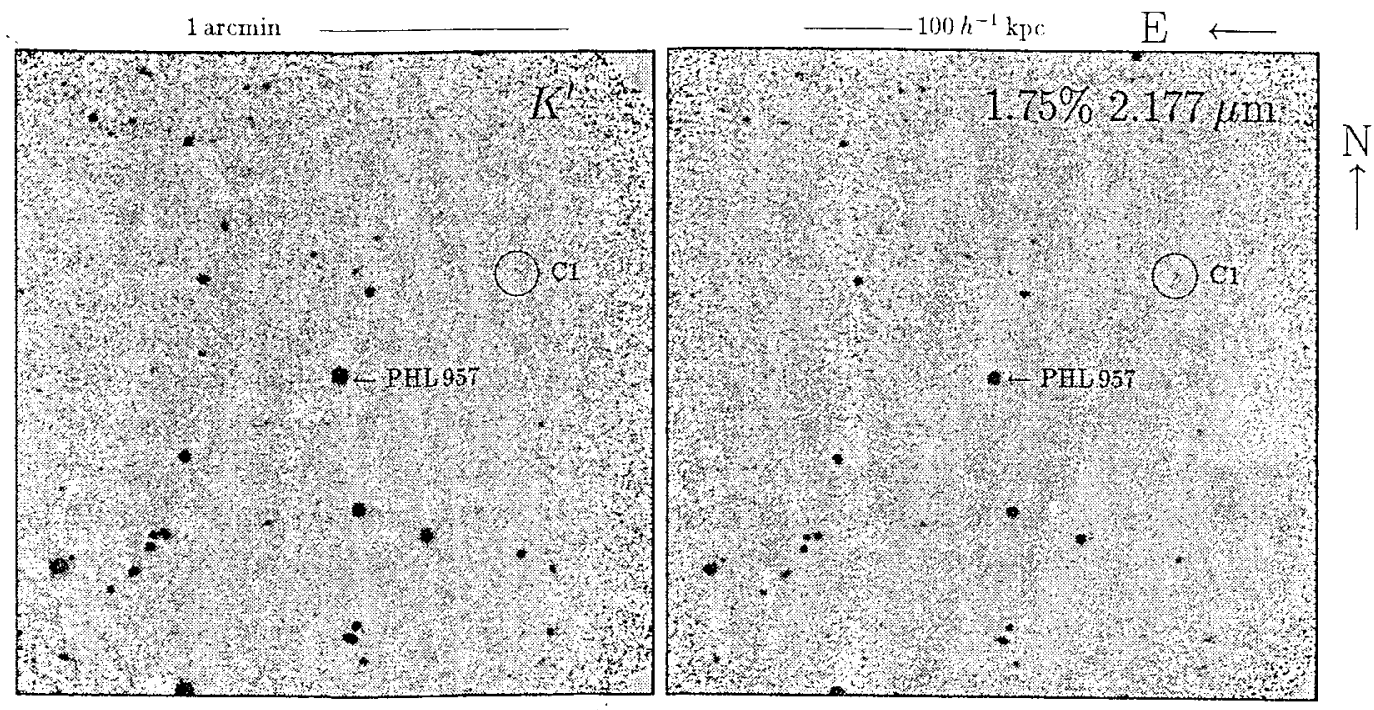

Figure 2. The field of the quasar PHL 957, imaged with the $256 \times 256$ array IRAC 2B on the ESO 2.2-m (Bunker et al. 1995). The narrowband filter is tuned to $\mathrm{H} \alpha$ at $z=2.31$, the redshift of the damped Ly $\alpha$ absorber (Wolfe et al. 1986). The broad-band $H^{\prime \prime}$ frame (left) reaches $0.8 \mathrm{mag}$ deeper than the narrow-band frame (right), but the object $\mathrm{C} 1$, a known galaxy at $z=2.313$ (Lowenthal et al. 1991, Hu et al. 1993), is brighter in the narrow-band frame due to the redshifted $\mathrm{H} \alpha+[\mathrm{N}$ II] line emission.

What should be the minimum survey volume to stand a realistic chance of intercepting several field galaxies at high- $z$ ? At a redshift of $z \approx 2.3$, where $\mathrm{H} \alpha$ appears in the middle of the $K$-window, a Ilarrow-band survey with a filter of width $\Delta \lambda / \lambda$ and a detector of area $\Omega$ will cover a co-moving volume of

$$
\Delta V_{\mathrm{CM}} \simeq 10.2 \frac{\Delta \lambda / \lambda}{0.01} \frac{\Omega}{1 \operatorname{arcmin}^{2}} h^{-3} \mathrm{Mpc}^{3}\left(z \approx 2.3, q_{0}=0.5\right) .
$$

Locally, the density of field galaxies is $\phi^{*}=0.015 h^{3} \mathrm{Mpc}^{-3}$ (Loveday et al. $1992)^{2}$. In the idealized case of no evolution in the co-moving number density, there should be on average one galaxy brighter than $0.3 L^{\star}$ in a volume of $1 / \phi^{*}$. In order to have a $95 \%$ chance of intercepting at least one such galaxy, a volume three times as large should be surveyed (i.e., $200 h^{-3} \mathrm{Mpc}^{3}$ ). This corresponds to $20 \mathrm{arcmin}^{2}$ with a $1 \%$ filter for $q_{0}=0.5$. The number of field galaxies brighter than $1 L^{*}$ and $0.1 L^{*}$ per square arcminute are plotted in Fig. 1 as a function of redshift. Any clustering or inchusion of volumes surveyed through higher-redshift (shorter rest-wavelength) lines serve only to increase the predicted numbers.

${ }^{2}$ This is the same number density as for the $z \approx 3-4$ Lyman break galaxies of Steidel et al., although this is probably coincidence: there is unlikely to be a one-to-one correspondence between today's $L^{*}$ galaxies and their presumed Lyman break progenitors, as merging will play a major rôle. 

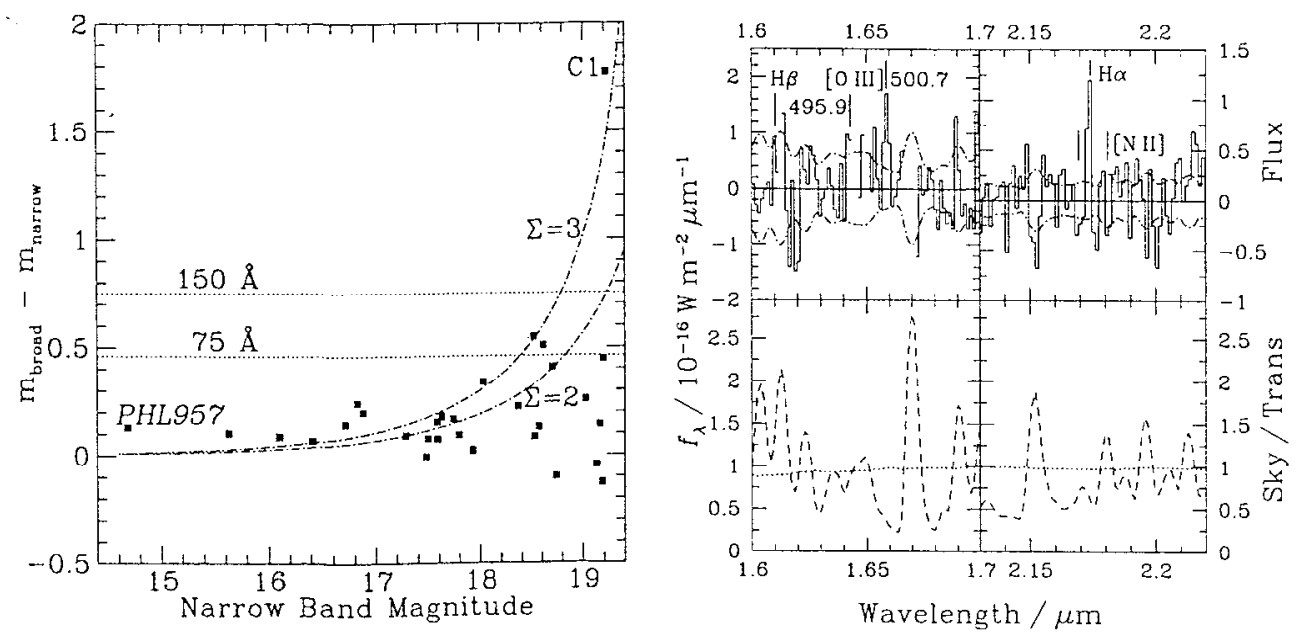

Figure 3. Left: Colour-magnitude diagram for the 30 objects detected at $S / N>4$ in the narrow-band image of the PHL 957 field by Bunker et al. (1995). The chain-dotted lines are lines of constant $\Sigma$, which is the number of standard deviations of the excess flux in the narrow band relative to the broad band. Also shown are lines of constant rest-frame equivalent width, for $\mathrm{H} \alpha$ at the redshift of the DLA $(z=2.313)$. Candidate high-redshift galaxies are objects with equivalent widths $E W_{\mathrm{rf}}>75 \AA$, and $\Sigma>2$. The line $\Sigma=2$ corresponds to a star formation rate of $11 h^{-2} M_{\odot} \mathrm{yr}^{-1}$. Note the extreme colour of the companion, $\mathrm{C} 1$ (Fig. 1), due to its $\mathrm{H} \alpha$ emission $\left(f \approx 2 \times 10^{-19} \mathrm{~W} \mathrm{~m}^{-2}\right)$. Right: The $\mathrm{H} \alpha$ (top-right panel) and [O III] $\lambda 500.7 \mathrm{~nm}$ emission (topleft panel) from C1 (UKIRT/CGS 4 spectroscopy from Bunker et al. 1999). The dot-dash lines are the $\pm 1 \sigma$ noise per pixel, and the lower panels show the fractional atmospheric transmission (dotted line) and the sky spectrum scaled down by a factor of 1000 (short-dash line).

What star formation rates/line fluxes should we expect? Deep redshift surveys (e.g., Lilly et al. 1996) suggest that $L^{*}$ galaxies have been mostly assembled by $z \approx 1$, with evolution since then mainly associated with lower-mass systems (the 'down-sizing' of Cowie et al. 1996). We concern ourselves here with the quest for the high-redshift progenitors of the present-day $\approx L^{*}$ galaxies, in the process of forming a large fraction of their stars: these used to be loosely referred to as 'primæval galaxies' (or 'proto-galaxies') before it was recognised that the star formation history of galaxies was actually a rather extended and non-coeval process.

At the current epoch, most of the luminous baryonic matter in the Universe is contained in galaxies with luminosities around $L^{*}$. If $L^{*}=10^{10} h^{-2} L_{\odot}$ and a typical stellar mass-to-light ratio is $W / L=5 \mathrm{M}_{\odot} / L_{\odot}$ (Faber \& Gallagher 1979) then to manufacture the mass in stars of an $L^{*}$ galaxy by the current epoch would require an average star formation rate of $\approx 25 h^{-2} M_{\odot} \mathrm{yr}^{-1}$ over $2 \mathrm{Gyr}$ (the Hubble time at $z \approx 1$ for $q_{0}=0.5$ ). This is an unobscured line flux of $f(\mathrm{H} \alpha) \sim 3 \times 10^{-19} \mathrm{~W} \mathrm{~m}^{-2}$ at $\approx \approx 2.3$ (Fig. 1). 
This model is, of course, rather simplistic - both $\phi^{*}$ and $L^{*}$ will evolve. If merging is important, then we might expect the high- $\sim$ progenitors of $L^{*}$ galaxies to be in many pieces. This hierarchical scenario would increase the surface density of galaxies, but reduce the average star formation rate per sub-unit which would favour depth rather than area as the pivotal survey consideration. However, if star formation at high- $z$ is episodic rather than continuous, then only a fraction of the population may be detectable in line emission at any given epoch. This has the effect of reducing the number density of actively star-forming systems, but because the star formation is concentrated in short bursts interspersed with quiescent phases, the star formation during a burst must be much greater than the average value calculated in the simplistic model. If the star formation history of a galaxy is indeed episodic, this would drive the survey considerations in the other direction - towards a larger area/volume, to maximize the chances of intercepting the luminous (but infrequent) star-bursts.

\subsection{The First Searches with $256^{2}$ Near-IR Arrays}

The early 1990s saw the emergence of $256^{2} \mathrm{InSb}$ and $\mathrm{HgCdTe}$ near-infrared arrays with low readout noise and background-limited performance even with narrow-band filters. With these, several groups undertook surveys which, for the first time, attained the flux limits and/or volumes required to test viable models of galaxy formation. An early 10-m Keck program with NIRC placed strong constraints (Pahre \& Djorgovski 1995), reaching $2 \times 10^{-19} \mathrm{~W} \mathrm{~m}^{-2}$ over $4 \mathrm{arcmin}^{2}$, but did not yield $z>2$ emission-line candidates. The first narrow-band imaging detections of line emission from high- $z$ objects came soon after. Bunker et al. (1995) imaged in $\mathrm{H} \alpha$ emission a companion of the $z=2.31$ damped Ly- $\alpha$ (DLA) QSO absorption system towards the quasar PHL 957 (Figs. 2\&3). Malkan, 'Teplitz \& McLean (1995) also detected $\mathrm{H} \alpha$ emission from the companion of another QSO absorption system at $z \approx 2.5$ (using NIRC/Keck) and subsequently found a cluster (Malkan, Teplitz \& McClean 1996). In both these cases, the line emission is unlikely to be solely due to star formation: the presence of strong high-ionization lines such as C IV $154.9 \mathrm{~nm}$ suggests an AGN contribution.

Figs. $4 \& 5$ show the constraints on galaxy evolution from the null results of a typical recent narrow-band survey (Bunker 1996). A wider-area search by Thompson, Mannucci \& Beckwith (1996) used the Calar Alto 3.5-m to survey $\approx 300 \mathrm{arcmin}^{2}$ around QSOs, finding one line-emission source to $3 \times 10^{-19} \mathrm{~W} \mathrm{~m}^{-2}$ - an unusual object with broad $\mathrm{H} \alpha$ of $\Delta v_{\mathrm{FWHM}} \sim 2000 \mathrm{~km} \mathrm{~s}^{-1}$, once again probably powered by an AGN (Beckwith et al. 1998). However, there seems to be large variance: undertaking a comparably-sized survey around absorber-line systems detected $\sim 20$ candidates (Mannucci et al. 1998). Using NIRC/Keck to reach fainter limiting fluxes $\left(\approx 10^{-19} \mathrm{~W} \mathrm{~m}^{-2}\right)$ but over a smaller area $\left(12 \operatorname{arcmin}^{2}\right)$, Teplitz, Malkan \& McLean (1998) report 13 further $z>2$ candidates.

Despite surveying $>500 \mathrm{arcmin}^{2}$, narrow-band searches have not as yet yielded a large population of star forming objects - there are $\approx 35$ candidates, of which $\approx 5$ have been spectroscopically confirmed so far. Over a similar àrea $\left(\sim 1000 \mathrm{arcmin}^{2}\right), U$-drop Lyman-break selection by Steidel and collaborators has yielded 750 spectroscopically-confirmed. Why is this? Firstly, only a thin sliver of redshift-space is sampled within a narrow-band, compared with that for broad-band colour selection; secondly, the infrared searches to date are at 
the "tip of the iceberg" in terms of the luminosity function if dust extinction is modest: for a typical galaxy in Steidel's sample $\left(M_{\mathrm{AB}}^{*} \approx-19.5\right.$ at $\lambda_{\text {rest }}=$ $1600 \AA$ ), the rest-UV would have to be suppressed by a factor of $\approx 7-8$ relative to $\mathrm{H} \alpha$ for it to be detected in $K^{-}$at $z \approx 2$ with the typical narrow-band flux limit of $3 \times 10^{-19} \mathrm{~W} \mathrm{~m}^{-2}$, corresponding to a fairly extreme obscuration of $A_{V} \approx 1.5 \mathrm{mag}$ $\left(A_{V} \lesssim 1 \mathrm{mag}\right.$ appears more typical). Given the small number of galaxies detected so far in $\mathrm{H} \alpha$ searches, there appears not to a large population of galaxies with moderately-heavy obscuration. However, narrow-band searches going a factor of $\approx 2$ deeper over a much wider area are required to comprehensively test this; the large-format near-infrared arrays on the new 8-m telescopes make this viable.
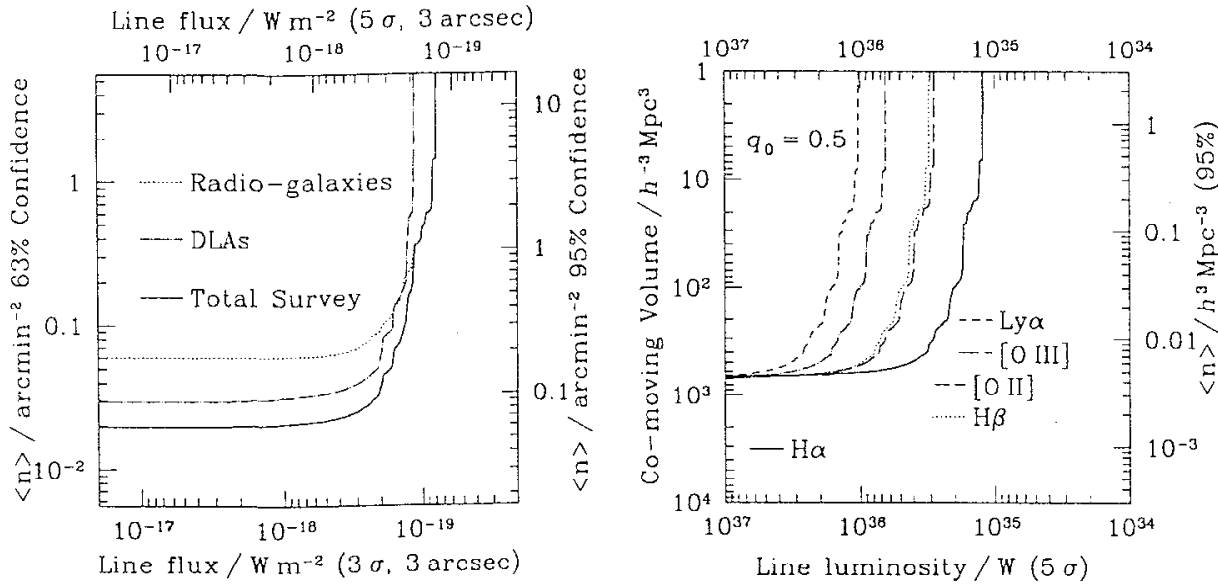

Figure 4. Left: the limits on emission line galaxies placed by the near-IR narrow-band survey of Bunker (1996), in terms of the observed quantities: the limiting flux is plotted as a function of the surface density to which this search is sensitive. Models occupying the region above and to the left of the line denoting the survey limit are excluded by the null results of the search. Both the $3 \sigma$ (lower axis) and $5 \sigma$ (upper axis) thresholds in a $3^{\prime \prime}$-aperture are shown. Surface densities ruled out at the $63 \%$ (left axis) and $95 \%$ (right axis) confidence levels are shown. The survey is divided into the total areas imaged around radio-galaxies and damped Ly $\alpha$ systems.

Right: the cumulative co-moving volumes sampled (left axis) and $5 \sigma$ limits on the line luminosities (bottom axis, assuming no extinction and $q_{0}=0.5$ ). The right axis plots the number density which would yield on average 3 galaxies in the survey volume: such models are excluded at $95 \%$ confidence if they lie above and to the left of the line denoting the survey limit for each line ( $\mathrm{H} \alpha$ at $\approx \approx 2.3 ;[\mathrm{O}$ III] \& $\mathrm{H} \beta$ at $z \approx 3.5$. [O II] at $z \sim 5$; and Ly $\alpha$ at $z \sim 16$ ). 

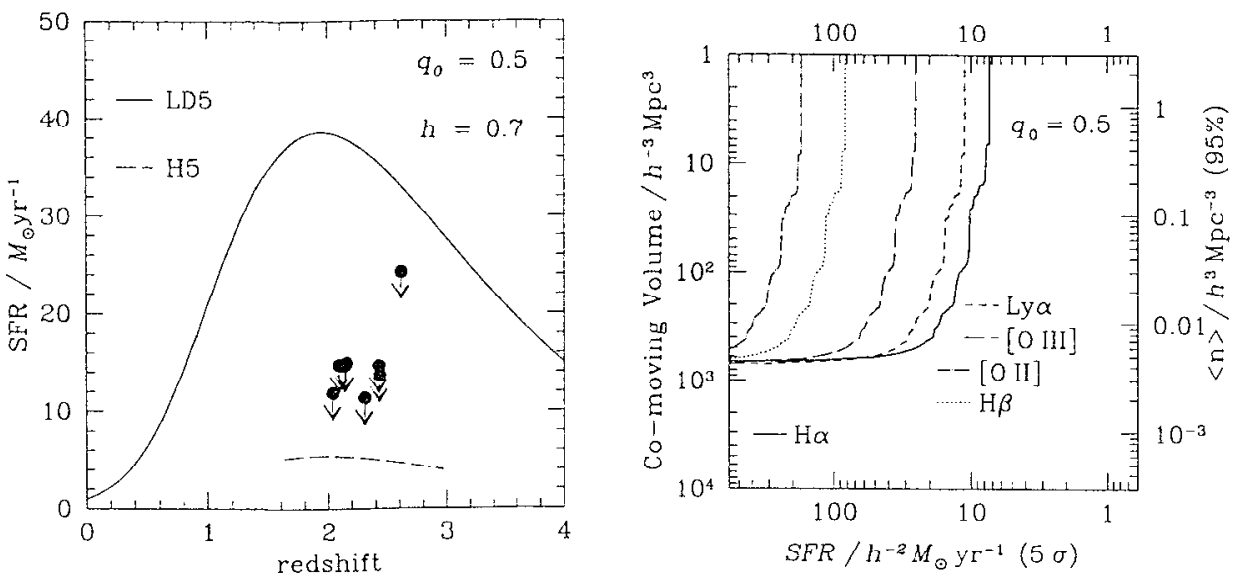

Figure 5. Left: comparison of observational $3 \sigma$ upper limits (downward arrows) to the SFRs in a sample of 8 DLAs (Bunker et al. 1999) against the predicted sample-averaged (i.e., cross-section weighted) $S F R s$ for the closed-box models of Pei \& Fall (1995). The data appear to rule out the hypothesis that DLAs are the large-disk progenitors of spiral galaxies in a $q_{0}=0.5$ cosmology (the upper limits fall below the 'LD5' solid curve). However, the curves plotted take no account of the possibility that the regions of star formation fall off the slit. The null detections are still consistent with a hierarchical picture (the dashed 'H5' curve) where the DLAs are sub-galactic units.

Right: The upper-limits on the star formation rates derived from the luminosity limits of various emission lines as a function of number density (see Fig. 4), from the narrow-band survey of Bunker (1996). Standard case B unextinguished line ratios have been adopted - Ly $\alpha$ is 8 times stronger than $\mathrm{H} \alpha$, so the star formation rates probed are comparable although the redshift is much higher $(z \approx 16$ vs. $z \approx 2.3)$.

\section{Near-Infrared Spectroscopy}

\subsection{Slitless Spectroscopy with HST/NICMIOS}

The installation of NICMOS on HST offered an unprecedented opportunity to chart star formation at $z>1$. The low-background in the $J$-and $H$-bands compared to ground-based observatories with their bright atmospheric $\mathrm{OH}$ airglow lines, coupled with wavelengths outside the usual atmospheric windows being accessible, made near-infrared slitless spectroscopy a truly effective tool for the first time. Comparable flux limits to the deeper narrow-band searches could be attained in a few orbits, but the comoving volume surveyed was much greater: the entire $H$-band was accessible in a single exposure (a range of $z \approx 0.8-1.8$ for $\mathrm{H} \alpha$ ). Pat McCarthy, Lin Yan and colleagues have analysed the many orbits of parallel-time data obtained with the grisms on the NIC 3 array $\left(52^{\prime \prime} \times 52^{\prime \prime}\right)$. Surveying several fields totalling $\approx 65$ arcmin $^{2}$. VcCarthy et al. (1999) found $\approx 30$ single-line emission objects down to a limiting flux of $0.4 \times 10^{-19} \mathrm{~W} \mathrm{~m}^{-2}$. Based on this, Yan et al. derive an SFR for $z=1.3 \pm 0.5$ from $\mathrm{H} \alpha$ which is a 
factor of 3 higher than that deduced from $280 \mathrm{~nm}$ continua, attributable to dust extinction (see Lin Yan's contribution to this proceedings for more details).

\subsection{Near-IR Spectroscopy of Photometrically-Selected 'Targets}

A parallel approach to emission-line searches to determine the global star formation history is to use photometric redshift estimates from deep multi-colour imaging, for example the Hubble Deep Fields, to preselect galaxies likely to be at a suitable redshift (particularly the 'redshift desert' in the optical at $1.3 \lesssim z \lesssim 2.7$ ), and then use near-infrared spectroscopy to target these and search for the rest-optical line emission. This technique is potentially very powerful, as spectroscopy is much more sensitive to line emission than the narrowband searches because of the finer resolution element.

With typical resolving powers of $\lambda / \lambda_{\text {FwHM }} \gtrsim 1000$, the latest near-IR spectrographs can resolve out the $\mathrm{OH}$ sky, enhancing the sensitivity between these lines (particularly in $H$-band, where the sky continuum is quite dark at wavelengths much shorter than the thermal infrared).

Glazebrook et al. (1999) targetted field galaxies of known redshift from the CFRS survey (Lilly et al. 1996) at $z \approx 1$, and from UKIRT/CGS $4 J$-band spectroscopy inferred a star formation rate from $\mathrm{H} \alpha \approx 3 \times$ higher than from the rest UV continuum, consistent with the NICMOS grism results (\$5.1.).

This technique of obtaining near-infrared spectroscopy of galaxies with previously-established redshifts has recently been extended to the Lyman-break selected population at $z \approx 3$. Pettini et al. (1998) obtained UKIRT/CGS 4 spectroscopy with UKIRT on five $U$-drops. The recent availability of NIRSPEC on Keck (e.g., James Larkin's paper in this volume), and the imminent appearance of similar instruments on the new $8-\mathrm{m}$ telescopes, have the potential to revolutionize this study: near-infrared spectroscopy of a sample of known $z \sim 3$ galaxies will shed light on their stellar populations, abundances, true star formation rates, dust content and kinematics.

The velocity widths measured from the nebular lines are likely to provide much more reliable kinematic information than Ly $\alpha$, which is resonantly broadened and typically exhibits a PCygni-like profile with the blue wing severely absorbed by outflowing neutral hydrogen. However, the profile of the nebular lines may not be broadened by the full gravitational potential of the host galaxy; there width may instead reflect just the velocity dispersion and outflows within the star-forming $\mathrm{H}$ il region.

\subsection{Near-Infrared Studies of Damped Absorbers}

An extension of the method of taking a near-infrared spectrum of a known high$\approx$ galaxy to determine its true star formation rate is to study QSO absorption line systems. We know a priori that there is a large gas column density at a particular redshift (causing Ly $\alpha$ absorption of the continuum of the background QSO which is presumably associated with a foreground galaxy. Therefore, a good strategy may be to target where you think a galaxy is. In this volume, Varsha Kulkarni describes a narrow-band search in $H$ with HST/NICMOS for line emission from a damped Ly $\alpha$ system. and several groups have used groundbased narrow-band imaging in $i$ to search for $\mathrm{H} \alpha$ emission from DLAs (e.g., Bunker et al. 1995; Mannucci et al. 1998). 
The measurement of quasar absorption lines allows an independent approach to studying the history of galaxies than the traditional flux-limited selection. The highest hydrogen column density absorbers seen in the spectra of background QSOs, the damped Ly $\alpha$ systems (DLAs), contain most of the neutral gas in the Universe at $z>1$ (Lanzetta et al. 1991). The global history of star formation in the Universe can be inferred from the evolution in the co-moving density of neutral gas (derived from the DLA statistics) as it is consumed in star formation; Pei \& Fall (1995) model this in a self-consistent manner accounting for dust pollution in the DLAs as star formation progresses. The average star" formation rate in each DLA depends then on their space density. One school of thought has $z>2$ DT.As being thick gaseous disks, the progenitors of massive spirals (e.g., Art Wolfe's contribution to this proceedings). Alternatively, DJAs could be more numerous gas-rich dwarfs, potentially sub-galactic building blocks. To differentiate between these, my colleagues and I have conducted a search for $\mathrm{H} \alpha$ emission from star formation in $z \approx 2.3$ damped systems (Bunker et al. 1999), using near-infrared spectroscopy with CGS 4 on UKIRT and building on the previous work of $H_{11}$ et al. (1993). The absence of any detectable emission at the faint fluxes probed runs counter to the predictions of the large disk hypothesis (Fig. 5) - adding further weight to hierarchical scenarios where today's massive galaxies were in pieces at high- $z$.

\subsection{The Next Frontier: Lyman- $\alpha$ at $z \gtrsim 10$ ?}

As we push to even greater redshifts, the optical becomes less and less useful: the opacity of the intervening $\mathrm{H}$ I absorbers effectively extinguishes most of the flux below $\lambda_{\text {rest }}=121.6 \mathrm{~nm}$ at $z>5$, forcing a move to the near-infrared. The continuum break at Ly $\alpha$ redshift to the near-IR is a potential way to get to $z>8$, although spectroscopy of the most promising ' $J$-drop' in the HDF-N was inconclusive (Dickinson et al. 1999). Despite its poor track-record, Ly $\alpha$ emission may be a better signature of star formation in the very early universe, when chemical enrichment and dust obscuration were less advanced. The varions $K$-band emission line searches ( $\$ 4$. and Figs. $4 \& 5$ ) already constrain star formation at immense redshift $(z \sim 16)$; as Avi Loeb's article in this volume suggests, deep integrations on a $10-\mathrm{m}$ may detect $\mathrm{Ly} \alpha$ even before the onset of the Gunn-Peterson effect, with the red-side of the resonantly-scattered line emission emerging unextinguished.

\section{Conclusions}

There are two primary considerations in the formation and evolution of galaxies: the assembly of mass (structure formation and the merging history); and the rate of conversion of neutral gas into stars (the star formation rate). Both of these are poorly understood, and may be regulated by various feedback mechanisms as well as being cloaked by dust. The current observational constraints are very weak at high redshift.

Detection of the rest-optical emission lines in galaxies at $z>2$ is important to measure the true star formation rates, to correct for dust and to eliminate systematics in the Madau diagram. This necessitates moving to the near-infrared $J_{-}, H$ - and $K^{-}$-bands. The brightness of the infrared sky background and the 
immature technology (compared to optical CCDs) has previously been a deterrent to using these windows. However, the advent of modern detectors with low read-noise and large format make near-infrared searches for 'primæval' galaxies viable. Near-infrared spectroscopy is about to be revolutionized through the imminent availability of instruments on $10 \mathrm{~m}$-class telescopes, and the latest generation of arrays with large fields-of-view mean that narrow-band searches may at last fulfill their potential. To push to $z \gtrsim 10-$ the next frontier in the Hy-redshift Universe - demands that we abandon the optical.

Acknowledgments. I am indebted to my mair collaborator, Steve Warren, for his many insights into the high-redshift Universe. Steve Rawlings, Mark Lacy, Gerry Williger, Paul Hewett \& Dave Clements have all been involved in the near-infrared searches undertaken by the Oxford group. I gratefully acknowledge enlightening discussions with Harry Teplitz, Lin Yan, Fillipo Mannucci, Mike Pahre \& Dave Thompson about their surveys. The hard work of George Djorgovski, Ivan King \& Daniel Stern made this meeting a reality, and what made it possible is (of course) the varied and influential research of Hyron Spinrad -- happy 65 th birthday, Hy!

\section{References}

Barger. A. J., Cowie, L. L., Sanders, D. B., et al. 1998, Nature, 394, 248

Beckwith S. V. W., Thompson, D., Mannucci F., \& Djorgorski, S. G. 1998, ApJ, $504,10 \tau$

Bunker, A. J., Warren, S. J., Hewett, P. C., \& Clements, D. L. 1995, MNRAS, 273,513

Bunker, A. J. 1996, D.Phil. Thesis, University of Oxford, "Searches for Distant Galaxies", abstract in 1997, PASP, 109, 628.

WTW address http://astro.berkeley, edu/ bunker/thesis.html

Bunker, A. J., Warren, S. J., Clements, D. L., Williger, G. M., \& Hewett, P. C. 1999, MNRAS, in press astro-ph/9906175

Chen, H. W., Lanzctta, K. M., \& Pascarelle, S. 1999, Nature, 398, 586

Chen, W. L., \& Neufeld, D. A. 1994, ApJ, 432, 467

de Propris, R., Pritchet, C. J., Hartwick, F. D. A., \& Hickson, P. 1993, AJ, 105, 1243

Collins, C. A., Parkes. I. M., \& Joseph, R. D. 1996, MNRAS, 282, 903

Cowie, L. I., Songaila. A., Hu, E. M., \& Cohen, J. G. 1996, A.J, 112,839

Dey, A., Spinrad, H., Stern, D., Graham, J. R., \& Chaffee, F. H. 1998, ApJ. 498. L93

Dickinson. M., Hanley, C., Elston, R., et al. 2000, ApJ, in press astro-ph/9908083

Faber, S. M., \& Gallagher, J. S. 1979, ARA\&A, 17, 132

Glazebrook. K., Blake, C.. Economou, F., Lilly, S., \& Colless, M. 1999, MNRAS, 306,843

Hu, E. W. Songaila, A., Cowie, L. L., \& Hodapp, K.-W. 1993, ApJ, 419, L13

Hu, E. M., Cowie, L. L. \& McMahon, R. G. 1998, Ap.J. 502, L99

Fennicutt. R. C. 1983. ApJ, 272, 54 
Kennicutt, R. C. 1992, ApJ, 388, 310

Koo, D. C. 1986, in Chiosi, C., \& Renzini, A., eds., 'Spectral Evolution of -Galaxies", Reidel - Dordrecht, p. 419

Kunth, D., Mas-Hesse, J. M., Terlevich, E., Terlevich, R., Lequeux, J., \& Fall, S. M. 1998, A\&A, 334, 11

Lanzetta, K. M., Wolfe, A. M., Turnshek, D. A., Lu, L., McMahon, R. G., \& Hazard, C., 1991, ApJS, 77, 1

Lilly, S. J., Le Fèvre, O., Hammer, F., \& Crampton, D. 1996, ApJ, 460, L1

Lowenthal, J. D., Hogan, C. J., Green, R. F., Caulet, A., Woodgate, B. E., Brown, T., \& Foltz, C. B. 1991, ApJ, 377, L7

Loveday, J., Peterson, B. A., Efstathion, G., \& Maddox, S. J. 1992, MNRAS, 390,338

Madau, P., Ferguson, H. C., Dickinson, M. E., Giavalisco, M., Steidel, C. C., \& Fruchter, A. 1996, MNRAS, 283, 1388

Malkan, M. A., Teplitz, H. I., \& McLean, I. S., 1995, ApJ, 448, L5

Malkan, M. A., Teplitz, H. I., \& McLean, I. S., 1996, ApJ, 468, L9

Mammucri F., \& Beckwith S. V. W. 1995, ApJ, 442, 569

Mannucci F., Thompson D., Beckwith S. V. W., \& Williger G. M. 1998, ApJ, $501, \mathrm{~L} 11$

McCarthy, P., Yan, L., Freudling, W., et al. 1999, Ap.J, 520, 548

Pahre, M. A., \& Djorgovshi, S. G. 1995, ApJ, 449, L1

Parkes, I. M., Collins, C. A., \& Joseph, R. D. 1994, MNRAS. 266, 983

Pei, Y. C., \& Fall, S. M. 1995, ApJ, 454, 69

Pettini, M., Kcllogg, M., Steidel, C. C., Dickinson, M., Adelberger, K. L., \& Giavalisco, M. 1998, ApJ, 508, 539

Pritchet, C. J., \& Hartwick, F. D. A. 1990, ApJ, 355, L11

Pritchet, C. J. 1994, PASP, 106, 1052

Steidel. C. C., Pettini, M., \& Hamilton, D. 1995, AJ, 110, 2519

Steidel, C. C., Giavalisco, M., Dickinson, M., \& Adelberger, A. 1996a, A.J, 112, 352

Steidcl, C. C., Giavalisco, M., Pettini, M., Dickinson, M., \& Adclberger, A. 1996b, ApJ, 462, L17

Steidel C. C., Adelberger K. L., Giavalisco M., Dichinson M. E., \& Pettini II. 1999, ApJ, 519, 1

Steidel C. C., Adelberger K. L., Shapley, A. E., Pettini M., Dickinson M. E., \& Giavalisco M. 2000, ApJ, in press astro-ph/9910144

Stern, D., Bunker, A. J., Spinrad, H., \& Dey, A. 2000, AJ, submitted

Teplitz, H. I., Malkan, M. A., \& McLean, T. S., 1998, ApJ, 506, 519

Thompson, D., Djorgovski, S. G., \& Beckwith. S. V. W. 1994, A.J, 10T, 1

Thompson, D., Djorgovski. S. G., \& Trauger, D. 1995, AJ, 110, 963

Thompson, D., Mannucci F., \& Beckwith S. V. W. 1996, AJ, 112, 1794

Yan, L. McCarthy, P., Freudling, W., et al. 1999, ApJ, 519, 47 
The Hy Redshift Universe

A SP Conference Series. Vol. 193, 1999

A. J. Bunker \& W. J. M. van Breugel, eds.

\title{
Star Forming Galaxies in the Moderately Hy-Redshift Universe
}

\author{
Charles C. Steidel and Kurt L. Adelberger \\ Palomar Observatory, California Institute of 'l'echnology, MS 105-24, \\ Pasadena, CA 91125
}

\begin{abstract}
We describe some recent results of several large surveys for star forming galaxies at high redshift. These "targeted" surveys use various forms of photometric pre-selection to maximize the efficiency with which spectroscopic large spectroscopic samples ban be compiled; the general aim is to address the connection between galaxy formation/evolution and the development of structure during the redshift range $1 \lesssim z \lesssim 4.5$. We also discuss the uses of Lyman $\alpha$ imaging for exploring the very high redshift universe, and the possible connection between star-forming galaxies selected in the UV and those selected in the sub-mm.
\end{abstract}

\section{What Do We Do When the Novelty Wears Off?}

The last several years have witnessed a renaissance of the classical "redshift race" (which Hy has himself won so many times) for distant objects, the effort to identify galaxies at ever higher redshift using whatever means and techniques are available. Photometric redshifts, or the use of photometric techniques in general, coupled with HST and large-aperture ground-based telescopes, have clearly made the difference, where unique spectrophotometric signatures are depended upon to isolate the relatively rare faint objects that might provide the next leap forward in redshift. So far the scientific rationale for such searches has been, as always, "because they are there", or perhaps, to "see if they are there". This simple justification of the quest is very powerful, and of course a lot of fun... but the field of galaxy formation and evolution is progressing, and we are running out of cosmic time (if not redshift!). Soon we will be forced to try to understand how this complex process works - the field is going to demand more quantitative results, and better connection to theory. 'This may involve considerable back-tracking!

In particular, given that such substantial progress has been made in establishing a paradigm for structure formation which develops naturally out of the physics of the early universe, and even possibly cosmological parameters that specify the details of the model, it would be nice to try to fit the observations of galaxies into that framework and understand galaxy formation as a natural consequence of the development of structure. We would like to have confidence that the universe we see reflected by observations of galaxies as a function of look-back time is the same one that produces large scale structure, the CMB, big bang nucleosynthesis, etc. In making this connection, we will learn a great deal about the complicated astrophysical properties that dictate what the observable 
galaxies will look like, and how those properties are influenced by environment, cosmic epoch, and the structure of the dark matter.

The key to making this kind of connection is constructing samples that are large enough to provide good statistics, and where the "selection function" can be characterized well enough to ensure that one is not comparing apples and oranges. One way of achieving both of these is to use photometric "culling" to isolate particular cosmic epochs and particular types of objects. We will discuss several examples of this type of survey below, where we (very) briefly discuss some ongoing work a.t "moderate" redshifts of $z \simeq 1-4$. We "backtrack" not because the very Hy-redshift universe is not interesting, but because it is just too hard! Instead, we concentrate on the universe's "middle age", where the necessary details and statistics are now observationally accessible.

\section{Lyman Break Galaxies}

The original intention of the set of color criteria we chose to flag candidate $\approx \sim 3$ galaxies was to find a region in color-space that would only be occupied by high redshift galaxies, should they exist (Steidel et al. 1995). This provided a. starting point for follow-up spectroscopy, which only became feasible when the combination of Keck and LRIS (Oke et al. 1995) became available. Over the past few years, we have been able to refine the color selection technique with the benefit of spectroscopic results; generally speaking, we have experimented with color selection beyond the boundaries of whatever our current set of criteria happened to be, to see if the efficiency remained high when a larger swath of color-color space was included. The current redshift histogram for objects satisfying our general $z \sim 3 \mathrm{LBG}$ color selection criteria is shown in Figure 1.

Photometric selection (as opposed to photometric redshifts) keys in on regions in color space that are unambiguous by design, providing a relatively "clean" sample of photometrically similar objects with minimal contamination. There are many applications where such samples are preferred, despite the fact that not every galaxy at a given redshift is necessarily included. To some extent, one pays a price for the simplicity of photometric selection using regions in colorcolor space in the form of increased complexity of the selection function. For example, with reference to Figure 1 (and also Figure 1 of S99), our fixed color criteria would select unreddened LBGs in the redshift range $2.9<z<3.7$, fairly heavily reddened objects in the range $2.6<z<3.1$, and "typical" $(E(B-V) \sim 0.15)$ objects in the range $2.7<z<3.4$. This means that the surveyed volume at a given redshift depends on the distribution of colors among the LBGs. To make full use of the fairly extensive statistics that now exist on the $z \sim 3$ galaxies, one must understand the selection function in detail, using a combination of the spectroscopic and photometric (i.e., LBG candidate) samples and extensive Monte Carlo simulations (599, Adelberger ct al. 2000).

The applications of the LBG samples to studies of large-scale structure have been discussed by Giavalisco at this mcting, and so we will not discuss them here.

In general, the application of photometric techniques to studies of the evolution of structure traced by galaxies will be extremely important. It is well known that different lypes of galaxies cluster differently in the local universe. 


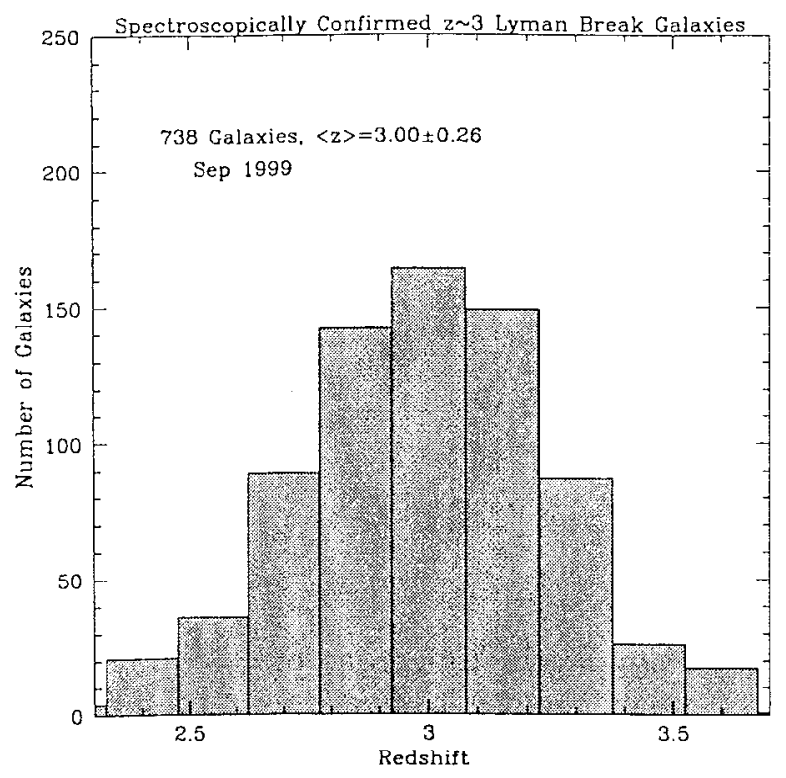

Figure 1. Redshift histogram for spectroscopically confirmed Lyman break galaxies selected using the color criteria $\mathcal{R}<=25.5, G-\mathcal{R}<1.2$, $U_{n} \quad G>1+(G-\mathcal{R})$.

and it is expected that the rate of change of clustering properties with redshift will be strongly dependent on observables such as as galaxy luminosity and color. Understanding this differential clustering evolution will probably be vital to connecting the galaxy formation/evolution process to the development of large scale structure. At present, spectroscopic samples of galaxies beyond the local universe are generally too small to provide robust mcasurements of clustering properties ( $c f$. Steidel 1998 and references therein), and projection effects for studies of the angular clustcring of faint galaxics wash out the information content of even the most precise measurements of $w(\theta)$. Photometric techniques allow the construction of very large samples over extensive solid angles that can be sorted according to galaxy luminosity and spectral type as a function of rcdshift. This is, in our view, where the real promise of photometric redshift techniques lies.

\section{How Many Galaxies is Enough?}

It is somewhat curious that most of the recent revitalization of the pursuit of photometric redshifts has been motivated by the Hubble Deep Field campaigns - curious because these data are hardly representative of typical photometry, and in fact were probably more expensive (in terms of dollars, certainly) than all of the ground-based spectroscopy put together! The sheer depth of the HDFs so far exceeds anything obtained from the ground that one is tempted to try to solve every problem in galaxy and structure formation using 2 small 5 square arc minute patches. This is potentially quite dangerous. 

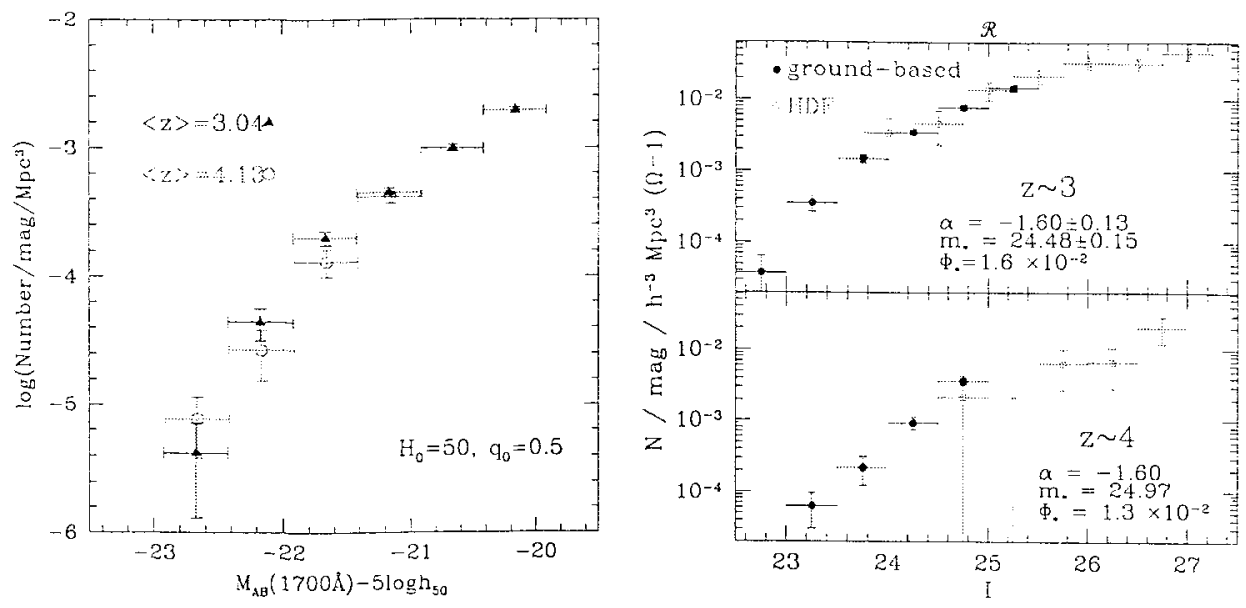

Tigure 2. Far-UV luminosity functions of $z \sim 3$ and $z \sim 4$ LBGs, from S99. The left panel shows a comparison of the bright ends of the LTs at the two redshifts, from LBG surveys optimized for diflerential comparison. The integrated luminosity densities are consistent with being the same over the range of luminosities in common. The right panel shows the LFs with data from the HDF-N included (the data have been reanalyzed by us using the distribution of continuum colors obtained from our spectroscopic LBG samples); the $\dot{z} \sim 3 \mathrm{LF}$ has been fitted with a Schechter function, while the curve shown for $z \sim 4$ is simply the $z \sim 3$ function shifted by the difference in distance modulus and with a small adjustment in normalization. The solid circles are from ground-based surveys covering $\sim 900$ square arc minutes, while the open circles are from $H D F-N$.

The important question is: how large a volume must be surveyed before one has a "fair" sample of the universe? This is not an issue of Poisson counting statistics, since galaxies are clustered on all scales to $\sim 100 h^{-1} \mathrm{Mpc}$, a scale two orders of magnitude larger than the transverse size of the HDH's. Perhaps the most vivid picture of the importance of sample variance from the recent literature is presented by Postman et al (1998), who performed an angular correlation function for faint galaxies selected in the I band over an unprecedentedly large field (degrees on a side). Uising the redshift distribution for 1-selected galaxies from the CFRS survey (Lilly et al. 1996), they found that their full survey yielded a co-moving correlation length that exceeded by a factor of two the correlation length obtained from the spectroscopic sample by the CFRS team (Le Fève et al 1996). The dispersion in the amplitude of the clustering found in the 256 independent fields comprising the full survey is striking. In general, for published redshift surveys, the correlation length measured at a given redshift exhibits a disturbing trend in that it appears to grow with the sample size!

Perhaps a more directly relevant cautionary tale involves the luminosity function and co-moving luminosity density of high redshift galaxies in the HDF: N. Madan et al (1996) analyzed samples of LBGs in coarse redshift bins in the HDF. and found that the luminosity density seemed to be dropping quickly with 
-redshift beyond $z \sim 3$, with a factor of $\sim 4$ (later revised to a factor of $\sim 2.5$ by Madau et al 1998) drop by $\approx \sim 4$. This prompted a great deal of excitement, since the "Madau plot" seemed to nicely show both the beginning and the end of the epoch of substantial star formation in galaxies. However, Figure 5 shows results based upon large area ground-based surveys that appeared in S99. A comparison of the $z \sim 4$ and $z \sim 3 \mathrm{LFs}$ showed no significant difference at the bright end measured by the ground-based data; we speculated that the HDF-N happened to be deficient in $z \sim 4$ galaxies relative to an average volume of the universe. This is not at all unexpected. High redshift galaxies in particular are very strongly clustered (S98, Adelberger et al 1998, Giavalisco et al 1998); the variance in LBG counts in angular cells the size of the HDF embedded in our relatively large survey fields is very large. The nature of sample variance is that one is more likely to measure a deficit than an over-density in a given cell.

There are a couple of important points to be emphasized here: first, fields the size of the HDF are not large enough to provide a fair sample of the universe, regardless of the quality of the data. ACS will provide an incremental improvement on the field of view, and it is likely that NGST will also be limited to fields of size a few arc minutes, although one gains at the highest redshifts $(z \gtrsim 10)$ because the same angular scale maps onto a larger co-moving transverse scale. A second point relevant to those interested in the evolution of the luminosity density with redshift: the faint end of the luminosity function is very steep at $\approx \sim 3$ and probably also at $z \sim 4$. This means that a considerable fraction of the luminosity density is contained in objects which are beyond the detection limit of even the HDF. Taken together, this implies that it will be a long time before a proper UV luminosity density is measured at very high redshifts. Given uncertainties about extinction (see below), it will be even longer before we understand the history of star formation. The good news is that there is plenty of work left to do!

\section{On the Uses of Lyman $\alpha$ Emission}

There has been recently renewed interest in using Lyman $\alpha$ emission as a means to detect galaxies at the highest redshifts, and for the first time even "blind" narrow band searches are turning up Lyman $\alpha$ emitters (Cowie \& Hu 1998; Hu, Cowie \& McMahon 1998, Pascarelle et al. 1998) by reaching sensitivity limits only a few times better than previous searches. Lyman $\alpha$, of course, has a long and somewhat sordid history; it suffers from resonant scattering and the resultant selective extinction if there is any dust in early galaxies, and in any case the emergent spectrum along any particular line of sight is subject to the vagaries of the geometry of the interstellar medium. Nevertheless, it can be a very prominent spectral feature, making the discovery of objects that are extremely faint in the continuum possible from the ground. If galaxy searches that depend on Lyman $\alpha$ are to be made quantitative, a relevant question is: what fraction of star-forming galaxies at high redshifts will have Lyman $\alpha$ emission strong enough to allow discovery using narrow-band imaging?

This question is easy to answer at $z \sim 3$, at least to $\mathcal{R}=25$, based on the spectroscopic sample of LBGs. Figure 3 shows the histogram of rest-frame Lyman $\alpha$ equivalent widths for a single LBG field in which $92 \%$ spectroscopic 


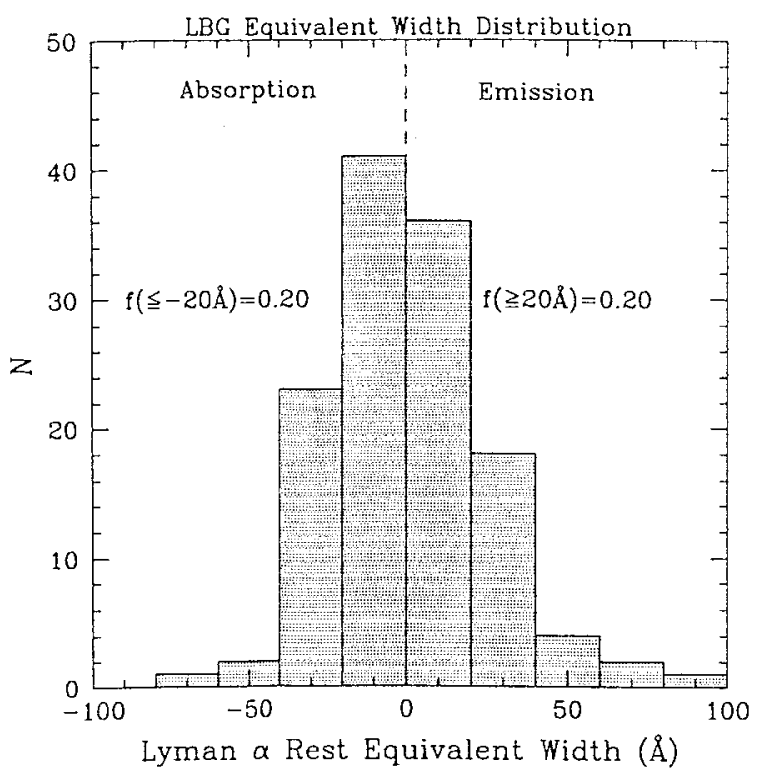

Figure 3. The distribution of rest equivalent width of the Lyman $\alpha$ line for continuum-selected Lyman break galaxies at $z \sim 3$ and with $\mathcal{R}_{A B} \leq 25.0$. The histogram is drawn from a ficld in which the spectroscopic completeness is $92 \%$, and so should be very close to the "true" distribution of Lyman alpha line strength. The mean value is zero, and the median is slightly smaller than zero (i.e., Lyman $\alpha$ is in absorption).

completeness has been achieved, to $\mathcal{R}_{A B}=25$. Note that the mean equivalent width for Lyman $\alpha$ is essentially zero, and the median is $-11 \AA$ ! The fraction of objects for which Lyman $\alpha$ emission exceeds $W_{\lambda}=20 \AA$ in the rest frame is $f=0.20$. Roughly speaking, this is the fraction of LBGs at a given redshift that would be recovered in a narrow band experiment. Of course, it is possible that the distribution of Lyman $\alpha$ equivalent widths changes significantly with redshift and/or continuum luminosity, although there is no indication from our sample of $z \sim 4$ LBGs that strong Lyman $\alpha$ emission is more common at the bright end of the LBG luminosity function than at $z \sim 3$ (see S99).

Possibly the most promising use of narrow-band imaging techniques (a form of photometric redshift, after all) is to use it to "map out" the distribution of galaxies over a large range of intrinsic luminosity within known or suspected structures at high redshift (cf. Campos et al. 1999, Steidel et al 2000). In this case, even if one were only seeing $20-25 \%$ of the galaxies, the number of objects found could be very large given the steep faint end of the luminosity function (see figure 2 above). We have recently obtained very deep Lyman $\alpha$ images of a region containing a significant over-density of galaxies at $z=3.09$ that was identified as part of our spectroscopic LBG survey (Steidel et al. 1998; S98). The spectroscopic sample contains 24 galaxies with $3.09 \pm 0.03$ in a field $\sim 9^{\prime}$ on a side, an enhancement relative to an average volume at $z \sim 3$ of a factor of 6 . The spectroscopic sample only reaches $\mathcal{R}=25.5$ and is far from complete; we were interested in whether narrow-band imaging, which is able to probe more than 


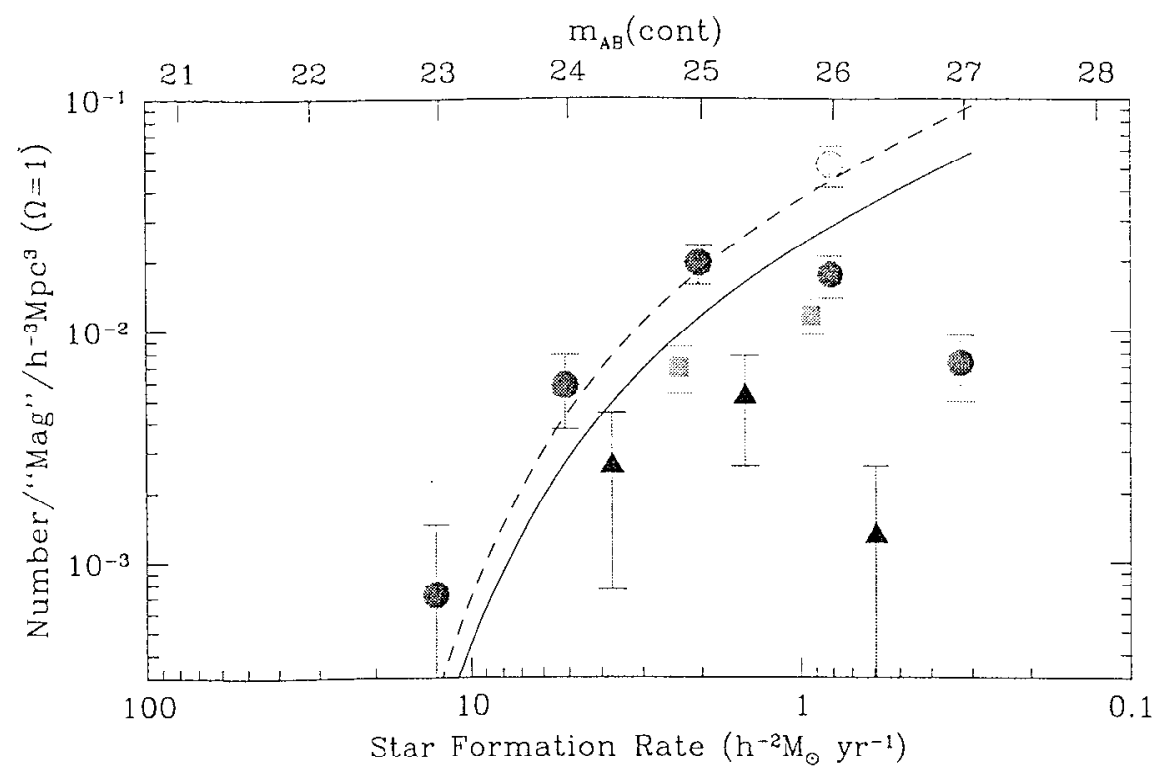

Figure 4. The LF of narrow-band emitters within the $z=3.09$ structure in SSA22a field (circles), together with the LBG "field" LF (solid curve); the triangles are from a "blank-field" survey by Cowie \& $\mathrm{Hu}$ (1998), and the squares are from a CMB decrement field presented by Campos et al. 1999. The open circle represents a correction to the $m_{A B}=26$ point that accounts for relative incompleteness with respect to emission line equivalent width assuming the distribution function shown in Figure 3. The dotted curve is the LBG field LF with a normalization increased by a factor of 1.5 . After accounting for the factor of 6 density density enhancement in the spike, this implies that about $1.5 / 6 \approx 25 \%$ of continumm-selected galaxies wonld be found using narrow-band Lyman $\alpha$ selection. (see S2000 for details)

2 magnitudes deeper in the luminosity function for strong-lined objects, would allow us to detect enough objects to allow a significant detection of substructure in a region that is almost certainly destined to become a rich cluster by the present epoch (cf. Governato et al. 1998, S98). Because this field is so overdense, we detect a factor of about 6 times higher surface density of Lyman $\alpha$ emitters than in comparable "blank field" samples (cf. Cowie \& Hu 1998), and so it also represents an interesting laboratory for comparing continuum and narrow-band selected objects at the same redshifts.

Figure 4 summarizes the continuum luminosity functions for Lyman $\alpha$ selected galaxies at $z \sim 3$ from the $z=3.09$ "spike" field, and from two other narrow-band experiments at similar redshift. The main point is that the LF of strong Lyman $\alpha$ emitters even at very faint continuum luminosities (after a correction is applied to make the sample sensitive to the same rest equivalent width threshold at each luminosity) mimics the field LBG luminosity function in shape: the implication is that there is no significant tendency for fainter continuum objects to have larger Lyman $\alpha$ equivalent widths. In fact, accounting 

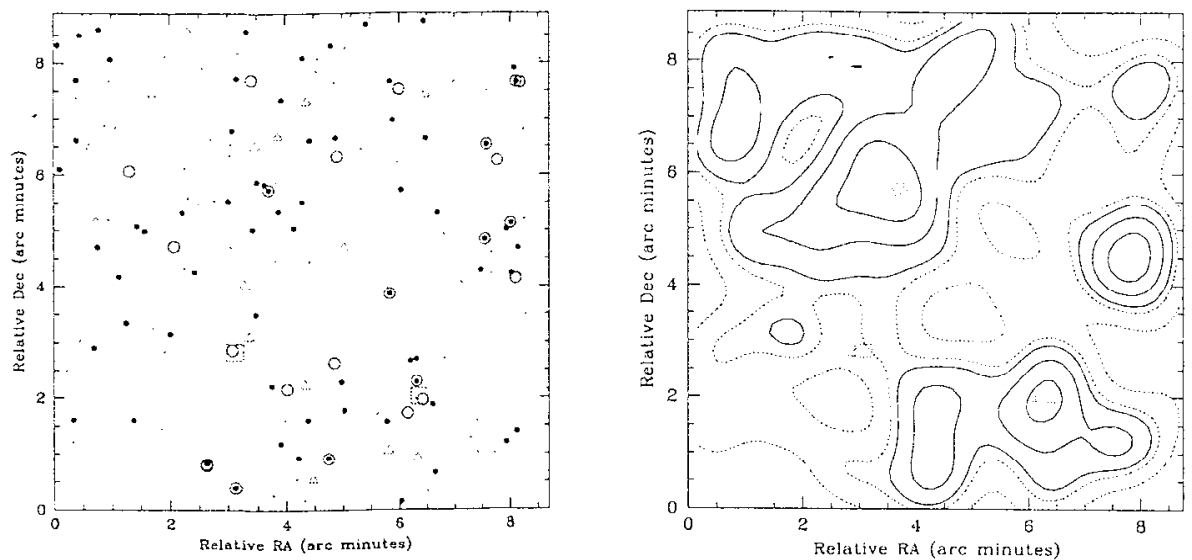

Figure 5. Maps of the $z=3.09$ "spike" on the plane of the sky. In the left panel, circles denote spectroscopically confirmed LBGs within the structure, triangles are narrow-band deficit objects that are also LBG candidates, and solid dots are narrow-band excess objects (smaller dots are fainter in the continuum). The large squares are the two giant $(\sim 20$ arc second) Lyman $\alpha$ Blobs discovered in the narrow-band images. The right panel is a surface density map smoothed on scales of 1 arc minute; the highest contours represent local density enhancements of a factor of 2 , or regions that are about 12 lines more dense than an average volume at $z \sim 3$. Note that one of these peaks is associated with a QSO (star symbol) and another with one of the two giant Lyman $\alpha$ blobs

for the factor of 6 density enhancement in the SSA22a field at $\langle z\rangle=3.09$, the fraction of Lyman $\alpha$ emitters with $W_{\lambda}>20 \AA$ appears to be $\sim 25 \%$, consistent with the estimate from the bright spectroscopic sample above. Details of this analysis can be found in Steidel et al. 2000 (S2000).

Figure 5 shows the result of combining the narrow-band selected galaxies in the $z=3.09 \pm 0.03$ "spike" with the existing spectroscopic Lyman break galaxy sample. There is a total of 162 objects in this relatively small region which are either known or strongly suspected to be within the large over-density of galaxies. The smoothed surface density map on the right yields an interesting result: while the overall distribution of objects within the volume is consistent with random. 2 of the 3 highest density peaks (all $3 \sigma$ peaks on comoving scales of $\sim 1 h^{-1} \mathrm{Mpc}$ ) are associated with unusual objects - one is associated with an $\mathcal{R}=21.7$ QSO, and the other is associated with one of the two giant Lyman $\alpha$ "Blobs" discovered in the narrow-band images (see S2000 for more information on this new class of object).

The bottom line: Lyman $\alpha$ imaging can be an effective means of finding objects at high redshift, particularly in regions of known density enhancement, so long as completeness with respect to continuum luminosity is not important. Our best guess about what fraction of high redshift galaxies will have strong enough 
line emission to be found using narrow-band techniques is $20-25 \%$, independent of continuum luminosity.

\section{The UV and Sub-mm Views of the High Redshift Universe: Any Connection?}

If high-redshift galaxies resemble rapidly star-forming galaxies in the local universe, the majority of the luminosity emitted by their massive stars will have been absorbed by dust and re-radiated at far-infrared wavelengths that cannot penetrate the earth's atmosphere. This simple fact has profound implications. It means, for example, that standard estimates of star-formation rates for highredshift galaxies based on the strength of their far-UV continua are likely to be serious underestimates, since most of luminosity of massive stars emerges from the galaxies in the far-IR not the far-UV (a galaxy of median UV color in our LBG sample would have only about $15-20 \%$ of its bolometric luminosity emerging in the UV - the rest presumably is re-radiated in the far-IR). It forces attempts to derive more accurate star-formation rates to rely on large and uncertain estimates of these galaxies' undetected far-IR luminosities. It also raises the possibility that significant populations of high-redshift galaxies may be missing from optical surveys altogether because too large a fraction of their massive stars' luminosities emerges in the far-IR instead of the far-UV (see Blain's contribution to these proceedings). Understanding the effects of dust on high-redshift galaxies is evidently crucial for interpreting the results of ever growing high-redshift surveys.

A useful tool in this regard is the relationship between local starburst galaxies' spectral slopes in the UV, $\beta$, and their ratios of far-IR to far-UV luminosity (see Meurer et al. 1999). This relationship can in principle let one estimate the (often) dominant dust-processed component of high-redshift galaxies' luminosities based on the (sometimes) trace amount of their UV radiation which escapes unabsorbed by dust. However, the $\beta /$ far-IR relationship has been shown to hold only for starburst galaxies in the local universe, and in order to use it to estimate with confidence the dust-corrected star-formation rates of high-redshift galaxies we need to show that these galaxies also obey the relationship. In Adelberger et al. (2000) we attempt to gather all the relevant evidence. These data, which consist of $850 \mu \mathrm{m}$ and $20 \mathrm{~cm}$ observations of Lyman-break galaxies at $z \sim 3$, of $15 \mu \mathrm{m}$ and $20 \mathrm{~cm}$ observations of Balmer-break galaxies at $z \sim 1$ (see $\S 6$ below), and of UV to radio observations of the sub-mm source SMMJ14011 at $z=2.565$ and the extremely red object HR10 at $\tilde{z}=1.44$, are largely consistent with the idea that the dust emission of star-forming galaxies at high-redshift can be reliably estimated with Meurer et al.'s $\beta /$ far-IR relation. The only clear exception among the galaxies in this sample is HR10, whose relatively large $850 \mu \mathrm{m}$ flux could not have been predicted from its faint UV continuum using the $\beta$ /far-IR relation.

If it is in fact true that UV-selected galaxies at high redshift obey the $\beta /$ far-IR relation, then we are in a position to estimate these galaries' total contribution to the $850 \mu \mathrm{m}$ background. Details of this calculation are presented in Adelberger \& Steidel (2000); the upshot, shown in Figure 6, is that known $[V$-selected populations at $1 \lesssim z \lesssim j$ could by themselves account for most of 


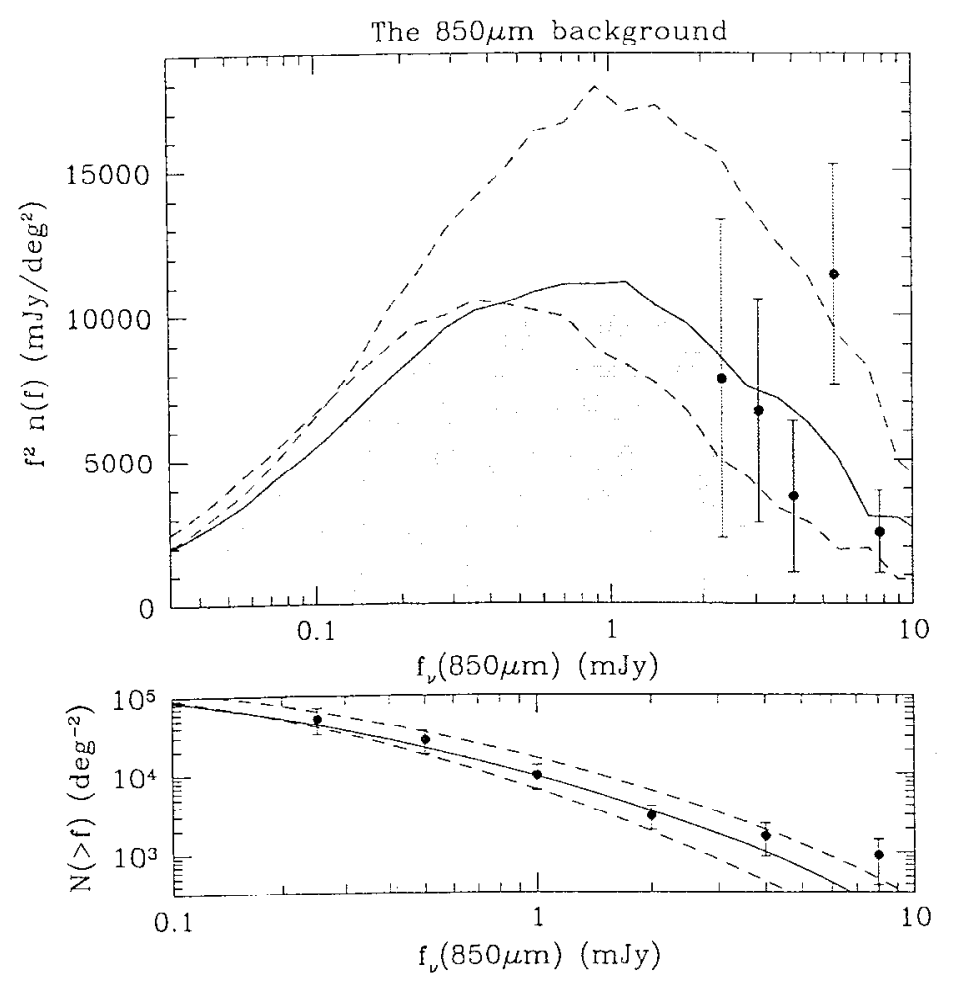

Figure 6. (Top) A comparison of the observed $850 \mu \mathrm{m}$ background to the estimated $850 \mu \mathrm{m}$ background produced by known UV-selected galaxy populations at $1<z<5$. The observed points from Barger, Cowie, \& Sanders (1999) are shown as points with error bars. EstiInaling total $850 \mu \mathrm{m}$ fluxes of these UV-selected galaxy populations is subject to significant uncertainties (see Adelberger \& Steidel (2000)), but it appears that these populations could be responsible for producing the bulk of the observed $850 \mu \mathrm{m}$ background - our maximum likelihood estimate (solid curve) produces $90 \%$ of the $850 \mu \mathrm{m}$ background measured by Fixsen et al. (1998). The bottom panel shows the UV-predicted sub-mm integral number counts (solid curve) together with the observations of Blain et al. 1999 (points with error bars). 


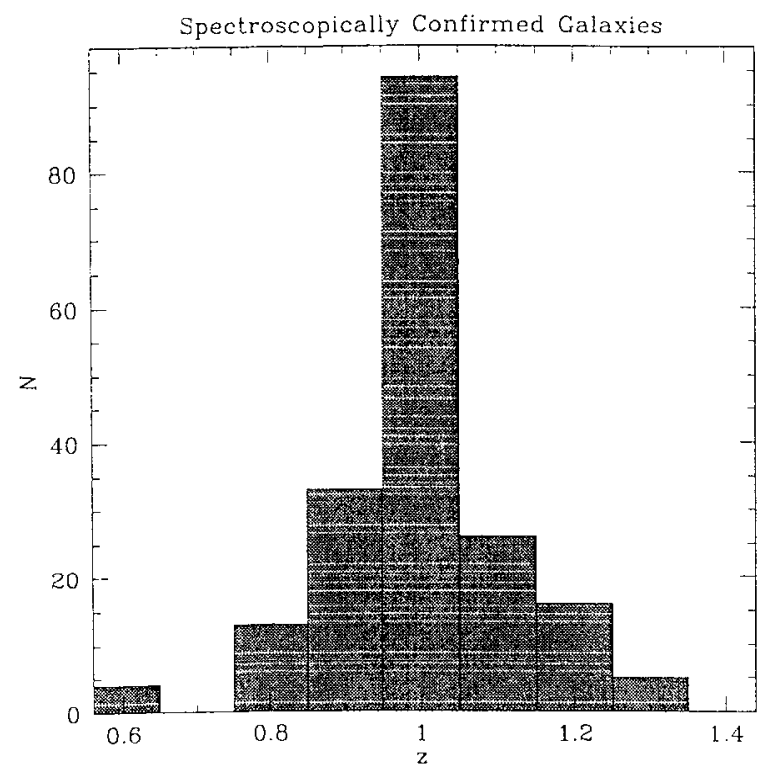

Figure 7 . The redshift distribution of photometrically preselected $z \sim 1$ "Balmer-break" galaxies observed in one night in March 1999.

the observed $850 \mu \mathrm{m}$ background, as well as the measured shape of the $850 \mu \mathrm{m}$ number counts brighter than $1 \mathrm{mJy}$, if they obeyed the $\beta /$ far-IR relation and had the same distribution of $\beta$ as observed in Lyman-break galaxies a.t $z \sim 3$. As far as we can tell, none of the current $850 \mu \mathrm{m}$ data require a significant fraction of star formation at high redshift to have occurred in objects that are too heavily reddened to be detected in UV-selected surveys. Moreover, the UV selected samples successfully "predict" the sub-mm and far-IR observations, and make further predictions that can be tested with future sub-mm/far-IR observations.

\section{6. $\quad z=1$ : The Next Frontier?}

Although galaxy samples at $z \sim 3$ can in principle teach one a great deal about galaxy formation, there is a limit to what can be learned from studying galaxies at this redshift. In particular, in order to understand better what these galaxies are, and how they are related to the ensemble of virialized dark matter halos predicted by models of structure formation, we would like to measure the sizes of their older stellar populations, their typical circular velocities, and the dependence of their star-formation rates and clustering properties upon their circular velocities and/or comoving number densities. Unfortunately the necessary observations range from difficult to impossible at $z \sim 3$. At $z \sim 1$, however, all these observations are relatively easy: the $H^{-}$band samples wavelengths red enough to be dominated by light from older stars, [OII] $\lambda 3727$ is still in the optical window, and objects of fixed luminosity are $\sim 2$ magnitudes brighter than at $\tilde{\sim} \sim 3$.

Fortunately it appears to be almost as easy to photometrically preselect galaxies at $z \sim 1$ as at $z \sim 3$. Adelberger et al. (2000) describe a technique 
for isolating galaxies at $z \sim 1$ from the dominant foreground and background populations on the basis of their $G \mathcal{R} I$ colors; the Balmer-break falls between the $\mathcal{R}$ and $I$ filters at this redshift, giving these galaxies their distinctive colors. The $\sim 400$ redshifts we have obtained for Balmer-break galaxies to date suggest that these selection criteria will provide an efficient way of finding star-forming galaxies at $z \sim 1$; the mean redshift of galaxies matching our photometric selection criteria is $\langle z\rangle \simeq 1.0$ with an rms spread of $\sigma_{z} \simeq 0.1$ (Figure 7 ).

Galaxies at $z \sim 1$ will match our selection criteria only if they are currently forming stars at rate similar to their average star-formation rate over the previous $\sim$ few hundred Myr. Data from deep redshift surveys in the Hubble Deep Field and in SSA22 suggest that about half of the known galaxies at $z \sim 1$ will satisfy this criterion. Our current sample of $z \sim 1$ Balmer-break galaxies consists of over 2000 photometric candidates in 0.2 square degrees; $\sim 400$ of these have measured redshifts $z \sim 1$. We anticipate learning much more about this sample in the near future.

\section{Summary}

We have described, through a few examples, how photometric techniques allow surveys covering very large volumes with the ability to slice samples by redshift, galaxy "type", and luminosity, and therefore are perfect for making sense of the development of structure, and the connection with galaxy evolution. The small fields available on space instruments like WFPC-2, ACS, and, later, NGST, will mean that sample variance may limit how accurately one can measure even the simplest global quantities (e.g., luminosity functions, luminosity density, gross clustering properties).

Photometric techniques also make a great deal of sense for improving the efficiency of spectroscopic surveys - one can use photometric selection to construct volume-limited samples at prescribed cosmic epochs (taking advantage of the ability to include a much vider range of luminosity at a given redshift than a survey selected by apparent magnitude), control the selection function to select only a particular class of object, and generally make every slit count. Such techniques will be essential for surveys over wide redshift ranges, as the ideal instrumental setup (e.g., UV/blue, optical, or near-IR, spectral resolution, etc.) will often depend strongly on redshift and galaxy color.

We expect that photometric techniques will make the biggest difference in the coming decade over the redshift range $0 \leq z \leq 4$ using wide field imagers and suitable follow-up spectroscopy from the ground. The information content at higher redshifts or at the faintest luminosities will depend on space-based data, which will be limited until the advent of NGST. However, the amount of work do be done in understanding galaxy formation/evolution at "modest" redshifts $z \lesssim 4$ is huge, and the potential for progress is very high.

Acknowledgments. We would like to thank our collaborators, Mark Dickinson, Mauro Giavalisco, Max Pettini. and Alice Shapley, for allowing us to present some results of joint work prior to publication, and the organizers for their patience in awaiting this written contribution. Finally, we would like to thank Hy Spinrad, the honoree of this meeting, for his boundless enthusiasm 
which rubs off on anyone who is lucky enough to spend some time in his general vicinity.

\section{References}

Adelberger, K.L., \& Steidel, C.C. 2000, Ap.J, submitted

Adelberger, K.L., Steidel, C.C., Giavalisco, M., Dickinson, M., Pettini, M.,\& Kellogg, M. 1998, ApJ, 505, 18

Barger, A.J., Cowie, L.L., \& Sanders, D.B. 1999, ApJ, 518, L5

Blain, A.W.B. 2000, these proceedings.

Blain, A.W.B., Smail, I.S., Ivison, R., \& Kneib, J.-P. 1999, MNRAS, 302,632

Calzetti, D. 1997, AJ, 113, 162

Campos, A., Yahil, A., Windhorst, R.A., Richards, E.A., Pascarelle, S., Impey, I., \& Petry, C. 1999, ApJ, 511, 1

Cowie, L.L., \& Hu, E.M. 1998, AJ, 115. 1319

Giavalisco, M., Adelberger, K.L., Steidel, C.C., Dichinson, M., \& Pettini, M. 2000 , in preparation

Giavalisco, M., Steidel, C.C., Adelberger, K.L., Dickinson, M., Pettini, M., \& Kellogg, M. 1998, A p.J, 503.543

Governato, F., Baugh, C.M., Frenk, C.S., Cole, S., Lacey, C.G., Quinn, T., \& Stadel, J. 1998, Nature, 394,241

Hu, E.M., Cowie, L.L., \& McWahon, R.G. 1998, ApJ, 502, L99

Le Fèvre, O., Hudon, D., Lilly, S.J., Crampton, D., Hammer, F., \& Tresse, L. 1996, ApJ, 461, 534

Lilly, S.J., Le Févre, O., Crampton, D.. Hammer, F., \& Tresse, L. 1995, ApJ, 455,50

Madau, P., Pozzetti, L., \& Dickinson, M. 1998, ApJ, 498, 106

Madau, P., Ferguson, H.C., Dickinson, M., Giavalisco, M., Steidel, C.C., \& Fruchter, A. 1996, MNRAS, 283. 1388

Meurer, G., Hechman, T.M., \& Calzetti, D. 1999, ApJ, in press

Oke, J.B., et al. 1995, PASP, 107, 3750

Pascarelle, S.M., Windhorst, R.A., \& Keel, W.C. 1998. AJ, 116, 2659

Postman, M., et al. 1998, ApJ, 506, 33

Shapley, A.E. et al. 2000, in preparation.

Steidel, C.C. 1998, in The Evolution of Large Scale Structure, in press (astro$\mathrm{ph} / 9811400)$

Steidel, C.C., Adelberger, K.L., Shapley, A.E., Pettini, M.. Dickinson, M., \& Giavalisco, M. 2000, ApJ, in press (astro-ph/9910144)

Steidel, C.C., Adelberger, K.L., Giavalisco, M., Dickinson, M., \& Pettini, M. 1999, Ap.J, 519, 1 (S99)

Steidel, C.C., Adelberger, K.L., Dickinson, M., Giavalisco, M., Pettini, M., \& Kellogg, M. 1998, Ap.J, 492, 428

Steidel, C.C., Pettini, M., \& Hamilton. D. 1995. AJ, 110, 2519 
. 
The Hy Redshift Universe

ASP Conference Series, Vol. 199, 19999

A. J. Bunker \& W. J. M. van Breugel, eds.

\title{
Cosmology and the Brightness of the Night Sky
}

\author{
Piero Madau \\ Institute of Astronomy, Madingley Road, Cambridge CB3 OHA, UK.
}

\begin{abstract}
I review the constraints imposed by the observed extragalactic background light (EBL) on the cosmic history of star formation and stellar mass density today. The logarithmic slope of the galaxy numbermagnitude relation from the Southern Hubble Deep Field imaging survey is flatter than 0.4 in all seven $U B V I J H K$ optical bandpasses, i.e. the light from resolved galaxies has converged from the UV to the near-IR. From the number counts one obtains a lower limit to the surface brightness of the optical extragalactic sky of about $15 \mathrm{nW} \mathrm{m}^{-2} \mathrm{sr}^{-1}$, comparable to the intensity of the far-IR background from COBE data. If the initial mass function has a Salpeter slope with a lower cutoff consistent with observations of M subdwarf disk stars, a lower limit of $\Omega_{g+s}>0.005 I_{50}$ (at Hubble constant $50 \mathrm{~km} \mathrm{~s}^{-1} \mathrm{Mpc}^{-1}$ ) can be derived for the visible mass density required to generate an EBL at a level of $50 I_{50} \mathrm{nW} \mathrm{m}^{-2} \mathrm{sr}^{-1}$. Motivated by the recent microlensing results of the MACHO collaboration, I also discuss the possibility that massive dark halos around spiral galaxies are composed of faint white dwarfs, and show that only a small fraction $(\lesssim 5 \%$ ) of the nucleosynthetic baryons can be locked in the remnants of intermediate-mass stars forming at $z_{F} \lesssim 5$, as the bright early phases of such halos would otherwise overproduce the observed EBL.
\end{abstract}

\section{Introduction}

Recent progress in our understanding of faint galaxy data made possible by the combination of $H S T$ deep imaging and $K e c k$ spectroscopy has vastly improved our understanding of the evolution of the stellar birthrate in optically-selected galaxies from the present-epoch up to $z \approx 4$ (Steidel et al. 1998; Madau et al. 1998). The large increase in the quantity of information available on the high-redshift universe at optical wavelengths has been complemented by measurements of the far-IR/sub-mm background by DIRBE and FIRAS onboard the COBE satellite (Hauser et al. 1998; Fixsen et al. 1998; Schlegel et al. 1998), and by theoretical progress made in understanding how intergalactic gas follows the dynamics dictated by dark matter halos until radiative, hydrodynamic. and star formation processes take over (Baugh et al. 1998; Somerville et al. 1999). Of perhaps equal importance for galaxy formation studies appear the recent findings of the microlensing experiments in the direction of the LMC, which suggest that between 20 and $100 \%$ of the dark matter in the Galactic halo is tied up in $0.5_{-0.2}^{+0.3} M_{\odot}$ objects (Alcock et al. 199T). The underlying goal of all these efforts is to understand the growth of cosmic structures, the internal properties of 
galaxies and their evolution, and ultimately to map the star formation history of the universe from the end of the cosinic 'dark age' to the present epoch.

In this talk I will discuss the implications for galaxy evolution studies of the observed galaxy number-apparent Inagnitude relation and its first moment, the integrated galaxy contribution to the extragalactic background light (EBL). The logarithmic slope of the differential galaxy counts is a remarkably simple cosmological probe of the history of stellar birth in galaxies, as it must drop below 0.4 to yield a finite value for the EBL. Together with the far-IR/sub-mm background, the optical EBL from both resolved and unresolved extragalactic sources is an indicator of the total luminosity of the universe, as the cumulative emission from young and evolved galactic systems, as well as from active galactic nuclei (AGNs), is recorded in this background. As such it can provide, for a given stellar mass function, a quantitative estimate of the baryonic mass that has been processed by stars throughout cosmic history.

Unless otherwise stated, an Einstein-de Sitter (EdS) cosmology $\left(\Omega_{M}=1\right.$, $\Omega_{\Lambda}=0$ ) will be adopted in the rest of this talk. The work presented here has been done in collaboration with L. Pozzetti (see Madau \& Pozzetti 1999).

\section{Galaxy counts from UV to near-IR}

The HDF-S dataset we have used includes deep near-IR NICMOS images and the deepest observations ever made with the STIS 50CCD imaging mode (details of the data reduction, source detection algorithm, and photometry can be found on ftp://archive.stsci.edu/pub/hdf.south/version1/.) Figure 1 shows the HDF$N$ and $-S$ galaxy counts compiled directly from the calalogs, as a function of $A B$ isophotal magnitudes in the $U B V I J H K$ bandpasses for all galaxies with signal-to-noise ratio $S / N>3$ within the band, together with a compilation of existing HST and ground-based data. No correction for detection completeness have been made.

One should note that different algorithms used for 'growing' the photometry beyond the outer isophotes of galdxies nnay significantly change the magnitude of faint galaxies. According to Bernstein et al. (1999) (see also Bernstein, this volume), roughly 50\% of the flux from resolved galaxies with $V>23$ mag lie outside the standard-sized apertures used by photometric packages. An extragalactic sky pedestal created by the overlapping wings of resolved galaxies appears to contribute significantly to the sky level, and is undetectable except by absolute surface photometry (Berislein el al. 1999). Also, at faint magnitude levels, distant objects which are brighter than the nominal depth of the catalog may be missed due to the $(1+z)^{4}$ dimming factor. All these systematic errors are inherent in faint-galaxy photometry; as a result, our estimates of the integrated fluxes from resolved galaxies will typically be too low, and must be strictly considered as lower limits.

\section{The brightness of the night sky}

The contribution of known galaxies to the optical EBL can be calculated directly by integrating the emitted flux times the differential number counts down to the 

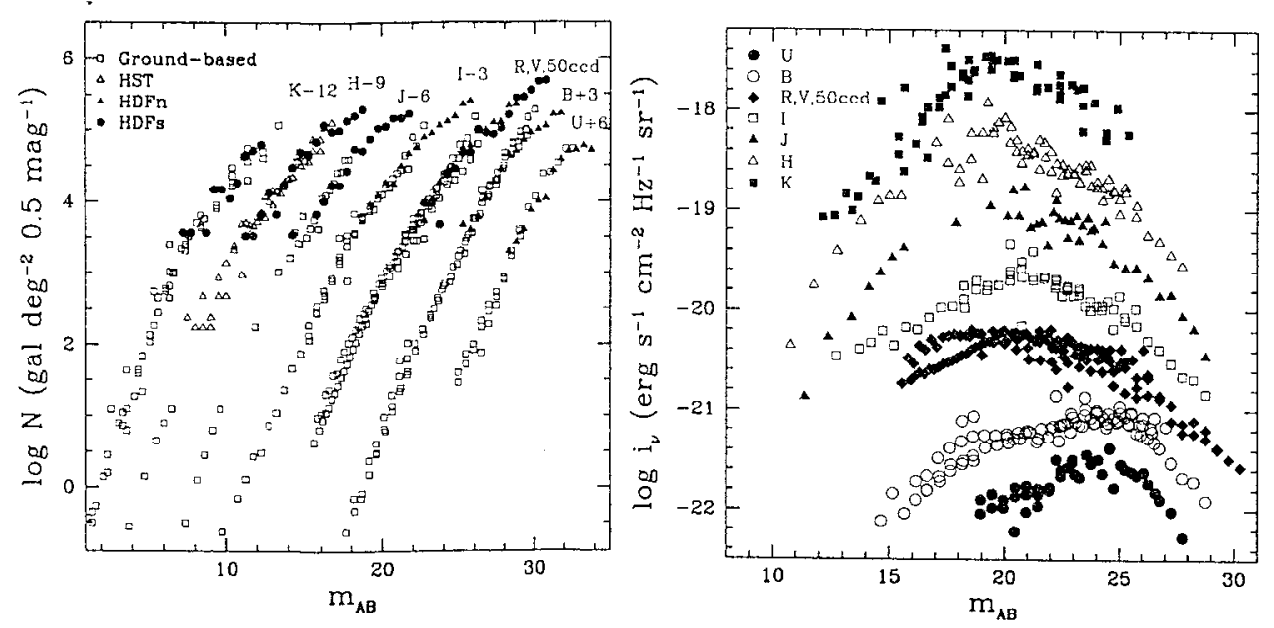

Figure 1. Left: Differential $U B V I J H K$ galaxy counts as a function of $A B$ magnitudes. Right: Extragalactic background light per magnitude bin, as a function of $U$ (filled circles), $B$ (open circles), $V$ (filled pentagons), $I$ (open squares), $J$ (filled triangles), $H$ (open triangles), and $K$ (filled squares) magnitudes. For clarity, the BVIJHK measurements have been multiplied by a factor of $2,6,15,50,150$, and 600 , respectively.

detection threshold. In all seven bands, the slope of the differential numbermagnitude relation is flatter than 0.4 above $m_{\mathrm{AB}} \sim 20$ (25) at near-IR (optical) wavelengths, and this flattening appears to be more pronounced at the shorter wavelengths. The leveling off of the counts is clearly seen in Figure 1, where the function $i_{\nu}=10^{-0.4\left(m_{A B}+48.6\right)} N(m)$ is plotted against apparent magnitude in all bands. While counts having a $\log$ arithmic slope $d \log N / d m \geq 0.40$ continue to add to the EBL at the faintest magnitudes, it appears that the HDF survey has achieved the sensitivity to capture the bulk of the near-ultraviolet, optical, and near-IR extragalactic light from discrete sources. The flattening at faint apparent magnitudes cannot be due to the reddening of distant sources as their Lyman break gets redshifted into the blue passband, since the fraction of Lyman-break galaxies at (say) $B \approx 25$ is small (Steidel et al. 1996; Pozzetti et al. 1998). Moreover, an absorption-induced loss of sources cannot explain the similar change of slope of the galaxy counts observed in the $V, I, J, H$, and $I$ bands. While this suggests that the surface density of optically luminous galaxies is leveling off beyond $z \sim 1.5$, one should caution against the possibility that a significant amount of light may be missed at faint magnitudes because of systematic errors.

The spectrum of the optical EBL is shown in Figure 2, together with the recent results from the $C O B E$ satellite. The values derived by integrating the galaxy counts down to very faint magnitude levels (because of the flattening of the number-magnitude relation most of the contribution to the optical EBL comes from relatively bright galaxies) imply a lower limit to the EBL intensity in 

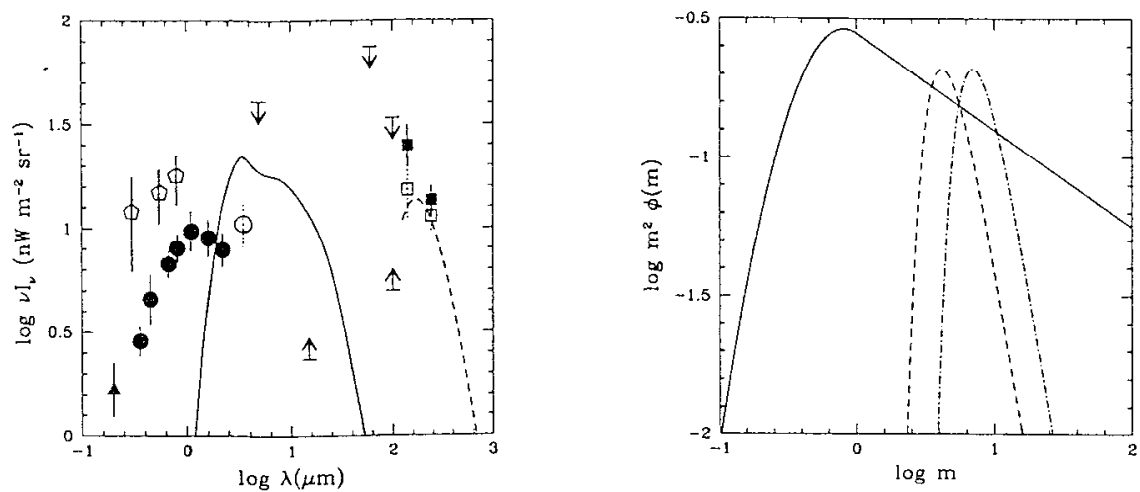

Figure 2. Left: Spectrum of the optical extragalactic background light from resolved sources as derived from a compilation (Madau \& Pozzetti 1999) of ground based and space-based galaxy counts in the UBVIJHK bands (filled dots), together with the FIRAS 125-5000 $\mu \mathrm{m}$ (dashed line) and DIRBE 140 and $240 \mu \mathrm{m}$ (filled/empty squares) detections. The empty pentagons at 3000,5500 , and $8000 \AA$ are Bernstein et al. (1999) measurements of the EBL from resolved and unresolved galaxies fainter than $V=23$ mag. The solid curve shows the synthetic EBL produced by a WD-progenitor dominated IMF with $\bar{m}=4$ and $\left(z_{F}, X, X_{\mathrm{WD}}\right)=(36,0.5,0.1)$, in the case of zero dust reddening. Right: Stellar initial mass functions, $\phi(m)$, multiplied by $m^{2}$. Solid line: Salpeter IMF, $\phi(m) \propto m^{-2.35}$ at high masses, matched to a GBF function at $m \leq 1$. Dotted line: WD-progenitor dominated IMF in galaxy halos, $\phi(m) \propto e^{-(\bar{m} / m)^{3}} m^{-5}$, with $\bar{m}=2.4$. Dot-dashed line: Same for $\bar{m}=4$. All IMFs have been normalized to unit mass.

the $0.2-2.2 \mu \mathrm{m}$ interval of $I_{\mathrm{opt}} \approx 15 \mathrm{nW} \mathrm{m}^{-2} \mathrm{sr}^{-1}$. Including the tentative detection a.t $3.5 \mu \mathrm{m}$ by Dwek \& Arendt (1999) would boost $I_{\text {opt }}$ to $\sim 19 \mathrm{nW} \mathrm{m}^{-2} \mathrm{sr}^{-1}$. Recent direct measurements of the optical EBL at 3000,5500, and $8000 \AA$ from absolute surface photometry by Bernstein et al. (1999) lie between a factor of 2.5 to 3 higher than the integrated light from galaxy counts. Applying this correction factor to the range $3000-8000 \AA$ gives a total optical EBL intensity in the range $25-30 \mathrm{nW} \mathrm{m}^{-2} \mathrm{sr}^{-1}$. This could become $\sim 45 \mathrm{nW} \mathrm{m}^{-2} \mathrm{sr}^{-1}$ if the same correction holds also in the near-IR. The COBE/FIRAS measurements yield $I_{\mathrm{FIR}} \approx 14 \mathrm{nW} \mathrm{m} \mathrm{m}^{-2} \mathrm{sr}^{-1}$ in the $125-2000 \mu \mathrm{m}$ range. When combined with the DIRBE points at 140 and $240 \mu \mathrm{m}$, one gets a far-IR background intensity of $I_{\mathrm{FIR}}(140-2000 \mu \mathrm{m}) \approx 20 \mathrm{nW} \mathrm{m}^{-2} \mathrm{sr}^{-1}$. The residual emission in the 3.5 to $140 \mu \mathrm{m}$ region is poorly known, but it is likely to exceed $10 \mathrm{nW} \mathrm{m}^{-2} \mathrm{sr}^{-1}$ (Dwek et al. 1998). A 'best-guess' estimate of the total EBL intensity observed today appears then to be

$$
I_{\mathrm{EBL}}=55 \pm 20 \mathrm{nWm} \mathrm{m}^{-2} \mathrm{sr}^{-1} \text {. }
$$

In the following. I will adopt a reference valne for the background light associated with star formation activity over the entire history of the universe of $I_{\mathrm{EBL}}=$ $50 I_{50} \mathrm{nW} \mathrm{m} \mathrm{Wr}^{-2} \mathrm{sr}^{-1}$. 


\section{EBL from quasar activity}

A direct measurement of the contribution of quasars to the EBL depends on poorly known quantities like the bolometric correction, the faint end of the luminosity function, and the space density of objects at high redshifts. Estimates range from 0.7 to $3 \mathrm{nW} \mathrm{m}^{-2} \mathrm{sr}^{-1}$ (Soltan 1982; Chokshi \& Turner 1992; Small $\&$ Blandford 1992). Another source of uncertainty is the possible existence of a distant population of dusty AGNs with strong intrinsic absorption, as invoked in many models for the X-ray background (e.g. Madau et al. 1994; Comastri et al. 1995). These Type II QSOs, while undetected at optical wavelengths, could contribute significantly to the far-IR background. It is in principle possible to bypass some of the above uncertainties by weighing the local mass density of black holes remnants (Soltan 1982), as recent dynamical evidence indicates that supermassive black holes reside at the center of most nearby galaxies (Richstone et al. 1998). The available data (about 40 objects) show a correlation (depicted in Fig. 3) between bulge and black hole mass, with $M_{\mathrm{BH}} \approx 0.006 M_{\mathrm{sph}}$ as a best-fit (Magorrian et al. 1998). The mass density in old spheroidal populations today is estimated to be $\Omega_{\mathrm{sph}} h=0.0018_{-0.00085}^{10.0012}$ (Fukugita et al. 1998, hereafter FHP), implying a mean mass density of quasar remnants today

$$
\rho_{\mathrm{BH}}=3 \pm 2 \times 10^{6} h \mathrm{M}_{\odot} \mathrm{Mpc}^{-3} .
$$

Since the observed (comoving) energy density from all quasars is equal to the emitted energy divided by the average quasar redshift, the total contribution to the EBL from accretion onto black holes can be estimated to be

$$
I_{\mathrm{BH}}=\frac{c^{3}}{4 \pi} \frac{\eta \rho_{\mathrm{BH}}}{\langle 1+z\rangle} \approx 4 \pm 2.5 \mathrm{nW} \mathrm{m}^{-2} \mathrm{sr}^{-1} \eta_{0.05} \frac{2.5}{\langle 1+z\rangle}
$$

$(h=0.5)$, where $\eta_{0.05}$ is the efficiency of accreted mass to radiation conversion in units of $5 \%$. Therefore, unless dust-obscured accretion onto supermassive black holes is a very efficient process $\left(\eta_{0.05} \gg 1\right)$, a population of quasars peaking at $z \sim 1.5-2$ is expected to make a contribution to the total brightress of the night sky not exceeding 10-20\% (Fabian \& Iwasawa 1998; Madau 1999).

\section{The stellar mass density today}

With the help of some simple stellar population synthesis tools we can now set a lower limit to the total stellar mass density that produced the observed EBL, and constrain the cosmic history of star birth in galaxies. One of the most serious uncertainties in this calculation is the lower cutoff, usually treated as a free parameter, of the initial mass function (IMF). Observations of $M$ subdwarfs stars with the HST have recently shed some light on this issue, showing that the IMF in the Galactic disk can be represented analytically over the mass range $0.1<$

$m<1.6$ (here $m$ is in solar units) by $\log \phi(m)=$ const $-2.33 \log m-1.82(\log m)^{2}$ (Gould et al. 1996, hereafter GBF). For $m>1$ this mass distribution agrees well with a Salpeter function, $\log \phi(m)=$ const $-2.35 \log m$. A shallow mass function (relative to the Salpeter slope) below $1 M_{\odot}$ has been measured in the Galactic bulge as well (Zoccali et al. 1999). Observations of normal Galactic star-forming 


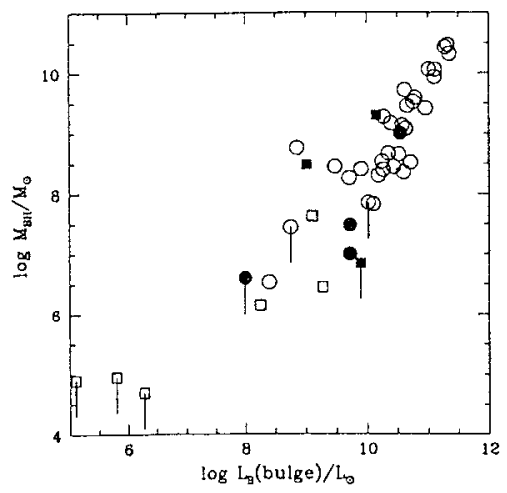

Figure 3. Black hole mass distribution against the bulge luminosity of their host galaxies (from Magorrian et al. 1998). Arrows indicate upper limits on $M_{\mathrm{BH}}$. The symbols denote different galaxy types: empty circles (E), filled squares (S0), filled circles (Sab), and empty squares (Sbc-Scd).

regions also show some convergence in the basic form of the IMF at intermediate and high masses, a power-law slope that is consistent with the Salpeter value (F.megreen 1998). In the following, I will use a "universal' IMF (shown in Figure 2) with the GBF form for $m<1$, matched to a Salpeter slope for $m \geq 1$; the mass integral of this function is 0.6 times that obtained extrapolating a Salpeter function down to $0.1 M_{\odot} \cdot{ }^{1}$

As shown in Figure 4, the bolometric luminosity as a function of age $\tau$ of a simple stellar population (a single generation of coeval, chemically homogeneous stars having total mass $M$, solar metallicity, and the above IMF) can be well approximated by

$$
L(\tau)= \begin{cases}1200 L_{\odot} \frac{M}{M \odot} & \tau \leq 2.6 \mathrm{Myr} \\ 0.7 L_{\odot} \frac{M}{M \odot}\left(\frac{\tau}{1 G \mathrm{Gr}}\right)^{-1.25} & 2.6 \leq \tau \leq 100 \mathrm{Myr} \\ 2.0 L_{\odot} \frac{M}{M M_{\odot}}\left(\frac{\tau}{1 \mathrm{Gyr}}\right)^{-0.8} & \tau>100 \mathrm{Myr}\end{cases}
$$

Over a timescale of $13 \mathrm{Gyr}$ (the age of the universe for an EdS cosmology with $h=0.5$ ), about $1.3 \mathrm{MeV}$ per stellar baryon will be radiated away. This number depends only weakly on the assumed metallicity of stars. In a stellar system with arbitrary star formation rate per comoving cosmological volume, $\dot{\rho}_{s}$, and formation epoch $t_{F}$, the bolonetric emissivity at time $t$ is given by the convolution integral

$$
\rho_{\mathrm{bol}}(t)=\int_{0}^{t} L(\tau) \dot{\rho}_{s}(t-\tau) d \tau
$$

\footnotetext{
${ }^{1}$ The bolometric light contributed by stars less massive than $1 M_{\odot}$ is very small for a "typical" IMF. The use of the GBF mass function at low masses instead of Salpeter then leaves the total radiated luminosity of a stellar population virtually unaffected.
} 

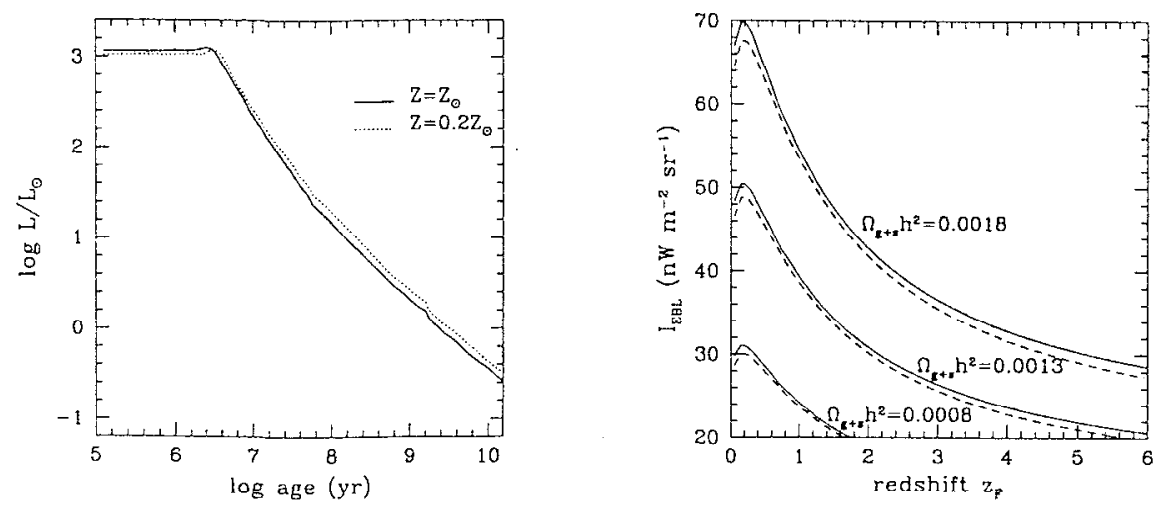

Figure 4. Left: Synthetic (based on an update of Bruzual \& Charlot's 1993 libraries) bolometric luminosity versus age of a simple stellar population having total mass $M=1 M_{\odot}$, metallicity $Z=Z_{\odot}($ solid line $)$ and $Z=0.2 Z_{\odot}$ (dotted line), and a GBF+Salpeter IMF. Right: EBL observed at Earth from the instantaneous formation at redshift $z_{F}$ of a stellar population having the same IMF $\left(Z=Z_{\odot}\right)$ and mass density $\Omega_{g+s} h^{2}=0.0018,0.0013$, and 0.0008 , as a function of $z_{F}$. Solid curves: EdS universe with $h=0.5\left(t_{H}=13 \mathrm{Gvr}\right)$. Dashed curves: $\Lambda$-dominated universe with $\Omega_{M}=0.3, \Omega_{\Lambda}=0.7$, and $h=0.65$ $\left(t_{H}=14.5 \mathrm{Gyr}\right)$.

The total background light observed at Earth $\left(t=t_{H}\right)$ is

$$
I_{\mathrm{EBL}}=\frac{c}{4 \pi} \int_{t_{F}}^{t_{H}} \frac{\rho_{\mathrm{bol}}(t)}{1+z} d t
$$

where the factor $(1+z)$ at the denominator is lost to cosmic expansion when converting from observed to radiated (comoving) luminosity density.

To set a lower limit to the present-day mass density, $\Omega_{g+s}$, of processed gas + stars (in units of the critical density $\rho_{\text {crit }}=2.77 \times 10^{11} h^{2} \mathrm{M}_{\odot} \mathrm{Mpc}^{-3}$ ), consider now a scenario where all stars are formed instantaneously at redshift $z_{F}$. The background light that would be observed at Earth from such an event is shown in Figure 4 as a function of $z_{F}$ for three values of $\Omega_{g+s} h^{2}$ corresponding to 4,7 , and 9 percent of the nucleosynthetic baryon density, $\Omega_{b} h^{2}=0.0193 \pm$ 0.0014 (Burles \& Tytler 1998). Two main results are worth stressing here: (1) the time evolution of the luminosity radiated by a simple stellar population makes the dependence of the observed EBL from $z_{F}$ much shallower than the $\left(1+z_{F}\right)^{-1}$ lost to cosmic expansion, as the energy output from stars is spread over their respective lifetimes; and (2) in order to generate an EBL at a level of $50 I_{50} \mathrm{nW} \mathrm{m}^{-2} \mathrm{sr}^{-1}$; one requires $\Omega_{g+s} h^{2}>0.0013 I_{50}$ for an EdS universe with $h=0.5$, hence a mean mass-to-blue light ratio today of $\left.\left\langle M / L_{B}\right\rangle_{g+s}\right\rangle$ $3.5 I_{50}$ (the total blue luminosity density at the present-epoch is $\mathcal{L}_{B}=2 \times$ $10^{8} \mathrm{hL} . \mathrm{Mpc}^{-3}$, Ellis et al. 1996). The dependence of these estimates on the cosmological model is rather weak. With the adopted IMF, about $30 \%$ of this mass will be returned to the interstellar medium in $10^{8} \mathrm{yr}$, after intermediate- 
mass stars eject their envelopes and massive stars explode as supernorae. This return fraction, $R$, becomes $50 \%$ after about 10 Gyr (e.g. Weidemann 198T).

A visible mass density at the level of the above lower limit, while able to explain the measured sky brightness, requires that most of the stars giving origin to the observed light must have formed at very low redshifts $\left(z_{F} \lesssim 0.5\right)$, a. scenario which appears to be ruled out by the observed evolution of the UV luminosity density (Madau 1999). For illustrative purposes, it is interesting to consider instead a model where the star formation rate per unit comoring volume stays approximately constant with cosmic time. In an EdS cosmology with $h=0.5$, one derives from equations $(4),(5)$, and (6)

$$
I_{\mathrm{EBL}}=1460 \mathrm{nW} \mathrm{m}{ }^{-2} \mathrm{sr}^{-1}\left\langle\frac{\dot{\rho}_{s}}{{\mathrm{M} \odot \mathrm{yr}^{-1} \mathrm{Mpc}^{-3}}}\right\rangle .
$$

The observed EBL therefore implies a 'fiducial' mean star formation density of $\left\langle\dot{\rho}_{s}\right\rangle=0.034 I_{50} \mathrm{M}_{\odot \mathrm{yr}^{-1}} \mathrm{Mpc}^{-3}$ (or a factor of 1.6 higher in the case of a Salpeter IMF down to $0.1 M_{\odot}$ ). Any value much greater than this over a sizeable fraction of the Hubble time will generate an EBL intensity well in excess of $50 \mathrm{nW} \mathrm{m}^{-2} \mathrm{sr}^{-1}$. Ignoring for the moment the recycling of returned gas into new stars, the visible mass density at the present epoch is simply $\rho_{g+s}=$ $\int_{0}^{t_{H}} \dot{\rho}_{s}(t) d t=4.4 \times 10^{8} I_{50} \mathrm{M}_{\odot} \mathrm{Mpc}^{-3}$, corresponding to $\Omega_{g+s} h^{2}=0.0016 I_{50}$.

Perhaps a more realistic scenario is one where the star formation density evolves as

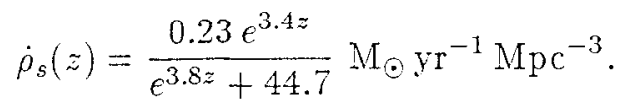

This fits reasonably well all measurements of the UV-continuum and $\mathrm{H} \alpha$ luminosity densities from the present-epoch to $z=4$ after an extinction correction of $A_{1500}=1.2 \mathrm{mag}\left(A_{2800}=0.55 \mathrm{mag}\right)$ is applied to the data (Madau 1999), and produce a total FBL of about the right magnitude $\left(I_{50} \approx 1\right)$. Since about half of the present-day stars are formed at $z>1.3$ in this model and their contribution to the FBL is redshifted away, the resulting visible mass density is $\Omega_{g+s} h^{2}=0.003 I_{50}\left(\left\langle M / L_{B}\right\rangle_{g+s}=8.5 I_{50}\right)$, almost twice as large as in the $\dot{\rho}_{s}=$ const approximation.

Depending on the star formation history and for the assumed IMF, the observed EBL then requires between $7 \%$ and $16 \%$ of the nucleosynthetic baryon density to be today in the forms of stars, processed gas, and their remnants. According to the most recent census of cosmic baryons, the mass density in stars and their remnants observed today is $\Omega_{s} h=0.00245_{-0.00088}^{+0.00125}$ (FHP), corresponding to a mean stellar mass-to-blue light ratio of $\left\langle M / L_{B}\right\rangle_{s}=3.4_{-1.3}^{+1.7}$ for $h=0.5$ (roughly $70 \%$ of this mass is found in old spheroidal populations). While this is about a factor of 2.5 smaller than the visible mass density predictcd by cquation (8), efficient recycling of ejected material into new star formation would tend to reduce the apparent discrepancy in the budget. Alternatively, the gas returned by stars may be ejected into the intergalactic medium. With an IMF-averaged yicld of returned metals of $y_{Z} \approx 1.5 Z_{\odot}{ }^{2}$ the predicted mean metallicity at

$\therefore{ }^{2}$ Here I have taken $y_{Z} \equiv \int m p_{z m} \phi(m) d m \times\left[\int m \phi(m) d m\right]^{-1}$, the stellar yields $p_{z m}$ of Tsujimoto et al. (1995), and a GBF + Salpeter IMF. 
the present epoch is $y_{Z} \Omega_{y+s} / \Omega_{b} \approx 0.25 Z_{\odot}$, in good agreement with the values inferred from cluster abundances (Renzini 1997).

\section{EBL from MACHOs}

One of the most interesting constraints posed by the observed brightness of the night sky concerns the possibility that a large fraction of the dark mass in present-day galaxy halos may be associated with faint white-dwarf (WD) remnlants of a population of intermediate-mass stars that formed at high redshifts. The results of the microlensing MACHO experiment towards the LMC indicates that $60 \pm 20 \%$ of the sought dark matter in the halo of the Milky Way may be in the form of $0.5_{-0.2}^{+0.3} M_{\odot}$ objects (Alcocl et al. 1997). The mass scale is a natural one for white dwarfs, a scenario also supported by the lack of a numerous spheroidal population of low-mass main sequence stars in the HDF (Gould et al. 1998). The total mass of MACHOs inferred within $50 \mathrm{kpc}$ is $2_{-0.7}^{+1.2} \times 10^{11} M_{\odot}$. implying a 'MACHO-to-blue light' ratio for the Milky Way in the range 5 to 25 solar (Fields et al. 1998). If these values were typical of the luminous universe as a whole, i.e. if MACHOs could be viewed as a new stellar population having similar properties in all disk galaxies, then the cosmological mass density of MACHOs today would be $\Omega_{\mathrm{MACHO}}=(5-25) f_{B} \mathcal{L}_{B} / \rho_{\text {crit }}=(0.0036-0.017) f_{B} h^{-1}$, a significant entry in the cosmic baryon budget (Fields et al. 1998). Here $f_{B} \approx 0.5$ is the fraction of the blue luminosity density radiated by stellar disks (FHP). Note thal if MACIIOs are halo WDs, the contribution of their progenitors to the mass density parameter is several times higher.

Halo IMFs which are very different from that of the solar neighborhood, i.e. which are heavily-biased towards WD progenitors and have very few stars forming with Inasses below $2 M_{\odot}$ (as these would produce bright WDs in the halo today that are not seen), and above $8 M_{\odot}$ (to avoid the overproduction of heary elements), have been suggested as a suitable mechanism for explaining the microlensing data (Adams \& Laughlin 1996; Chabrier et al. 1996). While the halo WD scenario may be tightly constrained by the observed rate of Type Ia SN in galaxies (Smecker \& Wyse 1991), the expected $\mathrm{C}$ and $N$ overenrichment of halo stars (Gibsun \& Mould 1997), and the number counts of faint galaxies in deep optical surveys (Charlot \& Silk 1995), here a potentially more direct method is explored, namely I will compute the contribution of WD progenitors in dark galaxy halos to the extragalactic background light.

Following Chabrier (1999), I will adopt a truncated power-law IMF,

$$
\phi(m)=\text { const } \times e^{-(\bar{m} / m)^{3}} m^{-5} .
$$

This form mimics a mass function strongly peaked at $0.84 \bar{m}$. To examine the dependence of the IMF on the results, let us consider two functions (shown in Fig. 3 ), $\bar{m}=2.4$ and $\bar{m}=4$ : both yield a present-day Galactic halo massto-light ratio $>100$ after a Hubble time, as required in the absence of a large non-baryonic component. I will further assume that a population of halo WD progenitors having mass density $X \Omega_{b} h^{2}=0.0193 X$ formed instantaneously at redshift $z F$ with this IMF and nearly primordial $\left(Z=0.02 Z_{\odot}\right)$ metallicity. The resulting EBL from such an event is huge, as shown in Figure 5 for $X=0.1,0.3$, and 0.6 and a $\Lambda$-dominated universe. 

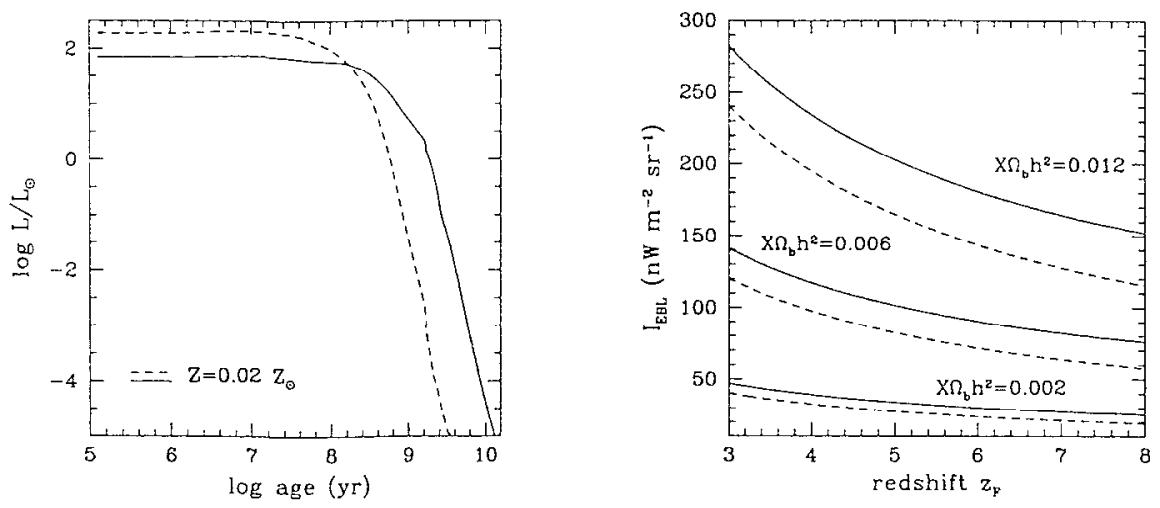

Figure 5. Left: Synthetic bolometric luminosity versus age of a simple stellar population having total mass $M=1 M_{\odot}$, metallicity $Z=0.02 Z_{\odot}$, and a WD progenitor dominated IMF with $\bar{m}=2.4$ (solid line) and $\bar{m}=4$ (dashed line). Right: EBL observed at Earth from the instantaneous formation at redishift $z_{F}$ of a stellar population having the same IMF and metallicity, and mass density $X \Omega_{b} h^{2}$ corresponding to 60,30 , and 10 per cent of the nucleosynthetic value, as a function of $z_{F}$. A $\Lambda$-dominated universe with $\Omega_{M}=0.3$, $\Omega_{\lambda}=0.7$, and $h=0.65$ has been assumed. Solid line: $\bar{m}=2.4$. Dashed line: $\bar{m}=4$.

Consider the $\bar{m}=2.4$ case first. With $z_{F}=3$ and $X=0.6$, this scenario would generate an EBL at a level of $300 \mathrm{nW} \mathrm{m}^{-2} \mathrm{sr}^{-1}$. Even if only $30 \%$ of the nucleosynthetic baryons formed at $z_{F}=5$ with a WD-progenitor dominated MF, the resulting background light at Earth would exceed the value of $100 \mathrm{nW} \mathrm{m}^{-2} \mathrm{sr}^{-1}$, the 'best-guess' upper limit to the observed EBL from the data plotted in Figure 2. The return fraction is $R \approx 0.8$, so only $20 \%$ of this stellar mass would be leftover as WDs, the rest being returned to the ISM. Therefore, if galaxy halos comprise $100 \%$ of the nucleosynthetic baryons, only a small fraction of their mass, $X_{\mathrm{WD}} \approx 0.2 \times 0.30=0.06$ could be in the form of white dwarfs. Pushing the peak of the IMF to more massive stars, $\bar{m}=4$, helps only marginally. With $\bar{m}=2.4$, the energy radiated per stellar baryon over a timescale of $13 \mathrm{Gyr}$ is equal to $2 \mathrm{MeV}$, corresponding to $10 \mathrm{MeV}$ per baryon in WD remnants. A similar value is obtained in the $\bar{m}=4$ case: because of the shorter lifetimes of more massive stars the expected EBL is reduced, but only by $20 \%$ or so (see Fig. 5). Moreover, the decreasing fraction of leftover WDs raises even more severe problems of metal galactic enrichment.

One should note that these limits are not necessarily in contrast with the microlensing results, as they may imply either that WDs are not ubiquitous in galaxy halos (i.e. the Milky Way is atypical), or that the bulk of the baryons are actually not galactic. One possible way to relax the above constraints on WD baryonic halos is to push their formation epoch to extreme redshifts $\left(z_{F}\right)$ 10), and hide the ensuing background light in the poorly constrained spectral region between: 5 and $100 \mu \mathrm{m}$. In Figure 2 I show the EBL produced by a WD-progenitor dominated IMF with $\bar{m}=4$ and $\left(z F, X, X_{\text {WD }}\right)=(36,0.5,0.1)$, 
assuming negligible dust reddening. While this model may be consistent with the observations if the large corrections factors inferred by Bernstein et al. (1999) extend into the near-IR, it is important to draw attention to the fact that even a tiny fraction of dust reprocessing in the (redshifted) far-IR would inevitably lead to a violation of the EIRAS background.

Acknowledgments. Partial support for this work was provided by NASA through grant AR-06337.10-94A from the Space Telescope Science Institute.

\section{References}

Alcock, C., et al. 1997, ApJ, 486, 697

Armand, C., Milliard, B., \& Deharveng, J.-M. 1994, A\&A, 284, 12

Baugh, C. M., et al. 1998, ApJ, 498, 504

Bernstein, R. A., Freedman, W. L., \& Madore, B. F. 1999, preprint

Bruzual, A. C., \& Charlot, S. 1993, ApJ, 405, 538

Burles, S., \& Tytler, D. 1998, ApJ, 499, 699

Chabrier, G. 1999, ApJ, in press (astro-ph/9901145)

Chabrier, G., Segretain, L., \& Mera, D. 1996, ApJ, 468, L21

Charlot, S., \& Silk, J. 1995, ApJ, 445, 124

Chokshi, A., \& Turner, E. L. 1992, MNRAS, 259, 421

Comastri, A., et al. 1995, A\&A, 296, 1

Dwch, E., \& Arendt, R. G. 1999, ApJ, in press (astro-ph/9809239)

Dwek, E., et al. 1998, ApJ, 508, 106

Ellis, R. S., et al. 1996, MNRAS, 280, 235

Elmegreen, B. G. 1998, in Unsolved Problems in Stellar Evolution, ed. M. Livio (Cambridge: Cambridge University Press), in press (astro-ph/9811289)

Fabian, A. C., \& Twasawa, K. 1999, MNRAS, in press

Fields, B.D., Freese, K., \& Graff, D. S. 1998, NewA, 3, 347

Fixsen, D. J., et al. 1998, AjJ, 508, 123

Fukugita, M., Hogan, C. J., \& Peebles, P. J. E. 1998, ApJ, 503, 518

Gibson, B., \& Mould, J. 1997, ApJ, 482, 98

Gould, A., Bahcall, J. N., \& Flynn, C. 1996, ApJ, 465, 759 (GBF)

Gould, A., Flynn, C., \& Bahcall, J. N. 1998, ApJ, 503, 798

Ilauser, M. G., et al. 1998, ApJ, 508, 25

Madau, P. 1999, in Proceedings of the Nobel Symposium on Particle Physics and the Universe, Physica Scripta, in press

Madau, P., Ghisellini, G., \& Fabian, A. C. 1994, MNR4S, 270, L17

Madau, P., \& Pozzetti, L. 1999, submitted to MNRAS (astro-ph/9907315) .

Madau, P., Pozzetti, L., \& Dickinson, M. 1998, ApJ, 498, 106

Magorrian, G., et al. 1998, AJ, 115; 2285

Pozzetti, L., et al. 1998, MNRAS, 298, 1133

Renzini, A. 1997, ApJ, 488, 35 
Richstone, D., et al. 1998, Nature, 395, 14

Schlegel, D. J., Finkbeiner, D. P., \& Davis, M. 1998. ApJ, 500, 525

Small, T. D., \& Blandford, R. D. 1992, MNRAS, 259, 725

Smecker, T. A., \& Wyse, R. 1991. ApJ, 372, 448

Soltan, A. 1982, MNRAS, 200, 115

Somerville, R. S., Prima.ck, J. R., \& Faber, S. M. 1999, MNRAS, in press (astro$\mathrm{ph} / 9802268$ )

Steidel, C. C., et al. 1998, in The Birth of Galaxies, ed. B. Guiderdoni, F. R. Bonchet, Trinh X. Thuan, \& Tran Thanh Van (Gif-sur-Yvette: Edition Frontieres), in press (astro-ph/981216 )

Steidel, C. C, et al. 1996, ApJ, 462, L17

Tsujimoto, T., et al. 1995, MNRAS, 277,945

Weidemann, V. 1987, A\&A, 188. 74

Zoccali, M., et al. 1999, ApJ, submitted (astro-ph/9906452) 
The Hy Redshift Universe

ASP Conference Series, Vol. 193. 1999

A. J. Bunker \& W. J. M. van Breugel, eds.

\title{
HST/LCO Measurements of the Optical Extragalactic Background Light
}

\author{
Rebecca A. Bernstein \\ Carnegie Observatories, 813 Santa Barbara St., Pasadena, CA, 91101
}

\begin{abstract}
.
We present the first detections of the mean flux of the optical extragalactic background light (EBL) at 3000,5500 , and $8000 \AA$, derived from coordinated data sets taken at Las Campanas Observatory and with $H S T$. In addition to detections in all three bands, we identify the minimum surface brightness contributed by resolved galaxies $(23<V<28$ AB mag) using a novel method of aperture photometry to which these data are uniquely suited. By comparing these results to the surface brightness from resolved galaxies measured using standard methods of galaxy photometry, we identify systemalic errors in the results of faint galaxy photometry and place limits on the EBL flux originating from undetected sources.
\end{abstract}

\section{Introduction}

The optical extragalactic background light (EBL) is the integrated flux from all extragalactic sources, both resolved and unresolved. The energy density of the EBL as a function of wavelength is directly related to the integrated star formation history of the universe, the total baryon fraction in stars, and the total metal mass produced by stellar nucleosynthesis. Although the spectral energy distribution (SED) of starbursting regions is dominated by UV emission from hot young stars, older stellar populations emit most of their energy at $1-1.5 \mu \mathrm{m}$. The optical EBL we detect in the range 2500-9000 $\AA$ therefore includes significant flux from older populations at low redshift as well as light from starbursting galaxies and younger populations out to $z \sim 9$, the redshift at which Lyman continuum absorption by intergalactic hydrogen redshifts to the wavelength of our reddest bandpass (F814W, $\lambda_{0} \sim 8000 \AA$ ). Further complicating interpretation of the EBL spectrum, dust in the emitting galaxies will absorb and re-radiate starlight at thermal infrared wavelengths. The energy density of the EBL over the full wavelength range of $\mathrm{UV}$ to sub-mm must therefore be included to obtain a complete census of the energy produced by stellar nucleosynthesis in the universe.

The cosmological significance of the EBL was first appreciated in the 1700's, when expectations of a infinite, static universe, uniformly filled with stars, led astronomers to puzzle over the fact that the nighttime sky is dark. The apparent conflict posed by the darkness of the night sky became known as Olbers' paradox. While it is now readily explained by the expansion of the universe, the finite 
speed of light, and, most importantly, the finite lifetimes of stars, Olbers' paradox stands as an excellent illustration of the power of background measurements to test our model of the universe. Our present picture of the star formation and chemical enrichment history of the universe is based on quite a diverse range of observational methods, among these are: redshift surveys, producing galaxy hminosity functions and measurements of the luminosity density from galaxies with $\approx$; spectroscopy of QSO absorption systems, producing estimates of the conversion rate of hydrogen into stars and the rate of metal enrichment with $z$; and stellar population synthesis modeling; producing estimates of the star formation rates when combined with local IR luminosity functions.

With new $10 \mathrm{~m}$-class telescopes and HST, the limits of these resolved-source methods are being extended to ever fainter levels; however, a measurement of the EBL remains an invaluable complement to observational methods which rely on resolved-source detections. Galaxies with low apparent surface brightness both intrinsically low surface brightness galaxies at low redshift and the majority of the galaxy luminosity function at high redshift - are easily missed in surfacebrightness-limited galaxy counts and in redshift surveys. Identification, not to mention photometry, of faint galaxies becomes strongly biased near detection limits. Even efforts to understand galary evolution, chemical enrichment, and star formation through QSO absorption line studies are now thought to be biased against the chcmically enriched, dustier systems, as these systems will obscurc QSOs which might lie behind them (Pei \& Fall 1995, Pettini et al. 1999). In contrast, a direct measurement of the EBL is a complete census of the cnergy produced by star formation and is immune to surface brightness selection effects.

In this contribution, we bricfly describe the first detection of the EBL a.t optical wavelengths and some of the implications of those results. On a practical note, because the results we discuss here are bascd on HST/WFPC2 observations, which have a $5 \mathrm{arcmin}^{2}$ field of view, galaxies brighter than $V=23 \mathrm{AB}$ mag are not statistically well represented in these data. Our measurement of the EBL is therefore defined as the flux from resolved and unresolved galaxies fainter than $Y=23 \mathrm{AB}$ mag. We use the abbreviation "EBL23" as a reminder of this bright magnitude cut-off. We combine EBL23 with galaxy counts at brighter magnitudes to obtain an estimate of the total EBL.

\section{Foreground Subtraction and Data Sets}

The key to any background measurement is the successful subtraction of foreground contamination. Figure 1 shows the surface brightnesses of foreground sources along lines of sight where they are faintest - at Galactic latitudes greater than 65 degrees and ecliptic latitudes greater than 30 degrees. For comparison, upper limits from previous attempts to measure the EBL are also shown, along with lower limits from integraling the flux in resolved galaxies (i.e., from galaxy counts). The cumulative flux from foreground sources is roughly 100 times the EBL fiux, so that roughly $1 \%$ accuracy in foreground renioval must be achieved in order to detect the EBL.

The brightest and most problematic foreground source is airglow, the rapidly varying molecular and atomic line emission produced by the Earth's own atmosphere. We have avoided this source at the wavelengths of our observations by 


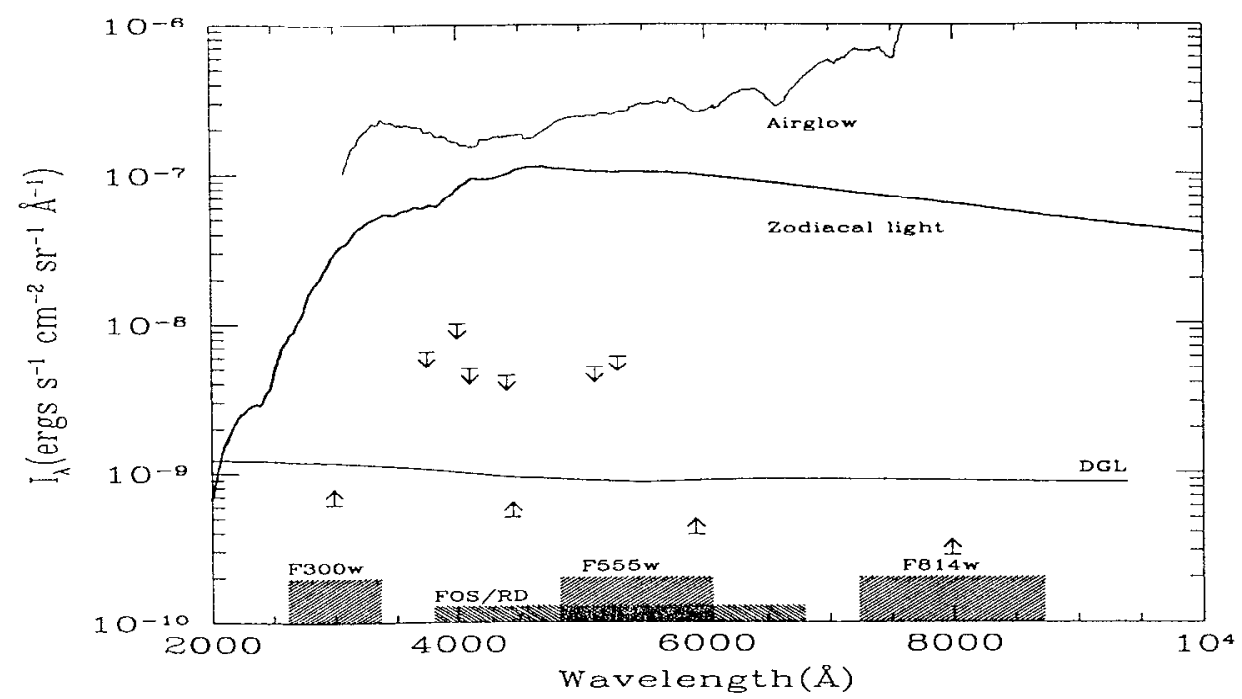

Figure 1. The surface brightness of airglow, zodiacal light, and diffuse galactic light are compared here to upper limits for the EBL from previous investigations downward arrows and lower limits from the cumulative surface brightness of sources with $V>23 \mathrm{AB}$ mag in the Hubble Deep Field (Williams et al. 1996). All foregrounds are shown at the flux levels we identify in this work. Hatched regions show the HST/WFPC2 and FOS band-passes.

using HST to measure the total night sky flux from above the atmosphere. The dominant foreground from HST is sunlight scattering off of the large ( $\geq 10 \mu \mathrm{m})$, rough, interplanetary dust grains which concentrate in the ecliptic plane zodiacal light (ZL). As the solar system is harder to escape than the Earth's atmosphere, it is fortunate that the scattering involved is well described by Mie theory and is only weakly wavelength dependent, with scattering becoming more efficient by $5 \%$ per $1000 \AA$ with increasing wavelength. The mean zodiacal light flux can thus be identified by the strength of solar absorption features (Fraunhofer lines) which are preserved in its spectrum (see Figure 2).

Stars in our own Galaxy can be resolved and subtracted relatively easily with modern optical CCD detectors. However, interstellar dust scatters incident starlight, producing diffuse Galactic light (DGL) at optical wavelengths. The dust column density and the interstellar radiation field strength, both of which determine the intensity of scattered DGL, are well correlated with the $100 \mu \mathrm{m}$ thermal emission from the dust along the line of sight. Our field was selected for its very low $100 \mu \mathrm{m}$ emission and therefore minimal DGL. The remaining lowlevel optical DGL can be estimated using simple scattering models, which are in

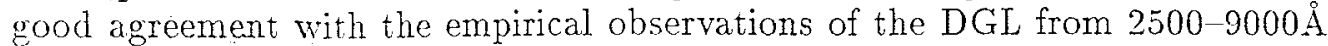
(see Witt et al. 1997 and references therein).

Previous efforts to measure the optical EBL have employed a variety of different approaches. In a pioneering effort, Mattila (1976) attempted to iso- 


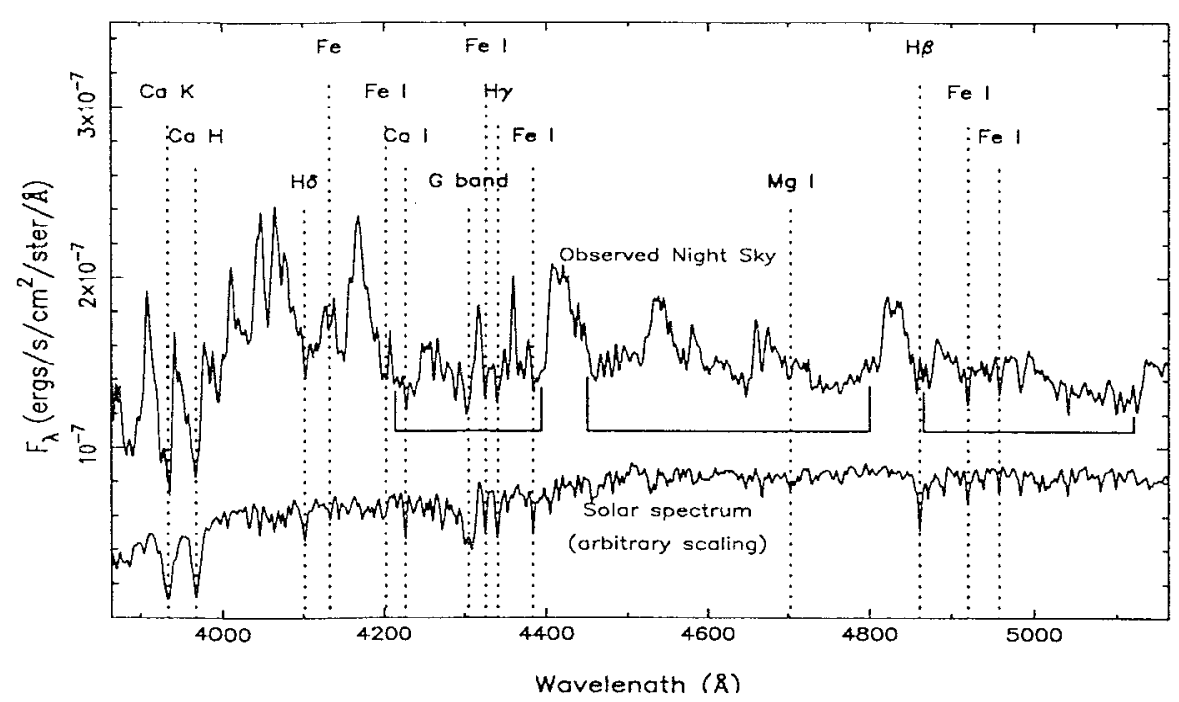

Figure 2. The spectrum of the night sky from Las Campanas Observatory taken simultaneously with the observations of the same field from HST. A solar spectrum is shown for comparison. Solar absorption features due to ZL in the night sky spectrum are readily identified.

late the EBL by differencing the flux on and off "dark" clouds at high Galactic latitude, using them as opaque screens to spatially isolate foregrounds from the EBL. This work identified the rapid temporal variability of terrestrial airglow and extinction and the spatial variability of diffuse Galactic light which proved to be the primary obstacles to early efforts to measure the EBL. Toller (1983) later attempted to avoid both atmospheric and zodiacal foregrounds using data from the Pioneer 10 spacecraft taken beyond the zodiacal dust cloud at a heliocentric distance of $3 \mathrm{AU}$ from the Sun. Poor spatial resolution $\left(2^{\circ}\right)$, however prevented the accurate subtraction of discrete Galactic stars, let alone the diffuse Galactic component from these data. Dube, Wilkes, \& Wilkinson (1979) made the first effort to measure and subtract foreground contributions explicitly based on geometrical modeling of airglow and Galactic foregrounds, and spectroscopic measurement of the ZL flux by the same technique which we have adopted. Rapid variability caused uncertainty in their airglow subtraction which dominated the errors in their results.

In this work, we isolate the EBL23 using three data sets obtained simultaneously: absolute surface photometry in $1000 \hat{A}$-wide bands centered at roughly 3000,5500 , and $8000 \AA$ from WFPC2; low $(\sim 300 \AA)$ resolution surface spectrophotometry at $4000-7000 \AA$ from the FOS; and moderate $(2.0 \AA)$ resolution surface spectrophotometry from the Boller and Chivens spectrograph on the 2.5m duPont telescope at Las Campanas. The HST data are used to measure the mean flux of the total background; while the LCO data are used to measure the ZL by the method described above. We estimate foreground DGL using scattering theory and empirical correlations between the optical DGL and ther- 


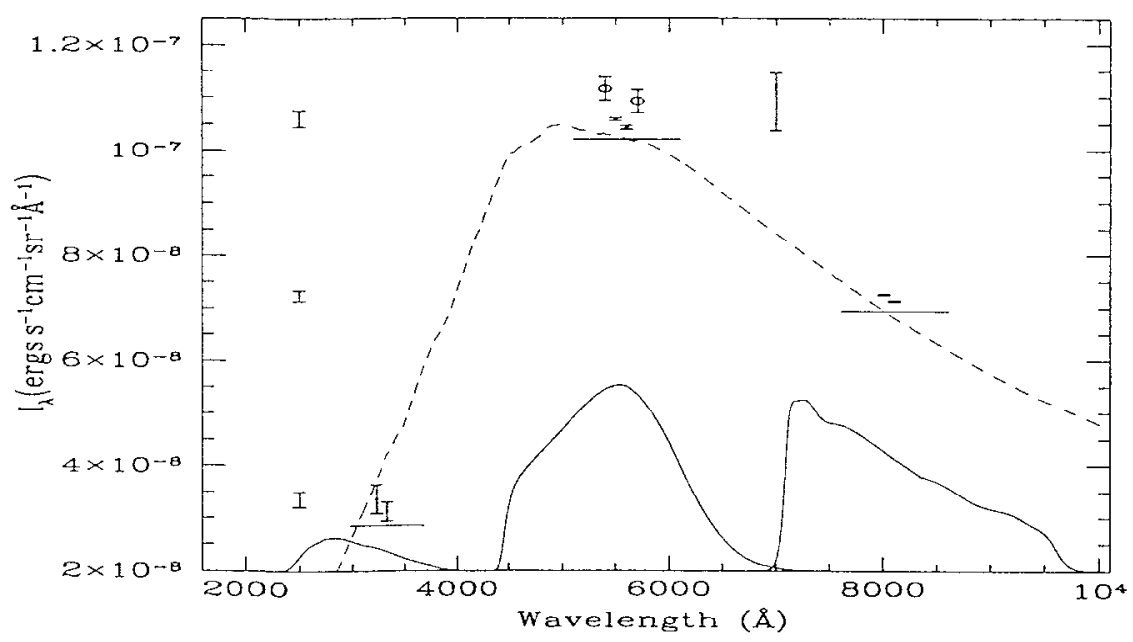

Figure 3. Total sky flux from WFPC2 (dots) and FOS (circles), November and December (offset $+100 \hat{\AA}$ ) data sets. The $2 \%$ difference in flux resulis from changes in ZL due to Earth's motion. Dashed line shows ZL spectrum. Horizontal bars show ZL convolved with WFPC2 filters. Shy flux at $\lambda>4000 \AA$ is $\sim 95 \% \mathrm{ZL}$. Error bars on all points indicate $1 \sigma$ RMS errors. Floating error bars at $2500 \AA$ show systematic uncertainties for WFPC2 result; the error bar at $7000 \AA$ shows the FOS uncertainty. Solid lines show WFPC2 band-passes.

mal $100 \mu \mathrm{m}$ emission from the same dust. The HST observations were scheduled in three visits of six orbits each, with one month between visits. This allowed us to look for and confirm the expected modulation in the ZL with the Earth's orbital position, to look for possible off-axis scattered light with the satellite oriented at different roll angles, and to safe-guard against unidentified photometric anomalies with the instrument.

Observations from LCO and HST must be simultaneous to ensure that the ZL measured from the ground is exactly the contribution seen by HST. Also, the HST and LCO data sets must be calibrated to the same absolute scale with $\sim 1 \%$ accuracy in order to detect the EBL. When necessary to achieve the required accuracy, original reduction procedures were developed and STScI calibrations were augmented with our own solutions. To eliminate stray light. HST observations were made only in the shadow of the Earth, with the Moon greater than 65 degrees from the optical axis of the telescope. We also selected the field to avoid $V>7 \mathrm{AB}$ mag stars within 3 degrees.

\section{Fesults on the EBL}

From each WFPC2 CCD image, we obtain a measurement of the night sky flux. As expected, we find variations in the sky flux between visits due to 
the varying $Z I$ contribution at different times of year. We find $n$ significant difierence between the results from the three WFPC2 chips, and no indication of stray light or photometric anomalies between visits. Final statistical errors are less than $1 \%$ in the two redder pass-bands. The statistical error in the meastrement at $3000 \AA$ is dominated by the accuracy with which we can subtract instrumental backgrounds, which are almost as bright as the total sky flux at this wavelength. Systematic errors are also of order $1 \%$, and are dominated by the aperture correction and point source calibration. The results are shown in Figure 3 . FOS results have a much large systematic uncertainty $(\sim 5 \%)$, due to uncertainty in the aperture solid angle and point spread function, and nonuniform sensitivity across the detector, which affects surface brightness results much more than point source measurements. The WFPC2 and FOS results are discussed in detail in Bernstein et al. (1999a).

We took spectra of blank sliy within the HST/WFPC2 field of view using the Boller and Chivens spectrograph at Las Campanas Observatory simultaneously with the November HST observations. We have used those spectra to measure the absolute flux of the $Z L$ at $1650 \AA$ with a precision of $0.8 \%$, and a systcmatic uncertainty of $<1 \%$, using the method outlined in $\$ 2$. That measurement is discussed in Bernstein et al. 1999b.

The optical DGL was estimated using a simple non-uniform, back-scattering model, $I_{\lambda}=j_{\lambda} \omega_{\lambda} \tau_{\lambda} S(g, b)$, in which $S(g, b)$ is the scattering phase function in terms of Galactic latitude, $b$, and the average phase function of the dust, $g$ (Jura 1079). The optical depth, $\tau_{\lambda}$, and the surface brightness of the interstellar radiation ficld, $j_{\lambda}$, are empirically determined. The effective albedo, $\omega$, and phase frinction, $g$, are based on extensive laboratory tests and modeling by Draine \& Lee (1980) and are in excellent agreement with both optical and IR observations. The predicted DGL for our field (galactic latitude $b \geq 50^{\circ}, 150^{\circ}$ from the galactic center) is in good agreement with prediction from scaling relations betwon the optical and UV scattering and the $100 \mu \mathrm{m}$ thermal emission from the galactic dust (see Witt et al. 1997 for a discussion of models and a review of results). The contribution from DGL is small enough that uncertainty in this estimate is an insignificant source of error for our final result.

Combining the HST and LCO results with a model of the DGL, we obtain the detections of the EBL23 shown in Figure 4. The errors are dominated by two systematic effects: undwoidable limitations in the flux calibration of independent. data sets to the same absolute scale; and uncertainty in the color of the ZL, which we combine with our absolute ZL flux measurement at $4650 \AA$ to obtain the ZL flux at each WFPC2 band. In comparison to the flux in individually detected galaxies in the HDF, the nominal detections plotted show roughly a factor of 5 higher flux: however, the counts are within the $3 \sigma$ error limits.

In order to identify the EBL flux coming from detectable galaxies - which one can regard as an absolute minimum value for the total $E B L$ - we have devcloped a method for simplified aperture photometry. Briefly, to identify the total flux from detectable sources we simply average the flux of every pixel in the frame to obtain the mean surface brightness of the foregrounds plus all extragalactic sources: this is a measure of the average surface brightness per pixel from "sky + objects." We then mask out all detectable objects, and again take the surface brightness of all remaining pixels to obtain the flux from what 


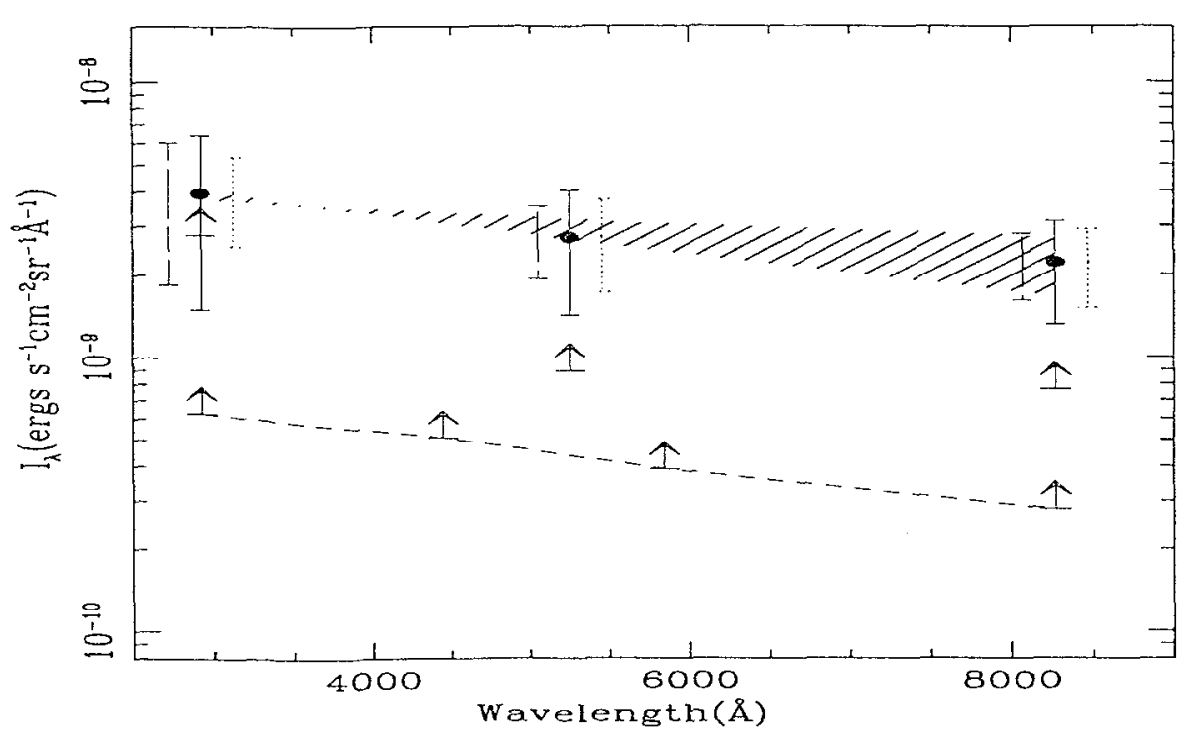

Figure 4. The detected EBL23 - EBL from galaxies with $V>$ $23 \mathrm{AB}$ mag. Solid error bars show $1 \sigma$ RMS errors; dotted error bars show systematic uncertainties. The hatch-marked region shows $(1 \sigma)$ uncertainty from ZL subtraction. The dashed line connects integrated galaxy counts from the HDF. The minimum EBL23 values we identify from galaxies $23<V \leq 28 \mathrm{AB}$ mag are shown by the unconnected lower limit arrows.

Inust be foregrounds only if one assumes that detectable galaxies account for the entire extragalactic signal: this is a measure of the average surface brightness per pixel from "sky." The difference between the surface brightness of "sky + objects" and "sky" is simply the flux recoverable from sources within the area of the masks we have used.

We repeat this measurement by masking the galaxies with masks that are the same size as the apertures used for individual source photometry by standard photometric packages (see, for example, Williams et al. 1996), and using masks which are 8 times that size. We find that roughly $15 \%$ of the flux from sources is in the region beyond the apertures used by SExtractor (Bertin \& Arnouts 1996) or FOCAS (Valdes 1982, Jarvis \& Tyson 1981). Furthermore, this flux is included by such photometry packages in estimates of the sky level. To characterize the lost flux by the central surface brightness $\left(\mu_{0}\right)$ of the individual sources, more than $50 \%$ of each galaxy's flux is not recovered by galaxy photometry for sources within 2 mag of the surface brightness limit of the data. This is an unsurprising result if one assumes that these galaxies have exponential profiles. As has been discussed extensively in the literature with reference to low surface brightness galaxy detection (e.g. Disney 1976, Disney \& Phillips 1983, Davies 1990, Dalcanton 1998), one should expect to recover less than $30 \%$ of the light from a galaxy which has $\mu_{0}$ within $1 \mathrm{mag}$ of the limiting isophot of the data. 


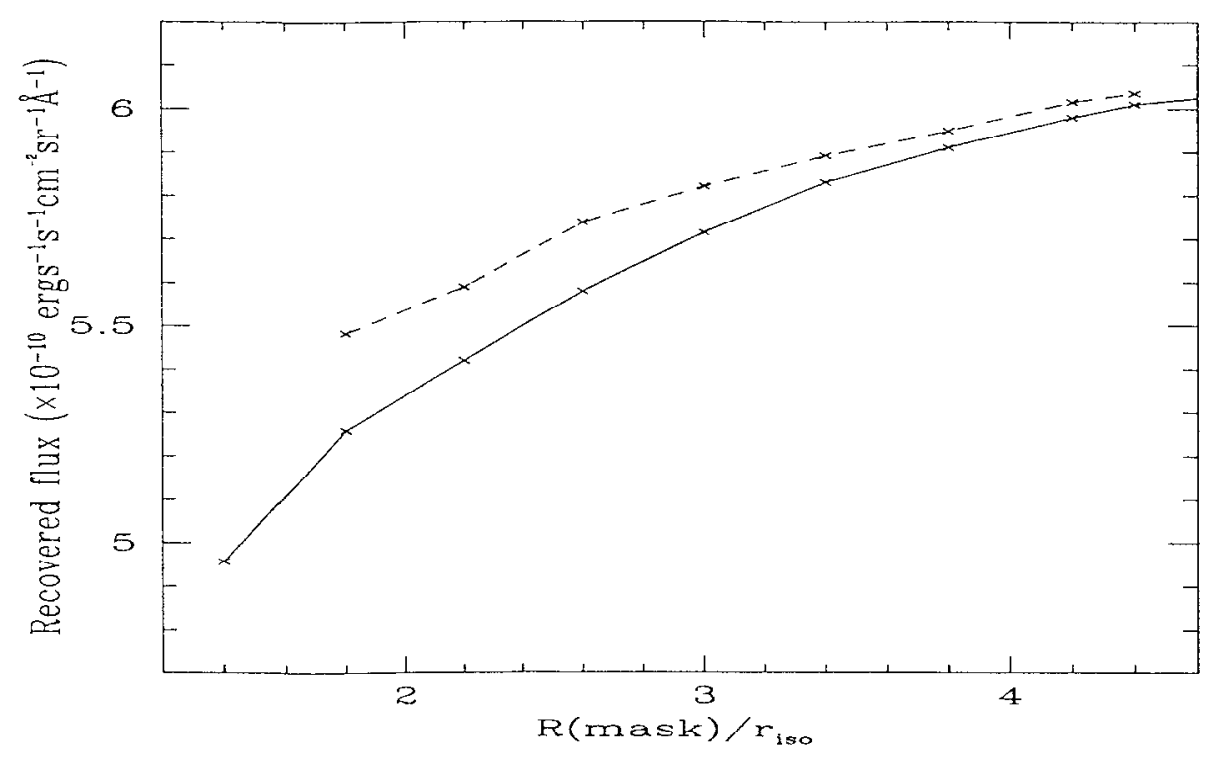

Figure 5. The cumlative flux recovered from galaxies fainter than $\mathrm{V}=23 \mathrm{AB}$ mag as a function of aperture radius for individual galaxies. Radius is given as the factor by which the limiting isophotal detection radius for each galaxy is multiplied. The solid line shows the flux recovered when isophotal detection radii are allowed to be smaller than three pixels, which is less than the $90 \%$ encircled energy radius for point sources in WFPC2 images. This reflects detection parameters similar to the isophotal detection radii used for the HDF catalog and most faint source photometry with HST. The dotted line shows the flux recovered when three pixels is the minimum allowed radius. This 3 pixel limit is not imposed in the tests discussed in the text and is shown here for comparison only.

There are important distinctions between this simplified aperture photometry and individual galaxy photometry. First, we obtain a single sky value for the entire frame. We can do so because the images in our data set are free of scattered light and are very accurately bias and dark corrected. Estimates of the "local" sky level from an aperture around a given object are more appropriate to data with significant scattered light (like the HDF-N images), or if the flux of an individual galaxy is the goal. Our goal is an accurate ensemble flux measurement. Also, the sky level is statistically very well determined in our method, as roughly $10^{5}$ pixels are used to estimate it. And, finally, if the aperture sizes (masks) we use are too small, our "sky" estimate will include galaxy light, as discussed above, but our "shy + object" estimate will be accurate. In both FOCAS and SExtractor, galaxy apertures will exclude some of each galaxy's flux,; .... and that flux will then also be included in the sky estimate and subtracted, so that individual galaxy photometry makes twice the flux error we identify above; $\sim 36 \%$ rather than $\sim 18 \%$. 


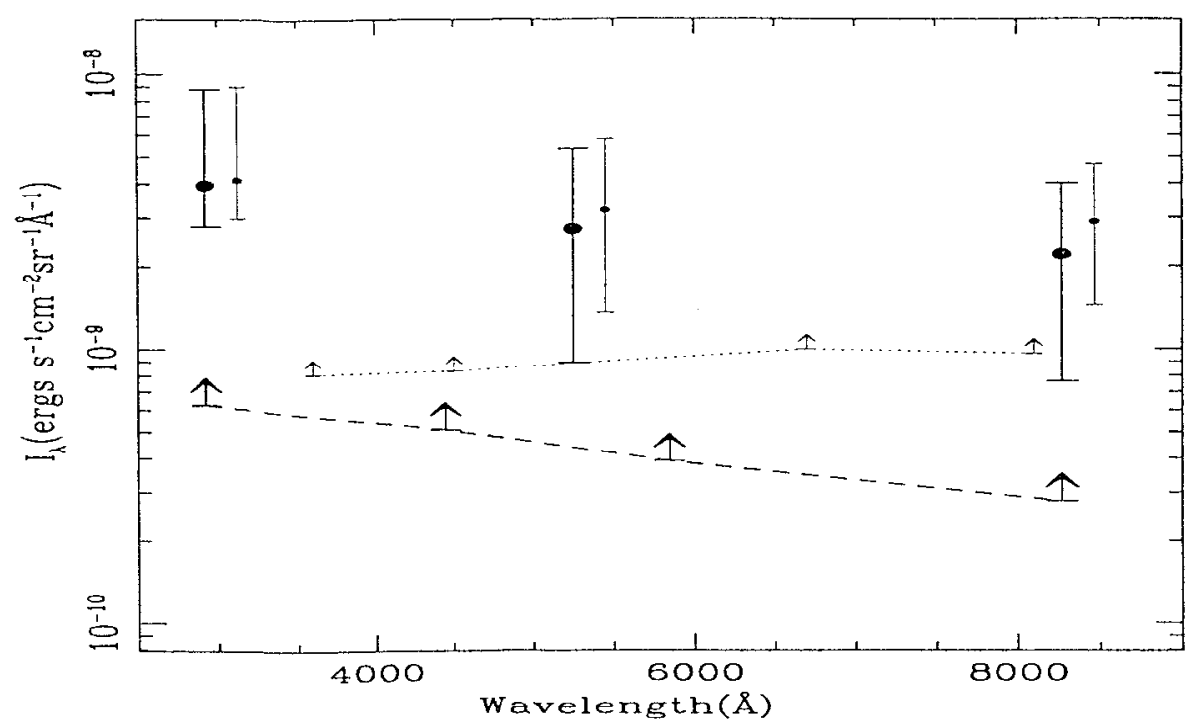

Figure 6. The large filled circles show the EBL23 from galaxies with $V>23 \mathrm{AB}$ mag. Error bars show the full $2 \sigma$ uncertainty: the upper bound show $2 \sigma$ upper limit on the detections, the lower bound shows the $2 \sigma$ lower limit of the flux recovered from galaxies in the range $23<V_{555}<28 \mathrm{AB}$ mag by the aperture photometry nethod described in the text. The dark dashed line and dark lower limit arrows show the integrated flux in the HDF catalug. The sinall filled circles show the total EBL: the detected EBL23 from sources with $V>23 \mathrm{AB}$ mag, plus the integrated ground-based counts at $V<23 \mathrm{AB}$ mag. The lower limit arrows connected by the light dotted line mark the flux in HDF counts plus ground-based counts for $V<23 \mathrm{AB}$ mag.

As can be seen in the growth curve shown in Figure 5, the flux recovered by our largest masks has not completely converged. At these aperture sizes, over $40 \%$ of the pixels are associated with detected sources, and the masks signifcantly overlap. Using light profiles measured from the HDF-N images and our own, we have modeled the flux from the wings of galaxies which contributes to the sky level beyond the range of our largest masks. We find that overlapping wings of detectable galaxies produce an extragalactic pedestal level which is is roughly $2 \times 10^{-10} \mathrm{ergs} \mathrm{s}^{-1} \mathrm{~cm}^{-2} \mathrm{sr}^{-1} \AA^{-1}$ in our data. This is a significant component and cannot be directly detected except by absolute surface photometry. Including this flux, the minimum EBL23 as a function of wavelength which we identify from detected galaxies is shown in Figure 4.

\section{Discussion}

The strongest limits we can place on the EBL23 are shown in Figure 6, which shows $2 \sigma$ upper and lower limits. The lower limits we identify are at least a factor of two higher than previous estimates of the flux from resolved galaxies 
fainter than $V=23 \mathrm{AB}$ mag (e.g. Pozzetti et al. 1998) due to errors in the absolute flux levels of galaxy photometry discussed above.

As the nominal flux values we detected for the EBT223 are higher than the minima by a factor of 2-3, it is interesting to consider what flux one might expect from galaxies which would escape detection in the HDF. In Bernstein et al. (1999c), we have estimated the flux coming from galaxies falling below the $\mu_{0}$ detection limits in the HDF-N images by modeling galaxy populations with redshift (including only passive luminosity evolution) and imposing detection limits appropriate to optical HST imaging for a 0.1" Gaussian PSF, and typical foreground sky levels. We have run models with and without the populations of LSBs discussed in the recent redshift surveys of Dalcanton et al. (1997), Sprayberry et al. (1997), and $\mathrm{O}^{\prime} \mathrm{Neil}$ et al. (1999), and we conclude that roughly $3-5 \times 10^{-10} \mathrm{crgs} \mathrm{s}^{-1} \mathrm{~cm}^{-2} \mathrm{sr}^{-1} \AA^{-1}$ should be expected based on the charac teristics of galaxy populations identified in the local universe. Furthermore, the fraction of the EBI which is coming from undetectable sources is larger at longer wavelengths. At wavelengths longer than $5500 \AA$, the majority of the undetected light is produced at $1.5<z<3$. At all wavelengths, $>90 \%$ of galaxics become undetectable due to $\mu_{0}$ detection limits at redshifts smaller than that at which the Lyman absorption edge shifts into the bandpass.

It should be appreciated that estimates of the star formation rate with redshift are typically based on UV LF's as a function of redshift, which have errors in total flux due to the errors in individual source photometry discussed above. The UV luminosity density (dominated by the flux of hot young stars) is then combined with estimates of the initial mass function (IMF) and SEDs of aging stellar populations to produce an estimate of the EBL. EBL estimates based on evolving LFs are tuned to match the galaxy counts by construction. as total galaxy volume densities are tuned to match galaxy counts. Estimates of the star formation rate as a function of redshift which are based on such UV LF models are low by more than a factor of two, as are estimates of the total baryon fraction processed through stars. The above comments are independent of dust considerations, which concern the fraction of light which does not escape the galaxies at optical wavelengths.

Based on our upper and lower limits, the recent DIRBE measurements of the IR EBL, and models of the EBL flux at $10-400 \mu \mathrm{m}$ derived from empirical SED of galaxies in the local universe, we estimate that the lotal energy density of the $\mathrm{EBL}$ is in the range $61-153 \mathrm{nW} \mathrm{m} \mathrm{m}^{-2} \mathrm{sr}^{-1}$. This range can be compared with the baryonic mass in stars and the total metal mass density found in clusters. Those comparisons depend on estimates of the IMF of stars, the SED of stars over their lifetimes, and the metal yield, all of which are uncertain by factors of at least $50-100 \%$. Nonetheless, for reasonable estimates of these quantities, the total background range we give is in agreement with observed cluster metalicities ( $\sim 1 / 3$ solar; Mushotzky \& Lowenstein 1997, Renzini 1997), estimates of the baryon fraction processed through stars $\left(0.004 \rho_{c}\right.$; Fukugita, Hogan \& Peebles 1998). For further discussion, see Bernstein et al (1999c), Dwek et al. (1998), and contributions in these proceedings (Dwek 1999, Madau 1999).

Acknowledgments. I would like to thank J. Dalcanton, E. Dwek, T. Small, and I. Smail for numerous helpful discussions. 


\section{References}

Bernstein, R.A., Freedman, W.L., Madore, B.F. 1999a, submitted

Bernstein, R.A.. Freedman, W.L., Madore. B.F. 1999b, submitted

Bernstcin, R.A., Freedman, W.L., Madorc, B.F. 1999c, submitted

Bertin, E. \& Arnouts, S. 1996, AAS, 117, 393

Dalcanton, J.J., Spergel, D.N., Gunn, J.E., Schmidt, M. \& Schneider, D.P. 1997, AJ, 114.635

Dalcanton, J.J. $1998 \mathrm{ApJ}, 495,251$

Disney, M. 1976 Nature, 263, 573

Disney; M., Phillips, S. 1983, MNRAS, 205, 1253

Draine, B.T. \& Lee. H.M. 1984, A\}J, 285, 89

Dube, R.R., Wickes, W.C. \& Wilkinson, D.T. 1979, ApJ, 232, 333

Dwek, E. et al. 1998, ApJ, 508, 106

Fukugita, M., Hogan, C.J., \& Peebles, P.J.E. 1998,ApJ, 503, 518

Jarvis, J.F. \& Tyson, J.A. 1981, A.J, 86, 476

Mattila, K. 1976, AA, 47, 77

Mushotzky, R.F. \& Loewenstein, M. 1997, ApJ, 481, L63

Pei, Y.C. \& Fall, S.M. 1995, ApJ, 454, 69

Pettini, M., Ellison, S.L., Steidel, C.C. \& Bowen, D.V. 1999, ApJ, 510, 576

Pozzetti, L., Madau, P., Zamorani, G., Ferguson, H.C., \& Bruzual, G.A. 1998, MNRAS, 298, 1133

Renzini, A. 1997, ApJ, 488, 35

Sprayberry, D.. Impey, C.D., Irwin, M.J. \& Bothun, G.D. 1997, ApJ, 482, 104

Toller, G.N. 1983, ApJ, 266, L79

Williams, R.E. et al. 1996, AJ, 112, 1335

Witt, A.N., Friedmann, B.C. \& Sasseen, T.P. 1997, A p.T, 481, 809 
. 
The Hy Redshift Universe

ASP Conference Series, Vol. 193, 1999

A. J. Bunker \& W. J. M. van Breugel, eds.

\title{
The Extragalactic Background Light: Detections and Limits and their Cosmological Implications
}

\author{
Eli Dwek \\ Laboratory for Astronomy and Solar Physics \\ NASA/Goddard Space Flight Center, Greenbelt, MD 20771 \\ eli.dweh@gsfc.nasa.gov
}

\begin{abstract}
The extragalactic background light (EBL) provides important constraints on models for the origin of galaxies, stars, metals, and dust in the universe. It is also a source of opacity for ultrahigh energy cosmic ray nuclei and photons. This contribution reviews the current observational status of the EBL in the 0.1 to $1000 \mu \mathrm{m}$ wavelength regime, and examines its cosmological implications.
\end{abstract}

\section{Introduction}

The extragalactic background light (EBL), limited here to the $0.1-1000 \mu \mathrm{m}$ wavelength regime, contains the record of all possible energy inputs in the universe since the epoch of recombination. Its intensity and spectral energy distribution (SED) contains therefore important information on the star formation and metal production rates in the universe, and the thermalization history of starlight and other photons by dust (Hanser et al. 1998, Dwek et al. 1998, Harwit 1999, Madau 1999, and Dwek 1996 for further references). It therefore provides important constraints on the cosmic history of galaxy formation and evolution. The EBL is also an important source of opacity for $\mathrm{TeV}$ photons (Stecker \& De Jager 1993), and ultrahigh energy cosmic rays (Stecker \& Salamon 1999) in the universe. Detection of the EBL can therefore place important constraints on the intrinsic spectrum of $\mathrm{TeV} \gamma$-ray sources, and hence on their photon production mechanism (Coppi \& Aharonian 1999, De Jager \& Dwek 1998).

Two major energy sources contribute to the observed EBL: nuclear and gravitational. The nuclear contribution consists of the cnergy released in stcllar nucleosynthetic processes. This energy is radiated predominantly at UV-visual wavelengths and either redshifted or absorbed and reradiated by dust into the infrared $(\lambda \gtrsim 1 \mu \mathrm{m})$ wavelength region. Gravitational potential energy dominates the energy released by brown dwarfs, accreting black holes, and gravitationally collapsing systems, and may make a significant contribution to the EBL (Bond, Carr, \& Hogan 1986, 1991). Black holes in active galactic nuclei (AGN) may contribute a significant fraction of the EBL in the mid-IR $(\approx 10-50 \mu \mathrm{m})$ wavelength region (Granato, Franceschini, \& Danese 1996), and AGN may be contributing to the IR energy released in starbursts as well. At far-IR wavelengths their contribution may be smaller. The estimated contribution of AGN to the 


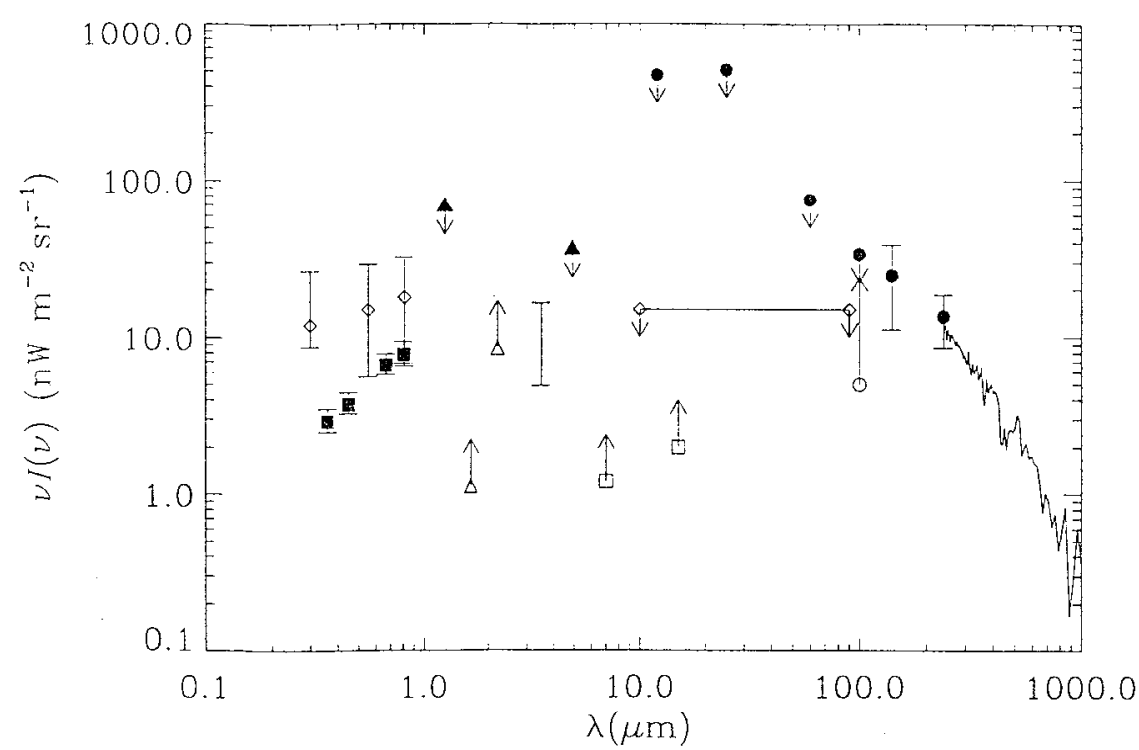

Figure 1. Observational constraints on the extragalactic bachground light: filled squares Pozzetti et al. 1997; open diamonds Bernstein (1999): filled circles Ilauser et al. 1998; solid line Fixsen et al. 1998; open diamonds 10-100 $\mu \mathrm{m}$ upper limits of Kashlinsky et al. 1996; open circle $100 \mu \mathrm{m}$ lower limit from Dwek et al. 1998; open squares ISO lower limits from Altieri et al. 1999; open triangles 1.65 and $2.2 \mu \mathrm{m}$ lower limits from Teplitz et al. 1998 and Bershady et al. 1998; solid triangles upper limits from Dwek \& Arendt 1998; $3.5 \mu \mathrm{m}$ error bar tentative detection by Dwek \& Arendt 1998

cosmic infrared background (CIB) at $850 \mu \mathrm{m}$ has recently been estimated to be about 10-20\% of the total intensity at that wavelength (Almaini et al. 1999). A third potential contributor to the EBL is the energy released by decaying relic particles (Bond, Carr, \& Hogan 1986, 1991).

\section{Observational Status of the EBL}

Figure 1 depicts the current detection and observational constraints on the EBL at UV to far-IR wavelengths.

Determination of the EBL from diffuse sky measurements is greatly hampered by the presence of foreground emission components. At infrared wavelengths these include scattered light from the interplanetary dust (IPD) clond, emission from discrete and unresolved stellar components in our Galaxy, and emission from dust in the Galactic interstellar medium (ISM). In a recent publication, Hauser et al. (1998) presented the results of the search for the cosmic infrared background (CIB) in the 1.25 to $240 \mu \mathrm{m}$ wavelength region that was conducted with the Diffuse Infrared Background Experiment (DIRBE) on the Cosmic Background Explorer ( $C O B E$ ) satellite. Careful subtraction of foreground emission from the IPD cloud (Kelsall et al. 1998) and from stellar and 
interstellar Galactic emission components (Arendt et al. 1998) revealed a residual emission component in the DIRBE skymaps that, after detailed analysis of the random and systematic uncertainties, was consistent with a positive signal at 100,140 , and $240 \mu \mathrm{m}$. Subsequent rigorous tests showed that only the 140 and $240 \mu \mathrm{m}$ signals were isotropic, a strict requirement for their extragalactic origin. Only upper limits for the CIB intensity were given for $\lambda=1.25-60 \mu \mathrm{m}$, where the CIB detection was hindered by residual emission from the IPD cloud. In the 1.25 to $4.9 \mu \mathrm{m}$ wavelength region, uncertainties in the subtraction of the Galactic stellar component contributed to the uncertainties as well. Analysis of the data obtained with the Far Infrared Absolute Spectrophotometer (FIRAS) instrument on the COBE resulted in the detection of the EBL in the 125$1000 \mu \mathrm{m}$ region as well (Puget et al. 1996, Fixsen et al. 1998). Independent analysis of the DIRBE data. (Schlegel, Finlibeiner, \& Davis 1997) gave results that are consistent with the Hauser et al. (1998) 140 and $240 \mu \mathrm{m}$ detections. Observations with the SCUBA array at the James Clark Maxwell Telescope resolred most of the EBL at $850 \mu \mathrm{m}$ into discrete sources (Hughes et al. 1998, Blain 1999).

Follow up analysis of the DIRBE data reduced the systematic uncertainties associated with the subtraction of the Galactic stellar emission, yielding somewhat lower upper limits on the 1.25 and $4.9 \mu \mathrm{m}$ EBL intensities, and resulting in a tentative detection of the EBL at $3.5 \mu \mathrm{m}$ (Dwek \& Arendt 1998).

Stricter upper limits on the EBL in the 10 to $100 \mu \mathrm{m}$ region, compared to those presented by Hauser et al.(1998), were derived from fluctuation analyses in the DIRBE 12 to $100 \mu \mathrm{m}$ shymaps (Kashlinsky, Mather, \& Odenwald 1996).

Galaxy counts provide strict lower limits on the EBL. The $\mathrm{K}(2.2 \mu \mathrm{m})$ band constraints are represented by a lower limit obtained from deep galaxy counts (Bershady, Lowenthal, \& Koo 1998). The lower limit in the $\mathrm{H}(1.65 \mu \mathrm{m})$ band was derived from images obtained by the Near-Infrared Camera and Multiobject Spectrometer (NICMOS) on board the Hubble Space Telescope (HST) (Teplitz et al. 1998). At 7 and $15 \mu \mathrm{m}$ the figure represents preliminary survey results obtained with the ISOCAM instrument on the Infrared Space Observatory (ISO) satellite (Altieri et al. 1999). The $100 \mu \mathrm{m}$ lower limit was derived from the DIRBE 140 and $240 \mu \mathrm{m}$ detections (Dwek et al. 1998).

The total integrated EBL intensity detected in the 0.2 to $3.5 \mu \mathrm{m}$ wavelength region is $\sim 30 \mathrm{nW} \mathrm{m} \mathrm{W} \mathrm{sr}^{-1}$ using the Bernstein (1999) detections. The total intensity detected in the 100 to $1000 \mu \mathrm{m}$ wavelength region is $\sim 20 \mathrm{nW} \mathrm{m} \mathrm{m}^{-2}$ $\mathrm{sr}^{-1}$, giving a total intensity of $\sim 50 \mathrm{nW} \mathrm{m}^{-2} \mathrm{sr}^{-1}$ in these two wavelength windows.

\section{The Extragalactic Background Light From Nucleosynthesis}

Most of the energy radiated by stars is liberated during the transmutation of protons into helium and heavier elements. The frequency-integrated EBL intensity, $I$, can therefore be related to $\Omega_{*}$, the fraction of the total critical mass density that has been processed through stars (Bond, Carr, \& Hogan 1986; Peebles 1993). For simplicity we will first assume that all the elements were instantaneously formed at some epoch corresponding to some redshift $z_{\epsilon}$. The intensity of the EBL consists of the energy released from the production of He 
that was not further processed into heavier elements, and of the energy released by the production of elements heavier than He. It can be written as:

$$
I=\left(\frac{c}{4 \pi}\right) \frac{\Omega_{\times} \rho_{c} c^{2}}{1+z_{e}}\left(\eta_{Y} \Delta Y+\eta_{Z} Z_{\times}\right) .
$$

The parameter $\eta_{Y}=0.0072$ is the energy conversion efficiency for the nuclear energy generating reactions $4 \mathrm{p} \rightarrow{ }^{4} \mathrm{He}, \eta_{Z}=0.0078$ is that for the $12 \mathrm{p} \rightarrow{ }^{12} \mathrm{C}$ reaction that leads to the production of metals, $Z_{*}$ is the current mass fraction of matter that was converted into metals, and $\Delta Y$ is the net enrichment in the ${ }^{4}$ He mass fraction due to stellar processing. For $Z_{\times}=Z_{\odot}=0.02$, the solar metallicity, and $\Delta Y=0.04$, which is lhe diflerence between the solar $(Y=0.28)$ and the primordial $(Y=0.24){ }^{4} \mathrm{He}$ mass fraction, most of the contribution to the EBL is due to the net enrichment of $\mathrm{He}$ in the universe, and

$$
\Omega_{\star}=1.1 \times 10^{-2} h_{50}^{-2}\left[\frac{I\left(n W m^{-2} s r^{-1}\right)}{50}\right]\left(1+z_{e}\right) .
$$

where $I$ is normalized to the observed EBL intensity. For an emission epoch of $\tilde{z}_{t}=1, \Omega_{\star} \approx 0.02$, lower than the upper limit of 0.15 derived from Big Bang nucleosynthesis arguments, and above the lower limit of 0.0014 implied from the amount of luminous matter currently locked up in stars. The EBL intensity therefore implies that at least $\sim 10 \%$, and possibly as much as $\sim 30 \%$ of the total baryonic mass density inferred from Big Bang nucleosynthesis was processed in stars into heavier elements.

In a more realistic treatment, we have to consider the continuous release of energy in the universe, rather than an instantaneous release at some redshift $\tilde{z}_{e}$. The intensity $I$ received per unit solid angle from such a continuous energy release is given by:

$$
I=\left(\frac{c}{4 \pi}\right) \int_{0}^{z *} \epsilon(z)\left|\frac{d t}{d z}\right| \frac{d z}{1+z}
$$

where $\epsilon(z)$ is the comoving luminosity density at redshift $z$, and $z_{*}$ represents the redshift when energy release first took place.

Assuming that the EBL arises only from energy released by nucleosynthesis, the value of $\epsilon(z)$ can be derived from the cosmic star formation rate (CSFR). Using the UV-optically determined CSFR presented by Madau, Pozzetti, \& Dickinson (1998) (see Figure 2), and integration the luminosity density over redshift for $\Omega_{0}=1$ and $\Omega_{\Lambda}=0$ gives a value of $I=30 \mathrm{nW} \mathrm{m} \mathrm{m}^{-2} \mathrm{sr}^{-1}$. This value is significantly below the detected value of $50 \mathrm{nW} \mathrm{m}^{-2} \mathrm{sr}^{-1}$. The UVO star formation rate therefore leaves no room for any expected EBL emission in the 3.5 to $100 \mu \mathrm{m}$ wavelength region, indicating that the UV-optically determined CSFR underestimates the actual rate of star formation in the universe. The magnitude of the underestimate is, however, still unresolved.

Rowan-Robinson et al. (1997) argued that ISO observations of the HDF suggest a constant star formation rate at redshifts $\gtrsim 1.5$, instead of a decreasing one. In this scenario the UV observations of the $\mathrm{HDF}$ represent only a fraction of the actual star-formation activities at these high redshifts. The SCUBA detection of highly shrouded galaxies at high redshift (Hughes et al. 1998) 


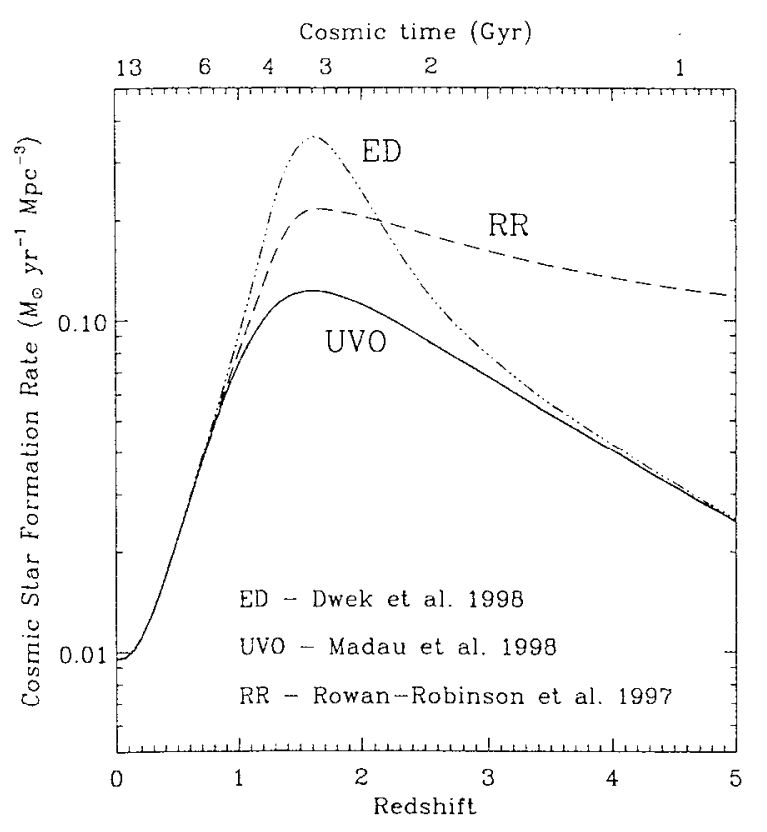

Figure 2. The cosmic star formation rate. The curve marked UVO is the UV-optically determined rate presented by Madau, Pozzetti, \& Dickinson (1998). The other curves are explained in the text.

supports this picture. The actual CSFR may have been underestimated at lower redshifts as well. Figure 2 depict two possible alternative cosmic star formation histories (labeled ED and RR) each given by a sum of two components: the first component is the UVO determined CSFR, and the second component is either sharply peaked at a redshift of $z=1.5$ (giving rise to the ED curve), or more gradually declining at high redshifts (giving rise to the RR curve). The EBL intensity predicted by the ED and $R R$ star formation rates are 49 and $46 \mathrm{nW}$ $\mathrm{m}^{-2} \mathrm{sr}^{-1}$, respectively, about cqual to tho detected intensity of the EBL.

\section{Spectral Energy Distribution of the EBL}

Additional constraints on the cosmic star formation histories can be derived from the comparison of the theoretically calculated spectral energy distribution of the EBL to the observational constraints. The specific intensity $I\left(\nu_{0}\right)$ of the EBL at the observed frequency $\nu_{0}$ is obtained by inlegrating the spectral luminosity density, $\epsilon(\nu, z)$, from the comoving volume elements at $z$, over redshifts:

$$
I\left(\nu_{0}\right)=\left(\frac{c}{4 \pi}\right) \int_{0}^{z *} \epsilon(1, z)\left|\frac{d t}{d z}\right| d z
$$

where $y=\nu_{0}(1+z)$ is the frequency in the rest frame of the comoving volume element. At each redshift, the spectral luminosity density can be written as the sum:

$$
\epsilon(\nu, z)=\epsilon_{s}(\nu, z)+\epsilon_{d}(\nu, z),
$$




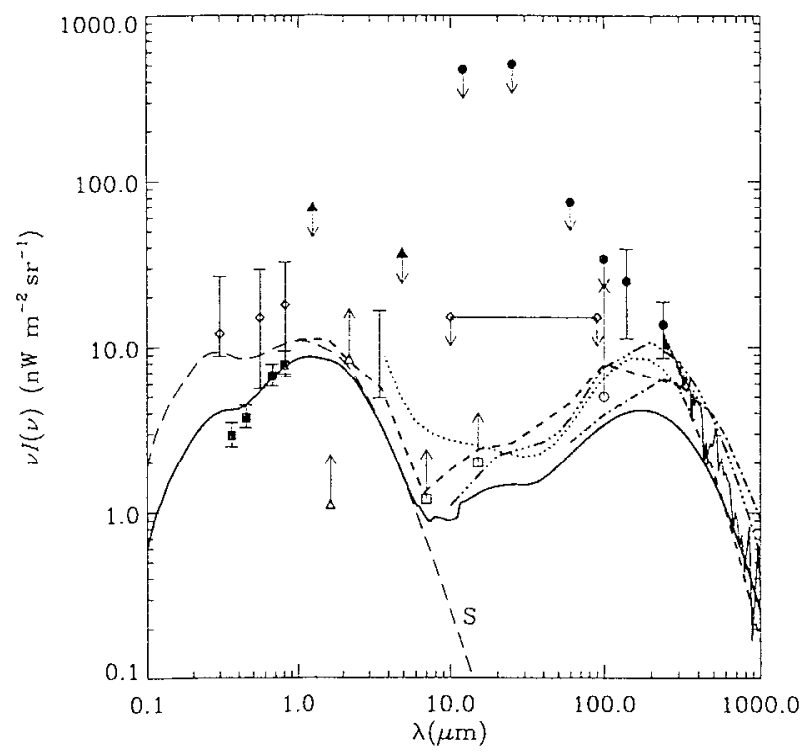

Figure 3. Comparison of model predictions with the observational constraints on the EBL. Models represented in the figures are: dott.ed line Malkan \& Stecker 1998; dashed-dotted line Beichman \& IIelou 1991; dashed line Franceschini et al. 1997; dashed-triple dotted line Guiderdoni et al. 1997; heavy solid line Dwek et al. 1998, model UVO.

where $\epsilon_{s}(\nu, z)$ and $\epsilon_{d}(\nu, z)$ are, respectively, the attenuated starlight and the dust contribution to the spectral luminosity density. The total, unattenuated, spectral luminosity density from starlight, $\epsilon(\nu, z)$, can be calculated from population synthesis models. Given the evolution of the CSFR as a function of time, the stellar mass spectrum, stellar evolutionary tracks and nucleosynthesis yields, and stellar atmosphere models as a function of metallicity, the spectral luminosity density can be uniquely determined as a function of time (Charlot, Worthy, \& Bressan 1996).

Figure 3 compares various recent models to the observational limits on the EBL. Older models are reviewed by (Lonsdale 1995, 1996). The curve labeled " $S$ " represents a "dust-free" model, calculated with the UVO star formation rate in which galactic starlight is allowed to escape into space unimpeded by dust. The curve illustrates the obvious, namely that in order to have any significant far-IR emission, a significant fraction of the starlight has to be reprocessed by dust. The heavy solid curve represents the EBL spectrum calculated by Dwek et al. (1998) with the UVO determined CSFR (see Figure 2). The discontinuities in the flux at $7.7,8.6$, and $11.3 \mu \mathrm{m}$ in the solid curve results from the cumulative contribution of the respective PAH emission lines to the EBL.

Very obvious in the figure is the failure of the UVO determined star formation rate to produce the observed EBL at far-IR wavelengths. Observationally this should not be too surprising. since the most intense star forming regions frequently manifest themselves primarily through their infrared, rather than their UV or optical emission. Furthermore, we have already shown that the total 
integrated EBL intensity predicted by the UVO model falls short of that of the detected EBL, leaving no room for any EBL intensity in the $\sim 3.5-100 \mu \mathrm{m}$ region of the spectrum. The calculated far-IR intensity cannot be increased by simply increasing the fraction of starlight that is reradiated in the model as thermal IR emission. This will require an increase in the extinction, causing the UV-optical part of the EBL to fall below the lower limits of Pozzetti et al. (1997). The figure also shows that all models (including the cosmic chemical evolution model prediction of Pei, Fall, \& Hauser 1999 - not shown in the figure) fall below the $2 \sigma$ lower limit of the $140 \mu \mathrm{m}$ detection. It seems clear that the excess IR emission needed to account for the detected EBL requires a star formation activity that escaped detection at UV and optical wavelengths.

These considerations have led Dwek et al. (1998) to consider alternative cosmic star formation scenarios for calculating the EBL. The model described in their paper uses a global CSFR to calculate the unattenuated stellar spectral luminosity density, $\epsilon(\nu, z)$, at each redshift using detailed population synthesis models. A simple dusty slab model is then used to calculate the attenuated stellar spectral luminosity density at each redshift:

$$
\epsilon_{s}(\nu, z)=\left[1-P_{a b s}(\nu)\right] \times \epsilon(\nu, z),
$$

where $P_{a b s}(\nu)$ is the probability that a photon of frequency $\nu$ is locally absorbed by the ambient dust. In this model $P_{a b s}(\nu)=1-\exp \left[-\tau_{a b s}(\nu)\right]$, independent of redshift, where $\tau_{a b s}$ is the dust absorption opacity, calculated for an average Galactic interstellar extinction law normalized to some ralue in the $\mathrm{V}$ band, with a wavelength independent albedo of 0.5 . The absorbed stellar radiation is reemitted by dust at IR. wavelengths with a spectrum given by the luminosity function-averaged SED of the IRAS galaxies. The local IR spectral energy density is shown in Fignre 4, and the details of its derivation are presented in Dwek et al. (1998). The spectral luminosity density of the dust at redshift $z$ can then be expressed in terms of its local spectral luminosity density, $\epsilon_{d}(\nu, 0)$, as:

$$
\epsilon_{d}(\nu, z)=\epsilon_{d}(\nu, 0) \frac{\int P_{a b s}(\nu) \epsilon(\nu, z) d \nu}{\int P_{a b s}(\nu) \epsilon(\nu, 0) d \nu}
$$

Figure 5 compares the EBL detections and constraints (represented here by the shaded area) with various model predictions using the UVO, ED, and RR cosmic star formation rates. The calculations show that the excess star formation in the $E D$ and $R R$ models must be produced by massive stars, otherwise the calculated $\mathrm{K}$-band luminosity density at $z=0$ would exceed the observed local value. Stars formed by the second component therefore release all their radiative output instantaneously into the ISM, where we assume it is totally absorbed by dust. To produce the observed excess IR background emission, over that predicted by the UVO model (see Figure 3), requires the rest frame spectra of these dusty, optically opaque, galaxies or star forming regions to peak shortwards of $\sim 100 \mu \mathrm{m}$. These optically opaque sources should therefore have spectra similar to that of ultraluminous IR galaxies (ULIRGs), peaking at wavelengths of about $80 \mu \mathrm{m}$.

'The figure shows that both, the $E D$ and the $R R$, models provide a significantly improved fit to the nominal DIRBE detections compared to other model 


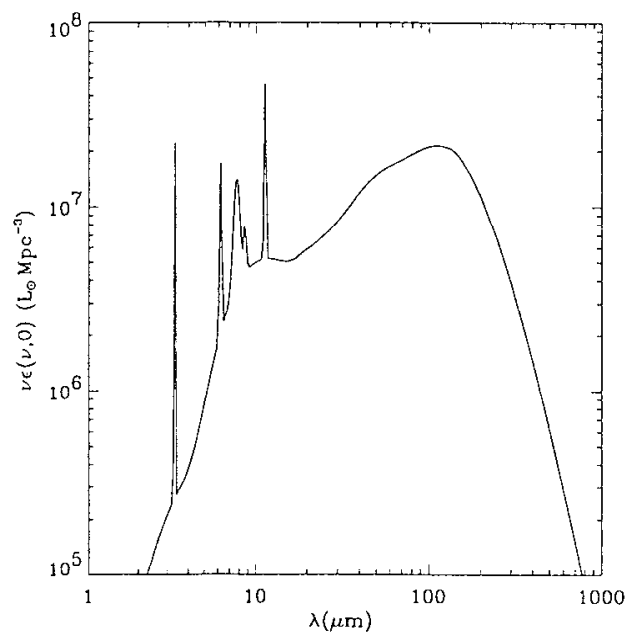

Figure 4. The luminosity function-averaged spectral luminosity density of IRAS galaxies.

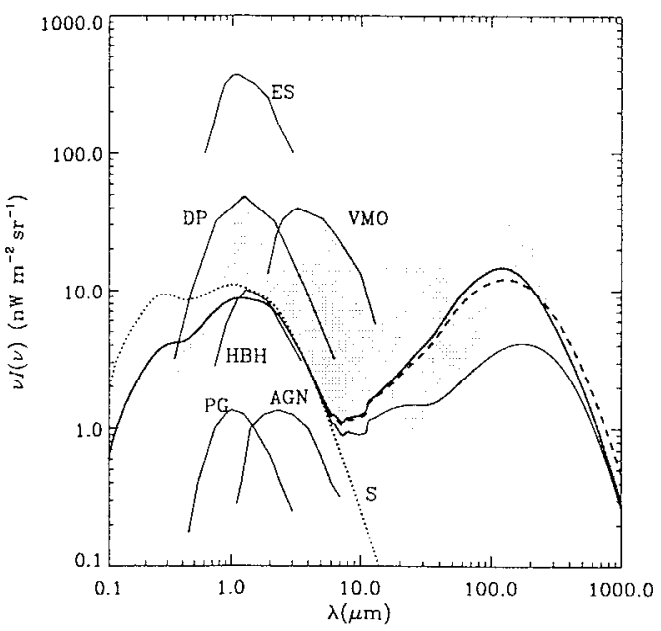

Figure 5. Comparison of model predictions with the observational constraints on the EBL, represented by the shaded area in the plot. Shown here are models $U V O$, thin solid line; $E D$, bold line; and $R R$, thick dashed line. Also shown are the predicted fluxes from various 'exotic' contributions: exploding stars (ES), decaying particles (DP), very massive objects (VMO), halo black holes ( $\mathrm{HBH}$ ), primeval galaxies (PG), and AGNs (Bond, Carr, \& Hogan 1986, 1991). 


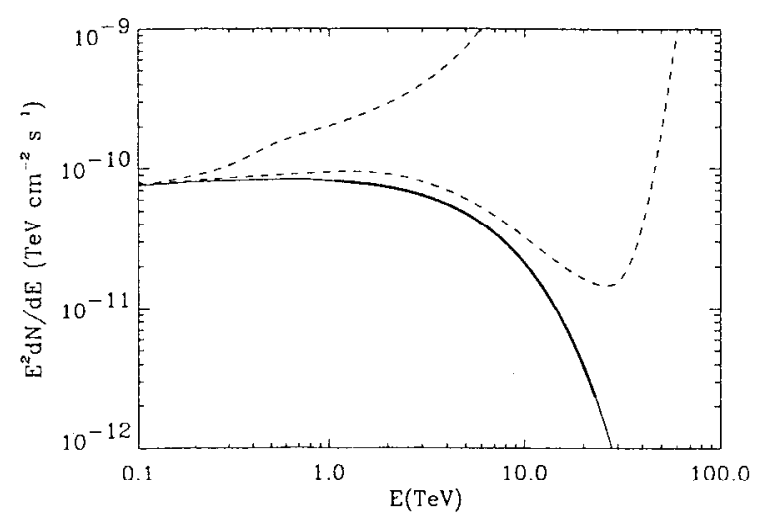

Figure 6. Observational constraints on the extragalactic background light.

predictions shown in Figure 3, and by construction, produce the local luminosity density at IR and UV-optical wavelengths. The $R R$ model predicts a somewhat larger IR background above $\sim 200 \mu \mathrm{m}$ than the $E D$ model, since a larger fraction of its star formation rate takes place at higher redshifts.

Figure 5 also shows the contribution of several unobscured "exotic" sources to the EBL. The contribution of some sources to the EBL can already be ruled out from the DIRBE upper limits in the 1.25 to $4.9 \mu \mathrm{m}$ wavelength region (Hanser et al. 1998; Dwek \& Arendt 1998). Complete obscuration of these sources by dust would move their spectra horizontally to longer wavelengths, with the location of the peak determined by the absorbing dust temperature. With some modification, these sources could contribute to the far--IR spectrum of the EBL. The possibility that some exotic and non-nuclear energy sources may contribute to the EBI, and the fact that the $E D$ and $R R$ star formation histories produce similar IR background spectra shows that there is no unique way to account for the EBT, spectrum. The current ambiguity in the magnitude and evolution of the cosmic star formation rate can only be resolved by future measurements of high redshift systems a.t infrared wavelengths.

\section{The Opacity to Local TeV $\gamma$-ray Sources}

The EBL is also a source of opacity to high energy $\gamma$-rays, which can be strongly attenuated by pair production via $\gamma+\gamma_{b} \rightarrow e^{+}+e^{-}$reactions. The interaction cross section has a peak value of $\sim 1.5 \times 10^{-25} \mathrm{~cm}^{2}$ at energies $E_{\gamma}(\mathrm{TeV}) / \lambda_{b}(\mu \mathrm{m})$ $\approx 0.4-1.2$, where $E_{\gamma}$ is the $\gamma$-ray energy, and $\lambda_{b}$ the wavelength of the EBL photon. Background photons in the $10-10^{3} \mu \mathrm{m}$ wavelength range are therefore a main sonce of opacity for $\sim 1-10^{3} \mathrm{TeV} \gamma$-ray sources, which should only be detectable up to distances of $\sim 50 \mathrm{Mpc}$ to $\sim 10 \mathrm{kpc}$, respectively (Gould \& Schréder 1966, Stecker 1998).

The attenuation, as much as it is a hindrance to high-energy astrophysics, can be used to probe the intensity of the CIB if a source of TeV photons just happens to be present at a relatively close distance (e.g. Stecker, De Jager, \& Salamon 1992; SDS92). Determination of the CIB by this method requires 
definitive evidence for intergalactic absorption in the observed source spectrum, and knowledge of the intrinsic spectrum of the source, which is a priori unknown. These two requirements are tightly coupled, and most limits on the CIB intensity are based on the assumption that the source spectrum is known and represented by a single power law (e.g. SDS92, Dwek \& Slavin 1994, Biller et al. 1995, Stanev \& Franceschini 1998). The shape of the source spectrum depends on the emission mechanism producing the $\mathrm{TeV}$ photons (e.g. Coppi \& Aharonian 1999), and may not be necessarily described by a single power law. It may therefore be more useful to use observational limits on the CIB to set limits on the intrinsic spectrum of the TeV $\gamma$-ray sources (De Jager \& Dwek 1998). Figure 6 depicts the limits on the intrinsic spectrum of Mrk 501, a TeV blazar located at a redshift of $z=0.034$. The heavy solid line depicts the analytical fit to the spectrum observed by the High Energy Gamma Ray Array (HEGRA) collaboration (Konopelko 1999), and the light solid line its extrapolation to lower and higher energies. The upper dashed line represents the intrinsic source spectrum obtained by propagating the observed spectrum backwards through an EBL characterized by the upper limits in Figure 1. The lower dashed line is the intrinsic source spectrum when the observed one is propagated through the lower limits of the EBL. The super exponential blowup in the intrinsic source spectrum at energies above $\sim 25 \mathrm{LeV}$ is clearly unphysical, and caused by the extrapolation of the observed source spectrum to energies above $\sim 20 \mathrm{TeV}$ where it is is probably completely absorbed by the cosmic microwave background. Currently, the spread between the two dashed curve is too large to provide any useful constraints on the intrinsic source spectrum. Much tighter limits on the CIB in the $10-100 \mu \mathrm{m}$ wavelength region are required for that purpose.

\section{References}

Almaini, O., Lawrence, A., \& Boyle, B. J. 1999, MNRAS, 305, L59

Altieri, B. et al. 1999, A\&A, 343, L65

Arendt, R. G. et al. 1998, ApJ, 508, 74

Beichman, C. A., \& Helou, G. 1991, ApJ, 370, L1

Bernstein, R. 1999, this conference

Bershady, M. A., Lowenthal, J. D., \& Koo, D. C. 1998, ApJ, 505, 50

Biller, S. D. et al. 1995, ApJ, 445, 227

Blain, A. W. 1999, this volume

Bond, J. R., Carr, B. J., \& Hogan, C. J. 1986, ApJ, 306, 428

Bond, J. R., Carr, B. J., \& Hogan, C. J. 1991, ApJ, 367, 420

Charlot, S., Worthey, G., \& Bressan, A. 1996, ApJ, 457, 625

Coppi, P. S., \& Aharonian, F. 1999, ApJ, 457, 625

De Jager, O. C., \& Dwek, E. 1998, Astroparticle Physics, submitted

Dwek, E, \& Slavin, J. 1994, ApJ, 436, 696

Dwek, E. 1996, ed. Unveiling the Cosmic Infrared Background, AIP Press

Dwek, E. et al. 1998, ApJ, 508, 106

Dwek, E., \& Arendt, R. G. 1998, ApJ, 508, L9 
Fall, S. M., Charlot, S., \& Pei, Y. C. 1996, ApJ, 464, L43

Fixsen, D. J. et al. 1998, ApJ, 508, 123

Franceschini, A., Granato, G., Mazzei, P., Danese, L., \& De Zotti, G. 1996, in Unveiling the Cosmic Infrared Background p.159, ed. E. Dwek, AIP Press

Gould, R., J., \& Schréder, G. 1966, Phys. Rev. Lett., 16, 252

Granato, G. L.. Franceschini, A., \& Danese, L. 1996, in Unveiling the Cosmic Infrared Background p. 226, ed. E. Dwek, AIP Press

Guiderdoni, B., et al. 1998, MNRAS, 295, 877

Hacking, P., B., \& Soifer, B. T. 1991, ApJ, 367, L49

Harwit, M. 1999, ApJ, 510, L83

Hauser, M. G. et al. 1998, ApJ, 508, 25

Hughes, D. H. et al. 1998, Nature, 394, 241

Kashlinsky, A., Mather, J. C., \& Odenwald, S. 1996, ApJ473 L9

Kelsall, T. J., et al. 1998, ApJ, 508, 44

Konopelko, A. 1999, astro-ph/9901093

Lonsdalc, C. 1995, in Extragalactic Background Radiation p. 145, cds. D. Calzetti, M. Livio, \& P. Madan, Cambridge University Press

Lonsdale, C. 1996, in Unveiling the Cosmic Infrared Backiground p. 14T, ed. E. Dwek, AIP Press

Madau, P., Pozzetti, L., \& Dickinson, M. 1998, ApJ, 498, 106

Madau, P. 1999, this conference proceedings (astro-ph/9907268)

Malkan, M. A., \& Stecker, F. W. 1997, ApJ, 496, 13

Peebles, J. E. 1993, Principles of Physical Cosmology, Princeton University Press

Pei, Y. C., \& Fall, S. M., \& Hauser, M. G. 1999, ApJ, 522, 000

Pozzetti, L., Madau, P., Ferguson, H. C., Zamorani, G., \& Bruzual, G. A. 199'،, MNRAS, 298, 1133

Puget, J. -L., Abergel, A., Bernard, J. -P, Boulangerm F., Burton, W. B., Désert, F. -X, \& Hartmann, D. 1996, å, 308, L5

Rowan-Robinson, M. et al. 1997, MNRAS, 289, 490

Schlegel, D. J., Finkbeiner, D. P., \& Davis, M. 1997, ApJ, 500, 525

Stanev, T., \& Franceschini, A. 1998, ApJ, 492, 219

Stecker, F. W., De Jager, O. C, \& Salamon M. H. 1993, ApJ, 390, L49

Stecker, F. W., De Jager, O. C. 1993, ApJ, 415, Li1

Stecker, F. W., Salamon, M. H. 1999, ApJ, 512, 521

Stecker, F. W. 1999, astro-ph/9812286

Teplitz, H. I. et al. 1998, ApJ, 507, L17 



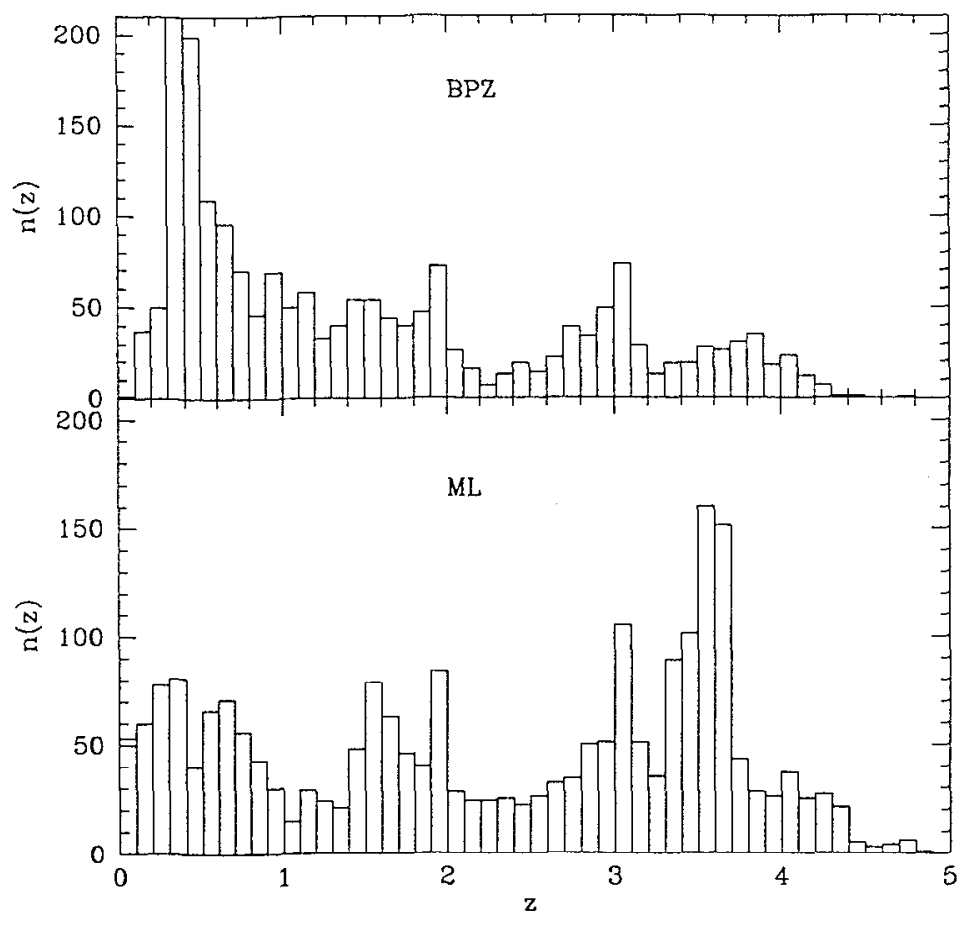

Figure 1. a)(bottom)Redshift histogram obtained by applying a maximum likelihood photometric redshift method to deep $B V R I$ observations of the CL0024+16 cluster field. The spurious redshift peak at $z \sim 3.5$ is caused by the color/redshift degeneracy between cluster members and high-z galaxies (see text). b) The top plot shows the effect of estimating the redshifts using BPZ (Benítez 1999). The high-z peak disappears and the cluster is clearly visible in the redshift distribution.

(Frye 1999), it is virtually impossible to obtain spectra for all or even for most of the $I>24$ objects present in those fields, and one has to resort to photometric redshifts.

We have obtained extremely deep $B V R I$ imaging of several $0.2-0.55$ galaxy clusters with LRIS at Keck II (magnitude limits $B<28.5, V<28, R<27.7, I<$ 26.9). Due to the lack of a blue arm in LRIS and the small angular size of NIRC, it is not practical to obtain matching $U$ and near-IR imaging at Keck. The extent of the color baseline is therefore very limited, what strongly hinders the estimation of photometric redshifts with traditional methods. Fig 1a. shows the $n(z)$ distribution resulting of applying standard maximum likelihood (ML) techniques to the Cl0024+16 field (Benítez \& Broadhurst 1999). It is obvious from this plot that many cluster members have been assigned erroneously high redshifts by the ML procedure, what it is not surprising since their optical colors are indeed very similar to those of high-z galaxies. This confusion renders the ML photo- $z$ virtually useless for most purposes. However, there is plenty of information which ML photo- $z$ estimation is not taking into account, like the conspicuous presence of a galaxy cluster in the field, the expected frac- 


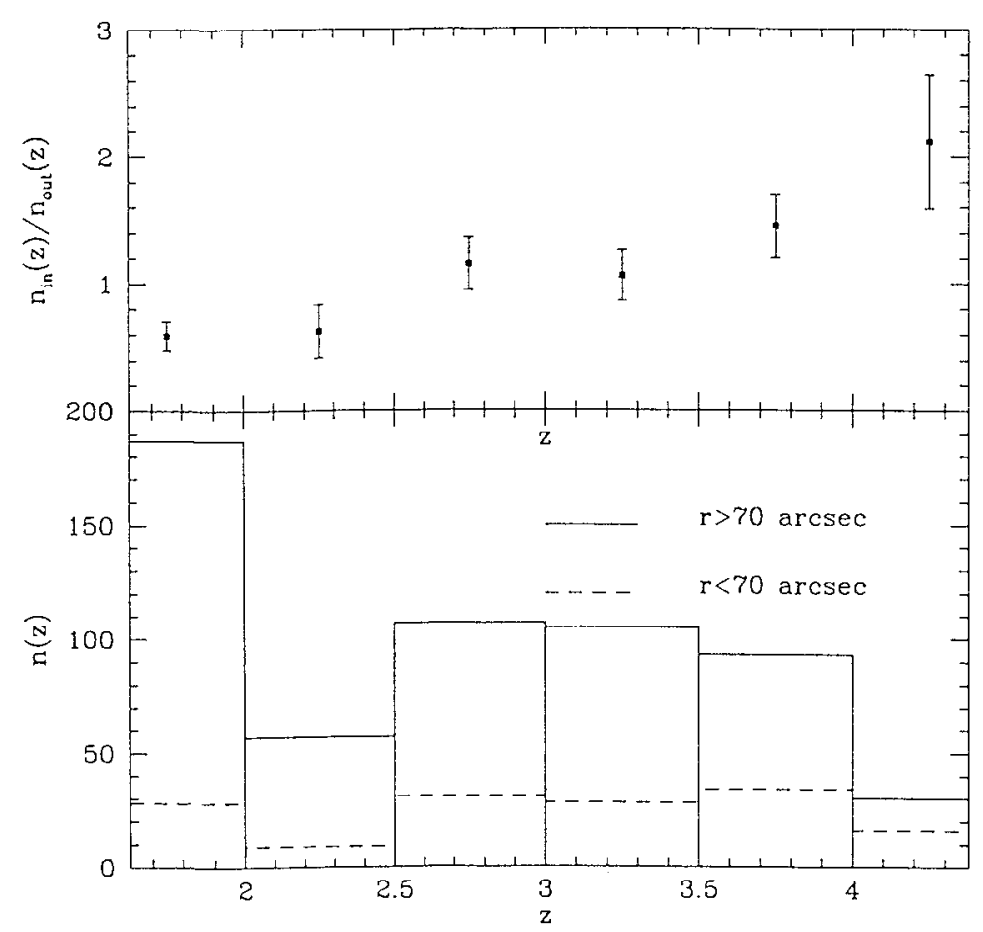

Figure 2. a)(bottom)Redshift distributions for background galaxies close to the cluster center (dashed line) and in the cluster outskirts (continuous line).b) (top) Normalized ratio of the above quantities.

tions of cluster and field spectroscopical types or the field redshift distribution. Bayesian photometric redshift estimation (BPZ, Benítez 1999) uses all those data, together with the galaxy colors, combining them in an optimal fashion. This significantly improves the redshift estimation, since many of the spurious peaks present in the redshift likelihood are eliminated in the final bayesian probability by using the appropriate prior. An example is shown in Fig. 1b, where a cluster prior as described in Benitez $1999 \mathrm{~b}$ has been used. There is also a very good agreement with spectroscopic redshifts. The cluster lensing effects have been tentatively taken into account by allowing for a certain amount of magnification in the central parts of the cluster. The photo-z estimation procedure is robust enough to be practically immune to errors of a factor of a few in the magnification estimation. Nevertheless, in the future it would be desirable to perform a simultaneous, iterative estimation of the cluster lensing properties and the photometric redshifts.

With the photometric redshifts thus obtained, it is possible to detect the 'shift' in the redshift distribution of bachground galaxies due to the cluster magnification (Broadhurst, Taylor \& Peacock 1994). If we set a magnitude limit and increase the redshift, we sample brighter and brighter parts of the luminosity function, and therefore the observed number counts as a function of redshift become steeper. Due to the magnification bias effect, there is an excess in the number of $z>3.5$ galaxies behind the cluster, as Fig 2. shows for for 
C10024+16 at $z=0.39$. A similar effect is observed in other clusters of our sample (Benítez \& Broadhurst 1999).

\section{References}

Benítez 1999a, astro-ph/9811189, accepted in ApJ

Benítez 1999b, in preparation

Benítez \& Broadhurst 1999, in preparation

Broadhurst, T. J., Taylor, A. N. \& Peacock, J. A. 1995, ApJ, 438, 49

Ellis, R. S. 1997, ARA\&A, 35, 380

Frye, B. 1999, Ph.D. thesis, UC Berkeley

Lanzetta, K. M., Yahil, A. \& Fernández-Soto, A. 1996, Nature, 381, 750

Gwyn, S. D. J. \& Hartwick, F. D. A. 1996, ApJ, 468, L77

Sawicki, M. J., Lin, H. \& Yee, H. K. C. 1997, AJ, 113, 1 
The Hy Redshift Universe

ASP Conference Series, Vol. 193, 1999

A. J. Burker \& W. J. M. van Breugel, eds.

\title{
Completeness and Confusion in the Identification of Lyman-Break Galaxies
}

\author{
Garret Cotter ${ }^{1}$, Toby Haynes ${ }^{1}$, Joanne C. Baker ${ }^{2}$, Michael E. Jones ${ }^{1}$ \\ and Richard Saunders ${ }^{1}$
}

\begin{abstract}
.
We have carried out a study to simulate distant clusters of galaxies in deep ground-based optical images. We find that when model galaxies are added to deep images obtained with the William Herschel Telescope, there is considerable scatter of the recovered galaxy colours away from the model values; this scatter is larger than that expected from photometric errors and is significantly affected by confusion, due to ground-based seeing, between objects in the field. In typical conditions of $\approx 1$-arcsec seeing, the combination of confusion and incompleteness causes a considerable underestimation of the true surface density of $z \approx 3$ galaxies. We argue that the actual surface density of $z \approx 3$ galaxies may be several times greater than that estimated by previons ground-based studies, consistent with the surface density of such objects found in the HDF.
\end{abstract}

\section{Introduction}

The field of the of the $z=3.8$ quasar pair PC1643+4631 A \& B contains a Cosmic Microwave Background decrement (Jones et al. 1997) which may be the Sunyaev-Zel'dovich effect of a cluster of galaxies at $z>>1$ (Saunders et al. 1997; Kneissl et al. 1998). In an attempt to detect such a cluster, we have carried out deep UGVRI imaging of the field. No cluster is immediately obvious in the images, so we carried out Monte-Carlo simulations to quantify our ability to detect a cluster of galaxies in our images (full details are given in Haynes et al. 1999 and Cotter et al. 1999).

\section{Model high $-z$ cluster galaxies}

Model clusters were created using simulated colours of evolving galaxies in the redshift range $0<z<4$, and added to our WHT images. We then used photometric redshift techniques to try to recover the simulated cluster. As the cluster redshift reached $z \approx 1$ and beyond, the lack of strong spectral features in the optical made the cluster increasingly difficult to detect. However, even at $\tilde{\sim} \sim 3$, where the characteristic Lyman-limit break became detectable, a large

${ }^{1}$ Astrophysics, Cavendish Laboratory, Madingley Road, Cambridge, CB3 0HE, UK

${ }^{2}$ Astronomy Department, University of California, Berkeley CA 94720, USA 


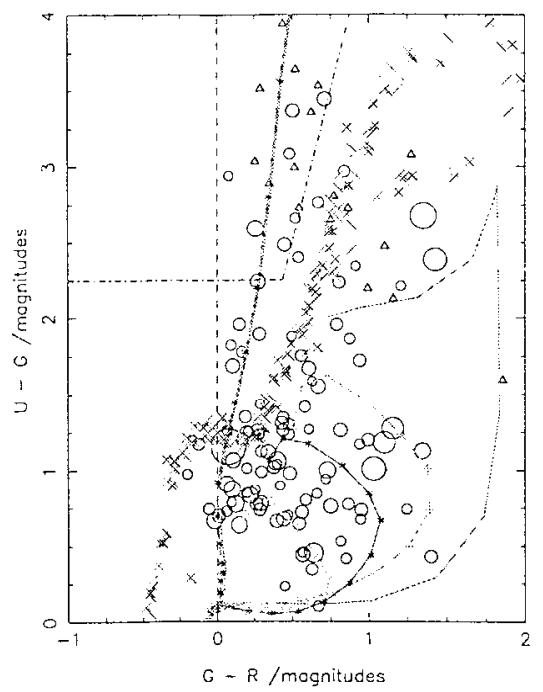

Figure 1. Recovered $U G R$ colours of simulated $2.5<z<3.5$ cluster galaxies. All objects shown have measured $R<25.5$ and $G>2 \sigma$. The triangles denote $1-\sigma$ lower limits in $U$ G. The crosses are stars from the Gunn \& Stryker (1983) database and the dot-dash line is our bound for selecting $z>3$ candidates. The tracks show the simulated galaxy colours at varions redshifts; the point at $U-G=2.2$ and $G-R=0.3$ corresponds to $z=3.0$, and points at larger $U-G$ are at higher redshift in steps of $\Delta z=0.1$. Note that the recovered colours are skewed towards the red in $G-R$.

fraction of the the fake cluster galaxies were still recovered from the simulation with ambiguous colours (Fig 1). Indeed, in our $z=3.0$ simulation, only one in five of the model cluster galaxies was identified as such by $U G R$ selection. The recovered colours were skewed towards the red in $G-R$; this is a result of confusion with other objects in the field.

\section{Recovery of model $z \approx 3$ galaxies}

Our original search for $\approx \approx 3$ Lyman-break galaxies (LBGs) in these images (Cotter \& Haynes 1998) had recovered a reasonable number of candidatesapproximately $1.1 \mathrm{arcmin}^{-2}$, similar to the findings of the surveys of Steidel et al. $(1996,1998)$. The fact that our recovery of simulated high-z cluster galaxies was so inefficient therefore prompted us to measure the effects of completeness and confusion specifically for $\approx \approx 3$ galaxies.

We ran 1000 simulations, each time adding ten fake LBGs to our images. Fake LBGs were drawn from a Schecter luminosity function with $R_{*}=24$ and $\alpha=1.06$ : all had input colours $G-R=2.2, U-G=0.3$. We used input half-light radii of $0.2-0.3^{\prime \prime}$ (Giavalisco et al. 1998).

$\therefore$ Then, using FOCAS, we attempted to recover the LBGs. Our selection $\ldots$ criteria are chosen to be as close as possible to that of Steidel et al. We select only those galaxies clearly detected with $R<25.5$ above the $3-\sigma$ isophote in $R$, measure magnitudes in $U$ and $G$ through this $R$-band isophote, and then impose 
a colour cut of $U-G>2, U-G>4(G-R)+0.5$, which is closely equivalent to the "robust" colour selection of Steidel et al. First, we find that $53 \%$ of galaxies with input $R<25.5$ are selected to the isophotal $R=25.5$ limit. Second, we find that, as for our fake cluster galaxies, a large fraction of the fake LBGs are scattered far away from their input colours.

In total, only $23 \%$ of the input LBGs with $R<25.5$ remain within the $z \geq 3$ region of the $U G R$ plane (Fig 2). Therefore, the true number of LBG candidates in our images may be four times greater than the $1.1 \operatorname{arcmin}^{-2}$ we measure. Again we stress that, while our $U G R$ filter set is slightly different from the $U_{n} G \mathcal{R}$ used by Steidel et al, our search for genuine LBG candidates in these images finds a surface density at least as great as that of Steidel et al.

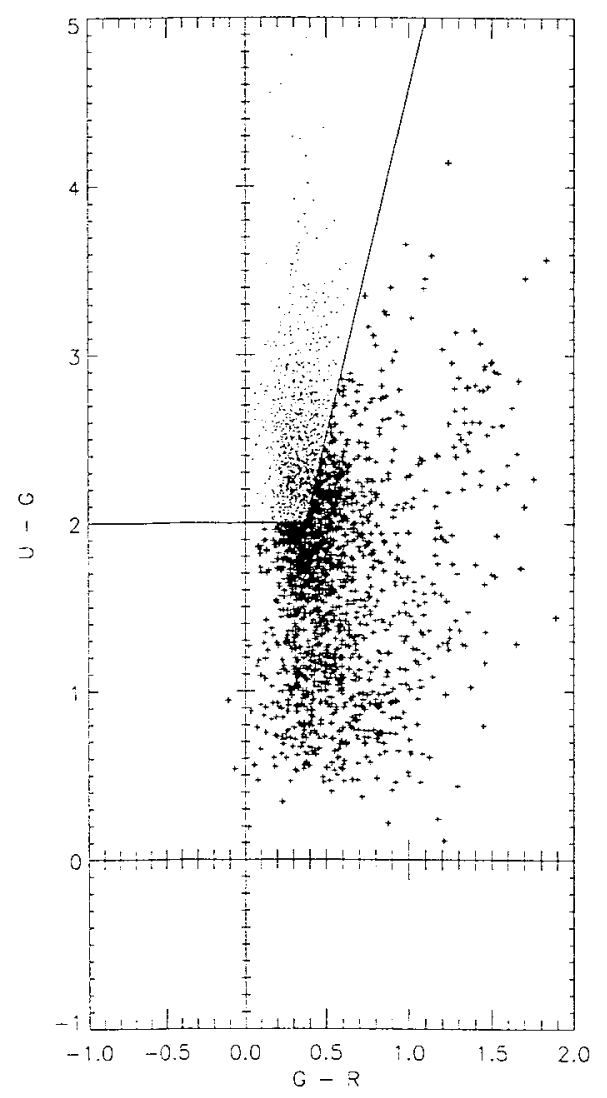

Figure 2. Recovered colours of fake $z=3.0$ LBGs; all have input colours $G-R=2.2, U-G=0.3$. All galaxies recovered to the $R=25.53-\sigma$ isophote are shown; crosses mark those outside the $z \geq 3$ region of colour-colour space defined by the solid line $(U-G>2, U-G>4(G-R)+0.5)$. Dots mark the $23 \%$ of the galaxies with input $R<25.5$ remaining in the $z \geq 3$ region.

These results suggest that ground-based $L G R$ selection. while extremely successful at identifying $z \sim 3$ galaxies. may miss a significant fraction of the population. This may have a bearing on the apparent discrepancy between the surface densities of LBGs measured in the Hubble Deep Field (HDF) and in ground-based surveys. There are 12 galaxies in the HDF which have spectro- 
scopic redshifts $2.8<z<3.5$ and $V_{606}<25.5$ (Dickinson 1997). These galaxies correspond to those which would be detected by the "robust" LBG candidate criteria of Steidel et al. (1998). However, one would expect, given the published surface-density of "robust" LBG candidates of $\approx 0.7 \mathrm{arcmin}^{-2}$ (Steidel et al. 1998), to find only three such LBGs in the HDF. Of course, cosmic variance will be significant for the HDF; but it is striking that the apparent overdensity of LBGs in the HDF corresponds with our estimate of the fraction of LBGs lost to incompleteness and confusion in the ground-based images.

\section{Conclusions}

We have carried out simulations to examine the effectiveness of searches for high-redshift galaxics in deep optical images typical of those obtaincd with $4-\mathrm{m}$ telescopes. We find that the scatter of the recovered colours of model galaxies away from their model colours is two to three times greater than that cxpcctcd from the photometric errors alone and arises as a result of confusion between the simulated galaxies and the real objects in the field. Because of the effects of incompleteness and confusion, the surface densities of LBGs based on groundbased imaging may be underestimated by a factor of four; this is consistent with the surface density of LBGs measured in the HDF.

To investigate these effects further, we will carry out more detailed simulations using our present data to investigate how the inferred luminosity function of the LBGs is affected by incompleteness and confusion. We also plan decp imaging programmes with the new generation of wider-field, high-image-quality instrumentation as it becomes available.

\section{References}

Cotter, G., Haynes, T., Baker, J. C., Jones, M. E., Saunders, R., 1999, MNRAS submitted

Cotter, G., Haynes, T., 1998, in The Young Universe, eds D'Odorico, S., Fontana, A., Giallongo, E., ASP

Dickinson, M., 1997, in The Hubble Deep Field: Proceedings of the Space Telescope Science Institute Symposium, eds Livio, M., Fall, S. M., Madau, P., Cambridge

Haynes, T., Cotter, G., Baker, J. C., Eales, S. A., Jones, M. E., Rawlings, S., Saunders, R., 1999, MNRAS submitted

Giavalisco, M., Steidel, C.C., Macchetto, F. D. 1996, ApJ, 470, 189

Gunn, J. E., Stryker, L. L. 1983, ApJS, 52, 121

Jones, M., et al. 1997 ApJ, 479, L1

Kineissl, R.. Sunyaev, R. A., White, S. D. M. 1998, MNRAS, 297, L29

Saunders, R., et al. 1997 ApJ, 4i9, L1

Steidel, C. C., Giavalisco, M., Pettini, M., Dickinson, M., Adelberger, K. 1996. ApJ, 462, $17 \mathrm{~L}$

Steidel, C. C., Adelberger, K., Dickinson, M., Giavalisco, M., Pettini, M., Kellogg, .N. 1998, ApJ, 492, 428 


\title{
Dust in High Redshift Starburst Galaxies
}

\author{
Karl D. Gordon ${ }^{1,2}$, Tracy L. Smith ${ }^{3}$ and Geoffrey C. Clayton ${ }^{1}$
}

\begin{abstract}
We have determined that the dust in starburst galaxies with $z=0$ to 3 in the North \& South Hubble Deep Fields is similar to the dust seen in the Small Magellanic Cloud star forming bar. Measuring the attenuation due to dust in individual galaxies with better than $1 \mathrm{mag}$ accuracy is not possible without combining $\mathrm{LV}$ and far-IR observations.
\end{abstract}

\section{Introduction}

To study galaxies, it is crucial to be able to separate the effects of the dust intrinsic to the galaxy from those associated with the galaxy's stellar age and metallicity. The accuracy of this separation directly affects the determination of a galaxy's physical properties (star formation rate, dust content. etc.). The importance of dust in galaxies has recently gained attention through investigations into the star formation rate as a function of redshift (e.g., Madau, Pozzetti, \& Dickinson 1998; Steidel et al. 1999).

For starburst galaxies, one important method to determining their star formation rate is to use their observed UV fluxes. The unattenuated UV flux of starburst galaxies is a good measure of their star formation rates as the majority of a starburst's flux is emitted in the UV. The main uncertainty in using the UV flux is determining the level of correction needed due to dust attenuation. In order to calculate the attenuation suffered by a galaxy, we need to know the type of dust in the galaxy (MW/SMC/other), the amount of dust, the global distribution of the stars/gas/dust, and the local distribution of the dust (clumpy/homogeneous).

\section{Dust Properties}

\subsection{Type of Dust}

The type of dust at a particular redshift can be determined using suitably chosen color-color plots. In Figure 1, we show the color-color plots which probe the presence/lack of the $2175 \AA$ bump in a galaxy's dust at $z=0,1,2, \& 3$. The $\mathrm{x}$ axis gives the color associated with the short wavelength side of the $2175 \mathrm{~A}$ bump and the y axis gives the color associated with the slope under the

\footnotetext{
${ }^{L}$ Department of Physics \& Astronomy. Louisiana State University, Baton Rouge, I.A 70803

${ }^{3}$ present address: Steward Observatory, University of Arizona, Tucson, AZ 85721

${ }^{3}$ Department of Physics \& Astronomy, University of Toledo. Toledo, OH 43606
} 

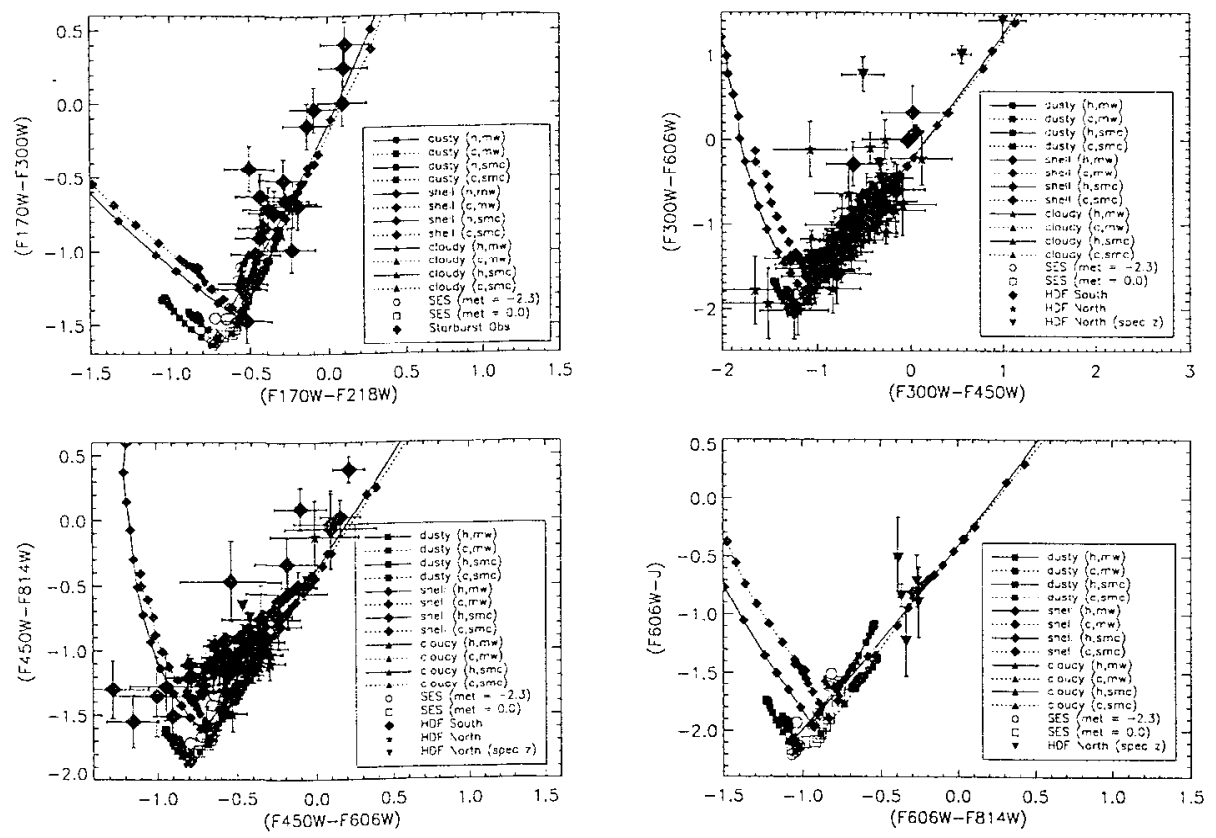

Figure 1. The color-color plots probing the presence/lack of the $2175 \AA$ bump are given for $z=0$ (upper left), 1 (upper right), 2 (lower left), and 3 (lower right).

bump. Fig. 2 gives the rest-frame location of various filters for different redshifts. The observational data for $z=0$ is from the IUE sample of starburst galaxies (Kinney et al. 1993). The data for $z=1,2, \& 3$ is from the Hubble Deep Fields (Fernández-Soto et al. 1999; Chen et al. 1999). The $z=1,2$, \& 3 observational points include galaxies with spectroscopic or photometric redshifts ranges of $0.9-1.1,1.9-2.1, \& 2.8-3.2$, respectively. The intrinsic colors of galaxies were determined using $1 \mathrm{Myr}$ to $10 \mathrm{Gyr}$ constant star formation PEGASE SES models (Fioc \& Rocca-Volmerange 1937). The reddening trajectories were determined using the DIRTY dust radiative transfer model (Witt \& Gordon 1996; 1999). Details of the calculations can be found in Witt \& Gordon (1999)

All four plots in Fig. 1 show that the dust in most starburst galaxies at redshifts between 0 and 3 lacks a $2175 \AA$ bump since their colors lie on the reddening trajectories associated with SMC-like dust, not MW-like dust. This is most likely due to processing of the dust in starburst galaxies due to the intense shock and radiative environment present in near starburst regions (Gordon, Calzetti. \& Witt 1997). Similar behaviors have been seen for dust extinction curves in the SMC (Gordon \& Clayton 1998), the LMC (Misselt, Clayton, \& Gordon 1999), and the Halo of the Milky Way (Clayton \& Gordon 1999). 


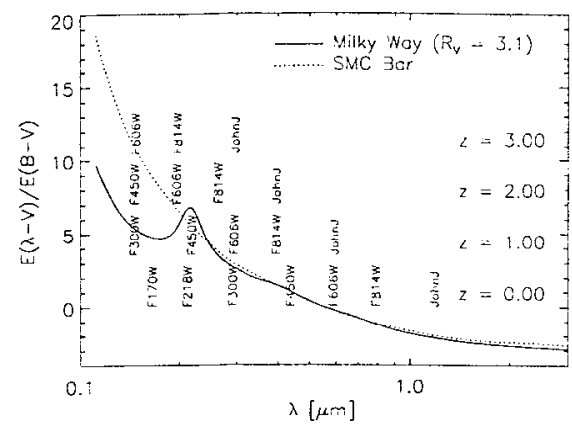

Figure 2. The rest-frame location of various WFPC2 and Johnson filters for $z=0,1,2, \& 3$ are shown along with the Milky Way and Small Magellanic Cloud Bar extinction curves.

\subsection{Effects of Dust}

The determination of the effects of dust in a galaxy (e.g., parameterized by the attenuation at $1600 \AA$ ) is not possible with the available data. Currently, the best available method is to use the slope of a galaxy's UV SED (parameterized by $\beta$ ) and an empirical calibration based on the observed properties of local starburst galaxies (Mewrer et al. 1999). This calibration is shown in Fig. 3a along with the results of our dust radiative transfer model (DIRTY). In the Meurer et al. empirical calibration, the $A t t(1600)$ for each galaxy was determined from the galaxy's $F(F I R) / F(1600)$. In Fig 3 b, we show the relationship between the $F(F I R) / F(1600)$ and $\beta$ for the galaxies used by Meurer et al. and our radiative transfer model runs. As can be seen from both figures, the uncertainty in using $\beta$ to determine Att(1600) is around $1 \mathrm{mag}$. This is due to not knowing the dust geometry or local distribution (i.e. dusty vs. shell and homogeneous vs. clumpy).

In order to determine the attenuation at $1600 \AA$ in starburst galaxies to better than $1 \mathrm{mag}$ uncertainty, UV and infrared observations are needed. This is illustrated in Fig. 4 which gives the relationship between $F(I R) / F(1600)$ and Att (1600) predicted using our radiative transfer model. $F(I R)$ is the integrated flux emitted by the dust in a galaxy. As can be seen from this figure, this relationship is only weakly dependent on the dust type, star/gas/dust geometry, or local dust distribution. This relationship is also only weakly dependent on the age or metallicity of the stellar population. This is because the majority of the flux which heats the dust (producing $F(I R)$ ) is absorbed in the UV. Thus, if UV and infrared observations can be obtained of a galaxy at any redshift, the galaxy's $A t t(1600)$ can be determined fairly accurately, and the star formation rate can be measured with a much reduced uncertainty.

\section{References}

Calzetti, D.. Kinney, A. L.. \& Storchi-Bergmann, T. 1994, Ap.J, 429, 582

Chen, H. et al. 1999, (astro-ph/9812339) 

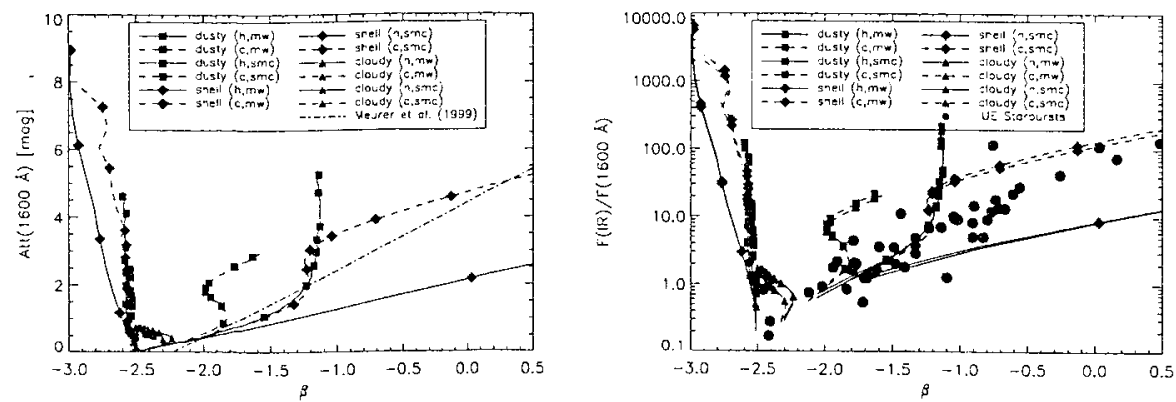

Figure 3. The relationship between $A t t(1600)$ and $\beta$ is shown in the graph on the left. The relationship between $F(F I R) / F(1600)$ and $\beta$ is shown on the right. The value of $\beta$ is determined by fitting the UV SED to $F(1200 \AA<\lambda<2400 \AA) \propto \lambda^{-3}$.

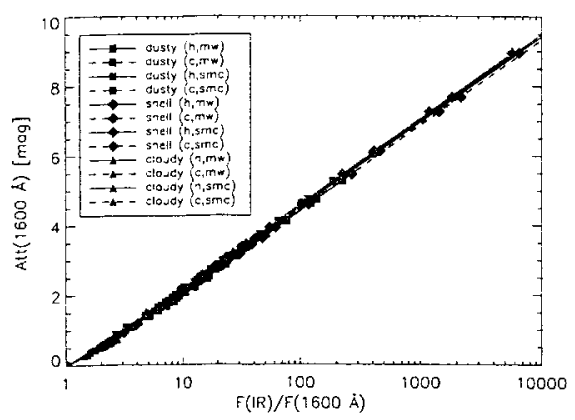

Figure 4. The relationship between $F(I R) / F(1600)$ is plotted.

Clayton, G. \& Gordon, K. 1999, in prep.

Condon, J. J. 1992, ARA\&A, 30, 575

Fernández-Soto, A. ct al. 1999, ApJ, 513, 34

Fioc, M. \& Rocca-Volmerange, B. 1997, A\&A, 326, 950

Gordon, K., Calzetti, D., \& Witt, A. 1997 , ApJ, 487, 625

Gordon, K. \& Clayton, G. 1998, 500, 816

Kinney, A. et al. 1993, ApJS, 86, 5

Madau, P., Pozzetti, L., \& Dickinson, M. 1998. ApJ, 498, 106

Meurer, G. R., et al. 1999, ApJ, 519, 1

Misselt, K., Clayton, G., \& Gordon, K. 1999. ApJ, 515, 128

Steidel. C., et al. 1999, ApJ, in press (astro-ph/9811399)

Witt, A. N. \& Gordon, K. D. 1996, ApJ, 463, 681

Witt, A. N. \& Gordon, K. D. 1999, ApJ, in press 
The Hy Redshift Universe

ASP Conference Series, Vol. 193, 1999

A. J. Bunker \& W. J. M. van Breugel, eds.

\title{
A Serendipitous Search for Hy-Redshift Ly $\alpha$ Emission: A Case Study of Two Sources at $z \simeq 3$
}

\author{
Curtis Manning, Daniel Stern, Hyron Spinrad and Andrew J. Bunker
}

Astronomy Department, University of California, Berkeley, CA 94720

email: cmanning@astro.berkeley.edu

\begin{abstract}
In the course of our on-going search for serendipitous highredshift Ly $\alpha$ emission in deep archival Keck spectra, we discovered two Ly $\alpha$ emission line candidates in a moderate dispersion $(\lambda / \Delta \lambda \simeq 1200)$ spectrogram. Both lines have high equivalent width $\left(W_{\lambda}^{\text {obs }} \geq 450 \AA\right.$ ), low velocity dispersions $\left(\sigma_{v} \sim 60 \mathrm{~km} \mathrm{~s}^{-1}\right)$, and deconvolved effective radii $r_{e} \approx 1.0 h_{50}^{-1} \mathrm{kpc}$. Their sizes and luminosities are suggestive of the primeval galaxy model of Lin \& Murray (1992), based on the selfsimilar collapse of an isothermal sphere. We argue that the line emission is $\mathrm{Ly} \alpha$, and it is stellar in origin. The sources are consistent with being primeval.
\end{abstract}

\section{Introduction}

Strong Ly $\alpha$ line emission has been proposed as a possible signature of primeval galaxies. Though early modeling conjectured that these emission lines would be highly luminous but diffuse, searches on $4 \mathrm{~m}$-class telescopes found no such emission lines (Pritchet 1994; Thompson \& Djorgovski 1995). Deep spectra at the Keck telescopes, however, regularly reveal serendipitous high-equivalent width, isolated emission lines (e.g., Stern et al. 1999). Indeed, the first confirmed galaxy at $z>5$ was discovered in this manner (Dey et al. 1998). We report on two sources with strong line emission identified on a single slitlet from deep slitmask observations of the SSA22 field, with equivalent widths $W_{\lambda}^{\text {obs }} \geq 375 \AA$. The source targeted by this slitlet is a color-selected Lyman-break galaxy (LBG); we analyze it in parallel for comparison. We adopt $\left(\Omega, \Lambda . H_{0}\right)=(1,0,50)$.

\section{Observations and Results}

We have obtained deep, moderate-dispersion spectra of $z \simeq 3$ LBGs in the SSA 22 field with the aim of detailed studies of the ages, kinematics, dust-content, and abundances of the ISBG population (Dey et al., in preparation). The data were taken with the Low Resolution Imaging Spectrometer (Oke et al. 1995) at the Keck II telescope on UT 1997 September 10, using the 600 lines $\mathrm{mm}^{-1}$ grating blazed at $5000 \hat{A}$. Slitlet widths were $1^{\prime \prime} \cdot 25$, resilting in a spectral resolution of $\sim 4.4 \AA$ (FWHV). Fonr 1800 s exposures (seeing FWHM $\simeq 0^{\prime \prime} 78$ ) are analyzed in this report. The data were processed using standard slit spectroscopy procedures (for details, see Manning et al.. in preparation). 


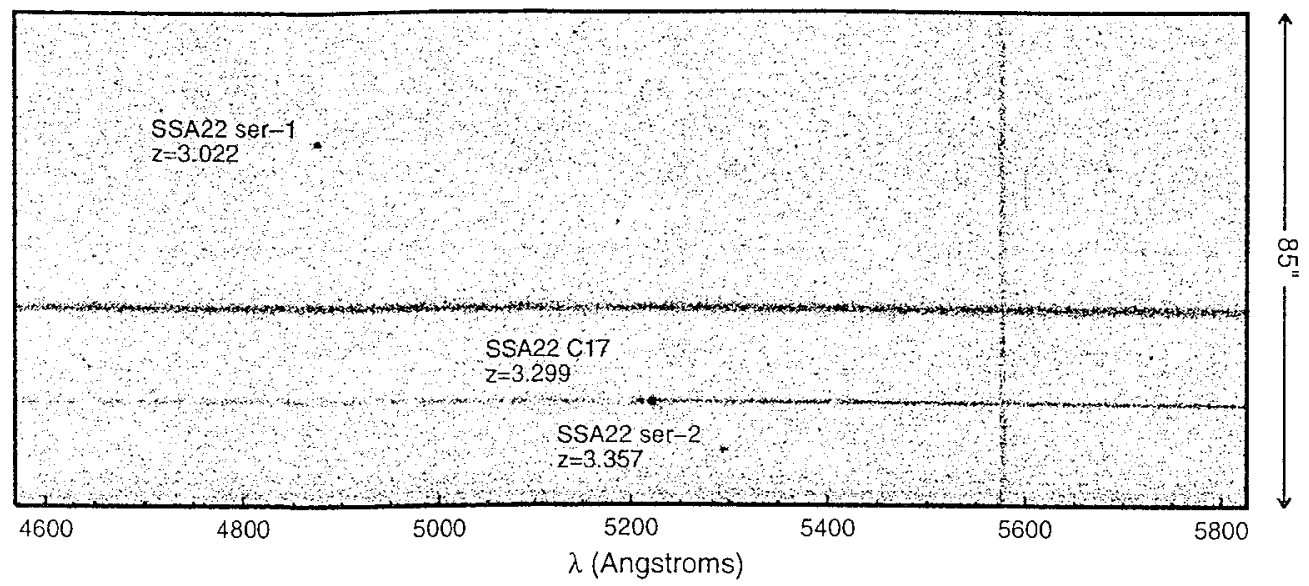

Figure 1. Two-dimensional spectrogram of slitlet in the SSA22 field targeting the LBG C17 at $z=3.299$. Two strong line emitters are serendipitously identified, ser-1 and ser-2. The high-equivalent widths, narrow velocity widths, and lack of secondary spectral features strongly argue that these are Ly $\alpha$ emitters at $z \simeq 3$. Note the foreground continuum source and residual of the $[\mathrm{O} \mathrm{r}] 5577 \mathrm{~A}$ skyline.

We find two serendipitous, isolated emission lines, ser-1 and ser-2, in a $100^{\prime \prime}$ long slitlet centered on the LBG, C1T ( $z=3.299$; see Fig. 1). Though only one of these serendipitously discovered sources (see Fig. 2) displays the obvious asymmetry characteristic of most high-redshift Ly $\alpha$ emission (e.g., Dey et al. 1398), we argue that Ly $\alpha$ is indeed the most likely interpretation for both. We performed a simulation of the $[\mathrm{O} \mathrm{II}]$ doublet under the $4.4 \AA$ resolution of our observations. We find that an [O $\mathrm{II}]$ identification is inconsistent with ser-1 and ser-2, since the doublet is marginally resolved and has significantly greater FWHM than our lines. The absence of associated emission argues against $\mathrm{H} \beta$, [O III] 4959 , or [O III] 5007 interpretations. Finally, the lines are shortward of $6563 \AA$, so $\mathrm{H} \alpha$ is not a viable identification. In the following, we assume what is most certainly the case - that these are in fact Ly $\alpha$ emission lines. Notably, neither spectrum displays perceptible continuum.

Measured continua were consistent with zero counts. We establish a $2 \sigma$ (95\% confidence) limit on the equivalent width, $W_{\lambda}^{\text {obs }} \geq 550 \AA$ (ser-1), and $\geq$ $474 \AA$ (ser-2). Rest values are tabulated in Table 1 . C17 has $W_{\lambda}^{\text {obs }}=35.6 \AA$.

Table 1. Emission Line Properties

\begin{tabular}{|c|c|c|c|c|c|c|c|c|}
\hline Object & $\lambda^{0 b_{5}}$ & $\mathrm{z}$ & $j-17$ & $L_{42}$ & $W_{i}^{\text {obs }}$ & $W_{i}^{\text {rest }}$ & $r_{e}$ & $\sigma_{v}$ \\
\hline $\begin{array}{ll}- \\
-\end{array}$ & $(\AA)$ & - & $\mathrm{cgs}$ & $\operatorname{cgs} s$ & $(\hat{A})$ & $(\hat{\AA})$ & $\left(h_{50}^{-1} \mathrm{kpc}\right)$ & $\left(\mathrm{kms}^{-1}\right)$ \\
\hline ser-1 & 4889.3 & 3.022 & 1.85 & 1.29 & $\geq 550$ & $\geq 137$ & $0.8 \%$ & 64 \\
\hline ser- 2 & 5296.8 & 3.357 & 1.35 & 1.19 & $\sum 474$ & $>109$ & 1.15 & 52 \\
\hline $\mathrm{C} 17$ & 5226.4 & 3.299 & 2.60 & 2.20 & $\overline{3} 5.6$ & 8.3 & 2.79 & 82 \\
\hline
\end{tabular}

Intrinsic source sizes are approximated by deconvolution of the observed spatial FWHM with the seeing, obtained from a quasar in an adjacent slitlet. 


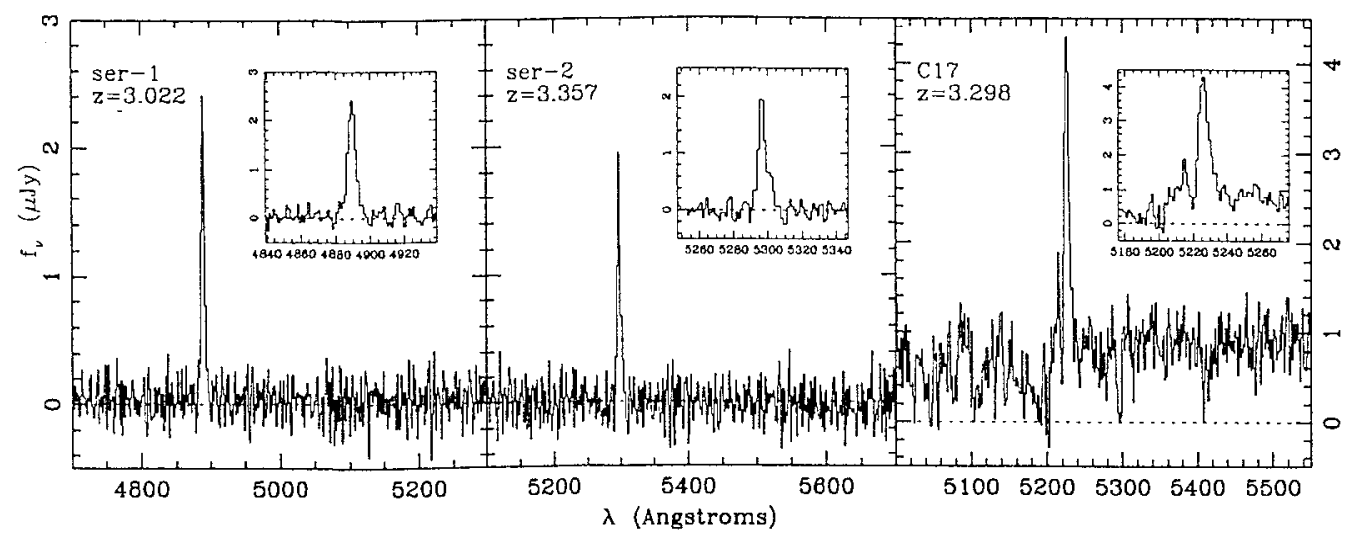

Figure 2. Extracted spectra of ser-1, ser-2 and the targeted LBG, C17. The ordinate on the left refers to the serendipitous sources, while the ordinate on the right refers to $\mathrm{C} 17$. Inserts illustrate the characteristic asymmetric Ly $\alpha$ profile in ser- 2 and $\mathrm{C} 17$.

The velocity widths have been deconvolved using the instrumental resolution, obtained from spectra of a NeAr lamp (see Table 1). The velocity dispersions of ser-2 and C17 are lower limits, as possible truncation of the blue-side of the emission line has not been accounted for.

\section{Discussion and Conclusions}

What is the physical origin of these isolated, high-equivalent width emission lines? The small velocity dispersions $\left(\sigma_{v} \simeq 60 \mathrm{~km} \mathrm{~s}^{-1}\right)$ and lack of associated $\mathrm{N} V$ or $\mathrm{C}$ IV emission argue against the presence of an $\mathrm{AGN}$, while the high surface brightness is inconsistent with photoionization by the metagalactic flux (e.g., Bunker et al. 1998). Thus we assume the lines are stellar in origin. Below we argue that they are primeval.

The starburst modeling of Charlot \& Fall (1993) suggest that the Ly $\alpha$ rest equivalent width may exceed $\sim 150 \AA$ for timescales of at most a few $\times 10^{7} \mathrm{yr}$. A model of primeval galaxies based on a collapsing isothermal cloud (Lin \& Murray 1992 ) predicts that primeval galaxies should have isophotal radii $r_{e} \sim 1 \mathrm{kpc}$, and luminosities of order $\sim \mathcal{L}^{*}$; strong star formation continues for $\sim 1.7 \mathrm{Gyr}$, fed by the continuing collapse of the cloud. Cloud mass may be tuned to fit observed luminosities. We suggest that this theory holds promise for the modeling of our serendipitous sources.

The Ly $\alpha$ luminosities imply lower limits to star formation rate (SFR) of $0.87 / 0.81 \mathrm{M}_{\odot} \mathrm{yr}^{-1}$ for ser-1/ser-2 (e.g., Dey et al. 1998), while non-detection of continua imply upper limits of $2.6 / 2.4 \mathrm{M}_{\odot} \mathrm{yr}^{-1}$. Assuming a SFR of $1 M_{\odot \mathrm{yr}^{-1}}$, and $W_{\lambda}^{\text {rest }}=150 \AA$; we project $V_{\mathrm{AB}} \simeq 28.0$ at $\approx=3$. Steidel et al. (1999) find a steep faint end slope for the luminosity function of star-forming galaxies at $\approx \sim 3(\alpha \approx-1.6)$, implying that a large fraction of the UV luminosity density is produced by galaxies fainter than the spectroscopic limits of photometric 
surveys. By virtue of the association of UV luminosity with SFR, this would also imply that a large fraction of the star formation density is unobserved. Because the Ly $\alpha$ lines of such as our serendipitous objects are likely to be observable only for a very small fraction of $1 \mathrm{Gyr}$, these two are tangible evidence for this manyfold larger population of slightly older, faint continuum, star forming galaxies with self-absorbed Ly $\alpha$, inaccessible to even the deepest spectroscopic or color selection surveys.

The evidence strongly suggests that ser- 1 and ser- 2 are primeval. In the near future, interesting conclusions may be possible regarding the degree of clustering and/or isolation of these low-luminosity emission line galaxies. The large surface density variance in Ly $\alpha$ emission line galaxy candidates between different fields in a coarse "narrow-band" ( $z \simeq 2.37$ ) HST study (Pascarelle et al. 1998), indicates that these compact galaxies are often at least loosly grouped. Clearly, the model of Lin \& Murray requires that primeval galaxies be born in relatively quiescent, yet gas-rich environments. However, as star-forming galaxies, their likely evolutionary antecedents, the LBGs, are often found clustered on large scales (e.g.. Steidel et al. 1998); perhaps they are protoclusters. The dynamics involved in the growth of large scale perturbations (i.e., infall and rising central densities) may be accompanied by a spreading "wave" (in the Lagrangian sense) of primeval galaxy formation, perhaps occurring at a characteristic overdensity - a scenario reminiscent of stellar "shell burning" - reproducing the insideout, older-to-younger galaxy age distribution seen in some recent studies of lower redshift fields and groups (e.g.. Loveday et al. 1999; Cohen et al. 1998).

Acknowledgments. We thank C. C. Steidel and A. Dey for useful discussions and assistance with the observations.

\section{References}

Bunker, A. J., Marleau, F. R., Graham, J. R. 1998, A.J, 116, 2086

Charlot, S.. \& Fall, S. M. 1993, Ap.J, 415, 580

Cohen, J., et al. 1998 submitted, ApJ, astro-ph/980906T

Dey, A. et al. 1998, ApJ, 498, L93

Hu, E. M., Cowie, L. L., \& McMahon, R. G., ApJ, 502, L99

Lin, D. N. C., \& Murray, S. D. 1992, ApJ, 394, 523

Loveday, J., Tresse, L., \& Maddox.S. J., 1999; astro-ph/9905386

Oke, J. B., et al. 1995, PASP, 107, 375

Pascarelle, S. M.. Windhorst, \& R. A., Ficcl, W. C. 1098, AJ, 116, 2659

Pritchet, C. J. 1994, PASP, 106, 1052

Steidel. C. C., et al. 1996, AJ, 112, 352

Steidel, C. C., et al. 1998, Ap.J, 492, 428

Steidel, C. C., et al. 1999, astro-ph/9811399

Stcrn, D., Bunker, A., Spinrad, H., \& Dey, A. 1909 Ap J, submitted

Thompson, D., Djorgovshi, S., \& Trauger, J. 1995, AJ, 110, 363 
The Hy Redshift Universe

ASP Conference Series, Vol. 193, 1999

A. J. Bunker \& W. J. M. van Breugel, eds.

\title{
The Halo Formation Rate and its Link to the Global Star Formation Rate
}

\author{
Will Percival, Lance Miller and Bill Ballinger \\ Astrophysics, Department of Physics, Keble Road, Oxford, OX1 $3 R H$
}

\begin{abstract}
The star formation history of the universe shows strong evolution with cosmological epoch. Although we know mergers between galaxies can cause luminous bursts of star formation, the relative importance of such mergers to the global star formation rate (SFR) is unknown. We present a simple analytic formula for the rate at which halos merge to form higher-mass systems, derived from Press-Schechter theory and confirmed by numerical simulations (for high halo masses). A comparison of the evolution in halo formation rate with the observed evolution in the global SFR indicates that the latter is largely driven by halo mergers at $\approx>1$. Recent numerical simulations by Kolatt et al. (1999) and Knebe \& Müller (1999) show how merging systems are strongly biased tracers of mass fluctuations, thereby explaining the strong clustering observed for Lyman-break galaxies without any need to assume that Lyman-break galaxies are associated only with the most massive systems at $z \sim 3$.
\end{abstract}

\section{Calculating the Halo Formation Rate}

In our analysis, a halo formation event is considered to have occurred when all the mass in the halo is assembled. Note that this is different to that previonsly used by some authors (Lacey \& Cole 1993). We wish to answer the question: Given a halo of mass $M$ forms at some time, what is the probability $P(t \mid M) \mathrm{dt}$ that it forms in the time interval $(\mathrm{t}, \mathrm{t}+\mathrm{dt})$ ?'

Standard PS theory calculates $P(M \mid t) d M$, the distribution of halo mass at fixed epoch, and we have shown that it is possible to calculate $P(t \mid M)$ dt from this using Bayes' theorem. We can also calculate the same formula using intrinsic properties of Brownian random walks involed in PS theory. The mass of halo a small mass element resides in at time $t$ is given by the first upcrossing of the line $\delta=\delta_{c}$ by a Brownian random walk in $\left(\delta, \sigma_{M}^{2}\right)$ space, where $\delta$ is a function of time and $\sigma_{M}^{2}$ is a function of mass. Lising the theory of random walks we can calculate the distribution of first upcrossing times at $\sigma_{M}^{2}, \mathrm{P}\left(\delta_{C} \mid \sigma_{M}^{2}\right)$, from which a simple change of variables can be used to obtain $P(t \mid M) d t$ :

$$
P(t \mid M) d t=\frac{\delta_{c}}{\sigma_{M}^{2}} \exp \left(-\frac{\delta_{c}^{2}}{2 \sigma_{M}^{2}}\right)\left|\frac{d \delta_{c}}{d t}\right| d t .
$$

This equation is in good agreement with Monte-Carlo realisations of Brownian random walks (Fig. 1). We have also run a large $\mathrm{N}$-body simulation. using 


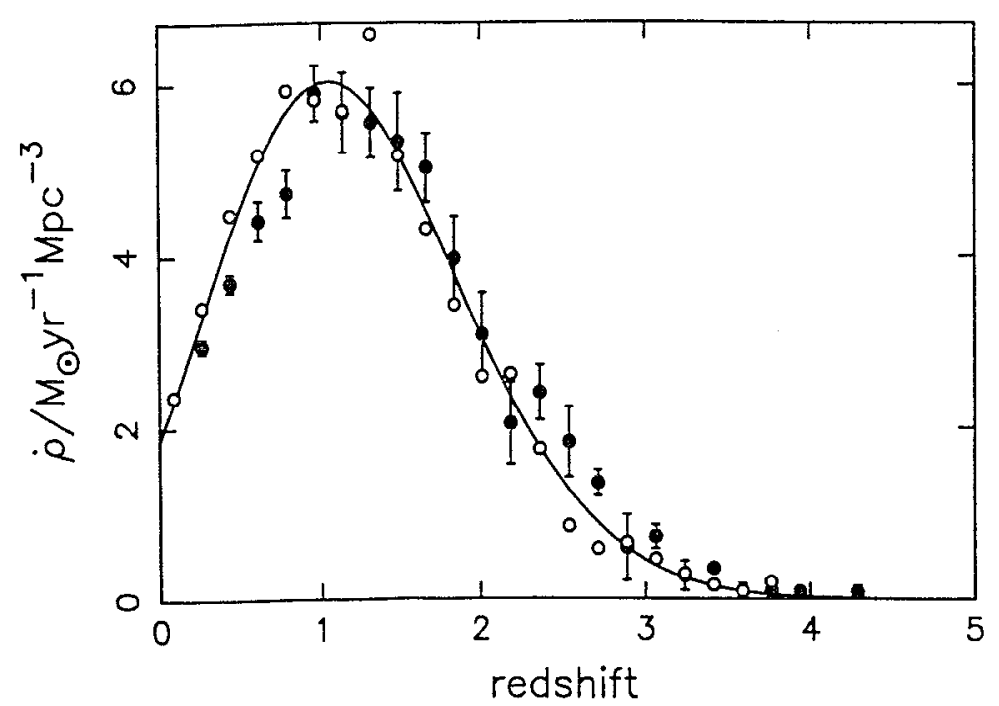

Figure 1. Comparison of N-body results (solid circles) with PressSchechter predictions of the halo formation rate for halos of mass $\sim$ $1.3 \times 10^{13} \mathrm{M}_{\odot}$. The curve shows the prediction of equation 1 at this mass with parameters as given in the text and normalised to the $\mathrm{N}$-body values. The distribution of $10^{4}$ 'formation events' at the required mass from Monte-Carlo realisations of random walks is also shown (open circles).

the Hydra N-body hydrodynamics code (Couchman, Thomas \& Pearce 1995). Groups of between 45 and 47 particles $\left(1.3 \times 10^{13} \mathrm{M}_{\odot}\right)$ were identified using a standard friends-of-friends algorithm at 362 output times. The number which could have formed in each time interval is compared to the expected distribution in Fig. 1.

Now suppose we are only interested in a subset of formation events - e.g. those which involve similar mass objects merging together. The formation rate from such mergers is the same as that derived above, because all walks which pass through a given point can be thought of as new walks starting from that point. Consequently the mass distribution of progenitors immediately prior to the formation event is independent of the formation epoch. Turning this argument around, placing constraints on the progenitors of halos immediately prior to their formation doesn't affect the distribution of formation times, although this might affect the bias (see later).

\section{The Star Formation Rate}

It is interesting to compare the halo formation rate with the observed global star formation rate. At $z<1$ the rate of halo formation falls off very rapidly with cosmic time, independently of halo mass, and it is likely that this effect is primarily responsible for the observed rapid evolution in the CFRS. Such 


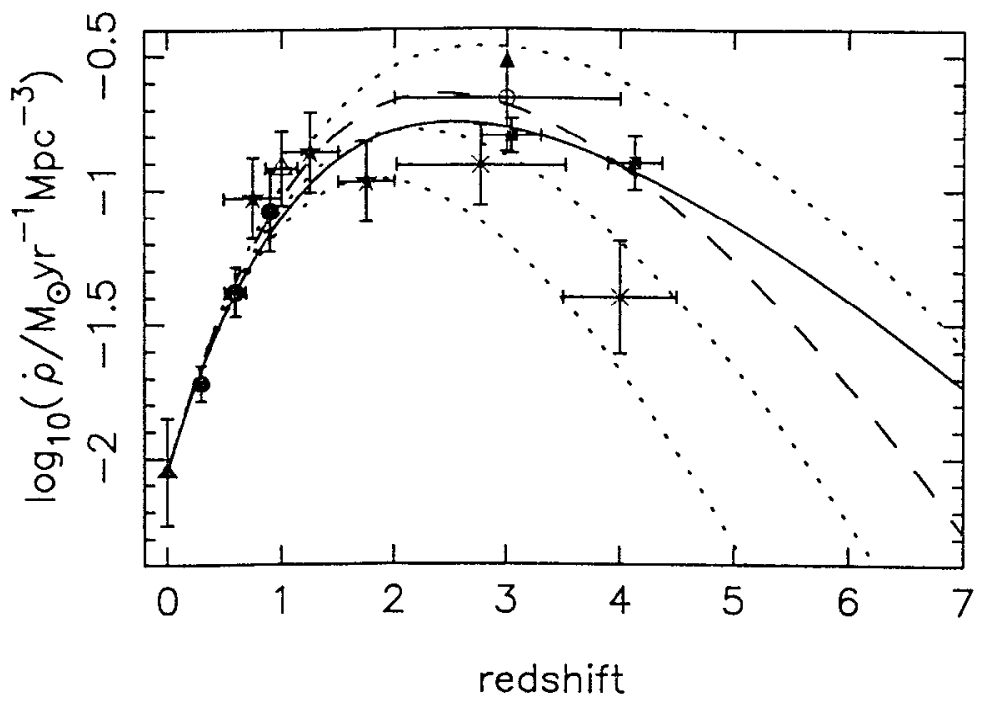

Figure 2. The observed mean comoving volume-averaged SFR as determined from the CFRS (Lilly et al. 1996) (solid circles), optical HDF data (Madau et al. 1996; Pettini et al. 1997) (crosses) and Connolly et al. (1997) (solid stars), extinction-corrected Lyman break galaxics (Steidel et al. 1998) (solid squares), sub-mm data (Hughes et al. 1998) (open circle), and $\mathrm{H} \alpha$ surveys (Gallego et al. 1995) (solid trianglc) and (Glazebrook et al. 1998) (open triangle). We assume a Salpeter IMF and flat $\Omega_{M}=1$ cosmology. Dotted lines are the predicted halo formation rates normalised to the local SFR (convolved with a nominal starburst lifetime of $0.6 \mathrm{Gyr}$, Bruzual et al. 1993) for masses of $10^{10.0}, 10^{11.0}, 10^{11.5} \mathrm{M}_{\odot}$ and for a mass of $10^{10.6} \mathrm{M}_{\odot}$ (dashed line). As an illustration, we combine the formation rate of different mass halos weighted by a Gaussian in dn/dlog M centred at a mass of $10^{10.6} \mathrm{M}_{\odot}$ (solid line). The low- $z$ evolntion is nnaffected, but an increasing contribution from lower-mass halos flattens the curve at high $\mathrm{z}$.

strong evolution is not seen in semi-analytic models that only include a quiescent component of star formation (Guiderdoni et al. 1998).

\section{The Clustering of Lyman-break Galaxies}

The strong clustering of Lyman-break galaxies has been explained as being dne to the high bias of the most massive overdensities, and consideration of the abundance of Iyman-break galaxies and their clustering leads to an interpretation of them as being associated with massive halos, $M \sim 8 \times 10^{11} h^{-1}-\mathrm{V}_{\odot}$ (Adelberger et al. 1998) for an $\Omega=0.3$ flat universe. If star formation is initiated after halo formation then these most massive halos form too late in the universe to reproduce the observed evolution in SFR in a simple way: we would require the efficiency of star-formation to evolve with redshift. Conversely, if the Lyman-break galaxies have lower mass but are associated with newly-formed. 
merging halos, then we might suppose that such mergers are also highly biased, and recent simulations indicate this to be the case (Kolatt et al. 1999, Knebe \& Vüller 1999).

\section{Conclusions}

We have presented the key points involved in deriving a simple formula for the rate of formation of new halos using Press-Schechter theory. It agrees with Monte-Carlo and I-body simulation results. We have argued that the strong cosmological evolution observed in the SFR is primarily driven by the cosmic variation in the rate of halo formation. Given that quiescent star formation does not provide enough evolution (Guiderdoni et al. 1998) we suggest that merger-induced starbursts are extremely important for star formation at $z \sim$ 1 and are perhaps the principal sites of the observed star formation at high redshifts. At high $z$, a more physically-motivated model is needed to decuce the relative contributions of a range of halo masses, but we have shown that a simple combination of such a range can produce evolution consistent with present data. Recent results indicate that such merging-halo systems are also sufficiently highly biased to explain the strong clustering of Lyman-break galaxies at $z \sim 3$.

The work highlighted here is more comprehensively covered in our recent paper available as astro-ph/9906204. We are also continuing to work on the bias of merging halos from numerical simulations.

\section{References}

Adelberger K., Steidel C., Giavalisco M., Dickinson M., Pettini M., Kellogg M., 1998, ApJ, 505, 18

Bruzual A. G., Charlot S., 1993, ApJ, 405, 538

Connolly A.J., Szalay A.S., Dickinson MI., SubbaRao M.V., Brunner R.J., 1997, ApJ, 486, L11

Couchman H. M. P., Thomas P.A., Pearce F.R., 1995, ApJ, 452, 797

Gallego J., Zamorano J., Aragon-Salamanca A., Rego M., 1995, Ap.J, 455, L1

Glazebrook K., Blake C., Economou F., Lilly S., Colless M., 1998, MNRAS submitted, astro-ph/9808276

Guiderdoni B., Hivon E., Bouchet F.R., Maffei B., 1998, MNRAS, 295, 877

Hughes D. et al., 1998, Nat, 394, 241

Kinebe, A. \& Müller, V., 1999. astro-ph/9809255

Kolatt T.S. et al., 1999. astro-ph/9906104

Lacey C., Cole S., 1993, MNRAS, 262.627

Lilly S.J.. Le Fevre O., Hammer F., Crampton D., 1996, Ap.J, 460, L1

Madau P., Pozzetti L., Dickinson M.. 1998, ApJ, 498, 106

Pettini M., Steidel C.C.. Adelberger K.L., Kellogg M., Dickinson M., Giavalisco M., 1997, 'Origins', Astron. Soc. Pacific Conference Series

Steidel C., Adelberger K., Giavalisco M., Dickinson M., Pettini M., 1998, ApJ submitted, astro-ph/9811399 
The Hy Redshift Universe

ASP Conference Series. Vol. 193, 1999

A. J. Bunker \& W. J. M. van Breugel, eds.

\title{
The Results From The NICMOS Parallel Imaging and Grism Survey
}

\author{
Lin Yan and Patrick McCarthy \\ The Carnegie Observatories, Pasadena, CA91108, email: lyan@ociw.edu
}

\begin{abstract}
We present the results of a strvey ntilizing the NICMOS Camera 3 Parallel grism and imaging observations. We have identified $33 \mathrm{H} \alpha$ emission-line galaxies at $0.75<z<1.9$. The inferred co-moving number density of these objects is $3.3 \times 10^{-4} h_{50}^{3} \mathrm{Mpc}^{-3}$, similar to that of the bright Lyman break objects at $z \sim 3$. The mean star formation rate of these galaxies is $21 \mathrm{M}_{\odot} \mathrm{yr}^{-1}$ for $\mathrm{H}_{0}=50 \mathrm{~km} / \mathrm{s} / \mathrm{Mpc}$. Using this sample, we derived the $H \alpha$ luminosity function $(\mathrm{LF})$ at $z-1.3$. The integrated $\mathrm{H} \alpha$ luminosity density at $z \sim 1.3$ is $1.64 \times 10^{40} \mathrm{~h}_{50}$ erg s $\mathrm{er}^{-1}$ $\mathrm{Mpc}^{-3}$, approximately 14 times greater than the local value reported by Gallego et al. (1995). The volume averaged star formation rate at $z=1.3 \pm 0.5$ is $0.13 \mathrm{M}_{\odot} \mathrm{yr}^{-1} \mathrm{Mpc}^{-3}$ without correction for extinction. The SFR derived at $\sim 6500 \AA$ is a factor of 3 higher than that deduced from $2800 \AA$ continua. We believe that this difference is largely due to dust extinction. The implied total extinction at $2800 \AA$ is in the range of $2-4$ magnitude. However, the precise determination of the total extinction is sensitive to the model assumptions.

Dcep ground-based VRI images of the NICMOS fields have revealed roughly a dozen of extremely red objects (EROs) with $\mathrm{R}-\mathrm{H}>5$ and $\mathrm{H}$ brighter than 20.6. The surface density of these objects is around 0.6 per square arcminutes.
\end{abstract}

\section{Introduction}

The NICMOS parallel imaging and grism observations were both made with Camera 3 with a field of view $\sim 52^{\prime \prime} \times 52^{\prime \prime}$. The imaging data were takcn with broad band filters F110W and F160W at $1.1 \mu$ ( $J$ band) and $1.6 \mu$ ( $\mathrm{H}$ band). The grism data has a spectral resolution of 200 per pixel and covers wavclength regions from $0.8 \mu$ to $1.2 \mu$ (G096) and $1.1 \mu$ to $1.9 \mu$ (G141). We have reduced and analysed the NIC3 parallel imaging data covcring $\sim 150 \mathrm{sq}$. arcminutes and the grism data in G141 grism $\sim 65 \mathrm{sq}$. arcminutes.

\section{Emission-line Galaxies and the $\mathbf{H} \alpha$ Luminosity Function at $z \sim 1.3$ from the NICMOS/HST Grism Parallel Observations}

The recent detections of dust enshrouded galaxies at $z>1$ at sub-millimeter wavelengths (Smail, Ivison \& Blain 1997; Hughes et al. 1998; Barger et al. 1998; Lilly et al. 1999) suggest that significant amounts of star formation activity at 
high redshifts may be obscured. Observations in the rest-frame UV wavelength suffer large uncertainties in extinction corrections. Furthermore, little is known about the properties of normal galaxies in the region between $1<z<2$, where neither the $4000 \mathrm{~A}$ break nor the Ly continuum break are easily accessible. The NICMOS data at the near-IR offers one means of accessing both redshift indicators and measures of star formation within this critical redshift range.

We have reduced and analysed the NIC3 parallel grism G141 data, covering $\sim 65 \mathrm{sq}$. arcminutes. The details of the data reduction can be found in McCarthy et al. (1999). We found a total of 33 emission line galaxies over an effective co-moving volume of $10^{5} h_{50}^{-3} \mathrm{Mpc}^{3}$ for $q_{0}=0.5$. The implied comoving number density of emission line galaxies in the range $0.75<z<1.9$ is $3.3 \times 10^{-4} h_{50}^{3} \mathrm{Mpc}^{-3}$, very similar to that of the bright Lyman break objects at $z \sim 3$. These objects have a median $\mathrm{H} \alpha$ luminosity of $2.7 \times 10^{42} \mathrm{erg} \mathrm{sec}^{-1}$. The most, if not all, of the emission lines detected are either $\mathrm{H} \alpha$ or unresolved blend of $\mathrm{H} \alpha+[\mathrm{NII}] 6583 / 6548$. This identification is mostly based on $\mathrm{H}$-band apparent magnitudes (median value of 20.5), the emission line equivalent widths and the lack of other detected lines within the G141 bandpass. The redshifts of 6 galaxies in our sample have been confirmed by detection of [OII] 3727 emission in the optical spectra using LRIS on the Keck 10m telescope (Teplitz et al. 1999; Nalkan et al. 1999). Figure 1 shows the spectra for a subset of galaxies in our sample.

We compute the $\mathrm{H} \alpha$ luminosity function (LF) based on this sample of emission-line galaxies. All of the detailed results are in Yan et al. (1999). Figure 2 shows our derived $\mathrm{H} \alpha \mathrm{LF}$ at $z=1.3$ and the local $\mathrm{H} \alpha \mathrm{LF}$ as measured by Gallego et al. (1995). This plot shows strong evolution in the $\mathrm{H} \alpha$ luminosity density from $z \sim 0$ to $z \sim 1.3$. This is no surprise given the evolution in the ultraviolet luminosity density, but our result provides an independent measure of evolution for $\mathrm{H} \alpha$ emission alone. The LF is well fit by a Schechter function over the range $6 \times 10^{41}<\mathrm{L}(H \alpha)<2 \times 10^{43} \mathrm{erg} \mathrm{sec}^{-1}$ with $\mathrm{L}^{*}=7 \times 10^{42} \mathrm{erg}$ $\mathrm{sec}^{-1}$ and $\phi^{*}=1.7 \times 10^{-3} \mathrm{Mpc}^{-3}$ for $\mathrm{H}_{0}=50 \mathrm{~km} \mathrm{~s}^{-1} \mathrm{Mpc}^{-1}$ and $\mathrm{q}_{0}=0.5$. The integrated $\mathrm{H} \alpha$ luminosity density at $z \sim 1.3$ (our median $z$ ) is $1.64 \times 10^{40} \mathrm{~h}_{50}$ erg $s^{-1} \mathrm{Mpc}^{-3}, \sim 14$ times greater than the local value reported by Gallego et al. (1995).

We compute the star formation rates from the integrated $\mathrm{H} \alpha$ and the LV continuum luminosity densities using the relations from Kennicutt (1999): $\operatorname{SFR}\left(\mathrm{M}_{\odot \mathrm{yr}^{-1}}\right)=7.9 \times 10^{-42} \mathrm{~L}(\mathrm{H} \alpha)\left(\mathrm{erg} \mathrm{s}^{-1}\right)$ and. $\operatorname{SFR}\left(\mathrm{M}_{\odot \mathrm{yr}}{ }^{-1}\right)=1.4 \times 10^{-28} \mathrm{~L}(1500-$ $2800 \AA)\left(\mathrm{erg} \mathrm{s}^{-1} \mathrm{~Hz}^{-1}\right)$. In Figure 3, we plot uncorrected published measurements of the volume-averaged global star formation rate at various epochs. The star formation rates shown in Figure 3 are calculated from the luminosity densities integrated over the entire luminosity functions, for both $\mathrm{H} \alpha$ and the UV continuum. The clear trend for the longer wavelength determinations of the star formation rate to exceed those based on UV continua is one of the pieces of evidence for significant extinction at intermediate and high redshifts. The amplitude of the extinction correction is quite uncertain Our measurement spans $0.7<z<1.9$, overlapping with the Connolly et al. photometric redshift sample and allowing a direct comparison between the observed $2800 \AA$ luminosity density and that inferred from $\mathrm{H} \alpha$. Our $\mathrm{H} \alpha$-based star formation rate is three times 

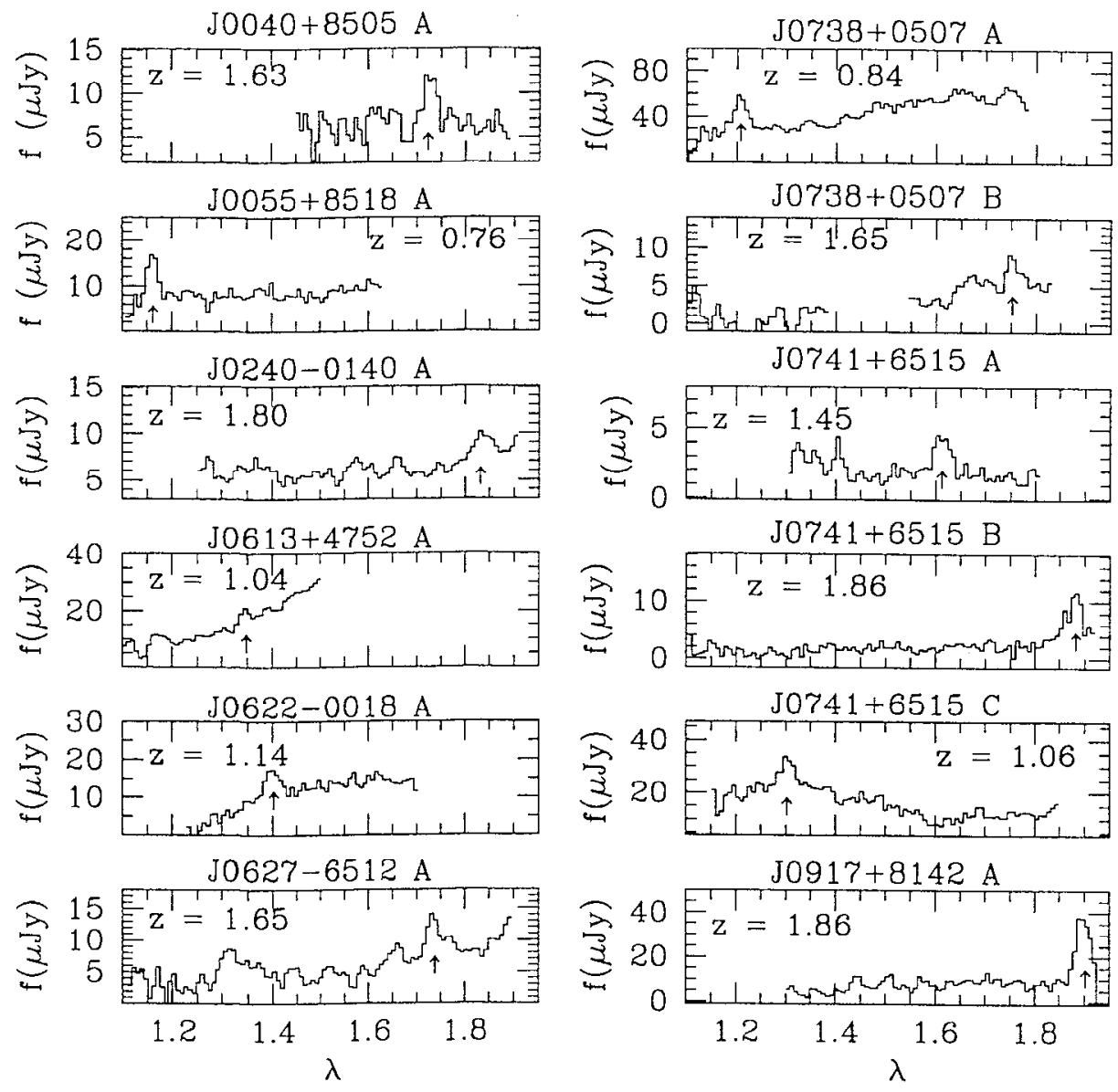

Figure 1. 1 D spectra of a subset of $33 \mathrm{H} \alpha$ emission linc galaxies. For each object we have marked our candidate emission-lines with arrows below the line. The redshifts, assuming an $\mathrm{H} \alpha$ identification for the line are given for each object. 

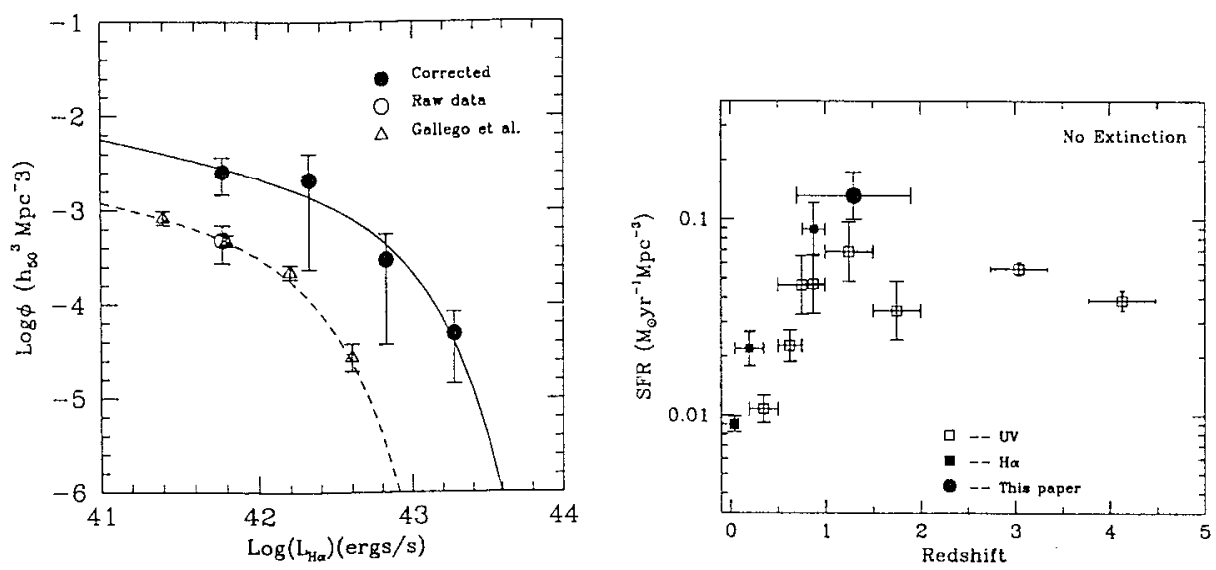

Figure 2. Left: $H \alpha$ luminosity function at $0.7<\tilde{z}<1.9$. The open circles represent our raw data and the filled circles are our points corrected for incompleteness. The open triangles show the local $\mathrm{H} \alpha$ luminosity function by Gallego et al. (1995). The solid and dashed lines are the best fits to the data at $\approx \sim 1.3$ and $z \sim 0$ respectively. Right: The global volume-averaged star formation rate as a function of redshift without any dust extinction correction. The oper squares represent measurements of the $2800 \AA$ or $1500 \AA$ continuum luminosity density by Lilly et al. (1996), Connolly et al. (1997) and Steidel et al. (1998), whereas the filled squares are the measurements using $\mathrm{H} \alpha 6563 \AA$ by Gallego et al. (1995), Tresse \& Maddox (1996) and Glazebrook et al. (1998). Our result is shown in the filled circle. 
larger than the average of the three redshift bins measured by Connolly et al. (1997).

If we attribute the entire difference to reddening, the total extinction corrections at $2800 \mathrm{~A}$ and $\mathrm{H} \alpha$ are large and model-dependent. The calculation is sensitive to the relative geometry of the stars, gas and dust, as well as the adopted reddening curve. In the extreme case of a homogeneous foreground screen and a MW or LMC reddening curve, we derive $A_{2800}=2.1$ magnitudes. If we adopt the Calzetti reddening law (Calzetti 1997), where stars, gas and dust are well mixed, onr estimate of the dust extinction at $2800 \mathrm{~A}$ is one to two magnitudes larger than in the simple screen case.

\section{Extremely Red Objects (EROs)}

We have obtained deep ground-based optical images of 27 NIC3 fields, yielding $\sim 20$ square arcminutes. Among these fields, we have identified abont a dozen of EROs with $\mathrm{R}-\mathrm{H}>5$ and $\mathrm{H}$ brighter than 20.6. The surface density of EROs with $\mathrm{H}<20.6$ and $\mathrm{R}-\mathrm{H}>5$ is roughly 0.6 per sq. arcminutes. We also found some evidence that EROs are highly clustered. Among 27 NIC3 fields, we found 2 clusters of EROs. The details of this work can be found in Yan et al. (1999).

\section{References}

Barger, A.J., Cowie, L.L., Sanders, D.B., et al. 1998, Nature, 394, 248

Connolly, A., Szalay, A. S., Dickinson, M., et al. 1997, ApJ, 487, L13

Calzetti, D., Kinney, A.L. \& Storchi-Bergmann, T. 1994, ApJ, 429, 582

Gallego, J., Zamorano, J., Aragon-Salamanca, A., Rego, M. 1995, ApJL, 459, 1

Hughes, D., et al. 1998, Nature, 394, 241.

Kennicutt, R.C. 1999, ARAA. in press. astro-ph/9807187

Lilly, S.J., Le Fevre, O., Hammer, F. \& Crampton, D. 1996, Ap.J, 460, L1

Lilly, S.J., Eales, S.A., et al. 1999, Ap.J, in press. astro-ph/9901047

McCarthy, P., Yan, L., et al. 1999, ApJ, august issue, in press, astro-ph/9902347

Steidel C., Dickinson, M., et al. 1998, ApJ, in press. astro-ph/9811399

Teplitz, H., et al. 1999, in prep.

Tresse, L., Maddox, S. 1998, ApJ, 495, 691

Yan, L. McCarthy, P., et al. 1999, ApJ, 519, 47

Yan, L. McCarthy, P. et al. 1999, ApJ, in prep. 

Section G. Formation of the First Objects 

The Hy Redshift Universe

ASP Conference Series, Vol. 193, 1999

A. J. Bunker \&g W. J. M. van Breugel, eds.

\title{
Galaxies at High Redshift $-1999^{1}$
}

\author{
Hyron Spinrad \\ Department of Astronomy, U.C. Berkeley
}

\begin{abstract}
We characterize the normal galaxies found recently at $z>4$ and in particular discuss the techniques used for their location. These most distant galaxies are found through photometric redshifts from broadband imaging, Ly- $\alpha$ emission-line searches with narrow-band filters, and by serendipity on long-exposure multislit spectrograms. We derive typical (un-obscured) SFR $\sim 10-15 \mathrm{M}_{\odot} \mathrm{yr}^{-1}$ from their rest-UV continua and Ly- $\alpha$ emission-lines.

The $z>4$ systems are often small, and fairly frequently show multi ple nuclei with kpc spacings - likely merger signs.

The star formation rate (SFR) density we derive from the spectra and the extant photometric redshifts at $z=5 \pm 0.5$ suggest a roughly constant co-moving SFR density from $z=3$ to $z \sim 5$.

Finally we speculate on several future observations and techniques which may lead to the detection of star forming galaxies to $z \sim 10$, entering the 'dark ages'.
\end{abstract}

\section{Introduction}

In this symposium which recognizes the extragalactic research efforts of my students, my post-docs and myself it seems appropriate to discuss the usual low $\mathrm{S} / \mathrm{N}$ spectra (and to some extent the better quality HST images) of the faint galaxies now safely measured to $z>4$ ( lately to at least $z=5.7$ ).

Until recently exploring non-AGN galaxies at $\approx>3$ seemed very difficult indeed; however the pioneering photometric research of Steidel \& Hamilton (1992) defined the class of Lyman-break galaxies as those with fairly active SF rates (to flatten their continua in $f_{\nu}$ ) and large enough redshift to place the Lyman limit well into the photometric $U$ band.

Following this lead. Cowie \& $\mathrm{Hu}$ (1998) and Hu, Cowie \& McMahon (1998) pushed their narrow-band imaging deep Hawaii fields sufficiently far down the emission-line luminosity function to finally extract a fairly high surface density of Lya emitters at $z>3$ ( and now $z=5.7$ ).

We are still using these techniques, or modest variants of them plus an eye toward "Serendipitous Discovery", to uncover and cventually study galaxies at $\approx>4$.

\footnotetext{
${ }^{2}$ Based in large part on observations made at the $W$. M. Keck Observatory.
} 


\section{Some Recent Spectroscopic Results}

A synthesis of these observations and recent work by Steidel et al. (1999) and by Manning et al. (1999 - this volume) suggests that the observed normal distant galaxies are generally small in angular and linear isophotal size - with diameters of only a few kpc. They seem to show a $L_{1500 \mathrm{~A}}$ luminosity function not too different from that derived for present day galaxics. They are actively forming new, massive stars - although clearly we are biased against even detecting those systems which have not partaken in active SF over the past $5 \times 10^{8}-1 \times 10^{9}$ years. The creation of a modest dust supply can easily absorb the scattered Lya line; thus we assume that if a starburst lasts for $>10^{9} \mathrm{yrs}$, that region of a young galaxy might not show Ly- $\alpha$ in emission - it could even have a localized 'damped' absorption line.

For the $z>4$ galaxies with photometric redshifts in the Hubble Deep Field HDF, Williams et al. 1996) we have been successful in obtaining spectroscopic redshifts to $R$ or $I=25^{m}$ (AB) with LRIS on the Keck telescopes. We find good spectroscopic accord with the photometric redshift predictions of Fernández-Soto et al. (1999).

Even without a strong Ly- $\alpha$ emission line we can determine robust redshifts for $I_{A B}^{814}=25^{m}$ galaxies. Figure 1 illustrates the "spectral dominance" of the strong $L y-\alpha$ and $\operatorname{Ly} \beta$ absorption 'edges' very near or at the systemic velocity of each galaxy. The spectrogram of HDF 4-473 (not shown here) does not have a continuum detection in its $\sim 5 \mathrm{hr}$ integration; this rcdshift is entirely based upon the strong Ly- $\alpha$ emission line and the extant NICMIOS and WF photometry (c.f., Weymann et al. 1998).

Note the amplitude of the $\mathrm{Ly}-\alpha$ and $L y-\beta$ discontinuities and the lack of measurable flux below rest wavelcngth $\lambda_{\text {rest }}=912 \AA$ (the Lyman limit). These three features show well in the spectrum of HDF 4-439, as does the strongest of the UV ISM absorptions, the Si II doublet at $1260 \AA$ (rest).

Can we do as well with the emission-line candidates and the serendipitous galaxies? The answers are also positive for these search techniques; Hu, Cowie \& McMahon in particular have been successful in locating and (to some degree) spectroscopically validating the strong cmission lines with the 'hoped-for' Ly$\alpha$ identifications. They used filters which transmit well only in $\mathrm{OH}$ sky band windows, corresponding to $z=3.4,4.5$ and now 5.7 (see Esther Hu's paper in these proceedings). The surface density of $z=4.5$ galaxies (assuming that $3 / 4$ of the candidates really show a $\mathrm{Ly}-\alpha$ line rather the common [O II] alternate) is $\sim 3 \operatorname{arcmin}^{-2}$, a bit higher than the surface density of $I<26.5^{m} z=5$ galaxies selected photometrically in the HDF (roughly a density of 1.2 there). Of course the narrow band emitters might be fainter in their $I$-band continua than our (arbitrary) $I=26.5^{m}$ limit.

The serendips at high redshift represent a novel and rich method to locate $\ddot{z}>4$ galaxies, especially those with moderate or strong Ly- $\alpha$ emission lines. Our first distant galaxy success at $z>5$ was discovered serendipitously by Dey et al. (1998); since then our UCB team has located three galaxies with $z 4.9$, one at $z=5.64$, a probable at $z=5.7$ and perhaps-one new serendip at $z=6.2$ if the emission line observed at $8787 \AA$ is really. $\mathrm{L} y-\alpha$ (the $[\mathrm{O}$ II] altermative would be $\tilde{i}=1.36$ ). 
In most cases the strength of the Ly- $\alpha$ line is consistent with an unobscured $\mathrm{SFR}$ of $10-15 \mathrm{M}_{\odot} \mathrm{yr}^{-1}$; the $1500 \AA$ (rest-frame) continuum also yields that level of SF. (c.f., Dey et al. 1998). Of course some dust may reduce both the Ly- $\alpha$ line and the ultraviolet continuum, as suggested by Pettini et al.

One the other hand, more spectacular and speculative case of a serendipitous galaxy discovery has been presented by Chen et al. (1999). These authors used the HST and STIS to obtain a deep slitless spectrogram near the HDF; the galaxy ' $A$ ' while quite small and faint, appears to show a probable Ly- $\alpha$ line with its associated redward continuum at $z=6.68$. However the observation is difficult and a confirmation by an independent group or a different technique would seem advisable.

Our serendips at high redshift are sufficiently numerous as to suggest a measure of their surface density. We find the surface density at approximate $I_{A B}<26.5^{m}$ and $4.5<z<5.5$ to be $2 \pm 1 \operatorname{arcmin}^{-2}$ (Stern \& Spinrad 1999). This leads to a remarkably high co-moving star formation rate density at $z=$ 5 , if the individual SFR in all the serendip galaxies (or the average one) is $\sim 10-15 M_{\odot} \mathrm{yr}^{-1}$ ! That would be the same (unobscured) individual galaxy SFR found by Dickinson (1998) and this then gives a density of SFR at $z=5$ similar to the Madau et al. (1998) and the Steidel et al. (1999) rate at $z=3$. There is no substantial decline.

\section{Morphologies of Some Distant Galaxies}

Of our six HDF galaxies with spectroscopic and photometric $z>4$ at least two show signs of recent likely merger events in their $I_{814}$ WFPC 2 images.

Most interesting is the 'double-core' of $\operatorname{HDF} 4-625(z=4.58)$ which has two distinct components, easily separated visually when one exams the WF $I_{814}$ image with an appropriate display stretch [see Fig. 2]. The two components are separated by $0^{\prime \prime} 17$; this corresponds to a $1.0 \mathrm{kpc}$ distance (in projection) for a $H_{0}=50 \mathrm{~km} \mathrm{~s}^{-1} \mathrm{Mpc}^{-1}, q_{0}=0.5$ cosmology. The companion nucleus is about 3.5 times fainter than the primary. This composite galaxy image seems to represent an excellent case for a merger seen late in its evolution; the picture seems roughly consistent with the theme of a recent paper by Kolatt et al. (1999) who used semi-analytic models to replicate young merging galaxies.

The picture for the HDF 3-951 (Spinrad et al. 1998) is very similar; there are three close objects, with the brightest nucleus a double again with $\sim 1 \mathrm{kpc}$ separation of the pair of nuclei. Interestingly neither galaxy (pair) shows strong Ly- $\alpha$ emission during these merger events when perhaps shock activity would be anticipated.

\section{Some Future Hopes/ Plans with Different Techniques}

a) Infrared spectroscopy of $z \approx 3$ galaxies has already proven quite useful for allowing us to observe diagnostics not available with the emitted ultraviolet region (c.f.. Pettini et al. 1998).

But it should be possible to push ahead to even fainter. more distant systems-perhaps at $z>0$. I speculate on the chance of detecting the Ly- $\alpha$ 
line and perhaps a large continuum discontinuity due to the Ly- $\alpha$ forest (and perhaps an additional Gunn-Peterson trough) through $J$ - and $H$-band spectroscopy with a 10-m class telescope and IR spectrometers like NIRSPEC at Keck II.

The first trials of NIRSPEC in 1999 suggest that an unresolved galaxy Ly- $\alpha$ line could be measured with a $S / N$ ratio $=5$ for a line of $f$ ux $10^{-17} \mathrm{ergs} \mathrm{cm}^{-2} \mathrm{~s}^{-1}$ at $1.2 \mu \mathrm{m}(z=9)$ in one hour. A more realistic case of a resolved galaxy and a spectrally resolved emission line would likely require $\sim 4 \mathrm{hr}$ in good conditions to observe the emission line profile; mere detection would be easier. A continuumbreak redshift, caused by the Ly- $\alpha$ forest and a putative Gunn-Peterson trough might be observed at $J$ band for $z=9.0(\lambda=1.216 \mu \mathrm{m})$. That would only yield a $S / N \sim 0.8$ per resolution element in $\sim 10^{4}$ secs of integration, for a hypothetical continuum level of $0.5 \mu \mathrm{Jy}$ redward of Ly- $\alpha$. Time may test this speculation.

b) The detection of the $\lambda=158 \mu \mathrm{m}$ [C II] cooling line at $z>6$ is likely to be possible early in the next millennium. This cool-gas emission line is observable in considerable strength in local SF regions; when the line redshifted sufficiently $(z>5.3$ to $z=11.6$ ) it will be observed in the $1 \mathrm{~mm}$ to $2 \mathrm{~mm}$ region where its accessible to $\mathrm{mm}$-interferometers and its detection will probably result in some rather useful line profile examples as well as the obvious value in either confirming or initiating a large redshift measurement.

Acknowledgments. I would like to thank the meeting's scientific and local organizers for an exciting Astronomical experience and several days of pleasant memories.

In particulas I thank Ivan King, Dan Stern, and Andy Bunker!

James Graham is thanked for NIRSPEC discussions. Some of this research has been supported by the NSF and by NASA (through HST grants).

\section{References}

Chen, H.W., Lanzetta, K.M., \& Pascarelle, S. 1999, Nature, 398, 586

Cowie, L.L., \& Hu, E.M. 1998, AJ, 115, 1319

Dey, A., et al. 1998, Ap.J, 498, L93

Dickinson, M.E. 1998, in "Hubble Deep Field", eds. M. Livio, S.M. Fall, \& P. Madau (Cambridge Univ. Press), p. 210

Fernandez-Soto, A., Lanzetta, K.M., \& Yahil, A. 1999, Ap.J, 513, 34

Hu, E.M., Cowie, L.L., and McMahon, R.G. 1998, ApJ, 502. L99

Kolatt, T.S., et al. 1999, MNRAS(in press) and astro-ph/9906104

Madau, P., et al. 1998, ApJ, 498, 106

Manning, C., et al. 1999 (this volume)

Pettini, M., et al. 1998. ApJ, 508, 539

Spinrad, H., et al. 1998, AJ, 116, 2617

Steidel, C.C, \& Hamilton. D. 1992, AJ, 104, 941

Steidel, C.C., et al. 1999, ApJ(in press) and astro-ph/981216

Stern. D., \& Spinrad, H. 1999, Publ. A.S.P. (in press) 


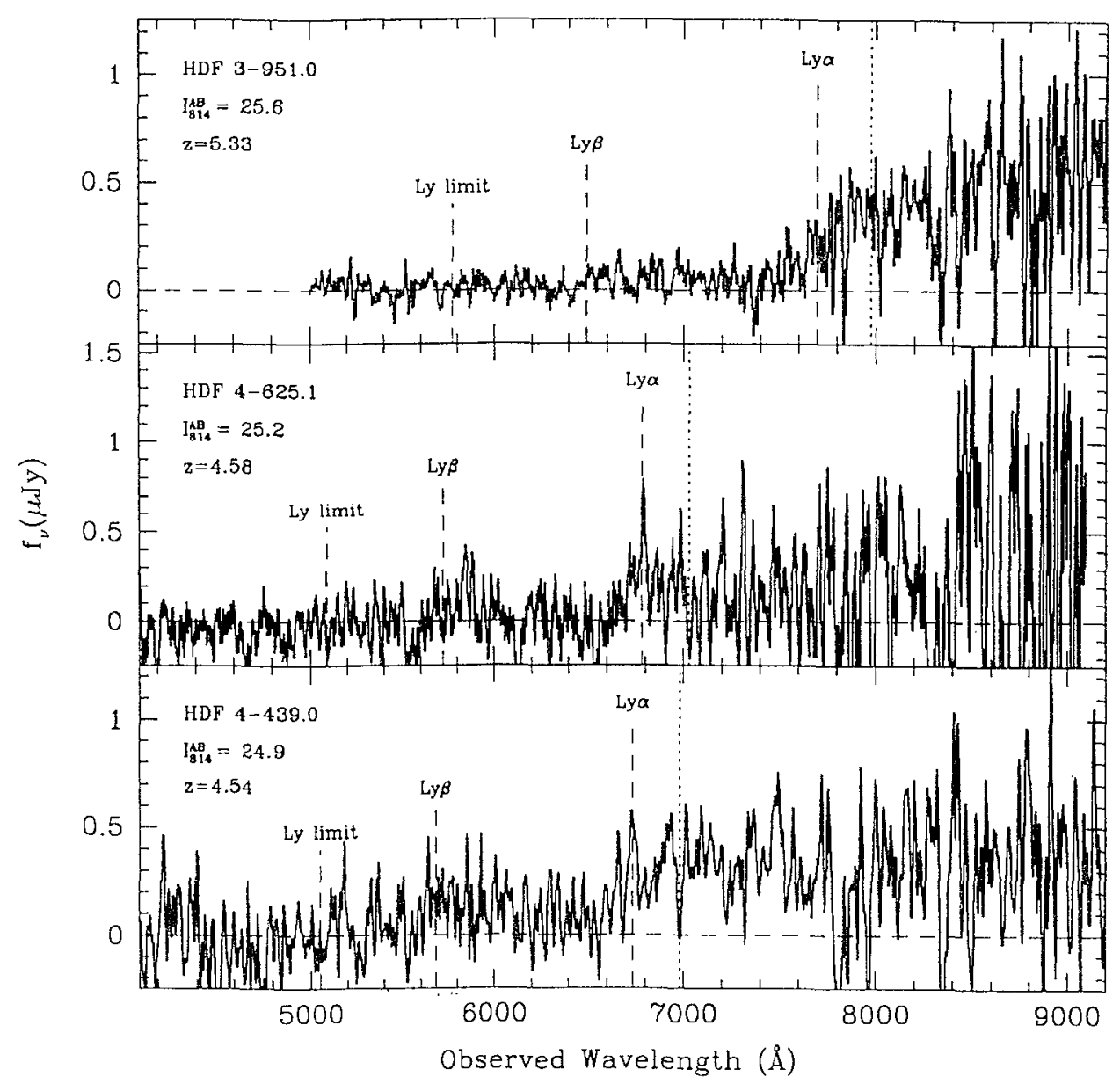

Figure 1. TRIS spectra of the $z>4$ galaxies photometrically selected in the HDF: note the discontinuities at the prominent Lyman-series lines and at the Lyman-limit (for HDF 4-439). The right hand dotted vertical lines indicate the position of the Si II 1260 Alend. The spectra represent long integrations from multiple observing scasons. 

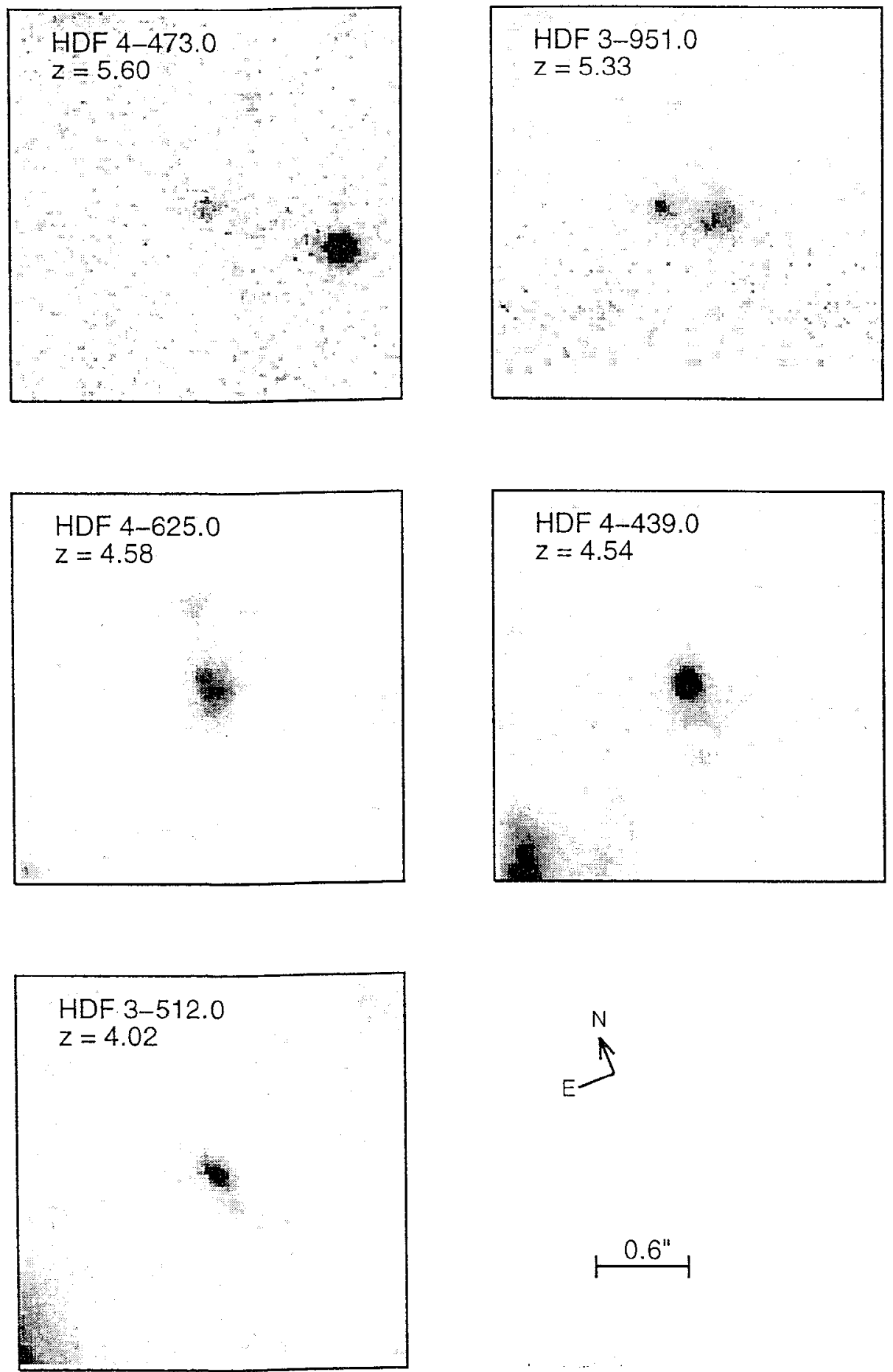

Figure 2. Images of $z>4$ HDF galaxies as seen in the $I_{814}$ band. Note the multiple nuclei of HDF 3-951 and HDF 4-625 and the tidaltails' possible for HDF 3-512 and HDF 4-439. Presumably both morphologies are result of previous close encounters or mergers. 
Weymann, R., et al. 1998, ApJ, 505, L95

Williams, R.E., Blacker, B., Dickinson, M., Dixon, W.V.D., Ferguson, H.C., - Levay, Z., Lucas, R.A., Mcelroy, D.B., Petro, L., \& Postman, M. 1996. AJ, 112,1335 

The Hy Redshift Universe

ASP Conference Series, Vol. 193, 1999

A. J. Bunker \& W. J. M. van Breugel, eds.

\title{
Galaxies of Redshift $z>5$ : The View from Stony Brook
}

\author{
Kenneth M. Lanzetta ${ }^{1}$,Hsiao-Wen Chen ${ }^{1}$, Alberto Fernández-Soto ${ }^{2}$, \\ Sebastian Pascarelle ${ }^{1}$, Noriaki Yahata ${ }^{1}$ and Amos Yahil ${ }^{1}$
}

\begin{abstract}
We report on some aspects of our efforts to establish properties of the extremely faint galaxy population by applying our photometric redshift technique to the HDF and HDF-S WFPC2 and NICMOS fields. We find that cosmological surface brightness dimming effects play a dominant role in setting what is observed at redshifts $\approx>2$, that the comoving number density of high intrinsic surface brightness regions increases monotonically with increasing redshift, and that previous estimates neglect a significant or dominant fraction of the ultraviolet luminosity density of the universe due to surface brightness effects. The ultraviolet luminosity density of the universe plausibly increases monotonically with increasing redshift to redshifts beyond $z=5$.
\end{abstract}

\section{Introduction}

We have over the past few years applied our photometric redshift technique to the Hubble Deep Field (HDF) and Hubble Deep Field South (HDF-S) WFPC2 and NICMOS fields (e.g. Lanzetta, Yahil, \& Fernández-Soto 1996, 1998; FernándezSoto, Lanzetta, \& Yahil 1999; Yahata et al. 2000). Our objective is to establish properties of the extremely faint galaxy population by identifying galaxies that are too faint to be spectroscopically identified by even the largest ground-based telescopes. Our experiences indicate that photometric redshift measurements are at least as robust and reliable as spectroscopic redshift measurements (and probably significantly more so). Specifically, comparison of photometric and reliable spectroscopic measurements in the HDF and HDF-S fields demonstrates that the photometric redshift measurements are accurate to within an RMS relative uncertainty of $\Delta z(1+z) \leqslant 10 \%$ and that there are no known examples of photometric redshift measurements that are in error by more than a few times the RMS uncertainty. These results apply at all redshifts $z<6$ that have yet been examined. It thus appears that the photometric redshift technique provides a means of obtaining redshift identifications of large samples of the faintest galaxies to the largest redshifts.

Here we report on some aspects of our efforts. Highlights of the results include the following:

\footnotetext{
'Department of Physics and Astronomy, State University of New York at Stony Brook. Stony Brook, NY 11794-3800, U.S.A.

2Department of Astrophysics and Optics, School of Physics, University of New South Wales, Kensington-Sydney, NSW 2052. AUSTRALIA
} 
1. We have identified nearly 3000 faint galaxies, of which nearly 1000 galaxies are of redshift $\approx>2$ and more than 50 galaxies are of redshift $z>5$ (ranging up to and beyond $z=10$ ). Further, we have fully characterized the survey area versus depth relationships, in terms of both energy flux density and surface brightness, in order to measure statistical properties of the very high redshift galaxy population.

2. We find that cosmological surface brightness dimming effects play a dominant role in setting what is observed at redshifts $z>2$. Most importantly, we find that it is more or less meaningless to interpret the galaxy luminosity function (or its moments) at high redslifis without explicitly taking account of surface brightness effects.

3. We find that the comoving number density of high intrinsic surface brightness regions (or in other words of high star formation rate density regions) increases monotonically with increasing redshift.

4. We find that previous estimates neglect a significant of dominant fraction of the ultraviolet luminosity density of the universe due to surface brightness effects and that the rest-frame ultraviolet luminosity density (or equivalently the cosmic star formation rate density) has not yet been measured at redshifts $z \gtrsim 2$. The ultraviolet luminosity density of the universe plausibly increases monotonically with increasing redshift to redshifts beyond $z=5$.

The most recent versions of our photometry and redshift catalogs of faint galaxies in the HDF and HDF-S fields can be found on our web site at:

$$
\text { http://ww.ess.sunysb.edu/astro/hdfs/. }
$$

Here and throughout we adopt a standard Friedmann cosmological model of dimensionless Hubble constant $h=H_{0} /\left(100 \mathrm{~km} \mathrm{~s}^{-1} \mathrm{Mpc}^{-1}\right)$ and deceleration parameter $q_{0}=0.5$.

\section{Observations and Analysis}

Our current observations and analysis differ from our previous observations and analysis in three important ways:

First, we have included all available public ground-and space-based imaging observations of the HDF, HDF-S WFPC2, and HDF-S NICMOS fields. Details of the current observations are summirized in Table 1.

Table 1

\begin{tabular}{|c|c|}
\hline Field & Filters \\
\hline HDE. & $\begin{array}{l}\text { F300W, F450W, F606W, F814W, } \\
\text { F110W, F160W, J, H, K }\end{array}$ \\
\hline HDF-S WFPC2 & $\begin{array}{l}\mathrm{F} 300 \mathrm{~W}, \mathrm{~F} 450 \mathrm{~W}, \mathrm{~F} 606 \mathrm{~W}, \mathrm{~F} 814 \mathrm{~W}, \\
U, B, V, R, I, J, H, K\end{array}$ \\
\hline $\mathrm{HDE}$ & $\begin{array}{l}\text { F110W, F160W, F222M, STIS, } \\
U, B, V, R, I\end{array}$ \\
\hline
\end{tabular}

Second. we have developed and applied a new quasi-optimal photometry technique based on fitting Inodels of the spatial profiles of the objects (which are obtained using a non-negative least squares image reconstruction method) 


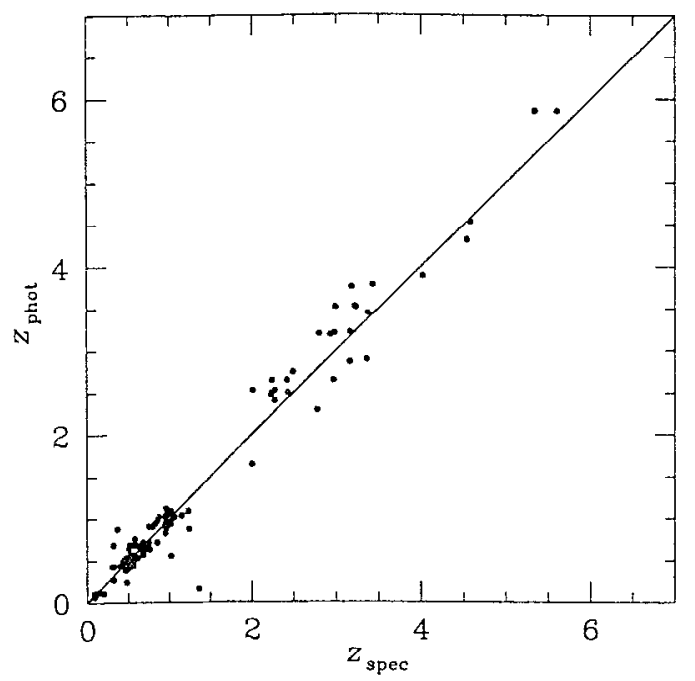

Figure 1. Comparison of 108 photometric and reliable spectroscopic measurements of galaxies in HDF and $\mathrm{HDF}-\mathrm{S}$. The RMS dispersion between the photometric and reliable spectroscopic measurements is $\approx 0.1$ at $z<2, \approx 0.3$ at $2<z<4$, and $\approx 0.15$ at $z>4$.

to the ground-and space-based images, according to the spatial profile fitting technique described previously by Fernández-Soto, Lanzetta, \& Yahil (1999). For faint objects, the signal-to-noise ratios obtained by this technique are larger than the signal-to-noise ratios obtained by aperture photometry techniques by typically a factor of two.

Third, we have measured photometric redshifts using a sequence of six spectrophotometric templates, including the four templates of our previous analysis (of E/SO, Sbc, Scd, and Irr galaxies) and two new templates (of star-forming galaxies). Inclusion of the two new templates eliminates the tendency of our previous analysis to systematically underestimate the redshifts of galaxies of redshift $2<z<3$ (by a redshift offset of roughly 0.3 ), in agreement with results found previously by Benítez et al. (1999).

The accuracy and reliability of the photometric redshift technique is illustrated in Figure 1, which shows the comparison of 108 photometric and reliable spectroscopic redshifts in HDF and HDF-S. (Note that a non-negligible fraction of published spectroscopic redshift measurements of galaxies in HDF and HDF$S$ have been shown to be in error and so must be excluded from consideration.) With the sequence of six spectrophotometric templates, the photometric redshifts are accurate to within an RMS relative uncertainty of $\Delta z /(1+z) \lesssim 10 \%$ and there are no known examples of photometric redshift measurements that are in error by more than a few times the RMS uncertainty. These results apply at all redshifts $z<6$ that have yet been examined. Details of some of our current observations and analysis are described by Yahata et al. (2000). 


\section{Stony Brook Faint Galaxy Redshift Survey}

Our analysis of the HDF and HDF-S WFPC2 and NICMOS fields constitutes a survey of galaxies to the faintest cncrgy flux density and surface brightness limits currently accessible. Properties of the redshift survey are as follows:

First, we have determined nine- or 12-band photometric redshifts of faint galaxies in three deep fields.

Second, we have selected galaxies at both optical and infrared wavelengths, in two or more of the F814W, F160W, $H$, and $K$ bands (depending on field). (We have related selection in different bands by adopting the spectral energy distribution of a star-forming galaxy).

Third, we have fully characterized the survey area versus depth relations, as functions of both energy flux density and surface brightness.

Fourth, we have established properties of the extremely faint galaxy population by using a maximum-likelihood parameter estimation technique and a bootstrap resampling parameter uncertainty estimation technique. The derived parameter uncertainties explicitly account for the effects of photometric error, sampling error, and cosmic dispersion with respect to the spectrophotometric templates.

The Stony Brook faint galaxy redshift survey includes nearly 3000 faint galaxies, of which nearly 1000 galaxies are of redshift $z>2$ and more than 50 galaxies are of redshift $z>5$ (ranging up to and beyond $z=10$ ). The depth and scope of the survey is summarized in Figure 2, which shows redshift distributions of all galaxies identified in the HDF and HDF-S WFPC2 and NICMOS fields. The redshift distributions of galaxies identified in the HDF and HDF-S WFPC2 field are characterized by broad peaks at redshift $\approx \approx 1$ and long tails extending to redshifts $z>5$. Further, the distributions are statistically different from one another (with the HDF $-\mathrm{S}$ WFPC2 field exhibiting a statistically significant excess of galaxies of redshift $z>2$ compared with the HDF), and both exhibit statistically significant large-scale fluctuations. The redshift distribution of galaxies identified in the HDF-S NICMOS field is characterized by a broad peak at redshift $z \approx 1$ and a long tail extending to redshifts $z>10$.

\section{Some High-Redshift Galaxies}

Examples of some high-redshift galaxies are shown in Figure 3, which plots observed and modeled spectral cnergy distributions and redshift likelihood func tions of galaxies identified in the HDF-S WFPC2 and NICMOS fields.

The top group of panels of Figure 3 shows four galaxics of redshift $3<\approx<4$ and near-infrared continuum magnitude $A B(8140) \approx 25$, and the middle group of panels of Figure 3 shows four galaxics of redshift $5<z<6$ and near-infrared continuum magnitude $A B(8140) \approx 26$. In each case, the spectral energy distribution shows unambiguous evidence of the Ly $\alpha$-forest and Lyman-limit decrements, and the redshift likelihood function is very sharply peaked, indicating that, of all the spectrophotometric models considered, the appropriately redshifted spectrophotometric template provides the only plausible fit to the observations. We believe that the redshifts indicated in the top and middle groups of panels of Figure 3 are established with essentially complete certainty--and with 

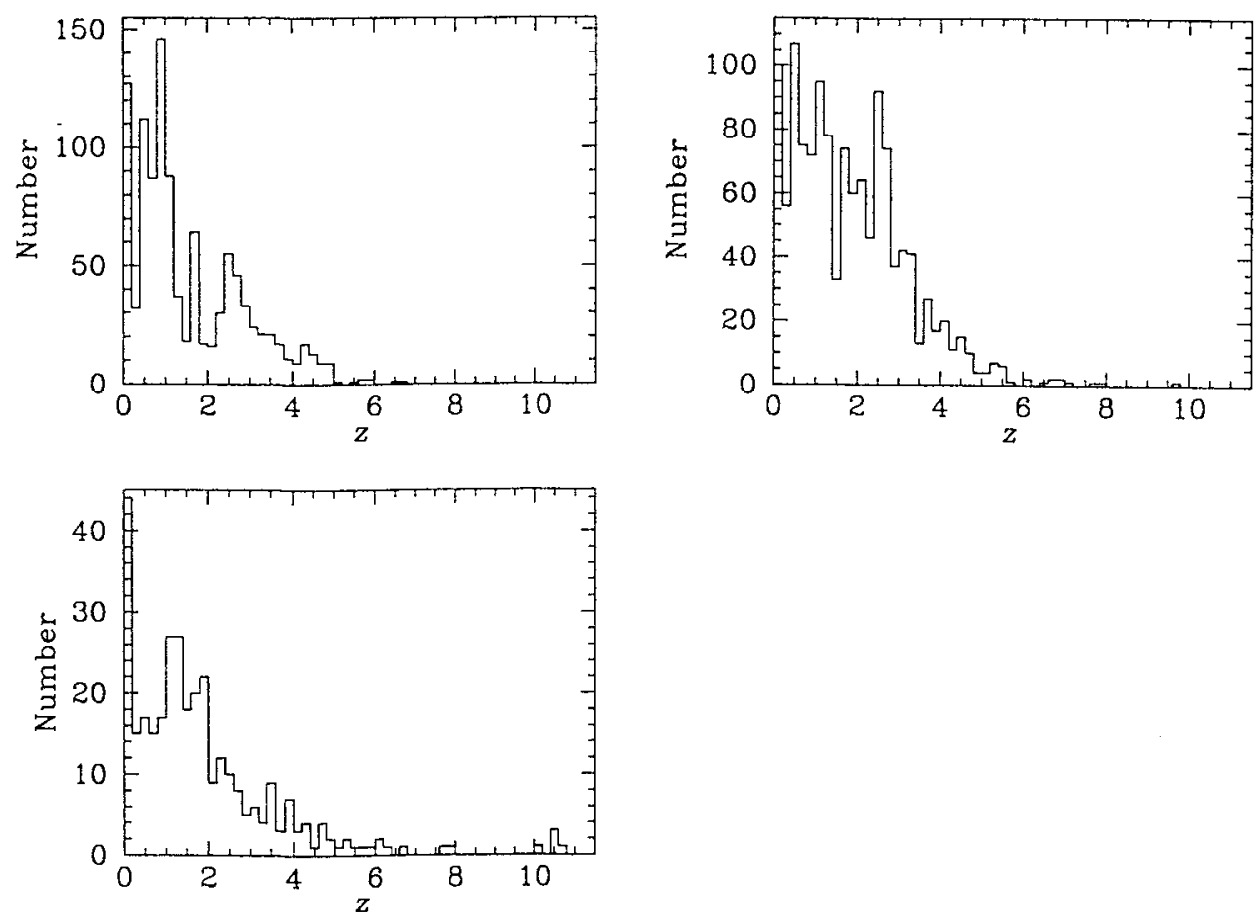

Figure 2. Redshift distributions of all galaxies identified in the HDF (upper left) and HDF-S WFPC2 (upper right) and NICMOS (lower left) fields.

substantially greater certainty than has been or could be achieved by means of spectroscopic observations of galaxies of the same redshifts and continuum magnitudes. The galaxies shown in the top and middle groups of panels of Figure 3 are unexceptional, and results shown for these galaxies are completely representative of results obtained for other similar galaxies.

Results of Figure 1 indicate that at redshifts $3<z<4$, the RMS measurement uncertainty of the photometric redshift technique is $\Delta z \approx 0.3$ or $\Delta z /(1+z) \approx 10 \%$, which we believe results primarily due to stochastic variations in the density of the Ly $\alpha$ forest among different lines of sight. Results of Figure 1 indicate (albeit with limited statistical certainty) that at redshifts $5<z<6$ the RMS measurement uncertainty of the photometric redshift technique is $\Delta z \approx 0.15$ or $\Delta z /(1+z) \approx 3 \%$, which we believe is superior to results at redshifts $3<z<4$ becanse almost complete absorption in the Ly $\alpha$ forest allows for less stochastic variations in the density of the Ly $\alpha$ forest among different lines of sight.

The bottom group of panels of Figure 3 shows four galaxies of best-fit photometric redshift measurement $z>6$ and near-infrared continuum magnitude $A B(16,000) \approx 2 T$, including two galaxies (galaxies $B$ and $C$ ) that we identified previously as candidate extremely high redshift galaxies on the basis of groundbased near-infrared measurements (Lanzetta, Yahil, \& Fernández-Soto 1998). At these redshifts and continum magnitudes, the redshift determinations are 

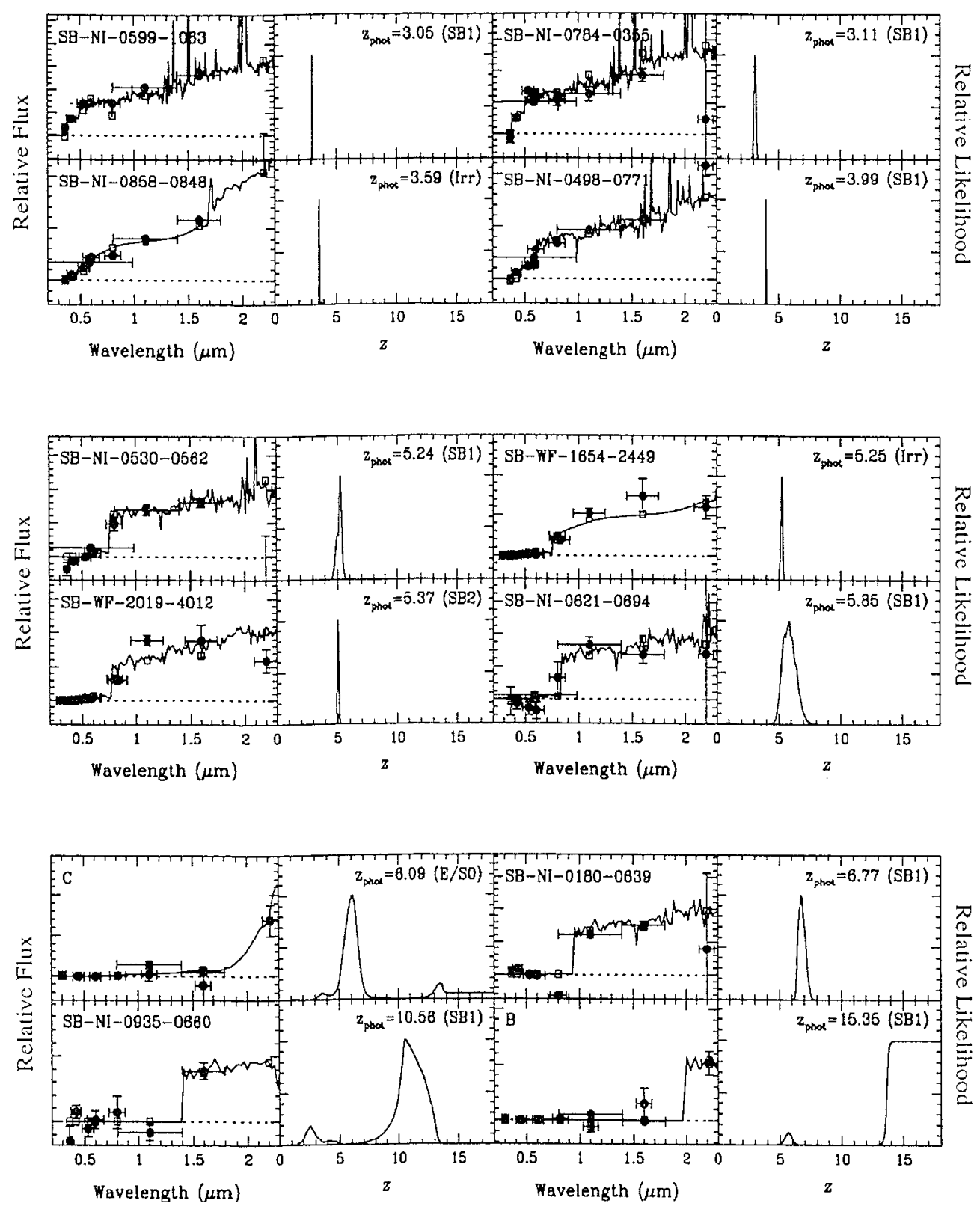

Figure 3. Observed and modeled spectral energy distributions (leftmost of each pair of panels) and redshift likelihood functions (rightmost of each pair of panels) of galaxies identified in the HDF-S. Top group of panels shows galaxies of redshift $3<z<4$, middle group of panels shows galaxies of redshift $\overline{5}<z<6$, and bottom group of panels shows galaxies of redshift $z>6$. Filled circles are measured fluxes and open squares are best-fit model fluxes. Vertical error bars indicate $1 \sigma$ uncertainties and horizontal error bars indicate filter FWHM. 

detection limits that will be required for working at higher redshifts. Fig. 4 from Thommes (1998) shows the expected surface number density of Ly $\alpha$ emitters for forr redshift intervals $(z=3.5,4.5,5.7$, and6.5) corresponding to spectral regions free of strong nightsky lines. Overplotted are the data from Cowie \& Hu (1998) for $z=3.4$, and other upper limits. It can be seen that, in agreement with the properties summarized above, surveys will need to exceed $5 \sigma$ detection limits of $10^{-17}$ ergs $\mathrm{cm}^{-2} \mathrm{~s}^{-1}\left(10^{-20} \mathrm{~W} \mathrm{~m}^{-2}\right)$, and preferably cover wide areas.

\section{6. $(R-Z)$ Color Selection}

Because SSA22-HCM1 is so bright in $Z$ it is interesting to examine color statistics for objects in $(R-Z)$ vs. $Z$. Fig. 5 shows the color distribution of objects in the HDF and SSA22 fields over a sample area of $75 \mathrm{arcmin}^{2}$. Magnitudes are measured on the $A B$ system using $2^{\prime \prime}$ diameter corrected apertures. The fiducial color boundary used here of $(R-Z)>2.75$ lies marginally above the range of possible $\mathrm{M}$ star contaminants, which might be distinguished by compactness criteria. At faint magnitudes the depth of the $R$ exposure dominates the errors in the $(R-Z)$ color measurement, which can be quite large. Filled symbols indicate known $z>5$ galaxies (or in the case of HDF 3-395.1 and 3-395.2, the summed magnitudes of the object pair). In the case of HDF 4-473.0, the colors are estimated from deep WFPC2 and NICMOS photometry (Weymann et al. 1998). The $5 \sigma Z$ band criterion is not sufficiently deep to include SSA22HCM1, the $z=5.60$ galaxy HDF $4-473.0$, or the $z=5.34$ emission-line galaxy RD1 (based on the estimated $I(A B)=26.1$ ), but the threshold is only a few tenths of a magnitude from reaching the brighter two objects. Thus, it appears that the use of deep $Z$-band filter imaging and $R-Z$ color selection on the large telescopes may be a possible way to extend methods for selecting candidates to higher $(z>5.7)$ redshifts for ground-based studies, although the background sky brightness near $9200 \AA$ is high. This could be used in combination with a near-IR color measurement to distinguish break objects from highly reddened galaxies.

\section{Evolution of the Continuum Break}

The new $z \sim 5$ quasars discovered by the Sloan Survey (Fan et al. 1999) provide a means of evaluating the expected continuum depression below the Ly $\alpha$ line at high redshifts. Because of the rapid increase in the gas density with redshift, it is expected thal, at the higher redshifts, there will be a sizable break. In Fig. 6 we show a spectrum of the newly discovered $z=5$ quasar, J033829.31+002156.3, for which we determine a factor of 4 break across the emission line. By comparison, Zhang et al. (1997) find, at $z=5$, for $H_{\mathrm{o}}=50 \mathrm{~km} \mathrm{~s}^{-1} \mathrm{Mpc}^{-1}, \Omega_{b}=0.06$ and a Haardt-Madau (1996) spectrum and ionizing flux, that the spectrum of a $z=5$ quasar will have an average flux in the region between $1050 \AA$ and $1170 \AA$ that is $10 \%$ of the continutum value which would be present in the absence of Lyman alpha scattering. A plot of the continuum break across Ly $\alpha$ for various $z>4$ quasars (Schneider et al. 1991; Kennefick et al. 1995) and galaxies is shown in 


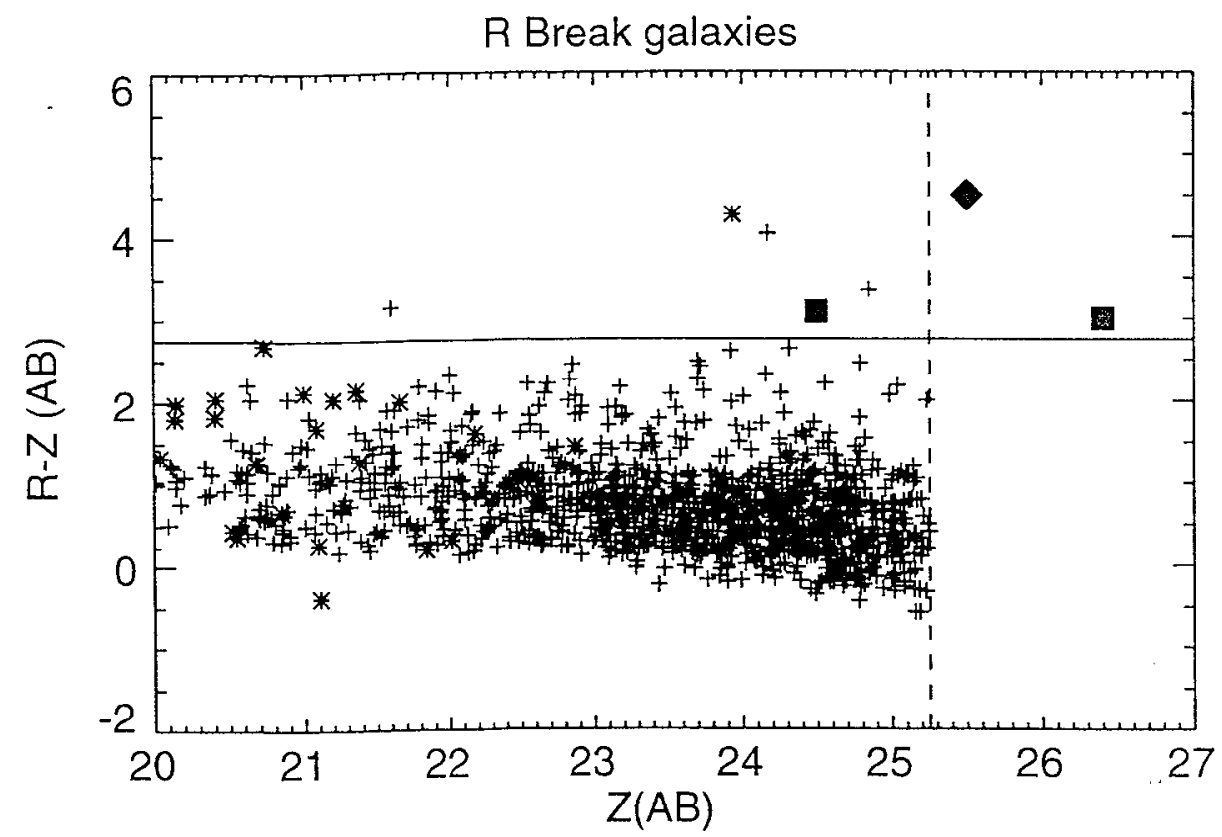

Figure 5. $(R-Z)$ colors of galaxies in the HDF and SSA22 fields. The dashed vertical line shows the $5 \sigma$ magnitude limit in $Z$. The filled squares show the colors of (both components of) HDF 3-395 and HDF 4-473.0, and the filled diamond shows the ineasured $(R-Z)$ color for SSA22-HCM1. The solid line at $(R-Z)=2.75$ provides an arbitrary divider for very red objects. Known stars (indicated with asterisks) can lie in the region above the line. Galaxies populating the upper part of the plot include both dust-reddened objects and high- $z$ continuum break objects.

Fig. 7. $D_{A}$ is the index (Oke \& Korycanski 1982)

$$
D_{A}=\left\langle 1-\frac{f_{\nu}(\text { observed })}{f_{\nu}(\text { continum })}\right\rangle
$$

in the rest-frame wavelength range $1050 \AA$ to $1170 \AA$.

\section{Future Investigations}

It is useful to consider possible directions for future studies. A number of $z>4$ galaxies have been found using cluster lens systems (e.g., Trager et al. 1997, Franx et al. 1997, Frye \& Broadhurst 1998, Pelló et al. 1999), where amplification of the source has increased its detectability. In general, such discoveries do not provide information on the statistics, continuum magnitudes, and base emissionline fluxes of the high-redshift galaxies found, but for very well-studied cluster lens "systems (e.g., Bézecourt et al. 1999) it may be possible to recover such information. Typical magnification is about 1 magnitude (Smail et al. 1999): and this may be a fruitful way to extend high-redshift searches.

- The availability of new IR spectrographs for the $8-10-\mathrm{m}$. class telescopes will make it possible to study [O II] associated with the $z<5$ systems (e.g. 


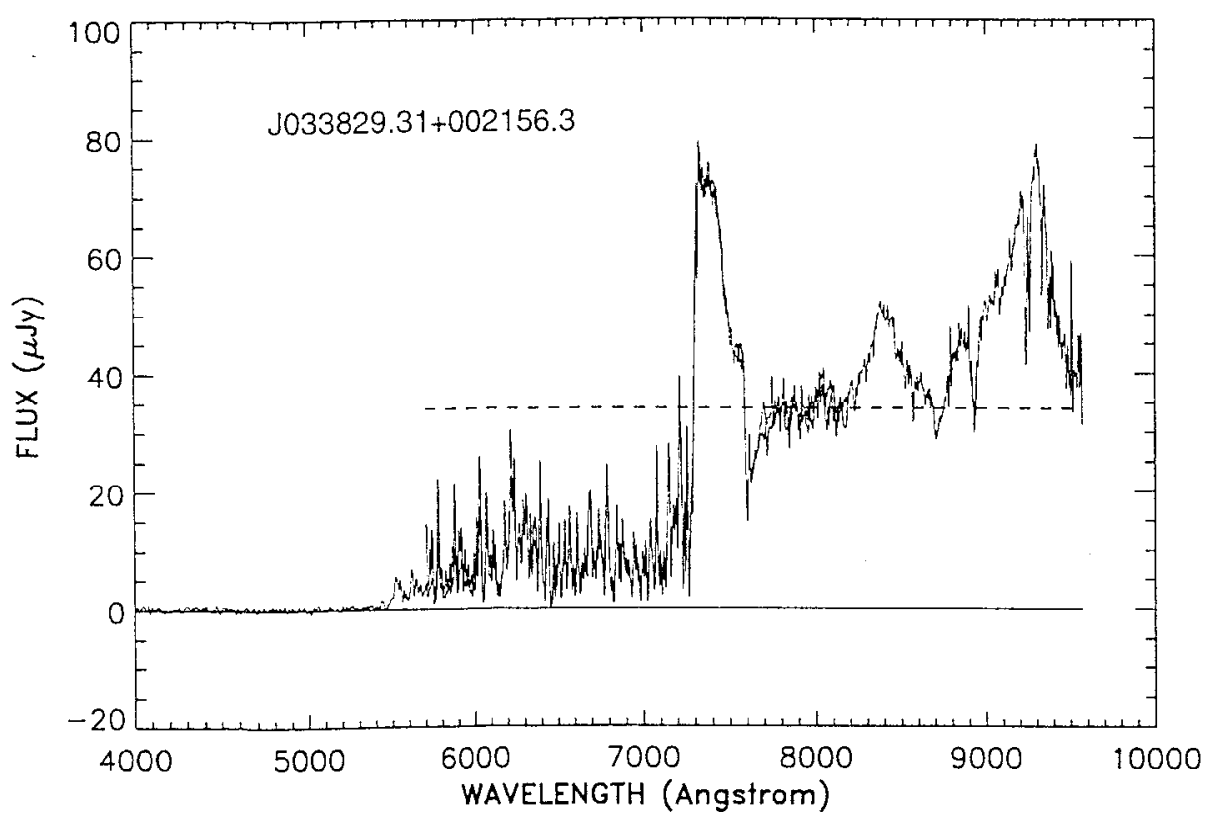

Figure 6. The Lyman break strength at high redshift is estimated to be a factor of 4 from LRIS spectra. (Songaila et al. 1999) of the $z=5.00$ Sloan Survey quasar (Fan et al. 1999).

Egami 1997). Such observations may provide more information on the degree of dust extinction in the high-redshift galaxies detected by their Ly $\alpha$. However, for $z>5$ systems [O II] lies in the thermal IR and cannot be readily studied from the ground.

For redshifts $z>5$, high equivalent width $\mathrm{H} \alpha$ emission from high ionization foreground extragalactic HII regions is a possible contaminant which can mimic Ly $\alpha$. Such systems may have no detectable continuum, and [N $\left.{ }^{I I}\right]$ suppressed to less than $2 \%$ of $\mathrm{H} \alpha$ (Stockton \& Ridgway 1998). The incidence of these systems is not known, but a few cases have been encountered in our $z \sim 5.7$ and $z \sim 6.5$ surveys - and identified by wide wavelength spectra covering the corresponding $[\mathrm{OII}]$ and $[\mathrm{OII}]$. Extensive wavelength coverage is required to verify sources as Ly $\alpha$ emitters.

Searches for yet higher $z$ galaxies are of necessity forced into the near IR since the light below $1216(1+z) \AA$ will be essentially extinguished by the strong Ly $\alpha$ forest blanketing or by H I Gunn-Peterson (Miralda-Escudé \& Rees 1998) at the redshifts where the IGM was neutral. We can look forward to detecting these objects with the Next Generation Space Telescope.

Acknowledgments. This work was supported in part by MASA grants GO-6626.01-95A, GO-7266.01-96A, and AR-6377.06-94A from Space Telescope Science Institute, which is operated by AURA. Inc. under NASA contract. Results reported here include observations made as a Visiting Astronomer at the W. M. Keck Observatory, which is jointly operated by the California Institute 


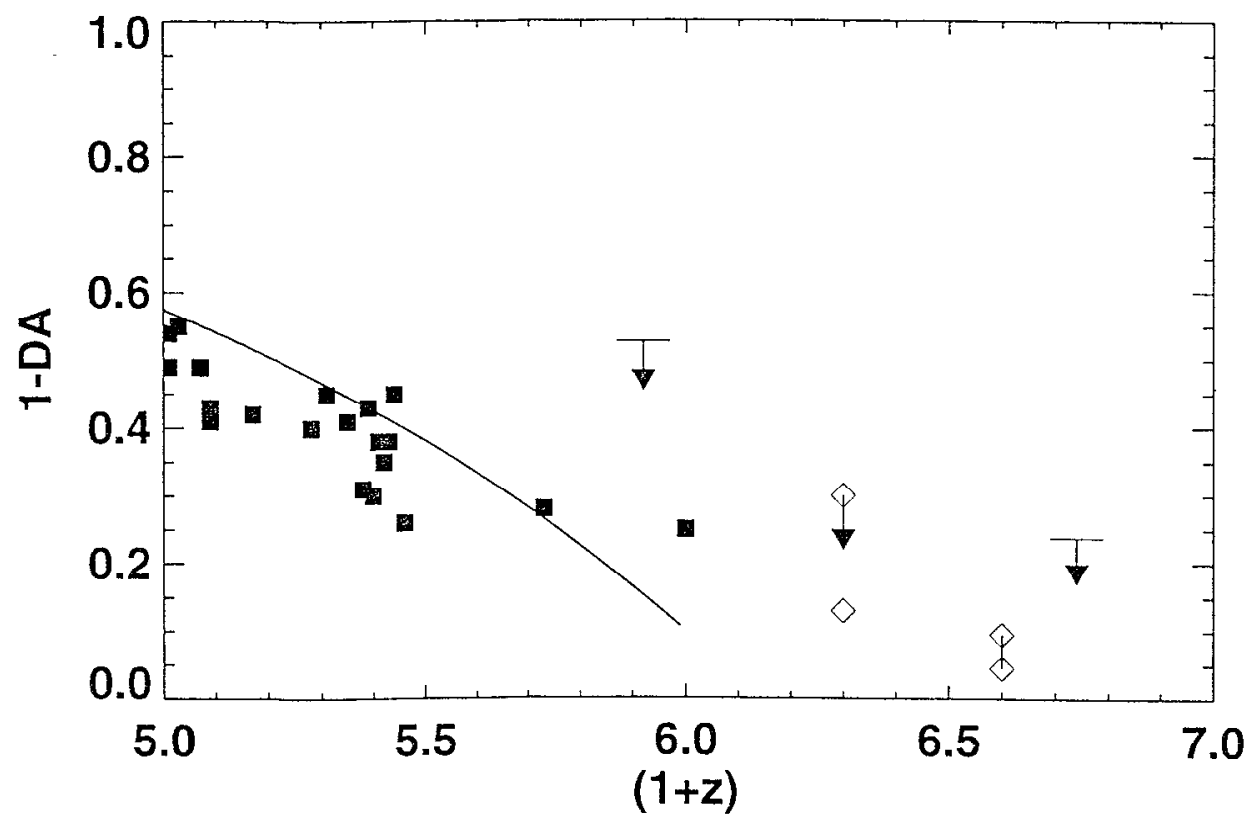

Figure 7 . The measured Lyman break strength $\left(1-D_{A}\right)$ at high redshifts for quasars ([filled squares] Schneider et al. 1991; Kennefick et al. 1995; Songaila et al. 1999) and high-redshift galaxies HDF 4-473.0 and 3-395(open diamonds). Upper limits are indicated for the $z=4.92$ lensed galdxy (Franx et al. 1997) and for SSA22-HCM1 at $z=5.74$ (Hu et al. 1999). The solid curve shows Zhang et al.'s (1997) extrapolation using a Haardt-Madau (1996) spectrum.

of Technology, the University of California, and the National Aeronautics and Space Administration.

\section{References}

Bézecourt et al. 1999, A\&A, 347, 21

Chen, H.-W., Lanzetta, K. M., \& Pascarelle, S. 1999, Nature, 398, Tד

Cowie, L. L. \& Hu, E. M. 1998, AJ, 115, 1319

Cowie, L. L., Songaila, A., Hu, E. M., \& Cohen, J. G. 1996, AJ, 112, 839

Dey, A., Spinrad, H., Stern, D., Graham, J. R., \& Chaffee, F. H. 1998, Ap.J, 498, L93

Egami, E. 1997, in The Ultraviolet Lniverse at Low and High Redshift, ed. W. H. Waller. M. N. Fanelli, J. E. Hollis, \& A. C. Danks (New York: AIP Press), 318

Fan, X. et al. 1999, AJ, 118, 1

Fernández-Soto, A., Lanzetta, K., \& Yahil, A. 1999, Ap.J, 513, 34 
Fontana, A., Cristiani, S., D'Odorico, S., Giallongo, E. \& Savaglio, S. 1996, MNRAS, 279, L27

Franx, M., Mlingworth, G. D., Kelson, D. D., van Dokkum, P. G., \&z Tran, K.-V. 1997, ApJ, 486, L75

Frye, B. \& Broadhurst, T. J. 1998, ApJ, 499, L115

Haiman, Z., \& Spaans, M. 1999, in After the Dark Ages: When Galaxies Were Young (The Universe at $2<z<5$ ), ed. S. S. Holt, \& E. P. Smith (New York: AIP Press), 63

Haardt, F. \& Madau, P. 1996, ApJ 461, 20

Hu, E. M. 1998, in The Young Universe: Galaxy Formation and Evolution at Intermediate and High Redshift, ed. S. D'Odorico, A. Fontana \& E. Giallongo, ASP Conf. Ser., 146, 148

Hu, E. M., Cowie, L. L., \& McMahon 1998, ApJ, 502, L99

Hu, E. M., Cowie, L. L., \& McMahon 1999, in proparation

Hu, E. M., McMahon, R. G., \& Cowie 1999, ApJ, 522, L9

Hu, E. M., \& McMahon, R. G. 1996, Nature, 382, 231

Hu, E. M., McMahon, R. G., \& Egami, E. 1996, ApJ, 459, Lõ3

Hu, E. M., McMahon, R. G., \& Egami, E. 1997, in HST and the High Redshift Universe, ed. N. Tanvir, A. Aragón-Salamanca, \& J. Wall (Singapore: World Scientific), p. 91

Kenncfick, J. et al. 1995, AJ, 110, 78

Meisenheimer, K. et al. 1998 in The Young Universe: Galaxy Formation and Evolution at Intermediate and High Redshift, ed. S. D'Odorico, A. Fontana $\&$ E. Giallongo, ASP Conf. Ser., 146, 134

Miralda-Escudé, J. \& Rees, M. 1998, ApJ, 497, 21

Oke, J. B. \& Korycanshi, D. G. 1982, ApJ, 255, 10

Pelló, R. et al. 1999, A\&A, 346, 359

Petitjean, P., Pécontal, E., Valls-Gaband, D. \& Charlot, S. 1996, Nature, 380, 411

Schneider, D. P., Schmidt, M. \& Gunn, J. E. 1991, AJ, 102, 837

Smail, I., Ivison, R. J., Blain, A. W., \& Kneib, J.-P. 1999, In After the Dark Ages: When Galaxies Were Young (The Universe at $2<z<5$ ), ed. S. S. Holt, \& E. P. Smith (New York: AIP Press), 312

Soifer, B. T., Neugebauer, G., Franx, M., Matthews, K., \& Mlingworth, G. D. 1998, ApJ, 501, L171

Songaila, A.. Hu, E. M., Cowie, L. L., \& McMahon, R. G. 1999, ApJ, 525, L5

Spinrad, H.. Stern, D., Bunker, A., Dey, A., Lanzetta, K., Yahil, A., Pascarelle, S., \& Fernández-Soto, A. 1998, AJ, 116, 2617

Steidel, C. C.. Adelberger, K. L., Giavalisco, M., Dickinson, M., \& Pettini. M. 1999, ApJ, 519, 1

Stockton, A. \& Ridgway, S. E., 1998, AJ, 115, 1340

Thommes, E. \& Meisenheimer, K. 1999, in preparation

Thommes, E. 1998, in From Stars to Galaxies to the Universe, ed. G. Boenner \& H. Mo, (Garching: Max-Planck-Institut für Astrophysik), astro-ph/9812223 
Thompson, D. \& Djorgovski, S. G. 1995, AJ, 110, 963

Trager, S. C., Faber, S. M., Dressler, A., \& Oemler, A. 1997, ApJ, 485, 92

van Breugel, W. J. M., de Breuck, C., Stanford, S. A., Stern, D., Röttgering, H., \& Miley, G. 1999, ApJ, 518, L61

Weymann, R. J., Stern, D., Bunker, A., Spinrad, H., Chaffee, F. H., Thompson, R. I., \& Storrie-Lombardi, L. J. 1998, ApJ, 505, L95

Williams, R. E. ct al. 1096, AJ, 112, 1335

Zhang, Y., Anninos, P., Norman, M. L., \& Meiksin, A. 1997, ApJ, 485, 496 
The Hy Redshift Universe

ASP Conference Series, Vol. 193, 1999

A. J. Bunker \& W. J. M. van Breugel, eds.

\title{
Searches for Quasars at $z>5$
}

\author{
Patrick S. Osmer \\ Department of Astronomy, The Ohio State University, 140. W. 18th \\ Ave., Columbus, $O H 43210$ USA, posmer@astronomy.ohio-state.edu
}

\begin{abstract}
.
Quasars continue to be the most luminous objects lnown in the universe but no longer have the largest redshifts. I review current techniques for finding quasars at $z>5$ and the status of current optical surveys. I compare the spectra of known quasars with $z \approx 5$ with the spectra of some recently discovered galaxies with $z>5$ to see what wc may expect in the future from surveys for high redshift quasars and galaxies. The prominent emission lines of quasars should make them casicr to detect and confirm spectroscopically than the $z>5$ galaxies discovered so far.
\end{abstract}

\section{Introduction and Background}

I am grateful to the Organizing Committee for the opportunity to speak on searches for high-redshift quasars at this meeting. Although I have not worked directly with $\mathrm{Hy}$, we have known each other for many years, and I have admired his research. I think three qualities stand out: 1) his determination and persistence at pushing telescopes and instruments to their limits, 2) his evident success at finding and studying successively more distant galaxies, and 3 ) his cheerfulness and optimism, which I think inspired his students and collaborators to be so successful over the many years of his career. I have also heard that $\mathrm{Hy}$ never liked quasars very much, so I feel honored that the topic made it on the program. Maybe the organizing committee decided it was safe to do so, now that galaxies have overtaken quasars as the objects of highest known redshift since 1997, when they displaced quasars for the first time since 1965.

Turning to the subject of this paper, the goals of surveys for high-redshift quasars include the observational determination of:

- The epoch of quasar formation

- The role of dust obscuration

- The relation of quasars to galaxy formation and evolution

- The contribution of quasars to the ionization of the intergalactic medium at high redshifts

According to the picture that quasars are powered by the accretion of matter onto massive black holes in the nuclei of galaxies, the formation and evolution 


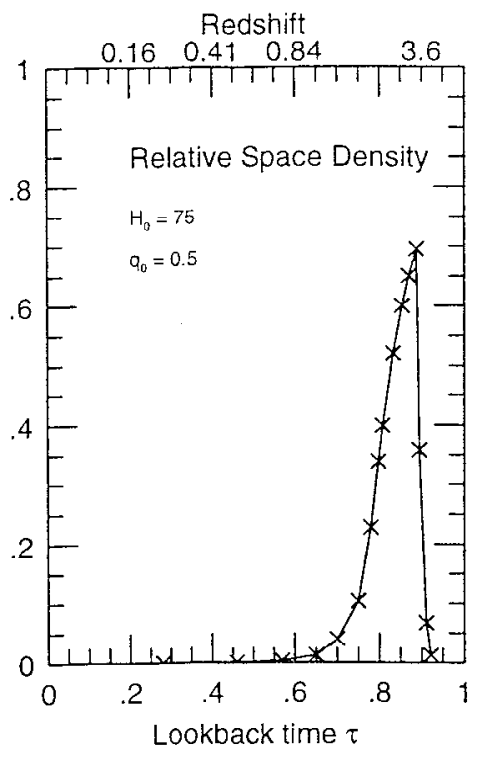

Figure 1. A linear plot of space density vs. lookback time for quasars with $M_{C}<-24.5$, based on the results of Warren et al. (1994). $M_{C}$ is the $A B$ continuum magnitude at the observed wavelength corresponding to Ly $\alpha$.

of black holes, quasars, and galaxies are closely related. The evolution of the space density of luminous quasars shows a strong peak at lookback times of 0.8 to 0.85 (Fig. 1). The evolution of galaxies is very much under debate, as we are hearing at this meeting, but it appears to extend over a broader range of redshift.

Among the questions we would like to address are: Which formed first, galaxies (or at least parts of galaxies), or quasars? What roles do interactions and mergers play in the fueling of quasars? and How do we account for the chemical abundances in high-redshift quasars, whose emission-line spectra look very similar to those at lower redshift?

We now have the observational capabilities to map the evolution of both quasars and galaxics to redshifts beyond 6 , when the universe was $5 \%$ or less of its present age. Hubble Space Telescope and $8-10$ - $\mathrm{m}$ ground-based telescopes have aiready enabled us to discover more objects at $z>5$ than seemed possible just a few years ago. Now the challenge is to carry out sufficiently large, systematic, and quantifiable survcys to answer the questions posed above.

In this paper I will discuss how to search for quasars at $\approx>5$, concentrating on optical methods. Next I will review the status of current surveys and then compare the spectra of the quasars and galaxies with $\approx>\approx 5$ that have been found to date. I will close by making-some speculations based on the current results and considering what we may expect in the future. 


\section{How to Search for Quasars at $z>5$ ?}

Since the discovery of the first quasar with $z>4$ in 1987 (Warren et al. 1987), more than 150 such objects have been found (G. Djorgovski and R. McMahon, private communications), and several search techniques are by now well developed. They include:

- Multi-color imaging

- Slitless Spectroscopy

- Deep Radio Surveys

- Deep X-Ray Surveys

The multicolor technique pioneered by Warren et al. (1987) and now applied to several surveys (see, e.g., Warren et al. 1991, Irwin et al. 1991, Kennefick et al. 1995, Djorgovski 1999) has contributed the majority of the known $z>4$ quasars. It uses the large $B-R$ color of quasars as a discriminant; the presence of Ly $\alpha$ emission in the $R$ filter band combined with the continuum depression caused by intervening Ly $\alpha$ absorption in the $B$ filter band makes quasars stand out from cool stars and yields an effective search criterion. The addition of $I$ band photometry, which is an indicator of the continuum level longward of Ly $\alpha$, aids in distinguishing high-redshift quasars from late-type stars. The multicolor technique was first used with photographic plates and Schmidt telescopes. The subsequent development of large format $C C D$ cameras is now enabling wideangle surveys to significantly fainter magnitudes, as discussed in more detail below.

At higher redshifts, e.g. $z>5$, the same principle applies, with Ly $\alpha$ shifting into the $I$ band. Howcver, as we shall see below, it is critical to have observations in the $Z$ band, which is in the continuum longward of Ly $\alpha$, to separate quasars from latc-type stars.

The slitless spectroscopy technique is an effective way to discover highredshift quasars through the direct detection of Ly $\alpha$ emission in low-resolution slitless spectra (Smith 1975, Osmer 1982) and has been used by Schmidt, Schneider, and Gunn (SSG, 1995 and refercnces thercin) in their Palomar Grism Surveys. The selection effects and survey efficiencies are more straightforward to model for slitless spectroscopy in many cases than they are for the multicolor technique. The SSG survey is one of the cornerstones of our knowledge of the evolution of optically selected quasars at $z>3$.

Deep radio surveys offer a different way to discover high-redshift radio galaxies and quasars. A key property of radio-selected objects is that they are significantly less affected by surrounding or intervening dust that blocks optical radiation. Although radio-loud quasars in general constitute about $10 \%$ of the total quasar population. the availability of deep radio surveys that cover a significant fraction of the sky offers the opportunity to find significant numbers of radio-loud quasars and radio galaxies at high redshift.

Current radio searches for high-redshift quasars make use of deep surveys for flat-spectrum sources (e.g. Hook and McMahon 1998) while searches for high-redshift radio galaxies focus on steep-spectrum sources (e.g. van Breugel 
et al. 1999, also this volume). In both cases, subsequent selection concentrates on optically faint objects to help weed out objects of lower redshift. Hook and McMahon (1998) select objects that are red in $B-R$ to enhance the selection of high-redshift objects. van Breugel et al. (1999) make use of the well-defined $K-z$ diagram for radio galaxies and concentrate on objects with faint $K$ magnitudes. The discoveries of the radio-selected quasar of highest known redshift, $z=4.72$ by Hook and McMahon (1998) and the radio galaxy of highest known redshift, $z=5.19$ by van Breugel et al. (1999) demonstrate the power of their approaches.

It may be argued that $\mathrm{X}$-ray emission is the key defining property of the global quasar/AGN population and should be the primary search criterion for discovering them. However, until recently, X-ray observatories have not had sufficient sensitivity to reach the objects of highest redshift. The ROSAT Deep Survey (Hasinger et al. 1998) is an indicator of the advances we may expect from CHANDRA (e.g., Mathur, this volume) and XMM as they come into operation; it has yielded the X-ray selected quasar of highest known redshift, $z=4.45$ (Schneider et al. 1998). The requirements are a limiting $\mathrm{X}$-ray sensitivity of $\approx 10^{-15} \mathrm{erg} \mathrm{s}^{-1} \mathrm{~cm}^{-2}(0.5-2 \mathrm{keV})$ and high accuracy, $\approx 1$ arcsec positions (to avoid confusion with foreground objects). Then follow-up optical imaging and spectroscopy, presumably concentrating on faint, red objects, should be an effective way of isolating high-redshift objects.

To summarize, all approaches to find $z>5$ quasars need to cover a wide area on the sky to faint limiting sensitivities because the objects are so rare. Optical surveys need to have wavelength coverage extending to $0.9 \mu$; indeed all approaches benefit from such coverage. Searches at even higher redshifts will push the requirements into the infrared $(\lambda>1 \mu)$.

\section{Status of Current Optical Surveys}

I would like to give a status report on two multicolor surveys my collaborators and $I$ are carrying out: the BT C40 and the BFQS. I will also describe two other major optical surveys, CADIS and SDSS, and note the important contributions they are making to the search for quasars at $z>5$.

The BTC40 is a large collaborative effort led by E. Falco and is aimed at finding gravitational lenses and distant galaxy clusters as well as high-redshift quasars. It is an optical, multicolor survey that makes use of the BTC camera and the CTIO 4-m telescope. Julia Kennefick is leading the quasar survey team, whose other members include Alberto Conti, Richard Greerl, Pat Hall, Eric Monier, Malcolm Smith, and myself. BTC40 has imaging data at high galactic latitude for $40 \mathrm{deg}^{2}$ in the $B, V, I, Z$ bands and reaches to $\approx 25$ th magnitude at its deepest limit. All the imaging data are in hand and are being analyzed. The goal is to select $z>5$ quasar candidates to $I=22$.

The BFQS survey is being led by Pat Hall in collaboration with the other team members mentioned above. It also uses the BTC camera and reaches to $m_{\text {lim }}=26.7$ over $7 \mathrm{deg}^{2}$. It uses the $B, R, I$ bands and is aimed at quasars with $3.3<z<5$ down to $L^{*}$ luminosities. The BFQS imaging data are also in hand and are being analyzed. 
Together, the BTC40 and BFQS surveys extend the multicolor technique to fainter magnitudes and wider areas than have been covered before.

The CADIS (Calar Alto Deep Imaging Survey, Meisenheimer et al. 1998) survey extends the multicolor concept to higher spectral resolution and broader wavelength coverage by using 13 medium-band filters over the 0.39 to $0.93 \mu$ range and 3 broad-band filters, including $h^{\prime}$. The filters are designed to give both improved sensitivity to the quasars being sought and improved rejection of non-quasars, such as emission-line galaxies (the veto filter concept). CADIS plans to cover 9 fields, each $10^{\prime} \times 10^{\prime}$ in area. To date the CADIS team (Wolf et al. 1999) have shown the effectiveness of their approach by finding 6 quasars with $2.2<z<3.7$ to $R-22 \mathrm{mag}$ in one field, about 6 times the expected number. The CADIS technique is more powerful than the original multicolor approaches because of its increased spectral resolution and greater number of filters. On the other hand, it requires more observing time per unit area on the sky to reach a given limit in sensitivity. Thus, it is a complementary and valuable addition to techniques for finding high-redshift quasars. It will be very important to see if the initial CADIS results on the surface density apply to the additional fields in their survey. Previous surveys have been subject to considerable field-to-field fluctuations, and it will be very interesting to see how the CADIS results come out. Our knowledge of the luminosity function of quasars to 22nd magnitude and fainter is still rudimentary.

The SDSS (Sloan Digital Sky Survey, Gunn \& Weinberg 1995) will be the definitive survey for high redshift quasars (and also for quasar clustering) down to $20 \mathrm{th}$ magnitude because of its large area coverage, $10,000 \mathrm{deg}^{2}$. SDSS is expected to yield $10^{5}$ quasars when completed. Already the initial results are very exciting. Fan et al. (1999) report the discovery of 15 quasars with $z>3.6$ in $140 \mathrm{dcg}^{2}$, including quasars with $z=4.9$ and $z=5.0$. They cxtend the high-redshift record for quasars for the first time since $1991^{1}$.

\subsection{The Importance of the $Z$ band}

Initial spectroscopic results from the $\mathrm{BTC} 40$ survey confirm the importance of using the $Z$ band for isolating $z>5$ quasar candidates. Unlike the situation at $4<z<5$, where quasars can be selected with reasonable efficiency solely on their large values of $B-R$, the analogous approach using $V-I$ does not work for $z>5$ quasars. Julia Kennefick found that samples selected on the basis of large $V-I$ are overwhelmed by late-type stars. The $Z$ band is necessary because it is a measure of the continuum level at wavelengths longward of the Ly $\alpha$ emission, and the behavior of late-type stars and $z>5$ quasars is significantly different in, for example, the $(I-Z)$ vs. $(V-I)$ two-color diagram, as Kennefick's simulations show (Fig. 2, left). We are now in the process of selecting $z>5$ candidates for follow up with spectroscopic observations.

The importance of the $Z$ band is amply demonstrated in the SDSS results (Fig. 2, right), where Fan et al. (1999) illustrate the stellar locus in the $\left(i^{*}-\right.$ $\left.z^{*}\right)$ v.s. $\left(r^{*}-i^{*}\right)$ plane and the location of their $z>4.5$ quasars, which are well

\footnotetext{
${ }^{1}$ After the meeting, Fan et al. (astro-ph/9909169)reported the discovery of a quasar at $z=5.03$.
} 


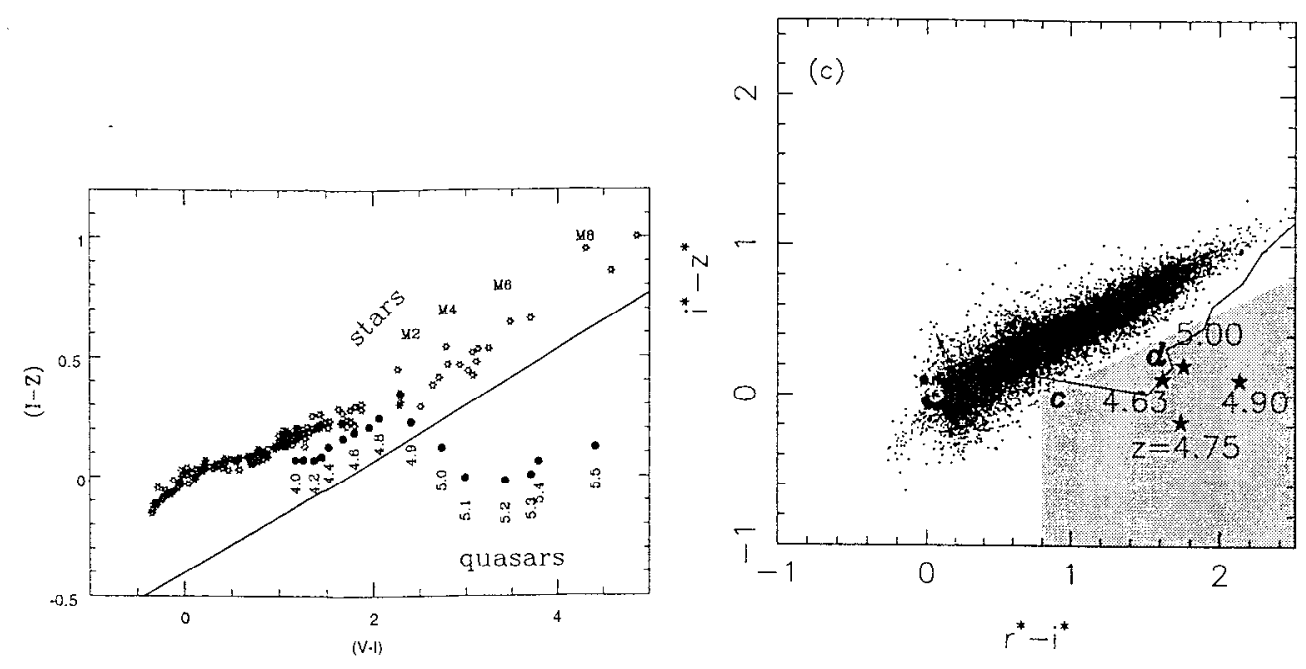

Figure 2. (Left) Simulation of stellar and quasar colors by J. Kennefick. (Right) The first SDSS results for stars and $z>4.5$ quasars (Fan et al. 1999)

separated from the stars. Their results confirm that we now have well established techniques for finding the most distant quasars.

\section{The Spectra of $z \approx 5$ Quasars Compared to $z>5$ Galaxies}

It is of interest to note that the spectra of the $z \approx 5$ quasars discovered to date (Fig. 3) show the characteristically strong emission features of Ly $\alpha, \mathrm{C} \mathrm{IV}$, $\mathrm{NV}$ and SI IV + O IV] seen in quasars at lower redshift and louk remarkably similar to them. Furthermore, the spectra provide information on the chemical abundances in the broad-line region that have important implications for the evolutionary history of the host galaxies of quasars (see review by Hamann \& Ferland 1999).

On the other hand, the spectra of the galaxies found so far at $z>5$ are very differenl from those of quasars (Figs. 4 and 5 ). For example, the Dey et al. (1998) galaxy at $z=5.34$ has a very strong but narrow Ly $\alpha$ emission line on a weak continuum. The Weymann et al. (1998) galaxy at $z=5.60$ and the van Breugel et al. (1999) radio galaxy show Lyo emission that is considerably weaker, while the Spinirad et al. (1998) galaxy pair at $z=5.34$ has very weak, if any, emission and is distinguished primarily by a break in the continuum.

Let us consider for a moment the faintiless of these galaxies and the large amount of spectroscopic observing time that has been dedicated by several groups of observers with the Keck telescopes to searching for faint galaxies at high redshift. It is surely impressive that galaxies with $z>5$ are being found. The: numerous discoveries exceed what I thought only a few years ago would occur. But the weakness of the spectral features of the galaxies shown in Figs: 4 and 5 compared to the strength of quasar emission lines in Fig. 3 do make one wonder if quasars/AGNs with broad-line spectra are truly very scarce at $z>5$. 

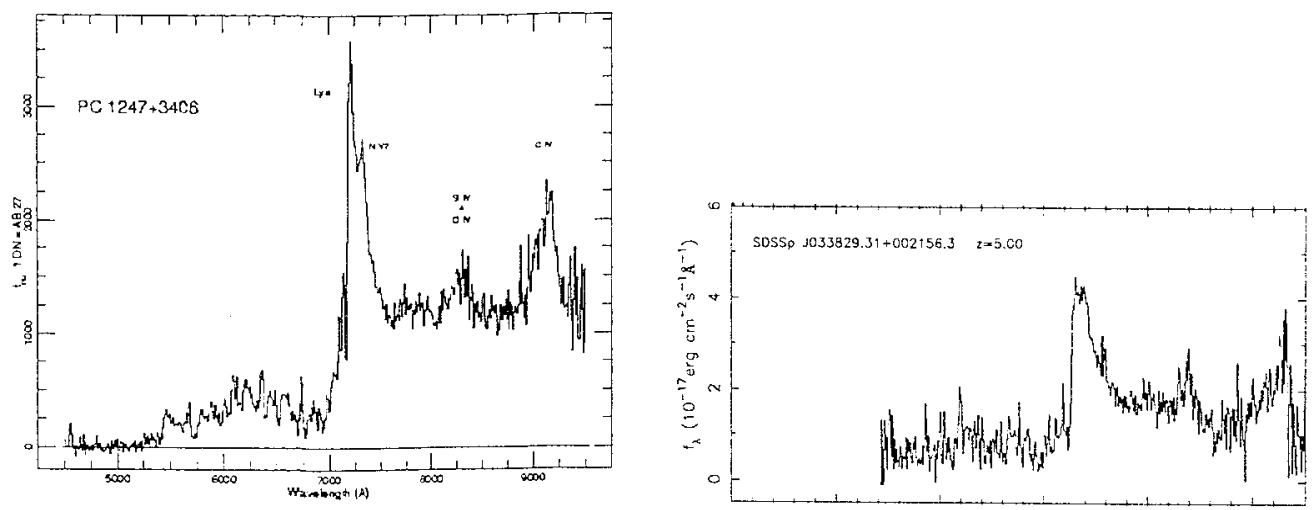

Figure 3. Spectra of PC1247+3406, $z=4.9$ (Schneider et al. 1991) and the $\operatorname{SDSS} z=5$ quasar (Fan et al. 1999)
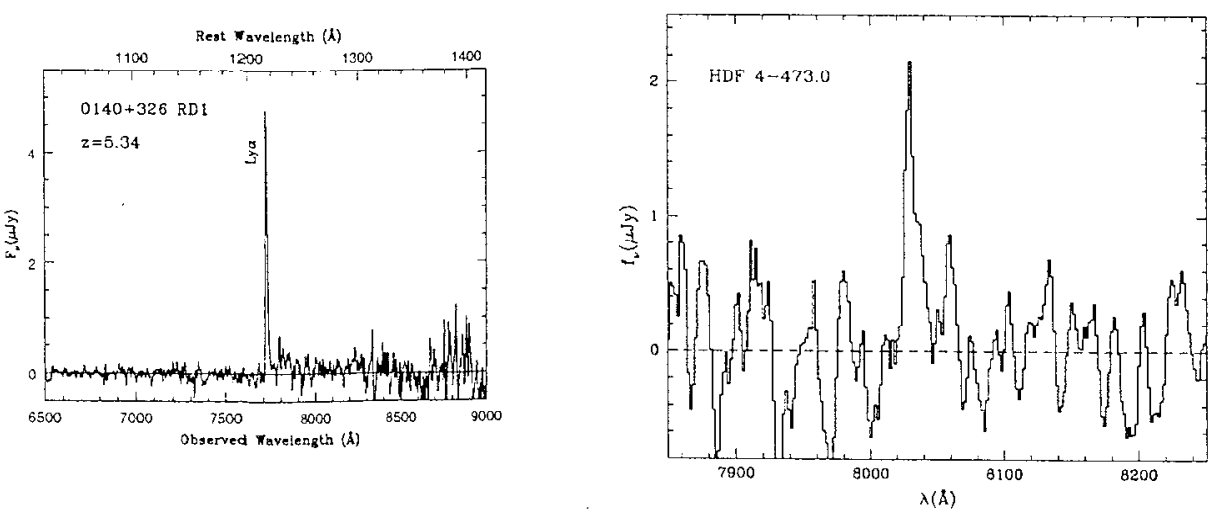

Figure 4. Spectra of the Dey et al. (1998) galaxy at $z=5.34$ (left) and the Weymann et al. (1998) galaxy at $z=5.60$ (right) 

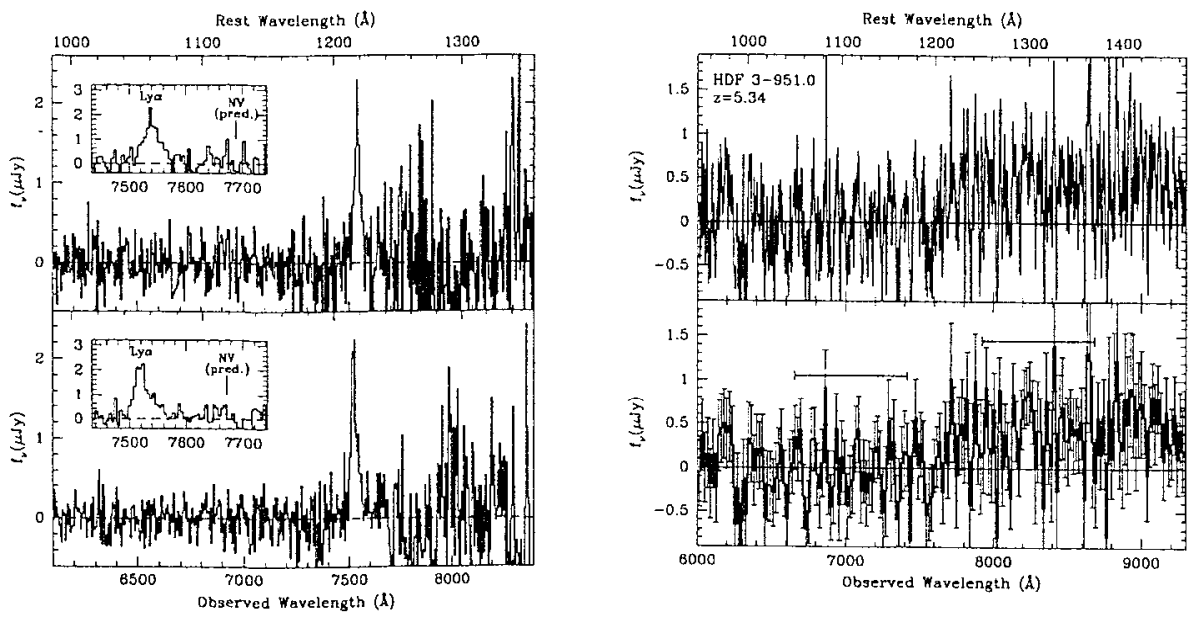

Figure 5. Spectra of the van Breugel et al. (1999) radio galaxy at $z=5.19$ (left) and the Spinrad et al. (1998) galaxy pair at $z=5.34$ (right)

Their spectra are much easier to identify than those of the galaxics seen to date. What is their absence telling us?

Of course, it may just be too early to tell, and we must pursue the spectroscopic follow up of the $z>5$ surveys described above. In any case, the observational opportunities that we now have to study the universc at $z>5$ are very exciting, and we may expect continued important discoveries in the near future. Let's meet again on the occasion of Hy's 70th birthday to scc what we have learned.

Acknowledgments. I am grateful to the Organizing Committee for the invitation to speak and for their financial support. This work has also been supported by the National Science Foundation under Grant AST-9802658.

\section{References}

Dey, A., Spinrad, H., Stern, D., Graham. J., \& Chaffee, F. 1998, ApJ, 498, L93 Djorgovski, S. 1999, DPOSS survey, http://astro.caltech.edu/ george

Fan, X. et al. 1999, AJ, 118, 1

Gunn, J. \& Weinberg, D. 1995, in Wide Field Spectroscopy and the Distant Universe, eds S. J. Maddox, A. Aragon-Salamanca (World Scientific: Singapore), p. 3

Hasinger, G., Burg, R.. Giacconi. R.. Schmidt, M., Trumper, J., \& Zamorani, G. 1998 A\&A. 329, 482

Hamann, F. \& Ferland, G. 1999, to appear in ARA\&A(astro-ph/9904223)

Hook, I. M. \& Mc.Mahon. R. G 1998. MNRAS, 294, LT

Irwin, M.. McMahon, R. G., \& Hazard. C. 1991, in The Space Distribution of Quasars, ed. D. Crampton, ASP Conf. Series, 21. 117 
Kennefick, J. D., Djorgovski, S. G., \& de Carvalho, 1995, AJ, 110, 2553

Meisenheimer, K., Beckwith, S., Fockenbrock, H., Huang, J., Leinert, Ch., Phleps, S., Roser, H.-J., Thommes, E., Thompson, D., Wolf, C., \& Chaffee, F. 1998, in The Young Universe, eds. S. D Odorico, A. Fontana, E. Giallongo, ASP Conf. Series, 146, 134

Osmer, P. S. 1982 Ap.J, 253, 28

Schmidt, M., Schneider, D. P. \& Gunn, J. E. 1995, AJ, 110, 68

Schneider, D. P., Schmidt, M., \& Gunn J. E. 1991, A.J102, 837

Schneider, D. P., Schmidt, M., Hasinger, G. Lehmann, I., Gunn, J. E., Giacconi, R., Trumper, J, \& Zamorani, G. 1998 AJ, 115, 1230

Smith, M. G. 1975 ApJ, 202, 591

Spinrad, H., Stern, D., Bunker, A., Dey, A., Lanzetta, F.. Yahil, A., Pascarellc, S., \& Fernandez-Soto, A. 1998, A.J, 116, 2617

van Breugel, W., De Breuck, C., Stanford, S., Stern, D., Rottgering, H., Miley, G. $1999, \mathrm{ApJ}, 518, \mathrm{~L} 61$

Warren, S. J., Hewett, P. C., Irwin, M. J. McMahon, R. G., \& Bridgeland, M. T. 1987, Nature, 325, 131

Warren, S. J., Hewett, P. C., \& Osmer, P. S. 1991, ApJS, 76, 1

Warren, S. J., Hewett, P. C., \& Osmer, P. S. 1994, ApJ, 421,412

Weymann, R. J, Stern, D., Bunker, A., Spinrad, H., Chaffee, F. H., Thompson, R. I., \& Storrie-Lombardi L. J. 1998, Ap.T, 505, L95

Wolf, C., Meisenheimer, K., Roser, H.-J., Beckwith, S. V. W., Fockenbrock, R., Hippelein, H., von Kuhlmann, B., Phleps, S., \& Thommes, E. 1999 A\&A, 343,399 
The Hy Redshift Universe

ASP Conference Series. Vol. 193, 1999

A. J. Bunker \& W. J. M. van Breugel, eds.

\title{
Kinematics of Ionized and Neutral Gas in Damped Ly $\alpha$ Systems
}

\author{
A.M. Wolfe ${ }^{1}$ \\ Department of Physics and CASS, University of California, San Diego \\ La Jolla, CA 92093-0424
}

\begin{abstract}
I investigate the kinematics of damped $\operatorname{Ly} \alpha$ systems with high resolution $\left(\approx 7 \mathrm{~km} \mathrm{~s}^{-1}\right)$ Keck spectra. The low-ion profiles comprise discrete velocity components within velocity intervals $\Delta v=(20,290)$ $\mathrm{km} \mathrm{s}^{-1}$. The $\Delta v$ distribution rules out semi-analytic CDM models in which the damped systems are rotating disks in dark-matter galaxy halos. The high-ion profiles also consist of discrete velocity components, but they do not line up with the low-ion components. Thus, the low-ion and high-ion gas are in separate kinematic subsystems. I describe MonteCarlo models which test whether the kinematics are consistent with the standard paradigm of the collapse of ionized gas to a rotating plane of neutral gas. None of the models, including those assuming galaxy disks to be in place at $z>4$, satisfy all the tests we have devised.
\end{abstract}

\section{Introduction}

So far, our discussion of the Hy-redshift universe has relied exclusively on emitting objects selected from flux-limited samples of galaxies and QSOs. This is not surprising at a meeting honoring Hy Spinrad who has achieved so much with these techniques. However, my approach is different. Rather than search for emission from the stellar components of protogalaxies, I have focused on the gas. Specifically my colleagues and I have searched for the cold ne11tral gas component by detecting the Ly $\alpha$ absorption line that is imprinted on the continuum emitted by background QSOs. To ensure the gas is neutral we select damped Ly $\alpha$ lines arising from gas with $N(\mathrm{H} \mathrm{I}) \geq 2 \times 10^{20} \mathrm{~cm}^{-2}$.

This technique has the disadvantage that it does not provide an image of the protogalaxy. But it has the advantage of probing further down the protogalactic mass function than is possible with flux limited surveys. This is indicated by the results of our investigations which show the damped Ly $\alpha$ systems contain sufficient baryons in cold neutral gas to account for the visible mass in current spiral galaxies (Wolfe 1998). As a result we can check a crucial prediction of CDM; namely, that the progenitors of current luminous galaxies were low-mass subunits at $z \sim 4$. In principal such objects are detectable in absorption, since according to the semi-analytic model of Mo et al. (1998) the probability for

\footnotetext{
${ }^{1}$ Visiting Astronomer, W. M. Keck Telescope. The Keck Observatory is a joint facility of the University of California and the California Institute of Technology
} 
intercepting the $\mathrm{HI}$ disks responsible for damped Ly $\alpha$ absorption along the line of sight increases with decreasing dark-halo mass. In fact the median halo mass of these interceptors is $\sim 10^{10} M_{\odot}$ at $z \sim 4$. By contrast if spiral galaxies were already in place, i.e., if the dark-matter halos and gaseous disks were in place by this redshift (e.g. Prochaska \& Wolfe 1997), a similar calculation suggests the median halo mass to be at least 10 times higher.

What is the nature of the damped Ly $\alpha$ systems? In this paper I discuss tests distinguishing models based on CDM from the null hypothesis (hereafter TF model) that the gravitational equilibrium of the damped systems has not changed since $z \sim 4$. In particular I will focus on kinematic observations and tests that can distinguish mass distributions weighted toward high-mass objects from those dominated by low-mass subunits. First I will discuss kinematics of the neutral gas, then I will discuss kinematics of the ionized gas, and finally the relationship between the two.

\section{Kinematics of Low Ions in Damped Ly $\alpha$ Systems}

Figure 1 shows our most recent compilation of optical depth profiles for 38 damped Lya systems acquired with the HIRES Echelle spectrograph on Keck I. The profiles are obtained from transitions such as Si II 1808 and Fe II 1608, i.e., singly-ionized species thal trace the neutral gas dominating the damped $L_{y} x$ systems (see Prochaska \& Wolfe 1996). The profiles, which are in the redshift interval $z=(1.2,4.4)$, have been ordered according to increasing velocity width, $\Delta v$, and are reflected so that the strongest component is at the lowest velocity. The figure shows that the gas is in narrow velocity components distributed over velocity intervals in the range $\Delta v=(20,290) \mathrm{km} \mathrm{s}^{-1}$. The components are not randomly distributed in velocity space. Rather, the component with largest optical depth is at the profile edge in $75 \%$ of the profiles with $\Delta v>40 \mathrm{~km} \mathrm{~s}^{-1}$

The velocity profiles add new information not obtainable from properties of gas content alone. Specifically, J. X. Prochaska and I used Monte Carlo techniques to test the semi-analytic CDM models of Kauffmann (1996). These models assume the damped systems arise in dark-matter halos modeled by singular isothermal spheres with circular velocities, $V_{\text {vir }}$, distributed according to Press-Schecter theory, where $V_{\text {vir }}$ is the circular velocity at the virial radius. We generated velocity profiles by letting sightlines penetrate randomly oriented disks. The models were tested by comparing empirical and synthetic distributions of test statistics such as $\Delta v$. Although the Kauffmann (1996) models are consistent with the $\Omega_{g}(z)$ relation, they cannot reproduce the $\approx 100 \mathrm{~km} \mathrm{~s}^{-1}$ median of the observed $\Delta v$ distribution, owing to the predominance of halos with $V_{\text {vir }}<100 \mathrm{~km} \mathrm{~s}^{-1}$ (Prochaska \& Wolfe 1997).

Results for new semi-analytic models are shown in Figure 2. These incorporate more realistic halo models (Navarro et al. 1997; Burkert 1995) and the disk models of Mo et al. (1998) which include cross-section weighted distributions of the spin parameter, $\lambda$, ignored previously. These additions result in larger $\Delta v$ for a given $V_{\text {vir }}$. The model labeled LCDM is the $\Lambda$ CDM model (see figure caption for details) and the results are typical of all the CDM models we computed. The $P_{K S}$ are the Kolmogorov-Smirnov probabilities that empirical and synthetic 
not unambiguous, and the high-redshift solutions are typically accompanied by lower-redshift solutions, of early-type galaxies of redshift $z \approx 3$. Additional deep imaging observations of these galaxies are needed to establish their redshifts with certainty.

\section{The Galaxy Luminosity Function at Redshifts $\approx>2$}

We have modeled the rest-frame $1500 \AA$ luminosity function of galaxies of redshift $z>2$ by adopting an evolving Schechter luminosity function

$$
\Phi(L, z)=\Phi_{*} / L_{*}(z)\left[L / L_{\star}(z)\right]^{-\alpha} \exp \left[-L / L_{\star}(z)\right]
$$

with

$$
L_{\star}(z)=L_{*}(z=3)\left(\frac{1+z}{4}\right)^{\beta} .
$$

The best-fit parameters for a simultaneous fit to the HDF and HDF-S WFPC2 and NICMIOS fields (where we have related selection in different bands by adopting the spectral energy distribution of a star-forming galaxy) are $\Phi_{x}=$ $0.004 \pm 0.001 h^{3} \mathrm{Mpc}^{-3}, L_{*}=2.7 \pm 0.3 \times 10^{28} h^{-2} \mathrm{erg} \mathrm{s}^{-1} \mathrm{~Hz}^{-1}, \alpha=1.49 \pm 0.03$, and $\beta=-1.2 \pm 0.3$. The best-fit model is compared with the observations in Figure 4 , which shows the cumulative galaxy surface density versus redshift and magnitude for galaxies selected in the F814W and F160W bands.

From a practical point of view, Figure 4 presents our best measurements and models of the empirical galaxy surface density versus redshift and near-infrared magnitude. The most striking result of Figure 4 is that galaxies identified by our analysis at the highest redshifts $z>7$ (which are detected only at the faintest F160W magnitudes $A B \gtrsim 28$ ) are predicted by a straightforward extrapolation of a plausible model of the high-redshift galaxy luminosity function. For our analysis to have uncovered no galaxies of redshift $z>7$ would have implied rapid evolution of the galaxy luminosity function at redshifts $z>6$.

\section{Effects of Cosmological Surface Brightness Dimming}

Results of the previous section indicate that the galaxy luminosity function evolves only mildly at redshifts $z>2$, i.e. as $(1+z)^{\beta}$ with $\beta \approx-1$. But due to $(1+z)^{3}$ cosmological surface brightness dimming, the measurcd luminosities of extended objects decrease rapidly with increasing redshift, even if the actual luminosities of the objects remain constant. For this reason, we consider it more or less meaningless to interpret the galaxy luminosity function (or its moments) over a redshift interval spanning $z=2$ through $z=10$ without explicitly taking account of surface brightness effects.

To make explicit the effects of cosmological surface brightness dimming on observations of high-redshift galaxies, we have constructed the "star formation rate intensity distribution function" $h(x)$. Specifically, we consider all pixels contained within galaxies on an individual pixel-by-pixel basis. Given the redshift of a pixel (which is set by the photometric redshift of the host galaxy), an empirical $k$ correction (which is set by the model spectral energy distribution of the host galaxy) and a cosmological model determine the rest-frame $1500 \AA$ 

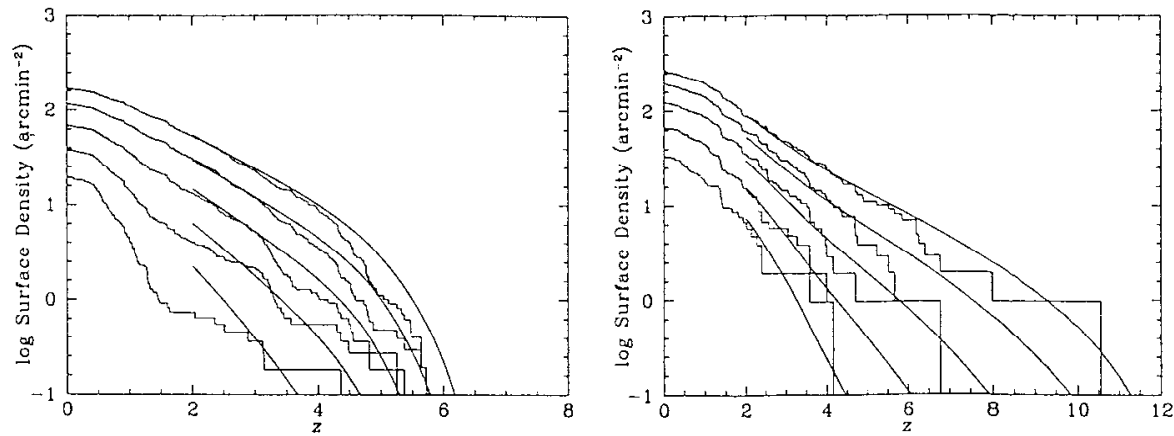

Figure 4. Logarithm of cumulative galaxy surface density versus redshift and magnitude (i.e. surface density of galaxies of redshift greater than a given redshift) for galaxies selected in the F814W (left panel) and F160W (right panel) bands. Smooth curves are best-fit model, and jagged curves are observations. Different curves show different magnitude thresholds, ranging from $A B=24$ (bottom curves) through $A B=28$ (top curves).

luminosity of the pixel, and an angular plate scale and a cosmological model determine the proper area of the pixel. Adopting a Salpeter initial mass function to convert the rest-frame $1500 \AA$ luminosity to the star formation rate and dividing the star formation rate by the proper area yields the "star formation rate intensity" $x$ of the pixel. Summing the proper areas of all pixels within given star formation rate intensity and redshift intervals, dividing by the star formation rate intensity interval, and dividing by the comoving volume then yields the "star formation rate intensity distribution function," which we designate as $h(x)$. The star formation rate intensity distribution function $h(x)$ is exactly analogous to the QSO absorption line systems column density distribution function $f(N)$ (as a function of neutral hydrogen colimm density $N$ ). In terms of the star formation rate intensity distribution function, the unobscured cosmir star formation rate density $\dot{\rho}_{s}$ (or equivalently the rest-frame ultraviolet luminosity density) is given by

$$
\dot{\rho}_{s}=\int_{0}^{\infty} x h(x) d x
$$

Results are shown in Figure 5, which plots the star formation rate intensity distribution function $h(x)$ versus star formation rate intensity $x$ determined from galaxies identified in the HDF and HDF-S NICMOS field. Several results are apparent on the basis of Figure 5: First, the star formation rate intensity threshold of the survey is an extremely strong function of redshift, ranging from

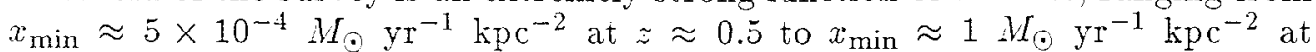
$\approx \approx 6$. We conclude that cosmological surface brightness dimming effects play a dominant role in setting what is observed at redshifts $z>2$. Second, the comoving number density of high intrinsic surface brightness regions increases monotonically with increasing redshift. We conclude that the comoving number density of high intrinsic surface brightness regions (or equivalently of high star formation rate density regions) increases monotonically with increasing redshift.: (See also Pascarelle, Lanzetta, \& Fernández-Soto 1998). Third, at redshifts 


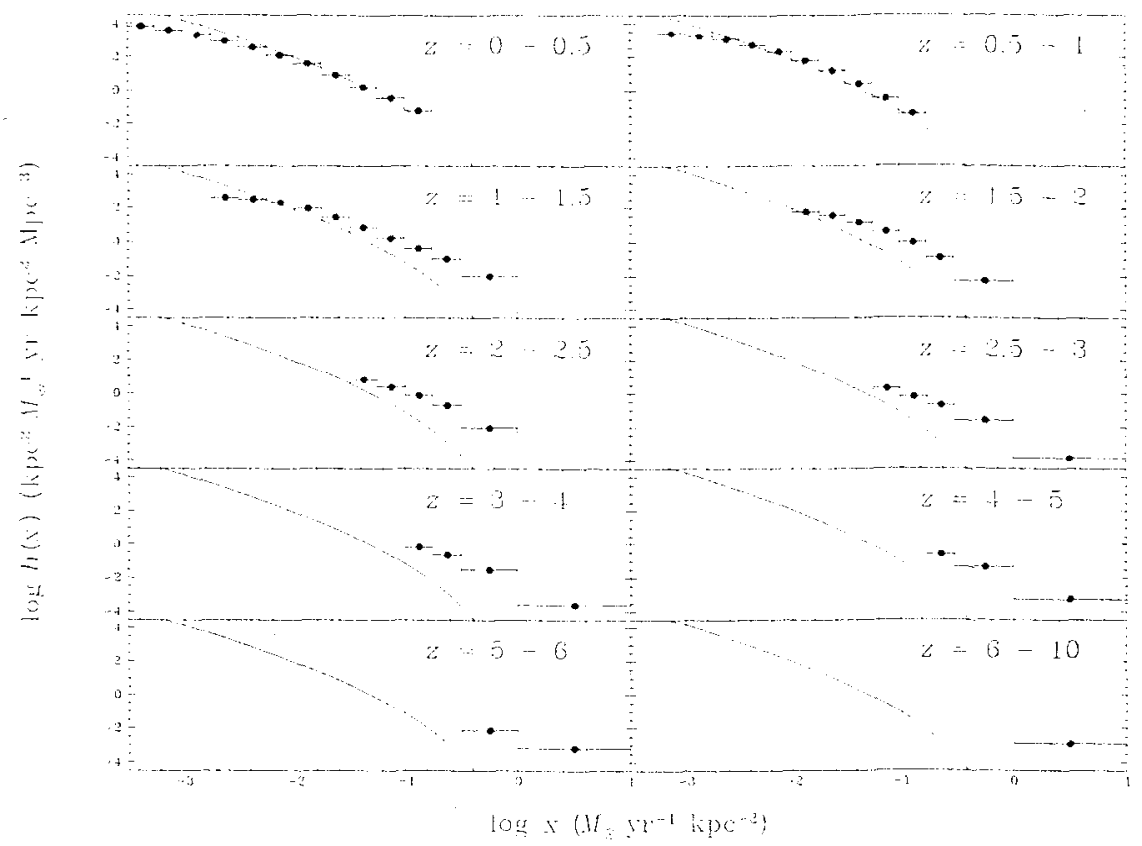

Figure 5. Logarithm of star formation rate intensity distribution function $h(x)$ versus logarithm of star formation rate intensity $x$, determined from galaxies identified in the HDF and HDF-S NICMIOS field. Different panels show different redshift intervals, ranging from $z=0$ through 10. Points show observations, with vertical error bars indicating $1 \sigma$ uncertainties and horizontal error bars indicating bin sizes. Smooth curves show a fiducial model (based on a bulge spatial profile) adjusted to roughly match the observations at $z=0-0.5$.

$z \lesssim 1.5$ [at which $h(x)$ is measured over a wide range in $x$ ], the distribution is characterized by a relatively shallow slope at log $x_{\sim}-1.5 \mathrm{M}_{\odot} \mathrm{yr}^{-1} \mathrm{kpc}^{-2}$ and by a relatively steep slope at $\log x_{\sim}^{>}-1.5 \mathrm{M}_{\odot} \mathrm{yr}^{-1} \mathrm{kpc}^{-2}$. These slopes are such that the bulk of the cosmic star formation rate density occurs at $\log x \approx-1.5 M_{\odot}$ $\mathrm{yr}^{-1} \mathrm{kpc}^{-2}$, which is measured only at redshifts $z<2$. We conclude that previous estimates neglecl a significunl or dominant fraction of the ultraviolet luminosity density of the universe due to surface brightness effects and that the rest-frame ultraviolel luminosity density (or equivalently the cosmic star formation rate density) has not yet been measured at redshifts $\approx \geq 2$.

This last point is illustrated in Figure 6 , which shows the ultraviolet luminosity density of the universe versus redshift measured to various intrinsic surface brightness thresholds. Specifically, Figure 6 shows the ultraviolet luminosity density of the universe versus redshift measured to intrinsic surface brightness thresholds that could be detected in the HDF at all redshifts to $z=5.0$, to $\dot{z}=3.4$, to $z=2.3$, to $z=1.6$, and to $z=1.1$. (Higher intrinsic surface brightness thresholds can be seen to higher redshifts, whereas lower intrinsic surface brightness thresholds can be seen only to lower redshifts.) Results of Figure 6 indicate that to any fixed intrinsic surface brightness threshold, the ultraviolet 


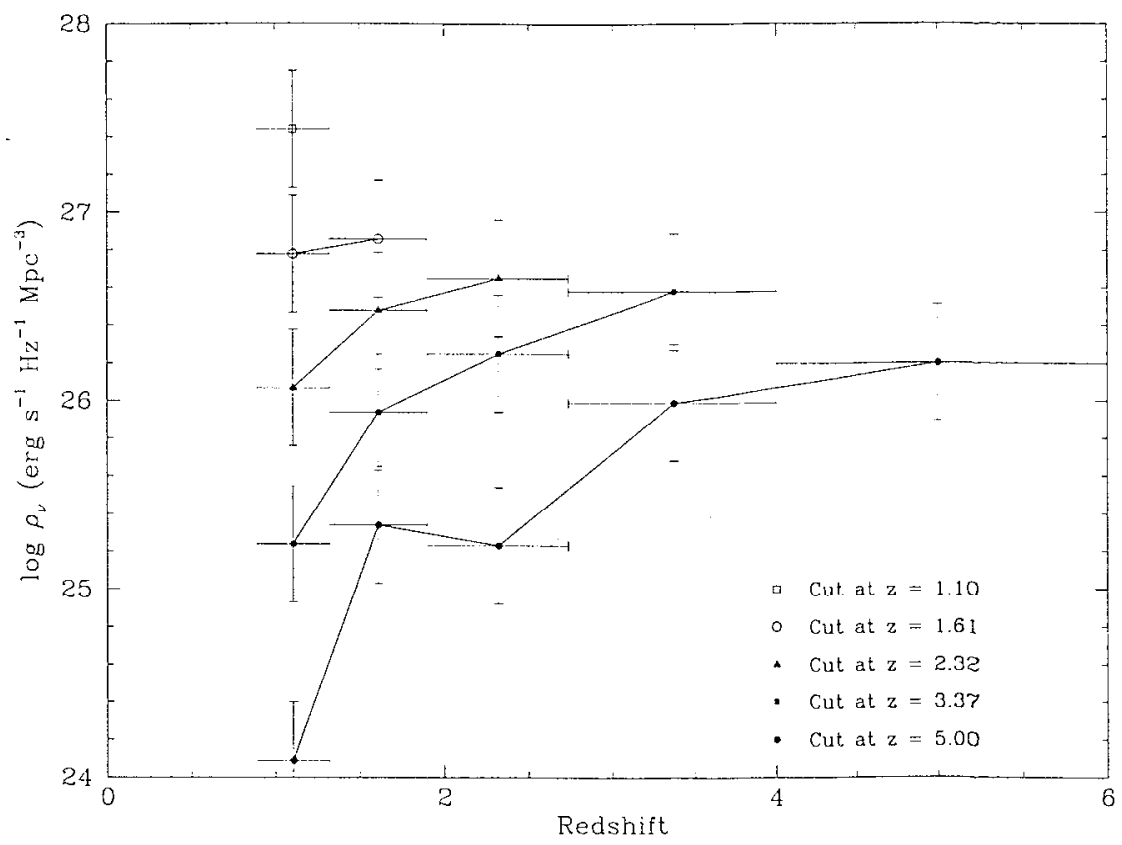

Figure 6. Logarithm of ultraviolet luminosity density versus redshift measured to various intrinsic surface brightness thresholds, determined from galaxies identified in the HDF.

luminosity density of the universe increases monotonically with increasing redshift. Apparently, the ultraviolet luminosity density of the universe plausibly increases monotonically with increasing redshift to redshifts beyond $z=5$.

Acknowledgments. We thank Hy Spinrad and Daniel Stern for providing spectroscopic redshift measurements in advance of publication and acknowledge Mark Dickinson and Roger Thompson for obtaining NICMOS observations of HDF. This research was supported by NASA grant NACW-4422 and NSE grant AST-9624216 and is based on observations with the NASA/ESA Hubble Space Telescope and on observations collected at the European Southern Observatory.

\section{References}

Benitez, N, Broadhurst, T., Bowwens, R., Silk, J., \& Rosati, P., 1999, ApJ, 515, L65

Fernández-Soto, A., Lanzetta, \& Yaliil, A. 1999, ApJ, 513, 34

Lanzetta, K. M., Yahil, A., \& Fernández-Soto, A. 1996, Nature, 381, 759 . 1998, AJ, 116, 1066

Pascarelle, S., Lanzetta, K. M., \& Fernández-Soto, A. 1998, ApJ, 508, L1

Yahata, N., Lanzetta, K. M., Chen, H.-W., Fernández-Soto, A., Pascarelle, S., Puetter, R., \& Yahil, A. 2000, Ap.J, submitted 
The Hy Redshift Universe

ASP Conference Series, Vol. 193,1999

A. J. Bunker \&f W. J. M. van Breugel, eds.

\title{
Galaxies at $z>5$ : The View from Hawaii
}

\author{
Esther M. Hu and Lennox L. Cowie \\ Institute for Astronomy, University of Hawaii, 2680 Woodlawn Drive, \\ Honolulu, HI 96822, USA \\ Richard G. McMahon \\ Institule of Astronomy, Madingley Road, Cambridge CBз OHA, UK
}

\begin{abstract}
We review the properties of $z>5$ galaxies studied with $H S T$ and with the Keck telescopes, and discuss the detectability of Ly $\alpha$ emission-line galaxies out to $z \sim 6.5$ based on these data and ongoing narrowband imaging surveys. The brightest sources may show $(R-Z)$ color breaks, although the high sky background at $Z\left(\lambda_{\text {eff }} \approx 9200 \AA\right)$, makes such observations challenging for typical faint sources. Keck LRIS observations of the $z=5$ SDSS quasar and $z>5$ galaxies observed with $H S T$ in the HDF show that the strength of the Lyman break is evolving more slowly than extrapolations from models at $z \sim 3$ would predict.
\end{abstract}

\section{Introduction}

Over the last year and a half, a number of very high redshift $(z>5)$ galaxies have been reported (Dey et al. 1998, Hu et al. 1998, Weymann et al. 1998, Spinrad et al. 1998, Chen et al. 1999, Hu et al. 1999), and our knowledge of the $z>4$ galaxy population (e.g., Hu et al. 1996, Petitjean et al. 1996, Fontana et al. 1996, Hu \& McMahon 1996, Trager et al. 1997, Franx et al. 1997, Hu et al. 1997, Frye \& Broadhurst 1998, Hu et al. 1998, Soifer et al. 1998, Pelló et al. 1999, Steidel et al. 1999, Hu et al. 1999) has been substantially increased. These samples have recently been joined by radio galaxy identifications which reach $z>5$ (van Breugel et al. 1999a, 1999b). At the highest redshifts now probed, identification of galaxies basically relies on two key diagnostics: the Lyman break across the continuum and the redshifted Ly $\alpha$ line. Both characteristics have been used to select and to verify high-z galaxy candidates.

The extremely faint nature of the high-z galaxy population in infrared contimuum light and the increasing strength and frequency of nightsky emission lines at very long wavelengths makes it clear that the contrast and detection of high-redshift galaxies from the ground is challenging, even for the new generation of 8 - to $10-\mathrm{m}$ class telescopes. The discovery of very high equivalent width emitters at $z=4.55$ ( $\mathrm{Hu} \&$ McMahon 1996) indicated the existance of a substantial population of strong Ly $\alpha$ emitters which could be observed to very high redshifts, beyond $z>5$. In this paper we report on the status of ongoing Ly $\alpha$ emission-line searches combined with color-selection data and consider the prospects for future identifications at the very high redshift end. 


\section{Ly $\alpha$ Searches}

The Ly $\alpha$ searches are designed to address issues of detectability of field galaxies at very high redshifts, using the increased contrast of the object against the background sky when viewed in the Ly $\alpha$ emission line. The contrast of emission features against the neighboring continuum is also enhanced by the $(1+z) \mathrm{mag}$ nification of line widths, producing high equivalent width signatures. Through the use of spectral regions free of strong night sky lines (e.g., Fig. 1), these studies are also designed to address object statistics over a range of different redshift intervals, since our ability to detect and reliably confirm very high-redshift galaxies is otherwise highly dependent on the location of emission features with respect to background nightsky lines. Narrowband Ly $\alpha$ searches combined with deep multi-color data on the Hawaii Survey Fields and HDF have been used as a training set (Cowie \& Hu 1998; Hu 1998) to test emission line identifications in terms of color and equivalent width diagnostics over an extensive spectroscopic database, starting at $z \sim 3.4$, where color selection is robust, and working out to successively higher redshifts $(z \sim 4.6,5.7,6.5)$. This allows studying continuity in properties of genuine Ly $\alpha$ emitters and both color-selected galaxies and lowredshift foreground emitters at progressively longer wavelengths, where higher sky backgrounds from the increased density of strong nightsky lines and fainter continua for the more distant galaxies are issues. A variant approach is to use a Fabry-Perot (Calar Alto Deep Imaging Survey, Meisenheimcr et al. 1998), which requires a high-level of precision in low-level light processing and tlat-fielding. The present Ly $\alpha$ surveys have reached $z \sim 5.7$ ( $\mathrm{Hu}$ et al. 1999), and $z \sim 6.5$ narrowband stidies have recently been started.

From the initial wide-field narrowband surveys for Lyo emitters at $z \sim 3.4$ and 4.5, Hu et al. (1998) estimated a surface density of Ly $\alpha$ emitters of $\sim$ $13,000 /$ unit $z / \square^{\circ}$ down to an emission flux of $\sim 10^{-17} \mathrm{ergs} \mathrm{cm}^{-2} \mathrm{sec}^{-1}$. These estimates were found to be consistent with a complementary deep spectroscopic study, where the slit provided limited spatial sampling, but wide wavelength coverage at somewhat greater sensitivity, and recovered 4 Ly $\alpha$ emitters with redshifts from $3.05 \ldots, 5.64$. Preliminary results from the $z \sim 5.7$ scarches suggest number densities perhaps a factor of 6 lower than the value for the lower redshift systems given by Hu et al. (1998).

\section{Spectroscopic Searches}

The surface number density estimates for Lyo emitters imply that sufficiently deep spectroscopic exposures may intercept some of these systems, at least over the lower redshift ranges. In fact, the first published $z>5$ galaxy, RD-1, was a serendipitously discovered Ly $\alpha$ emitter at $z=5.34$ reported by Dey et al. (1998) during deep long-slit observations of a neighboring object in the field, with both emission line and break identified in the spectra, and with the break confirmed by broad-band filter imaging. For RD-1, estimates of the continuum magnitude above the Lyman break are $\sim 26.3 \mathrm{AB}$ mags using the broad-band imaging data, with emission-line flux $=3.5 \times 10^{-17} \mathrm{ergs} \mathrm{cm}^{-2} \mathrm{~s}^{-1}, W_{\lambda}=600 \AA$ (Dey et al. 1998). The success of the spectroscopic searches depends on the surface number density at the redshifts and fluxes being probed. Equivalent width estimates, 


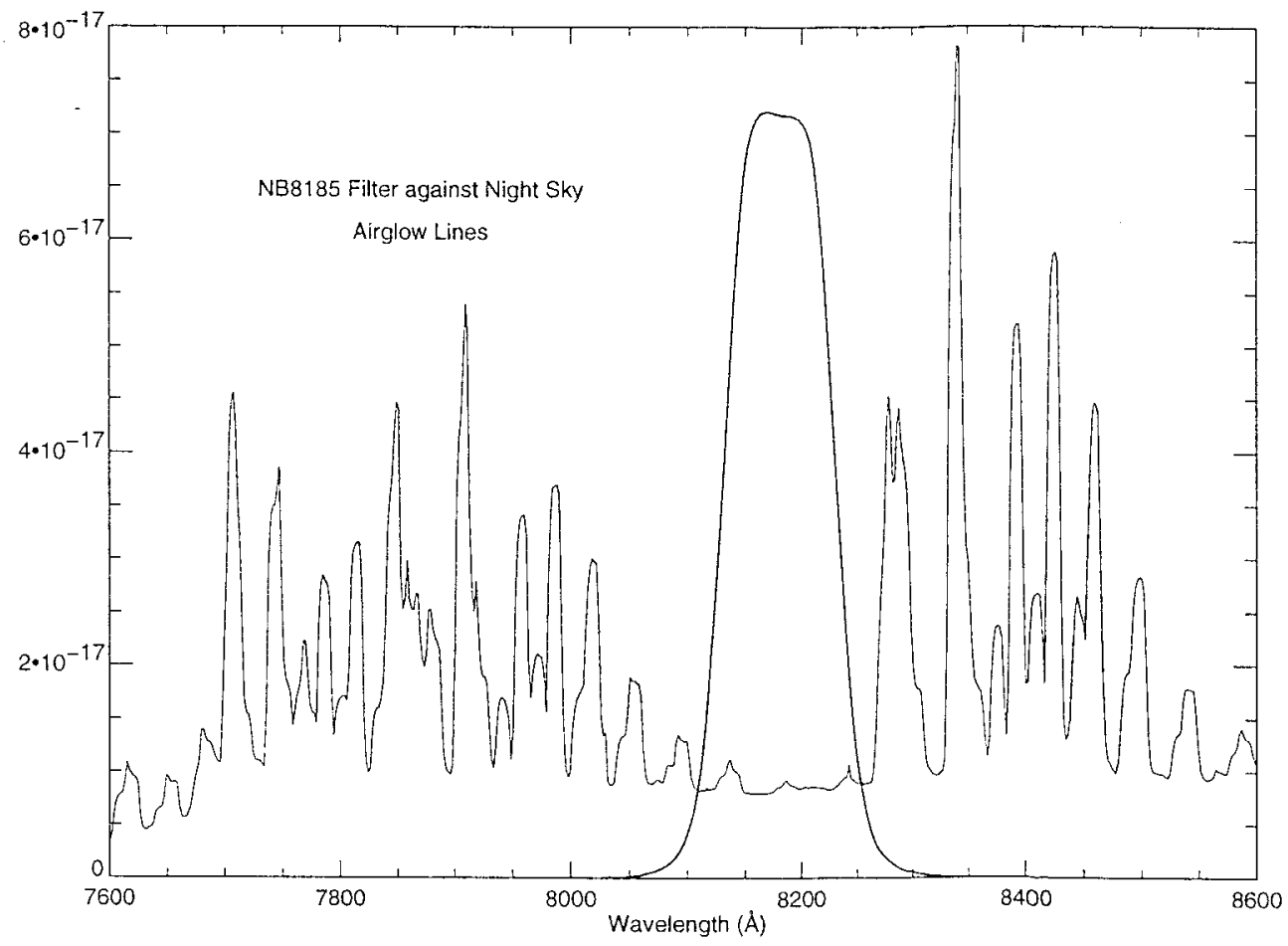

Figure 1. Narrowband filter at $8185 \AA$ and fluxed background night sky lines.

and particularly continuum magnitudes for faint objects measured in dispersed modes have larger associated errors than estimates tied to standard broadband filter photometry. Higher sensitivities trade off against small area coverage, and for slit spectroscopy there is the additional issue of whether with chance superpositions sample objects fall only partly within the slit. The additional problem in using this method as a search technique is that it is difficult to assess the fraction of emitters missed becanse they lie in regions of strong nightsky lines, and that for such cases confirming observations can be extremely hard to make.

An interesting variant of this search technique is to use slitless spectroscopy (Chen et al. 1999). Incomplete object sampling by a slit is removed, but the general problem of overlapping galaxy spectra must be dealt with. Using extremely deep STIS parallel exposures from HST, Chen et al. (1999) identify a Ly $\alpha$ emitter at a probable redshift of 6.68. At $z=6.68$ Ly $\alpha$ falls in regions of strong $\mathrm{OH}$ lines, which makes confirmation with ground-based observations extremely challenging.

\section{Color Breaks}

The strong depression of the continuum below the redshifted Lyman break caused by the numerous neutral hydrogen absorbers dubbed "the Ly $\alpha$ forest" is the most notable feature in the galaxy continuum light of very high redshift 

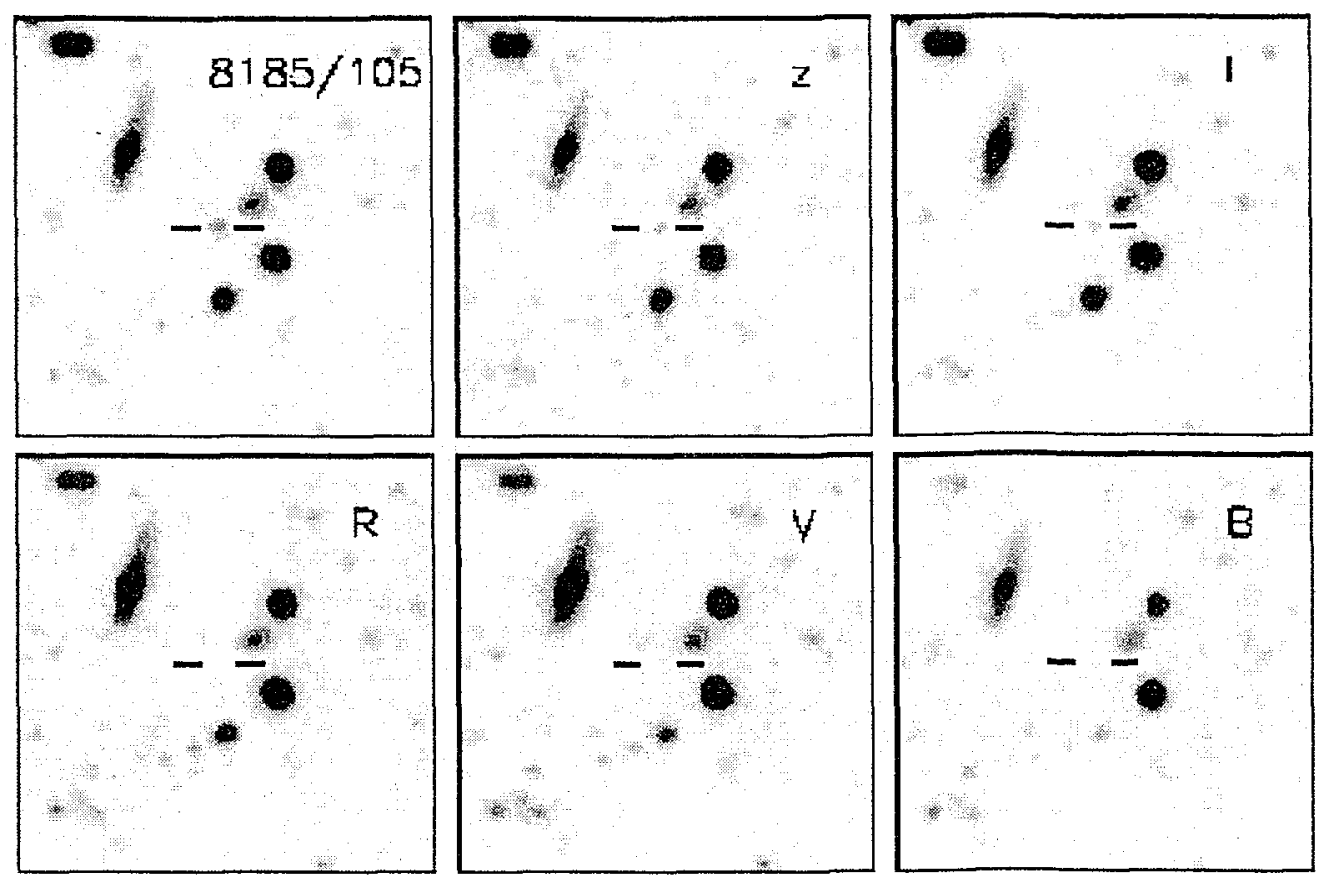

Figure 2. Multicolor $B, V, R, I, Z$, and narrowband $8185 / 105 \AA$ images taken with LRIS on Keck of the $z=5.74$ object, SSA22-HCM1. Each panel is $30^{\prime \prime}$ on a side. The strong contrast of this object in the $8185 \AA$ narrowband filter can be seen, in comparison with the $Z$ band, which samples the continuum in a region free of emission. SSA22-HCM1 is an $R$ 'dropout' and the object is absent at $B, V$, and $R$ in these extremely deep Keck LRIS images.

systems, where the extreme faintness of these objects, typically $>26$ mags $(A B)$ above the break, precludes the use of detailed absorption features to estimate the redshift. This method has been most successfully applied (e.g., FernándezSoto et al. 1999) in the Hubble Deep Field (HDF; Williams et al. 1996), where the high precision of the photometric measurements permits robust detection of a break signature, and where the availability of photometric data at longer wavelengths (NICMOS infrared observations) allows discrimination of Lyman break galaxies from red objects. HDF 4-473.0 (Weymann et al. 1998), shown to be a ' $V$ dropout' by the absence of detectable flux in the WFPC2 F606W filter, combined with a roughly flat $f_{\nu}$ spectrum out through $1.6 \mu \mathrm{m}$, was confirmed as a $z=5.60$ galaxy through deep LRIS spectra on Keck. An emission line with flux $\sim 10^{-17}$ ergs $\mathrm{cm}^{-2} \mathrm{~s}^{-1}$ and $W_{\lambda}=300 \AA$, identified as Ly $\alpha$, was used to establish the redshift. The magnitude above the break is $\sim 26.6 \mathrm{AB}$ mags.

A second color selected target, HDF 3-951.1 and 3-951.2, was identified by the color break, and placed at an estimated redshift $z=5.34$ (Spinrad et al. 1998) on the basis of the break location. For this close pair, no emission features are detected (Fig. 2 of Spinrad et al. 1998), with upper limits on possible Ly $\alpha$ more than a factor of 10 below the observed flux of HDF 4-473.0 Spinrad et al. (1998) consider the possibility that the absence of Ly $\alpha$ emission might be associated with its brighter continum. Because studies at this depth and 


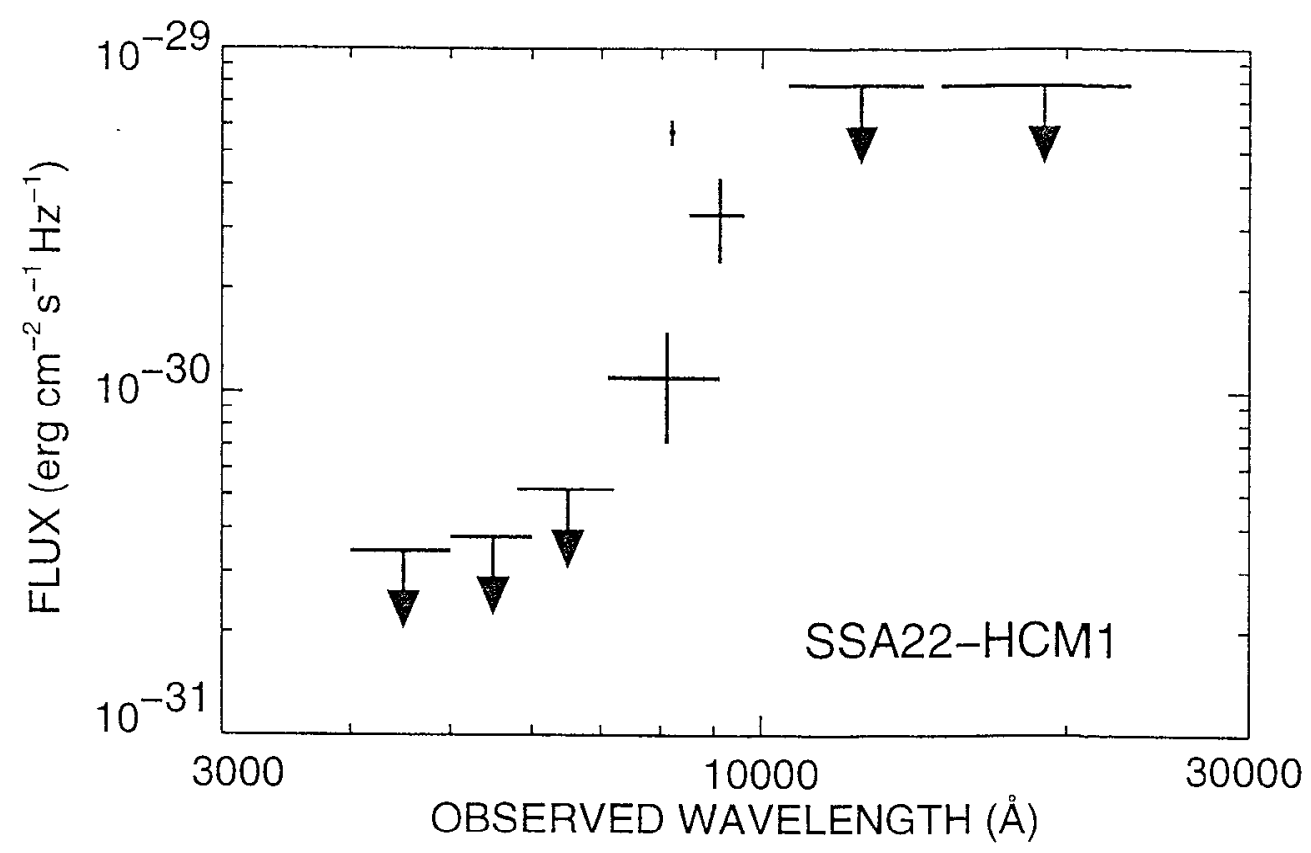

Figure 3. Spectral energy distribution for SSA22-HCM1 obtained using LRIS, and showing the bandwidths and errors for measurements in $B, V, R$, $I$, narrowband $8185 / 105 \AA$ (emission), RG850 (line-free continuum longwards of the emission), $J$, and $H+K^{\prime}$. Significantly, the object has no flux in the $R$ band, where strong Lyman $\alpha$ forest absorption is expected to be present. The strong break ( $2 \sigma$ upper limit of 0.23 across the line) combined with the strong emission line is a signature for $\operatorname{Ly} \alpha$.

precision of color measurement are only available for a small region of sky ( $5 \operatorname{arcsec}^{2}$ each for the HDF and HDF South), key questions one would like to address from the HDF color-break selected galaxies are: (1) How typical are these properties of the high-redshift galaxies; what are the consequences for future detections? (2) Are the bright high-redshift galaxies devoid of Lyo emission?

\section{Properties of the $z>5$ Galaxies}

Figs. 2 and 3 show images and a spectral energy distribution (SED) for the $z=$ 5.74 galaxy, SSA22-HCM1 ( $\mathrm{Hu}$ et al. 1999). This object is arl ' $R$ ' dropout, and is notably absent in deep Feck LRIS images in $B, V$, and $R$, which reach $1 \sigma$ limits of $B-28.3, V=28.2$, and $R=27.8$ for a $2^{\prime \prime}$ diameter aperture. The SED shows both the strong Lyman break and the Ly $\alpha$ emission feature (flux $=1.75 \times 10^{-1} i$ ergs $\mathrm{cm}^{-2} \mathrm{~s}^{-1} ; W_{\lambda}=175 \AA$ ), which was confirmed with deep LRIS spectroscopy. The increased contrast in the appearance of the object as detected in the $8185 \AA$ narrowband filter compared with the line-free $Z$ band filter around $9200 A$ may be noted. For these measurements we use fluxes measured in the narrowband filter, instead of values recovered from spectroscopic extractions, for greater precision. Aperture $1 \sigma$ errors are estimated from laying down random apertures 


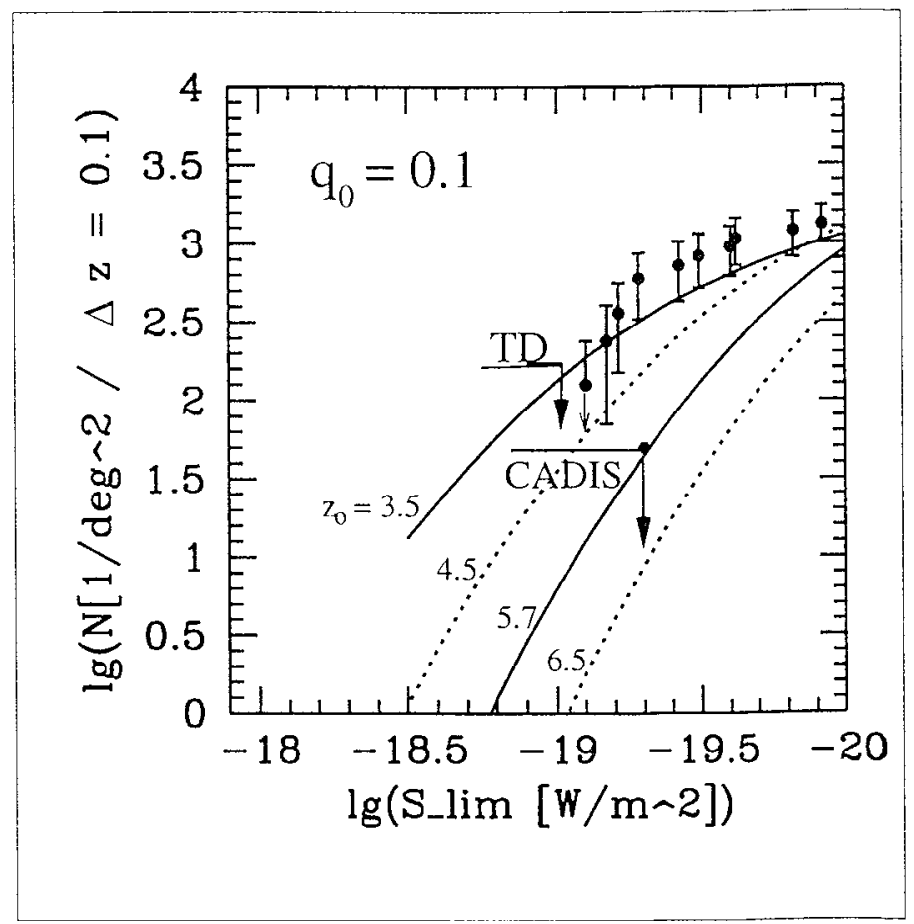

Figure 4. Theoretical models (Thommes \& Meisenheimer 1999, in preparation) for the surface density of high- $z$ Ly $\alpha$ emitters as a function of flux and redshift. The plotted points show the fits to the $z=3.43$ emitters (Cowie \& Hu 1998). Arrows show limits from non-detections by earlier long-slit surveys of Thompson \& Djorgovski (1995) and of the current limits for the Fabry-Perot surveys by the CADIS group (Thommes et al. 1998). The main qualitative points to note are: (1) the surface density of emitters falls off dramatically as a function of limiting survey flux and (2) reaching the redshift $z>6$ population critically requires getting down to below $10^{-17} \mathrm{erg} \mathrm{cm}^{-2} \mathrm{~s}^{-1}$. At current sensitivities coverage of fairly wide areas is desired.

away from identified sources, since in the deep exposures magnitude limits are set by the background faint source population (Fig. 2). The measured continuum above the break, $25.5 \mathrm{mags}(\mathrm{AB})$, is a magnitude higher than the Weymann et al. (1998) observed estimate for HDF 4-473.0, and lies between our estimated continuum magnitudes of 24.9 and 25.7 (based on our deep $Z$ band observations of the HDF) for the 3-951 pair. SSA22-HCM1 is the brightest of the $z=5.7$ Ly $\alpha$ emitters surveyed to date, and in contrast to 3-351 has strong emission. However, it appears that more typical emitters at these redshifts have properties like 4-473.0 ( $\mathrm{Hu}$ et al. 1999, in preparation). We can summarize the expected properties for L $y \alpha$-emitting galaxies above $z \sim 6$, based on the current $z \sim 5 . \bar{\imath}$ survey, as: Ly $\alpha$ fluxes $\sim 10^{-17} \mathrm{ergs} \mathrm{cm}^{-2} \mathrm{~s}^{-1}$, equivalent widths of a few $100 \AA$, and continuum magnitudes fainter than $\sim 26.5$.

Efforts to model the high-redshift emitters have begun (e.g., Thommcs 1998, Haiman \& Spaans 1999), and we can use these as a starting point to estimate the 


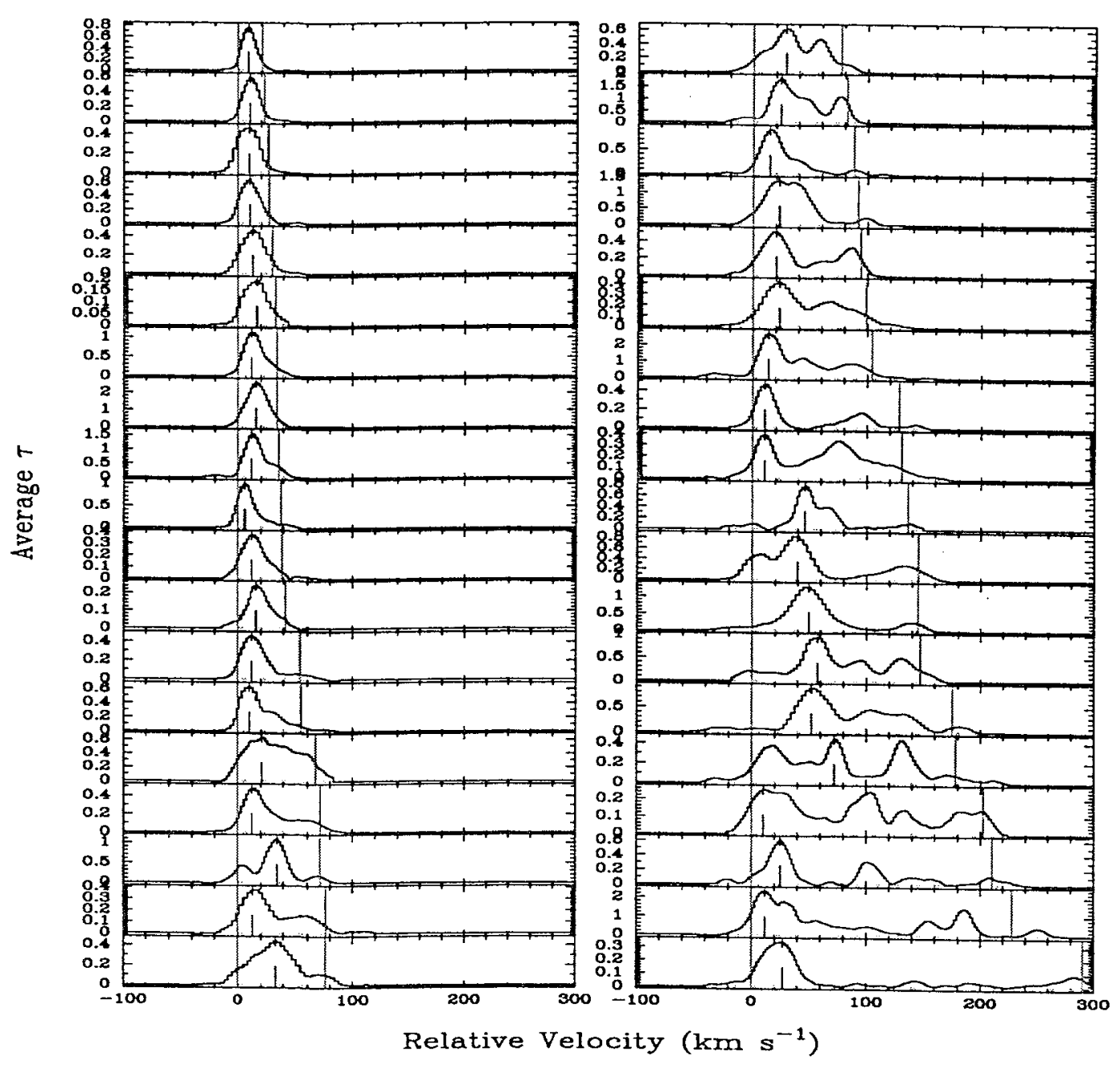

Figure 1. Optical Depth of Low-ion transitions vs. velocity. The data are binned over intervals of $\sim 10 \mathrm{~km} \mathrm{~s}^{-1}$. 
(a) Velocity Test

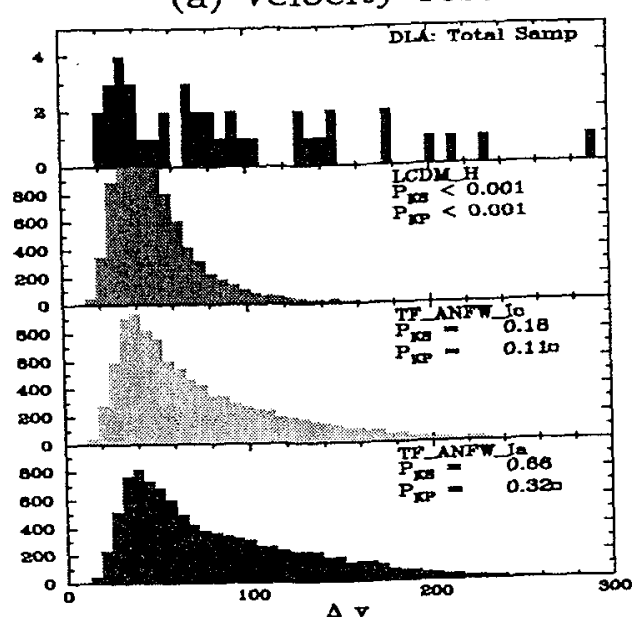

(c) Edge-Leading Test

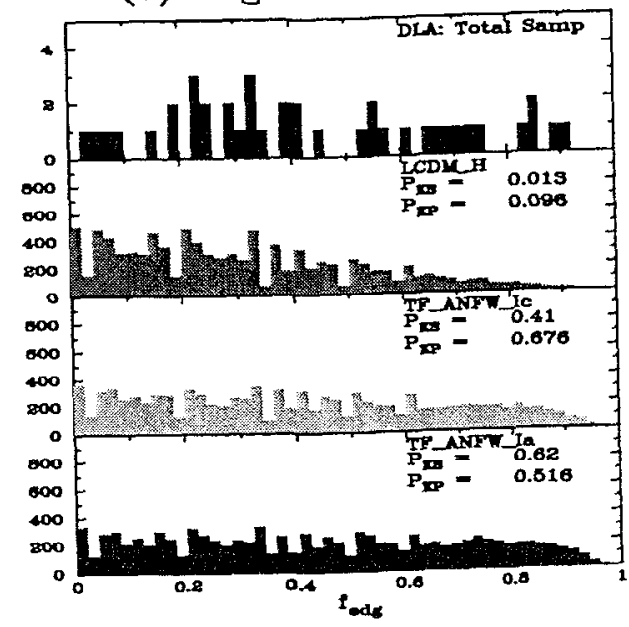

(b) Mean-Median Test

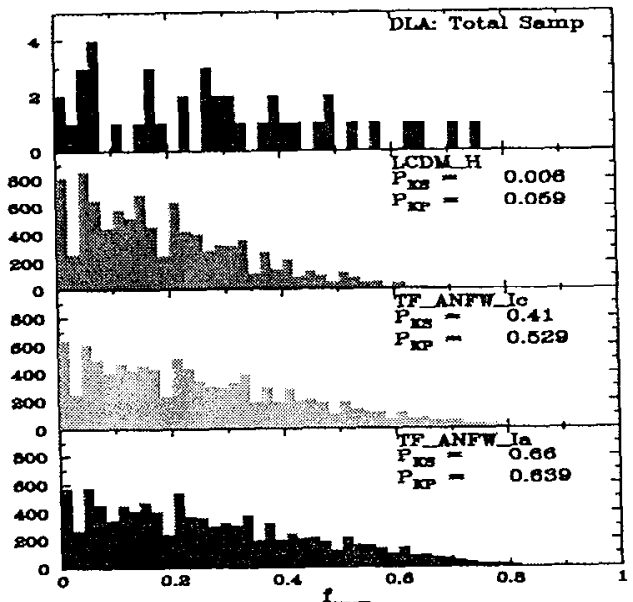

(d) Two-Peak Test

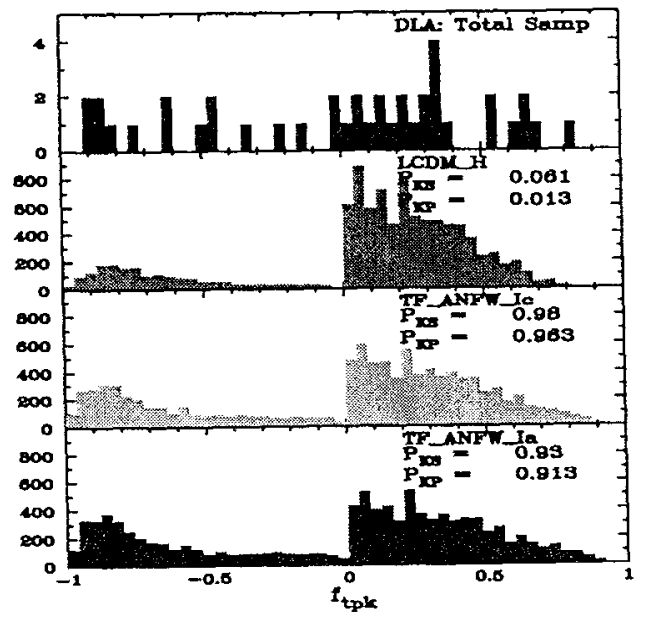

Figure 2. Comparison of empirical and synthetic distributions of test statistics, $\Delta v, f_{m m}, f_{e d g}$, and $f_{t p k}$ (Prochaska \& Wolfe 1997). The empirical distributions are in the top panel in every case. The model parameters for LCDM, TFc, and TFa are $\Omega_{M}=0.3, \Omega_{\Lambda}=0.7, h=$ 0.7 , and $\sigma_{8}=1.0$ for the CDM models. The input $V_{\text {vir }}$ vary between 30 and $300 \mathrm{~km} \mathrm{~s}^{-1}$ for the LCDN and TFC models and 100 and 300 $\mathrm{km} \mathrm{s}^{-1}$ for TFa model.

distributions are drawn from the same parent population. For this and other CDM models we combined the input power spectra with Press-Schecter theory to generate cross-section weighted distributions of $V_{v i r}$. Despite the interception of larger gradients in rotation speed than in the Kauffmann (1996) models, none of the "adiabatic" CDN models reproduce the $\Delta v$ distribution. Late formation of high-mass halos still puts too much power in slowly rotating disks. While the 
non-Gaussian character of the linear density field in isocurvature models (Peebles 1999) results in earlier formation of massive halos, the fraction of high $V_{\text {vir }}$ is still too low to reproduce the observed $\Delta v$ distribution. The only plausible model incorporating single rotating disks is the null hypothesis which assumes the disks of present galaxies to be in place at redshifts higher than observed for damped Ly $\alpha$ systems. In this case the $V_{\text {vir }}$ are inferred from the Tully-Fisher relationship with an assumed rotation curve. In all models the lower limits on $V_{v i r}$ are set by the masses below which gas escapes from dark-matter halos. Outflow is driven by pressure exerted by photoionized gas (Kepner et al. 1997) in the $\Lambda C D M$ and TFC models, and by supernova blast waves in the TFa model (Dekel \& Silk 1986). Notice that models compatible with the $\Delta v$ test are also compatible with the asymmetry tests.

\section{High Ions vs. Low Ions}

\subsection{Data}

I am currently focusing on the kinematics of ionized gas in damped Ly $\alpha$ systems. Ionized gas is important because prior to dissipative collapse, gas in protogalaxies was mainly ionized. During the merger events predicted by CDM, baryons are shock heated to $T \sim 10^{5}$ to $10^{6} \mathrm{~K}$, the virial temperatures of halos destined to become normal galaxies. Subsequently the hot gas cools, producing a two-phase medium of cooler $\left(T \sim 10^{4} \mathrm{~K}\right)$ denser clouds in pressure equilibrium with the low-density hot gas. Gas in both phases is ionized. As it cools the hot gas undergoes an inward-cooling flow at velocities far below the adiabatic sound speed, while the clouds experience rapid radial infall at supersonic speeds (Mo \& Miralda Escudé 1996). Because the cloud infall velocities are influenced by the masses of the dark-matter halos, studies of cloud kinematics may help to determine the halo mass distribution. Such studies are feasible because these clouds are predicted to be photoionized and to exhibit detectable absorption by $\mathrm{C}^{+3}$ and $\mathrm{Si}^{+3}$ ions (Mo \& Miralda-Escudé 1996)

We have collected a sample of C IV 1548 1550, Si IV 1393 1402, and Al II 18541862 HIRES velocity profiles for damped systems in which we have acquired low-ion profiles (Wolfe \& Prochaska 1999). Figure 3 presents an example of our findings. Here I compare C IV and low-ion velocity profiles for a sample of 32 damped Ly $\alpha$ systems. The profiles leave the following impressions: (1) In common with the low ions, the C IV profiles exhibit a multi-component structure comprising several narrow components spanning a wide range of velocity intervals. (2) The C IV and low-ion profiles appear to be kinematically disjoined. In many cases strong C IV components are at velocities at which low-ion absorption is weak or absent. In other cases, C IV absorption is weak or absent at velocities where strong low-ion absorption components are present. (3) Despite their differences, the C IV and low-ion velocity profiles overlap in velocity space in such a way that the low-ion profiles generally lie within the high-ion profiles. We also find (4) the C IV and Si IV profiles appear to be strongly correlated, and (5) the Al III and low-ion profiles appear to be strongly correlated. (6) In regions of velocity space where C IV absorption is clear of low-ion absorption. no Al III absorption is detected. 


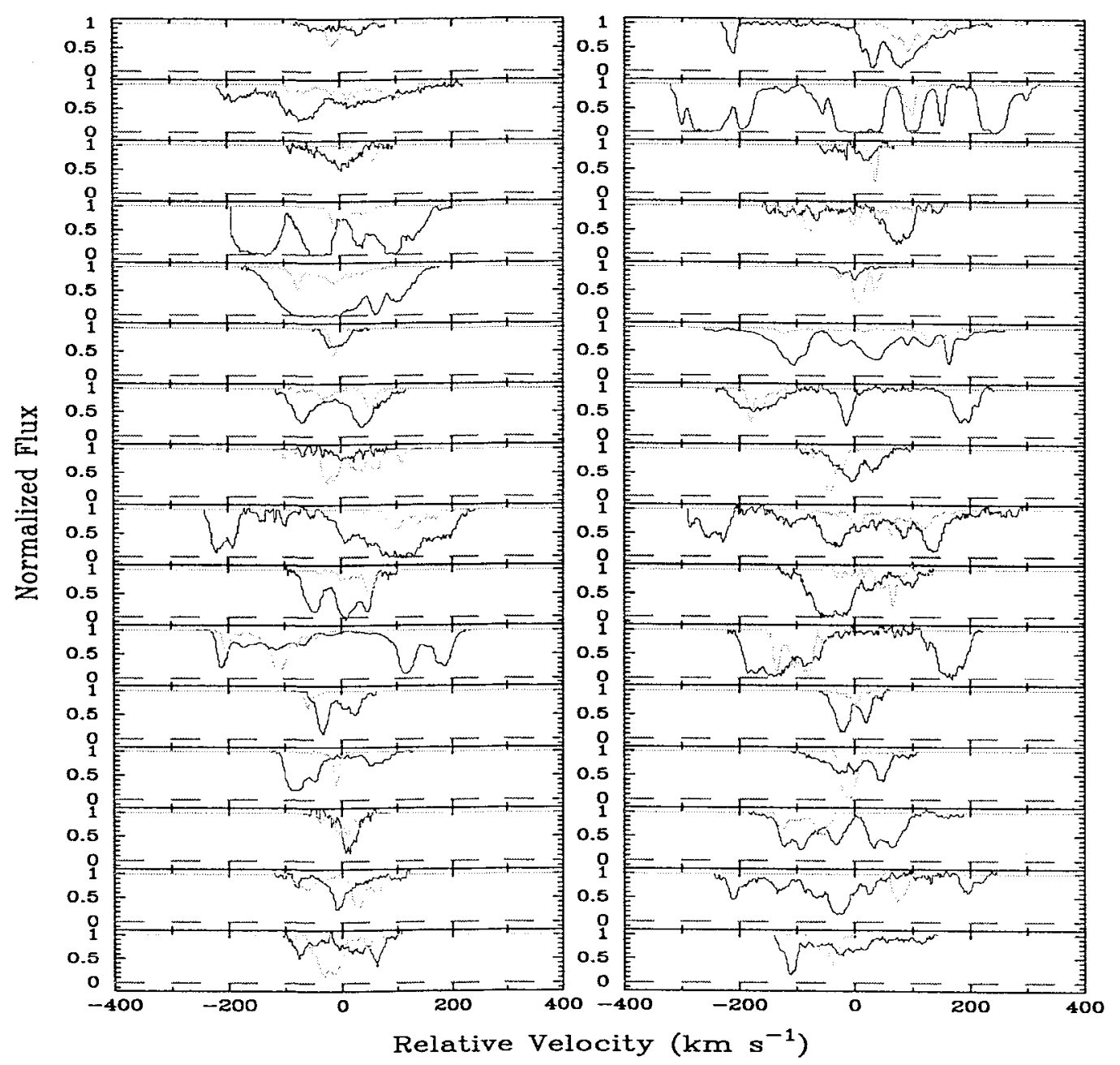

Figure 3. Comparison between C IV 1548 or 1551 (solid lines) and Low-ion profiles (dotted lines) in 32 damped Ly $\alpha$ systems. 

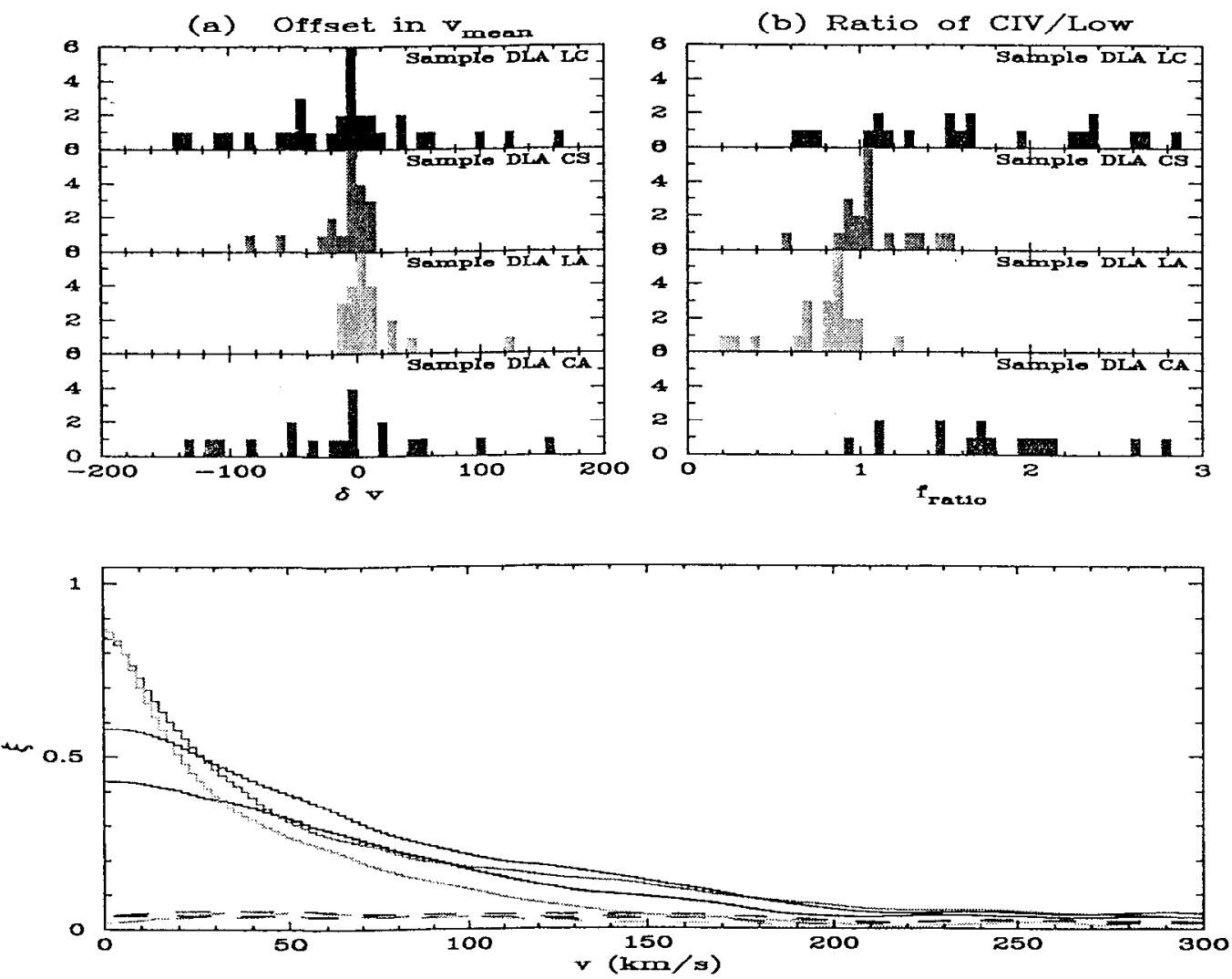

Figure 4. (a) Distribution of difference in mean velocities of the $\mathrm{C}$ IV vs. low-ion, C IV vs. Si IV, Al III vs. low-ion, and C IV vs. Al III profiles. (b) Distribution of ratio of profile widths, $\Delta v$ for same ion pairs. (c) Cross-correlation functions and $1 \sigma$ errors for same ion pairs. Curves with $\xi(0) \approx 0.85$ are Al III vs. low (light) and C IV vs. Si IV (dark). Curve with $\xi(0) \approx 0.6$ is C IV vs. Al III and $\xi(0) \approx 0.4$ is $\mathrm{C}$ IV vs. low.

The impressions are confirmed by quantitative tests. For example, the probability that the distribution of C IV and low-ion $\Delta v$ are drawn from the same parent population, $P_{K S}=0.002$. This suggests the low-ion and high-ion gas are in distinct kinematic subsystems. Yet tests examining relative properties of the subsystems indicate they are still interrelated. First, consider the gross kinematic properties. Figure ta shows the distribution of $\delta v$, the difference between the means of the (i) low-ion and C IV profiles, (ii) C IV and Si IV profiles, (iii) low-ion and Al III profiles, and (iv) C IV and Al III profiles. Not surprisingly, the distribution widths are narrower for C IV vs. Si IV and Al III vs. low-ion than for C IV vs. low-ion and C IV vs. Al III. This just tells us that the high-ion subsystem incorporates both $\mathrm{Si}^{+3}$ and $\mathrm{C}^{+3}$ ions, but no intermediate ions such as $\mathrm{Al}^{+2}$ which are only associated with the low-ion subsystem. Although the dispersion of the C IV vs. low-ion distribution is relatively high 
$\left(\sigma_{\delta v}=6 T \pm 12 \mathrm{~km} \mathrm{~s}^{-1}\right)$, we shall see that it is low enough to place crucial restrictions on most models. Figure $4 \mathrm{~b}$ shows the distribution of the $f_{\text {ratio }}$, the $\Delta v$ ratios for the same ion pairs. Notice how $f_{\text {ratio }} \equiv \Delta v_{C I V} / \Delta v_{\text {low-ion }} \geq 1$ for 30 out of 32 systems; i.e., the low-ion velocity widths act as a floor to the high-ion velocity widths. While not a correlation, this systematic effect points to a global interaction between the high-ion and low-ion subsystems that must be accounted for by dynamical models of damped Ly $\alpha$ systems. In Figure $4 c$ we plot $\xi(v)$, the cross-correlation functions between the four ion pairs, where $v$ is lag velocity. These functions measure the correlation between the detailed velocity structures of the profiles. The strong correlation between the low-ion and Al III profiles and C IV and Si IV profiles confirms the rather accurate one-to-one alignment between the velocity components of these ion pairs. By contrast, $\xi(v)$ for the $\mathrm{C}$ IV and low-ions and C IV and Al III exhibits significantly lower, but statistically significant, amplitudes. The lower amplitudes reflect the misalignment between the low-ion (and Al III) and C IV components in many of the profiles. In this case, correlation amplitude arises from overlap in velocity space between the ion pairs. This interpretation is supported by the $\sim 80 \mathrm{~km} \mathrm{~s}^{-1}$ half-width of the cross-correlation function which more closely resembles the coherence lengths of the large-scale structures in the C IV profiles than the $\sim 30 \mathrm{~km} \mathrm{~s}^{-1}$ half-widths of the individual C IV components.

\subsection{Models}

Wo have applied these tests to models in which halos consist of virialized hot gas in pressure equilibrium with radially infalling cooler clouds that produce detectablc C IV absorption. We compute the C IV velocity profiles by extending sightlines that penetrate low-ion disks (see $\S 2.1$ ) through the halo gas. None of the models satisfics all the tests. In Figure 5 we compare empirical and model distributions of $\Delta v$ for the C IV lines (the asymmetry test statistics are not crucial for the high ions). The ACDM model ( 5 a) fails because the median $\Delta v$ is low compared to the data while the TFa model ( $5 \mathrm{c}$ ) fails because it is too high. Only the TFc model (5b) works.

In Figure $6 a$ we compare the $\delta v$ distributions for $\mathrm{C}$ IV relative to the lowions. In this case the $\Lambda \mathrm{CDM}$ model succceds while the other models fail: although the deeper potential wells of the TF model produce the required large $\Delta v$, they also produce more rapidly rotating disks which result in $\delta v$ distributions that are too wide. The $f_{\text {ratio }}$ tests are shown in Figure $6 \mathrm{~b}$. Although the TFC model cannot be ruled out entirely, none of the models work very well because they predict median $f_{\text {ratio }}$ that are too large. Figure $6 \mathrm{c}$ shows that none of the models is compatible with the empirical cross-correlation functions. In every case there is too much misalignment between low-ion and high-ion velocity components.

\section{Conclusions}

The accurate HIRES velocity profiles have provided the first view of gas kinematics in protogalaxies. Althongh a fully self-consistent. picture has not emerged, the value of the kinematic studies is that they identify the ingredients of a successful model. The first such ingredient is the assumption that gravity dominates gas 


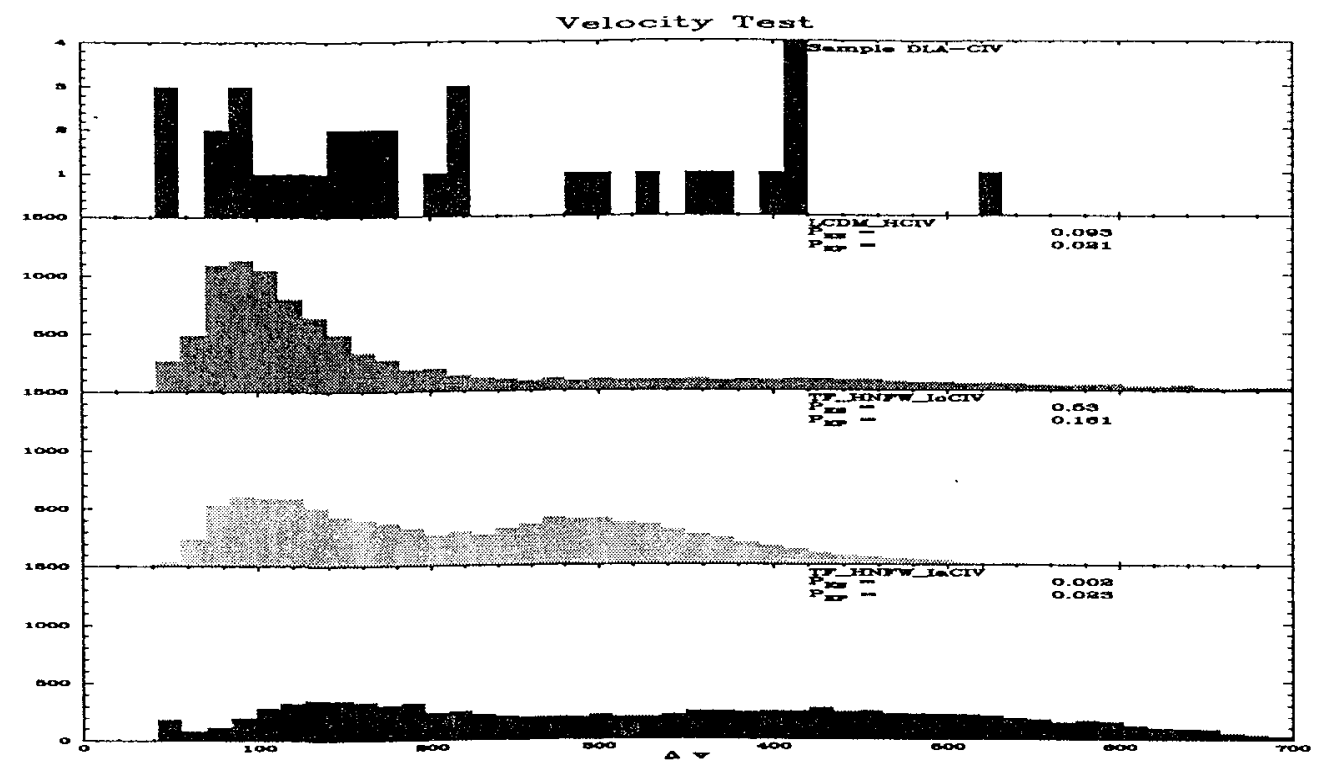

Figure 5. Comparison between model and empirical distributions of test statistics. Models are the same as in Fig. 2. $P_{K S}=0.093,0.53$, and 0.002 for $\Lambda \mathrm{CDM}, \mathrm{TFc}$, and TFa models respectively

motions in damped Ly $\alpha$ systems. Acceleration of ionized gas by the same gravitational field accelerating neutral gas is a natural way to explain the coupling between the two kinematic subsystems. By contrast, we find that explosive motions from single or multiple sources do not reproduce the coupling in a natural way. Thus, as in the ISM, feedback due to star formation, while undoubtedly present, should contribute little to the resultant velocity field. Otherwise systematic effects such as the relatively low $\sigma_{\delta v}$, the ratio $\Delta v_{C I V} / \Delta v_{l o w} \geq 1$, and statistically significant $\xi$ for $C$ IV vs. low-ions are difficult to reproduce. A promising mechanism for coupling the subsystems is to include angular momentum of the radially infalling C IV clouds: the differences between low-ion and high-ion velocities decrease as the clouds spin up and approach co-rotation with the disk. As a result $\sigma_{\delta v}$ might decrease and $\xi$ might increase to acceptable levels. This mechanism might also reduce the $\Delta v_{C I V} / \Delta v_{l o w}$ ratios which presently are too large.

The production of large $\Delta v$ for both low and high ions is a second ingredient for a successful model. This is naturally achieved by radial infall of ionized gas and rotation of neutral gas in the deep potential wells of the TF model. Despite the large $\Delta v$, the $\sigma_{\delta v}$ and $\Delta v_{C I V} / \Delta v_{\text {low }}$ might reduce to observable values if angular momentum of the radially infalling gas is included as discussed above. Another way to increase $\Delta v$ of the low ions is to let the infalling gas add to the $\Delta v$ contributed by the rotating disk. This might be achieved if the ionization level of the infalling gas decreases as it falls to the disk. However. the lowamplitude of the cross-correlation function for C IV vs. AI III profiles implies the C IV gas does not contain ions at significantly lower ionization levels; i.e. the gas is optically thin at the Lyman limit. As a result the infalling gas wonld 

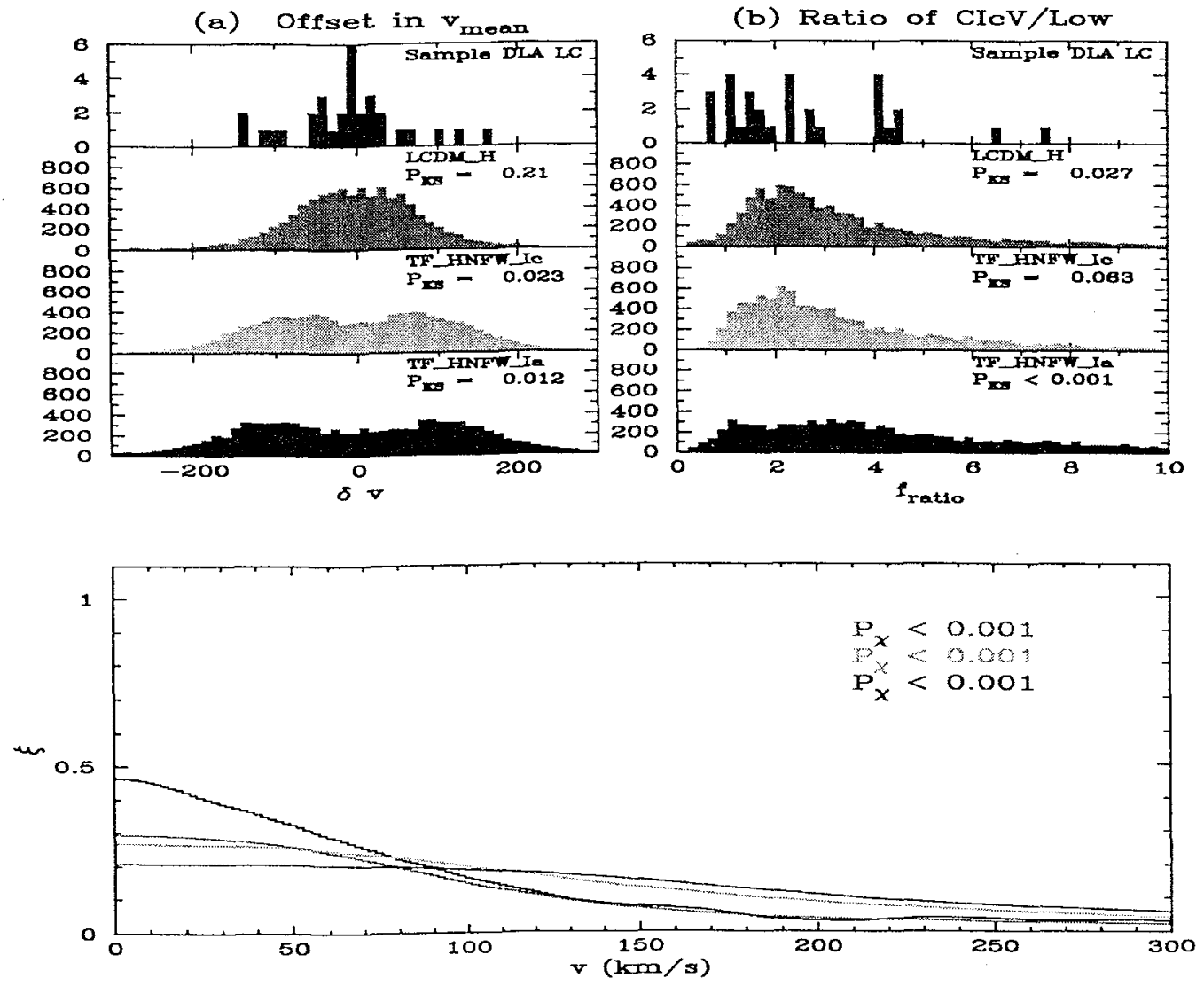

Figure 6. Comparison between model and empirical distributions of the $\Delta v, f_{\text {ratio }}$, and $\xi_{a b}$ statistics for C IV vs. low-ions. $P_{\chi}$ are $\chi^{2}$ probabilities for the model fits to empirical $\xi$. Models are the same as in Fig. 2. $\xi$ data is dark curve in Figure 6c 
be highly ionized until it merged with low-ion gas in the disk, and thus could not contribute to the low-ion $\Delta v$.

We are exploring other ideas. Haehnelt et al. (1998) describe how large $\Delta v$ might also be attained when sightlines through damped Ly $\alpha$ systems suffer multiple impacts with protogalactic clumps predicted by numerical simulations of CDM. In this case the $\Delta v$ are produced by infall and random motions of separate clumps comprising dark matter and gas rather than by rotation of gaseous disks in dark-matter halos. While multiple impacts may also keep $\sigma_{\delta v}$, $\xi$, and $\Delta v_{C I V} / \Delta v_{\text {low }}$ within observational limits, the challenge is to explain how multiple impacts maintain the edge-leading asymmetry of the low-ion profiles in Figure 1. We (Prochaska, Pen, Shi, \& Wolfe) are using Pen's moving mesh hydrodynamical code (Pen 1998) to re-examine this problem. Using a largr comoving volume than Haehnelt et al. (1998) our simulations should surpass the statistical power of this work which was limited to only eight density clumps. We are also examining the idea of McDonald \& Miralda-Escudé (1999), who obtained agreement with the observed $\Delta v$ and asymmetry of the low-ions by letting the low-ion clouds move randomly in dark-matter halos. They required the low-ion clouds to be at smaller galactic radii than the high-ion clouds. But in that case the latter would have smaller $\Delta v$ than the former, in disagreement with observation. Moreover, the low-ion asymmetry was achieved by modeling the low-ion profiles with only 2 or 3 velocity components, whereas model fitting requires many more. We are performing extensive tests of this hypothesis.

Acknowledgments. T wish to thank my collaborator Jason Prochaska for permission to report on our unpublished results. Partial support was received from NASA grant NAGW-2119 and NSF grant AST 86-942044.

\section{References}

Burkert, A., 1995, ApJ, 447, L25

Dekel, A., \& Silk, J., 1986, ApJ, 303, 39

Haehnelt, M. G., Steinmetz, M., \& Rauch 1998, Ap.J, 495, 647

Kauffmann, G. 1996, MNRAS 281, 475

Kepner, J. V., Babul, A., \& Spergel, D. N. 1997, ApJ, 487, 61

McDonald, P., \& Miralda-Escudé, J., 1999, ApJ, 519,486

Mo, H.J., Mao, S., \& White, S.D.M., 1998, MNRAS, 295, 319

Mo, H.J., \& Miralda-Escudé, J., 1006, Ap.J, 469, 589

Navarro. J. F.. Frenk, C. S., \& White, S. D. M., 1097, Ap.J, 490, 493

Peebles, P. J. E. 1999, ApJ, 510, 531

Pen U. 1998, ApJS, 115, 19

Prochaska, J. X., \& Wolfe, A. M. 1996, Ap.J 474, 140

Prochaskia, J. X., \& Wolfe, A. M. 1997, Ap. 487, 73

Wolfe, A. M. 1998, in Critical Dialogues in Cosmology, ed. N. Turok, Singapore: World Scientific Press, 500.

Wolfe, A. M., \& Prochaska, J. X. 1999, Ap.J. submitted for publication 

The Hy Redshift Universe

ASP Conference Series, Vol. 193, 1999

A. J. Bunker \& W. J. M. van Breugel, eds.

\title{
The First Sources of Light in the Universe
}

\author{
Abraham Loeb \\ Astronomy Department, Harvard University, Cambridge, MA 02138
}

\begin{abstract}
The formation of the first stars and quasars marks the transition between the smooth initial state and the clumpy current state of the Universe. In popular CDM cosmologies, the first sources started to form at a redshift $z \sim 30$ and ionized most of the hydrogen in the Universe by $z \sim 8$. Current observations are at the threshold of probing the reionization epoch. The study of high-redshift sources is likely to attract major attention in observational and theoretical cosmology over the next decade.
\end{abstract}

\section{Preface}

It is a special privilege for me to contribute to the celebration of Hy Spinrad's 65th birthday. Although I am not an observer, I can empathise with the $\mathrm{Hy}-\mathrm{z}$ experience that was described so often at this meeting. About seven years ago, when I started constructing theoretical models for sources at high redshifts, there was little interest in this problem among my fellow theorists, with a few notable exceptions. It now appears in retrospect that I could have made more friends among the observers. Thanks to the pioneering work of $\mathrm{Hy}$ and his colleagues, this field has not only matured over the past several years, but might actually come to dominate the research in cosmology over the next decade.

\section{Introduction}

The detection of cosmic microwave background (CMB) anisotropies (Bennet et al. 1996) confirmed the notion that the present structure in the Universe originated from small density fluctuations at early times. The gravitational collapse of overdense regions could explain the present-day abundance of bound objects, such as galaxies or X-ray clusters, under the appropriate extrapolation of the detected large-scale anisotropies to smaller scales (e.g., Baugh et al. 1997). Recent deep observations with the Hubble Space Telescope (Steidel et al. 1996; Madau et a1. 1996; Chen et al. 1998; Clements et al. 1999) and ground-based telescopes, such as Keck (Lowenthal et al. 1996; Dey et al. 1999; Hu et al. 1998. 1999: Spinrad et al. 1999: Steidel et al. 1999), have constrained considerably the evolution of galaxies and their stellar content at $z \lessgtr 5$. However, in the bottomup hierarchy of the popular Cold Dark Matter (CDM) cosmologies, galaxies were assembled out of building blocks of smaller mass. The elementary building blocks, i.e. the first gaseous objects to have formed, acquired a total mass of 


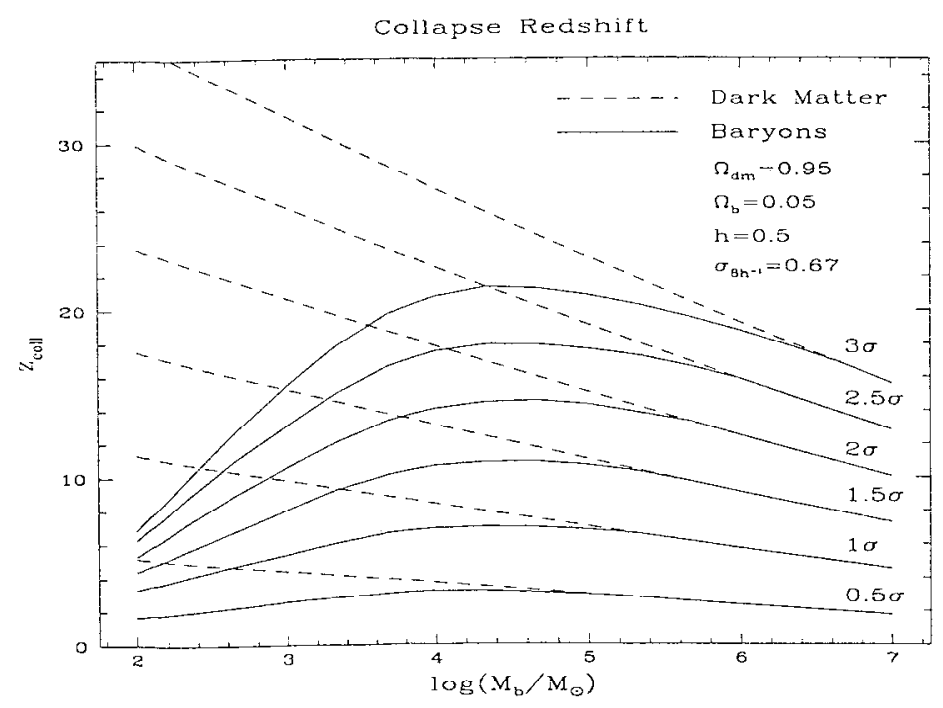

Figure 1. Collapse redshift, $\tilde{z}_{c o l l}$, for cold dark matter (dashed lines) and baryons (solid lines) in spheres of various baryonic masses, $M_{\mathrm{b}}$, and initial overdensities. The overdensities are in units of the rms amplitude of fluctuations $\sigma(M)$ for a standard CDM power-spectrum with $\sigma_{8 h-1}=0.67$. (This cosmological model was chosen only for illustration purposes.) The collapse of the baryons is delayed relative to the dark matter due to gas pressure. The curves were obtained by following the motion of the baryonic and dark matter shells with a spherically symmetric, Lagrangian hydrodynamics code (Haiman \& Loeb 1997).

order the Jeans mass $\left(\sim 10^{6} M_{\odot}\right)$, below which gas pressure opposed gravity and prevented collapse (Haiman \& Loeb 1997; Ostriker \& Gnedin 1997). In variants of the standard CDM cosmology, these basic building blocks formed at $z \sim 10-30$ (see Fig. 1).

The first light from stars and quasars ended the "dark ages" of the Universe and initiated a "renaissance of enlightenment" in the otherwise fading glow of the big bang. It is easy to see why the mere conversion of trace announts of gas into stars or black holes at this early epoch could have had a dramatic effect on the ionization state and temperature of the rest of the gas in the Universe. Nuclear fusion releases $\sim 7 \times 10^{6} \mathrm{eV}$ per hydrogen atom, and thin-disk accretion onto a Schwarzschild black hole releases ten times more energy; however, the ionization of hydrogen requires only $13.6 \mathrm{eV}$. It is therefore sufficient to convert a small fraction of $\sim 10^{-5}$ of the lolal baryonic inass into stars or black holes in order to ionize the rest of the Universe. (The actual required fraction is higher because only some of the emitted photons are above the ionilation threshold of $13.6 \mathrm{eV}$ and because each hydrogen atom could recombine more than once at $\approx z T$ ).

Calculations of structure formation in popular CDM cosmologies imply that the Universe was ionized at $z \sim 8-12$ (Haiman \& Loeb 1998, 1999b,c; Gnedin \& Ostriker 1998). The free electrons produced during reionization scatter the mi- 
crowave backgronnd and smooth its anisotropies on angular scales below the size of the horizon at the reionization epoch $\left(\sim 10^{\circ}\right.$ for reionization at $\left.z \sim 10\right)$. The fractional decrement in the anisotropy amplitude is of order the optical depth of the intergalactic medium to Thomson scattering, i.e. a few percent. The forthcoming MAP and PLANCK satellites will thus be able to constrain the reionization redshift (Zaldarriaga, Seljak, \& Spergel 1997). Secondary anisotropies are also produced during this epoch on smaller angular scales ( $H u$ 1999).

A variety of CDM models that are all consistent with both the COBE anisotropies $\left(z \approx 10^{3}\right)$ and the abundance of objects today $(z-0)$ differ appreciably in their initial amplitude of density fluctuations on small scales. The reionization history of the Universe is determined by the collapse redshift of the smallest objects $\left(\sim 10^{6}-10^{9} \mathrm{M}_{\odot}\right)$ and is therefore ideally suited to discriminate between these models.

\section{Formation of the First Galaxies}

Current observations reveal the existence of galaxies out to redshifts as high as $\approx \sim 6.7$ (Chen et al. 1999; Weymann et al. 1998; Dey et al. 1998; Spinrad et al. 1998; Hu et al. 1998, 1999) or possibly even higher (Clements et al. 1999), and bright quasars out to $z \sim 5$ (Fan et al. 1999). Based on sources for which high resolution spectra are available, the intergalactic medium appears to be predominantly ionized at this epoch, implying the existence of ionizing sources at even higher redshifts (Madau 1999; Madau, Haardt, \& Rees 1999; Haiman \& Loeb 1998, 1999c; Gnedin \& Ostriker 1997).

The Next Generation Space Telescope (NGST), the successor to the Hubble Space Telescope, is scheduled for launch in 2008, and is expected to reach an imaging scnsitivity better than $1 \mathrm{nJy}$ in the infrared. Its main scientific goal is to probe directly the first galaxies (see, http://ngst.gsfc.nasa.gov/ for more details).

How many sources will NGST see? Figure 2 shows the predicted number of quasars and star clusters expected per field of view of NGST, based on semianalytic modeling of a hierarchical CDM cosmology (Haiman \& Loeb 1999c). In this calculation, a fraction of the gas in each dark matter halo forms stars, and a. much smaller fraction assembles into a massive central black hole. The star formation efficiency was calibrated based on the inferred metallicity range of the Ly $\alpha$ forest (Songaila \& Cowie 1996; Tytler et al. 1995) while the characteristic quasar lightcurve was calibrated in Eddington units so as to fit simultanleously the observed luminosity function of bright quasars at $z \sim 2-4$, and the black hole mass function in the local universe (Magorrian et al. 1998). Both populations of sources were extrapolated to high redshifts and low luminosities using the Press-Schechter formalism (for more details, see Haiman \& Loeb 1997, 1998, 1999c).

Typically, there should be of order tens of sources at redshifts $z>10$ per field of view of NGST. The lack of point source detection in the Hubble Deep Field is consistent with a low-mass cutoff for luminous matter in halos with circular velocities $\lesssim 50-75 \mathrm{~km} \mathrm{~s}^{-1}$, due to photoionization heating (Haiman, Madall, \& Loeb 1999). The redshift of early sources can be easily identified 


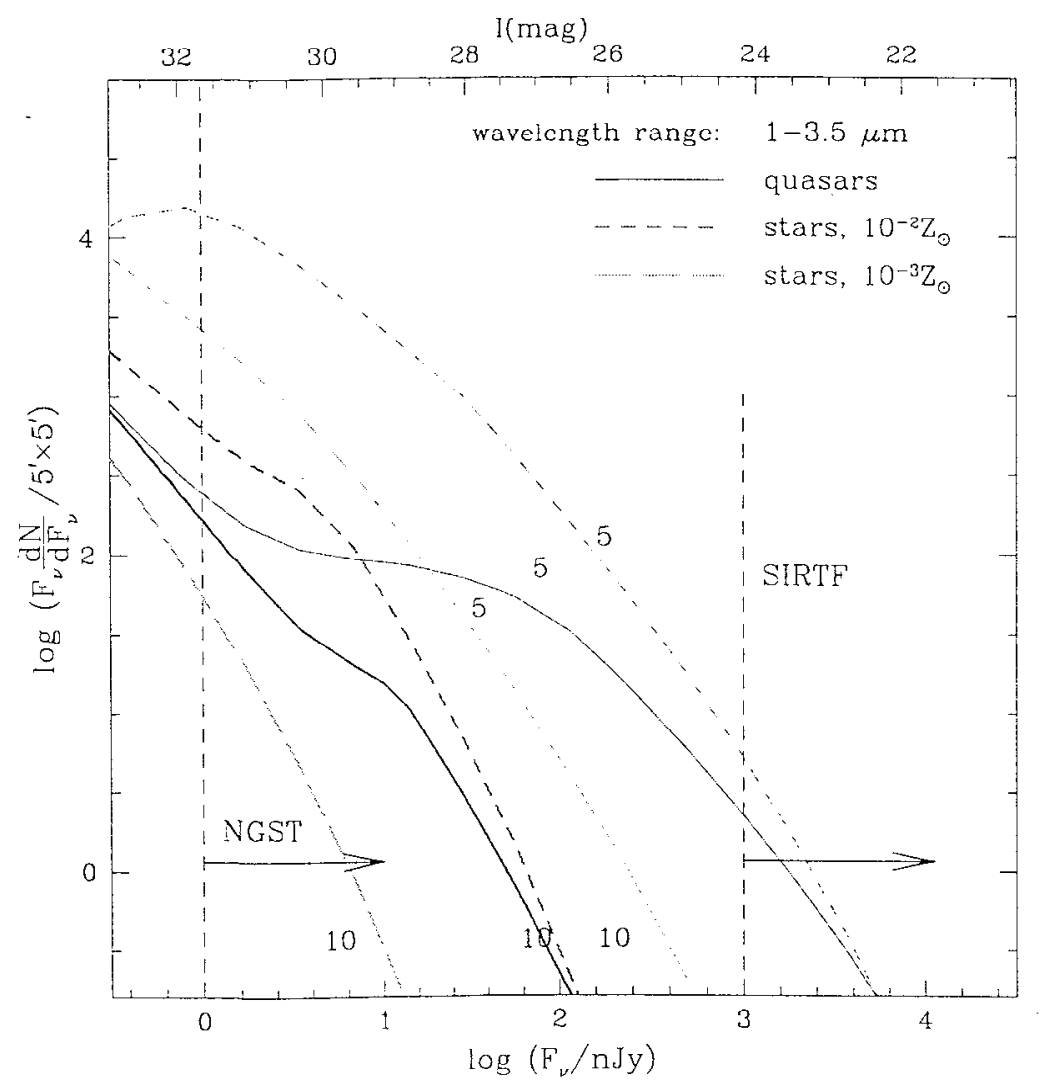

Figure 2. Predicted number counts per $5^{\prime} \times 5^{\prime}$ field of view per logarithmic flux interval in the NGST wavelength range of $1-3.5 \mu \mathrm{m}$. The numbers of quasars and star clusters were calculated for a $\Lambda$ CDM cosmology with $\left(\Omega_{\mathrm{M}}, \Omega_{\Lambda}, \Omega_{\mathrm{b}}, h, \sigma_{8 h^{-1}}, n\right)=$ $(0.35,0.65,0.04,0.65,0.8 T, 0.96)$. The lowest mass scale of virialized baryonic objects was chosen consistently with the photoionization feedback due to the UV background. The star formation efficiency was calibrated so as to bracket the possible values for the average metallicity of the Universe at $z \sim 3$, namely between $10^{-3} Z_{\odot}$ and $10^{-2} Z_{\odot}$. The thick lines, labeled " 10 ", correspond to objects located at redshifts $z>10$, and the thin lines, labeled " 5 ", correspond to objects with $z>5$. The upper labels on the horizontal axis correspond to Johnson I magnitude (from Haiman \& Loeb 1999c). 


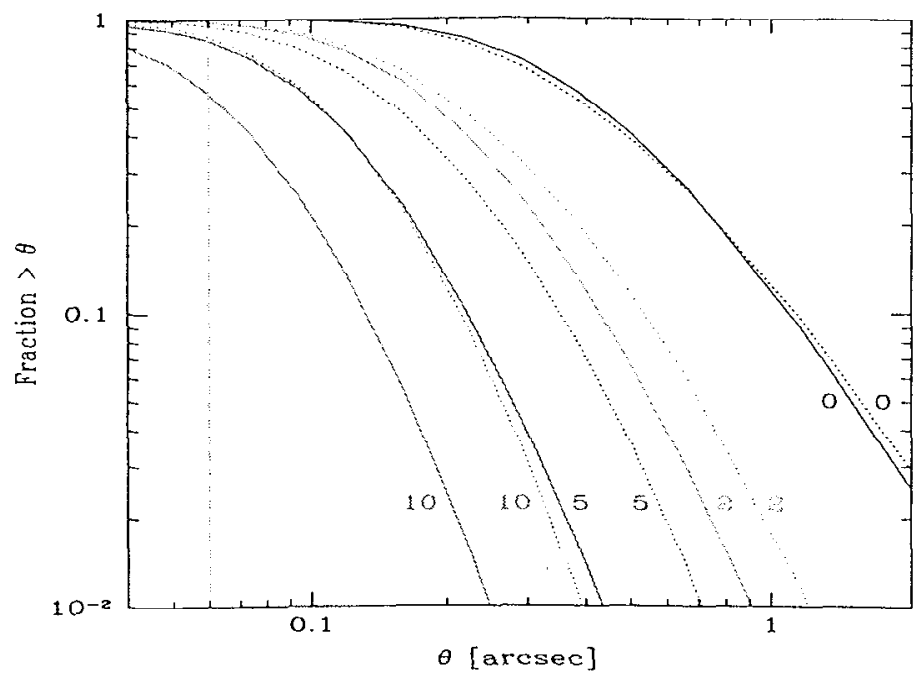

Figure 3. Predicted distribution of galactic disk sizes in various redshift intervals, in the $\triangle$ CDM model. Given $\theta$ in arcseconds, each curve shows the fraction of the total number counts contributed by sources larger than $\theta$. The diameter $\theta$ is measured out to one exponential scale length. The calculation assumed either a high efficiency $(\eta=20 \%$, solid curves) or a low efficiency ( $\eta=2 \%$, dotted curves) of converting cold gas into stars within each galactic disk. Each curve is marked by the lower limit of the corresponding redshift interval. Hence, ' 0 ' indicates sources with $0<z<2$, and similarly for sources with $2<z<5$, $5<z<10$, and $z>10$. All curves include a minimum circular velocity of galactic halos of $V_{\text {circ }}=50 \mathrm{~km} \mathrm{~s}^{-1}$ and a limiting point source flux of $1 \mathrm{nJy}$. The vertical dashed line indicates the NGST resolution of 0'06 (from Barkana \& Loeb 1999b).

photometrically based on thcir Lya trough. Figurc 2 demonstrates that NGST will play a dominant role in exploring the reionization epoch and in bridging between the initial and current states of the Univcrse. Existing telescopes are just starting to probe this epoch now.

The expected size distribution of high-redshift galaxies was calculated semianaly tically by Barkana \& Loeb (1999). Figure 3 shows that most of the galaxies are more extended than the resolution limit of NGST, $\sim 0^{\prime \prime} 06$. Despite the cosmological $(1+z)^{-4}$ dimming in surface brightness, galaxies at $z \sim 10$ are predicted to have an observed surface brightness which is comparable to their $\tilde{z} \sim 3$ counterparts. This follows from the decline in the proper size of galactic disks with increasing redshift (which is caused by the higher density of the Universe and the lower masses of the galaxies at high redshifts). Due to the compactness of high-redshift galaxies, only $\lesssim 1 \%$ of the sky is expected to be covered by galactic disks at $z z 5$ and only $\lesssim 10^{-3}$ by galaxies at $z \gtrsim 10$. Hence, deep high-resolution observations of galaxies at high-redshifts (e.g., with IGST) are not expected to be confusion limited or miss considerable levels of 


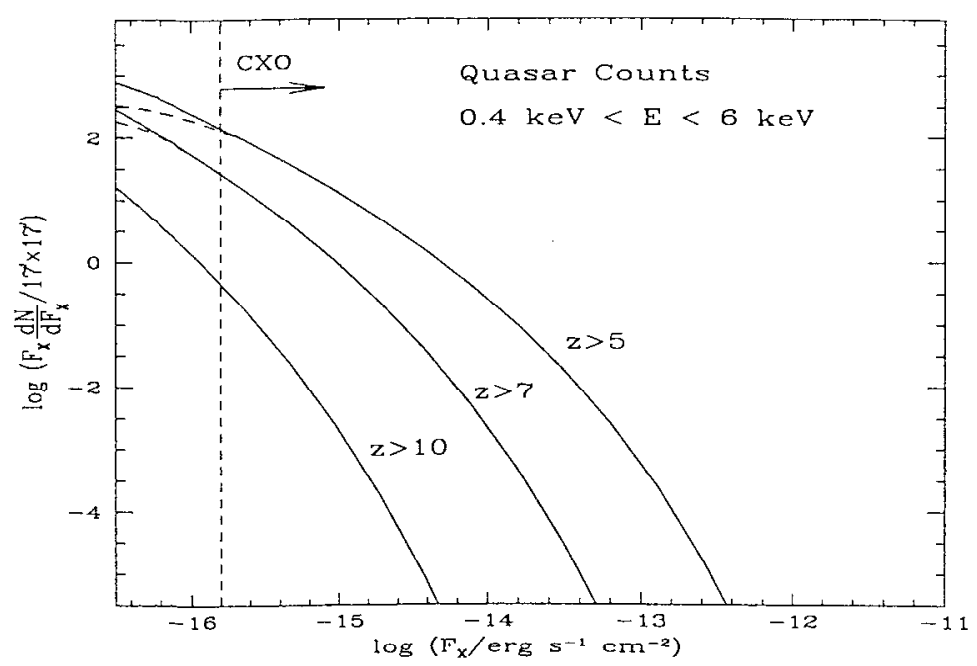

Figure 4. The predicted surface density of quasars with redshift exceeding $z=5, z=\tau$, and $z=10$ as a function of observed X-ray flux in the CXO detection band. The solid curves correspond to a cutoff in circular velocity for the host halos of $v_{\text {circ }} \geq 50 \mathrm{~km} \mathrm{~s}^{-1}$, the dashed curves to a cutoff of $v_{\text {circ }} \geq 100 \mathrm{~km} \mathrm{~s}^{-1}$. The vertical dashed line show the $\mathrm{CXO}$ sensitivity for a $5 \sigma$ detection of a point source in an integration time of $5 \times 10^{5}$ seconds (from Haiman \& Loeb 1999b).

star formation due to surface brightness limitations. The radiation produced by the first sources might however get reprocessed through galactic and intergalactic dust and contribute to the diffuse infrared background (Haiman \& Loeb 1998; Toeb \& Haiman 1997).

Barkana \& Loeb (1999) also predicted that about 5\% of these galaxies will be gravitationally lensed by foreground galaxies. Lensing would bring into view sources which are otherwise below the detection threshold. Lensed sources would be multiply imaged and hence appear to be composed of multiple components; their redshift identification requires sub-arcsecond resolution, since it might be otherwise compromised by blending of background light from the lensing galaxy.

Which sources triggered reionization? It is currently unknown whether the Universe was reionized by quasars or stars at $z \gtrsim 5$. Haiman \& Loeb (1999b) pointed out that quasars can be best distinguished from stellar sources by thcir $\mathrm{X}$-ray emission. Based on simple semi-analytic extension of the observed quasar luminosity function, we have shown that deep X-ray imaging with CXO will likely reveal $\sim 100$ quasars per $17^{\prime} \times 17^{\prime}$ field of view from redshift $\approx z 5$ at the flux threshold of $\sim 2 \times 10^{-16} \mathrm{erg} \mathrm{s}^{-1} \mathrm{~cm}^{-2}$ (see Fig. 4). The redshifts of these faint point-sources could be identified by follow-up infrared observations from the ground or with NGST. By summing-up the UV emission from these $z z 5$ quasars, one could determine whether they triggered reionization. The X-ray selection of these quasars is not influenced by dust obscuration. 
STAGES OF STELLAR REIONIZATIONOFTHE UNIVERSE

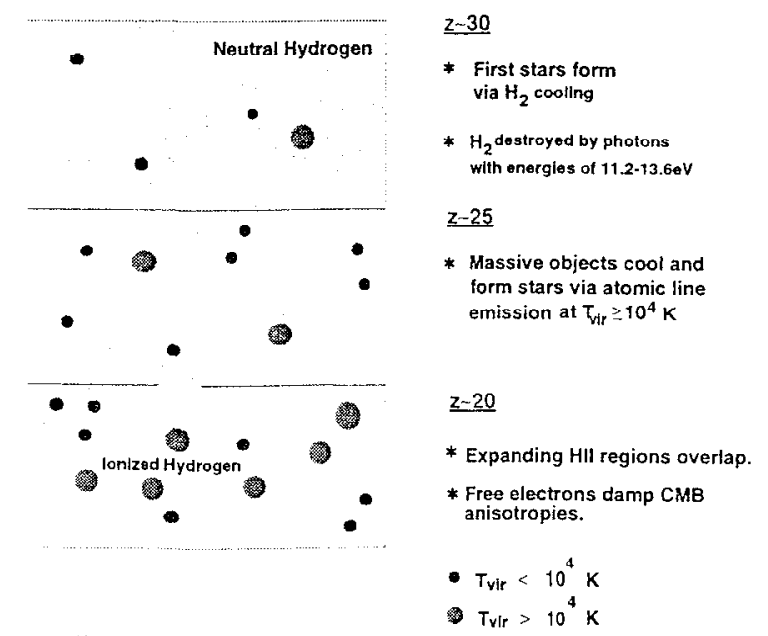

Figure 5.

\section{Feedback on the Intergalactic Medium (IGM)}

\subsection{Reionization}

The stages in the reionization history of the Universe are illustrated schematically in Figure 5. This sequence follows the collapse redshift history of baryonic objects shown in Figure 1, which was calculated with a spherically-symmetric code for the gas and the dark matter dynamics (Haiman, Thoul, \& Loeb 1996). For objects with baryonic masses $23 \times 10^{4} M_{\odot}$, gravity dominates and results in the characteristic bottom-up hierarchy of CDM cosmologies; at lower masses, gas pressure delays the cullapse. The first objects lo collapse are located at the "knee" that separates the above regimes. Such objects reach virial temperatures of several hundred degrees and could fragment into stars only through cooling by molecular hydrogen [see Haiman et al. (1996) or Tegmark et al. (1997), for details regarding the chemistry network leading to the formation of $\mathrm{H}_{2}$ in a primordial gas].

However, molecular hydrogen $\left(\mathrm{H}_{2}\right)$ is fragile and could easily be photodissociated by photons with energies of $11.2-13.6 \mathrm{eV}$, to which the Universe is transparent even before it gets ionized. Haiman, Rees, \& Loeb (1997) showed that a UV flux of $\lesssim 1 \mathrm{erg} \mathrm{cm}^{-2} \mathrm{~s}^{-1} \mathrm{~Hz}^{-1} \mathrm{sr}^{-1}$ is capable of dissociating $\mathrm{H}_{2}$ throughout the collapsed environments in the Universe (see also Haiman, Abel, \& Rees 1999). This flux is lower by more than two orders of magnitude than the minimum flux necessary to ionize the Universe, which amounts to one $\mathrm{CV}^{\mathrm{V}}$ photon per baryon. The inevitable conclusion is that soon after the first stars form, the formation of additional stars due to $\mathrm{H}_{2}$ cooling is suppressed. Further fragmentation is possible only through atomic line cooling, which is effective in objects with high virial temperatures, $T_{\text {vir }} \gtrsim 10^{4} \mathrm{~K}$. Such objects correspond to a total mass $z 10^{8} M_{\odot}[(1+z) / 10]^{-3 / 2}$. Figure 5 illustrates this sequence of events by describing two classes of objects: those with $T_{\text {vir }}<10^{4} \mathrm{~K}$ (small dots) and 


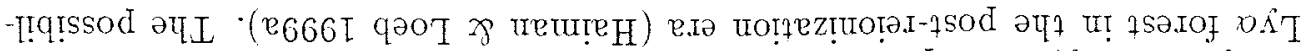

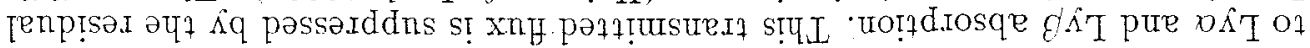

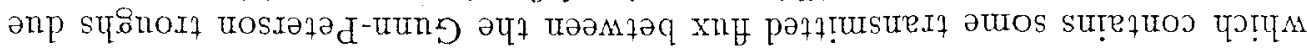

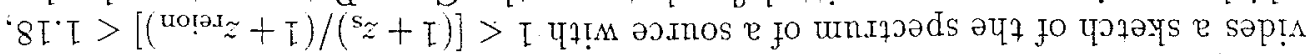

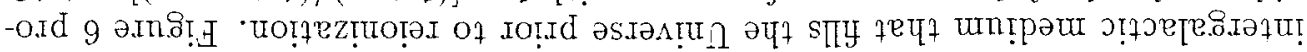

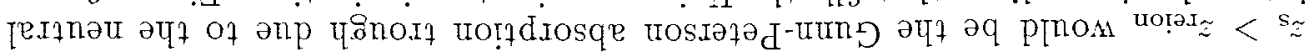

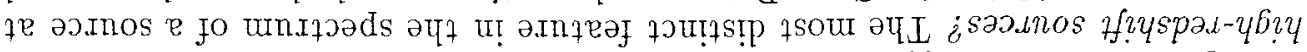

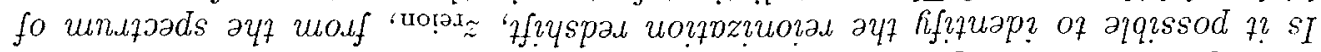

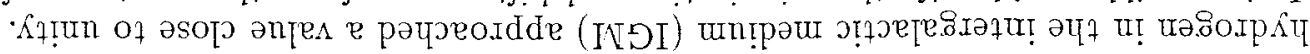

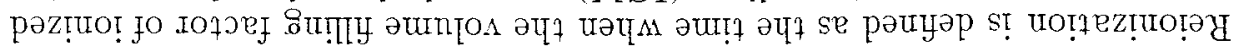

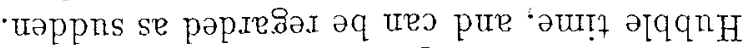

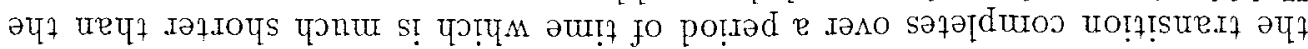

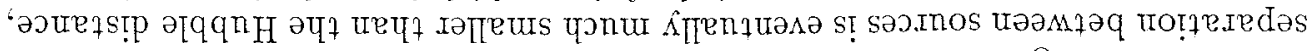

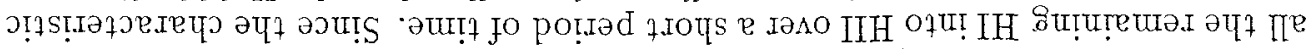

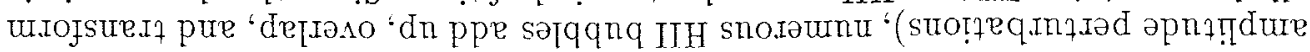

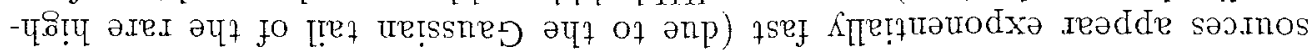

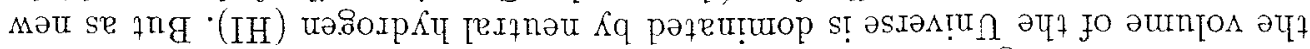

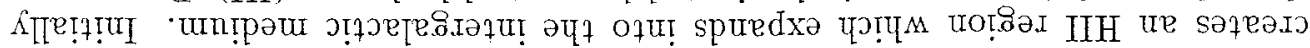

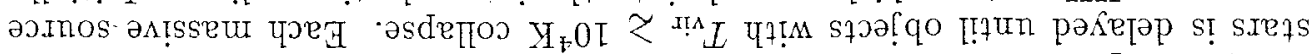

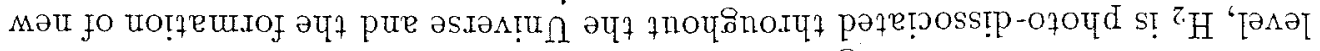

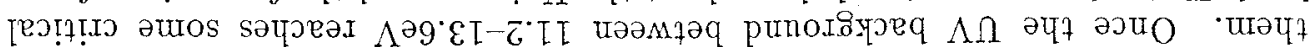

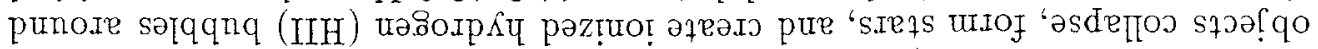

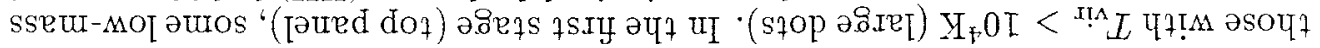

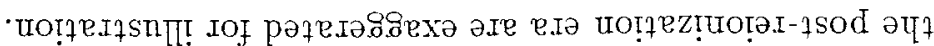

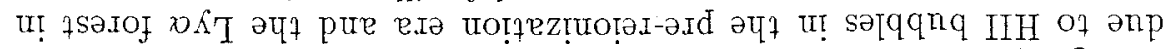

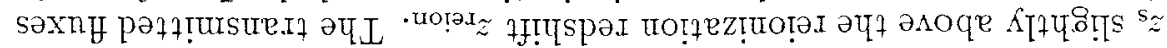

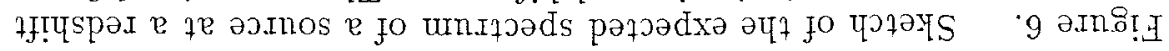
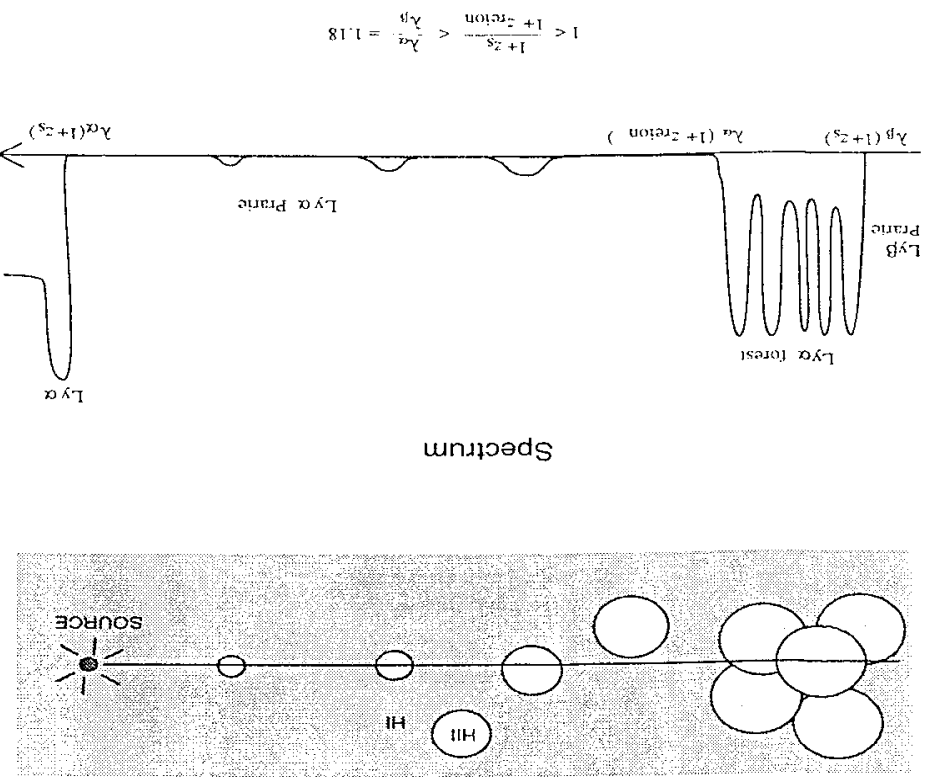

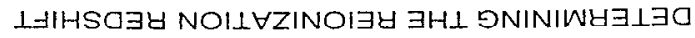




\section{Ly $\alpha$ SOLRCE BEFORE REIONIZATION}
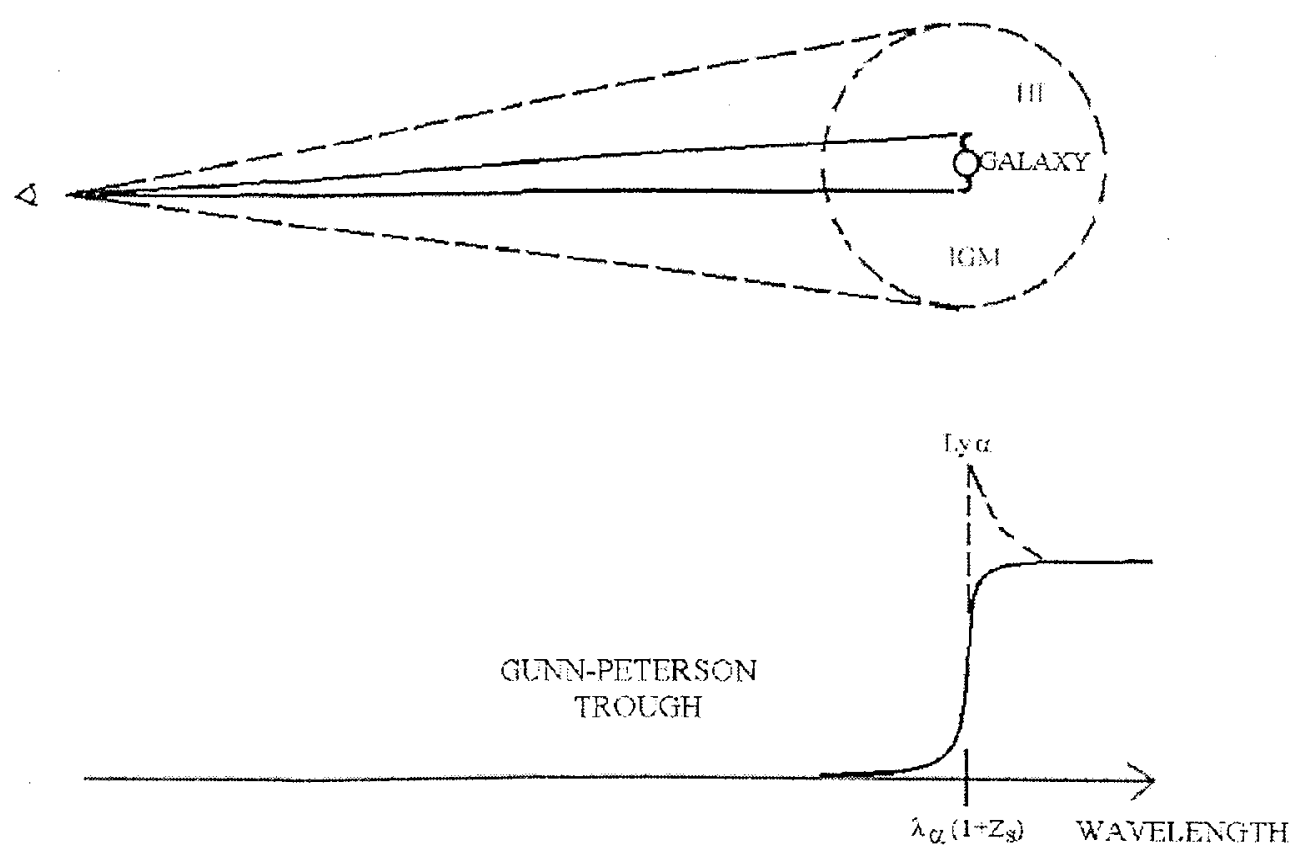

Figure 7 . Scattering of Ly $\alpha$ line photons from a galaxy embedded in the neutral intergalactic medium prior to reionization. The line photons diffuse in frequency due to the Hubble expansion of the surrounding medium and eventually redshift out of resonance and escape to infinity. A distant observer sees a Ly $\alpha$ halo surrounding the source which constitutes an asymmetric line profile. The observed line should be broadened and redshifted by about a thousand $\mathrm{km} \mathrm{s}^{-1}$ relative to other lines (such as, $\mathrm{H}_{\alpha}$ ) emitted by the galaxy (see Loeb \& Rybicki 1999, for quantitative details).

ity of identifying $z_{\text {reion }}$ from the damping wing of the Gunn-Peterson trough (Miralda-Escudé 1997) suffers from potential confusion with damped Ly $\alpha$ absorption along the line of sight and from ambiguities due to peculiar velocities and the proximity effect. An alternative method makes use of the superposition of the spectra of many sources. In the absence of the Ly $\alpha$ forest this superposition should result in the sawtooth template spectrum (Haiman, Rees, \& Loeb 1997).

The reionization redshift can also be inferred from a direct detection of intergalactic HI. Loeb \& Rybicki (1999) have shown that the existence of a neutral IGM before reionization can be inferred from narrow-band imaging of embedded Ly $\alpha$ sources. The spectra of the first galaxies and quasars in the Eniverse should be strongly absorbed shortward of their rest-frame Ly $\alpha$ wavelength by neutral hydrogen in the intervening intergalactic medium. However, the Ly $\alpha$ 
line photons emitted by these sources are not eliminated but rather scatter until they redshift out of resonance and escape due to the Hubble expansion of the surrounding intergalactic HI (see Fig. 7 ). Typically, the Ly $\alpha$ photons emitted by a source at $\tilde{z}_{\mathrm{s}} \sim 10$ scatter over a characteristic angular radius of $\sim 15^{\prime \prime}$ around the source and compose a line which is broadened and redshifted by $\sim 10^{3} \mathrm{~km} \mathrm{~s}{ }^{1}$ relative to the source. The scattered photons are highly polarized (Rybicki \& Loeb 1999). Detection of the diffuse Ly $\alpha$ halos around high redshift sources would provide a unique tool for probing the neutral intergalactic medium before the epoch of reionization. The Lyo sources serve as lampposts which illuminate the surrounding HI fog. On sufficiently large scales where the Hubble flow is smooth and the gas is neutral, the Ly $\alpha$ brightness distribution can be used to determine the cosmological mass densities of baryons and matter. NGST might be able to detect the Ly $\alpha$ halos around sources as bright as the galaxy discovered by $\mathrm{Hu}$ et al. (1999) at $z=5.74$, even if such a galaxy is moved out to $z \sim 10$.

Loeb \& Rybicki (1999) explored the above effect for a uniform, fully-neutral IGM in a pure Hubble flow. It would be useful to extend their analysis to more realistic cases of sources embedded in an inhomogeneous IGM, which is partially ionized by the same sources. One could extract particular realizations of the perturbed IG.M around massive galaxies from hydrodynamic simulation, and apply a suitable radiative transfer code to propagate the Ly $\alpha$ photons from the embedded galaxies. Observations of Ly $\alpha$ halos could in principle be used to map the pecluiar velocity and density fields of the neutral IGM during the reionization epoch.

\subsection{Metal Enrichment}

In addition to altering the ionization state of hydrogen in the Universe, the first galaxies enriched the IGM with metals. Because the potential wells of the first dwarf galaxies are relatively shallow $\left(\sim 10 \mathrm{~km} \mathrm{~s}^{-1}\right)$, supernova-driven winds are likely to have expelled the metal-rich gas out of these systems and mixed it with the intergalactic medium. Incomplete mixing could have led to the observed order-of-magnitude scatter in the $\mathrm{C} / \mathrm{H}$ ratio along lines-of-sight to different quasars (Rauch, Haehnelt, \& Steinmetz 1997; Hellsten et al. 1998). It is an interesting coincidence that the supernova energy output associated with a metal enrichment of $\sim 1 \% Z_{\odot}$ corresponds to $\sim 10 \mathrm{eV}$ per hydrogen atom, which is just above the binding energy of these early star clusters. Supernova feedback in these objects could have therefore dictated the average metallicity observed in the Ly $\alpha$ forest. Direct observations of these supernovae might be feasible in the future (Miralda-Escudé \& Rees 1997).

The rise of the UV background during reionization is also expected to boil the gas out of shallow potential wells. Barkana \& Loeb (1998) have shown that a dominant fraction of the virialized gas in the Universe at $z \sim 10$ will likely reside in potential wells with circular velocity of $\lesssim 15 \mathrm{~km} \mathrm{~s}^{-1}$ and evaporate shortly after reionization. This process could also enrich the intergalactic medium with metals.

Acknowledgments. I thank my collaborators, Rannan Barkana, Zoltan Haiman, Martin Rees, and George Rybicki for many illuminating discussions on 
the topics described in this review. This work was supported in part by the NASA grants NAG 5-7039 and NAG 5-7768.

\section{References}

Barkana, R., \& Loeb, A. 1999a, ApJ, in press, astro-ph/9901114

Barkana, R., \& Loeb, A. 1999b, ApJ, submitted, astro-ph/9906398

Baugh, C. M., Cole, S., Frenk, C. S. \& Lacey, C. G. 1997, astro-ph/9703111

Bennett, C. L. et al. 1996, ApJ, 464, L1

Chen, H.-W., Lanzetta, K., \& Pascarelle, S. 1999, Nature, 398, 586

Dey, A., et al. 1998, ApJ, 498, L93

Ean, X. et al. (SDSS collaboration) 1999, AJ, in press, astro-ph/9903237

Gnedin, N. Y., \& Ostriker J. P. 1997, ApJ, 486, 581

IIaiman, Z.. \& Loeb, A. 1997, ApJ, 483, 21

Haiman, Z., \& Loeb, .A. 1398, ApJ, 503, 505

Haiman, Z., \& Loeb, A. 1999a, ApJ, in press, astro-ph/9807070

$$
\text { - 1999b, ApJ, in press, astro-ph/9904340 }
$$

1999c, in "After the Dark Ages: When Galaxies Were

Young (the Universe at $2<z<5$ )", Eds. S. S. Holt \& E. P. Smith, AIP

Conf. Proc. 470, (AIP: New-York), pp. 34-47, astro-ph/9811395

Maiman, Z., Abel, T., \& Rees, M. J. 1999, ApJ, submitted, astro-ph/9903336

Haiman, Z., Madau, P., \& Loeb, A. 1999, ApJ, 514, 535

Haiman. Z., Thoul, A., \& Loeb, A. 1996, ApJ, 464, 523

Haiman, Z.. Rees, M. J., \& Loeb, A. 1996, ApJ, 467, 522

- $1997, \mathrm{ApJ}, 476,458$

Ilellsten, U., Hernquist, L., Katz, N., \& Weinberg, D. II., 1998, ApJ, 499,172

Hu, W. 1999, ApJ, submitted, astro-ph/9907103

Hu, E. M., Cowie, L. L. \& McMahon, R. G. 1998, ApJ, 502, L99

Hu, E. M., McMahon, R. G., \& Cowie, L. L. 1999, astro-ph/9907079

Loeb, A., \& Haiman, Z. 1997, ApJ, 490, 571

Loeb, A., \& Rybicki, G. 1999, ApJ, in press, astro-ph/9902180

Lowenthal, J. D., Koo, D. C., Guzman, R., Gallego, J., Phillips, A. C., Faber, S. M., Vogt, N. P., Ellingworth, G. D., \& Gronwall, C. 1996. Ap.J, in press, astro-ph/9612239

Madau, P. 1999, in Physica Scripta, Proceedings of the Nobel Symposium. Particle Physics and the Universe (Enkoping, Sweden, August 20-25, 1998). astro-ph/9002228

Madau, P.. Ferguson, H. C.. Dickinson, M., Giavalisco, M., Steidel, C. C., \& Fruchter, A. 1996. MNRAS, 283. 1388

Wadan, P., Haardt, F., \& Rees, M. J. 1999, ApJ, 514, 648

Magorrian. J., et al. 1998, AJ. 115, 2285

Viralda-Escudé, J. 1997. ApJ, 501, 15 
Miralda-Escudé, J., \& Rees, M. 1997, ApJL, 478, L57

Ostriker, J. P., \& Gnedin 1996, N. Y. 1996, 472, L63

Rauch, M., Haehnelt, M. G. \& Steinmetz M. 1997, ApJ, 481, 601

Rybicki, G., \& Loeb, A. 1999, ApJL, in press, astro-ph/9903291

Songaila, A., \& Cowie, L. L. 1996, AJ, 112, 335

Spinrad, H. et al. 1998, AJ, 116, 2617

Steidel, C. C., Giavalisco, M., Dickinson, M., Adelberger, K. L. 1996, AJ, 112, 352

Tegmark, M. et al., 1997, Ap.J, 474, 1

Tytler, D. et al. 1995, in QSO Absorption Lines, ed. G. Meylan ed., Springer, p. 289

Weymann, R. J., et al. 1998, Ap.J, 505, L95

Zaldarriaga, M., Spergel, D. N., \& Seljak, U. 1997, ApJ, 488, 1 
The Hy Redshift Universe

ASP Conference Series, Vol. 193, 1999

A. J. Bunker 8 W. J. M. van Breugel, eds.

\title{
Reionization of the Universe
}

\author{
Nick Gnedin \\ University of Colorado at Boulder
}

\begin{abstract}
Numerical simulations of the cosmological reionization which for the first time incorporate the full 3D radiative transfer arc prescnted. The implications of the reionization for the Lyman- $\alpha$ forest at lower redshifts are discussed.
\end{abstract}

\section{Introduction}

Reionization is the single most important event in the thermal history of the universe since recombination at $z \sim 1200$. Its aftermath determines the properties of the intergalactic medium (IGM) at lower redshifts, which manifests itself in the Lyman-alpha forest of numerous absorption lines.

Thus, understanding how reionization proceeds and what features it imprints in the IGM is of utmost importance for studying the evolution of the IGM at lower redshifts.

Progress in understanding reionization in recent years focused along two separate approaches. Semi-analytical models (of which Valageas \& Silk 1999 is the most comprehensive study up to date) attempt to describe the evolution of ionizing radiation based on ad hoc assumptions. While these models are useful in describing some of the important physical processes, they lack predictive power and often miss key physical ingredients.

At the same time, numerical simulations performed so far (Gnedin \& Ostriker 1997) were not able to incorporate all the relevant physics processes, mainly the full three-dimensional radiative transfer.

This poster presents the most recent numerical simulations that now include a full (albeit approximate) treatment of the three-dimensional radiative transfer.

\section{Approximate Radiative 'Transfer}

The adopted approach for incorporating the full radiative transfer into a cosmological simulation is based on the following simple observation: in the optically thin regime the radiative transfer in cosmology simply reduces to counting Huxes from all the sources present in a simulation, which is equivalent to computing a potential with a $1 / r^{2}$ law. This is entirely analogous to gravity calculation, and thus can be done with a $\mathrm{P}^{3} \mathrm{M}$ technique.

On the other hand, computing the optical depth $\tau$ precisely presents a currently untrackable problem. However, a plausible approximation can be developed, because the high precision in evaluating $\tau$ is not required: if the optical 
depth is small, it does not matter much whether it is $10^{-3}$ or $10^{-5}$. If it is large, it again does not matter much whether it is $10^{3}$ or $10^{5}$. Only when $\tau \sim 1$ it needs to be computed accurately, but the volume of space where this condition is achieved is very small, so at the end no large error is introduced even if $\tau$ is computed with only an order-of-magnitude accuracy.

'The approximation adopted in this work assigns the value for the optical depth according to the "Local Optical Depth ansatz":

$$
\tau_{\nu}\left(x^{i}\right)=\sum_{\alpha} \sigma_{\nu}^{(\alpha)} n^{(\alpha)}\left(x^{i}\right) L\left(x^{i}\right)
$$

where index $\alpha$ runs over the list of species (in our case $\mathrm{H} \mathrm{I}, \mathrm{Her}$, and HeIr), and $L$ is a characteristic length, which is taken to be the characteristic length of the density distribution,

$$
L=\left(\alpha|\nabla \log \rho|^{2}+\beta \rho|\Delta \log \rho|\right)^{-1 / 2}
$$

where $\rho$ is the baryon density, and $\alpha$ and $\beta$ are constants chosen to reproduce the correct result for the column density of a $1 /\left(r^{2}+r_{c}^{2}\right)$ density distribution in the limits $r \rightarrow 0$ and $r \rightarrow \infty$.

Figure 1 shows the comparison between the cxact spherically symmetric solution (which can be done with existing computers because of the symmetry) of the propagation of the ionization front down the $1 / r^{2}$ density distribution versus the approximate full 3D simulation. The ionization front in an approximate scheme is not as sharp as in the exact solution, and there arc also some other defects, but the front speed is reproduced correctly.

\section{Simulations}

Simulations of a representative $\mathrm{CDM}+\Lambda$ cosmological model with $\Omega_{0}=0.3$ and $h=0.7$ were performed with the high-resolution SLH-P $\mathrm{P}^{3} \mathrm{M}$ code, which now includes:

- dark matter ( $\mathrm{P}^{3} \mathrm{M}$; Gnedin \& Bertschinger 1996, ApJ, 470, 115);

- gas dynamics (SLH; Gnedin 1995, ApJS, 97, 231);

- star formation phenomenology (Gnedin 1996, 456, 1);

- stellar feedback including UV and X-ray emission, supernova, and (equilibrium) metal cooling;

- exact atomic and molecular physics of $\mathrm{H}$ and He plasma;

- approximate full 3D radiative transfer (this work);

Simulations had the total mass resolution of $10^{6.4} h^{-1} \mathrm{M} \odot$ and the baryonic Inass resolution of $10^{5.5} h^{-1} \mathrm{M}_{\odot}$, and the spatial comoving resolution of $1.5 h^{-1} \mathrm{kpc}$ in a $2 h^{-1} \mathrm{Mpc}$ box. Larger simulations are on the way.

Because scales above a few Mpc cannot be simulated with enough resolution with the existing numerical schemes, only stellar sources were included in the simulations. Quasars, which can ionize regions many tens of Mpc in size. cannot be simulated properly yet. However, there exist good reasons to assume that quasars cannot reionize the universe (Madau, Haardt, \& Rees 1999, Ap.J, 514, $648)$. 


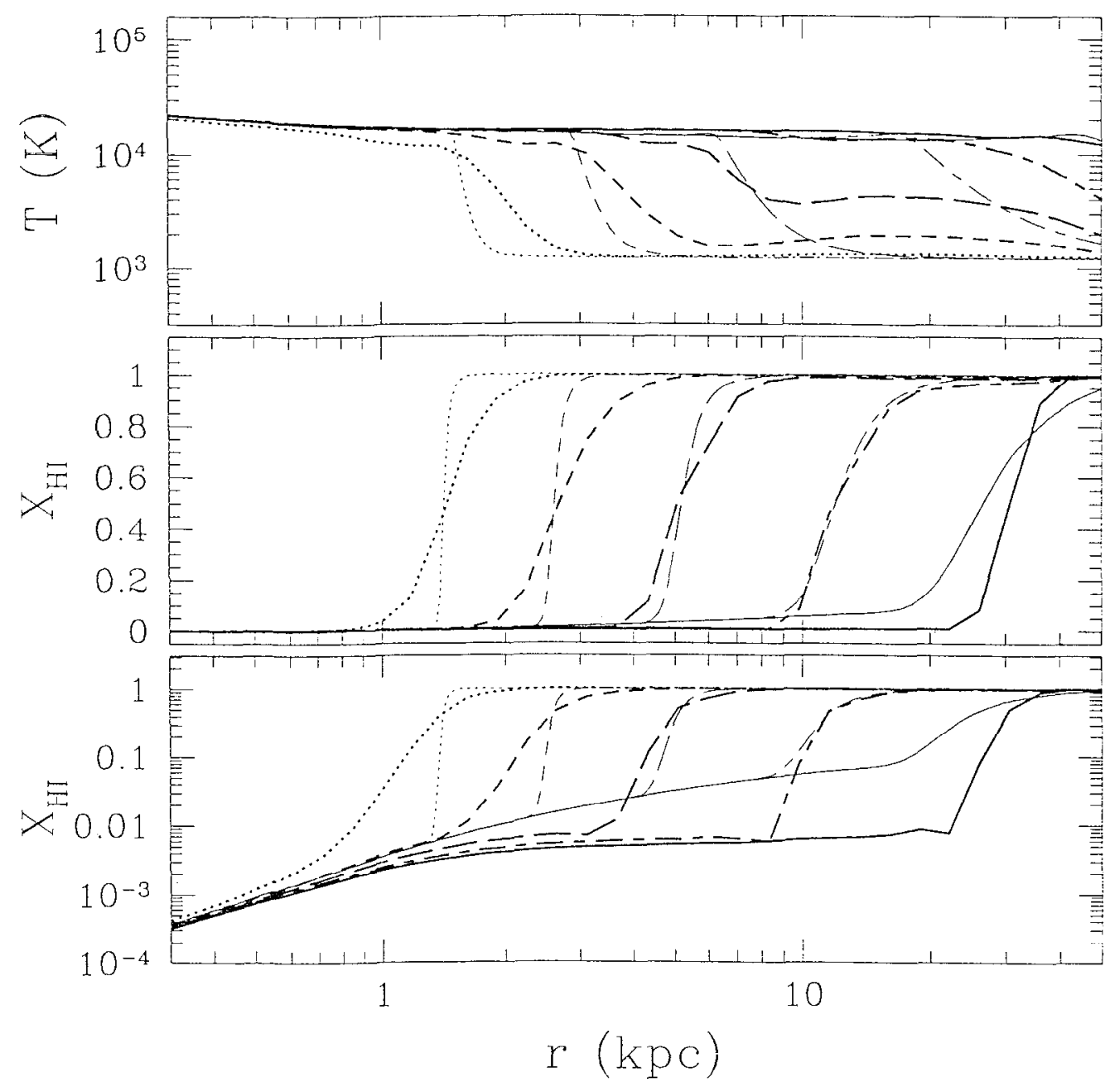

Figure 1. Distribution of the temperature (top panel) and neutral hydrogen fraction on linear (middle panel) and logarithmic (bottom panel) scale at five different times (as labcled by different lines) in the exact spherically symmetric solution (thin lines) and the approximate 3D solution (thick lines). 


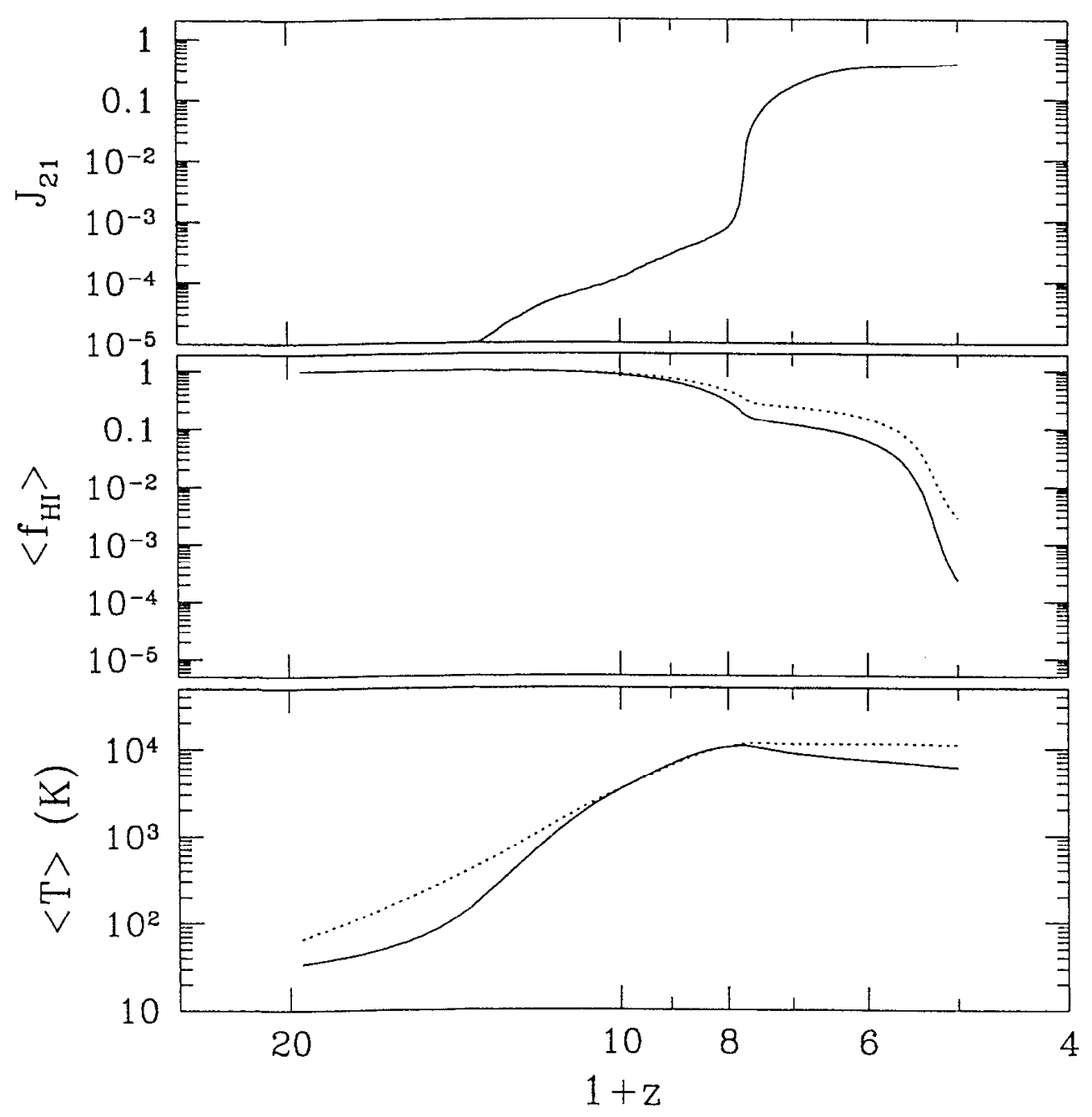

Figure 2. Evolution of the radiation intensity $J_{21}$ (upper panel), average neutral hydrogen fraction $f_{\mathrm{H}}$ (middle panel), and the average temperature (lower panel) as a function of redshift. Solid lines show the volume average and dotted lines show the mass average for the temperature and neutral hydrogen fraction. 


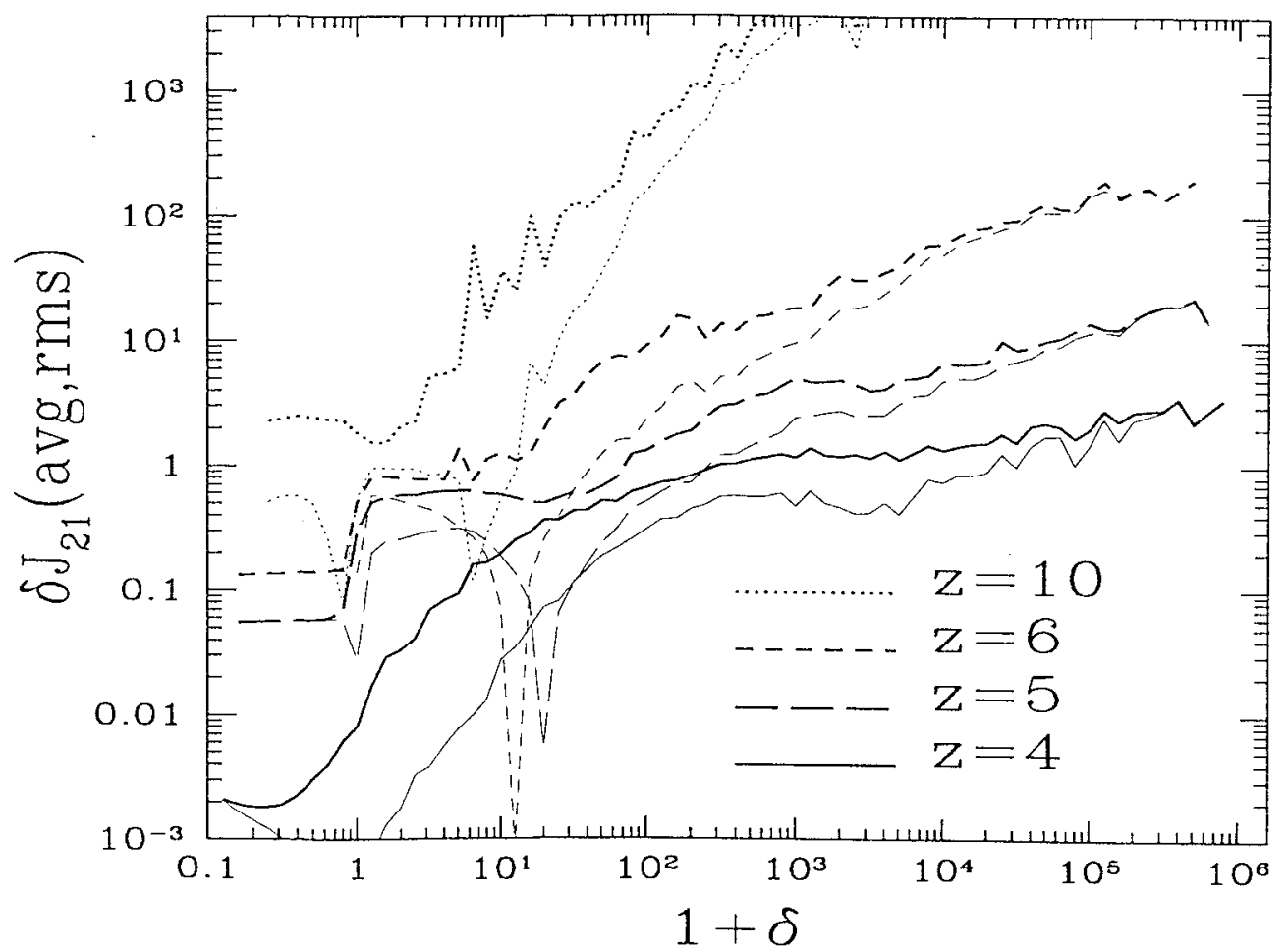

Figure 3. Rms (thick lines) and average (thin lines) fluctuation of the ionizing intensity $J_{21}$ as a function of gas density at four different redshifts.

\section{Results}

Figure 2 shows in three panels evolution of the ionizing intensity $J_{21}$, neutral hydrogen fraction $X_{\mathrm{HI}}$, and the average temperature of the universe. The sharp jump in $J_{21}$ corresponds to the moment of reionization at $z \sim \tau$.

Figure 3 presents the main result of this work, the spatial fluctuations in the ionizing intensity $\delta J_{21}=J_{21} / \vec{J}_{21}-1$ as a function of gas density. Even at $\tilde{z}=4$ the fluctuations are large for gas overdensity greater than about 10 . Thus, models and simulations of the Lyman-alpha forest that assume uniform ionizing radiation (i.e. all of them up to now) can only reproduce the forest reliably for $\delta<10$. which translates into the upper limit on the column density $V_{\mathrm{H} 1} \lesssim 10^{14} \mathrm{~cm}^{-2}$.

\section{References}

Valageas, P., \& Silk, J. 1999, A\&A, in press (astro-ph/9903411)

Gnedin, X. Y., \& Ostriker. J. P. 1997, ApJ 486, 581 

The Hy Redshift Universe

ASP Conference Series, Vol. 193, 1999

A. J. Bunker \& W. J. M. van Breugel, eds.

\title{
NICMOS Imaging of Damped Ly- $\alpha$ Absorbers at $z=2$
}

Varsha P. Kulkarni ${ }^{1}$, John M. Hill, Glenn Schneider,

Steward Observatory, University of Arizona, Tucson, AZ 85721

Ray J. Weymann, Lisa J. Storrie-Lombardi

Carnegie Observatories, Pasadena, CA 01101

Marcia J. Rieke, Rodger I. Thompson,

Steward Observatory, University of Arizona, Tucson, AZ 85721

Buell Jannuzi

National Optical Astronomy Observatories, Tucson, AZ 85726

\begin{abstract}
We report results from deep NICMOS imaging of the fields of two damped Lyman- $\alpha$ (DLA) quasar absorbers at $z_{D L A}=1.83$ and $z_{D I A}=1.86$. The images were obtained in the broad filter $\mathrm{F} 160 \mathrm{~W}$ and the narrow filter F190N or F187N with camera 2 on NICMOS, with the goal of detecting the rest-frame optical continuum and $\mathrm{H}-\alpha$ emission from the DLA absorbers. The broad band images put sensitive constraints on the sizes of the DLAs, while the narrow band images put the tightest existing limits on the star formation rates in DLAs. These results have important implications for the physical nature of DLA absorbers.
\end{abstract}

\section{Introduction}

Damped Lyman-alpha (DLA) absorbcrs in spectra of high-redshift quasars are believed to trace the precursors of present-day galaxies. But the nature of absorbing galaxies is not yet understood. DLAs have been variously thought to arise in large rotating proto-disks (e.g., Wolfe et al. 1986, Prochaska \& Wolfe 1997) or dwarf galdxies (e.g.. York et al. 1986, Matteucci et al. 1997). Urlfortunately, most previous efforts to directly image the objects causing high-z DLAs have been unsuccessful. Most of these previous searches altempled to detect the redshifted Ly- $\alpha$ emission from the DLAs. However, these nondetections cannot directly constrain the star formation rate (SFR) in DLAs, since the Ly- $\alpha$ line can easily get extinguished by even small amounts of dust, owing to resonant scattering. Here we report HST NICMOS observations of the fields of the DLAs toward LBQS $1210+1731\left(z_{D L .4}=1.892 . \approx_{Q 5 O}=2.543\right)$ and

\footnotetext{
${ }^{1}$ Current Address: Clemson University, Dept. of Physics and Astronomy, Clemson, SC 29634
} 
Q1244 $+3443\left(z_{D L A}=1.859, z_{Q S O}=2.48\right)$, aimed at detecting redshifted $\mathrm{H}-\alpha$ and optical continuum emission from the DLAs.

\section{Observations, Data Reduction, PSF Subtraction}

Broad band images were obtained with NICMOS camera 2, using filter F160W $\left(\lambda_{\text {central }}=1.5940 \mu \mathrm{m}, \mathrm{FWHM}=0.4030 \mu \mathrm{m}\right)$, dithered in steps of 7.5 pixels. Narrow band images were also obtained using dither steps of 7.5 pixels, in filter F190N $\left(\lambda_{\text {central }}=1.9005 \mu \mathrm{m}, \mathrm{FWHM}=0.0174 \mu \mathrm{m}\right)$ for LBQS $1210+1731$ and in filter F187N $\left(\lambda_{\text {central }}=1.8740 \mu \mathrm{m}, \mathrm{FWHM}=0.0192 \mu \mathrm{m}\right)$ for $\mathrm{Q} 1244+3443$. These filters should contain the redshifted $\mathrm{H}-\alpha$ lines at $z=1.892$ and $z=1.859$, respectively. Finally, coronagraphic F160W images were also obtained for each QSO (See Kulkarni et al. 1999a, 1999b). Data were reduced using the IRAF package Nicred 1.8 (McLeod 1997). Reference PSFs for quasar PSF subtraction were obtained from observations of stars in the same filter / aperture combinations. The PSF star images were chosen to match as closely as possible the telescope "breathing" focus values for our quasar observations. For the coronagraphic images, more importance was given to getting close match between the exact positions in the coronagraphic hole where the quasar and the PSF star were located. The PSF star observations were analyzed in exactly the same manner as the quasar observations. The reduced PSF stars were registered and scaled to match the quasar and then subtracted from the quasar.

\section{Searching for the DLA near LBQS $1210+1731$}

\subsection{Non-coronagraphic F160W and F190N images}

About $99 \%$ of the flux in the non-coronagraphic F160W and F190N images comes from the quasar point source and disappears after PSF subtraction. A feature to the "lower right" of the center at about 0.26 " from the quasar ("O1") is the main asymmetric residual left behind in the images (Fig. 1). We have carried out several tests to determine whether $\mathrm{O} 1$ is real or an artifact arising from HST breathing, color differences between quasar and PSF star, differences in focus settings, asymmetries in the PSF core etc. (Kulkarni et al. 1999a). The best-fitting PSF and several others with reasonably close breathing values suggest that $\mathrm{O} 1$ is real, given the significant excess over a number of pixels. We cannot completely rule out that $\mathrm{O} 1$ is some artifact arising for reasons we have not been able to account for. If $\mathrm{O} 1$ is associated with the DLA, then it is 2.4 $\mathrm{h}_{0.7}^{-1} \mathrm{kpc}$ long for $q_{0}=0.5$ or $3.2 \mathrm{~h}_{0.7}^{-1} \mathrm{kpc}$ long for $q_{0}=0.1$. O1 has a flux of $9.8 \pm 2.4 \mu \mathrm{Jy}$ in the F160W filter and $10.6 \pm 1.5 \mu \mathrm{Jy}$ in the F190N filter. On estimating the continuum contribution to the F190N flux, there is no evidence for statistically significant redshifted $\mathrm{H}-\alpha$ line flux. Given the low dust-to-gas ratios inferred for DLAs (e.g. Pei et al. 1991; Pettini et al. 1997 and references therein), the nondetection of $\mathrm{H}-\alpha$ emission is likely to be because of low SFR. Assuming no dust and using the $\mathrm{H}-\alpha$ luminosity-SFR conversion of Kennicutt (1983), the $3 \sigma$ upper limit on the SFR is $4.0 \mathrm{~h}_{0.7}^{-2} \mathrm{M}_{\odot \mathrm{yr}} \mathrm{yr}^{-1}$ for $q_{0}=0.5$. This is the tightest existing constraint on the SFR in DLAs. 

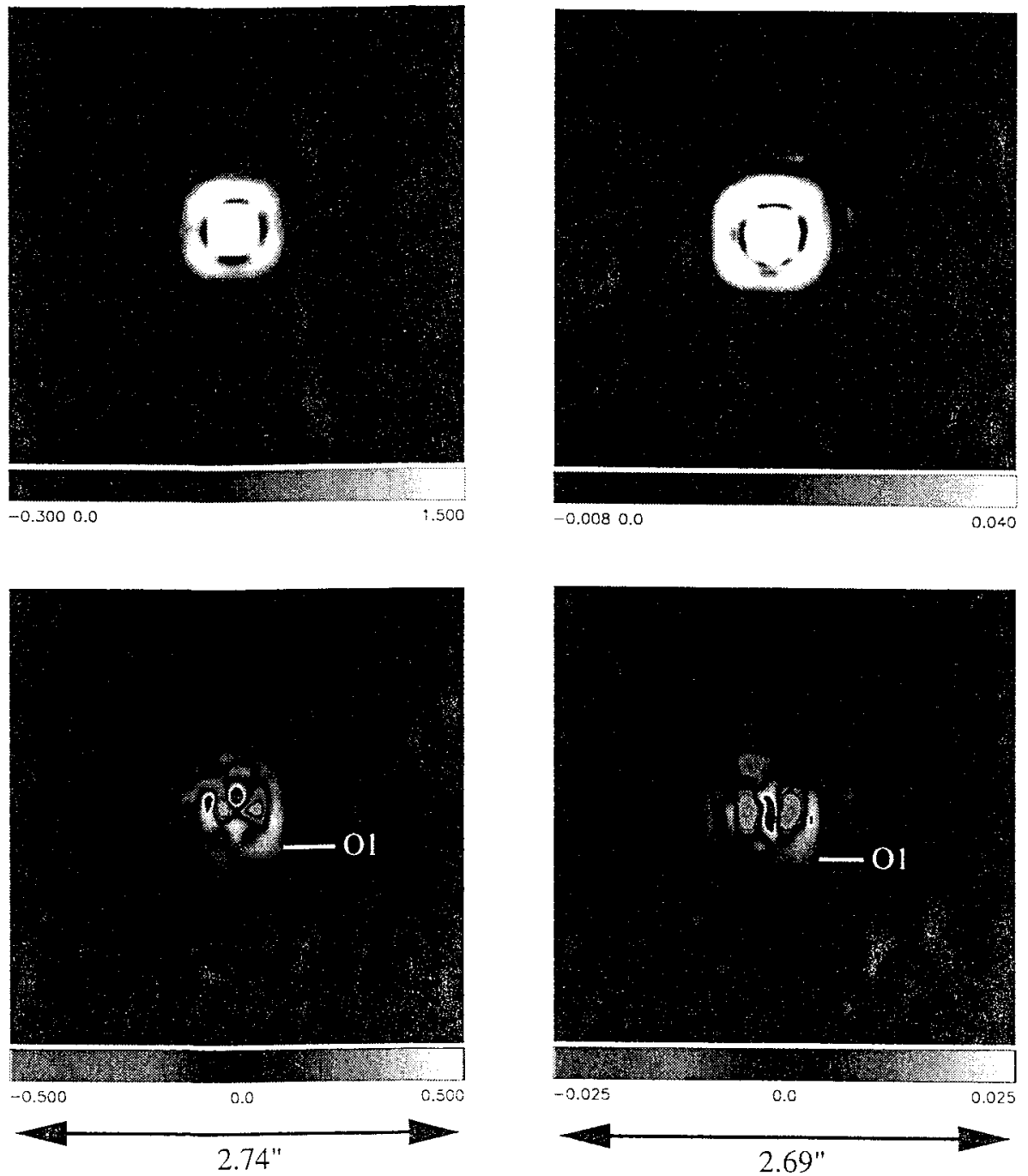

Figure 1. Left: Zoomed-in 2.74" $\times 2.71^{\prime \prime}$ region of the NICMOS camera 2 non-coronagraphic $1.6 \mu \mathrm{m}$ broad-band image of Q1210+1731, (a) before (top) and (b) after (bottom) PSF subtraction (bottom). Right: Zoomed-in 2.69" $\times 2.66^{\prime \prime}$ region of the NICMOS camera 2 noncoronagraphic $1.9 \mu \mathrm{m}$ narrow-band image of Q1210+1731, (c) before (top) and (d) after (bottom) PSF subtraction. 

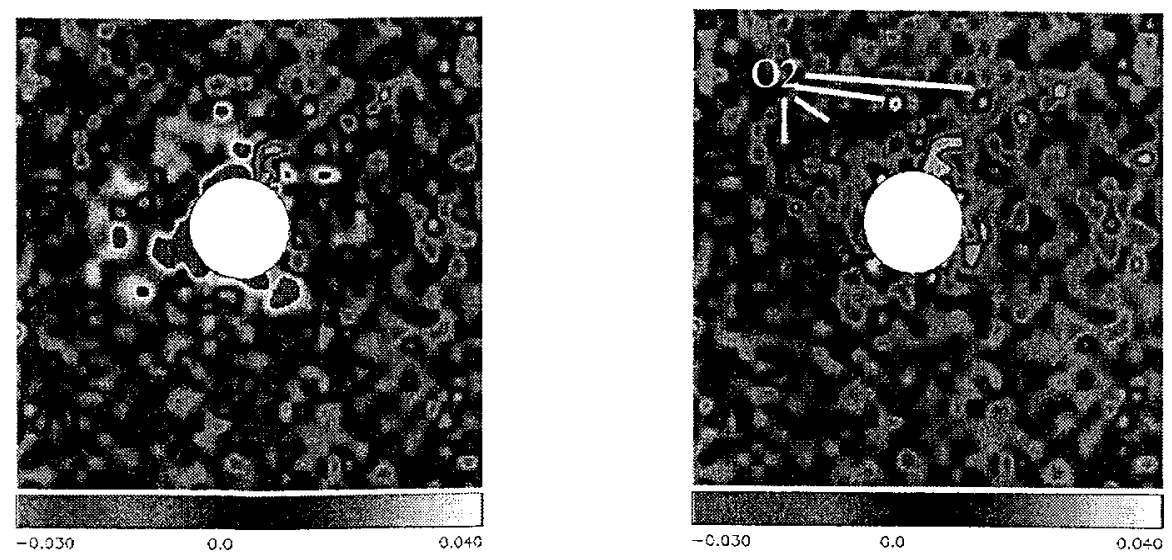

Figure 2. Zoomed-in $2.89^{\prime \prime} \times 2.81^{\prime \prime}$ regions of coronagraphic $1.6 \mu \mathrm{m}$ image of Q1210+1731, (a) before (left) and (b) after (right) PSF subtraction

\subsection{Coronagraphic Image of LBQS1210+1731}

The NICMOS coronagraph reduces the scattered and diffracted cncrgy from the occulted target's PSF core by factors of 4-6, compared to direct imaging (Schneider et al. 1998; Lowrance et al. 1998). Most of the flux in the coronagraphic images comes from small amount of scattered light from the quasar and glints from the edge of the hole. These disappear almost completely after PSF subtraction. But a weak feature consisting of several knots (object "O2") remains at about 0.7 "from the quasar center (Fig. 2). There are no known artifacts in the coronagraphic F160W image at the positions of the $\mathrm{O} 2$ knots. If $\mathrm{O} 2$ is at the redshift of the DLA, it has a total size of $4-5 \mathrm{~h}_{0.7}^{-1} \mathrm{kpc}$ for $q_{0}=0.5$.

\section{The DLA toward Q1244+3443}

The results for this DLA are similar to those for the DLA toward Q1210+1731 (Fig. 3). Note the possible presence of a compact object below the quasar center in the F160V image, at a separation of only $0.16 "$. The narrow-band image reveals no strong extended emission from this or any other source, suggesting a low SFR. Further details are discussed by Kulkarni et al. (1999b). Thus, our observations of both the DLAs suggest compact or low surface brightness objects with a few continuum emission knots, and low star formation rates.

Acknowledgments. This work was supported by NASA grant NAG5-3042. 

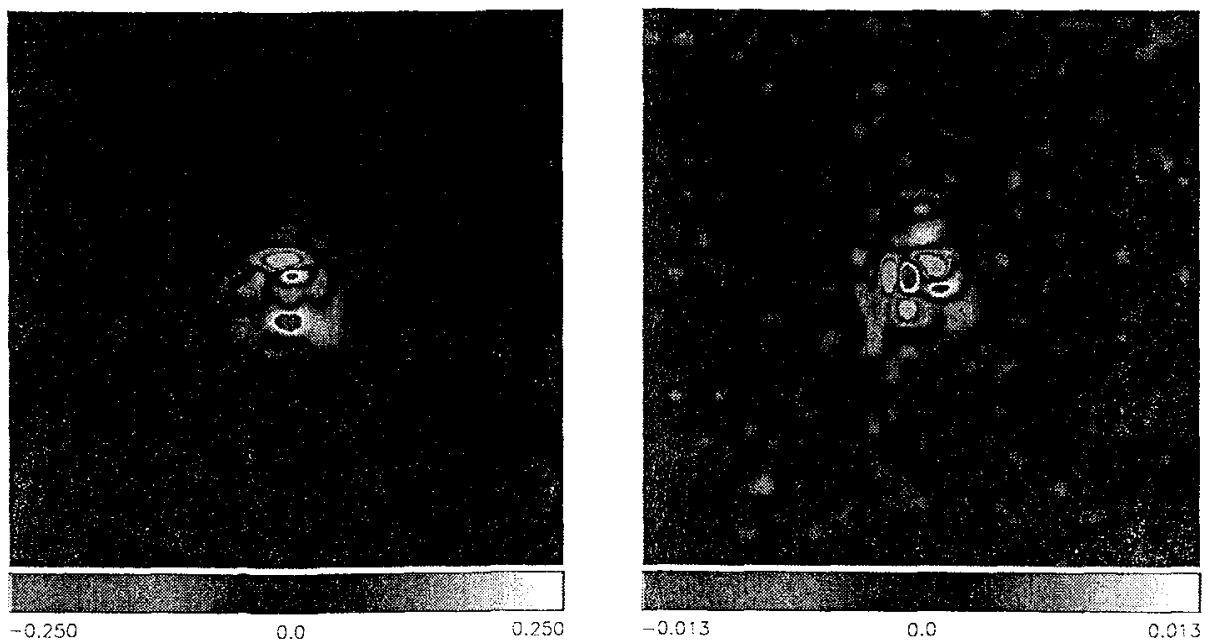

Figure 3. Zoomed-in $2.81^{\prime \prime} \times 2.79^{\prime \prime}$ regions of the NICMOS camera 2 non-coronagraphic (a) $1.6 \mu \mathrm{m}$ (left) and (b) $1.87 \mu \mathrm{m}$ (right) images of the field of Q1244+3443, after PSF subtraction

\section{References}

Kennicutt, R. C. 1983, ApJ, 272, 54

Kulkarni, V. P., Hill, J. M., Schneider, G., Weymann, R. J., Storrie-Lombardi, L. J., Rieke, M. J., Thompson, R. I., \& Jannuzi, B. 1999a, ApJ, submitted

Kulkarni, V. P., Hill, J. M., Schneider, G., Weymann, R. J., Storrie-Lombardi. L. J., Rieke, M. J., Thompson, R. I., \& Jannuzi, B. 1999b, in preparation Lowrance, P. et al. 1998, in Proc. 'NICMOS and the VLT' (ESO), 96

Matteucci, F., Molaro, P., \& Vladilo, G. 1997, A\&A, 321, 45

McLeod, B. 1997, in Proc. 1997 HST Calibration Workshop, eds. S. Casertano et al. (STSCI), 281

Pei, Y. C., \& Fall, S. M. 1995, ApJ, 454, 69

Prochaska, J. X., \& Wolfe, A. M. 1997, ApJ, 487, 73

Pettini, M., King, D. L., Smith, L. J., \& Hunstead, R. W. 1997, ApJ, 478, 536

Schneider, G., Thompson, R. I., Smith, B. 1., \& Terrile, R. J. 1098, SPIE Conf. Proc. Ser.. 3356

Wolfe, A. M., Turnshek, D. A., Smith, L. J., Cohen, R. D. 1986, Ap.JS, 61, 249

York, D. G., Dopita, M., Green, R., \& Bechtold, J. 1986, Ap.J, 311, 610 

The Hy Redshift Universe

ASP Conference Series, Vol. 193. 1999

A. J. Bunker \&' W. J. M. van Breugel, eds.

\title{
Damped Ly $\alpha$ Systems in Semi-Analytic Models: Sensitivity to dynamics and disk properties.
}

\author{
Ariyeh Maller \\ Physics Department, University of California, Santa Cruz, CA 90564 \\ Rachel S. Somerville \\ Racah Institute of Physics, The Hebrew University, Jerusalem, 91904 , \\ Israel
}

Jason X. Prochaska

Observatories of the Carnegie Institution of Washington, Pasadena. CA 91101

Joel R. Primack

Physics Department, University of California, Santa Cruz, CA 90564

\begin{abstract}
Previously (Maller et al. 1999, hereafter MSPP) we have shown that it is possible to account for the kinematic properties of damped Lyman alpha systems (DLAS) as measured by Prochaska \& Wolfe (1997, 1998, hereafter PW97 and PW98) in the context of semi-analytic models (S.AMs) (Somerville \& Primack 1999, hereafter SP99). In these models, hierarchical structure formation is approximated by constructing a merger tree for each dark matter halo. A natural consequence is that every virialized halo may contain not only a central galaxy, but also a number of satellite galaxies as determined by its merging history. Thus the kinematics of the DLAS arise from the combined effects of the in ternal rotation of gas disks and the motions between gas disks within a common halo. Here we investigate the sensitivity of this model to some of the assumptions made in MSPP, including the modeling of satellite dynamics, the scale height of the gas, and the cosmology.
\end{abstract}

\section{Satellite Dynamics}

In the SAMs, a merger tree represents a halo's growth through the meroers of smaller halos (see Somerville 1997). When halos merge, the central galaxy of the largest progenitor halo becomes the central galaxy of the new halo, and all other galaxies become satellites. These satellites then fall in towards the central galary due to dynamical friction, and eventually merge with it (see SP99). Becanse the treatment of the dynamics of the satellites is necessarily simplified in the usual semi-analytic spirit, and since the kinematics of the DLAS arise from both the rotation of disks and the motions of satellite galaxies in the common halo. it is important to test whether our results are sensitive to the details of our modeling. 

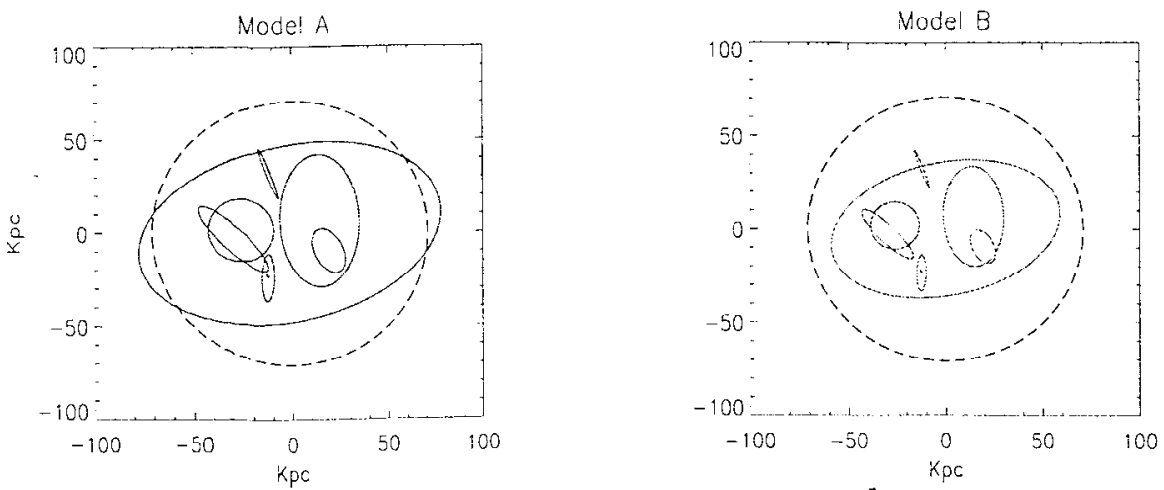

Figure 1. A halo of circular velocity $156 \mathrm{~km} \mathrm{~s}^{-1}$ with its satellite galaxies. The ellipses mark where the cold gas is truncated, in Model A (left) with thin disks and Model B (right) with thicker disks. The dashed line is the virial radius of the parent halo.

In the models presented in MSPP, all satellites were assumed to start falling in from the virial radius of the newly formed halo, and all satellites were assumed to be on circular orbits. These assumptions will tend to maximize the dynamical friction timescale of the satellites, and correspond to the assumptions made in some earlier versions of the SAMs (e.g. Somerville 1997). In the models presented here, we have modifed these assumptions in two ways. We initially place the satellites at one half of the virial radins, and assign the orbits from a random distribution as observed in N-body simulations by Navarro, Frenk, \& White (1995). They found that the "circularity" parameter $\epsilon$ has a flat distribution, where $\epsilon \equiv J / J_{c}$ is defined as the ratio of the angular momentum of the satellite to that of a circular orbit with the same energy. The dynamical friction time scale is then scaled by a factor $\epsilon^{0.78}$ (Lacey \& Cole 1993). This causes some of the satellites to fall in faster than in our previous modeling. In addition, we have changed from the SCDM cosmology used in MSPP to a more fashionable $\Omega_{0}=0.4, \Omega_{\Lambda}=0.6$ cosmology. These ingredients are compatible with the models that were shown to produce good agreement with both local galaxy observations (SP99) and the high redshift Lyman-break galaxies (Somerville, Primack, \& Faber 1999).

We find that the combined effect of these changes has a negligible effect on our results. Although we do see fewer satellites within a halo of a given mass, the number of satellites in the immer part of the halo is similar. These are the satellites most likely to give rise to multiple hits, which as we argued in MSPP is the crucial factor in matching the observed kinematics of the DLAS. As in MSPP, we still find that the gaseous disks must have very large radial extents in order to match the observations.

\section{Disk Thickness}

Another important feature of the models is the assumed distribution of the gas within the disks. In MSPP, we investigated several radial profiles and obtained 


\begin{tabular}{ccccccc}
\hline Model & vertical scale height & normalization & $\Delta V$ & $\mathrm{f}_{m m}$ & $\mathrm{f}_{\mathrm{edg}}$ & $\mathrm{f}_{t p k}$ \\
\hline \hline $\mathrm{A}$ & $.1 R_{*}$ & $\log N_{t}=19.3$ & 0.35 & 0.10 & 0.56 & 0.26 \\
$\mathrm{~B}$ & $.5 R_{*}$ & $\log N_{t}=19.6$ & 0.41 & 0.24 & 0.54 & 0.71 \\
\hline
\end{tabular}

I'able 1. The properties of our two models and the KS probabilities for the four statistics of PW97.

the best results with an assumed gas distribution of the form

$$
n(R, z)=\frac{N_{t}}{2 h_{z}} \frac{R_{t}}{R} \exp \frac{-|z|}{h_{z}} \quad\left(R<R_{t}\right)
$$

where the truncation density $N_{t}$ is an adjustable parameter. The vertical scale height of the gas disks is another uncertainty. In MSPP we assumed it to be one tenth of the stellar disk scale radius, i.e. $h_{z}=0.1 R_{*}$ where $R_{*}$ is the stellar disk scale length as given by the SAMs (see SP99). Because the gas disks in the successful models tend to have a large radial extent, much larger theil the stellar disk, this leads to very thin gaseous disks. When the scale height is incrcased to half the stellar scalc radius $\left(h_{z}=0.5 R_{*}\right)$, we are able to use more physically plausible values for the truncation density $N_{t}$ (see Table 1 ), and the gas disks are now typically contained within the truncation radius of the dark matter sub-halos surrounding the satellites. This model therefore seems to be morc physical, yet still produces good agrecment with all four of the diagnostic statistics of PW97 (see Table 1). Note that PW98 have shown that the effect of including a warp in the gas disk is the same as increasing its thickness, so our exploration of thicker disks can also be thought of as including warps in the disk.

Figure 2 shows the distribution of thicknesses and inclinations for the disks that contribute to DLAS in the two models. We find that the disks that produce damped systems in model B are more likely to have a face-on geometry. With thinncr disks, more of the cross section comes from an inclined geometry as $n \sim h_{x}^{-1}$. The total cross section in the two models is roughly the same; in model A, more of it comes from extended highly inclined disks, while in model B. denser, face-on disks are more important.

Thus the need for gas disks with large radial extent can be reduced by using thicker disks, though not by a large amount. Increasing the thickness by a factor of five only reduces the radial truncation value by $30 \%$. The gaseous disks in model B are still quite large compared to the stellar component.

\section{Conclusions}

In MSPP we argued that the observed kinematics of DLAS can be reproduced in hierarchical models if a significant fraction of the lines of sight pass through multiple disks orbiting within a common dark matter halo. However, we found that in order to obtain a high enough cross section for multiple hits, we had to assume very large radial extents for the gaseous dishs in our models. We have tested the robustness of these conclusions by modifying several of the uncertain ingredients of our models. We find that modifying the dynamical friction 

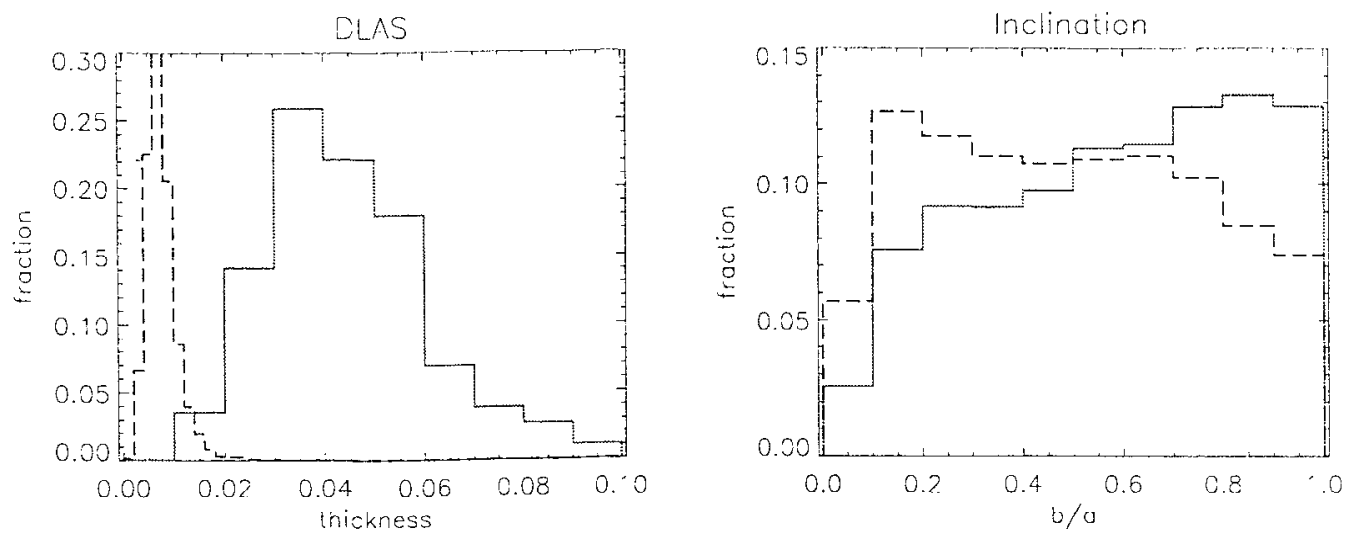

Figure 2. The distribution of thicknesses (left panel) and inclinations (right panel) of the disks that produce DLAS from our two models; Model A (dashed), Model B (solid). We characterize the thickness as $3 h_{z} / R_{t}$. The DLAS in Model A are produced by more inclined disks then in Model B.

timescale of the satellites by assuming a different intial radius or orbit has a small effect on our results. Similarly, our results do not seem to be sensitive to the assumed cosmology. Increasing the vertical scale height of the disks has a larger effect and leads to models that are more physically plausible and still produce good agreement with the diagnostic statistics of PW. Our results suggest that in order to reconcile the observed kinematics of DLAS with hierarchical theories of structure formation, gaseous disks at high redshift must be large in radial extent and thickened or warped. The physical cause of these properties remains obscure, however we speculate that tidal encounters or outflows could be responsible.

\section{References}

Lacey, C. \& Cole, S. 1993, MNRAS, 262, 627

Maller, A. H., Somerville, R. S., Prochaska, J. X., \& Primack, J. R. 1999, in After the Dark Ages: When Galaxies were Young, ed. S. Holt \& E. Smith (AIP Press), 102

Navarro, J. F., Frenk, C. S., \& White, S. D. M. 1995, MNRAS, 275, 56

Prochaska, J. X. \& Wolfe, A. M. 199

-. 1998, ApJ, 507, 113

Somerville, R. S. 1997, PhD thesis, Univ. California, Santa Cruz

Somerville. R. S. \& Primack, J. R. 1999, MNRAS, accepted, astro-ph/9802268

Somerville, R. S., Primack, J. R., \& Faber, S. M. 1990, MNRAS, accepted; astro-ph/9802268 
The Hy Redshift Universe

ASP Conference Series, Vol. 193, 1999

A. J. Bunker \& W. J. M. van Breugel, eds.

\section{ChaMP and the High Redshift Quasars in X-rays}

S. Mathur ${ }^{1}$

Harvard Smithsonian Center for Astrophysics Cambridge, MA 02138

H. Marshall ${ }^{1}$

MIT, Cambridge, MA 02139

N. Evans ${ }^{1}$, P. Green ${ }^{1}$ and B. Wilkes ${ }^{1}$

Harvard Smithsonian Center for Astrophysics Cambridge, MA 02138

Abstract. Chandra X-ray Obscrvatory, (formerly known as AX.AF), will observe down to the flux limit of $2 \times 10^{-16} \mathrm{erg} \mathrm{s}^{-1} \mathrm{~cm}^{-2}$. In its first year of operation Chandra's CCD detcctors will observe over 1500 quasars serendipitously in the soft $(0.5-3.5 \mathrm{keV})$ band. Over 200 quasars will be detected in $\mathrm{X}$-rays in the redshift range $3<z<4$ and over 400 quasars in $2<z<3$. This will enable us to determine the high redshift $\mathrm{X}$-ray luminosity function. This is the contribution by unabsorbed sources only. The total numbers would be larger by $\sim 60 \%$.

\section{Introduction}

NASA's Chandra X-ray Observatory was launched on July 23, 1999. The Chandra Multiwavelength Project (ChaMP) will combine radio to X-ray observations of serendipitous Chandra sources, with emphasis on optical identification. The ChaMP is superior to previous X-ray surveys because of (1) unprecedented X-ray positional accuracy $\left(\sim 1^{\prime \prime}\right),(2)$ X-ray flux limits 20 times deeper than current wide area surveys (down to $\left.f(0.5-3.5 \mathrm{keV}) \sim 2 \times 10^{-16} \mathrm{erg} \mathrm{s}^{-1} \mathrm{~cm}^{-2}\right)$, (3) larger sky coverage $\left(\sim 8 \mathrm{deg}^{2}\right)$ per year than current deep surveys.

\section{Prediction of Redshift Distribution of Quasars in ChaMP Fields}

The $\mathbf{X}$-ray Luminosity Function at $z=0$ is described as

$$
\Phi\left(L_{X}\right)=\Phi_{1}^{\star} L_{44}^{-\gamma 1} \quad \text { for } L<L^{\star}(0)
$$

\footnotetext{
${ }^{1}$ The Chandra Multiwavelength Project (ChaMP) is an independent scientific collaboration for followup studies of serendipitous X-ray sources in Chandra X-ray images. The ChaMP Web site is http://hea-www.harvard.edu/CHAMP.
} 


$$
\Phi\left(L_{X}\right)=\Phi_{2}^{\star} L_{44}^{-\gamma^{2}} \quad \text { for } L>L^{\star}(0)
$$

where $\mathrm{T}_{44}$ is the $\mathrm{X}$-ray luminosity in $10^{44} \mathrm{erg} \mathrm{s}^{-1}$. The redshift evolution of the luminosity function is characterized by

$$
L_{X}(z)=L_{X}(0)(1+z)^{k}
$$

Continuity of the luminosity function at the break luminosity requires that

$$
\Phi_{1}^{\star}=\Phi_{2}^{k} L_{44}^{\star \gamma 1-\gamma 2}
$$

The total number $N$ of quasars in the sample is obtained by integrating the luminosity function over luminosity and volume, i.e.,

$$
N=\iint \Phi\left(L_{X}, z\right) \Omega\left(L_{X}, z\right) d V(z) d L_{X}
$$

Here $\Omega\left(L_{X}, z\right)$ is the solid angle covered by the survey as a function of redshift and luminosity. The parameters of the $\mathrm{X}$-ray luminosity function determined by Boyle et al. (1993) are as follows: $\gamma 1=1.7 \pm 0.2, \gamma 2=3.4 \pm 0.1$, $\log L^{\star}(0)=43.84, \Phi_{1}^{\star}=5.7 \times 10^{-7} \mathrm{Mpc}^{-3}\left(10^{44} \mathrm{erg} \mathrm{s}^{-1}\right)^{\gamma 1-1}$. Following Comastri et al. (1995), we have used $k=2.6$ and increased the the normalization $\Phi_{1}^{\star}$ by $20 \%$.

The $\mathrm{X}$-ray $\log \mathrm{N}$ - $\log \mathrm{S}$ Curve: Using the above luminosity function we derived the number density of quasars as a function of observed flux. The luminosity function was integrated over the luminosity range $10^{42}<L_{X}<10^{48}$ erg $\mathrm{s}^{-1}$ and the redshift range $0<z<4 . \mathrm{H}_{0}=50$ and $\mathrm{q}_{0}=0$ were assumed throughout. The predicted $\log N-\log S$ curve is shown in figure 1.

Since the unabsorbed sources dominate at the faint end in the soft $\mathrm{X}$-ray range, and since they are likely to be observed at high redshift, in the present analysis we will concentrate on unabsorbed sources only. The absorbed sources would contribute an additional $\sim 60 \%$ (Comastri et al. 1995), making the total number consistent with the extrapolation of the empirical determination of $\log \mathrm{N}$ $\log \mathrm{S}$ (Hasinger et al. 1993). The flux of unabsorbed quasars is given by $f \propto E^{-\alpha}$ and in the soft $\mathrm{X}$-ray band, $\alpha$ is typically 1.3 .

The ChaMP Sky Coverage: The ChaMP Cycle 1 consists of 85 extragalactic fields, $-\mathrm{b} \rightarrow 20^{\circ}$. From all the Chandra cycle 1 fields we have excluded (1) deep fields of PI survey observations, (2) fields with extended sources \& planetary targets, (3) ACIS sub-arrays and continuous clocking modes. See figure 2 for ChaMP sky coverage as a function of flux limit.

Cumulative Number Distribution in ChaMP: Inlegrating the predicted $\log \mathrm{N}-\log \mathrm{S}$ over the ChallP shy coverage, we obtained the cumulative number distribution of quasars in the ChaMP fields (figure 3). The total number in soft band is expected to be over 1500 for unabsorbed sources and over 2500 total.

Predicted Redshift Distribution: The histogram (figure 4) shows the predicted number distribution of quasars in ChaMP fields. Over 200 quasars will be detected in the redshift range $3<z<4$ and over 400 quasars in $2<z<3$. 


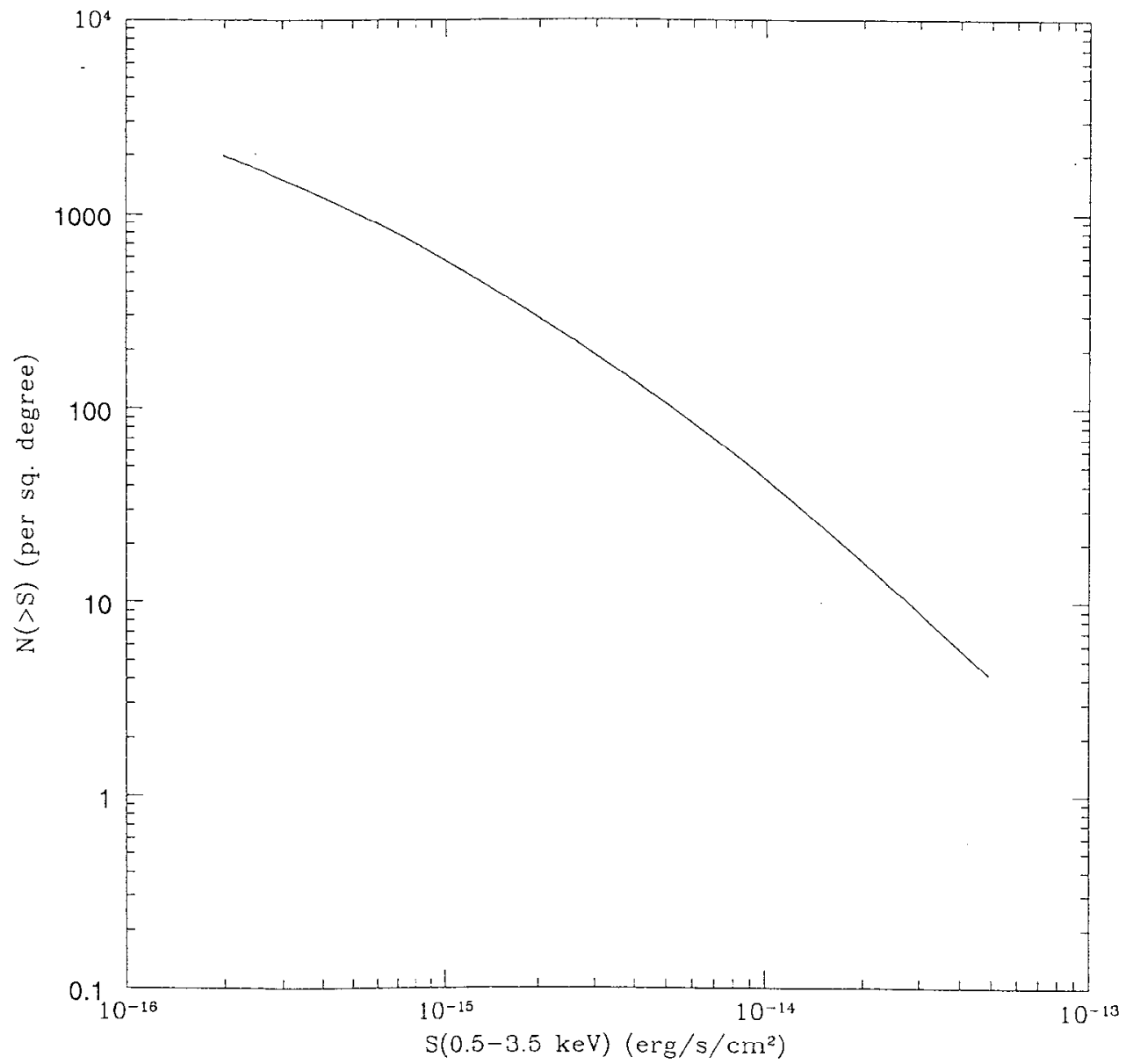

Figure 1. The predicted number counts in the soft band for unabsorbed quasars.

\section{Comparison with Previous X-ray Surveys}

We will be able to determine the X-ray luminosity function and its redshift evolution with unprecedented accuracy.

\section{References}

Boyle, B., Griffiths, R., Shanks, T., Stewart, G., \& Georgantopoulus, I. 1993. MNRAS, 260, 49

Comstri, A., Setti, G., Zamorani. G., \& Hasinger. G. 1995, A\&A, 296, 1

Gioia et al. 1990, ApJS. i2. 56 T 


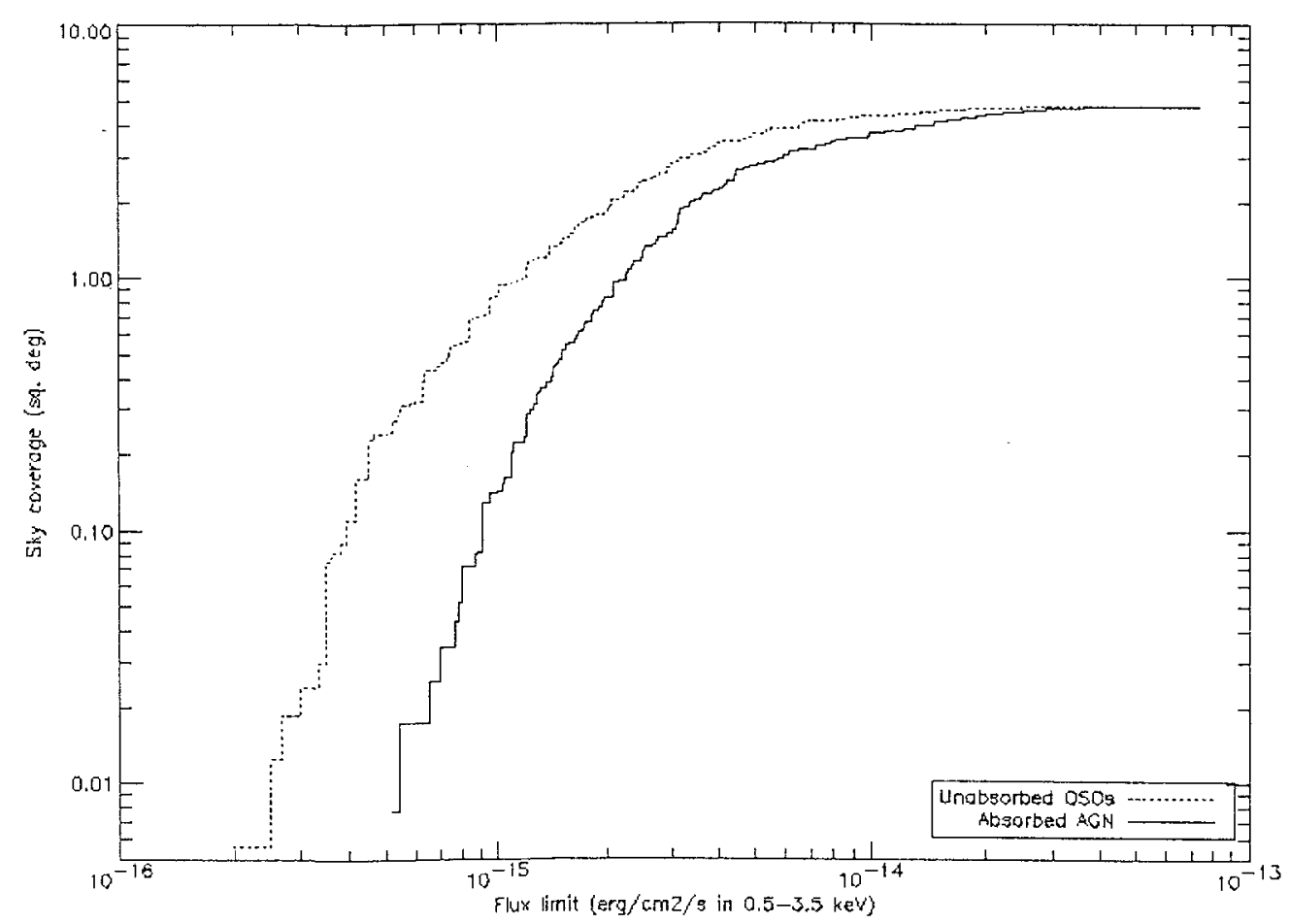

Figure 2. The total sky coverage of ChaimP fields as a function of flux limit in the soft band.

Hasinger, G. et al. 1993, A\&A, 275, 1

It's my pleasure (SM) to thank A. Comastri for useful discussions. This work is supported in parts by NASA grant NAG5-3249 (LTSA). 


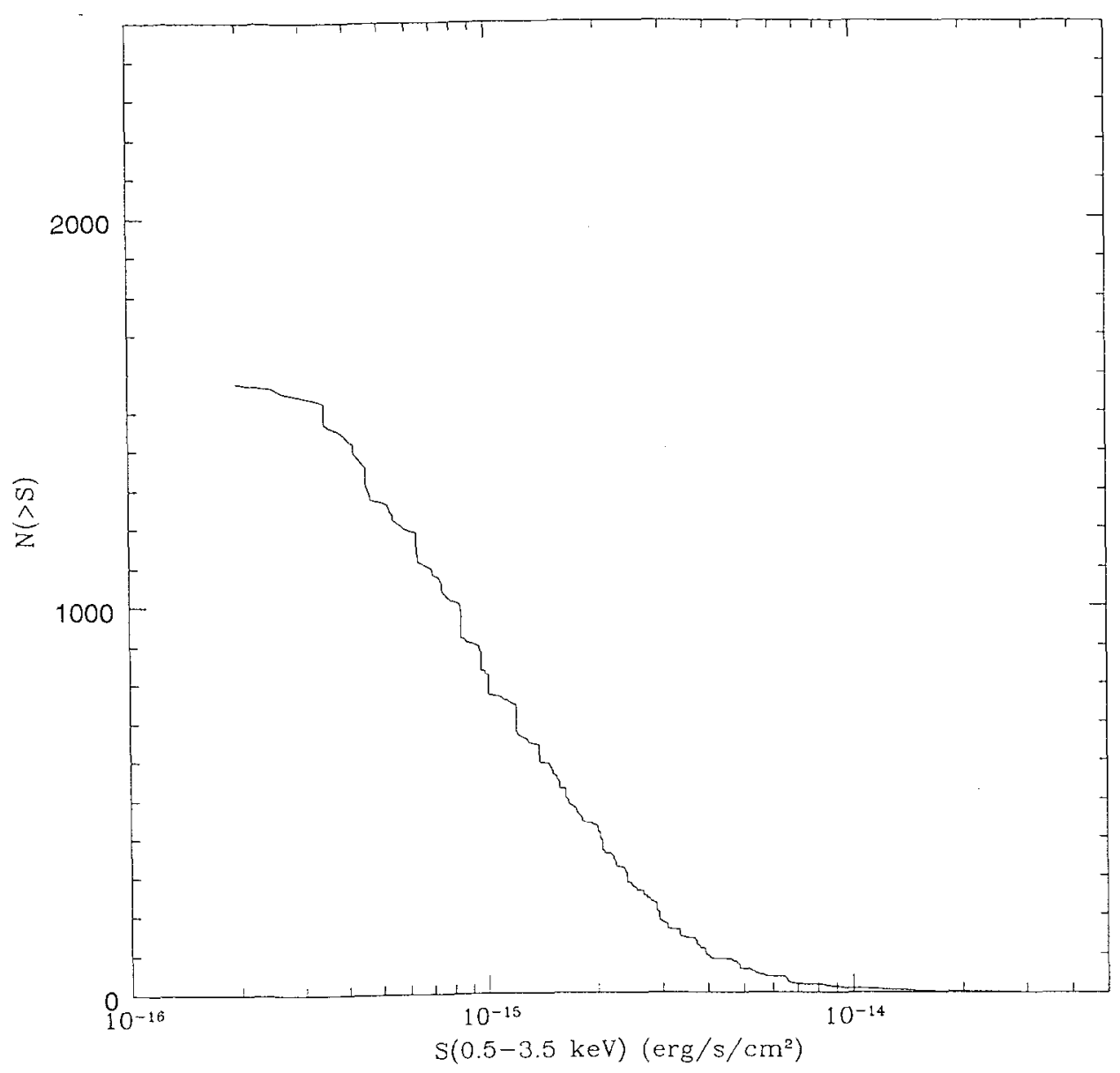

Figure 3. Expected cumulative source counts. Unabsorbed sources only.

\begin{tabular}{|lrr|}
\hline \hline Survey & Total number of Sources & Quasars at $z>2$ \\
\hline EMSS & 835 & $<5$ \\
(Gioia et al.) & 661 & 12 \\
ROSAT Deep & & $<10$ \\
(Hasinger et al.) & 89 & $>600$ \\
$\begin{array}{l}\text { ROSAT } \\
\text { (Boyle et al.) }\end{array}$ & $>1500$ & \\
$\begin{array}{l}\text { ChaMP } \\
\text { (soft band, unabsorbed) }\end{array}$ & & \\
\hline
\end{tabular}




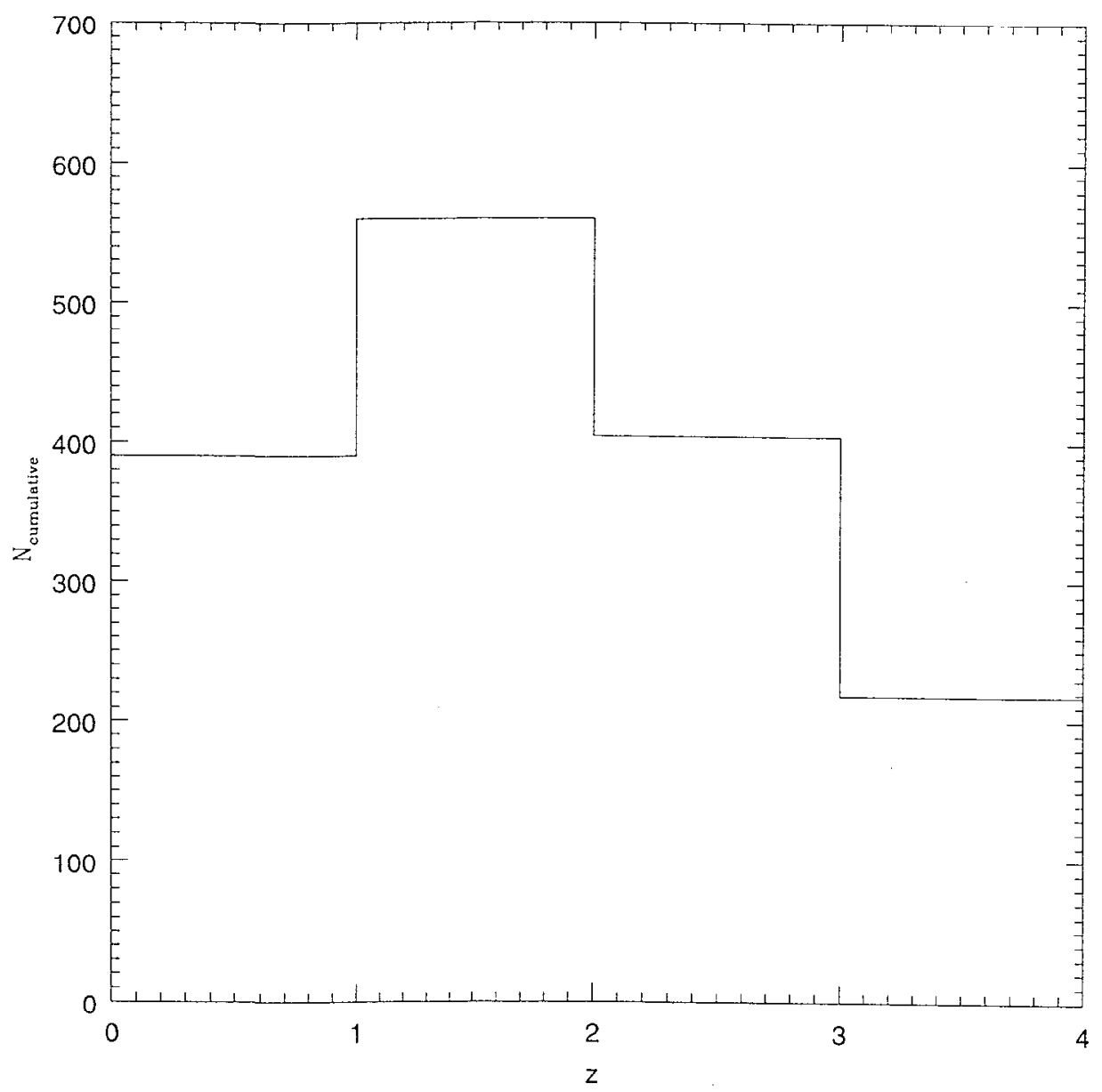

Figure 4. Redshift distribution of the unabsorbed sources expected to be detected in ChaMP fields. 
The Hy Redshift Universe

ASP Conference Series, Vol. 193, 1999

A. J. Bunker \& W. J. M. van Breugel, eds.

\title{
3D Modelling of the Ly $\alpha$ Radiation from (Proto-) Galaxies
}

\author{
R. Wehrse \\ Institute $f$. Theoret. Astrophys, Heidelberg University, Tiergartenstr. \\ 15, D 69121 Heidelberg, Germany, \\ Interdisciplin. Center $f$. Scientific Computing, Heidelberg University, \\ INF 368, D 69120 Heidelberg, Germany
}

\section{Introduction}

Lyman- $\alpha$ emission is one of the first indications of a new-born galaxy. Its strength and profile contain information about the density distribution, excitation condition, optical depth, and velocity field in the galaxy and possibly its surroundings. In first place, however, the line is influenced by the intra-galactic dust which may weaken it super-exponentially. It could therefore - at least in principle - be used as a main source for an empirical study of the physical state of a young galaxy.

Unfortunately, there are several effects and difficulties that have inhibited the full use of the information content of the line: (i) the strong dependence on the dust makes it virtually undetectable in many cases; (ii) it may have a very high optical depth so that radiative transfer effects (including redistribution) may play a primary role; (iii) it certainly originates from a medium of nonsimple geometry (perhaps even from a strongly inhomogeneous medium); the velocity fields may involve both motions to and from the galaxy (in- and outflow of matter) in addition to rotation. It is therefore not surprising that in spite of a wealth of observations available (cf. Steidel, this volume) a comprehensive modelling is not yet available.

In this paper we concentrate on the radiative transfer aspects of the problem: in the next section we present the appropriate transfer equation and briefly summarize an algorithm used for its solution. In the subsequent chapter the example of a flattened ellipsoid surrounded by low density gas is given to demonstrate the complexity that can result from even quite simple density and velocity distributions. On the other hand, the calculations also show the potentials of diagnostics of accurately observed brightness distributions that have good spatial and frequency resolution.

\section{The Radiative Transfer Equation and its Solution}

When velocities $\beta(x) \ll 1$ and velocity gradients $\partial \beta / \partial s \ll \chi_{\text {cont }}(\partial s=$ path element in ray direction, $\chi_{\text {cont }}=$ continuous extinction coefficient) the radiative transfer equation for the comoving frame can be written

$$
\mathrm{n} \cdot \nabla I(\mathbf{x}, \mathbf{n}, \xi)+w(\mathbf{x}, \mathbf{n}) \frac{\partial I(\mathbf{x}, \mathbf{n}, \xi)}{\partial \xi}=\cdots \chi(\mathbf{x}, \xi)(I(\mathbf{x}, \mathbf{n}, \xi)-S(\mathbf{x}, \mathbf{n}, \xi))
$$


with $\mathrm{n}=\left(n_{x}, n_{y}, n_{z}\right)$ unit vector in ray direction, $\mathrm{x}=$ vector comprising spatial variables, $\xi=\ln \lambda, \lambda=$ wavelength,$w(\mathbf{x}, \mathbf{n})=\mathbf{n} \cdot \nabla(\mathbf{n} \cdot \beta(\mathbf{x})=$ velocity gradient in ray direction, $\chi(x, \xi)=\chi_{l}(\mathrm{x}, \xi)+\chi_{d}(\mathrm{x})=$ total extinction coefficient $=$ line plus dust extinction coefficient, $\epsilon_{l}, \epsilon_{d}=$ ratio of absorption to extinction for the line and the dust, $p\left(\mathbf{n}, \mathbf{n}^{\prime}\right)=$ phase function for the dust, $R\left(\mathbf{x}, \mathbf{n}, \mathbf{n}^{\prime}, \xi, \xi^{\prime}\right)=$ redistribution function for the line, $\chi_{l}^{0}(\mathrm{x})=\int_{-\infty}^{\infty} \chi(\mathrm{x}, \xi) d \xi, B_{l}(\mathrm{x}, \xi)=$ Planck function for the gas. The source function given is in our case by

$$
\begin{aligned}
S(\mathbf{x}, \mathbf{n} . \xi) & =\frac{1}{\chi(\mathbf{x}, \xi)}\left(\epsilon_{l} \chi_{l}(\mathrm{x}, \xi) B_{l}(\mathbf{x}, \xi)\right. \\
& +\frac{1-\epsilon_{d}}{4 \pi} \int_{4 \pi} p\left(\mathrm{n}, \mathbf{n}^{\prime}\right) I\left(\mathbf{x}, \mathbf{n}^{\prime}, \xi\right) d \mathbf{n}^{\prime} \\
& \left.+\frac{1-\epsilon_{l}}{4 \pi} \chi_{l}^{0}(\mathbf{x}) \int_{-\infty}^{\infty} \int_{4 \pi} R\left(\mathbf{x}, \mathbf{n}, \mathbf{n}^{\prime}, \xi, \xi^{\prime}\right) d \mathbf{n}^{\prime} d \xi^{\prime}\right) .
\end{aligned}
$$

In Eq. 1 we assume that the velocities and their gradients are so small that aberration, advection as well as boosting terms can be neglected. The hydrogen atoms are assumed have no memory so that the redistribution function is given by $R=\phi(\xi) \cdot \phi\left(\xi^{\prime}\right)$ ("complete redistribution"). Furthermore, we assume that the radiation field of the Ly $\alpha$ line can be described in the two-level approximation (in view of the fact that $\mathrm{Ly} \alpha$ is a resonance line and the hydrogen levels not involved in the transition are well separated in energy, this seems to be the least critical assumption).

Except for the special case of $\epsilon=1$ (when for a sufficiently simple velocity field the transfer equation can be determined by the method of characteristics) and the limiting case of a plane-parallel medium, Eq. 1 has to be solved fully numerically. Since the problem involves 6 dimensions ( 3 spatial, 2 angular, 1 frequency) the solution is always extremely cpu-intensive and memory-demanding. Effectively, only the following methods of solution are presently available: (i) Monte Carlo approach; (ii) short and long characteristics; (iii) finite differences; (iv) finite elements.

In order to keep the numerics as simple as possible we use in this paper the method of long characteristics. By means of Mathematica this algorithm can be implemented in a 20- to 30-line code. Although for efficient use it has to be assumed that the source function does not differ too much from the local Planck function, it is expected that the emergent fluxes show already qualitatively the correct behavior.

\section{Examples}

In order to demonstrate the effects of velocity fields we approximate the galaxy with a density distribution $\rho(\mathrm{x})=\rho_{0} \exp \left(-(\mathrm{x} \cdot(1 / a, 1 / a, 1 / 2 a))^{2}\right)$, i.e. by a rotational ellipsoid that is located at the center of a box. The galaxy is assumed to be homogeneous with a dust temperature much lower than the gas temperature and it is seen under angles $\theta=\phi=\pi / 4$. The velocity field is given by

$$
\beta(\mathbf{x})=b_{\exp _{0}} \frac{\sqrt{\mathrm{x} \cdot \mathrm{x}}}{1+(\mathrm{x} \cdot \mathrm{x})^{5 / 2}} \mathbf{x}+b_{\text {rot }_{0}} \frac{\sqrt{x^{2}+y^{2}}}{1+(x \cdot x+y \cdot y)^{5 / 2}}(-y, x, 0)^{t}
$$



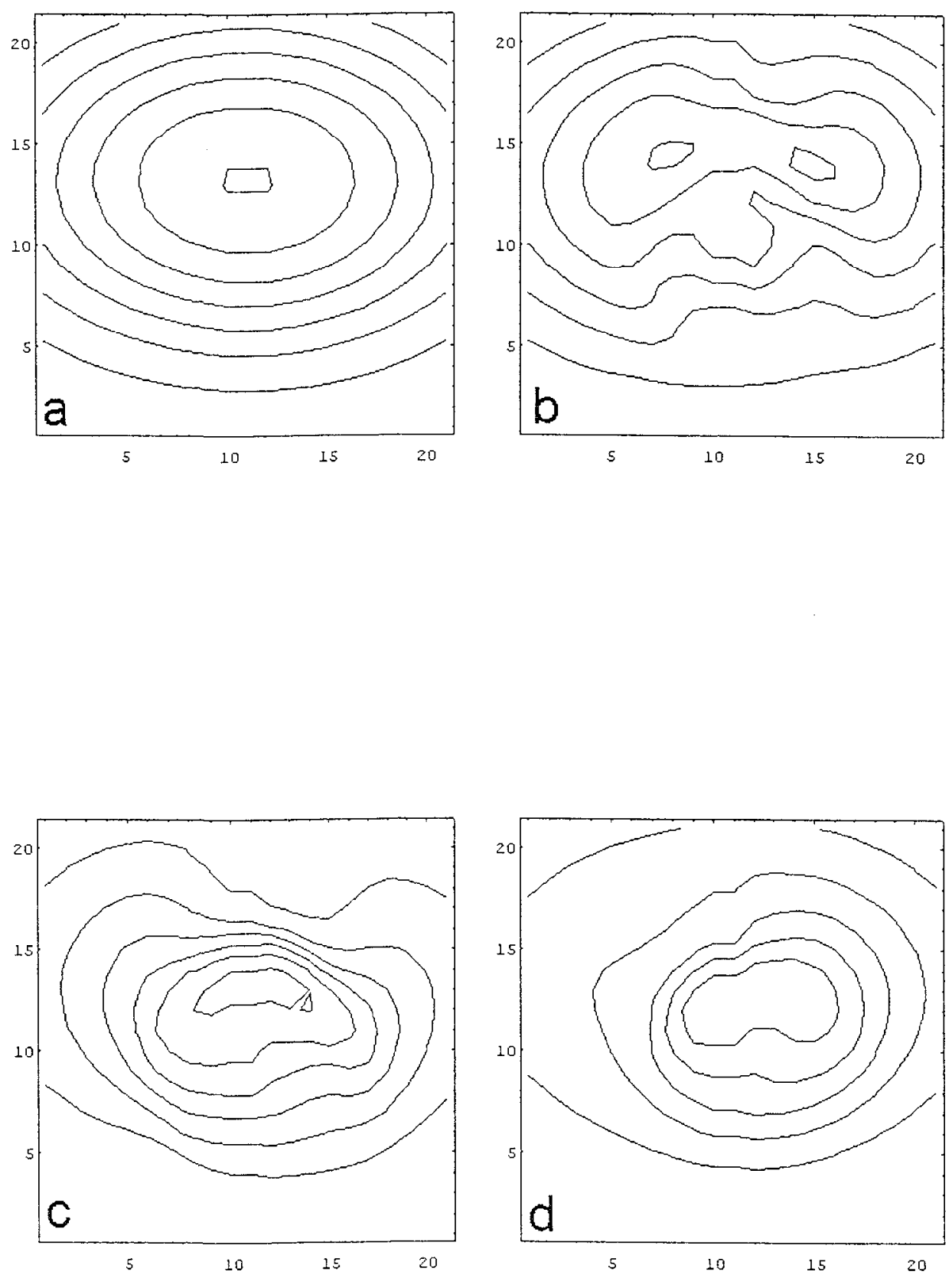

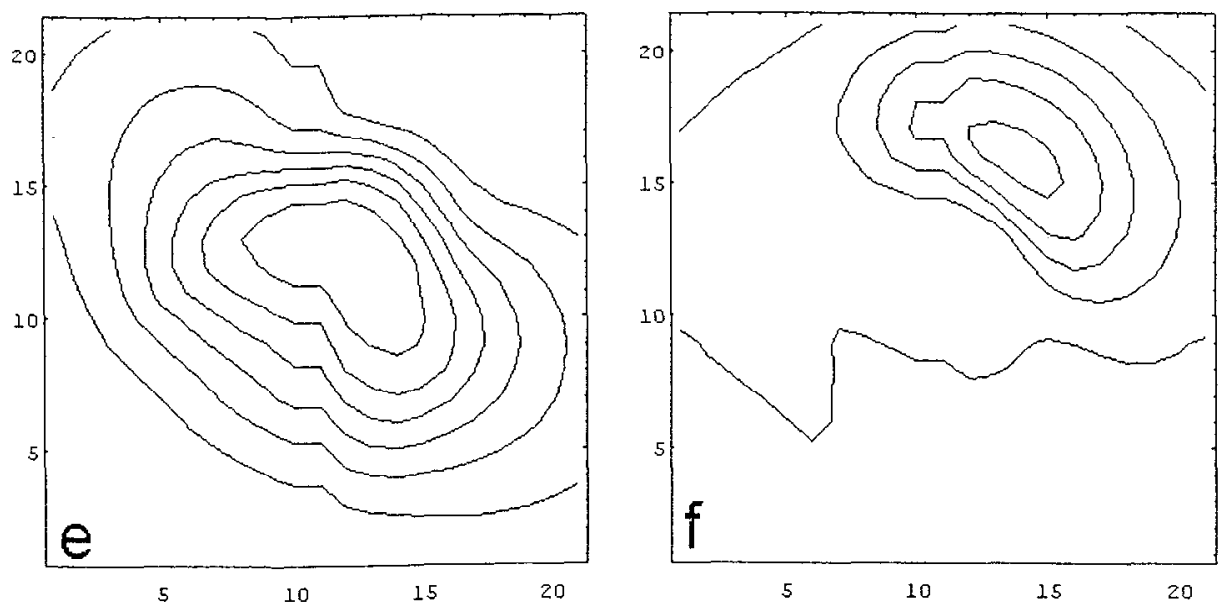

Figure 1. Examples for calculated brightness distributions in the Ly $\alpha$ line from isothermal ellipsoidal galaxies: a) no motion, line center, b) $b_{\text {rot }_{0}}=b_{\exp _{0}}=3 \xi_{D}$, line center, c) same as b) but $\Delta \xi=\xi_{D}$, d) $b_{\text {exp }}=3 \xi_{D}, b_{\text {rot }}=0, \Delta \xi=\xi_{D}$, e) $b_{e^{x p p_{0}}}=0, b_{\text {rot }}=3 \xi_{D}, \Delta \xi=x i_{D}$, f) same as e) but $\Delta \xi=-\xi_{D}$

The space of the box outside the galaxy is filled by a dilute gas that follows the same velocity law. The line profile is everywhere a Gaussian of width $\xi_{D}$.

In Fig. 1 a few examples are given for resulting spatially resolved brightness distributions that could be compared directly with observations. It is seen that the shapes of the isophotes vary strongly with wavelength and velocity. Due to specific combinations of Doppler shifts and densities, even double structures may occur.

\section{Discussion and Outlook}

In this paper we have briefly studied the frequency and spatial dependence of the flux emitted in the Ly $\alpha$ line from an extremely simple model galaxy that rotates and has an ontflow. Already in this case, the resulting isophotes may be quite complex. It is expected that the proper inclusion of scattering, excitation. density, and temperature effects will complicate the situation even more. They will be dealt with in a separate paper. On the other hand, it is evident that observations of the Ly $\alpha$ line (and possibly additional lines of different optical depths and excitation conditions) with good spatial and frequency resolution contain a lot of information that can constrain the structure of galaxies at highredshift.

Acknowledgments. This work has been supported by the Deutsche Forschungsgemeinschaft (SFB 439, project A4). 
Section H. Future Prospects 
The Hy Redshift Universe

ASP Conference Series, Vol. 193, 1999

A. J. Bunker \&s W. J. M. van Breugel, eds.

\title{
Future Ground-Based Radio and Submillimeter Observations
}

\author{
R.B. Partridge \\ Haverford College
}

Abstract. Radio and submillimeter observations will offer excellent opportunities to locate and characterize star-forming galaxies at high redshift, especially when a new generation of instruments comes on line. These opportunities are assessed here.

\section{Introduction}

This volume celebrates the present, Hy Spinrad's 65th birthday. But I'd like to devote my remarks to a brief recollection of the past, and a more detailed look at the future, in particular the future of ground-based microwave and submillimeter observations of high redshift objects.

\section{The Past}

In the summer of 1972, I spent a couple of very pleasant months in Berkeley. I came with two projects to work on, one of them a search for high redshift, star-forming galaxies, the kind of objects Jim Peebles and I had called "young galaxies" (Partridge and Peebles, 1967). That interest brought me into contact with Hy. Everyone in the department treated me with great kindness and provided real intellectual stimulus, and I spent many enjoyable hours talking with $\mathrm{Hy}$; as well as George Field, Iran King and many others about the high redshift Universe. The research that summer was the first of many less than successful attempts to find "young galaxies" (Partridge, 1974; see also Davis and Wilkinson, 1974). We were looking for objects that were large, red, symmetric and at $z \sim 10$ : it appears we should have been looking for objects that were small, blue, lumpy and at $z \sim 1$. Those were not our only mistakes. We also ignored the effect of dust absorption.

Despite this less than auspicious beginning, the idea of "young" or primeval galaxies provides a useful context for the question: What will present and planned. gronnd-based, radio and submillimeter facilities tell is about the early stages of galaxy formation? We'll begin by considering the properties of highredshift, star-forming sources in the radio and far infrared (FIR). Next. IM1 catalog some properties of planned and present facilities. And finally, I'll hazard some predictions about what these facilities will find. 


\section{Sources}

The observer's dream source is one with strong, unique and easily measured spectral lines and a continuum that rises as a positive power of frequency. The former allows redshifts to be determined; the latter makes the cosmological $\mathrm{H}_{\text {- }}$ correction positive. Jim Peebles and I didn't try to estimate the actual radio properties of "young galaxies," but if we had, we'd have predicted synchrotron emission from the supernova of massive stars and free-free emission from HII regions (see e.g., Condon, 1992). The former has radio flux $\propto \nu^{-0.7}$ typically, and the latter has flux $\propto \nu^{-0.1}$. Hence for both, the $\mathrm{K}$-correction is negative, in the sense that objects with this spectrum become more difficult to detect at high redshift. That is one explanation for the fact reported here by Rogier Windhorst that the deepest radio image of the HDF (Richards et al., 1998) detects only a handful of the optical galaxies, despite roughly comparable observing time.

Neither the synchrotron nor the free-free spectrum has any useful characteristic features, so they offer no hope of measuring redshifts. Worse, star formation is not the only way to produce synchrotron radiation: AGN's and their associated jet phenomena are also synchrotron emitters. Thus radio spectra alone cannot distinguish between star formation and $\mathrm{AGN}$. Only the radio morphology can (e.g., Condon et al., 1991; Crawford et al., 1996; Hammer, et al. 1995).

\subsection{Why Radio Observations May in Fact be Useful}

Thus far it appears the radio band is a poor place to look for high redshift young galaxies. But there are some pleasant surprises. The first is the work of Chambers, Miley, Van Breugel, and Hy himself. They were able to find high redshift galaxies associated with steep-spectrum radio sources in which the radio emission was entirely dominated by AGN's (Chambers, Miley and Van Breugel, 1987; see Chambers, et al., 1996 and Longair here). In these cases, of course, the radio emission has nothing to do with the radio-wavelength flux produced by star formation. Next, CO rotational line emission from high redshift objects is surprisingly strong (I'll return to this point later). The last surprise is just now emerging (Richards et al., 2000; Waddington et al., 2000). It is that $\sim 20 \%$ of the radio sources detected in deep surveys (e.g., of the HDF) are unidentified optically. As both Waddington and Windhorst show here, these radio sources may be very dusty objects at high $z$.

\subsection{The Crucial Role of Dust Re-emission}

It turns out that the dust, which we ignored in our initial picture of young galaxies, is the key to their detection in the radio and FIR window. The dust, at temperatures 20-40 K, reemits strongly in the far infrared. At the long wavelength end, the emissivity is strongly frequency dependent, so the dust spectrum at long wavelengths is almost always steeper than the $\nu^{2}$ RayleighJeans law, typically going as $\nu^{3}-\nu^{4}$. Thus, as many have recognized, there is a very strong positive $\mathrm{K}$-correction. As a consequence, emission from warm dust is nearly as visible at a redshift of 10 as it is at a redshift of 1 (see Cowie's report here). The combined radio-submillimeter spectrum for a dusty, star-forming galary is indicated in Fig.1. 


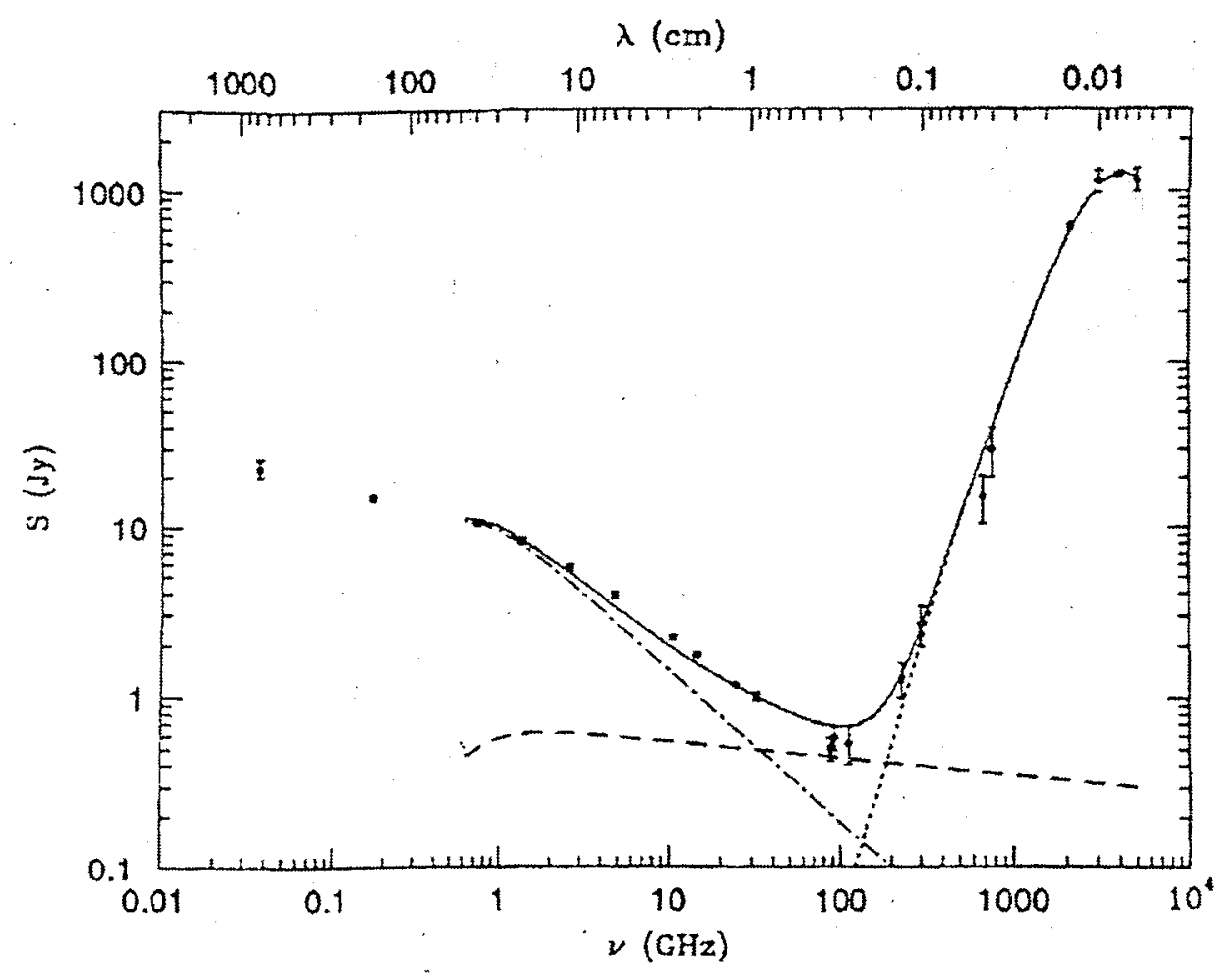

Figure 1. The rest-frame spectrum of a typical star-forming galaxy, M82, in this case. Three emission mechanisms are responsible for the flux: synchrotron (dash-dot), free-free (dash) and dust re-emission (short dash). From Condon (1992). 
Note that there is a sort of "valley of death" in the millimeter to centimeter range (this of course is the valley of life for microwave background observations, where searches for fluctuations in the microwave background are least likely to be troubled by foreground sources). On the high frequency side of the valley, the $\mathrm{K}$-correction is positive, and our best hope for the detection of high redshift, star-forming objects is found. Hence the intense excitement when deep surveys were made with the SCUBA instrument a year or so ago. SCUBA and other current instruments lack angular resolution, making identifications diffcult as Len Cowie notes here. However, the clearly detected SCUBA sources may be at substantially higher redshift than the radio-detected sources in the HDF. Unfortunately, those two issues are intertwined. There is some dispute (Richards, 1999; Downes et al., 1999) over the optical and radio identifications of the SCUBA sources. The original claim by Hughes et al.,(1998) that some were identified with faint optical objects at $z \sim 3$ may in part have resulted from some misidentifications (see technical note below). These comments should not detract from the general enthusiasm we all feel for submillimeter observationsonce angular resolution is improved on the high frequency side of the "valley of death," we will have an immensely powerful tool for the detection of high redshift, star-forming objects. In addition, the characteristic profile of the valley of death can provide approximate redshift information (see Carilli and Yun, 1999), since the ratio of $1.4 \mathrm{GHz}$ to $350 \mathrm{GHz}$ flux changes, as illustrated in Fig.2.

\subsection{Centimeter Wave Flux and Star Formations}

I want to add a brief coda to this section on the connection between radio flux, submillimeter flux and star formation. It has been known for nearly 15 years (eg., Helou et al., 1985) that radio flux and far infrared flux are strongly correlated. For instance, the ratio of $21 \mathrm{~cm}$ radio flux to $60 \mu$ far infrared emission is 0.00457 over a wide range of luminosities. Thus radio flux and far infrared flux can be used as proxies for one another, provided the redshift of the source is known to allow the $\mathrm{K}$-corrections which are radically different at the two wavelengths (e.g., Haarsma and Partridge, 1998). In addition, both fluxes are proportional to the star formation rate (e.g., Condon, 1992). Since stellar emission warms the dust, the correlation between star formation rate (SFR) and FIR emission is fairly evident. But the less well understood radio/FIR correlation also allows us to argue (Condon, 1992) that radio flux, if not corrupted by unrelated AGN emission, is also proportional to the star formation rate.

So radio observations do in fact offer some real benefits: they correlate directly with SFR (and no correction for obscuration by dust is needed); high frequency instruments are already capable of seeing ultraluminous young galaxies to $z \sim 10$; and redshift information can be found, roughly from the ratio of radio to submillimeter $\mathrm{flux}$ and precisely from $\mathrm{CO}$ (or other) molecular lines. 


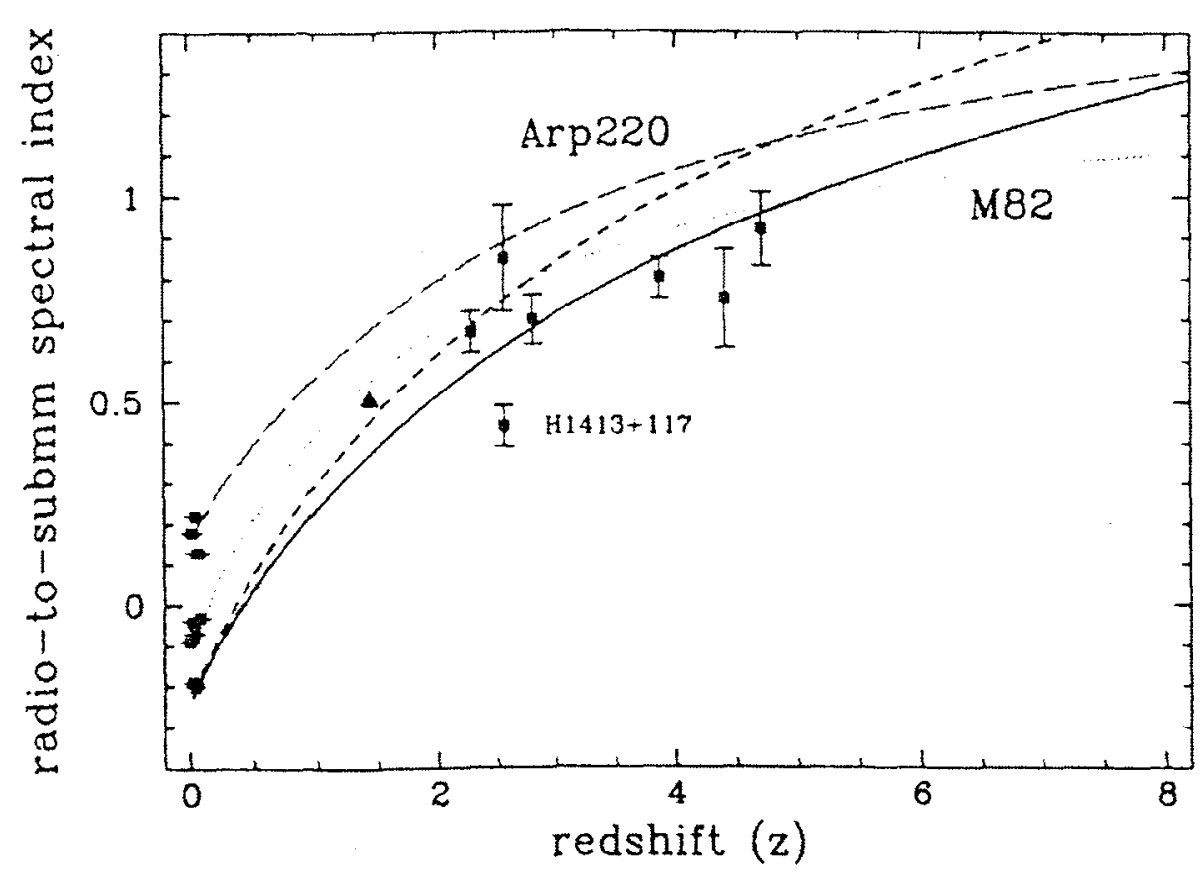

Fignre 2. Models for the $1.4 \mathrm{GHz}$ to $350 \mathrm{GHz}(850 \mu)$ spectral index from Carilli and Yun (1998). The strong dependence of $\propto_{1.4}^{350}$ is a result of the opposite sign of the $\mathrm{K}$-correction as a spectrum like that in Fig.1 is redshifted through the two observing bands. See Carilli \& Yun for details on the observational points. 


\section{Some General Properties of Radio/Submillimeter Instruments}

In centimeter wavelength radio astronomy, where superheterodyne, coherent detectors are used, neither spectral resolution ${ }^{1}$ nor spatial resolution presents any problem (see Table 1). For instance, the VLBA operated by NRAO rontinely achieves resolutions of milliarcseconds (mas) on strong radio sources.

Table 1. Some Current Ground-Based Radio and Submillimeter Instruments

\begin{tabular}{|c|c|c|c|c|}
\hline Name & Description & Wavelength & $\sim 5 \sigma$ sensitivity $\dagger$ & resolution etc. $\hat{\dagger}$ \\
\hline VLA & $\begin{array}{l}\text { interferometer } \\
2725 \text {-m dishes }\end{array}$ & $21,6,3.6 \mathrm{~cm}$ & $40,20,10 \mu J y$ & depends on config., $3^{\prime \prime}$ easy \\
\hline VLBA & $\begin{array}{c}\text { continental interferometer } \\
1025 \text {-m dishes }\end{array}$ & down to $7 \mathrm{~mm}$ & $\sim 150 \mu J y$ & down to $0.3 \mathrm{mas}$ \\
\hline Merlin & UK interferometer & down to $1.3 \mathrm{~cm}$ & $50 \mu J_{y}$ & 50 mas at $5 \mathrm{GHz}$ \\
\hline OVRO ${ }^{*}$ & $610-\mathrm{m}$ dishes & $2.7,1.3 \mathrm{~mm}$ & $0.3,1 m J_{y}$ & to $1^{\prime \prime}$ \\
\hline BIMA* & $106-\mathrm{m}$ dishes & & $\sim 1 \mathrm{~m} J_{y}$ & to $11 / 2^{\prime \prime}$ \\
\hline IRAM & $515-\mathrm{m}$ dishes & " & $0.3 \mathrm{~m} J y$ at $1.3 \mathrm{~mm}$ & to $1 / 2^{\prime \prime}$ \\
\hline SCUBA & $\begin{array}{c}\text { 91-element bolometer } \\
\text { array }\end{array}$ & 850 or $450 \mu$ & $\sim 3 m J y$ at $850 \mu$ & $15^{\prime \prime}$ pixels at $850 \mu$ \\
\hline
\end{tabular}

\footnotetext{
*To be combined to form CARMA.

${ }^{\dagger}$ Lest anyone get upset: these are not necessarily calculated for the same observing time or optimum conditions, but for reasonable values.
}

In addition, detector sensitivity is quite high, reaching to within a factor of few above the fundamental quantum limit of detector noise. High resolution is achieved by the use of interferometry or aperture synthesis. That technique has now been extended to the millimeter wave regime by two arrays operating in California, OVRO and BIMA, and one in France (IRAM), all precursors to the joint European-US ALMA array to be constructed over the next decade in Chile. I will have more to say about ALMA below. At submillimeter wavelengths, coherent detection has not been much used to date, because bolometers now offer higher sensitivity in the continuum. Bolometers are not suited to either interferometry or high spectral resolution. A major advance in the last few years has been the move from single element submillimeter detectors to dense-packed bolometer arrays, like SCUBA. Each pixel in a SCUBA map is a measurement made by an independent detector, just as for an optical CCD image. The problem, of course, is that the much longer wavelengths involved in submillimeter astronomy constrain the resolution available. Ideally, one would like to employ interferometric techniques on the high frequency side of the "valley of death," and that is the promise of ALMA.

What angular resolution is needed for the task of detecting high redshift galaxies? Our work (Windhorst et al. 1993) shows the median angular scale of galaxies as measured in the radio to be $\lesssim 1^{\prime \prime}$ for faint sonrces, in reasonable.

\footnotetext{
${ }^{1}$ Frequency agility is a problem, since radio detectors function only over a restricted frequency
} range. 
agreement with the size of the high redshift objects seen in high resolution optical images, e.g., the HDF. So resolution of the order of $0.1-0.3^{\prime \prime}$ would be ideal. Resolution of the order of $1^{\prime \prime}$ will be necessary simply to permit unambiguous identification with optical objects. See Table 2 for design goals.

\subsection{The Green Bank Telescope (GBT)}

The first new instrument I will discuss is a 100 meter diameter filled aperture (continuous collection area) telescope potentially usable to frequencies as high as $115 \mathrm{GHz}(\lambda \sim 3 \mathrm{~mm})$. The angular resolution at the highest frequency will be $\tau^{\prime \prime}$, or $15-18^{\prime \prime}$ at $\lambda=7 \mathrm{~mm}$, where it will first operate. The huge collecting area will make it very sensitive, but images will need to be built up point by point. However, since it is not an interferometer, extremely wide bandwidths can be used in the receivers, increasing its overall flux sensitivity. It will initially be equipped with a 4 -horn array operating at $\sim 7 \mathrm{~mm}$ followed by a 2 -horn receiver at 26-40 GHz. These receivers will be able to detect the $J=1-0$ line of $\mathrm{CO}$ at redshifts 1.9-3.4, covering part of a relatively "dead" zone for optical redshift determinations. GBT will survey too small a solid angle to find many high redshift objects, but will be very powerful in determining their redshift.

Table 2. Some Future Ground-Based Radio and Sub-MM Instruments

\begin{tabular}{|c|c|c|c|c|}
\hline Name & Description & Wavelength & sensitivity $\dagger$ & resolution etc. $\dagger$ \\
\hline Upgraded VLA & $\begin{array}{l}\text { up to } 35 \\
25-\mathrm{m} \text { dishes }\end{array}$ & $6 \mathrm{~m}>\lambda>6 \mathrm{~mm}$ & $\begin{array}{l}\text { see text; } \\
\sim 5 / t . I y\end{array}$ & $\begin{array}{c}\text { depends on } \lambda \text { and config., } \\
1^{\prime \prime} \text { easy }\end{array}$ \\
\hline GBT & 100-m dish & down to $3 \mathrm{~mm}$; & $\sim 10 \mu J_{y}$ & $\sim 20^{\prime \prime}(\lambda / \mathrm{cm})$ \\
\hline SMA & $86-m$ dishes & $\lambda \sim 0.4-1.5 \mathrm{~mm}$ & $\sim 1 \mathrm{~mJ} J$ & to $0.2^{\prime \prime}$ \\
\hline ALMA & $\begin{array}{c}\text { up to } 60 \\
10-12-m \text { dishes }\end{array}$ & $\begin{array}{c}\text { all atmospheric } \\
\text { windows } \\
1 \mathrm{~cm}>\lambda>450 \mu\end{array}$ & $\begin{array}{l}\text { see text; } \\
\sim 10 \mu J y\end{array}$ & $\begin{array}{c}\text { depends on } \nu \text { and config. } \\
0.2^{\prime \prime} \text { easy }\end{array}$ \\
\hline BOLOCAM & $\begin{array}{c}1.51 \text { element } \\
\text { bolometer array }\end{array}$ & $\lambda \sim 1-2 m m$ & $\sim 200 \mu J y$ & $30^{\prime \prime}$ pixels on $\mathrm{CSO}$ \\
\hline
\end{tabular}

${ }^{\dagger}$ For reasonable choices of frequency, configuration and time integration.

\subsection{The VLA (Now and in the Future)}

The VLA, an interferometer of twenty-seven 25-meter elements, is the Keck telescope of radio astronomy. The VLA offers good sensitivity at a limited number of frequencies (current surveys, for instance, can detect star-forming galaxies with $\mathrm{SFR}=100 \mathrm{M} \odot / \mathrm{yr}$ to $\mathrm{z}>2$ ), and excellent resolution and positional accuracy (recall that the absolute astrometry of the HDF image [Williams et al., 1996] relied on our VLA observations [Fomalont et al,, 1997]). But VLA technology dates from the 1970 's. There are now ambitious plans to upgrade the VLA by increasing its angular resolution, the range of frequencies at which it can operate and its sensitivity. The last point is of particular importance here. The sensitivity of the VLA is limited in part by the bandwidth employed (now $50 \mathrm{MHz}$ ) and in part by the technology of its receivers (only those operating 
at $8.5 \mathrm{GHz}$ are approximately state of the art, though new receivers are slowly being added at $43 \mathrm{GHz}$ ).

Technical Note: I remind you that the sensitivity of an interferometric array can be written as follows if we neglect atmospheric absorption:

$$
S_{\min } \propto \frac{T_{r e c}}{A \sqrt{\Delta \nu \Delta t N(N-1)}}
$$

where $\Delta \nu$ is the bandwidth of the correlator employed, $\Delta t$ the integration time, A the area of the telescopes and $N$ the number of elements in the array. At present, $\Delta \nu$ is limited to $50 \mathrm{MHz}$ at the VLA; for observations at GHz frequencies, that could easily be increased by a factor of 10 , and will be in the VLA upgrade. $T_{\text {rec }}$ is the receiver noise temperature, a measure of the quality of the receiver. As a rule of thumb take $T_{\text {rec }}=30\left(\frac{\nu}{100 G H z}\right)^{\frac{1}{2}} \pi$ at best.

The upgraded VLA will offer better resolution and positional accuracy than the current instrument, essentially complete frequency coverage from $50 \mathrm{MHz}$ to $50 \mathrm{GHz}$, and at many frequencies up to 10 times higher sensitivity. The NRAO staff planning for the VLA upgrade have estimated its sensitivity to star-forming galaxies if measured at $1.4 \mathrm{GHz}$. For a star formation rate of $10 M_{\odot} / \mathrm{yr}$. (as for M82), the renewed VLA could detect galaxies to $z \sim 2.5$; for $100 M_{\odot} / y r$. to $z \sim 5$ (current sensitivity allows a similar search to $z \sim 2$; Richards, et al., 1999). Since we believe the bulk of star formation occurs at redshifts $z<5$, and probably $z<2$, the upgraded VLA will basically be able to detect the great majority of star-forming galaxies and to determine their position to high precision.

\subsection{BOLOCAM}

BOLOCAM, like SCUBA and other submm instruments currently in use, is an array of bolometric detectors. Bolometers owe their sensitivity to the large bandwidth they can employ. The flip side of that statement is that their sensitivity per unit bandwidth is often substantially less than that of coherent receivers, so they are not well suited to spectroscopy. BOLOCAM's strength lies in its ability to detect dusty, star-forming galaxies at large redshift (see its Web page, http://www-lmt.phast.umass.edu/ins/continuum/bolocam.html). Its 151-pixel array covers a far larger portion of the sky than SCUBA; when used at the Caltech Submillimeter Observatory, and at its shortest operating wavelength of $1.1 \mathrm{~mm}$, each pixel will have $\sim 30^{\prime \prime}$ diameter on the sky. Planned sensitivity per pixel is high, $\sim 200 \mu \mathrm{Jy}$, or several times lower than SCUBA.

I would like to make a cautionary remark about BOLOCAM (and indeed other bolometric arrays as well). They will be very good at finding objects, but less good at identifying objects, because of their limited angular resolution. Thus whenever BOLOCAM identifies an interesting $1.1 \mathrm{~mm}$ source, follow-up observations will be needed to refine its position and to detcrminc its spectrum. I hasten to add that this is not a great drawback: in a sense, the same process was fruitfully followed with the SCUBA observations of Hughes ct al. (1998) and Barger et al. (1998), as Blain mentions here. As this cautionary remark suggests. the ideal instrument for the detection of high redshift, dusty objects would be one that combines high sensitivity at high frequency with high resolution. That is ALMA. 


\subsection{ALMA}

A major ingredient in the planning for the US MMA and the European LSA was high redshift studies. The two planned millimeter arrays have now been merged to form ALMA, an immensely promising instrument that combines high resolution, high sensitivity and good performance at submm wavelengths where star-forming systems are bright. It will operate in all the atmospheric windows from $\lambda=1 \mathrm{~cm}$ to $\sim 450 \mu$ with sensitivity close to that planned for the upgraded VLA at much longer wavelengths.

Next, let's look at its ability to detect high-z galaxies. Fig. 3. shows a simulation of an ALMA deep survey by Min Yun of NRAO. As it shows, ALMA should detect $\sim 100$ sources per arcmin ${ }^{2}$ or $\sim 100$ times the surface density seen by SCUBA. Onr confidence in these simulations is bolstered by the promising early counts from SCUBA (Hughes et al., 1998; Barger et al., 1999).

Not only will ALMA find high-z galaxies (and with much better positional accuracy than bolometer arrays), it will be able to measure redshifts. Silk and Spaans (1997) have shown that even high order rotational lines of CO (e.g., J = 6-5) will be strong enough in starburst galaxies to be detected at $z=10$ or more in one or another of ALMA's frequency bands. The only difficulty will be in determining which $\mathrm{CO}$ line one has detected (and for that the approximate redshift given by the radio/submillimeter flux ratio may suffice). At high redshifts, the $158 \mu$ carbon line may be a useful indicator as well.

Finally, a wrinkle optical observers may not fully apprecia.te. Interferometers can produce 2-d images of arbitrary spectral resolution (no need for slits, fibers or masks). An excellent example is the $\mathrm{J}=3-2 \mathrm{CO}$ line seen in the redshift 2.8 galaxy SMM02399-0136 (Frayer et al., 1998). Fig.4a shows a spectrum of this galaxy as you're used to seeing spectra (and pretty shabby it is). Fig. 4 b. shows images at different frequency slices around $(345 \mathrm{GHz}) /(z+1)$-much more convincing and informative. Spertral images like this will pour out of ALMA. This ability to determine redshifts without recourse to optical astronomy may be a crucial element in the characterization of high-z, star-forming galaxies.

\section{What Will These Instruments See?}

I have emphasized the ability of radio and submillimeter instruments to detect continuum and even line sources to high redshifts. But there is little point in arguing that an instrument can detect a starburst galaxy at $z=10$ if there are no galaxies at such high redshifts. We need to ask, for instance, what fraction of sources at radio and submillimeter wavelengths have we already detected? If, as others have noted here, the bulk of the FIR background has already been resolved, the scientific yield from the new instruments may be less than we hope. On the other hand, there is the equally important question: What fraction of the sources we may discover in the radio and submillimeter regime are entirely invisible at the optical?

How close have radio and submillimeter surveys come to seeing the bulk of all sources? More formally, at what flux density do the source counts begin to converge, that is to have $d N / d S$ going slower than $S^{-1}$ ? Windhorst in his paper here presents some arguments for this convergence; see also Haarsma and Partridge (1998), in which we argue that the convergence will be reached at 


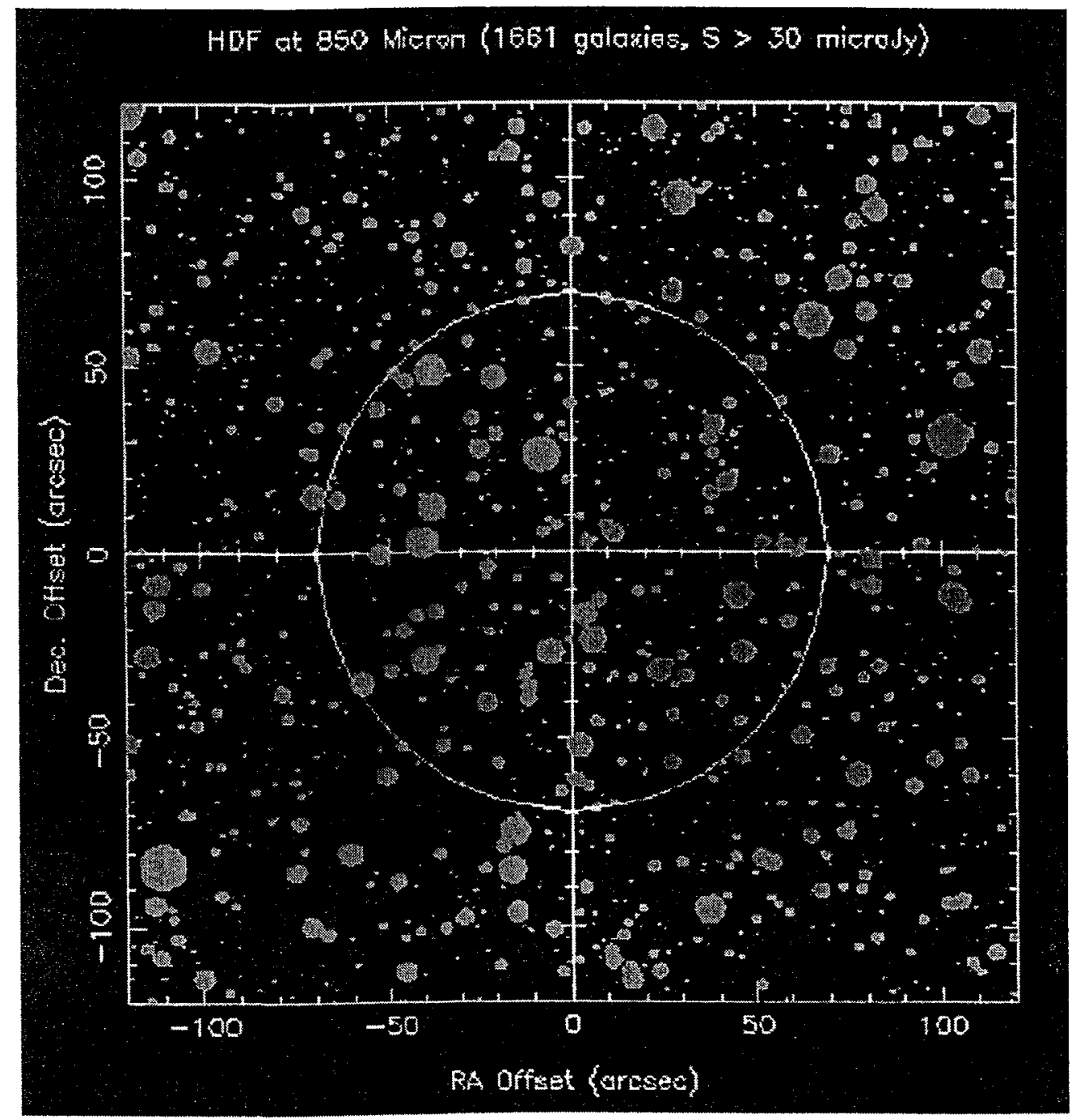

Figure 3. Simulation of the Hubble Deep Field as ALMA would see it a. $850 \mu$ (Min Yun, private communication). Spot size is image brightness (log scale). 

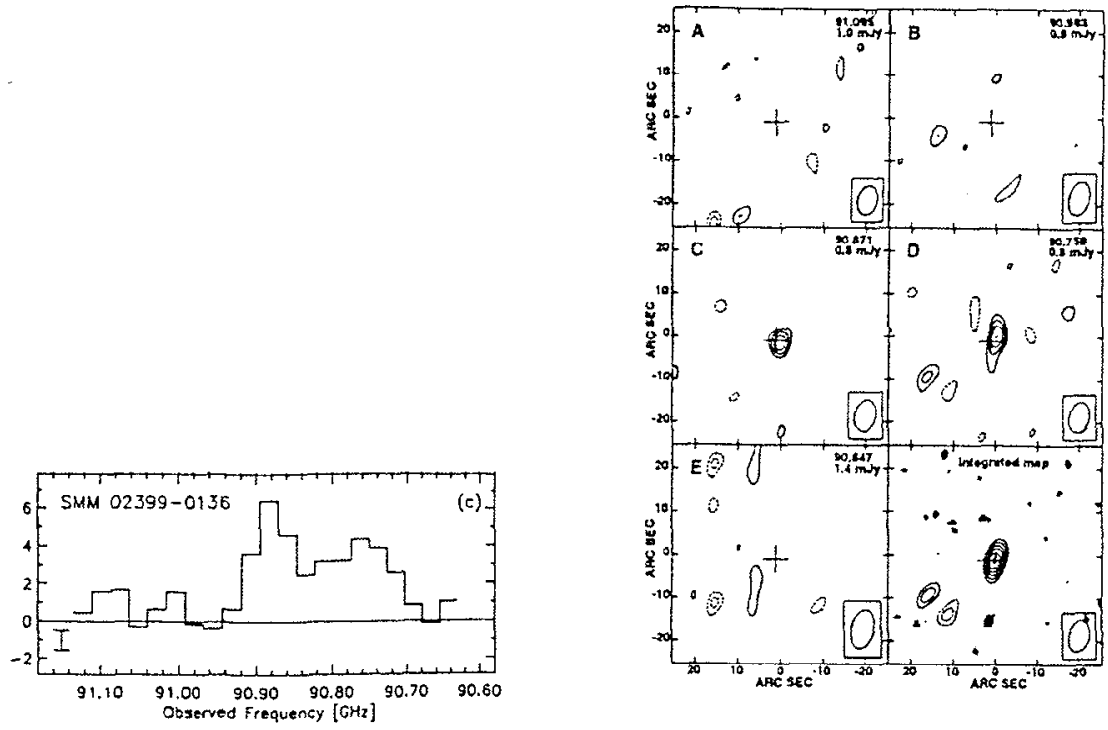

Figure 4. (a) Conventional spectrum of the $\mathrm{CO}(3 \rightarrow 2)$ line of SMM02399 at $z=2.8$. (b) Frequency slices of the image of the source in $\sim 100 \mathrm{MHz}$ steps about the frequency $345 \mathrm{GHz} /(z+1)$. Both from Frayer et al.,(1999).

$\mathrm{S} \sim 1 \mu \mathrm{Jy}$ at $8.5 \mathrm{GHz}$, corresponding to $\mathrm{S} \sim 2 \mu \mathrm{Jy}$ at $1.4 \mathrm{GHz}$ in the radio. The argument we use is that if the source counts converge at a much lower value of flux, the surface brightness of the radio sky would be too high. A similar argument can, of course, be made in the submillimeter regime. Haarsma and I do so, suggesting that counts at $\lambda \sim 200 \mu$ must converge by about $1 \mathrm{mJy}$. We based our argument on the correlation in flux between radio and FIR fluxes. However, the same argument for convergence can be made directly from the submillimeter source counts (e.g., Puget et al., 1999, who suggest convergence must set in by $\mathrm{S} \sim 10 \mathrm{mJy}$ at $\lambda=175 \mu$, a stronger constraint).

Before I leave this topic, I want to emphasize that the statement that radio source counts converge at $\sim 1 \mu \mathrm{Jy}$ does not mean that no interesting radio sources will be detected at lower flux values. The argument is a weaker one: that by the time we reach $\sim 1 \mu \mathrm{Jy}$, we will have essentially entirely resolved the radio background into discrete sources. It is worth asking what the areal density of sources at the convergence point will be. It is $\sim 3 \times 10^{8}$ per steradian, or an average separation of $\sim 14^{\prime \prime}$ if Haarsma and I (1998) are right. The upgraded VLA will have the sensitivity and the angular resolution both to detect and to resolve radio sources down to this convergence limit.

To me. a more interesting question is whether there are a substantial number of radio and submillimeter sources entirely invisible in the optical with present technology. We know that some radio sources detected at 8.5 or $1.4 \mathrm{GHz}$ are unidentified even in deep Hubble exposures. Windhorst has discussed this point 
in more detail in this volume. The same claim is made for some of the SCUBA sources (see papers by Blain and Cowie here).

Technical Notc: As a salient example, the optical identification of the strongest SCUBA source in the HDF is still subject to some uncertainty (see the preprint by Downcs et al., 1999). The SCUBA team (Hughes et al., 1998) identified their source HDF 850.1 with a galaxy at redshift of 3.36 , in part on the plausible basis that the ratio of fluxes at their two wavelengths, $850 \mu$ and $450 \mu$ suggested a high redshift. That identification was later disputed by Richards (1999) who suggested an ISO VLA source at $z=0.3$. Using IRAM, Downes et al. (1999) were able to pinpoint the position of HDF 850.1 and argued that it is associated with a faint arc lilic pair of optical sources $0.8^{\prime \prime}$ away from the radio position. In fact, as their image shows, even that identification could be questioned-is it possiblc that the single brightest $850 \mu$ source in the HDF remains optically unidentified?

Given the important role of dust obscuration, it is entirely plansible that some radio and submillimeter sources might not be visible in the optical. Two consequences follow. The first is that estimates of the star formation rate as a function of redshift (Madau et al., 1996) based on optical measurements may well underestimate the total amounl of star formation, particularly at high redshifts where rest-wavelength $\mathrm{UV}$ is being observed. This of course is a well-known issue and attempts have been made to correct for dust obscuration (Madau et al.,1998; Calzetti and Heckman, 1999; Steidel et al.,1999; also Dwek's paper here). Here I want to point out the advantage of using radio observations, which are entirely unaffected by dust obscuration, to recalculate the Madau diagram; iny postdoc, Debbie Haarsma, has done just that using radio fluxes and redshifts for the HDF and other regions where sensitive radio surveys are available. Her results, at least for low redshifts, are shown in Fig. 5. They agree well with other estimates of the star formation rate for $z<2$, but tend to lie somewhat higher, as we might expect from the argument given above.

In addition, our normal reliance on optical astronomers to determine redshifts miay not work if the sources are invisible or barely visible in the optical. Hy can speak to that, having tried to determine the redshift for one of our "unidentified" HDF radio sources. It took all Hy's skill (and Dan Stern's) and a hefty amount of Keck time to find a redshift for this source. Both photons agree with $z=4.4$ (Waddington, et al., 2000). In the future, it may be that we radio astronomers will have to solve our own redshift problems, for instance by using $\mathrm{CO}$ lines as noted above.

Acknowledgments. Much of my work reported here was part of a long and fruitful collaboration with Ed Fomalont, Ken Kellermann, Eric Richards and Rogier Windhorst (see his paper). Recently, Debbie Haarsma has made major contributions. Her research and mine is supported in part by NSF grant AST 96-1697 to Haverford and by the Keck Northeast Astronomy Consortium.

\section{References}

Barger, A.J. et al., 1998, Nature 394, 248

Calzetti, D., and Heckman, T.M., 1999, ApJ, 519, 27 


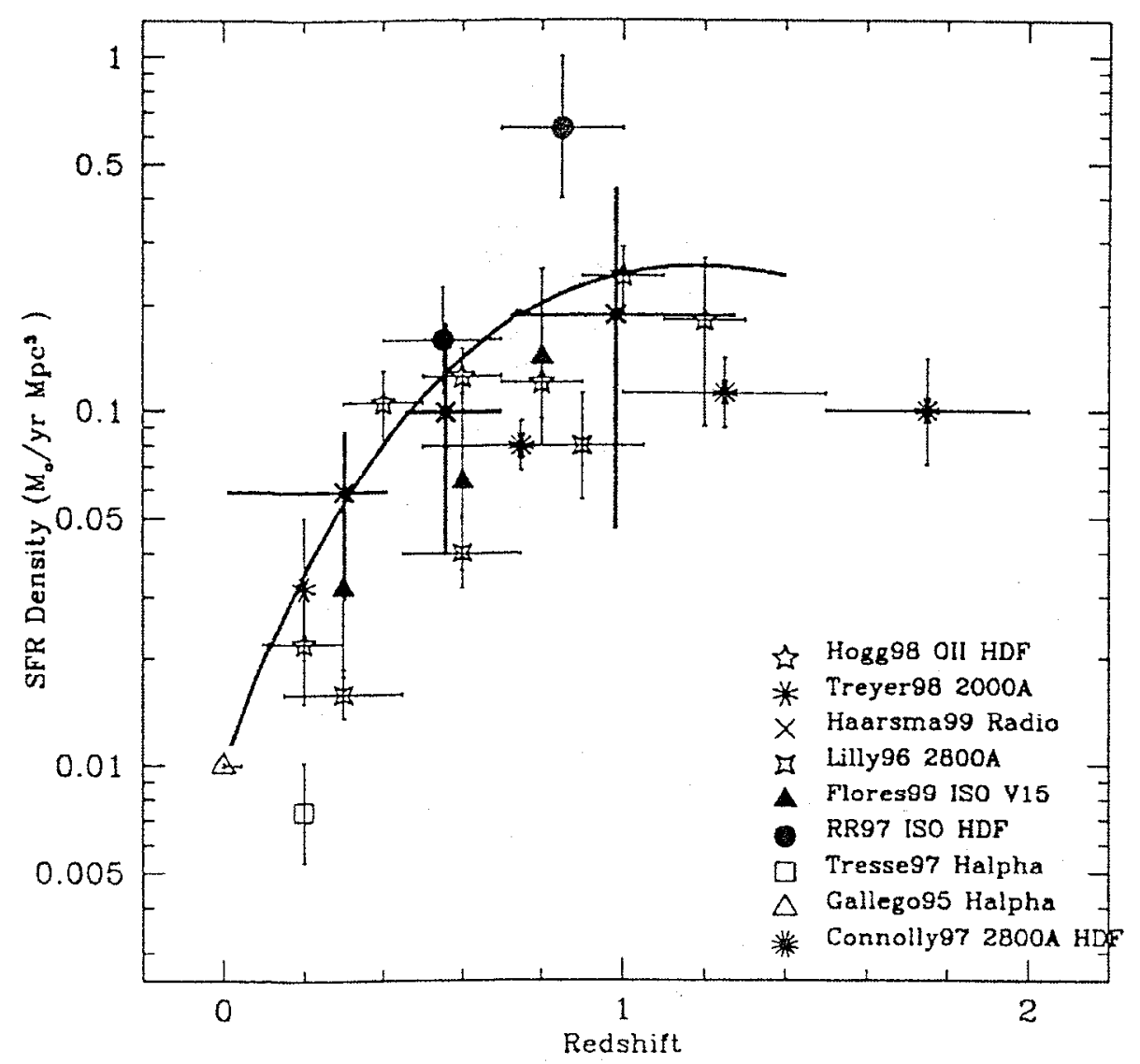

Figure 5. Star formation history derived from optical measurements and the radio measurements analyzed by Haarsma et al.,(2000) heavy crosses. The solid line is a fit derived from an evolutionary model described in Haarsma et al. 
Carilli, C. L., and Yun, M.S., ApJ, 513, L13

Chambers, K.C., Miley, G.K., and van Breugel, W.J.M. 1987, Nature 329, 604

Chambers, K.C., Miley, G.K., van Breugel, W.J.M., and Huang, J-S. 1996 ApJSuppl. 106, 215

Condon, J.J. 1992, Ann. Rev. A\&A., 30, 575

Condon, J.J., Anderson, M.L., and Helou, G. 1991, ApJ, 376, 95

Crawford, 'I', Partridge, R.B., Marr, J., and Strauss, M.A. 1996, ApJ, 460, 225

Davis, M., and Wilkinson, D.T. 1974 ApJ, 192, 251

Downes, D. et al., 1999 A\&A, in press

Fomalont, E.B., Kellermann, K.I., Richards, E.A., Windhorst, R.A., and Par tridge, R.B. 1997, ApJ, 475, L5

Frayer, D.T., et al., 1998 ApJ, 506, LT

Haarsma, D.B., and Partridge, R.B. 1998, ApJ, 503, L5

Haarsma, D.B. Partridge, R.B., Windhorst, R.A., and Richards, E.A., 2000, submitted to ApJ

Hammer, F., Crampton, D., Lilly, S.D., Le Fèvre, O., and Kenet, T. 1995, MNRAS, 276, 1085

Helou, G., Soifer, B.T., and Rowan Robinson, M. 1985, ApJ, 298, L7

Hughes, D.et al., 1998, Nature, 394, 241

Madau, P., Ferguson, H.C. Dickson, M.E., Giavalisco, M., Steidel, C.C., and Fruchter, A. 1996, MNRAS, 283, 1388

Madau, P., Pozzetti. L., and Dickson, M. 1998, ApJ, 498, 106

Partridge, R.B. 1974 ApJ, 192, 241

Partridge, R.B., and Peebles, P.J.E. 1967, ApJ, 147, 868; also ApJ, 148, 377

Puget, J.L. et. al., 1999, A\&A, in press; see Astrophy/98 12039

Richards, E. A. 1999, ApJ, 513, L9

Richards, E.A., Kellermann, K.I., Fomalont, E.B., Windhorst, R.A., and Partridge, R.B. 1998, AJ, 116, 1039

Richards, E.A. 2000, in preparation

Silk, J. and Spaans, M. 1997, Ap.J, 488, L79

Steidel, C.C., Adelberger, K.L., Giavalisco., M., Dickinson, M., and Pettini, M. 1999, ApJ, 519

Waddington, I., Windhorst, R.A., Cohen, S.H., Partridge, R.B., Spinrad, H., and Stern, D. 2000, submitted to ApJ

Williams, R.E. et al., 1996 AJ, 112, 1335

Windhorst, R.A., Fomalont, E.B., Partridge, R.B., and Lowenthal, J.D. 1993, ApJ, 405, 498 
The Hy Redshift Universe

ASP Conference Series, Vol. 193, 1999

A. J. Bunker \&\& W. J. M. van Breugel, eds.

\title{
Future Observations with SIRTF and Other Mid-Infrared/Sub-mm Missions
}

\author{
Peter Eisenhardt \\ Jet Propulsion Laboratory, California Institute of Technology, MS \\ 169-327, 4800 Oak Grove Drive, Pasaderua, CA 91109
}

\begin{abstract}
.
Infrared observations are crucial for studies of high redshift galaxies. I summarize the capabilities of three future infrared space observatories, and give a few examples of applications to the study of galaxy formation and evolution. SIRTF, with its $85 \mathrm{~cm}, 5.5 \mathrm{~K}$ telescope, will be launched in December 2001, providing natural background limited imaging from $3-180 \mu \mathrm{m}$ and spectroscopy from $5-40 \mu \mathrm{m}$ over its expected 5 year lifetime. The Next Generation Sky Survey was a proposed (but not selected) Medium Explorer surveying the entire sky at 3.5, 4.7, 12 and $23 \mu \mathrm{m}$ with a thousand to a million times better sensitivity than previous all-sky infrared surveys. The larger $(3.5 \mathrm{~m})$ but warmer $(70 \mathrm{~K})$ Far Infrared and Sub-mm Telescope is planned for launch in 2007, providing imaging and spectroscopy from $80-670 \mu \mathrm{m}$ over its 3 year lifetime.
\end{abstract}

\section{Introduction}

Hy Spinrad's career intersects with my own life in several important ways: my parents met in International House, the site of this conference, in the year $\mathrm{Hy}$ came to Berkeley as a freshman. In fact the only time in $\mathrm{Hy}^{\prime}$ 's astronomical career away from Berkeley was a 2 year stint at JPL, when he was one of the first three people hired into the JPL astrophysics group - the group in which I plan observations of high redshift galaxies with the Space Infrared Telescope Facility (SIRTF) today. I began working on SIRTF with Hy's sponsorship at Berkeley a dozen vears ago.

I performed some archival research on Hy's years at JPL and came across the image shown in Fignre 1. The data were obtained in 1963, making the lookback time over half Hy's current age. It is evident that Hy is among the more luminous members of the astronomical community, since he's evolved relatively little since then. All the major features seem to be in place, although the bar structures are more pronounced.

\section{Infrared Observations and Galaxy Formation}

There are several fundamental reasons why infrared observations are crucial for studies of galaxy formation and evolution. These include the cosmological' redshift, the ubiquity of the $\mathrm{H}^{-}$ion as a dominant opacity source in stellar 


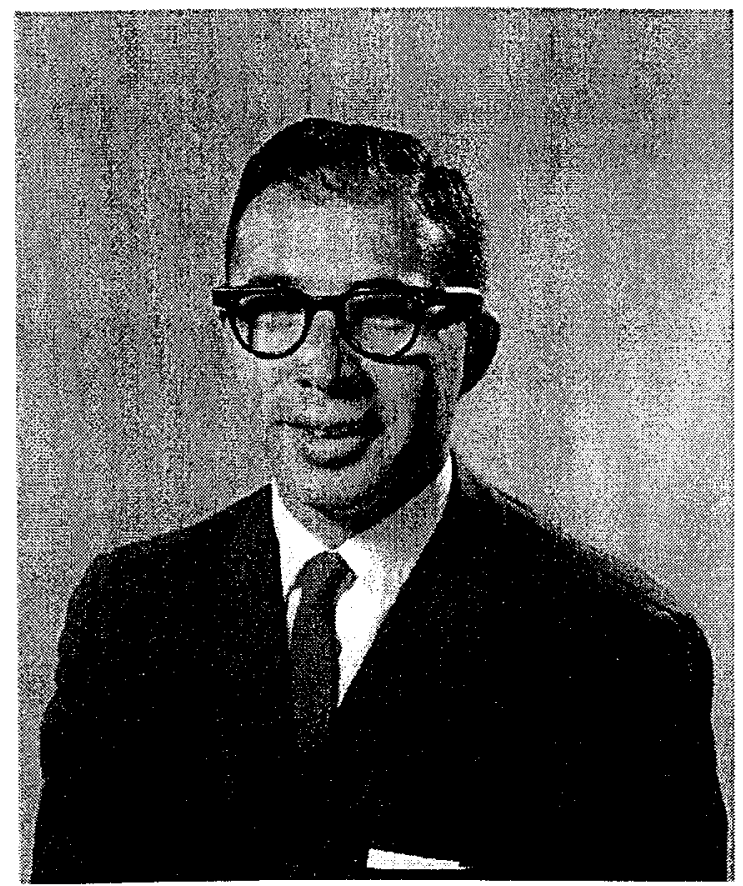

Figure 1. Hyron Spinrad at JPL in 1963

populations, and the importance of dust in modulating the spectral energy distribution of star forming regions. Such considerations have motivated a wide variety of planned and proposed space infrared missions, including the three I discuss here: SIRTF, NGSS, and FIRST.

It is almost a tautology to state that infrared data are necessary to understand evolutionary effects in high redshift galaxies. Comparisons of galaxies at different lookback time must be made at constant rest frame wavelengths to be meaningful. For starlight the most relevant wavelengths are from the Iiyman break at 912.4 to the $\mathrm{CO}$ absorption bands at $2.3 \mu \mathrm{m}$.

The Lyman break technique has now been extended to the point $(z z 7)$ that galaxies at the high redshift frontier are undetectable without infrared observations (Lanzetta et al. 1998, Dickinson et al. 1999). The Lyman break technique samples rest frame UV light emitted by the hottest stars and hence is an excellent guide to unobscured star-forming populations. Extending this technique to even higher redshifts is likely to require NGST.

But the spectra of the garden variety stars and galaxies we know most about peak in the near infrared, because of the $\mathrm{H}^{-}$opacity minimum at $1.6 \mu \mathrm{m}$ (John 1988). Identifying large samples of high redshift galaxies in the rest frame near IR has been one of the defining scientific programs for SIR'L' (see Figure 2).

Finally, star formation, at least locally, is strongly associated with dust and hence with high levels of UV extinction. Meurer, Heckman, \& Calzetti (1999) and Steidel et al. (1999) have used the correlation of UV spectral index with far IR emission to estimate that extinction corrections to estimates of the global star formation rate are a factor of 5 . Recent far-infrared detections 


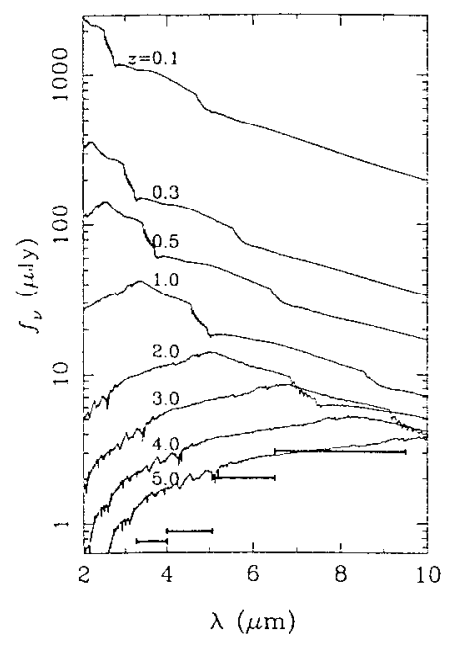

Figure 2. Model spectra as a function of redshift for a maximally old $L^{*}$ galaxy in which all stars formed in an instantaneous burst at $z=\infty$, and evolve passively thereafter, in an $H_{0}=50, q_{o}=0.1$ cosmology. The flux is normalized to $W_{K}=-25.1$ today (Gardner et al. 1997). Also shown are the IRAC sensitivities ( $1 \sigma$ in 500 seconds).

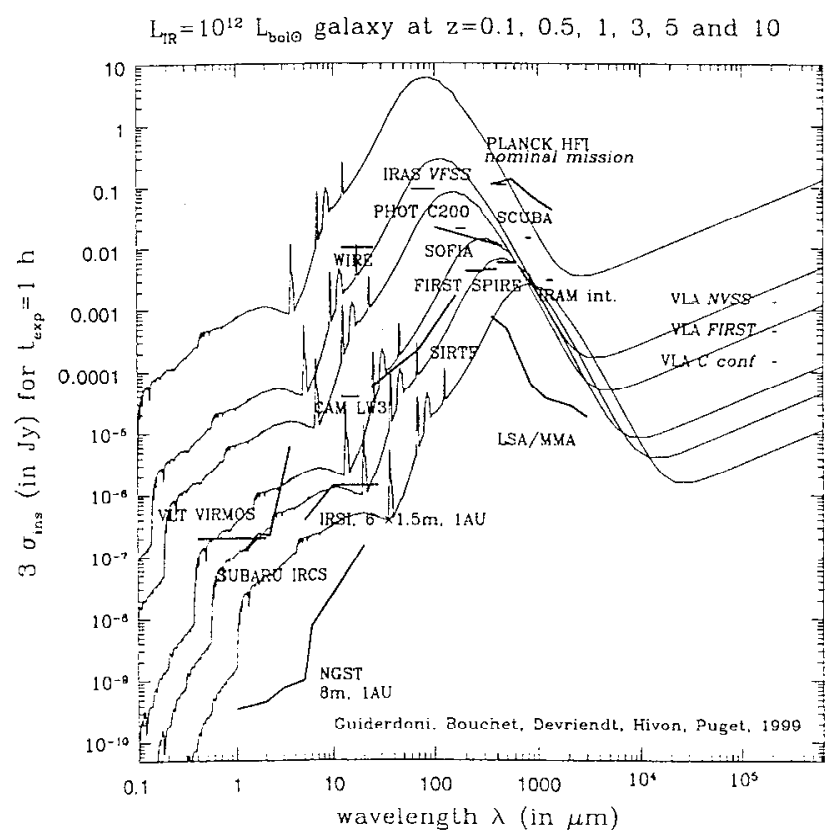

Figure 3. Model spectra of an IR luminous galaxy at various redshifts, from Guiderdoni et al. (1999). Also plotted are the sensitivities of various existing and proposed instruments. 
of the cosmic background (Hauser 1998), and far-infrared and submillimeter detections of field galaxies with ISO and SCUBA (Kawara at al. 1998, Puget et al. 1999, Barger et al. 1998, Hughes et al. 1998) also hint at a heavily dust enshrouded starburst population in the early universe. Whether these sources are in fact starbursts or are powered by $A G N$, what their luminosities are, and what their redshift distribution is, are very much open questions. The identification and characterization of distant ultra-luminous infrared galaxies (ULIRG's - see Figure 3) requires space infrared missions which directly sample the bulk of their bolometric luminosity.

\section{SIRTF}

Studies of galaxy formation and evolution have been one of the chief motivations for SIRTF since its inception. After many years of conceptual design studies, SIRTF has entered its final construction phase (Fanson et al. 1998). SIRTF will consist of a 0.85 -meter cryogenically-cooled telescope and three science instruments capable of performing imaging and spectroscopy in the $3-180 \mu \mathrm{m}$ wavelength band. With launch planned for December 2001, SIRTF will complete NASA's family of Great Observatories. Large format infrared detector arrays, coupled with innovative choices in orbit and system architecture will give SIRTF a large increase in sensitivity across its wavelength range, compared to previous missions such as IRAS and ISO. Over $75 \%$ of the observing time during its 2.5 year-minimum (5 year goal) lifetime will be awarded to general investigators. A call for Legacy Proposals (large projects of both immediate scientific interest and lasting archival value, and with no proprietary data period) is planned for July of 2000 . For the most up-to-date information on SIRTF, see http://sirtf.caltech.edu.

Several innovative design features have enabled SIRTF to retain the majority of the originally envisioned science capability at a fraction of the original cost and mass. SIRTF's 85 -cm aperture Cassegrain telescope is cooled by helium vapor to $5.5 \mathrm{~K}$. The telescope primary and secondary mirrors are constructed of beryllium, and the f-ratio of the system is $\mathrm{f} / 12$. The telescope is a RitcheyChretien design, diffraction limited at a wavelength of $6.5 \mu \mathrm{m}$. Three science instruments share the 32 arcminute diameter focal plane, and are located in a chamber cooled to $1.4 \mathrm{~K}$ by superfluid helium. A major technical development of the SIRTF mission has been the implementation of a "warm-launch architecture," in which SIRTF's telescope assembly is launched at ambient temperature and allowed to cool radiatively (passively) to $\approx 30 \mathrm{~K}$, and only then thermally connected to the helium tank. Only the focal-plane instruments and the compact liquid helium cryostat are enclosed in a vacuum shell. This has led to a dramatic reduction in the volume of liquid cryogen required (360 liters) for the 5 -year mission.

A Delta $7920-\mathrm{H}$ rocket will be used to launch SIRTE directly into an Earthtrailing heliocentric orbit, which drifts away from the Earth at approximately 0.1 AU per year. To orbit corrections or adjustments are envisaged. This orbit removes the thermal load and viewing constraints imposed by the Earth, providing much simpler operations than are possible in an Earth orbit. SIRTE's window of visibility on the celestial sky will form an annulus, perpendicular to 
the ccliptic plane, with allowed solar elongations ranging from 80 to 120 degrees. All regions of the sky will be visible to SIRTF twice a year, for a minimum of $\approx 40$ days each period (at the ecliptic equator). The visibility periods increase to $\gtrsim 200$ days per year at an ecliptic latitude of $60^{\circ}$, and constant viewing is possible within $10^{\circ}$ of the ecliptic poles. About a third of the sky will be instantaneously visible to SIRTF at any given time.

\subsection{SIRTF Instruments}

The SIRTF instruments are the Infrared Array Camera (IRAC - G. Fazio, SAO, PI, Fazio et al. 1998); the Infrared Spectrograph (IRS - J. Houck, Cornell, PI, Roellig et al. 1998); and the Multiband Imaging Photometer for SIRTF (MIPS G. Rieke, U. Arizona, PI, Heim et al. 1998).

IRAC provides imaging at $3.6,4.5,5.8$, and $8 \mu \mathrm{m}$, with predicted point source sensitivities ( $5 \sigma \mathrm{in} 500 \mathrm{sec}$ ) of $\approx 3,4,10$, and $15 \mu \mathrm{Jy}$ respectively. All bands use arrays with $256^{2} 1^{\prime \prime} .2$ pixels, with InSb detectors for the two shorter wavelengths, and Si:As IBC detectors for the two longer longer wavelengths. Dichroic beam-splitters allow the 3.6 and $5.8 \mu \mathrm{m}$ arrays to view the same $5.1 \times 5 ! 1$ field while an adjacent field is simultaneously imaged at 4.5 and $8 \mu \mathrm{m}$. A shutter allows dark current and absolute sky brightness measurements, and its mirrored inner surface can also be illuminated by calibration sources.

The IRS uses a $128^{2}$ pixel Si:As IBC array and a $128^{2}$ pixel Si:Sb IBC array to provide low resolution $(R \approx 50)$ long-slit $\left(1^{\prime}-2 ! 5\right)$ spectroscopy from $5-40 \mu \mathrm{m}$. A second pair of these arrays provides moderate resolution $(R \approx 600)$ echelle spectroscopy with $12^{\prime \prime}-22^{\prime \prime}$ slits from $10-40 \mu \mathrm{m}$. The low resolution sensitivity is $\sim 1 \mathrm{mJy}$ ( $5 \sigma$ in $500 \mathrm{sec}$ ), while the line sensitivity in the echelle mode is $\approx 3 \times 10^{-18} \mathrm{~W} / \mathrm{m}^{2}$. Part of the low resolution Si:As array is used to obtain "peak-up" images over two $1^{\prime} \times 1.2$ fields: one covering $13-18 \mu \mathrm{m}$, the other $18-26 \mu \mathrm{m}$. Onboard centroids are determined from these peak-up images to offset the source directly onto the selected slit with sub-arcsecond accuracy.

The MIPS provides imaging over a 5.3 field using a $128^{2}$ pixel Si:As IBC array at $24 \mu \mathrm{m}$, and a $32^{2}$ pixel Ge:Ga photoconductor array at $100 \mu \mathrm{m}$. A $2 \times 20$ pixel stressed Ge:Ga photoconductor array images a $0.5 \times 5.3$ field at $160 \mu \mathrm{m}$. Predicted $5 \sigma$ in 500 second sensitivities are $0.37,1.4$, and $22.5 \mathrm{mJy}$ respectively in the three bands (the $160 \mu \mathrm{m}$ value is limited by confusion). A scan mirror derived from the design proven in ISO's SWS instrument allows sources to be chopped on and off the detectors. The scan mirror also enables MIPS to efficiently survey large areas, by freezing an image of the sky on the three detectors for several seconds while the observatory continuously scans in the opposite direction at a constant rate. Other settings of the scan mirror enable a fully-sampled, higher magnification mode at $70 \mu \mathrm{m}$, and an $\mathrm{R} \approx 15$ spectral energy distribution mode from $52-99 \mu \mathrm{m}$.

\section{NGSS}

The Next Generation Sky Survey (NGSS) was a proposed NASA Medinm Explorer to survey the entire sky at 3.5 and $4.7 \mu \mathrm{m}$ with a million times better sensitivity than COBE, and at 12 and $23 \mu \mathrm{m}$ with a thousand times better sensitivity than IRAS. E.L. Wright of UCLA was the PI. The minimum $5 \sigma$ point source 
sensitivities expected for NGSS were $55,60,320$, and $1120 \mu \mathrm{Jy}$ respectively at these four wavelengths, and the image quality was specified as $5^{\prime \prime}$ FWHM ( $10^{\prime \prime}$ at $23 \mu \mathrm{m})$. NGSS would have $>2 \times$ better sensitivity over the 2500 square degrees nearest the ecliptic poles due to the lower zodiacal background and increased number of passes. NGSS planned to scan the sky in a polar, sun-synchronous orbit with a $50 \mathrm{~cm}$ telescope, using the MIPS scan mirror to freeze a $34^{\prime}$ field onto four $1024^{2}$ arrays cooled with a solid hydrogen cryostat similar to WIRE. Although it was not selected in the current round of Medium Explorers for launch in $2003 / 2004$, NGSS may be reproposed in the future. For further information see http://www.astro.ucla.edu/ wright/NGSS.

\section{FIRST}

The Far Infrared and Sub-mm Telescope (FIRST) is cornerstone number 4 of the European Space Agency's 'Horizon 2000' science plan. FIRST will perform photometry and spectroscopy in the $80-670 \mu \mathrm{m}$ range. Using an architecture reminiscent of SIRTF, NASA will supply the $3.5 \mathrm{~m}$ diameter Cassegrain telescope which is passively cooled to $<70 \mathrm{~K}$, while the three science instruments are housed in a supcrfluid helium cryostat. Launch of FIRST on an Ariane 5 into an orbit around the $\mathrm{L} 2$ point $(1.5$ million $\mathrm{km}$ from the Earth in the anti-solar direction) is planned for 2007. FIRST will be a general purpose observatory, with a minimum operational lifetime of 3 years.

The FIRST instruments are the Heterodyne Instrument for FIRST (HIFI - Th. de Grauuw, SRON, PI); the Photoconductor Array Camera and Spectrometer (PACS - A. Poglitsch, MPE, PI); and the Spectral and Photometric Imaging REceiver (SPIRE - M. Griffin, QMW, PI). HIFI offers R $=10^{3}-10^{6}$ heterodyne spectroscopy over the $110-625 \mu \mathrm{m}$ range, using SuperconductorInsulator-Superconductor (SIS) and Hot Electron Bolometer (HEB) mixers. The line sensitivity is $\sim 10^{-17} \mathrm{~W} / \mathrm{m}^{2}$ ( $5 \sigma$ in 1 hour). PACS uses two $25 \times 16 \mathrm{Ge}: \mathrm{Ga}$ arrays simultaneously covering $80-130 \mu \mathrm{m}$ and $130-210 \mu \mathrm{m}$ with full sampling at 90 and $180 \mu \mathrm{m}$ respectively, providing $\sim 5 \mathrm{mJy}$ point source detections. As a spectrometer PACS covers $\sim 1500 \mathrm{~km} / \mathrm{sec}$ at $\mathrm{R} \sim 150$, with a line sensitivity of $\sim 2 \times 10^{-18} \mathrm{~W} / \mathrm{m}^{2}$. SPIRE will use bolometer arrays cooled to $0.3 \mathrm{~K}$ to image a $4^{\prime} \times 4^{\prime}$ field simultaneously at 250,350 , and $500 \mu \mathrm{m}$ to $\sim 3 \mathrm{mJy}$. The arrays contain $32^{2}, 24^{2}$, and $16^{2}$ pixels respectively, and are refrigerated by a closed-cycle

${ }^{3}$ He sorption cooler. SPIRE also includes a Fourier Transform Spectrometer operating over a $2^{\prime} \times 2^{\prime}$ field with adjustable resolution from $0.04-2 \mathrm{~cm}^{-1}$, corresponding to $\mathrm{R}=20-1000$ at $250 \mu \mathrm{m}$. Figure 4 summarizes the performance of the FIRST instruments. Additional information about FIRST can be found at http://astro.estec.esa.nl/First.

\section{Future Space Missions and Galaxy Formation and Evolution}

Relative to ISO, SIRTE's detector arrays provide typically $1-2$ orders of magnitude better sensitivity, coupled with $1-2$ orders of magnitude more pixels. The gains of NGSS and FIRST are even more substantial. These gains are sufficient to bring vast numbers of distant galaxies at large lookback times within the grasp of the new generation of space infrared missions. 

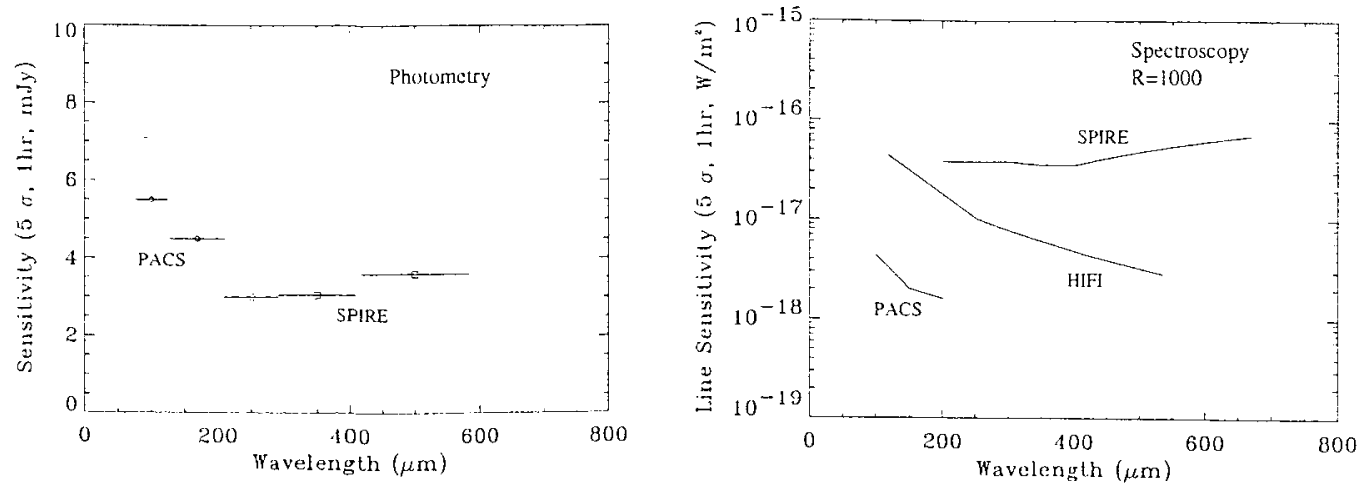

Figure 4. Photometric and spectroscopic sensitivity of FIRST instruments.

\subsection{Redshifted Starlight}

One of the defining scientific programs for SIRTF is the study of galaxies to $z>3$ by means of deep surveys at $3-10 \mu \mathrm{m}$. This limit was selected because it is apparently beyond the peak in the space density of luminous quasars (Schmidt, Schneider, \& Gunn 1995). Not only will IRAC's excellent sensitivity in this warelength region allow such galaxies to be detected (Figure 2), but the $\mathrm{H}^{-}$oparity minimum at $1.6 \mu \mathrm{m}$ (John 1988 ) is experted to be a major tool in photometric redshift determination at $1<z<5$ (Wright, Eisenhardt, \& Fazio 1994; Simpson \& Eisenhardt 1999), since it is a ubiquitous feature of stellar atmospheres.

UV-bright examples of such galaxies have a.lready been detected by means of the Lyman break technique (Steidel et al. 1996, 1999), and they play an important role in the overall star formation history of the Universe (Madau et al. 1996). By detecting galaxies on the strength of their UV emission, however, LBG samples are necessarily biased in favor of those with both active star formation and relatively modest extinction. Such samples will not reveal if there is an underlying population of galaxies which have alrearly assembled the bulk of their stellar mass. In the absence of ongoing star formation, even massive galaxies will be too faint in the rest-frame ultraviolet to be picked up by optical surveys. The stellar mass already present at an early epoch constrains the star formation rate to that point, a quantity which is still uncertain due to the possibility of significant dust extinction. Hence an accurate picture of the star formation history of the universe can only be determined by making an accurate census of all galaxies, not just star-forming ones with low extinction. Since the luminosity in the rest-frame near-infrared correlates linearly with mass (Gavazzi, Pierini, $\&$ Boselli 1996) and is relatively unaffected by dust obscuration, this is clearly the spectral region in which to make such a census.

Figure 2 shows that IRAC sensitivity is sufficient to sample around the rest frame $1.6 \mu \mathrm{m}$ peak to $z>3$. NGSS could construct a similar sample at $z=1$ over 2500 square degrees around the ecliptic poles. Scaling from existing If selected samples, Simpson \& Eisenhardt (1999) estimate that IR.LC could generate a sample containing roughly one thousand $z=3 L^{*}$ galaxies in 100 hours of observation. Beyond generating a sample selected primarily on mass. 


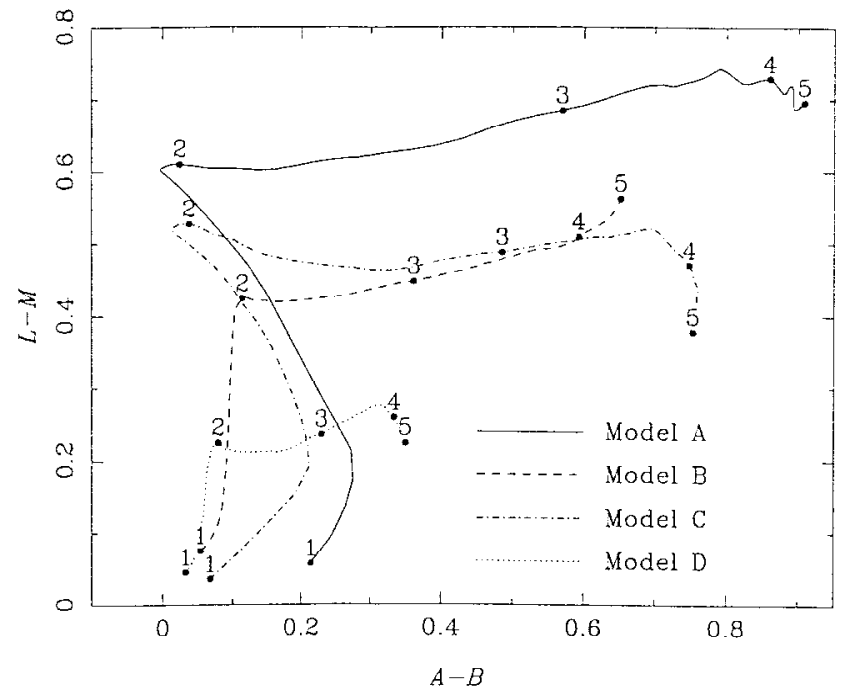

Figure 5. Loci of the four galaxy models discussed in [23] in the $L-M$ vs $A_{p}-B_{p}(3.6-4.5 \mu \mathrm{m}$ vs. $5.6-8.0 \mu \mathrm{m})$ color-color diagram. The locations of the models at $z=1,2,3,4,5$ are indicated.

IRAC sampling of the $1.6 \mu \mathrm{m}$ peak can be used to obtain photometric redshifts. The 5.8 and $8 \mu \mathrm{m}$ IRAC filters were optimized for photometric redshifts of $z \approx 3$ galaxies (Simpson \& Eisenhardt 1999). If supplemented by comparably deep ground-based $K$ data (e.g. with the planned UKIRT wide field camera), NGSS could use a similar approach to identify many thousands of $z \sim 1$ galaxy clusters.

As an illustration of how photometric redshifts can be derived from IRAC data, Figure 5 shows a $3.6-4.5 \mu \mathrm{m}$ vs. $5.6-8.0 \mu \mathrm{m}$ color-color plot for the galaxy models considered in Simpson \& Eisenhardt (1999) in the redshift range $1<z<5$. It can be seen that the color in the two Si:As filters is generally able to provide an excellent measurement of the galaxy redshift for $z z 2$. For $1 \lesssim z \lesssim 2$, the $3.6-4.5 \mu \mathrm{m}$ color provides most of the photometric redshift signal. Only Model D has a very limited range of colors which might hamper andylysis, since we are observing the Rayleigh-Jeans tail of a recent starburst with only weak metal line blanketing; however, this is exactly the sort of UV-bright galaxy which would be detected in surveys for UV dropouts, and so this does not pose a problem.

\subsection{Infrared Luminous Galaxies}

In the local universe, $30 \%$ or more of the bolometric energy of galaxies is emitted at $>10 \mu \mathrm{m}$ (Lonsdale 1999, Sanders \& Mirabel 1996). The ratio of IR to UV/optical luminosity increases with star formation rate and luminosity, and can exceed 100:1 in extreme cases. As derived from UV luminosity, both the incidence of high SFR galaxies and the global SFR were much higher at $z \geq 1$ than today (Madau et al. 1996). Hence it is plausible to expect that ultraluminous IR starburst galaxies such as Arp 220 were also much more comnion at high redshift. 

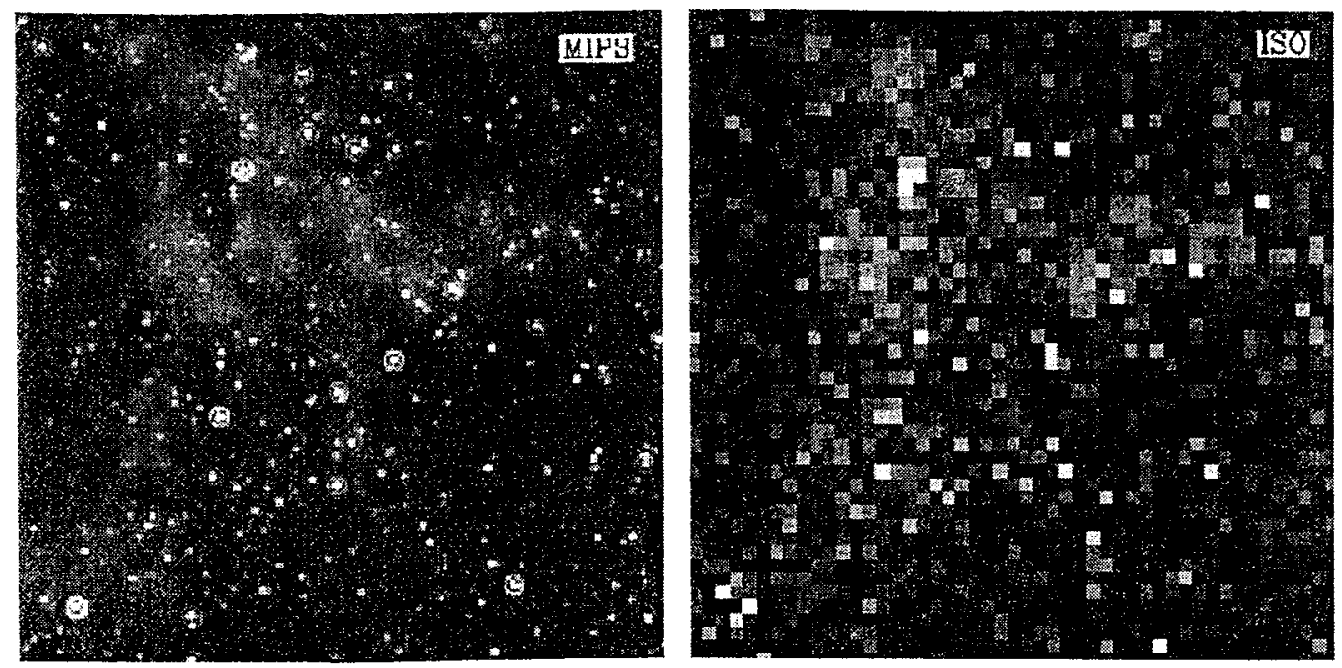

Figure 6. Simulated $70 \mu \mathrm{m}$ map of $35 \times 35$ ' region with MIPS (left) vs. ISO (right).

The detection of the cosmic infrared background (CIB) at 140 and $240 \mu \mathrm{m}$ by DIRBE (Hauser et al. 1998) demonstrates that at least half of the bolometric energy integrated over the age of the Universe is found in the IR-submm range (Dwek et al. 1998). Deep observations with SCUBA reveal a population of $850 \mu \mathrm{m}$ sources with fluxes $>1 \mathrm{mJy}$ which can account for much of the CIB (Hughes et al. 1998, Barger et al. 1998), while ISO surveys to $100 \mathrm{mJy}$ at $175 \mu \mathrm{m}$ reveal a population which accounts for roughly $10 \%$ of the CIB (Reach et al. 1999).

Figure 3 from Guiderdoni et al. (1999) plots the spectrum of an IR starburst galaxy with luminosity similar to Arp 220 at various redshifts. The sensitivity of a number of existing, planned, and proposed instruments is shown for comparison.

SIRTF's MIPS instrument can obtain photometry for sources similar to Arp 220 to $z \sim 2$, and should be able to identify the bulk of the discrete sources comprising the CIB at $160 \mu \mathrm{m}$. FIRST's SPIRE instrument will extend this to $500 \mu \mathrm{m}$ with better angular resolution, probing more deeply into the confusion expected at these wavelengths. Targeted far-IR observations of luminous LBG galaxies on the one hand, and of SCUBA sources on the other will allow the construction of complete spectral energy distributions for these objects, and show whether there is any significant overlap between objects which contribute to the UV/optical cosmic background and those which make up the CIB.

The MIPS scan mirror enables large areas to be surveved efficiently. Figure 6 illustrates the type of data expected from 24 hours of LIPS observation, as compared to the same time with ISO. With its very large arrays, NGSS would survey even more efficiently: the mosaic shown in Figure 6 corresponds to a single NGSS field. although only at wavelengths $\leq 23 \mu \mathrm{m}$. Surveys such as these will allow the identification of large enough samples to search for rare and perhaps presently unknown classes of objects. 

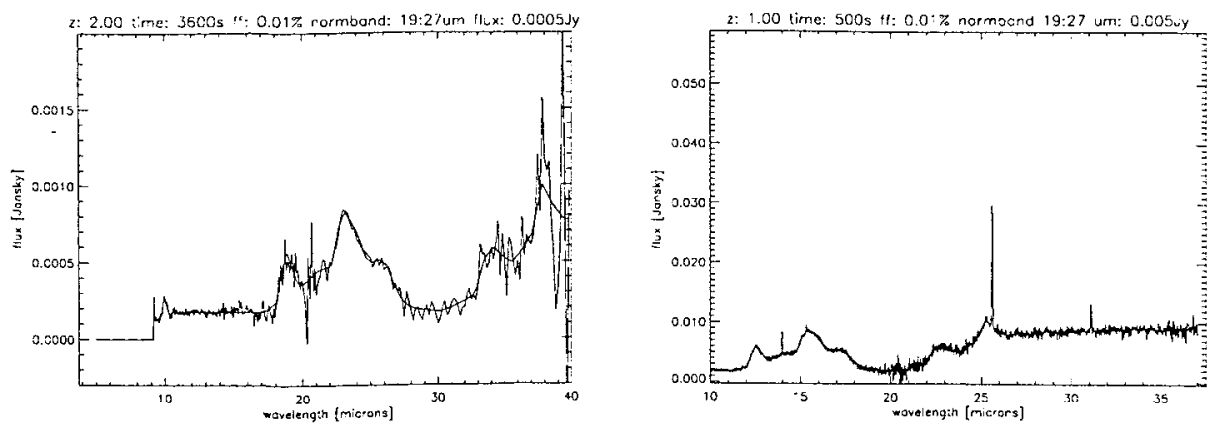

Figure 7. Simulated low-resolution IRS spectrum of an ultraluminous IR galaxy at $z=2$ (left), and high-resolution spectrum at $z=1$ (right).

\subsection{Starbursts vs. AGN}

While surveys can reveal previously unsuspected populations and determine global parameters, spectroscopy is necessary to understand the physical nature of the infrared luminous objects IR surveys will discover. For heavily extincted objects with extreme ratios of IR to UV/optical luminosity, the IRS is likely to be the only tool to obtain the redshift until the advent of NGST and FIRST. Figure 7 shows simulated IRS spectra of an Arp 220-like ULIRG with the low resolution modules at $z=2$ and with the high resolution modules at $z=1$. The complete low resolution spectrum would require a few hours of IRS observing, while the high resolution spectrum corresponds to 1000 seconds of integration.

One of the fundamental questions regarding ULIRG's is whether they are powered by starbursts or by AGN. The broad peak at an observed wavelength of $23 \mu \mathrm{m}$ in the low resolution spectrum is the rest-frame $7.7 \mu \mathrm{m}$ PAH feature. ISO observations have established that the strength of this feature provides good discrimination between the AGN and starburst mechanisms (Lutz et al. 1998). More luminous systems tend to have a higher incidence of AGN (and weak or non-existent $7.7 \mu \mathrm{m}$ PAH emission).

More quantitative information about excitation conditions and abundances can be obtained by measuring the strengths of the gas-phase emission lines visible in the high resolution spectrum in Figure 7 . In particular ratios of the forbidden neon lines including [NeII] $12.8 \mu \mathrm{m},[\mathrm{NeV}] 14.3 \mu \mathrm{m}$, and [NeIII] $15.6 \mu \mathrm{m}$ can readily distinguish starbursts, shocks, and $A G N$, and are very insensitive to extinction (Voit 1992). The high ratio of [NeII]/[NeIII] and $[\mathrm{NeIII}] /[\mathrm{NeV}]$ in Figure $T$ is clear evidence for a starburst.

Finally, for sources with known redshift, PACS will measure features to $>200 \mu \mathrm{m}$, and for sources with adequate flux, HIFI will resolve the velocity structure of lines such as [CII] $158 \mu \mathrm{m}$, which is thought to be the primary cooling line in star-forming galaxies. 


\section{Conclusions}

SIRTF's and FIRST's advances over previous IR capabilities will enable dramatic progress to be made on some of today's most pressing questions regarding galaxy formation and evolution. In the broadest terms, they will provide the data needed to understand the connection between the starlight making up the UV/Optical cosmic background and the dust responsible for the CIB.

Surveys with IRAC will enable the generation of field galaxy samples selected on the rest-frame $1.6 \mu \mathrm{m}$ peak out to $z=4$. Such samples are complementary to those based on the Lyman break, because they are relatively insensitive to dust and to the current star formation rate. These samples will be selected approximately on mass, assuming the local linear scaling of the $1.6 \mu \mathrm{m}$ peak with dynamical mass (i.e., the Tully-Fisher relation) continues to hold at high redshift. By sampling around the rest frame $1.6 \mu \mathrm{m}$ peak, we expect that IRAC will be able to estimate photometric redshifts to $10 \%$ for galaxies as bright as $L^{*}$ to $z>3$. MIPS and SPIRE observations will allow the study of ULIRG's to $z \sim 3$ and beyond out to $>100 \mu \mathrm{m}$ in the rest frame. The resulting spectral energy distributions will define the bolometric luminosities of sources recently found by SCUBA, and establish the degree of overlap between the sub-mm and UV selected populations. IRS observations will provide redshifts for populations too red to measure using ground-based telescopes, and reveal the extent to which AGN vs. starbursts are responsible for the ULIRG component as a function of lookback time. High resolution spectra of more luminous sources with HIFI will reveal the temperature, density and kinematics of the high redshift ISMI.

Finally, with these advances in the capability, it is highly likely that previously unsuspected phenomena will be discovered. The first opportunity for the astronomical community to make such discoveries is fast approaching: the first proposals for SIRTF time will be due in September 2000, with launch a little over a year later.

Acknowledgments. This work was carried out at the Jet Propulsion Laboratory, California Institute of Technolngy, under a contract with the National Aeronautics and Space Administration. Mark Garcia, Lee Armus, Chris Simp-

son, and Mark Dickinson provided assistance with the figures. Giovanni Fazio and Michael Werner provided useful summaries of the SIRTF mission and instruments, and Mike Seiffert provided information and figures for FIRST.

\section{References}

Barger, A. J., Cowie, L. L., Sanders, D. B., Fulton, E., Taniguchi. Y., Sato, Y., Kawara, K.. \& Okuda, H. 1998, Nature, 394, 248

Dickinson, M., et al. 1999, ApJ, in press

Dwek, E., et al. 1998, ApJ, 508, 106

Fanson, J., Fazio, G., Houck, J., Kelly, T., Rieke, G., Tenerelli, D.. \& Whitten. M. 1998, in Space Telescopes und Instruments V p. 478. Proc. SPIE 3356

Eazio, G., et al. 1998, Proc. SPIE 3354, 1024

Gardner, J. P., Sharples, R. M., Frenk, C. S., \& Carrasco, B. E. 1997, Ap., 490, L99 
Gavazzi, G., Pierini, D., \& Boselli, A. 1996, A\&A, 312, 397

Guiderdoni, B., Bouchet, F.R., Devriendt, J., Hivon, E., \& Puget, J. L. 1999, in - The Birth of Galaxies in press, eds B. Guiderdoni et al, astro-ph 9902141

Hauser, M. G., et al. 1998, ApJ, 508, 25

Heim, G. B., et al. 1998, Proc. SPIE 3356, 985

Hughes, D. H. et al. 1998, Nature, 394, 241

John, T. L. 1988, A\&A, 193, 189

Kawara, K., et al. 1998, A\&A, 336, L9

Lanzetta, K. M., Yahil, A., \& Fernández-Soto, A. 1998, AJ, 116, 1066

Lonsdalc, C. J. 1900, in Astrophysics with Infrared Surveys p. 24, eds Bicay ct al., ASP Conference Series 177

Lutz, D., Spoon, H. W. W., Rigopoulou, D., Moorwood, A. F. M., \& Gerizel, R. 1998, ApJ 505, L103

Madau, P., Ferguson, H. C., Dickinson, M. E., Giavalisco, M., Steidel, C. C., \& Fruchter, A. 1996, MNRAS, 283, 1388

Meurer, G. R., Heckman, T. M., \& Calzetti, D. 1999, ApJ, 521, 64

Puget, J. L., et al. 1999, A\&A, 345, 29P

Reach, W. T., Puget, J.-L., Lagache, G., Clements, D., \& Dole, H. 1999, in Astrophysics with Infrared Surveys p. 116, eds Bicay et al., ASP Conference Series $17 T$

Roellig, T. L., et al. 1998, Proc. SPIE 3354, 1192

Sanders, D., \& Mirabel, F. 1996, ARA\&A, 34, 749

Schmidt, M., Schneider, D. P., \& Gunn, J. E. 1995, AJ, 110, 68

Simpson, C., and Eisenhardt, P. 1999, PASP, 111, 691

Steidel, C. C., Giavalisco, M., Pettini, M., Dickinson, M., \& Adelberger, K. L. 1996, Ap.J, 462, L17

Steidel, C. C., Adelberger, K. L., Giavalisco, M., Dickinson, M., \& Pettini, M. $1999, \mathrm{ApJ}, 519,1$

Voit, G. M. 1992, ApJ, 399, 495

Wright, E. L., Eisenhardt, P., \& Fazio, G. 1994, BAAS, 26, 893 
The Hy Redshift Universe

ASP Conference Series, Vol. 193, 1999

A. J. Bunker \& W. J. M. van Breugel, eds.

\title{
The Future Hy-Redshift Universe - A Hy-E Perspective
}

\author{
Richard Mushotzky \\ Goddard Space Flight Center
}

\begin{abstract}
Deep imaging x-ray observations are probably the best way of detecting high-redshift active galaxies which are, presumably, embedded in high-redshift galaxies. We review the present $x$-ray data on faint sources and the capabilities of Chandra and XMM for the high-redshift universe. We briefly discuss the exciting possibility of directly detecting the formation of galaxies by observing the cooling of gas in potential wells.
\end{abstract}

\section{Introduction}

Why should one be interested in using high energy astrophysics to study the high redshift universe? The answer is clear - there are several fundamental problems that are best studied using $\mathrm{x}$-ray data and a few for which $\mathrm{x}$-ray astronomy is the only way to obtain reliable results.

\subsection{The first quasars? - Massive Black Hole (MBH) formation and evolution}

It is now thought that MBH (massive black holes) and galaxies "grow up together". This realization that the formation and evolution of massive galaxies and massive black holes is intimately connected is not 'new' (Canuto 1977; Meszaros 1975 ) but the impact of recent discoveries (Magurrian et al. 1998; van der Maerl 1999) has rejuvenated the field. To quote Roger Blandford (1999) "...although nuclear activity has hitherto been regarded as a sideshow it now seems distinctly possible that the formation of a black hole is linked to the early evolution of its surrounding galax'y." However it is not yet clear what is the chicken and what is the egg - that is does the galaxy or the massive black hole form 'first'. In some sense it is unimportant since searching for the first MBH is probably also searching for the first galaxy. The question of how to look for these 'early' MBHs has received considerable recent effort (Djorgovshi 1999, this symposium).

If these objects can be found and are of sufficient brightness then these $\mathrm{Hy}-\mathrm{z}$ $A G N$ can also be used as probes of the IGM out to the highest redshift. It is likely that the evolution of MBHs has a strong influence on the IGM heating and ionization and the high energy component maybe crucial (Madau \& Efstathiou 1999). The effects due to MBHs may also be critically important for the feedback between energy injection and the cooling so crucial for models of galaxy formation (Haehnelt et al. 1999) and in being a major contributor to 
the total energy budget of the universe (if for example a major fraction of the luminous SCUBA sources are AGN - see contributions by Cowie and Blain in this symposium).

\subsection{Why bother to use X-rays to find High- $z$ AGN?}

The $\mathrm{x}$-ray emission from $A G N$ is ubiquitous and $\sim 10 \%$ of the bolometric luminosity appears in the $\mathrm{x}$-ray band, with the fraction of energy in the $\mathrm{x}$-rays increasing in less luminous objects. There do not appear to be AGN that do not shine in the $2-20 \mathrm{keV}$ band (Laor et al. 1997). The 'contrast' between the nucleus and galaxy in the x-ray band is extremely high, even for low-luminosity $A G N$ and thus $x$-ray emission from the nucleus is easy to see even for objects as low in total luminosity as $3 \times 10^{39} \mathrm{ergs} \mathrm{s}^{-1}$ (at lower luminosities there is the possibility of confusion from luminous $\mathrm{x}$-ray binaries and stellar-mass black holes). As opposed to the optical (where stellar light is a major contributor), the $\mathrm{UV}$ (where light from young massive stars often dominates), the IR (where dust re-radiation from massive stars dominates) or the radio (where emission from $\mathrm{H}$ II regions, young supernova and other indications of rapid star formation are often very important), there are very few sources of radiation that can confuse the issue in the $\mathrm{x}$-ray. Again to quote from Blandford "...most of the growth of lower mass black holes could have been rendered invisible by the presence of stellar light."

Point-like $\mathrm{x}$-ray emission is much easier to recognize as being caused by 'low' luminosity AGN in the $\mathrm{x}$-ray band than in the IR/optical/radio. Using our surveys of the low redshift universe as a guideline, if the total integrated $\mathrm{x}$-ray luminosity of a small ( $<20 \mathrm{kpc}$ in size) object is greater than $10^{43} \mathrm{ergs} \mathrm{s}^{-1}$ the object is almost certainly an AGN. There are, in the low-redshift universe, no galaxies with a total (non-AGN) luminosity exceeding this level. Thus even without detailed x-ray spectra or imaging the "identification" of the nature of the source is 'clear'.

$\mathrm{X}$-rays are also rather penetrating, column densities corresponding to $A_{V}=$ $5\left(N_{\mathrm{HI}} \approx 10^{22}\right.$ atoms $\left.\mathrm{cm}^{2}\right)$ only reduce fluxes by $\sim 3$ in the Chandra and XMM bands. This is easily seen in 'hard' $(2-10 \mathrm{keV}) \mathrm{x}$-ray surveys where $\sim 1 / 2$ of the brightest objects are highly reddened in the optical and often invisible in the UV. At $\approx \sim 10$ the absorber has to be Compton thick $\left(A_{V} \sim 2000\right.$ !) to 'kill' the $\mathrm{x}$-ray flux (Fig. 1). Thus there are no dark ages for very high redshift $A G N$ in the X-ray band caused by the Gunn-Peterson effect. X-rays have a 'reverse' Ly- $\alpha$ forest effect - redshifting reduces the effects of absorption. Thus for a fixed flux and column density, high-redshift quasars are easier to detect. This effect is similar in sign to (but of smaller magnitude thanl) that seen for the sub-rnm sources.

The space density of $\mathrm{x}$-ray selected $A G N$ is not dropping at $z \gg 3$ while that of optical and radio selected AGN is dropping sharply (Miyaji et al. 1998 see below). The fundamental properties of black holes should not be functions of metallicity or environment, but only of mass, accretion rate and black hole spin. Since the $x$-ray flux originates from very close to the event horizon, the x-ray properties of high redshift 'primordial' black holes should be very similar to that of lower redshift objects. This allows a reasonable calculation of their observable properties (Haiman \& Loeb 1999). 


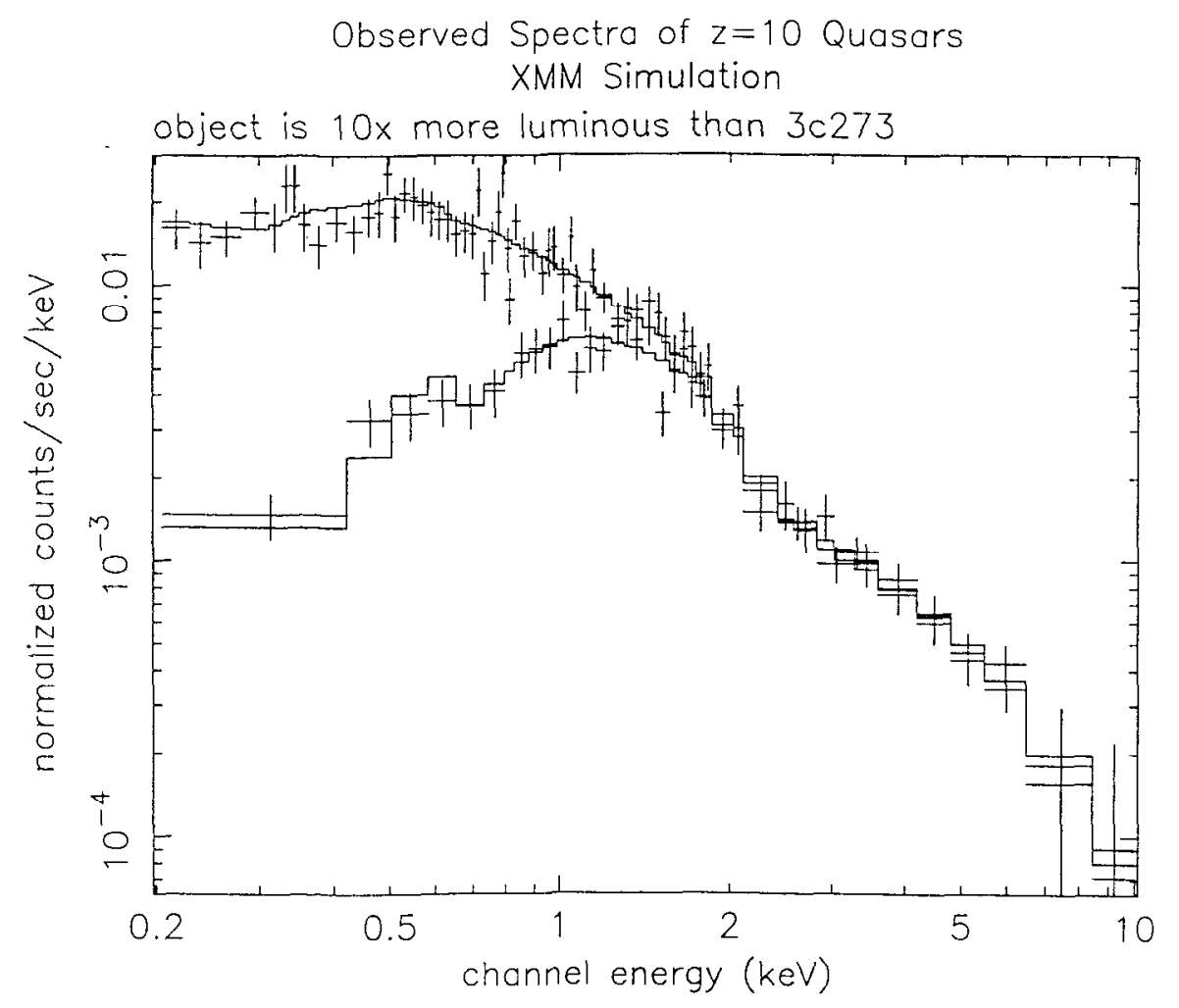

Figure 1. X-ray spectra of two AGN at $z \sim 10$, one with no absorption and the other with a line of sight column density of $\sim$ $10^{24}$ atorns $\mathrm{cm}^{-2}$. Notice that at energies greater than $2 \mathrm{keV}$ (observer's frame) that there is no reduction in the source flux. The effects at lower energies are much larger.

The combination of these properties allows sensitive searches in the $x$-ray for 'primordial' black holes and may make the $x$-ray band the 'best' place to search for these objects. Since primordial black holes are, almost certainly, in primordial galaxies the $x$-ray band may be the 'best' way to search for these objects.

\section{What do we/should we have known by now from the present generation of $\mathrm{x}$-ray surveys?}

While the apparent redshift distribution of serendipitous $\mathrm{x}$-ray sources shows a cutoff at $z \approx 4$ the distribution of flux $v s$. $z$ indicates that this cutoff is artificial (Fig. 2). Based on the observed distribution of redshift vs. flux there should be MANY $z>4$ quasars in the Rosat archives. Many of the faint Rosat sources are not 'classical' AGN and often have redder colors, and narrow Balmer lines (Schmidt et al. 1998). It is the presence of weak high ionization lines, like $\mathrm{Ne} \mathrm{V}$, that indicate that the optical flux is originating in an active nucleus. The areal density of these objects is rather high, exceeding $900 \mathrm{deg}^{-2}$ 


\section{Deep Rosat survey AGN Redshift Distribution}

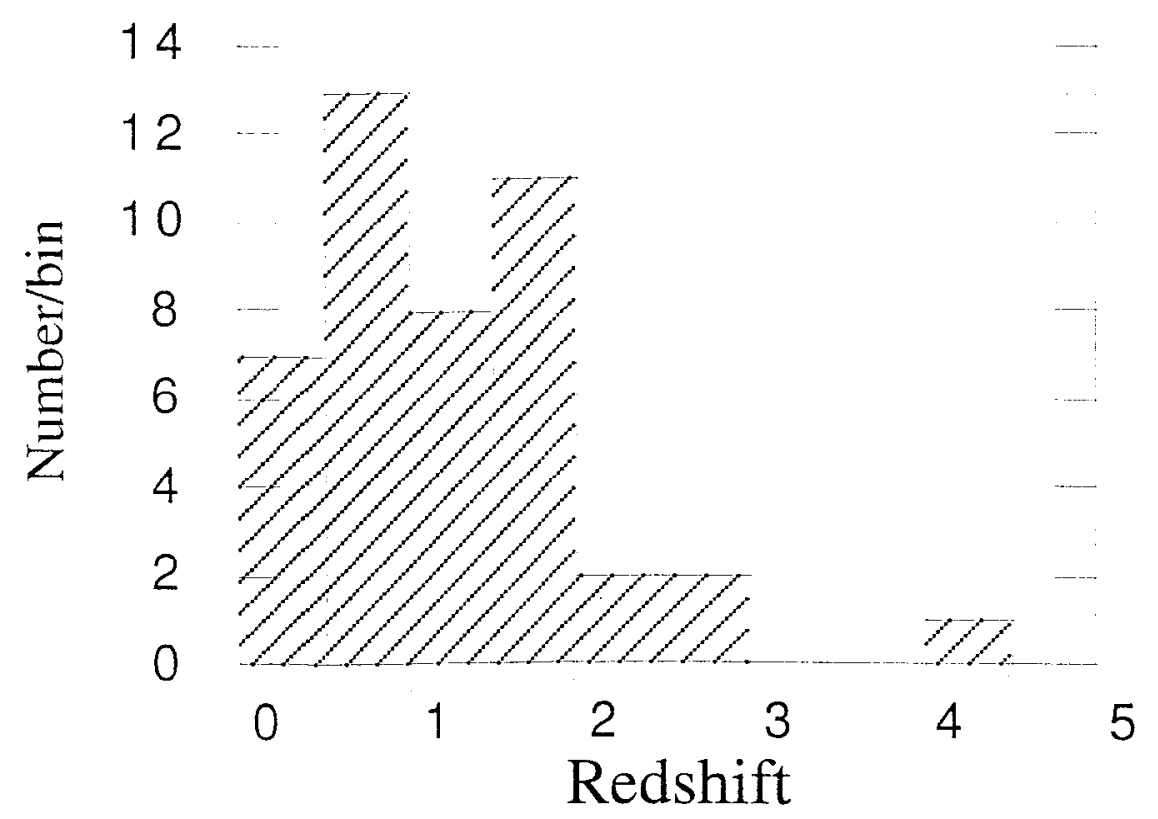

Figure 2. Redshift distribution in the published samples from deep Rosat fields.

at $F(x) \sim 10^{-15}$ ergs Deep 0. Rasat surgey. 1998). If most of these objectsare indeed $A G N$ then this deep Rosat sample has the highest areal density of active galaxies ever ound - for example at $21^{\text {st }}$ magnitude color-selected QSO samples find $\sim 20$ object de ave in the Hubble

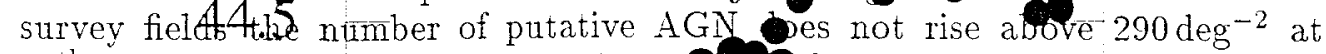
$24^{\text {th }}$ mag (Graham et al. 1999;Beck-Wir 10 Anderson 1999). The fainter Rusat sourcesqute very red with $(B-9)$ - and the optical counterparts have $R>24$ (Hasinger et al. 190). $K$ ) color selection does not seem to be a gegd way $\theta^{f}$ selectio candian So far the $U B V$ dropout technique has not been uset. 8 help id of thources. The redshift distribution is very broad, showing a rough peak at $z<1.5$ and only a very weak dependence of $F(x)$ on $z$. Ehore are \$Beral brigt, Rdio loud $z \approx 4$ x-ray detected AGN.(Fig. 3).

Oreliminary results from the Rosat deep surveys (Miyaji et al. 1998) show no cutoff in quasar space density at $z>3$ - contrary to optical and radio samples. This ' is to be distinguished from the apparent cutoff in the raw data: the Miyaji et al. papererforms a proper calculation of true space density and - its evolution 120 le Figs. $2 \& 3$ just present the observed distributions. This lack of a icutoff is probably caused by (1) the 'anti-absorption' effect: in the x-rayband - as ziquasesthe effect of photo-electric absorption is reduced, contrary to the situation in the UV and optical; and/gr (2) the hioh contrast of AGN in

\section{redshift}


$\checkmark$

0

1

$\stackrel{3}{2}{ }^{3}$

The Future Hy-Redshift Universe - A Hy-E Perspective

655

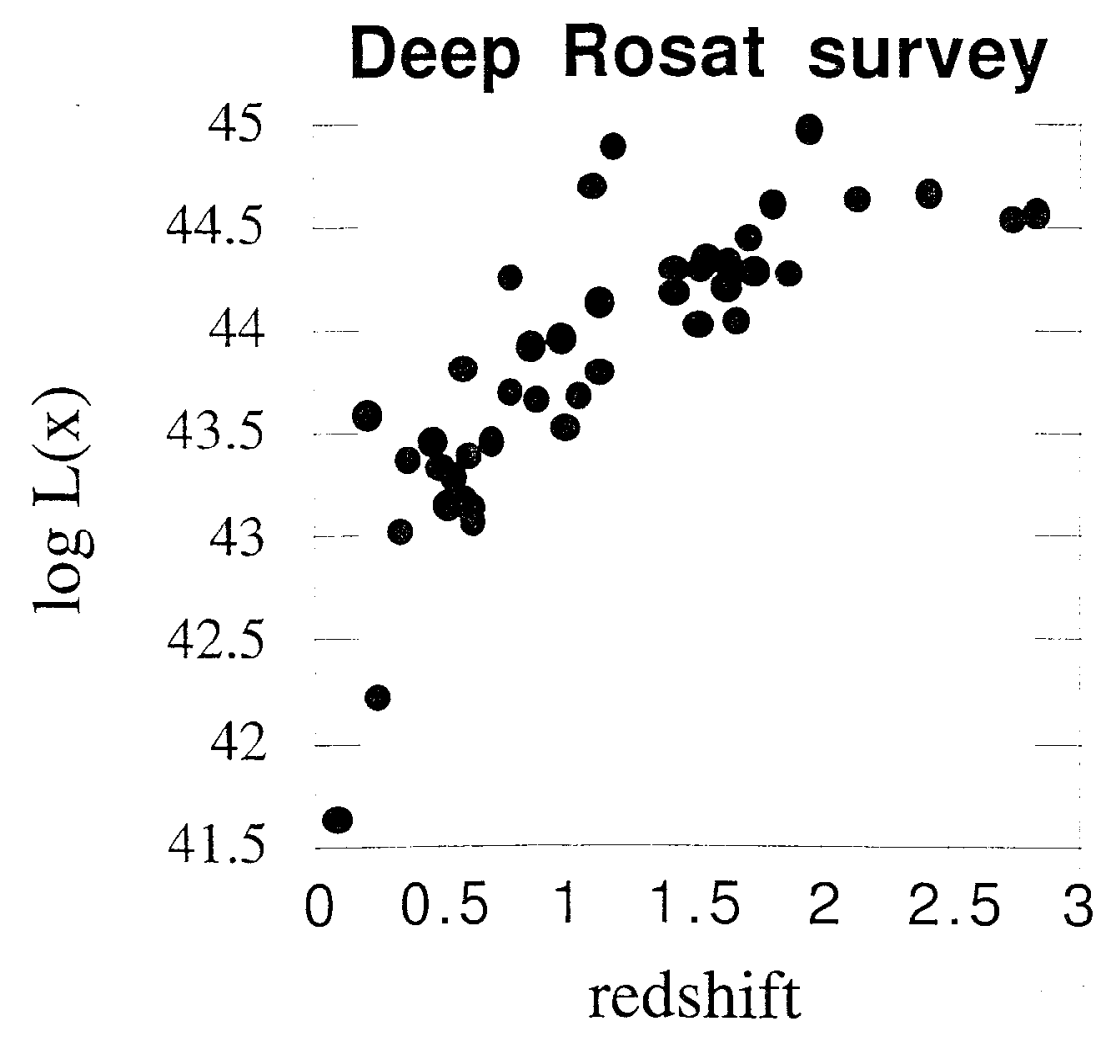

Figure 3. Luminosity vs redshift of serendipitous active galaxies in the Rosat deep surveys. All of these objects are less luminous that $3 \mathrm{C} 273\left(L(x) \approx 10^{45.5} \mathrm{ergs} \mathrm{s}^{-1}\right)$.

the $x$-ray band and the lack of numerous populations of $x$-ray bright stars and galaxies. At the flux levels of these surveys most $(\approx 80 \%)$ of the sources are AGN rather than stars or galaxies, but the optical colors of these objects are not unusual and thus they are not found in color-selected UV/optical surveys. The vast majority are not radio sources, however the strong correlation between radio, optical and $x$-ray flux allows easy selection of $\mathrm{Bl}$ Lac objects in these surveys.

These results are obtained primarily from the deepest Rosat observations, which reach $F(x) \approx 3 \times 10^{-15} \mathrm{ergs} \mathrm{cm}^{-2} \mathrm{~s}^{-1}$ and which use both of the Rosat detectors - the PSPC and the HRI. This flux level is roughly the confusion limit for the PSPC (which is the more efficient of the two Rosat detectors). For the HRI (which has better spatial resolution, but a smaller ficld of view, higher background and poorer quantum efficiency than the PSPC) the required exposure to reach this sensitivity limit is over $10^{6}$ secs. The effective solid angle of the HRI surveys is only $\approx 700 \mathrm{arcmin}^{2}$. At these high space densities the HRI data is required to properly identify the $\sim 10-20 \%$ of the PSPC sources which are confused and to discriminate extended from point-like sources. Surveys in the hardcr 2 - $10 \mathrm{keV}$ band have not gone nearly as dcop bccause of the 
poorer PSF of the ASCA and SAX telescopes used for these surveys. However, with a higher areal density ${ }^{1}$, the number of sources at a given flux level is larger. The identification of these $2-10 \mathrm{keV}$ selected sources is proceeding slowly (Georgantopoulos et al. 1999; Ueda et al. 1999), with a fair fraction of the proposed identifications being Seyfert II or "narrow line" galaxies mostly at low redshifts $(z<1)$.

\section{The Next Generation}

How sensitive are Chandra (launched on July 23 1999!) and XMM (December 1999 launch) compared to Rosat? With a HPD beam of $\approx 14^{\prime \prime}$ but a net collecting area $\sim 10 \times$ that of Rosat and factor of five larger bandwidth XMM reaches the Rosat PSPC confusion 'limit' of $\approx 3 \times 10^{-15} \mathrm{ergs} \mathrm{cm}^{-2} \mathrm{~s}^{-1}$ in exposures of $<10 \mathrm{ksec}$; however it is not clear (depending on the poorly known $\log N-\log S$ law in the $2-10 \mathrm{keV}$ band) how faint XMIM can go (Fig. 4), while with a $1.3^{\prime \prime}$ $90 \%$ encircled power Chandra is 'never' confusion limited and its sensitivity limit is $\sim 10^{-16} \mathrm{ergs} \mathrm{cm}^{-2} \mathrm{~s}^{-1}$ in cxposures of $\approx 200 \mathrm{kscc}$ (depending on the detector background). Thus in almost every exposure XMM will reach the Rosat deep survey limit and provide coarse spectral data on thesc objects over a $30^{\prime}$ diameter field of view. In the very first Chandra deep surveys (to be done in the early fall of 1999) the $x$ ray $\log N-\log S$ will go an order of magnitude deeper than with Rosat. With the absolute position uncertainty of $<1^{\prime \prime}$ and a relative error of $<0.25^{\prime \prime}$ the corrospondence between Chandra sourccs and those in other wavelength bands should be 'easy'. The flux limits are given in Table 1.

Table 1. X-ray luminosity limits for two different cosmologies at $z=$ 5 and $H_{0}=65 \mathrm{~km} \mathrm{~s}^{-1} \mathrm{Mpc}^{-1}$

\begin{tabular}{llc}
$\Omega_{M}=0.2, \Lambda_{0}=0.8$ & $\Omega_{M}=0.05, \Lambda_{0}=0$ & satellite \\
\hline $2.7 \times 10^{45} \mathrm{ergs} \mathrm{s}^{-1}$ & $9.3 \times 10^{45} \mathrm{ergs} \mathrm{s}^{-1}$ & XNM 'confusion' limit \\
$1.8 \times 10^{44} \mathrm{ergs} \mathrm{s}^{-1}$ & $6.3 \times 10^{44} \mathrm{ergs} \mathrm{s}^{-1}$ & Chandra $100 \mathrm{ks}$ limit
\end{tabular}

For reference the Seyfert galaxy NGC5548 has a x-ray luminosity of $10^{44} \mathrm{ergs}^{-1}$ and the quasar $3 \mathrm{C} 273 \sim 10^{46}$ ergs s$^{-1}$.

At redshifts of $z=3$ Chandra can detect objects 50 times less luminous than those shown in Figure 3. Based on the Rosat optical to $x$-ray flux correlation the optical counterparts of the Chandra sources will have $R \approx 28 \mathrm{mag}(\approx 20 \mathrm{nJy})$ and require NGST for spectroscopy (since they would be invisible at $\lambda<1 \mu \mathrm{m}$ ). Most of the optical counterparts to the x-ray detected objects are expected to be very red, as in deep Rosat observations, and thus these limits are misleading. It is not entirely clear what the magnitudes and colors of the Chandra sources should be. but they will clearly be at the limits of ground based work. At the

\footnotetext{
${ }^{1}$ For a E-2 photon index spectrum typical of low redshift AGN there are roughly three times as
} many sources per unit solid angle in $2-10 \mathrm{keV}$ than in the $0 . \overline{5}-2 \mathrm{keV}$ band. 


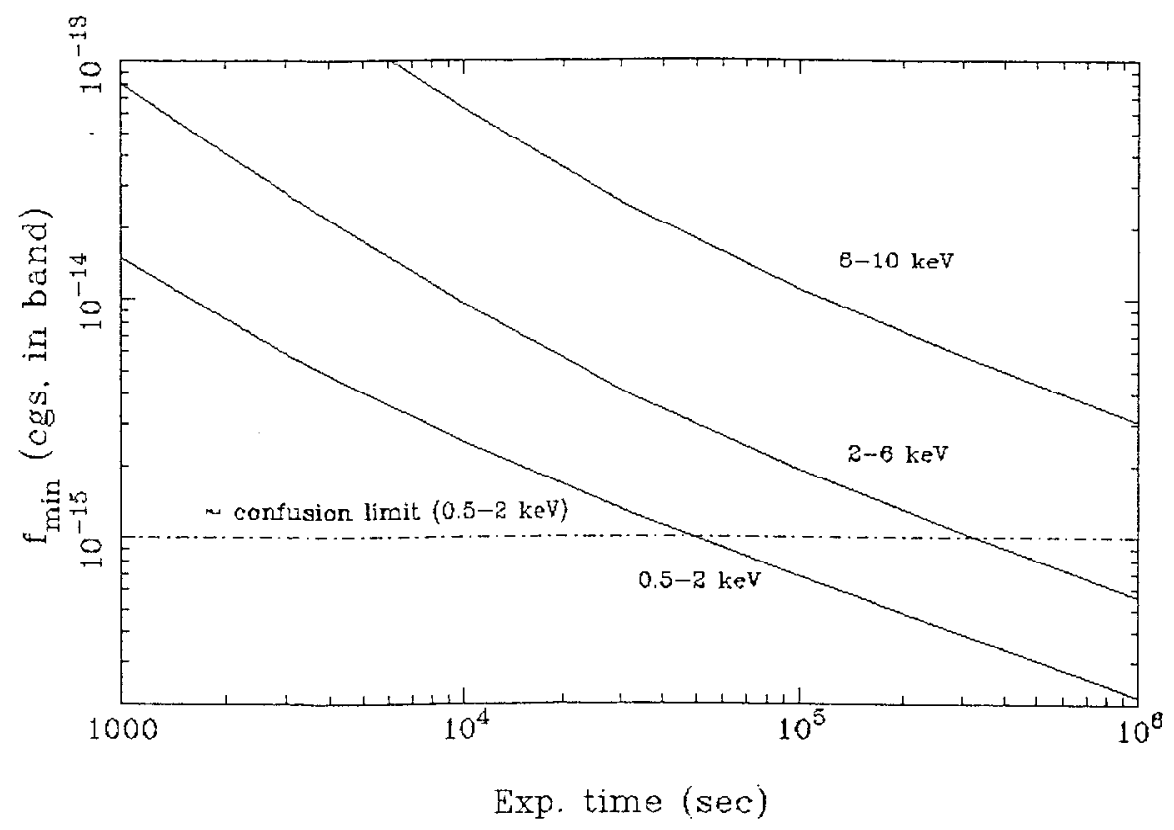

Figure 4. XMM sensitivity in different spectral bands as a function of exposure time (XMM SSC 1999). The horizontal dotted line is one estimate of the source confusion limit.

masscs cxpected for high- $z \mathrm{MBH}$ formation of $M \sim 10^{7}-10^{8} M_{\odot}$, Chandra can detect these objects at $z \sim 5$ if they are radiating at even $0.1-0.01$ of the Eddington limit.

Angular Accuracy: Chandra should have relative positions $<0.25^{\prime \prime}$ and absolute positions $<0.5^{\prime \prime}$ over its $17^{\prime} \times 1 \gamma^{\prime}$ FOV and XMM should have relative positions $<0.25^{\prime \prime}$ and absolute positions $<2^{\prime \prime}$ thus allowing unique counterparts to be identified in all wavelength bands. The Chandra positions will allow placement inside high- $z$ active galaxies to examine whether the earliest massive black holes are at the centers of the optical images. The Chandra PSF with a FWHM of $\approx 1 / 4 "$ and the extremely low background rate (roughly 1 count/day per PSF) means that only 10 photons are required for source detection and positioning. XMM will also have a UV-sensitive optical monitor which can reach $23^{\text {rd }}$ magnitude which will allow better absolute positions to be determined for the $\mathrm{x}$-ray sources.

What can we learn about these 'putative' objects? At a fl1x threshold of $10^{-14} \mathrm{ergs} \mathrm{cm}^{-2} \mathrm{~s}^{-1}$ : XMM should detect roughly 100 objects/sq degree, and can derive the $\mathrm{x}$-ray gross spectral parameters of these objects such as the power law index and the effective column density of absorbing material. The observed column density depends on the (possibly unknown) redshift, and $x$-ray spectral data of sufficient signal to noise for large column densities $\left(>6 \times 10^{22}\right.$ atoms $\left.\mathrm{cm}^{-2}\right)$ can estimate the redshift at $z \sim 10$ to $\approx \pm 5 \%$ accuracy. Since the transformation of effective $x$-ray column density to hydrogen column density is metallicity dependent and the redshift estimate depends on the presence of oxygen and iron $K$-shell 
absorption edges, information on the abundances in the absorbing gas can be obtained. For the detection of $x$-ray emission lines and precise $x$-ray redshifts we will have to wait for Constellation- $X$ for these weak very high- $z$ objects. However brighter objects at a flux of $\approx 10^{-13} \mathrm{ergs} \mathrm{cm}^{-2} \mathrm{~s}^{-1}$, if they have x-ray spectral features similar to that of low redshift Seyfert galaxies, can have their redshift determined to $\Delta z= \pm 0.04$ at $z=0.3$ in $50 \mathrm{ksec}$ exposures with XMM. A search for optical counterparts will be difficult, especially if the objects are absorbed and the sub-mm may be preferred if accurate position are possible. With the large expected samples (there should be over $\sim 1000 \mathrm{objects} / \mathrm{deg}^{2}$ for Chandra) the space density, luminosity function and evolution can be estimated reliably and Chandra and XMM will provide the first detailed census of AGN to $\approx>5$ with minimal selection effects.

While $x$-rays are a good way of finding and studying active galaxies at all redshifts, they are a 'relatively' poor way of finding high-redshift star formation. since the $\mathrm{x}$-ray to optical ratio of rapidly star forming galaxies is $100-10^{4}$ less than for AGN (Iwasawa 1999) and thus $\mathrm{X}$-ray selected samples of starbursts tend to find 'hidden' AGN instead (e.g., Ptak \& Griffiths 1999 for the spectacular discovery of an AGN in the extremely well-studied galaxy M82). Therefore the combination of far-IR and x-ray samples allow the 'splitting' of the observed energy budget into AGN and star formation which is not easily accessible in any other way. Since the only other way of determining the distribution of absorbed $A G N$ is in the radio, the fact that only $10 \%$ of $A G N$ are "radio-loud" while essentially $100 \%$ are $x$-ray loud, makes surveys for $A G N$ in the radio, necessarily, incomplete. In fact, if $\sim 10 \%$ of the SCUBA sources are indeed radio-loud AGN (Yun, Reddy \& Condon 1999 in prep) this may indicate that the majority of the sources are AGN!! If indeed these objects are AGN or superstar bursts detailed spectroscopy with Constellation- $X$

(http://constellation.gsfc.nasa.gov/booklet/execsum.html)

will determine their character. It seems likely that many of these objects will be composite (like the famous AGN/starbursts in the Circinus galaxy and NGC 4945) and thus the combination of X-ray spectroscopy with Constellation-X and IR imaging and spectroscopy is the only way of understanding these objects.

\section{Clusters and Groups of Galaxies}

For extended sources the flux limits that Chandra and XMM will attain are similar to those of the point sources as long as the size is less than $1^{\prime}$. However cosmological evolution should make massive clusters much rarer at high- $z$ than AGN. In a hierarchical $\Omega_{M}=0.3$ universe, massive clusters do not collapse until $z<2$ and should not exist at all at $z>4$. From an observational point of view it is difficult to detect $z>1$ clusters in almost any wavelength band (Mushotzliy $1999)$ but much progress has been made in the $0.8<z<1.3$ redshift range (Tanaka et al. 1999; Yee. Gladders \& Lopez-Cruz 1999). However at present the number of confirmed clusters at $z>1.3$ is ZERO (but see Dickinson et al. 1999 for a possible $\approx \approx 1.7$ object). Preliminary Rosat results (Hasinger 1999 priv comm.) indicate a possible $z \approx 1.6$ cluster based on the existence of an extended Rosat HRI source in the Lockman Hole deep survey field (Hasinger et al. 1998) and the colors of the faint galaxies in the error box. However no spectroscopic 
redshifts are available. Given the small solid angle of the Rosat deep survey and the relative insensitivity of the Rosat HRI to extended sources this may indicate that high redshift clusters are rather common! If this is true XMM and Chandra can find clusters at $z \gg 2$ since both of these missions are much more sensitive to extended emission than is the Rosat HRI (by factors of $10-100$ depending on source size). It is not clear how one can "get really $\mathrm{Hy}$ " (e.g., $z>4$ ) with direct detection of $x$-ray cluster emission unless the universe is a lot different than presently thought since these massive objects are not expected to exist at $\approx \gg 2 ! !$

However $\mathrm{X}$-ray (and Sunyaev-Zeldovich) surveys will provide the largest and most robust samples of massive objects to the highest redshifts and determine the evolution of the most massive objects in the universe. Because of the large volumes one needs to find rare objects like massive clusters, targeted surveys of suspected high- $z$ clusters can be much more efficient than 'blind' surveys. For example, both XMM and Chandra can 'easily' survey for clusters similar to 3C 324 (Dickinson et al. 1999) to $z>2$ in exposures of $\sim 100 \mathrm{ksec}$. In such a program XMM's speed allows a fast search of targeted objects and Chandra's angular resolution allows immediate identification. If such objects are found one needs the high throughput of Constellation-X to perform detailed spectroscopy of the systems discovered by XMMI and Chandra in the high- $z$ universe. Constellation- $X$ will measure the abundances of Fe at arbitrary redshifts and determine the abundances of the alpha process elements to redshifts $z>1$ (see http://constellation.gsfc.nasa.gov/booklet/missionsci.html\#icm). Constellation- $X$ will also allow detection of a hot IGM in emission and absorption along a reasonable number of lines of sight to high redshift active galaxies. The hot IGM is likely to be a low-redshift phenomenon $(z<1)$ but is the natural evolution of the high-redshift Lyman- $\alpha$ forest.

\section{Direct Observations of the Earliest Systems to Collapse}

One of the "Holy Grails" of astronomy is to find direct evidence for the formation of galaxies. Since the main physical process is the cooling of gas in the dark matter potential well it seems as if the search for the signal of cooling is fundamental. If massive systems (e.g., galaxies and groups with $M \sim 10^{11}-10^{13} M_{\odot}$ and $T \sim 10^{7} \mathrm{~K}$ ) are among the earliest objects in the universe to collapse, they should be visible via their $x$-ray emission and $x$-ray astronomy may be an important step to very high redshifts. In fact, it has been argued (Fabian et al. 1986) that cooling flows seen at low redshift are the analog of galaxy formation at high redshift.

Calculations of galaxy formation via cooling give luminosities of $5 \times 10^{43} / \mathrm{tg}_{9} \mathrm{ergs} \mathrm{s}^{-1}$ (where $t_{9}$ is the cooling time in units of $10^{9}$ years) for objects of mass $M \sim$ $10^{13} M_{-}$if the gas cools from the virial temperature of the dark matter halo (see fig. 2 in White \& Frenk 1991; Valageas, Silk \& Schaeffer 1999) with the luminosity scaling with the mass. Since the virial temperature of galaxies through clusters range from $10^{6}-10^{8} \mathrm{~K}$ the emission should appear in the $\mathrm{x}$-ray band. Of course, at higher redshifts the objects will be cooler (the virial temperature is lower for higher-z objects of a fixed mass) and appear to be cooler (because of the redshift) and for objects at $z>5$ their flux will be strongly affected by 
galactic absorption. If these objects are forming at $z \sim 3$ they should be detectable by Chandra in long exposures and appear as slightly extended sources with no reasonable visible counterpart! To detect this emission at $z \sim 5$ requires a sensitivity $10-30 \times$ better than that achieved by Chandra and XMM, e.g., fluxes of $10^{-17} \mathrm{ergs} \mathrm{cm}^{-2} \mathrm{~s}^{-1}$. This is technically possible and requires a collecting area of $\sim 5 \mathrm{~m}^{2}$ and an angular resolution of $\sim 2^{\prime \prime}$. In the U.S. $\mathrm{x}$-ray program working decadal report (Murray 1999) this mission is called "The Early Universe Observer" and XEUS (XEUS team report) in the ESA program.

While the theoretical situation is rather unclear (for example it could be that the gas is dense enough that it cools before it reaches the virial temperature), it is clearly possible, experimentally, to detect such objects. These future large missions will also allow detailed spectroscopy of the highest-z groups, clusters and $A G N$ and will observe the hot IGM in absorption on many lines of sight. The sensitivity of these missions is sufficient to detect every galaxy at $z<3$, determine the star formation rate in the universe from measurement of the direct metal injection in galactic winds, and find the earliest black holes.

Acknowledgments. Thanks to the organizing committee for an enjoyable meeting, and to Andrew Bunker for his help in assembling this manuscript.

\section{References}

Beck-Winchatz, R. \& Anderson, S. 1999 astro-ph/9901210

Blandford, R. 1999 astro-ph/9906025

Canuto, V. 1976 Nuovo Cimento Rivesta, 3, vol 2 pg 1

Dickinson, M. et al. 1999 ApJ, submitted

Fabian, A. C., Arnaud, K. A., Nulsen. P. E. J, \& Mushotzky, R. F. 1986, ApJ, 305,9

Georgantopoulos, I., Almaini, O., Shanks, T., Stewart, G., Griffiths, R., Boyle, B., \& Gunn, K., 1998 astro-ph/9810413

Graham et al. 1999, ApJ, 512, 69

Haehnelt, M., Natarajan, P., \& Rees, M. 1998, MNRAS, 300, 817

Haiman, Z., \& Loeb, A. 1999, astro-ph/9904340

Hasinger, G., Giacconi, R., Gunn. J. E., Lehmann. I., Schmidt, M., Schneider, D. P, Truemper, J., Wambsganss, J., Woods, D., \& Zamorani, G. 1998, A\&A, 340, L27

Hasinger, G. et al. 1999, astro-ph/9901103

Hasinger, G. et al. 1998, A\&A, 329, 482

Iwasawa, K. 1999, MNRAS, 302, 96

Laor, A., Fiore, F., Elvis, M., Wilkes, B., \& Mcdowell, J. 1997, Ap.J, 477, 93

Madau, P., \& Efstathiou, G. 1999. astro-ph/9902080

Wagorrian, J. et al. 1998, AJ, 115. 2285

Mivaji, T., Hasinger, G., \& Schmidt, G. 1998, astro-ph/9809398

Meszaros, P. 1075, A\&A, 38, 5

Wushotzky, R. 1999 in "After the Dark Ages", AIP Conference 470, p155 
Murray, S. et al. "X-ray Program working group report" http://hea-www.harvard.edu/XAPWG/

Ptak, A., \&c Griffiths, R. 1999, ApJ, 517, L85

Schmidt, M., Hasinger, G., Gunn, J., Schneider, D., Burg, R., Giacconi, R., Lehmann, I., Mackenty, J., Trumper, J., \& Zamorani, G. 1998, A\&A, 329,495

Tanaka, I., et al. 1999, astro-ph/9907437

Ueda, Y., Takahashi, T., Ishisaki, Y., Ohashi, T., \& Makishima, K. 1999. astro-ph/9908128

Valageas, P., Schaeffer, R ., \& Silk , J. 1999, astro-ph/9903388

Van Der Marel, R. 1999, AJ, 117, 744

Whitc, S. D. M., \& Frenk, C. 1991, ApJ, 379, 52

Yee, H. K. C., Gladders, M. D., \& Lopez-Cruz, O. 1999, astro-ph/9908001

Yun, M., Reddy \& Condon 1999 in prep.

XEUS report ftp://astro.estec.esa.nl/pub/mbeijers/xeus/xeus.html 
The Hy Redshift Universe

ASP Conference Series. Vol. 193, 1999

A. J. Bunker \& W. J. M. van Breugel, eds.

\title{
The Future: Prospects for Observational Cosmology (and Cosmogony) - Ground-Based Optical-IR.
}

\author{
Garth Mllingworth \\ UCO/Lick Observatory, University of California, Santa Cruz, CA \\ 95064
}

\begin{abstract}
With 14-16 large telescopes about to come on line in the next 5 years, the future for astronomy looks bright. When one combines their capabilities with major $(>10 \times)$ gains in HST's capabilities, new submm facilities like ALMA, space missions like SIRTF and the CMB anisotropy probes MAP and Planck, it is clear that the next decade will be one where quantum advances in astronomical knowledge are certain to occur. Many long standing major problems arc, in a broad sense, likely to be solved. The nature of the universe from $z=0$ out to $z \sim 1$, the extent to which dust cffects our view of galaxy development at high redshifts, and accurate values for cosmological parameters are all within reach within the next decade. But for those whose are looking to a research career extending beyond the next $5-10$ years, one should not despair - the galaxy formation puzzle will not be finished - too many loose cnds will remain (e.g., the physics of star formation, the epoch and characteristics of the earliest stars and metal production, the detailed impact of dust, details of the reionization epoch, the mass scales of objects at high redshifts, etc.).
\end{abstract}

\section{Introduction}

During the last five years we have witnessed explosive growth in the number of papers dealing with the universe at intermediate $(z<1)$ and high redshift $(z>$ 2). By dedicating large blocks of 4-m class telescope time, a number of surveys have provided quantitative insight into the $z<1$ universe (the Canada-france Redshift Survey (CFRS - http://www.astro.utoronto.ca/lilly/CFRS - is a key example). But it is with the new facilities, like HST with the WFPC2 camera (http://www.stsci.edu), and Keck, with its efficient low resolution spectrograph (LRIS - http://www.keck.hawaii.edu), plus the recent submm imaging capability given by SCUBA (http://www.jach.hawaii.edu/JACpublic/JCMT/scuba) on the JCMT, that have dramatically changed the observational environment in which we now work. Since this telescope/instrumental revolution is in its infancy, we can expect even greater progress in future.

We have gone from a period before the middle of this decade where the high resolution imaging and routine spectroscopy of redshift $z>1$ galaxies was impractical, or impossible, to one where it is almost rontine. It is astonishing to realize that the latter half of this decade, a mere five years, has seen the number of galaxies known at redshifts $\tilde{z}>2$ expand from a handful to over 900 . Steidel. 
and his collaborators, using the Lyman-limit photometric detection technique originally pioneered for QSO detections at high redshift, alone have assembled a sample of approximately 750 galaxies around $z \sim 2.5-3.5$.

\section{Science Issues for the Next Decade}

The next five years should bring even greater gains, and it is likely that our view of the universe at all redshifts will be revised before the next decade draws to a close, to the point where the issues, and the hey questions, are quite different from those that face us now. To exemplify this let us consider four areas which together pose a major challenge to how well we can understand the nature of galaxies at high redshift, and how they formed and evolved. They are: (i) the properties of galaxy at $z=0$; (ii) the values of the cosmological parameters; (iii) the impact of dust on our observations of galaxies, particularly at high redshift; and (iv) the properties of galaxies when the universe was about $50 \%$ of its current age, i.e., at $z \sim 1$. I expect that our knowledge base, both observationally and theoretically, will be vastly inproved as we near the end of the next decade in each of these areas.

For example, our incomplete kilowledge of (i) the properties of galaxies at $z=0$ remains a major stumbling block for establishing the degree and type of evolutionary changes seen from higher redshift, and particularly from $z \sim 1$, to the present day. Many issues remain surprisingly uncertain, given decades of work on nearby galaxies. For example, the form of the luminosity function by type, the distributions of scale lengths and velocity widths, and the resulting mass scales, the extent and magnitude of dust extinction in galaxies, are all inadequately known if we are to establish in detail the evolutionary process from $z>1$ to the present day. While many redshift surveys are underway at the present time, the two largest, the Sloan Digital Sky Survey (SDSS - see http://www.sdss.org), and the southern AAT 2dF survey (http://mso.anu.edu.au colless/2dF) should finally characterize, in a statistically robust way, the properties of galaxies in the local universe. A very important imaging survey that will provide additional, and complementary, data for galaxies in the local universe in the near-IR is the 2MASS survey (http://www.ipac.caltech.edu/2mass).

Another constraint that complicates progress is our still uncertain knowledge of (ii), the key cosmological parameters. The resulting uncertainty in timescales, for example, makes it difficult to utilize effectively the independent results from stellar evolutionary models. Recent work has resulted in a much better definition of the Hubble Constant $I_{0}$, and the recent trend to a milore open (or even lambda-dominated) geometry has eased many of the timescale problems associated with an $\Omega_{m}-1$ Einstein-de Sitter universe, but substantial uncertainties remain. Continuing work on distant supernovae, gravitational lensing, etc, plus the results from the CMB anisotropy-mapping spacecraft MAP (http://map.gsfc.nasa.gov) and Planck (http://sci.esa.int/planck) should remove cosmology as an issue for galaxy formation and evolution within the next decade - unless these new missions cause us to rethink our view of the universe.

A topic that has been swept under the carpet for many years is that of dust, item (iii) in our list. While many UV-optical observers have begun to try and 
understand (and correct) for the impact of dust, the scale of the problem has been made clear by the recent submm SCUBA results from the JCMT. We may be missing a very substantial fraction of the star formation in the universe at early times. The star-forming regions of many (most?) high-redshift galaxies, like some local starbursting galaxies (e.g., Arp 220), may be enshrouded and largely invisible in the optical, with the short wavelength flux redistributed to the submm region. Thus these objects will be missed in the typical optical/UV survey for high redshift galaxies. Furthermore, recent results also suggest that a significant fraction of the submm sources may even be too faint to study at all in the optical, and thus many of the sophisticated tools that we have developed for analysis of optical/near-IR images and spectra of high redshift galaxies may not be usable for these dust-enshrouded, starbursting (and/or AGN enhanced??) submm sources. Nonetheless, a number of new submm arrays (e.g., the OVROBIMA - http://bima.astro.umd.edu/ and http://www.ovro.caltech.edu/mm/ and ALMA, the Atacama Large Millimeter Array - http://www.alma.nrao.edu), and new instruments on existing facilities (the next generation SCUBA-like detectors), will allow us to probe this part of the observational parameter space in much more detail. These new observational capabilities will allow us, in all likelihood, to establish the enshrouded star-formation rate as a function of redshift over the next decade.

Finally, there is accumulating evidence that (iv) the galaxy population at redshifts higher than $z \sim 1$ is significantly different to that at the present day. It is striliing how similar many galaxies are at $z \sim 0.5-1$, especially the luminous ( $\sim L^{*}$ and brighter) population, yet beyond $z \sim 1-1.5$ the characteristic properties begin to cvolve away from those at lower redshift. While this is probably not an artifact of two well-known observational effects, namely the $(1+z)^{4}$ cosmological surface brightness dimming, and the shift in the observational restframe towards the UV, suitable observational checks of the impact of these effects do nced to be made. The devclopment of near IR imaging and spectroscopic capability on the ground (e.g., http://www.eso.org and http://www.gemini.edu), as well as the IR channcl of the new HST WFC3 instrument (http://www.stsci.edu/instruments/wfc3), plus major surveys like the UCSC DEEP survey (http://www.ucolick.org/deep/home.html) survey using the DEIMOS spectrograph (http://www.ucolick.org/loen/Deimos/deimos.html) at the Keck Observatory, will provide the means to establish much more quantitatively the nature of the $z \sim 1$ galaxy population and how it differs from that at the present epoch.

\section{Facilities in the Next Decade}

Later, I will address some science issues which I think will remain largely unsolved, even with the new observational capabilities that are listed below, but for now let me summarize the new facilities that (hopefully) will enable us to put these problems largely behind us.

The gains in general purpose capabilities that will surely be used for the above problems are:

(1) An order-of-magnitude increase in the number of large ground-based telescopes, from the two Kieck 10-m telescopes used to date plus, in the near- 
future, the four ESO VLT 8-m telescopes (http://www.eso.org) to some 14-16 large $(\geq 6.5 \mathrm{~m}) \mathrm{O} / \mathrm{IR}$ telescopes (roughly evenly split north and south), with efficient optical and near-IR $(\sim 1-2 \mu \mathrm{m})$ imagers and multiobject spectrographs. Many of these telescopes will also have adaptive optical systems, with laser guide stars. The potential of adaptive optics for studying distant galaxies is substantial and is part of the focus of a major new adaptive optics center at UCSC funded by the NSF - the Center for Adaptive Optics (CfAO - look for a new website that can be found through http://www.ucsc.edu).

(2) A substantial increase in HST's capabilities through the development of new instruments - the typical gains over current instrumentation are $>10 \times$. These instruments are the wide-field CCD Advanced Camera (ACS http://adcam.pha.jhu.edu) with its SDSS filter set (as well as a large complement of narrow-band and other broad-band filters - the ACS will be launched on the HST servicing mission SM-3B in 2001), the WFC3/IR UV-IR Imager (http://www.stsci.edu/instruments/wfc3) that will give us a wide-field IR imaging capability in $\mathrm{J}$ and $\mathrm{H}$ (for launch on the HST servicing mission SM-4 in 2004), and the UV Cosmic Origins Spectrograph (COS - http://cos.colorado.edu - also for SM-4 in 2004).

(3) Further developments in submm imaging on the JCMT with updated SCUBA-like imagers, and with interferometers, particularly ALMA, the Atacama Large Millimeter Array (http://www.alma.nrao.edu; http://www.eso.org), which will be an extremely powerful telescope for studying dusty objects over a large range of redshifts.

Additional capabilities and programs that are explicitly directed at the scientific issues listed above are:

(1) For the properties of galaxies in the local universe at $z=0$, the SDSS (Sloan Digital Sky Survey - http://www.sdss.org) and the AAT $2 \mathrm{dF}$ (http://mso.anu.edu.au/colless/2dF) survey will provide a variety of data on $\sim 10^{6} z=0$ galaxies. Many other redshift and imaging surveys that will complement (and supplement) these two surveys can also be found via the SDSS website links (http://www.sdss.org/links.html). Of the many surveys being carried out, two near-IR imaging surveys will likely be of particular value for dealing with dust and extinction issues for interpreting data from distant galaxies. These are the 2MASS JHK imaging survey (http://www.ipac.caltech.edu/2mass), and the DENIS IJK southern sky survey (http://cdsweb.u-strasbg.fr/denis.html).

(2) Much more accurate cosmological parameters will be obtained from the general observational capabilities listed above, as well as through specialized missions, particularly those that will provide measurements of the anisotropy in the cosmic microwave background (CMB) through new space missions, as well as through further developments of ground-based and balloon detectors. For example, supernovae studies will be well-served by the increasing numbers of large telescope spectrographs, and the additional wide-field imaging capabilities of HST with the Advanced Camera, the ACS (http://adcam.pha.jhu.edu). The NASA MAP (late 2000 or early 2001 launch) Microwave Anisotropy Probe (http://map.gsfc.nasa.gov) plus the more powerful Planck mission from ESA in 2007 (http://astro.estec.esa.nl/SA-general/Projects/Planck) will provide a wealth of data and constraints from C.IB anisotropies. 
(3) The effects of dust should be much better understood by utilizing the capabilities of SIRTF (http:/sirtf.caltech.edu), following its launch in late 2001, and combining these data with results from the OVRO-BIMA submm array, and ultimately from ALMA (http://www.alma.nrao.edu). However, it may well be that the Next Generation Space Telescope NGST (http://www.ngst.stsci.edu and http://ngst.gsfc.nasa.gov) will be needed to bridge the gap at large redshift between the universe of galaxies as observed in the optical/near-IR, and the universe as observed in the submm. Nonetheless, the combined capabilities of large ground-based telescopes in the near-IR, plus SIRTF plus the submm arrays will advance our knowledge of (dusty) galaxies immensely.

(4) Several spectrographs are being built specifically for doing surveys of large numbers of intermediate redshift galaxies on $6.5-10 \mathrm{~m}$ class telescopes. The ESO VLT VIRMOS and NIRMOS (http://www.eso.org) spectrographs, as well as the Keck DEIMOS spectrograph (http://www.keck.hawaii.edu), are examples of multiobject multi-slit spectrographs designed explicitly for such surveys. The Keck DEEP project (http://www.ucolick.org/deep) was originally developed by a group at Lick Observatory in collaboration with the Berkeley Center for Particle Astrophysics (CfPA - http://cfpa.berkeley.edu:80), but has now expanded to encompass many other collaborators, to the point that the team now expects to be able to obtain redshifts for $\sim 10^{5} z \sim 1$ galaxies. Many of the DEEP fields are also likely to be imaged with HST with the ACS following its launch in 2001, and so this database will be a remarkable resource for galaxy properties and large-scale structure at $\approx \sim 1$. Ultimately this, and the corresponding ESO surveys using VIRMOS and NIRMIOS, should provide a view of the universe at $\approx \sim 1$, at $\sim 50 \%$ of its present age, that will be comparable to that resulting from the $2 \mathrm{dF}$ survey of the $z \sim 0$ universe.

This is a remarkable list of new telescopes and substantial upgrades to existing telescopes, and provides credence for the view expressed above that we will make very major gains in understanding in a number of key scientific areas in a relatively short period. Another way to look at this is to contrast the expected capabilities that will come into operation in the next 5-10 years with what we have achieved in the last few years with the first generation cameras and instruments on just a few telescopes, HST, Keck and the JCMT (submm).

\section{High Altitude Telescopes}

There remains an area which has considerable potential for studies of distant galaxies in the near-mid infrared that is not widely considered in discussions of future instrumental/telescope capability - and that is through telescopes at high altitude on long-duration balloon flights or tethered aerostats. Given current technology it appears to be quite practical to launch (lightweight) 2.5 - 4-m class telescopes to elevations approaching $35 \mathrm{~km}$. The advantages are many compared to telescopes at altitudes of $4-5 \mathrm{~km}$. It is a cold $(-50 \mathrm{C})$ environment, with exceptionally high transparency, and very large $r_{0} \sim 20 \mathrm{~m}$. These are clearly ideal conditions for a diffraction-limited, high-sensitivity IR telescope. A number of groups have discussed such telescopes over the years. For example. Holland Ford. Pierre Bely and Mike Dopita proposed several years ago a mission called POST (Polar Stratospheric Telescope - see e.g.. 
http://mso.anu.edu.au/mad/madpubls.html) which utilized a tethered aerostat to fly a 4-m lightweight telescope in Antarctica to $\sim 12 \mathrm{~km}$ for carrying out observations in the $2-7 \mu \mathrm{m}$ spectral range. More recent concepts involve balloon flights with which higher elevations can be obtained.

\section{The Unsolved Problems?}

Clearly we are on the edge of an even more dramatic outpouring of results on distant galaxies. However, there are five areas which are important for establishing a clear picture of the formation and evolution of galaxies that I suspect will still be shrouded in some degree of uncertainty for some time to come probably until NGST is lannched, and even beyond.

These include (i) providing a good understanding of the physics of star formation, (ii) establishing the details of the reionization epoch, and (iii) characterizing the mass spectrum and mass scales of high redshift objects. Continued uncertainty about the exact nature of the dust (iv) in high redshift objects will also complicate efforts to understand galaxy formation and evolution at high redshift. Finally, observations of the first generations of metal-producing stars (v) will not be easy. Each of these is central to a complete picture of galaxy formation in its entirety, but each poses significant observational (and theoretical challenges) that will leave us with many uncertainties five years hence, and probably even 10 years hence.

Establishing (i) how stars form is a problem of daunting complexity, and observational and theoretical challenges, and yet it is central to a full understanding of the question of galaxy formation, and their evolution to the presentday galaxy population. The timescale, and the environment in the reionization epoch (ii) at $z>5$ is also a key issue, and one which may not be amenable to extensive investigation with the facilities available in the next decade before NGST.

Since theoretical models in their most parameter-free form give mass scales, it is obviously desirable to measure mass distributions and mass scale sizes (iii) for high redshift objects. However, this lias proved to be quite difficult, but in retrospect, not unsurprisingly so. While many constraints will likely be placed on the masses of distant objects, I suspect that this will remain a difficult task to do in a convincing manner, largely because the objects we are measuring at high redshifts are compact star-forming regions, or compact dust-enshrouded star-forming regions, and the interpretation of the resulting velocities is greatly complicated by several effects relating to the energy input from the massive stars and supernovae, and the attendant and ubiquitous dust. The energy input into the ISM results in substantial non-gravitational motions that are reflected in the observed line velocity structure, while lines of sight into the object(s) under study are limited by dust distributions, further complicating the interpretation of the absorption or emission line velocity structure. An additional problem is that the very compact nature of the star forming regions also may result in velocity widths that are not representative of the potential well of the galaxy. Together these pose significant observational challenges that will leave us uncertain about masses for a long time to come. 
While observations using the new submm capabilities will provide substantial gains in our understanding of distant, dusty galaxies, there are related, but more difficult, aspects of this topic (iv) which we must also deal with. The first is the derivation of the form of the reddening law(s) in star-bursting galaxies, both at lower redshift and at high redshift. The second issue is whether simple screen models which appear to be usable in low redshift, starburst galaxies are appropriate for high redshift, lower metallicity, clumpy, starbursting objects. The growing recognition that these are crucial issues, and their attendant study with IR telescopes and instruments will lead to much greater sophistication in how we all deal with dust in galaxies at all redshifts. While substantial progress will be made, it is not clear, though, that these more detailed and fundamental issues will be fully dealt with during the next 5-10 years, and so they may well remain for the NGST and even beyond.

The redshift of the first generation of stars $(v)$, and the epoch and processes by which the first metals were generated, is unlikely to be well-established in the near future. The redshifts involved push much of the flux into the mid-IR, and so are not observable from the ground because of the thermal background, nor from space with the small IR telescopes of the coming decade. Furthermore, dust is probably not plentiful at these earliest epochs, and so the submm becomes less useful. Tone of our current facilities can or will probe this epoch very thoroughly since it is at $z>6-8$, and maybe even at $z>10-15$ ! This issue is very clearly one for NGST, and is in many ways the raison d'etre for NGST.

\section{Summary}

The remarkable increase in the number of facilities and in their breadth of observational capabilities will lead us to characterize a number of key issues at the present time as "solved", in a big picture sense, though of course many important detailed questions will remain. Howcver, the bleakness of a "problem solved" future is not on the immediate horizon. Ten years from now a number of fundamental questions will remain (c.g., the physics of star formation, the epoch and characteristics of the earliest stars and metal production, details of the reionization epoch, the mass scales of objects at high redshifts, a full characterization of the properties of dust in star-forming objects at high redshift). These should provoke our current gencration of young scientists to work towards getting even more powerful telescopes and instruments than those we are currently considering, and because of their importance for some of the most fundamental questions that we can ask, provide the rationale for doing so.

Acknowledgments. I would like to acknowledge many valuable discussions with numerous colleagues - the boundless imagination of many of our small community makes it a delight to be part of this field. I am particularly grateful to those in our funding agencies and national centers who work. often under great stress, to bring us the facilities and funding that allows our community to reach far and to do it so rapidly. 

The Hy Redshift Universe

ASP Conference Series, Vol. 193, 1999

A. J. Bunker \& W. J. M. van Breugel, eds.

\title{
Probing $z>5$ with the Multiband Imaging Photometer for SIRTF (MIPS)
}

\author{
Dean C. Hines, George H. Rieke, Frank J. Low and Chad W. \\ Engelbracht \\ Steward Observatory, The University of Arizona, Tucson, Arizona
}

\begin{abstract}
Because of thcir extreme luminosities, unique spectral energy distributions and probable number densities, the Hyperluminous Infrared Galaxies (HIGs) may be the dominant objects detected by the Multiband Imaging Photometer for SIRTF (MIPS) at high redshift. Herein we briefly summarizc HIG properties, and present a simple method to determine their identity and redshift. Samples of HIGs over a range of redshifts, and especially for $z>5$ should provide important clues to the formation and evolution of both galaxies and supermassive black holes.
\end{abstract}

\section{Introduction}

Scheduled for launch aboard the Space Infrared Telescope Facility (SIRTF), the Multiband Imaging Photometer for SIRTF (MIPS) will provide imaging in three wide bands $24,70 \& 160 \mu \mathrm{m}$, as well as low resolution $(\mathrm{R} \sim 20)$ spectroscopy from 52 to $99 \mu \mathrm{m}$. The extraordinary sensitivity afforded by MIPS should en able us to detect and identify extremely luminous objects to redshifts of 10 , if they exist (Low \& Hines 1999). In particular, the Hyperluminous Infrared Galaxies (HIGs: Low et al. 1989; Cutri et al. 1994), with extreme luminosities $\log \left(\mathrm{L} / \mathrm{L}_{\odot}\right) \gtrsim 13$ and warm far-IR spectral energy distributions [SEDs: $0.25 \leq$ $\mathrm{F} \nu(25 \mu \mathrm{m}) / \mathrm{F} \nu(60 \mu \mathrm{m}) \leq 3]$, may provide a powerful probe for redshifts beyond $\tilde{z}=5$ (e.g., Hines \& Low 1999).

HIGs have an advantage over QSOs and Arp 220-like objects as probes of the high redshift universe. Above $z=5$, the 'cool' slope of the Arp 220-likc SED drops below the $24 \mu \mathrm{m}$ detection threshold even for objects that are 20 times more luminous than Arp 220. On the other hand, the flat or 'hot' SED of QSOs does not provide a sensitive indication of redshift, even though they could be easily detected. Furthermore, HIGs may outnumber QSOs by a factor of 2-4 in the local universe (e.g. Wills \& Hines 1997; Hines et al. 1999a: Cutri et al. 1999), so they may be the dominant class of objects detcctable by SIRTF at high redshift.

\section{HIGs}

HIGs typically show high, non-time-variable polarization $(p \approx 5 \%-20 \%)$ caused by scattering (Hines \& Wills 1993; Hines et al. 1995; Wills \& Hines 1997), and extended ionization and polarized "bicones" are observed in at least two HIGs 
(Hines et al. 1999a,b). This morphology implies a bright nucleus surrounded by an optically and geometrically thick torus which blocks our direct view, but allows light to escape at the poles to ionize gas and be scattered (thus polarized) into our line of sight.

The spectrum of scattered light within the bicones reveals a power-law UV/optical continuum and broad permitted emission lines, both typical of average luminous QSOs. Therefore, the scattering material and ionized gas view a powerful QSO nucleus directly. Counting the number of ionizing photons required to produce the observed narrow-line flux, we find that the intrinsic UV/optical luminosities are 10 - 50 times larger than we observe directly. Thus, the QSO nuclei are sufficient to power the majority of the far-IR emission. Viewed along the polar axis of the bicones, HIGs would be indistinguishable from objects like the Palomar-Green QSOs. Therefore, large numbers of QSOs have likely been missed by optical surveys (e.g. Wills \& Hines 1997; Cutri et al. 1999).

\section{Identification and Photometric Redshifts from Surveys with.MIPS}

Because HIGs emit the bulk of their luminosity in the MIPS bands, and may outnumber traditional QSOs by factors of $?-4$, they could constitute the primary population detected by MIPS for $5 \leq z \leq 10$. Figure 1a shows the regimes occupied QSOs, HIGs and Arp 220s in a MIPS color-color diagram $\left[\mathrm{F}_{\nu}(70 \mu \mathrm{m}) / \mathrm{F}_{\nu}(160 \mu \mathrm{m})\right.$ vs. $\left.\mathrm{F}_{\nu}(70 \mu \mathrm{m}) / \mathrm{F}_{\nu}(24 \mu \mathrm{m})\right]$. The three classes are well separated except for a degeneracy between $z \sim 5$ Arp 220 s and $z \sim 10$ HIGs. The color-color diagram also enables crude redshift estimates, especially for the HIGs and Arp 220s. However, the "hotter" (flat.) SED of the QSOs makes even crude redshift determination very uncertain.

More refined redshift estimates will come from supplementary ground-based observations. The flux densities at $850 \mu \mathrm{m}$ and $1.1 \mathrm{~mm}$ are nearly constant for HIGs above $z \approx 1$, while other bands decrease as usual. Therefore, ratios of other bands against the $850 \mu \mathrm{m}$ and $1.1 \mathrm{~mm}$ flux density can be quite sensitive to redshift (e.g. Sanders 1999; Blain 1999 and references therein). From Figure $1 \mathrm{~b}$ we see that the $850 \mu \mathrm{m} / 70 \mu \mathrm{m}$ and $1.1 \mathrm{~mm} / 70 \mu \mathrm{m}$ ratios for HIGs vary by more than four orders of magnitude between $z=1-10$. In combination with other photometry, including $3.6 \mathrm{~cm}$ radio (e.g. Carilli \& Yun 1999), photometric redshifts good to a fow percent should be possible for HIGs.

\section{Concluding Remarks}

Samples of Hyperluminous Infrared Galaxies identified in upcoming surveys with MIPS aboard SIRTF may provide important constraints about the high redshift universe. In particular, HIGs are potentially important probes of the formation and evolution of galaxies and SMBHs.

Acknowledgments. We have benefited from discussions with and contributions by B. Wills, G. Schmidt, P. Smith, R. Cutri and L. Armus. 


\section{References}

Blain, A. 1999, MNRAS, in press, astro-ph/ /9906438

Carilli, C. \& Yun, M. 1999, ApJ, 513, L13

Cutri, R.M. et al. 1994, ApJ, 424, L65

Cutri, R.M. et al. 1999, in prep.

Hines, D.C. \& Wills, B.J. 1993, Ap.J, 415, 82

Hines, D.C. \& Low, F.J. 1999, in the proceedings of the Workshop on Photometric Redshifts and High Redshift Galaxies, eds., R.J. Weymann, L. Storrie-Lombardi, M. Sawicki, \& R. Brunner, ASP Conference Series, in press

Hines, D.C. et al. 1995, Ap.J, 450, L1

Hines, D.C. et al. 1998a, ApJ, 512, 145

Hines, D.C. et al. 1998b, ApJ, submitted

Low, F.J. et al. 1989, ApJ, 340, L1

Low, F.J. \& Hines, D.C. 1999, in Astrophysics with Infrared Surveys: A Prelude to SIRTF, ed. M. Bicay, R. M. Cutri and B. F. Madore, ASP Conference Series, in press

Sanders, D.B. et al. 1988, ApJ, 325, 74

Sanders, D.B. 1999, in "Space Infrared Telescopes and Related Science," 32nd COSPAR workshop, Nagoya, Japan 1998, ed. T. Matsumoto, T. de Graauw, astro-ph/9904292

Wills, B.J., \& Hines, D.C. 1997, in Mass Ejection from AGN, ASP Conf. Series, Vol. 128, Arav, Shlosman, \& Weymann, eds., p. 99 

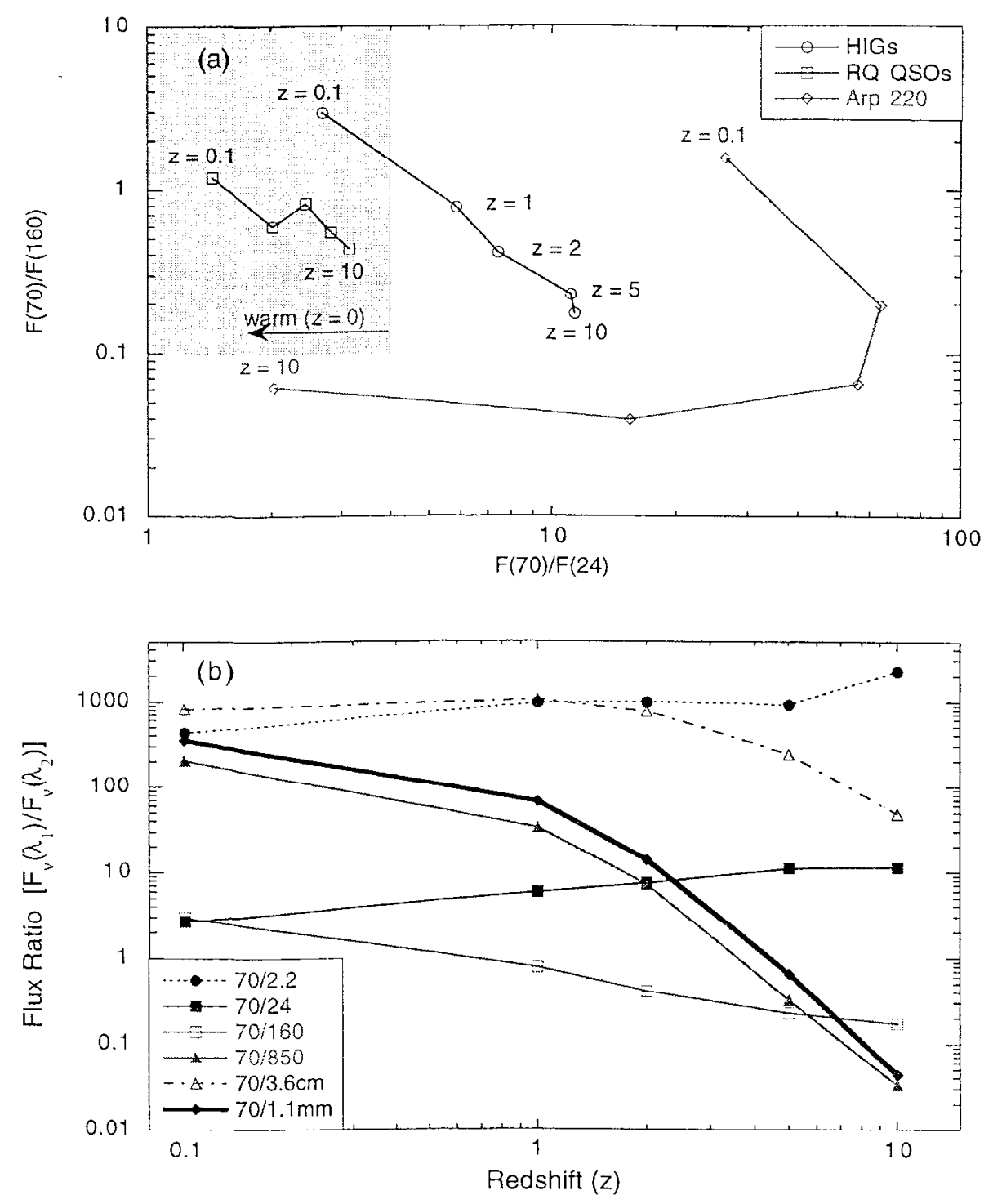

Figure 1. (a) Selection of QSOs, HIGs \& Arp 220-like objects by their "hot," "warm," and "cool" spectral slopes. The MIPS colors allow a crude determination of redshift. However, there is a possible degeneracy between $\approx \sim 5$ Arp 220's and $\approx \sim 10$ HIGs. The arrow delineates the $z \sim 0$ IRAS warm color criterion. (b) Flux ratios as a function of redshift. Note the strong redshift sensitivity of the $70 \mu \mathrm{m} / 850 \mu \mathrm{m}$ and $70 \mu \mathrm{m} / 1.1 \mathrm{~mm}$ ratio (after Hines \& Low 1999). 


\section{Author Index}

Adelberger, K. L. 344,462

Allington-Smith. J. 110

Andrews, T. B. 407

Armus, L. 86

Aussel, H. 254, 437

Baker, J. C. 79,513

Ballinger, W. 525

Banas, K. 411

Barger, A. J. 213

Becklin, E. E. 98

Benítez, N. 509

Bernstein, R. A. 487

Best, P. N. 71

Blain, A. W. 246, 425

Blundell, K. M. 75, 90

Bond, J. R. 275

Brainerd, T. G. 411

Brauher, J. 411

Bremer, M. N. 336

Broadhurst, T. J. 509

Brown, T. 167, 185

Bruzual A., G. 121

Bunker, A. J. 262, 448, 521

Carilli, C. 44

Carlberg, R. G. 292, 377

Cesarsky, C. J. 254, 437

Chen, H. 544

Cimatti, A. 102, 312

Clayton, G. C. 517

Clements, D. L. 275

Cohen, M. H. 102

Cohen, S. 419

Corbin, M. R. 250

Cotter, G. 513

Cowie, L. L. 213, 554

Crampton, D. 275

Crawford, C. S. $\mathbf{3 3 6}$

Danese, L. 437

Davis, M. 353

The DEEP Team 171

De Breuck, C. 44

de Pater, I. 1

de Silva, E. 79

De Young, D. S. 83
Désert, F. X. 437

Dey, A. 34, 114, 258, 344

di Serego Alighieri, S. 102

Dickey, J. 179

Dickinson, M. 110, 114, 234, 267

Djorgovski, S. G. 114, 397, 411

Dune, L. 275

Dunlop, J. S. 133

Dwek, E. 498

Eales, S. 90, 275

Egami, E. 86

Eisenhardt, P. 114, 639

Elbaz, D. 254, 437

Ellingson, E. 292

Ellis, R. S. 110

Engelbracht, C. W. 669

Evans, A. S. 86,415

Evans, N. 612

Fabian, A. C. 336

Fadda, D. 437

Feltzing, S. 181

Fernández-Soto, A. 544

Fèvre, O. L. 275

Figer, D. F. 98

Flores, H. 437

Fosbury, R. A. E. 102

Franceschini, A. 437

Franx, M. 340

Gear, W. 275

Giavalisco, M. 388

Gilbert, A. M. 98

Gilmore, G. 181

Glassman, T. M. 98

Gnedin, N. 598

Gordon, K. D. 517

Goto. M. 348

Graham, J. R. 98, 262, 344

Green, P. 612

Hall, P. B. 37T, 415

Hammer, F. 275

Hanley, C. 267

Hartwick, F. D. A. 415

Harwit, M. 437 
Hasinger, G. 336

Hata, R. 348

Haynes, T. 513

Heap, S. R. 167, 185

Hill, J. M. 603

Hines, D. C. 669

Hogg, D. W. 224

Honma, M. 179

Hopkins, A. 55

Houdashelt, M. 181

$\mathrm{Hu}$, E. M. 554

Huberly, I. 167, 185

Hunstead, R. 79

Ichikawa, T. 179

Illingworth, G. D. 340,662

Im, M. 171

Ivison, R. J. 246, 425

Iwamuro, F. 348

Jannuzi, B. T. 258, 603

Jarvis, M. J. 90

Jones, M. L. 513

Kajisawa, M. 348

Kazes, I. 179

Keel, W. C. 419

Kelson, D. D. 340

King, I. R. 1

Kneib, J. -P. 246, 425

Koo, D. C. 203, 437

Kulkarni, V. P. 603

Lacy, M. 90, 94

Landsman, W. 185

Lanz, T. M. 167, 185

Lanzetta, K. M. 544

Larkin, J. E. 98

Law, C. J. 411

Lehmann, I. 336

Levenson, N. A. 98

Liebert, J. W. 1

Lilly, S. 191, 275

Lin, H. 292, $37 \tau$

Liu, M. C. 344

Loeb, A. 586

Longair, M. S. 11, 71

Low, F. J. 669

Madau, P. 475

Maihara, T. 348
Maller, A. 608

Mandolesi, R. 437

Manning, C. 521

Marleau, F. R. 262

Marshall, H. 612

Mathur, S. 612

McCarthy, P. J. 23, 114, 529

McLean, I. S. 98

McMahon, R. G. 554

Miley, G. 44

Miller, L. 525

Minniti, D. 44

Morris, S. L. 377

Motohara, K. 348

Murphy Jr., T. W. 86

Mushotzky, R. 323, 651

Neugebaner, G. 86

Nolan, L. A. 175

Oemler Jr., A. 110, 281

O'Neil, E. 250

Osmer, P. S. 566

Pahrc, M. A. 156

Papovich, C. 267

Partridge, R. B. 625

Pascarelle, S. 419, 544

Patton, D. 377

Percival, W. $\mathbf{5 2 5}$

Primack, J. R. 608

Pritchet, C. J. 415

Prochaska, J. X. 608

Puget, J. L. 437

Rawlings, S. 75, 90

Renzini, A. 312

Richards, E. A. 55

Rieke, G. H. 669

Riehe, M. J. 603

Röttgering, H. J. 44, 71

Saunders, R. 79, 513

Sawichi, M. 377, 415

Schneider, G. 603

Shepherd, C. W. 37T

Silk, J. 367

Smail, I. 246, 425

Smith, T. L. 517

Sofue, Y. 179

Soifer, B. T. 86 
Somerville, R. S. 608

Spinrad, H. 114, 521, $\mathbf{5 3 7}$

Stanford, S. A. 44, 114, 304

Starck, J. L. 437

Steidel, C. C. 344,462

Stern, D. 44, 521

Storrie-Lombardi, L. J. 271, 603

Sweigart, A. 185

Taguchi, T. 348

Tanabe, H. 348

Tanaka, I. 348

Teplitz, H. 98

Terada, H. 348

Thacker, R. J. 353

Thompson, R. I. 250, 271, 603

Tutui, Y. 179

Vacca, W. D. 250

van Breugel, W. 44, 114

van Dokkum, P. G. 340

Vernet, J. 102

Villar-Martín, M. 102

Vogt, N. P. 145

Waddington, I. 55, 106, 419

Wakamatsu, K. 179

Webb, T. 275

Wehrse, R. 618

Weymann, R. J. 271, 603

Wilcox, M. K. 98

Wilkes, B. 612

Willott, C. J. 90

Windhorst, R. A. 55, 419

Wolfe, A. M. 575

Wu, W. 419

Wyse, R. F. G. 181

Yahata, N. 544

Yahil, A. 544

Yamada, T. 348

Yan, L. 529

Yee, H. K. C. 292, 377

Yi, S. 185

Zirbel, E. L. 110

Zirm, A. 114 



\section{Subject Index}

absorption lines, 262, 575, 603, 608 abundances, 1, 102, 323, 575, 651 active galaxies, $11,23,34,44,71$, $75,83,86,90,94,98,102$, $106,110,114,267,348,612$

atmospheres, 167

biographies, 1

brown dwarfs, 181

clusters, 110, 281, 292, 304, 312, $323,336,336,340,344,348$, $397,411,415,419,509,651$

comets, 1

compact galaxies, 521

cooling flows, $323,336,651$

cosmology, 44, 133, 167, 171, 185, $213,224,246,258,271,275$, $353,367,377,388,397,407$, $411,425,462,475,487,498$, $509,513,521,525,529,544$, $554,586,598$

dark matter, 181,475

diffuse radiation, $262,275,475,487$, 498,586

distances and redshifts, 1, 106, 179, $191,203,224,312,407,509$, $529,537,544$

dust, $254,437,517,618$

dwarf galaxies, 181

early universe, 234, 271, 498, 554, 586,598

elliptical, 407

elliptical and lenticular, $c D$ galaxies, $133,156,171,185,312$, 344

emission lines, 79

evolution of galaxies, $55,75,79.94$, $106,121,133,145,156,167$. $171,175,185,191,203,213$, 224, 234, 246, 250, 254, 258, $267,275,281,292,304,340$, $344,348,377,388,397,419$, $425,437,462,475,513,521$, $537,544,554,575$

extinction, $254,437,517$ formation of galaxies, $44,83,86$, $133,185,191,203,213,246$, $250,271,312,353,367,388$, $397,425,462,521,525,529$, $537,554,566,575,586,598$, $608,618,625,639,662$

fundamental parameters, 156,171 , 224,340

galaxies, 1, 11, 23, 34, 44, 55, 71, $75,79,83,86,90,94,98$, $102,106,110,114,121,133$, $145,156,167,171,175,179$, $181,185,191,203,213,224$, $234,246,250,254,258,267$, $271,275,281,292,304,312$, $323,336,340,344,348,353$, $36 \tau, 377,388,397,407,411$, $415,419,425,437,448,462$, $475,487,509,513,517,521$, $525,529,537,544,554,566$, $575,586,598,603,608,612$, $618,625,639,651,662,669$

Galaxy, 475

galaxies: individual ( $4 \mathrm{C}, 40.36$ ), 86 galaxies: individual (DMS 2139.0$0405), 262$

galaxies: individual (LBDS 53W091), 167,185

galaxies: infrared, $98,106,114,156$, $234,250,254,267,344,415$, $437,529,603,639,662,669$

general, $1,1,11,23,55,75,79,102$, $175,191,246,281,304,312$, $336,340,344,397,397,411$, $415,419,419,425,566,586$, $612,662,669$

gravitational lensing, 246, 425, 509

halo, 475

instruments, 639

interactions of galaxies, 411,525

intergalartic medium, 262, 554

irregular galaxies, 191

ISM, 11, 23, 71, 102, 517, 618, 618

jets, $34,75,79,83$ 
kinematics and dynamics of galaxies, 98, 145, 191, 203, 608, 618

large scale structure of universe, 224 , $258,353,377,388,411$

line profiles, 521,618

low-mass stars, 181

luminosity function, 90, 171, 181

mass function, $90,171,181$

methods, 353, 598

miscellaneous, 133,475

n-body simulations, 353

new physics, 407

numerical, 598

observations, $44,133,167,171,185$, $213,224,246,271,397,411$, $425,462,498,513,521,529$, $544,554,586$

photometric, 487

photometric redshifts, 509

photometry, 156, 234, 487, 513

Pioneer spacecraft, 407

polarization, 34, 83, 102

profiles (emission line), 521, 618

quasars, 11, 23, 55, 75, 79, 98, 102, $175,262,336,397,415,419$, $566,575,586,603,608,612$, 669

radiative transfer, 598

radio continuum, $11,23,44,55,75$, $90,106,114,267,625$

radio lines, 179

relativity, 407

shock waves, $11,23,71,114$

solar system, 1

space vehicles, 639

spiral, 145,179

starburst, 397, 517, 521, 525, 529, 537

stars, 1, 167, 181, 181

static universe model, 407

stellar content, $34,121,133,156$, $175,185,281,344,537$

structure of galaxies, $98,110,133$, 281

submillimeter, $133,275,625$ supernovæ, 407

surveys, 224, 258, 566

techniques, 487

telescopes, 662

theory, 367, 525, 598

time, 407

time-dilation, 407

ultraviolet, 102, 167

$\mathrm{X}$-rays, $323,336,612,651$ 